\title{
Rerouting the Organocatalytic Benzoin Reaction toward Aldehyde Deuteration
}

Santosh C. Gadekar, Vasudevan Dhayalan, Ashim Nandi, Inbal L. Zak, Meital Shema Mizrachi, Sebastian Kozuch and Anat Milo*

Department of Chemistry, Ben-Gurion University of the Negev, Beer-Sheva 84105, Israel

*Correspondence can be addressed to anatmilo@bgu.ac.il

Supporting Information

\section{Table of Contents}

1. GENERAL INFORMATION 2

2. SYNTHESIS OF N-HETEROCYCLIC CARBENE (NHC) PRECATALYSTS

2.1 SYNTHESIS OF IMIDAZOLE BASED NHCS

$\begin{array}{ll}\text { 2.2 SYNTHESIS OF PYRROLIDINE BASED TRIAZOLIUM NHCS } & 7\end{array}$

3. SYNTHESIS OF 2 AND 3-SUBSTITUTED BENZALDEHYDE DERIVATIVES 15

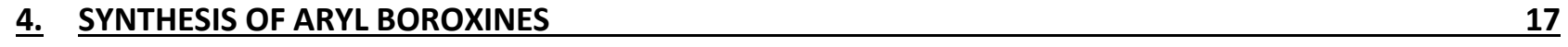

5. CATALYTIC PROCEDURES AND COMPOUND CHARACTERIZATION DATA

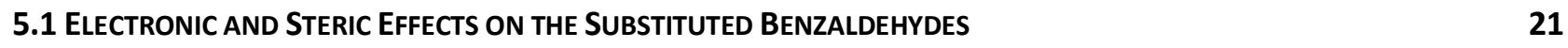

5.2 OPTIMIZATION WITH 2-METHYL BeNZALDEHYDE

5.3 OPTIMIZATION OF 4-(TRIFLUOROMETHYL)BENZALDEHYDE AND BOROXINE EFFECT 24

5.4 EfFect Of Different Aryl Boroxines on DeUteration of Aromatic Aldehydes 26

5.5 SUBSTRATE SCOPE WITH 2, 3 AND 4-SUBSTITUTED BENZALDEHYDES

5.6 SUBSTRATE SCOPE WITH HETEROCYCLES AND BIOLOGICALly ACtiVe MOleCULES

6. INITIAL REACTION RATES $\quad 76$

7. REACTIONS WITH DIFFERENT TRIAZOLIUM NHCS $\quad 90$

8. EXPERIMENTS WITH ALCOHOLS $\quad 92$

8.1 REACTIONS WITH 3A AND R-OH (5 EQ.)

8.2 REACTIONS WITH 3B AND R-OH (5 EQ.)

8.3 Reactions With 3B AND Methanol (DifFeRent Concentrations) 95

8.4 REACTIONS WITH 3B AND R-OH (0.10 MMOL) 97

9. LARGE SCALE REACTIONS 100

10. MECHANISTIC STUDIES $\quad 103$

$\begin{array}{ll}10.1 \text { REACTION WITH BENZOIN } & 103\end{array}$

$\begin{array}{ll}\text { 10.2 REACTIONS WITH 2,6-METHOXYPHENYL BORONIC-ACID } & 103\end{array}$

11. HRMS AND NMR SPECTRA $\quad 107$

\begin{tabular}{ll} 
12. REFERENCES & 491 \\
\hline
\end{tabular} 


\section{General Information}

Reagents were purchased from commercial suppliers and used without further purification. All of the aldehydes were distilled before use. Anhydrous solvents (toluene, chloroform, tetrahydrofuran, dichloromethane, methanol, diethyl ether, acetone, acetonitrile and chlorobenzene) were prepared by storing commercial grade solvents over $20 \% \mathrm{~m} / \mathrm{v} 3 \AA$ molecular sieves (activated by microwave for 6-7 minutes followed by flame activation under vacuum) for at least 48h under argon atmosphere. Thin-layer chromatography (TLC) analysis of crude reaction mixtures (RM) was performed using Merck silica gel 60 F254 TLC plates and visualized under UV or by staining with $\mathrm{KMnO}_{4}$. Column chromatography was performed on Merck Silica Gel $60 \AA$, 230 X 400 mesh. Nuclear magnetic resonance (NMR) spectra were recorded using Bruker DPX400 or DMX500 instruments. ${ }^{1} \mathrm{H}$ and ${ }^{13} \mathrm{C}$ chemical shifts are reported in ppm downfield of tetramethylsilane and referenced to the residual solvent peak $\left(\mathrm{CHCl}_{3} ; \delta \mathrm{H}=7.26 \mathrm{ppm}\right.$ and $\delta \mathrm{C}=77.0 \mathrm{ppm}, \mathrm{DMSO} ; \delta \mathrm{H}=2.50 \mathrm{ppm}$ and $\delta \mathrm{C}$ $=39.5$ ppm, THF; $\delta \mathrm{H}=1.72,3.58 \mathrm{ppm}$, toluene; $\delta \mathrm{H}=2.08,6.97,7.01,7.09 \mathrm{ppm},\left(\mathrm{CH}_{3}\right)_{2} \mathrm{CO} ; \delta \mathrm{H}=2.05$ ppm, $\mathrm{CH}_{3} \mathrm{CN} ; \delta \mathrm{H}=1.94 \mathrm{ppm}, \mathrm{CH}_{3} \mathrm{OH} ; \delta \mathrm{H}=3.31 \mathrm{ppm}$ ). Multiplicities are reported using the following abbreviations: $\mathrm{s}=$ singlet, $\mathrm{d}=$ doublet, $\mathrm{t}=$ triplet, $\mathrm{q}=$ quartet, $\mathrm{qnt}=$ quintet, $\mathrm{sxt}=$ sextet, $\mathrm{spt}=$ septet, $\mathrm{m}=$ multiplet, $\mathrm{br}=$ broad resonance. Solvent abbreviations are reported as follows: EtOAc $=$ ethyl acetate, $\mathrm{Hex}=$ hexanes, $\mathrm{DCM}=$ dichloromethane, $\mathrm{Et}_{2} \mathrm{O}=$ diethyl ether, $\mathrm{MeOH}=$ methanol, $\mathrm{THF}=$ tetrahydrofuran, $\mathrm{DIPEA}=$ diisopropylethylamine, $\mathrm{CH}(\mathrm{OEt})_{3}=$ Triethyl orthoformate, $\mathrm{PhCl}=$ Chlorobenzene. HR-MS data were obtained using a Thermo Scientific ${ }^{T M} \mathrm{Q}$ Exactive $^{\mathrm{TM}}$ Focus Hybrid Quadrupole-Orbitrap ${ }^{\mathrm{TM}}$ Mass Spectrometer. Unless otherwise stated, all operations were performed using high-vacuum standard Schlenk techniques under nitrogen/argon atmosphere. 


\section{Synthesis of N-Heterocyclic Carbene (NHC) Precatalysts}

\subsection{Synthesis of Imidazole Based NHCs}

Catalysts $\mathbf{3 a}$ and $\mathbf{3 b}$ were prepared according to the reaction sequence depicted in Scheme S1.<smiles>[R2]c1cc([R])c(N)c([R])c1</smiles><smiles>[R2]c1cc([R])c(N2C=[N+](c3c([R])cc([R2])cc3[R])CC2)c([R])c1</smiles>

3<smiles>[R]c1cc([R])c(N=CC=Nc2c([R])cc([R])cc2[R])c([R])c1</smiles>

1<smiles></smiles><smiles>[R]c1cc([R])c(NCCNc2c([R])cc([R])cc2[R])c([R])c1</smiles>

2

a, $\mathrm{R}_{1}={ }^{\text {sec }} \mathrm{C}_{3} \mathrm{H}_{7}, \mathrm{R}_{2}=\mathrm{H}$

b, $\mathrm{R}_{1}=\mathrm{CH}_{3}, \mathrm{R}_{2}=\mathrm{CH}_{3}$

Scheme 1. Reaction pathway for NHC (3a and 3b) synthesis

$N, N^{\prime}$-Bis(2,6-diisopropylphenyl)ethane-1,2-diimine (1a):<smiles>CC(C)c1cccc(C(C)C)c1N</smiles>

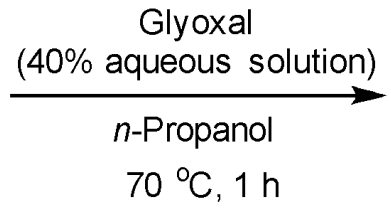<smiles>CC(C)c1cccc(C(C)C)c1N=CC=Nc1c(C(C)C)cccc1C(C)C</smiles>

1a

Scheme 2. Synthesis of $N, N^{\prime}$-Bis(2,6-diisopropylphenyl)ethane-1,2-diimine

Catalyst precursor $1 \mathbf{a}$ was prepared according to a literature procedure. ${ }^{1} \mathrm{~A}$ dry, argon flushed two neck Schlenk flask equipped with a magnetic stir bar and a septum was charged with $40 \%$ aqueous solution of glyoxal ( $3.60 \mathrm{~mL}, 25 \mathrm{mmol}, 1$ equiv.) in $n$-propanol (40 mL), 2,6-diisopropylphenylamine (10.56 mL, $56 \mathrm{mmol}, 2.24$ equiv.), $4 \mathrm{~mL}$ of $n$-propanol and $10 \mathrm{~mL}$ of water were added. After stirring

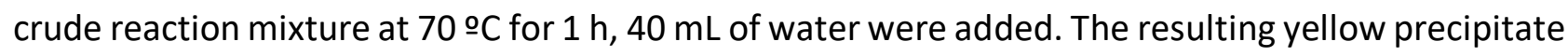


was collected by filtration and dried under vacuo for $12 \mathrm{~h}$. The crude product, $1 \mathrm{a}(8.90 \mathrm{~g}, 95 \%)$ was used in the next step without further purification.

$N, N$-Bis(2,6-diisopropylphenyl)ethylenediamine (2a):

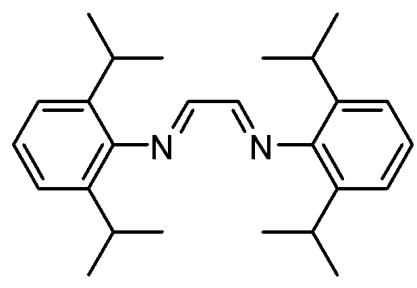

$1 a$

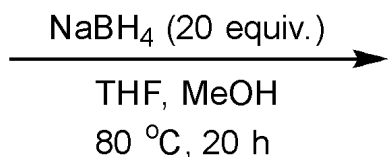

$80{ }^{\circ} \mathrm{C}, 20 \mathrm{~h}$

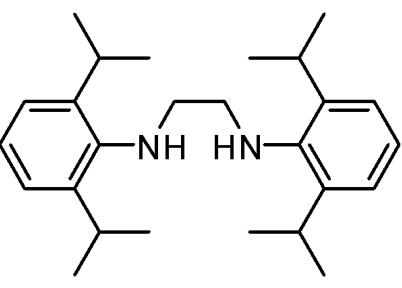

$2 a$

Scheme 3. Synthesis of $N, N^{\prime}$-Bis(2,6-diisopropylphenyl)ethylenediamine

Catalyst precursor 3a was prepared using a modified procedure based on previous reports. ${ }^{2} \mathrm{~A}$ dry, argon flushed two neck Schlenk flask equipped with a magnetic stir bar and a septum was charged with $N, N^{\prime}$-Bis(2,6-diisopropylphenyl)ethane-1,2-diimine, 1a (3.00 g, $8 \mathrm{mmol}, 1$ equiv.) in anhydrous THF (36 mL) and anhydrous $\mathrm{MeOH}(32 \mathrm{~mL})$. Sodium borohydride (4.50 g, $119.6 \mathrm{mmol}, 15$ equiv.) was slowly added portion wise (added over $20 \mathrm{~min}$.) at $0 \stackrel{\circ}{\circ}$ and then warmed to room temperature and the crude reaction mixture was stirred at $80 \stackrel{\circ}{ } \mathrm{C}$ for $5 \mathrm{~h}$ under argon. After cooling down crude reaction mixture to room temperature, sodium borohydride $(1.50 \mathrm{~g}, 39.90 \mathrm{mmol}, 5.00$ eq) was added portion wise at room temperature and the mixture was stirred at $80 \stackrel{\circ}{ }$ overnight under argon. The crude reaction mixture was then brought to room temperature and a saturated solution of ammonium chloride $\left(\mathrm{NH}_{4} \mathrm{Cl}\right)$ was added. The aqueous phase was extracted three times with EtOAc and combined, the organic layer was washed with brine $(100 \mathrm{~mL})$, dried with sodium sulfate $\left(\mathrm{Na}_{2} \mathrm{SO}_{4}\right)$, and concentrated under vacuum to afford product $2 \mathrm{a}(3.00 \mathrm{~g}, 99 \%)$.

\section{1,3-bis(2,6-diisopropylphenyl)-4,5-dihydro-1H-imidazol-3-ium chloride (3a):}

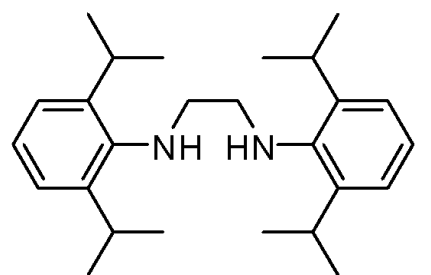

$2 a$

$$
\begin{gathered}
\underset{\mathrm{NH}_{4} \mathrm{Cl}(1.1 \text { equiv. })}{\left.\left(\mathrm{C}_{2} \mathrm{H}_{5} \mathrm{O}\right)_{3} \mathrm{CH} \text { (8 equiv. }\right)} \\
120^{\circ} \mathrm{C}, 4 \mathrm{~h}
\end{gathered}
$$

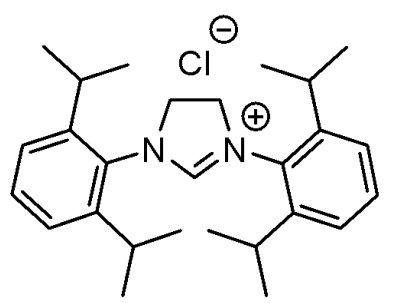

$3 a$

Scheme 4. Synthesis of 3a 
Catalyst SIPr.HCl was prepared according to previous reports. ${ }^{2}$ A dry, argon flushed two neck Schlenk flask equipped with a magnetic stir bar and a septum was charged with N,N'-Bis(2,6diisopropylphenyl)ethylenediamine, $2 \mathrm{a}(6.00 \mathrm{~g}, 15.78 \mathrm{mmol}, 1.00 \mathrm{eq})$, ammonium chloride (928 $\mathrm{mg}$, $17.35 \mathrm{mmol}, 1.10 \mathrm{eq})$ and triethyl orthoformate $(21.00 \mathrm{~mL}, 126.00 \mathrm{mmol}, 8.00 \mathrm{eq})$ and stirred at 120 으 for $4 \mathrm{~h}$. After cooling the crude reaction mixture to room temperature, diethyl ether was added. The resulting suspension was filtered and washed with excess of diethyl ether to give a white solid. The solid was dried under vacuum to afford 3a, SIPr·HCl $(5.00 \mathrm{~g}, 89 \%)$. NMR spectra are given on pages 96-97.

${ }^{1} \mathrm{H}$ NMR $\left(400 \mathrm{MHz}, \mathrm{CDCl}_{3}\right) \delta(\mathrm{ppm})=8.37(\mathrm{~s}, 1 \mathrm{H}), 7.47(\mathrm{t}, \mathrm{J}=8.0 \mathrm{~Hz}, 2 \mathrm{H}), 7.27(\mathrm{~d}, \mathrm{~J}=8.0 \mathrm{~Hz}, 4 \mathrm{H})$, 4.85 (s, 4 H), 3.01 (spt, J = 8.0 Hz, 4 H), 1.39 (d, J = 4.0 Hz, 12 H), 1.25 (d, J = 8.0 Hz, 12 H). ${ }^{13} \mathrm{C} \mathrm{NMR}$ $\left(100 \mathrm{MHz}, \mathrm{DMSO}-d_{6}\right) \delta(\mathrm{ppm})=158.3,146.2,131.7,129.4,125.1,55.6,29.4,25.6,23.9$.

$N, N^{\prime}$-dimesitylethane-1,2-diimine (1b):<smiles>Cc1cc(C)c(N)c(C)c1</smiles>

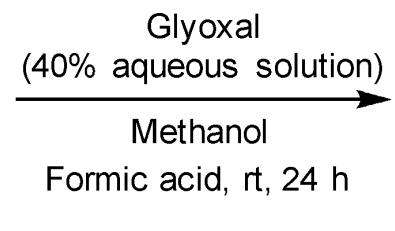

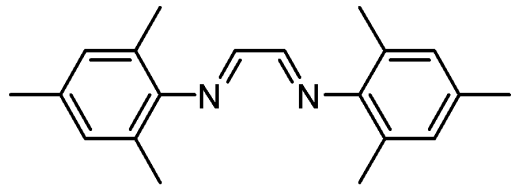

$1 \mathrm{~b}$

Scheme 5. Synthesis of $N, N^{\prime}$-dimesitylethane-1,2-diimine

Catalyst precursor $\mathbf{1 b}$ was prepared according to a literature procedure. ${ }^{3} \mathrm{~A}$ dry, argon flushed two neck Schlenk flask equipped with a magnetic stir bar and a septum was charged with $40 \%$ aqueous solution of glyoxal (4.23 mL, $37 \mathrm{mmol}, 1$ equiv.) in $\mathrm{MeOH}(40 \mathrm{~mL}), 2,4,6$-trimethylphenylamine (10.40 mL, $74 \mathrm{mmol}, 2.00$ equiv.) and three drops of formic acid were added. The crude reaction mixture was stirred at room temperature for $24 \mathrm{~h}$. The resulting yellow suspension was collected by filtration, washed with a minimum amount of methanol then with diethyl ether and dried under vacuo for $12 \mathrm{~h}$. The crude product, $\mathbf{1 b}(8.42 \mathrm{~g}, 78 \%)$ was used in the next step without further purification.

$N, N$ '-dimesitylethane-1,2-diamine (2b):

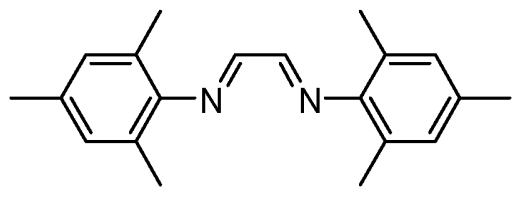

$1 \mathrm{~b}$

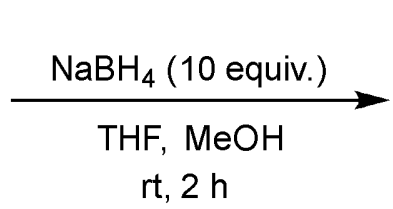

$\mathrm{rt}, 2 \mathrm{~h}$

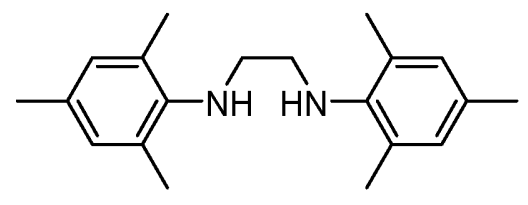

$2 \mathbf{b}$ 
Scheme 6. Synthesis of $N, N^{\prime}$-dimesitylethane-1,2-diamine

Catalyst precursor $\mathbf{2} \mathbf{b}$ was prepared according to previous reports. ${ }^{2} \mathrm{~A}$ dry, argon flushed two neck Schlenk flask equipped with a magnetic stir bar and a septum was charged with $N, N^{\prime}-$ dimesitylethane-1,2-diamine, $\mathbf{1 b}(3.00 \mathrm{~g}, 10.25 \mathrm{mmol}, 1$ equiv.) in anhydrous THF (36 mL) and anhydrous $\mathrm{MeOH}$ (32 mL). Sodium borohydride (3.90 g, $102 \mathrm{mmol}, 10$ equiv.) was slowly added at room temperature and the crude reaction mixture was stirred for $2 \mathrm{~h}$ under argon. Then, a saturated solution of $\mathrm{NH}_{4} \mathrm{Cl}$ was added and aqueous phase was extracted three times with with diethyl ether. The combined organic layers were washed with brine $(100 \mathrm{~mL})$, dried with $\mathrm{Na}_{2} \mathrm{SO}_{4}$, and concentrated under vacuum to afford product $\mathbf{2 b}$ (3.00 g, 99\%).

\section{1,3-dimesityl-4,5-dihydro-1H-imidazol-3-ium chloride (3b):}

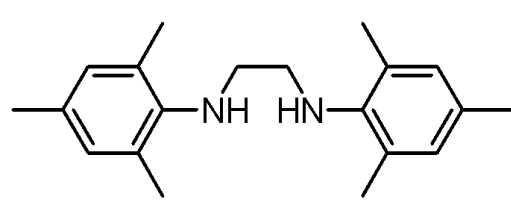

$2 \mathbf{b}$

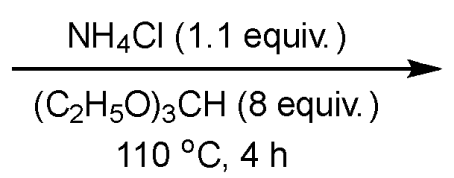

$110^{\circ} \mathrm{C}, 4 \mathrm{~h}$

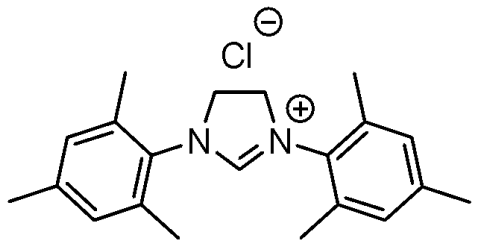

$3 \mathbf{b}$

SIMes $\cdot \mathrm{HCl}$

\section{Scheme 7. Synthesis of $\mathbf{3 b}$}

Catalyst 3b was prepared according to previous reports. ${ }^{2}$ A dry, argon flushed two neck Schlenk flask equipped with a magnetic stir bar and a septum was charged with N,N'-dimesitylethane-1,2diamine, 2 b (3.00 g, $10.12 \mathrm{mmol}, 1.00 \mathrm{eq}$ ), ammonium chloride (595 mg, $11.13 \mathrm{mmol}, 1.10 \mathrm{eq}$ ) and triethyl orthoformate $(13.48 \mathrm{~mL}, 80.96 \mathrm{mmol}, 8.00 \mathrm{eq})$ and stirred at 110 으 for $4 \mathrm{~h}$. After cooling crude reaction mixture to room temperature, diethyl ether was added. The resulting suspension was filtered and the formed white solid was washed with excess of diethyl ether. The solid was dried under vacuum to afford 3b, SIMes· $\mathbf{H C l}$ (3.15 g, 91\%). NMR spectra are given on pages 98-99.

${ }^{1} \mathrm{H}$ NMR $\left(400 \mathrm{MHz}, \mathrm{CDCl}_{3}\right) \delta(\mathrm{ppm})=9.31(\mathrm{~s}, 1 \mathrm{H}), 6.97(\mathrm{~s}, 4 \mathrm{H}), 4.61(\mathrm{~s}, 4 \mathrm{H}), 2.41(\mathrm{~s}, 12 \mathrm{H}), 2.29(\mathrm{~s}, 6$ H). ${ }^{13} \mathrm{C}$ NMR $\left(100 \mathrm{MHz}\right.$, DMSO- $\left.d_{6}\right) \delta(\mathrm{ppm})=160.0,140.6,135.1,130.3,130.1,52.1,21.2,18.1$ 


\subsection{Synthesis of Pyrrolidine Based Triazolium NHCs}

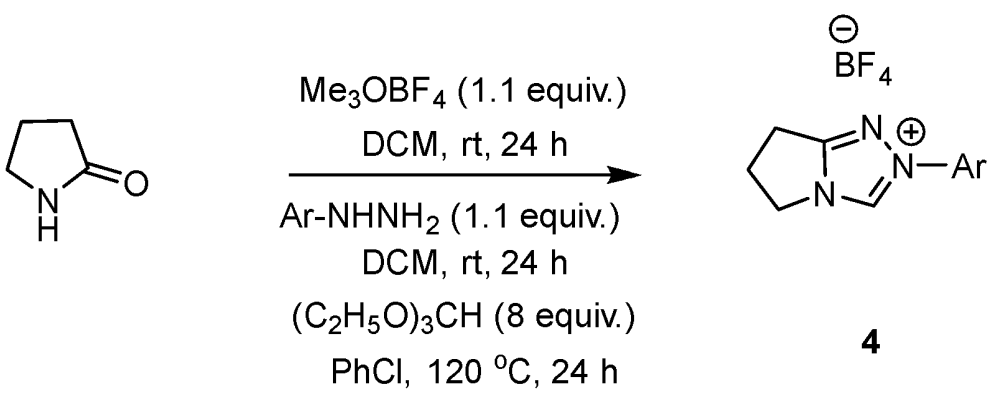

Scheme 8. Synthesis of 4

Pyrrolidine based triazolium catalysts 4 were prepared according to previous reports. ${ }^{4} \mathrm{~A}$ dry, argon flushed two neck Schlenk flask equipped with a magnetic stir bar and a septum was charged with 2-pyrrolidone (1.75 g, $20.6 \mathrm{mmol}, 1$ eq.) in anhydrous dichloromethane ( $120 \mathrm{~mL})$, trimethyloxonium tetrafluoroborate ( $3.34 \mathrm{~g}, 22.6 \mathrm{mmol}, 1.1 \mathrm{eq}$.) was added and the crude reaction mixture was stirred at room temperature for $24 \mathrm{~h}$. Aryl hydrazine ( $22.6 \mathrm{mmol}, 1.1 \mathrm{eq}$.$) was added to the solution and$ the crude reaction mixture was further stirred at room temperature for $24 \mathrm{~h}$. The solvent was removed in vacuo and dried well under high vacuum for $12 \mathrm{~h}$ and the product was used without further purification. Triethyl orthoformate $(26.6 \mathrm{~mL}, 164.8 \mathrm{mmol}, 8$ eq.) and anhydrous chlorobenzene $(120 \mathrm{~mL})$ were added and the crude reaction mixture was heated at $120 \cong \mathrm{C}$ for $24 \mathrm{~h}$ under argon atmosphere. The solvent was removed in vacuo and the crude product was purified by washing with solvent or recrystallization.

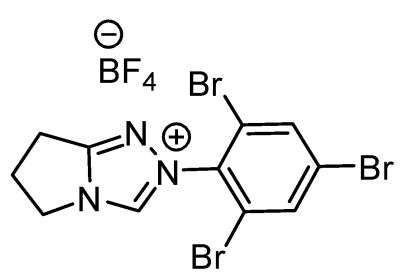

$4 a$

2-Pyrrolidone (562 mg, $6.60 \mathrm{mmol}, 1$ eq.), anhydrous dichloromethane (40 mL), trimethyloxonium tetrafluoroborate $(1.10 \mathrm{~g}, 7.26 \mathrm{mmol}, 1.1 \mathrm{eq}$.$) , were prepared according to the general procedure$ (page 7). Then, 2,4,6-tribromophenyl hydrazine ( $2.50 \mathrm{~g}, 7.26 \mathrm{mmol}, 1.1 \mathrm{eq}$.) was added to the solution and stirred at room temperature for $2 \mathrm{~h}$. In the final step, triethyl orthoformate $(8.80 \mathrm{~mL}$, $52.80 \mathrm{mmol}, 8$ eq.) and anhydrous chlorobenzene $(40 \mathrm{~mL})$ were prepared according to the general procedure given for pyrrolidine based triazolium NHC precatalysts (page 7). Catalyst was recrystallized using ethyl acetate solvent. Reaction yield $=45 \%$ (1.49 g). Spectral data for product 4a have been previously reported. ${ }^{5}$ NMR spectra are given on pages 100-101. 
${ }^{1} \mathrm{H}$ NMR $\left(500 \mathrm{MHz}, \mathrm{DMDO}-d_{6}\right) \delta(\mathrm{ppm})=10.50(\mathrm{~s}, 1 \mathrm{H}), 8.39(\mathrm{~s}, 2 \mathrm{H}), 4.56(\mathrm{t}, \mathrm{J}=10 \mathrm{~Hz}, 2 \mathrm{H}), 3.28(\mathrm{t}$, $J=10 \mathrm{~Hz}, 2 \mathrm{H}), 2.80-2.74(\mathrm{~m}, 2 \mathrm{H}) .{ }^{13} \mathrm{C}$ NMR $\left(125 \mathrm{MHz}, \mathrm{DMDO}-d_{6}\right) \delta(\mathrm{ppm})=163.8,143.1,135.4$, $133.5,126.9,123.2,48.2,26.5,21.4$

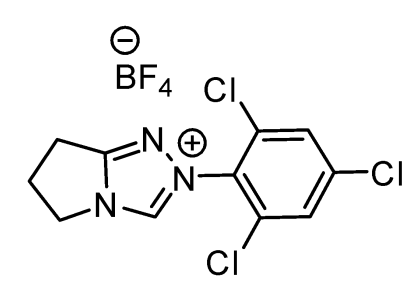

4b

2-Pyrrolidone ( $851 \mathrm{mg}, 10.00 \mathrm{mmol}, 1$ eq.), anhydrous dichloromethane (60 mL), trimethyloxonium tetrafluoroborate (1.63 g, $11.00 \mathrm{mmol}, 1.1$ eq.), 2,4,6-trichlorophenyl hydrazine (2.33 g, 11.00 mmol, 1.1 eq.), triethyl orthoformate $(13.32 \mathrm{~mL}, 80.00 \mathrm{mmol}, 8$ eq.) and anhydrous chlorobenzene $(60 \mathrm{~mL})$ were prepared according to the general procedure given for pyrrolidine based triazolium NHC precatalysts (page 7). Catalyst was recrystallized using ethyl acetate:methanol (9:1) solvent combination. Reaction yield $=37 \%(1.40 \mathrm{~g})$. Spectral data for product $\mathbf{4 b}$ have been previously reported. ${ }^{6}$ NMR spectra are given on pages 102-103.

${ }^{1} \mathrm{H}$ NMR $\left(500 \mathrm{MHz}, \mathrm{DMDO}-d_{6}\right) \delta(\mathrm{ppm})=10.46(\mathrm{~s}, 1 \mathrm{H}), 8.16(\mathrm{~s}, 2 \mathrm{H}), 4.53(\mathrm{t}, \mathrm{J}=10 \mathrm{~Hz}, 2 \mathrm{H}), 3.26(\mathrm{t}$, $J=10 \mathrm{~Hz}, 2 \mathrm{H}$ ), 2.77 (qnt, $J=10 \mathrm{~Hz}, 2 \mathrm{H}) .{ }^{13} \mathrm{C}$ NMR $\left(125 \mathrm{MHz}, \mathrm{DMDO}-d_{6}\right) \delta(\mathrm{ppm})=164.0,143.5$, $138.1,133.6,130.4,129.4,48.2,26.5,21.5$

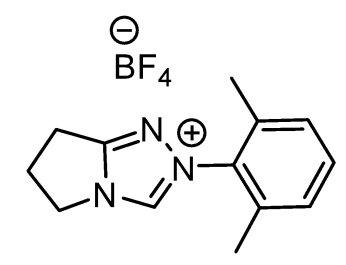

4c

2-Pyrrolidone (851 mg, $10.00 \mathrm{mmol}, 1$ eq.), anhydrous dichloromethane (60 mL), trimethyloxonium tetrafluoroborate $(1.63 \mathrm{~g}, 11.00 \mathrm{mmol}, 1.1 \mathrm{eq}$.), 2,6-dimethylphenyl hydrazine $(1.50 \mathrm{~g}, 11.00 \mathrm{mmol}$, 1.1 eq.), triethyl orthoformate $(13.32 \mathrm{~mL}, 80.00 \mathrm{mmol}, 8$ eq.) and anhydrous chlorobenzene $(60 \mathrm{~mL})$ were prepared according to the general procedure given for pyrrolidine based triazolium NHC precatalysts (page 7). Obtained catalyst was washed with ethyl acetate. Reaction yield $=83 \%(2.50$ g). NMR spectra are given on pages 103-104.

${ }^{1} \mathrm{H}$ NMR $\left(500 \mathrm{MHz}, \mathrm{DMDO}-d_{6}\right) \delta(\mathrm{ppm})=10.29(\mathrm{~s}, 1 \mathrm{H}), 7.49(\mathrm{t}, J=5 \mathrm{~Hz}, 1 \mathrm{H}), 7.35(\mathrm{~d}, J=5 \mathrm{~Hz}, 2 \mathrm{H})$, $4.46(t, J=10 \mathrm{~Hz}, 2 \mathrm{H}), 3.20(\mathrm{t}, J=10 \mathrm{~Hz}, 2 \mathrm{H}), 2.77$ (qnt, $J=10 \mathrm{~Hz}, 2 \mathrm{H}), 2.12(\mathrm{~s}, 6 \mathrm{H}) .{ }^{13} \mathrm{C}$ NMR $(125$ 
$\left.\mathrm{MHz}, \mathrm{DMDO}-d_{6}\right) \delta(\mathrm{ppm})=163.3,141.6,135.4,134.4,131.3,128.8,47.2,26.4,21.4,17.0$. HRMS(ESI) calcd for $\mathrm{C}_{13} \mathrm{H}_{16} \mathrm{~N}_{3}{ }^{+}(\mathrm{M})^{+}:$214.1339, Found: 214.1339

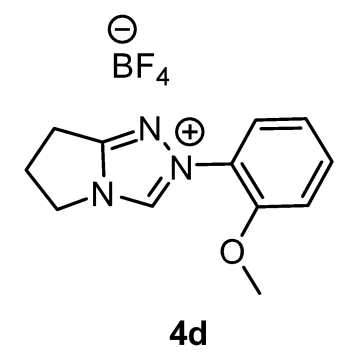

2-Pyrrolidone ( $851 \mathrm{mg}, 10.00 \mathrm{mmol}, 1$ eq.), anhydrous dichloromethane (60 $\mathrm{mL}$ ), trimethyloxonium tetrafluoroborate $(1.63 \mathrm{~g}, 11.00 \mathrm{mmol}, 1.1 \mathrm{eq}$.$) , 2-methoxyphenyl hydrazine (1.52 g, 11.00 \mathrm{mmol}$, 1.1 eq.), triethyl orthoformate ( $13.32 \mathrm{~mL}, 80.00 \mathrm{mmol}, 8$ eq.) and anhydrous chlorobenzene (60 $\mathrm{mL})$ were prepared according to the general procedure given for pyrrolidine based triazolium NHC precatalysts (page 7). Obtained catalyst was washed with ethyl acetate. Reaction yield $=66 \%(2.00$ g). NMR spectra are given on pages 105-106.

${ }^{1} \mathrm{H}$ NMR $\left(500 \mathrm{MHz}, \mathrm{DMDO}-d_{6}\right) \delta(\mathrm{ppm})=10.42(\mathrm{~s}, 1 \mathrm{H}), 7.75(\mathrm{dd}, J=10 \mathrm{~Hz}, 1 \mathrm{H}), 7.62(\mathrm{td}, J=10 \mathrm{~Hz}$, $1 \mathrm{H}), 7.42(\mathrm{~d}, J=10 \mathrm{~Hz}, 1 \mathrm{H}), 7.22(\mathrm{t}, J=10 \mathrm{~Hz}, 1 \mathrm{H}), 4.42(\mathrm{t}, J=10 \mathrm{~Hz}, 2 \mathrm{H}), 3.95(\mathrm{~s}, 3 \mathrm{H}), 3.19$ (t, J= $10 \mathrm{~Hz}, 2 \mathrm{H}), 2.74$ (qnt, $J=10 \mathrm{~Hz}, 2 \mathrm{H}) .{ }^{13} \mathrm{C}$ NMR $\left(125 \mathrm{MHz}, \mathrm{DMDO}-d_{6}\right) \delta(\mathrm{ppm})=162.3,151.3,141.0$, 132.0, 125.0, 124.4, 121.3, 113.4, 56.5, 47.2, 26.5, 21.3. HRMS(ESI) calcd for $\mathrm{C}_{12} \mathrm{H}_{14} \mathrm{~N}_{3} \mathrm{O}^{+}(\mathrm{M})^{+}$: 216.1131, Found: 216.1137

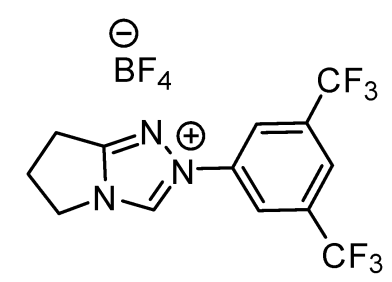

$4 e$

2-Pyrrolidone (851 mg, $10.00 \mathrm{mmol}, 1$ eq.), anhydrous dichloromethane (60 mL), trimethyloxonium tetrafluoroborate (1.63 g, $11.00 \mathrm{mmol}, 1.1$ eq.), 3,5-bis(trifluoromethyl)phenyl hydrazine (2.69 g, $11.00 \mathrm{mmol}, 1.1$ eq.), triethyl orthoformate (13.32 mL, $80.00 \mathrm{mmol}, 8$ eq.) and anhydrous chlorobenzene $(60 \mathrm{~mL})$ were prepared according to the general procedure given for pyrrolidine based triazolium NHC precatalysts (page 7). Catalyst was recrystallized using methanol:diethyl ether (1:9) solvent combination. Reaction yield $=47 \%$ (2.00 g). NMR spectra are given on pages 106-108.

${ }^{1} \mathrm{H}$ NMR $\left(500 \mathrm{MHz}, \mathrm{DMDO}-d_{6}\right) \delta(\mathrm{ppm})=10.98(\mathrm{~s}, 1 \mathrm{H}), 8.62(\mathrm{~s}, 2 \mathrm{H}), 8.45(\mathrm{~s}, 1 \mathrm{H}), 4.49(\mathrm{t}, J=10 \mathrm{~Hz}$, $2 \mathrm{H}$ ), 3.26 (t, $J=10 \mathrm{~Hz}, 2 \mathrm{H}), 2.79$ (qnt, $J=10 \mathrm{~Hz}, 2 \mathrm{H}) .{ }^{13} \mathrm{C}$ NMR $\left(125 \mathrm{MHz}, \mathrm{DMDO}-d_{6}\right) \delta(\mathrm{ppm})=$ 
163.4, 140.2, 137.0, 132.0 (q, $J=34 \mathrm{~Hz}$ ), 124.0, 122.6 (q, $J=271 \mathrm{~Hz}$ ), 122.1, 47.3, 26.7, 21.3. ${ }^{19} \mathrm{~F}$ NMR $\left(376 \mathrm{MHz}, \mathrm{DMDO}-d_{6}\right) \delta(\mathrm{ppm})=-61.5(\mathrm{~s}, 6 \mathrm{~F}),-148.7(\mathrm{~s}, 4 \mathrm{~F})$. HRMS(ESI) calcd for $\mathrm{C}_{13} \mathrm{H}_{10} \mathrm{~F}_{6} \mathrm{~N}_{3}{ }^{+}(\mathrm{M})^{+}$: 322.0773, Found: 322.0782

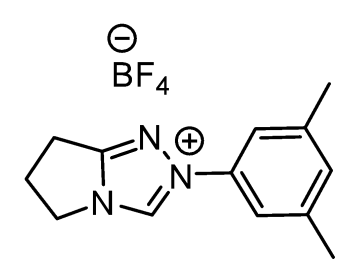

$4 f$

2-Pyrrolidone ( $851 \mathrm{mg}, 10.00 \mathrm{mmol}, 1$ eq.), anhydrous dichloromethane (60 mL), trimethyloxonium tetrafluoroborate $(1.63 \mathrm{~g}, 11.00 \mathrm{mmol}, 1.1 \mathrm{eq}$ ), 3,5-dimethylphenylhydrazine $(1.50 \mathrm{~g}, 11.00 \mathrm{mmol}$, 1.1 eq.), triethyl orthoformate $(13.32 \mathrm{~mL}, 80.00 \mathrm{mmol}, 8$ eq.) and anhydrous chlorobenzene $(60 \mathrm{~mL})$ were prepared according to the general procedure given for pyrrolidine based triazolium NHC precatalysts (page 7). Obtained catalyst was washed with ethyl acetate. Reaction yield $=72 \%(2.16$ g). NMR spectra are given on pages 108-109.

${ }^{1} \mathbf{H}$ NMR $\left(500 \mathrm{MHz}, \mathrm{CDCl}_{3}\right) \delta(\mathrm{ppm})=9.43(\mathrm{~s}, 1 \mathrm{H}), 7.31(\mathrm{~d}, J=10 \mathrm{~Hz}, 1 \mathrm{H}), 7.13(\mathrm{~s}, 1 \mathrm{H}), 7.07(\mathrm{~d}, J=5$ $\mathrm{Hz}, 1 \mathrm{H}), 4.53(\mathrm{t}, J=10 \mathrm{~Hz}, 2 \mathrm{H}), 3.16(\mathrm{t}, J=10 \mathrm{~Hz}, 2 \mathrm{H}), 2.79$ (qnt, $J=10 \mathrm{~Hz}, 2 \mathrm{H}), 2.37(\mathrm{~s}, 3 \mathrm{H}), 2.22$ $(\mathrm{s}, 3 \mathrm{H}) .{ }^{13} \mathrm{C}$ NMR $\left(125 \mathrm{MHz}, \mathrm{DMDO}-d_{6}\right) \delta(\mathrm{ppm})=162.6,141.3,140.8,133.4,132.6,132.1,127.7$, 126.1, 46.9, 26.5, 21.3, 20.7, 17.0. HRMS(ESI) calcd for $\mathrm{C}_{13} \mathrm{H}_{16} \mathrm{~N}_{3}{ }^{+}(\mathrm{M})^{+}:$: 214.1339, Found: 214.1345

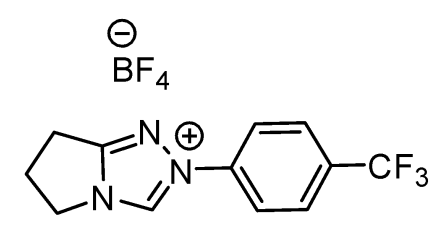

$4 \mathrm{~g}$

2-Pyrrolidone (851 mg, $10.00 \mathrm{mmol}, 1$ eq.), anhydrous dichloromethane (60 mL), trimethyloxonium tetrafluoroborate (1.63 g, $11.00 \mathrm{mmol}, 1.1 \mathrm{eq}$ ), 4-(trifluoromethyl)phenyl hydrazine (1.94 g, 11.00 mmol, 1.1 eq.), triethyl orthoformate (13.32 mL, $80.00 \mathrm{mmol}, 8$ eq.) and anhydrous chlorobenzene $(60 \mathrm{~mL})$ were prepared according to the general procedure given for pyrrolidine based triazolium $\mathrm{NHC}$ precatalysts (page 7). Obtained catalyst was washed with ethyl acetate. Reaction yield $=55 \%$ (1.87 g). Spectral data for product $4 \mathrm{~g}$ have been previously reported. ${ }^{5}$ NMR spectra are given on pages $110-111$. 
${ }^{1} \mathrm{H}$ NMR $\left(500 \mathrm{MHz}, \mathrm{DMDO}-d_{6}\right) \delta(\mathrm{ppm})=10.83(\mathrm{~s}, 1 \mathrm{H}), 8.14-8.09(\mathrm{~m}, 4 \mathrm{H}), 4.44(\mathrm{t}, \mathrm{J}=10 \mathrm{~Hz}, 2 \mathrm{H})$, $3.23\left(\mathrm{t}, J=10 \mathrm{~Hz}, 2 \mathrm{H}\right.$ ), 2.76 (qnt, $J=10 \mathrm{~Hz}, 2 \mathrm{H}$ ). ${ }^{13} \mathrm{C}$ NMR $\left(125 \mathrm{MHz}, \mathrm{DMDO}-d_{6}\right) \delta$ (ppm) = 163.4, 139.3, 138.5, $130.3(q, J=33 \mathrm{~Hz}), 127.6,123.7$ (q, $J=271 \mathrm{~Hz}), 121.5,47.1,26.7,21.3 .{ }^{19} \mathrm{~F}$ NMR $(376$ $\left.\mathrm{MHz}, \mathrm{DMDO}-d_{6}\right) \delta(\mathrm{ppm})=-61.3(\mathrm{~s}, 3 \mathrm{~F}),-148.6(\mathrm{~s}, 4 \mathrm{~F})$.

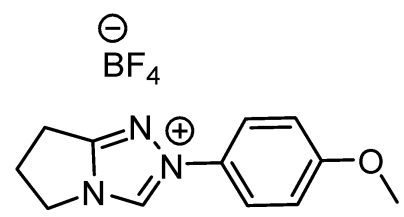

4h

2-Pyrrolidone (851 mg, $10.00 \mathrm{mmol}, 1$ eq.), anhydrous dichloromethane (60 mL), trimethyloxonium tetrafluoroborate $(1.63 \mathrm{~g}, 11.00 \mathrm{mmol}, 1.1 \mathrm{eq}$.), 4-methoxyphenyl hydrazine $(1.52 \mathrm{~g}, 11.00 \mathrm{mmol}$, 1.1 eq.), triethyl orthoformate ( $13.32 \mathrm{~mL}, 80.00 \mathrm{mmol}, 8$ eq.) and anhydrous chlorobenzene (60 $\mathrm{mL})$ were prepared according to the general procedure given for pyrrolidine based triazolium NHC precatalysts (page 7). Obtained catalyst was washed with ethyl acetate. Reaction yield $=61 \%$ (1.85 g). Spectral data for product $4 \mathrm{~h}$ have been previously reported. ${ }^{7}$ NMR spectra are given on pages 112-113.

${ }^{1} \mathrm{H}$ NMR $\left(500 \mathrm{MHz}, \mathrm{DMDO}-d_{6}\right) \delta(\mathrm{ppm})=10.55(\mathrm{~s}, 1 \mathrm{H}), 7.79(\mathrm{~d}, J=10 \mathrm{~Hz}, 2 \mathrm{H}), 7.22(\mathrm{~d}, J=10 \mathrm{~Hz}, 2$ H), $4.40(t, J=10 \mathrm{~Hz}, 2 \mathrm{H}), 3.85(\mathrm{~s}, 3 \mathrm{H}), 3.19(\mathrm{t}, J=10 \mathrm{~Hz}, 2 \mathrm{H}), 2.74$ (qnt, $J=10 \mathrm{~Hz}, 2 \mathrm{H}) .{ }^{13} \mathrm{C}$ NMR $\left(125 \mathrm{MHz}, \mathrm{DMDO}-d_{6}\right) \delta(\mathrm{ppm})=162.8,160.5,137.8,128.8,122.6,115.2,55.8,46.9,26.5,21.3$

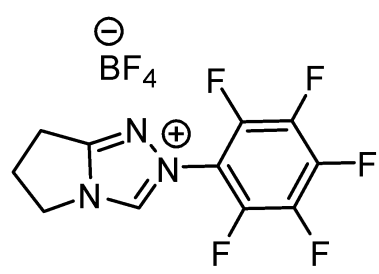

$4 \mathbf{i}$

2-Pyrrolidone (1.70 g, $20.00 \mathrm{mmol}, 1$ eq.), anhydrous dichloromethane (120 mL), trimethyloxonium tetrafluoroborate $(3.26 \mathrm{~g}, 22.00 \mathrm{mmol}, 1.1 \mathrm{eq}$.$) , pentafluorophenyl hydrazine (4.36 g, 22.00 \mathrm{mmol}$, 1.1 eq.), were prepared according to the general procedure given for pyrrolidine based triazolium NHC precatalysts (page 7). Then, triethyl orthoformate $(26.64 \mathrm{~mL}, 160.00 \mathrm{mmol}, 8$ eq.) and

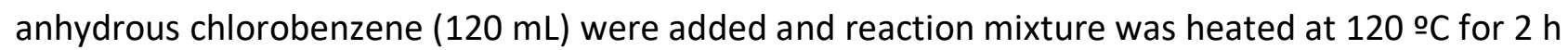
under argon atmosphere. Obtained catalyst was washed with ethyl acetate. Reaction yield $=66 \%$ (4.80 g). Spectral data for product 4i have been previously reported. ${ }^{8}$ NMR spectra are given on pages 114-115. 
${ }^{1} \mathbf{H}$ NMR $\left(500 \mathrm{MHz}, \mathrm{DMDO}-d_{6}\right) \delta(\mathrm{ppm})=10.52(\mathrm{~s}, 1 \mathrm{H}), 4.48(\mathrm{t}, J=10 \mathrm{~Hz}, 2 \mathrm{H}), 3.24(\mathrm{t}, J=10 \mathrm{~Hz}, 2$ H), 2.76 (qnt, $J=10 \mathrm{~Hz}, 2 \mathrm{H}) .{ }^{13} \mathrm{C}$ NMR $\left(125 \mathrm{MHz}, \mathrm{DMDO}-d_{6}\right) \delta(\mathrm{ppm})=164.4,144.0,143.6$ (d, $J=11$ $\mathrm{Hz}), 141.9(\mathrm{t}, J=13 \mathrm{~Hz}), 141.5(\mathrm{~d}, J=11 \mathrm{~Hz}), 138.7(\mathrm{t}, J=11 \mathrm{~Hz}), 136.6(\mathrm{t}, J=13 \mathrm{~Hz}), 111.4(\mathrm{t}, J=13$ $\mathrm{Hz}), 48.2,26.6,21.5 .{ }^{19} \mathrm{~F} \mathrm{NMR}\left(376 \mathrm{MHz}, \mathrm{DMDO}-d_{6}\right) \delta(\mathrm{ppm})=-146.0(\mathrm{~d}, J=19 \mathrm{~Hz}, 2 \mathrm{~F}),-148.5(\mathrm{~m}, 1$ F), $-148.8(\mathrm{~s}, 4 \mathrm{~F}),-160.3(\mathrm{~m}, 2 \mathrm{~F})$.

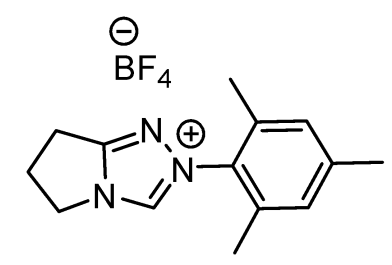

\section{4j}

2-Pyrrolidone (255 mg, $3.00 \mathrm{mmol}, 1$ eq.), anhydrous dichloromethane (20 mL), trimethyloxonium tetrafluoroborate (488 mg, $3.30 \mathrm{mmol}, 1.1$ eq.), mesityl hydrazine (496 mg, $3.30 \mathrm{mmol}, 1.1$ eq.), triethyl orthoformate $(4.00 \mathrm{~mL}, 24.00 \mathrm{mmol}, 8$ eq.) and anhydrous chlorobenzene $(20 \mathrm{~mL})$ were prepared according to the general procedure given for pyrrolidine based triazolium NHC precatalysts (page 7). Obtained catalyst was washed with ethyl acetate. Reaction yield $=79 \%$ (750 $\mathrm{mg})$. Spectral data for product $\mathbf{4} \mathbf{j}$ have been previously reported. ${ }^{9}$ NMR spectra are given on pages 116-117.

${ }^{1} \mathrm{H}$ NMR $\left(500 \mathrm{MHz}, \mathrm{DMDO}-d_{6}\right) \delta(\mathrm{ppm})=10.24(\mathrm{~s}, 1 \mathrm{H}), 7.15(\mathrm{~s}, 2 \mathrm{H}), 4.46(\mathrm{t}, \mathrm{J}=10 \mathrm{~Hz}, 2 \mathrm{H}), 3.20(\mathrm{t}$, $J=10 \mathrm{~Hz}, 2 \mathrm{H}$ ), 2.77 (qnt, $J=10 \mathrm{~Hz}, 2 \mathrm{H}), 2.34(\mathrm{~s}, 3 \mathrm{H}), 2.07(\mathrm{~s}, 6 \mathrm{H}) .{ }^{13} \mathrm{C} \mathrm{NMR}\left(125 \mathrm{MHz}, \mathrm{DMDO}-d_{6}\right) \delta$ $(p p m)=163.2,141.6,141.2,135.0,132.0,129.2,47.2,26.4,21.4,20.6,16.9$

$\Theta$ $\mathrm{BF}_{4}$<smiles></smiles>

4k

2-Pyrrolidone (851 mg, $10.00 \mathrm{mmol}, 1$ eq.), anhydrous dichloromethane (60 mL), trimethyloxonium tetrafluoroborate (1.63 g, $11.00 \mathrm{mmol}, 1.1 \mathrm{eq}$.), phenyl hydrazine (1.19 g, $11.00 \mathrm{mmol}, 1.1$ eq.), triethyl orthoformate $(13.32 \mathrm{~mL}, 80.00 \mathrm{mmol}, 8 \mathrm{eq}$.) and anhydrous chlorobenzene $(60 \mathrm{~mL})$ were prepared according to the general procedure given for pyrrolidine based triazolium NHC precatalysts (page 7). Obtained catalyst was washed with methanol. Reaction yield $=84 \%$ (2.30 g). Spectral data for product $\mathbf{4 k}$ have been previously reported. ${ }^{10}$ NMR spectra are given on pages 118 119. 
${ }^{1} \mathrm{H}$ NMR $\left(500 \mathrm{MHz}, \mathrm{DMDO}-d_{6}\right) \delta(\mathrm{ppm})=10.68(\mathrm{~s}, 1 \mathrm{H}), 7.89(\mathrm{~d}, J=5 \mathrm{~Hz}, 1 \mathrm{H}), 7.70(\mathrm{t}, J=10 \mathrm{~Hz}, 2 \mathrm{H})$, $7.63(\mathrm{t}, J=5 \mathrm{~Hz}, 1 \mathrm{H}), 4.42(\mathrm{t}, J=10 \mathrm{~Hz}, 2 \mathrm{H}), 3.21(\mathrm{t}, J=10 \mathrm{~Hz}, 2 \mathrm{H}), 2.75$ (qnt, $J=10 \mathrm{~Hz}, 2 \mathrm{H}) .{ }^{13} \mathrm{C}$ NMR $\left(125 \mathrm{MHz}, \mathrm{DMDO}-d_{6}\right) \delta(\mathrm{ppm})=163.0,138.4,135.6,130.4,130.3,120.7,46.9,26.6,21.3$

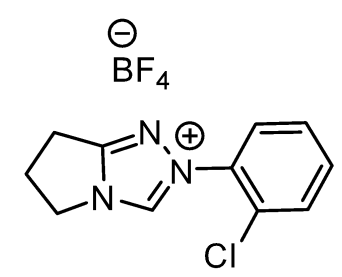

4I

2-Pyrrolidone ( $851 \mathrm{mg}, 10.00 \mathrm{mmol}, 1$ eq.), anhydrous dichloromethane (60 mL), trimethyloxonium tetrafluoroborate $(1.63 \mathrm{~g}, 11.00 \mathrm{mmol}, 1.1 \mathrm{eq}$.), 2-chlorophenyl hydrazine (1.57 g, $11.00 \mathrm{mmol}, 1.1$ eq.), triethyl orthoformate (13.32 mL, $80.00 \mathrm{mmol}, 8$ eq.) and anhydrous chlorobenzene (60 mL) were prepared according to the general procedure given for pyrrolidine based triazolium NHC precatalysts (page 7). Catalyst was recrystallized using ethyl acetate:methanol (9:1) solvent combination. Reaction yield =33\% (1.00 g). NMR spectra are given on pages 119-120.

${ }^{1} \mathrm{H}$ NMR $\left(500 \mathrm{MHz}, \mathrm{DMDO}-\mathrm{d}_{6}\right) \delta(\mathrm{ppm})=10.50(\mathrm{~s}, 1 \mathrm{H}), 7.86(\mathrm{dd}, J=10 \mathrm{~Hz}, 1 \mathrm{H}), 7.79-7.73(\mathrm{~m}, 2 \mathrm{H})$, $7.68(\mathrm{td}, J=10 \mathrm{~Hz}, 1 \mathrm{H}), 4.46(\mathrm{t}, J=10 \mathrm{~Hz}, 2 \mathrm{H}), 3.22(\mathrm{t}, J=10 \mathrm{~Hz}, 2 \mathrm{H}), 2.76$ (qnt, $J=10 \mathrm{~Hz}, 2 \mathrm{H}) .{ }^{13} \mathrm{C}$ NMR $\left(125 \mathrm{MHz}, \mathrm{DMDO}-d_{6}\right) \delta(\mathrm{ppm})=163.0,141.9,133.2,133.1,130.9,128.9,128.7,128.6,47.4$, 26.6, 21.3. HRMS(ESI) calcd for $\mathrm{C}_{11} \mathrm{H}_{11} \mathrm{ClN}_{3}{ }^{+}(\mathrm{M})^{+}:$:220.0636, Found: 220.0639

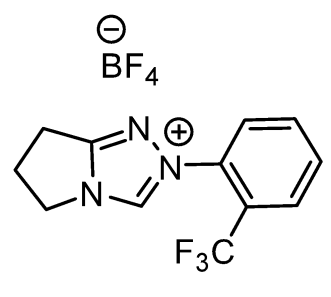

$4 m$

2-Pyrrolidone (851 mg, $10.00 \mathrm{mmol}, 1$ eq.), anhydrous dichloromethane (60 mL), trimethyloxonium tetrafluoroborate (1.63 g, $11.00 \mathrm{mmol}, 1.1 \mathrm{eq}$ )), 2-(trifluoromethyl)phenyl hydrazine (1.94 g, 11.00 $\mathrm{mmol}, 1.1$ eq.), triethyl orthoformate $(13.32 \mathrm{~mL}, 80.00 \mathrm{mmol}, 8$ eq.) and anhydrous chlorobenzene $(60 \mathrm{~mL})$ were prepared according to the general procedure given for pyrrolidine based triazolium NHC precatalysts (page 7). Obtained catalyst was washed with ethyl acetate. Reaction yield $=42 \%$ (1.60 g). NMR spectra are given on pages 121-122.

${ }^{1} \mathrm{H}$ NMR $\left(500 \mathrm{MHz}, \mathrm{DMDO}-d_{6}\right) \delta(\mathrm{ppm})=10.50(\mathrm{~s}, 1 \mathrm{H}), 8.12(\mathrm{~d}, J=5 \mathrm{~Hz}, 1 \mathrm{H}), 8.04(\mathrm{t}, J=5 \mathrm{~Hz}, 1 \mathrm{H})$, $7.98(t, J=5 \mathrm{~Hz}, 1 \mathrm{H}), 7.86(\mathrm{~d}, J=5 \mathrm{~Hz}, 1 \mathrm{H}), 4.49(\mathrm{t}, J=10 \mathrm{~Hz}, 2 \mathrm{H}), 3.23(\mathrm{t}, J=10 \mathrm{~Hz}, 2 \mathrm{H}$ ), 2.77 (qnt, 
$J=10 \mathrm{~Hz}, 2 \mathrm{H}) .{ }^{13} \mathrm{C}$ NMR $\left(125 \mathrm{MHz}, \mathrm{DMDO}-d_{6}\right) \delta(\mathrm{ppm})=163.0,142.3,134.5,132.8,132.5,129.8$, 127.9, 125.0 (q, $J=31 \mathrm{~Hz}), 122.5(q, J=271 \mathrm{~Hz}), 47.5,26.6,21.2 .{ }^{19}$ F NMR (376 MHz, DMDO- $\left.d_{6}\right) \delta$ $(\mathrm{ppm})=-57.7(\mathrm{~s}, 3 \mathrm{~F}),-148.6(\mathrm{~s}, 4 \mathrm{~F})$. HRMS(ESI) calcd for $\mathrm{C}_{12} \mathrm{H}_{11} \mathrm{~F}_{3} \mathrm{~N}_{3}{ }^{+}(\mathrm{M})^{+}:$254.0900, Found: 254.0896

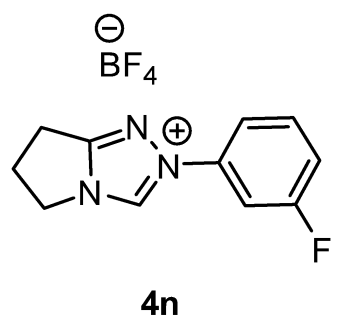

2-Pyrrolidone (851 mg, $10.00 \mathrm{mmol}, 1$ eq.), anhydrous dichloromethane (60 mL), trimethyloxonium tetrafluoroborate $(1.63 \mathrm{~g}, 11.00 \mathrm{mmol}, 1.1 \mathrm{eq}$ ), 3-fluorophenyl hydrazine (1.39 g, $11.00 \mathrm{mmol}, 1.1$ eq.), triethyl orthoformate (13.32 mL, $80.00 \mathrm{mmol}, 8$ eq.) and anhydrous chlorobenzene (60 mL) were prepared according to the general procedure given for pyrrolidine based triazolium NHC precatalysts (page 7). Catalyst was recrystallized using ethyl acetate:benzene (1:1) solvent combination. Reaction yield $=65 \%$ (1.95 g). NMR spectra are given on pages 123-125.

${ }^{1}$ H NMR $\left(500 \mathrm{MHz}\right.$, DMDO- $\left.d_{6}\right) \delta(\mathrm{ppm})=10.73(\mathrm{~s}, 1 \mathrm{H}), 7.85-7.73(\mathrm{~m}, 3 \mathrm{H}), 7.55-7.48(\mathrm{~m}, 1 \mathrm{H}), 4.43$ $(\mathrm{t}, J=10 \mathrm{~Hz}, 2 \mathrm{H}), 3.22$ (t, $J=10 \mathrm{~Hz}, 2 \mathrm{H}), 2.76$ (qnt, $J=10 \mathrm{~Hz}, 2 \mathrm{H}) .{ }^{13} \mathrm{C}$ NMR $\left(125 \mathrm{MHz}, \mathrm{DMDO}-d_{6}\right) \delta$ $(\mathrm{ppm})=163.1,162.2(\mathrm{~d}, J=245 \mathrm{~Hz}), 138.9,136.7(\mathrm{~d}, J=10 \mathrm{~Hz}), 132.4(\mathrm{~d}, J=9 \mathrm{~Hz}), 117.3$ (d, J = 21 $\mathrm{Hz}), 116.8,108.3(\mathrm{~d}, J=26 \mathrm{~Hz}), 47.1,26.6,21.3 .{ }^{19} \mathrm{~F} \mathrm{NMR}\left(376 \mathrm{MHz}, \mathrm{DMDO}-d_{6}\right) \delta(\mathrm{ppm})=-109.8(\mathrm{~s}$, $1 \mathrm{~F}),-148.6$ (s, 4 F). HRMS(ESI) calcd for $\mathrm{C}_{11} \mathrm{H}_{11} \mathrm{FN}_{3}{ }^{+}(\mathrm{M})^{+}:$204.0932, Found: 204.0930 


\section{Synthesis of 2 and 3-Substituted Benzaldehyde Derivatives}

Synthesis of 2-" ${ }^{n}$ utoxybenzaldehyde:<smiles>O=Cc1ccccc1O</smiles><smiles>CCCCOc1ccccc1C=O</smiles>

Scheme 9: Synthesis of $2-{ }^{n}$ Butoxybenzaldehyde

2- ${ }^{n}$ butoxybenzaldehyde was prepared according to a literature procedure. ${ }^{11} \mathrm{~A}$ dry, argon flushed two neck round bottom flask equipped with a magnetic stir bar and a septum was charged with salicylaldehyde (3.20 mL, $30 \mathrm{mmol}, 1$ eq.) and $\mathrm{K}_{2} \mathrm{CO}_{3}(4.96 \mathrm{~g}, 36 \mathrm{mmol}, 1.2 \mathrm{eq}$ ), $\mathrm{N}, \mathrm{N}$ dimethylformamide $(60 \mathrm{~mL})$ and 1-bromobutane $(3.90 \mathrm{~mL} .36 \mathrm{mmol}, 1.2$ eq.) was added. subsequently. After stirring reaction at $90 \circ \mathrm{C}$ for $18 \mathrm{~h}$, the mixture was poured into ice cold distilled water $(600 \mathrm{~mL})$ and extracted with dichloromethane $(3 \times 60 \mathrm{~mL})$. The combined organic phases were dried over $\mathrm{Na}_{2} \mathrm{SO}_{4}$, concentrated and the crude product was purified by silica gel column chromatography using Hex:EtOAc (90:10) combination to yield colorless liquid (5.52 g, 100\%). This aldehyde was distilled under vacuum before use. NMR spectra are given on pages 126-127.

${ }^{1} \mathrm{H}$ NMR $\left(400 \mathrm{MHz}, \mathrm{CDCl}_{3}\right) \delta(\mathrm{ppm})=10.52(\mathrm{~s}, 1 \mathrm{H}), 7.83(\mathrm{dd}, J=4 \mathrm{~Hz}, 1 \mathrm{H})$, 7.55-7.50 (m, $\left.1 \mathrm{H}\right)$, 7.02$6.97(\mathrm{~m}, 2 \mathrm{H}), 4.08(\mathrm{t}, J=4 \mathrm{~Hz}, 2 \mathrm{H}), 1.87-1.80(\mathrm{~m}, 2 \mathrm{H}), 1.58-1.48(\mathrm{~m}, 2 \mathrm{H}), 0.99(\mathrm{t}, J=4 \mathrm{~Hz}, 3 \mathrm{H}) .{ }^{13} \mathrm{C}$ NMR $\left(100 \mathrm{MHz}, \mathrm{CDCl}_{3}\right) \delta(\mathrm{ppm})=190.1,161.7,136.0,128.4,125.1,120.6,112.6,68.4,31.3,19.4$, 13.9

Synthesis of 2-( ${ }^{\text {sec }}$ Butoxy)benzaldehyde:<smiles>O=Cc1ccccc1O</smiles>

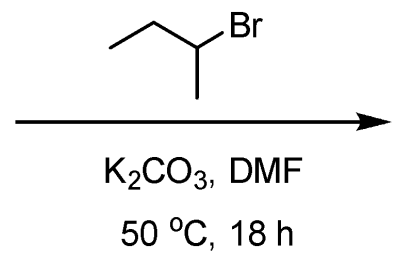<smiles>CCC(C)Oc1ccccc1C=O</smiles>

Scheme 10: Synthesis of 2-( $\left.{ }^{\sec B u t o x y}\right)$ benzaldehyde

The procedure given above for the synthesis of $2-{ }^{n}$ butoxybenzaldehyde was modified for the synthesis of this derivative. A dry, argon flushed two neck round bottom flask equipped with a magnetic stir bar and a septum was charged with salicylaldehyde $(3.20 \mathrm{~mL}, 30 \mathrm{mmol}, 1$ eq.) and $\mathrm{K}_{2} \mathrm{CO}_{3}(4.96 \mathrm{~g}, 36 \mathrm{mmol}, 1.2 \mathrm{eq}$ ) $, \mathrm{N}, \mathrm{N}$-dimethylformamide $(60 \mathrm{~mL})$ and 2-bromobutane $(3.90 \mathrm{~mL}$. $36 \mathrm{mmol}, 1.2$ eq.) were added. After stirring at 90 으 for $18 \mathrm{~h}$, the reaction mixture was allowed to 
cool down to room temperature, 2-bromobutane (3.90 mL. $36 \mathrm{mmol}, 1.2$ eq.) was added and the reaction mixture was stirred for 2 days at 90 ㄷ․ Then the mixture was poured into ice cold distilled water $(600 \mathrm{~mL})$ and extracted with dichloromethane $(3 \times 60 \mathrm{~mL})$. The combined organic phases were dried over $\mathrm{Na}_{2} \mathrm{SO}_{4}$, concentrated and the crude product was purified by silica gel column chromatography using Hex:EtOAc (90:10) combination to yield colorless liquid (4.94 g, 94\%). This aldehyde was distilled under vacuum before use. NMR spectra are given on pages 128-129.

${ }^{1} \mathrm{H}$ NMR $\left(400 \mathrm{MHz}, \mathrm{CDCl}_{3}\right) \delta(\mathrm{ppm})=10.51(\mathrm{~s}, 1 \mathrm{H}), 7.83(\mathrm{dd}, J=4 \mathrm{~Hz}, 1 \mathrm{H}), 7.53-7.49(\mathrm{~m}, 1 \mathrm{H})$, 7.00$6.96(\mathrm{~m}, 2 \mathrm{H}), 4.46$ (sxt, J = $4 \mathrm{~Hz}, 1 \mathrm{H}), 1.87-1.65(\mathrm{~m}, 2 \mathrm{H}), 1.36$ (d, J = $4 \mathrm{~Hz}, 3 \mathrm{H}), 1.01$ (t, J = $4 \mathrm{~Hz}, 3$ H). ${ }^{13} \mathrm{C} \mathrm{NMR}\left(100 \mathrm{MHz}, \mathrm{CDCl}_{3}\right) \delta(\mathrm{ppm})=190.3,161.1,135.9,128.4,125.9,120.5,114.0,76.1,29.3$, 19.3, 9.8. HRMS(ESI) calcd for $\mathrm{C}_{11} \mathrm{H}_{14} \mathrm{O}_{2}{ }^{+}(\mathrm{M}+\mathrm{Na})^{+}: 201.0886$, Found: 201.0886

\section{Synthesis of 3-(tert-butoxy)benzaldehyde:}<smiles>O=Cc1cccc(O)c1</smiles>

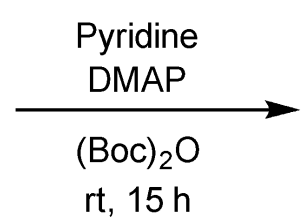<smiles>CC(C)(C)Oc1cccc(C=O)c1</smiles>

Scheme 11: Synthesis of tert-butyl (3-formylphenyl) carbonate

A dry, argon flushed two neck round bottom flask equipped with a magnetic stir bar and a septum was charged with 3-hydroxybenzaldehyde ( $2 \mathrm{~g}, 10 \mathrm{mmol}, 1$ eq.) in anhydrous acetonitrile (40 mL). Then, pyridine (1.74 g, $20 \mathrm{mmol}, 2$ eq.) and 4-dimethylaminopyridine ( $122 \mathrm{mg}, 1 \mathrm{mmol}, 0.1 \mathrm{eq}$.) were added, followed by addition of Boc anhydride $(2.4 \mathrm{~g}, 11.0 \mathrm{mmol}, 1.1 \mathrm{eq}$.). After stirring at 20 oC for $15 \mathrm{~h}$, the solvent was removed under reduced pressure. The obtained crude reaction mixture was dissolved in dichloromethane $(20 \mathrm{~mL})$ and washed by aqueous sodium bicarbonate $(3 \mathrm{X} 20 \mathrm{~mL})$ and distilled water $\left(2 \times 100 \mathrm{~mL}\right.$ ). The combined organic phases were dried over $\mathrm{Na}_{2} \mathrm{SO}_{4}$, concentrated and the crude product was purified by silica gel column chromatography using Hex:EtOAc (80:20) combination to yield colorless oil (2.18 g, 98\%). NMR spectra are given on pages 130-131.

${ }^{1} \mathrm{H}$ NMR $\left(400 \mathrm{MHz}, \mathrm{CD}_{3} \mathrm{CN}\right) \delta(\mathrm{ppm})=9.99(\mathrm{~s}, 1 \mathrm{H}), 7.80(\mathrm{~d}, J=8 \mathrm{~Hz}, 1 \mathrm{H}), 7.69(\mathrm{~s}, 1 \mathrm{H}), 7.62(\mathrm{t}, J=8$ $\mathrm{Hz}, 1 \mathrm{H}), 7.47$ (d, $J=8 \mathrm{~Hz}, 1 \mathrm{H}), 1.53(\mathrm{~s}, 9 \mathrm{H}) .{ }^{13} \mathrm{C}$ NMR $\left(100 \mathrm{MHz}, \mathrm{CD}_{3} \mathrm{CN}\right) \delta(\mathrm{ppm})=192.7,152.8$, 152.5, 139.0, 131.4, 128.5, 128.3, 122.5, 84.8, 27.8. 


\section{Synthesis of Aryl Boroxines}

Aryl boronic acids received from commercial suppliers contain a mixture of boronic acid, boroxine and a dimer of boronic acid. We found that using such a complex mixture for the synthesis of boroxine leads to an impure product. Therefore, for all of the following aryl boronic acids the mixture (boronic acid, boroxine and dimer) was completely converted to boronic acid by heating at $90 \stackrel{\circ}{ }$ in distilled water for $12 \mathrm{~h}$. The obtained solid was filtred off and dried under Drierite for $12 \mathrm{~h}$. The pure boronic acid was then converted to boroxine by the following procedure.<smiles>[R]c1ccc(B(O)O)cc1</smiles><smiles>[R9][R4](=O)CCC</smiles><smiles>[R]c1ccc(B2OB(c3ccc([R])cc3)OB(c3cc([R])ccc3OCC=[R])O2)cc1</smiles>

Scheme 12: Synthesis of Aryl Boroxines

\section{General Procedure for Synthesis of Aryl Boroxines:}

Aryl Boroxines were prepared by refluxing the aryl boronic acids in toluene. ${ }^{12} \mathrm{~A}$ dry, argon flushed Dean-Stark apparatus equipped with a magnetic stir bar and a septum was charged with aryl boronic acid (16.4 mmol) and anhydrous toluene $(70 \mathrm{~mL})$. Reaction was refluxed for $24 \mathrm{~h}$ and after cooling to room temperature, the crude reaction mixture was evaporated under vacuum to give final product.

\section{Phenyl Boroxine:}<smiles>c1ccc(B2OB(c3ccccc3)OB(c3ccccc3)O2)cc1</smiles>

Phenyl boronic acid $(2.00 \mathrm{~g}, 16.4 \mathrm{mmol})$ in dry toluene $(70 \mathrm{~mL})$ was refluxed for $24 \mathrm{~h}$ according to the general procedure given for boroxines synthesis ${ }^{13}$. Reaction yield $=99 \%$ (1.70 g). NMR spectra are given on pages 132-133. 
${ }^{1} \mathbf{H}$ NMR $\left(500 \mathrm{MHz}\right.$, DMSO-d $\left.d_{6}\right) \delta(\mathrm{ppm})=7.90-7.89(\mathrm{~m}, 6 \mathrm{H}), 7.40-7.36(\mathrm{~m}, 9 \mathrm{H}) .{ }^{13} \mathrm{C} \mathrm{NMR}(125 \mathrm{MHz}$, DMSO- $\left.d_{6}\right) \delta(p p m)=138.4,133.4,129.5,127.4$

4-Methylphenyl Boroxine (4-Me-Ph):<smiles>Cc1ccc(B2OB(c3ccc(C)cc3)OB(c3ccc(C)cc3)O2)cc1</smiles>

4-Methylphenyl boronic acid $(2.23 \mathrm{~g}, 16.4 \mathrm{mmol})$ in dry toluene $(70 \mathrm{~mL})$ was refluxed for $24 \mathrm{~h}$ according to the general procedure given for boroxines synthesis ${ }^{13}$. Reaction yield $=98 \%(1.90 \mathrm{~g})$. NMR spectra are given on pages 134-135.

${ }^{1} \mathrm{H}$ NMR $\left(500 \mathrm{MHz}, \mathrm{DMSO}-d_{6}\right) \delta(\mathrm{ppm})=7.78(\mathrm{~d}, J=5 \mathrm{~Hz}, 6 \mathrm{H}), 7.19(\mathrm{~d}, J=5 \mathrm{~Hz}, 6 \mathrm{H}), 2.32(\mathrm{~s}, 9 \mathrm{H})$. ${ }^{13} \mathrm{C}$ NMR $\left(125 \mathrm{MHz}, \mathrm{DMSO}-d_{6}\right) \delta(\mathrm{ppm})=138.6,135.1,133.6,128.1,21.2$

\section{4-(Trifluoromethyl)phenyl Boroxine (4-CF $-\mathrm{Ph})$ :}<smiles>FC(F)(F)c1ccc(B2OB(c3ccc(C(F)(F)F)cc3)OB(c3ccc(C(F)(F)F)cc3)O2)cc1</smiles>

4-(Trifluoromethyl)phenyl boronic acid $(3.12 \mathrm{~g}, 16.4 \mathrm{mmol})$ in dry toluene $(70 \mathrm{~mL})$ was refluxed for $24 \mathrm{~h}$ according to the general procedure given for boroxines synthesis ${ }^{14}$. Reaction yield $=99 \%(2.78$ g). NMR spectra are given on pages 136-137.

${ }^{1} \mathrm{H}$ NMR $\left(500 \mathrm{MHz}\right.$, DMSO- $\left.d_{6}\right) \delta(\mathrm{ppm})=8.09(\mathrm{~d}, J=10 \mathrm{~Hz}, 6 \mathrm{H}), 7.73(\mathrm{~d}, J=10 \mathrm{~Hz}, 6 \mathrm{H}) .{ }^{13} \mathrm{C} \mathrm{NMR}$ $\left(125 \mathrm{MHz}, \mathrm{DMSO}-d_{6}\right) \delta(\mathrm{ppm})=143.0,134.0,129.9(\mathrm{q}, J=39 \mathrm{~Hz}), 124.5(\mathrm{q}, J=339 \mathrm{~Hz}), 124.1 .{ }^{19} \mathbf{F}$ NMR $\left(376 \mathrm{MHz}, \mathrm{DMDO}-d_{6}\right) \delta(\mathrm{ppm})=-61.2(\mathrm{~s}, 9 \mathrm{~F})$ 
4-Methoxyphenyl Boroxine (4-OMe-Ph):<smiles>COc1ccc(B2OB(c3ccc(OC)cc3)OB(c3ccc(OC)cc3)O2)cc1</smiles>

4-Methoxyphenyl boronic acid $(2.49 \mathrm{~g}, 16.4 \mathrm{mmol})$ in dry toluene $(70 \mathrm{~mL})$ was refluxed for $24 \mathrm{~h}$ according to the general procedure given for boroxines synthesis ${ }^{13}$. Reaction yield $=99 \%(2.18 \mathrm{~g})$. NMR spectra are given on pages 138-139.

${ }^{1} \mathrm{H}$ NMR $\left(500 \mathrm{MHz}, \mathrm{DMSO}-d_{6}\right) \delta(\mathrm{ppm})=7.77(\mathrm{~d}, J=10 \mathrm{~Hz}, 6 \mathrm{H}), 6.69(\mathrm{~d}, J=10 \mathrm{~Hz}, 6 \mathrm{H}), 3.72(\mathrm{~s}, 9 \mathrm{H})$. ${ }^{13} \mathrm{C}$ NMR $\left(125 \mathrm{MHz}, \mathrm{DMSO}-d_{6}\right) \delta(\mathrm{ppm})=160.6,135.1,129.9,113.1,54.9$ 


\section{Catalytic Procedures and Compound Characterization Data}

\section{General Procedure for Deuteration of Aromatic Aldehydes:}
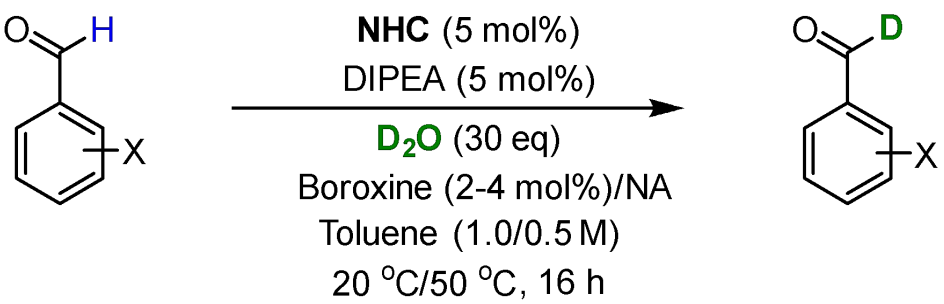

Scheme 13: General Scheme for Deuteration Aromatic Aldehydes (NA = without boroxine)

Deuteration reactions were run in $8 \mathrm{~mL}$ vials with a screw cap septa and stirred at $1000 \mathrm{rpm}$. A dry, argon flushed $8 \mathrm{~mL}$ vial equipped with a magnetic stir bar was charged with NHC $(0.055 \mathrm{mmol}, 0.05$ eq.), phenyl boroxine (0.022-0.044 mmol, 0.02-0.04 eq.) or without phenyl boroxine and flushed with argon followed by the addition of $500 \mu \mathrm{L}(1 \mathrm{M})$ or $1.60 \mathrm{~mL}(0.5 \mathrm{M})$ anhydrous toluene. DIPEA (0.055 mmol, 0.05 eq.), $D_{2} \mathrm{O}(600 \mu \mathrm{L}, 33 \mathrm{mmol}, 30$ eq.) and then aryl aldehyde (1.1 mmol) were added. The reaction vial was closed with a screw cap then wrapped with teflon tape and electrical

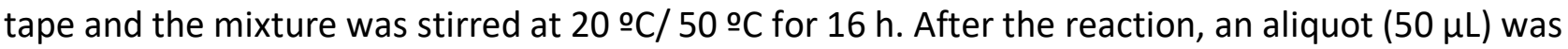
taken for NMR measurments (delay time D1 = 50 seconds was used in all studies). The crude reaction mixture was directly transferred on silica gel (dried silica gel which was neutralized with $1 \%$ triethyl amine was used for column chromatography) and purified using $\mathrm{Hex}_{\mathrm{Et}} \mathrm{O}$ combination. Deuterium incorporation and reaction yield after purification are included below for each substrate (the deuterium incorporation for the crude reaction mixture is given in brackets). We observed that some of the aldehydes (mostly liquid substrates) evaporate under reduced pressure/high vacuum.

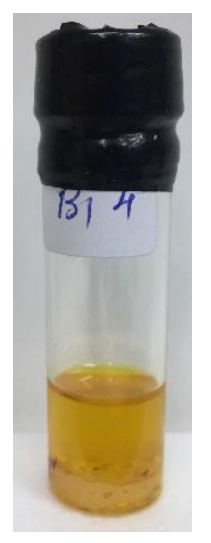

Figure 1: Reaction vial closed with screw cap and wrapped with teflon and electrical tape The following NMR spectra and HRMS traces are appended on pages (96-473). 


\subsection{Electronic and Steric Effects on the Substituted Benzaldehydes}

These reactions were performed according to the catalytic general procedure given in section 5 (page 20). Results for \%deuterium incorporation (\%CDO), \%Aldehyde and \%Benzoin formed are summarized in the table 1 and figure 2 below.<smiles>[X]c1ccccc1C=O</smiles>

$\mathrm{Ar}-\mathrm{CHO}$

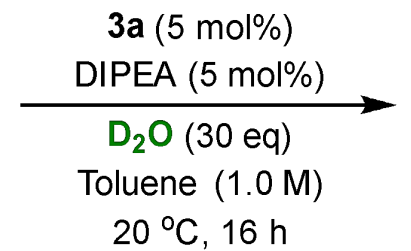

$20{ }^{\circ} \mathrm{C}, 16 \mathrm{~h}$<smiles>[X]c1ccccc1C([2H])=O</smiles>

$$
\mathrm{X}=2-\mathrm{CF}_{3}, 3-\mathrm{CF}_{3}, 4-\mathrm{CF}_{3}, \mathrm{H}, 2-{ }^{\mathrm{n}} \mathrm{OBu}, 2-\mathrm{OMe}, 3-\mathrm{OMe}, 4-\mathrm{OMe}
$$

Scheme 14: Reactivity of 2/3/4-electron donating and withdrawing aromatic aldehydes under catalytic reaction condition

Table 1: Results with 2/3/4-electron donating and withdrawing aromatic aldehydes

\begin{tabular}{|c|c|c|c|c|c|c|c|}
\hline \multirow[b]{2}{*}{ Entry } & \multirow[b]{2}{*}{$\mathrm{Ar}-\mathrm{CHO}$} & \multicolumn{3}{|c|}{ Aldehyde } & \multirow{2}{*}{$\begin{array}{c}\text { Benzoin } \\
\text { Aromati } \\
\text { c } \\
\mathrm{CH}\end{array}$} & \multirow[b]{2}{*}{$\begin{array}{c}\% \\
\text { Aldehyde }\end{array}$} & \multirow[b]{2}{*}{$\begin{array}{c}\% \\
\text { Benzoi } \\
\mathrm{n}\end{array}$} \\
\hline & & $\begin{array}{l}\mathrm{CHO} \\
(1 \mathrm{H})\end{array}$ & $\begin{array}{c}\text { \%Deuterium } \\
\text { Incorporatio } \\
n\end{array}$ & $\begin{array}{c}\text { Aromatic } \\
\mathrm{CH}\end{array}$ & & & \\
\hline 1 & $2-\mathrm{CF}_{3}$ & 0.24 & 76 & 1 & - & 100 & - \\
\hline $1^{\prime}$ & $2-\mathrm{CF}_{3}$ & 0.24 & 76 & 1 & - & 100 & - \\
\hline 2 & $3-\mathrm{CF}_{3}$ & 0.00 & 100 & 1 & 3.07 & 25 & 75 \\
\hline 2 ' & $3-\mathrm{CF}_{3}$ & 0.00 & 100 & 1 & 3.72 & 21 & 79 \\
\hline 3 & $4-\mathrm{CF}_{3}$ & 0.00 & 100 & 1 & 2.98 & 25 & 75 \\
\hline $3^{\prime}$ & $4-\mathrm{CF}_{3}$ & 0.00 & 100 & 1 & 2.80 & 26 & 74 \\
\hline 4 & $\mathrm{H}$ & 0.02 & 98 & 1 & 0.25 & 80 & 20 \\
\hline $4^{\prime}$ & $\mathrm{H}$ & 0.02 & 98 & 1 & 0.23 & 81 & 19 \\
\hline 5 & $2-\mathrm{O}^{n} \mathrm{Bu}$ & 0.42 & 58 & 1 & - & 100 & - \\
\hline 5 & $2-\mathrm{O}^{n} \mathrm{Bu}$ & 0.41 & 59 & 1 & - & 100 & - \\
\hline 6 & 2-OMe & 0.20 & 80 & 1 & - & 100 & - \\
\hline 6 & 2-OMe & 0.20 & 80 & 1 & - & 100 & - \\
\hline 7 & 3-OMe & 0.01 & 99 & 3 & - & 100 & - \\
\hline 7 ' & 3-OMe & 0.02 & 98 & 3 & - & 100 & - \\
\hline 8 & 4-OMe & 0.02 & 98 & 2 & - & 100 & - \\
\hline 8 & 4-OMe & 0.02 & 98 & 2 & - & 100 & - \\
\hline
\end{tabular}

Clean aldehyde, benzoin and reaction mixture spectrums were stacked for comparison. Aldehyde proton $(\mathrm{CHO})$ was used as a reference here. NMR spectra are given on pages 140-150. 


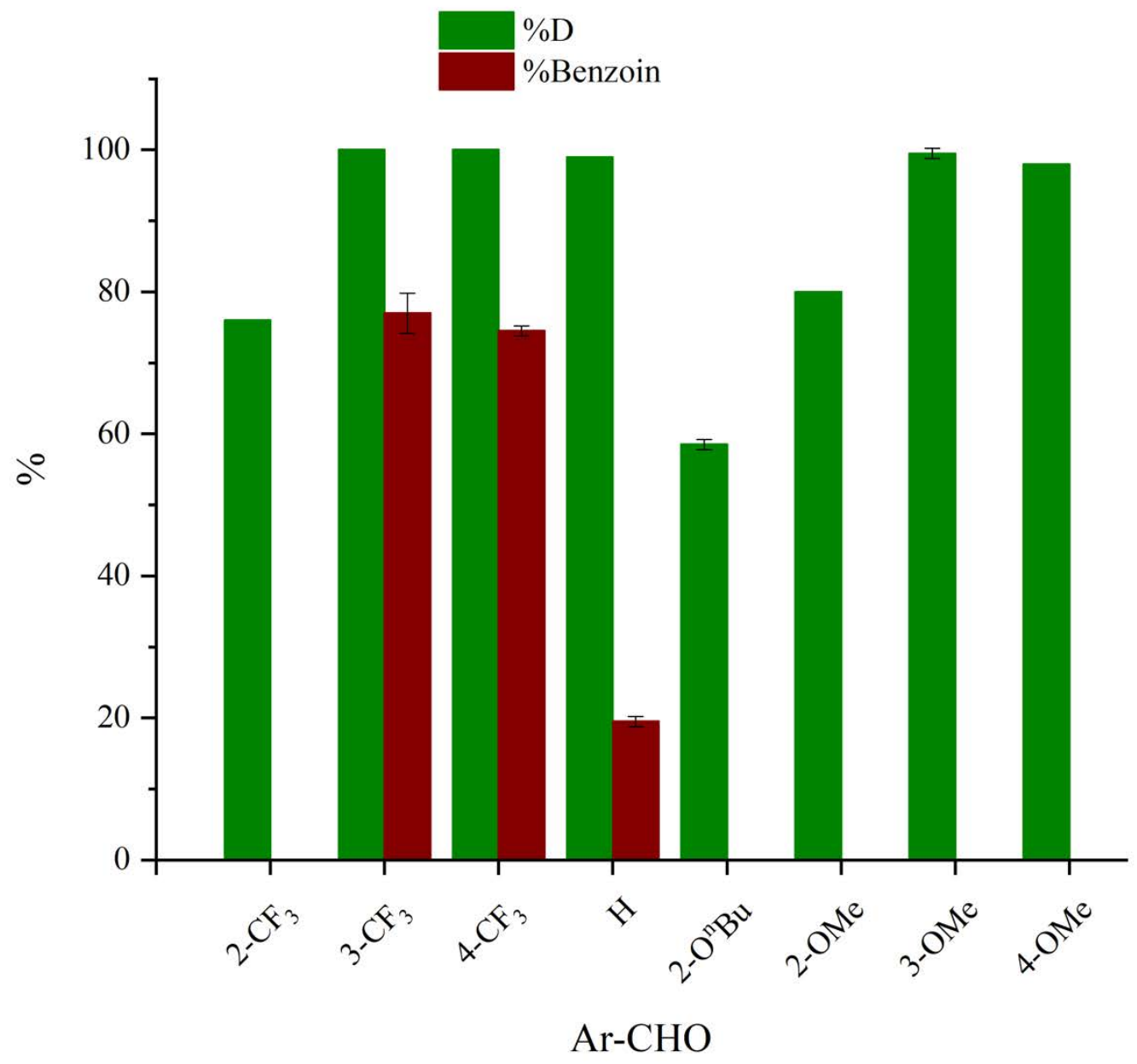

Figure 2: Results with 2/3/4-electron donating and withdrawing aromatic aldehydes 


\subsection{Optimization with 2-Methyl Benzaldehyde}

These reactions were performed according to the catalytic general procedure given in section 5 (page 20). Optimization of reaction conditions are carried out with 2-methyl benzaldehyde in differernt anhydrous organic solvents (deuterated or non-deuterated).<smiles>Cc1ccccc1C=O</smiles>

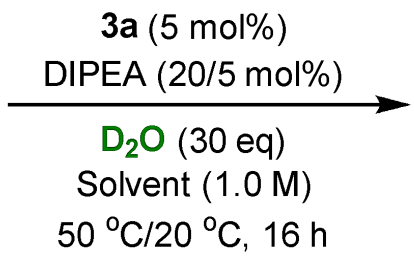<smiles>[2H]C(=O)c1ccccc1C</smiles>

Scheme 15: Optimization with 2-methyl benzaldehyde

Table 2: Optimization table for 2-Methyl Benzaldehyde (\%D = \%Deuterium Incorporation)

\begin{tabular}{|c|c|c|c|c|c|c|c|}
\hline \multirow{2}{*}{ Entry } & \multirow{3}{*}{$\begin{array}{c}\text { Reaction } \\
\text { Solvent } \\
\text { Acetonitrile- } d_{3}\end{array}$} & \multirow{3}{*}{$\begin{array}{c}\text { DIPEA } \\
\text { (mol\%) } \\
20\end{array}$} & \multirow{3}{*}{$\begin{array}{c}\text { Ph- } \\
\text { Boroxine } \\
\text { (mol\%) } \\
-\end{array}$} & \multirow{3}{*}{$\begin{array}{l}\text { Reaction } \\
\text { Time } \\
16 \mathrm{~h}\end{array}$} & \multirow{3}{*}{$\begin{array}{c}\text { Reaction } \\
\text { Temperatur } \\
\text { e } \\
50 \stackrel{\mathrm{C}}{ }\end{array}$} & \multicolumn{2}{|c|}{ Aldehyde } \\
\hline & & & & & & $\mathrm{CHO}$ & $\% \mathrm{D}$ \\
\hline 1 & & & & & & 0.97 & 3 \\
\hline 2 & $\begin{array}{c}\text { Tetrahydrofuran- } \\
d_{8}\end{array}$ & 20 & - & $16 \mathrm{~h}$ & $50 \stackrel{\circ}{C}$ & 0.81 & 19 \\
\hline 3 & Aetone- $d_{6}$ & 20 & - & $16 \mathrm{~h}$ & 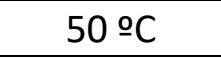 & 0.98 & 2 \\
\hline 4 & Methanol- $d_{4}$ & 20 & - & $16 \mathrm{~h}$ & $50 \div \mathrm{C}$ & 0.95 & 5 \\
\hline 5 & Chloroform-d & 20 & - & $16 \mathrm{~h}$ & $50 \div \mathrm{C}$ & 0.55 & 45 \\
\hline 6 & Toluene- $d_{8}$ & 20 & - & $16 \mathrm{~h}$ & $50 \div C$ & 0.10 & 90 \\
\hline 7 & Toluene & 5 & - & $16 \mathrm{~h}$ & $50 \div C$ & 0.11 & 89 \\
\hline 8 & Toluene & 5 & - & $16 \mathrm{~h}$ & $20 \div C$ & 0.01 & 99 \\
\hline 9 & Toluene & 5 & 2 & $16 \mathrm{~h}$ & $20 \div C$ & 0.36 & 64 \\
\hline 10 & Toluene & 5 & 4 & $16 \mathrm{~h}$ & $20 \div \mathrm{C}$ & 0.87 & 13 \\
\hline
\end{tabular}

NMR spectra are given on pages 151-155. 


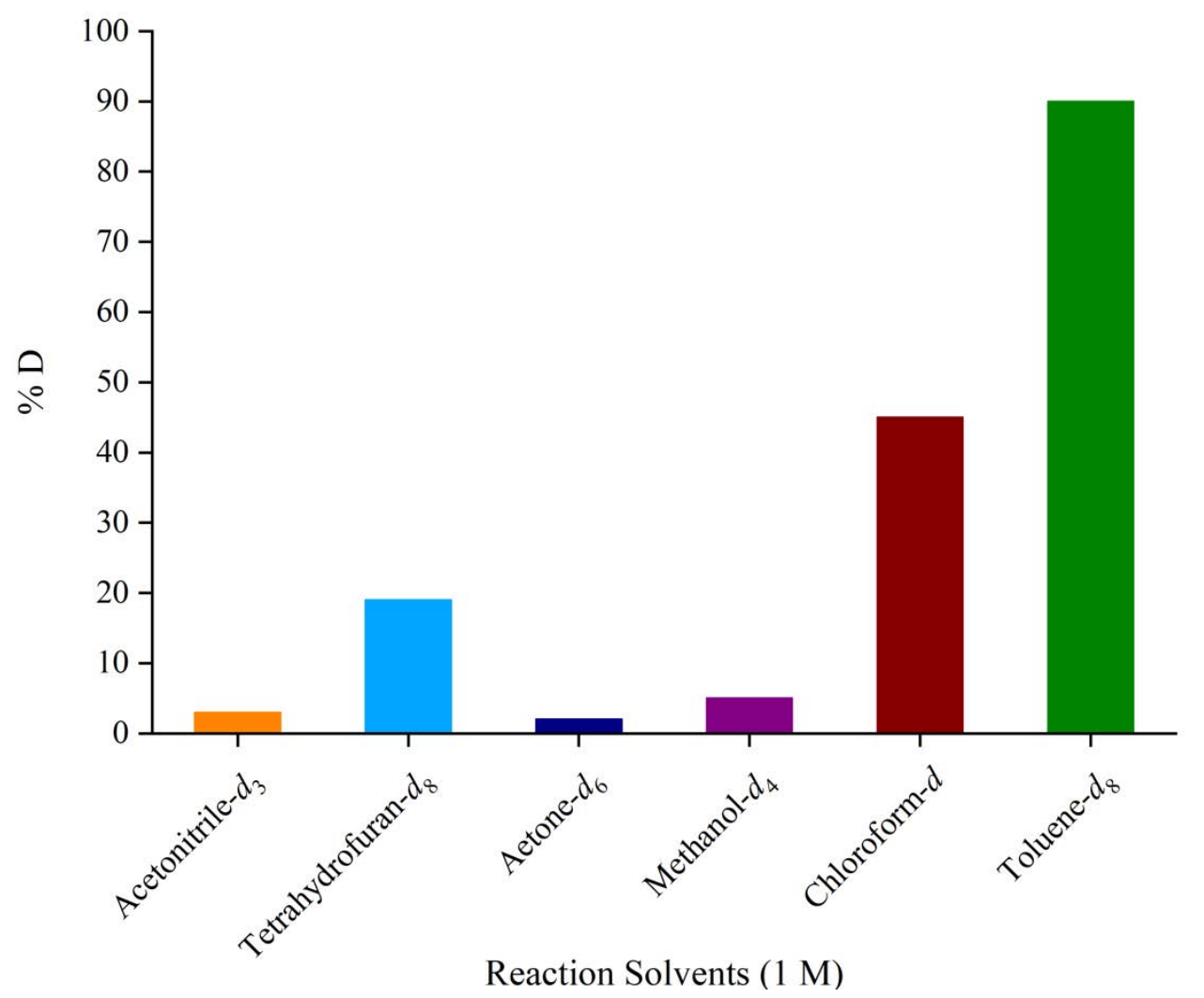

Figure 3: Effect of solvent on deutaration of 2-methyl benzaldehyde

\subsection{Optimization of 4-(Trifluoromethyl)benzaldehyde and Boroxine Effect}

The above optimized reaction condition has to be modified when working with 3/4-substituted electron withdrawing aromatic aldehydes (for example, 4-(trifluoromethyl)benzaldehyde) due to formation of benzoin product. These reactions were performed according to the catalytic general procedure given in section 5 (page 20).<smiles>O=Cc1ccc(C(F)(F)F)cc1</smiles>
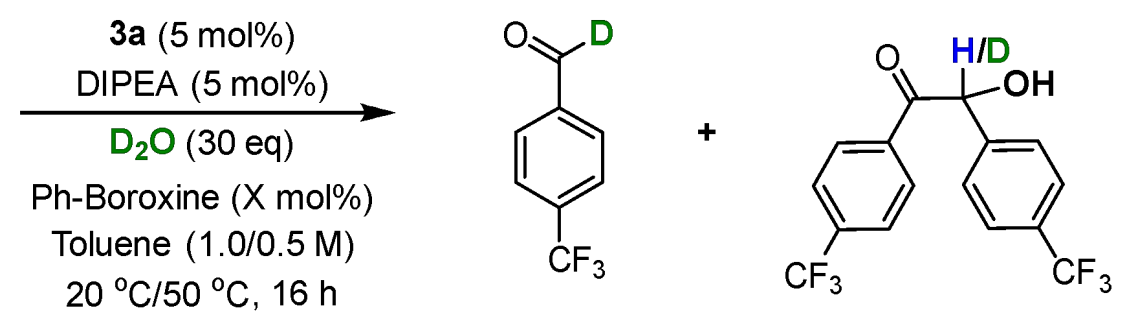

Scheme 16: Optimization reaction with 4-(trifluoromethyl)benzaldehyde 
Table 3: Optimization table ( $\%$ Deuterium Incorporation $=\% C D O)$

\begin{tabular}{|c|c|c|c|c|c|c|c|c|c|}
\hline \multirow{2}{*}{$\begin{array}{c}\text { Entr } \\
\mathrm{y}\end{array}$} & \multirow{3}{*}{$\begin{array}{c}\text { Ph- } \\
\text { Boroxin } \\
\text { e } \\
(\mathrm{mol} \%) \\
-\end{array}$} & \multirow{3}{*}{$\begin{array}{c}\text { Reactio } \\
\text { n } \\
\text { Conc. } \\
(\mathrm{M}) \\
1.00\end{array}$} & \multirow{3}{*}{$\begin{array}{c}\text { Reactio } \\
\text { n } \\
\text { Temp. } \\
20^{\circ} \mathrm{C}\end{array}$} & \multicolumn{3}{|c|}{ Aldehyde } & \multirow{2}{*}{$\begin{array}{c}\begin{array}{c}\text { Benzoi } \\
\text { n }\end{array} \\
\mathrm{CH} \\
(2 \mathrm{H})\end{array}$} & \multirow{2}{*}{$\begin{array}{c}\% \\
\text { Aldehyd } \\
\text { e }\end{array}$} & \multirow{2}{*}{$\begin{array}{c}\% \\
\text { Benzoi } \\
\mathrm{n}\end{array}$} \\
\hline & & & & $\begin{array}{l}\mathrm{CHO} \\
(1 \mathrm{H})\end{array}$ & $\% \mathrm{D}$ & $\begin{array}{c}\mathrm{CH} \\
(2 \mathrm{H})\end{array}$ & & & \\
\hline 1 & & & & 0.00 & 100 & 2 & 5.95 & 25 & 75 \\
\hline 2 & - & 0.50 & $20^{\circ} \mathrm{C}$ & 0.00 & 100 & 2 & 7.90 & 20 & 80 \\
\hline 3 & 0.5 & 0.50 & $20^{\circ} \mathrm{C}$ & 0.00 & 100 & 2 & 6.75 & 23 & 77 \\
\hline 4 & 2.0 & 0.50 & $20^{\circ} \mathrm{C}$ & 0.04 & 96 & 2 & 0.05 & 98 & 2 \\
\hline 5 & 2.0 & 0.50 & $50^{\circ} \mathrm{C}$ & 0.00 & 100 & 2 & 0.00 & 100 & 0 \\
\hline 6 & 2.0 & 1.00 & $20^{\circ} \mathrm{C}$ & 0.49 & 51 & 2 & 0.05 & 98 & 2 \\
\hline 7 & 2.0 & 1.00 & $50^{\circ} \mathrm{C}$ & 0.01 & 99 & 2 & 0.20 & 91 & 9 \\
\hline 8 & 4.0 & 0.50 & $20^{\circ} \mathrm{C}$ & 0.32 & 68 & 2 & 0.00 & 100 & 0 \\
\hline 9 & 4.0 & 0.50 & $50^{\circ} \mathrm{C}$ & 0.00 & 100 & 2 & 0.00 & 100 & $\mathbf{0}$ \\
\hline 10 & 4.0 & 1.00 & $20^{\circ} \mathrm{C}$ & 0.74 & 26 & 2 & 0.00 & 100 & 0 \\
\hline 11 & 4.0 & 1.00 & $50^{\circ} \mathrm{C}$ & 0.05 & 95 & 2 & 0.00 & 100 & 0 \\
\hline
\end{tabular}

NMR spectra are given on pages 156-161.

$\% \mathrm{D} \quad \%$ Aldehyde $\square$ \%enzoin

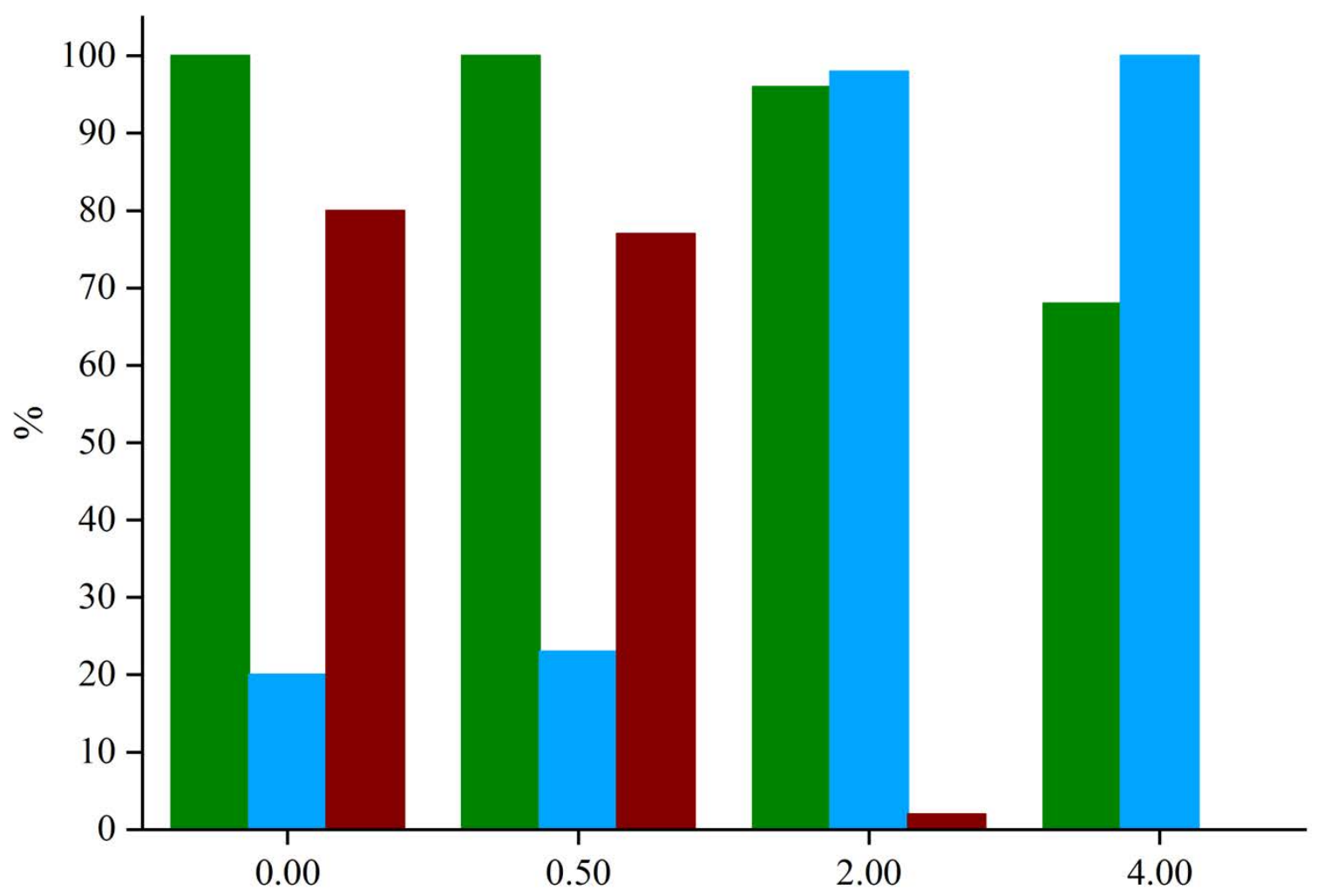

Phenyl boroxine (mol\%) 
Figure 4: Effect of phenyl boroxine (data used in the above plot was with $0.50 \mathrm{M}$ reaction concentration at $20^{\circ} \mathrm{C}$ )

\subsection{Effect of Different Aryl Boroxines on Deuteration of Aromatic Aldehydes}

These reactions were performed in chloroform- $d$ with different aryl boroxines according to the catalytic general procedure given in section 5 (page 20). Results are summarized as below.<smiles>COc1ccc(C=O)cc1</smiles>

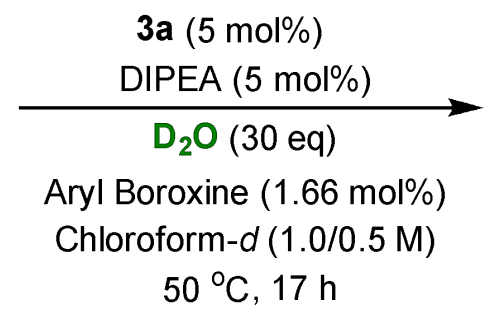<smiles>[2H]C(=O)c1ccc(OC)cc1</smiles>

Scheme 17: Effect of Different Aryl Boroxines on Deuteration of Aromatic Aldehydes

Table 4: Effect of Aryl Boroxines on Deuteration of Aromatic Aldehydes

\begin{tabular}{|c|c|c|c|c|}
\hline Entry & $\begin{array}{c}\text { Ph-Boroxine } \\
\text { (mol\%) }\end{array}$ & $\begin{array}{c}\text { Reaction Conc. } \\
(\mathrm{M})\end{array}$ & \%D (RM) & $\begin{array}{c}\% \\
\text { Aldehyde }\end{array}$ \\
\hline 1 & $4-\mathrm{CF}_{3}-\mathrm{Ph}$ & 1.0 & 21 & 100 \\
\hline 2 & $4-\mathrm{OMe}-\mathrm{Ph}$ & 1.0 & 20 & 100 \\
\hline 3 & $4-\mathrm{Me}-\mathrm{Ph}$ & 1.0 & 19 & 100 \\
\hline 4 & $4-\mathrm{CF}_{3}-\mathrm{Ph}$ & 0.5 & 57 & 100 \\
\hline 5 & $4-\mathrm{OMe}-\mathrm{Ph}$ & 0.5 & 56 & 100 \\
\hline 6 & $4-\mathrm{Me}-\mathrm{Ph}$ & 0.5 & 57 & 100 \\
\hline
\end{tabular}

NMR spectra are given on pages 162-167. 


\subsection{Substrate Scope with 2, 3 and 4-Substituted Benzaldehydes}

We have summarized below conditions for deuteration of 52 different aromatic aldehydes. All of the substrate give excellent deuterium incorporation (>95\%) and high reaction yield. Most of the substrates (40 examples) work under 3a (SIPr) catalyst reaction condition and some of the aldehydes require use of the pyrrolidine based triazolium catalysts $4 \mathbf{a}\left(\mathrm{TAC}_{\mathrm{Br}}\right)$ or $4 \mathbf{i}\left(T A C_{F}\right)$. Below each substrate we provide the reaction conditions and results summarized in a table. Reactions are performed in duplicates (for example, entry $1^{\prime}$ is the duplicate entry 1 ).

\section{Condition 1:}
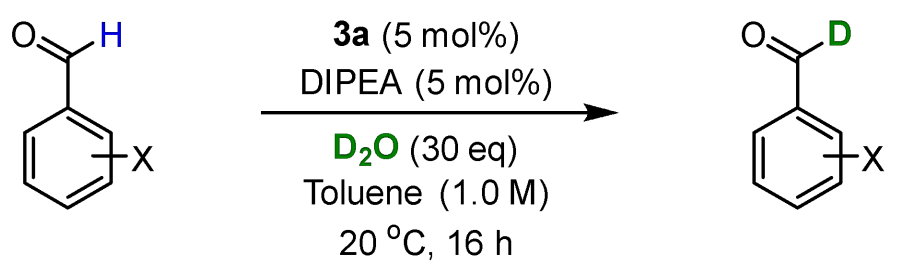

Scheme 18: Reaction condition 1

\section{2-Methylbenzaldehyde-d}<smiles>[2H]C(=O)c1ccccc1C</smiles>

3a (23.5 mg, $0.055 \mathrm{mmol})$, DIPEA $(10 \mu \mathrm{L}, 0.055 \mathrm{mmol}), \mathrm{D}_{2} \mathrm{O}(600 \mu \mathrm{L}, 33 \mathrm{mmol})$ and 2methylbenzaldehyde $(127 \mu \mathrm{L}, 1.1 \mathrm{mmol})$ in anhydrous toluene $(500 \mu \mathrm{L})$ were prepared according to the general procedure given in section 5 (page 20) and stirred at $20^{\circ} \mathrm{C}$. Crude residue was purified by flash-column chromatography using Hex:Et ${ }_{2} \mathrm{O}$ (97:3) combination to give colorless liquid. Spectral data for this product have been previously reported. ${ }^{15}$

Deuterium incorporation = 98\% (crude: 99\%, 98\%), Reaction yield = 92\% (123 mg). NMR spectra are given on pages $168-170$.

${ }^{1} \mathbf{H}$ NMR $\left(500 \mathrm{MHz}, \mathrm{CDCl}_{3}\right) \delta(\mathrm{ppm})=10.41(\mathrm{~s}, 0.02 \mathrm{H}), 7.95-7.92(\mathrm{dd}, J=5 \mathrm{~Hz}, 1 \mathrm{H}), 7.63-7.59(\mathrm{dt}, J=$ $5 \mathrm{~Hz}, 1 \mathrm{H}), 7.51-7.48(\mathrm{~m}, 1 \mathrm{H}), 7.41-7.39(\mathrm{~m}, 1 \mathrm{H}), 2.81(\mathrm{~s}, 3 \mathrm{H}) .{ }^{13} \mathrm{C} \mathrm{NMR}\left(125 \mathrm{MHz}, \mathrm{CDCl}_{3}\right) \delta(\mathrm{ppm})=$ $192.6(t, J=33 \mathrm{~Hz}), 140.8,134.2,133.8,132.1,131.9,126.5,19.7$

\begin{tabular}{|c|c|c|c|c|c|c|c|c|c|c|}
\hline \multirow[t]{2}{*}{ Entry } & \multirow{2}{*}{$\begin{array}{c}3 a \\
(\mathrm{~mol} \%)\end{array}$} & \multirow{2}{*}{$\begin{array}{l}\text { DIPEA } \\
\text { (mol\%) }\end{array}$} & \multirow{2}{*}{$\begin{array}{l}\mathrm{D}_{2} \mathrm{O} \\
\text { (eq. } \\
\text { ) }\end{array}$} & \multirow{2}{*}{$\begin{array}{c}\text { Ph- } \\
\text { Boroxine } \\
\text { (mol\%) }\end{array}$} & \multirow{2}{*}{$\begin{array}{l}\text { Reactio } \\
\text { n Conc. } \\
\text { (M) }\end{array}$} & \multirow{2}{*}{$\begin{array}{l}\text { Reactio } \\
\text { n Temp. }\end{array}$} & \multirow{2}{*}{$\begin{array}{c}\% \mathrm{D} \\
\text { (RM) }\end{array}$} & \multirow{2}{*}{$\begin{array}{c}\% \\
\text { Aldehyd } \\
\mathrm{e}\end{array}$} & \multicolumn{2}{|c|}{$\begin{array}{c}\text { After } \\
\text { Purification }\end{array}$} \\
\hline & & & & & & & & & $\% \mathrm{D}$ & \%Yield \\
\hline 1 & 5 & 5 & 30 & - & 1 & $20^{\circ} \mathrm{C}$ & 99 & 100 & 98 & 92 \\
\hline $1^{\prime}$ & 5 & 5 & 30 & - & 1 & $20^{\circ} \mathrm{C}$ & 98 & 100 & - & - \\
\hline
\end{tabular}




\section{2-Chlorobenzaldehyde-d}<smiles>[2H]C(=O)c1ccccc1Cl</smiles>

3a (23.5 mg, $0.055 \mathrm{mmol})$, DIPEA $(10 \mu \mathrm{L}, 0.055 \mathrm{mmol}), \mathrm{D}_{2} \mathrm{O}(600 \mu \mathrm{L}, 33 \mathrm{mmol})$ and 2chlorobenzaldehyde $(124 \mu \mathrm{L}, 1.1 \mathrm{mmol})$ in anhydrous toluene $(500 \mu \mathrm{L})$ were prepared according to the general procedure given in section 5 (page 20) and stirred at $20^{\circ} \mathrm{C}$. Crude residue was purified by flash-column chromatography using Hex: $\mathrm{Et}_{2} \mathrm{O}$ (98:2) combination to give colorless liquid. Spectral data for this product have been previously reported. ${ }^{15}$

Deuterium incorporation $=97 \%$ (crude: $99 \%, 98 \%)$, Reaction yield = 89\% (139 mg). NMR spectra are given on pages 171-173.

${ }^{1} \mathrm{H}$ NMR $\left(400 \mathrm{MHz}, \mathrm{CDCl}_{3}\right) \delta(\mathrm{ppm})=10.49(\mathrm{~s}, 0.03 \mathrm{H})$, 7.94-7.92 $(\mathrm{m}, 1 \mathrm{H})$, 7.55-7.51 $(\mathrm{m}, 1 \mathrm{H})$, 7.46$7.44(\mathrm{~m}, 1 \mathrm{H}), 7.40-7.37(\mathrm{~m}, 1 \mathrm{H}) .{ }^{13} \mathrm{C}$ NMR $\left(100 \mathrm{MHz}, \mathrm{CDCl}_{3}\right) \delta(\mathrm{ppm})=189.6(\mathrm{t}, J=22 \mathrm{~Hz}), 138.1$, $135.3,132.5,130.7,129.5,127.4$

\begin{tabular}{|c|c|c|c|c|c|c|c|c|c|c|}
\hline Entry & $\begin{array}{c}3 a \\
\text { (mol\%) }\end{array}$ & $\begin{array}{c}\text { DIPEA } \\
\text { (mol\%) }\end{array}$ & $\begin{array}{c}\mathrm{D}_{2} \mathrm{O} \\
\text { (eq. } \\
\text { (m) }\end{array}$ & $\begin{array}{c}\text { Boroxine } \\
\text { (mol\%) }\end{array}$ & $\begin{array}{c}\text { Reactio } \\
\mathrm{n} \text { Conc. } \\
\text { (M) }\end{array}$ & $\begin{array}{c}\text { Reactio } \\
\mathrm{n} \text { Temp. }\end{array}$ & $\begin{array}{c}\text { \%D } \\
(\mathrm{RM})\end{array}$ & $\begin{array}{c}\% \\
\text { Aldehyd } \\
\mathrm{e}\end{array}$ & \multicolumn{2}{|c|}{$\begin{array}{c}\text { After } \\
\text { Purification }\end{array}$} \\
\hline 1 & 5 & 5 & 30 & - & 1 & $20^{\circ} \mathrm{C}$ & 99 & 100 & 97 & $\%$ Yield \\
\hline $1^{\prime}$ & 5 & 5 & 30 & - & 1 & $20^{\circ} \mathrm{C}$ & 98 & 100 & - & - \\
\hline
\end{tabular}

\section{2-Bromobenzaldehyde-d}<smiles>[2H]C(=O)c1ccccc1Br</smiles>

3a (23.5 mg, $0.055 \mathrm{mmol})$, DIPEA $(10 \mu \mathrm{L}, 0.055 \mathrm{mmol}), \mathrm{D}_{2} \mathrm{O}(600 \mu \mathrm{L}, 33 \mathrm{mmol})$ and 2bromobenzaldehyde $(128 \mu \mathrm{L}, 1.1 \mathrm{mmol})$ in anhydrous toluene $(500 \mu \mathrm{L})$ were prepared according to the general procedure given in section 5 (page 20) and stirred at $20^{\circ} \mathrm{C}$. Crude residue was purified by flash-column chromatography using Hex:Et ${ }_{2} \mathrm{O}$ (98:2) combination to give colorless liquid. Spectral data for this product have been previously reported. ${ }^{15}$

Deuterium incorporation = 97\% (crude: 99\%, 98\%), Reaction yield = 92\% (188 mg). NMR spectra are given on pages $174-176$. 
${ }^{1} \mathrm{H}$ NMR $\left(500 \mathrm{MHz}, \mathrm{CDCl}_{3}\right) \delta(\mathrm{ppm})=10.37(\mathrm{~s}, 0.03 \mathrm{H})$, 7.93-7.91 (m, $\left.1 \mathrm{H}\right)$, 7.66-7.64 $(\mathrm{m}, 1 \mathrm{H})$, 7.477.42 (m, 2 H). ${ }^{13} \mathrm{C}$ NMR $\left(125 \mathrm{MHz}, \mathrm{CDCl}_{3}\right) \delta(\mathrm{ppm})=191.7$ (t, $\left.J=28 \mathrm{~Hz}\right), 135.5,134.0,133.6,130.0$, $128.1,127.3$

\begin{tabular}{|c|c|c|c|c|c|c|c|c|c|c|}
\hline Entry & $\begin{array}{c}\text { 3a } \\
\text { (mol\%) }\end{array}$ & $\begin{array}{c}\text { DIPEA } \\
\text { (mol\%) }\end{array}$ & $\begin{array}{c}\mathrm{D}_{2} \mathrm{O} \\
\text { (eq. }\end{array}$ & $\begin{array}{c}\text { Ph- } \\
\text { Boroxine }\end{array}$ & $\begin{array}{c}\text { Reactio } \\
\text { (mol\%) }\end{array}$ & $\begin{array}{c}\text { Ronc. } \\
\text { (M) }\end{array}$ & $\begin{array}{c}\text { Reactio } \\
\text { n Temp. }\end{array}$ & $\begin{array}{c}\text { \%D } \\
\text { (RM) }\end{array}$ & $\begin{array}{c}\text { \% } \\
\text { Aldehyd } \\
\text { e }\end{array}$ & \multicolumn{2}{|c|}{$\begin{array}{c}\text { After } \\
\text { Purification }\end{array}$} \\
\hline 1 & 5 & 5 & 30 & - & 1 & $20^{\circ} \mathrm{C}$ & 99 & 100 & 97 & 92 \\
\hline $1^{\prime}$ & 5 & 5 & 30 & - & 1 & $20^{\circ} \mathrm{C}$ & 98 & 100 & - & - \\
\hline
\end{tabular}

\section{3-Methoxybenzaldehyde-d}<smiles>[2H]C(=O)c1cccc(OC)c1</smiles>

3a (23.5 mg, $0.055 \mathrm{mmol})$, DIPEA $(10 \mu \mathrm{L}, 0.055 \mathrm{mmol}), \mathrm{D}_{2} \mathrm{O}(600 \mu \mathrm{L}, 33 \mathrm{mmol})$ and 3methoxybenzaldehyde (134 $\mu \mathrm{L}, 1.1 \mathrm{mmol})$ in anhydrous toluene $(500 \mu \mathrm{L})$ were prepared according to the general procedure given in section 5 (page 20) and stirred at $20^{\circ} \mathrm{C}$. Crude residue was purified by flash-column chromatography using Hex:Et ${ }_{2} \mathrm{O}$ (93:7) combination to give colorless liquid. Spectral data for this product have been previously reported. ${ }^{15}$

Deuterium incorporation = 97\% (crude: 99\%, 98\%), Reaction yield = 88\% (133 mg). NMR spectra are given on pages 177-179.

${ }^{1} \mathrm{H}$ NMR $\left(400 \mathrm{MHz}, \mathrm{CDCl}_{3}\right) \delta(\mathrm{ppm})=9.97(\mathrm{~s}, 0.03 \mathrm{H}), 7.46-7.42(\mathrm{~m}, 2 \mathrm{H}), 7.38-7.39(\mathrm{~m}, 1 \mathrm{H})$, 7.18$7.16(\mathrm{~m}, 1 \mathrm{H}), 3.86(\mathrm{~s}, 3 \mathrm{H}) .{ }^{13} \mathrm{C} \mathrm{NMR}\left(100 \mathrm{MHz}, \mathrm{CDCl}_{3}\right) \delta(\mathrm{ppm})=191.8(\mathrm{t}, \mathrm{J}=27 \mathrm{~Hz}), 160.2,137.8(\mathrm{t}$, $J=4 \mathrm{~Hz}), 130.1,123.5,121.6,112.0,55.5$

\begin{tabular}{|c|c|c|c|c|c|c|c|c|c|c|}
\hline \multirow{2}{*}{ Entry } & \multirow{2}{*}{$\begin{array}{c}3 \mathbf{a} \\
(\mathrm{mol} \%)\end{array}$} & \multirow{2}{*}{$\begin{array}{l}\text { DIPEA } \\
\text { (mol\%) }\end{array}$} & \multirow{2}{*}{$\begin{array}{c}\mathrm{D}_{2} \mathrm{O} \\
\text { (eq. } \\
\text { ) }\end{array}$} & \multirow{2}{*}{$\begin{array}{c}\text { Ph- } \\
\text { Boroxine } \\
\text { (mol\%) }\end{array}$} & \multirow{2}{*}{$\begin{array}{l}\text { Reactio } \\
\text { n Conc. } \\
\text { (M) }\end{array}$} & \multirow{2}{*}{$\begin{array}{l}\text { Reactio } \\
\text { n Temp. }\end{array}$} & \multirow{2}{*}{$\begin{array}{c}\% \mathrm{D} \\
\text { (RM) }\end{array}$} & \multirow{2}{*}{$\begin{array}{c}\% \\
\text { Aldehyd } \\
\mathrm{e}\end{array}$} & \multicolumn{2}{|c|}{$\begin{array}{c}\text { After } \\
\text { Purification }\end{array}$} \\
\hline & & & & & & & & & $\% \mathrm{D}$ & \%Yield \\
\hline 1 & 5 & 5 & 30 & - & 1 & $20^{\circ} \mathrm{C}$ & 99 & 100 & 97 & 88 \\
\hline $1^{\prime}$ & 5 & 5 & 30 & - & 1 & $20^{\circ} \mathrm{C}$ & 98 & 100 & - & - \\
\hline
\end{tabular}




\section{4-Methoxybenzaldehyde-d}<smiles>[2H]C(=O)c1ccc(OC)cc1</smiles>

3a (23.5 mg, $0.055 \mathrm{mmol})$, DIPEA $(10 \mu \mathrm{L}, 0.055 \mathrm{mmol}), \mathrm{D}_{2} \mathrm{O}(600 \mu \mathrm{L}, 33 \mathrm{mmol})$ and 4methoxybenzaldehyde (134 $\mu \mathrm{L}, 1.1 \mathrm{mmol})$ in anhydrous toluene $(500 \mu \mathrm{L})$ were prepared according to the general procedure given in section 5 (page 20) and stirred at $20^{\circ} \mathrm{C}$. Crude residue was purified by flash-column chromatography using $\mathrm{Hex}_{\mathrm{Et}} \mathrm{O}$ (93:7) combination to give colorless liquid. Spectral data for this product have been previously reported. ${ }^{15}$

Deuterium incorporation $=97 \%$ (crude: $98 \%, 98 \%)$, Reaction yield $=87 \%$ (131 mg). NMR spectra are given on pages $180-182$.

${ }^{1} \mathrm{H}$ NMR $\left(400 \mathrm{MHz}, \mathrm{CDCl}_{3}\right) \delta(\mathrm{ppm})=9.88(\mathrm{~s}, 0.03 \mathrm{H}), 7.85-7.82(\mathrm{~m}, 2 \mathrm{H}), 7.02-6.98(\mathrm{~m}, 2 \mathrm{H}), 3.88(\mathrm{~s}$, $3 \mathrm{H}) \cdot{ }^{13} \mathrm{C} \mathrm{NMR}\left(100 \mathrm{MHz}, \mathrm{CDCl}_{3}\right) \delta(\mathrm{ppm})=190.6(\mathrm{t}, J=26 \mathrm{~Hz}), 164.8,132.1,130.0(\mathrm{t}, \mathrm{J}=3 \mathrm{~Hz}), 114.5$, 55.7

\begin{tabular}{|c|c|c|c|c|c|c|c|c|c|c|}
\hline Entry & $\begin{array}{c}3 a \\
\text { (mol\%) }\end{array}$ & $\begin{array}{c}\text { DIPEA } \\
\text { (mol\%) }\end{array}$ & $\begin{array}{c}\mathrm{D}_{2} \mathrm{O} \\
\text { (eq. } \\
\text { ) }\end{array}$ & $\begin{array}{c}\text { Ph- } \\
\text { Boroxine } \\
\text { (mol\%) }\end{array}$ & $\begin{array}{c}\text { Reactio } \\
\mathrm{n} \text { Conc. } \\
\text { (M) }\end{array}$ & $\begin{array}{c}\text { Reactio } \\
\text { n Temp. }\end{array}$ & $\begin{array}{c}\% \mathrm{D} \\
\text { (RM) }\end{array}$ & $\begin{array}{c}\% \\
\text { Aldehyd } \\
\mathrm{e}\end{array}$ & \multicolumn{2}{|c|}{$\begin{array}{c}\text { After } \\
\text { Purification }\end{array}$} \\
\hline 1 & 5 & 5 & 30 & - & 1 & $20^{\circ} \mathrm{C}$ & 98 & 100 & 97 & \%Yield \\
\hline $1^{\prime}$ & 5 & 5 & 30 & - & 1 & $20^{\circ} \mathrm{C}$ & 98 & 100 & - & - \\
\hline
\end{tabular}

\section{Benzo[d][1,3]dioxole-4-carbaldehyde-d}<smiles>[2H]C(=O)c1cccc2c1OCO2</smiles>

3a (23.5 mg, $0.055 \mathrm{mmol})$, DIPEA $(10 \mu \mathrm{L}, 0.055 \mathrm{mmol}), \mathrm{D}_{2} \mathrm{O}(600 \mu \mathrm{L}, 33 \mathrm{mmol})$ and benzo[d][1,3]dioxole-4-carbaldehyde $(165 \mathrm{mg}, 1.1 \mathrm{mmol})$ in anhydrous toluene $(500 \mu \mathrm{L})$ were prepared according to the general procedure given in section 5 (page 20) and stirred at $20^{\circ} \mathrm{C}$. Crude residue was purified by flash-column chromatography using Hex:Et ${ }_{2} \mathrm{O}(83: 17)$ combination to give colorless solid. Spectral data for this product have been previously reported. ${ }^{15}$

Deuterium incorporation $=98 \%$ (crude: $99 \%, 98 \%)$, Reaction yield $=98 \%$ (163 mg). NMR spectra are given on pages 183-185. 
${ }^{1} \mathrm{H}$ NMR $\left(500 \mathrm{MHz}, \mathrm{CDCl}_{3}\right) \delta(\mathrm{ppm})=10.11(\mathrm{~s}, 0.02 \mathrm{H})$, 7.28-7.26 (m, $\left.1 \mathrm{H}\right)$, 7.02-7.01 $(\mathrm{m}, 1 \mathrm{H})$, 6.94$6.91(\mathrm{~m}, 1 \mathrm{H}), 6.12(\mathrm{~s}, 2 \mathrm{H}) .{ }^{13} \mathrm{C}$ NMR $\left(125 \mathrm{MHz}, \mathrm{CDCl}_{3}\right) \delta(\mathrm{ppm})=187.9(\mathrm{t}, J=28 \mathrm{~Hz}), 149.4,149.0$, $121.9,121.3,119.5,113.6,102.7$

\begin{tabular}{|c|c|c|c|c|c|c|c|c|c|c|}
\hline Entry & $\begin{array}{c}\text { 3a } \\
\text { (mol\%) }\end{array}$ & $\begin{array}{c}\text { DIPEA } \\
\text { (mol\%) }\end{array}$ & $\begin{array}{c}\mathrm{D}_{2} \mathrm{O} \\
\text { (eq. }\end{array}$ & $\begin{array}{c}\text { Ph- } \\
\text { Boroxine } \\
\text { (mol\%) }\end{array}$ & $\begin{array}{c}\text { Reactio } \\
\mathrm{n} \text { Conc. } \\
\text { (M) }\end{array}$ & $\begin{array}{c}\text { Reactio } \\
\text { n Temp. }\end{array}$ & $\begin{array}{c}\text { \%D } \\
\text { (RM) }\end{array}$ & $\begin{array}{c}\text { \% } \\
\text { Aldehyd } \\
\text { e }\end{array}$ & \multicolumn{2}{|c|}{$\begin{array}{c}\text { After } \\
\text { Purification }\end{array}$} \\
\hline 1 & 5 & 5 & 30 & - & 1 & $20^{\circ} \mathrm{C}$ & 99 & 100 & 98 & \%Yield \\
\hline $1^{\prime}$ & 5 & 5 & 30 & - & 1 & $20^{\circ} \mathrm{C}$ & 98 & 100 & - & - \\
\hline
\end{tabular}

\section{1-Naphthaldehyde-d}<smiles>[2H]C(=O)c1cccc2ccccc12</smiles>

3a (23.5 mg, $0.055 \mathrm{mmol})$, DIPEA (10 $\mu \mathrm{L}, 0.055 \mathrm{mmol}), \mathrm{D}_{2} \mathrm{O}(600 \mu \mathrm{L}, 33 \mathrm{mmol})$ and 1-naphthaldehyde (149 $\mu \mathrm{L}, 1.1 \mathrm{mmol})$ in anhydrous toluene $(500 \mu \mathrm{L})$ were prepared according to the general procedure given in section 5 (page 20) and stirred at $20{ }^{\circ} \mathrm{C}$. Crude residue was purified by flashcolumn chromatography using $\mathrm{Hex}_{\mathrm{Et}} \mathrm{O}$ (93:7) combination to give colorless liquid. Spectral data for this product have been previously reported. ${ }^{15}$

Deuterium incorporation = 99\% (crude: 99\%, 98\%), Reaction yield = 98\% (169 mg). NMR spectra are given on pages $186-188$.

${ }^{1} \mathrm{H}$ NMR $\left(500 \mathrm{MHz}, \mathrm{CDCl}_{3}\right) \delta(\mathrm{ppm})=10.40(\mathrm{~s}, 0.01 \mathrm{H}), 9.27(\mathrm{~d}, J=5 \mathrm{~Hz}, 1 \mathrm{H}), 8.10(\mathrm{~d}, J=10 \mathrm{~Hz}, 1 \mathrm{H})$, $7.99(\mathrm{dd}, J=10 \mathrm{~Hz}, 1 \mathrm{H}), 7.93(\mathrm{dd}, J=5 \mathrm{~Hz}, 1 \mathrm{H}), 7.71-7.68(\mathrm{~m}, 1 \mathrm{H}), 7.64-7.58\left(\mathrm{~m}, 2 \mathrm{H} .{ }^{13} \mathrm{C}\right.$ NMR (125 $\left.\mathrm{MHz}, \mathrm{CDCl}_{3}\right) \delta(\mathrm{ppm})=193.3(\mathrm{t}, J=28 \mathrm{~Hz}), 136.7,135.4,133.9,131.5,130.7,129.2,128.6,127.1$, 125.0

\begin{tabular}{|c|c|c|c|c|c|c|c|c|c|c|}
\hline Entry & $\begin{array}{c}3 a \\
\text { (mol\%) }\end{array}$ & $\begin{array}{c}\text { DIPEA } \\
\text { (mol\%) }\end{array}$ & $\begin{array}{c}\mathrm{D}_{2} \mathrm{O} \\
\text { (eq. } \\
\text { ) }\end{array}$ & $\begin{array}{c}\text { Boroxine } \\
\text { (mol\%) }\end{array}$ & $\begin{array}{c}\text { Reactio } \\
\text { n Conc. } \\
\text { (M) }\end{array}$ & $\begin{array}{c}\text { Reactio } \\
\text { n Temp. }\end{array}$ & $\begin{array}{c}\% \mathrm{D} \\
\text { (RM) }\end{array}$ & $\begin{array}{c}\% \\
\text { Aldehyd } \\
\text { e }\end{array}$ & \multicolumn{2}{|c|}{$\begin{array}{c}\text { After } \\
\text { Purification }\end{array}$} \\
\hline 1 & 5 & 5 & 30 & - & 1 & $20{ }^{\circ} \mathrm{C}$ & 99 & 100 & 99 & 98 \\
\hline $1^{\prime}$ & 5 & 5 & 30 & - & 1 & $20^{\circ} \mathrm{C}$ & 98 & 100 & - & - \\
\hline
\end{tabular}




\section{2-(Trifluoromethoxy)benzaldehyde-d}<smiles>[2H]C(=O)c1ccccc1OC(F)(F)F</smiles>

3a (23.5 mg, $0.055 \mathrm{mmol})$, DIPEA $(10 \mu \mathrm{L}, 0.055 \mathrm{mmol}), \mathrm{D}_{2} \mathrm{O}(600 \mu \mathrm{L}, 33 \mathrm{mmol})$ and 2(trifluoromethoxy)benzaldehyde $(209 \mu \mathrm{L}, 1.1 \mathrm{mmol}$ ) in anhydrous toluene $(500 \mu \mathrm{L})$ were prepared according to the general procedure given in section 5 (page 20) and stirred at $20^{\circ} \mathrm{C}$. Crude residue was purified by flash-column chromatography using Hex: $\mathrm{Et}_{2} \mathrm{O}(93: 7)$ combination to give colorless liquid.

Deuterium incorporation $=98 \%$ (crude: 99\%, 98\%), Reaction yield = 98\% (206 mg). NMR spectra are given on pages 189-191.

${ }^{1} \mathrm{H}$ NMR $\left(500 \mathrm{MHz}, \mathrm{CDCl}_{3}\right) \delta(\mathrm{ppm})=10.38(\mathrm{~s}, 0.02 \mathrm{H}), 7.97(\mathrm{dd}, J=5 \mathrm{~Hz}, 1 \mathrm{H})$, 7.68-7.64 (m, $\left.1 \mathrm{H}\right)$, 7.45-7.42 (m, $1 \mathrm{H}), 7.37-7.35(\mathrm{~m}, 1 \mathrm{H}) .{ }^{13} \mathrm{C} \mathrm{NMR}\left(125 \mathrm{MHz}, \mathrm{CDCl}_{3}\right) \delta(\mathrm{ppm})=187.4(\mathrm{t}, J=28 \mathrm{~Hz})$, $151.0,135.8,129.1,128.5,127.5,121.5,120.5(q, J=259 \mathrm{~Hz}) .{ }^{19} \mathrm{~F}$ NMR $\left(376 \mathrm{MHz}, \mathrm{CDCl}_{3}\right) \delta(\mathrm{ppm})=$ -57.70 (s, 3 F). HRMS(ESI) calcd for $\mathrm{C}_{9} \mathrm{H}_{4} \mathrm{D}_{2} \mathrm{O}^{+}(\mathrm{M}+\mathrm{H})^{+}: 192.0377$, Found: 192.0373

\begin{tabular}{|c|c|c|c|c|c|c|c|c|c|c|}
\hline \multirow[t]{2}{*}{ Entry } & \multirow{2}{*}{$\begin{array}{c}3 a \\
(\mathrm{~mol} \%)\end{array}$} & \multirow{2}{*}{$\begin{array}{l}\text { DIPEA } \\
\text { (mol\%) }\end{array}$} & \multirow{2}{*}{$\begin{array}{l}\mathrm{D}_{2} \mathrm{O} \\
\text { (eq. } \\
\text { ) }\end{array}$} & \multirow{2}{*}{$\begin{array}{c}\text { Ph- } \\
\text { Boroxine } \\
\text { (mol\%) }\end{array}$} & \multirow{2}{*}{$\begin{array}{l}\text { Reactio } \\
\text { n Conc. } \\
\text { (M) }\end{array}$} & \multirow{2}{*}{$\begin{array}{l}\text { Reactio } \\
\text { n Temp. }\end{array}$} & \multirow{2}{*}{$\begin{array}{c}\% \mathrm{D} \\
\text { (RM) }\end{array}$} & \multirow{2}{*}{$\begin{array}{c}\% \\
\text { Aldehyd } \\
\text { e }\end{array}$} & \multicolumn{2}{|c|}{$\begin{array}{c}\text { After } \\
\text { Purification }\end{array}$} \\
\hline & & & & & & & & & $\% \mathrm{D}$ & \%Yield \\
\hline 1 & 5 & 5 & 30 & - & 1 & $20^{\circ} \mathrm{C}$ & 99 & 100 & 98 & 98 \\
\hline $1^{\prime}$ & 5 & 5 & 30 & - & 1 & $20^{\circ} \mathrm{C}$ & 98 & 100 & - & - \\
\hline
\end{tabular}

\section{2-Ethynylbenzaldehyde-d}<smiles>[2H]C#Cc1ccccc1C(=O)O</smiles>

3a (23.5 mg, $0.055 \mathrm{mmol})$, DIPEA $(10 \mu \mathrm{L}, 0.055 \mathrm{mmol}), \mathrm{D}_{2} \mathrm{O}(600 \mu \mathrm{L}, 33 \mathrm{mmol})$ and 2ethynylbenzaldehyde (143 mg, $1.1 \mathrm{mmol})$ in anhydrous toluene $(500 \mu \mathrm{L})$ were prepared according to the general procedure given in section 5 (page 20) and stirred at $20^{\circ} \mathrm{C}$. Crude residue was purified by flash-column chromatography using Hex: $\mathrm{Et}_{2} \mathrm{O}$ (98:2) combination to give colorless solid.

Deuterium incorporation $=95 \%$ (crude: $95 \%, 94 \%)$, at ethynyl group deuterium incorporation $=$ 91\%, Reaction yield = 95\% (138 mg). NMR spectra are given on pages 192-194. 
${ }^{1} \mathbf{H}$ NMR $\left(500 \mathrm{MHz}, \mathrm{CDCl}_{3}\right) \delta(\mathrm{ppm})=10.53(\mathrm{~s}, 0.05 \mathrm{H})$, 7.94-7.92 (m, $\left.1 \mathrm{H}\right)$, 7.62-7.60 (m, $\left.1 \mathrm{H}\right)$, 7.58$7.54(\mathrm{~m}, 1 \mathrm{H}), 7.49-7.46(\mathrm{~m}, 1 \mathrm{H}), 3.36(\mathrm{~s}, 0.09 \mathrm{H}) .{ }^{13} \mathrm{C} \mathrm{NMR}\left(125 \mathrm{MHz}, \mathrm{CDCl}_{3}\right) \delta(\mathrm{ppm})=191.2(\mathrm{t}, J=$ $28 \mathrm{~Hz}$ ), 136.6, 134.0, 133.8, 129.3, 127.4, 125.6, 84.4, 78.9 ( $\mathrm{t}, J=8 \mathrm{~Hz}$ ). HRMS(ESI) calcd for $\mathrm{C}_{9} \mathrm{H}_{4} \mathrm{D}_{2} \mathrm{O}^{+}$ $(\mathrm{M}+\mathrm{H})^{+}:$133.0617, Found: 133.0612

\begin{tabular}{|c|c|c|c|c|c|c|c|c|c|c|}
\hline \multirow[t]{2}{*}{ Entry } & \multirow{2}{*}{$\begin{array}{c}3 a \\
(\mathrm{~mol} \%)\end{array}$} & \multirow{2}{*}{$\begin{array}{l}\text { DIPEA } \\
\text { (mol\%) }\end{array}$} & \multirow{2}{*}{$\begin{array}{l}\mathrm{D}_{2} \mathrm{O} \\
\text { (eq. } \\
\text { ) }\end{array}$} & \multirow{2}{*}{$\begin{array}{c}\text { Ph- } \\
\text { Boroxine } \\
\text { (mol\%) }\end{array}$} & \multirow{2}{*}{$\begin{array}{l}\text { Reactio } \\
\text { n Conc. } \\
\text { (M) }\end{array}$} & \multirow{2}{*}{$\begin{array}{l}\text { Reactio } \\
\text { n Temp. }\end{array}$} & \multirow{2}{*}{$\begin{array}{l}\% \mathrm{D} \\
\text { (RM) }\end{array}$} & \multirow{2}{*}{$\begin{array}{c}\% \\
\text { Aldehyd } \\
\mathrm{e}\end{array}$} & \multicolumn{2}{|c|}{$\begin{array}{c}\text { After } \\
\text { Purification }\end{array}$} \\
\hline & & & & & & & & & $\% \mathrm{D}$ & \%Yield \\
\hline 1 & 5 & 5 & 30 & - & 1 & $20^{\circ} \mathrm{C}$ & 95 & 100 & 95 & 95 \\
\hline $1^{\prime}$ & 5 & 5 & 30 & - & 1 & $20^{\circ} \mathrm{C}$ & 94 & 100 & - & - \\
\hline
\end{tabular}




\section{Condition 2:}
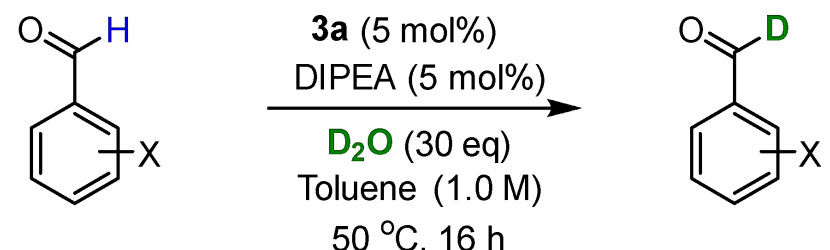

$50{ }^{\circ} \mathrm{C}, 16 \mathrm{~h}$

Scheme 19: Reaction condition 2

\section{2-Hydroxybenzaldehyde-d}<smiles>[2H]C(=O)c1ccccc1O</smiles>

3a (23.5 mg, $0.055 \mathrm{mmol})$, DIPEA $(10 \mu \mathrm{L}, 0.055 \mathrm{mmol}), \mathrm{D}_{2} \mathrm{O}(600 \mu \mathrm{L}, 33 \mathrm{mmol})$ and 2hydroxybenzaldehyde (117 $\mu \mathrm{L}, 1.1 \mathrm{mmol})$ in anhydrous toluene $(500 \mu \mathrm{L})$ were prepared according to the general procedure given in section 5 (page 20) and stirred at $50 \stackrel{\circ}{ }$. Crude residue was purified by flash-column chromatography using $\mathrm{Hex}_{\mathrm{Et}} \mathrm{O}$ (93:7) combination to give colorless liquid. Spectral data for this product have been previously reported. ${ }^{15}$

Deuterium incorporation $=96 \%$ (crude: 97\%, 97\%), Reaction yield $=84 \%$ (114 mg). NMR spectra are given on pages 195-198.

${ }^{1} \mathrm{H}$ NMR $\left(500 \mathrm{MHz}, \mathrm{CDCl}_{3}\right) \delta(\mathrm{ppm})=11.04(\mathrm{~s}, 0.04 \mathrm{H}), 9.90(\mathrm{~s}, 1 \mathrm{H})$, 7.57-7.51 (m, $\left.2 \mathrm{H}\right)$, 7.04-6.98 $(\mathrm{m}$ , $2 \mathrm{H}) .{ }^{13} \mathrm{C}$ NMR $\left(125 \mathrm{MHz}, \mathrm{CDCl}_{3}\right) \delta(\mathrm{ppm})=196.4(\mathrm{t}, J=28 \mathrm{~Hz}), 161.8,137.2,133.8,120.7,120.0$, 117.8 .

\begin{tabular}{|c|c|c|c|c|c|c|c|c|c|c|}
\hline \multirow{2}{*}{ Entry } & \multirow{2}{*}{$\begin{array}{c}3 a \\
(\mathrm{~mol} \%)\end{array}$} & \multirow{2}{*}{$\begin{array}{l}\text { DIPEA } \\
\text { (mol\%) }\end{array}$} & \multirow{2}{*}{$\begin{array}{l}\mathrm{D}_{2} \mathrm{O} \\
\text { (eq. } \\
\text { ) }\end{array}$} & \multirow{2}{*}{$\begin{array}{c}\text { Ph- } \\
\text { Boroxine } \\
\text { (mol\%) }\end{array}$} & \multirow{2}{*}{$\begin{array}{l}\text { Reactio } \\
\text { n Conc. } \\
\text { (M) }\end{array}$} & \multirow{2}{*}{$\begin{array}{l}\text { Reactio } \\
\text { n Temp. }\end{array}$} & \multirow{2}{*}{$\begin{array}{c}\% \mathrm{D} \\
\text { (RM) }\end{array}$} & \multirow{2}{*}{$\begin{array}{c}\% \\
\text { Aldehyd } \\
\mathrm{e}\end{array}$} & \multicolumn{2}{|c|}{$\begin{array}{c}\text { After } \\
\text { Purification }\end{array}$} \\
\hline & & & & & & & & & $\% \mathrm{D}$ & \%Yield \\
\hline 1 & 5 & 5 & 30 & - & 1 & $20^{\circ} \mathrm{C}$ & 59 & 100 & - & - \\
\hline $1^{\prime}$ & 5 & 5 & 30 & - & 1 & $20^{\circ} \mathrm{C}$ & 59 & 100 & - & - \\
\hline 2 & 5 & 5 & 30 & - & 1 & $50 \stackrel{\circ}{ }$ & 97 & 100 & 96 & 84 \\
\hline $2^{\prime}$ & 5 & 5 & 30 & - & 1 & $50 \stackrel{\circ}{ }$ & 97 & 100 & - & - \\
\hline
\end{tabular}

\section{3-Methylbenzaldehyde-d}<smiles>[2H]C(=O)c1cccc(C)c1</smiles> 
3a (23.5 mg, $0.055 \mathrm{mmol})$, DIPEA $(10 \mu \mathrm{L}, 0.055 \mathrm{mmol}), \mathrm{D}_{2} \mathrm{O}(600 \mu \mathrm{L}, 33 \mathrm{mmol})$ and 3methylbenzaldehyde $(130 \mu \mathrm{L}, 1.1 \mathrm{mmol})$ in anhydrous toluene $(500 \mu \mathrm{L})$ were prepared according to the general procedure given in section 5 (page 20) and stirred at 50 ㅇ. C. Crude residue was purified by flash-column chromatography using Hex: $\mathrm{Et}_{2} \mathrm{O}$ (97:3) combination to give colorless liquid. Spectral data for this product have been previously reported. ${ }^{15}$

Deuterium incorporation $=96 \%$ (crude: $98 \%, 98 \%)$, Reaction yield $=81 \%$ (108 mg). NMR spectra are given on pages 199-201.

${ }^{1} \mathbf{H}$ NMR $\left(400 \mathrm{MHz}, \mathrm{CDCl}_{3}\right) \delta(\mathrm{ppm})=9.99(\mathrm{~s}, 0.04 \mathrm{H}), 7.70-7.68(\mathrm{~m}, 2 \mathrm{H}), 7.46-7.40(\mathrm{~m}, 2 \mathrm{H}), 2.44(\mathrm{~s}$, $3 \mathrm{H}) .{ }^{13} \mathrm{C}$ NMR $\left(100 \mathrm{MHz}, \mathrm{CDCl}_{3}\right) \delta(\mathrm{ppm})=192.4(\mathrm{t}, J=21 \mathrm{~Hz}), 139.1,136.5,135.4,130.1,129.0$, $127.4,21.3$

\begin{tabular}{|c|c|c|c|c|c|c|c|c|c|c|}
\hline \multirow[t]{2}{*}{ Entry } & \multirow{2}{*}{$\begin{array}{c}3 a \\
(\mathrm{~mol} \%)\end{array}$} & \multirow{2}{*}{$\begin{array}{l}\text { DIPEA } \\
\text { (mol\%) }\end{array}$} & \multirow{2}{*}{$\begin{array}{l}\mathrm{D}_{2} \mathrm{O} \\
\text { (eq. } \\
\text { ) }\end{array}$} & \multirow{2}{*}{$\begin{array}{c}\text { Ph- } \\
\text { Boroxine } \\
\text { (mol\%) }\end{array}$} & \multirow{2}{*}{$\begin{array}{l}\text { Reactio } \\
\text { n Conc. } \\
\text { (M) }\end{array}$} & \multirow{2}{*}{$\begin{array}{l}\text { Reactio } \\
\text { n Temp. }\end{array}$} & \multirow{2}{*}{$\begin{array}{c}\% \mathrm{D} \\
\text { (RM) }\end{array}$} & \multirow{2}{*}{$\begin{array}{c}\% \\
\text { Aldehyd } \\
\text { e }\end{array}$} & \multicolumn{2}{|c|}{$\begin{array}{c}\text { After } \\
\text { Purification }\end{array}$} \\
\hline & & & & & & & & & $\% \mathrm{D}$ & \%Yield \\
\hline 1 & 5 & 5 & 30 & - & 1 & $50 \stackrel{\circ}{C}$ & 98 & 100 & 96 & 81 \\
\hline $1^{\prime}$ & 5 & 5 & 30 & - & 1 & $50 \cong \mathrm{C}$ & 98 & 100 & - & - \\
\hline
\end{tabular}

\section{4-(Diethoxymethyl)benzaldehyde-d}<smiles>[2H]C(=O)c1ccc(C(OCC)OCC)cc1</smiles>

3a (23.5 mg, $0.055 \mathrm{mmol})$, DIPEA $(10 \mu \mathrm{L}, 0.055 \mathrm{mmol}), \mathrm{D}_{2} \mathrm{O}(600 \mu \mathrm{L}, 33 \mathrm{mmol})$ and 4(diethoxymethyl)benzaldehyde $(219 \mu \mathrm{L}, 1.1 \mathrm{mmol})$ in anhydrous toluene $(500 \mu \mathrm{L})$ were prepared according to the general procedure given in section 5 (page 20) and stirred at 50 ㄷ. Crude residue was purified by flash-column chromatography using Hex:Et ${ }_{2} \mathrm{O}(93: 7)$ combination to give colorless liquid.

Deuterium incorporation $=98 \%$ (crude: $98 \%, 98 \%)$, Reaction yield = 85\% (196 mg). NMR spectra are given on pages 202-204.

${ }^{1} \mathrm{H}$ NMR $\left(400 \mathrm{MHz}, \mathrm{CDCl}_{3}\right) \delta(\mathrm{ppm})=10.02(\mathrm{~s}, 0.02 \mathrm{H}), 7.88(\mathrm{~d}, J=8 \mathrm{~Hz}, 2 \mathrm{H}), 7.65(\mathrm{~d}, J=8 \mathrm{~Hz}, 2 \mathrm{H})$, $5.56(\mathrm{~s}, 1 \mathrm{H}), 3.65-3.52(\mathrm{~m}, 4 \mathrm{H}), 1.25(\mathrm{t}, J=8 \mathrm{~Hz}, 6 \mathrm{H}) .{ }^{13} \mathrm{C} \mathrm{NMR}\left(100 \mathrm{MHz}, \mathrm{CDCl}_{3}\right) \delta(\mathrm{ppm})=191.9(\mathrm{t}$, $J=27 \mathrm{~Hz}), 145.8,136.4(\mathrm{t}, J=4 \mathrm{~Hz}), 129.8,127.5,100.9,61.4,15.3$. HRMS(ESI) calcd for $\mathrm{C}_{12} \mathrm{H}_{15} \mathrm{DO}_{3}{ }^{+}$ $(\mathrm{M}+\mathrm{Na})^{+}:$232.1054, Found: 232.1052 


\begin{tabular}{|c|c|c|c|c|c|c|c|c|c|c|}
\hline \multirow{2}{*}{ Entry } & \multirow{2}{*}{$\begin{array}{c}3 a \\
(\mathrm{~mol} \%)\end{array}$} & \multirow{2}{*}{$\begin{array}{l}\text { DIPEA } \\
\text { (mol\%) }\end{array}$} & \multirow{2}{*}{$\begin{array}{c}\mathrm{D}_{2} \mathrm{O} \\
\text { (eq. } \\
\text { ) }\end{array}$} & \multirow{2}{*}{$\begin{array}{c}\text { Ph- } \\
\text { Boroxine } \\
\text { (mol\%) }\end{array}$} & \multirow{2}{*}{$\begin{array}{l}\text { Reactio } \\
\text { n Conc. } \\
\text { (M) }\end{array}$} & \multirow{2}{*}{$\begin{array}{l}\text { Reactio } \\
\text { n Temp. }\end{array}$} & \multirow{2}{*}{$\begin{array}{c}\% \mathrm{D} \\
\text { (RM) }\end{array}$} & \multirow{2}{*}{$\begin{array}{c}\% \\
\text { Aldehyd } \\
\text { e }\end{array}$} & \multicolumn{2}{|c|}{$\begin{array}{c}\text { After } \\
\text { Purification }\end{array}$} \\
\hline & & & & & & & & & $\% \mathrm{D}$ & \%Yield \\
\hline 1 & 5 & 5 & 30 & - & 1 & $50 \stackrel{\circ}{ }$ & 98 & 100 & 98 & 85 \\
\hline $1^{\prime}$ & 5 & 5 & 30 & - & 1 & $50 \stackrel{\circ}{ }$ & 98 & 100 & - & - \\
\hline
\end{tabular}

\section{3-Hydoxybenzaldehyde-d}<smiles>[2H]C(=O)c1cccc(O)c1</smiles>

Due to solubility issues 3-hydroxy benzaldehyde could not directly be deuterated, thus, the hydroxy group was Boc protected and following deuteration the protecting group was removed in situ. Boc protection was performed according to the procedure on page 16 above.

One-pot deuteration and de-protection of 3-OBoc benzaldehyde: $3 a(23.5 \mathrm{mg}, 0.055 \mathrm{mmol})$, DIPEA $(10 \mu \mathrm{L}, 0.055 \mathrm{mmol}), \mathrm{D}_{2} \mathrm{O}(600 \mu \mathrm{L}, 33 \mathrm{mmol})$ and 3-OBoc benzaldehyde (244 $\left.\mathrm{mg}, 1.1 \mathrm{mmol}\right)$ in anhydrous toluene $(500 \mu \mathrm{L})$ were prepared according to the general procedure given in section 5

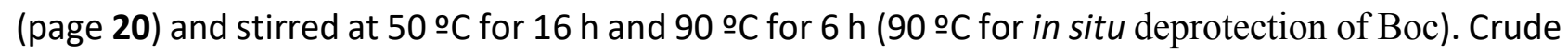
residue was purified by flash-column chromatography using $\mathrm{Hex} \mathrm{Et}_{2} \mathrm{O}(60: 40)$ combination to give colorless solid.

Deuterium incorporation $=98 \%$ (crude: $99 \%, 98 \%)$, Reaction yield $=92 \%$ (125 mg). NMR spectra are given on pages $205-210$.

${ }^{1} \mathbf{H}$ NMR $\left(500 \mathrm{MHz}, \mathrm{CD}_{3} \mathrm{CN}\right) \delta(\mathrm{ppm})=9.92(\mathrm{~s}, 0.02 \mathrm{H}), 7.44-7.39(\mathrm{~m}, 2 \mathrm{H}), 7.30-7.27(\mathrm{~m}, 2 \mathrm{H})$, 7.15$7.10(\mathrm{~m}, 1 \mathrm{H}) .{ }^{13} \mathrm{C}$ NMR $\left(125 \mathrm{MHz}, \mathrm{CD}_{3} \mathrm{CN}\right) \delta(\mathrm{ppm})=193.2(\mathrm{t}, J=34 \mathrm{~Hz}), 158.5,139.1(\mathrm{t}, J=5 \mathrm{~Hz})$, 131.4, 122.8, 122.6, 115.7. HRMS(ESI) calcd for $\mathrm{C}_{7} \mathrm{H}_{5} \mathrm{DO}_{2}{ }^{+}(\mathrm{M}+\mathrm{H})^{+}: 124.0509$, Found: 124.0509

\begin{tabular}{|c|c|c|c|c|c|c|c|c|c|c|}
\hline \multirow{3}{*}{$\begin{array}{c}\text { Entr } \\
\mathrm{y}\end{array}$} & \multirow{3}{*}{$\begin{array}{c}\mathbf{3 a} \\
(\mathrm{mol} \%)\end{array}$} & \multirow{3}{*}{$\begin{array}{l}\text { DIPEA } \\
(\mathrm{mol} \%)\end{array}$} & \multirow{3}{*}{$\begin{array}{l}\mathrm{D}_{2} \mathrm{O} \\
\text { (eq.) }\end{array}$} & \multirow{3}{*}{$\begin{array}{l}\text { Reaction Temp. } \\
\text { and time }\end{array}$} & \multirow{3}{*}{$\begin{array}{c}\% \mathrm{D} \\
(\mathrm{RM} \\
)\end{array}$} & \multirow{3}{*}{$\begin{array}{c}\% \\
\text { Aldehyde }\end{array}$} & \multirow{2}{*}{\multicolumn{2}{|c|}{$\begin{array}{c}\begin{array}{c}\text { After } \\
\text { Purification }\end{array} \\
\text { OBoc }\end{array}$}} & \multirow{2}{*}{\multicolumn{2}{|c|}{$\begin{array}{c}\begin{array}{c}\text { After } \\
\text { Purification }\end{array} \\
\mathrm{OH}\end{array}$}} \\
\hline & & & & & & & & & & \\
\hline & & & & & & & $\begin{array}{l}\% \\
\mathrm{D}\end{array}$ & $\%$ Yield & $\begin{array}{l}\% \\
\mathrm{D}\end{array}$ & $\%$ Yield \\
\hline 1 & 5 & 5 & 30 & $20^{\circ} \mathrm{C}$ for $16 \mathrm{~h}$ & 57 & 100 & - & - & - & - \\
\hline 2 & 5 & 5 & 30 & $50^{\circ} \mathrm{C}$ for $16 \mathrm{~h}$ & 100 & 100 & 98 & 61 & 98 & 37 \\
\hline 3 & 5 & 5 & 30 & $\begin{array}{c}50^{\circ} \mathrm{C} \text { for } 16 \mathrm{~h} \text {, } \\
90^{\circ} \mathrm{C} \text { for } 6 \mathrm{~h}\end{array}$ & 99 & 100 & - & - & 98 & 92 \\
\hline 3 , & 5 & 5 & 30 & $\begin{array}{c}50^{\circ} \mathrm{C} \text { for } 16 \mathrm{~h} \text {, } \\
90^{\circ} \mathrm{C} \text { for } 6 \mathrm{~h}\end{array}$ & 98 & 100 & - & - & - & - \\
\hline 4 & 5 & 5 & 30 & $90^{\circ} \mathrm{C}$ for $16 \mathrm{~h}$ & 93 & 100 & - & - & 93 & 90 \\
\hline
\end{tabular}




\section{Condition 3:}
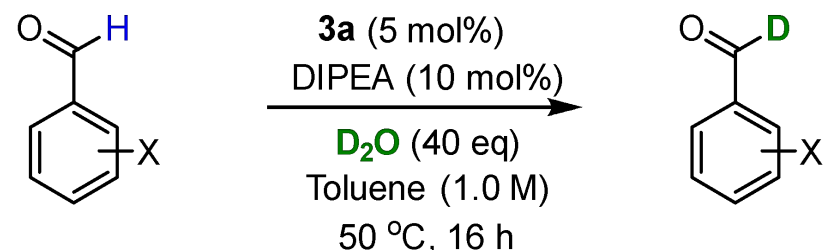

$50{ }^{\circ} \mathrm{C}, 16 \mathrm{~h}$

Scheme 20: Reaction condition 3

\section{2-(Trifluoromethyl)benzaldehyde-d}<smiles>[2H]C(=O)c1ccccc1C(F)(F)F</smiles>

3a (23.5 mg, $0.055 \mathrm{mmol})$, DIPEA $(20 \mu \mathrm{L}, 0.11 \mathrm{mmol}), \mathrm{D}_{2} \mathrm{O}(600 \mu \mathrm{L}, 33 \mathrm{mmol})$ and 2(trifluoromethyl)benzaldehyde $(145 \mu \mathrm{L}, 1.1 \mathrm{mmol})$ in anhydrous toluene $(500 \mu \mathrm{L})$ were prepared according to the general procedure given in section 5 (page 20) and stirred at $50 \stackrel{\circ}{ }{ }^{\circ}$. Crude residue was purified by flash-column chromatography using $\mathrm{Hex}_{\mathrm{Et}} \mathrm{O}(97: 3)$ combination to give colorless liquid. Spectral data for this product have been previously reported. ${ }^{16}$

Deuterium incorporation $=97 \%$ (crude: 99\%, 98\%), Reaction yield $=95 \%$ (183 mg). NMR spectra are given on pages 211-216.

${ }^{1} \mathbf{H}$ NMR $\left(500 \mathrm{MHz}, \mathrm{CDCl}_{3}\right) \delta(\mathrm{ppm})=10.40(\mathrm{~s}, 0.03 \mathrm{H})$, 8.14-8.13 $(\mathrm{m}, 1 \mathrm{H})$, 7.80-7.78 $(\mathrm{m}, 1 \mathrm{H})$, 7.74$7.69(\mathrm{~m}, 2 \mathrm{H}) .{ }^{13} \mathrm{C}$ NMR $\left(125 \mathrm{MHz}, \mathrm{CDCl}_{3}\right) \delta(\mathrm{ppm})=188.8(\mathrm{t}, J=28 \mathrm{~Hz}), 133.8,132.5,131.2(\mathrm{q}, J=33$ $\mathrm{Hz}), 129.2,126.2(\mathrm{~d}, J=5 \mathrm{~Hz}), 123.9(\mathrm{q}, J=274 \mathrm{~Hz}) .{ }^{19} \mathrm{~F} \mathrm{NMR}\left(376 \mathrm{MHz}, \mathrm{CDCl}_{3}\right) \delta(\mathrm{ppm})=-55.6(\mathrm{~s}, 3$ F)

\begin{tabular}{|c|c|c|c|c|c|c|c|c|c|c|}
\hline \multirow[t]{2}{*}{ Entry } & \multirow{2}{*}{$\begin{array}{c}3 a \\
(\mathrm{~mol} \%)\end{array}$} & \multirow{2}{*}{$\begin{array}{l}\text { DIPEA } \\
\text { (mol\%) }\end{array}$} & \multirow{2}{*}{$\begin{array}{l}\mathrm{D}_{2} \mathrm{O} \\
\text { (eq. } \\
\text { ) }\end{array}$} & \multirow{2}{*}{$\begin{array}{c}\text { Ph- } \\
\text { Boroxine } \\
\text { (mol\%) }\end{array}$} & \multirow{2}{*}{$\begin{array}{l}\text { Reactio } \\
\text { n Conc. } \\
\text { (M) }\end{array}$} & \multirow{2}{*}{$\begin{array}{l}\text { Reactio } \\
\text { n Temp. }\end{array}$} & \multirow{2}{*}{$\begin{array}{c}\% \mathrm{D} \\
\text { (RM) }\end{array}$} & \multirow{2}{*}{$\begin{array}{c}\% \\
\text { Aldehyd } \\
\text { e }\end{array}$} & \multicolumn{2}{|c|}{$\begin{array}{c}\text { After } \\
\text { Purification }\end{array}$} \\
\hline & & & & & & & & & $\% \mathrm{D}$ & \%Yield \\
\hline 1 & 5 & 5 & 30 & - & 1 & $20^{\circ} \mathrm{C}$ & 76 & 100 & - & - \\
\hline $1^{\prime}$ & 5 & 5 & 30 & - & 1 & $20^{\circ} \mathrm{C}$ & 76 & 100 & - & - \\
\hline 2 & 5 & 5 & 30 & - & 1 & $50 \stackrel{\circ}{C}$ & 95 & 100 & - & - \\
\hline $2^{\prime}$ & 5 & 5 & 30 & - & 1 & $50 \stackrel{\circ}{ }$ & 92 & 100 & - & - \\
\hline 3 & 5 & 10 & 30 & - & 1 & $50 \stackrel{\circ}{ }$ & 98 & 100 & - & - \\
\hline $3^{\prime}$ & 5 & 10 & 30 & - & 1 & $50 \stackrel{\circ}{ }$ & 98 & 100 & - & - \\
\hline 4 & 5 & 10 & 40 & - & 1 & $50 \stackrel{\circ}{ }$ & 99 & 100 & 97 & 95 \\
\hline $4^{\prime}$ & 5 & 10 & 40 & - & 1 & $50 \stackrel{\circ}{ } \mathrm{C}$ & 98 & 100 & - & - \\
\hline
\end{tabular}




\section{2-Methoxybenzaldehyde-d}<smiles>[2H]C(=O)c1ccccc1OC</smiles>

3a (23.5 mg, $0.055 \mathrm{mmol})$, DIPEA $(10 \mu \mathrm{L}, 0.055 \mathrm{mmol}), \mathrm{D}_{2} \mathrm{O}(600 \mu \mathrm{L}, 33 \mathrm{mmol})$ and 2methoxybenzaldehyde $(150 \mathrm{mg}, 1.1 \mathrm{mmol})$ in anhydrous toluene $(500 \mu \mathrm{L})$ were prepared according to the general procedure given in section 5 (page 20) and stirred at $50 \circ \mathrm{C}$. Crude residue was purified by flash-column chromatography using $\mathrm{Hex}_{\mathrm{Et}} \mathrm{O}(91: 9)$ combination to give colorless solid. Spectral data for this product have been previously reported. ${ }^{15}$

Deuterium incorporation $=98 \%$ (crude: $98 \%, 98 \%)$, Reaction yield = 92\% (139 mg). NMR spectra are given on pages $217-221$.

${ }^{1} \mathbf{H}$ NMR $\left(500 \mathrm{MHz}, \mathrm{CDCl}_{3}\right) \delta(\mathrm{ppm})=10.48(\mathrm{~s}, 0.02 \mathrm{H})$, 7.85-7.83 (m, $\left.1 \mathrm{H}\right)$, 7.57-7.54 (m , $\left.1 \mathrm{H}\right)$, 7.05$6.99(\mathrm{~m}, 2 \mathrm{H}), 3.93(\mathrm{~s}, 3 \mathrm{H}) .{ }^{13} \mathrm{C}$ NMR $\left(125 \mathrm{MHz}, \mathrm{CDCl}_{3}\right) \delta(\mathrm{ppm})=189.6(\mathrm{t}, J=28 \mathrm{~Hz}), 162.0,136.1$, $128.7,124.9,120.8,111.7,55.8$

\begin{tabular}{|c|c|c|c|c|c|c|c|c|c|c|}
\hline \multirow{2}{*}{ Entry } & \multirow{2}{*}{$\begin{array}{c}\mathbf{3 a} \\
(\mathrm{mol} \%)\end{array}$} & \multirow{2}{*}{$\begin{array}{l}\text { DIPEA } \\
\text { (mol\%) }\end{array}$} & \multirow{2}{*}{$\begin{array}{c}\mathrm{D}_{2} \mathrm{O} \\
\text { (eq. } \\
\text { ) }\end{array}$} & \multirow{2}{*}{$\begin{array}{c}\text { Ph- } \\
\text { Boroxine } \\
\text { (mol\%) }\end{array}$} & \multirow{2}{*}{$\begin{array}{c}\text { Reactio } \\
\text { n Conc. } \\
\text { (M) }\end{array}$} & \multirow{2}{*}{$\begin{array}{l}\text { Reactio } \\
\text { n Temp. }\end{array}$} & \multirow{2}{*}{$\begin{array}{c}\% \mathrm{D} \\
\text { (RM) }\end{array}$} & \multirow{2}{*}{$\begin{array}{c}\% \\
\text { Aldehyd } \\
\text { e } \\
\end{array}$} & \multicolumn{2}{|c|}{$\begin{array}{c}\text { After } \\
\text { Purification }\end{array}$} \\
\hline & & & & & & & & & $\% \mathrm{D}$ & \%Yield \\
\hline 1 & 5 & 5 & 30 & - & 1 & $20^{\circ} \mathrm{C}$ & 80 & 100 & - & - \\
\hline $1^{\prime}$ & 5 & 5 & 30 & - & 1 & $20^{\circ} \mathrm{C}$ & 80 & 100 & - & - \\
\hline 2 & 5 & 5 & 30 & - & 1 & $50 \circ \mathrm{C}$ & 88 & 100 & - & - \\
\hline 3 & 5 & 10 & 30 & - & 1 & $50 \stackrel{\circ}{ }$ & 93 & 100 & - & - \\
\hline $3^{\prime}$ & 5 & 10 & 30 & - & 1 & $50 \stackrel{\circ}{ }$ & 94 & 100 & - & - \\
\hline 4 & 5 & 10 & 40 & - & 1 & $50 \stackrel{\circ}{ }$ & 98 & 100 & 98 & 92 \\
\hline $4^{\prime}$ & 5 & 10 & 40 & - & 1 & $50 \cong \mathrm{C}$ & 98 & 100 & - & - \\
\hline
\end{tabular}

\section{4-Methylbenzaldehyde-d}<smiles>[2H]C(=O)c1ccc(C)cc1</smiles>

3a (23.5 mg, $0.055 \mathrm{mmol})$, DIPEA $(10 \mu \mathrm{L}, 0.055 \mathrm{mmol}), \mathrm{D}_{2} \mathrm{O}(600 \mu \mathrm{L}, 33 \mathrm{mmol})$ and 4methylbenzaldehyde $(134 \mu \mathrm{L}, 1.1 \mathrm{mmol})$ in anhydrous toluene $(500 \mu \mathrm{L})$ were prepared according to the general procedure given in section 5 (page 20) and stirred at $50 \circ \mathrm{C}$. Crude residue was purified 
by flash-column chromatography using $\mathrm{Hex}_{\mathrm{Et}} \mathrm{O}$ (97:3) combination to give colorless liquid. Spectral data for this product have been previously reported. ${ }^{15}$

Deuterium incorporation $=97 \%$ (crude: $98 \%, 98 \%)$, Reaction yield = 87\% (116 mg). NMR spectra are given on pages $222-226$.

${ }^{1} \mathrm{H}$ NMR $\left(400 \mathrm{MHz}, \mathrm{CDCl}_{3}\right) \delta(\mathrm{ppm})=9.96(\mathrm{~s}, 0.03 \mathrm{H}), 7.78(\mathrm{~d}, J=8 \mathrm{~Hz}, 2 \mathrm{H}), 7.33(\mathrm{~d}, J=8 \mathrm{~Hz}, 2 \mathrm{H})$, $2.43(\mathrm{~s}, 3 \mathrm{H}) .{ }^{13} \mathrm{C}$ NMR $\left(100 \mathrm{MHz}, \mathrm{CDCl}_{3}\right) \delta(\mathrm{ppm})=191.7(\mathrm{t}, J=26 \mathrm{~Hz}), 145.6,134.2(\mathrm{t}, J=4 \mathrm{~Hz})$, $129.9,129.7,21.9$

\begin{tabular}{|c|c|c|c|c|c|c|c|c|c|c|}
\hline \multirow[t]{2}{*}{ Entry } & \multirow{2}{*}{$\begin{array}{c}3 a \\
\text { (mol\%) }\end{array}$} & \multirow{2}{*}{$\begin{array}{l}\text { DIPEA } \\
\text { (mol\%) }\end{array}$} & \multirow{2}{*}{$\begin{array}{c}\mathrm{D}_{2} \mathrm{O} \\
\text { (eq. } \\
\text { ) }\end{array}$} & \multirow{2}{*}{$\begin{array}{c}\text { Ph- } \\
\text { Boroxine } \\
\text { (mol\%) }\end{array}$} & \multirow{2}{*}{$\begin{array}{l}\text { Reactio } \\
\text { n Conc. } \\
\text { (M) }\end{array}$} & \multirow{2}{*}{$\begin{array}{l}\text { Reactio } \\
\text { n Temp. }\end{array}$} & \multirow{2}{*}{$\begin{array}{l}\% \mathrm{D} \\
\text { (RM) }\end{array}$} & \multirow{2}{*}{$\begin{array}{c}\% \\
\text { Aldehyd } \\
\mathrm{e}\end{array}$} & \multicolumn{2}{|c|}{$\begin{array}{c}\text { After } \\
\text { Purification }\end{array}$} \\
\hline & & & & & & & & & $\% \mathrm{D}$ & \%Yield \\
\hline 1 & 5 & 5 & 30 & - & 1 & $50 \stackrel{\circ}{C}$ & 94 & 100 & - & - \\
\hline $1^{\prime}$ & 5 & 5 & 30 & - & 1 & 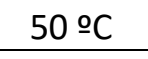 & 93 & 100 & - & - \\
\hline 2 & 5 & 10 & 30 & - & 1 & 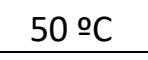 & 98 & 100 & - & - \\
\hline $2^{\prime}$ & 5 & 10 & 30 & - & 1 & $50 \stackrel{\circ}{C}$ & 97 & 100 & - & - \\
\hline 3 & 5 & 10 & 40 & - & 1 & $50 \stackrel{\circ}{C}$ & 98 & 100 & 97 & 87 \\
\hline $3^{\prime}$ & 5 & 10 & 40 & - & 1 & $50 \circ \mathrm{C}$ & 98 & 100 & - & - \\
\hline
\end{tabular}

\section{4-Isopropylbenzaldehyde-d}<smiles>[2H]C(=O)c1ccc(C(C)C)cc1</smiles>

3a (23.5 mg, $0.055 \mathrm{mmol})$, DIPEA $(10 \mu \mathrm{L}, 0.055 \mathrm{mmol}), \mathrm{D}_{2} \mathrm{O}(600 \mu \mathrm{L}, 33 \mathrm{mmol})$ and 4isopropylbenzaldehyde (166 $\mu \mathrm{L}, 1.1 \mathrm{mmol})$ in anhydrous toluene $(500 \mu \mathrm{L})$ were prepared according to the general procedure given in section 5 (page 20) and stirred at $50 \stackrel{\circ}{ }{ }^{\circ}$. Crude residue was purified by flash-column chromatography using Hex: $\mathrm{Et}_{2} \mathrm{O}$ (93:7) combination to give colorless liquid. Spectral data for this product have been previously reported. ${ }^{17}$

Deuterium incorporation $=98 \%$ (crude: $98 \%, 98 \%)$, Reaction yield $=97 \%$ (159 mg). NMR spectra are given on pages $227-231$.

${ }^{1} \mathrm{H}$ NMR $\left(400 \mathrm{MHz}, \mathrm{CDCl}_{3}\right) \delta(\mathrm{ppm})=9.97(\mathrm{~s}, 0.02 \mathrm{H}), 7.81(\mathrm{~d}, J=8 \mathrm{~Hz}, 2 \mathrm{H}), 7.39(\mathrm{~d}, J=8 \mathrm{~Hz}, 2 \mathrm{H})$, 2.99 (spt, $J=8 \mathrm{~Hz}, 1 \mathrm{H}), 1.28(\mathrm{~d}, J=8 \mathrm{~Hz}, 6 \mathrm{H}) .{ }^{13} \mathrm{C} \mathrm{NMR}\left(100 \mathrm{MHz}, \mathrm{CDCl}_{3}\right) \delta(\mathrm{ppm})=191.8(\mathrm{t}, J=27$ $\mathrm{Hz}), 156.4,134.6(\mathrm{t}, J=4 \mathrm{~Hz}), 130.1,127.3,34.6,23.8$ 


\begin{tabular}{|c|c|c|c|c|c|c|c|c|c|c|}
\hline \multirow[t]{2}{*}{ Entry } & \multirow{2}{*}{$\begin{array}{c}3 a \\
(\mathrm{~mol} \%)\end{array}$} & \multirow{2}{*}{$\begin{array}{l}\text { DIPEA } \\
\text { (mol\%) }\end{array}$} & \multirow{2}{*}{$\begin{array}{c}\mathrm{D}_{2} \mathrm{O} \\
\text { (eq. } \\
\text { ) }\end{array}$} & \multirow{2}{*}{$\begin{array}{c}\text { Ph- } \\
\text { Boroxine } \\
\text { (mol\%) }\end{array}$} & \multirow{2}{*}{$\begin{array}{l}\text { Reactio } \\
\text { n Conc. } \\
\text { (M) }\end{array}$} & \multirow{2}{*}{$\begin{array}{l}\text { Reactio } \\
\text { n Temp. }\end{array}$} & \multirow{2}{*}{$\begin{array}{c}\% \mathrm{D} \\
\text { (RM) }\end{array}$} & \multirow{2}{*}{$\begin{array}{c}\% \\
\text { Aldehyd } \\
\text { e }\end{array}$} & \multicolumn{2}{|c|}{$\begin{array}{c}\text { After } \\
\text { Purification }\end{array}$} \\
\hline & & & & & & & & & $\% \mathrm{D}$ & \%Yield \\
\hline 1 & 5 & 5 & 30 & - & 1 & $50 \stackrel{\circ}{C}$ & 96 & 100 & - & - \\
\hline $1^{\prime}$ & 5 & 5 & 30 & - & 1 & $50 \stackrel{\circ}{ }$ & 89 & 100 & - & - \\
\hline 2 & 5 & 10 & 30 & - & 1 & $50 \stackrel{\circ}{ }$ & 98 & 100 & - & - \\
\hline $2^{\prime}$ & 5 & 10 & 30 & - & 1 & $50 \stackrel{\circ}{C}$ & 98 & 100 & - & - \\
\hline 3 & 5 & 10 & 40 & - & 1 & $50 \stackrel{\circ}{ }$ & 98 & 100 & 98 & 97 \\
\hline $3^{\prime}$ & 5 & 10 & 40 & - & 1 & $50 \cong \mathrm{C}$ & 98 & 100 & - & - \\
\hline
\end{tabular}




\section{Condition 4:}
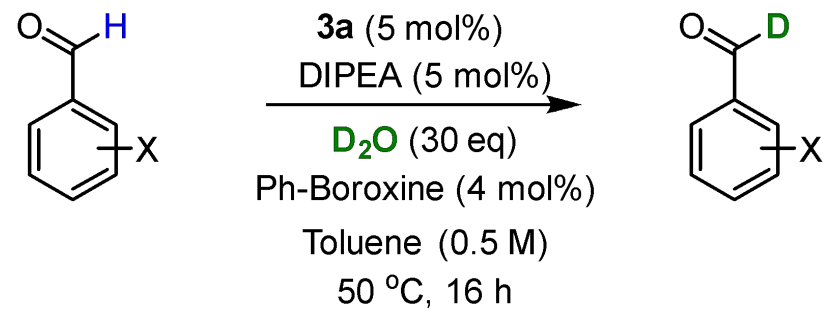

Scheme 21: Reaction condition 4 (Ph = Phenyl)

\section{3-Fluorobenzaldehyde-d}<smiles>[2H]C(=O)c1cccc(F)c1</smiles>

3a (23.5 mg, $0.055 \mathrm{mmol})$, DIPEA $(10 \mu \mathrm{L}, 0.055 \mathrm{mmol}), \mathrm{D}_{2} \mathrm{O}(600 \mu \mathrm{L}, 33 \mathrm{mmol})$, phenyl boroxine (13.7 $\mathrm{mg}, 0.044 \mathrm{mmol})$ and 3-fluorobenzaldehyde $(117 \mu \mathrm{L}, 1.1 \mathrm{mmol})$ in anhydrous toluene (1.60 $\mathrm{mL}$ ) were prepared according to the general procedure given in section 5 (page 20) and stirred at 50 ㅇ.C. Crude residue was purified by flash-column chromatography using Hex:Et ${ }_{2} \mathrm{O}$ (98:2) combination to give colorless liquid.

Deuterium incorporation $=98 \%$ (crude: 99\%, 99\%), Reaction yield = 92\% (127 mg). NMR spectra are given on pages $232-234$.

${ }^{1} \mathbf{H}$ NMR $\left(500 \mathrm{MHz}, \mathrm{CDCl}_{3}\right) \delta(\mathrm{ppm})=10.00(\mathrm{~s}, 0.02 \mathrm{H})$, 7.69-7.67 (m, $\left.1 \mathrm{H}\right)$, 7.58-7.51 (m, $\left.2 \mathrm{H}\right)$, 7.36$7.32(\mathrm{~m}, 1 \mathrm{H}) .{ }^{13} \mathrm{C}$ NMR $\left(125 \mathrm{MHz}, \mathrm{CDCl}_{3}\right) \delta(\mathrm{ppm})=190.7(\mathrm{t}, J=28 \mathrm{~Hz}), 163.3(\mathrm{~d}, J=250 \mathrm{~Hz}), 138.5$, $130.9(\mathrm{~d}, J=6 \mathrm{~Hz}), 126.2,121.7(\mathrm{~d}, J=21 \mathrm{~Hz}), 115.5(\mathrm{~d}, J=21 \mathrm{~Hz}) .{ }^{19} \mathbf{F}$ NMR $\left(376 \mathrm{MHz}, \mathrm{CDCl}_{3}\right) \delta(\mathrm{ppm})$ $=-61.5(\mathrm{~s}, 1 \mathrm{~F}) . \mathrm{HRMS}(\mathrm{ESI})$ calcd for $\mathrm{C}_{7} \mathrm{H}_{4} \mathrm{DFO}^{+}(\mathrm{M}+\mathrm{H})^{+}: 126.0460$, Found: 126.0458

\begin{tabular}{|c|c|c|c|c|c|c|c|c|c|c|}
\hline \multirow[t]{2}{*}{ Entry } & \multirow{2}{*}{$\begin{array}{c}3 a \\
(\mathrm{~mol} \%)\end{array}$} & \multirow{2}{*}{$\begin{array}{l}\text { DIPEA } \\
\text { (mol\%) }\end{array}$} & \multirow{2}{*}{$\begin{array}{l}\mathrm{D}_{2} \mathrm{O} \\
\text { (eq. } \\
\text { ) }\end{array}$} & \multirow{2}{*}{$\begin{array}{c}\text { Ph- } \\
\text { Boroxine } \\
\text { (mol\%) }\end{array}$} & \multirow{2}{*}{$\begin{array}{l}\text { Reactio } \\
\text { n Conc. } \\
\text { (M) }\end{array}$} & \multirow{2}{*}{$\begin{array}{l}\text { Reactio } \\
\text { n Temp. }\end{array}$} & \multirow{2}{*}{$\begin{array}{l}\% \mathrm{D} \\
\text { (RM) }\end{array}$} & \multirow{2}{*}{$\begin{array}{c}\% \\
\text { Aldehyd } \\
\text { e }\end{array}$} & \multicolumn{2}{|c|}{$\begin{array}{c}\text { After } \\
\text { Purification }\end{array}$} \\
\hline & & & & & & & & & $\% \mathrm{D}$ & \%Yield \\
\hline 1 & 5 & 5 & 30 & 4 & 0.5 & $50 \cong \mathrm{C}$ & 99 & 100 & 98 & 92 \\
\hline $1^{\prime}$ & 5 & 5 & 30 & 4 & 0.5 & $50 \stackrel{\circ}{ }$ & 99 & 100 & - & - \\
\hline
\end{tabular}




\section{3-Chlorobenzaldehyde-d}<smiles>[2H]C(=O)c1cccc(Cl)c1</smiles>

3a (23.5 mg, $0.055 \mathrm{mmol})$, DIPEA (10 $\mu \mathrm{L}, 0.055 \mathrm{mmol}), \mathrm{D}_{2} \mathrm{O}(600 \mu \mathrm{L}, 33 \mathrm{mmol})$, phenyl boroxine (13.7 $\mathrm{mg}, 0.044 \mathrm{mmol})$ and 3-chlorobenzaldehyde (124 $\mu \mathrm{L}, 1.1 \mathrm{mmol})$ in anhydrous toluene (1.60 $\mathrm{mL}$ ) were prepared according to the general procedure given in section 5 (page 20) and stirred at 50 으. Crude residue was purified by flash-column chromatography using Hex:Et ${ }_{2} \mathrm{O}$ (98:2) combination to give colorless liquid. Spectral data for this product have been previously reported. ${ }^{15}$ Deuterium incorporation $=98 \%$ (crude: $99 \%, 99 \%)$, Reaction yield = 87\% (135 mg). NMR spectra are given on pages $235-237$.

${ }^{1} \mathrm{H}$ NMR $\left(400 \mathrm{MHz}, \mathrm{CDCl}_{3}\right) \delta(\mathrm{ppm})=9.98(\mathrm{~s}, 0.02 \mathrm{H})$, 7.86-7.85 (m, $\left.1 \mathrm{H}\right)$, 7.78-7.75 (m, $\left.1 \mathrm{H}\right)$, 7.61$7.59(\mathrm{~m}, 1 \mathrm{H}), 7.50-7.46(\mathrm{~m}, 1 \mathrm{H}) .{ }^{13} \mathrm{C}$ NMR $\left(100 \mathrm{MHz}, \mathrm{CDCl}_{3}\right) \delta(\mathrm{ppm})=190.6(\mathrm{t}, \mathrm{J}=26 \mathrm{~Hz}), 137.9(\mathrm{t}$, $J=4 \mathrm{~Hz}), 135.6,134.5,130.5,129.4,128.1$

\begin{tabular}{|c|c|c|c|c|c|c|c|c|c|c|}
\hline \multirow{2}{*}{ Entry } & \multirow{2}{*}{$\begin{array}{c}3 a \\
(\mathrm{~mol} \%)\end{array}$} & \multirow{2}{*}{$\begin{array}{l}\text { DIPEA } \\
\text { (mol\%) }\end{array}$} & \multirow{2}{*}{$\begin{array}{c}\mathrm{D}_{2} \mathrm{O} \\
\text { (eq. } \\
\text { ) }\end{array}$} & \multirow{2}{*}{$\begin{array}{c}\text { Ph- } \\
\text { Boroxine } \\
\text { (mol\%) }\end{array}$} & \multirow{2}{*}{$\begin{array}{l}\text { Reactio } \\
\text { n Conc. } \\
\text { (M) }\end{array}$} & \multirow{2}{*}{$\begin{array}{l}\text { Reactio } \\
\text { n Temp. }\end{array}$} & \multirow{2}{*}{$\begin{array}{c}\% \mathrm{D} \\
\text { (RM) }\end{array}$} & \multirow{2}{*}{$\begin{array}{c}\% \\
\text { Aldehyd } \\
\text { e }\end{array}$} & \multicolumn{2}{|c|}{$\begin{array}{c}\text { After } \\
\text { Purification }\end{array}$} \\
\hline & & & & & & & & & $\% \mathrm{D}$ & \%Yield \\
\hline 1 & 5 & 5 & 30 & 4 & 0.5 & $50 \stackrel{\circ}{C}$ & 99 & 100 & 98 & 87 \\
\hline $1^{\prime}$ & 5 & 5 & 30 & 4 & 0.5 & $50 \div \mathrm{C}$ & 99 & 100 & - & - \\
\hline
\end{tabular}

\section{4-Chlorobenzaldehyde-d}<smiles>[2H]C(=O)c1ccc(Cl)cc1</smiles>

3a (23.5 mg, $0.055 \mathrm{mmol})$, DIPEA $(10 \mu \mathrm{L}, 0.055 \mathrm{mmol}), \mathrm{D}_{2} \mathrm{O}(600 \mu \mathrm{L}, 33 \mathrm{mmol})$, phenyl boroxine $(13.7 \mathrm{mg}, 0.044 \mathrm{mmol})$ and 4-chlorobenzaldehyde $(155 \mathrm{mg}, 1.1 \mathrm{mmol})$ in anhydrous toluene (1.60 $\mathrm{mL}$ ) were prepared according to the general procedure given in section 5 (page 20) and stirred at 50 으. Crude residue was purified by flash-column chromatography using Hex:Et ${ }_{2} \mathrm{O}$ (98:2) combination to give colorless solid. Spectral data for this product have been previously reported. ${ }^{15}$ Deuterium incorporation $=97 \%$ (crude: $100 \%, 99 \%)$, Reaction yield $=83 \%$ (129 mg). NMR spectra are given on pages $238-240$. 
${ }^{1} \mathbf{H}$ NMR $\left(400 \mathrm{MHz}, \mathrm{CDCl}_{3}\right) \delta(\mathrm{ppm})=9.99(\mathrm{~s}, 0.03 \mathrm{H}), 7.85-7.81(\mathrm{~m}, 2 \mathrm{H}), 7.53-7.50(\mathrm{~m}, 2 \mathrm{H}) .{ }^{13} \mathrm{C} \mathrm{NMR}$ $\left(100 \mathrm{MHz}, \mathrm{CDCl}_{3}\right) \delta(\mathrm{ppm})=190.7(\mathrm{t}, J=26 \mathrm{~Hz}), 141.1,134.8(\mathrm{t}, J=4 \mathrm{~Hz}), 131.0,129.6$

\begin{tabular}{|c|c|c|c|c|c|c|c|c|c|c|}
\hline \multirow[t]{2}{*}{ Entry } & \multirow{2}{*}{$\begin{array}{c}3 a \\
(\mathrm{~mol} \%)\end{array}$} & \multirow{2}{*}{$\begin{array}{l}\text { DIPEA } \\
\text { (mol\%) }\end{array}$} & \multirow{2}{*}{$\begin{array}{l}\mathrm{D}_{2} \mathrm{O} \\
\text { (eq. } \\
\text { ) }\end{array}$} & \multirow{2}{*}{$\begin{array}{c}\text { Ph- } \\
\text { Boroxine } \\
\text { (mol\%) }\end{array}$} & \multirow{2}{*}{$\begin{array}{l}\text { Reactio } \\
\text { n Conc. } \\
\text { (M) }\end{array}$} & \multirow{2}{*}{$\begin{array}{l}\text { Reactio } \\
\text { n Temp. }\end{array}$} & \multirow{2}{*}{$\begin{array}{c}\% \mathrm{D} \\
\text { (RM) }\end{array}$} & \multirow{2}{*}{$\begin{array}{c}\% \\
\text { Aldehyde }\end{array}$} & \multicolumn{2}{|c|}{$\begin{array}{c}\text { After } \\
\text { Purification }\end{array}$} \\
\hline & & & & & & & & & $\% D$ & \%Yield \\
\hline 1 & 5 & 5 & 30 & 4 & 0.5 & $50 \stackrel{\circ}{ }$ & 100 & 100 & 97 & 83 \\
\hline $1^{\prime}$ & 5 & 5 & 30 & 4 & 0.5 & 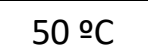 & 99 & 100 & - & - \\
\hline
\end{tabular}

\section{3,5-Dichlorobenzaldehyde-d}<smiles>[2H]C(=O)c1cc(Cl)cc(Cl)c1</smiles>

3a $(23.5 \mathrm{mg}, 0.055 \mathrm{mmol})$, DIPEA $(10 \mu \mathrm{L}, 0.055 \mathrm{mmol}), \mathrm{D}_{2} \mathrm{O}(600 \mu \mathrm{L}, 33 \mathrm{mmol})$, phenyl boroxine (13.7 $\mathrm{mg}, 0.044 \mathrm{mmol})$ and 3,5-dichlorobenzaldehyde $(192.5 \mathrm{mg}, 1.1 \mathrm{mmol})$ in anhydrous toluene $(1.60 \mathrm{~mL})$ were prepared according to the general procedure given in section 5 (page 20) and stirred at $50 \stackrel{\circ}{ } \mathrm{C}$. Crude residue was purified by flash-column chromatography using Hex:Et ${ }_{2} \mathrm{O}$ (91:9) combination to give colorless solid.

Deuterium incorporation = 98\% (crude: 99\%, 98\%), Reaction yield = 94\% (182 mg). NMR spectra are given on pages $241-243$.

${ }^{1} \mathrm{H}$ NMR $\left(500 \mathrm{MHz}, \mathrm{CDCl}_{3}\right) \delta(\mathrm{ppm})=9.92(\mathrm{~s}, 0.02 \mathrm{H}), 7.74(\mathrm{~d}, J=2 \mathrm{~Hz}, 2 \mathrm{H}), 7.60(\mathrm{t}, J=2 \mathrm{~Hz}, 1 \mathrm{H}) .{ }^{13} \mathrm{C}$ NMR $\left(125 \mathrm{MHz}_{1} \mathrm{CDCl}_{3}\right) \delta(\mathrm{ppm})=189.2(\mathrm{t}, J=28 \mathrm{~Hz}), 138.6,136.3,134.2,127.9$. HRMS(ESI) calcd for $\mathrm{C}_{7} \mathrm{H}_{4} \mathrm{DCl}_{2} \mathrm{O}^{+}(\mathrm{M}+\mathrm{H})^{+}$: 175.9775 , Found: 175.9775

\begin{tabular}{|c|c|c|c|c|c|c|c|c|c|c|}
\hline Entry & $\begin{array}{c}3 a \\
\text { (mol\%) }\end{array}$ & $\begin{array}{c}\text { DIPEA } \\
\text { (mol\%) }\end{array}$ & $\begin{array}{c}\mathrm{D}_{2} \mathrm{O} \\
\text { (eq. }\end{array}$ & $\begin{array}{c}\text { Ph- } \\
\text { Boroxine }\end{array}$ & $\begin{array}{c}\text { Reactio } \\
\text { (mol\%) }\end{array}$ & $\begin{array}{c}\text { Conc. } \\
\text { (M) }\end{array}$ & $\begin{array}{c}\text { Reactio } \\
\text { n Temp. }\end{array}$ & $\begin{array}{c}\% \mathrm{D} \\
\text { (RM) }\end{array}$ & $\begin{array}{c}\% \\
\text { Aldehyd } \\
\text { e }\end{array}$ & \multicolumn{2}{|c|}{$\begin{array}{c}\text { After } \\
\text { Purification }\end{array}$} \\
\hline 1 & 5 & 5 & 30 & 4 & 0.5 & 50 \% & 99 & 100 & 98 & 94 \\
\hline $1^{\prime}$ & 5 & 5 & 30 & 4 & 0.5 & 50 OC & 98 & 100 & - & - \\
\hline
\end{tabular}

\section{3-Bromobenzaldehyde-d}<smiles>[2H]C(=O)c1cccc(Br)c1</smiles> 
3a $(23.5 \mathrm{mg}, 0.055 \mathrm{mmol})$, DIPEA $(10 \mu \mathrm{L}, 0.055 \mathrm{mmol}), \mathrm{D}_{2} \mathrm{O}(600 \mu \mathrm{L}, 33 \mathrm{mmol})$, phenyl boroxine (13.7 $\mathrm{mg}, 0.044 \mathrm{mmol})$ and 3-bromobenzaldehyde $(128 \mu \mathrm{L}, 1.1 \mathrm{mmol})$ in anhydrous toluene (1.60 $\mathrm{mL}$ ) were prepared according to the general procedure given in section 5 (page 20) and stirred at 50 으. Crude residue was purified by flash-column chromatography using Hex:Et ${ }_{2} \mathrm{O}$ (98:2) combination to give colorless liquid. Spectral data for this product have been previously reported. ${ }^{15}$ Deuterium incorporation = 98\% (crude: 99\%, 99\%), Reaction yield = 95\% (194 mg). NMR spectra are given on pages $244-246$.

${ }^{1} \mathrm{H}$ NMR $\left(400 \mathrm{MHz}, \mathrm{CDCl}_{3}\right) \delta(\mathrm{ppm})=9.96(\mathrm{~s}, 0.02 \mathrm{H}), 8.02(\mathrm{~m}, 1 \mathrm{H}), 7.83-7.81(\mathrm{~m}, 1 \mathrm{H})$, 7.77-7.75 (m, $1 \mathrm{H})$, 7.44-7.41 (m, $1 \mathrm{H}) .{ }^{13} \mathrm{C}$ NMR $\left(100 \mathrm{MHz}, \mathrm{CDCl}_{3}\right) \delta(\mathrm{ppm})=190.5(\mathrm{t}, J=27 \mathrm{~Hz}), 138.1$ (t, J = $\left.3 \mathrm{~Hz}\right)$, $137.4,132.5,130.8,128.5,123.5$

\begin{tabular}{|c|c|c|c|c|c|c|c|c|c|c|}
\hline \multirow[t]{2}{*}{ Entry } & \multirow{2}{*}{$\begin{array}{c}3 a \\
(\mathrm{~mol} \%)\end{array}$} & \multirow{2}{*}{$\begin{array}{l}\text { DIPEA } \\
\text { (mol\%) }\end{array}$} & \multirow{2}{*}{$\begin{array}{l}\mathrm{D}_{2} \mathrm{O} \\
\text { (eq. } \\
\text { ) }\end{array}$} & \multirow{2}{*}{$\begin{array}{c}\text { Ph- } \\
\text { Boroxine } \\
\text { (mol\%) }\end{array}$} & \multirow{2}{*}{$\begin{array}{l}\text { Reactio } \\
\text { n Conc. } \\
\text { (M) }\end{array}$} & \multirow{2}{*}{$\begin{array}{l}\text { Reactio } \\
\text { n Temp. }\end{array}$} & \multirow{2}{*}{$\begin{array}{c}\% \mathrm{D} \\
\text { (RM) }\end{array}$} & \multirow{2}{*}{$\begin{array}{c}\% \\
\text { Aldehyd } \\
\text { e }\end{array}$} & \multicolumn{2}{|c|}{$\begin{array}{c}\text { After } \\
\text { Purification }\end{array}$} \\
\hline & & & & & & & & & $\% \mathrm{D}$ & \%Yield \\
\hline 1 & 5 & 5 & 30 & 4 & 0.5 & 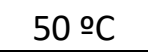 & 99 & 100 & 98 & 95 \\
\hline $1^{\prime}$ & 5 & 5 & 30 & 4 & 0.5 & $50 \cong \mathrm{C}$ & 99 & 100 & - & - \\
\hline
\end{tabular}

\section{4-Bromobenzaldehyde-d}<smiles>[2H]C(=O)c1ccc(Br)cc1</smiles>

3a (23.5 mg, $0.055 \mathrm{mmol})$, DIPEA (10 $\mu \mathrm{L}, 0.055 \mathrm{mmol}), \mathrm{D}_{2} \mathrm{O}(600 \mu \mathrm{L}, 33 \mathrm{mmol})$, phenyl boroxine (13.7 $\mathrm{mg}, 0.044 \mathrm{mmol})$ and 4-bromobenzaldehyde (204 mg, $1.1 \mathrm{mmol})$ in anhydrous toluene (1.60 $\mathrm{mL}$ ) were prepared according to the general procedure given in section 5 (page 20) and stirred at 50 으. Crude residue was purified by flash-column chromatography using Hex:Et ${ }_{2} \mathrm{O}$ (98:2) combination to give colorless solid. Spectral data for this product have been previously reported. ${ }^{15}$ Deuterium incorporation $=97 \%$ (crude: $99 \%, 99 \%)$, Reaction yield $=90 \%$ (184 mg). NMR spectra are given on pages $247-249$.

${ }^{1} \mathbf{H}$ NMR $\left(400 \mathrm{MHz}, \mathrm{CDCl}_{3}\right) \delta(\mathrm{ppm})=9.98(\mathrm{~s}, 0.03 \mathrm{H}), 7.77-7.74(\mathrm{~m}, 2 \mathrm{H}), 7.70-7.67(\mathrm{~m}, 2 \mathrm{H}) .{ }^{13} \mathrm{C} \mathrm{NMR}$ $\left(100 \mathrm{MHz}, \mathrm{CDCl}_{3}\right) \delta(\mathrm{ppm})=190.9(\mathrm{t}, J=27 \mathrm{~Hz}), 135.2(\mathrm{t}, J=4 \mathrm{~Hz}), 132.6,131.1,129.9$

\begin{tabular}{|c|c|c|c|c|c|}
\hline \multirow[t]{2}{*}{ Entry } & \multirow{2}{*}{$\begin{array}{c}3 \mathbf{a} \\
(\mathrm{mol} \%)\end{array}$} & \multirow{2}{*}{$\begin{array}{l}\text { DIPEA } \\
\text { (mol\%) }\end{array}$} & \multirow{2}{*}{$\begin{array}{c}\mathrm{D}_{2} \mathrm{O} \\
\text { (eq } \\
\text { ) }\end{array}$} & & \multirow{2}{*}{$\begin{array}{c}\text { Ph- } \\
\text { Boroxine } \\
\text { (mol\%) }\end{array}$} \\
\hline & & & & & \\
\hline
\end{tabular}




\begin{tabular}{|c|c|c|c|c|c|c|c|c|c|c|}
\cline { 2 - 11 } & 5 & 5 & 30 & 4 & 0.5 & $50 \cong \mathrm{C}$ & 99 & 100 & 97 & 90 \\
\hline $1^{\prime}$ & 5 & 5 & 30 & 4 & 0.5 & $50 \cong \mathrm{C}$ & 99 & 100 & - & - \\
\hline
\end{tabular}




\section{3,5-Dibromobenzaldehyde-d}<smiles>[2H]C(=O)c1cc(Br)cc(Br)c1</smiles>

3a (23.5 mg, $0.055 \mathrm{mmol})$, DIPEA (10 $\mu \mathrm{L}, 0.055 \mathrm{mmol}), \mathrm{D}_{2} \mathrm{O}(600 \mu \mathrm{L}, 33 \mathrm{mmol})$, phenyl boroxine (13.7 $\mathrm{mg}, 0.044 \mathrm{mmol}$ ) and 3,5-dibromobenzaldehyde $(290.3 \mu \mathrm{L}, 1.1 \mathrm{mmol}$ ) in anhydrous toluene $(1.60 \mathrm{~mL}$ ) were prepared according to the general procedure given in section 5 (page 20) and stirred at $50 \stackrel{\circ}{ } \mathrm{C}$. Crude residue was purified by flash-column chromatography using $\mathrm{Hex}^{\mathrm{Et}} \mathrm{t}_{2} \mathrm{O}$ (93:7) combination to give colorless solid.

Deuterium incorporation = 95\% (crude: 96\%, 95\%), Reaction yield = 91\% (264 mg). NMR spectra are given on pages $250-252$.

${ }^{1} \mathrm{H}$ NMR $\left(500 \mathrm{MHz}, \mathrm{CDCl}_{3}\right) \delta(\mathrm{ppm})=9.90(\mathrm{~s}, 0.05 \mathrm{H}), 7.94(\mathrm{~d}, J=2 \mathrm{~Hz}, 2 \mathrm{H}), 7.91(\mathrm{t}, J=2 \mathrm{~Hz}, 1 \mathrm{H}) .{ }^{13} \mathrm{C}$ NMR $\left(125 \mathrm{MHz}, \mathrm{CDCl}_{3}\right) \delta(\mathrm{ppm})=189.0(\mathrm{t}, J=34 \mathrm{~Hz}), 139.7,139.0(\mathrm{t}, J=5 \mathrm{~Hz}), 131.3,124.1$. HRMS(ESI) calcd for $\mathrm{C}_{7} \mathrm{H}_{3} \mathrm{DBr}_{2} \mathrm{O}^{+}(\mathrm{M}+\mathrm{H})^{+}: 265.8744$, Found: 265.8742

\begin{tabular}{|c|c|c|c|c|c|c|c|c|c|c|}
\hline \multirow{2}{*}{ Entry } & \multirow{2}{*}{$\begin{array}{c}3 \mathbf{a} \\
(\mathrm{mol} \%)\end{array}$} & \multirow{2}{*}{$\begin{array}{l}\text { DIPEA } \\
\text { (mol\%) }\end{array}$} & \multirow{2}{*}{$\begin{array}{l}\mathrm{D}_{2} \mathrm{O} \\
\text { (eq. } \\
\text { ) }\end{array}$} & \multirow{2}{*}{$\begin{array}{l}\text { Ph- } \\
\text { Boroxine } \\
\text { (mol\%) }\end{array}$} & \multirow{2}{*}{$\begin{array}{l}\text { Reactio } \\
\text { n Conc. } \\
\text { (M) }\end{array}$} & \multirow{2}{*}{$\begin{array}{l}\text { Reactio } \\
\text { n Temp. }\end{array}$} & \multirow{2}{*}{$\begin{array}{c}\% \mathrm{D} \\
\text { (RM) }\end{array}$} & \multirow{2}{*}{$\begin{array}{c}\% \\
\text { Aldehyd } \\
\mathrm{e}\end{array}$} & \multicolumn{2}{|c|}{$\begin{array}{c}\text { After } \\
\text { Purification }\end{array}$} \\
\hline & & & & & & & & & $\% \mathrm{D}$ & \%Yield \\
\hline 1 & 5 & 5 & 30 & 4 & 0.5 & $50 \stackrel{\circ}{ }$ & 96 & 100 & 95 & 91 \\
\hline $1^{\prime}$ & 5 & 5 & 30 & 4 & 0.5 & 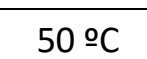 & 95 & 100 & - & - \\
\hline
\end{tabular}

\section{3-lodobenzaldehyde-d}<smiles>[2H]C(=O)c1cccc(I)c1</smiles>

3a (23.5 mg, $0.055 \mathrm{mmol})$, DIPEA $(10 \mu \mathrm{L}, 0.055 \mathrm{mmol}), \mathrm{D}_{2} \mathrm{O}(600 \mu \mathrm{L}, 33 \mathrm{mmol})$, phenyl boroxine (13.7 mg, $0.044 \mathrm{mmol}$ ) and 3-iodobenzaldehyde ( $255 \mathrm{mg}, 1.1 \mathrm{mmol}$ ) in anhydrous toluene (1.60 mL) were prepared according to the general procedure given in section 5 (page 20) and stirred at 50 ㅇ. Crude residue was purified by flash-column chromatography using $\mathrm{Hex}_{\mathrm{Et}} \mathrm{O}$ (90:10) combination to give colorless solid. Spectral data for this product have been previously reported. ${ }^{18}$

Deuterium incorporation = 97\% (crude: 99\%, 99\%), Reaction yield = 94\% (241 mg). NMR spectra are given on pages $253-255$. 
${ }^{1} \mathrm{H}$ NMR $\left(400 \mathrm{MHz}, \mathrm{CDCl}_{3}\right) \delta(\mathrm{ppm})=9.93(\mathrm{~s}, 0.03 \mathrm{H}), 8.22(\mathrm{t}, J=4 \mathrm{~Hz}, 1 \mathrm{H}), 7.96(\mathrm{dt}, J=4 \mathrm{~Hz}, 1 \mathrm{H})$, $7.85(\mathrm{dt}, J=4 \mathrm{~Hz}, 1 \mathrm{H}), 7.29(\mathrm{t}, J=8 \mathrm{~Hz}, 1 \mathrm{H}) .{ }^{13} \mathrm{C} \mathrm{NMR}\left(100 \mathrm{MHz}, \mathrm{CDCl}_{3}\right) \delta(\mathrm{ppm})=190.5(\mathrm{t}, J=27$ $\mathrm{Hz}), 143.3,138.6,138.1(\mathrm{t}, J=4 \mathrm{~Hz}), 130.9,129.0,94.8$.

\begin{tabular}{|c|c|c|c|c|c|c|c|c|c|c|}
\hline \multirow{2}{*}{ Entry } & \multirow{2}{*}{$\begin{array}{c}3 a \\
\text { (mol\%) }\end{array}$} & \multirow{2}{*}{$\begin{array}{l}\text { DIPEA } \\
\text { (mol\%) }\end{array}$} & \multirow{2}{*}{$\begin{array}{l}\mathrm{D}_{2} \mathrm{O} \\
\text { (eq. } \\
\text { ) }\end{array}$} & \multirow{2}{*}{$\begin{array}{c}\text { Ph- } \\
\text { Boroxine } \\
\text { (mol\%) }\end{array}$} & \multirow{2}{*}{$\begin{array}{l}\text { Reactio } \\
\text { n Conc. } \\
(\mathrm{M})\end{array}$} & \multirow{2}{*}{$\begin{array}{l}\text { Reactio } \\
\text { n Temp. }\end{array}$} & \multirow{2}{*}{$\begin{array}{c}\% \mathrm{D} \\
\text { (RM) }\end{array}$} & \multirow{2}{*}{$\begin{array}{c}\% \\
\text { Aldehyd } \\
\text { e }\end{array}$} & \multicolumn{2}{|c|}{$\begin{array}{c}\text { After } \\
\text { Purification }\end{array}$} \\
\hline & & & & & & & & & $\% \mathrm{D}$ & \%Yield \\
\hline 1 & 5 & 5 & 30 & 4 & 0.5 & $50 \stackrel{\circ}{ }$ & 99 & 100 & 97 & 94 \\
\hline $1^{\prime}$ & 5 & 5 & 30 & 4 & 0.5 & $50 \stackrel{\circ}{ }$ & 99 & 100 & - & - \\
\hline
\end{tabular}

\section{4-lodobenzaldehyde-d}<smiles>[2H]C(=O)c1ccc(I)cc1</smiles>

3a (23.5 mg, $0.055 \mathrm{mmol})$, DIPEA (10 $\mu \mathrm{L}, 0.055 \mathrm{mmol}), \mathrm{D}_{2} \mathrm{O}(600 \mu \mathrm{L}, 33 \mathrm{mmol})$, phenyl boroxine (13.7 $\mathrm{mg}, 0.044 \mathrm{mmol}$ ) and 4-iodobenzaldehyde ( $255 \mathrm{mg}, 1.1 \mathrm{mmol})$ in anhydrous toluene (1.60 $\mathrm{mL})$ were prepared according to the general procedure given in section 5 (page 20) and stirred at 50 o C. Crude residue was purified by flash-column chromatography using $\mathrm{Hex}_{\mathrm{Et}} \mathrm{O}$ (92:8) combination to give colorless solid. Spectral data for this product have been previously reported. ${ }^{19}$

Deuterium incorporation $=97 \%$ (crude: $100 \%, 99 \%)$, Reaction yield $=91 \%$ (233 mg). NMR spectra are given on pages $256-258$.

${ }^{1} \mathrm{H}$ NMR $\left(400 \mathrm{MHz}, \mathrm{CDCl}_{3}\right) \delta(\mathrm{ppm})=9.96(\mathrm{~s}, 0.03 \mathrm{H}), 7.91(\mathrm{~d}, J=8 \mathrm{~Hz}, 2 \mathrm{H}), 7.59(\mathrm{~d}, J=8 \mathrm{~Hz}, 2 \mathrm{H}) .{ }^{13} \mathrm{C}$ $\operatorname{NMR}\left(100 \mathrm{MHz}, \mathrm{CDCl}_{3}\right) \delta(\mathrm{ppm})=191.2(\mathrm{t}, J=27 \mathrm{~Hz}), 138.6,135.6(\mathrm{t}, J=4 \mathrm{~Hz}), 130.9,103.0$

\begin{tabular}{|c|c|c|c|c|c|c|c|c|c|c|}
\hline \multirow[t]{2}{*}{ Entry } & \multirow{2}{*}{$\begin{array}{c}3 a \\
(\mathrm{~mol} \%)\end{array}$} & \multirow{2}{*}{$\begin{array}{l}\text { DIPEA } \\
\text { (mol\%) }\end{array}$} & \multirow{2}{*}{$\begin{array}{c}\mathrm{D}_{2} \mathrm{O} \\
\text { (eq. } \\
\text { ) }\end{array}$} & \multirow{2}{*}{$\begin{array}{c}\text { Ph- } \\
\text { Boroxine } \\
\text { (mol\%) }\end{array}$} & \multirow{2}{*}{$\begin{array}{l}\text { Reactio } \\
\text { n Conc. } \\
\text { (M) }\end{array}$} & \multirow{2}{*}{$\begin{array}{l}\text { Reactio } \\
\text { n Temp. }\end{array}$} & \multirow{2}{*}{$\begin{array}{l}\% \mathrm{D} \\
\text { (RM) }\end{array}$} & \multirow{2}{*}{$\begin{array}{c}\% \\
\text { Aldehyd } \\
\text { e }\end{array}$} & \multicolumn{2}{|c|}{$\begin{array}{c}\text { After } \\
\text { Purification } \\
\end{array}$} \\
\hline & & & & & & & & & $\% \mathrm{D}$ & \%Yield \\
\hline 1 & 5 & 5 & 30 & 4 & 0.5 & $50 \stackrel{\circ}{C}$ & 100 & 100 & 97 & 91 \\
\hline $1^{\prime}$ & 5 & 5 & 30 & 4 & 0.5 & $50 \stackrel{\circ}{ }$ & 99 & 100 & - & - \\
\hline
\end{tabular}




\section{Methyl 3-formylbenzoate-d}<smiles>[2H]C(=O)c1cccc(C(=O)OC)c1</smiles>

3a (23.5 mg, $0.055 \mathrm{mmol})$, DIPEA $(10 \mu \mathrm{L}, 0.055 \mathrm{mmol}), \mathrm{D}_{2} \mathrm{O}(600 \mu \mathrm{L}, 33 \mathrm{mmol})$, phenyl boroxine (13.7 $\mathrm{mg}, 0.044 \mathrm{mmol}$ ) and methyl 3-formylbenzoate $(181 \mathrm{mg}, 1.1 \mathrm{mmol})$ in anhydrous toluene $(1.60 \mathrm{~mL}$ ) were prepared according to the general procedure given in section 5 (page 20) and stirred at 50 o. C. Crude residue was purified by flash-column chromatography using Hex:Et ${ }_{2} \mathrm{O}$ (91:9) combination to give colorless solid. Spectral data for this product have been previously reported. ${ }^{15}$ Deuterium incorporation $=95 \%$ (crude: $97 \%, 97 \%)$, Reaction yield = 94\% (171 mg). NMR spectra are given on pages $259-261$.

${ }^{1} \mathrm{H}$ NMR $\left(400 \mathrm{MHz}, \mathrm{CDCl}_{3}\right) \delta(\mathrm{ppm})=10.07(\mathrm{~s}, 0.05 \mathrm{H}), 8.53-8.52(\mathrm{~m}, 1 \mathrm{H}), 8.29(\mathrm{dt}, J=4 \mathrm{~Hz}, 1 \mathrm{H})$, $8.08(\mathrm{dt}, J=4 \mathrm{~Hz}, 1 \mathrm{H}), 7.64-7.60(\mathrm{~m}, 1 \mathrm{H}), 3.96(\mathrm{~s}, 3 \mathrm{H}) .{ }^{13} \mathrm{C} \mathrm{NMR}\left(100 \mathrm{MHz}, \mathrm{CDCl}_{3}\right) \delta(\mathrm{ppm})=191.2$ $(\mathrm{t}, J=27 \mathrm{~Hz}), 166.1,136.6(\mathrm{t}, J=4 \mathrm{~Hz}), 135.3,133.2,131.4,131.3,129.4,52.6$

\begin{tabular}{|c|c|c|c|c|c|c|c|c|c|c|}
\hline \multirow[t]{2}{*}{ Entry } & \multirow{2}{*}{$\begin{array}{c}3 a \\
(\mathrm{~mol} \%)\end{array}$} & \multirow{2}{*}{$\begin{array}{l}\text { DIPEA } \\
\text { (mol\%) }\end{array}$} & \multirow{2}{*}{$\begin{array}{l}\mathrm{D}_{2} \mathrm{O} \\
\text { (eq. } \\
\text { ) }\end{array}$} & \multirow{2}{*}{$\begin{array}{c}\text { Ph- } \\
\text { Boroxine } \\
\text { (mol\%) }\end{array}$} & \multirow{2}{*}{$\begin{array}{c}\text { Reactio } \\
\text { n Conc. } \\
\text { (M) }\end{array}$} & \multirow{2}{*}{$\begin{array}{l}\text { Reactio } \\
\text { n Temp. }\end{array}$} & \multirow{2}{*}{$\begin{array}{l}\% \mathrm{D} \\
\text { (RM) }\end{array}$} & \multirow{2}{*}{$\begin{array}{c}\% \\
\text { Aldehyd } \\
\text { e }\end{array}$} & \multicolumn{2}{|c|}{$\begin{array}{c}\text { After } \\
\text { Purification }\end{array}$} \\
\hline & & & & & & & & & $\% \mathrm{D}$ & \%Yield \\
\hline 1 & 5 & 5 & 30 & 4 & 0.5 & 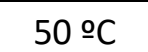 & 97 & 100 & 95 & 94 \\
\hline $1^{\prime}$ & 5 & 5 & 30 & 4 & 0.5 & $50 \stackrel{\circ}{C}$ & 97 & 100 & - & - \\
\hline
\end{tabular}

\section{Methyl 4-formylbenzoate-d}<smiles>[2H]C(=O)c1ccc(C(=O)OC)cc1</smiles>

3a (23.5 mg, $0.055 \mathrm{mmol})$, DIPEA (10 $\mu \mathrm{L}, 0.055 \mathrm{mmol}), \mathrm{D}_{2} \mathrm{O}(600 \mu \mathrm{L}, 33 \mathrm{mmol})$, phenyl boroxine (13.7 $\mathrm{mg}, 0.044 \mathrm{mmol}$ ) and methyl 4-formylbenzoate (181 $\mathrm{mg}, 1.1 \mathrm{mmol}$ ) in anhydrous toluene $(1.60 \mathrm{~mL}$ ) were prepared according to the general procedure given in section 5 (page 20) and stirred at $50 \stackrel{\circ}{ } \mathrm{C}$. Crude residue was purified by flash-column chromatography using Hex: $\mathrm{Et}_{2} \mathrm{O}$ (91:9) combination to give colorless solid. Spectral data for this product have been previously reported. ${ }^{15}$ 
Deuterium incorporation = 97\% (crude: 99\%, 99\%), Reaction yield = 83\% (151 mg). NMR spectra are given on pages $262-264$.

${ }^{1} \mathbf{H}$ NMR $\left(400 \mathrm{MHz}, \mathrm{CDCl}_{3}\right) \delta(\mathrm{ppm})=10.11(\mathrm{~s}, 0.03 \mathrm{H}), 8.22-8.19(\mathrm{~m}, 2 \mathrm{H}), 7.97-7.94(\mathrm{~m}, 2 \mathrm{H}), 3.97$ (s, 3H). ${ }^{13} \mathrm{C}$ NMR $\left(100 \mathrm{MHz}, \mathrm{CDCl}_{3}\right) \delta(\mathrm{ppm})=191.5(\mathrm{t}, J=27 \mathrm{~Hz}), 166.2,139.2(\mathrm{t}, J=4 \mathrm{~Hz}), 135.3$, $130.3,129.7,52.7$

\begin{tabular}{|c|c|c|c|c|c|c|c|c|c|c|}
\hline \multirow[t]{2}{*}{ Entry } & \multirow{2}{*}{$\begin{array}{c}3 a \\
(\mathrm{~mol} \%)\end{array}$} & \multirow{2}{*}{$\begin{array}{l}\text { DIPEA } \\
\text { (mol\%) }\end{array}$} & \multirow{2}{*}{$\begin{array}{c}\mathrm{D}_{2} \mathrm{O} \\
\text { (eq. } \\
\text { ) }\end{array}$} & \multirow{2}{*}{$\begin{array}{c}\text { Ph- } \\
\text { Boroxine } \\
\text { (mol\%) }\end{array}$} & \multirow{2}{*}{$\begin{array}{l}\text { Reactio } \\
\text { n Conc. } \\
\text { (M) }\end{array}$} & \multirow{2}{*}{$\begin{array}{l}\text { Reactio } \\
\text { n Temp. }\end{array}$} & \multirow{2}{*}{$\begin{array}{c}\% \mathrm{D} \\
\text { (RM) }\end{array}$} & \multirow{2}{*}{$\begin{array}{c}\% \\
\text { Aldehyd } \\
\text { e }\end{array}$} & \multicolumn{2}{|c|}{$\begin{array}{c}\text { After } \\
\text { Purification }\end{array}$} \\
\hline & & & & & & & & & $\% \mathrm{D}$ & \%Yield \\
\hline 1 & 5 & 5 & 30 & 4 & 0.5 & 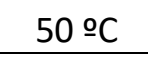 & 99 & 100 & 97 & 83 \\
\hline $1^{\prime}$ & 5 & 5 & 30 & 4 & 0.5 & $50 \stackrel{\circ}{C}$ & 99 & 100 & - & - \\
\hline
\end{tabular}

\section{3-(Trifluoromethyl)benzaldehyde-d}<smiles>[2H]C(=O)c1cccc(C(F)(F)F)c1</smiles>

3a (23.5 mg, $0.055 \mathrm{mmol})$, DIPEA $(10 \mu \mathrm{L}, 0.055 \mathrm{mmol}), \mathrm{D}_{2} \mathrm{O}(600 \mu \mathrm{L}, 33 \mathrm{mmol})$, phenyl boroxine $(13.7 \mathrm{mg}, 0.044 \mathrm{mmol})$ and 3-(trifluoromethyl)benzaldehyde $(147 \mu \mathrm{L}, 1.1 \mathrm{mmol})$ in anhydrous toluene $(1.60 \mathrm{~mL}$ ) were prepared according to the general procedure given in section 5 (page 20) and stirred at $50 \stackrel{\circ}{ } \mathrm{C}$. Crude residue was purified by flash-column chromatography using Hex:Et ${ }_{2} \mathrm{O}$ (98:2) combination to give colorless liquid. Spectral data for this product have been previously reported. ${ }^{20}$

Deuterium incorporation $=98 \%$ (crude: $100 \%, 99 \%)$, Reaction yield $=90 \%$ (173 mg). NMR spectra are given on pages $265-267$.

${ }^{1} \mathrm{H}$ NMR $\left(500 \mathrm{MHz}, \mathrm{CDCl}_{3}\right) \delta(\mathrm{ppm})=10.08(\mathrm{~s}, 0.02 \mathrm{H}), 8.15(\mathrm{~s}, 1 \mathrm{H}), 8.08(\mathrm{~d}, J=10 \mathrm{~Hz}, 1 \mathrm{H}), 7.89(\mathrm{~d}$, $J=10 \mathrm{~Hz}, 1 \mathrm{H}), 7.69(\mathrm{t}, J=10 \mathrm{~Hz}, 1 \mathrm{H}) .{ }^{13} \mathrm{C}$ NMR $\left(125 \mathrm{MHz}^{\mathrm{C}} \mathrm{CDCl}_{3}\right) \delta(\mathrm{ppm})=190.6(\mathrm{t}, J=26 \mathrm{~Hz}), 136.8$, $132.8,131.9(q, J=34 \mathrm{~Hz}), 130.9,129.9,126.5,123.6(q, J=271 \mathrm{~Hz}) .{ }^{19} \mathrm{~F}$ NMR $\left(376 \mathrm{MHz}, \mathrm{CDCl}_{3}\right) \delta$ $(\mathrm{ppm})=-63.0(\mathrm{~s}, 3 \mathrm{~F})$

\begin{tabular}{|c|c|c|c|c|c|c|c|c|c|c|}
\hline Entry & $\begin{array}{c}3 a \\
\text { (mol\%) }\end{array}$ & $\begin{array}{c}\text { DIPEA } \\
\text { (mol\%) }\end{array}$ & $\begin{array}{c}\mathrm{D}_{2} \mathrm{O} \\
\text { (eq. } \\
\text { ) }\end{array}$ & $\begin{array}{c}\text { Ph- } \\
\text { Boroxine } \\
\text { (mol\%) }\end{array}$ & $\begin{array}{c}\text { Reactio } \\
\mathrm{n} \text { Conc. } \\
\text { (M) }\end{array}$ & $\begin{array}{c}\text { Reactio } \\
\text { n Temp. }\end{array}$ & $\begin{array}{c}\% \mathrm{D} \\
\text { (RM) }\end{array}$ & $\begin{array}{c}\% \\
\text { Aldehyd } \\
\text { e }\end{array}$ & \multicolumn{2}{|c|}{$\begin{array}{c}\text { After } \\
\text { Purification }\end{array}$} \\
\hline 1 & 5 & 5 & 30 & 4 & 0.5 & $50 \cong \mathrm{C}$ & 100 & 100 & 98 & 90 \\
\hline $1^{\prime}$ & 5 & 5 & 30 & 4 & 0.5 & $50 \cong \mathrm{C}$ & 99 & 100 & - & - \\
\hline
\end{tabular}




\section{4-(Trifluoromethyl)benzaldehyde-d}<smiles>[2H]C(=O)c1ccc(C(F)(F)F)cc1</smiles>

3a (23.5 mg, $0.055 \mathrm{mmol})$, DIPEA $(10 \mu \mathrm{L}, 0.055 \mathrm{mmol}), \mathrm{D}_{2} \mathrm{O}(600 \mu \mathrm{L}, 33 \mathrm{mmol})$, phenyl boroxine (13.7 $\mathrm{mg}, 0.044 \mathrm{mmol})$ and 4-(trifluoromethyl)benzaldehyde $(150 \mu \mathrm{L}, 1.1 \mathrm{mmol})$ in anhydrous toluene $(1.60 \mathrm{~mL}$ ) were prepared according to the general procedure given in section 5 (page 20) and stirred at $50 \stackrel{\circ}{ } \mathrm{C}$. Crude residue was purified by flash-column chromatography using $\mathrm{Hex}: \mathrm{Et}_{2} \mathrm{O}$ (98:2) combination to give colorless liquid. Spectral data for this product have been previously reported. ${ }^{15}$

Deuterium incorporation $=98 \%$ (crude: $100 \%, 100 \%)$, Reaction yield $=94 \%$ (181 mg). NMR spectra are given on pages $268-270$.

${ }^{1} \mathrm{H}$ NMR $\left(500 \mathrm{MHz}, \mathrm{CDCl}_{3}\right) \delta(\mathrm{ppm})=10.10(\mathrm{~s}, 0.02 \mathrm{H}), 8.01(\mathrm{~d}, J=8 \mathrm{~Hz}, 2 \mathrm{H}), 7.81(\mathrm{~d}, J=8 \mathrm{~Hz}, 2 \mathrm{H})$.

${ }^{13} \mathrm{C}$ NMR $\left(125 \mathrm{MHz}, \mathrm{CDCl}_{3}\right) \delta(\mathrm{ppm})=190.9(\mathrm{t}, J=28 \mathrm{~Hz}), 138.7,135.8(\mathrm{q}, J=33 \mathrm{~Hz}), 130.1,126.3$, $123.6(\mathrm{q}, J=271 \mathrm{~Hz}) .{ }^{19} \mathrm{~F} \mathrm{NMR}\left(376 \mathrm{MHz}, \mathrm{CDCl}_{3}\right) \delta(\mathrm{ppm})=-63.2(\mathrm{~s}, 3 \mathrm{~F})$

\begin{tabular}{|c|c|c|c|c|c|c|c|c|c|c|}
\hline Entry & $\begin{array}{c}3 a \\
\text { (mol\%) }\end{array}$ & $\begin{array}{c}\text { DIPEA } \\
\text { (mol\%) }\end{array}$ & $\begin{array}{c}\mathrm{D}_{2} \mathrm{O} \\
\text { (eq. }\end{array}$ & $\begin{array}{c}\text { Ph- } \\
\text { Boroxine } \\
\text { (mol\%) }\end{array}$ & $\begin{array}{c}\text { Reactio } \\
\text { n Conc. } \\
\text { (M) }\end{array}$ & $\begin{array}{c}\text { Reactio } \\
\text { n Temp. }\end{array}$ & $\begin{array}{c}\text { \%D } \\
\text { (RM) }\end{array}$ & $\begin{array}{c}\% \\
\text { Aldehyd } \\
\text { e }\end{array}$ & \multicolumn{2}{|c|}{$\begin{array}{c}\text { After } \\
\text { Purification }\end{array}$} \\
\hline 1 & 5 & 5 & 30 & 4 & 0.5 & 50 \% & 100 & 100 & 98 & 94 \\
\hline $1^{\prime}$ & 5 & 5 & 30 & 4 & 0.5 & $50 \cong \mathrm{C}$ & 100 & 100 & - & - \\
\hline
\end{tabular}

\section{4-Cyanobenzaldehyde-d}<smiles>[2H]C(=O)c1ccc(C#N)cc1</smiles>

3a (23.5 mg, $0.055 \mathrm{mmol})$, DIPEA (10 $\mu \mathrm{L}, 0.055 \mathrm{mmol}), \mathrm{D}_{2} \mathrm{O}(600 \mu \mathrm{L}, 33 \mathrm{mmol})$, phenyl boroxine $(13.7 \mathrm{mg}, 0.044 \mathrm{mmol})$ and 4-cyanobenzaldehyde (144 $\mathrm{mg}, 1.1 \mathrm{mmol})$ in anhydrous toluene (1.60 $\mathrm{mL}$ ) were prepared according to the general procedure given in section 5 (page 20) and stirred at 50 으. Crude residue was purified by flash-column chromatography using Hex:Et ${ }_{2} \mathrm{O}$ (91:9) combination to give colorless solid. Spectral data for this product have been previously reported. ${ }^{15}$ 
Deuterium incorporation $=95 \%$ (crude: $100 \%, 100 \%)$, Reaction yield $=82 \%$ (119 mg). NMR spectra are given on pages $271-273$.

${ }^{1} \mathbf{H}$ NMR $\left(400 \mathrm{MHz}, \mathrm{CDCl}_{3}\right) \delta(\mathrm{ppm})=10.10(\mathrm{~s}, 0.05 \mathrm{H}), 8.01-7.99(\mathrm{~m}, 2 \mathrm{H}), 7.86-7.83(\mathrm{~m}, 2 \mathrm{H}) .{ }^{13} \mathrm{C}$ $\operatorname{NMR}\left(100 \mathrm{MHz}, \mathrm{CDCl}_{3}\right) \delta(\mathrm{ppm})=190.4(\mathrm{t}, J=27 \mathrm{~Hz}), 138.8(\mathrm{t}, J=4 \mathrm{~Hz}), 133.0,130.0,117.9,117.8$

\begin{tabular}{|c|c|c|c|c|c|c|c|c|c|c|}
\hline \multirow{2}{*}{ Entry } & \multirow{2}{*}{$\begin{array}{c}\mathbf{3 a} \\
(\mathrm{mol} \%)\end{array}$} & \multirow{2}{*}{$\begin{array}{l}\text { DIPEA } \\
\text { (mol\%) }\end{array}$} & \multirow{2}{*}{$\begin{array}{c}\mathrm{D}_{2} \mathrm{O} \\
\text { (eq. } \\
\text { ) }\end{array}$} & \multirow{2}{*}{$\begin{array}{c}\text { Ph- } \\
\text { Boroxine } \\
\text { (mol\%) }\end{array}$} & \multirow{2}{*}{$\begin{array}{l}\text { Reactio } \\
\text { n Conc. } \\
\text { (M) }\end{array}$} & \multirow{2}{*}{$\begin{array}{l}\text { Reactio } \\
\text { n Temp. }\end{array}$} & \multirow{2}{*}{$\begin{array}{c}\% \mathrm{D} \\
\text { (RM) }\end{array}$} & \multirow{2}{*}{$\begin{array}{c}\% \\
\text { Aldehyd } \\
\text { e }\end{array}$} & \multicolumn{2}{|c|}{$\begin{array}{c}\text { After } \\
\text { Purification }\end{array}$} \\
\hline & & & & & & & & & $\% \mathrm{D}$ & \%Yield \\
\hline 1 & 5 & 5 & 30 & 4 & 0.5 & $50 \stackrel{\circ}{ }$ & 100 & 100 & 95 & 82 \\
\hline $1^{\prime}$ & 5 & 5 & 30 & 4 & 0.5 & $50 \stackrel{\circ}{ } \mathrm{C}$ & 100 & 100 & - & - \\
\hline
\end{tabular}




\section{Condition 5:}
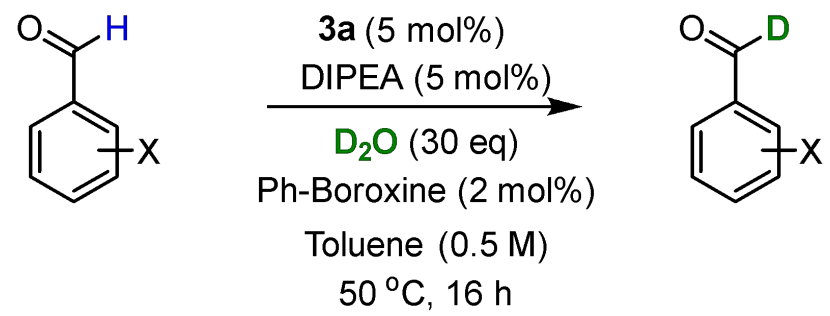

Scheme 22: Reaction condition $5(\mathrm{Ph}=$ Phenyl)

\section{3-Cyanobenzaldehyde-d}<smiles>[2H]C(=O)c1cccc(C#N)c1</smiles>

3a (23.5 mg, $0.055 \mathrm{mmol})$, DIPEA ( $10 \mu \mathrm{L}, 0.055 \mathrm{mmol}), \mathrm{D}_{2} \mathrm{O}(600 \mu \mathrm{L}, 33 \mathrm{mmol})$, phenyl boroxine (6.9 $\mathrm{mg}, 0.022 \mathrm{mmol}$ ) and 4-cyanobenzaldehyde $(144 \mathrm{mg}, 1.1 \mathrm{mmol})$ in anhydrous toluene $(1.60 \mathrm{~mL})$ were prepared according to the general procedure given in section 5 (page 20) and stirred at 50 ㅇ․ Crude residue was purified by flash-column chromatography using $\mathrm{Hex}_{\mathrm{E}} \mathrm{Et}_{2} \mathrm{O}$ (91:9) combination to give colorless solid. Spectral data for this product have been previously reported..$^{15}$

Deuterium incorporation $=95 \%$ (crude: $98 \%, 97 \%)$, Reaction yield $=83 \%$ (121 mg). NMR spectra are given on pages $274-277$.

${ }^{1} \mathrm{H}$ NMR $\left(400 \mathrm{MHz}, \mathrm{CDCl}_{3}\right) \delta(\mathrm{ppm})=10.05(\mathrm{~s}, 0.05 \mathrm{H}), 8.18(\mathrm{t}, J=4 \mathrm{~Hz}, 1 \mathrm{H}), 8.13(\mathrm{dt}, J=4 \mathrm{~Hz}, 1 \mathrm{H})$, $7.92(\mathrm{dt}, J=4 \mathrm{~Hz}, 1 \mathrm{H}), 7.70(\mathrm{t}, J=4 \mathrm{~Hz}, 1 \mathrm{H}) .{ }^{13} \mathrm{C} \mathrm{NMR}\left(100 \mathrm{MHz}, \mathrm{CDCl}_{3}\right) \delta(\mathrm{ppm})=189.7(\mathrm{t}, J=27$ $\mathrm{Hz}), 137.4,136.9(\mathrm{t}, J=4 \mathrm{~Hz}), 133.4,133.3,130.2,117.7,113.9$

\begin{tabular}{|c|c|c|c|c|c|c|c|c|c|c|}
\hline Entry & $\begin{array}{c}3 a \\
\text { (mol\%) }\end{array}$ & $\begin{array}{c}\text { DIPEA } \\
\text { (mol\%) }\end{array}$ & $\begin{array}{c}\mathrm{D}_{2} \mathrm{O} \\
\text { (eq. } \\
\text { ) }\end{array}$ & $\begin{array}{c}\text { Boroxine } \\
\text { (mol\%) }\end{array}$ & $\begin{array}{c}\text { Reactio } \\
\text { n Conc. } \\
\text { (M) }\end{array}$ & $\begin{array}{c}\text { Reactio } \\
\text { n Temp. }\end{array}$ & $\begin{array}{c}\text { \%D } \\
\text { (RM) }\end{array}$ & $\begin{array}{c}\% \\
\text { Aldehyd } \\
\text { e }\end{array}$ & \multicolumn{2}{|c|}{$\begin{array}{c}\text { After } \\
\text { Purification }\end{array}$} \\
\hline 1 & 5 & 5 & 30 & 4 & 0.5 & $50 \cong \mathrm{C}$ & 92 & 100 & - & \%Yield \\
\hline $1^{\prime}$ & 5 & 5 & 30 & 4 & 0.5 & $50 \cong \mathrm{C}$ & 88 & 100 & - & - \\
\hline 2 & 5 & 5 & 30 & 2 & 0.5 & $50 \cong \mathrm{C}$ & 98 & 100 & 95 & 83 \\
\hline $2^{\prime}$ & 5 & 5 & 30 & 2 & 0.5 & $50 \cong \mathrm{C}$ & 97 & 100 & - & - \\
\hline
\end{tabular}




\section{4-Fluorobenzaldehyde-d}<smiles>[2H]C(=O)c1ccc(F)cc1</smiles>

3a (23.5 mg, $0.055 \mathrm{mmol})$, DIPEA (10 $\mu \mathrm{L}, 0.055 \mathrm{mmol}), \mathrm{D}_{2} \mathrm{O}(600 \mu \mathrm{L}, 33 \mathrm{mmol})$, phenyl boroxine (6.9 $\mathrm{mg}, 0.022 \mathrm{mmol})$ and 4-fluorobenzaldehyde $(118 \mu \mathrm{L}, 1.1 \mathrm{mmol})$ in anhydrous toluene $(1.60 \mathrm{~mL})$ were prepared according to the general procedure given in section 5 (page 20) and stirred at 50 ㅇ․ Crude residue was purified by flash-column chromatography using $\mathrm{Hex}_{\mathrm{Et}} \mathrm{O}$ (98:2) combination to give colorless liquid. Spectral data for this product have been previously reported. ${ }^{15}$

Deuterium incorporation $=98 \%$ (crude: 100\%, 100\%), Reaction yield $=97 \%$ (133 mg). NMR spectra are given on pages $278-282$.

${ }^{1} \mathrm{H}$ NMR $\left(500 \mathrm{MHz}, \mathrm{CDCl}_{3}\right) \delta(\mathrm{ppm})=9.96(\mathrm{~s}, 0.02 \mathrm{H}), 7.93-7.89(\mathrm{~m}, 2 \mathrm{H}), 7.23-7.18(\mathrm{~m}, 2 \mathrm{H}) .{ }^{13} \mathrm{C}$ NMR $\left(125 \mathrm{MHz} \mathrm{CDCl}_{3}\right) \delta(\mathrm{ppm})=190.3(\mathrm{t}, J=28 \mathrm{~Hz}), 166.7(\mathrm{~d}, J=255 \mathrm{~Hz}), 133.0,132.4(\mathrm{~d}, J=9 \mathrm{~Hz}), 116.5$ $(\mathrm{d}, J=23 \mathrm{~Hz}) .{ }^{19} \mathrm{~F}$ NMR $\left(376 \mathrm{MHz}, \mathrm{CDCl}_{3}\right) \delta(\mathrm{ppm})=-102.4(\mathrm{~s}, 1 \mathrm{~F})$

\begin{tabular}{|c|c|c|c|c|c|c|c|c|c|c|}
\hline \multirow{2}{*}{ Entry } & \multirow{2}{*}{$\begin{array}{c}3 \mathbf{a} \\
(\mathrm{mol} \%)\end{array}$} & \multirow{2}{*}{$\begin{array}{c}\text { DIPEA } \\
\text { (mol\%) }\end{array}$} & \multirow{2}{*}{$\begin{array}{c}\mathrm{D}_{2} \mathrm{O} \\
\text { (eq. } \\
\text { ) }\end{array}$} & \multirow{2}{*}{$\begin{array}{c}\text { Ph- } \\
\text { Boroxine } \\
\text { (mol\%) }\end{array}$} & \multirow{2}{*}{$\begin{array}{l}\text { Reactio } \\
\text { n Conc. } \\
\text { (M) }\end{array}$} & \multirow{2}{*}{$\begin{array}{l}\text { Reactio } \\
\text { n Temp. }\end{array}$} & \multirow{2}{*}{$\begin{array}{c}\% \mathrm{D} \\
\text { (RM) }\end{array}$} & \multirow{2}{*}{$\begin{array}{c}\% \\
\text { Aldehyd } \\
\text { e }\end{array}$} & \multicolumn{2}{|c|}{$\begin{array}{c}\text { After } \\
\text { Purification }\end{array}$} \\
\hline & & & & & & & & & $\% \mathrm{D}$ & \%Yield \\
\hline 1 & 5 & 5 & 30 & 4 & 0.5 & $50 \stackrel{\circ}{C}$ & 91 & 100 & - & - \\
\hline $1^{\prime}$ & 5 & 5 & 30 & 4 & 0.5 & $50 \stackrel{\circ}{ } \mathrm{C}$ & 88 & 100 & - & - \\
\hline 2 & 5 & 5 & 30 & 2 & 0.5 & $50 \div \mathrm{C}$ & 100 & 100 & 98 & 97 \\
\hline $2^{\prime}$ & 5 & 5 & 30 & 2 & 0.5 & $50 \stackrel{\circ}{ }$ & 100 & 100 & - & - \\
\hline
\end{tabular}

\section{2-Fluorobenzaldehyde-d}<smiles>[2H]C(=O)c1ccccc1F</smiles>

3a (23.5 mg, $0.055 \mathrm{mmol})$, DIPEA (10 $\mu \mathrm{L}, 0.055 \mathrm{mmol}), \mathrm{D}_{2} \mathrm{O}(600 \mu \mathrm{L}, 33 \mathrm{mmol})$, phenyl boroxine (6.9 $\mathrm{mg}, 0.022 \mathrm{mmol})$ and 2-fluorobenzaldehyde (116 $\mu \mathrm{L}, 1.1 \mathrm{mmol})$ in anhydrous toluene $(1.60 \mathrm{~mL})$ were prepared according to the general procedure given in section 5 (page 20) and stirred at 50 ㅇ․ Crude residue was purified by flash-column chromatography using $\mathrm{Hex}_{\mathrm{Et}} \mathrm{O}$ (98:2) combination to give colorless liquid. 
Deuterium incorporation = 98\% (crude: 99\%, 99\%), Reaction yield = 89\% (122 mg). NMR spectra are given on pages $283-287$.

Note: For this specific substrate the benzoin and aldehyde peaks merge. In the NMR spectra (for entry $1,1^{\prime}$ and 2 that contain large quantities of the benzoin product), the aldehyde signal appeard at $7.54 \mathrm{ppm}$ with integral value 1. The benzoin and aldehyde signal merge at $7.89 \mathrm{ppm}$ with a total integral value 6.74 (for entry 1). Therefore, the total integral value (6.74) was subtracted from the aldehyde integral value (1) for calculation of \%Benzoin.

${ }^{1} \mathbf{H}$ NMR $\left(500 \mathrm{MHz}, \mathrm{CDCl}_{3}\right) \delta(\mathrm{ppm})=10.37(\mathrm{~s}, 0.02 \mathrm{H}), 7.90-7.86(\mathrm{~m}, 1 \mathrm{H}), 7.64-7.58(\mathrm{~m}, 1 \mathrm{H}), 7.29-$ $7.25(\mathrm{~m}, 1 \mathrm{H}), 7.20-7.15(\mathrm{~m}, 1 \mathrm{H}) .{ }^{13} \mathrm{C} \mathrm{NMR}\left(125 \mathrm{MHz}, \mathrm{CDCl}_{3}\right) \delta(\mathrm{ppm})=187.0(\mathrm{tt}, J=28 \mathrm{~Hz}), 164.8(\mathrm{~d}$, $J=256 \mathrm{~Hz}), 136.5(\mathrm{~d}, J=9 \mathrm{~Hz}), 128.8,124.8,124.2,116.6$ (d, $J=20 \mathrm{~Hz}) .{ }^{19} \mathrm{~F} \mathrm{NMR}\left(376 \mathrm{MHz}, \mathrm{CDCl}_{3}\right) \delta$ $(p p m)=-122.0(s, 1 \mathrm{~F})$. HRMS(ESI) calcd for $\mathrm{C}_{7} \mathrm{H}_{4} \mathrm{DFO}^{+}(\mathrm{M}+\mathrm{H})^{+}:$126.0460, Found: 126.0457

\begin{tabular}{|c|c|c|c|c|c|c|c|c|c|c|}
\hline \multirow{3}{*}{$\begin{array}{c}\text { Entry } \\
1 \\
\end{array}$} & \multirow{2}{*}{$\begin{array}{c}3 \mathbf{a} \\
(\mathrm{mol} \%)\end{array}$} & \multirow{3}{*}{$\begin{array}{c}\mathrm{D}_{2} \mathrm{O} \\
\text { (eq. } \\
\text { ) } \\
30\end{array}$} & \multirow{3}{*}{$\begin{array}{c}\text { Ph- } \\
\text { Boroxine } \\
\text { (mol\%) } \\
-\end{array}$} & \multirow{3}{*}{$\begin{array}{c}\text { Reactio } \\
\text { n Conc. } \\
\text { (M) } \\
1\end{array}$} & \multirow{3}{*}{$\begin{array}{l}\text { Reactio } \\
\text { n } \\
\text { Temp. } \\
20^{\circ} \mathrm{C} \\
\end{array}$} & \multirow{3}{*}{$\begin{array}{c}\% \mathrm{D} \\
(\mathrm{RM}) \\
100 \\
\end{array}$} & \multirow{2}{*}{$\begin{array}{c}\% \\
\text { Aldehyd } \\
\mathrm{e}\end{array}$} & \multirow{2}{*}{$\begin{array}{c}\% \\
\text { Benzoin }\end{array}$} & \multicolumn{2}{|c|}{$\begin{array}{c}\text { After } \\
\text { Purification }\end{array}$} \\
\hline & & & & & & & & & $\% D$ & \%Yield \\
\hline & 5 & & & & & & 15 & 85 & - & - \\
\hline $1^{\prime}$ & 5 & 30 & - & 1 & $20^{\circ} \mathrm{C}$ & 100 & 10 & 90 & - & - \\
\hline 2 & 5 & 30 & 2 & 1 & $20^{\circ} \mathrm{C}$ & 97 & 87 & 13 & - & - \\
\hline 3 & 5 & 30 & 4 & 1 & $20^{\circ} \mathrm{C}$ & 16 & 100 & - & - & - \\
\hline 4 & 5 & 30 & 2 & 0.5 & $50 \stackrel{\circ}{ }$ & 99 & 100 & - & 98 & 89 \\
\hline $4^{\prime}$ & 5 & 30 & 2 & 0.5 & $50 \div \mathrm{C}$ & 99 & 100 & - & - & - \\
\hline
\end{tabular}




\section{Condition 6:}<smiles>O=Cc1ccccc1I</smiles>

$$
\begin{gathered}
4 \mathbf{i}(5 \mathrm{~mol} \%) \\
\underset{\text { DIPEA }(5 \mathrm{~mol} \%)}{\stackrel{\mathrm{D}_{2} \mathrm{O}(30 \mathrm{eq})}{\longrightarrow}} \\
\text { Ph-Boroxine }(4 \mathrm{~mol} \%) \\
\text { Toluene }(1.0 \mathrm{M}) \\
20^{\circ} \mathrm{C}, 16 \mathrm{~h}
\end{gathered}
$$<smiles>[2H]C(=O)c1ccccc1I</smiles>

Scheme 23: Reaction condition $6(\mathrm{Ph}=$ Phenyl)

\section{2-lodobenzaldehyde-d}<smiles>[2H]C(=O)c1ccccc1I</smiles>

4i (20 mg, $0.055 \mathrm{mmol})$, DIPEA (10 $\mu \mathrm{L}, 0.055 \mathrm{mmol}), \mathrm{D}_{2} \mathrm{O}(600 \mu \mathrm{L}, 33 \mathrm{mmol})$, phenyl boroxine (13.7 $\mathrm{mg}, 0.044 \mathrm{mmol}$ ) and 2-iodobenzaldehyde $(255 \mathrm{mg}, 1.1 \mathrm{mmol})$ in anhydrous toluene $(500 \mu \mathrm{L})$ were prepared according to the general procedure given in section 5 (page 20) and stirred at $20^{\circ} \mathrm{C}$. Crude residue was purified by flash-column chromatography using Hex: $\mathrm{Et}_{2} \mathrm{O}$ (91:9) combination to give colorless solid. Spectral data for this product have been previously reported. ${ }^{21}$

\begin{tabular}{|c|c|c|c|c|c|c|c|c|c|c|}
\hline \multirow{3}{*}{$\begin{array}{c}\text { Entry } \\
1 \\
\end{array}$} & \multirow{2}{*}{$\begin{array}{c}\mathrm{NHC} \\
\text { (mol\%) }\end{array}$} & \multirow{3}{*}{$\begin{array}{c}\mathrm{D}_{2} \mathrm{O} \\
\text { (eq. } \\
\text { ) } \\
30\end{array}$} & \multirow{3}{*}{$\begin{array}{c}\text { Ph- } \\
\text { Boroxine } \\
\text { (mol\%) } \\
-\end{array}$} & \multirow{3}{*}{$\begin{array}{c}\text { Reactio } \\
\text { n Conc. } \\
\text { (M) } \\
1\end{array}$} & \multirow{3}{*}{$\begin{array}{l}\text { Reactio } \\
\text { n } \\
\text { Temp. } \\
20^{\circ} \mathrm{C}\end{array}$} & \multirow{3}{*}{$\begin{array}{c}\% \mathrm{D} \\
\text { (RM) } \\
70\end{array}$} & \multirow{3}{*}{$\begin{array}{c}\% \\
\begin{array}{c}\text { Aldehyd } \\
\text { e }\end{array} \\
100\end{array}$} & \multirow{3}{*}{$\begin{array}{c}\% \\
\text { Benzoin } \\
-\end{array}$} & \multicolumn{2}{|c|}{$\begin{array}{c}\text { After } \\
\text { Purification }\end{array}$} \\
\hline & & & & & & & & & $\% \mathrm{D}$ & \%Yield \\
\hline & $3 a(5)$ & & & & & & & & - & - \\
\hline $1^{\prime}$ & $3 a(5)$ & 30 & - & 1 & $20^{\circ} \mathrm{C}$ & 69 & 100 & - & - & - \\
\hline 2 & $3 a(5)$ & 30 & - & 1 & $50 \stackrel{\circ}{C}$ & 60 & 100 & - & - & - \\
\hline $2^{\prime}$ & $3 a(5)$ & 30 & - & 1 & $50 \stackrel{\circ}{C}$ & 60 & 100 & - & - & - \\
\hline 3 & $4 i(5)$ & 30 & - & 1 & $20^{\circ} \mathrm{C}$ & 98 & 83 & 17 & - & - \\
\hline $3^{\prime}$ & $4 i(5)$ & 30 & - & 1 & $20^{\circ} \mathrm{C}$ & 98 & 79 & 21 & - & - \\
\hline 4 & $4 i(5)$ & 30 & 4 & 1 & $20^{\circ} \mathrm{C}$ & 98 & 100 & - & 97 & 94 \\
\hline $4^{\prime}$ & $4 i(5)$ & 30 & 4 & 1 & $20^{\circ} \mathrm{C}$ & 98 & 100 & - & - & - \\
\hline
\end{tabular}

Deuterium incorporation $=97 \%$ (crude: 98\%, 98\%), Reaction yield $=94 \%$ (241 mg). NMR spectra are given on pages $288-293$.

${ }^{1} \mathbf{H}$ NMR $\left(500 \mathrm{MHz}, \mathrm{CDCl}_{3}\right) \delta(\mathrm{ppm})=10.07(\mathrm{~s}, 0.03 \mathrm{H}), 7.96-7.94(\mathrm{~m}, 1 \mathrm{H})$, 7.90-7.88 $(\mathrm{m}, 1 \mathrm{H})$, 7.48$7.45(\mathrm{~m}, 1 \mathrm{H}), 7.31-7.26(\mathrm{~m}, 1 \mathrm{H}) .{ }^{13} \mathrm{C}$ NMR $\left(125 \mathrm{MHz}, \mathrm{CDCl}_{3}\right) \delta(\mathrm{ppm})=195.5(\mathrm{t}, J=28 \mathrm{~Hz}), 140.8$, $135.6,135.2,130.4,128.9,100.8$ 


\section{Condition 7:}
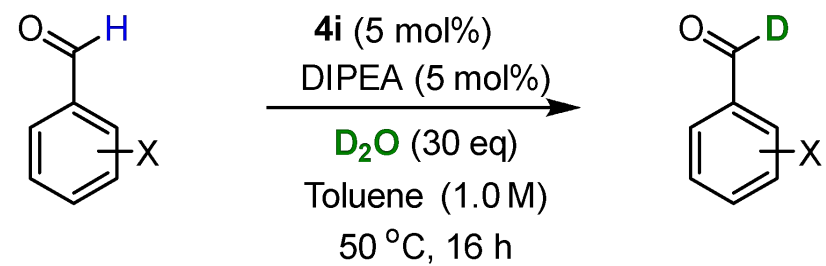

Scheme 24: Reaction condition 7

\section{2- ${ }^{n}$ Butoxybenzaldehyde- $d$}<smiles>[2H]C(=O)c1ccccc1OCCCC</smiles>

4i (20 mg, $0.055 \mathrm{mmol}$ ), DIPEA (10 $\mu \mathrm{L}, 0.055 \mathrm{mmol}), \mathrm{D}_{2} \mathrm{O}(600 \mu \mathrm{L}, 33 \mathrm{mmol})$ and 2("butoxy)benzaldehyde ( $200 \mu \mathrm{L}, 1.1 \mathrm{mmol}$ ) in anhydrous toluene $(500 \mu \mathrm{L})$ were prepared according to the general procedure given in section 5 (page 20) and stirred at $50 \stackrel{\circ}{ }$. Crude residue was purified by flash-column chromatography using $\mathrm{Hex}: \mathrm{Et}_{2} \mathrm{O}(95: 5)$ combination to give colorless liquid.

Deuterium incorporation $=98 \%$ (crude: $99 \%, 99 \%)$, Reaction yield $=91 \%$ (179 mg). NMR spectra are given on pages $294-298$.

${ }^{1} \mathrm{H}$ NMR $\left(500 \mathrm{MHz}, \mathrm{CDCl}_{3}\right) \delta(\mathrm{ppm})=10.51(\mathrm{~s}, 0.02 \mathrm{H}), 7.83(\mathrm{dd}, J=5 \mathrm{~Hz}, 1 \mathrm{H}), 7.54-7.51(\mathrm{~m}, 1 \mathrm{H})$, 7.01-6.96 (m, 2 H), 4.08 (t, J = 5 Hz, 2 H), 1.86-1.80 (m, 2 H), 1.56-1.49 (m, 2 H), 0.99 (t, J = 5 Hz, 3 H). ${ }^{13} \mathrm{C} \mathrm{NMR}\left(125 \mathrm{MHz}, \mathrm{CDCl}_{3}\right) \delta(\mathrm{ppm})=189.7(\mathrm{t}, J=28 \mathrm{~Hz}), 161.7,136.0,128.3,124.9,120.6,112.6$, 68.3, 31.2, 19.4, 13.9. HRMS(ESI) calcd for $\mathrm{C}_{11} \mathrm{H}_{13} \mathrm{DO}_{2}{ }^{+}(\mathrm{M}+\mathrm{H})^{+}:$: 180.1129, Found: 180.1130

\begin{tabular}{|c|c|c|c|c|c|c|c|c|c|c|}
\hline \multirow[t]{2}{*}{ Entry } & \multirow{2}{*}{$\begin{array}{c}\mathrm{NHC} \\
(\mathrm{mol} \%)\end{array}$} & \multirow{2}{*}{$\begin{array}{c}\text { DIPEA } \\
\text { (mol\%) }\end{array}$} & \multirow{2}{*}{$\begin{array}{c}\mathrm{D}_{2} \mathrm{O} \\
\text { (eq. } \\
\text { ) }\end{array}$} & \multirow{2}{*}{$\begin{array}{c}\text { Ph- } \\
\text { Boroxine } \\
\text { (mol\%) }\end{array}$} & \multirow{2}{*}{$\begin{array}{c}\text { Reactio } \\
\text { n Conc. } \\
\text { (M) }\end{array}$} & \multirow{2}{*}{$\begin{array}{l}\text { Reactio } \\
\text { n } \\
\text { Temp. }\end{array}$} & \multirow{2}{*}{$\begin{array}{c}\% \mathrm{D} \\
\text { (RM) }\end{array}$} & \multirow{2}{*}{$\begin{array}{c}\% \\
\text { Aldehyd } \\
\text { e }\end{array}$} & \multicolumn{2}{|c|}{$\begin{array}{c}\text { After } \\
\text { Purification }\end{array}$} \\
\hline & & & & & & & & & $\% \mathrm{D}$ & \%Yield \\
\hline 1 & $3 a(5)$ & 5 & 30 & - & 1 & $20^{\circ} \mathrm{C}$ & 58 & 100 & - & - \\
\hline $1^{\prime}$ & $3 a(5)$ & 5 & 30 & - & 1 & $20^{\circ} \mathrm{C}$ & 59 & 100 & - & - \\
\hline 2 & $4 i(5)$ & 5 & 30 & - & 1 & $20^{\circ} \mathrm{C}$ & 84 & 100 & - & - \\
\hline $2^{\prime}$ & $4 i(5)$ & 5 & 30 & - & 1 & $20^{\circ} \mathrm{C}$ & 81 & 100 & - & - \\
\hline 3 & $4 i(5)$ & 5 & 30 & - & 1 & $50 \stackrel{\circ}{C}$ & 99 & 100 & 98 & 91 \\
\hline $3^{\prime}$ & $4 i(5)$ & 5 & 30 & - & 1 & $50 \cong \mathrm{C}$ & 99 & 100 & - & - \\
\hline
\end{tabular}




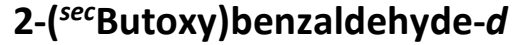<smiles>[2H]C(=O)c1ccccc1OC(C)CC</smiles>

4i (20 mg, $0.055 \mathrm{mmol})$, DIPEA $(10 \mu \mathrm{L}, 0.055 \mathrm{mmol}), \mathrm{D}_{2} \mathrm{O}(600 \mu \mathrm{L}, 33 \mathrm{mmol})$ and 2( ${ }^{\text {sec }}$ butoxy)benzaldehyde $(200 \mu \mathrm{L}, 1.1 \mathrm{mmol})$ in anhydrous toluene $(500 \mu \mathrm{L})$ were prepared according to the general procedure given in section 5 (page 20) and stirred at $50^{\circ} \mathrm{C}$. Crude residue was purified by flash-column chromatography using $\mathrm{Hex}_{\mathrm{Et}} \mathrm{O}(94: 6)$ combination to give colorless liquid.

Deuterium incorporation $=98 \%$ (crude: $99 \%, 99 \%)$, Reaction yield = 92\% (181 mg). NMR spectra are given on pages 299-302.

${ }^{1} \mathbf{H}$ NMR $\left(500 \mathrm{MHz}, \mathrm{CDCl}_{3}\right) \delta(\mathrm{ppm})=10.51(\mathrm{~s}, 0.02 \mathrm{H}), 7.83(\mathrm{dd}, J=5 \mathrm{~Hz}, 1 \mathrm{H}), 7.53-7.49(\mathrm{~m}, 1 \mathrm{H})$, 7.00-6.97 (m, $2 \mathrm{H}), 4.46(\mathrm{sxt}, J=5 \mathrm{~Hz}, 1 \mathrm{H}), 1.86-1.66(\mathrm{~m}, 2 \mathrm{H}), 1.36(\mathrm{~d}, J=5 \mathrm{~Hz}, 3 \mathrm{H}), 1.01(\mathrm{t}, J=4 \mathrm{~Hz}$, $3 \mathrm{H}) .{ }^{13} \mathrm{C}$ NMR $\left(125 \mathrm{MHz}, \mathrm{CDCl}_{3}\right) \delta(\mathrm{ppm})=189.9$ (t, $\left.J=28 \mathrm{~Hz}\right), 161.1,135.9,128.4,125.8,120.4$, 114.0, 76.1, 29.3, 19.3, 9.8. HRMS(ESI) calcd for $\mathrm{C}_{11} \mathrm{H}_{13} \mathrm{DO}_{2}{ }^{+}(\mathrm{M}+\mathrm{H})^{+}: 180.1129$, Found: 180.1129

\begin{tabular}{|c|c|c|c|c|c|c|c|c|c|c|}
\hline \multirow{2}{*}{ Entry } & \multirow{2}{*}{$\begin{array}{c}\mathrm{NHC} \\
\text { (mol\%) }\end{array}$} & \multirow{2}{*}{$\begin{array}{l}\text { DIPEA } \\
\text { (mol\%) }\end{array}$} & \multirow{2}{*}{$\begin{array}{l}\mathrm{D}_{2} \mathrm{O} \\
\text { (eq. } \\
\text { ) }\end{array}$} & \multirow{2}{*}{$\begin{array}{c}\text { Ph- } \\
\text { Boroxine } \\
\text { (mol\%) }\end{array}$} & \multirow{2}{*}{$\begin{array}{l}\text { Reactio } \\
\text { n Conc. } \\
\text { (M) }\end{array}$} & \multirow{2}{*}{$\begin{array}{c}\text { Reactio } \\
\text { n } \\
\text { Temp. }\end{array}$} & \multirow{2}{*}{$\begin{array}{c}\% \mathrm{D} \\
\text { (RM) }\end{array}$} & \multirow{2}{*}{$\begin{array}{c}\% \\
\text { Aldehyd } \\
\text { e }\end{array}$} & \multicolumn{2}{|c|}{$\begin{array}{c}\text { After } \\
\text { Purification }\end{array}$} \\
\hline & & & & & & & & & $\% \mathrm{D}$ & \%Yield \\
\hline 1 & $3 a(5)$ & 5 & 30 & - & 1 & $20^{\circ} \mathrm{C}$ & 18 & 100 & - & - \\
\hline $1^{\prime}$ & $3 a(5)$ & 5 & 30 & - & 1 & $20^{\circ} \mathrm{C}$ & 18 & 100 & - & - \\
\hline 2 & $4 i(5)$ & 5 & 30 & - & 1 & $50 \stackrel{\circ}{C}$ & 99 & 100 & 98 & 92 \\
\hline $2^{\prime}$ & $4 i(5)$ & 5 & 30 & - & 1 & $50 \stackrel{\circ}{ } \mathrm{C}$ & 99 & 100 & - & - \\
\hline
\end{tabular}

\section{[1,1'-biphenyl]-2-carbaldehyde-d}<smiles>[2H]C(=O)c1ccccc1-c1ccccc1</smiles>

4i (20 mg, $0.055 \mathrm{mmol})$, DIPEA (10 $\mu \mathrm{L}, 0.055 \mathrm{mmol}), \mathrm{D}_{2} \mathrm{O}(600 \mu \mathrm{L}, 33 \mathrm{mmol})$ and [1,1'-biphenyl]-2carbaldehyde (200 mg, $1.1 \mathrm{mmol})$ in anhydrous toluene $(500 \mu \mathrm{L})$ were prepared according to the

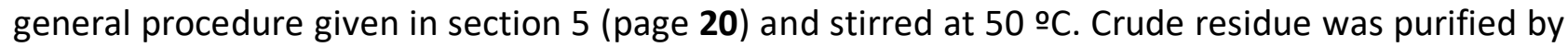
flash-column chromatography using $\mathrm{Hex}_{\mathrm{Et}} \mathrm{O}$ (91:9) combination to give colorless solid. Spectral data for this product have been previously reported. ${ }^{19}$

Deuterium incorporation $=98 \%$ (crude: $99 \%, 98 \%$ ), Reaction yield = 97\% (195 mg). NMR spectra are given on pages 303-306. 
${ }^{1} \mathrm{H}$ NMR $\left(500 \mathrm{MHz}, \mathrm{CDCl}_{3}\right) \delta(\mathrm{ppm})=9.99(\mathrm{~s}, 0.02 \mathrm{H}), 8.04(\mathrm{dd}, J=5 \mathrm{~Hz}, 1 \mathrm{H}), 7.65(\mathrm{tt}, J=10 \mathrm{~Hz}, 1 \mathrm{H})$, 7.52-7.43 (m, $5 \mathrm{H}), 7.40-7.38(\mathrm{~m}, 2 \mathrm{H}) .{ }^{13} \mathrm{C}$ NMR (125 MHz, $\left.\mathrm{CDCl}_{3}\right) \delta(\mathrm{ppm})=192.3(\mathrm{t}, J=28 \mathrm{~Hz})$, $146.1,137.9,133.7,130.9,130.2,128.6,128.3,127.9,127.7$

\begin{tabular}{|c|c|c|c|c|c|c|c|c|c|c|}
\hline \multirow[t]{2}{*}{ Entry } & \multirow{2}{*}{$\begin{array}{c}\mathrm{NHC} \\
\text { (mol\%) }\end{array}$} & \multirow{2}{*}{$\begin{array}{l}\text { DIPEA } \\
\text { (mol\%) }\end{array}$} & \multirow{2}{*}{$\begin{array}{l}\mathrm{D}_{2} \mathrm{O} \\
\text { (eq. } \\
\text { ) }\end{array}$} & \multirow{2}{*}{$\begin{array}{l}\text { Ph- } \\
\text { Boroxine } \\
\text { (mol\%) }\end{array}$} & \multirow{2}{*}{$\begin{array}{l}\text { Reactio } \\
\text { n Conc. } \\
\text { (M) }\end{array}$} & \multirow{2}{*}{$\begin{array}{l}\text { Reactio } \\
\text { n } \\
\text { Temp. }\end{array}$} & \multirow{2}{*}{$\begin{array}{c}\% \mathrm{D} \\
\text { (RM) }\end{array}$} & \multirow{2}{*}{$\begin{array}{c}\% \\
\text { Aldehyd } \\
\text { e }\end{array}$} & \multicolumn{2}{|c|}{$\begin{array}{c}\text { After } \\
\text { Purification }\end{array}$} \\
\hline & & & & & & & & & $\% \mathrm{D}$ & \%Yield \\
\hline 1 & $3 a(5)$ & 5 & 30 & - & 1 & $20^{\circ} \mathrm{C}$ & 1 & 100 & - & - \\
\hline $1^{\prime}$ & $3 a(5)$ & 5 & 30 & - & 1 & $20^{\circ} \mathrm{C}$ & 0 & 100 & - & - \\
\hline 2 & 4i (5) & 5 & 30 & - & 1 & $50 \stackrel{\circ}{C}$ & 99 & 100 & 98 & 97 \\
\hline $2^{\prime}$ & 4i (5) & 5 & 30 & - & 1 & 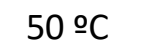 & 98 & 100 & - & - \\
\hline
\end{tabular}

\section{Phenanthrene-9-carbaldehyde-d}

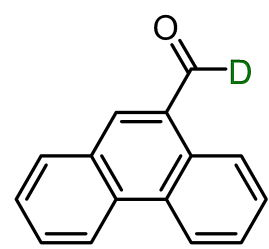

4i (10 mg, $0.0275 \mathrm{mmol})$, DIPEA ( $5 \mu \mathrm{L}, 0.0275 \mathrm{mmol}), \mathrm{D}_{2} \mathrm{O}(300 \mu \mathrm{L}, 17 \mathrm{mmol})$ and phenanthrene-9carbaldehyde (113 mg, $0.55 \mathrm{mmol})$ in anhydrous toluene $(250 \mu \mathrm{L})$ were prepared according to the

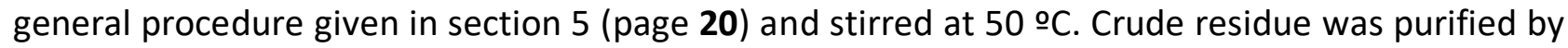
flash-column chromatography using Hex:Et ${ }_{2} \mathrm{O}(89: 11)$ combination to give colorless solid.

Deuterium incorporation $=97 \%$ (crude: $99 \%, 98 \%$ ), Reaction yield $=86 \%$ (98 mg). NMR spectra are given on pages 307-311.

${ }^{1} \mathbf{H}$ NMR $\left(500 \mathrm{MHz}, \mathrm{CDCl}_{3}\right) \delta(\mathrm{ppm})=10.35(\mathrm{~s}, 0.03 \mathrm{H}), 9.38-9.35(\mathrm{~m}, 1 \mathrm{H}), 8.69-8.64(\mathrm{~m}, 2 \mathrm{H}), 8.21$ (s, 1 H), 8.01-7.99 (m, 1 H), 7.81-7.78 (m, 1 H), 7.74-7.71 (m, 2 H), 7.67-7.64 (m, 1 H). ${ }^{13}$ C NMR (125 $\left.\mathrm{MHz} \mathrm{CDCl}_{3}\right) \delta(\mathrm{ppm})=193.4(\mathrm{t}, J=28 \mathrm{~Hz}), 141.3,133.1,130.7,130.5,130.3,130.2,128.3,127.7$, 127.4, 126.1, 123.0, 122.8. HRMS(ESI) calcd for $\mathrm{C}_{15} \mathrm{H}_{9} \mathrm{DO}^{+}(\mathrm{M}+\mathrm{H})^{+}:$208.0867, Found: 208.0866

\begin{tabular}{|c|c|c|c|c|c|c|c|c|c|c|}
\hline \multirow{2}{*}{ Entry } & \multirow{2}{*}{$\begin{array}{c}\mathrm{NHC} \\
(\mathrm{mol} \%)\end{array}$} & \multirow{2}{*}{$\begin{array}{l}\text { DIPEA } \\
\text { (mol\%) }\end{array}$} & \multirow{2}{*}{$\begin{array}{c}\mathrm{D}_{2} \mathrm{O} \\
\text { (eq. } \\
\text { ) }\end{array}$} & \multirow{2}{*}{$\begin{array}{c}\text { Ph- } \\
\text { Boroxine } \\
\text { (mol\%) }\end{array}$} & \multirow{2}{*}{$\begin{array}{l}\text { Reactio } \\
\text { n Conc. } \\
\text { (M) }\end{array}$} & \multirow{2}{*}{$\begin{array}{c}\text { Reactio } \\
\mathrm{n} \\
\text { Temp. }\end{array}$} & \multirow{2}{*}{$\begin{array}{c}\% \mathrm{D} \\
\text { (RM) }\end{array}$} & \multirow{2}{*}{$\begin{array}{c}\% \\
\text { Aldehyd } \\
\text { e }\end{array}$} & \multicolumn{2}{|c|}{$\begin{array}{c}\text { After } \\
\text { Purification }\end{array}$} \\
\hline & & & & & & & & & $\% \mathrm{D}$ & \%Yield \\
\hline 1 & $3 a(5)$ & 5 & 30 & - & 1 & $20^{\circ} \mathrm{C}$ & 49 & 100 & - & - \\
\hline $1^{\prime}$ & $3 a(5)$ & 5 & 30 & - & 1 & $20^{\circ} \mathrm{C}$ & 45 & 100 & - & - \\
\hline 2 & $3 a(5)$ & 5 & 30 & - & 1 & $50 \stackrel{\circ}{C}$ & 85 & 100 & - & - \\
\hline 3 & $4 i(5)$ & 5 & 30 & - & 1 & $20^{\circ} \mathrm{C}$ & 65 & 100 & - & - \\
\hline 4 & $4 i(5)$ & 5 & 30 & - & 1 & $50 \stackrel{\circ}{ }$ & 99 & 100 & 97 & 86 \\
\hline $4^{\prime}$ & $4 i(5)$ & 5 & 30 & - & 1 & $50 \stackrel{\circ}{ }$ & 98 & 100 & - & - \\
\hline
\end{tabular}


Condition 8: Due to poor solubility of 4-hydoxybenzaldehyde in toluene, chloroform was used.<smiles>O=Cc1ccc(O)cc1</smiles>

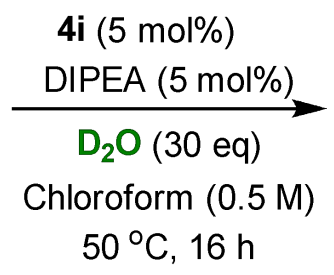<smiles>[2H]C(=O)c1ccc(O)cc1</smiles>

Scheme 25: Reaction condition 8

\section{4-Hydoxybenzaldehyde-d}<smiles>[2H]C(=O)c1ccc(O)cc1</smiles>

4i $(20 \mathrm{mg}, 0.055 \mathrm{mmol})$, DIPEA $(10 \mu \mathrm{L}, 0.055 \mathrm{mmol}), \mathrm{D}_{2} \mathrm{O}(600 \mu \mathrm{L}, 33 \mathrm{mmol})$ and 4hydoxybenzaldehyde $(134 \mathrm{mg}, 1.1 \mathrm{mmol})$ in anhydrous chloroform $(1.60 \mathrm{~mL}$ ) were prepared according to the general procedure given in section 5 (page 20) and stirred at $50 \stackrel{\circ}{ }$. Crude residue was purified by flash-column chromatography using Hex: $\mathrm{Et}_{2} \mathrm{O}$ (50:50) combination to give white solid. Spectral data for this product have been previously reported. ${ }^{20}$

Deuterium incorporation = 96\% (crude: $97 \%, 97 \%)$, Reaction yield = 92\% (125 mg). NMR spectra are given on pages 312-316.

${ }^{1} \mathbf{H}$ NMR $\left(400 \mathrm{MHz}, \mathrm{DMSO}-d_{6}\right) \delta(\mathrm{ppm})=10.58(\mathrm{~s}, 1 \mathrm{H}), 9.79(\mathrm{~s}, 0.04 \mathrm{H}), 7.76(\mathrm{~d}, J=8 \mathrm{~Hz}, 2 \mathrm{H}), 6.93$ $(\mathrm{d}, J=8 \mathrm{~Hz}, 2 \mathrm{H}) .{ }^{13} \mathrm{C}$ NMR $\left(100 \mathrm{MHz}, \mathrm{DMSO}-\mathrm{d}_{6}\right) \delta(\mathrm{ppm})=190.7(\mathrm{t}, J=27 \mathrm{~Hz}), 163.3,132.1,128.3(\mathrm{t}$, $J=3 \mathrm{~Hz}), 115.8$

\begin{tabular}{|c|c|c|c|c|c|c|c|c|c|c|}
\hline \multirow{3}{*}{$\begin{array}{c}\text { Entry } \\
1 \\
\end{array}$} & \multirow{2}{*}{$\begin{array}{c}\mathrm{NHC} \\
\text { (mol\%) }\end{array}$} & \multirow{3}{*}{$\begin{array}{l}\mathrm{D}_{2} \mathrm{O} \\
\text { (eq. } \\
\text { ) } \\
30\end{array}$} & \multirow{3}{*}{$\begin{array}{c}\text { Ph- } \\
\text { Boroxine } \\
\text { (mol\%) } \\
-\end{array}$} & \multirow{3}{*}{$\begin{array}{c}\text { Reactio } \\
\text { n Conc. } \\
\text { (M) } \\
1\end{array}$} & \multirow{3}{*}{$\begin{array}{l}\text { Reactio } \\
\text { n } \\
\text { Temp. } \\
20^{\circ} \mathrm{C}\end{array}$} & \multirow{3}{*}{$\begin{array}{l}\text { Reaction } \\
\text { Solvent } \\
\text { Toluene }\end{array}$} & \multirow{3}{*}{$\begin{array}{c}\% \mathrm{D} \\
\text { (RM) } \\
3\end{array}$} & \multirow{2}{*}{$\begin{array}{c}\% \\
\text { Aldehyde }\end{array}$} & \multicolumn{2}{|c|}{$\begin{array}{c}\text { After } \\
\text { Purification }\end{array}$} \\
\hline & & & & & & & & & $\% \mathrm{D}$ & \%Yield \\
\hline & $3 a(5)$ & & & & & & & 100 & - & - \\
\hline $1^{\prime}$ & $3 a(5)$ & 30 & - & 1 & $20^{\circ} \mathrm{C}$ & Toluene & 0 & 100 & - & - \\
\hline 2 & $3 a(5)$ & 30 & - & 1 & $20^{\circ} \mathrm{C}$ & Chloroform-d & 0 & 100 & - & - \\
\hline 3 & $3 a(5)$ & 30 & - & 1 & $50 \stackrel{\circ}{C}$ & Chloroform-d & 1 & 100 & - & - \\
\hline 4 & $4 i(5)$ & 30 & - & 0.5 & $20^{\circ} \mathrm{C}$ & Chloroform-d & 84 & 100 & - & - \\
\hline 5 & $4 i(5)$ & 30 & - & 0.5 & $50 \stackrel{\circ}{C}$ & Chloroform & 97 & 100 & 96 & 92 \\
\hline $5^{\prime}$ & $4 i(5)$ & 30 & - & 0.5 & $50 \stackrel{\circ}{C}$ & Chloroform & 97 & 100 & - & - \\
\hline
\end{tabular}




\subsection{Substrate Scope with Heterocycles and Biologically Active Molecules}

The information gained above with aromatic aldehydes are applied to deuteration heterocyclic and biologically active molecules.

\section{Thiophene-2-carbaldehyde-d}<smiles>[2H]C(=O)c1cccs1</smiles>

3a (23.5 mg, $0.055 \mathrm{mmol})$, DIPEA (10 $\mu \mathrm{L}, 0.055 \mathrm{mmol}), \mathrm{D}_{2} \mathrm{O}(600 \mu \mathrm{L}, 33 \mathrm{mmol})$, phenyl boroxine (6.9 $\mathrm{mg}, 0.022 \mathrm{mmol}$ ) and thiophene-2-carbaldehyde (103 $\mu \mathrm{L}, 1.1 \mathrm{mmol})$ in anhydrous toluene $(1.60 \mathrm{~mL})$ were prepared according to the general procedure given in section 5 (page 20) and stirred at 50 ㅇ. . Crude residue was purified by flash-column chromatography using $\mathrm{Hex}_{\mathrm{Et}} \mathrm{O}$ (87:13) combination to give colorless liquid. Spectral data for this product have been previously reported. ${ }^{22}$

Deuterium incorporation $=98 \%$ (crude: $100 \%, 98 \%)$, Reaction yield $=93 \%$ (116 mg). NMR spectra are given on pages 317-320.

${ }^{1} \mathrm{H}$ NMR $\left(500 \mathrm{MHz}, \mathrm{CDCl}_{3}\right) \delta(\mathrm{ppm})=9.93(\mathrm{~s}, 0.02 \mathrm{H}), 7.78-7.75(\mathrm{~m}, 2 \mathrm{H}), 7.21(\mathrm{~d}, J=4 \mathrm{~Hz}, 1 \mathrm{H}) .{ }^{13} \mathrm{C}$ $\operatorname{NMR}\left(125 \mathrm{MHz}, \mathrm{CDCl}_{3}\right) \delta(\mathrm{ppm})=182.8(\mathrm{t}, \mathrm{J}=28 \mathrm{~Hz}), 144.1,136.4,135.2,128.4$.

\begin{tabular}{|c|c|c|c|c|c|c|c|c|c|c|}
\hline \multirow{3}{*}{$\begin{array}{l}\text { Entry } \\
1\end{array}$} & \multirow{2}{*}{$\begin{array}{c}3 a \\
(\mathrm{~mol} \%)\end{array}$} & \multirow{3}{*}{$\begin{array}{l}\mathrm{D}_{2} \mathrm{O} \\
\text { (eq. } \\
\text { ) } \\
30\end{array}$} & \multirow{3}{*}{$\begin{array}{c}\text { Ph- } \\
\text { Boroxine } \\
\text { (mol\%) } \\
-\end{array}$} & \multirow{3}{*}{$\begin{array}{c}\text { Reactio } \\
\text { n Conc. } \\
\text { (M) } \\
1\end{array}$} & \multirow{3}{*}{$\begin{array}{l}\text { Reactio } \\
\text { n } \\
\text { Temp. } \\
20^{\circ} \mathrm{C}\end{array}$} & \multirow{3}{*}{$\begin{array}{c}\% \mathrm{D} \\
\text { (RM) } \\
99\end{array}$} & \multirow{2}{*}{$\begin{array}{c}\% \\
\text { Aldehyd } \\
\text { e }\end{array}$} & \multirow{2}{*}{$\begin{array}{c}\% \\
\text { Benzoin }\end{array}$} & \multicolumn{2}{|c|}{$\begin{array}{c}\text { After } \\
\text { Purification }\end{array}$} \\
\hline & & & & & & & & & $\% \mathrm{D}$ & \%Yield \\
\hline & 5 & & & & & & 67 & 33 & - & - \\
\hline $1^{\prime}$ & 5 & 30 & - & 1 & $20^{\circ} \mathrm{C}$ & 99 & 78 & 22 & - & - \\
\hline 2 & 5 & 30 & 2 & 1 & $20^{\circ} \mathrm{C}$ & 38 & 100 & - & - & - \\
\hline 3 & 5 & 30 & 4 & 1 & $20^{\circ} \mathrm{C}$ & 32 & 100 & - & - & - \\
\hline 4 & 5 & 30 & 2 & 0.5 & $50 \cong \mathrm{C}$ & 100 & 100 & - & 98 & 93 \\
\hline $4^{\prime}$ & 5 & 30 & 2 & 0.5 & $50 \stackrel{\circ}{C}$ & 98 & 100 & - & - & - \\
\hline
\end{tabular}

\section{Indole-4-carbaldehyde-d}

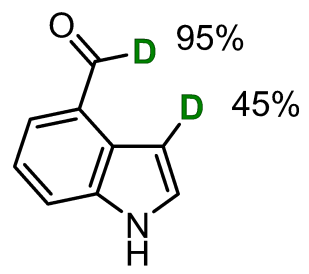

4i (10 mg, $0.0275 \mathrm{mmol})$, DIPEA $(5 \mu \mathrm{L}, 0.0275 \mathrm{mmol}), \mathrm{D}_{2} \mathrm{O}(300 \mu \mathrm{L}, 17 \mathrm{mmol})$ and indole-4carbaldehyde ( $80 \mathrm{mg}, 0.55 \mathrm{mmol}$ ) in anhydrous toluene $(250 \mu \mathrm{L})$ were prepared according to the

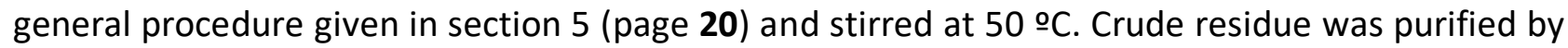


flash-column chromatography using $\mathrm{Hex}_{\mathrm{Et}} \mathrm{O}(50: 50)$ combination to give colorless solid. Spectral data for this product have been previously reported. ${ }^{16}$

Deuterium incorporation $=95 \%$ (crude: $97 \%, 96 \%)$, Reaction yield $=91 \%$ (73 mg). NMR spectra are given on pages 321-323.

${ }^{1} \mathrm{H}$ NMR $\left(500 \mathrm{MHz}, \mathrm{CDCl}_{3}\right) \delta(\mathrm{ppm})=10.27(\mathrm{~s}, 0.05 \mathrm{H}), 8.76(\mathrm{~s}, 1 \mathrm{H})$, 7.70-7.65 (m, $\left.2 \mathrm{H}\right), 7.44(\mathrm{~s}, 1 \mathrm{H})$, 7.38-7.33 (m, 1.55 H). ${ }^{13} \mathrm{C} \mathrm{NMR}\left(125 \mathrm{MHz}, \mathrm{CDCl}_{3}\right) \delta(\mathrm{ppm})=193.2(\mathrm{t}, J=26 \mathrm{~Hz}), 136.7,128.4,127.9$, $127.7,125.9,121.5,117.7,103.1$

\begin{tabular}{|c|c|c|c|c|c|c|c|c|c|c|}
\hline \multirow{2}{*}{ Entry } & \multirow{2}{*}{$\begin{array}{c}\mathrm{NHC} \\
(\mathrm{mol} \%)\end{array}$} & \multirow{2}{*}{$\begin{array}{l}\text { DIPEA } \\
\text { (mol\%) }\end{array}$} & \multirow{2}{*}{$\begin{array}{l}\mathrm{D}_{2} \mathrm{O} \\
\text { (eq. } \\
\text { ) }\end{array}$} & \multirow{2}{*}{$\begin{array}{c}\text { Ph- } \\
\text { Boroxine } \\
\text { (mol\%) }\end{array}$} & \multirow{2}{*}{$\begin{array}{l}\text { Reactio } \\
\text { n Conc. } \\
\text { (M) }\end{array}$} & \multirow{2}{*}{$\begin{array}{l}\text { Reactio } \\
\text { n } \\
\text { Temp. }\end{array}$} & \multirow{2}{*}{$\begin{array}{c}\% \mathrm{D} \\
\text { (RM) }\end{array}$} & \multirow{2}{*}{$\begin{array}{c}\% \\
\text { Aldehyd } \\
\mathrm{e}\end{array}$} & \multicolumn{2}{|c|}{$\begin{array}{c}\text { After } \\
\text { Purification }\end{array}$} \\
\hline & & & & & & & & & $\% \mathrm{D}$ & \%Yield \\
\hline 1 & $3 a(5)$ & 5 & 30 & - & 1 & $20^{\circ} \mathrm{C}$ & 8 & 100 & - & - \\
\hline 2 & $4 i(5)$ & 5 & 30 & - & 1 & $50 \stackrel{\circ}{ }$ & 97 & 100 & 95 & 91 \\
\hline $2^{\prime}$ & $4 i(5)$ & 5 & 30 & - & 1 & $50 \stackrel{\circ}{ }$ & 96 & 100 & - & - \\
\hline
\end{tabular}

\section{Quinoline-4-carbaldehyde-d}<smiles>[2H]C(=O)c1ccnc2ccccc12</smiles>

3a (23.5 mg, $0.055 \mathrm{mmol})$, DIPEA (10 $\mu \mathrm{L}, 0.055 \mathrm{mmol}), \mathrm{D}_{2} \mathrm{O}(600 \mu \mathrm{L}, 33 \mathrm{mmol})$, phenyl boroxine (6.9 $\mathrm{mg}, 0.022 \mathrm{mmol}$ ) and quinoline-4-carbaldehyde $(172 \mathrm{mg}, 1.1 \mathrm{mmol})$ in anhydrous toluene $(1.60 \mathrm{~mL})$ were prepared according to the general procedure given in section 5 (page 20) and stirred at 50 ㅇ. . Crude residue was purified by flash-column chromatography using $\mathrm{Hex}_{\mathrm{Et}} \mathrm{O}$ (77:23) combination to give colorless solid. Spectral data for this product have been previously reported. ${ }^{15}$

Deuterium incorporation = 97\% (crude: 99\%, 98\%), Reaction yield = 95\% (165 mg). NMR spectra are given on pages 324-327.

${ }^{1} \mathrm{H}$ NMR $\left(500 \mathrm{MHz}, \mathrm{CDCl}_{3}\right) \delta(\mathrm{ppm})=10.50(\mathrm{~s}, 0.03 \mathrm{H}), 9.19(\mathrm{~d}, J=4 \mathrm{~Hz}, 1 \mathrm{H}), 9.02-9.00(\mathrm{~m}, 1 \mathrm{H}), 8.22-$ $8.20(\mathrm{~m}, 1 \mathrm{H})$, 7.83-7.78 (m, $2 \mathrm{H}), 7.74-7.71(\mathrm{~m}, 1 \mathrm{H}) .{ }^{13} \mathrm{C} \mathrm{NMR}\left(125 \mathrm{MHz}, \mathrm{CDCl}_{3}\right) \delta(\mathrm{ppm})=192.7(\mathrm{t}$, $J=28 \mathrm{~Hz}), 150.6,149.4,136.8,130.3,130.2,129.5,125.9,124.5,124.0$ 


\begin{tabular}{|c|c|c|c|c|c|c|c|c|c|c|}
\hline \multirow[t]{2}{*}{ Entry } & \multirow{2}{*}{$\begin{array}{c}3 a \\
(\mathrm{~mol} \%)\end{array}$} & \multirow{2}{*}{$\begin{array}{c}\text { DIPEA } \\
\text { (mol\%) }\end{array}$} & \multirow{2}{*}{$\begin{array}{c}\mathrm{D}_{2} \mathrm{O} \\
\text { (eq. } \\
\text { ) }\end{array}$} & \multirow{2}{*}{$\begin{array}{c}\text { Ph- } \\
\text { Boroxine } \\
\text { (mol\%) }\end{array}$} & \multirow{2}{*}{$\begin{array}{l}\text { Reactio } \\
\text { n Conc. } \\
\text { (M) }\end{array}$} & \multirow{2}{*}{$\begin{array}{c}\text { Reactio } \\
\text { n } \\
\text { Temp. }\end{array}$} & \multirow{2}{*}{$\begin{array}{c}\% \mathrm{D} \\
\text { (RM) }\end{array}$} & \multirow{2}{*}{$\begin{array}{c}\% \\
\text { Aldehyd } \\
\mathrm{e}\end{array}$} & \multicolumn{2}{|c|}{$\begin{array}{c}\text { After } \\
\text { Purification }\end{array}$} \\
\hline & & & & & & & & & $\% \mathrm{D}$ & \%Yield \\
\hline 1 & 5 & 5 & 30 & - & 1 & $50 \stackrel{\circ}{C}$ & 98 & 100 & - & - \\
\hline $1^{\prime}$ & 5 & 5 & 30 & - & 1 & $50 \stackrel{\circ}{ }$ & 98 & 100 & - & - \\
\hline 2 & 5 & 5 & 30 & 2 & 0.5 & $50 \stackrel{\circ}{ }$ & 99 & 100 & 97 & 95 \\
\hline $2^{\prime}$ & 5 & 5 & 30 & 2 & 0.5 & $50 \stackrel{\circ}{ } \mathrm{C}$ & 98 & 100 & - & - \\
\hline
\end{tabular}

\section{Benzo[b]thiophene-2-carbaldehyde-d}<smiles>O=C(O)c1cc2ccccc2s1</smiles>

3a (11.8 mg, $0.0275 \mathrm{mmol})$, DIPEA (5 $\mu \mathrm{L}, 0.0275 \mathrm{mmol}), \mathrm{D}_{2} \mathrm{O}(300 \mu \mathrm{L}, 17 \mathrm{mmol})$, phenyl boroxine (3.4 $\mathrm{mg}, 0.011 \mathrm{mmol}$ ) and benzo[b]thiophene-2-carbaldehyde $(89 \mathrm{mg}, 0.55 \mathrm{mmol}$ ) in anhydrous toluene $(800 \mu \mathrm{L})$ were prepared according to the general procedure given in section 5 (page 20) and

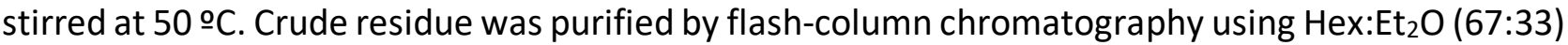
combination to give colorless solid. Spectral data for this product have been previously reported. ${ }^{15}$ Deuterium incorporation $=97 \%$ (crude: $99 \%, 98 \%)$, Reaction yield $=90 \%$ (81 mg). NMR spectra are given on pages 328-332.

${ }^{1} \mathbf{H}$ NMR $\left(500 \mathrm{MHz}, \mathrm{CDCl}_{3}\right) \delta(\mathrm{ppm})=10.11(\mathrm{~s}, 0.03 \mathrm{H}), 8.04(\mathrm{~s}, 1 \mathrm{H})$, 7.96-7.94 $(\mathrm{m}, 1 \mathrm{H})$, 7.92-7.89 $(\mathrm{m}$, $1 \mathrm{H}), 7.53-7.49(\mathrm{~m}, 1 \mathrm{H}), 7.46-7.43(\mathrm{~m}, 1 \mathrm{H}) .{ }^{13} \mathrm{C}$ NMR $\left(125 \mathrm{MHz}^{\mathrm{C} C D C l}\right)_{3} \delta(\mathrm{ppm})=184.6(\mathrm{t}, J=28 \mathrm{~Hz})$, $143.4,142.8,138.7,134.6,128.3,126.4,125.4,123.5$

\begin{tabular}{|c|c|c|c|c|c|c|c|c|c|c|}
\hline \multirow{3}{*}{$\begin{array}{c}\text { Entry } \\
1 \\
\end{array}$} & \multirow{3}{*}{$\begin{array}{c}\begin{array}{c}3 a \\
(\mathrm{~mol} \%)\end{array} \\
5 \\
\end{array}$} & \multirow{3}{*}{$\begin{array}{c}\text { DIPEA } \\
\text { (mol\%) } \\
5 \\
\end{array}$} & \multirow{3}{*}{$\begin{array}{c}\text { Ph- } \\
\text { Boroxine } \\
\text { (mol\%) } \\
-\end{array}$} & \multirow{3}{*}{$\begin{array}{c}\text { Reactio } \\
\text { n Conc. } \\
\text { (M) } \\
1 \\
\end{array}$} & \multirow{3}{*}{$\begin{array}{c}\text { Reactio } \\
\text { n } \\
\text { Temp. } \\
50 \cong \mathrm{C}\end{array}$} & \multirow{3}{*}{$\begin{array}{c}\% \mathrm{D} \\
(\mathrm{RM}) \\
97 \\
9\end{array}$} & \multirow{3}{*}{$\begin{array}{c}\% \\
\begin{array}{c}\% \\
\text { Aldehyd } \\
\text { e }\end{array} \\
69 \\
\end{array}$} & \multirow{3}{*}{$\begin{array}{c}\begin{array}{c}\% \\
\text { Benzoin }\end{array} \\
31 \\
\end{array}$} & \multicolumn{2}{|c|}{$\begin{array}{c}\text { After } \\
\text { Purification }\end{array}$} \\
\hline & & & & & & & & & $\% \mathrm{D}$ & \%Yield \\
\hline & & & & & & & & & - & - \\
\hline 2 & 5 & 5 & 2 & 1 & $50 \stackrel{\circ}{ } \mathrm{C}$ & 91 & 100 & - & - & - \\
\hline 3 & 5 & 5 & 4 & 1 & 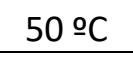 & 92 & 100 & - & - & - \\
\hline 4 & 5 & 10 & 2 & 1 & 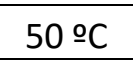 & 95 & 100 & - & - & - \\
\hline $4^{\prime}$ & 5 & 10 & 2 & 1 & $50 \stackrel{\circ}{ } \mathrm{C}$ & 95 & 100 & - & - & - \\
\hline 5 & 5 & 5 & 2 & 0.5 & $50 \stackrel{\circ}{C}$ & 99 & 100 & - & 97 & 90 \\
\hline $5^{\prime}$ & 5 & 5 & 2 & 0.5 & 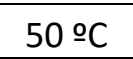 & 98 & 100 & - & - & - \\
\hline
\end{tabular}




\section{${ }^{t}$ Butyl-3-formyl-indole-1-carboxylate-d}<smiles>[2H]C(=O)c1cn(C(=O)OC(C)(C)C)c2ccccc12</smiles>

4i (10 mg, $0.0275 \mathrm{mmol})$, DIPEA ( $5 \mu \mathrm{L}, 0.0275 \mathrm{mmol}), \mathrm{D}_{2} \mathrm{O}(300 \mu \mathrm{L}, 17 \mathrm{mmol})$, phenyl boroxine (85.8 $\mathrm{mg}, 0.275 \mathrm{mmol}$ ) and tbutyl-3-formyl-indole-1-carboxylate $(135 \mathrm{mg}, 0.55 \mathrm{mmol}$ ) in anhydrous toluene $(800 \mu \mathrm{L}$ ) were prepared according to the general procedure given in section 5 (page 20) and stirred at 50 ㄷ. C Crude residue was purified by flash-column chromatography using $\mathrm{Hex}_{\mathrm{Et}} \mathrm{E} \mathrm{O}(80: 20)$ combination to give colorless solid. Spectral data for this product have been previously reported. ${ }^{23}$ Deuterium incorporation = 98\% (crude: 99\%, 100\%), Reaction yield = 90\% (122 mg). NMR spectra are given on pages 333-338.

${ }^{1} \mathrm{H}$ NMR $\left(500 \mathrm{MHz}\right.$, DMSO- $\left.d_{6}\right) \delta(\mathrm{ppm})=10.07(\mathrm{~s}, 0.02 \mathrm{H}), 8.63(\mathrm{~s}, 1 \mathrm{H})$, 8.15-8.09 (m, $\left.2 \mathrm{H}\right)$, 7.45-7.36 $(\mathrm{m}, 2 \mathrm{H}), 1.66$ (s, $9 \mathrm{H}) .{ }^{13} \mathrm{C}$ NMR $\left(125 \mathrm{MHz}, \mathrm{DMSO}-d_{6}\right) \delta(\mathrm{ppm})=186.8(\mathrm{t}, J=28 \mathrm{~Hz}), 148.3,138.3$, $135.3,125.9,125.6,124.5,121.4,120.6,115.0,85.5,27.5$

\begin{tabular}{|c|c|c|c|c|c|c|c|c|c|c|}
\hline \multirow{3}{*}{$\begin{array}{l}\text { Entry } \\
1\end{array}$} & \multirow{2}{*}{$\begin{array}{c}\mathrm{NHC} \\
(\mathrm{mol} \%)\end{array}$} & \multirow{3}{*}{$\begin{array}{l}\mathrm{D}_{2} \mathrm{O} \\
\text { (eq. } \\
\text { ) } \\
30\end{array}$} & \multirow{3}{*}{$\begin{array}{c}\text { Ph- } \\
\text { Boroxine } \\
\text { (mol\%) } \\
-\end{array}$} & \multirow{3}{*}{$\begin{array}{c}\text { Reactio } \\
\text { n Conc. } \\
\text { (M) } \\
1\end{array}$} & \multirow{3}{*}{ 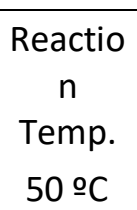 } & \multirow{3}{*}{$\begin{array}{c}\% \mathrm{D} \\
(\mathrm{RM}) \\
58\end{array}$} & \multirow{2}{*}{$\begin{array}{c}\% \\
\text { Aldehyd } \\
\text { e }\end{array}$} & \multirow{2}{*}{$\begin{array}{c}\% \\
\text { Benzoin }\end{array}$} & \multicolumn{2}{|c|}{$\begin{array}{c}\text { After } \\
\text { Purification }\end{array}$} \\
\hline & & & & & & & & & $\% D$ & \%Yield \\
\hline & $3 a(5)$ & & & & & & 100 & - & - & - \\
\hline 2 & $4 \mathbf{i}(5)$ & 30 & - & 1 & $20^{\circ} \mathrm{C}$ & 99 & 52 & 48 & - & - \\
\hline 3 & $4 i(5)$ & 30 & - & 1 & $50 \stackrel{\circ}{C}$ & 100 & 56 & 44 & - & - \\
\hline 4 & $4 i(5)$ & 30 & 4 & 0.5 & $50 \stackrel{\circ}{C}$ & 100 & 61 & 39 & - & - \\
\hline 5 & $4 i(5)$ & 30 & 10 & 0.5 & $50 \stackrel{\circ}{C}$ & 100 & 64 & 36 & - & - \\
\hline 6 & $4 i(5)$ & 30 & 30 & 0.5 & $50 \stackrel{\circ}{C}$ & 100 & 83 & 17 & - & - \\
\hline 7 & $4 i(5)$ & 30 & 50 & 0.5 & $50 \stackrel{\circ}{C}$ & 99 & 90 & 10 & 98 & 90 \\
\hline $7^{\prime}$ & $4 i(5)$ & 30 & 50 & 0.5 & 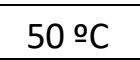 & 100 & 89 & 11 & - & - \\
\hline
\end{tabular}

\section{1-Methyl-indole-3-carbaldehyde-d}<smiles>[2H]C(=O)c1cn(C)c2ccccc12</smiles> 
4a (28 mg, $0.055 \mathrm{mmol})$, DIPEA (10 $\mu \mathrm{L}, 0.055 \mathrm{mmol}), \mathrm{D}_{2} \mathrm{O}(600 \mu \mathrm{L}, 33 \mathrm{mmol})$ and 1-methyl-indole3-carbaldehyde (175 mg, $1.1 \mathrm{mmol}$ ) in anhydrous toluene $(500 \mu \mathrm{L})$ were prepared according to the general procedure given in section 5 (page 20) and stirred at $20^{\circ} \mathrm{C}$. Crude residue was purified by flash-column chromatography using $\mathrm{Hex}_{\mathrm{Et}} \mathrm{O}$ (67:33) combination to give colorless solid.

Deuterium incorporation $=97 \%$ (crude: $98 \%, 97 \%)$, Reaction yield $=91 \%$ (160 mg). NMR spectra are given on pages 339-343.

${ }^{1} \mathrm{H}$ NMR $\left(500 \mathrm{MHz}, \mathrm{CDCl}_{3}\right) \delta(\mathrm{ppm})=9.98(\mathrm{~s}, 0.03 \mathrm{H}), 8.32-8.30(\mathrm{~m}, 1 \mathrm{H}), 7.66(\mathrm{~s}, 1 \mathrm{H})$, 7.37-7.31 (m, $3 \mathrm{H}) .{ }^{13} \mathrm{C}$ NMR $\left(125 \mathrm{MHz}, \mathrm{CDCl}_{3}\right) \delta(\mathrm{ppm})=184.3(\mathrm{t}, J=26 \mathrm{~Hz}), 139.3,138.0,125.4,124.2,123.1$, 122.2, 118.1, 110.0, 33.8. HRMS(ESI) calcd for $\mathrm{C}_{10} \mathrm{H}_{8} \mathrm{DNO}^{+}(\mathrm{M}+\mathrm{H})^{+}:$161.0820, Found: 161.0817

\begin{tabular}{|c|c|c|c|c|c|c|c|c|c|c|}
\hline \multirow{3}{*}{$\begin{array}{c}\text { Entry } \\
1\end{array}$} & \multirow{2}{*}{$\begin{array}{c}\mathrm{NHC} \\
\text { (mol\%) }\end{array}$} & \multirow{2}{*}{$\begin{array}{l}\text { DIPEA } \\
\text { (mol\%) }\end{array}$} & \multirow{2}{*}{$\begin{array}{c}\mathrm{D}_{2} \mathrm{O} \\
\text { (eq. } \\
\text { ) }\end{array}$} & \multirow{2}{*}{$\begin{array}{c}\text { Ph- } \\
\text { Boroxine } \\
\text { (mol\%) }\end{array}$} & \multirow{2}{*}{$\begin{array}{l}\text { Reactio } \\
\text { n Conc. } \\
\text { (M) }\end{array}$} & \multirow{2}{*}{$\begin{array}{c}\text { Reactio } \\
\text { n } \\
\text { Temp. }\end{array}$} & \multirow{2}{*}{$\begin{array}{l}\% \mathrm{D} \\
\text { (RM) }\end{array}$} & \multirow{2}{*}{$\begin{array}{c}\% \\
\text { Aldehyd } \\
\mathrm{e}\end{array}$} & \multicolumn{2}{|c|}{$\begin{array}{c}\text { After } \\
\text { Purification }\end{array}$} \\
\hline & & & & & & & & & $\% \mathrm{D}$ & \%Yield \\
\hline & $3 a(5)$ & 5 & 30 & - & 1 & $20^{\circ} \mathrm{C}$ & 15 & 100 & - & - \\
\hline 2 & $3 a(5)$ & 5 & 30 & - & 1 & $50 \stackrel{\circ}{C}$ & 19 & 100 & - & - \\
\hline 3 & $4 i(5)$ & 5 & 30 & - & 1 & $20^{\circ} \mathrm{C}$ & 36 & 100 & - & - \\
\hline 4 & $4 \mathbf{i}(5)$ & 5 & 30 & - & 1 & 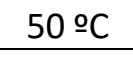 & 69 & 100 & - & - \\
\hline 5 & $4 a(5)$ & 5 & 30 & - & 1 & $20^{\circ} \mathrm{C}$ & 98 & 100 & 97 & 91 \\
\hline $5^{\prime}$ & $4 a(5)$ & 5 & 30 & - & 1 & $20^{\circ} \mathrm{C}$ & 97 & 100 & - & - \\
\hline
\end{tabular}

\section{9-Ethyl-carbazole-3-carbaldehyde-d}<smiles>[2H]C(=O)c1ccc2c(c1)c1ccccc1n2CC</smiles>

4i (20 mg, $0.055 \mathrm{mmol})$, DIPEA (10 $\mu \mathrm{L}, 0.055 \mathrm{mmol}), \mathrm{D}_{2} \mathrm{O}(600 \mu \mathrm{L}, 33 \mathrm{mmol})$, phenyl boroxine (13.7 $\mathrm{mg}, 0.044 \mathrm{mmol}$ ) and 9-ethyl-carbazole-3-carbaldehyde (246 mg, $1.1 \mathrm{mmol}$ ) in anhydrous toluene $(1.6 \mathrm{~mL})$ were prepared according to the general procedure given in section 5 (page 20) and stirred at $50 \stackrel{\circ}{ } \mathrm{C}$. Crude residue was purified by flash-column chromatography using Hex:Et $\mathrm{O}_{2} \mathrm{O}$ (50:50) combination to give colorless solid. Spectral data for this product have been previously reported. ${ }^{15}$ Deuterium incorporation = 98\% (crude: 99\%, 98\%), Reaction yield = 97\% (239 mg). NMR spectra are given on pages 344-347.

${ }^{1} \mathbf{H}$ NMR $\left(500 \mathrm{MHz}, \mathrm{CDCl}_{3}\right) \delta(\mathrm{ppm})=10.09(\mathrm{~s}, 0.02 \mathrm{H}), 8.61(\mathrm{~s}, 1 \mathrm{H}), 8.17-8.15(\mathrm{~m}, 1 \mathrm{H}), 8.02(\mathrm{dd}, J=$ $4 \mathrm{~Hz}, 1 \mathrm{H}), 7.56-7.53(\mathrm{~m}, 1 \mathrm{H}), 7.48-7.45(\mathrm{~m}, 1 \mathrm{H}), 7.35-7.31(\mathrm{~m}, 2 \mathrm{H}), 4.40$ (q, J = $4 \mathrm{~Hz}, 2 \mathrm{H}), 1.47$ (q, 
$J=4 \mathrm{~Hz}, 3 \mathrm{H}) .{ }^{13} \mathrm{C}$ NMR $\left(125 \mathrm{MHz}, \mathrm{CDCl}_{3}\right) \delta(\mathrm{ppm})=191.6(\mathrm{t}, J=26 \mathrm{~Hz}), 143.7,140.8,128.6,127.3$, $126.9,124.1,123.3,123.2,120.9,120.4,109.3,108.8,38.1,14.0$

\begin{tabular}{|c|c|c|c|c|c|c|c|c|c|c|}
\hline \multirow{3}{*}{$\begin{array}{l}\text { Entry } \\
1\end{array}$} & \multirow{2}{*}{$\begin{array}{c}3 \mathbf{a} \\
(\mathrm{mol} \%)\end{array}$} & \multirow{2}{*}{$\begin{array}{c}\mathrm{D}_{2} \mathrm{O} \\
\text { (eq. } \\
\text { ) }\end{array}$} & \multirow{2}{*}{$\begin{array}{c}\text { Ph- } \\
\text { Boroxine } \\
\text { (mol\%) }\end{array}$} & \multirow{2}{*}{$\begin{array}{l}\text { Reactio } \\
\text { n Conc. } \\
\text { (M) }\end{array}$} & \multirow{3}{*}{$\begin{array}{l}\text { Reactio } \\
\text { n } \\
\text { Temp. } \\
50 \cong \mathrm{C}\end{array}$} & \multirow{3}{*}{$\begin{array}{c}\% \mathrm{D} \\
(\mathrm{RM}) \\
77\end{array}$} & \multirow{2}{*}{$\begin{array}{c}\% \\
\text { Aldehyd } \\
\mathrm{e}\end{array}$} & \multirow{2}{*}{$\begin{array}{c}\% \\
\text { Benzoin }\end{array}$} & \multicolumn{2}{|c|}{$\begin{array}{c}\text { After } \\
\text { Purification }\end{array}$} \\
\hline & & & & & & & & & $\% \mathrm{D}$ & \%Yield \\
\hline & 5 & 30 & - & 1 & & & 100 & - & - & - \\
\hline 2 & 5 & 30 & - & 1 & $50 \stackrel{\circ}{ } \mathrm{C}$ & 98 & 91 & 9 & - & - \\
\hline 3 & 5 & 30 & 4 & 1 & $50 \stackrel{\circ}{C}$ & 98 & 84 & 16 & - & - \\
\hline 4 & 5 & 30 & 4 & 0.5 & $50 \stackrel{\circ}{C}$ & 99 & 95 & 5 & 98 & 97 \\
\hline $4^{\prime}$ & 5 & 30 & 4 & 0.5 & $50 \stackrel{\circ}{C}$ & 98 & 95 & 5 & - & - \\
\hline
\end{tabular}

\section{Pyrrole-2-carbaldehyde-d}<smiles>[2H]C(=O)c1ccc[nH]1</smiles>

4a (28 mg, $0.055 \mathrm{mmol})$, DIPEA (10 $\mu \mathrm{L}, 0.055 \mathrm{mmol}), \mathrm{D}_{2} \mathrm{O}(600 \mu \mathrm{L}, 33 \mathrm{mmol})$ and pyrrole-2carbaldehyde (105 mg, $1.1 \mathrm{mmol})$ in anhydrous toluene $(500 \mu \mathrm{L})$ were prepared according to the general procedure given in section 5 (page 20) and stirred at $50 \stackrel{\circ}{\circ}$. Crude residue was purified by flash-column chromatography using $\mathrm{Hex}_{\mathrm{Et}} \mathrm{O}$ (63:38) combination to give colorless solid. Spectral data for this product have been previously reported. ${ }^{24}$

Deuterium incorporation $=95 \%$ (crude: $99 \%, 97 \%)$, Reaction yield $=74 \%$ (78 mg). NMR spectra are given on pages 348-351.

${ }^{1} \mathrm{H}$ NMR $\left(500 \mathrm{MHz}, \mathrm{CDCl}_{3}\right) \delta(\mathrm{ppm})=10.73(\mathrm{~s}, 0.05 \mathrm{H}), 9.51(\mathrm{~s}, 1 \mathrm{H})$, 7.20-7.19 (m, $\left.1 \mathrm{H}\right)$, 7.03-7.01 (m, $1 \mathrm{H}), 6.36-6.34(\mathrm{~m}, 1 \mathrm{H}) .{ }^{13} \mathrm{C}$ NMR $\left(125 \mathrm{MHz}, \mathrm{CDCl}_{3}\right) \delta(\mathrm{ppm})=179.4(\mathrm{t}, J=26 \mathrm{~Hz}), 132.9,127.3$, 122.2, 111.5.

\begin{tabular}{|c|c|c|c|c|c|c|c|c|c|c|}
\hline \multirow[t]{2}{*}{ Entry } & \multirow{2}{*}{$\begin{array}{c}\mathrm{NHC} \\
\text { (mol\%) }\end{array}$} & \multirow{2}{*}{$\begin{array}{l}\text { DIPEA } \\
\text { (mol\%) }\end{array}$} & \multirow{2}{*}{$\begin{array}{l}\mathrm{D}_{2} \mathrm{O} \\
\text { (eq. } \\
\text { ) }\end{array}$} & \multirow{2}{*}{$\begin{array}{c}\mathrm{Ph}- \\
\text { Boroxine } \\
\text { (mol\%) }\end{array}$} & \multirow{2}{*}{$\begin{array}{l}\text { Reactio } \\
\text { n Conc. } \\
\text { (M) }\end{array}$} & \multirow{2}{*}{$\begin{array}{l}\text { Reactio } \\
\text { n } \\
\text { Temp. }\end{array}$} & \multirow{2}{*}{$\begin{array}{c}\% \mathrm{D} \\
\text { (RM) }\end{array}$} & \multirow{2}{*}{$\begin{array}{c}\% \\
\text { Aldehyd } \\
\text { e }\end{array}$} & \multicolumn{2}{|c|}{$\begin{array}{c}\text { After } \\
\text { Purification }\end{array}$} \\
\hline & & & & & & & & & $\% \mathrm{D}$ & \%Yield \\
\hline 1 & $3 a(5)$ & 5 & 30 & - & 1 & $20^{\circ} \mathrm{C}$ & 3 & 100 & - & - \\
\hline $1^{\prime}$ & $3 a(5)$ & 5 & 30 & - & 1 & $20^{\circ} \mathrm{C}$ & 3 & 100 & - & - \\
\hline 2 & 4a (5) & 5 & 30 & - & 1 & $50 \stackrel{\circ}{ }$ & 99 & 100 & 95 & 74 \\
\hline $2^{\prime}$ & $4 a(5)$ & 5 & 30 & - & 1 & $50 \stackrel{\circ}{ }$ & 97 & 100 & - & - \\
\hline
\end{tabular}




\section{1-(phenylsulfonyl)-pyrrole-2-carbaldehyde-d}<smiles>O=C(O)c1cccn1S(=O)(=O)c1ccccc1</smiles>

4a (14 mg, $0.0275 \mathrm{mmol})$, DIPEA ( $5 \mu \mathrm{L}, 0.0275 \mathrm{mmol}), \mathrm{D}_{2} \mathrm{O}(300 \mu \mathrm{L}, 17 \mathrm{mmol}$ ) and 1-(phenylsulfonyl)pyrrole-2-carbaldehyde $(130 \mathrm{mg}, 0.55 \mathrm{mmol})$ in anhydrous toluene $(250 \mu \mathrm{L})$ were prepared according to the general procedure given in section 5 (page 20) and stirred at 50 ㄷ. Crude residue was purified by flash-column chromatography using Hex: $\mathrm{Et}_{2} \mathrm{O}(50: 50)$ combination to give colorless solid.

Deuterium incorporation $=98 \%$ (crude: $98 \%, 98 \%)$, Reaction yield $=92 \%$ (120 mg). NMR spectra are given on pages 352-355.

${ }^{1} \mathrm{H}$ NMR $\left(500 \mathrm{MHz}, \mathrm{CDCl}_{3}\right) \delta(\mathrm{ppm})=9.94(\mathrm{~s}, 0.02 \mathrm{H})$, 7.93-7.91 (m, $\left.2 \mathrm{H}\right)$, 7.65-7.62 (m, $\left.2 \mathrm{H}\right)$, 7.55$7.51(\mathrm{~m}, 2 \mathrm{H}), 7.17(\mathrm{q}, J=5 \mathrm{~Hz}, 1 \mathrm{H}), 6.41(\mathrm{t}, J=5 \mathrm{~Hz}, 1 \mathrm{H}) .{ }^{13} \mathrm{C} \mathrm{NMR}\left(125 \mathrm{MHz}, \mathrm{CDCl}_{3}\right) \delta(\mathrm{ppm})=178.6$ $(t, J=28 \mathrm{~Hz}), 138.3,134.7,133.6,129.6,127.5,124.9,112.7$. HRMS(ESI) calcd for $\mathrm{C}_{11} \mathrm{H}_{8} \mathrm{DNO}_{3} \mathrm{~S}^{+}$ $(\mathrm{M}+\mathrm{H})^{+}:$237.0439, Found: 237.0437

\begin{tabular}{|c|c|c|c|c|c|c|c|c|c|c|}
\hline \multirow{3}{*}{$\begin{array}{l}\text { Entry } \\
1 \\
\end{array}$} & \multirow{2}{*}{$\begin{array}{c}\mathrm{NHC} \\
(\mathrm{mol} \%)\end{array}$} & \multirow{3}{*}{$\begin{array}{c}\mathrm{D}_{2} \mathrm{O} \\
\text { (eq. } \\
\text { ) } \\
30 \\
\end{array}$} & \multirow{3}{*}{$\begin{array}{c}\text { Ph- } \\
\text { Boroxine } \\
\text { (mol\%) } \\
-\end{array}$} & \multirow{3}{*}{$\begin{array}{c}\text { Reactio } \\
\text { n Conc. } \\
\text { (M) } \\
1 \\
\end{array}$} & \multirow{3}{*}{$\begin{array}{l}\text { Reactio } \\
\text { n } \\
\text { Temp. } \\
50 \stackrel{\circ}{ }=0\end{array}$} & \multirow{3}{*}{$\begin{array}{c}\% \mathrm{D} \\
\text { (RM) } \\
46 \\
\end{array}$} & \multirow{2}{*}{$\begin{array}{c}\% \\
\text { Aldehyd } \\
\mathrm{e}\end{array}$} & \multirow{2}{*}{$\begin{array}{c}\% \\
\text { Benzoin }\end{array}$} & \multicolumn{2}{|c|}{$\begin{array}{c}\text { After } \\
\text { Purification }\end{array}$} \\
\hline & & & & & & & & & $\% \mathrm{D}$ & \%Yield \\
\hline & $3 a(5)$ & & & & & & 100 & - & - & - \\
\hline 2 & $4 \mathbf{i}(5)$ & 30 & - & 1 & $50 \div C$ & 100 & 34 & 66 & - & - \\
\hline 3 & $4 a(5)$ & 30 & - & 1 & 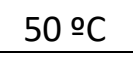 & 98 & 94 & 6 & 98 & 92 \\
\hline $3^{\prime}$ & $4 a(5)$ & 30 & - & 1 & $50 \stackrel{\circ}{C}$ & 98 & 94 & 6 & - & - \\
\hline
\end{tabular}

\section{Dibenzo[b,d]furan-1-carbaldehyde-d}<smiles>[2H]C(=O)c1cccc2oc3ccccc3c12</smiles>

3a (11.8 mg, $0.0275 \mathrm{mmol})$, DIPEA (5 $\mu \mathrm{L}, 0.0275 \mathrm{mmol}), \mathrm{D}_{2} \mathrm{O}(300 \mu \mathrm{L}, 17 \mathrm{mmol})$ and dibenzo[b,d]furan-1-carbaldehyde $(108 \mathrm{mg}, 0.55 \mathrm{mmol})$ in anhydrous toluene $(250 \mu \mathrm{L})$ were prepared according to the general procedure given in section 5 (page 20) and stirred at 50 ㄷ. Crude 
residue was purified by flash-column chromatography using Hex:Et ${ }_{2} \mathrm{O}(89: 11)$ combination to give colorless solid.

Deuterium incorporation $=98 \%$ (crude: $98 \%, 98 \%)$, Reaction yield = 98\% (106 mg). NMR spectra are given on pages $356-358$.

${ }^{1} \mathrm{H}$ NMR $\left(500 \mathrm{MHz}, \mathrm{CDCl}_{3}\right) \delta(\mathrm{ppm})=10.57(\mathrm{~s}, 0.02 \mathrm{H}), 8.16(\mathrm{~d}, J=5 \mathrm{~Hz}, 1 \mathrm{H}), 7.97-7.93(\mathrm{~m}, 2 \mathrm{H}), 7.67$ (d, $J=10 \mathrm{~Hz}, 1 \mathrm{H}), 7.52(\mathrm{t}, J=10 \mathrm{~Hz}, 1 \mathrm{H}), 7.45(\mathrm{t}, J=10 \mathrm{~Hz}, 1 \mathrm{H}), 7.40$ (t, J=10 Hz, $1 \mathrm{H}) .{ }^{13} \mathrm{C} \mathrm{NMR}$ $\left(125 \mathrm{MHz}, \mathrm{CDCl}_{3}\right) \delta(\mathrm{ppm})=188.2(\mathrm{t}, J=28 \mathrm{~Hz}), 156.7,156.0,128.2,127.5,126.8,126.1,123.6$, 123.0, 122.9, 121.3, 120.9, 112.3. HRMS(ESI) calcd for $\mathrm{C}_{13} \mathrm{H}_{7} \mathrm{DO}_{2}{ }^{+}(\mathrm{M}+\mathrm{H})^{+}:$198.0660, Found: 198.0657

\begin{tabular}{|c|c|c|c|c|c|c|c|c|c|c|}
\hline \multirow[t]{2}{*}{ Entry } & \multirow{2}{*}{$\begin{array}{c}3 a \\
(\mathrm{~mol} \%)\end{array}$} & \multirow{2}{*}{$\begin{array}{l}\text { DIPEA } \\
\text { (mol\%) }\end{array}$} & \multirow{2}{*}{$\begin{array}{c}\mathrm{D}_{2} \mathrm{O} \\
\text { (eq. } \\
\text { ) }\end{array}$} & \multirow{2}{*}{$\begin{array}{c}\text { Ph- } \\
\text { Boroxine } \\
\text { (mol\%) }\end{array}$} & \multirow{2}{*}{$\begin{array}{l}\text { Reactio } \\
\text { n Conc. } \\
\text { (M) }\end{array}$} & \multirow{2}{*}{$\begin{array}{l}\text { Reactio } \\
\text { n } \\
\text { Temp. }\end{array}$} & \multirow{2}{*}{$\begin{array}{c}\% \mathrm{D} \\
\text { (RM) }\end{array}$} & \multirow{2}{*}{$\begin{array}{c}\% \\
\text { Aldehyd } \\
\text { e }\end{array}$} & \multicolumn{2}{|c|}{$\begin{array}{c}\text { After } \\
\text { Purification }\end{array}$} \\
\hline & & & & & & & & & $\% \mathrm{D}$ & \%Yield \\
\hline 1 & 5 & 5 & 30 & - & 1 & $50 \stackrel{\circ}{ } \mathrm{C}$ & 98 & 100 & 98 & 98 \\
\hline $1^{\prime}$ & 5 & 5 & 30 & - & 1 & $50 \stackrel{\circ}{ } \mathrm{C}$ & 98 & 100 & - & - \\
\hline
\end{tabular}

\section{Ibuprofen Derivative-d}<smiles>[2H]C(=O)c1cccc(OC(=O)[C@H](C)c2ccc(CC(C)C)cc2)c1</smiles>

3a (11.8 mg, $0.0275 \mathrm{mmol})$, DIPEA ( $5 \mu \mathrm{L}, 0.0275 \mathrm{mmol}), \mathrm{D}_{2} \mathrm{O}(300 \mu \mathrm{L}, 17 \mathrm{mmol}$ ) and $S(+)$ ibuprofen derivative (171 $\mathrm{mg}, 0.55 \mathrm{mmol}, 92 \% \mathrm{ee})$ in anhydrous toluene $(250 \mu \mathrm{L})$ were prepared according to the general procedure given in section 5 (page 20) and stirred at $50 \stackrel{\circ}{ } \mathrm{C}$. Crude residue was purified by flash-column chromatography using Hex: $\mathrm{Et}_{2} \mathrm{O}(91: 9)$ combination to give colorless solid. Spectral data for this product have been previously reported. ${ }^{17}$

Deuterium incorporation $=98 \%$ (crude: 98\%, 97\%), Reaction yield = 98\% (168 mg), starting material ee $=92 \%$, deuterated product $\mathbf{e e}=91 \%$. NMR spectra are given on pages 359-363.

${ }^{1} \mathrm{H}$ NMR $\left(500 \mathrm{MHz}, \mathrm{CDCl}_{3}\right) \delta(\mathrm{ppm})=9.96(\mathrm{~s}, 0.02 \mathrm{H}), 7.71(\mathrm{~d}, J=10 \mathrm{~Hz}, 1 \mathrm{H}), 7.52-7.48(\mathrm{~m}, 2 \mathrm{H})$, 7.29$7.24(\mathrm{~m}, 3 \mathrm{H}), 7.15$ (d, J = 5 Hz, 2 H), 3.95 (q, J = $10 \mathrm{~Hz}, 1 \mathrm{H}), 2.47$ (d, J = 5 Hz, 2 H), 1.86 (spt, $J=5 \mathrm{~Hz}$, $1 \mathrm{H}), 1.61(\mathrm{~d}, J=5 \mathrm{~Hz}, 3 \mathrm{H}), 0.90(\mathrm{~d}, J=5 \mathrm{~Hz}, 6 \mathrm{H}) .{ }^{13} \mathrm{C} \mathrm{NMR}\left(125 \mathrm{MHz}, \mathrm{CDCl}_{3}\right) \delta(\mathrm{ppm})=191.0$ (t, J= $26 \mathrm{~Hz}), 173.1,151.6,141.2,137.7,137.0,130.2,129.8,127.8,127.3,122.3,45.4,45.2,30.3,22.5$, 18.6

\begin{tabular}{|c|c|c|c|c|c|c|c|c|c|}
\hline Entry & $\begin{array}{c}\text { DIPEA } \\
(\text { mol\%) }\end{array}$ & $\mathrm{D}_{2} \mathrm{O}$ & $\begin{array}{c}\text { Ph- } \\
\text { Boroxine }\end{array}$ & $\begin{array}{c}\text { Reactio } \\
\mathrm{n} \text { Conc. }\end{array}$ & $\begin{array}{c}\text { Reactio } \\
\mathrm{n}\end{array}$ & $\begin{array}{c}\% \mathrm{D} \\
(\mathrm{RM})\end{array}$ & $\%$ & $\begin{array}{c}\text { After } \\
\text { Purification }\end{array}$ \\
\hline
\end{tabular}




\begin{tabular}{|c|c|c|c|c|c|c|c|c|c|c|}
\hline & $\begin{array}{c}\text { 3a } \\
\text { (mol\%) }\end{array}$ & & $\begin{array}{c}\text { (eq. } \\
\text { ) }\end{array}$ & $\begin{array}{c}\text { (mol\%) } \\
\text { (M) }\end{array}$ & Temp. & $\begin{array}{c}\text { Aldehyd } \\
\mathrm{e}\end{array}$ & \%D & \%Yield \\
\hline 1 & 5 & 5 & 30 & - & 1 & $50 \cong \mathrm{C}$ & 98 & 100 & 98 & 98 \\
\hline $1^{\prime}$ & 5 & 5 & 30 & - & 1 & $50 \cong \mathrm{C}$ & 97 & 100 & - & - \\
\hline
\end{tabular}

\section{Menthol Derivative-d}<smiles>[2H]C(=O)c1cccc(C(=O)O[C@H]2C[C@H](C)CC[C@H]2C(C)C)c1</smiles>

3a (11.8 mg, $0.0275 \mathrm{mmol})$, DIPEA ( $5 \mu \mathrm{L}, 0.0275 \mathrm{mmol}), \mathrm{D}_{2} \mathrm{O}(300 \mu \mathrm{L}, 17 \mathrm{mmol})$ and L(-) menthol $(1 R, 2 S, 5 R)$ derivative (159 $\mathrm{mg}, 0.55 \mathrm{mmol}, 99 \% \mathrm{ee})$ in anhydrous toluene $(250 \mu \mathrm{L})$ were prepared according to the general procedure given in section 5 (page 20) and stirred at $50 \stackrel{\circ}{ }$. Crude residue was purified by flash-column chromatography using Hex: $\mathrm{Et}_{2} \mathrm{O}(91: 9)$ combination to give colorless solid. Spectral data for this product have been previously reported. ${ }^{17}$

Deuterium incorporation $=98 \%$ (crude: $98 \%, 98 \%)$, Reaction yield = 91\% (144 mg), starting material ee $=99 \%$, deuterated product $\mathbf{e e}=99 \%$. NMR spectra are given on pages 364-368.

\begin{tabular}{|c|c|c|c|c|c|c|c|c|c|c|}
\hline \multirow[t]{2}{*}{ Entry } & \multirow{2}{*}{$\begin{array}{c}3 a \\
(\mathrm{~mol} \%)\end{array}$} & \multirow{2}{*}{$\begin{array}{l}\text { DIPEA } \\
\text { (mol\%) }\end{array}$} & \multirow{2}{*}{$\begin{array}{c}\mathrm{D}_{2} \mathrm{O} \\
\text { (eq. } \\
\text { ) }\end{array}$} & \multirow{2}{*}{$\begin{array}{c}\text { Ph- } \\
\text { Boroxine } \\
\text { (mol\%) }\end{array}$} & \multirow{2}{*}{$\begin{array}{l}\text { Reactio } \\
\text { n Conc. } \\
\text { (M) }\end{array}$} & \multirow{2}{*}{$\begin{array}{l}\text { Reactio } \\
\text { n } \\
\text { Temp. }\end{array}$} & \multirow{2}{*}{$\begin{array}{c}\% \mathrm{D} \\
\text { (RM) }\end{array}$} & \multirow{2}{*}{$\begin{array}{c}\% \\
\text { Aldehyd } \\
\text { e }\end{array}$} & \multicolumn{2}{|c|}{$\begin{array}{c}\text { After } \\
\text { Purification }\end{array}$} \\
\hline & & & & & & & & & $\% \mathrm{D}$ & \%Yield \\
\hline 1 & 5 & 5 & 30 & - & 1 & $50 \stackrel{\circ}{ } \mathrm{C}$ & 98 & 100 & 98 & 91 \\
\hline $1^{\prime}$ & 5 & 5 & 30 & - & 1 & $50 \stackrel{\circ}{ } \mathrm{C}$ & 98 & 100 & - & - \\
\hline
\end{tabular}

${ }^{1} \mathrm{H}$ NMR $\left(500 \mathrm{MHz}, \mathrm{CDCl}_{3}\right) \delta(\mathrm{ppm})=10.09(\mathrm{~s}, 0.02 \mathrm{H}), 8.52(\mathrm{t}, J=5 \mathrm{~Hz}, 1 \mathrm{H}), 8.31(\mathrm{dt}, J=10 \mathrm{~Hz}, 1 \mathrm{H})$, $8.08(\mathrm{dt}, J=10 \mathrm{~Hz}, 1 \mathrm{H}), 7.62(\mathrm{~d}, J=10 \mathrm{~Hz}, 1 \mathrm{H}), 4.99(\mathrm{td}, J=10 \mathrm{~Hz}, 1 \mathrm{H}), 2.15-2.09(\mathrm{~m}, 1 \mathrm{H}), 1.98-$ $1.90(\mathrm{~m}, 1 \mathrm{H}), 1.77-172(\mathrm{~m}, 2 \mathrm{H}), 1.62-1.52(\mathrm{~m}, 2 \mathrm{H}), 1.19-1.09(\mathrm{~m}, 2 \mathrm{H}), 0.94-0.91(\mathrm{~m}, 6 \mathrm{H}), 0.80(\mathrm{~d}$, $J=5 \mathrm{~Hz}, 3 \mathrm{H}) .{ }^{13} \mathrm{C}$ NMR $\left(125 \mathrm{MHz}, \mathrm{CDCl}_{3}\right) \delta(\mathrm{ppm})=191.3(\mathrm{t}, J=26 \mathrm{~Hz}), 165.1,136.6,135.4,133.1$, $132.1,131.3,129.3,75.7,47.3,41.1,34.4,31.6,26.7,23.7,22.2,20.9,16.6$

\section{Naproxen Derivative-d}<smiles>[2H]C(=O)c1cccc(OC(=O)[C@H](C)c2ccc3cc(OC)ccc3c2)c1</smiles>

3a (11.8 mg, $0.0275 \mathrm{mmol})$, DIPEA (5 $\mu \mathrm{L}, 0.0275 \mathrm{mmol}), \mathrm{D}_{2} \mathrm{O}(300 \mu \mathrm{L}, 17 \mathrm{mmol})$ and naproxen derivative (184 $\mathrm{mg}, 0.55 \mathrm{mmol})$ in anhydrous toluene $(250 \mu \mathrm{L})$ were prepared according to the 
general procedure given in section 5 (page 20) and stirred at $50 \circ \mathrm{C}$. Crude residue was purified by flash-column chromatography using $\mathrm{Hex}_{\mathrm{Et}} \mathrm{O}$ (50:50) combination to give colorless solid.

Deuterium incorporation = 98\% (crude: 99\%, 98\%), Reaction yield = 96\% (177 mg). NMR spectra are given on pages $369-371$.

${ }^{1} \mathrm{H}$ NMR $\left(500 \mathrm{MHz}, \mathrm{CDCl}_{3}\right) \delta(\mathrm{ppm})=9.95(\mathrm{~s}, 0.02 \mathrm{H}), 7.78-7.74(\mathrm{~m}, 3 \mathrm{H}), 7.72(\mathrm{dt}, J=5 \mathrm{~Hz}, 1 \mathrm{H}), 7.54-$ $7.48(\mathrm{~m}, 3 \mathrm{H}), 7.27-7.25(\mathrm{~m}, 1 \mathrm{H}), 7.19-7.15(\mathrm{~m}, 2 \mathrm{H}), 4.13(\mathrm{q}, J=5 \mathrm{~Hz}, 1 \mathrm{H}), 3.93(\mathrm{~s}, 3 \mathrm{H}), 1.72(\mathrm{~d}, J=$ $5 \mathrm{~Hz}, 3 \mathrm{H}) .{ }^{13} \mathrm{C} \mathrm{NMR}\left(125 \mathrm{MHz}, \mathrm{CDCl}_{3}\right) \delta(\mathrm{ppm})=191.0(\mathrm{t}, J=26 \mathrm{~Hz}), 173.0,158.0,151.5,137.7,134.9$, $134.3,130.2,129.5,129.1,127.7,127.6,127.4,126.3,126.1,122.3,119.4$, 105.8, 55.5, 45.7, 18.6. HRMS(ESI) calcd for $\mathrm{C}_{21} \mathrm{H}_{17} \mathrm{DO}_{4}{ }^{+}(\mathrm{M}+\mathrm{H})^{+}: 336.1341$, Found: 336.1334

\begin{tabular}{|c|c|c|c|c|c|c|c|c|c|c|}
\hline \multirow[t]{2}{*}{ Entry } & \multirow{2}{*}{$\begin{array}{c}3 a \\
(\mathrm{~mol} \%)\end{array}$} & \multirow{2}{*}{$\begin{array}{l}\text { DIPEA } \\
\text { (mol\%) }\end{array}$} & \multirow{2}{*}{$\begin{array}{l}\mathrm{D}_{2} \mathrm{O} \\
\text { (eq. } \\
\text { ) }\end{array}$} & \multirow{2}{*}{$\begin{array}{c}\text { Ph- } \\
\text { Boroxine } \\
\text { (mol\%) }\end{array}$} & \multirow{2}{*}{$\begin{array}{c}\text { Reactio } \\
\text { n Conc. } \\
\text { (M) }\end{array}$} & \multirow{2}{*}{$\begin{array}{c}\text { Reactio } \\
\text { n } \\
\text { Temp. }\end{array}$} & \multirow{2}{*}{$\begin{array}{c}\% \mathrm{D} \\
\text { (RM) }\end{array}$} & \multirow{2}{*}{$\begin{array}{c}\% \\
\text { Aldehyd } \\
\text { e }\end{array}$} & \multicolumn{2}{|c|}{$\begin{array}{c}\text { After } \\
\text { Purification }\end{array}$} \\
\hline & & & & & & & & & \%D & \%Yield \\
\hline 1 & 5 & 5 & 30 & - & 1 & 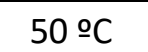 & 99 & 100 & 98 & 96 \\
\hline $1^{\prime}$ & 5 & 5 & 30 & - & 1 & $50 \stackrel{\circ}{ } \mathrm{C}$ & 98 & 100 & - & - \\
\hline
\end{tabular}

\section{Epiandrosterone Derivative-d}

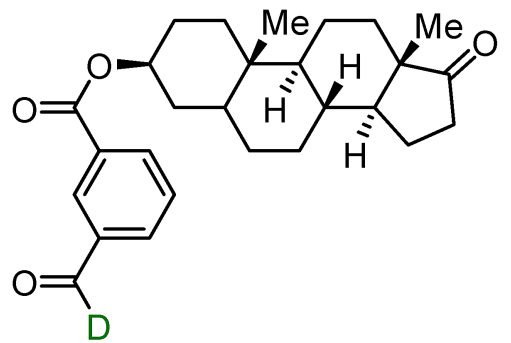

3a (6 mg, $0.0138 \mathrm{mmol})$, DIPEA (2.5 $\mu \mathrm{L}, 0.0138 \mathrm{mmol}), \mathrm{D}_{2} \mathrm{O}(150 \mu \mathrm{L}, 8.50 \mathrm{mmol})$, phenyl boroxine $(3.4 \mathrm{mg}, 0.011 \mathrm{mmol})$ and epiandrosterone derivative $(118 \mathrm{mg}, 0.28 \mathrm{mmol})$ in anhydrous toluene $(400 \mu \mathrm{L})$ were prepared according to the general procedure given in section 5 (page 20) and stirred at $50 \stackrel{\circ}{ }$. Crude residue was purified by flash-column chromatography using Hex:Et ${ }_{2} \mathrm{O}$ (63:37) combination to give colorless solid. ${ }^{19}$

Deuterium incorporation $=98 \%$ (crude: $100 \%, 100 \%)$, Reaction yield $=97 \%$ (115 mg). NMR spectra are given on pages $372-375$.

${ }^{1} \mathbf{H}$ NMR $\left(400 \mathrm{MHz}, \mathrm{CDCl}_{3}\right) \delta(\mathrm{ppm})=10.08(\mathrm{~s}, 0.02 \mathrm{H}), 8.51(\mathrm{~s}, 1 \mathrm{H}), 8.29(\mathrm{~d}, J=8 \mathrm{~Hz}, 1 \mathrm{H}), 8.07(\mathrm{~d}, J$ $=4 \mathrm{~Hz}, 1 \mathrm{H}), 7.61(\mathrm{t}, J=8 \mathrm{~Hz}, 1 \mathrm{H}), 4.98(\mathrm{~m}, 1 \mathrm{H}), 2.44(\mathrm{~m}, 1 \mathrm{H}), 2.07(\mathrm{~m}, 1 \mathrm{H}), 1.94(\mathrm{~m}, 2 \mathrm{H}), 1.74(\mathrm{~m}$, $6 \mathrm{H}), 1.52(\mathrm{~m}, 3 \mathrm{H}), 1.32(\mathrm{~m}, 6 \mathrm{H}), 1.06(\mathrm{~m}, 2 \mathrm{H}), 0.91(\mathrm{~s}, 3 \mathrm{H}), 0.86(\mathrm{~s}, 3 \mathrm{H}), 0.75(\mathrm{~m}, 1 \mathrm{H}) .{ }^{13} \mathrm{C}$ NMR $\left(100 \mathrm{MHz}, \mathrm{CDCl}_{3}\right) \delta(\mathrm{ppm})=221.3,191.3(\mathrm{t}, J=27 \mathrm{~Hz}), 165.1,136.5,135.3,133.2,132.1,131.2$, 
$129.3,74.9,54.5,51.5,47.9,44.8,36.9,36.0,35.8,35.2,34.1,31.7,30.9,28.4,27.6,21.9,20.6$, 14.0, 12.4. 


\begin{tabular}{|c|c|c|c|c|c|c|c|c|c|c|}
\hline \multirow[t]{2}{*}{ Entry } & \multirow{2}{*}{$\begin{array}{c}3 a \\
(\mathrm{~mol} \%)\end{array}$} & \multirow{2}{*}{$\begin{array}{l}\text { DIPEA } \\
\text { (mol\%) }\end{array}$} & \multirow{2}{*}{$\begin{array}{c}\text { Ph- } \\
\text { Boroxine } \\
\text { (mol\%) }\end{array}$} & \multirow{2}{*}{$\begin{array}{l}\text { Reactio } \\
\text { n Conc. } \\
\text { (M) }\end{array}$} & \multirow{2}{*}{$\begin{array}{l}\text { Reactio } \\
\text { n } \\
\text { Temp. }\end{array}$} & \multirow{2}{*}{$\begin{array}{c}\% \mathrm{D} \\
\text { (RM) }\end{array}$} & \multirow{2}{*}{$\begin{array}{c}\% \\
\text { Aldehyd } \\
\text { e }\end{array}$} & \multirow{2}{*}{$\begin{array}{c}\% \\
\text { Benzoin }\end{array}$} & \multicolumn{2}{|c|}{$\begin{array}{c}\text { After } \\
\text { Purification }\end{array}$} \\
\hline & & & & & & & & & $\% \mathrm{D}$ & \%Yield \\
\hline 1 & 5 & 5 & - & 1 & $50 \stackrel{\circ}{C}$ & 98 & 91 & 9 & - & - \\
\hline $1^{\prime}$ & 5 & 5 & - & 1 & $50 \stackrel{\circ}{C}$ & 98 & 92 & 8 & - & - \\
\hline 2 & 5 & 5 & 4 & 0.5 & 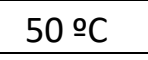 & 100 & 100 & - & 98 & 97 \\
\hline $2^{\prime}$ & 5 & 5 & 4 & 0.5 & 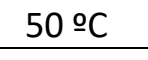 & 100 & 100 & - & - & - \\
\hline
\end{tabular}

\section{Pregnenolone Derivative-d}

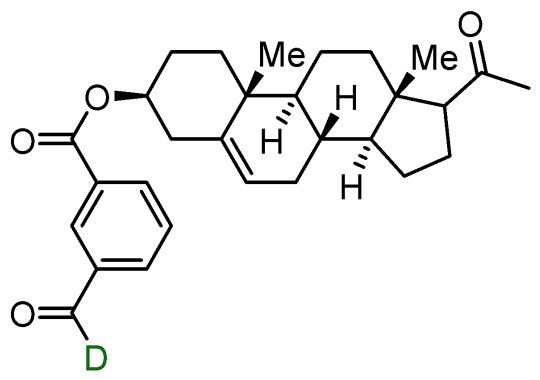

3a (11.8 mg, $0.0275 \mathrm{mmol}$ ), DIPEA (5 $\mu \mathrm{L}, 0.0275 \mathrm{mmol}), \mathrm{D}_{2} \mathrm{O}(300 \mu \mathrm{L}, 17 \mathrm{mmol}$ ) and pregnenolone derivative $(246 \mathrm{mg}, 0.55 \mathrm{mmol})$ in anhydrous toluene $(250 \mu \mathrm{L})$ were prepared according to the general procedure given in section 5 (page 20) and stirred at $50 \stackrel{\circ}{ }$. Crude residue was purified by flash-column chromatography using $\mathrm{Hex} \mathrm{Et}_{2} \mathrm{O}$ (67:33) combination to give colorless solid. ${ }^{19}$

Deuterium incorporation $=98 \%$ (crude: 99\%, 98\%), Reaction yield $=93 \%$ (230 mg). NMR spectra are given on pages $376-379$.

${ }^{1} \mathrm{H}$ NMR $\left(400 \mathrm{MHz}, \mathrm{CDCl}_{3}\right) \delta(\mathrm{ppm})=10.08(\mathrm{~s}, 0.02 \mathrm{H}), 8.52(\mathrm{t}, J=4 \mathrm{~Hz}, 1 \mathrm{H}), 8.30(\mathrm{dt}, J=8 \mathrm{~Hz}, 1 \mathrm{H})$, $8.05(\mathrm{dt}, J=8 \mathrm{~Hz}, 1 \mathrm{H}), 7.62(\mathrm{t}, J=8 \mathrm{~Hz}, 1 \mathrm{H}), 5.43(\mathrm{~d}, J=4 \mathrm{~Hz}, 1 \mathrm{H}), 4.90(\mathrm{~m}, 1 \mathrm{H}), 2.52(\mathrm{~m}, 3 \mathrm{H}), 2.19$ (m, 1 H), $2.12(\mathrm{~s}, 3 \mathrm{H}), 2.00(\mathrm{~m}, 4 \mathrm{H}), 1.71(\mathrm{~m}, 5 \mathrm{H}), 1.50(\mathrm{~m}, 3 \mathrm{H}), 1.21(\mathrm{~m}, 3 \mathrm{H}), 1.05(\mathrm{~m}, 4 \mathrm{H}), 0.64$ $(\mathrm{s}, 3 \mathrm{H}) .{ }^{13} \mathrm{C}$ NMR $\left(100 \mathrm{MHz}, \mathrm{CDCl}_{3}\right) \delta(\mathrm{ppm})=209.5,191.2$ (t, J = $\left.27 \mathrm{~Hz}\right), 164.9,139.5,136.5,135.3$, 133.1, 132.0, 131.2, 129.3, 122.8, 75.2, 63.7, 56.9, 50.0, 44.1, 38.9, 38.2, 37.1, 36.7, 31.9, 31.6, 27.9, 24.6, 22.9, 21.1, 19.4, 13.3.

\begin{tabular}{|c|c|c|c|c|c|c|c|c|c|c|}
\hline \multirow{2}{*}{ Entry } & \multirow{2}{*}{$\begin{array}{c}3 a \\
(\mathrm{~mol} \%)\end{array}$} & \multirow{2}{*}{$\begin{array}{l}\text { DIPEA } \\
\text { (mol\%) }\end{array}$} & \multirow{2}{*}{$\begin{array}{l}\mathrm{D}_{2} \mathrm{O} \\
\text { (eq. } \\
\text { ) }\end{array}$} & \multirow{2}{*}{$\begin{array}{c}\text { Ph- } \\
\text { Boroxine } \\
\text { (mol\%) }\end{array}$} & \multirow{2}{*}{$\begin{array}{l}\text { Reactio } \\
\text { n Conc. } \\
\text { (M) }\end{array}$} & \multirow{2}{*}{$\begin{array}{l}\text { Reactio } \\
\text { n } \\
\text { Temp. }\end{array}$} & \multirow{2}{*}{$\begin{array}{l}\% \mathrm{D} \\
\text { (RM) }\end{array}$} & \multirow{2}{*}{$\begin{array}{c}\% \\
\text { Aldehyd } \\
\text { e }\end{array}$} & \multicolumn{2}{|c|}{$\begin{array}{c}\text { After } \\
\text { Purification }\end{array}$} \\
\hline & & & & & & & & & $\% \mathrm{D}$ & \%Yield \\
\hline 1 & 5 & 5 & 30 & - & 1 & $50 \div \mathrm{C}$ & 92 & 100 & - & - \\
\hline $1^{\prime}$ & 5 & 5 & 30 & - & 1 & $50 \stackrel{\circ}{ } \mathrm{C}$ & 92 & 100 & - & - \\
\hline 2 & 5 & 10 & 30 & - & 1 & $50 \stackrel{\circ}{ } \mathrm{C}$ & 99 & 100 & 98 & 93 \\
\hline $2^{\prime}$ & 5 & 10 & 30 & - & 1 & $50 \stackrel{\circ}{ } \mathrm{C}$ & 98 & 100 & - & - \\
\hline
\end{tabular}


<smiles>[2H]C(=O)c1cccc(C(=O)O[C@H]2C3OC(C)(C)O[C@@H]3O[C@@H]2C2COC(C)(C)O2)c1</smiles>

3a (6 mg, $0.0138 \mathrm{mmol})$, DIPEA (2.5 $\mu \mathrm{L}, 0.0138 \mathrm{mmol}), \mathrm{D}_{2} \mathrm{O}(150 \mu \mathrm{L}, 8.50 \mathrm{mmol})$, phenyl boroxine (3.4 $\mathrm{mg}, 0.011 \mathrm{mmol}$ ) and diacetone-glucose derivative $(108 \mathrm{mg}, 0.28 \mathrm{mmol})$ in anhydrous toluene $(400 \mu \mathrm{L})$ were prepared according to the general procedure given in section 5 (page 20) and stirred at $50 \stackrel{\circ}{ }{ }^{\circ}$. Crude residue was purified by flash-column chromatography using $\mathrm{Hex} \mathrm{Et}_{2} \mathrm{O}$ (67:33) combination to give colorless solid. ${ }^{19}$

Deuterium incorporation $=99 \%$ (crude: $100 \%, 100 \%)$, Reaction yield $=91 \%(100 \mathrm{mg})$. NMR spectra are given on pages $380-383$.

${ }^{1} \mathrm{H}$ NMR $\left(400 \mathrm{MHz}, \mathrm{CD}_{3} \mathrm{CN}\right) \delta(\mathrm{ppm})=10.08(\mathrm{~s}, 0.01 \mathrm{H}), 8.47(\mathrm{~s}, 1 \mathrm{H}), 8.28(\mathrm{~d}, J=8 \mathrm{~Hz}, 1 \mathrm{H}), 8.16(\mathrm{~d}$, $J=8 \mathrm{~Hz}, 1 \mathrm{H}), 7.73(\mathrm{t}, J=8 \mathrm{~Hz}, 1 \mathrm{H}), 5.98(\mathrm{~d}, J=4 \mathrm{~Hz}, 1 \mathrm{H}), 5.39(\mathrm{~d}, J=4 \mathrm{~Hz}, 1 \mathrm{H}), 4.69(\mathrm{~d}, J=4 \mathrm{~Hz}, 1$ H), $4.37(\mathrm{~m}, 2 \mathrm{H}), 4.10(\mathrm{~m}, 2 \mathrm{H}), 1.51(\mathrm{~s}, 3 \mathrm{H}), 1.36(\mathrm{~s}, 3 \mathrm{H}), 1.29(\mathrm{~s}, 3 \mathrm{H}), 1.22(\mathrm{~s}, 3 \mathrm{H}) .{ }^{13} \mathrm{C}$ NMR $(100$ $\left.\mathrm{MHz}, \mathrm{CD}_{3} \mathrm{CN}\right) \delta(\mathrm{ppm})=192.6(\mathrm{t}, J=27 \mathrm{~Hz}), 165.4,137.9,135.9,134.7,131.6,131.4,130.8,113.0$, $109.9,106.3,84.2,80.7,78.2,73.6,67.6,27.0,26.9,26.4,25.4$.

\begin{tabular}{|c|c|c|c|c|c|c|c|c|c|c|}
\hline \multirow{3}{*}{$\begin{array}{l}\text { Entry } \\
1 \\
\end{array}$} & \multirow{2}{*}{$\begin{array}{c}3 a \\
(\mathrm{~mol} \%)\end{array}$} & \multirow{3}{*}{$\begin{array}{c}\text { DIPEA } \\
\text { (mol\%) } \\
5\end{array}$} & \multirow{3}{*}{$\begin{array}{c}\text { Ph- } \\
\text { Boroxine } \\
\text { (mol\%) } \\
-\end{array}$} & \multirow{3}{*}{$\begin{array}{c}\text { Reactio } \\
\text { n Conc. } \\
\text { (M) } \\
1 \\
\end{array}$} & \multirow{3}{*}{$\begin{array}{c}\text { Reactio } \\
\text { n } \\
\text { Temp. } \\
50 \cong \mathrm{C}\end{array}$} & \multirow{3}{*}{$\begin{array}{c}\% \mathrm{D} \\
\text { (RM) } \\
99\end{array}$} & \multirow{2}{*}{$\begin{array}{c}\% \\
\text { Aldehyd } \\
\mathrm{e} \\
\end{array}$} & \multirow{2}{*}{$\begin{array}{c}\% \\
\text { Benzoin }\end{array}$} & \multicolumn{2}{|c|}{$\begin{array}{c}\text { After } \\
\text { Purification }\end{array}$} \\
\hline & & & & & & & & & $\% \mathrm{D}$ & \%Yield \\
\hline & 5 & & & & & & 88 & 12 & - & - \\
\hline $1^{\prime}$ & 5 & 5 & - & 1 & $50 \stackrel{\circ}{C}$ & 99 & 73 & 27 & - & - \\
\hline 2 & 5 & 5 & 4 & 0.5 & $50 \stackrel{\circ}{ } \mathrm{C}$ & 100 & 100 & - & 99 & 91 \\
\hline $2^{\prime}$ & 5 & 5 & 4 & 0.5 & 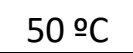 & 100 & 100 & - & - & - \\
\hline
\end{tabular}




\section{Initial Reaction Rates}<smiles>[X]c1ccccc1C=O</smiles>

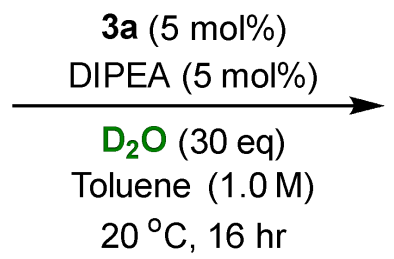<smiles>[X]c1ccccc1C([2H])=O</smiles>

$20^{\circ} \mathrm{C}, 16 \mathrm{hr}$

Scheme 26: General scheme for reaction rate

Initial reaction rates were obtained with 2-substituted benzaldehyde derivatives. Reactions were performed according to the catalytic general procedure given in section 5 (page 20) but the scale of the reactions was decreased to one third $(0.37 \mathrm{mmol}$ of aldehyde). Reactions were run in $2 \mathrm{~mL} \mathrm{GC}$ vials with a screw cap septa and stirred at $1000 \mathrm{rpm}$. A dry, argon flushed $2 \mathrm{~mL} \mathrm{GC}$ vial equipped with a magnetic stir bar was charged with $3 a(7.9 \mathrm{mg}, 0.0185 \mathrm{mmol}, 0.05$ eq.) and flushed with argon followed by the addition of $170 \mu \mathrm{L}$ anhydrous toluene. DIPEA (3.3 $\mu \mathrm{L}, 0.0185 \mathrm{mmol}, 0.05$ eq.), $\mathrm{D}_{2} \mathrm{O}(200 \mu \mathrm{L}, 11.10 \mathrm{mmol}, 30$ eq.) and then 2-substituted benzaldehyde (0.37 mmol, 1 eq.) were added. Reaction vial was closed with screw cap then wrapped teflon tape and mixture was stirred at 20 o $\mathrm{C}$ for a fixed amount of time (depending on the rate of deuteration for each substrate, for example, the reactions with 2-fluorobenzladehyde were performed for 1, 2, 3, 4 and 5 minutes). Then the crude reaction mixture was diluted with anhydrous chloroform-d (500 $\mu \mathrm{L})$ and NMR measurments (delay time D1 = 50 seconds) and analysis were carried out for the organic layer.

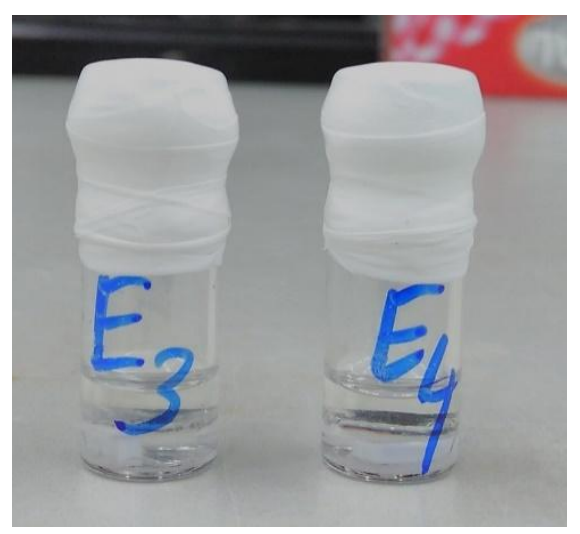

Figure 5: Reaction vial closed with screw cap and wrapped with teflon tape

Five points were collected for each substrate and each entry indicates a separate reaction with duplicate (set $B$ is a duplicate of set $A$ and each entry is a separate reaction to eliminate sampling errors). Concentration of aldehyde- $d$ formed was plotted against the reaction time. 11 different 2substituted aldehydes were used to obtain initial rates of reaction. To ensure adequate accuracy in initial rate calculations for each substrates the $R^{2}$ was $>96 \%$ and intercept was $\leq 11 \%$ for all of these substrate. Results are summarized below for each substrate. 
Initial Reaction Rate for 2-Fluorobenzaldehyde:

Table 5: Initial Reaction Rate for 2-Fluorobenzaldehyde

\begin{tabular}{|c|c|c|c|c|c|c|c|c|}
\hline $\begin{array}{c}\text { Reaction } \\
\text { Time } \\
\left(\begin{array}{c}\text { minutes } \\
)\end{array}\right.\end{array}$ & $\begin{array}{c}\text { Set } \\
\text { A } \\
(\% \mathrm{D} \\
)\end{array}$ & $\begin{array}{c}\text { Set } \\
\mathrm{B} \\
(\% \mathrm{D}\end{array}$ & $\begin{array}{c}\text { Set A } \\
\text { mmols } \\
(\text { Aldehyde- } d)\end{array}$ & $\begin{array}{c}\text { Set B } \\
\text { mmols } \\
(\text { Aldehyde- } d)\end{array}$ & $\begin{array}{c}\text { Set A } \\
\text { Molarit } \\
\mathrm{y} \\
(\mathrm{M})\end{array}$ & $\begin{array}{c}\text { Set B } \\
\text { Molarit } \\
\mathrm{y} \\
(\mathrm{M})\end{array}$ & $\begin{array}{c}\text { Average } \\
\text { Molarit } \\
\mathrm{y} \\
(\mathrm{M})\end{array}$ & $\begin{array}{c}\text { Standard } \\
\text { Deviatio } \\
\mathrm{n}\end{array}$ \\
\hline 1 & 18 & 15 & 0.0666 & 0.0555 & 0.1815 & 0.1512 & 0.1663 & 0.0214 \\
\hline 2 & 28 & 30 & 0.1036 & 0.1110 & 0.2823 & 0.3025 & 0.2924 & 0.0143 \\
\hline 3 & 39 & 39 & 0.1443 & 0.1443 & 0.3932 & 0.3932 & 0.3932 & 0.0000 \\
\hline 4 & 48 & 48 & 0.1776 & 0.1776 & 0.4839 & 0.4839 & 0.4839 & 0.0000 \\
\hline 5 & 52 & 58 & 0.1924 & 0.2146 & 0.5243 & 0.5847 & 0.5545 & 0.0428 \\
\hline
\end{tabular}

NMR spectra are given for set A from pages 384-386.

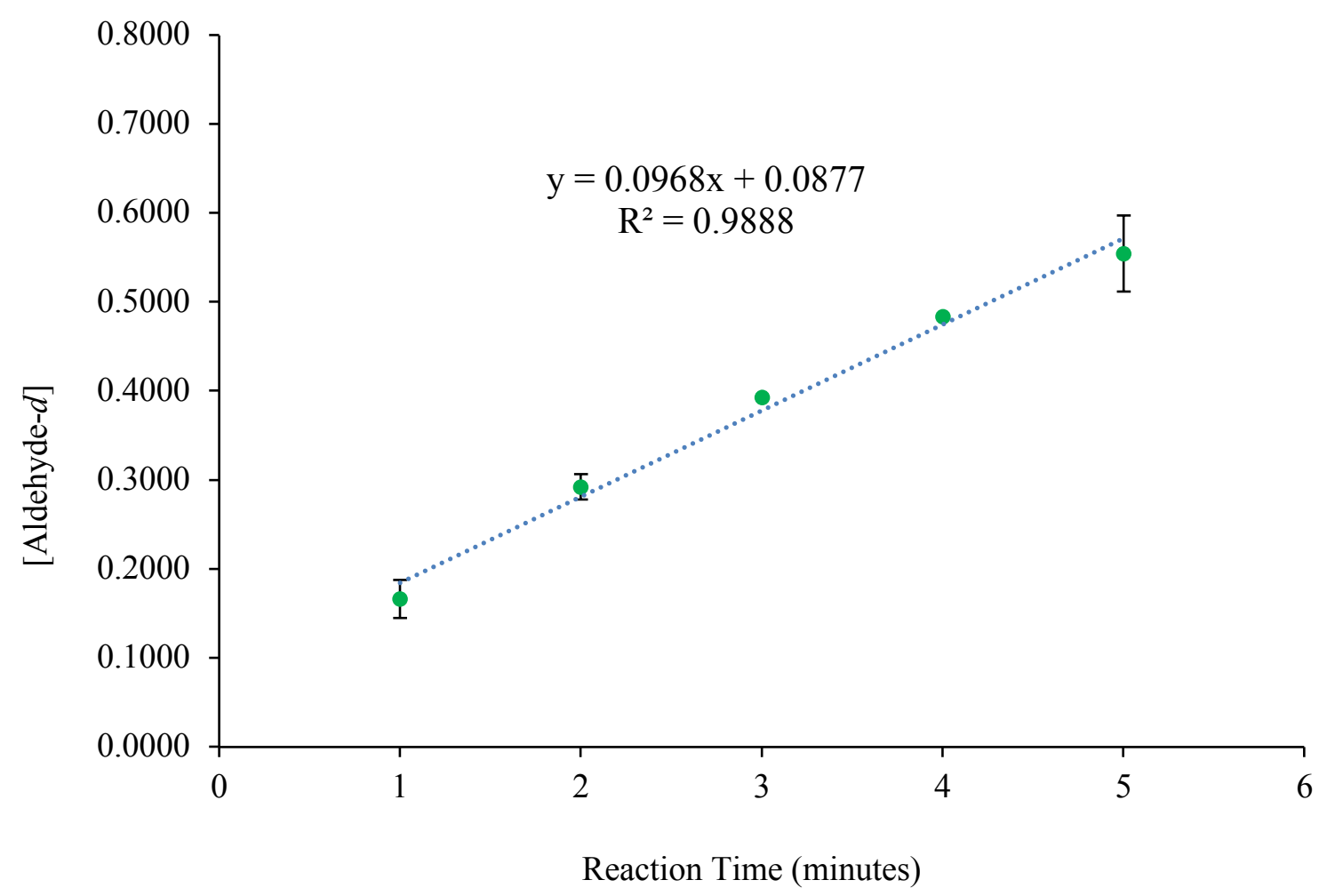

Figure 6: Plot of [Aldehyde-d] formation for 2-Fluorobenzaldehyde against reaction time 
Initial Reaction Rate for 2-Chlorobenzaldehyde:

Table 6: Initial Reaction Rate for 2-Chlorobenzaldehyde

\begin{tabular}{|c|c|c|c|c|c|c|c|c|}
\hline $\begin{array}{c}\text { Reaction } \\
\text { Time } \\
\left(\begin{array}{c}\text { minutes } \\
)\end{array}\right.\end{array}$ & $\begin{array}{c}\text { Set } \\
\text { A } \\
(\% \mathrm{D} \\
)\end{array}$ & $\begin{array}{c}\text { Set } \\
\mathrm{B} \\
(\% \mathrm{D}\end{array}$ & $\begin{array}{c}\text { Set A } \\
\text { mmols } \\
(\text { Aldehyde- } d)\end{array}$ & $\begin{array}{c}\text { Set B } \\
\text { mmols } \\
(\text { Aldehyde- } d)\end{array}$ & $\begin{array}{c}\text { Set A } \\
\text { Molarit } \\
\mathrm{y} \\
(\mathrm{M})\end{array}$ & $\begin{array}{c}\text { Set B } \\
\text { Molarit } \\
\mathrm{y} \\
(\mathrm{M})\end{array}$ & $\begin{array}{c}\text { Average } \\
\text { Molarit } \\
\mathrm{y}(\mathrm{M})\end{array}$ & $\begin{array}{c}\text { Standard } \\
\text { Deviatio } \\
\mathrm{n}\end{array}$ \\
\hline 5 & 19 & 20 & 0.0703 & 0.0740 & 0.1916 & 0.2016 & 0.1966 & 0.0071 \\
\hline 10 & 36 & 34 & 0.1332 & 0.1258 & 0.3629 & 0.3428 & 0.3529 & 0.0143 \\
\hline 15 & 40 & 39 & 0.1480 & 0.1443 & 0.4033 & 0.3932 & 0.3982 & 0.0071 \\
\hline 20 & 53 & 53 & 0.1961 & 0.1961 & 0.5343 & 0.5343 & 0.5343 & 0.0000 \\
\hline 25 & 62 & 59 & 0.2294 & 0.2183 & 0.6251 & 0.5948 & 0.6099 & 0.0214 \\
\hline
\end{tabular}

NMR spectra are given for set A from pages 387-389.

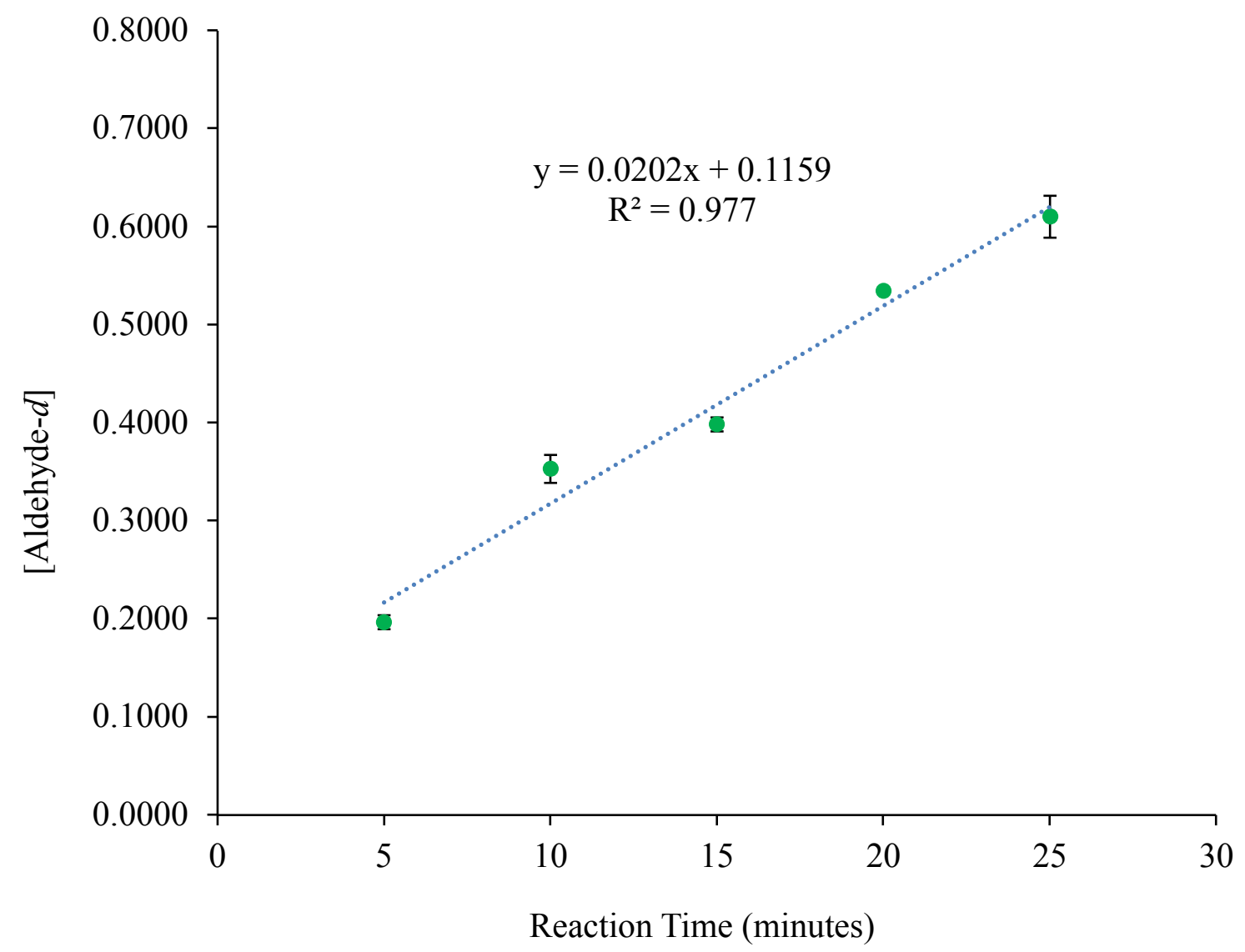

Figure 7: Plot of [Aldehyde-d] formation for 2-chlorobenzaldehyde against reaction time 
Initial Reaction Rate for 2-Bromobenzaldehyde:

Table 7: Initial Reaction Rate for 2-Bromobenzaldehyde

\begin{tabular}{|c|c|c|c|c|c|c|c|c|}
\hline $\begin{array}{c}\text { Reaction } \\
\text { Time } \\
(\text { minutes } \\
)\end{array}$ & $\begin{array}{c}\text { Set } \\
\text { A } \\
(\% \mathrm{D} \\
)\end{array}$ & $\begin{array}{c}\text { Set } \\
\mathrm{B} \\
(\% \mathrm{D}\end{array}$ & $\begin{array}{c}\text { Set A } \\
\mathrm{mmols} \\
(\text { Aldehyde- } d)\end{array}$ & $\begin{array}{c}\text { Set B } \\
\text { mmols } \\
(\text { Aldehyde- } d)\end{array}$ & $\begin{array}{c}\text { Set A } \\
\text { Molarity } \\
(\mathrm{M})\end{array}$ & $\begin{array}{c}\text { Set B } \\
\text { Molarit } \\
\mathrm{y} \\
(\mathrm{M})\end{array}$ & $\begin{array}{c}\text { Average } \\
\text { Molarit } \\
\mathrm{y}(\mathrm{M})\end{array}$ & $\begin{array}{c}\text { Standard } \\
\text { Deviatio } \\
\mathrm{n}\end{array}$ \\
\hline 5 & 13 & 12 & 0.0481 & 0.0444 & 0.1311 & 0.1210 & 0.1260 & 0.0071 \\
\hline 10 & 20 & 19 & 0.0740 & 0.0703 & 0.2016 & 0.1916 & 0.1966 & 0.0071 \\
\hline 15 & 26 & 25 & 0.0962 & 0.0925 & 0.2621 & 0.2520 & 0.2571 & 0.0071 \\
\hline 20 & 32 & 32 & 0.1184 & 0.1184 & 0.3226 & 0.3226 & 0.3226 & 0.0000 \\
\hline 25 & 39 & 39 & 0.1443 & 0.1443 & 0.3932 & 0.3932 & 0.3932 & 0.0000 \\
\hline
\end{tabular}

NMR spectra are given for set A from pages 390-392.

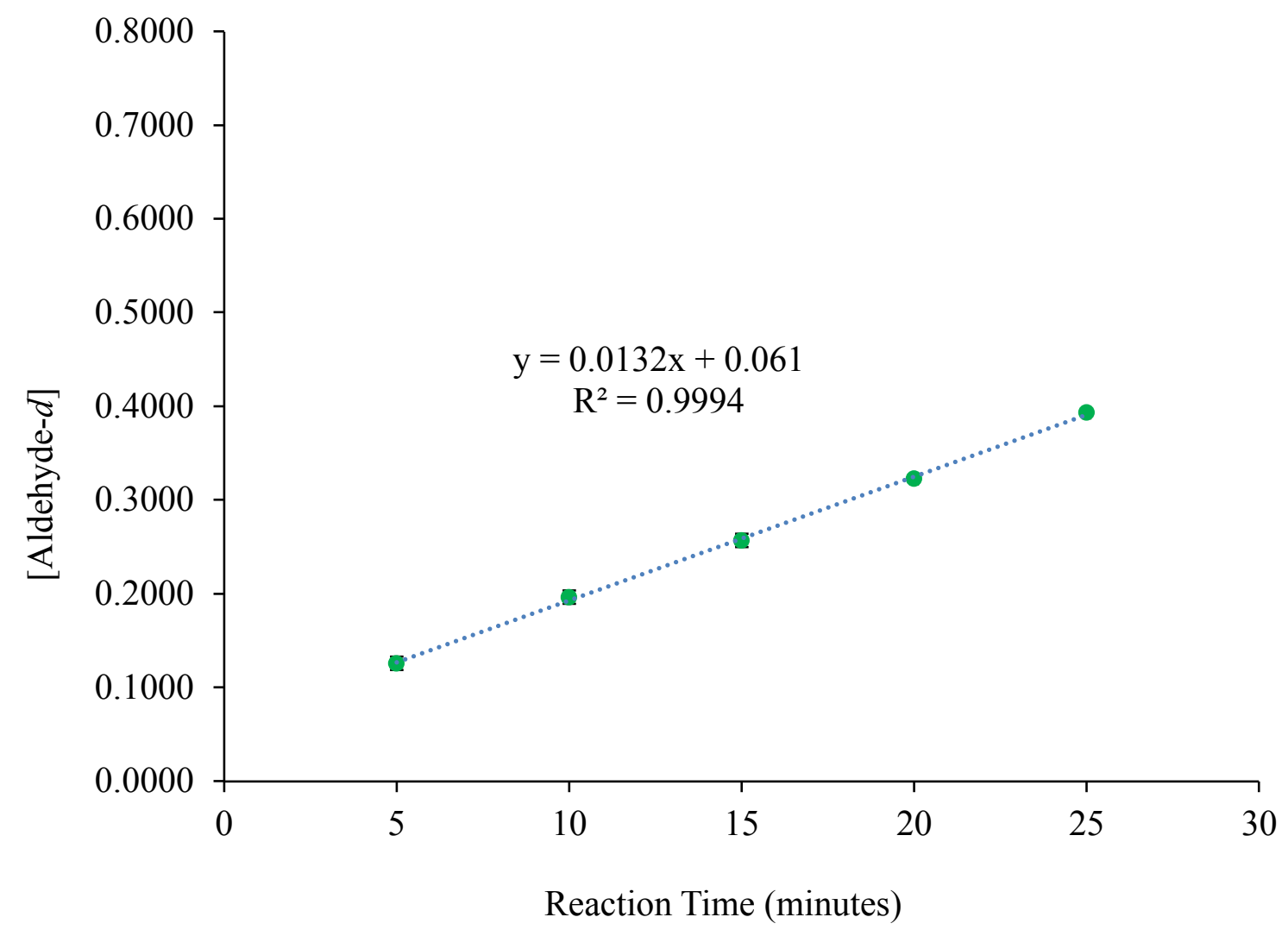

Figure 8: Plot of [Aldehyde- $d$ ] formation for 2-bromobenzaldehyde against reaction time 
Initial Reaction Rate for 2-Methylbenzaldehyde:

Table 8: Initial Reaction Rate for 2-Methylbenzaldehyde

\begin{tabular}{|c|c|c|c|c|c|c|c|c|}
\hline $\begin{array}{c}\text { Reaction } \\
\text { Time } \\
\left(\begin{array}{c}\text { minutes } \\
)\end{array}\right.\end{array}$ & $\begin{array}{c}\text { Set } \\
\text { A } \\
(\% \mathrm{D} \\
)\end{array}$ & $\begin{array}{c}\text { Set } \\
\mathrm{B} \\
(\% \mathrm{D}\end{array}$ & $\begin{array}{c}\text { Set A } \\
\text { mmols } \\
(\text { Aldehyde- } d)\end{array}$ & $\begin{array}{c}\text { Set B } \\
\text { mmols } \\
(\text { Aldehyde- } d)\end{array}$ & $\begin{array}{c}\text { Set A } \\
\text { Molarit } \\
\mathrm{y} \\
(\mathrm{M})\end{array}$ & $\begin{array}{c}\text { Set B } \\
\text { Molarit } \\
\mathrm{y} \\
(\mathrm{M})\end{array}$ & $\begin{array}{c}\text { Average } \\
\text { Molarit } \\
\mathrm{y}(\mathrm{M})\end{array}$ & $\begin{array}{c}\text { Standard } \\
\text { Deviatio } \\
\mathrm{n}\end{array}$ \\
\hline 5 & 12 & 10 & 0.0444 & 0.0370 & 0.1210 & 0.1008 & 0.1109 & 0.0143 \\
\hline 10 & 15 & 14 & 0.0555 & 0.0518 & 0.1512 & 0.1411 & 0.1462 & 0.0071 \\
\hline 15 & 18 & 19 & 0.0666 & 0.0703 & 0.1815 & 0.1916 & 0.1865 & 0.0071 \\
\hline 20 & 22 & 24 & 0.0814 & 0.0888 & 0.2218 & 0.2420 & 0.2319 & 0.0143 \\
\hline 25 & 24 & 25 & 0.0888 & 0.0925 & 0.2420 & 0.2520 & 0.2470 & 0.0071 \\
\hline
\end{tabular}

NMR spectra are given for set A from pages 393-395.

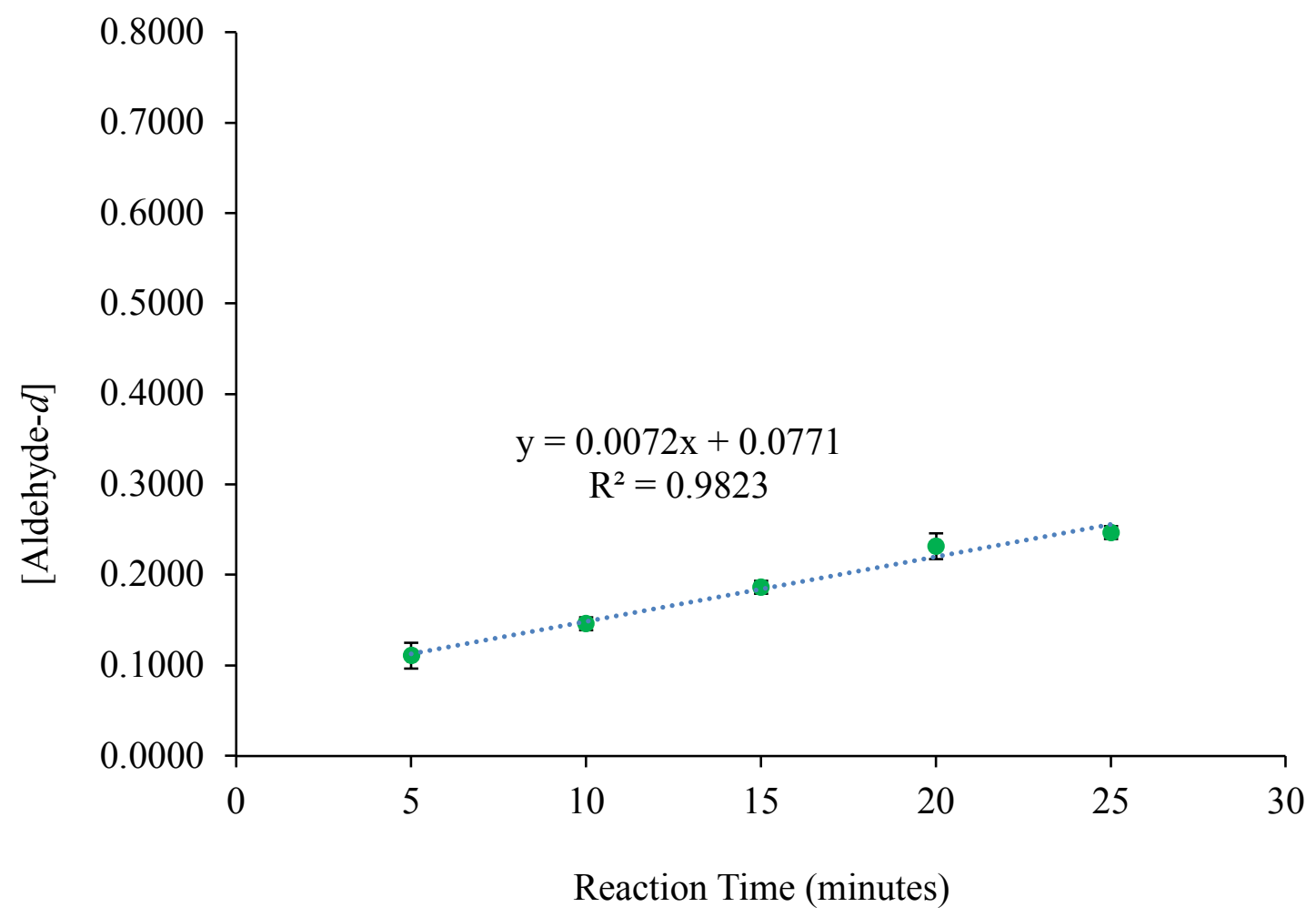

Figure 9: Plot of [Aldehyde- $d$ ] formation for 2-methylbenzaldehyde against reaction time 
Initial Reaction Rate for 2-Methoxybenzaldehyde:

Table 9: Initial Reaction Rate for 2-Methoxybenzaldehyde

\begin{tabular}{|c|c|c|c|c|c|c|c|c|}
\hline $\begin{array}{c}\text { Reaction } \\
\text { Time } \\
\left(\begin{array}{c}\text { minutes } \\
)\end{array}\right.\end{array}$ & $\begin{array}{c}\text { Set } \\
\text { A } \\
(\% \mathrm{D} \\
)\end{array}$ & $\begin{array}{c}\text { Set } \\
\mathrm{B} \\
(\% \mathrm{D}\end{array}$ & $\begin{array}{c}\text { Set A } \\
\text { mmols } \\
(\text { Aldehyde- } d)\end{array}$ & $\begin{array}{c}\text { Set B } \\
\text { mmols } \\
(\text { Aldehyde- } d)\end{array}$ & $\begin{array}{c}\text { Set A } \\
\text { Molarit } \\
\mathrm{y} \\
(\mathrm{M})\end{array}$ & $\begin{array}{c}\text { Set B } \\
\text { Molarit } \\
\mathrm{y} \\
(\mathrm{M})\end{array}$ & $\begin{array}{c}\text { Average } \\
\text { Molarit } \\
\mathrm{y}(\mathrm{M})\end{array}$ & $\begin{array}{c}\text { Standard } \\
\text { Deviatio } \\
\mathrm{n}\end{array}$ \\
\hline 30 & 7 & 6 & 0.0259 & 0.0222 & 0.0706 & 0.0605 & 0.0655 & 0.0071 \\
\hline 60 & 7 & 10 & 0.0259 & 0.0370 & 0.0706 & 0.1008 & 0.0857 & 0.0214 \\
\hline 90 & 14 & 13 & 0.0518 & 0.0481 & 0.1411 & 0.1311 & 0.1361 & 0.0071 \\
\hline 120 & 17 & 18 & 0.0629 & 0.0666 & 0.1714 & 0.1815 & 0.1764 & 0.0071 \\
\hline 150 & 22 & 24 & 0.0814 & 0.0888 & 0.2218 & 0.2420 & 0.2319 & 0.0143 \\
\hline
\end{tabular}

NMR spectra are given for set A from pages 396-398.

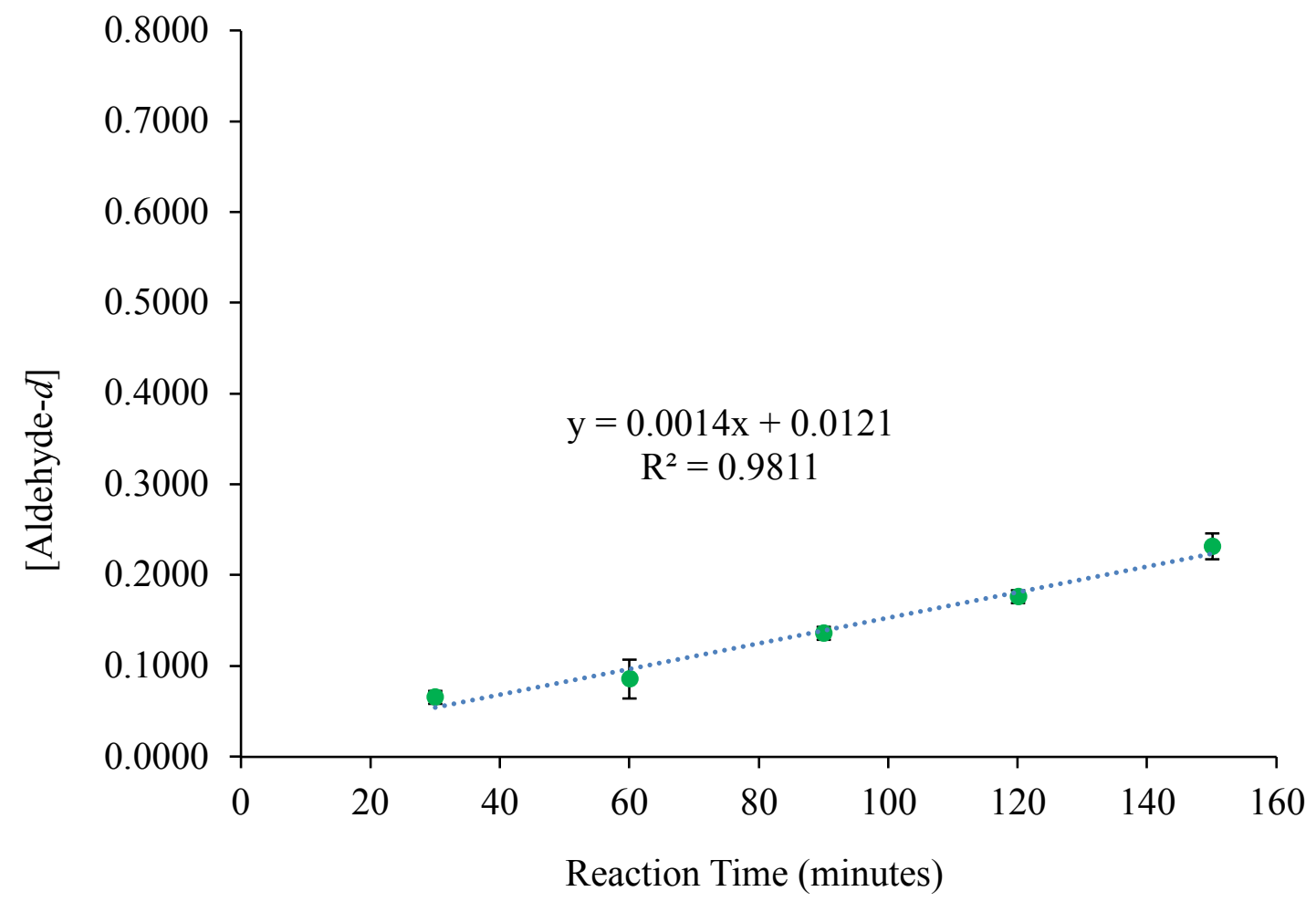

Figure 10: Plot of [Aldehyde- $d$ ] formation for 2-methoxybenzaldehyde against reaction time 
Initial Reaction Rate for 1-Naphthaldehyde:

Table 10: Initial Reaction Rate for 1-Naphthaldehyde

\begin{tabular}{|c|c|c|c|c|c|c|c|c|}
\hline $\begin{array}{c}\text { Reaction } \\
\text { Time } \\
\text { (minutes } \\
\text { ) }\end{array}$ & $\begin{array}{c}\text { Set A } \\
(\% \mathrm{D})\end{array}$ & $\begin{array}{c}\text { Set } \\
\mathrm{B} \\
(\% \mathrm{D})\end{array}$ & $\begin{array}{c}\text { Set A } \\
\text { mmols } \\
\text { (Aldehyde- } d)\end{array}$ & $\begin{array}{c}\text { Set B } \\
\text { mmols } \\
\text { (Aldehyde-d) }\end{array}$ & $\begin{array}{c}\text { Set A } \\
\text { Molarity } \\
(\mathrm{M})\end{array}$ & $\begin{array}{c}\text { Set B } \\
\text { Molarity } \\
(\mathrm{M})\end{array}$ & $\begin{array}{c}\text { Average } \\
\text { Molarit } \\
\mathrm{y}(\mathrm{M})\end{array}$ & $\begin{array}{c}\text { Standard } \\
\text { Deviatio } \\
\mathrm{n}\end{array}$ \\
\hline 5 & 18 & 19 & 0.0666 & 0.0703 & 0.1815 & 0.1916 & 0.1865 & 0.0071 \\
\hline 10 & 33 & 32 & 0.1221 & 0.1184 & 0.3327 & 0.3226 & 0.3277 & 0.0071 \\
\hline 15 & 46 & 47 & 0.1702 & 0.1739 & 0.4638 & 0.4738 & 0.4688 & 0.0071 \\
\hline 20 & 56 & 56 & 0.2072 & 0.2072 & 0.5646 & 0.5646 & 0.5646 & 0.0000 \\
\hline 25 & 66 & 67 & 0.2442 & 0.2479 & 0.6654 & 0.6755 & 0.6704 & 0.0071 \\
\hline
\end{tabular}

NMR spectra are given for set A from pages 399-401.

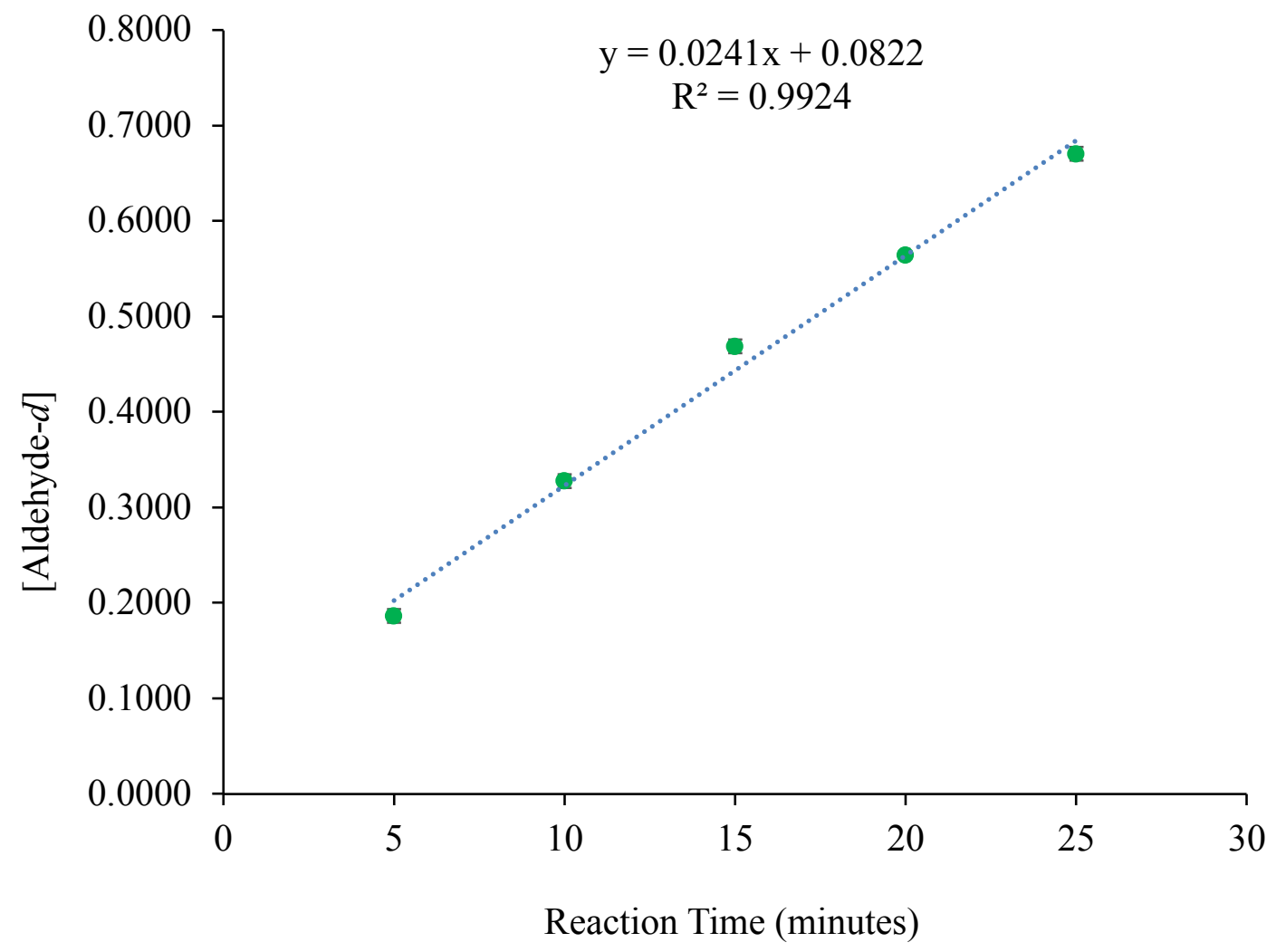

Figure 11: Plot of [Aldehyde- $d$ ] formation for 1-naphthaldehyde against reaction time 
Initial Reaction Rate for Thiophene-2-carboxaldehyde:

Table 11: Initial Reaction Rate for Thiophene-2-carboxaldehyde

\begin{tabular}{|c|c|c|c|c|c|c|c|c|}
\hline $\begin{array}{c}\text { Reaction } \\
\text { Time } \\
\left(\begin{array}{c}\text { minutes } \\
)\end{array}\right.\end{array}$ & $\begin{array}{c}\text { Set } \\
\text { A } \\
(\% \mathrm{D} \\
)\end{array}$ & $\begin{array}{c}\text { Set } \\
\mathrm{B} \\
(\% \mathrm{D}\end{array}$ & $\begin{array}{c}\text { Set A } \\
\text { mmols } \\
(\text { Aldehyde- } d)\end{array}$ & $\begin{array}{c}\text { Set B } \\
\text { mmols } \\
(\text { Aldehyde- } d)\end{array}$ & $\begin{array}{c}\text { Set A } \\
\text { Molarit } \\
\mathrm{y} \\
(\mathrm{M})\end{array}$ & $\begin{array}{c}\text { Set B } \\
\text { Molarit } \\
\mathrm{y} \\
(\mathrm{M})\end{array}$ & $\begin{array}{c}\text { Average } \\
\text { Molarit } \\
\mathrm{y} \\
(\mathrm{M})\end{array}$ & $\begin{array}{c}\text { Standard } \\
\text { Deviatio } \\
\mathrm{n}\end{array}$ \\
\hline 2.5 & 14 & 12 & 0.0518 & 0.0444 & 0.1411 & 0.1210 & 0.1311 & 0.0143 \\
\hline 5 & 14 & 25 & 0.0518 & 0.0925 & 0.1411 & 0.2520 & 0.1966 & 0.0784 \\
\hline 7.5 & 18 & 27 & 0.0666 & 0.0999 & 0.1815 & 0.2722 & 0.2268 & 0.0642 \\
\hline 10 & 22 & 32 & 0.0814 & 0.1184 & 0.2218 & 0.3226 & 0.2722 & 0.0713 \\
\hline 12.5 & 25 & 34 & 0.0925 & 0.1258 & 0.2520 & 0.3428 & 0.2974 & 0.0642 \\
\hline
\end{tabular}

NMR spectra are given for set A from pages 402-404.

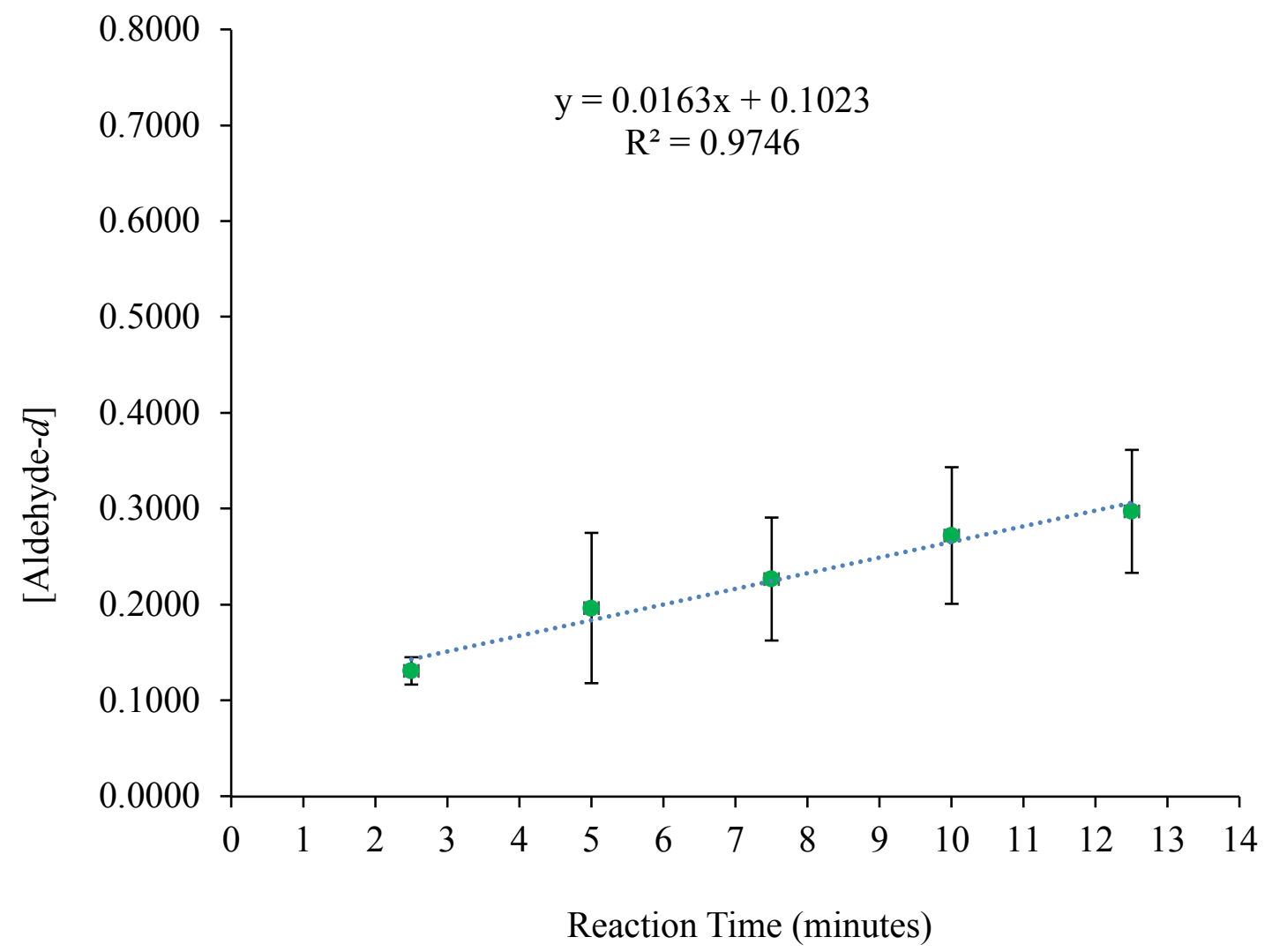

Figure 12: Plot of [Aldehyde- $d$ ] formation for thiophene-2-carboxaldehyde against reaction time 
Initial Reaction Rate for 2-- ${ }^{n}$ Butoxybenzaldehyde:

Table 12: Initial Reaction Rate for $2-^{n}$ Butoxybenzaldehyde

\begin{tabular}{|c|c|c|c|c|c|c|c|c|}
\hline $\begin{array}{c}\text { Reaction } \\
\text { Time } \\
\left(\begin{array}{c}\text { minutes } \\
)\end{array}\right.\end{array}$ & $\begin{array}{c}\text { Set } \\
\text { A } \\
(\% \mathrm{D} \\
)\end{array}$ & $\begin{array}{c}\text { Set } \\
\mathrm{B} \\
(\% \mathrm{D}\end{array}$ & $\begin{array}{c}\text { Set A } \\
\text { mmols } \\
(\text { Aldehyde- } d)\end{array}$ & $\begin{array}{c}\text { Set B } \\
\text { mmols } \\
(\text { Aldehyde- } d)\end{array}$ & $\begin{array}{c}\text { Set A } \\
\text { Molarit } \\
\mathrm{y} \\
(\mathrm{M})\end{array}$ & $\begin{array}{c}\text { Set B } \\
\text { Molarit } \\
\mathrm{y} \\
(\mathrm{M})\end{array}$ & $\begin{array}{c}\text { Average } \\
\text { Molarit } \\
\mathrm{y}(\mathrm{M})\end{array}$ & $\begin{array}{c}\text { Standard } \\
\text { Deviatio } \\
\mathrm{n}\end{array}$ \\
\hline 60 & 8 & 7 & 0.0296 & 0.0259 & 0.0807 & 0.0706 & 0.0756 & 0.0071 \\
\hline 120 & 18 & 16 & 0.0666 & 0.0592 & 0.1815 & 0.1613 & 0.1714 & 0.0143 \\
\hline 180 & 27 & 25 & 0.0999 & 0.0925 & 0.2722 & 0.2520 & 0.2621 & 0.0143 \\
\hline 240 & 32 & 33 & 0.1184 & 0.1221 & 0.3226 & 0.3327 & 0.3277 & 0.0071 \\
\hline 300 & 46 & 44 & 0.1702 & 0.1628 & 0.4638 & 0.4436 & 0.4537 & 0.0143 \\
\hline
\end{tabular}

NMR spectra are given for set A from pages 405-407.

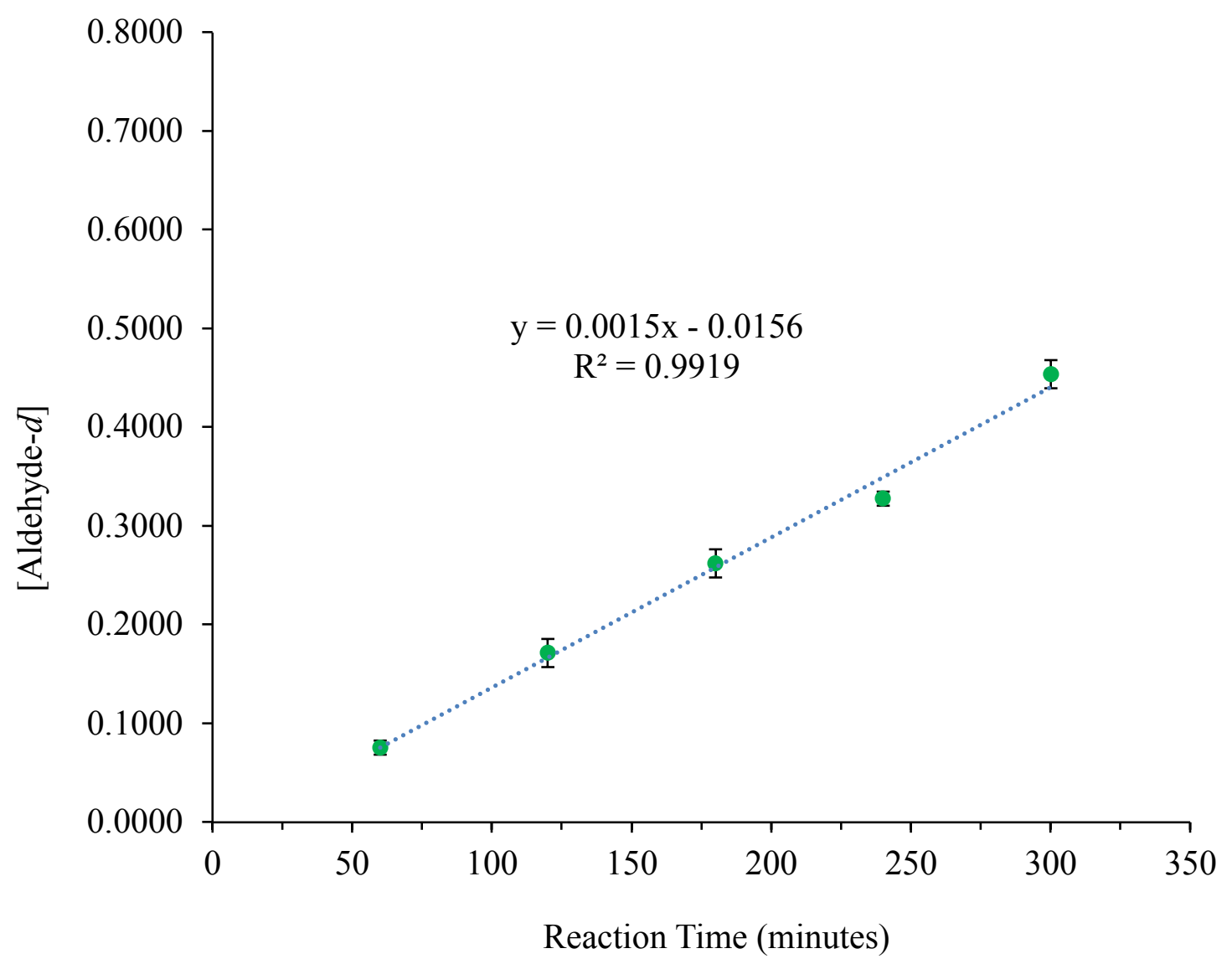

Figure 13: Plot of [Aldehyde- $d$ ] formation for $2-{ }^{n}$ butoxybenzaldehyde against reaction time 
Initial Reaction Rate for 2-( ${ }^{5 e c}$ Butoxy)benzaldehyde:

Table 13: Initial Reaction Rate for 2-( $\left.{ }^{\left({ }^{2 e c} B u t o x y\right)}\right)$ benzaldehyde

\begin{tabular}{|c|c|c|c|c|c|c|c|c|}
\hline $\begin{array}{c}\text { Reaction } \\
\text { Time } \\
\left(\begin{array}{c}\text { minutes } \\
)\end{array}\right.\end{array}$ & $\begin{array}{c}\text { Set } \\
\mathrm{A} \\
(\% \mathrm{D} \\
)\end{array}$ & $\begin{array}{c}\text { Set } \\
\mathrm{B} \\
(\% \mathrm{D}\end{array}$ & $\begin{array}{c}\text { Set A } \\
\text { mmols } \\
(\text { Aldehyde- } d)\end{array}$ & $\begin{array}{c}\text { Set B } \\
\text { mmols } \\
(\text { Aldehyde- } d)\end{array}$ & $\begin{array}{c}\text { Set A } \\
\text { Molarit } \\
\mathrm{y} \\
(\mathrm{M})\end{array}$ & $\begin{array}{c}\text { Set B } \\
\text { Molarit } \\
\mathrm{y} \\
(\mathrm{M})\end{array}$ & $\begin{array}{c}\text { Average } \\
\text { Molarit } \\
\mathrm{y}(\mathrm{M})\end{array}$ & $\begin{array}{c}\text { Standard } \\
\text { Deviatio } \\
\mathrm{n}\end{array}$ \\
\hline 60 & 3 & 3 & 0.0111 & 0.0111 & 0.0302 & 0.0302 & 0.0302 & 0.0000 \\
\hline 120 & 7 & 6 & 0.0259 & 0.0222 & 0.0706 & 0.0605 & 0.0655 & 0.0071 \\
\hline 180 & 10 & 10 & 0.0370 & 0.0370 & 0.1008 & 0.1008 & 0.1008 & 0.0000 \\
\hline 240 & 12 & 12 & 0.0444 & 0.0444 & 0.1210 & 0.1210 & 0.1210 & 0.0000 \\
\hline 300 & 14 & 15 & 0.0518 & 0.0555 & 0.1411 & 0.1512 & 0.1462 & 0.0071 \\
\hline
\end{tabular}

NMR spectra are given for set A from pages 408-410.

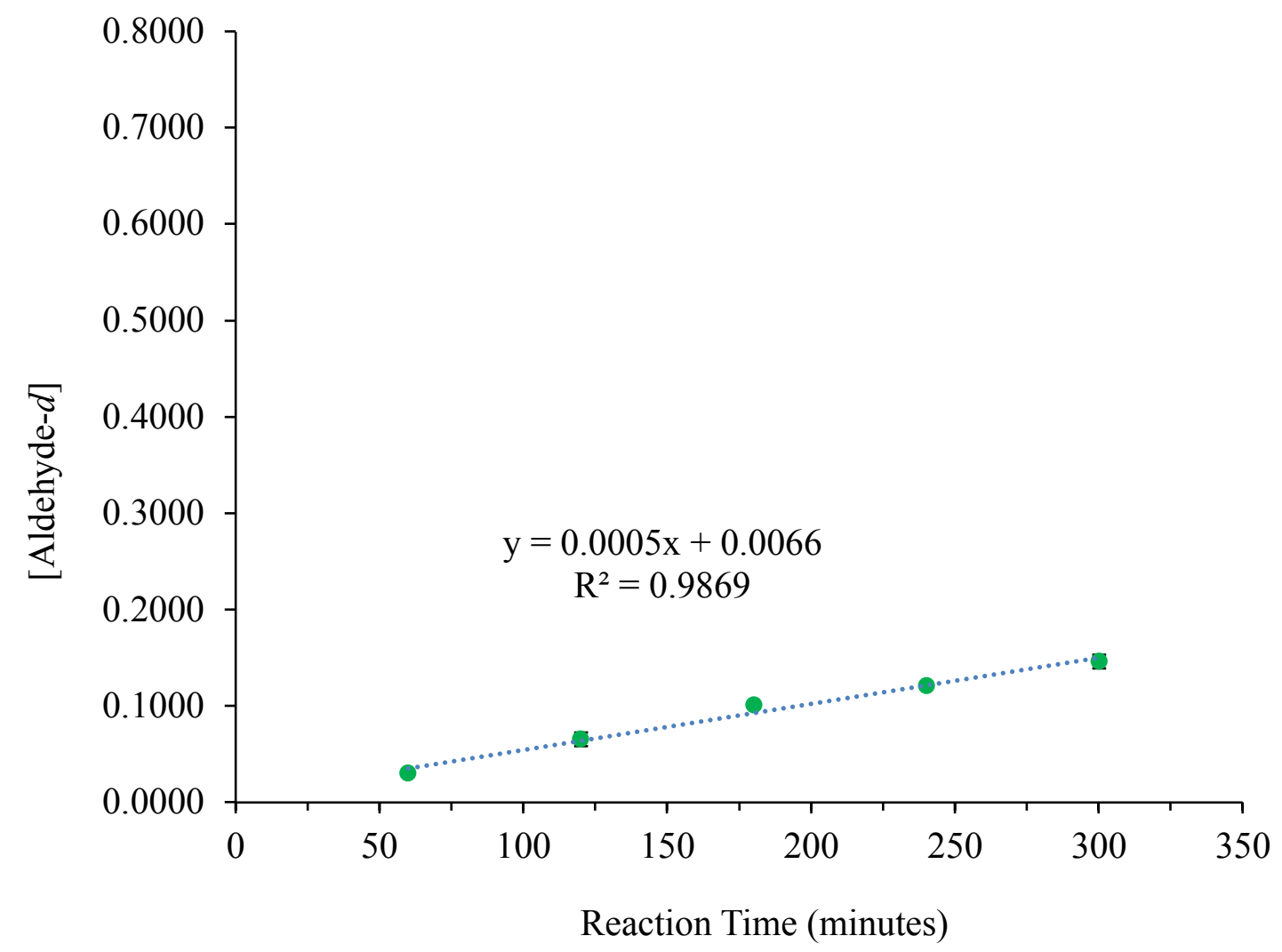

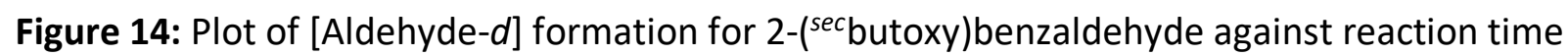


Initial Reaction Rate for 2-Ethynylbenzaldehyde:

Table 14: Initial Reaction Rate for 2-Ethynylbenzaldehyde

\begin{tabular}{|c|c|c|c|c|c|c|c|c|}
\hline $\begin{array}{c}\text { Reaction } \\
\text { Time } \\
\left(\begin{array}{c}\text { minutes } \\
)\end{array}\right.\end{array}$ & $\begin{array}{c}\text { Set } \\
\text { A } \\
(\% \mathrm{D} \\
)\end{array}$ & $\begin{array}{c}\text { Set } \\
\mathrm{B} \\
(\% \mathrm{D}\end{array}$ & $\begin{array}{c}\text { Set A } \\
\text { mmols } \\
(\text { Aldehyde- } d)\end{array}$ & $\begin{array}{c}\text { Set B } \\
\text { mmols } \\
(\text { Aldehyde- } d)\end{array}$ & $\begin{array}{c}\text { Set A } \\
\text { Molarit } \\
\mathrm{y} \\
(\mathrm{M})\end{array}$ & $\begin{array}{c}\text { Set B } \\
\text { Molarit } \\
\mathrm{y} \\
(\mathrm{M})\end{array}$ & $\begin{array}{c}\text { Average } \\
\text { Molarit } \\
\mathrm{y}(\mathrm{M})\end{array}$ & $\begin{array}{c}\text { Standard } \\
\text { Deviatio } \\
\mathrm{n}\end{array}$ \\
\hline 30 & 12 & 11 & 0.0444 & 0.0407 & 0.1210 & 0.1109 & 0.1159 & 0.0071 \\
\hline 60 & 18 & 13 & 0.0666 & 0.0481 & 0.1815 & 0.1311 & 0.1563 & 0.0356 \\
\hline 90 & 21 & 20 & 0.0777 & 0.0740 & 0.2117 & 0.2016 & 0.2067 & 0.0071 \\
\hline 120 & 31 & 21 & 0.1147 & 0.0777 & 0.3125 & 0.2117 & 0.2621 & 0.0713 \\
\hline 150 & 34 & 33 & 0.1258 & 0.1221 & 0.3428 & 0.3327 & 0.3377 & 0.0071 \\
\hline
\end{tabular}

NMR spectra are given for set A from pages 411-413.

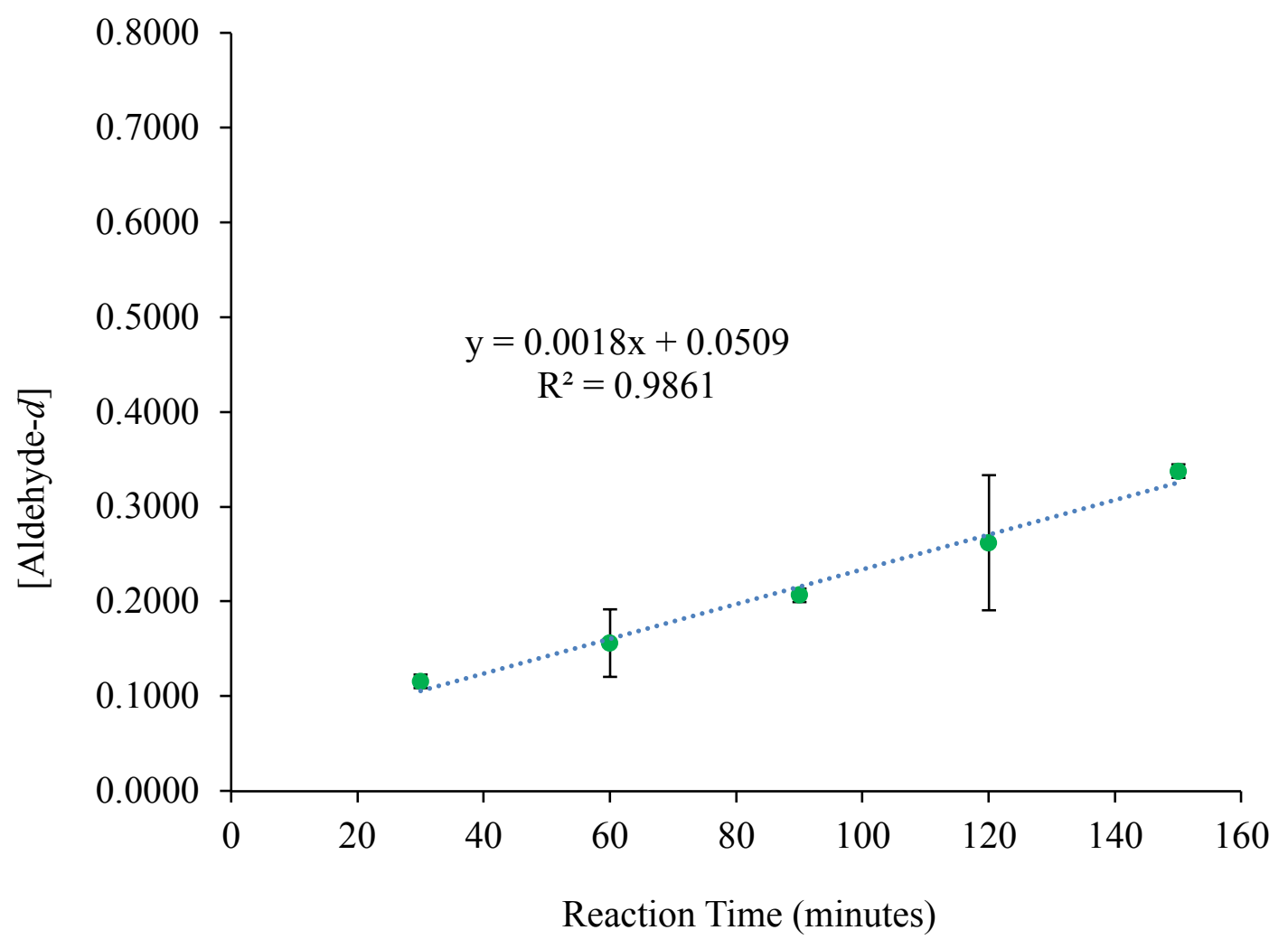

Figure 15: Plot of [Aldehyde- $d$ ] formation for 2-ethynylbenzaldehyde against reaction time 
Initial Reaction Rate for 2-(Trifluoromethyl)benzaldehyde:

Table 15: Initial Reaction Rate for 2-(Trifluoromethyl)benzaldehyde (Ald- $d=$ Aldehyde- $d$ )

\begin{tabular}{|c|c|c|c|c|c|c|c|c|c|c|c|c|c|c|}
\hline $\begin{array}{l}\text { Reaction } \\
\text { Time } \\
\text { (minutes) }\end{array}$ & $\begin{array}{c}\text { Set } \\
\text { A } \\
(\% \mathrm{D} \\
)\end{array}$ & $\begin{array}{c}\text { Set } \\
\text { B } \\
(\% \mathrm{D} \\
)\end{array}$ & $\begin{array}{c}\text { Set } \\
\mathrm{C} \\
(\% \mathrm{D} \\
)\end{array}$ & $\begin{array}{c}\text { Set } \\
\mathrm{D} \\
(\% \mathrm{D} \\
)\end{array}$ & $\begin{array}{l}\text { Set A } \\
\text { mmols } \\
\text { (Ald-d) }\end{array}$ & $\begin{array}{l}\text { Set B } \\
\text { mmols } \\
(\text { Ald-d) }\end{array}$ & $\begin{array}{l}\text { Set C } \\
\text { mmols } \\
(\text { Ald- } d)\end{array}$ & $\begin{array}{l}\text { Set D } \\
\text { mmols } \\
(\text { Ald- } d)\end{array}$ & $\begin{array}{c}\text { Set A } \\
\text { Molarit } \\
\mathrm{y} \\
(\mathrm{M})\end{array}$ & $\begin{array}{c}\text { Set B } \\
\text { Molarit } \\
\mathrm{y} \\
(\mathrm{M})\end{array}$ & $\begin{array}{c}\text { Set C } \\
\text { Molarit } \\
y \\
(\mathrm{M})\end{array}$ & $\begin{array}{c}\text { Set D } \\
\text { Molarit } \\
\mathrm{y} \\
(\mathrm{M})\end{array}$ & $\begin{array}{c}\text { Average } \\
\text { Molarit } \\
\text { y (M) }\end{array}$ & $\begin{array}{l}\text { Standard } \\
\text { Deviation }\end{array}$ \\
\hline 5 & 3 & 4 & 5 & 4 & 0.0111 & 0.0148 & 0.0185 & 0.0148 & 0.03025 & 0.04033 & 0.05041 & 0.04033 & 0.04033 & 0.00823 \\
\hline 10 & 4 & 5 & 5 & 6 & 0.0148 & 0.0185 & 0.0185 & 0.0222 & 0.04033 & 0.05041 & 0.05041 & 0.06049 & 0.05041 & 0.00823 \\
\hline 15 & 6 & 9 & 6 & 7 & 0.0222 & 0.0333 & 0.0222 & 0.0259 & 0.06049 & 0.09074 & 0.06049 & 0.07057 & 0.07057 & 0.01426 \\
\hline 20 & 7 & 7 & 8 & 9 & 0.0259 & 0.0259 & 0.0296 & 0.0333 & 0.07057 & 0.07057 & 0.08065 & 0.09074 & 0.07813 & 0.00965 \\
\hline 25 & 9 & 9 & 8 & 10 & 0.0296 & 0.0333 & 0.0296 & 0.037 & 0.08065 & 0.09074 & 0.08065 & 0.10082 & 0.08822 & 0.00965 \\
\hline
\end{tabular}

NMR spectra are given for set A from pages 414-416.

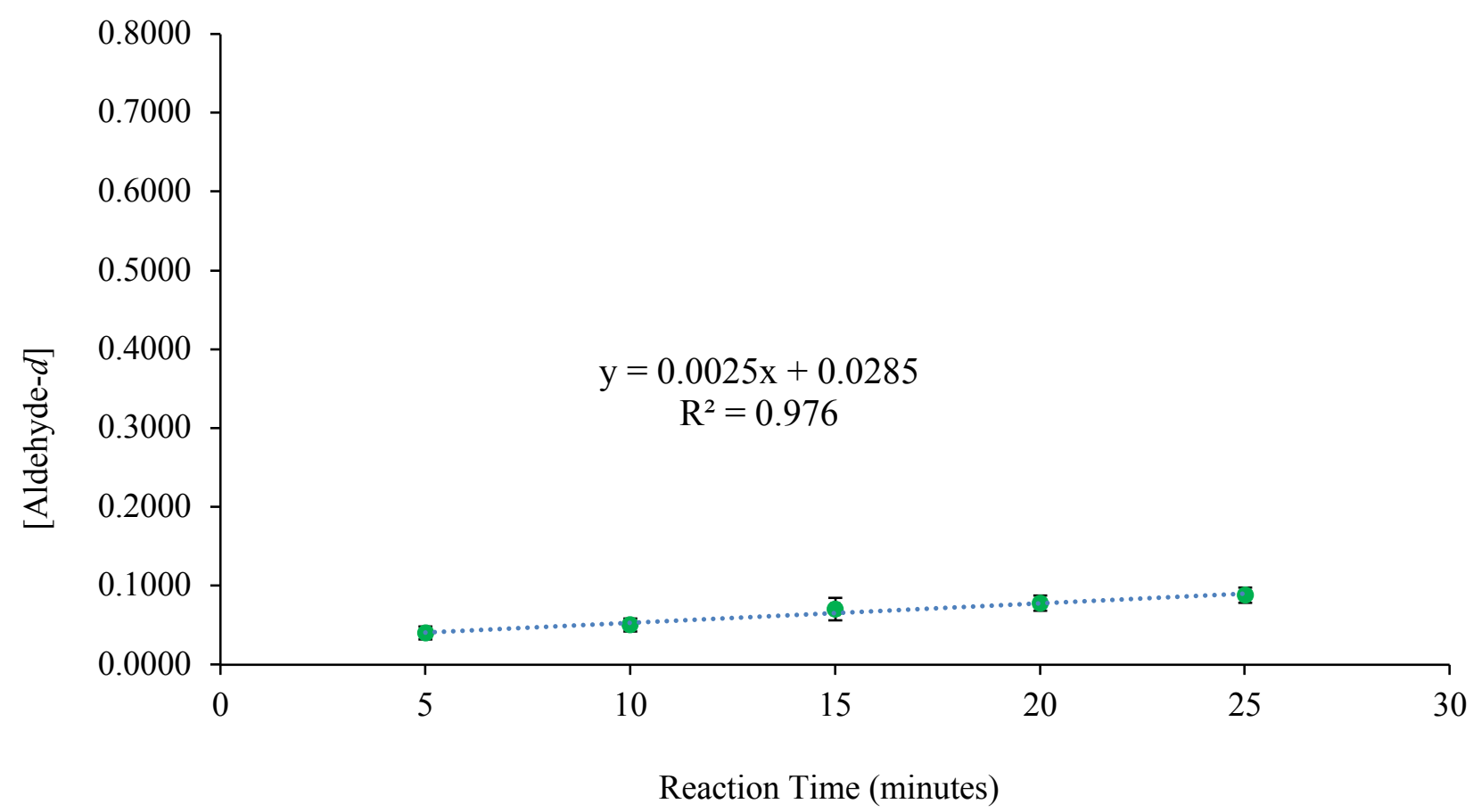


Figure 16: Plot of [Aldehyde- $d$ ] formation for 2-(trifluoromethyl)benzaldehyde against reaction time 


\section{Kinetic Isotope Effect:}

To calculate KIE value, we used reaction rate of 2-chlorobenzaldehyde (page 71) and 2chlorobenzaldehyde- $d$ with $\mathrm{H}_{2} \mathrm{O}$ (given below).

2-Chlorobenzaldehyde rate, $\mathrm{k}_{H}=0.0202 \mathrm{M} / \mathrm{min}$

2-Chlorobenzaldehyde- $d$ rate, $\mathrm{k}_{D}=0.0125 \mathrm{M} / \mathrm{min}$

$\mathrm{KIE}=\mathrm{k}_{H} / \mathrm{k}_{D}=0.0202 / 0.0125=1.62$

\section{Initial Reaction Rate for 2-Chlorobenzaldehyde-d:}

Table 16: Initial Reaction Rate for 2-Chlorobenzaldehyde-d

\begin{tabular}{|c|c|c|c|c|c|c|c|c|}
\hline $\begin{array}{l}\text { Reaction } \\
\text { Time } \\
\text { (minutes } \\
\text { ) }\end{array}$ & $\begin{array}{c}\text { Set } \\
\text { A } \\
(\% \mathrm{H})\end{array}$ & $\begin{array}{l}\text { Set } \\
\text { B } \\
(\% \mathrm{H})\end{array}$ & $\begin{array}{c}\text { Set A } \\
\text { mmols } \\
\text { (Aldehyde-d) }\end{array}$ & $\begin{array}{c}\text { Set B } \\
\text { mmols } \\
\text { (Aldehyde- } d \text { ) }\end{array}$ & $\begin{array}{c}\text { Set A } \\
\text { Molarity } \\
\text { (M) }\end{array}$ & $\begin{array}{c}\text { Set B } \\
\text { Molarity } \\
\text { (M) }\end{array}$ & $\begin{array}{c}\text { Average } \\
\text { Molarit } \\
\text { y (M) }\end{array}$ & $\begin{array}{c}\text { Standard } \\
\text { Deviatio } \\
\mathrm{n}\end{array}$ \\
\hline 5 & 14 & 13 & 0.0518 & 0.0481 & 0.1411 & 0.1311 & 0.1361 & 0.0071 \\
\hline 10 & 22 & 23 & 0.0814 & 0.0851 & 0.2218 & 0.2319 & 0.2268 & 0.0071 \\
\hline 15 & 28 & 29 & 0.1036 & 0.1073 & 0.2823 & 0.2924 & 0.2873 & 0.0071 \\
\hline 20 & 33 & 34 & 0.1221 & 0.1258 & 0.3327 & 0.3428 & 0.3377 & 0.0071 \\
\hline 25 & 38 & 40 & 0.1406 & 0.1480 & 0.3831 & 0.4033 & 0.3932 & 0.0143 \\
\hline
\end{tabular}

NMR spectra are given for set A from pages 417-419.

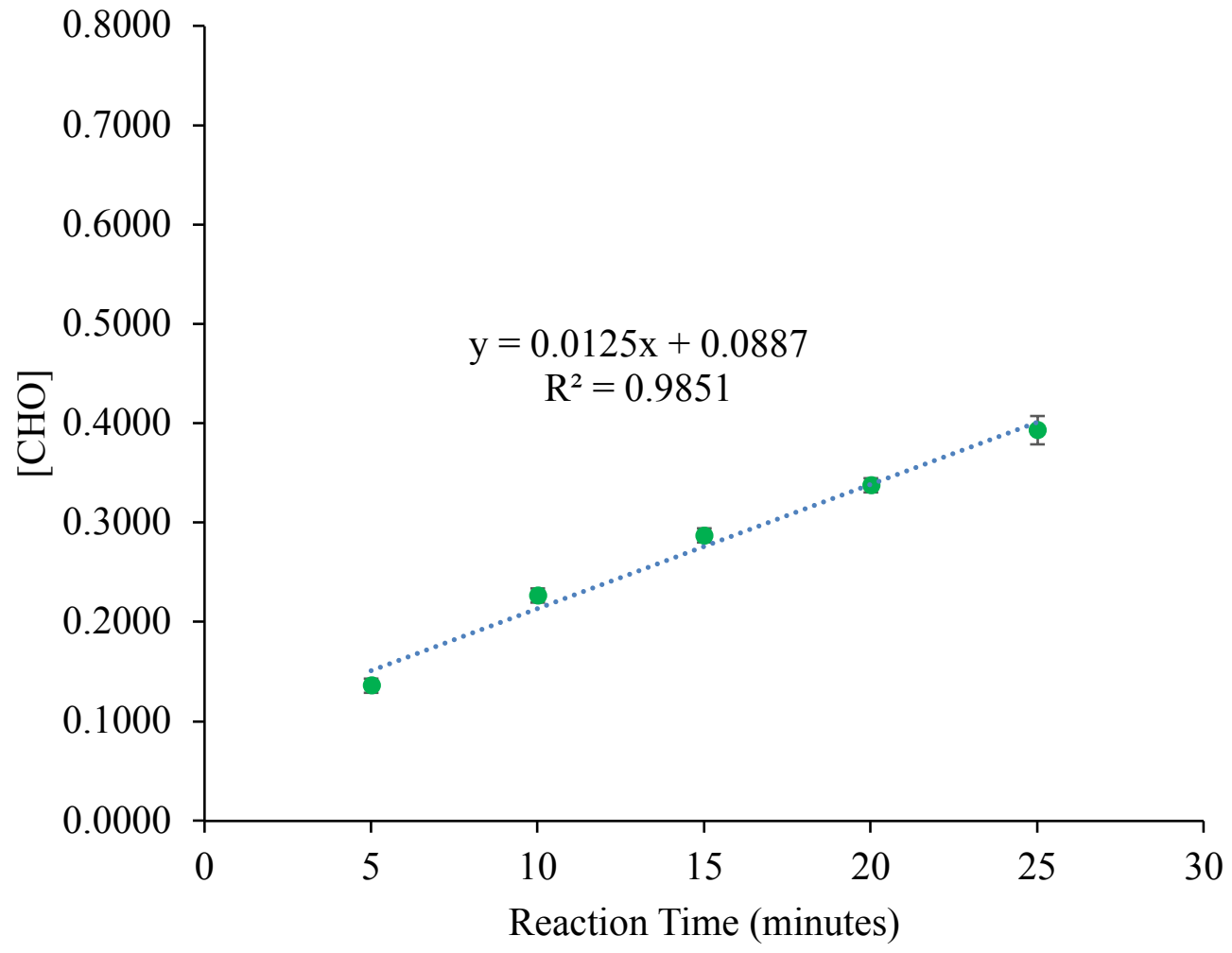


Figure 17: Plot of [Aldehyde- $d$ ] formation for 2-chlorobenzaldehyde- $d$ against reaction time

7. Reactions with different triazolium NHCs<smiles>CCCCOc1ccccc1C=O</smiles>

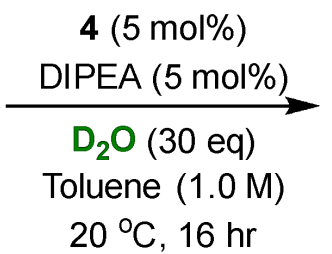<smiles>[2H]C(=O)c1ccccc1OCCCC</smiles>

Scheme 27: Reactions with triazolium NHCs

These reactions were performed according to the catalytic general procedure given in section 5 (page 20) but the scale of the reactions was decreased to one third $(0.37 \mathrm{mmol}$ of 2 ${ }^{n}$ butoxybenzaldehyde). Reactions were run in $2 \mathrm{~mL} \mathrm{GC}$ vials with a screw cap septa and stirred at $1000 \mathrm{rpm}$. A dry, argon flushed $2 \mathrm{~mL} \mathrm{GC}$ vial equipped with a magnetic stir bar was charged with pyrrolidine based triazolium catalyst $(0.0185 \mathrm{mmol}, 0.05$ eq.) and flushed with argon followed by the addition of $170 \mu \mathrm{L}$ anhydrous toluene. DIPEA ( $3.3 \mu \mathrm{L}, 0.0185 \mathrm{mmol}, 0.05$ eq.), $\mathrm{D}_{2} \mathrm{O}(200 \mu \mathrm{L}, 11.10$ mmol, 30 eq.) and then $2{ }^{n}{ }^{n}$ butoxybenzaldehyde ( $66 \mu \mathrm{L}, 0.37 \mathrm{mmol}, 1$ eq.) was added. Reaction vial was closed with screw cap then wrapped teflon tape and mixture was stirred at $20 \stackrel{\circ}{ }$ for $16 \mathrm{~h}$. Then the crude reaction mixture was diluted with anhydrous chloroform-d (500 $\mu \mathrm{L}$ ) and NMR measurments (D1 = 50 seconds) and analysis were carried out for the organic layer.

Reactions were performed in duplicate with each catalyst (for example, $1^{\prime}$ is duplicate of 1 ) and results are summarized below. 
Table 17: Results with triazolium NHCs

\begin{tabular}{|c|c|c|}
\hline $\begin{array}{c}\text { Entr } \\
\mathrm{y}\end{array}$ & NHCs & $\begin{array}{c}\text { \%Aldehyde- } \\
d \\
(\mathrm{RM})\end{array}$ \\
\hline 1 & 2,4,6-tri-Br-Phenyl (4a) & 98 \\
\hline 1 ' & 2,4,6-tri-Br-Phenyl (4a) & 98 \\
\hline 2 & 2,4,6-tri-Cl-Phenyl (4b) & 98 \\
\hline 2 ' & 2,4,6-tri-Cl-Phenyl (4b) & 98 \\
\hline 3 & 2,6-di-Me-Phenyl (4c) & 23 \\
\hline $3^{\prime}$ & 2,6-di-Me-Phenyl (4c) & 25 \\
\hline 4 & 2-OMe-Phenyl (4d) & 6 \\
\hline 4 ' & 2-OMe-Phenyl (4d) & 8 \\
\hline 5 & 3,5-di-CF - -Phenyl (4e) & 67 \\
\hline 5 , & 3,5-di-CF - -Phenyl (4e) & 65 \\
\hline 6 & 3,5-di-Me-Phenyl (4f) & 8 \\
\hline 6 ' & 3,5-di-Me-Phenyl (4f) & 9 \\
\hline 7 & 4-CF 3 -Phenyl (4g) & 56 \\
\hline 7 & 4-CF - -Phenyl (4g) & 58 \\
\hline 8 & 4-OMe-Phenyl (4h) & 14 \\
\hline $8^{\prime}$ & 4-OMe-Phenyl (4h) & 14 \\
\hline 9 & $\mathrm{~F}_{5}$-Phenyl (4i) & 81 \\
\hline 9' & F5-Phenyl (4i) & 84 \\
\hline 10 & Mesityl (4j) & 18 \\
\hline $10^{\prime}$ & Mesityl (4j) & 17 \\
\hline 11 & Phenyl (4k) & 29 \\
\hline $11^{\prime}$ & Phenyl (4k) & 31 \\
\hline 12 & 2-Cl-Phenyl (4I) & 34 \\
\hline $12^{\prime}$ & 2-Cl-Phenyl (4I) & 34 \\
\hline 13 & 2- $\mathrm{CF}_{3}-$ Phenyl (4m) & 42 \\
\hline $13^{\prime}$ & 2-CF - -Phenyl (4m) & 41 \\
\hline 14 & 3-F-Phenyl (4n) & 58 \\
\hline $14^{\prime}$ & 3-F-Phenyl (4n) & 59 \\
\hline
\end{tabular}

NMR spectra are given on pages 420-433. 


\section{Experiments with Alcohols}

2-Chlorobenzaldehyde- $d$ (deuterium incorporation $=98 \%$ ) was synthesized using the procedure given below in section 9 (large scale reactions).

\subsection{Reactions with 3a and R-OH (5 eq.)}<smiles>[2H]C(=O)c1ccccc1Cl</smiles>
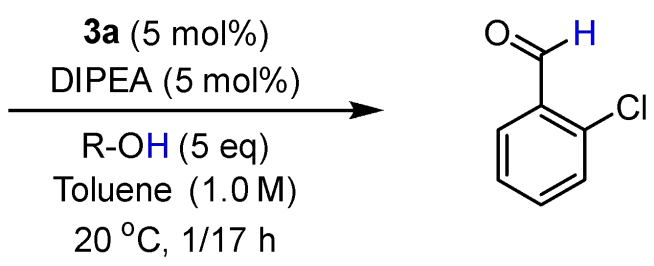

Scheme 28: Reactions with 3a and R-OH (5 eq.)

These reactions were performed according to the catalytic general procedure given in section 5 (page 20) but the scale of the reactions was decreased to one third $(0.37 \mathrm{mmol}$ of 2chlorobenzaldehyde- $d$ ). Reactions were run in $2 \mathrm{~mL} \mathrm{GC}$ vials with a screw cap septa and stirred at 1000 rpm. A dry, argon flushed 2 mL GC vial equipped with a magnetic stir bar was charged with 3a (7.9 $\mathrm{mg}, 0.0185 \mathrm{mmol}, 0.05$ eq.) and flushed with argon followed by the addition of $370 \mu \mathrm{L}$ anhydrous toluene. DIPEA (3.3 $\mu \mathrm{L}, 0.0185 \mathrm{mmol}, 0.05$ eq.), R-OH (1.85 mmol, 5 eq.) and then 2chlorobenzaldehyde- $d$ (42 $\mu \mathrm{L}, 0.37 \mathrm{mmol}, 1$ eq.) was added. Reaction vial was closed with screw cap then wrapped teflon tape and mixture was stirred at 20 으 for 1 or $17 \mathrm{~h}$. Then crude reaction mixture was diluted with anhydrous chloroform-d $(500 \mu \mathrm{L})$ and NMR measurments (D1 = 50 seconds) and analysis were carried out with organic layer.

Reactions are performed in duplicate with each alcohol (for example, $1^{\prime}$ is duplicate of 1 ) and results are summarized below. 
Table 18: 1 Hour reaction time with $3 a$

\begin{tabular}{|c|c|c|c|}
\hline $\begin{array}{c}\text { Entr } \\
y\end{array}$ & R-OH (5 eq.) & $\begin{array}{l}\text { Reaction Time } \\
\text { (hours) }\end{array}$ & $\begin{array}{c}\text { \%Loss of Deuterium } \\
\text { (RM) }\end{array}$ \\
\hline 1 & Water $\left(\mathrm{H}_{2} \mathrm{O}\right)$ & 1 & 32 \\
\hline 1 ' & Water $\left(\mathrm{H}_{2} \mathrm{O}\right)$ & 1 & 32 \\
\hline 2 & Methanol $\left(\mathrm{CH}_{3}-\mathrm{OH}\right)$ & 1 & 0 \\
\hline 2 ' & Methanol $\left(\mathrm{CH}_{3}-\mathrm{OH}\right)$ & 1 & 0 \\
\hline 3 & Ethanol $\left(\mathrm{C}_{2} \mathrm{H}_{5}-\mathrm{OH}\right)$ & 1 & 0 \\
\hline $3^{\prime}$ & Ethanol $\left(\mathrm{C}_{2} \mathrm{H}_{5}-\mathrm{OH}\right)$ & 1 & 0 \\
\hline 4 & Phenol (Ph-OH) & 1 & 0 \\
\hline 4 ' & Phenol (Ph-OH) & 1 & 0 \\
\hline 5 & 4-Methylphenol (4-Me-Ph-OH) & 1 & 0 \\
\hline 5 & 4-Methylphenol (4-Me-Ph-OH) & 1 & 0 \\
\hline 6 & Pentafluorophenol (F5-Ph-OH) & 1 & 0 \\
\hline 6 & Pentafluorophenol ( $\left.\mathrm{F}_{5}-\mathrm{Ph}-\mathrm{OH}\right)$ & 1 & 0 \\
\hline 7 & 4-Fluorophenol (4-F-Ph-OH) & 1 & 0 \\
\hline 7 & 4-Fluorophenol (4-F-Ph-OH) & 1 & 0 \\
\hline
\end{tabular}

NMR spectra are given on pages 434-437.

Table 19: 17 Hour reaction time with 3a

\begin{tabular}{|c|c|c|c|}
\hline $\begin{array}{c}\text { Entr } \\
y\end{array}$ & R-OH (5 eq.) & $\begin{array}{c}\text { Reaction Time } \\
\text { (hours) }\end{array}$ & $\begin{array}{c}\text { \%Loss of Deuterium } \\
\text { (RM) }\end{array}$ \\
\hline 1 & Water $\left(\mathrm{H}_{2} \mathrm{O}\right)$ & 17 & 89 \\
\hline $1^{\prime}$ & Water $\left(\mathrm{H}_{2} \mathrm{O}\right)$ & 17 & 89 \\
\hline 2 & Methanol $\left(\mathrm{CH}_{3}-\mathrm{OH}\right)$ & 17 & 0 \\
\hline $2^{\prime}$ & Methanol $\left(\mathrm{CH}_{3}-\mathrm{OH}\right)$ & 17 & 0 \\
\hline 3 & Ethanol $\left(\mathrm{C}_{2} \mathrm{H}_{5}-\mathrm{OH}\right)$ & 17 & 0 \\
\hline $3^{\prime}$ & Ethanol $\left(\mathrm{C}_{2} \mathrm{H}_{5}-\mathrm{OH}\right)$ & 17 & 0 \\
\hline 4 & Phenol $\left(\mathrm{Ph}_{-}-\mathrm{OH}\right)$ & 17 & 0 \\
\hline $4{ }^{\prime}$ & Phenol $(\mathrm{Ph}-\mathrm{OH})$ & 17 & 0 \\
\hline 5 & 4-Methylphenol $(4-\mathrm{Me}-\mathrm{Ph}-\mathrm{OH})$ & 17 & 0 \\
\hline 5 & 4-Methylphenol $(4-\mathrm{Me}-\mathrm{Ph}-\mathrm{OH})$ & 17 & 0 \\
\hline 6 & Pentafluorophenol $\left(\mathrm{F}_{5}-\mathrm{Ph}-\mathrm{OH}\right)$ & 17 & 0 \\
\hline 6 & Pentafluorophenol $\left(\mathrm{F}_{5}-\mathrm{Ph}-\mathrm{OH}\right)$ & 17 & 0 \\
\hline 7 & 4-Fluorophenol $(4-\mathrm{F}-\mathrm{Ph}-\mathrm{OH})$ & 17 & 0 \\
\hline $7^{\prime}$ & 4-Fluorophenol $(4-\mathrm{F}-\mathrm{Ph}-\mathrm{OH})$ & 17 & \\
\hline & & & \\
\hline
\end{tabular}

NMR spectra are given on pages 438-441. 
8.2 Reactions with $3 \mathrm{~b}$ and $\mathrm{R}-\mathrm{OH}(5$ eq.)<smiles>[2H]C(=O)c1ccccc1Cl</smiles>

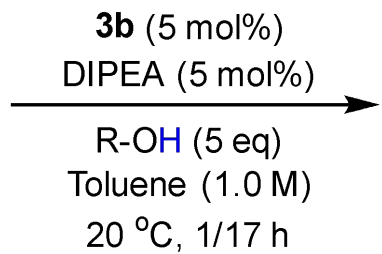<smiles>O=Cc1ccccc1</smiles>

Scheme 29: Reactions with $3 \mathbf{b}$ and R-OH (5 eq.)

These reactions were performed according to the catalytic general procedure given in section 5 (page 20) but the scale of the reactions was decreased to one third $(0.37 \mathrm{mmol}$ of 2chlorobenzaldehyde- $d$ ). Reactions were run in $2 \mathrm{~mL} \mathrm{GC}$ vials with a screw cap septa and stirred at $1000 \mathrm{rpm}$. A dry, argon flushed $2 \mathrm{~mL} \mathrm{GC}$ vial equipped with a magnetic stir bar was charged with pyrrolidine based $\mathbf{3 b}(6.34 \mathrm{mg}, 0.0185 \mathrm{mmol}, 0.05 \mathrm{eq}$.) and flushed with argon followed by the addition of $370 \mu \mathrm{L}$ anhydrous toluene. DIPEA ( $3.3 \mu \mathrm{L}, 0.0185 \mathrm{mmol}, 0.05 \mathrm{eq}$ ), alcohol ( $1.85 \mathrm{mmol}$, 5 eq.) and then 2-chlorobenzaldehyde- $d(42 \mu \mathrm{L}, 0.37 \mathrm{mmol}, 1$ eq.) was added. Reaction vial was closed with screw cap then wrapped teflon tape and mixture was stirred at $20 \stackrel{\circ}{ } \mathrm{C}$ for 1 or $17 \mathrm{~h}$. Then crude reaction mixture was diluted with anhydrous chloroform- $d(500 \mu \mathrm{L}$ ) and NMR measurments (D1 $=50$ seconds) and analysis were carried out with organic layer.

Reactions are performed in duplicate with each alcohol (for example, $1^{\prime}$ is duplicate of 1 ) and results are summarized below.

Table 20: 1 Hour reaction time with $\mathbf{3 b}$

\begin{tabular}{|c|c|c|c|}
\hline $\begin{array}{c}\text { Entr } \\
\text { y }\end{array}$ & R-OH (5 eq.) & $\begin{array}{c}\text { Reaction Time } \\
\text { (hours) }\end{array}$ & $\begin{array}{c}\text { \%Loss of Deuterium } \\
\text { (RM) }\end{array}$ \\
\hline 1 & Methanol $\left(\mathrm{CH}_{3}-\mathrm{OH}\right)$ & 1 & 0 \\
\hline 1 & Methanol $\left(\mathrm{CH}_{3}-\mathrm{OH}\right)$ & 1 & 0 \\
\hline 2 & Ethanol $\left(\mathrm{C}_{2} \mathrm{H}_{5}-\mathrm{OH}\right)$ & 1 & 0 \\
\hline 2 & Ethanol $\left(\mathrm{C}_{2} \mathrm{H}_{5}-\mathrm{OH}\right)$ & 1 & 0 \\
\hline 3 & Phenol $(\mathrm{Ph}-\mathrm{OH})$ & 1 & 0 \\
\hline 3 & Phenol $(\mathrm{Ph}-\mathrm{OH})$ & 1 & 0 \\
\hline 4 & 4-Methylphenol $(4-\mathrm{Me}-\mathrm{Ph}-\mathrm{OH})$ & 1 & 0 \\
\hline 4 & 4-Methylphenol $(4-\mathrm{Me}-\mathrm{Ph}-\mathrm{OH})$ & 1 & 0 \\
\hline 5 & Pentafluorophenol (F5-Ph-OH) & 1 & 0 \\
\hline 5 & Pentafluorophenol ( $\left.\mathrm{F}_{5}-\mathrm{Ph}-\mathrm{OH}\right)$ & 1 & 0 \\
\hline
\end{tabular}

NMR spectra are given on pages 442-444. 
Table 21: 17 Hour reaction time with $\mathbf{3 b}$

\begin{tabular}{|c|c|c|c|}
\hline $\begin{array}{c}\text { Entr } \\
\mathrm{y}\end{array}$ & $\mathrm{R}-\mathrm{OH}$ (5 eq.) & $\begin{array}{l}\text { Reaction Time } \\
\text { (hours) }\end{array}$ & $\begin{array}{l}\% \text { Loss of Deuterium } \\
\text { (RM) }\end{array}$ \\
\hline 1 & Water & 17 & 80 \\
\hline 1 ' & Water & 17 & 80 \\
\hline 2 & Methanol $\left(\mathrm{CH}_{3}-\mathrm{OH}\right)$ & 17 & 4 \\
\hline 2 & Methanol $\left(\mathrm{CH}_{3}-\mathrm{OH}\right)$ & 17 & 4 \\
\hline 3 & Ethanol $\left(\mathrm{C}_{2} \mathrm{H}_{5}-\mathrm{OH}\right)$ & 17 & 3 \\
\hline $3^{\prime}$ & Ethanol $\left(\mathrm{C}_{2} \mathrm{H}_{5}-\mathrm{OH}\right)$ & 17 & 3 \\
\hline 4 & Trifluoroethanol & 17 & 0 \\
\hline 4 ' & Trifluoroethanol & 17 & 0 \\
\hline 5 & Phenol (Ph-OH) & 17 & 0 \\
\hline 5 & Phenol (Ph-OH) & 17 & 0 \\
\hline 6 & 4-Methylphenol (4-Me-Ph-OH) & 17 & 0 \\
\hline 6 & 4-Methylphenol (4-Me-Ph-OH) & 17 & 0 \\
\hline 7 & Pentafluorophenol ( $\left.\mathrm{F}_{5}-\mathrm{Ph}-\mathrm{OH}\right)$ & 17 & 0 \\
\hline 7 & Pentafluorophenol ( $\left.\mathrm{F}_{5}-\mathrm{Ph}-\mathrm{OH}\right)$ & 17 & 0 \\
\hline 8 & 4-Fluorophenol & 17 & 0 \\
\hline 8, & 4-Fluorophenol & 17 & 0 \\
\hline
\end{tabular}

NMR spectra are given on pages 444-448.

\subsection{Reactions with 3b and Methanol (Different Concentrations)}<smiles>[2H]C(=O)c1ccccc1Cl</smiles>

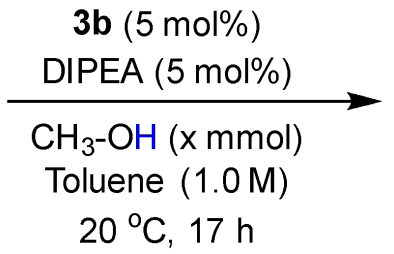<smiles>O=Cc1ccccc1Cl</smiles>

Scheme 30: Reactions with 3b and $\mathrm{CH}_{3}-\mathrm{OH}$ (Different Concentrations)

Experiments were performed as per procedure given on page $\mathbf{8 0}$ (Section 8.2 Reactions with $\mathbf{3 b}$ and $\mathrm{R}-\mathrm{OH}$ ) by changing concentration of methanol in the reaction. Reactions are performed in duplicate with each alcohol (for example, $1^{\prime}$ is duplicate of 1 ) and results are summarized below. 
Table 22: 17 Hour reaction time with different mmols of methanol $\left(\mathrm{CH}_{3}-\mathrm{OH}\right)$ and $\mathbf{3 b}$

\begin{tabular}{|c|c|c|}
\hline $\begin{array}{c}\text { Entr } \\
\mathrm{y}\end{array}$ & $\mathrm{mmol}$ of $\mathrm{CH}_{3}-\mathrm{OH}(\mathrm{M})$ & $\%$ Loss of Deuterium (RM) \\
\hline 1 & $0.00999(0.027)$ & 12 \\
\hline 1 ' & $0.00999(0.027)$ & 12 \\
\hline 2 & $0.037(0.10)$ & 17 \\
\hline 2 ' & $0.037(0.10)$ & 17 \\
\hline 3 & $0.06(0.162)$ & 20 \\
\hline $3^{\prime}$ & $0.06(0.162)$ & 20 \\
\hline 4 & $0.08(0.216)$ & 22 \\
\hline $4 '$ & $0.08(0.216)$ & 21 \\
\hline 5 & $0.10(0.270)$ & 25 \\
\hline 5 , & $0.10(0.270)$ & 24 \\
\hline 6 & $0.12(0.324)$ & 24 \\
\hline 6 ' & $0.12(0.324)$ & 24 \\
\hline 7 & $0.14(0.378)$ & 24 \\
\hline $7^{\prime}$ & $0.14(0.378)$ & 23 \\
\hline 8 & $0.16(0.432)$ & 23 \\
\hline 8 ' & $0.16(0.432)$ & 23 \\
\hline 9 & $0.20(0.541)$ & 20 \\
\hline 9' & $0.20(0.541)$ & 19 \\
\hline 10 & $0.30(0.811)$ & 14 \\
\hline $10^{\prime}$ & $0.30(0.811)$ & 14 \\
\hline 11 & $0.40(1.081)$ & 8 \\
\hline $11^{\prime}$ & $0.40(1.081)$ & 8 \\
\hline 12 & $0.60(1.622)$ & 6 \\
\hline $12^{\prime}$ & $0.60(1.622)$ & 6 \\
\hline 13 & $0.90(2.432)$ & 5 \\
\hline $13^{\prime}$ & $0.90(2.432)$ & 4 \\
\hline 14 & $1.20(3.243)$ & 5 \\
\hline $14^{\prime}$ & $1.20(3.243)$ & 4 \\
\hline 15 & $1.60(4.324)$ & 2 \\
\hline $15^{\prime}$ & $1.60(4.324)$ & 2 \\
\hline 16 & $1.85(5.000)$ & 4 \\
\hline $16^{\prime}$ & $1.85(5.000)$ & 4 \\
\hline
\end{tabular}

NMR spectra are given on pages 449-456. 


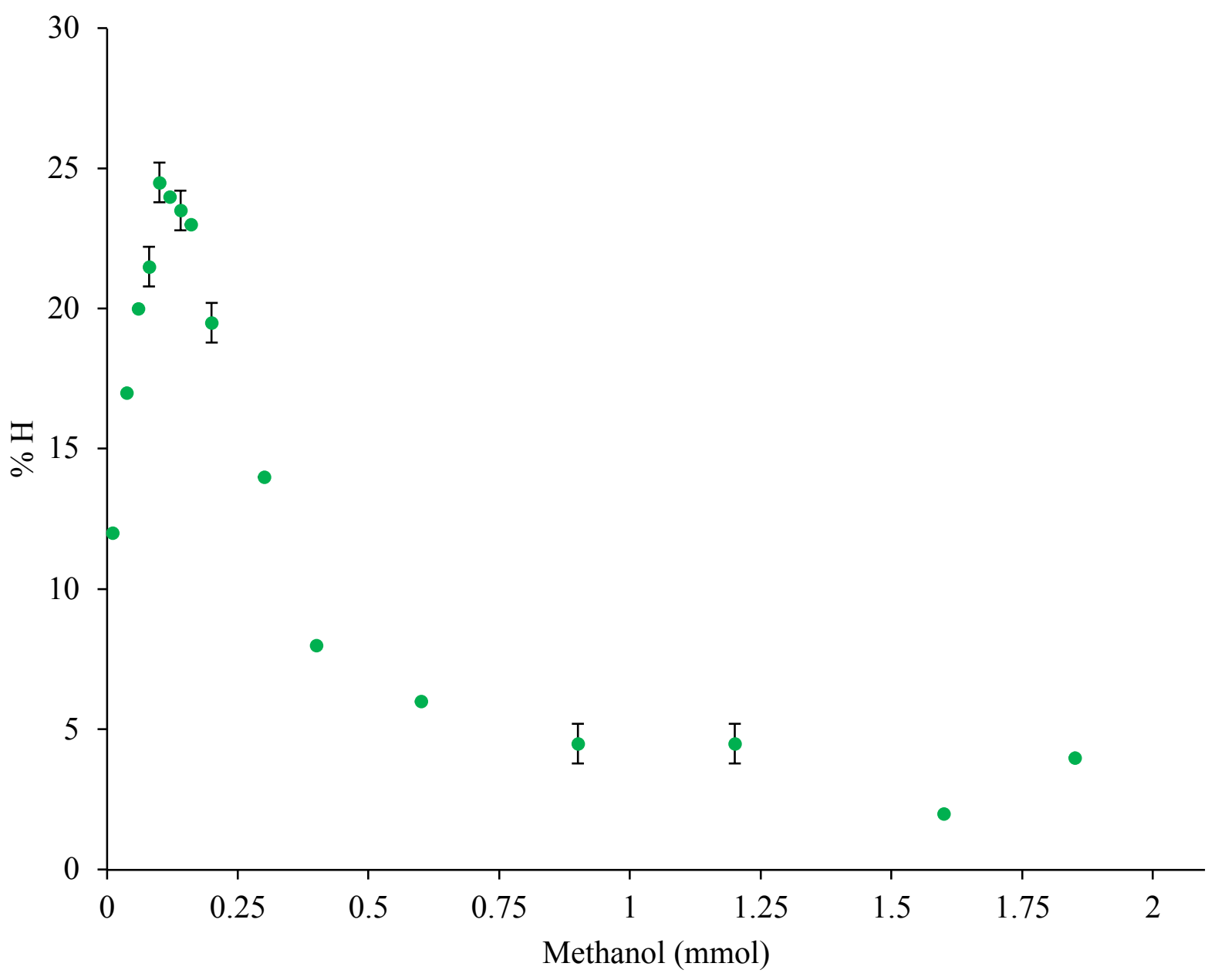

Figure 18: Plot of methanol (mmol) against \%Loss of Deuterium

\subsection{Reactions with $3 \mathrm{~b}$ and R-OH $(0.10 \mathrm{mmol})$}<smiles>[2H]C(=O)c1ccccc1Cl</smiles>

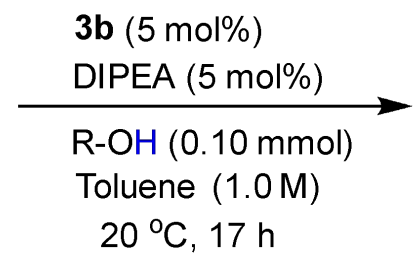<smiles>O=Cc1ccccc1Cl</smiles>

Scheme 31: Reactions with 3b and R-OH (0.10 mmol)

Experiments were performed as per procedure given on page $\mathbf{8 0}$ (8.2 Reactions with $\mathbf{3 b}$ and R-OH) by keeping concentration of alcohols $(0.10 \mathrm{mmol})$ in the reaction. Reactions are performed in duplicate with each alcohol (for example, $1^{\prime}$ is duplicate of 1 ) and results are summarized below. 
Table 23: 17 Hour reaction time with $0.10 \mathrm{mmol}(0.27 \mathrm{M})$ alcohols $(\mathrm{R}-\mathrm{OH})$ and $\mathbf{3 b}$

\begin{tabular}{|c|c|c|}
\hline $\begin{array}{c}\text { Entr } \\
\text { y }\end{array}$ & R-OH (0.10 mmol) & \%Loss of Deuterium (RM) \\
\hline 1 & Water & 48 \\
\hline $1^{\prime}$ & Water & 50 \\
\hline 2 & Ethanol & 26 \\
\hline $2^{\prime}$ & Ethanol & 27 \\
\hline 3 & Trifluoroethanol & 14 \\
\hline 3 & Trifluoroethanol & 15 \\
\hline 4 & Phenol & 11 \\
\hline $4^{\prime}$ & Phenol & 11 \\
\hline 5 & 4-Methylphenol & 14 \\
\hline 5 & 4-Methylphenol & 13 \\
\hline 6 & Pentafluorophenol & 0 \\
\hline 6 & Pentafluorophenol & 0 \\
\hline 7 & 4-Fluorophenol & 6 \\
\hline 7 & 4-Fluorophenol & 6 \\
\hline
\end{tabular}

NMR spectra are given on pages 457-460. 
Reactions with 3-Chlorobenzaldehyde with $3 a$ and $D_{2} O$ (5 eq.):<smiles>O=Cc1ccccc1Cl</smiles>

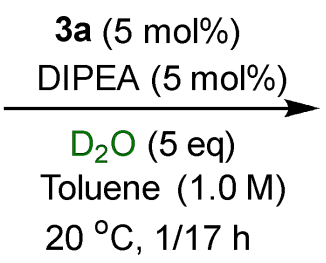<smiles>[2H]C(=O)c1ccccc1Cl</smiles>

Scheme 32: 1 and 17 Hour reaction of 3-Chlorobenzaldehyde with $\mathrm{D}_{2} \mathrm{O}$ (5 eq.)

Experiments were performed as per procedure given on page $\mathbf{8 0}$ (Section 8.1 Reactions with 3a and $\mathrm{R}-\mathrm{OH}$ (5 eq.)). Results are summarized below.

Table 24: Result for 1 and 17 Hour reaction of 3-Chlorobenzaldehyde with $\mathrm{D}_{2} \mathrm{O}$ (5 eq.)

\begin{tabular}{|c|c|c|}
\hline $\begin{array}{c}\text { Entr } \\
\mathrm{y}\end{array}$ & $\begin{array}{c}\text { Reaction Time } \\
\text { (hours) }\end{array}$ & \%D (RM) \\
\hline 1 & 1 & 66 \\
\hline $1^{\prime}$ & 1 & 64 \\
\hline 2 & 17 & 90 \\
\hline $2^{\prime}$ & 17 & 90 \\
\hline
\end{tabular}

NMR spectra are given on pages 461. 


\section{Large Scale Reactions}<smiles>O=Cc1ccccc1Cl</smiles>

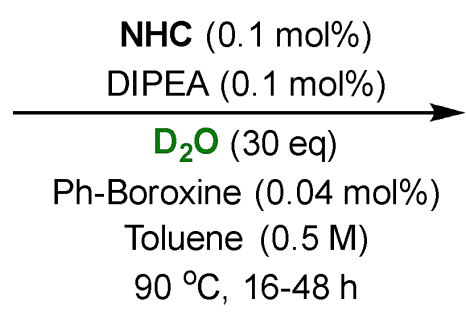

Scheme 33: Large scale reactions

Deuteration reactions were run in $25 \mathrm{~mL}$ round bottom flask and stirred at $1200 \mathrm{rpm}$. A dry, argon flushed $25 \mathrm{~mL}$ round bottom flask equipped with a magnetic stir bar was charged with NHC $(0.1$ mol\%, 0.001 eq.), phenyl boroxine (1.37 mg, 0.04 mol\%, 0.0004 eq.) and flushed with argon followed by the addition of $16 \mathrm{~mL}(0.5 \mathrm{M})$ anhydrous toluene. DIPEA ( $2 \mu \mathrm{L}, 0.1 \mathrm{~mol} \%, 0.001$ eq. $), \mathrm{D}_{2} \mathrm{O}(6.00$ $\mathrm{mL}, 330 \mathrm{mmol}, 30$ eq.) and then 2-chlorobenzaldehyde (1.24 mL, $11 \mathrm{mmol}, 1$ eq.) were added. The reaction flask was closed with screw cap then wrapped with teflon tape and electrical tape and the mixture was stirred at $90{ }^{\circ} \mathrm{C}$ for $16-48 \mathrm{~h}$. After the reaction, an aliquot (50 $\left.\mu \mathrm{L}\right)$ was taken for NMR measurments (delay time D1 = 50 seconds was used in all studies). The crude reaction mixture was directly transferred on silica gel and purified using Hex:Et ${ }_{2} \mathrm{O}$ (98:2) combination. Deuterium incorporation and reaction yield after purification are included below.

NMR spectra are given on pages 462-475. 
Table 25: Condition optimization

\begin{tabular}{|c|c|c|c|c|c|c|c|c|c|c|c|c|}
\hline \multirow{2}{*}{ Entry } & \multirow{2}{*}{$\begin{array}{l}\text { Aldehyd } \\
\text { e (mmol) }\end{array}$} & \multirow{2}{*}{$\begin{array}{c}\text { NHC } \\
(\mathrm{mol} \%)\end{array}$} & \multirow{2}{*}{$\begin{array}{l}\text { DIPEA } \\
\text { (mol\%) }\end{array}$} & \multirow{2}{*}{$\begin{array}{l}\mathrm{D}_{2} \mathrm{O} \\
\text { (eq.) }\end{array}$} & \multirow{2}{*}{$\begin{array}{l}\text { Ph-Boroxine } \\
\text { (mol\%) }\end{array}$} & \multirow{2}{*}{$\begin{array}{l}\text { Reaction } \\
\text { time }(\mathrm{h})\end{array}$} & \multirow{2}{*}{$\begin{array}{l}\text { Reaction } \\
\text { Conc. (M) }\end{array}$} & \multirow{2}{*}{$\begin{array}{l}\text { Reaction } \\
\text { Temp. }\end{array}$} & \multirow{2}{*}{$\begin{array}{c}\% \mathrm{D} \\
\text { (RM) }\end{array}$} & \multirow{2}{*}{$\begin{array}{c}\% \\
\text { Aldehyde }\end{array}$} & \multicolumn{2}{|c|}{ After Purification } \\
\hline & & & & & & & & & & & $\% \mathrm{D}$ & \%Yield \\
\hline 1 & 11 & $3 a(5)$ & 5 & 30 & - & 16 & 1 & $20 \div \mathrm{C}$ & 98 & 100 & 97 & 88 \\
\hline $1^{\prime}$ & 11 & $3 a(5)$ & 5 & 30 & - & 16 & 1 & $20 \stackrel{\circ}{C}$ & 98 & 100 & - & - \\
\hline 2 & 11 & $3 a(5)$ & 5 & 10 & - & 16 & 1 & $20 \div \mathrm{C}$ & 94 & 100 & - & - \\
\hline 3 & 11 & $3 a(5)$ & 5 & 15 & - & 24 & 1 & $20 \stackrel{\circ}{C}$ & 96 & 100 & 95 & 91 \\
\hline $3^{\prime}$ & 11 & $3 a(5)$ & 5 & 15 & - & 24 & 1 & $20 \cong \mathrm{C}$ & 96 & 100 & - & - \\
\hline 4 & 11 & $3 a(1)$ & 1 & 30 & - & 24 & 1 & 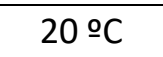 & 83 & 100 & - & - \\
\hline 5 & 11 & $3 a(1)$ & 1 & 30 & - & 24 & 1 & $50 \stackrel{\circ}{C}$ & 98 & 100 & 97 & 97 \\
\hline $5^{\prime}$ & 11 & $3 a(1)$ & 1 & 30 & - & 24 & 1 & $50 \stackrel{\circ}{C}$ & 98 & 100 & - & - \\
\hline 6 & 11 & $3 a(1)$ & 1 & 15 & - & 24 & 1 & $50 \stackrel{\circ}{C}$ & 95 & 100 & - & - \\
\hline 7 & 11 & $3 a(0.1)$ & 0.1 & 30 & - & 16 & 1 & $90 \div \mathrm{C}$ & 8 & 100 & - & - \\
\hline 8 & 11 & $3 a(0.1)$ & 0.1 & 15 & - & 16 & 1 & $90 \div \mathrm{C}$ & 9 & 100 & - & - \\
\hline 9 & 11 & $4 i(0.1)$ & 0.1 & 15 & - & 24 & 0.5 & $50 \stackrel{\circ}{C}$ & 38 & 100 & - & - \\
\hline 10 & 11 & $4 \mathbf{i}(0.1)$ & 0.1 & 15 & 0.04 & 24 & 0.5 & $50 \stackrel{\circ}{C}$ & 46 & 100 & - & - \\
\hline 11 & 11 & $4 \mathbf{i}(0.1)$ & 0.1 & 15 & - & 24 & 2 & $50 \stackrel{\circ}{C}$ & 26 & 100 & - & - \\
\hline 12 & 11 & $4 a(0.1)$ & 0.1 & 30 & 0.04 & 24 & 0.5 & $90 \stackrel{\circ}{ } \mathrm{C}$ & 99 & 100 & 98 & 95 \\
\hline $12^{\prime}$ & 11 & $4 a(0.1)$ & 0.1 & 30 & 0.04 & 24 & 0.5 & $90 \stackrel{\circ}{C}$ & 98 & 100 & - & - \\
\hline 13 & 11 & $4 a(0.1)$ & 0.1 & 30 & 0.04 & 24 & 0.5 & $50 \stackrel{\circ}{C}$ & 50 & 100 & - & - \\
\hline 14 & 11 & $4 a(0.1)$ & 0.1 & 15 & 0.04 & 24 & 0.5 & $90 \stackrel{\circ}{C}$ & 96 & 100 & - & - \\
\hline $14^{\prime}$ & 11 & $4 a(0.1)$ & 0.1 & 15 & 0.04 & 24 & 0.5 & 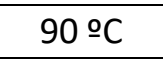 & 96 & 100 & - & - \\
\hline 15 & 11 & $4 a(0.1)$ & 0.1 & 15 & 0.04 & 48 & 0.5 & $90 \stackrel{\circ}{C}$ & 97 & 100 & 96 & 92 \\
\hline 16 & 11 & $4 a(0.1)$ & 0.1 & 30 & - & 24 & 0.5 & $90 \div \mathrm{C}$ & 98 & 100 & 98 & 95 \\
\hline $16^{\prime}$ & 11 & $4 a(0.1)$ & 0.1 & 30 & - & 24 & 0.5 & $90 \stackrel{\circ}{C}$ & 97 & 100 & - & - \\
\hline 17 & 11 & $4 a(0.1)$ & 0.1 & 30 & - & 16 & 0.5 & $90 \div \mathrm{C}$ & 98 & 100 & 98 & 96 \\
\hline $17^{\prime}$ & 11 & $4 a(0.1)$ & 0.1 & 30 & - & 16 & 0.5 & $90 \div \mathrm{C}$ & 98 & 100 & - & - \\
\hline 18 & 11 & $4 a(0.1)$ & 0.1 & 15 & - & 24 & 0.5 & $90 \stackrel{\circ}{C}$ & 96 & 100 & 96 & 93 \\
\hline $18^{\prime}$ & 11 & $4 a(0.1)$ & 0.1 & 15 & - & 24 & 0.5 & $90 \div \mathrm{C}$ & 96 & 100 & - & - \\
\hline
\end{tabular}




\section{Mechanistic studies}

\subsection{Reaction with benzoin}

In order to verify that the boronic acid additives funnel reactivity away from the benzoin adduct rather than facilitate the retro-benzoin process, the following expirement was conducted:<smiles>O=C(c1cccc(Cl)c1)C(O)c1ccccc1</smiles>

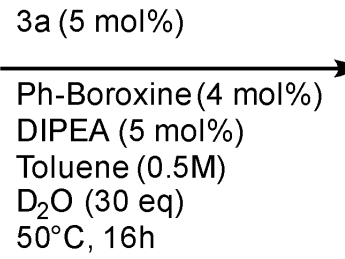<smiles>O=Cc1cccc(Cl)c1</smiles>

Scheme 34: Deuteration of 3-Chlorobenzoin to deutorated aldehyde

The reaction was run in an $8 \mathrm{~mL}$ vial and stirred at $1000 \mathrm{rpm}$. A dry, argon flushed $8 \mathrm{~mL}$ vial equipped with a magnetic stir bar was charged with 3-chlorobenzoin ( $0.65-0.73 \mathrm{mmol}, 0.5$ eq.), $3 \mathrm{a}$ (5 mol\%, 0.05 eq.), phenyl boroxine ( 4 mol\%, 0.04 eq.) and flushed with argon followed by the addition of anhydrous toluene $(0.5 \mathrm{M})$. DIPEA (5 mol\%, 0.05 eq.) and $\mathrm{D}_{2} \mathrm{O}$ (30 eq.) were added. The reaction lask was closed with screw cap then wrapped with teflon tape and electrical tape and the mixture was stirred at $50^{\circ} \mathrm{C}$ for $16 \mathrm{~h}$. After the reaction, an aliquot $(50 \mu \mathrm{L})$ was taken for NMR measurments (delay time D1 = 50 seconds was used in all studies) and HPLC.

No aldehyde was formed during the reaction. NMR and HPLC spectra are given on pages 479.

\subsection{Reactions with 2,6-Methoxyphenyl Boronic-acid}

To test wether boronic acid might be serving as a proton/deuteron reservoir in the reaction we were required to either fully deuterate boronic acids or use protonated ones with a deuterated substrate that would undergo protonation. For technical ease we selected to work with a deuterated aldehyde. Fully protonating phenyl bronic acid is fairly difficult due to its equilibrium with boroxine. As we knew that changes to the structure of aryl boronic acid had limited influence on the reaction, we selected to perform a set of reactions using 2,6-methoxyphenyl boronic acid, which does not readily form boroxine.

\section{2 eq. and 0.12 eq. of 2,6-Methoxyphenyl Boronic-acid per substrate with water}

In order to probe how 2,6-methoxyphenyl boronic acid functions under our reaction conditions, 0.12 eq were used, which are equal to 0.04 boroxine taking into account 3 boronic acid molecules per one boroxine. We also ran a reaction with 0.2 eq of 2,6-methoxyphenyl boronic acid to test the effect of an increased quantity of boronic acid on the reaction. 


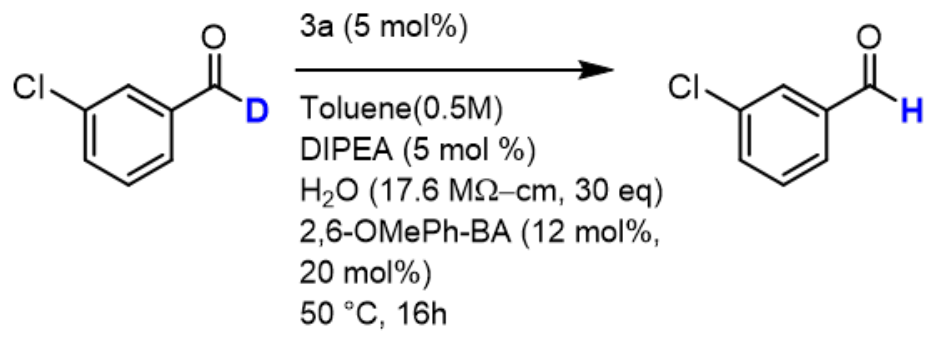

Scheme 35: Protonation of 3-Chlorobenzaldehyde

Protonation reactions were run in $1 \mathrm{~mL}$ vial and stirred at $1000 \mathrm{rpm}$. A dry, argon flushed $1 \mathrm{~mL}$ vial equipped with a magnetic stir bar was charged with 3a (7.9 mg, $0.0185 \mathrm{mmol}, 0.05$ eq.), phenyl boroxine ( $8.1 \mathrm{mg}, 0.044 \mathrm{mmol}, 0.012 \mathrm{eq}$ or $13.5 \mathrm{mg}, 0.074 \mathrm{mmol}, 0.2 \mathrm{eq}$ ) and flushed with argon followed by the addition of anhydrous toluene $(590 \mu \mathrm{L}, 0.45 \mathrm{M})$. DIPEA (3.2 $\mu \mathrm{L}, 0.0185 \mathrm{mmol}, 0.05$ eq.), $\mathrm{D}_{2} \mathrm{O}(222 \mu \mathrm{L}, 33$ eq.) and 3-chlorobenzaldehyde-D 98\% (42 $\mu \mathrm{L}, 0.37 \mathrm{mmol}, 0.5$ eq.) were added. The reaction flask was closed with screw cap then wrapped with teflon tape and electrical tape and the mixture was stirred at $50{ }^{\circ} \mathrm{C}$ for $16 \mathrm{~h}$. After the reaction, an aliquot $(50 \mu \mathrm{L})$ was taken for NMR measurments (delay time D1 $=50$ seconds was used in all studies).

Proton incorporation is given below, NMR spectra after evaporation are given on pages 480-481.

\begin{tabular}{|c|c|c|}
\hline $\begin{array}{c}\text { Entr } \\
\mathrm{y}\end{array}$ & $\begin{array}{c}\text { Eq. of } \\
\text { Boronic acid }\end{array}$ & $\% \mathrm{H}$ \\
\hline 1 & 0.12 & 93 \\
\hline 1 & 0.12 & 99 \\
\hline 2 & 0.20 & 96 \\
\hline $2^{\prime}$ & 0.20 & 95 \\
\hline
\end{tabular}

\section{Different percentage of 2,6-Methoxyphenyl Boronic-acid without water}

In order to isolate the impact of the boronic acid itself other proton sources were removed (water was not added and the vial was kept dry under argon).

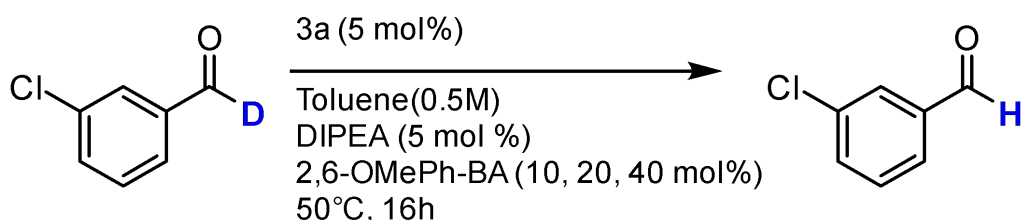

Scheme 36: Protonation of 3-Chlorobenzaldehyde with boronic acid as the only hydrogen source 
The reactions were run in a $1 \mathrm{~mL}$ vial and stirred at $1000 \mathrm{rpm}$. A dry, argon flushed $1 \mathrm{~mL}$ vial equipped with a magnetic stir bar was charged with $3 \mathrm{a}(7.9 \mathrm{mg}, 0.0185 \mathrm{mmol}, 0.05$ eq.), phenyl boroxine $(6.7 \mathrm{mg}, 0.044 \mathrm{mmol}, 0.12$ eq or $13.5 \mathrm{mg}, 0.074 \mathrm{mmol}, 0.2 \mathrm{eq}$ or $20.2 \mathrm{mg}, 0.111 \mathrm{mmol}, 0.3$ eq) and flushed with argon followed by the addition of anhydrous toluene (538 $\mu \mathrm{L}, 0.5 \mathrm{M})$. DIPEA (3.2 $\mu \mathrm{L}, 0.0185 \mathrm{mmol}, 0.05$ eq.) and 3-chlorobenzaldehyde-D 98\% (42 $\mu \mathrm{L}, 0.37 \mathrm{mmol}, 1$ eq.) were added. The reaction flask was closed with screw cap then wrapped with teflon tape and electrical tape and the mixture was stirred at $50{ }^{\circ} \mathrm{C}$ for $16 \mathrm{~h}$. After the reaction, an aliquot $(50 \mu \mathrm{L})$ was taken for NMR measurments (delay time D1 $=50$ seconds was used in all studies).

\begin{tabular}{|l|c|c|c|}
\hline $\begin{array}{c}\text { Entr } \\
\mathrm{y}\end{array}$ & $\begin{array}{c}\text { Eq. of } \\
\text { Boronic acid }\end{array}$ & $\% \mathrm{H}$ & $\begin{array}{c}\text { Percentage } \\
\text { of protons } \\
\text { donated } \\
{[\%]}\end{array}$ \\
\hline 1 & 0.1 & 21 & 100 \\
\hline $1{ }^{\prime}$ & 0.1 & 20 & 100 \\
\hline 2 & 0.2 & 33 & 82.5 \\
\hline $2^{\prime}$ & 0.2 & 32 & 80 \\
\hline 3 & 0.4 & 43 & 53.75 \\
\hline 3 & 0.4 & 39 & 48.75 \\
\hline
\end{tabular}

Proton incorporation is presented in the table below. NMR spectra after evaporation are given on pages $481-482$. 


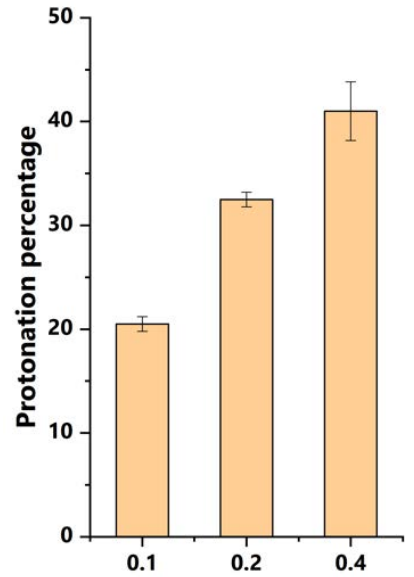

a

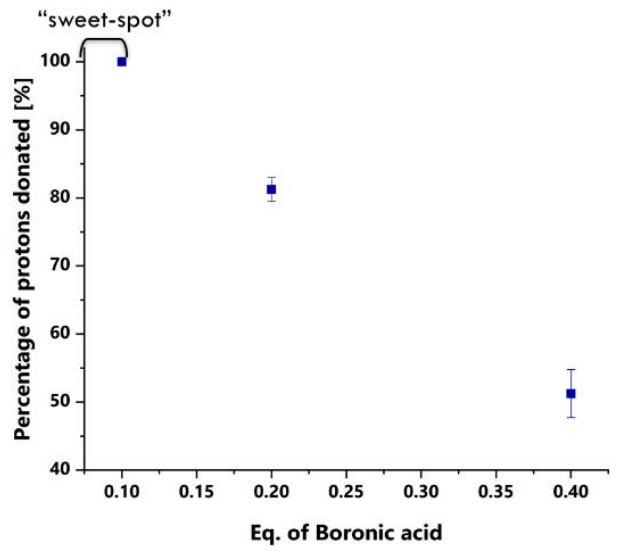

b

Figure 19: a. protonation percentage according to the amount of BA. b. percentage of protons donated in each experiment from the boronic acid. 


\section{HRMS and NMR Spectra}

Raw data files are provided online: https://github.com/Milo-group/Interception

Synthesis of Imidazol based NHCs:

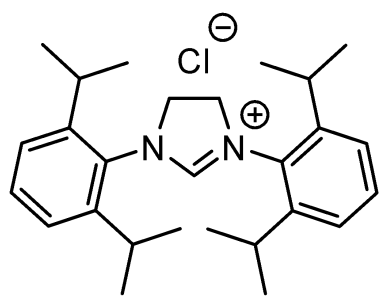

$3 a$

SCG-SIPr-31122019.1. fid

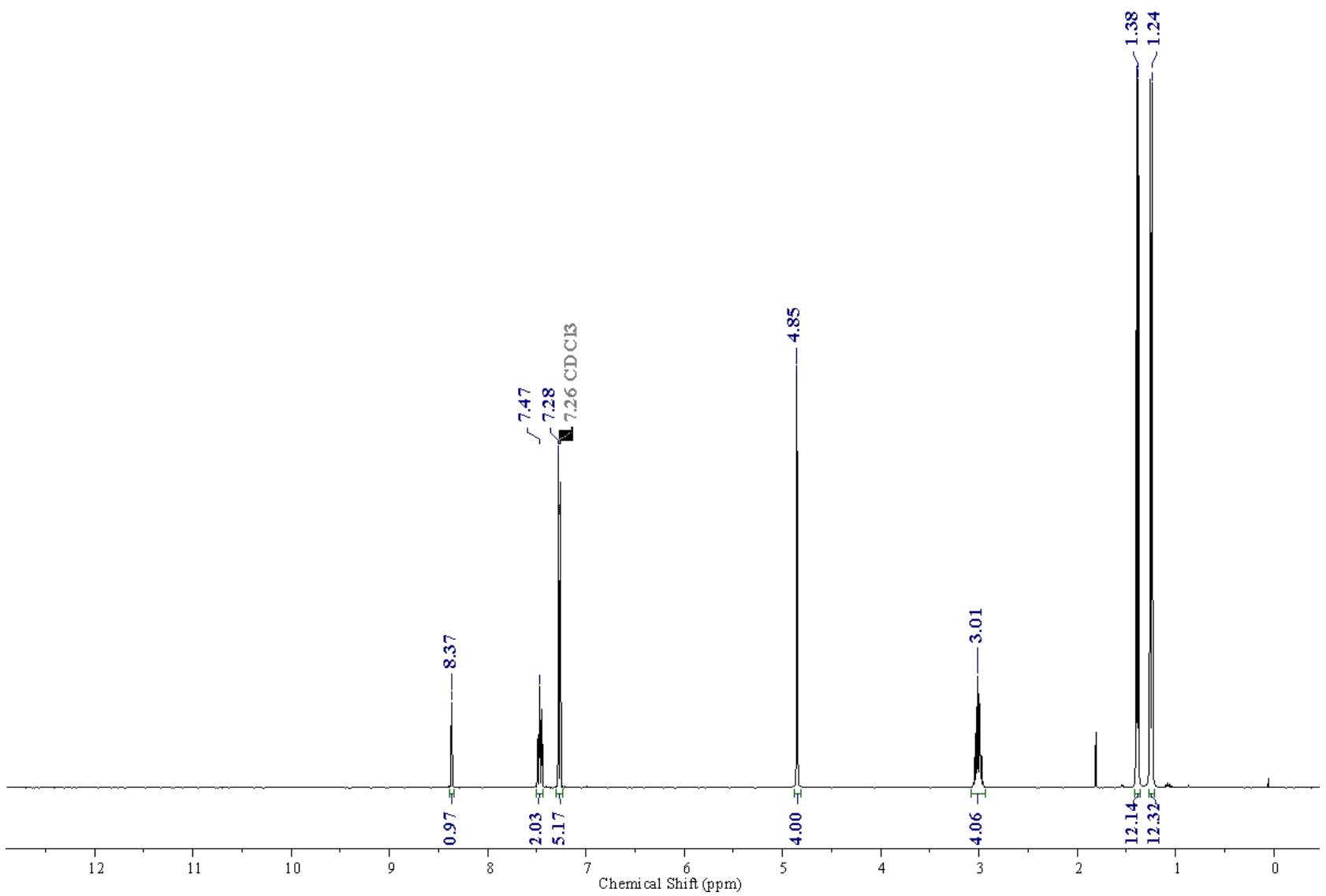




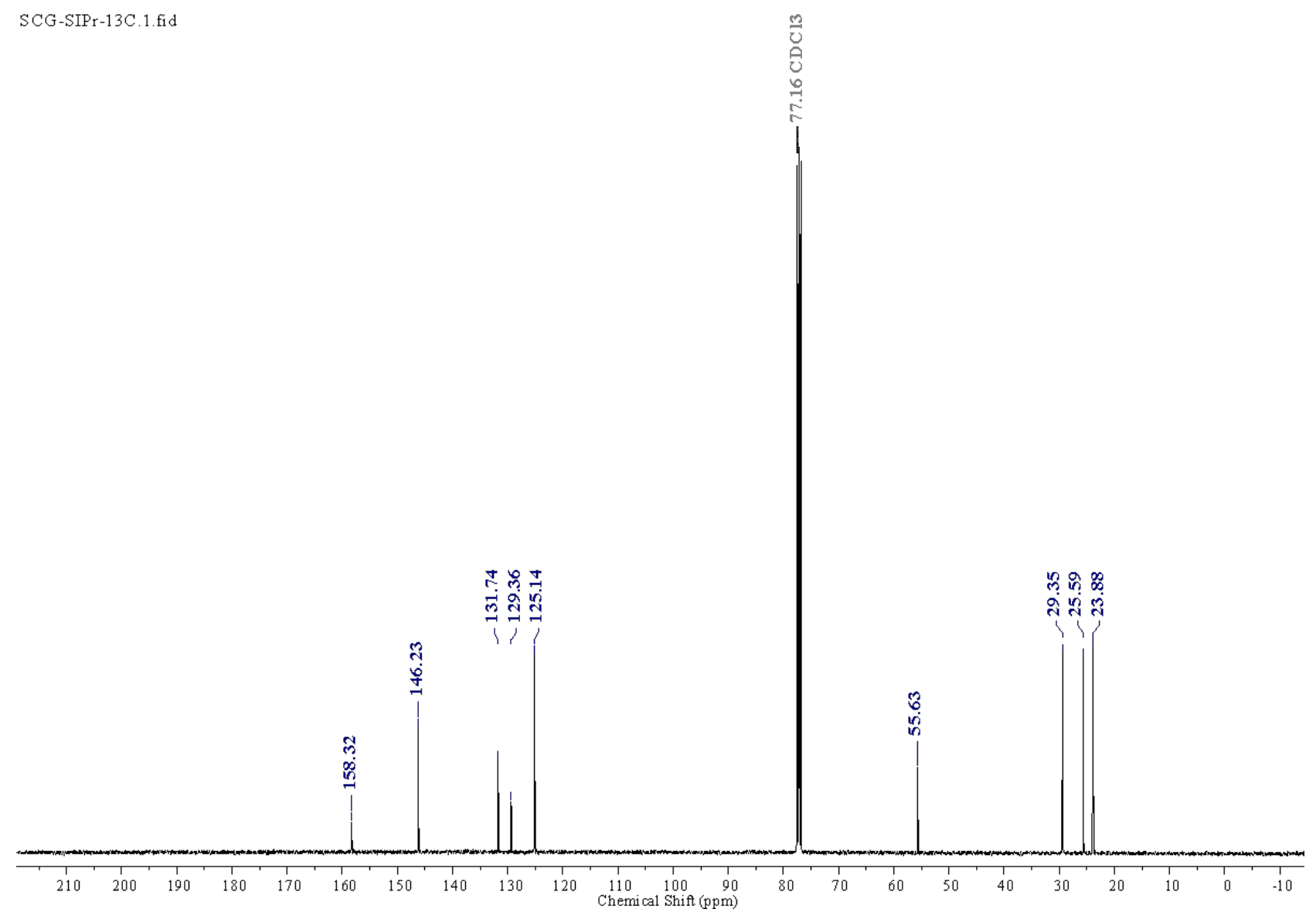




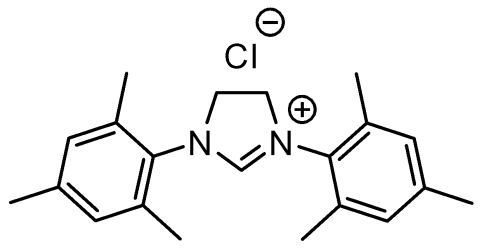

$3 b$

SCG-SIMes-21112019

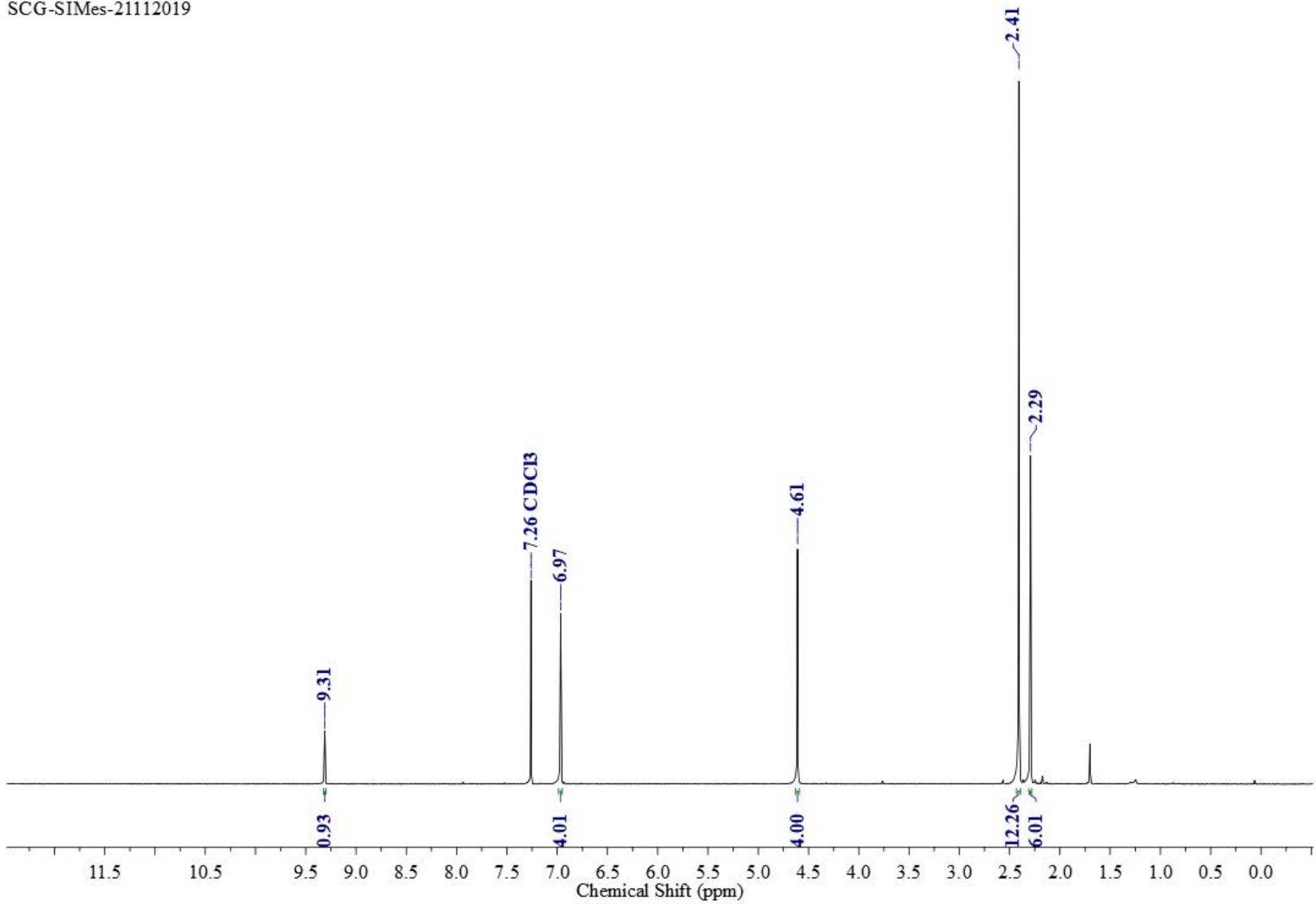



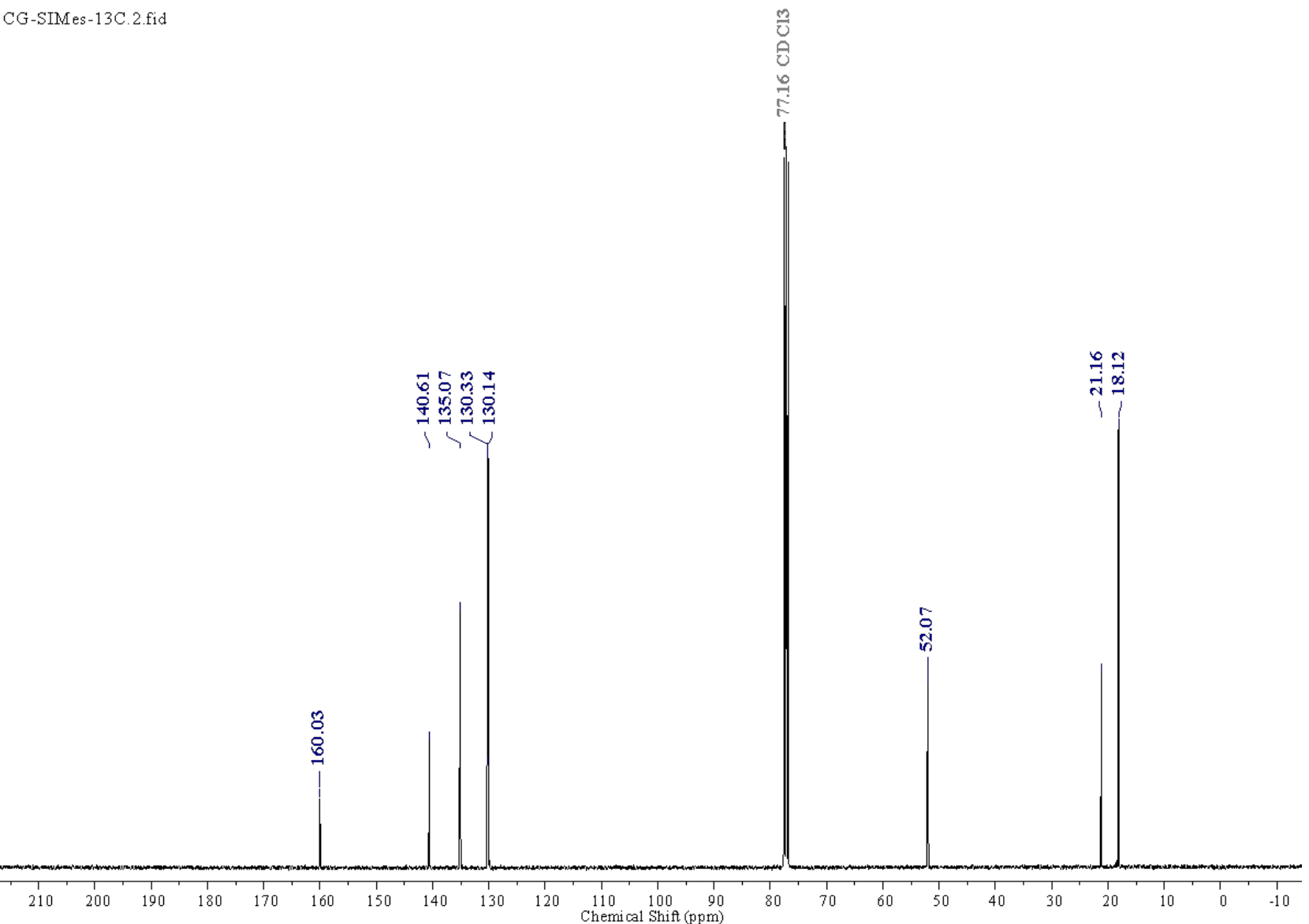
Synthesis of Pyrrolidine Based Triazolium NHCs:

$\Theta$<smiles>O=C(F)c1nn2c(c1Br)CCC2</smiles>

4a

VDOS-NHC $2.4,6 \mathrm{Br}$ VP final, $2.4 \mathrm{fid}$

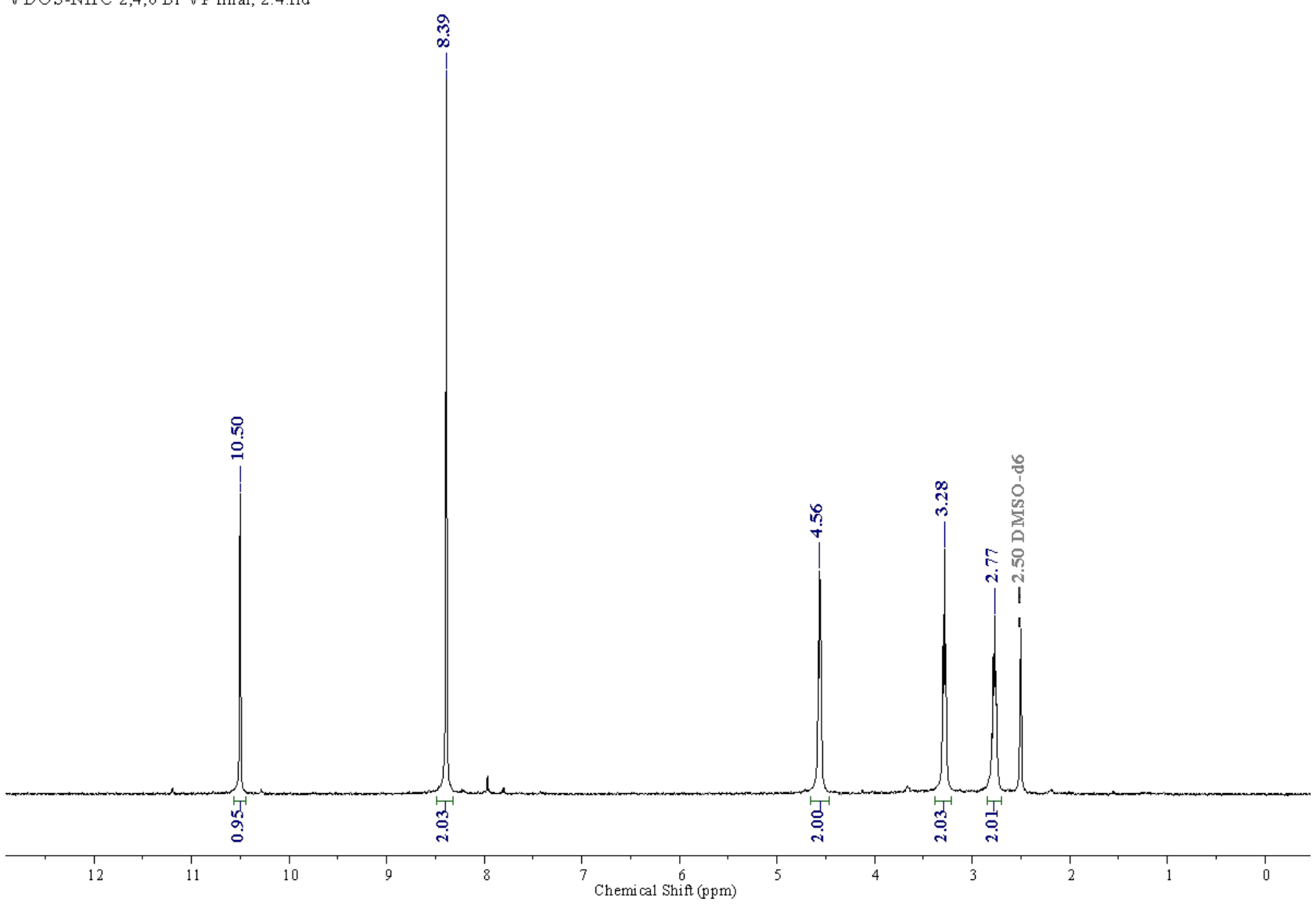




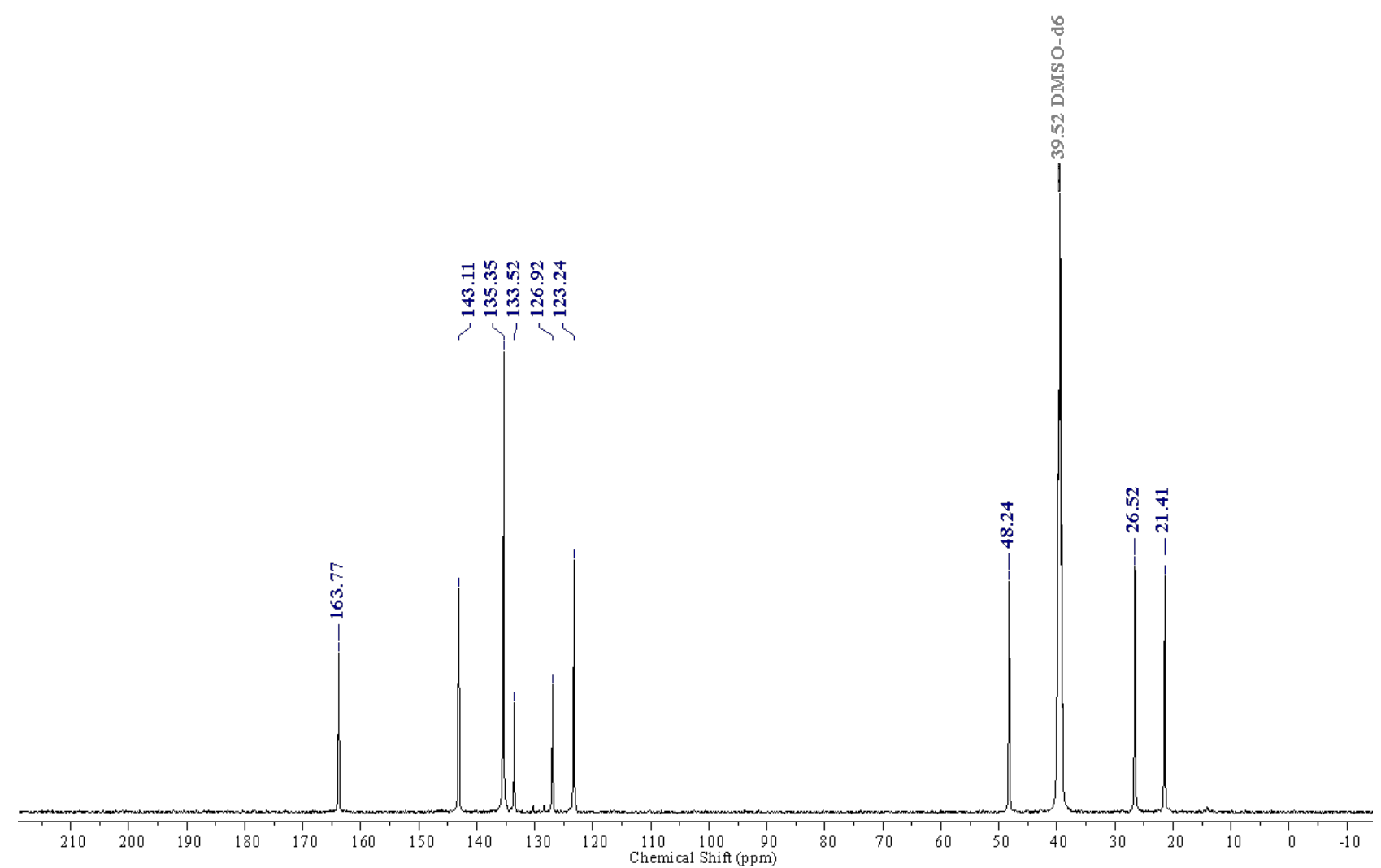




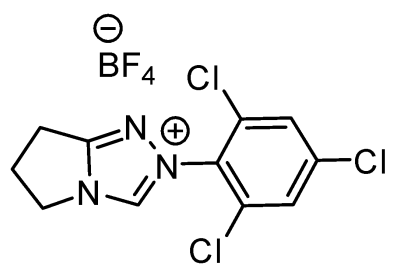

4b

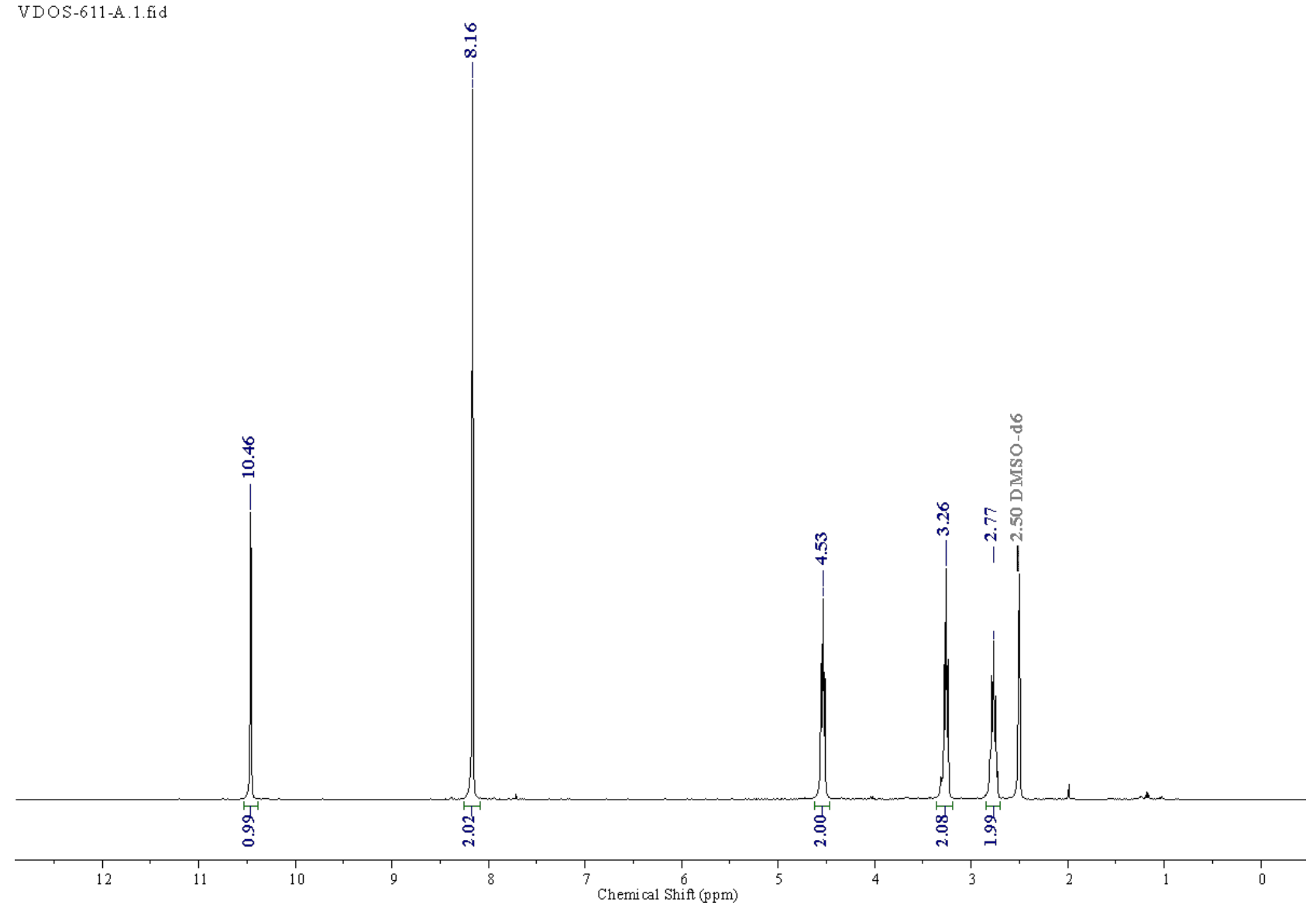



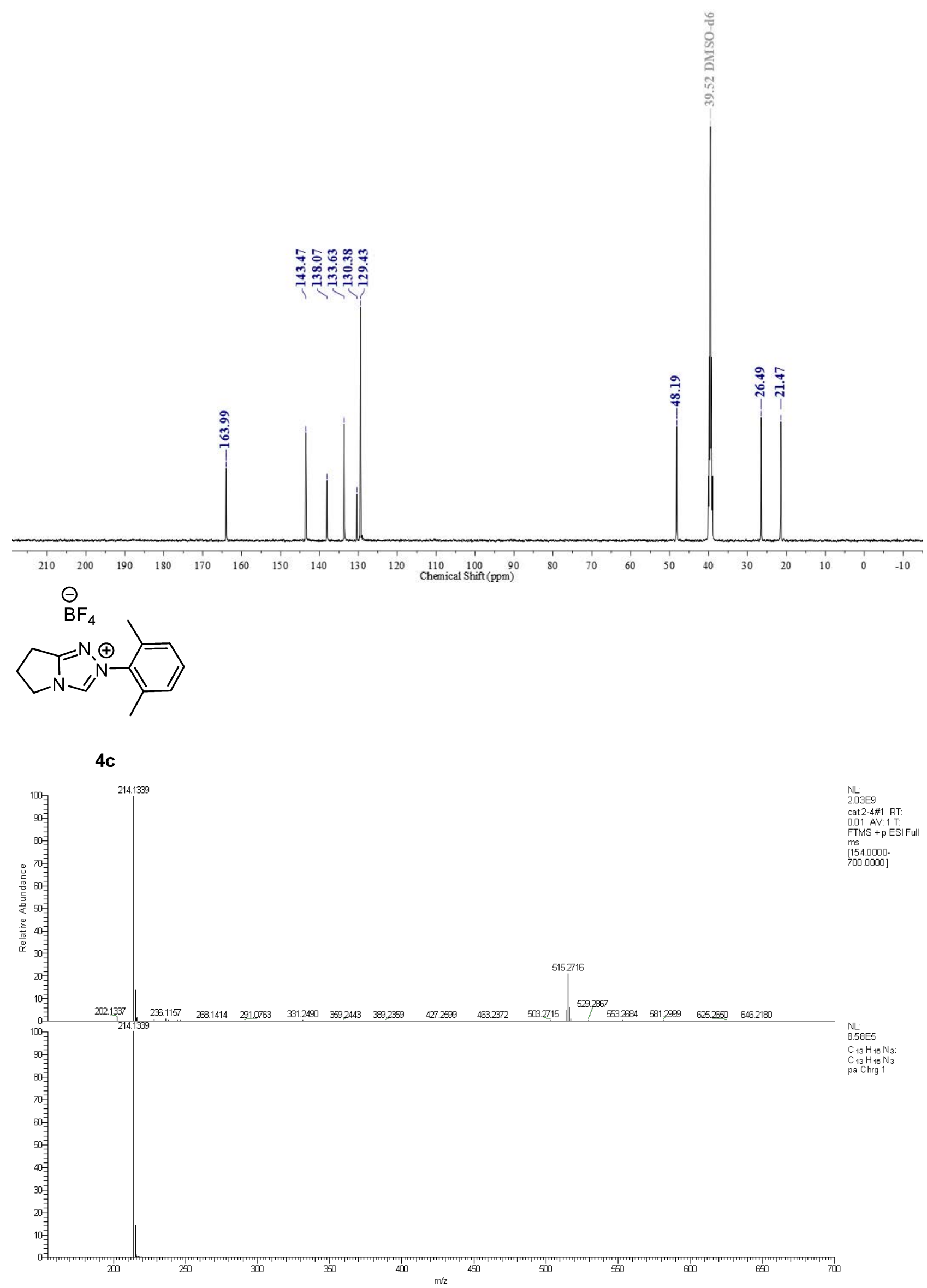

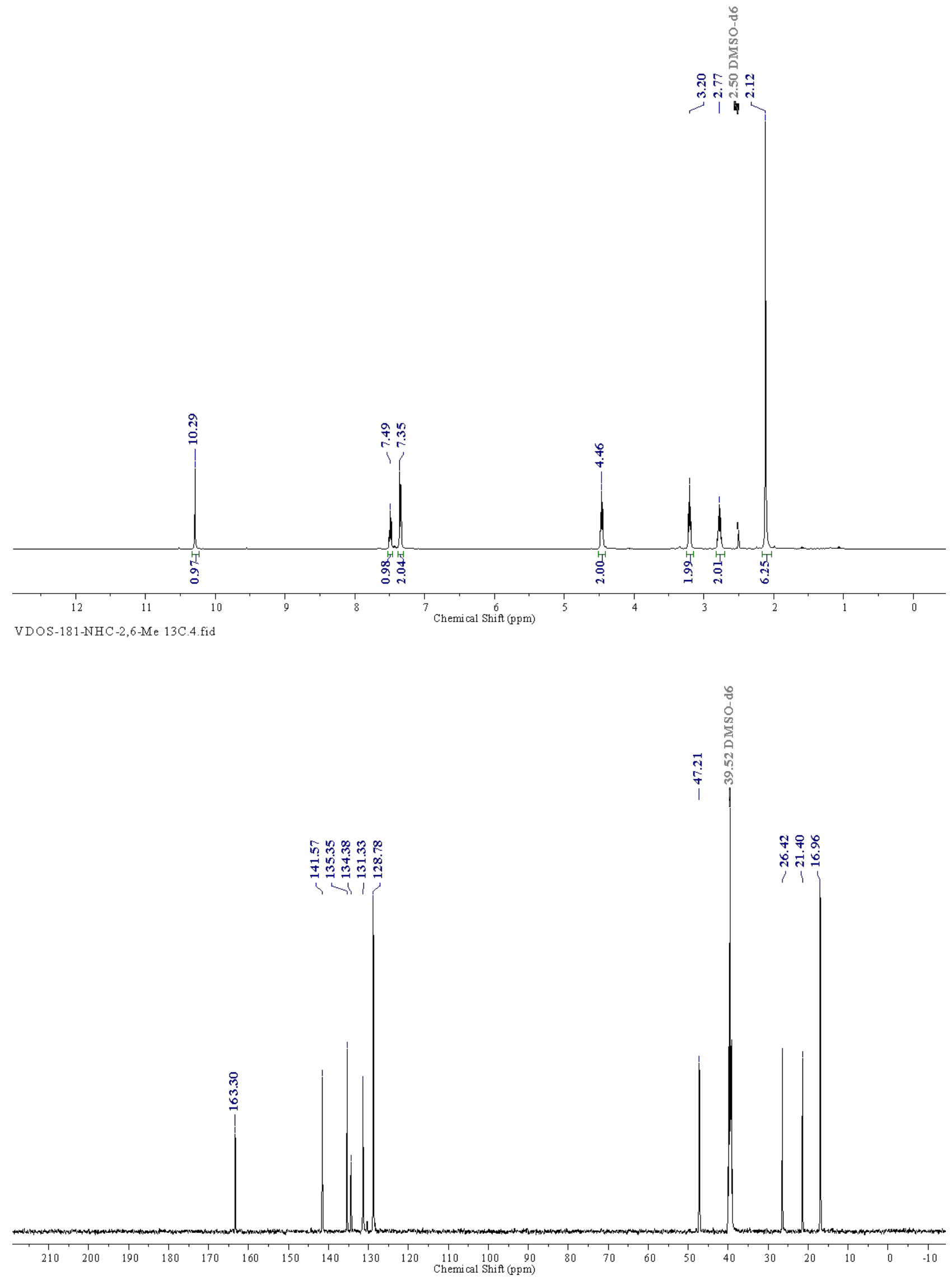
$\ominus$

$\mathrm{BF}_{4}$

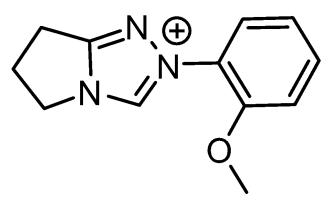

4d

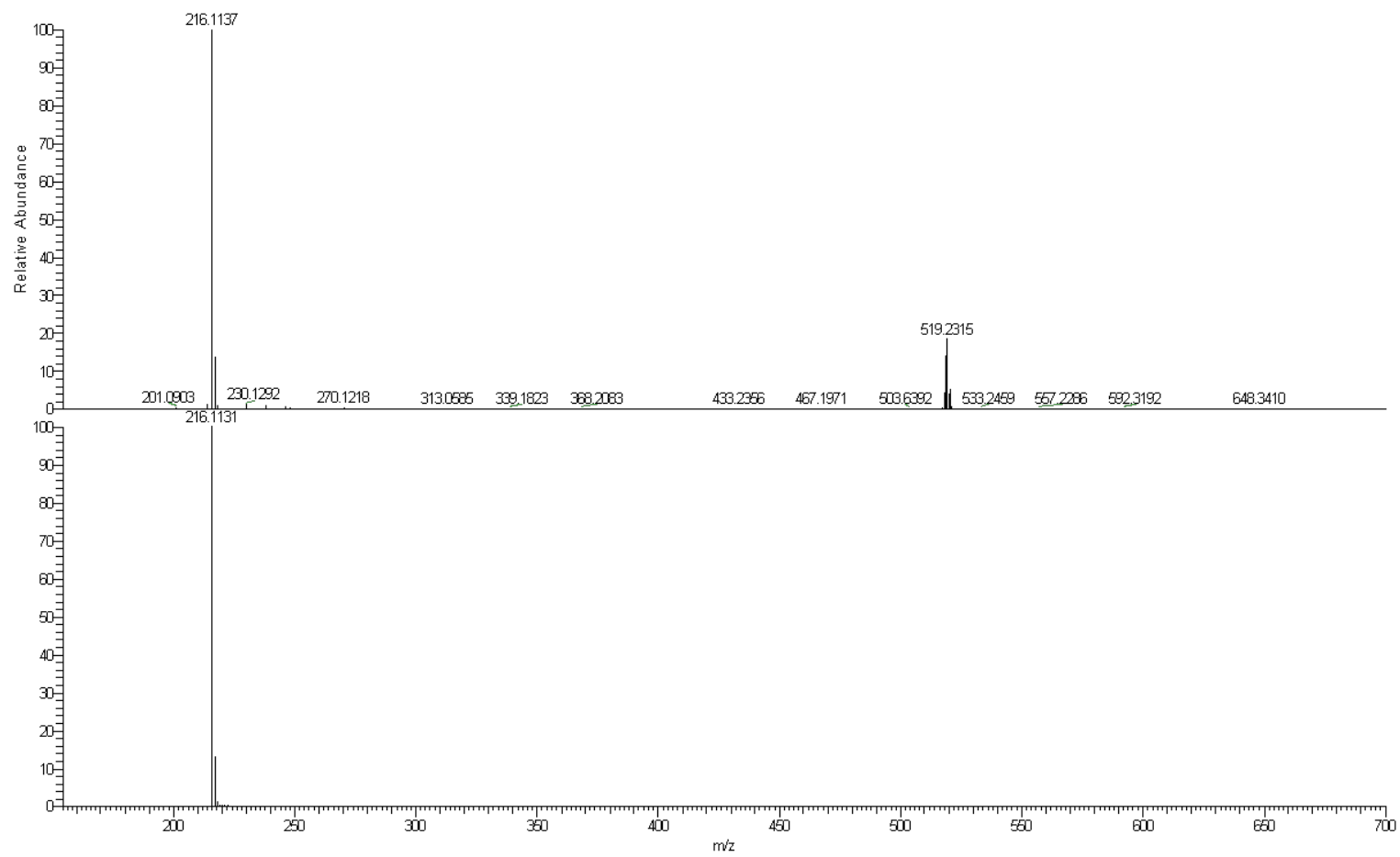

9.04 E9

cat3-1\#1 RT:

FTMS + p ESI Full

ms 1540000 -

700.0000

VDOS-180-NHC-2-OMe.2.fid

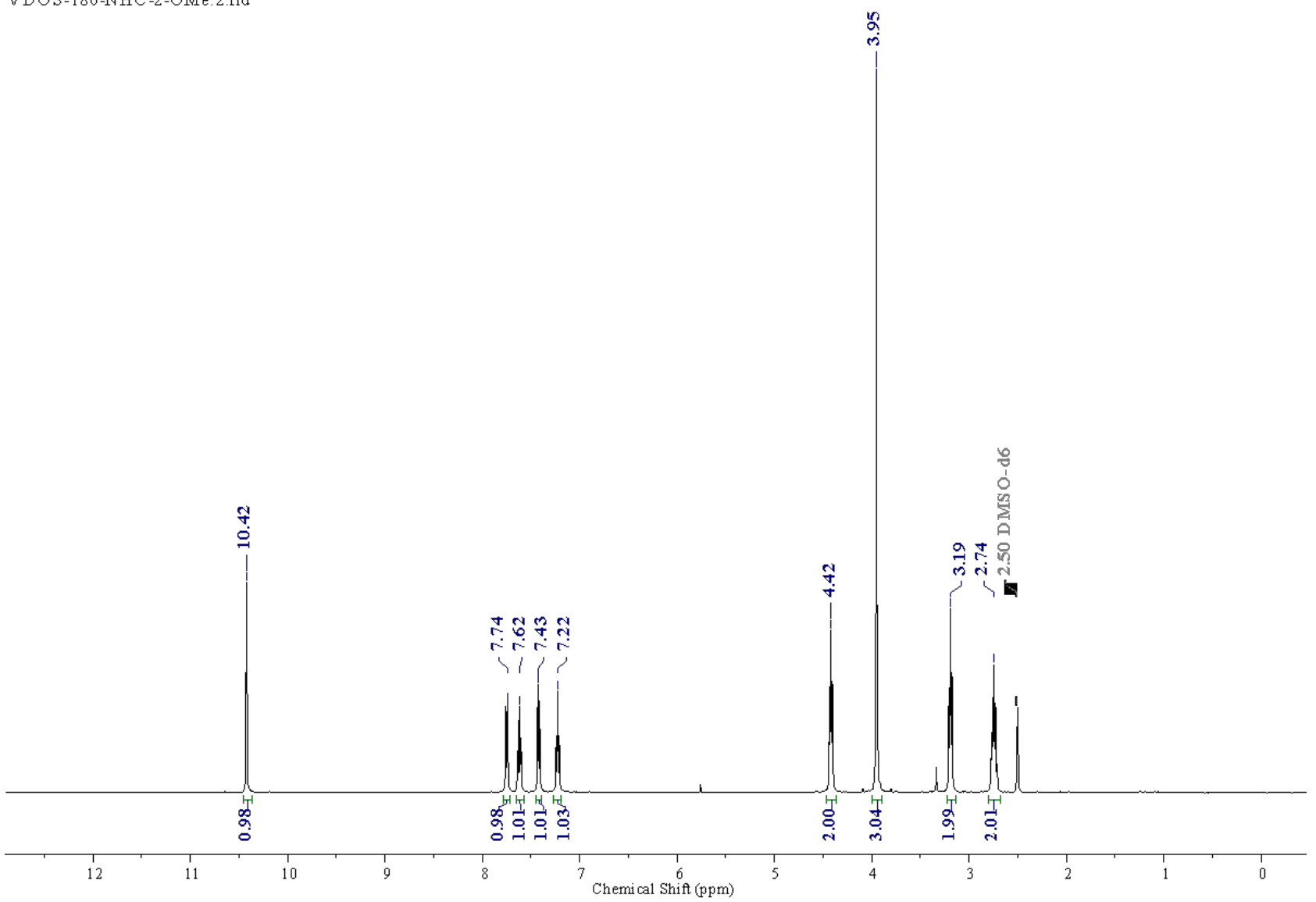



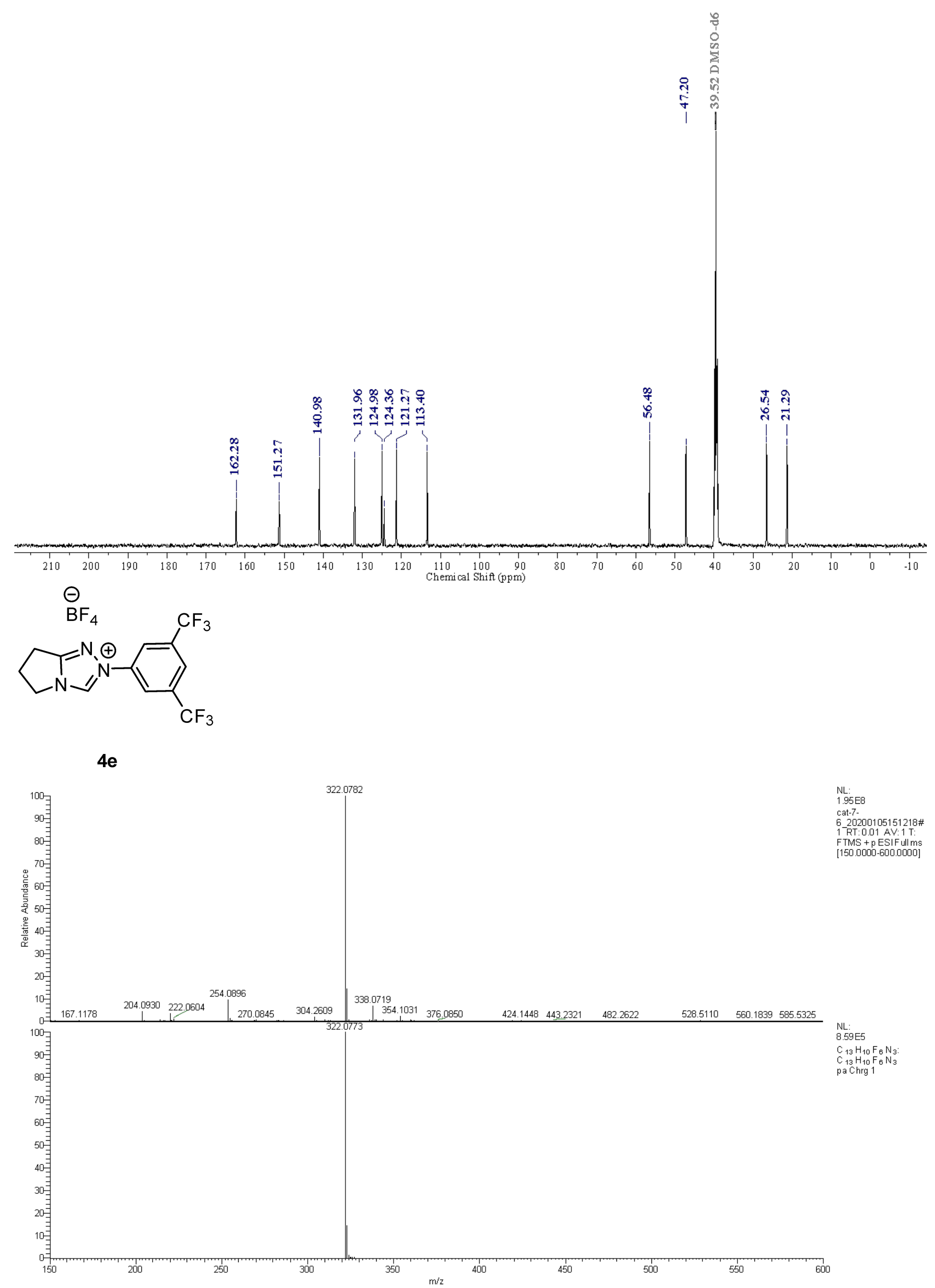


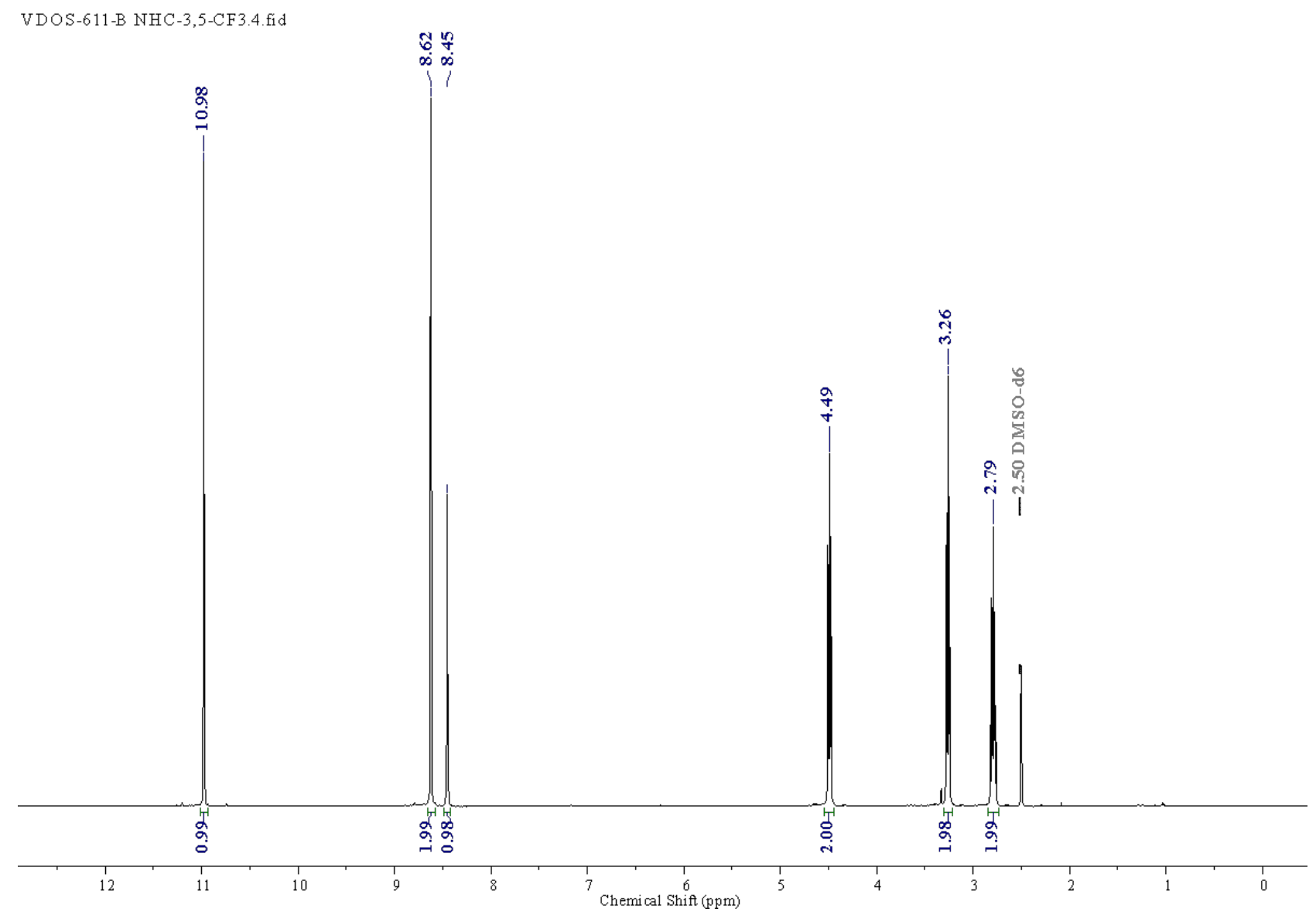

VDOS-611-B NHC-3,5-CF3 13C-2.5.fid

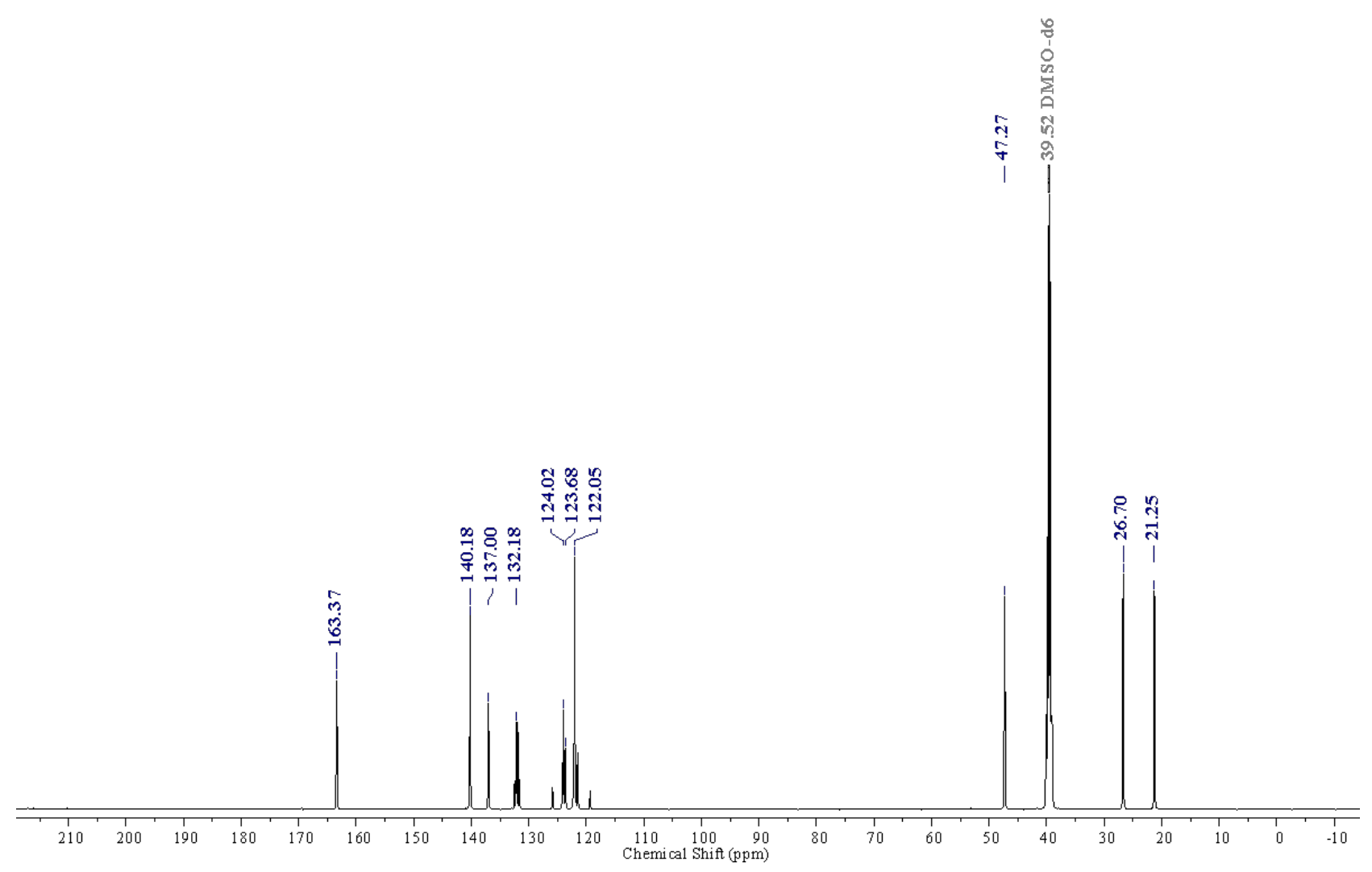



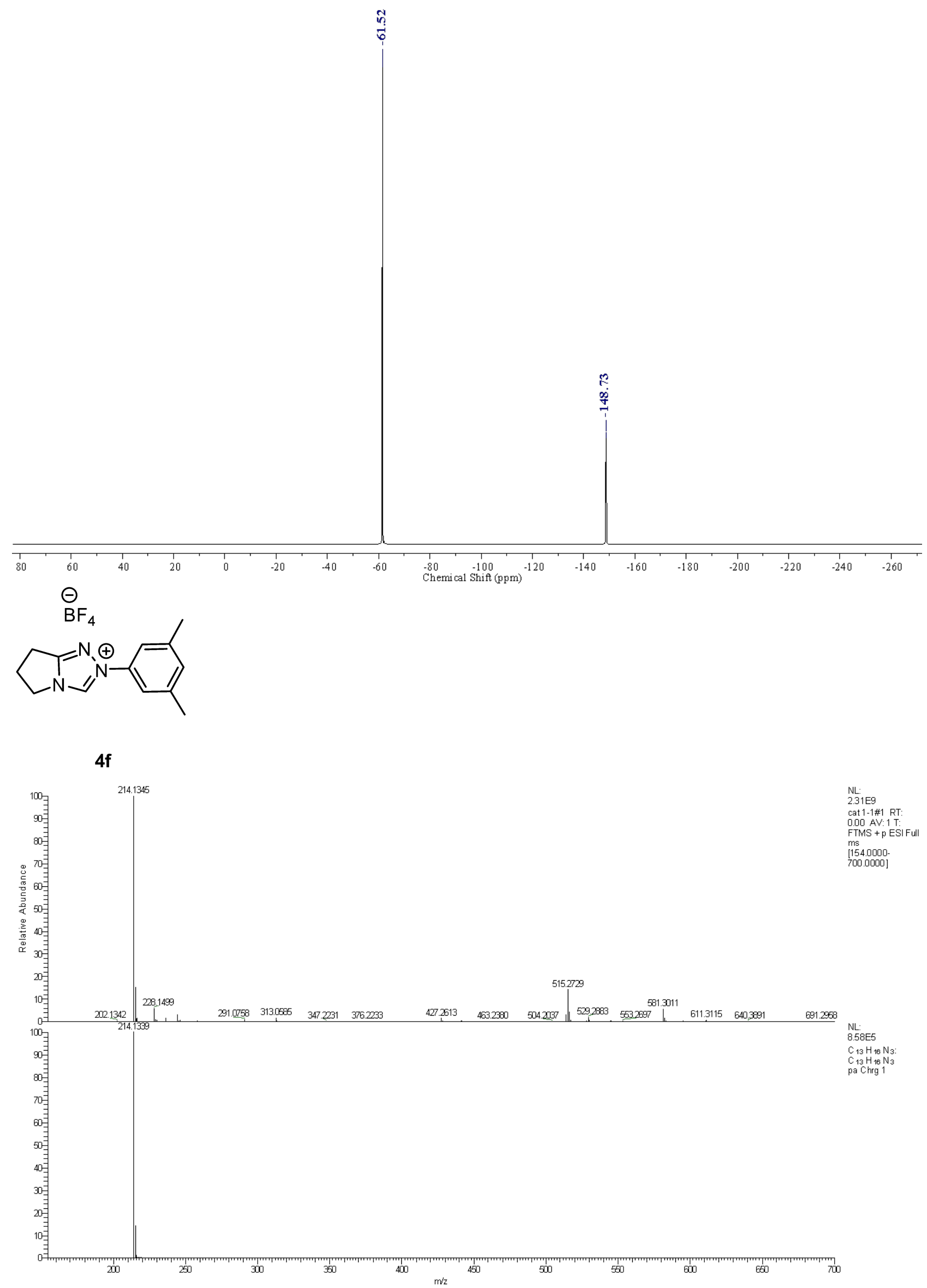


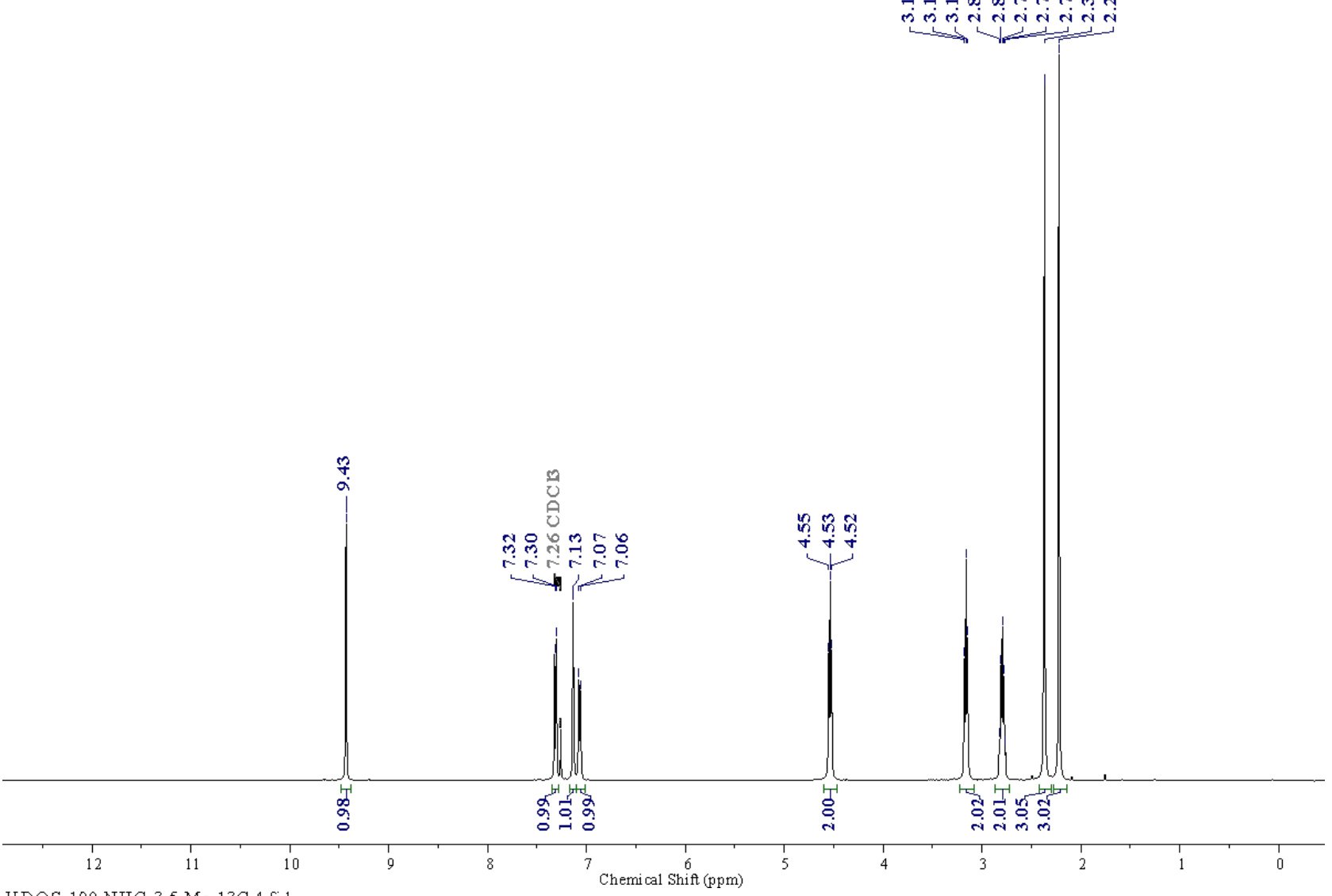

VDOS-190-NHC-3,5-Me 13C.4.fid

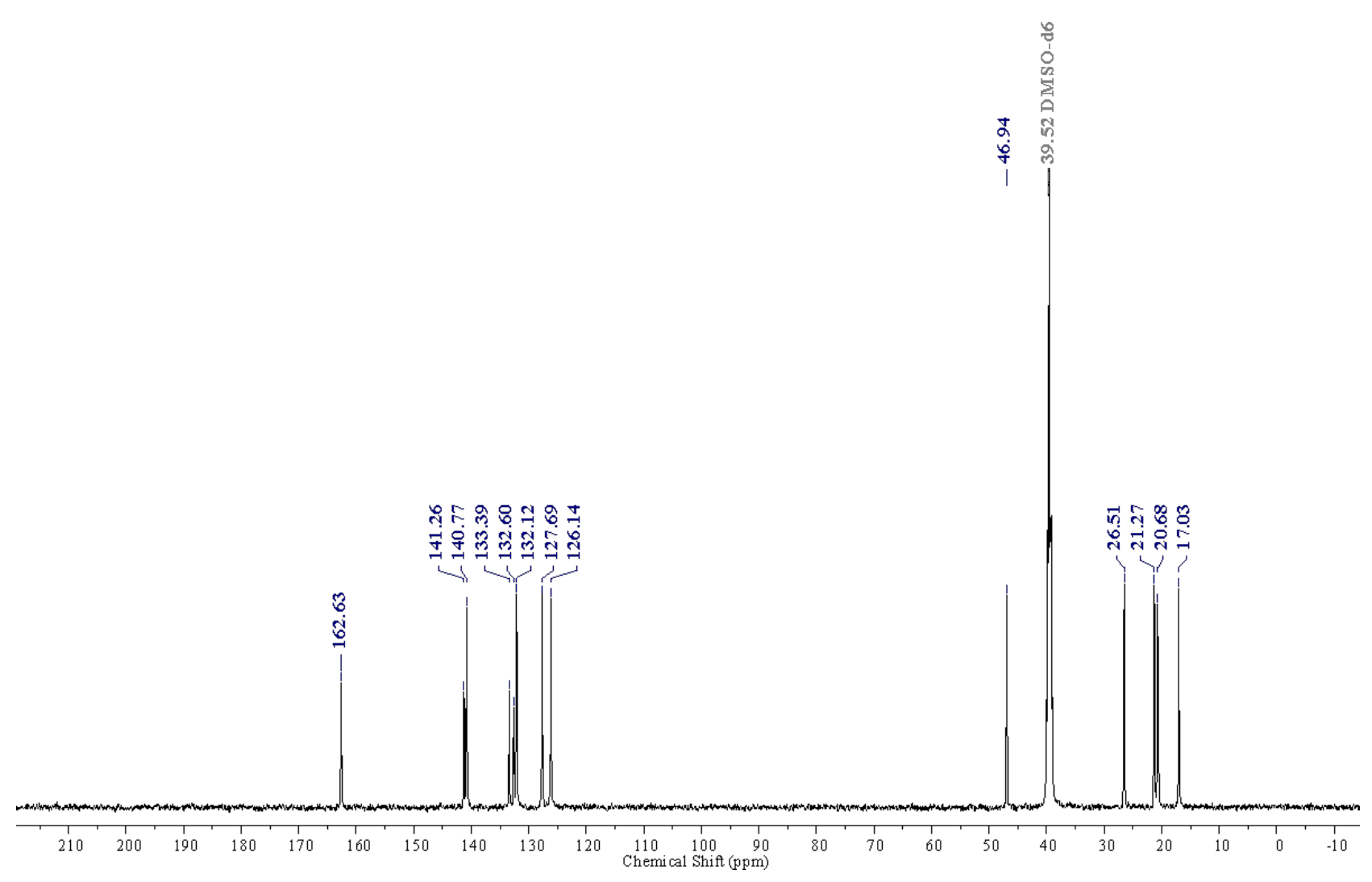




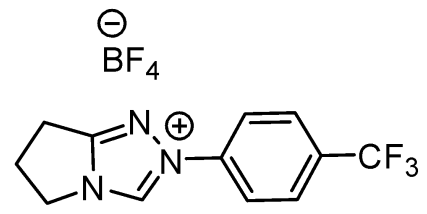

$4 g$

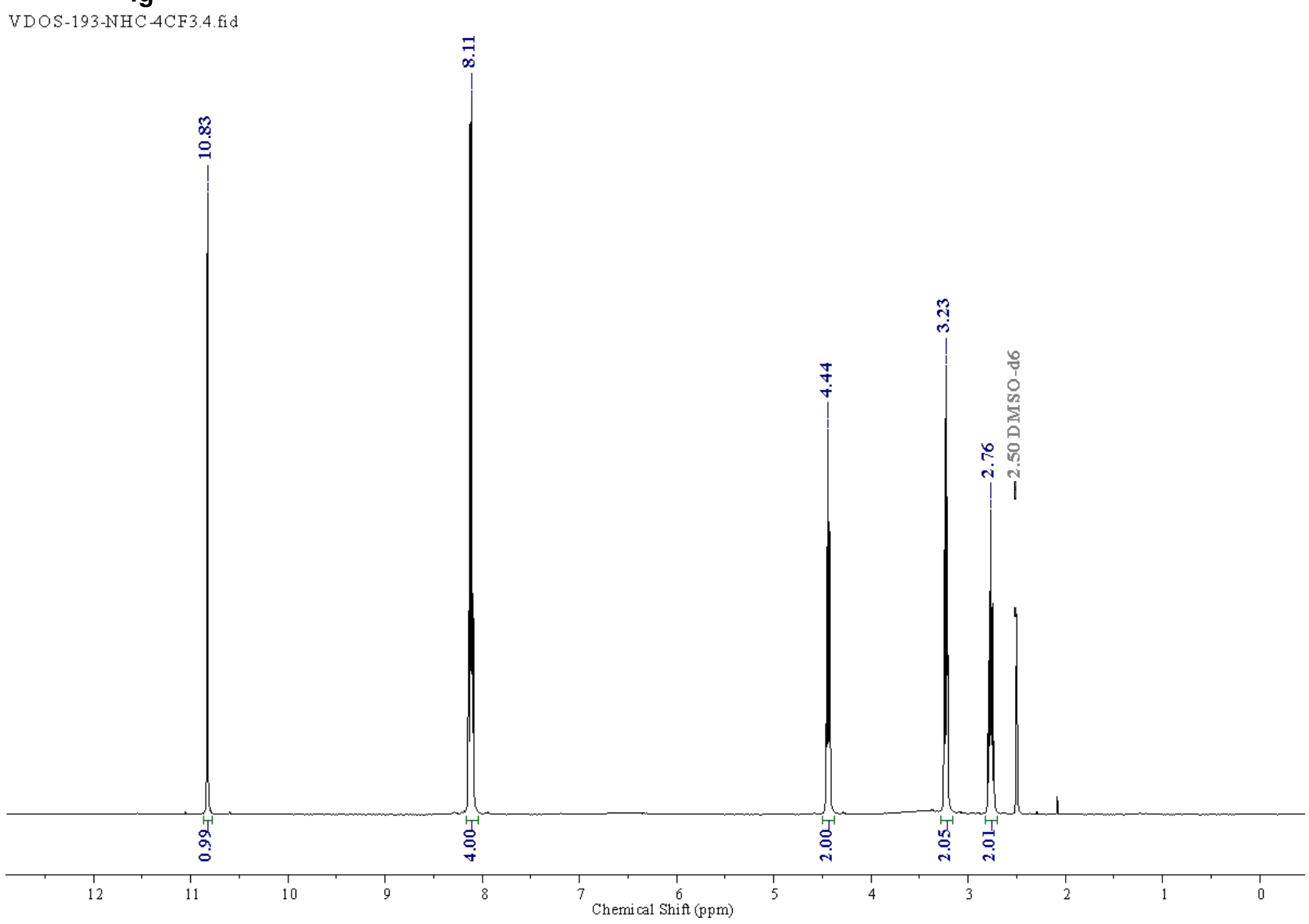

121 

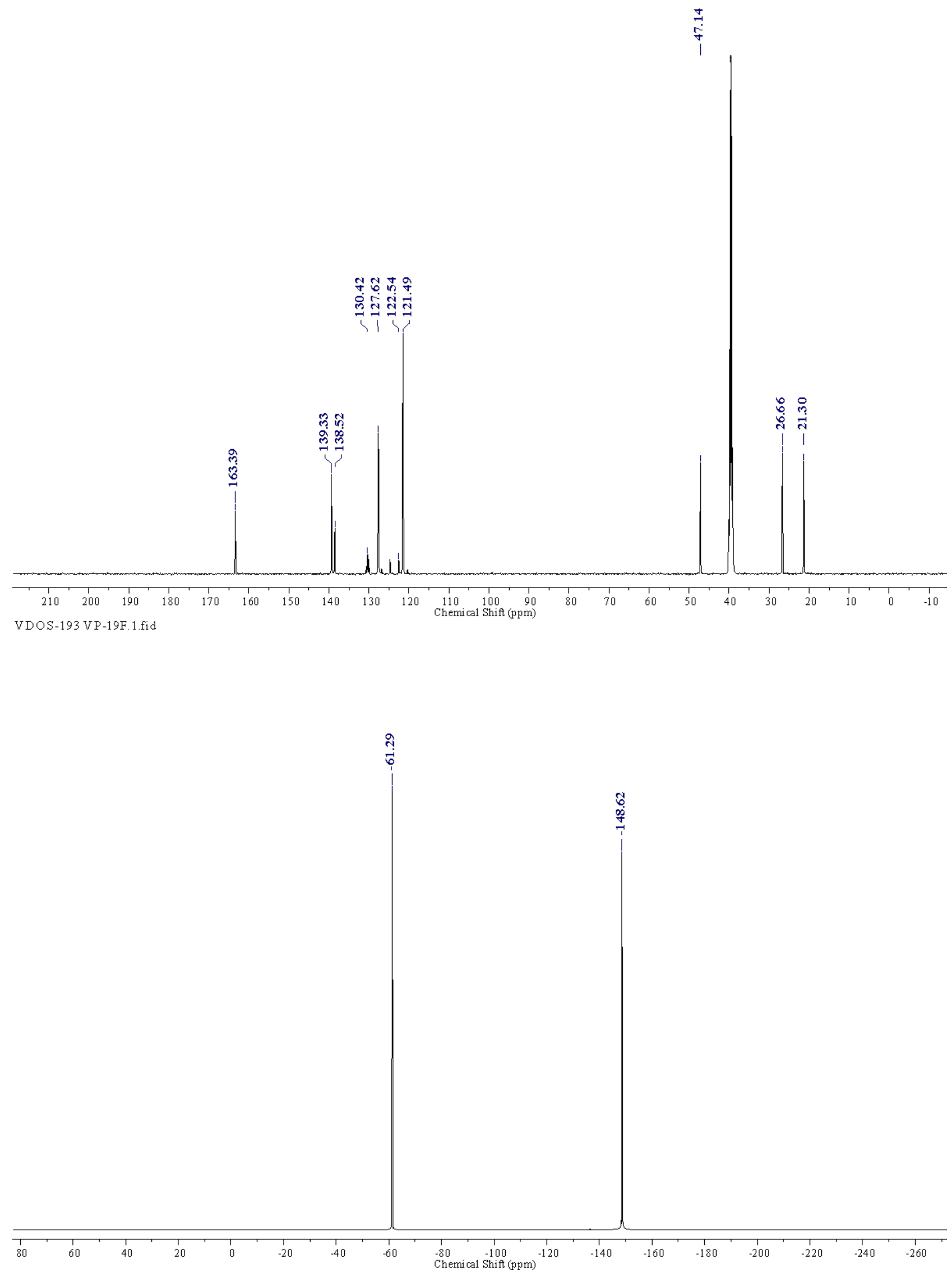


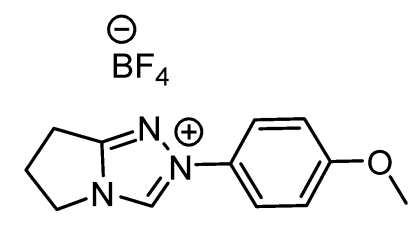

4h

VDOS-177-NHC $40 \mathrm{Me} 3 \mathrm{fid}$

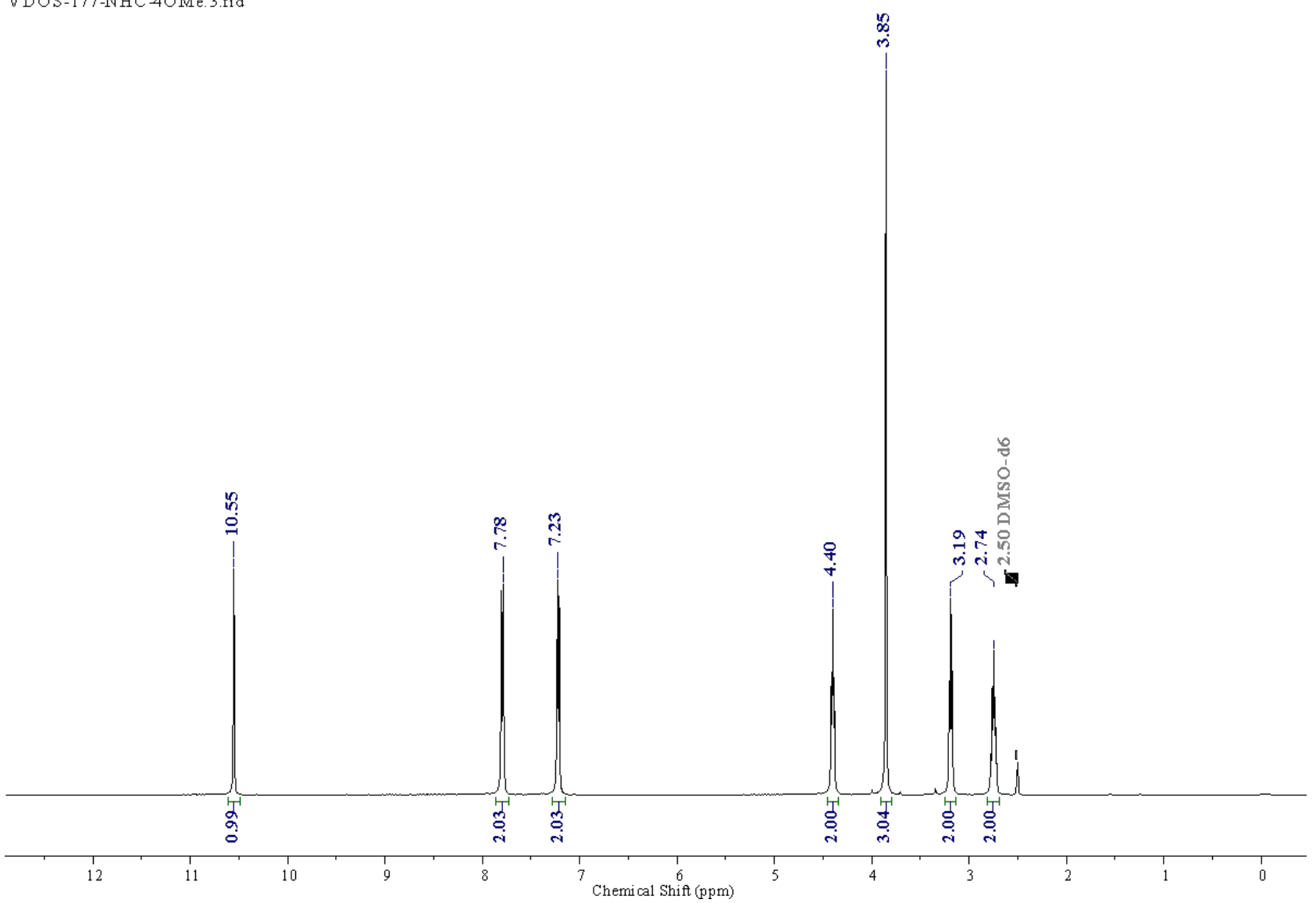




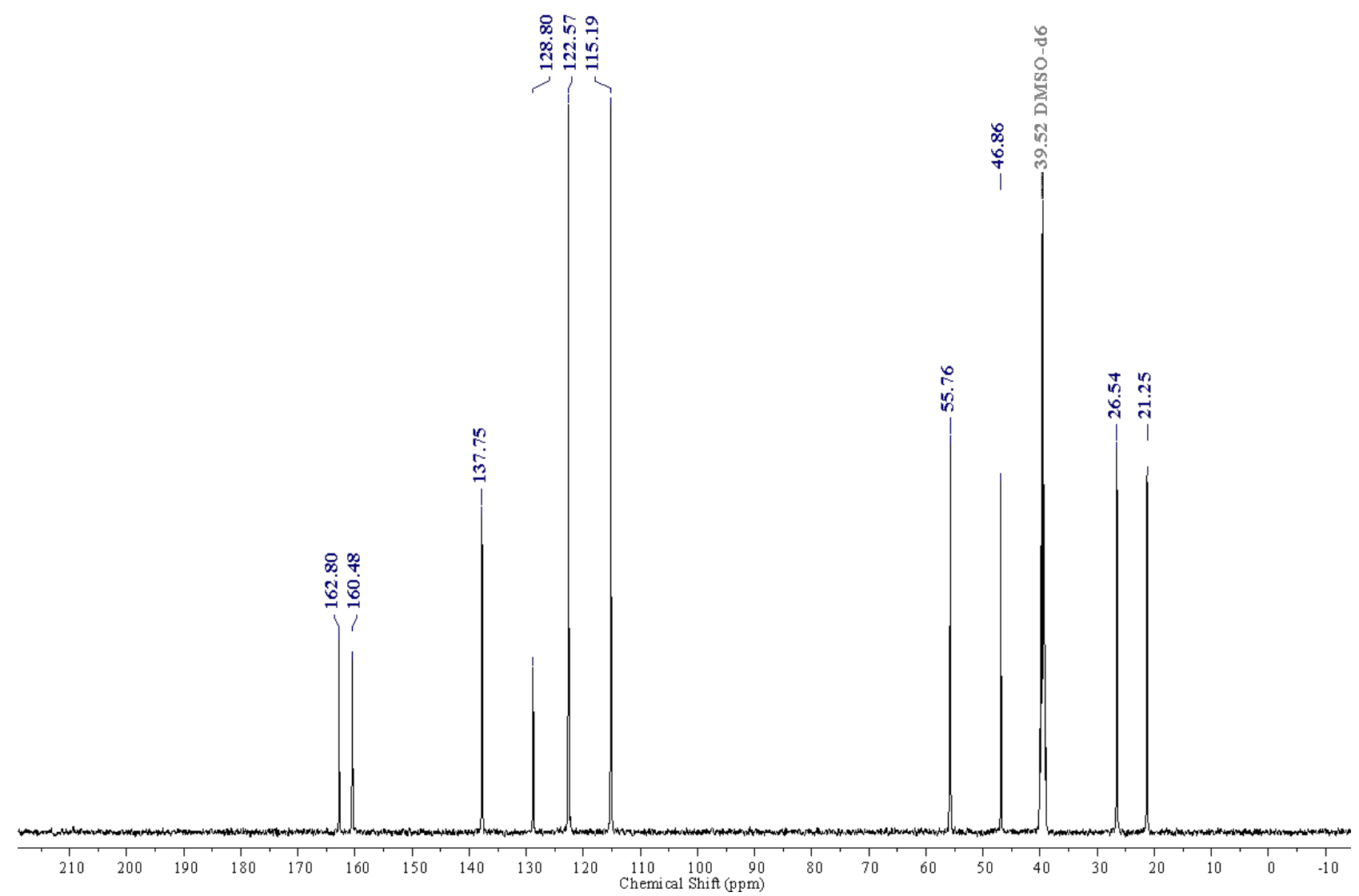




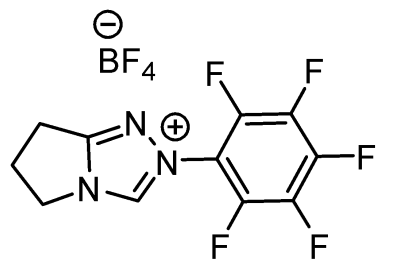

$4 \mathbf{i}$

VDOS-79VP-1H.1.fid

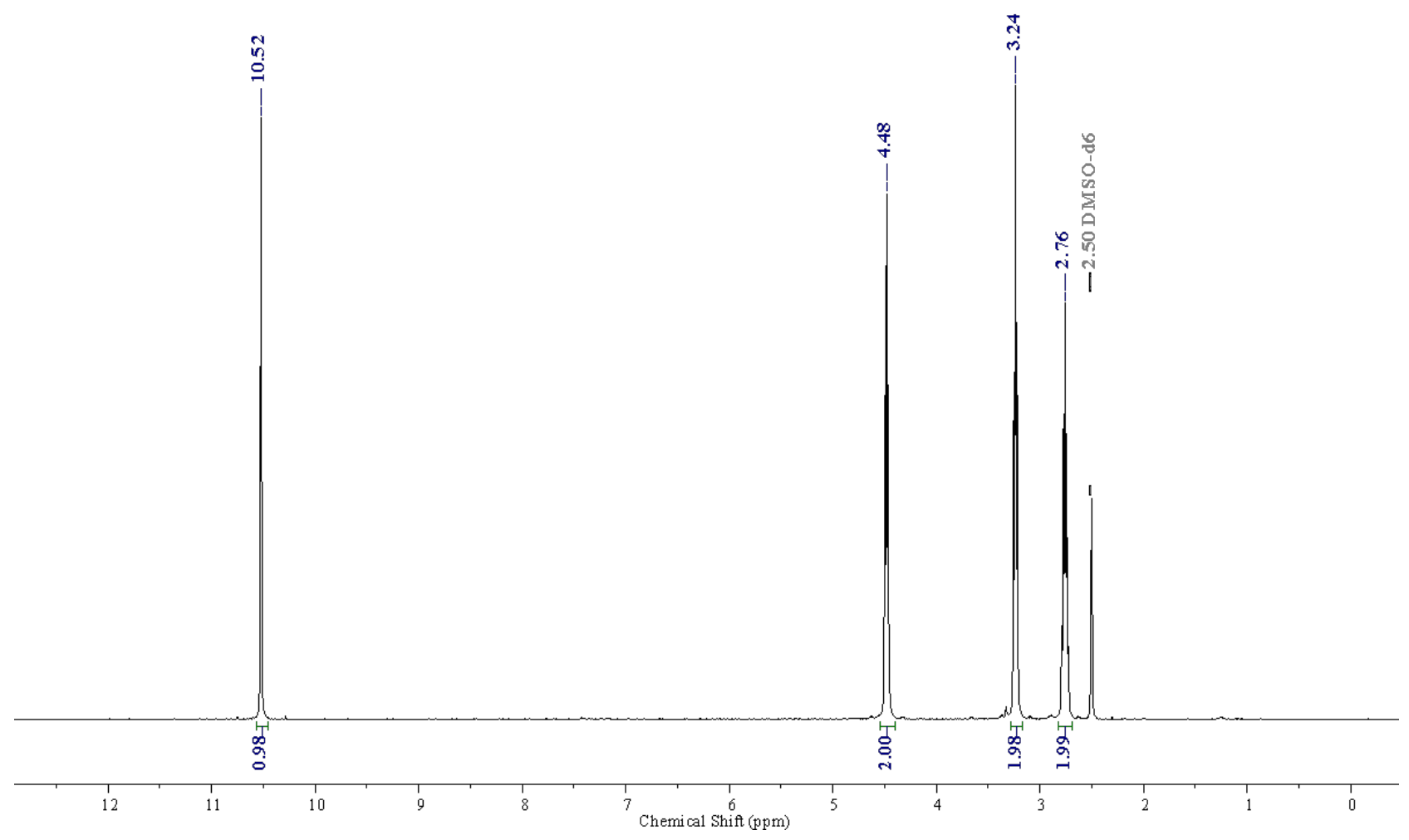



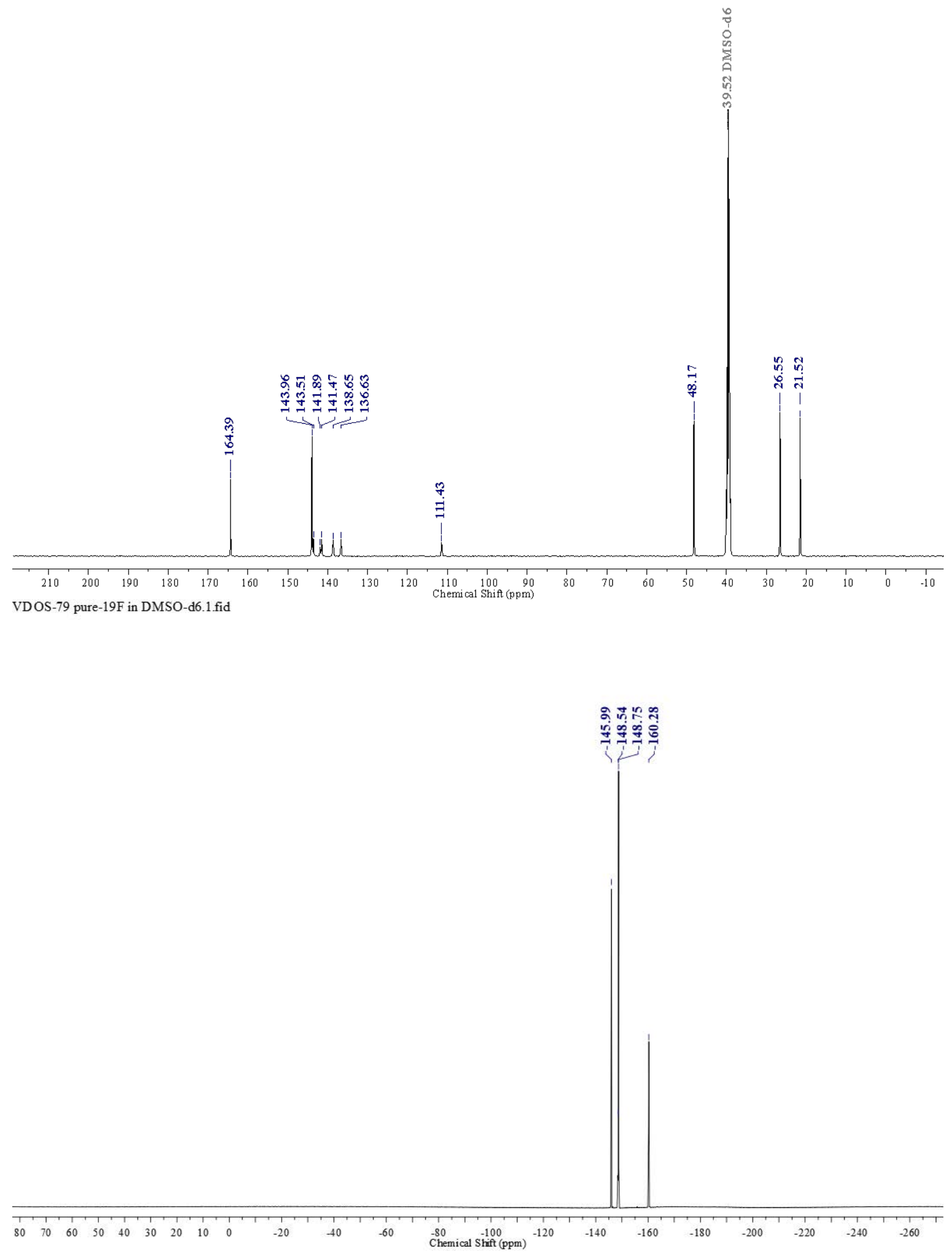


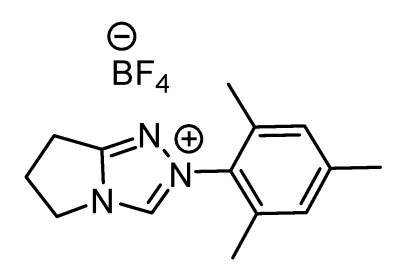

4j

VDOS-NH C-AC-Mesity1. 1. fid 


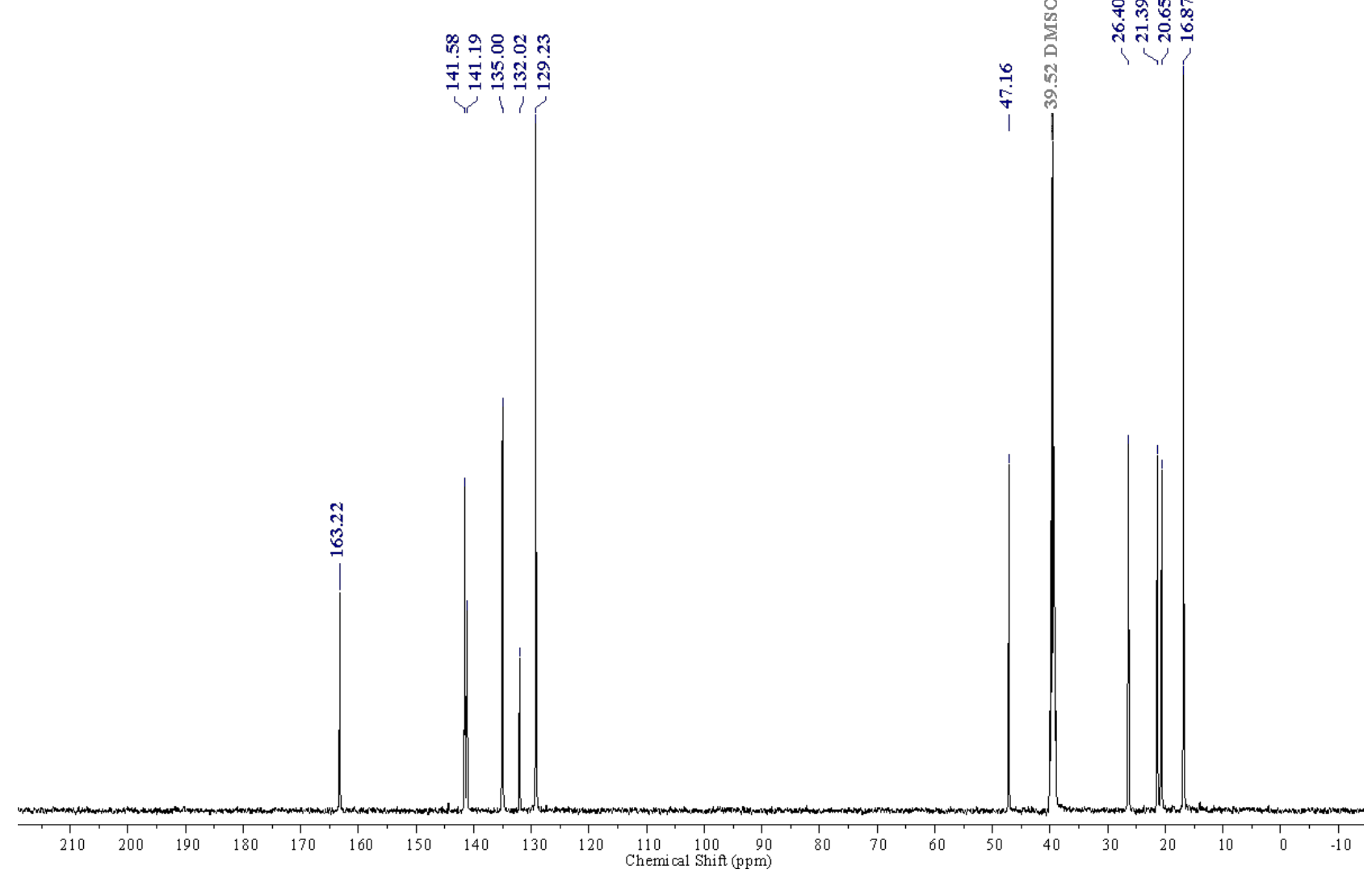


${\stackrel{\ominus}{B F_{4}}}$

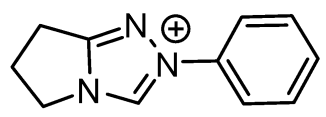

$4 \mathbf{k}$

VDOS-NHC-AC-Ph.2.fid

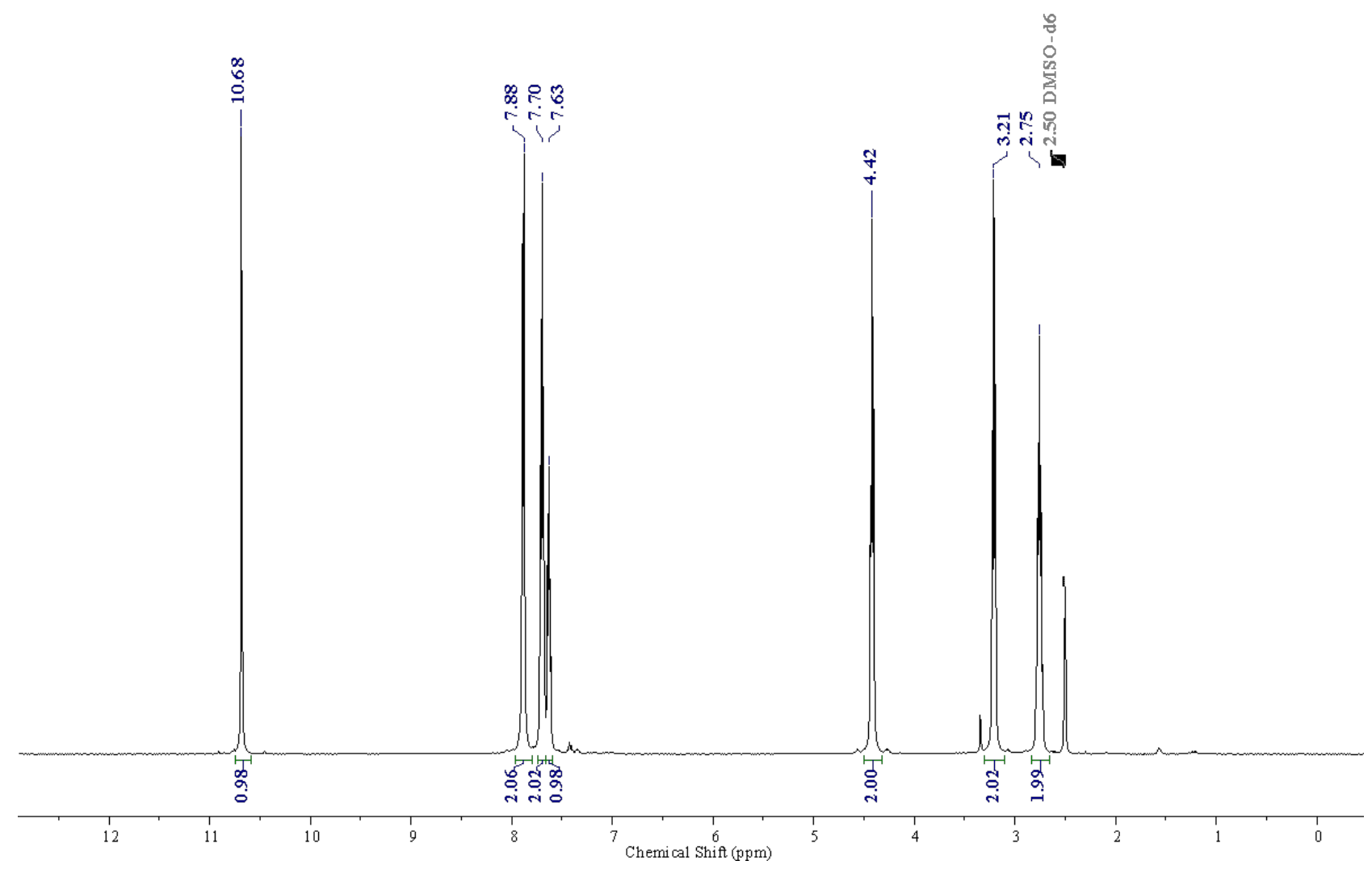



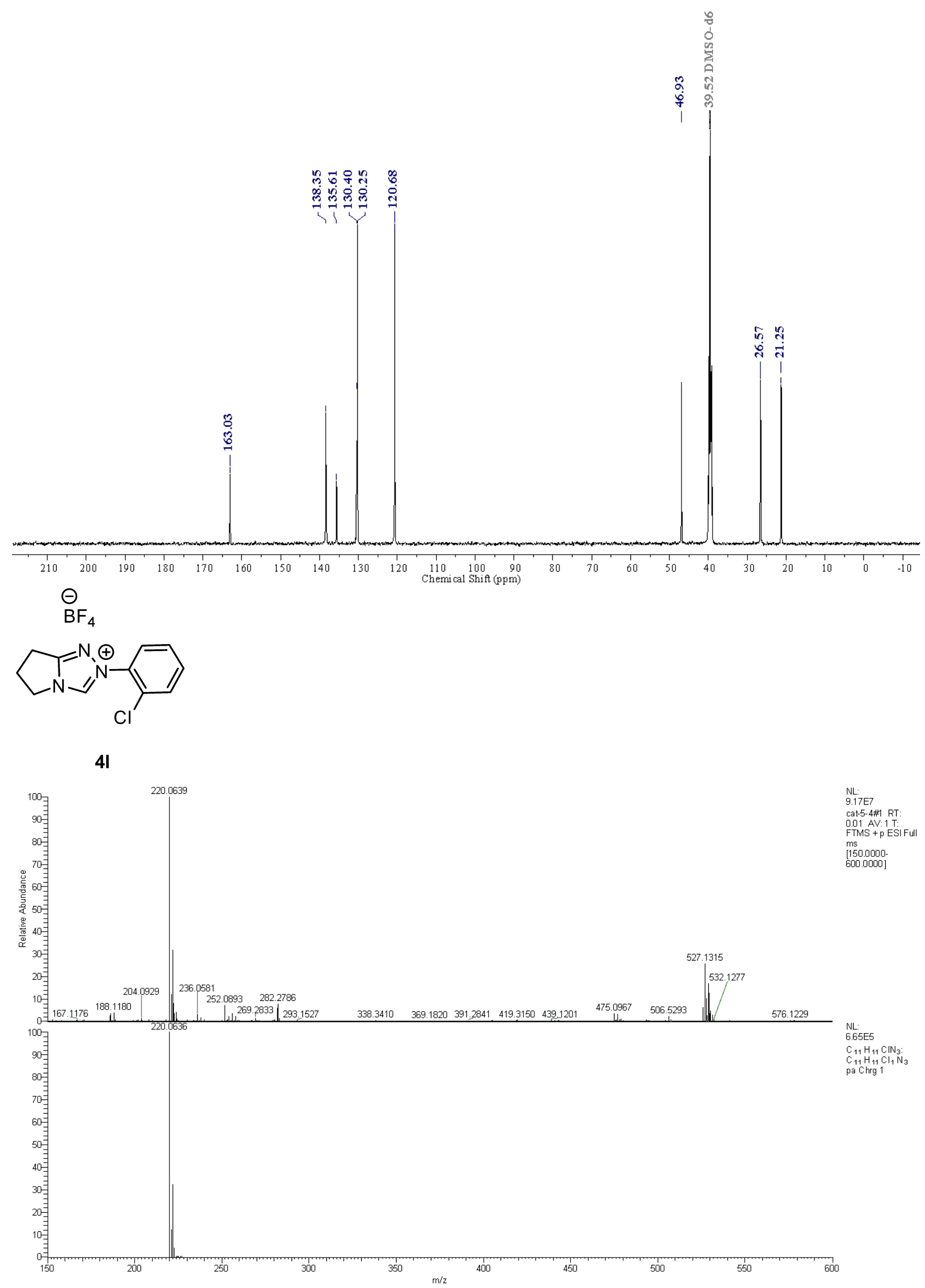

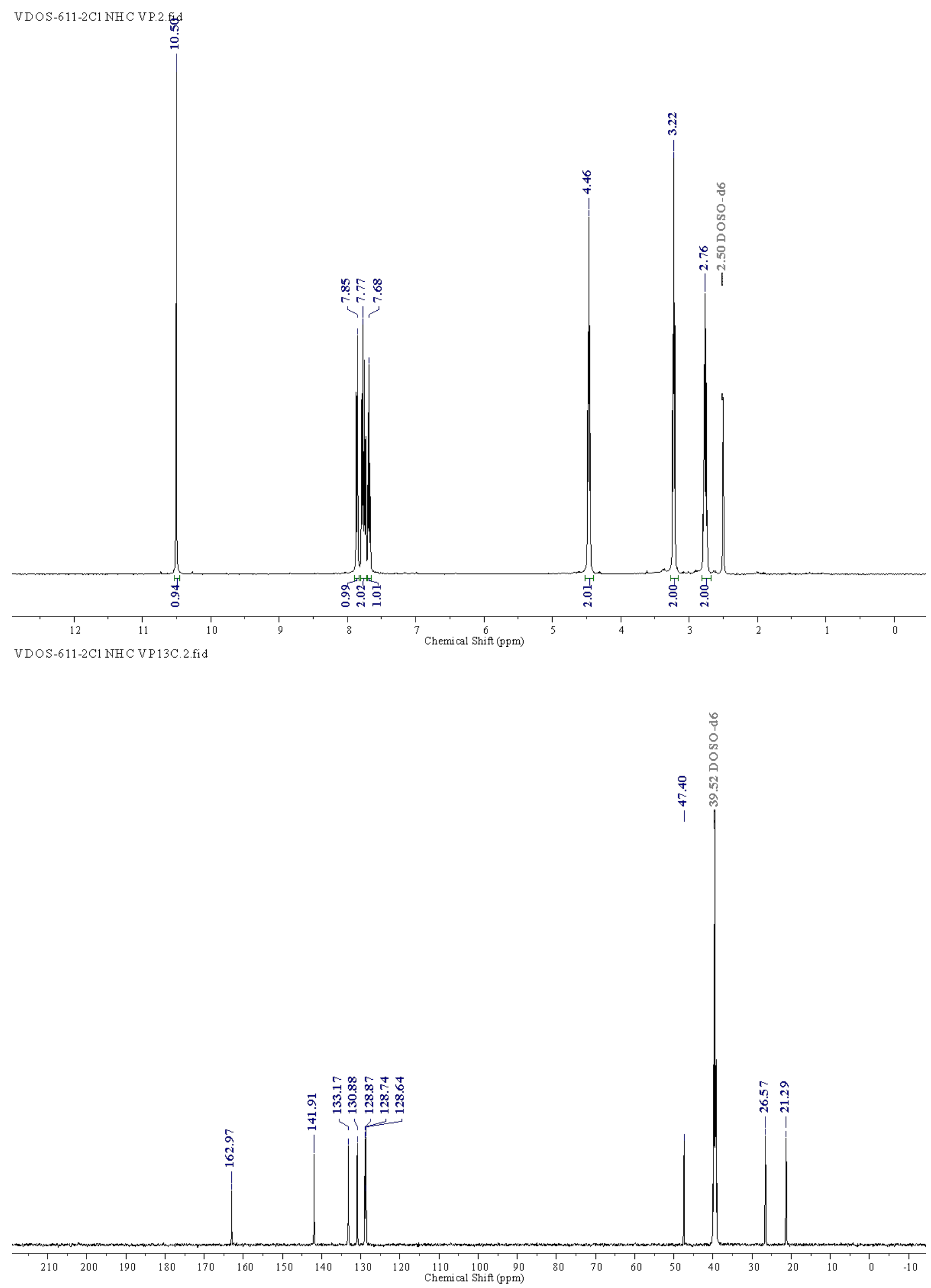

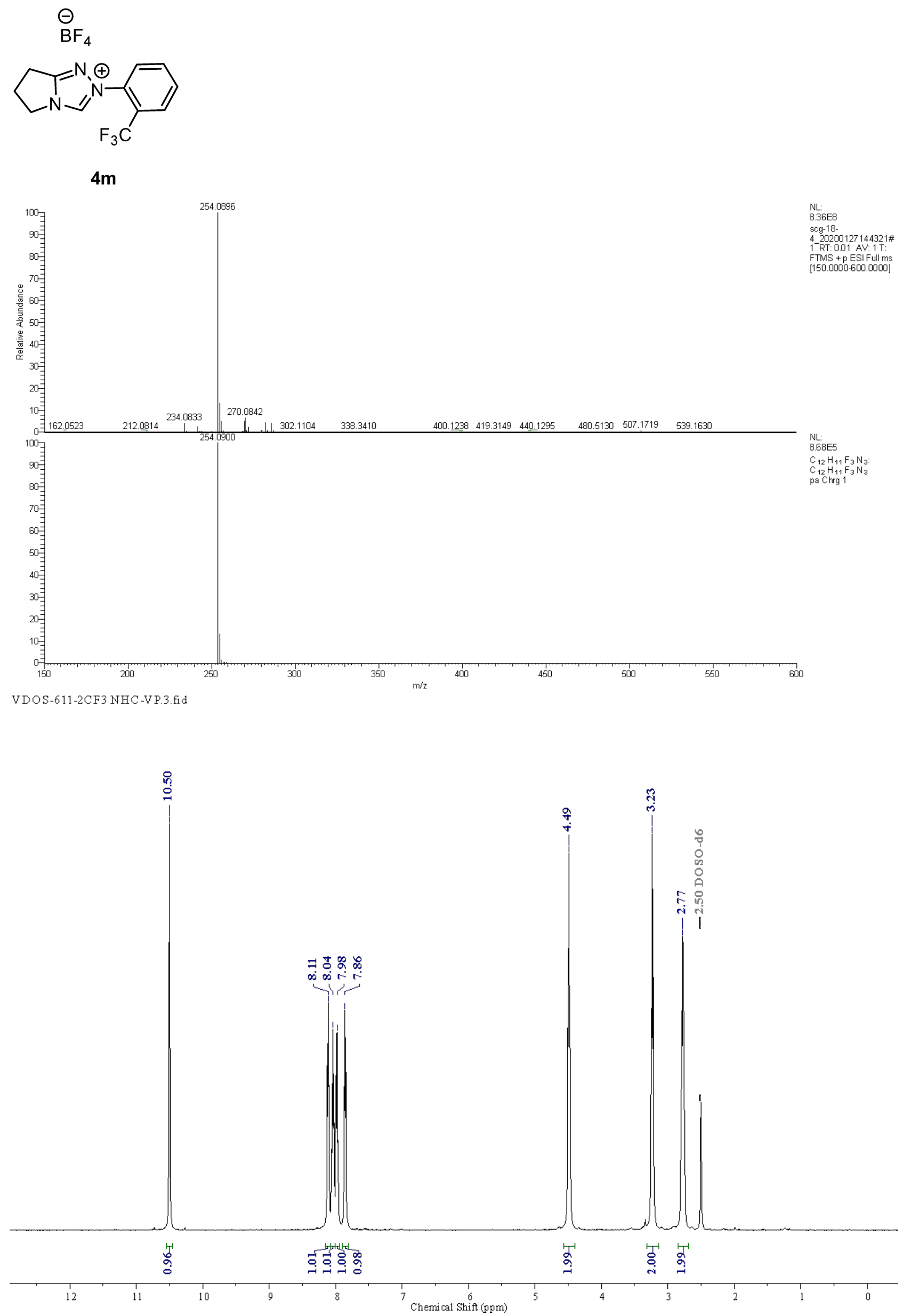


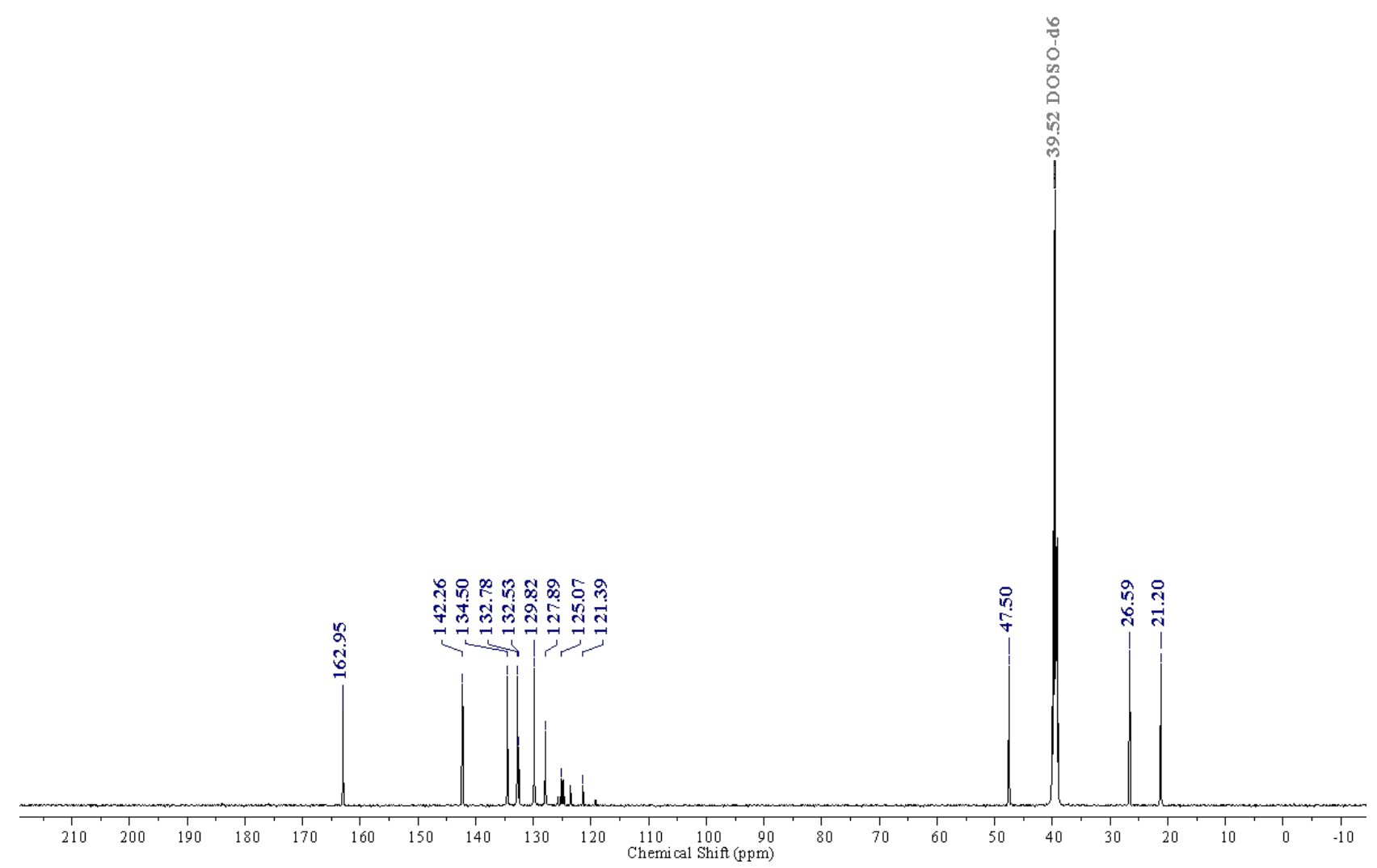

VDOS-611-2CF3 VP-19F. 1.fid

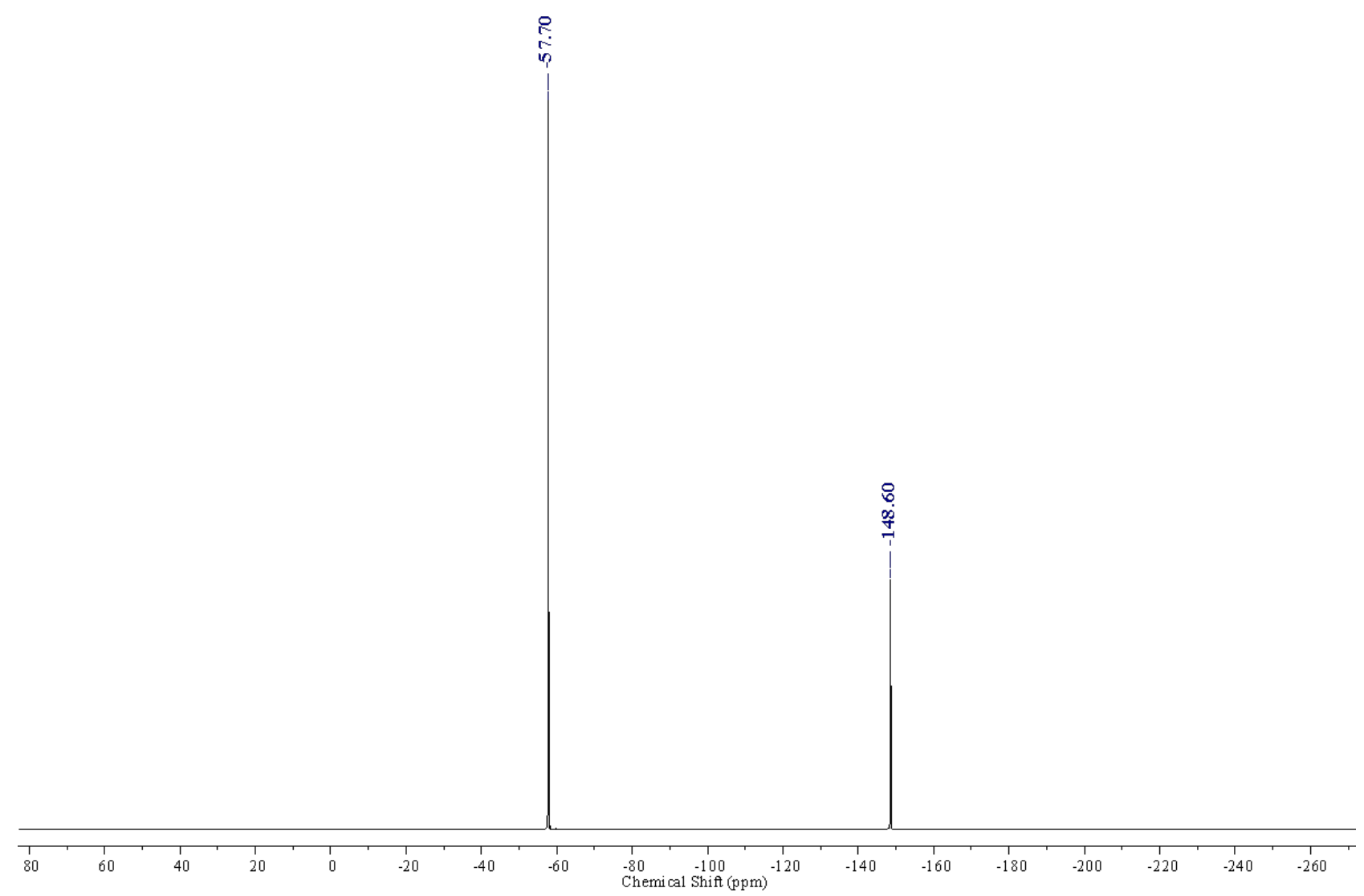


$\Theta$

$\mathrm{BF}_{4}$

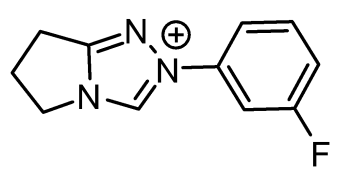

$4 n$

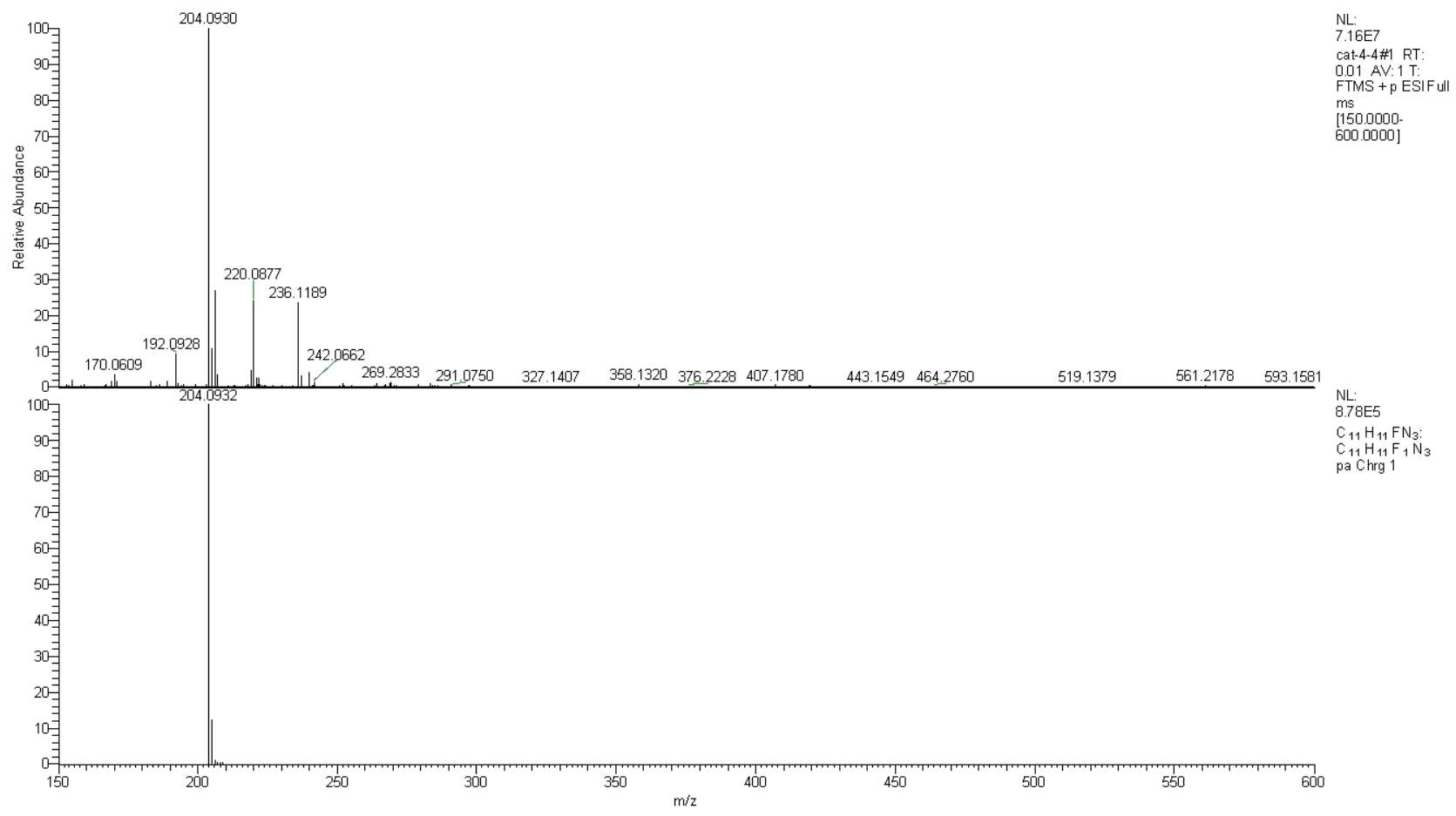




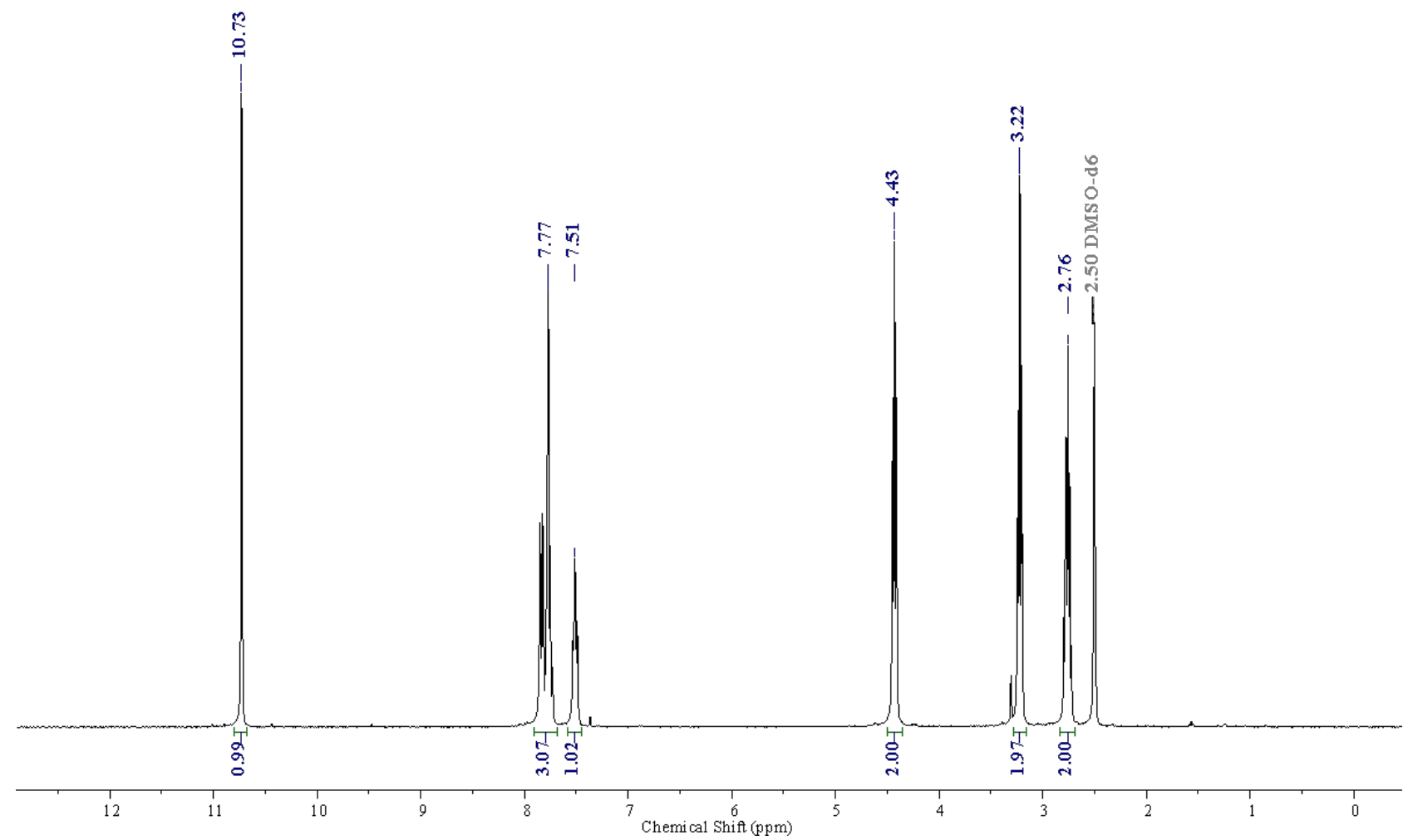

VDOS-611-C pure-13C.8.fid

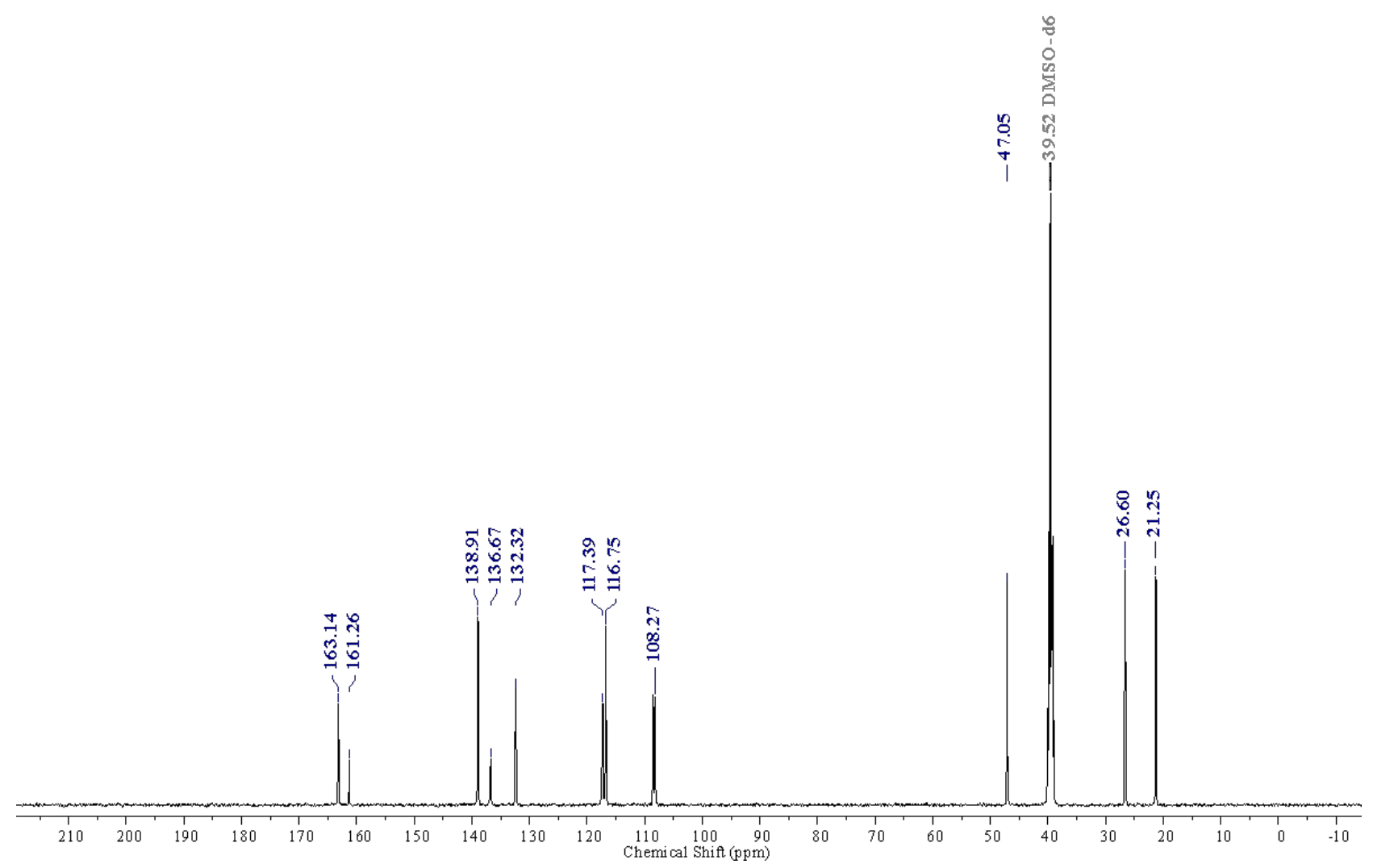




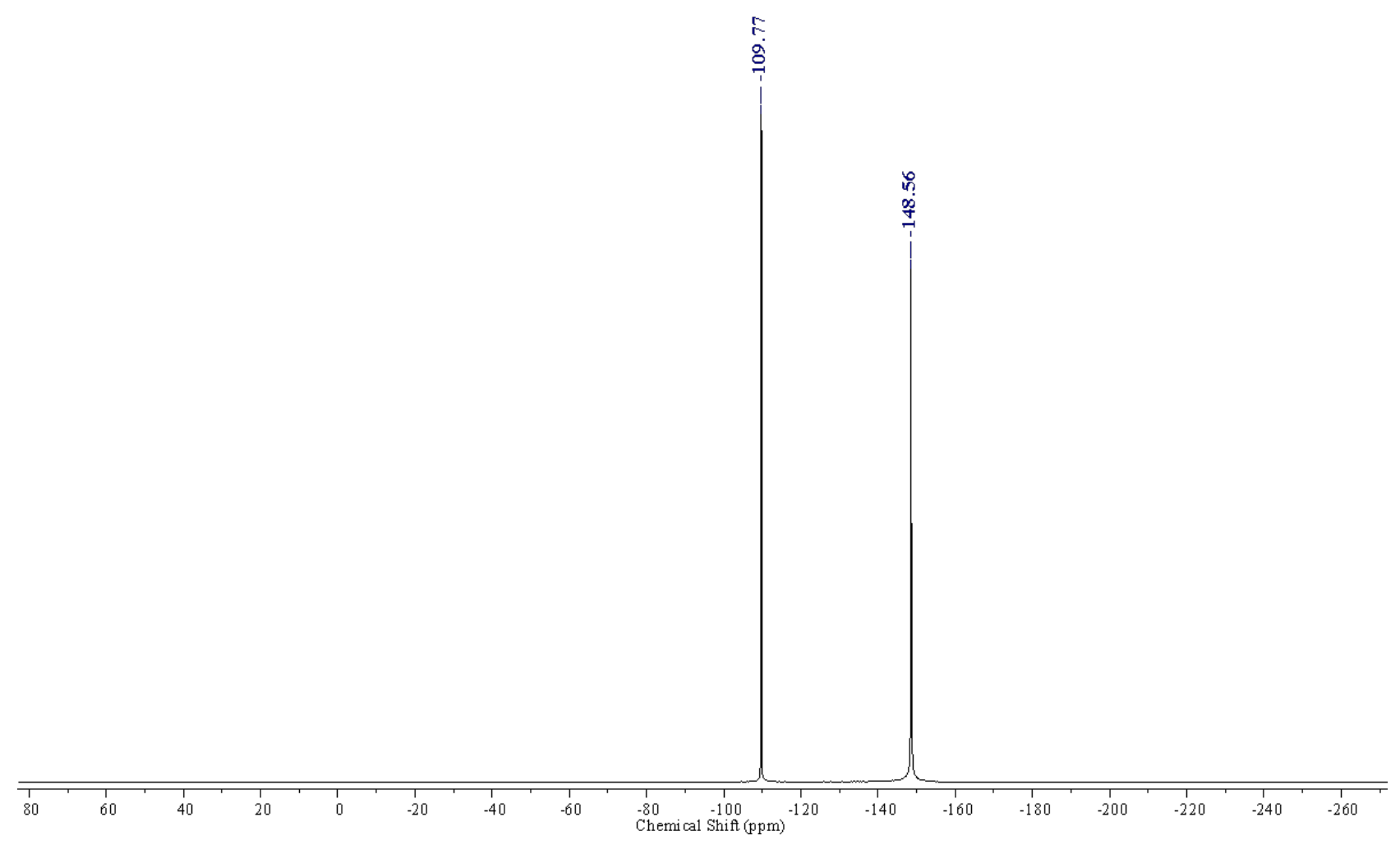


Synthesis of 2-Substituted Benzaldehyde Derivatives:

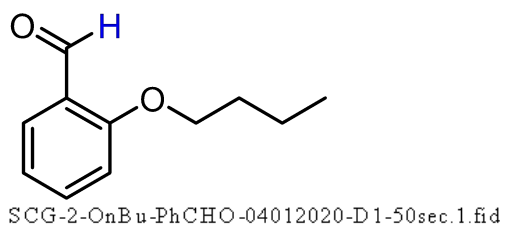

SCG-2-OnBu-PhCHO-04012020-D1-50sec 1 fid

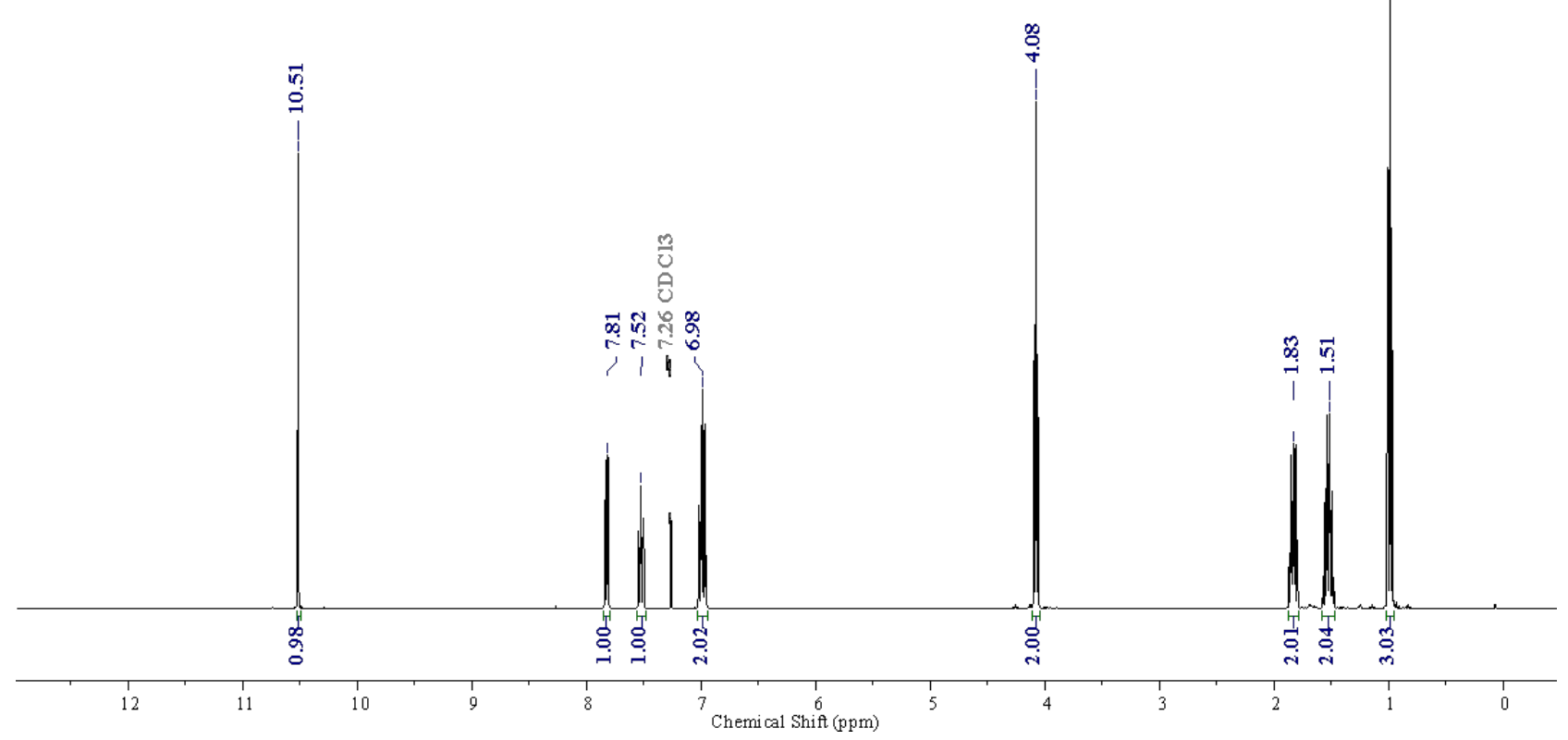




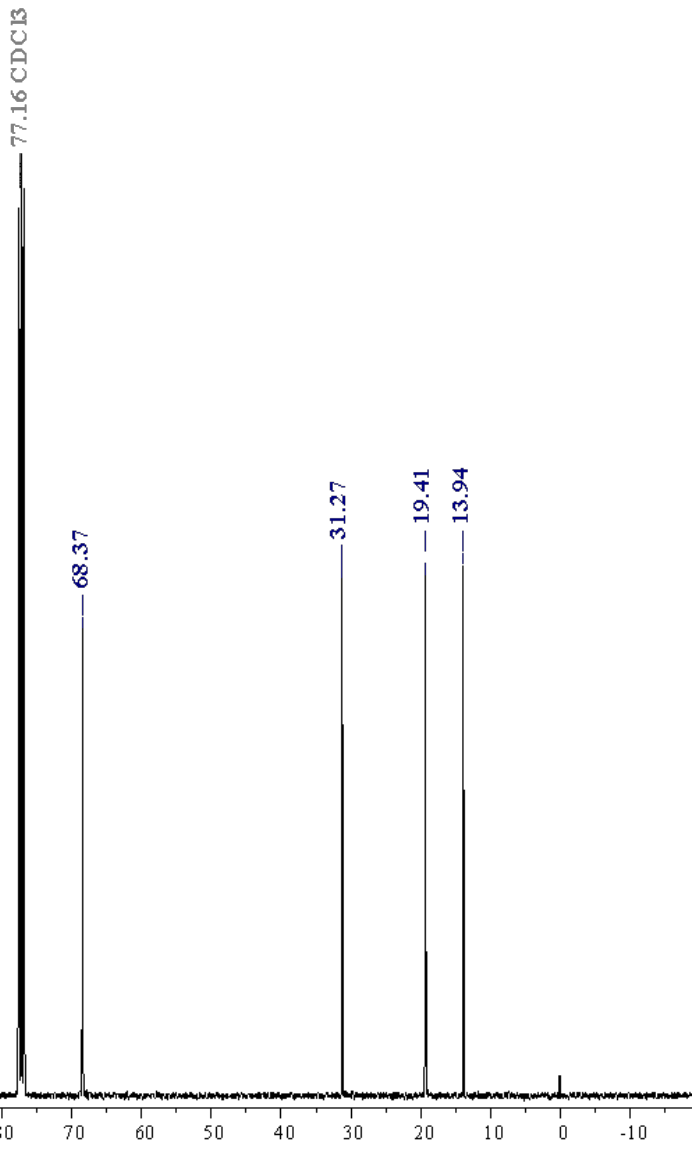



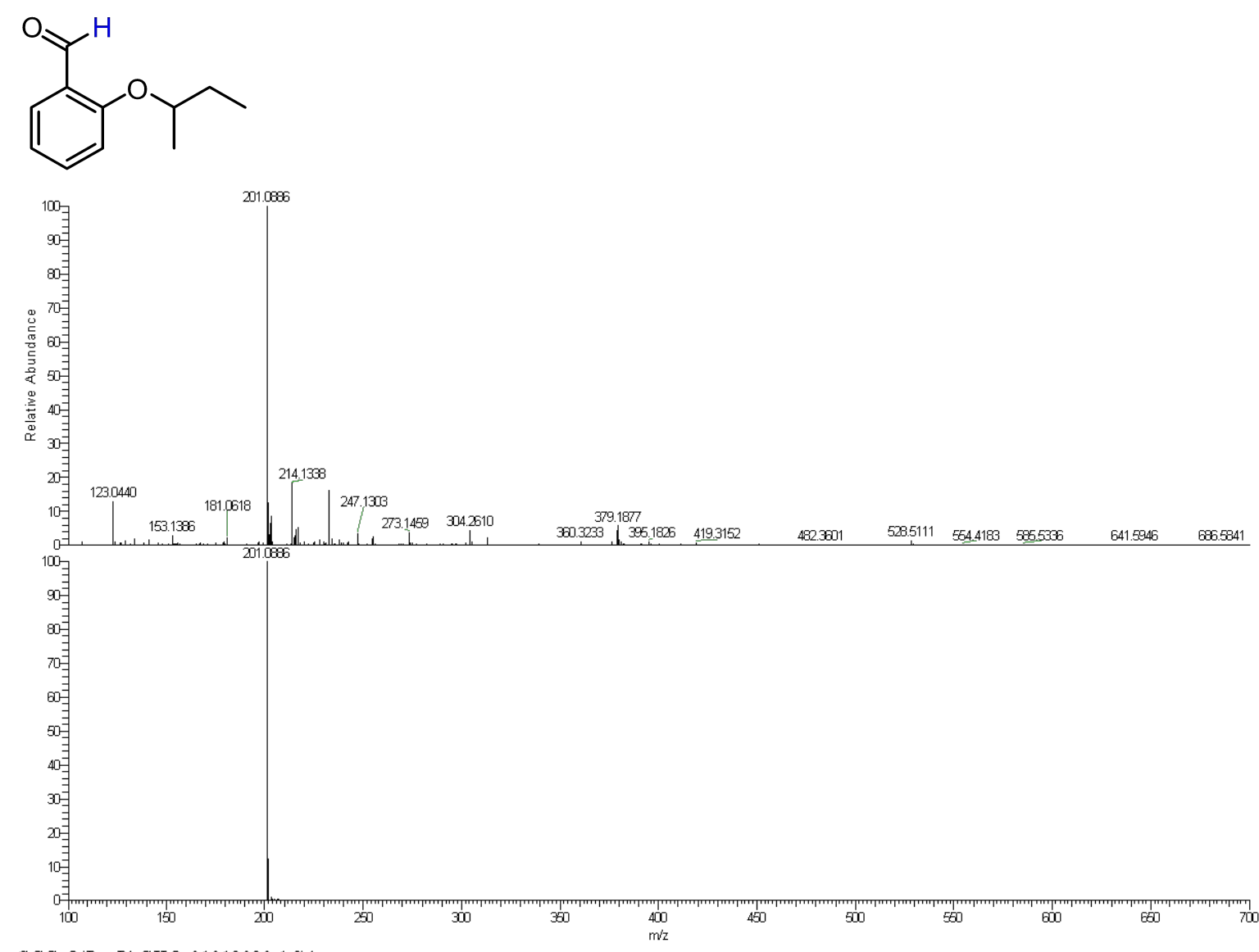

\subsection{8}

OBU-3-4 A RT: FTMS + $p$ ESI Full ms

SCG-OiBu-PhCHO-01012020.1 fid

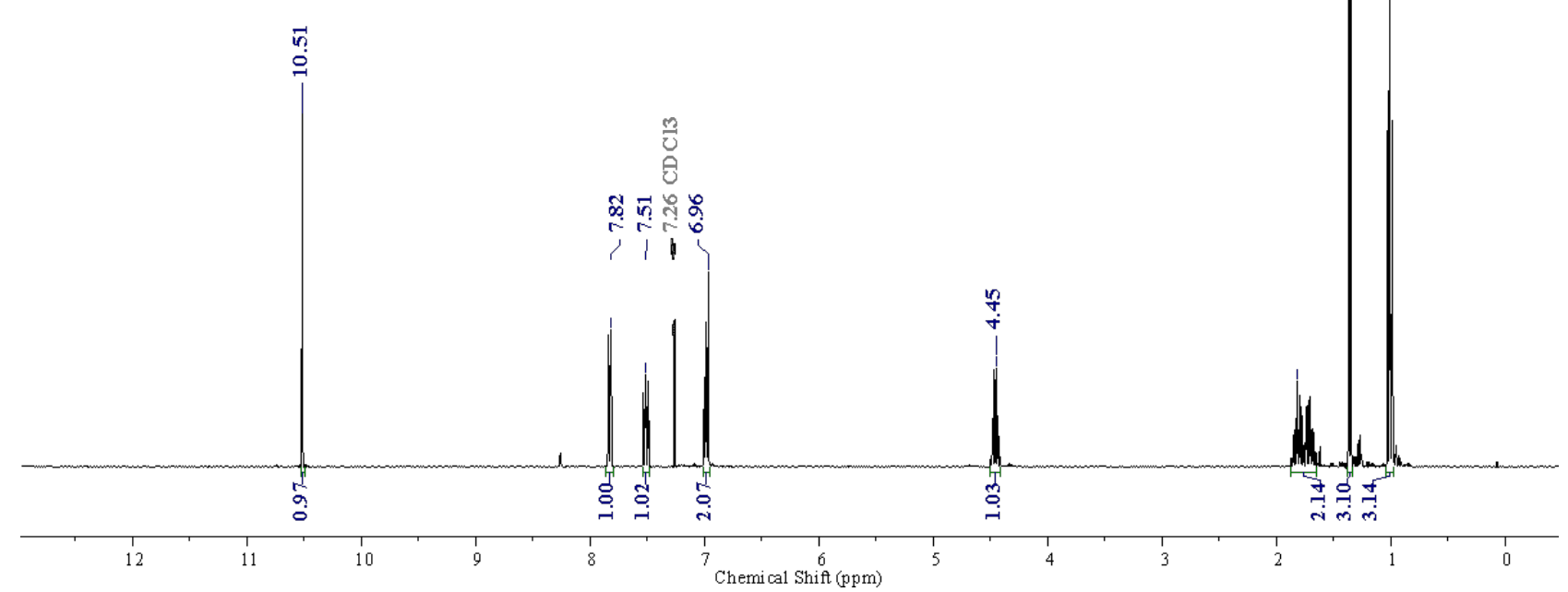




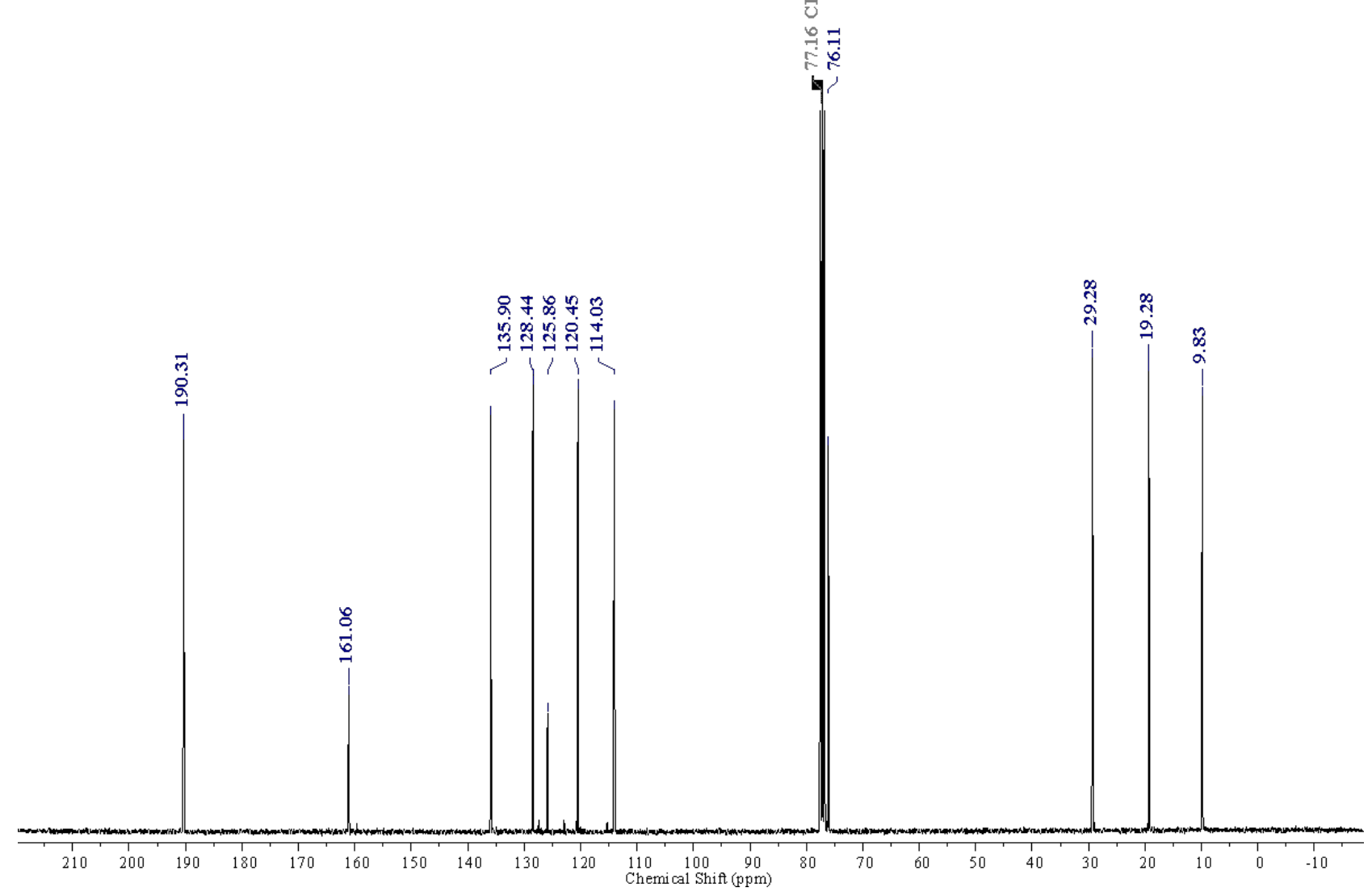




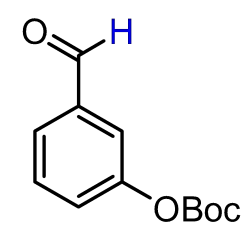

VDOS-695-SM Boc CD3CN

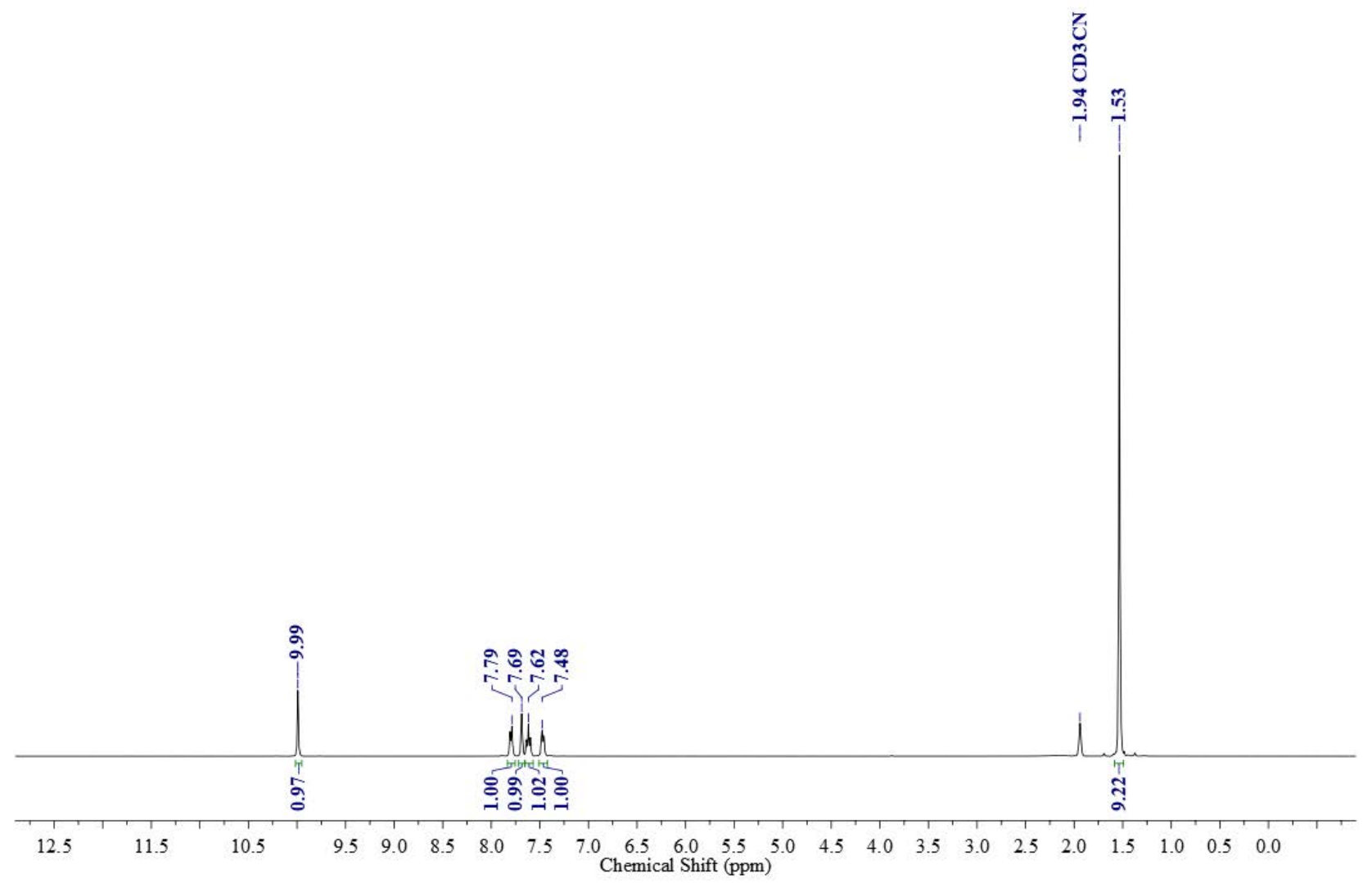


VDOS-695-SM Boc CD3CN-13CNMR

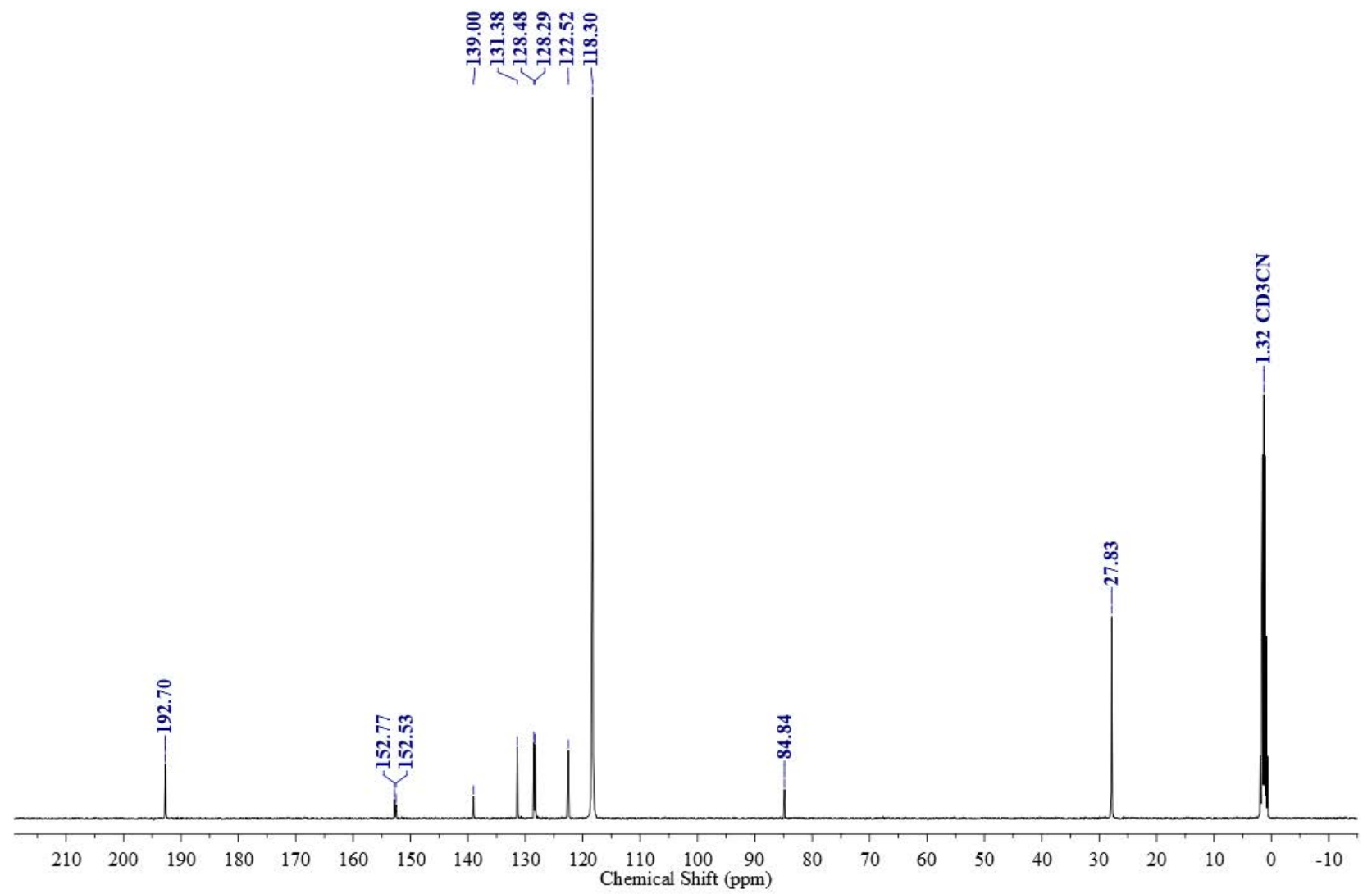


Synthesis of Aryl Boroxines:

Phenyl Boroxine:<smiles>c1ccc(B2OB(c3ccccc3)OB(c3ccccc3)O2)cc1</smiles>

VDOS-Ph-B oroxin VP 1H.4. fid

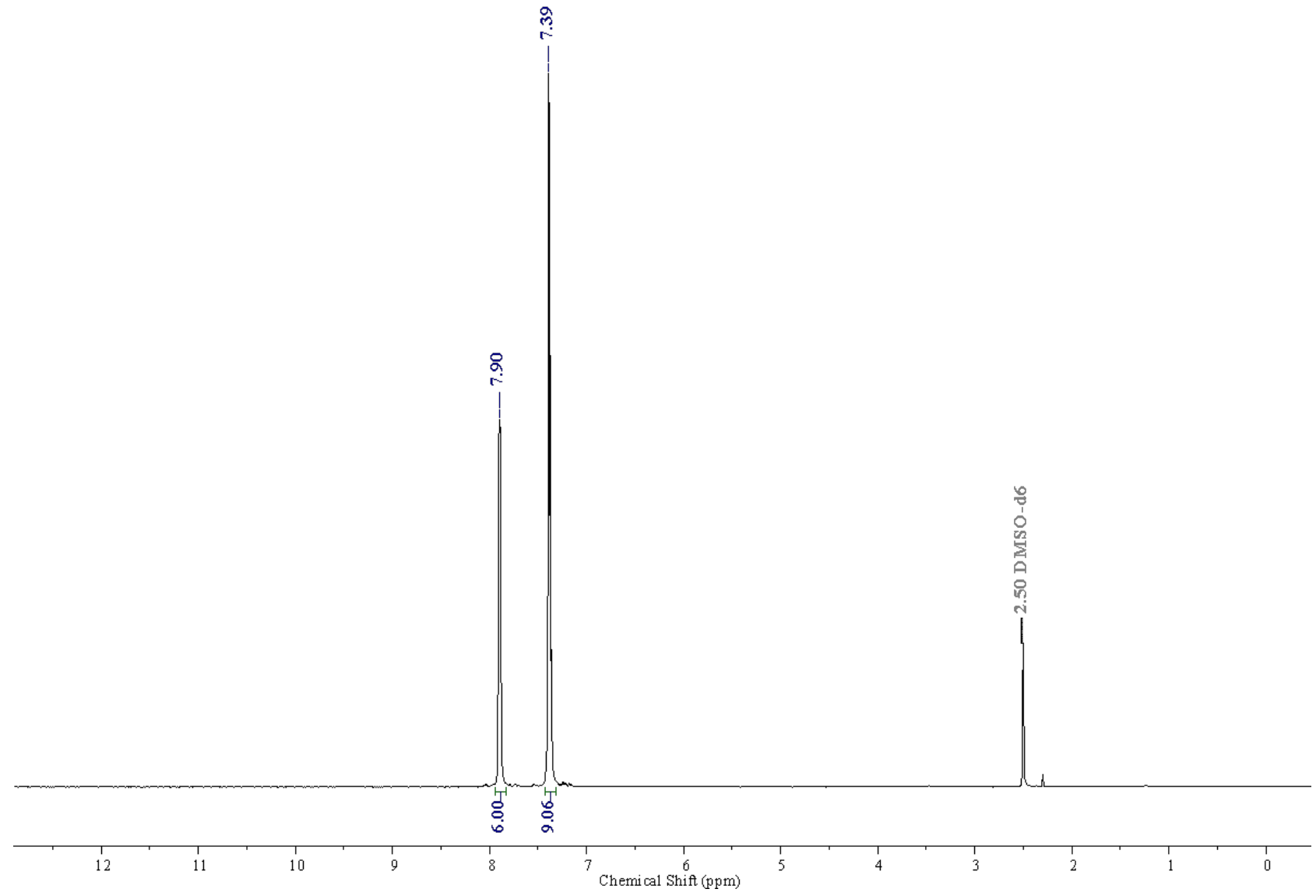




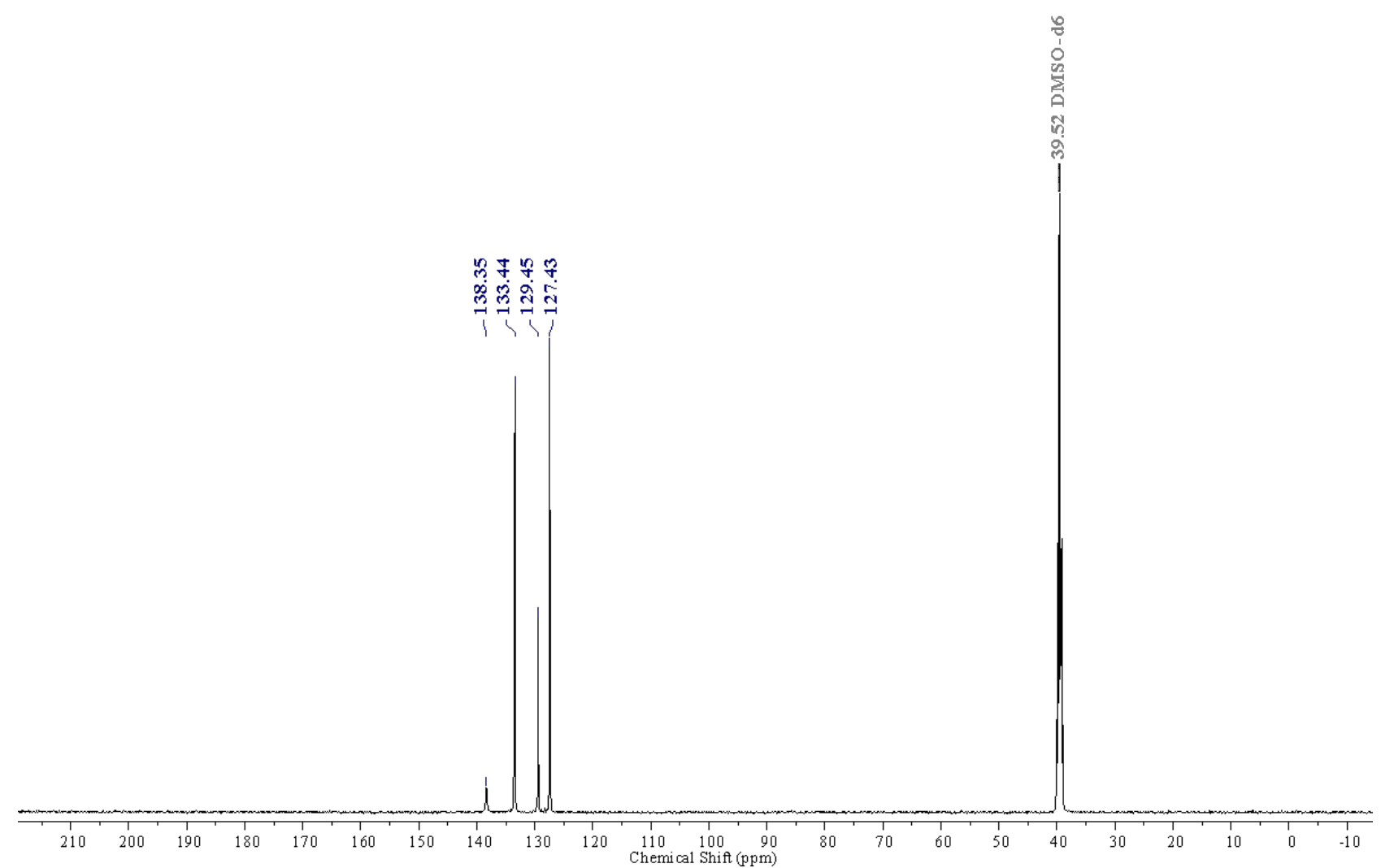


4-Methylphenyl Boroxine:<smiles>Cc1ccc(B2OB(c3ccc(C)cc3)OB(c3ccc(C)cc3)O2)cc1</smiles>

VDOS-4Me Ph B oraxin VP-2.5 fid

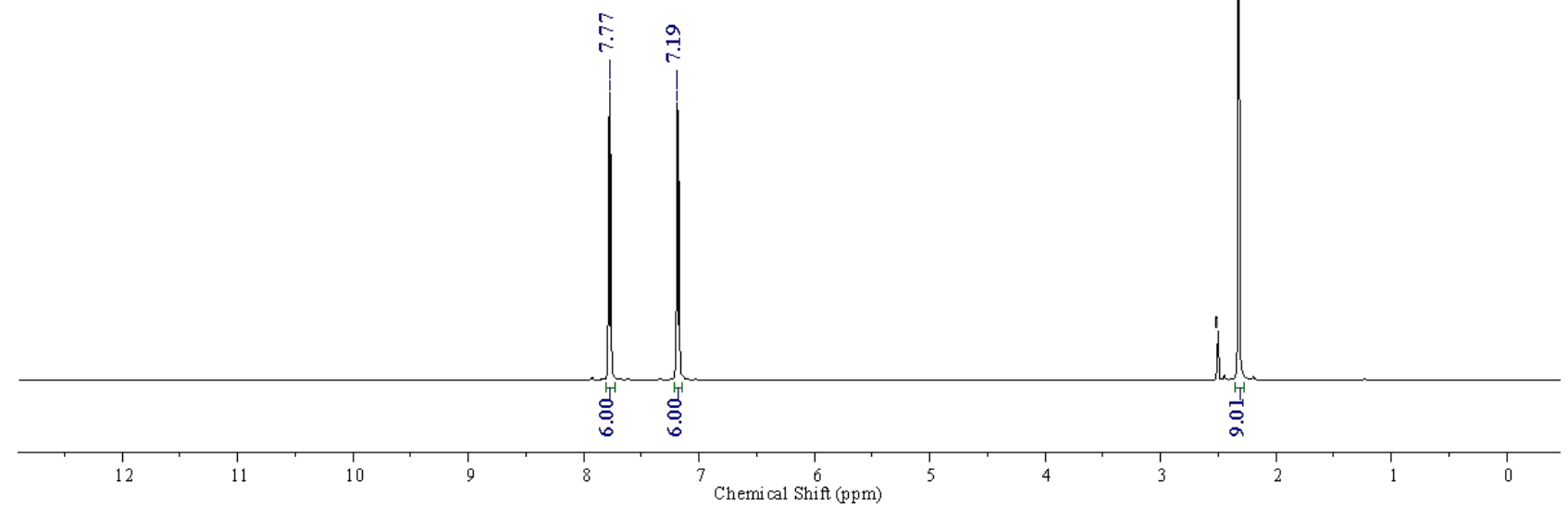




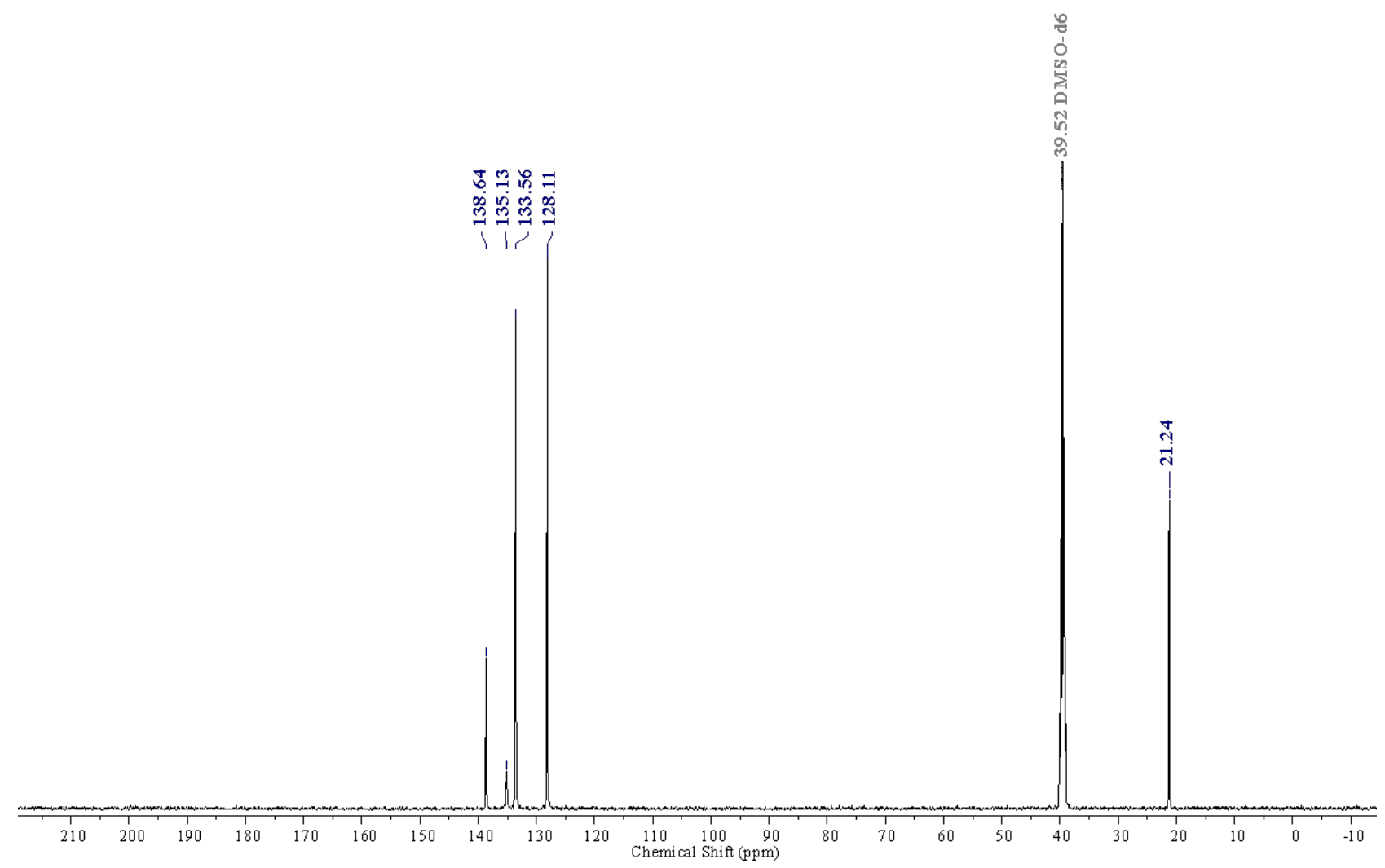


4-(Trifluoromethyl)phenyl Boroxine:

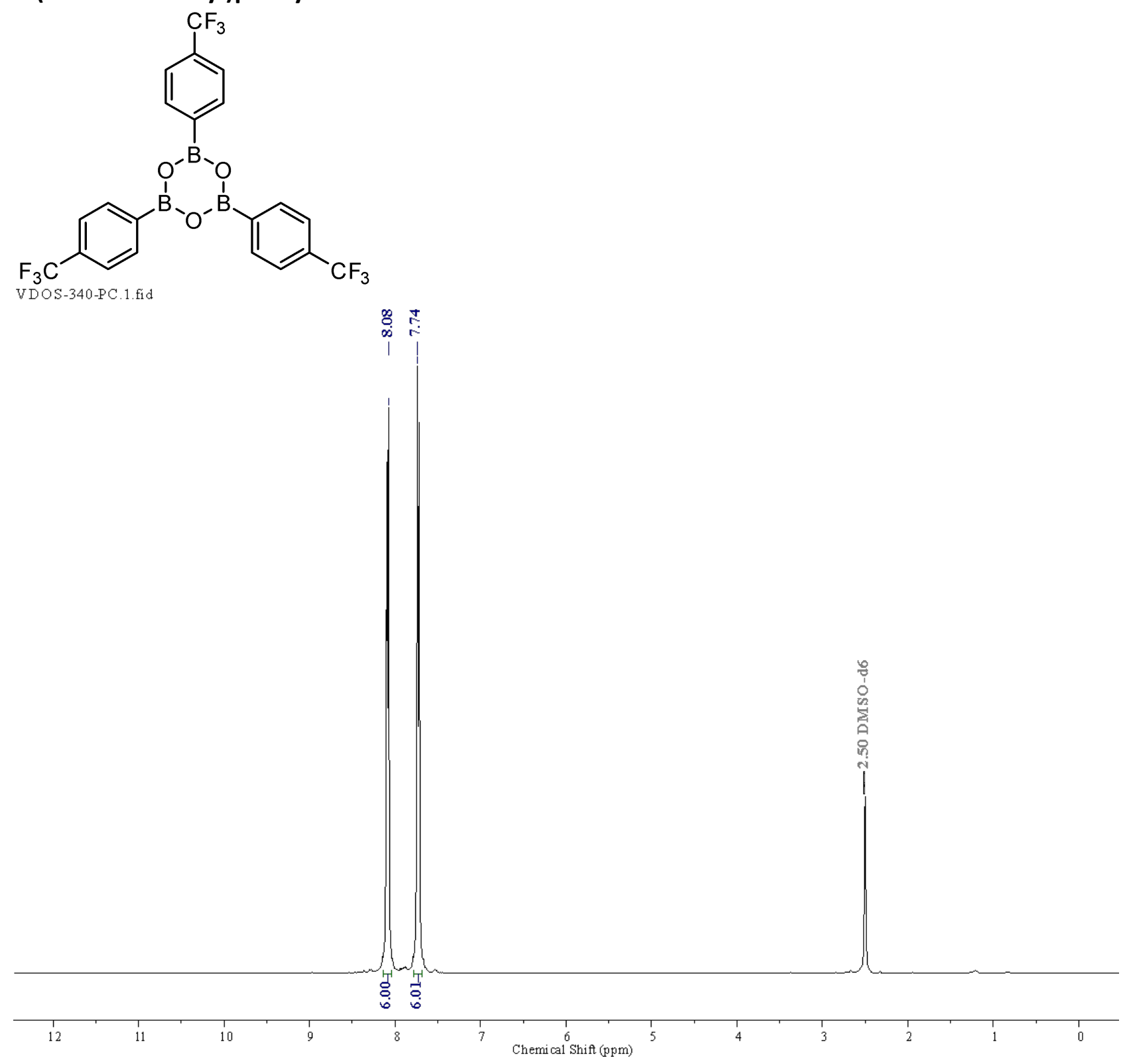



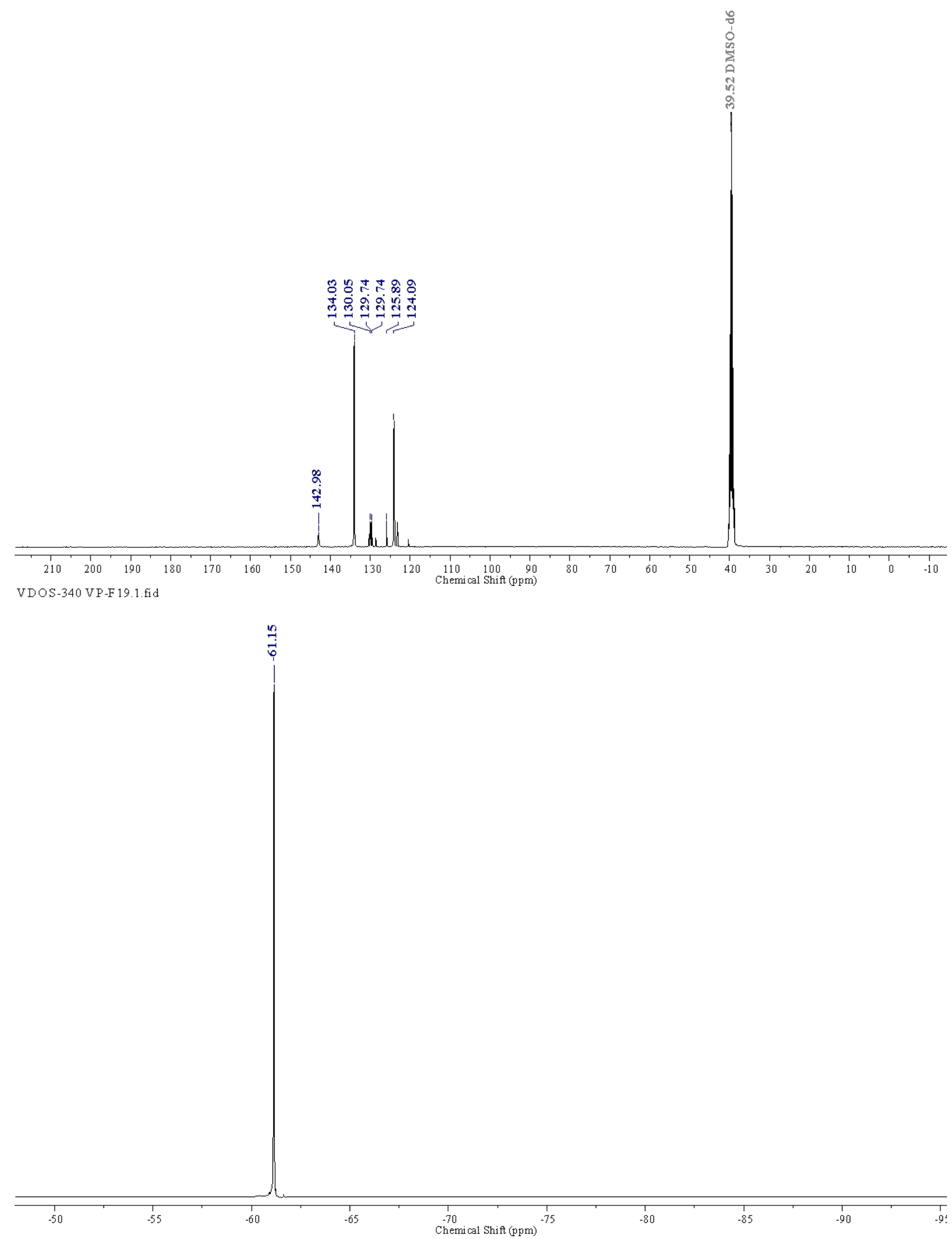
4-Methoxyphenyl Boroxine:<smiles>COc1ccc(B2OB(c3ccc(OC)cc3)OB(c3ccc(OC)cc3)O2)cc1</smiles>

VDOS-4OMe B oraxin VP.3.fid

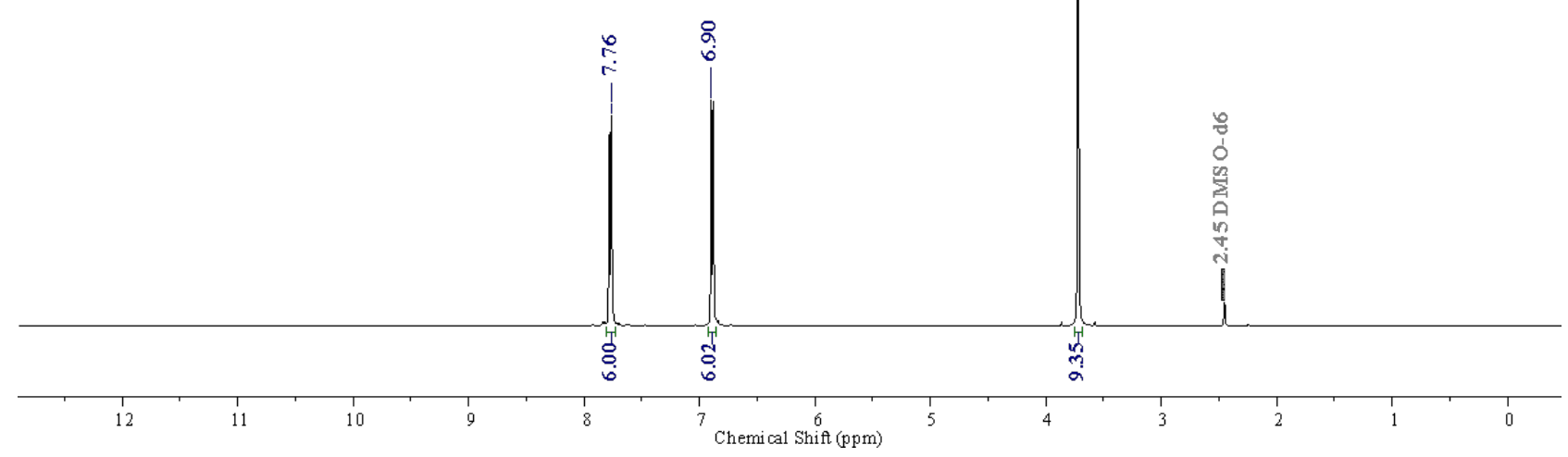




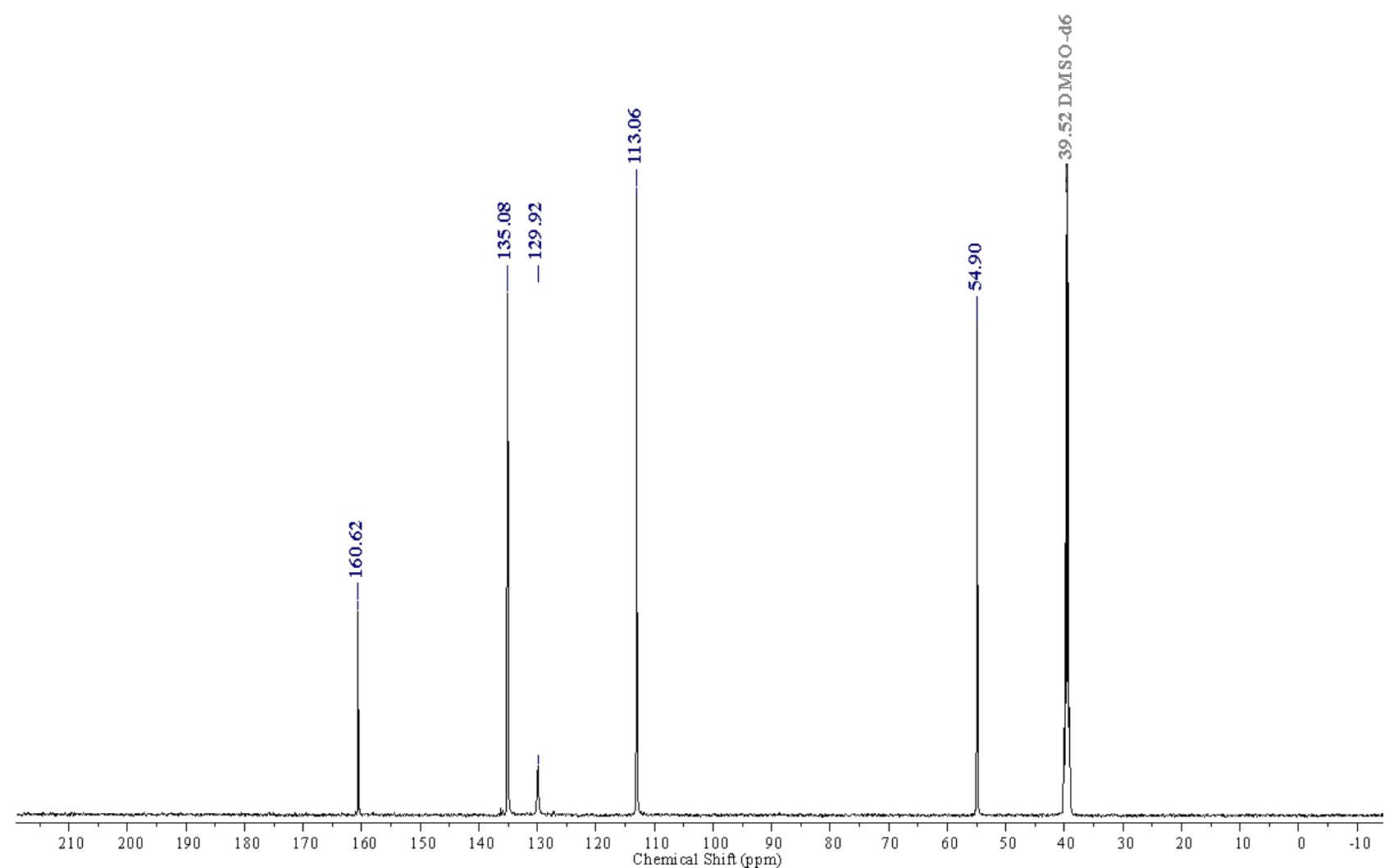


Electronic and Steric Effects on the Substituted Benzaldehydes:

Entry 1: With 2- $\mathrm{CF}_{3}-\mathrm{PhCHO}$

SCG-309-K1-16hr-D1-50sec.1. fid

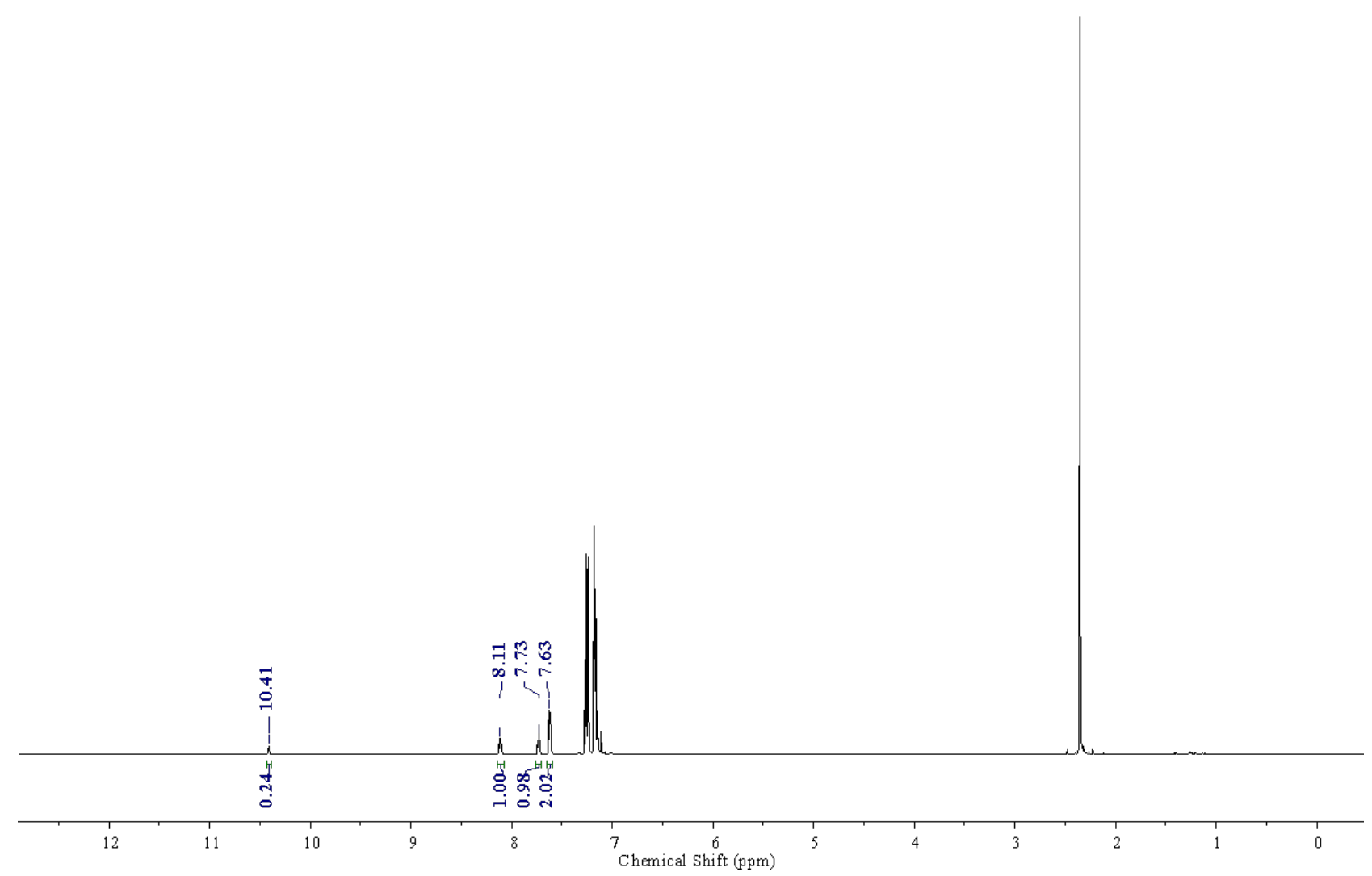

Entry 1': With 2-- $\mathrm{CF}_{3}-\mathrm{PhCHO}$, Duplicate SCG-309-K2-16hr-D1-50sec.1.fid

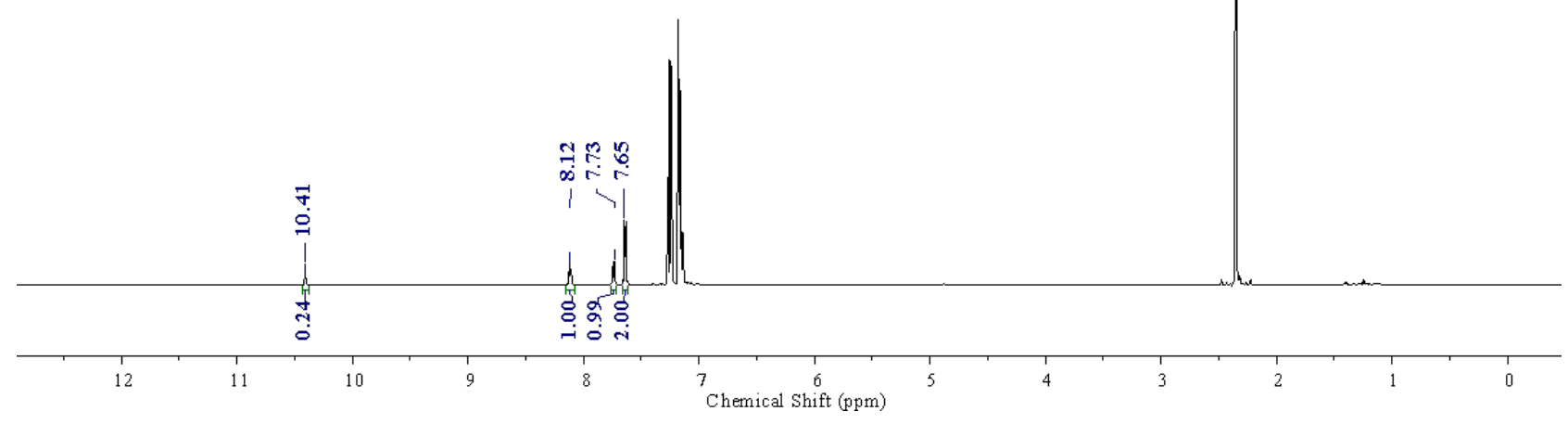


2-Hydroxy-1,2-bis (3-(trifluoromethyl)phenyl)ethanone:<smiles>O=C(c1cccc(C(F)(F)F)c1)C(O)c1cccc(C(F)(F)F)c1</smiles>

VDOS-3CF3 benzoin-CDCL3-VPF-H 6.1 fid

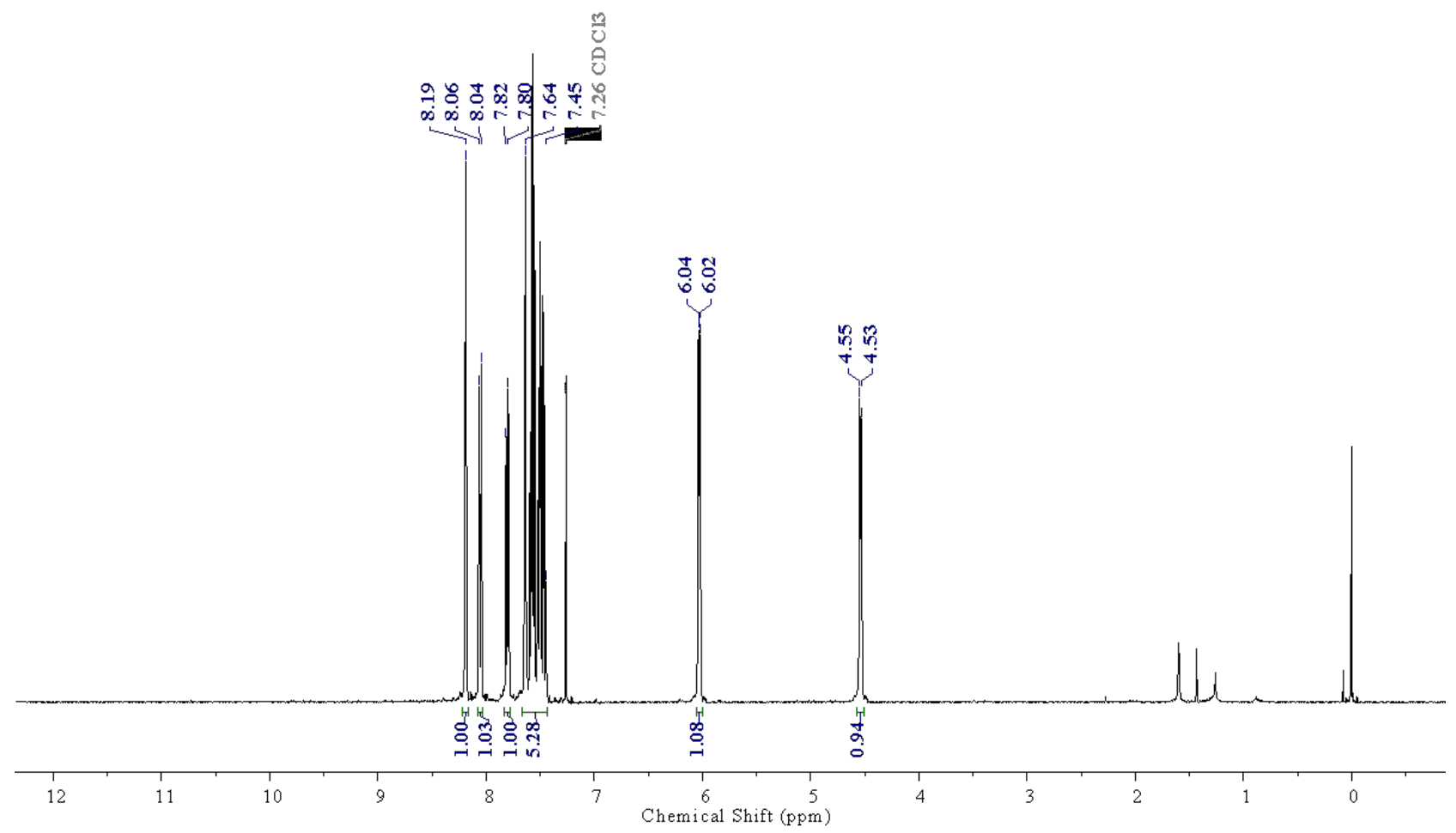


Entry 2: With 3-- $\mathrm{CF}_{3}-\mathrm{PhCHO}$

SCG-319-B 4-16hr-D 1-50sec.1.fid
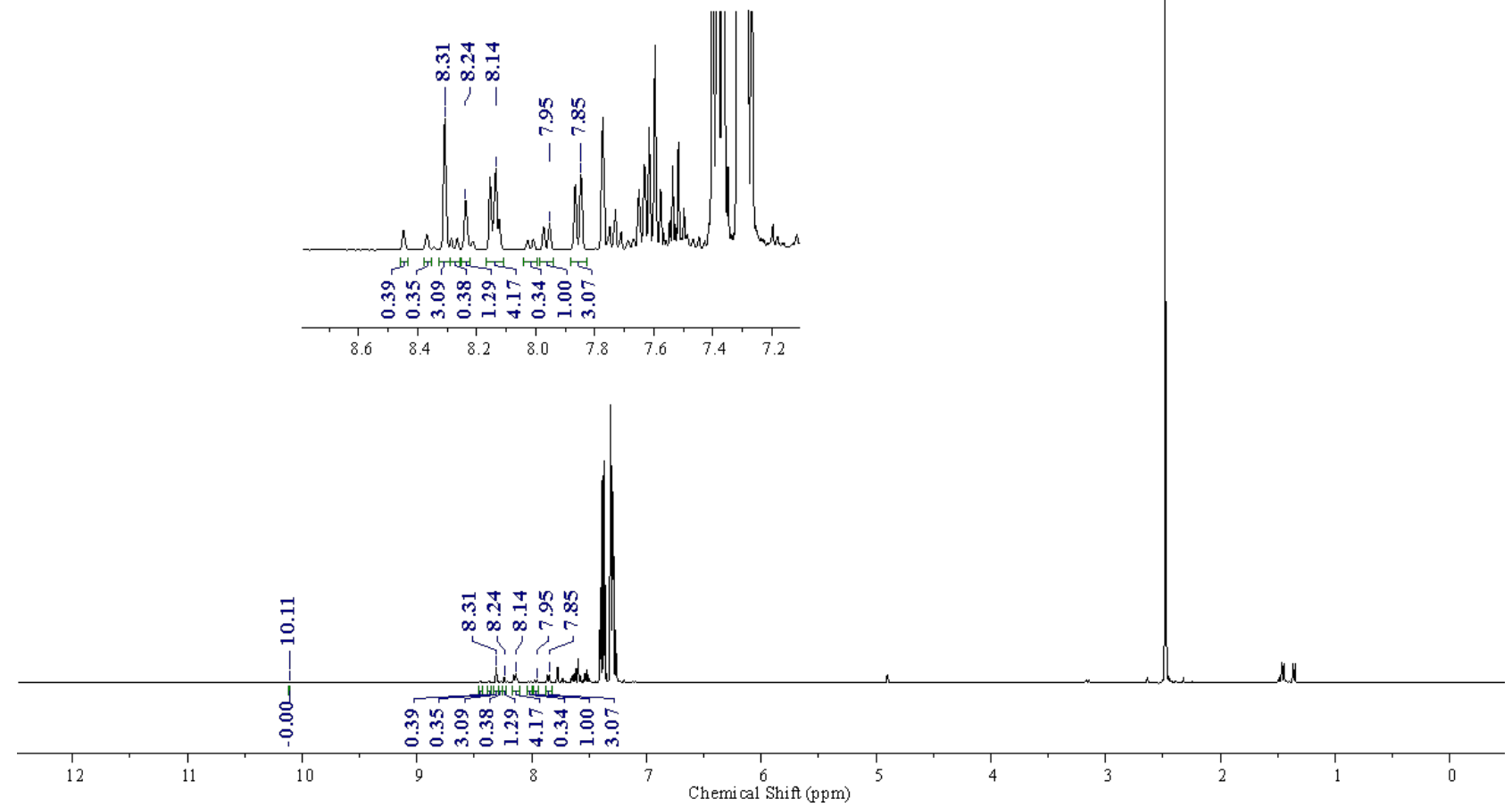

Entry 2': With 3- $\mathrm{CF}_{3}-\mathrm{PhCHO}$, Duplicate

SCG-319-B 5-16hr-D 1-50sec.1.fid
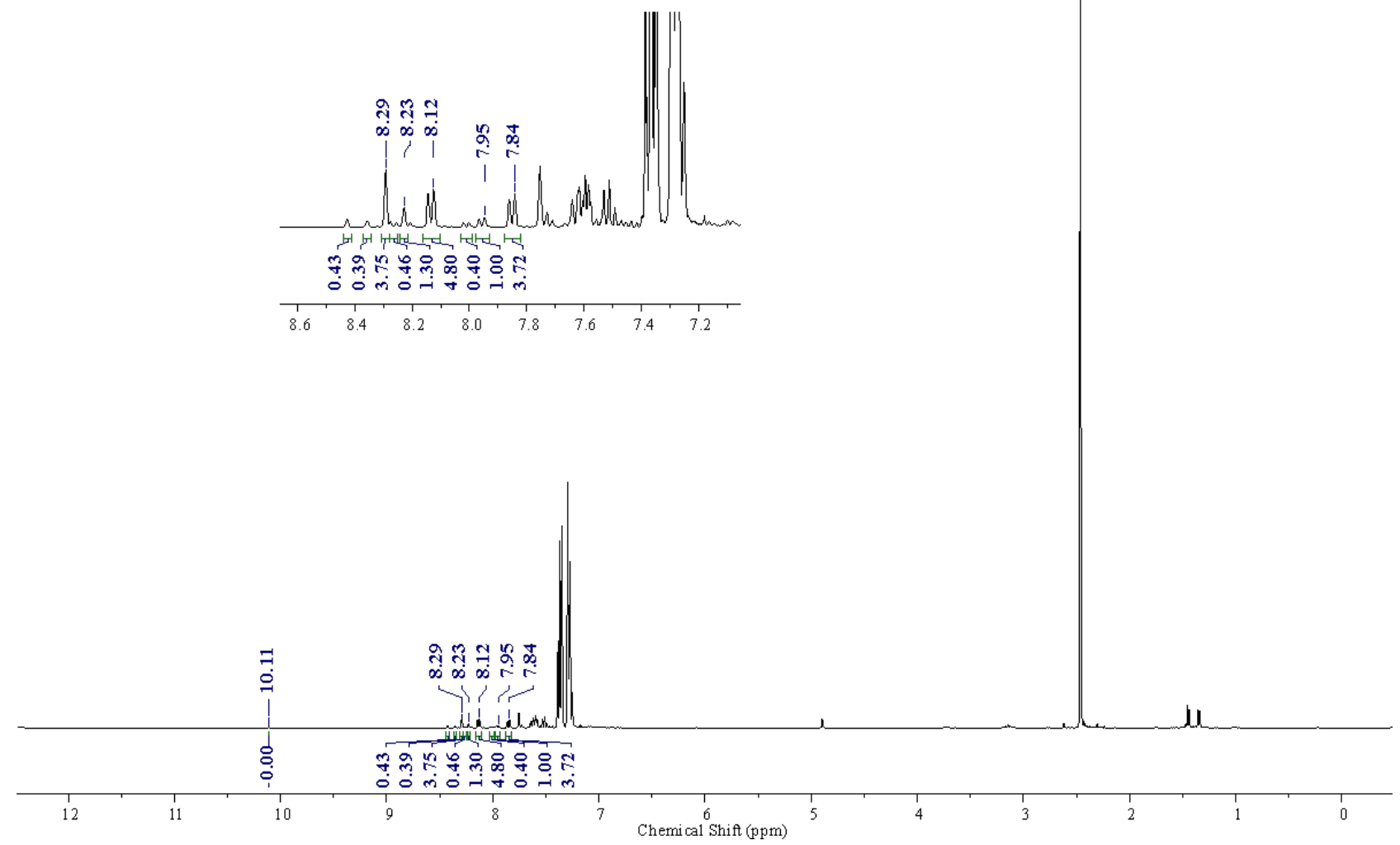
2-hydroxy-1,2-bis(4-(trifluoromethyl)phenyl)ethan-1-one:

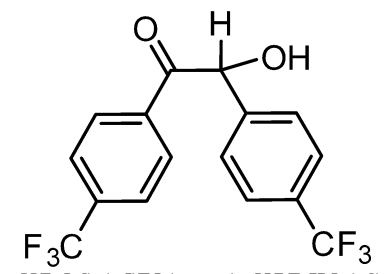

VDOS-4-CF 3 benzoin VPF-H 3.1 fid

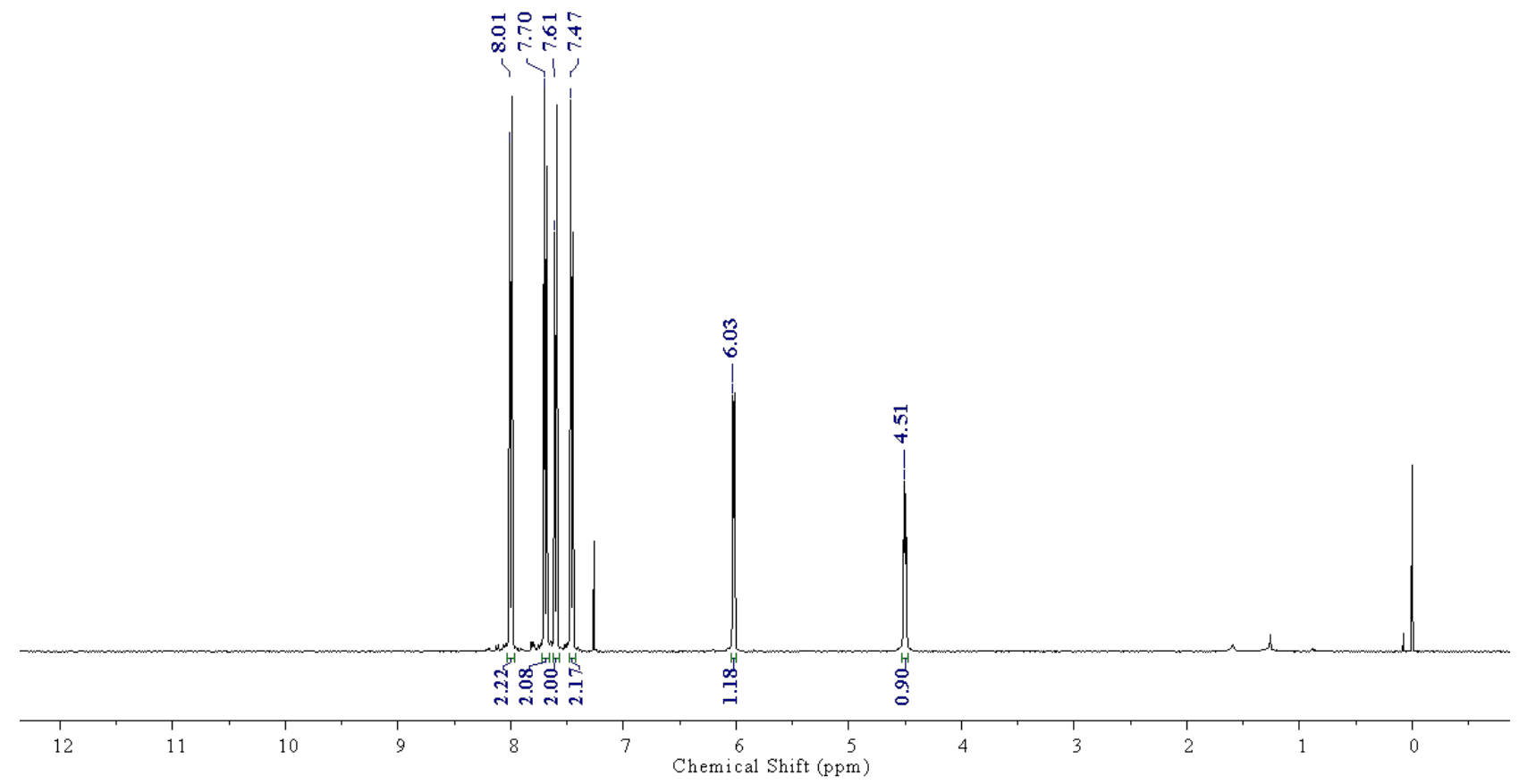


Entry 3: With 4-- $\mathrm{CF}_{3}-\mathrm{PhCHO}$

SCG-319-A3-16hr-D 1-50sec.1.fid
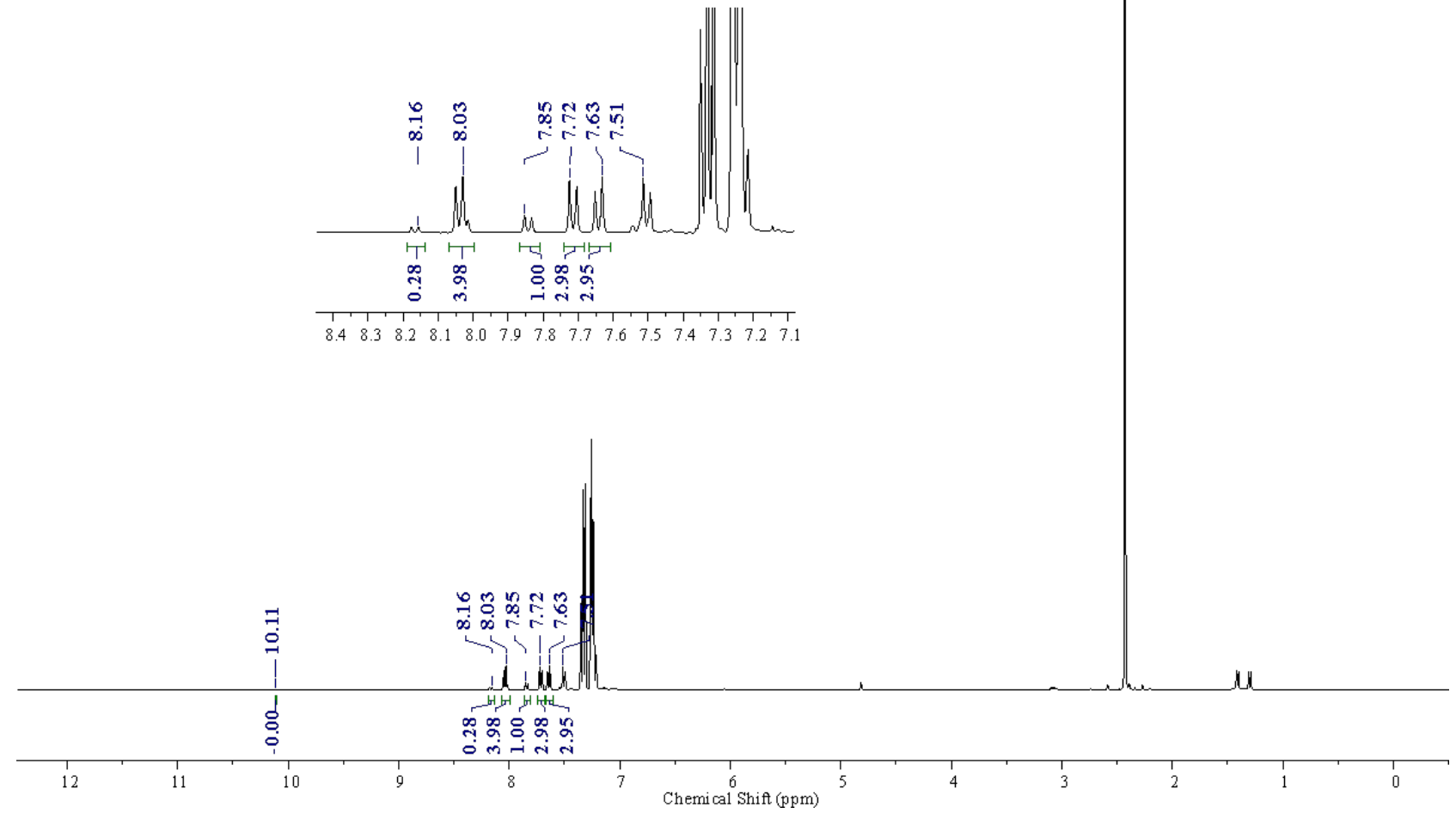

Entry 3': With 4-- $\mathrm{CF}_{3}-\mathrm{PhCHO}$, Duplicate

SCG-319-A4-16hr-D 1-50sec.1.fid
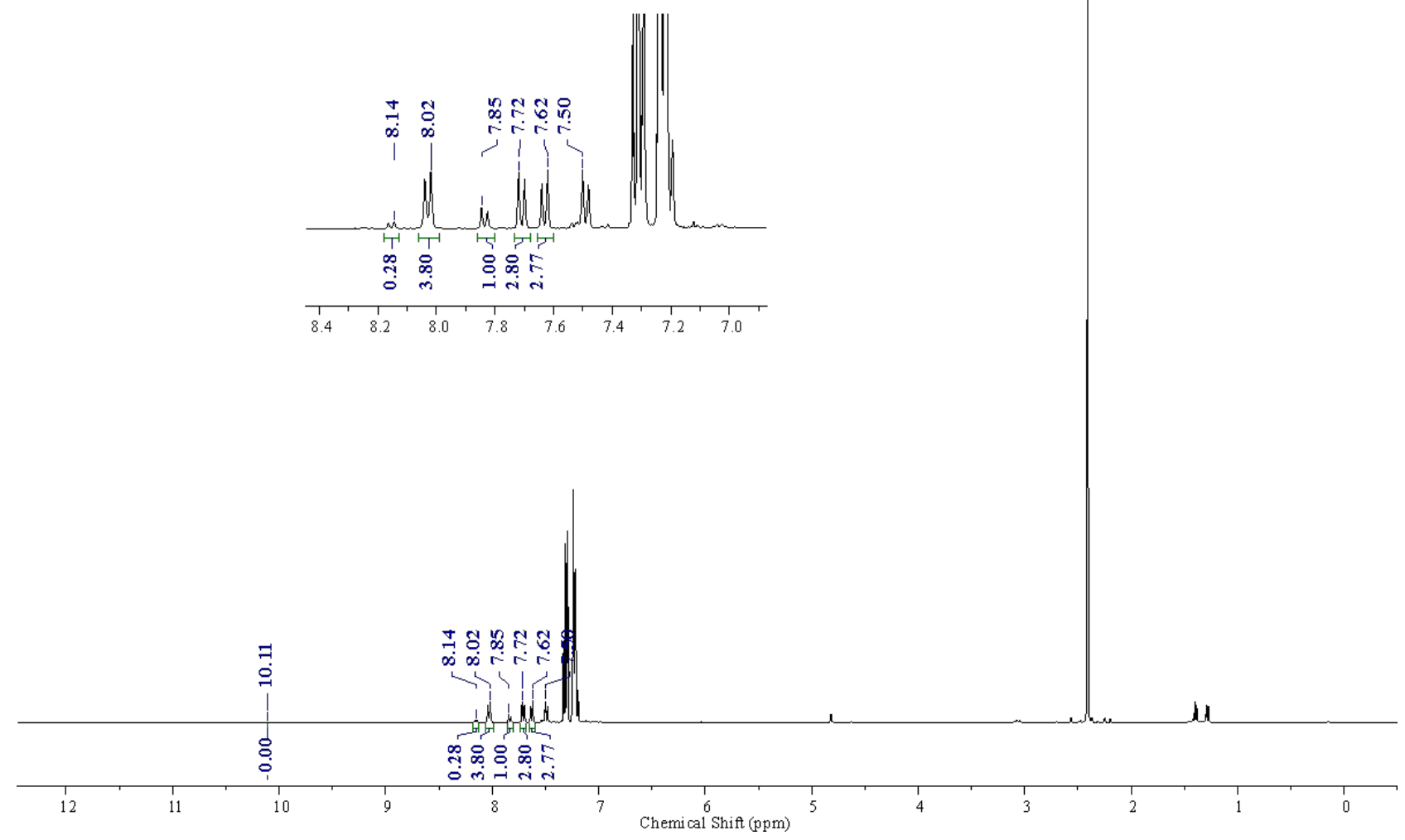
2-Hydroxy-1,2-diphenylethan-1-one:<smiles>O=C(c1ccccc1)C(O)c1ccccc1</smiles>

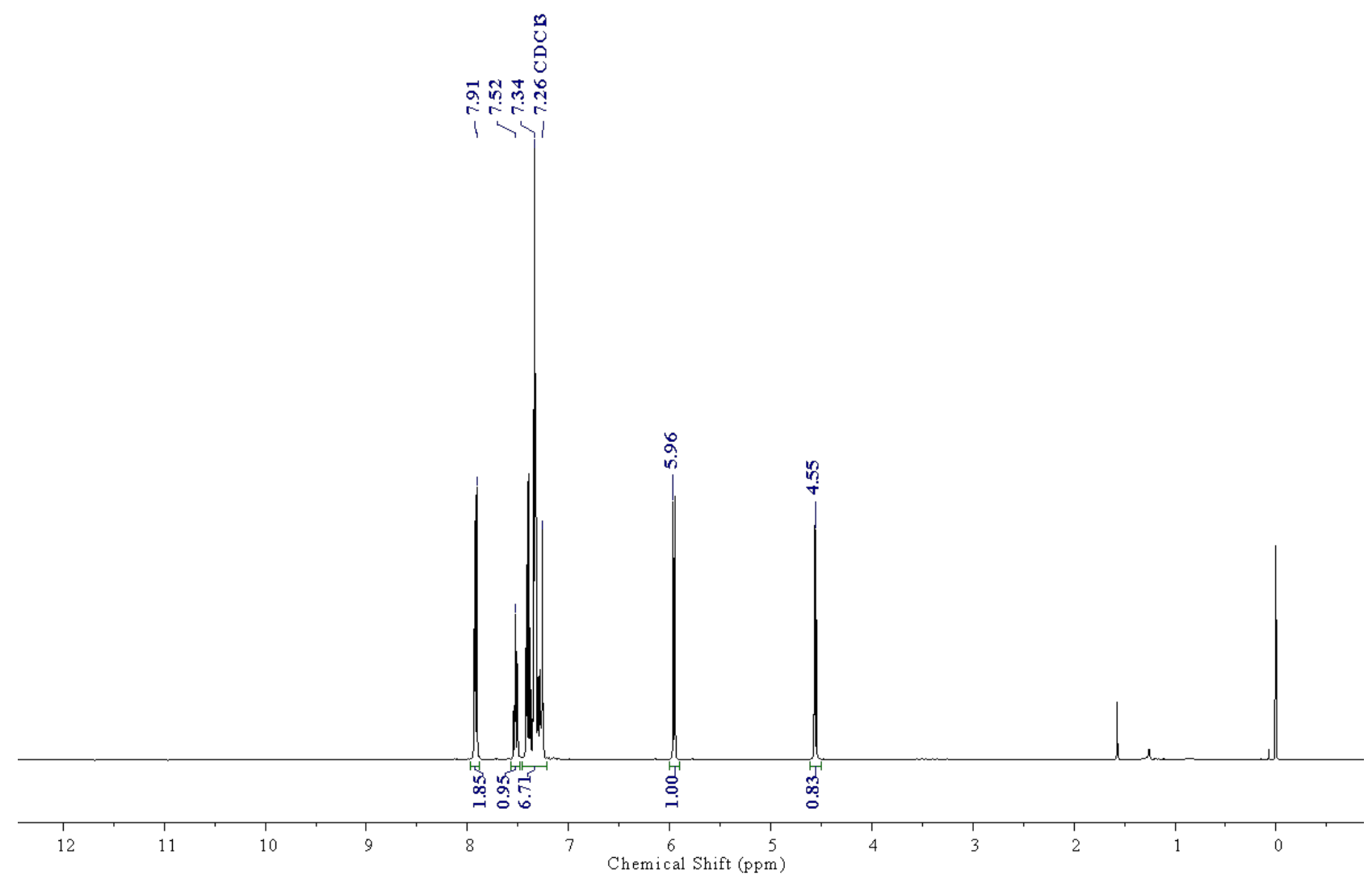


Entry 4: With PhCHO

SCG-319-K 1-16hr-D 1-50sec.1. fid
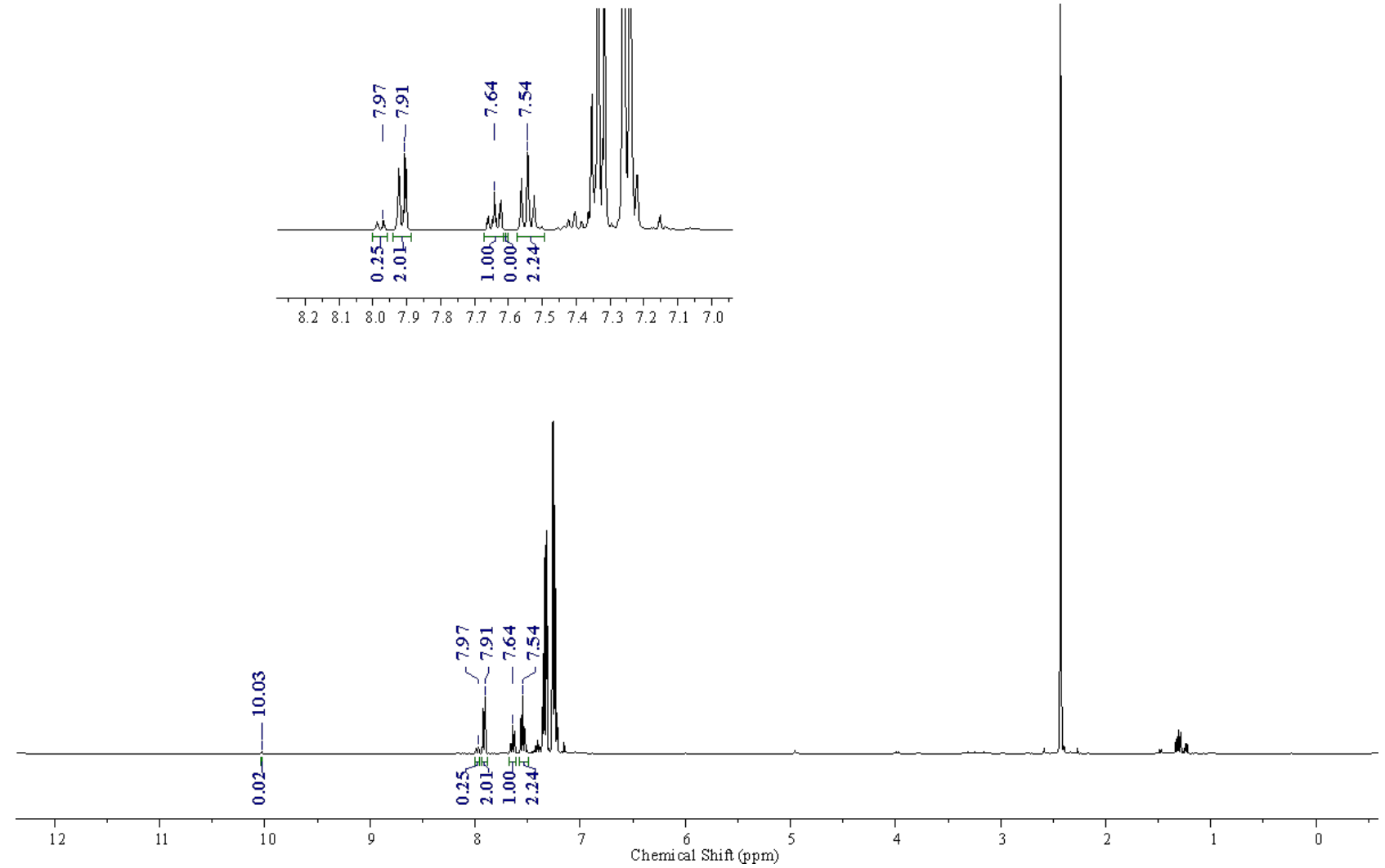

Entry 4': With PhCHO, Duplicate

SCG-319-K2-16hr-D 1-50sec.1.fid
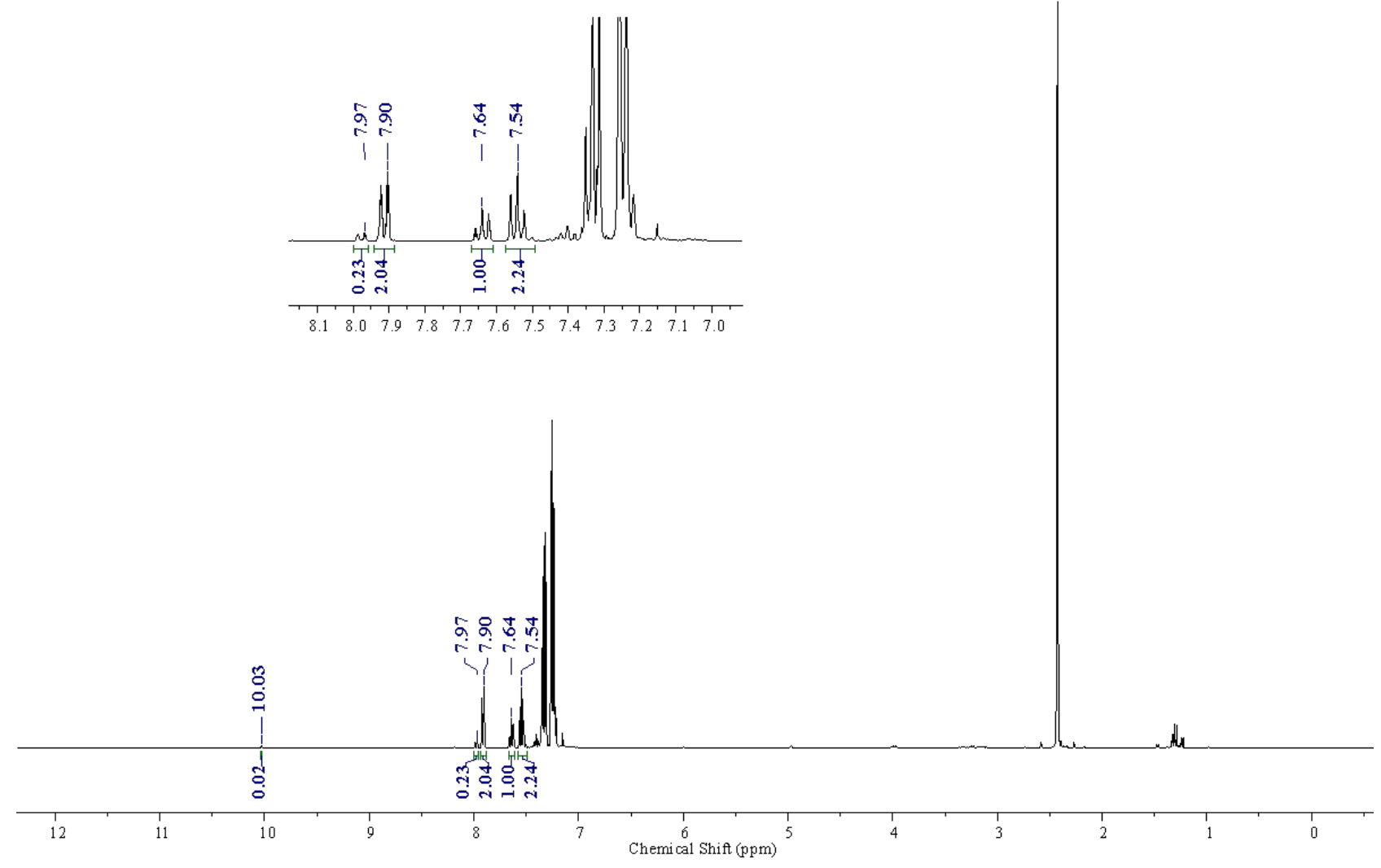
Entry 5: With 2-O $\mathrm{O}^{n} \mathrm{Bu}-\mathrm{PhCHO}$

SCG-348-G1-16hr-D 1-50sec.1. fid

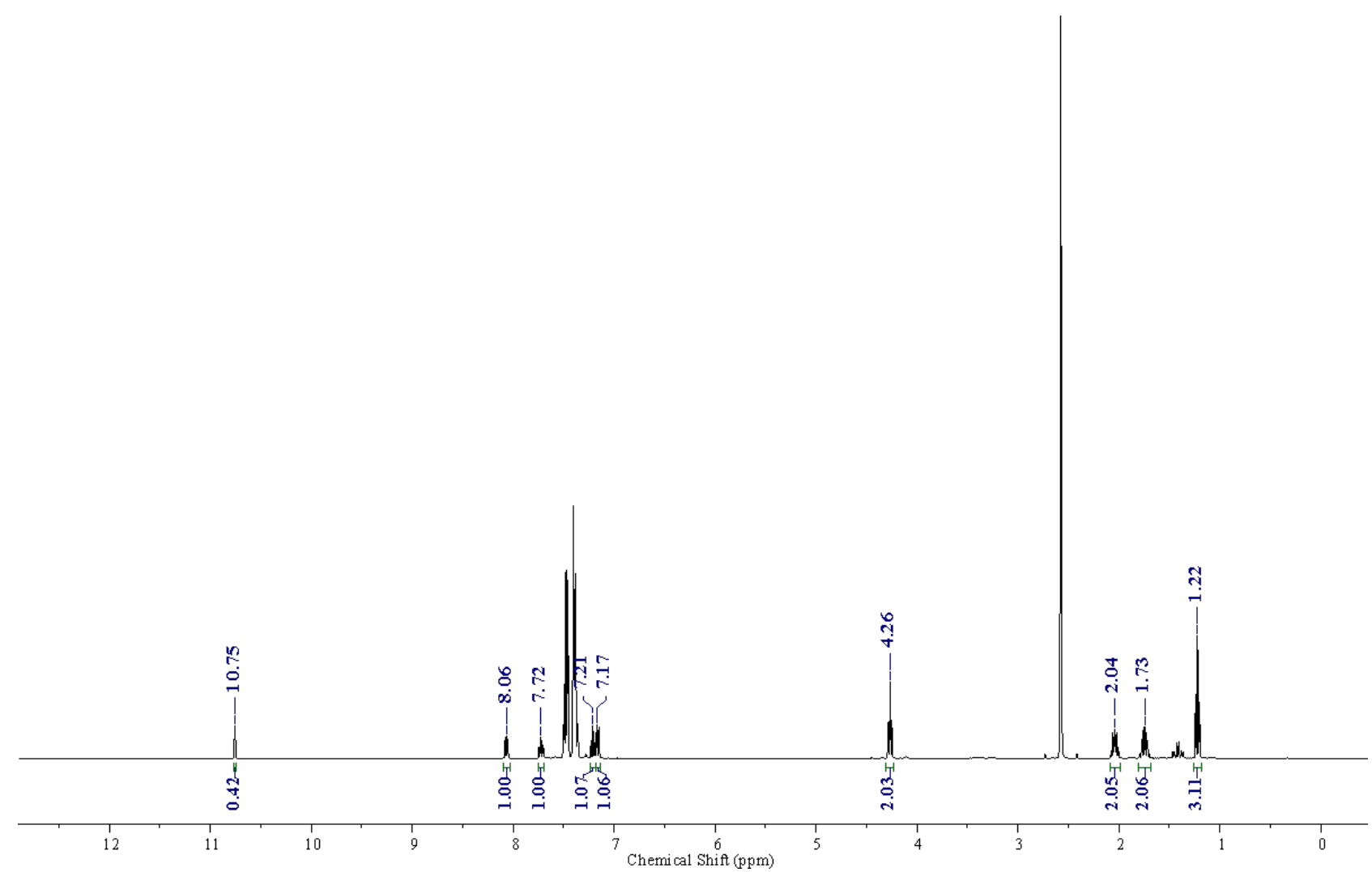

Entry 5': With 2-On Bu-PhCHO, Duplicate

SCG-348-G2-16hr-D 1-50sec.1. fid

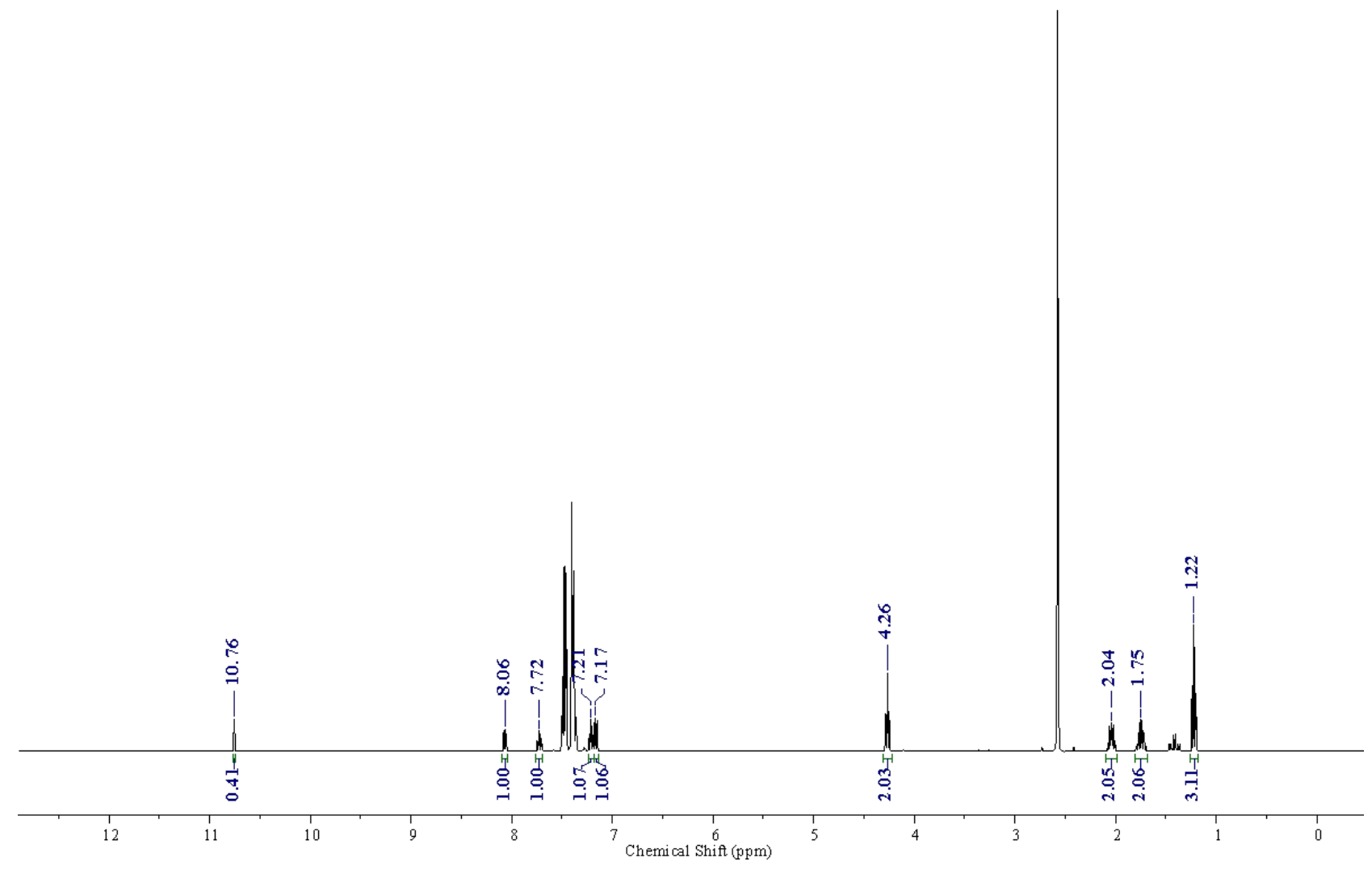


Entry 6: With 2-OMe-PhCHO

SCG-307-A2-16hr-D1-50sec.1. fid

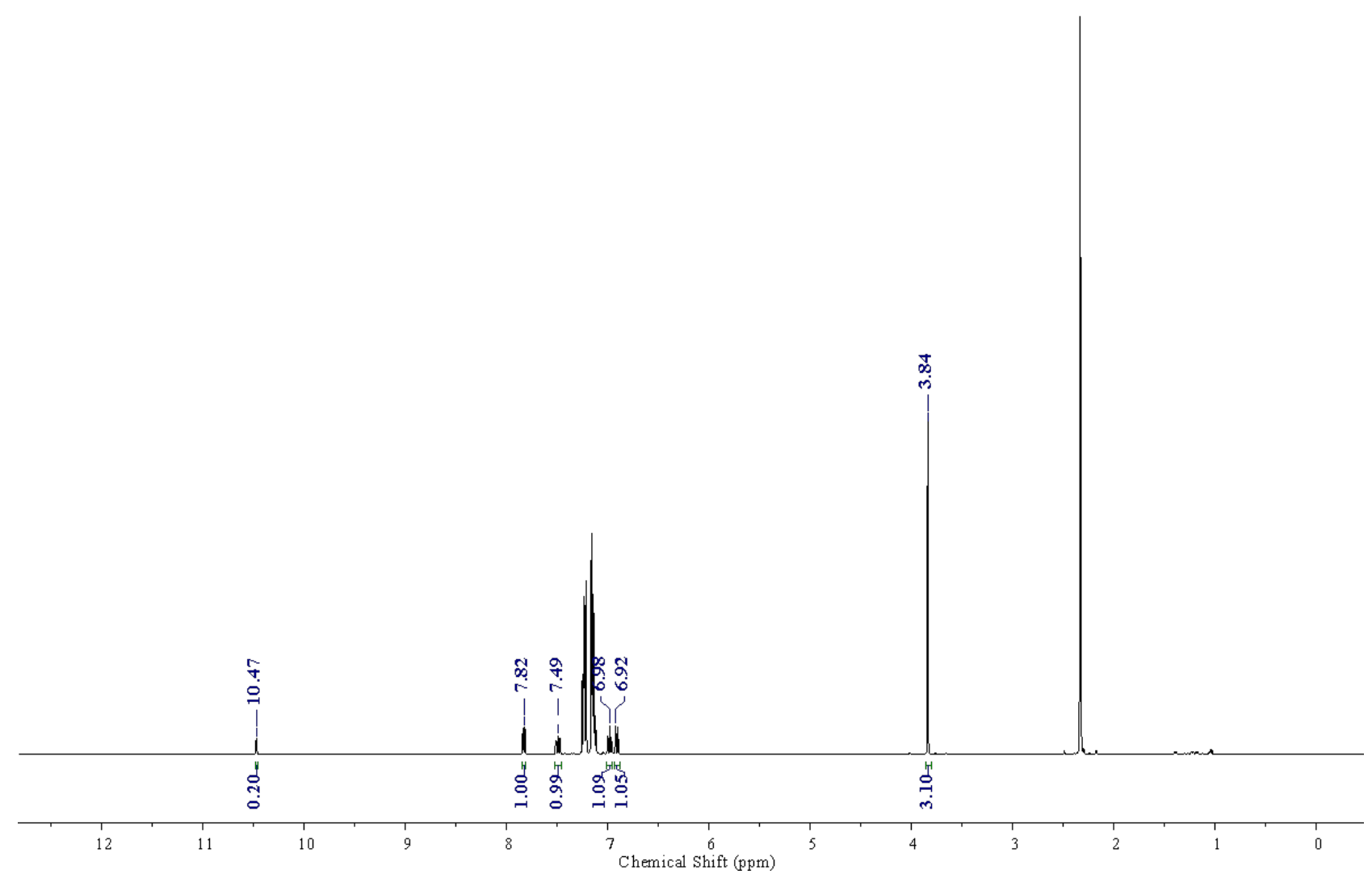

Entry 6': With 2-OMe-PhCHO, Duplicate

SCG-307-A3-16hr-D1-50sec.1. fid

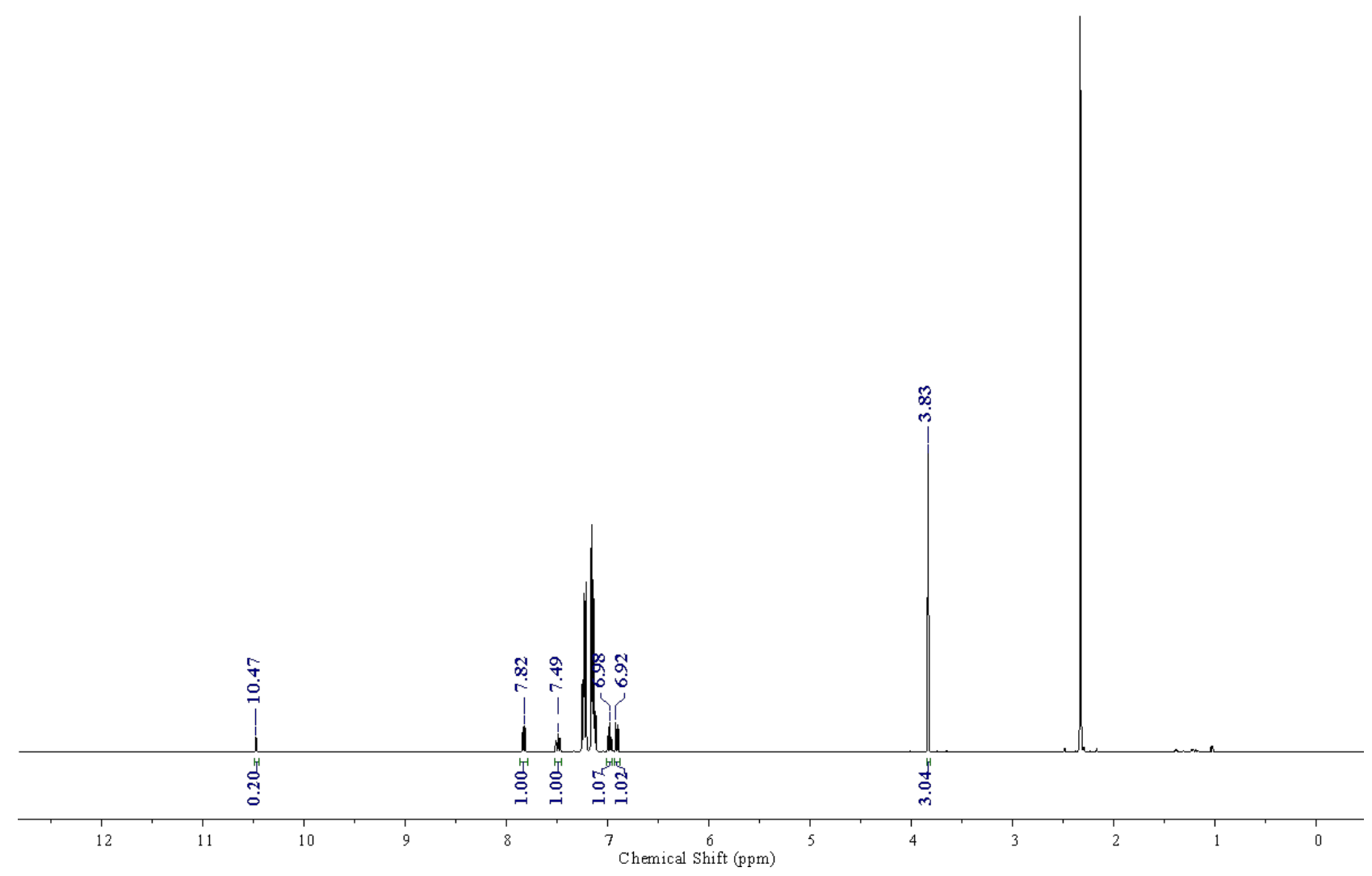


Entry 7: With 3-OMe-PhCHO

SCG-312-U 1-16hr-D 1-50sec

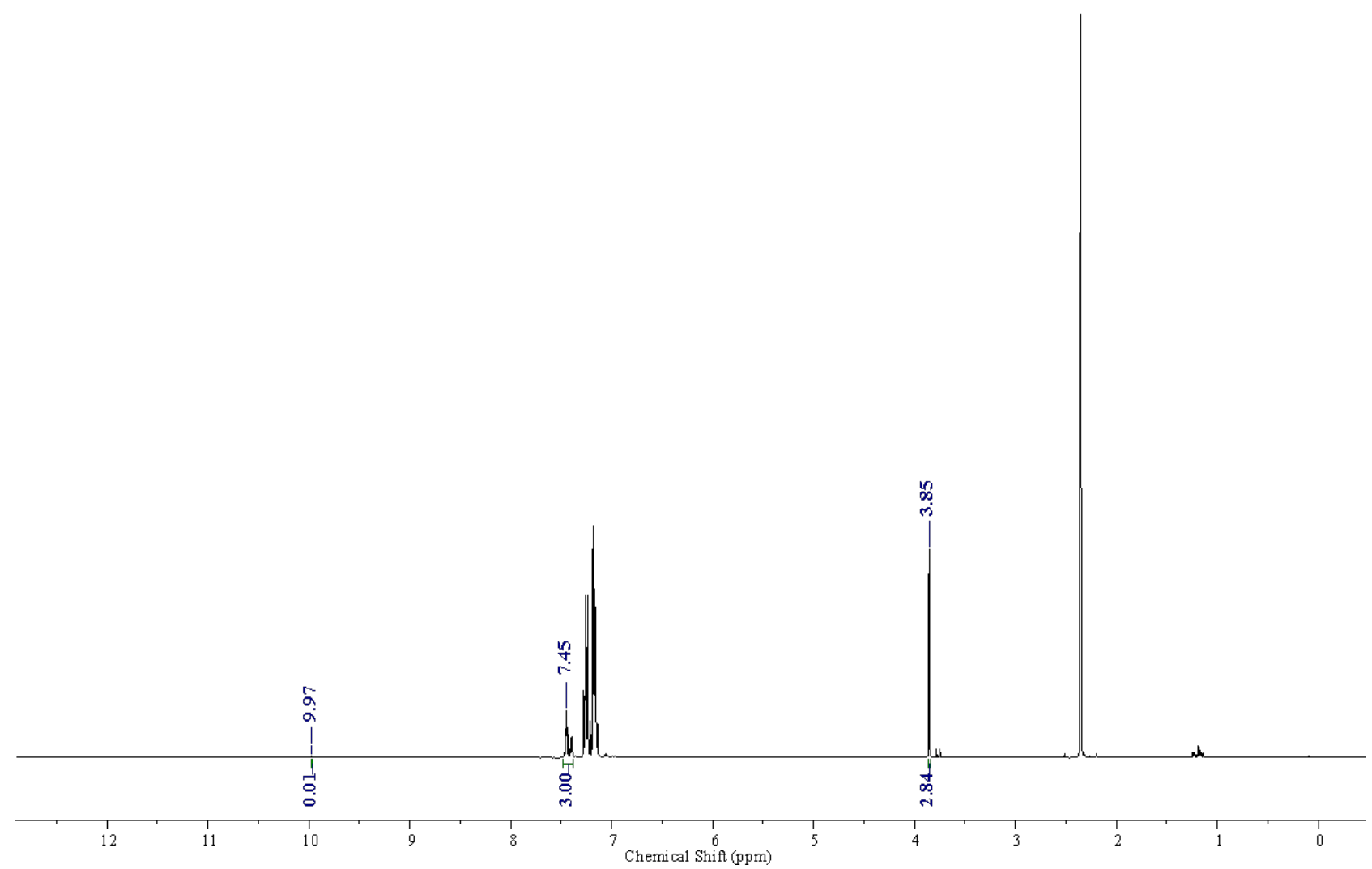

Entry 7': With 3-OMe-PhCHO, Duplicate

SCG-312-U2-16hr-D 1-50sec

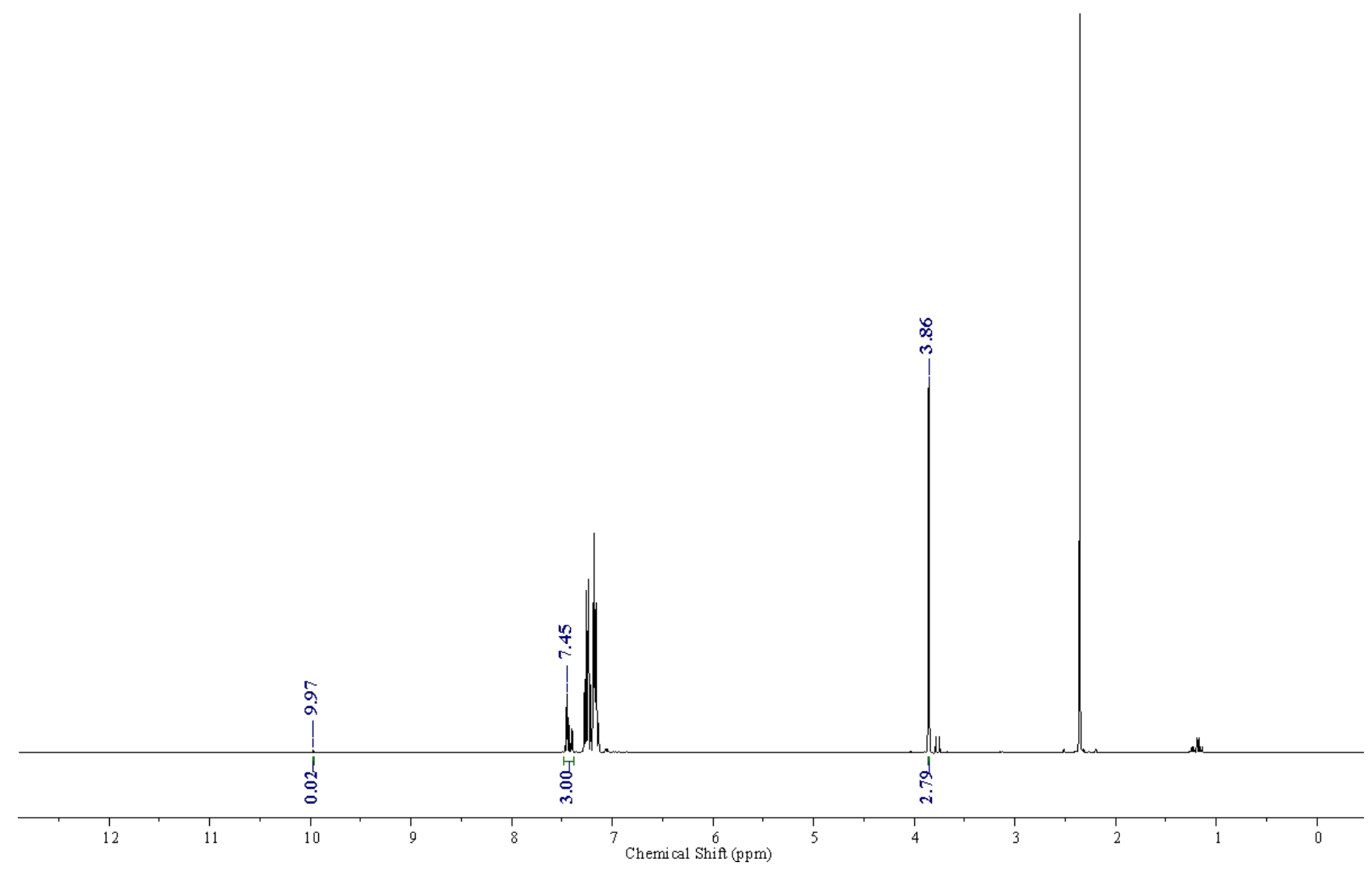


Entry 8: With 4-OMe-PhCHO

SCG-312-R1-16hr-D 1-50sec.1.fid

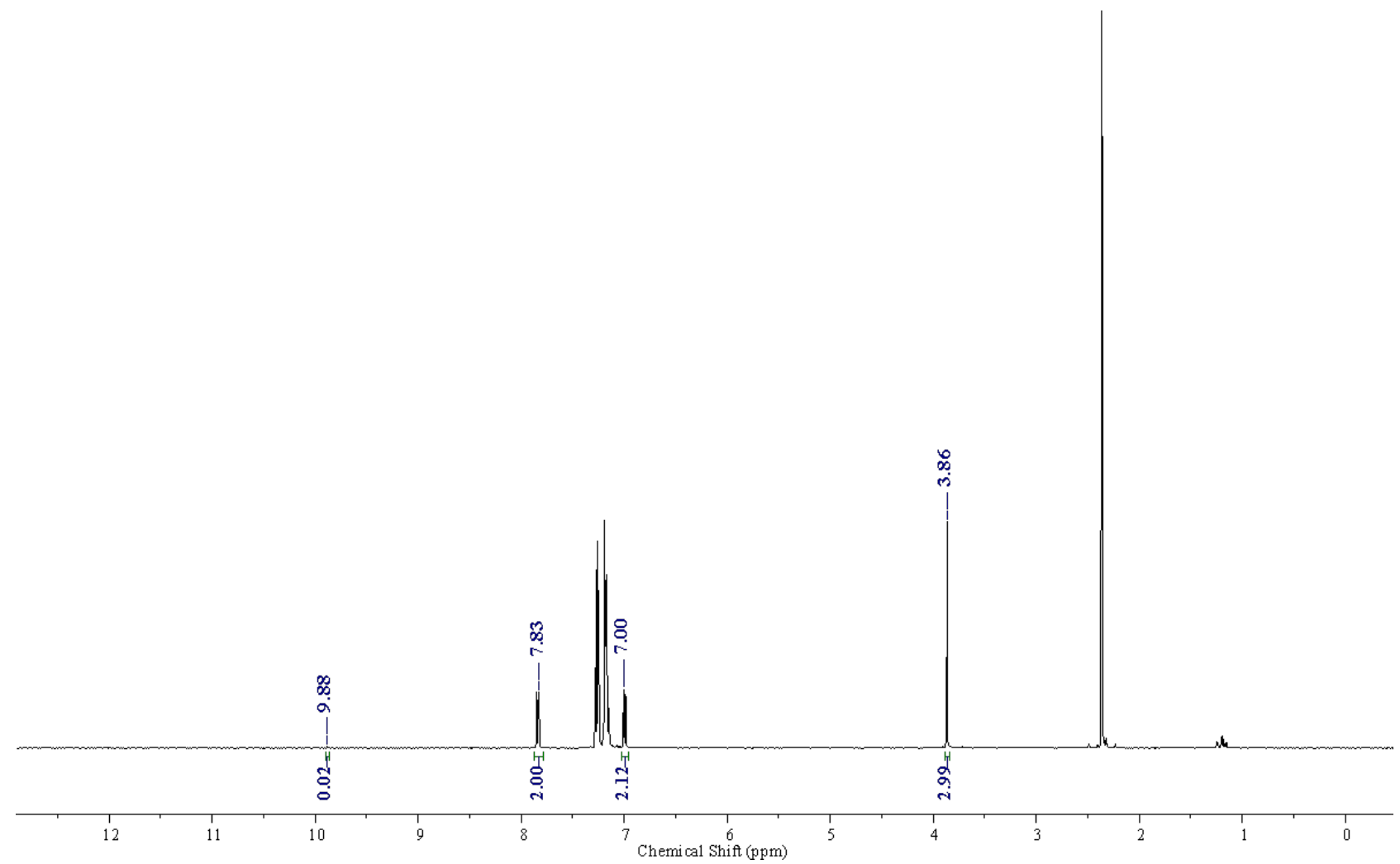

Entry 8': With 4-OMe-PhCHO, Duplicate

SCG-312-R2-16hr-D 1-50sec.1.fid

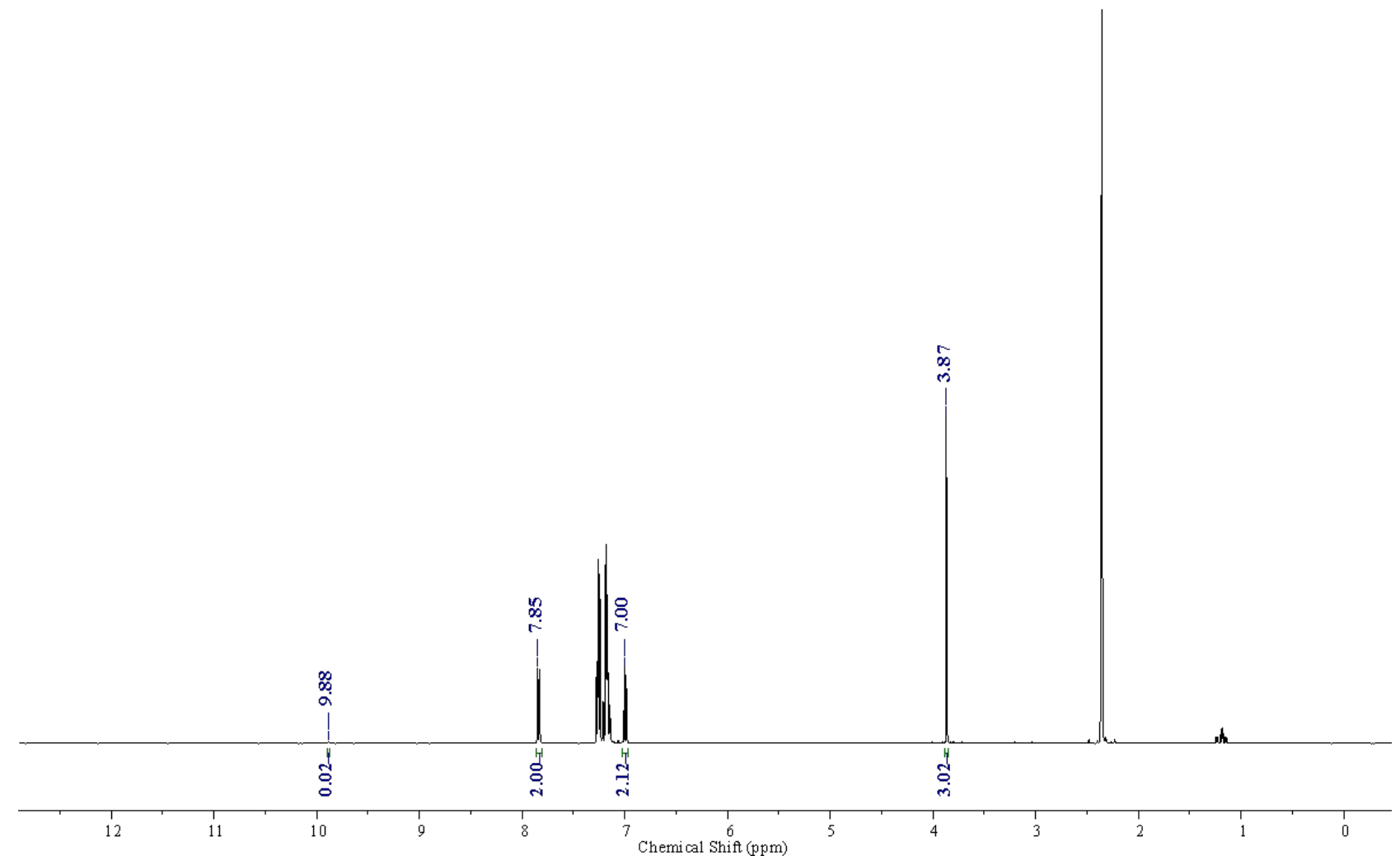


Optimization with 2-Methyl Benzaldehyde:

Entry 1: Reaction in Acetonitrile- $d_{3}$

SCG-289-B-16hr-D1-50sec.1. fid

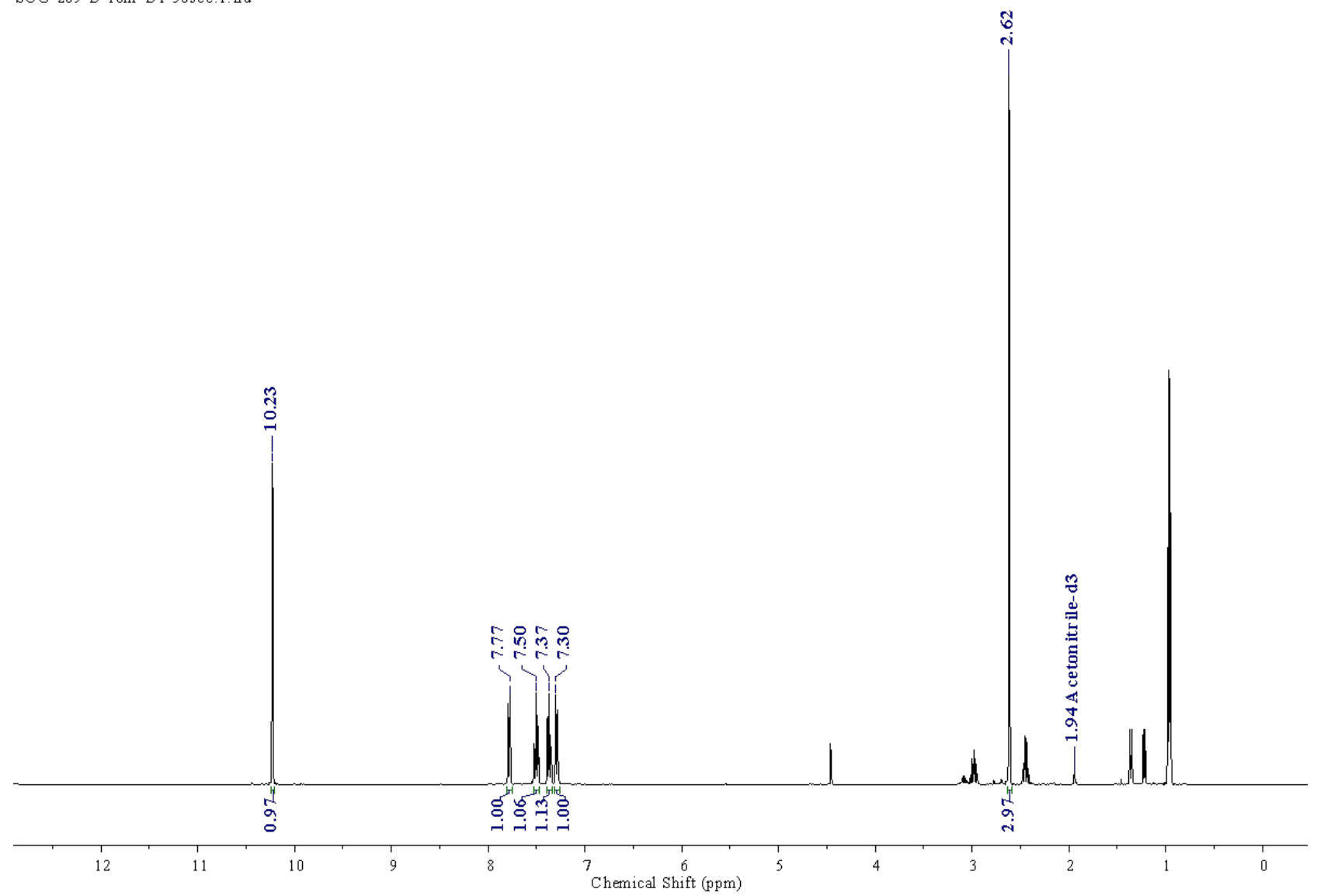

Entry 2: Reaction in Tetrahydrofuran- $d_{8}$

SCG-289-C-16hr-D1-50sec.1. fid

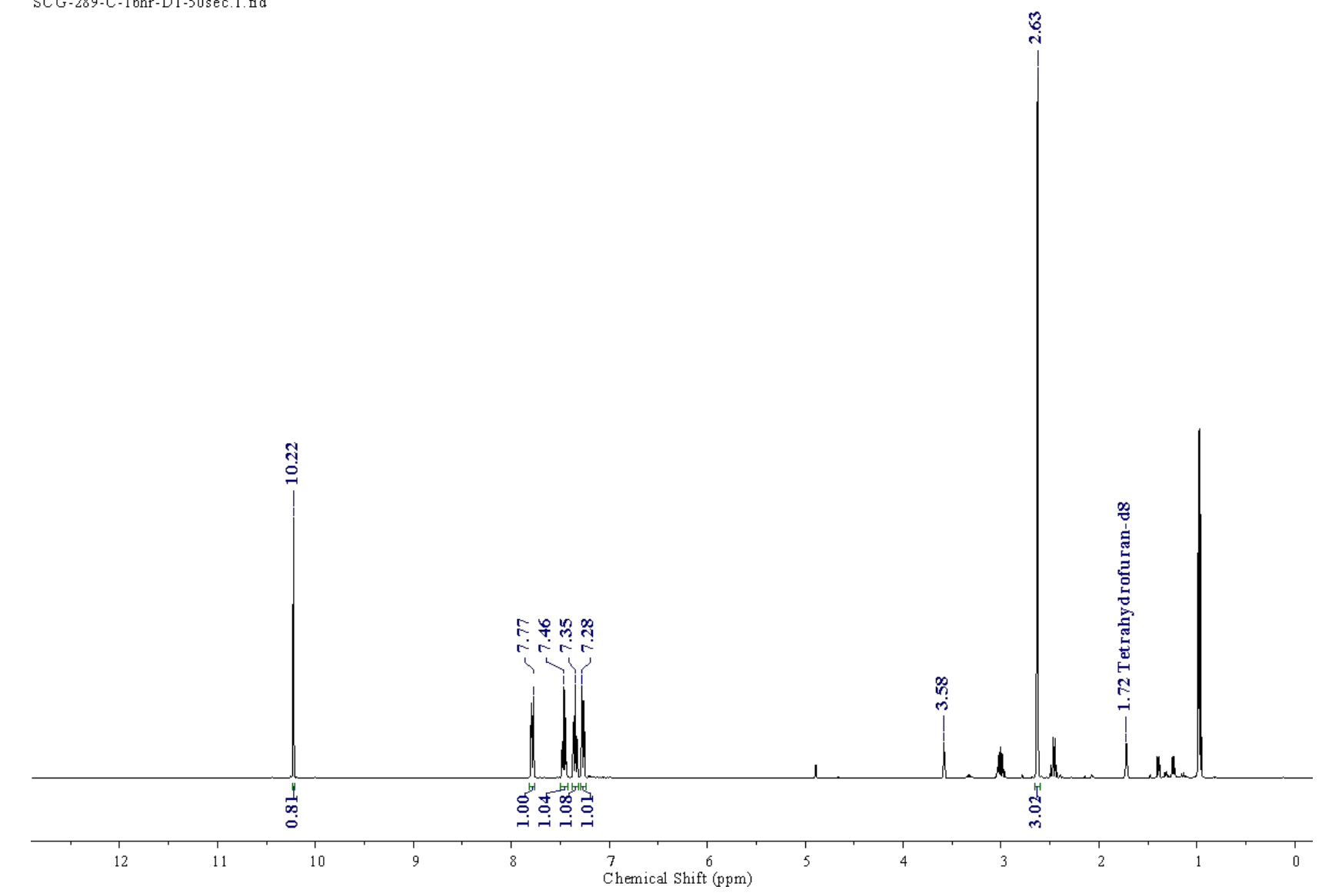


Entry 3: Reaction in Aetone- $d_{6}$

SCG-289-D-16hr-D1-50sec.1. fid

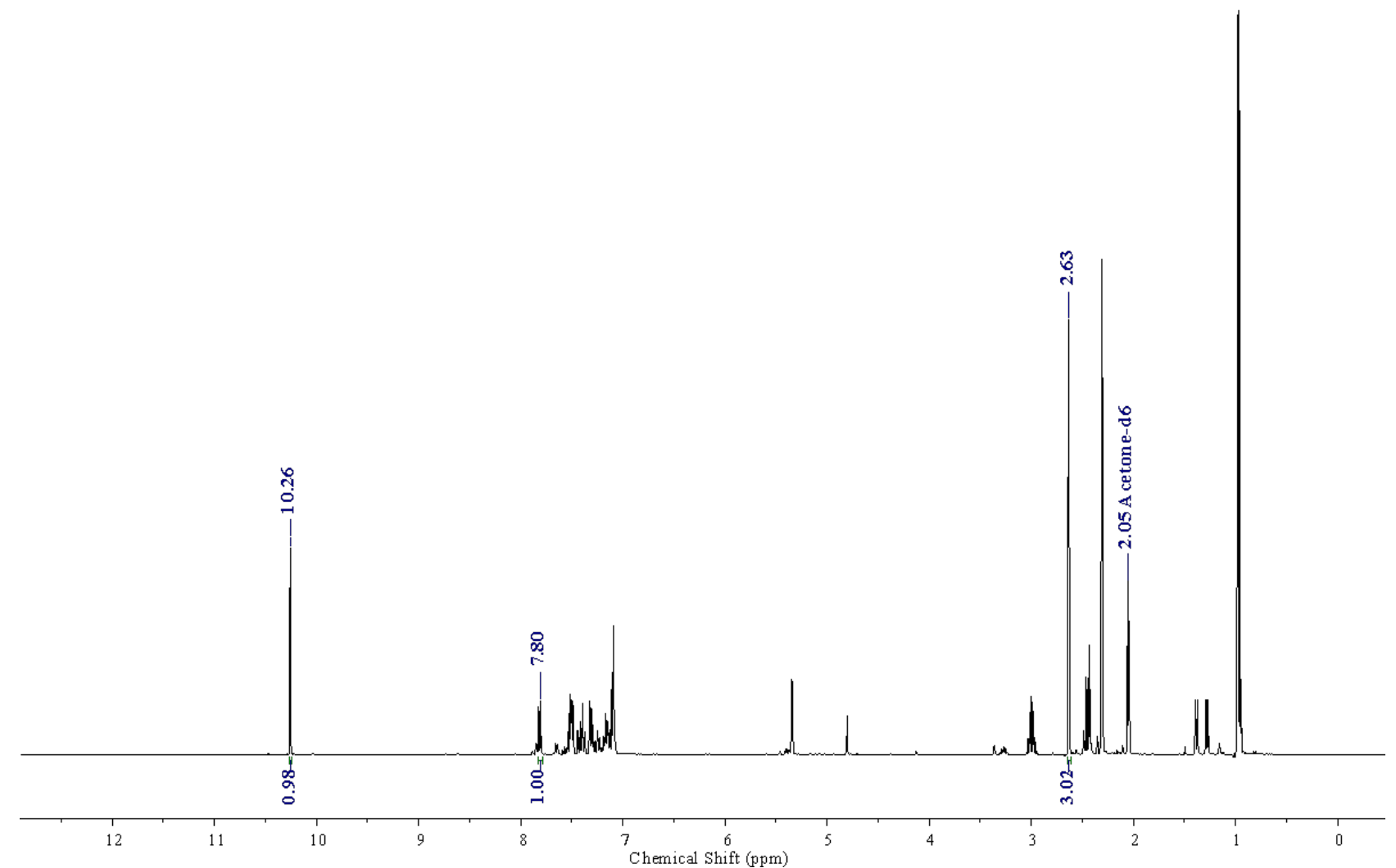

Entry 4: Reaction in Methanol- $d_{4}$

SCG-289-E-16hr-D 1-50sec.1 fid

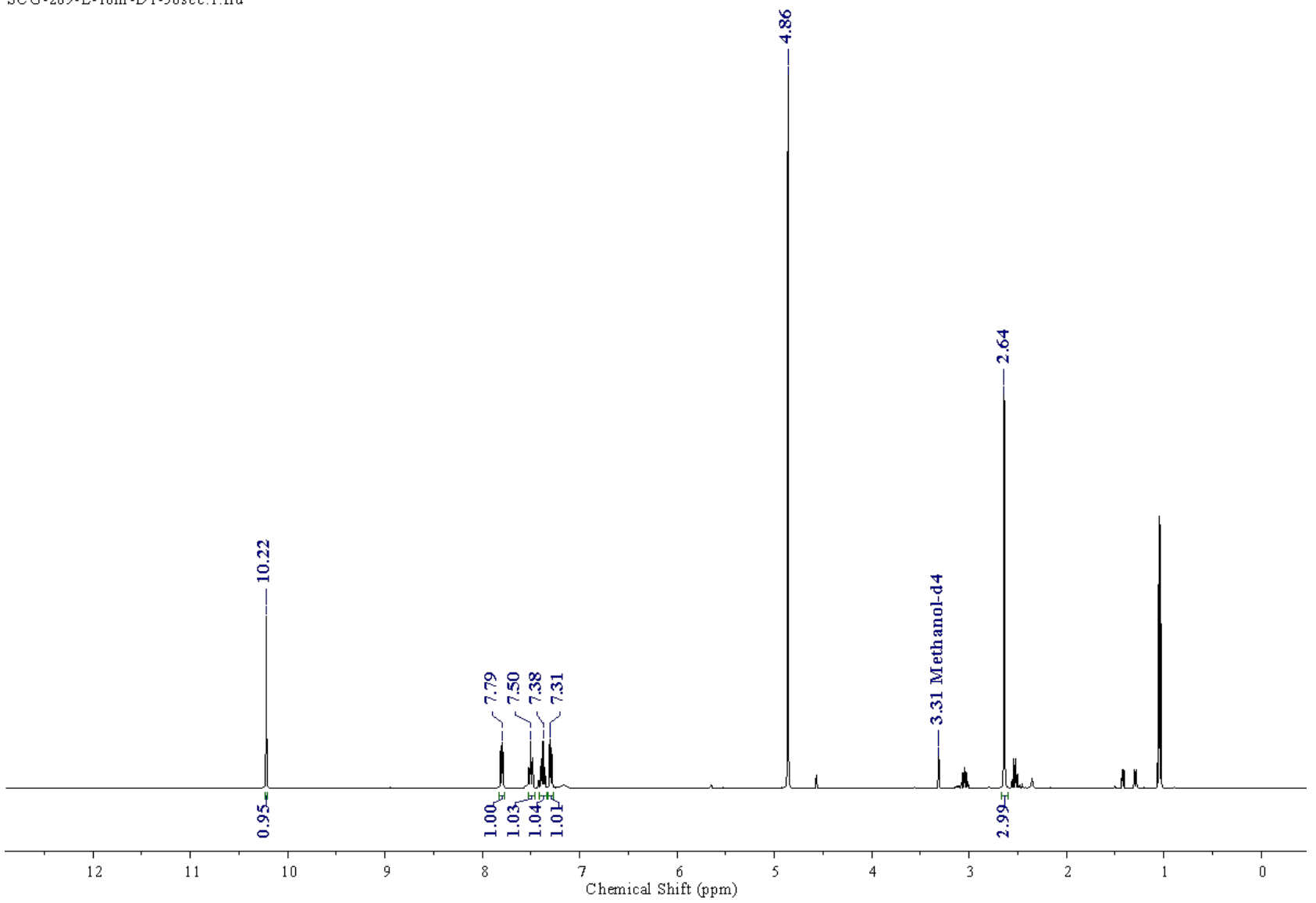


Entry 5: Reaction in Chloroform-d

SCG-283-H2-17hr-D1-50sec.1. fid

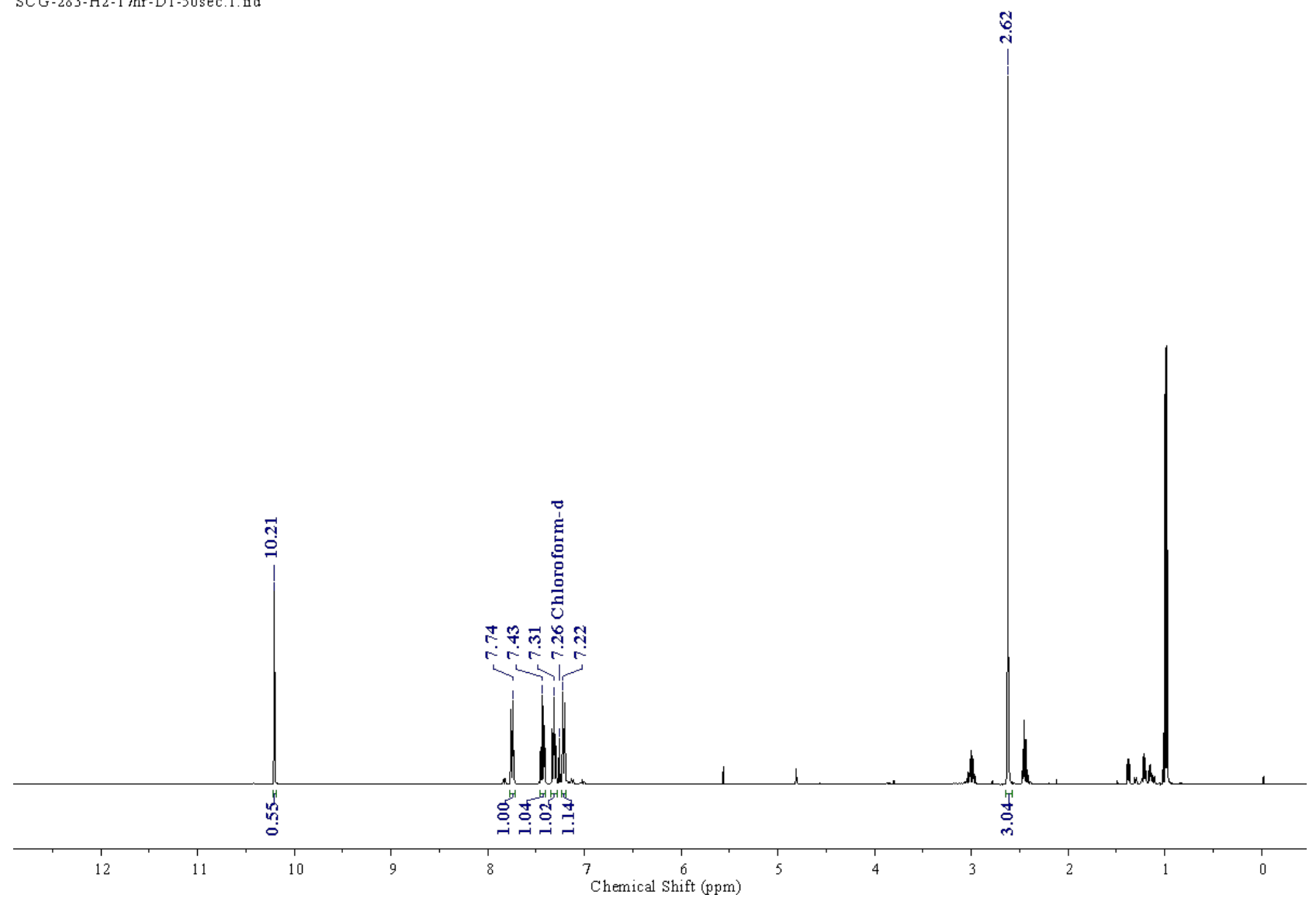

Entry 6: Reaction in Toluene- $d_{8}$

SCG-289-A-16hr-D1-50sec.1. fid

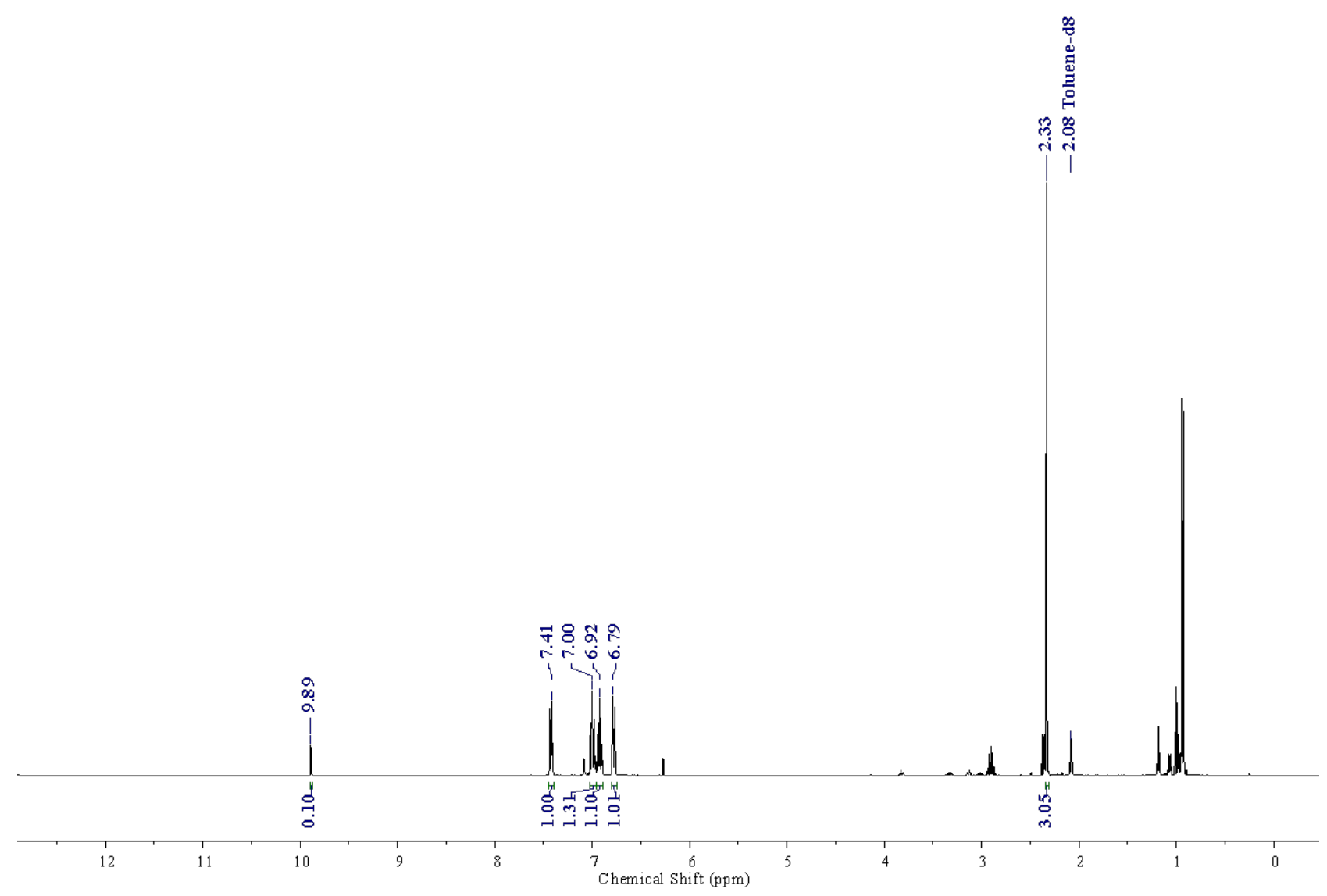


Entry 7: Reaction in Toluene

SCG-291-A2-16hr-D 1-50sec.1.fid

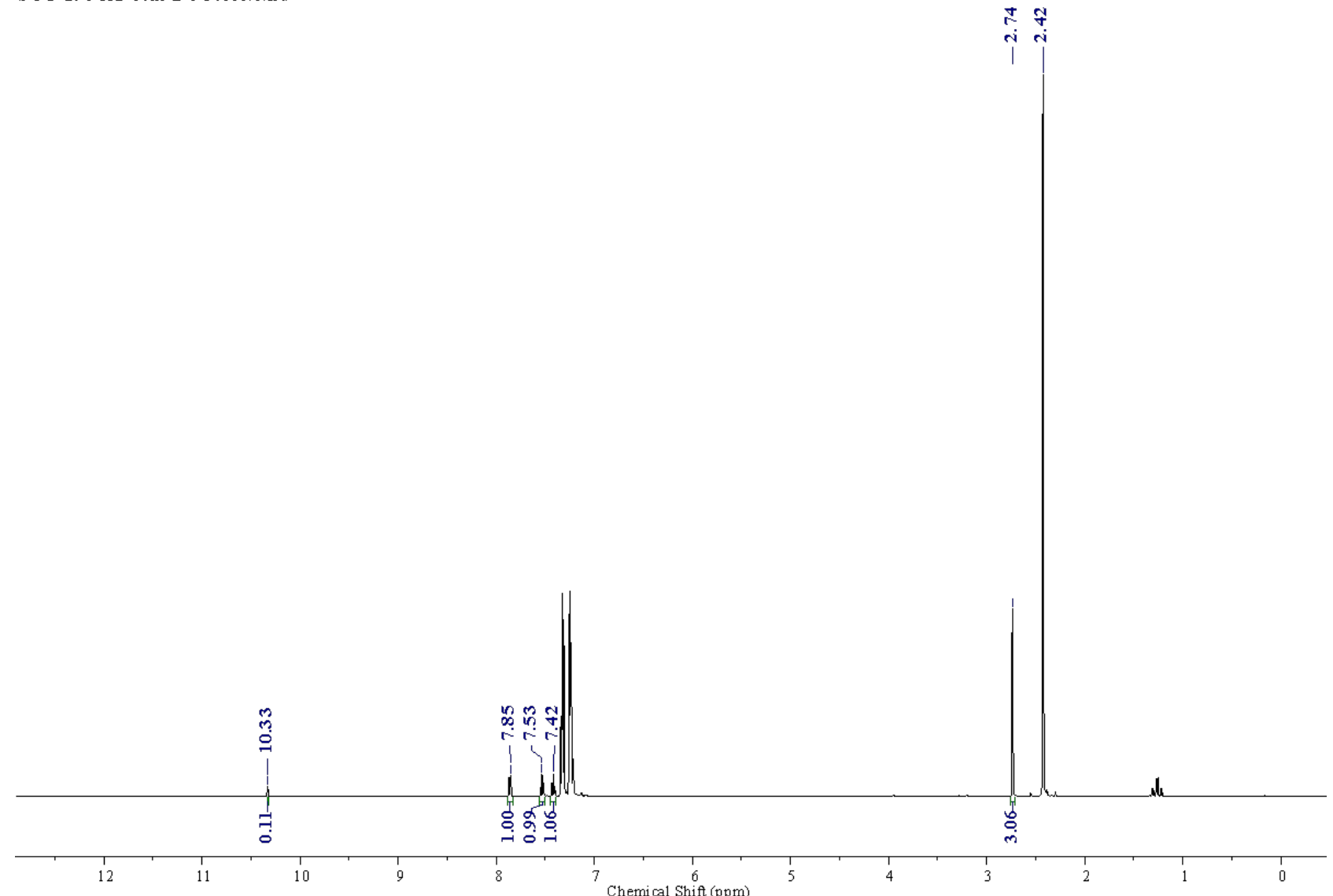

Entry 8:

SCG-307-E1-16hr-D 1-50sec.1. fid

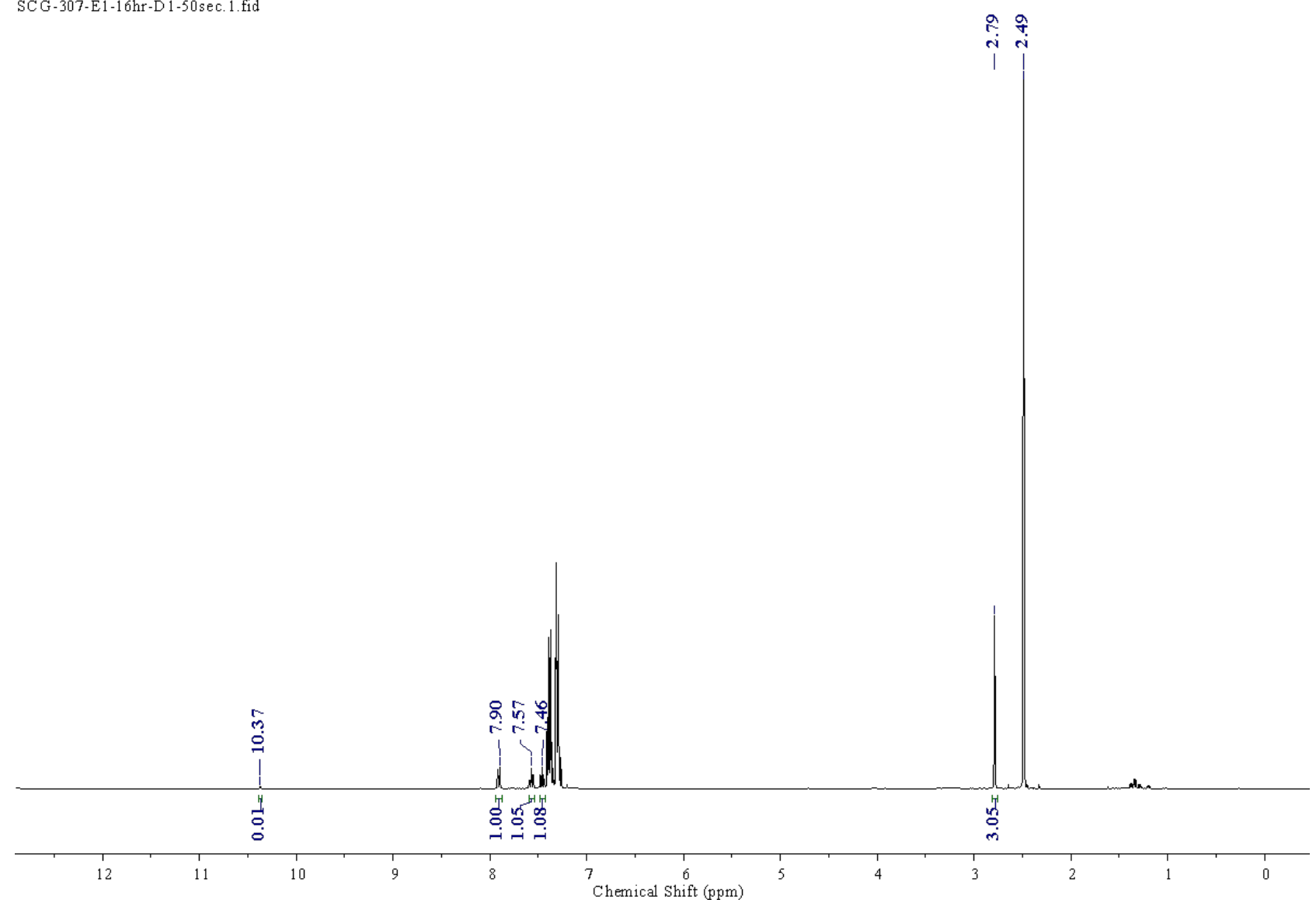


Entry 9:

SCG-294-K-16hr-D1-50sec.1. fid

$\overrightarrow{0}$

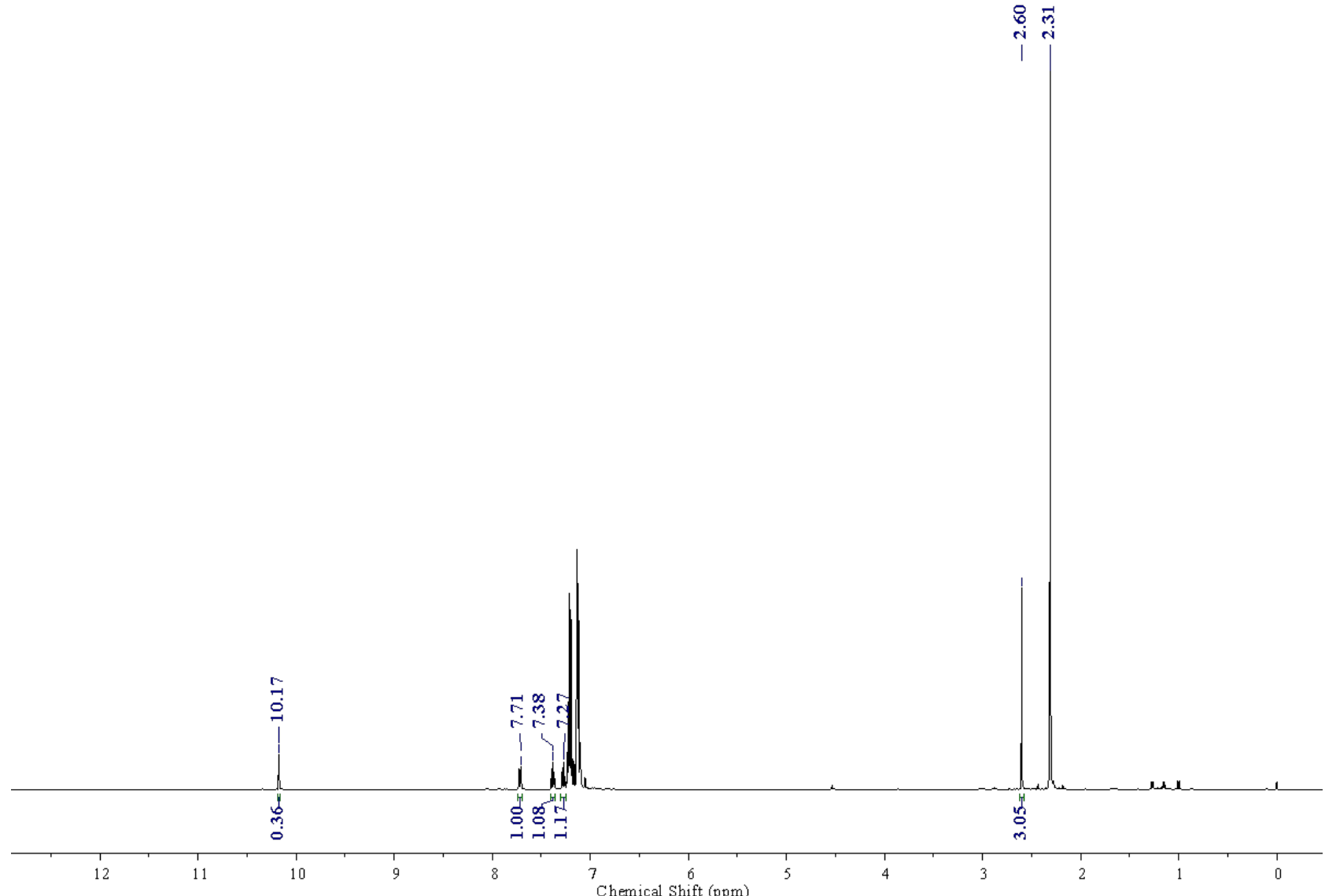

Entry 10:

SCG-294-J-1 6hr-D1-50sec.1. fid

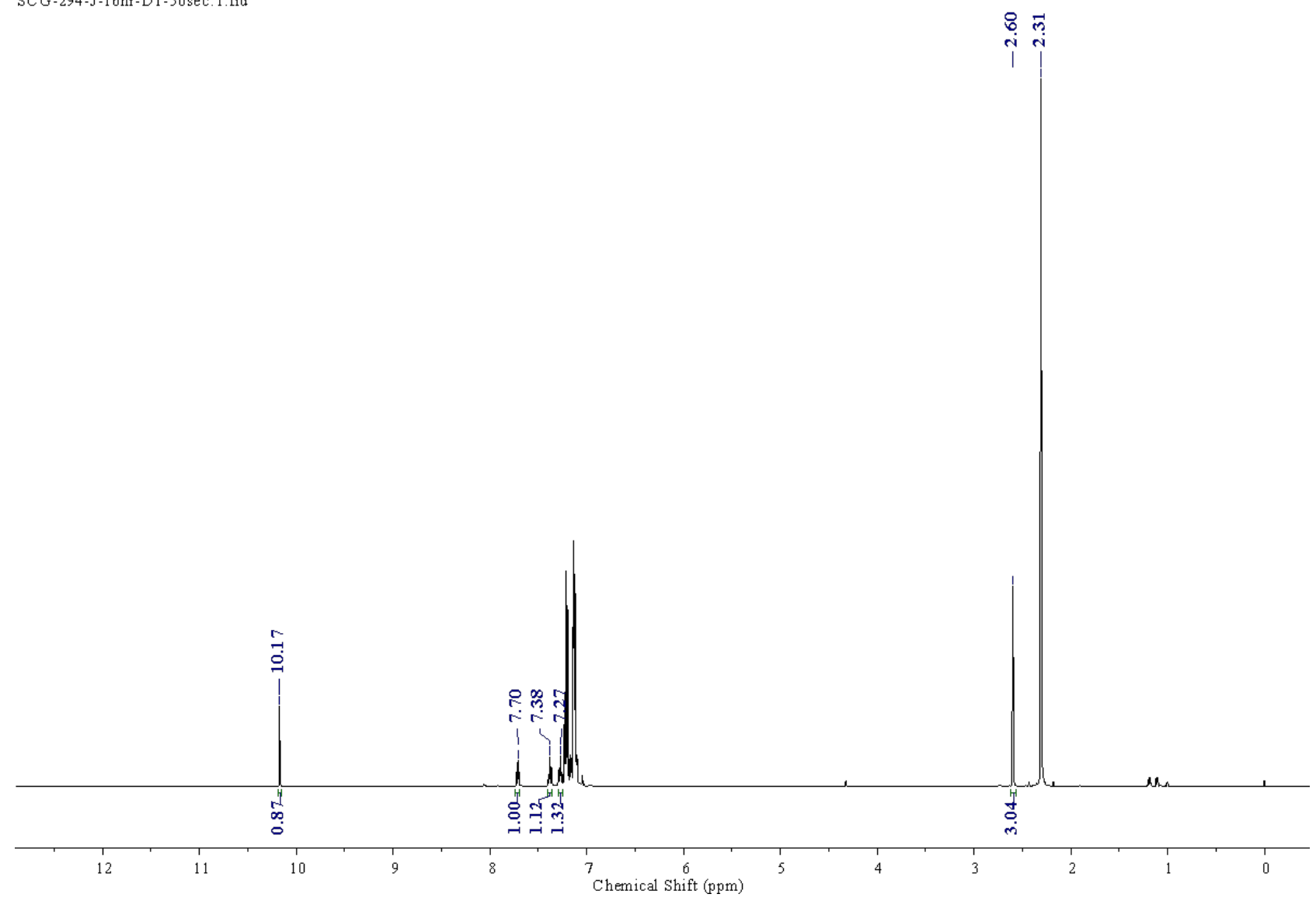


Optimization with 4-(Trifluoromethyl)benzaldehyde and Effect of Phenyl Boroxine:

Entry 1:

SCG-319-A3-16hr-D1-50sec.1 fid

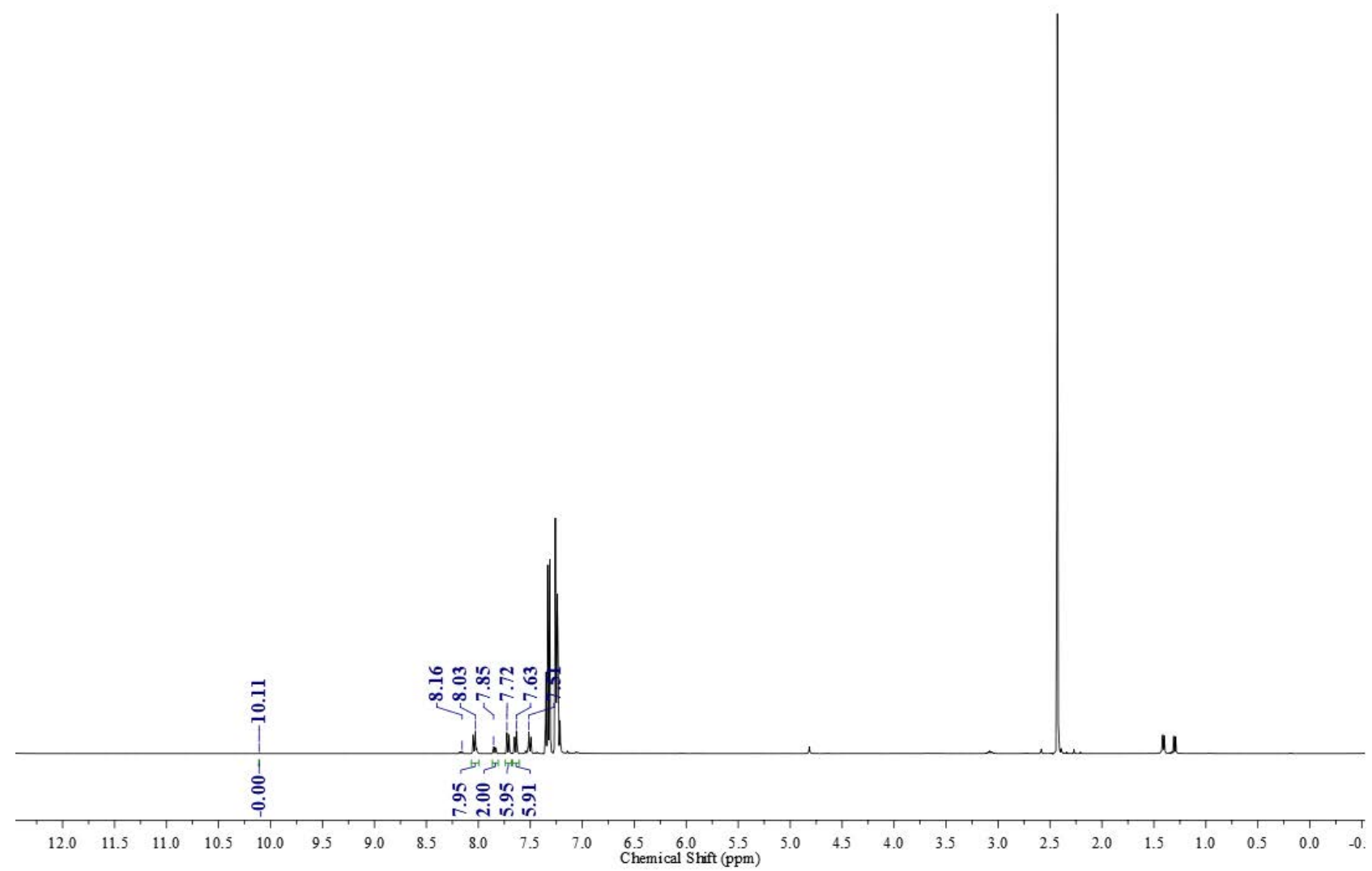

Entry 2:

SCG-292-F2-16hr-D 1-50sec. 1.fid

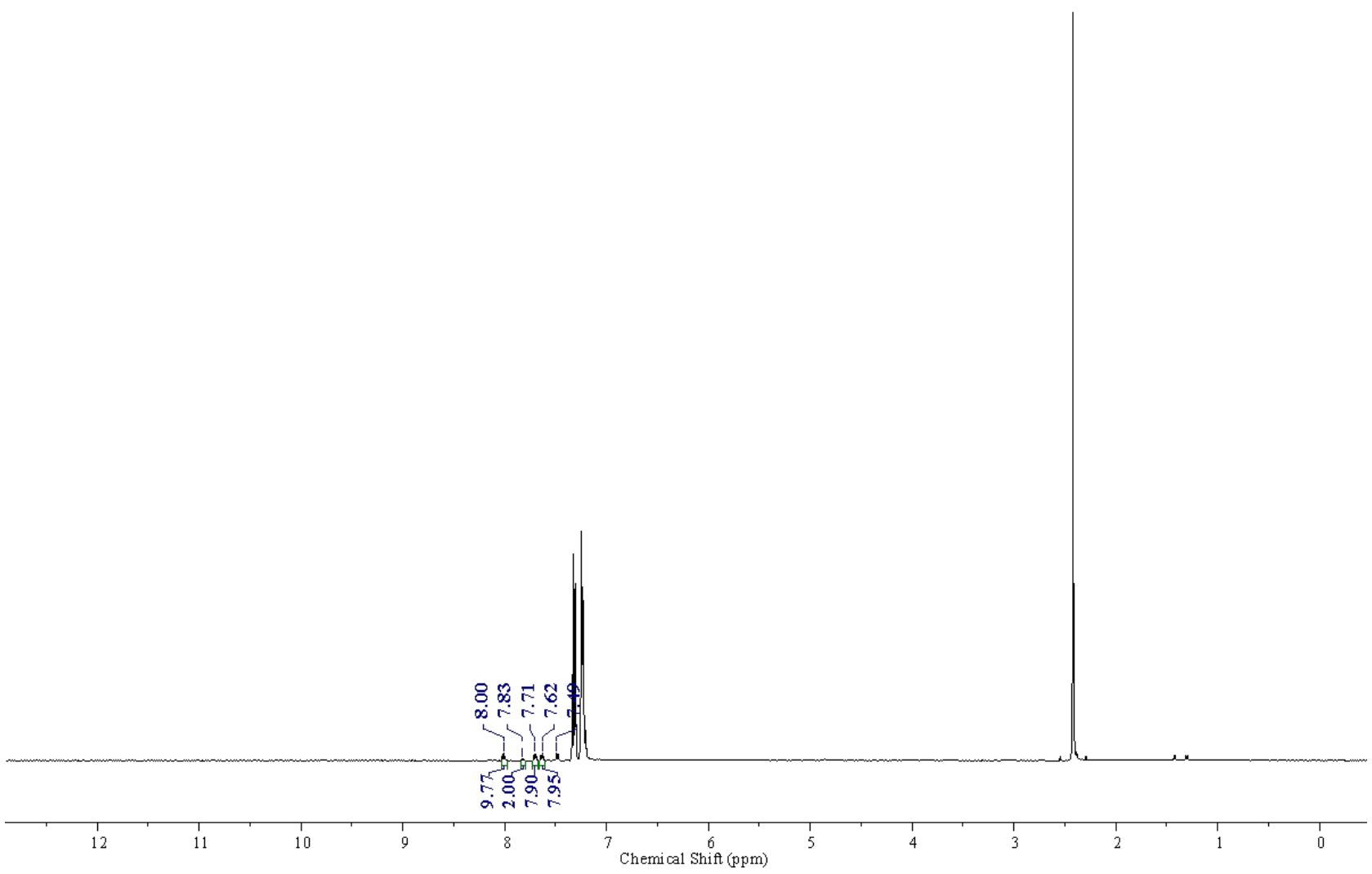


Entry 3:

SCG-293-M3-16hr-D 1-50sec.1.fid

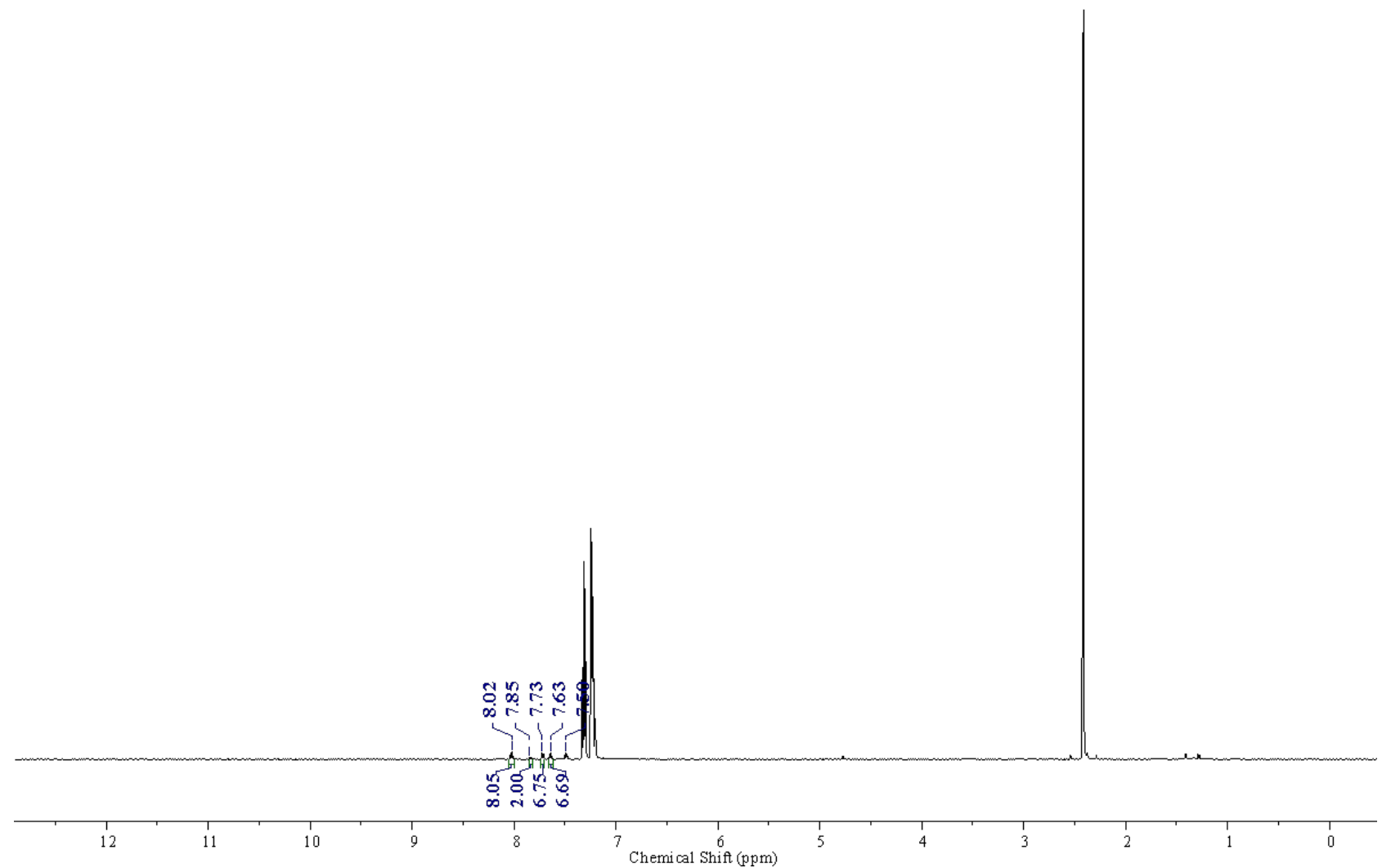

Entry 4:

SCG-293-M-16hr-D1-50sec.1.fid

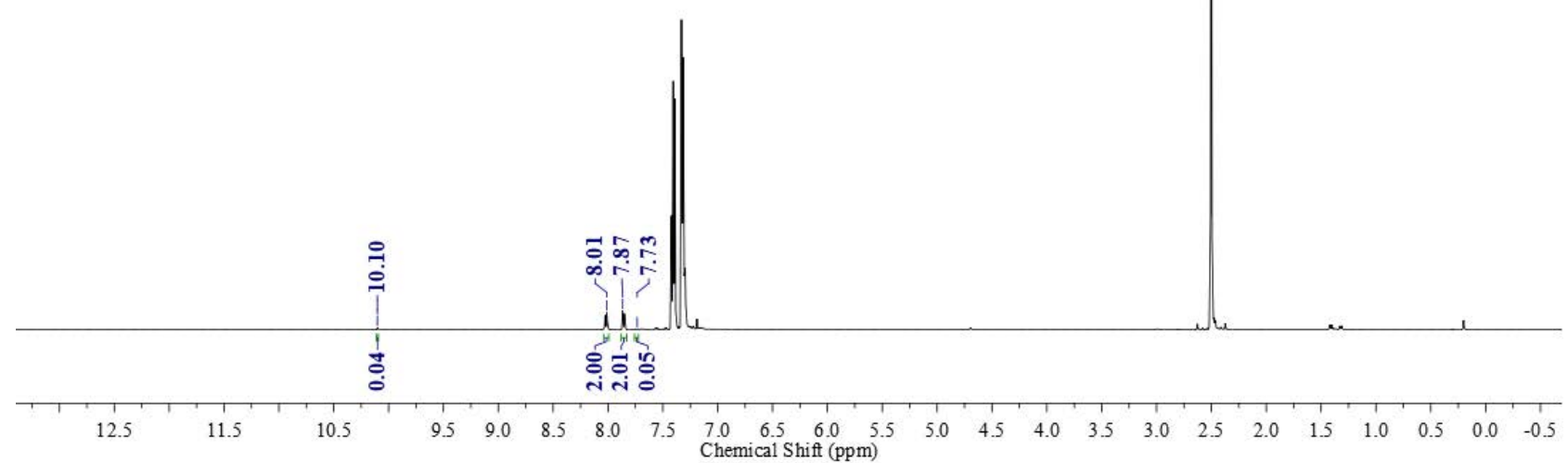


Entry 5:

SCG-293-N-16hr-D1-50sec.1. fid

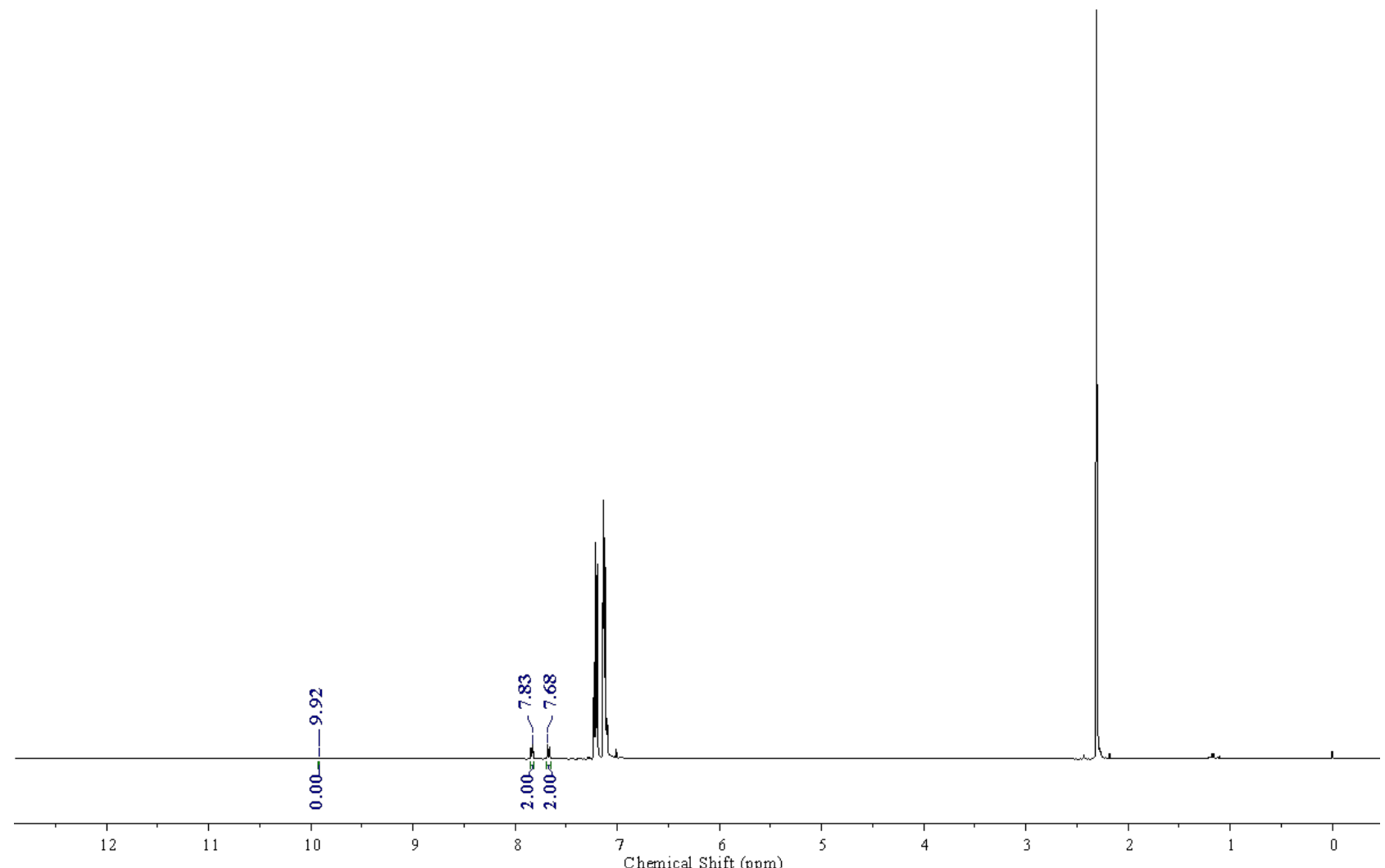

Entry 6:

SCG-293-W-16hr-D1-50sec.1.fid

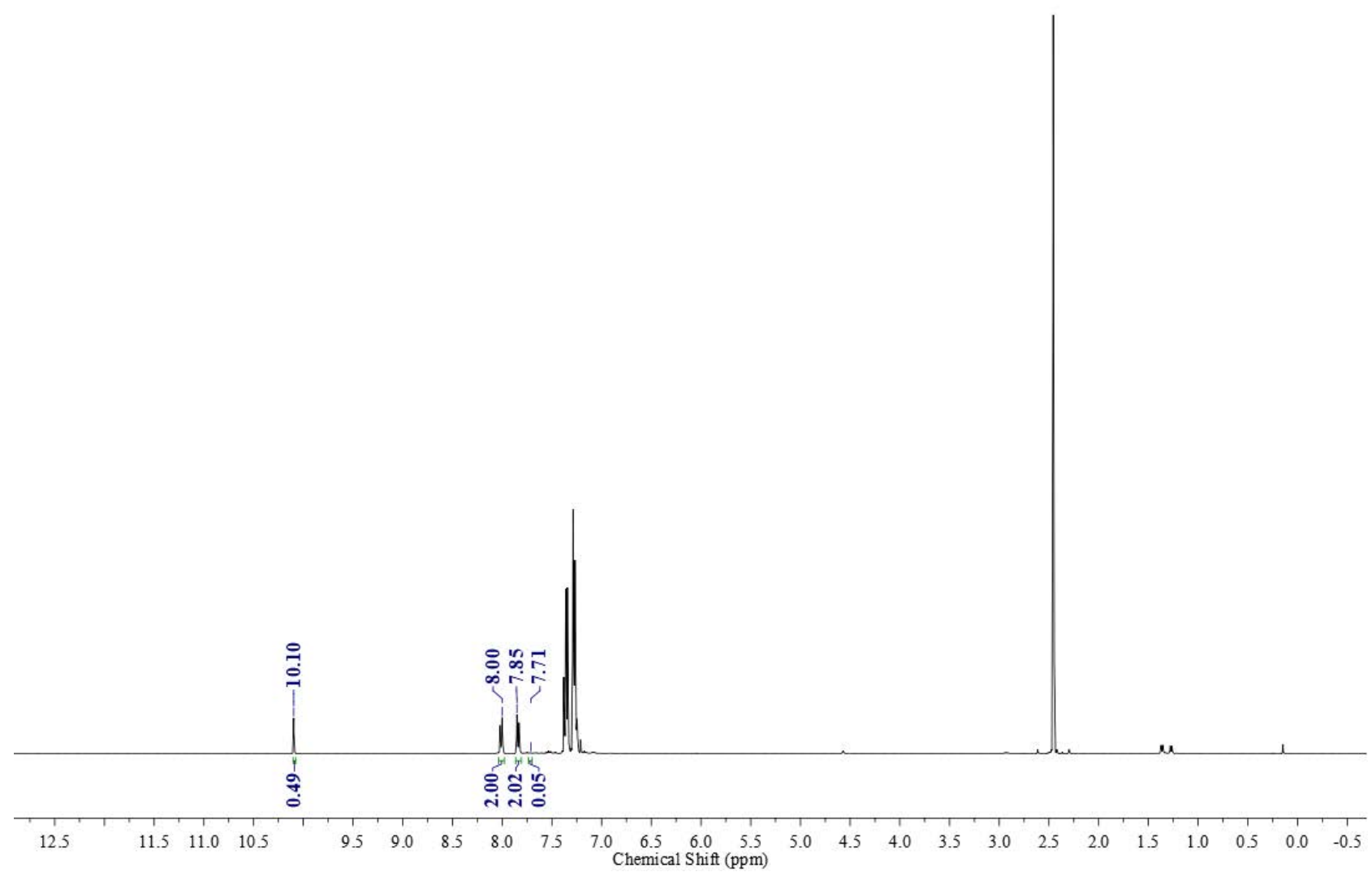


Entry 7:

SCG-293-V-16hr-D1-50sec.1.fid

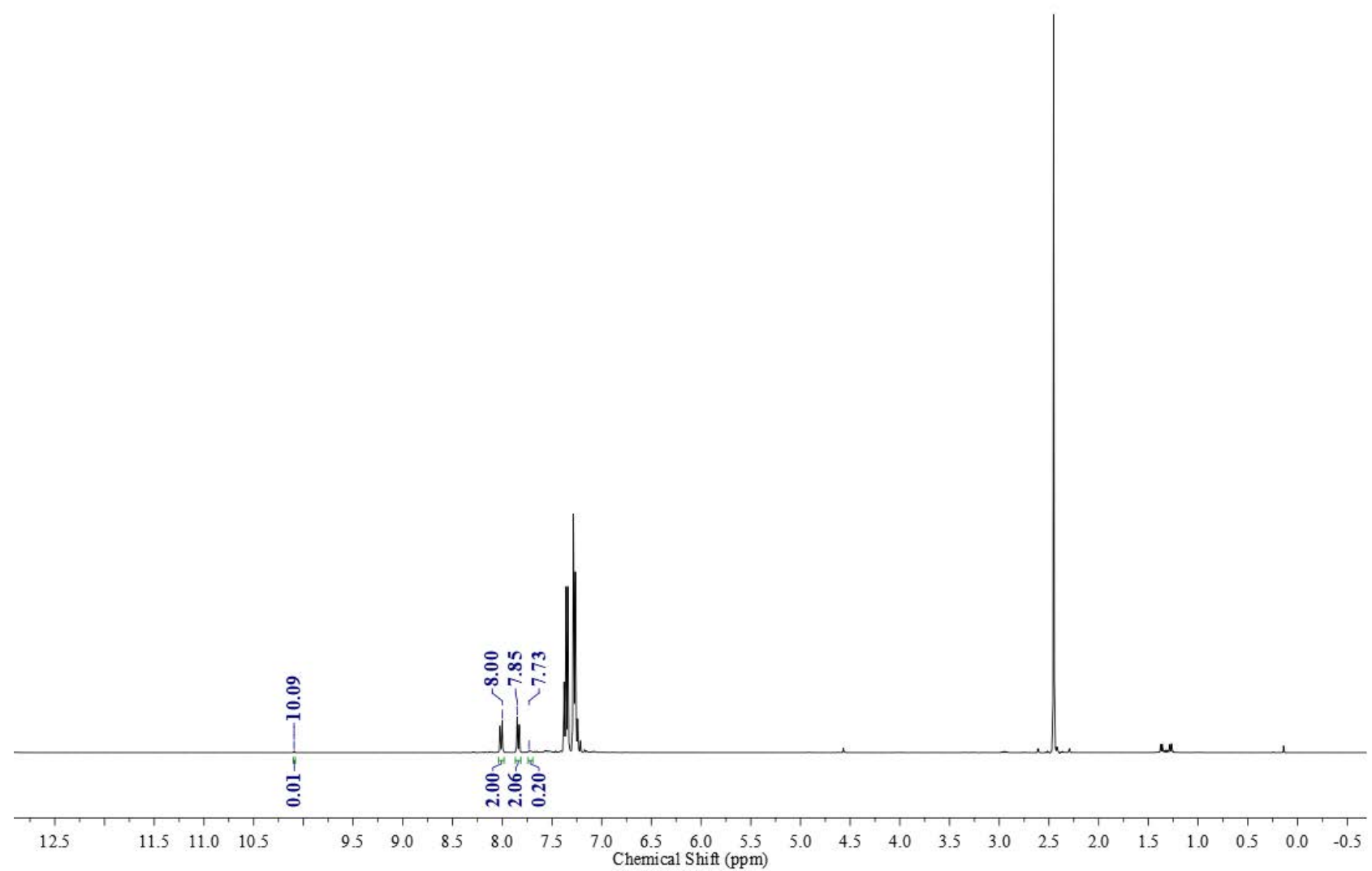

Entry 8:

SCG-292-G-16hr-D1-50sec.1 fid

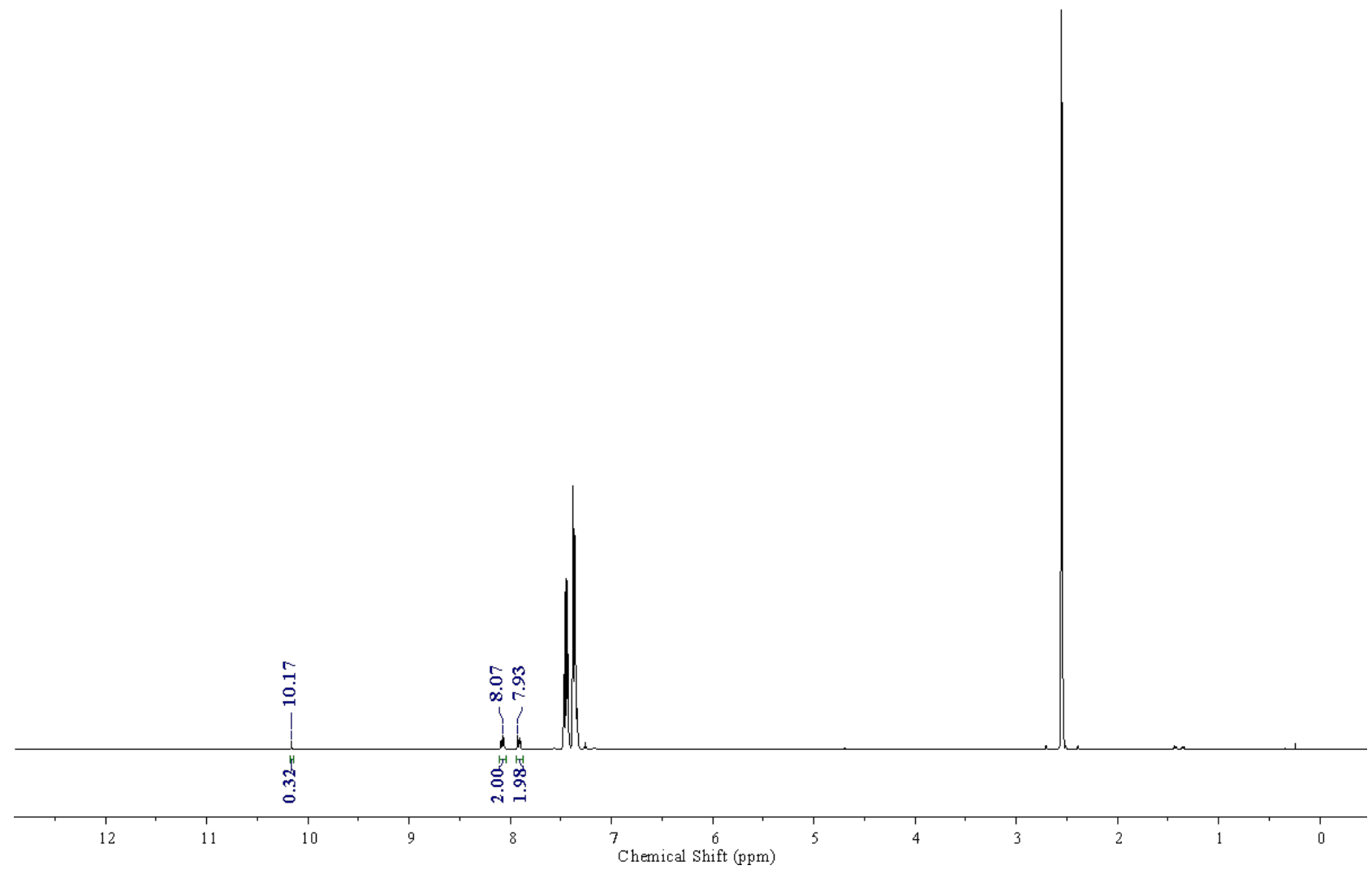


Entry 9:

SCG-293-H-16hr-D 1-50sec .1. fid

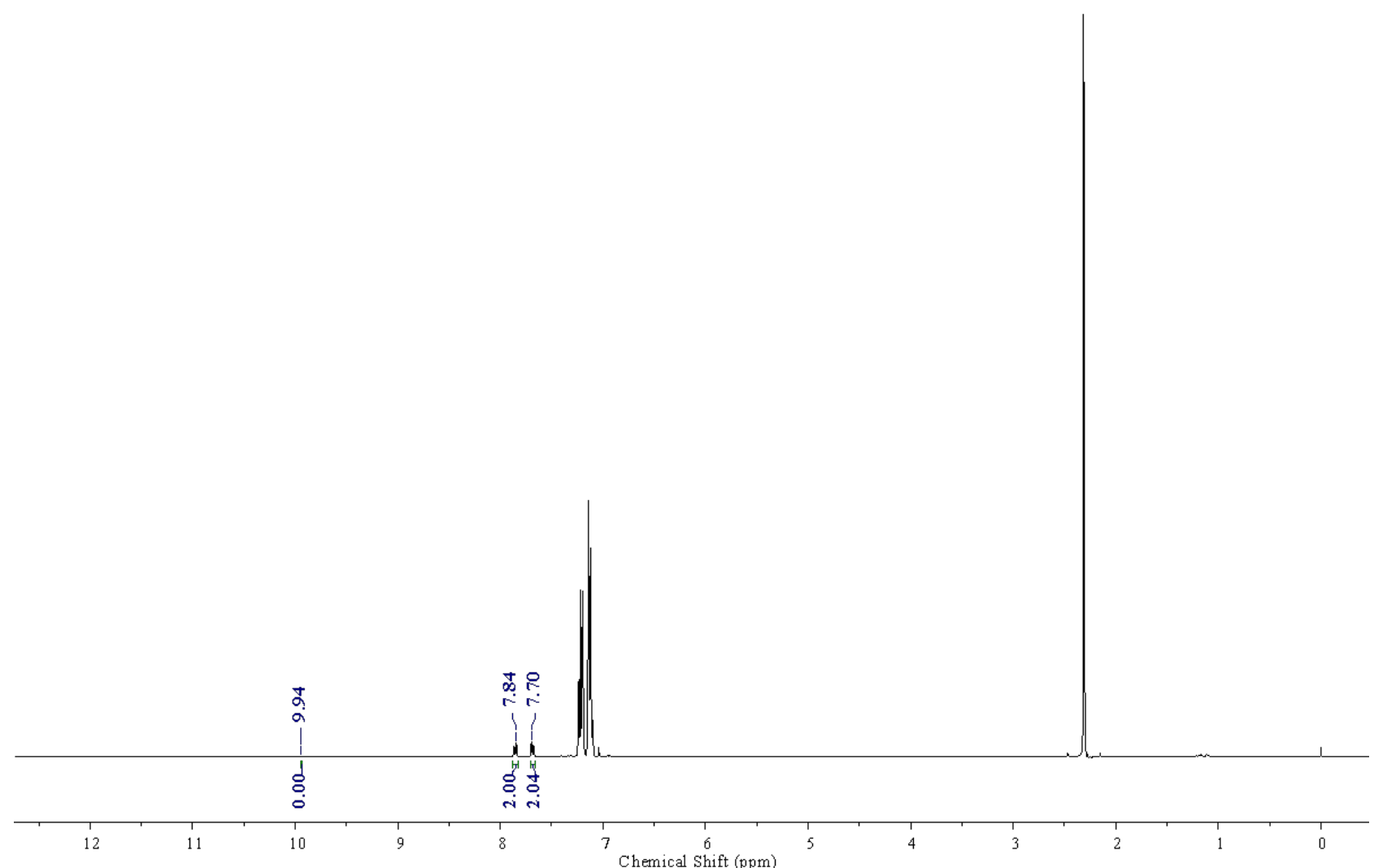

Entry 10:

SCG-293-G-16hr-D1-50sec.1 fid

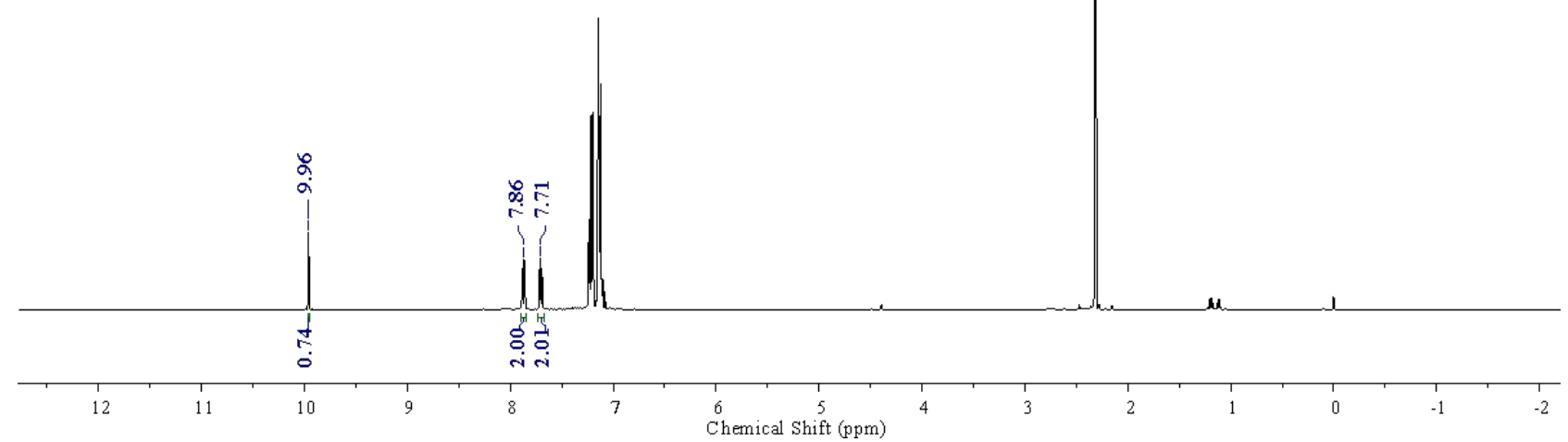


Entry 11:

SCG-293-U-16hr-D1-50sec.1. fid

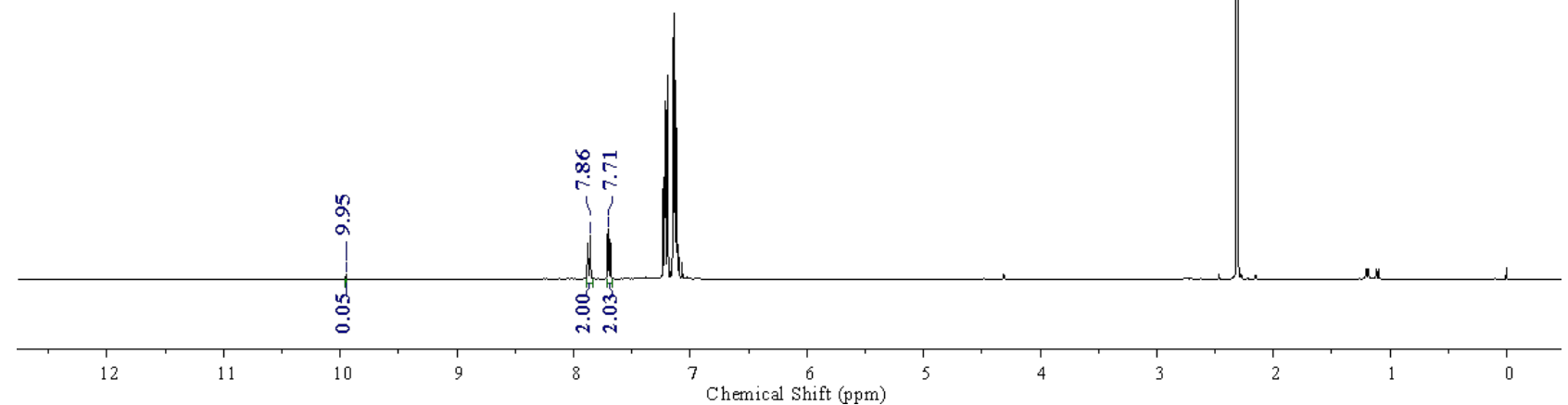


Effect of Different Aryl Boroxines on Deuteration of Aromatic Aldehydes:

Entry 1: With 4-(trifluoromethyl)phenyl boroxine, Reaction concentration $=1.0 \mathrm{M}, \mathrm{NMR}$ for Crude reaction mixture

SCG-259-A2-1 7hr-D 1-50sec.1. fid

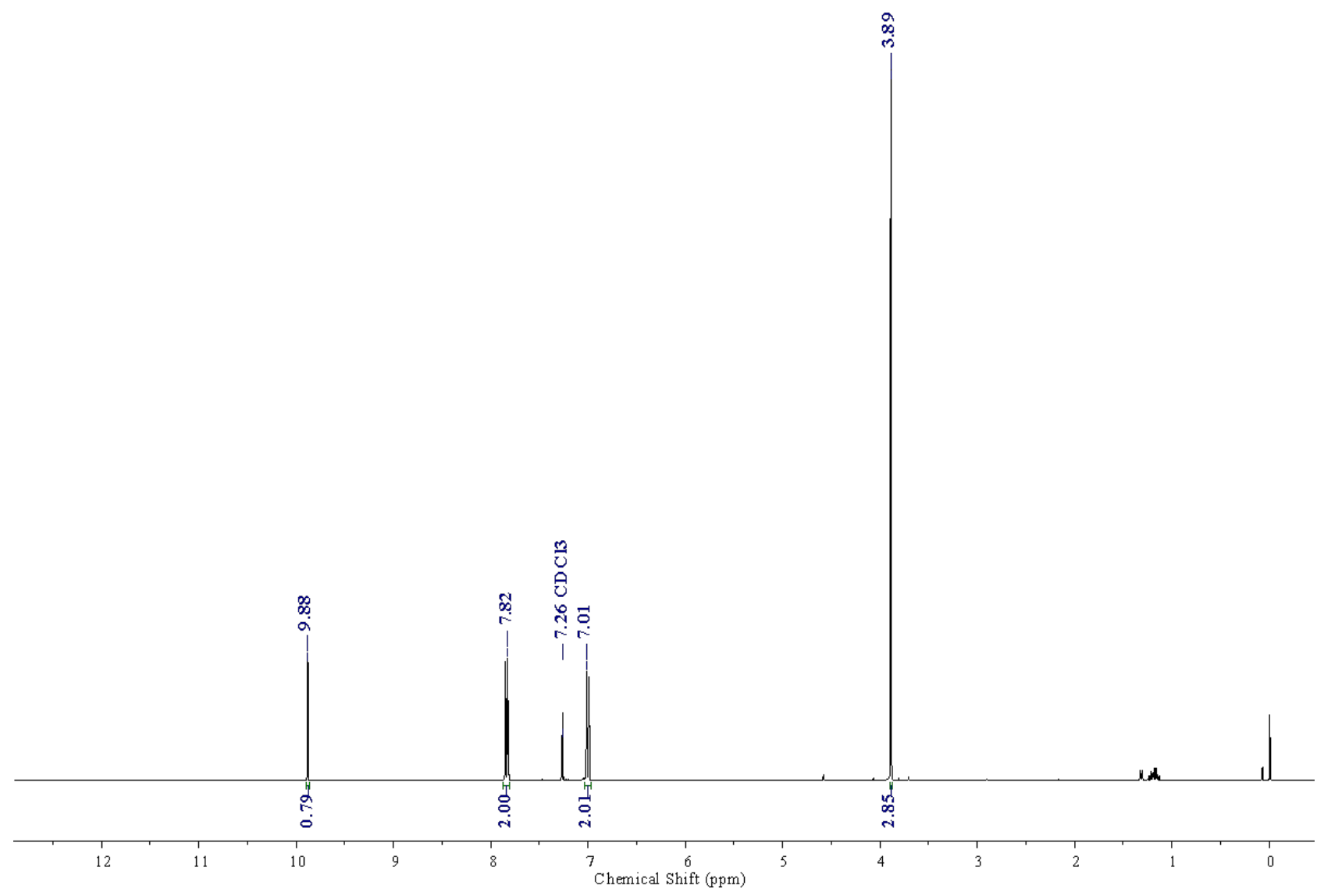


Entry 2: With 4-methoxyphenyl boroxine, Reaction concentration $=1.0 \mathrm{M}, \mathrm{NMR}$ for Crude reaction mixture

SCG-259-B2-17hr-D 1-50sec.1.fid

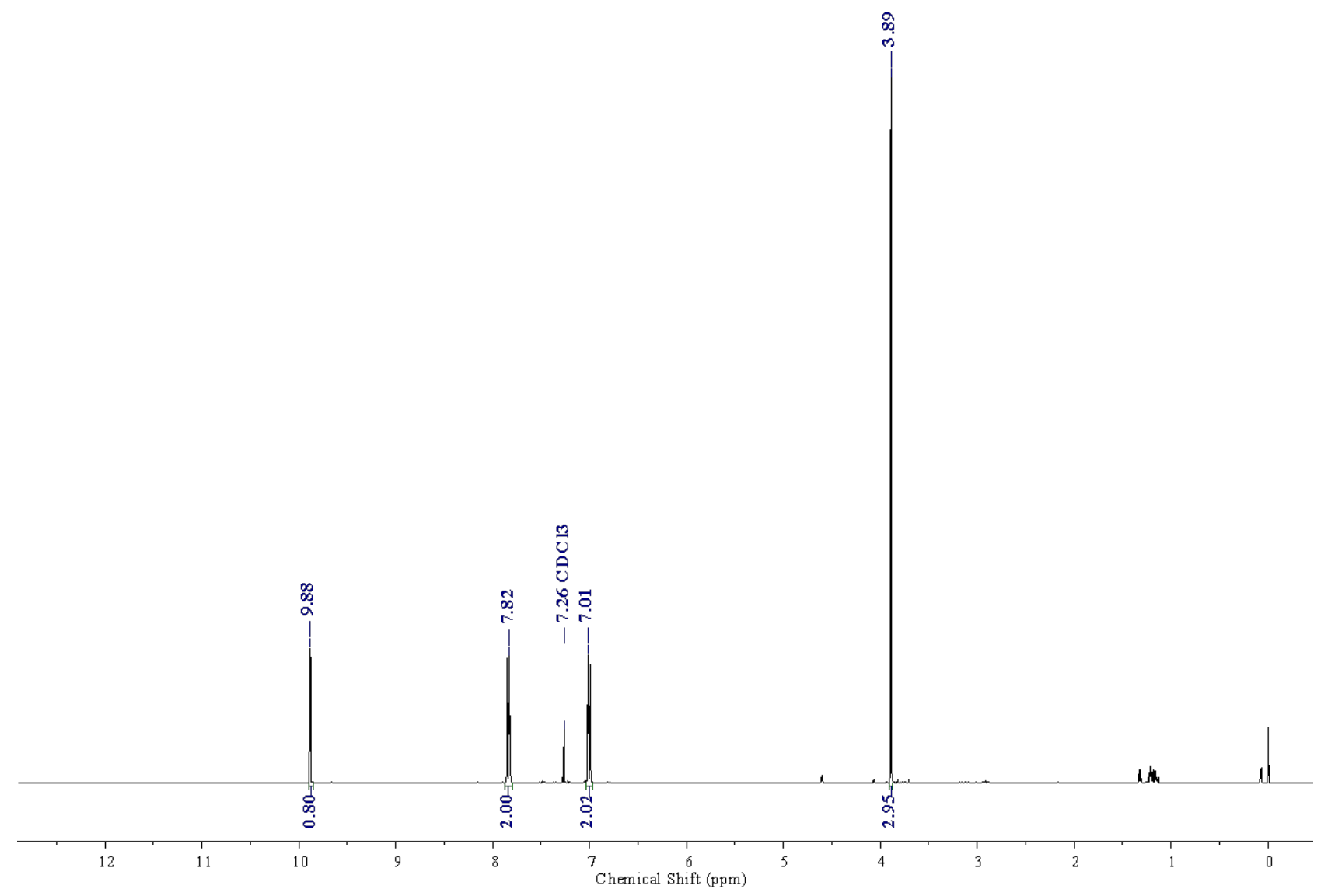


Entry 3: With 4-methylphenyl boroxine, Reaction concentration $=1.0 \mathrm{M}, \mathrm{NMR}$ for Crude reaction mixture

SCG-259-C2-17hr-D 1-50sec.1.fid

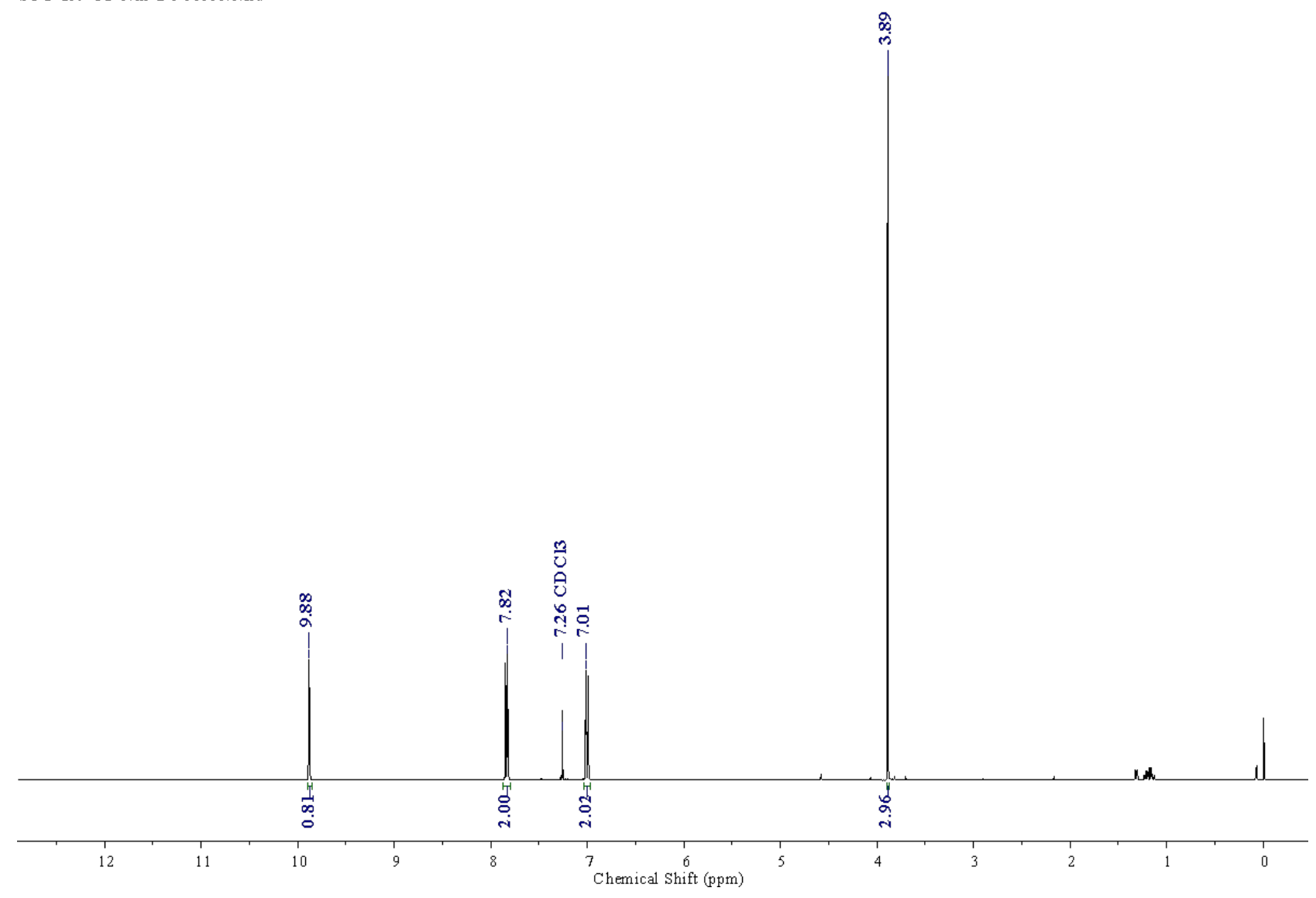


Entry 4: With 4-(trifluoromethyl)phenyl boroxine, Reaction concentration $=0.5 \mathrm{M}, \mathrm{NMR}$ for Crude reaction mixture

SCG-259-A1-1 thr-D1-50sec.1. fid

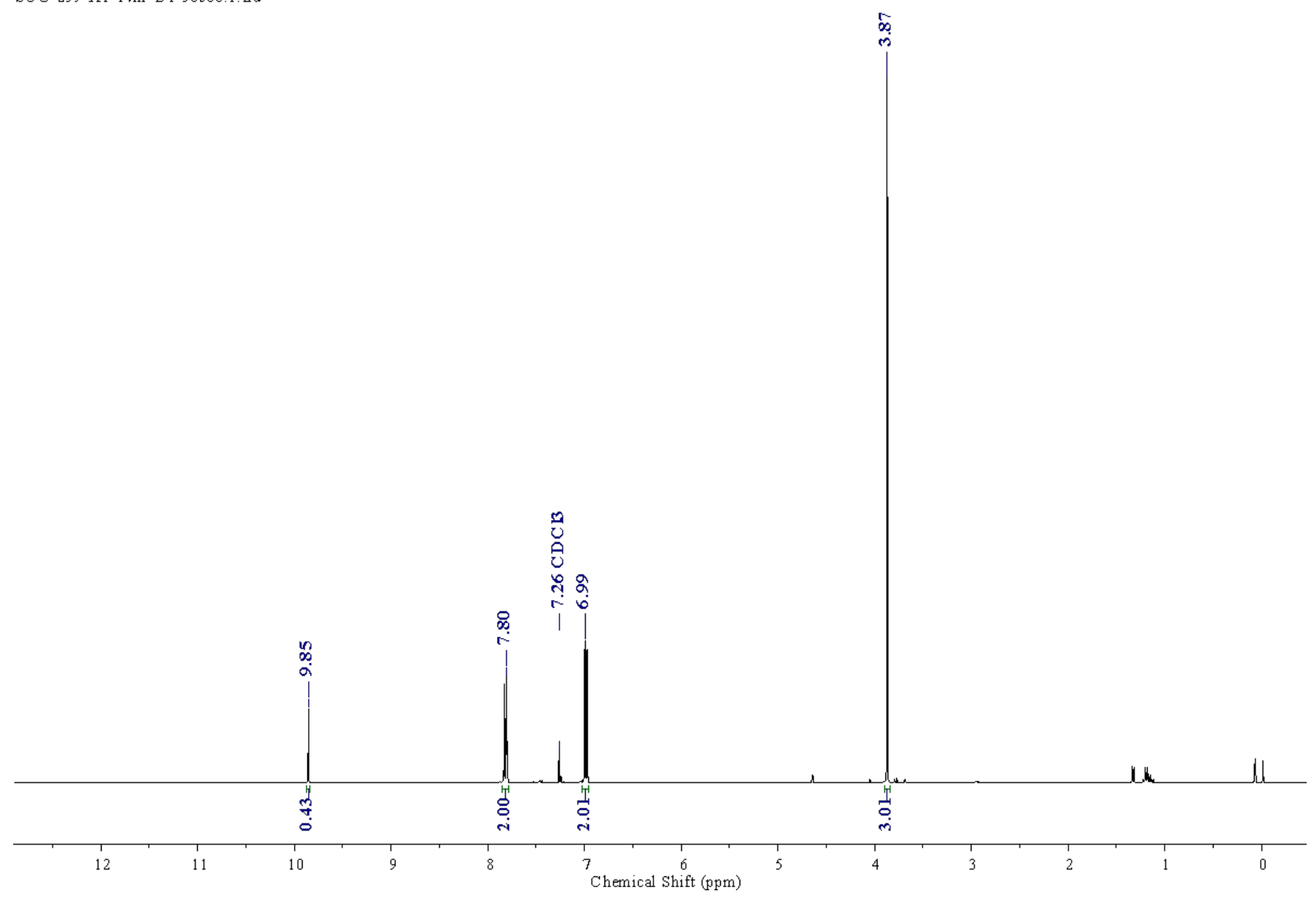


Entry 5: With 4-methoxyphenyl boroxine, Reaction concentration $=0.5 \mathrm{M}, \mathrm{NMR}$ for Crude reaction mixture

SCG-259-B1-17hr-D 1-50sec.1.fid

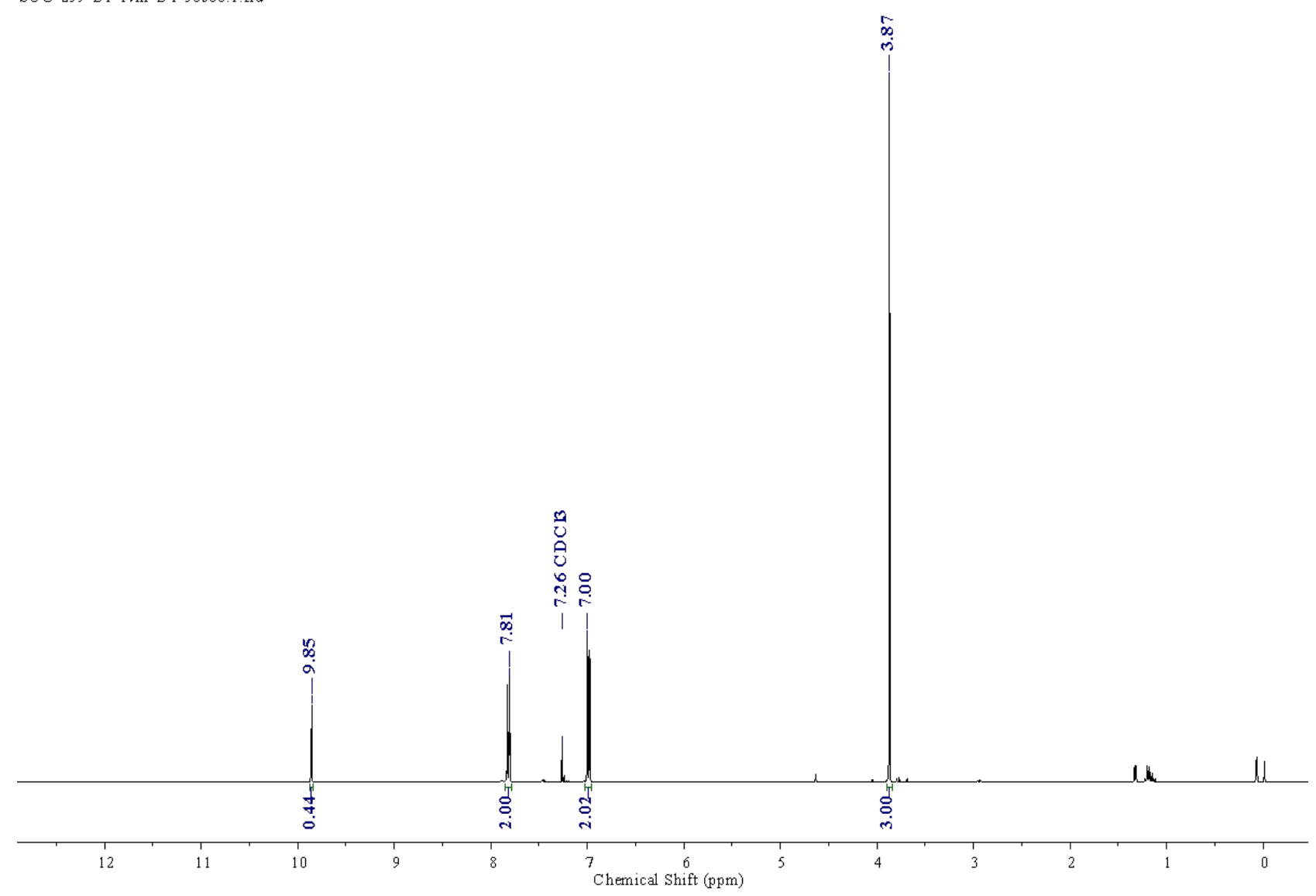


Entry 6: With 4-methylphenyl boroxine, Reaction concentration $=0.5 \mathrm{M}, \mathrm{NMR}$ for Crude reaction mixture

SCG-259-C1-17hr-D 1-50sec.1.fid

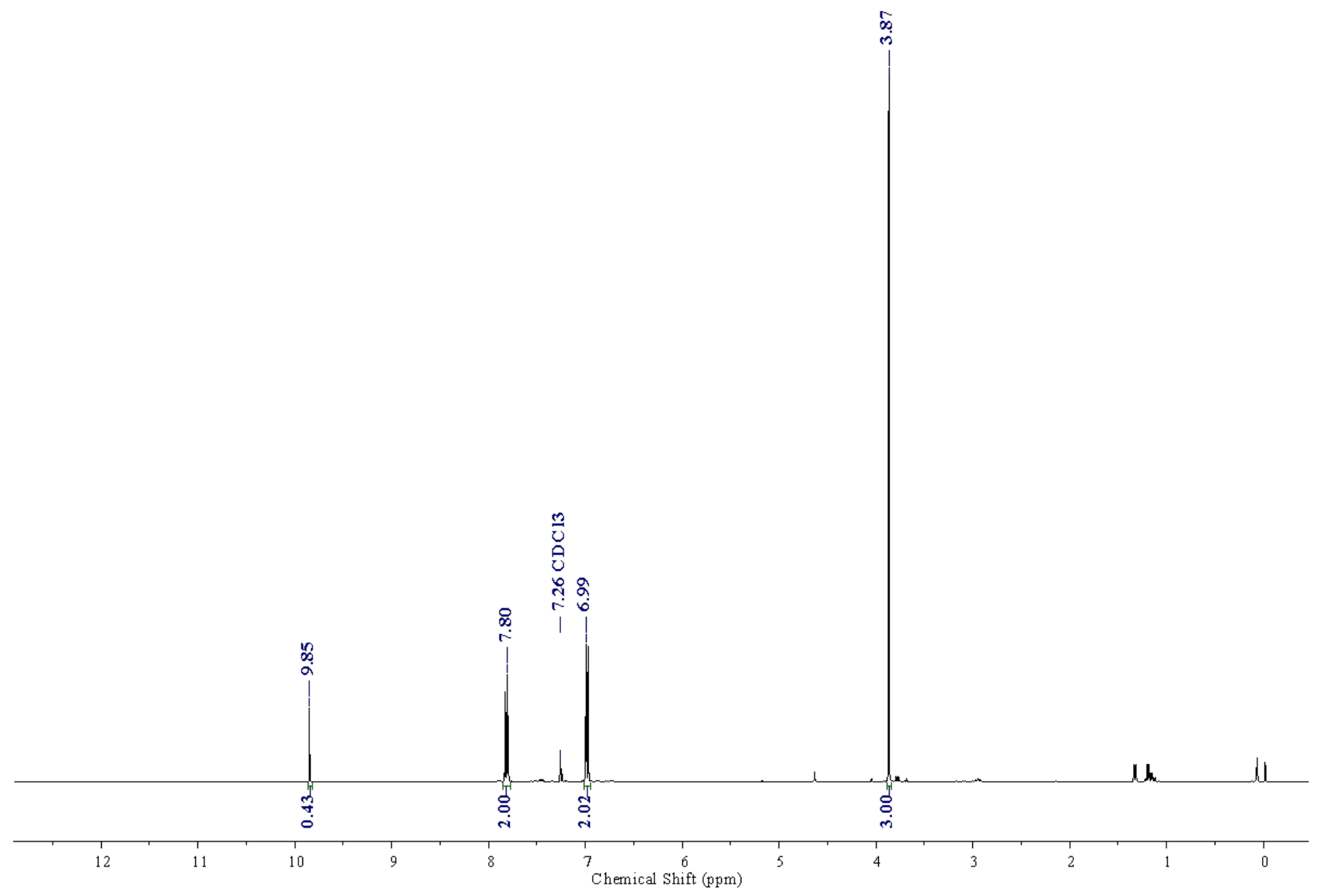


Substrate Scope with 2, 3 and 4-Substituted Benzaldehydes:

1. 2-Methylbenzaldehyde-d<smiles>[2H]C(=O)c1ccccc1C</smiles>

Entry 1: NMR for Crude reaction mixture VDOS-597-A 1.3.fid

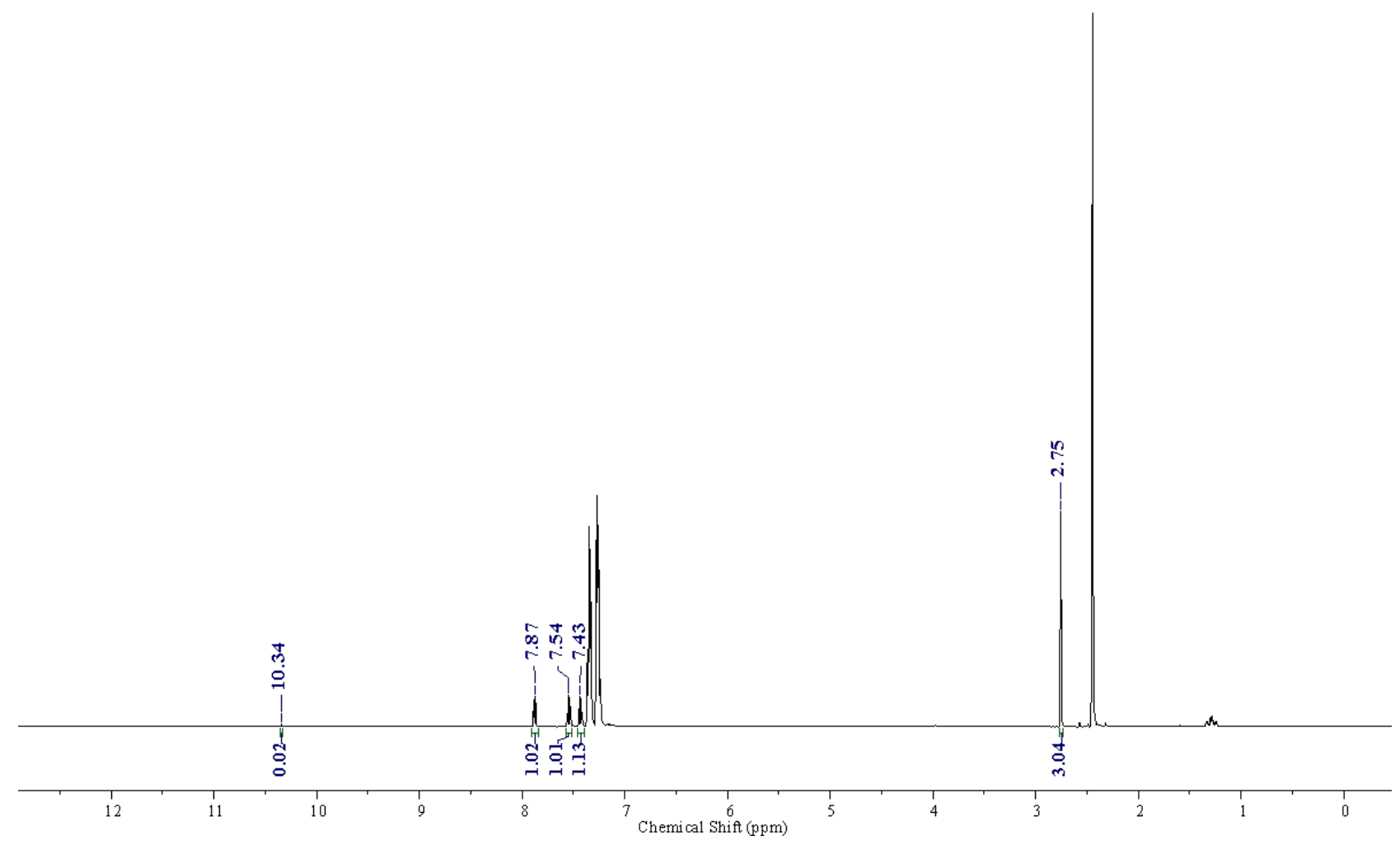


Entry 1': NMR for Crude reaction mixture, Duplicate

VDOS-597-A.2.4.fid

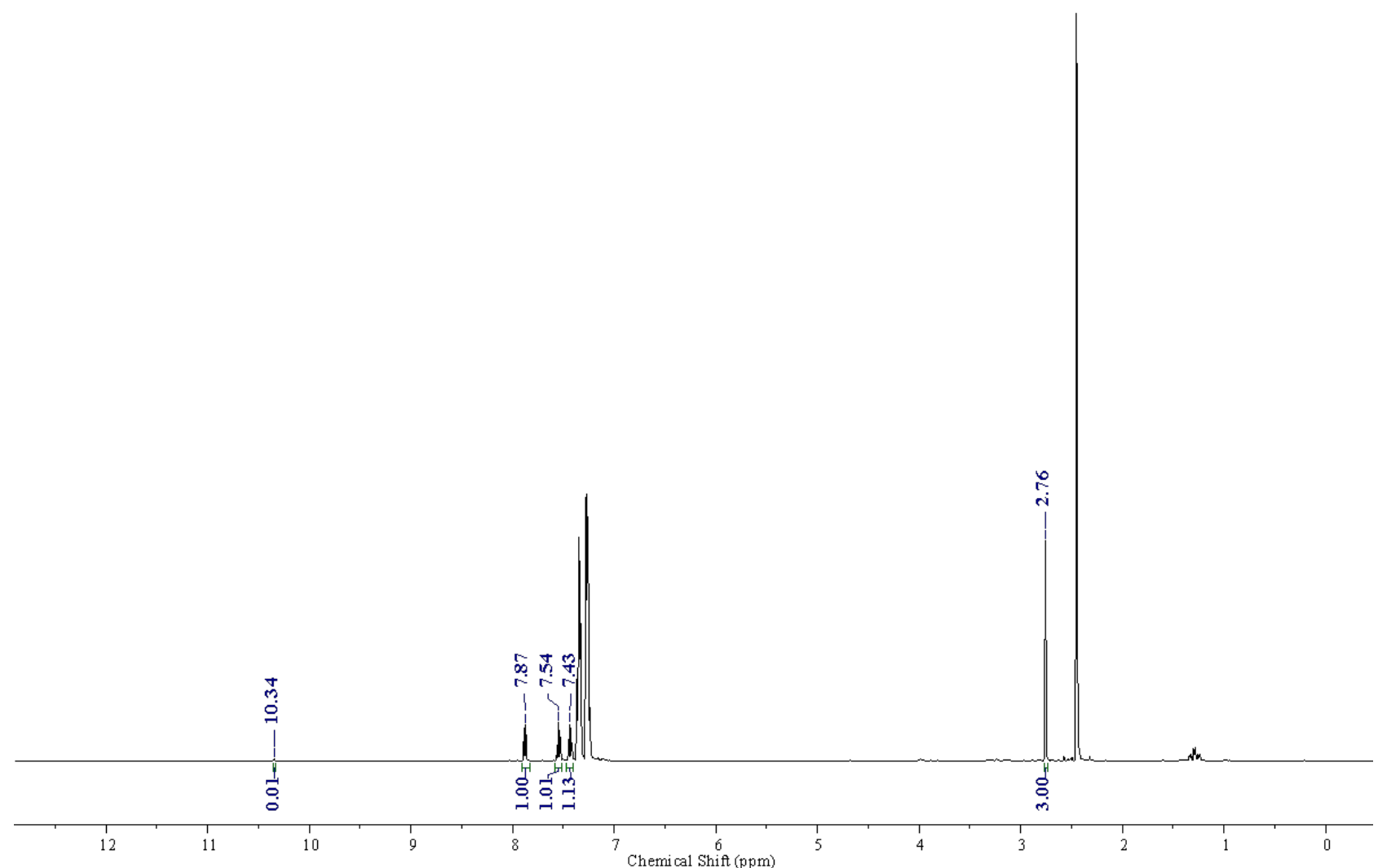

NMR after purification:

VDOS-597-A 2 pure. 1.fid

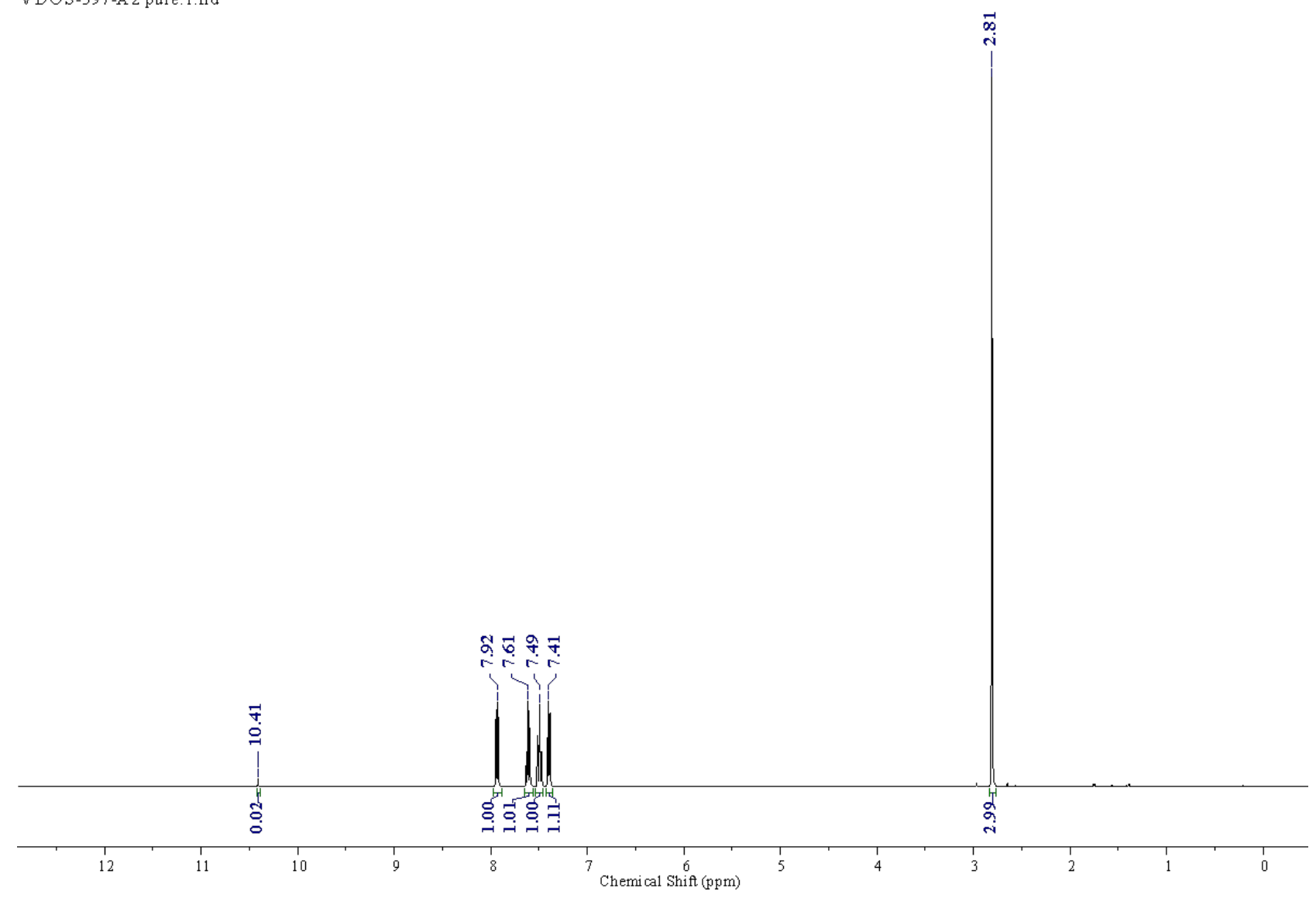




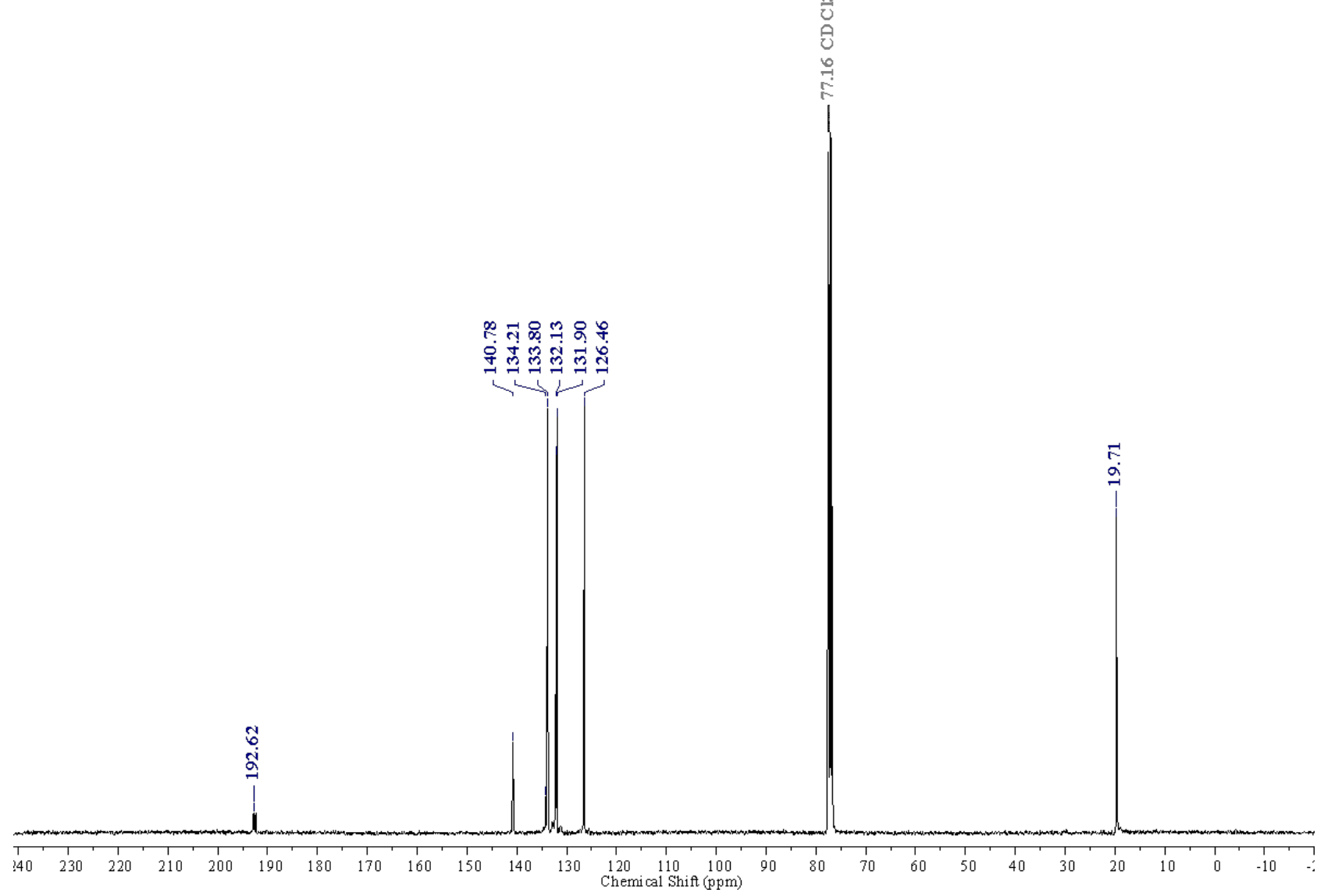


2. 2-Chlorobenzaldehyde-d<smiles>[2H]C(=O)c1ccccc1Cl</smiles>

Entry 1: NMR for Crude reaction mixture SCG-324-A.1-17hr-D 1-50sec.1.fid

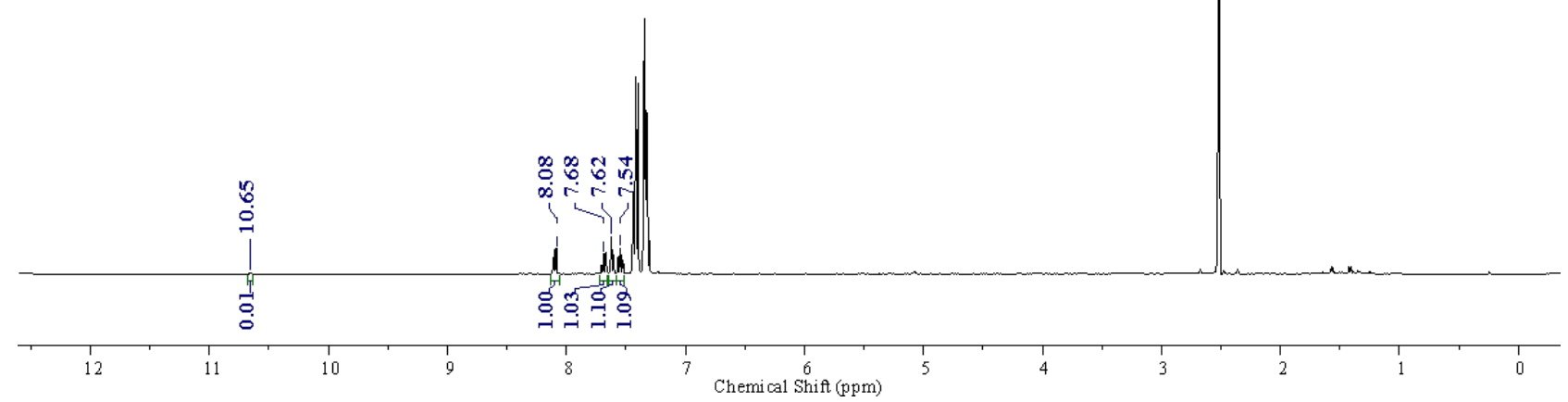


Entry 1': NMR for Crude reaction mixture, Duplicate VDOS-587-A1.19. fid

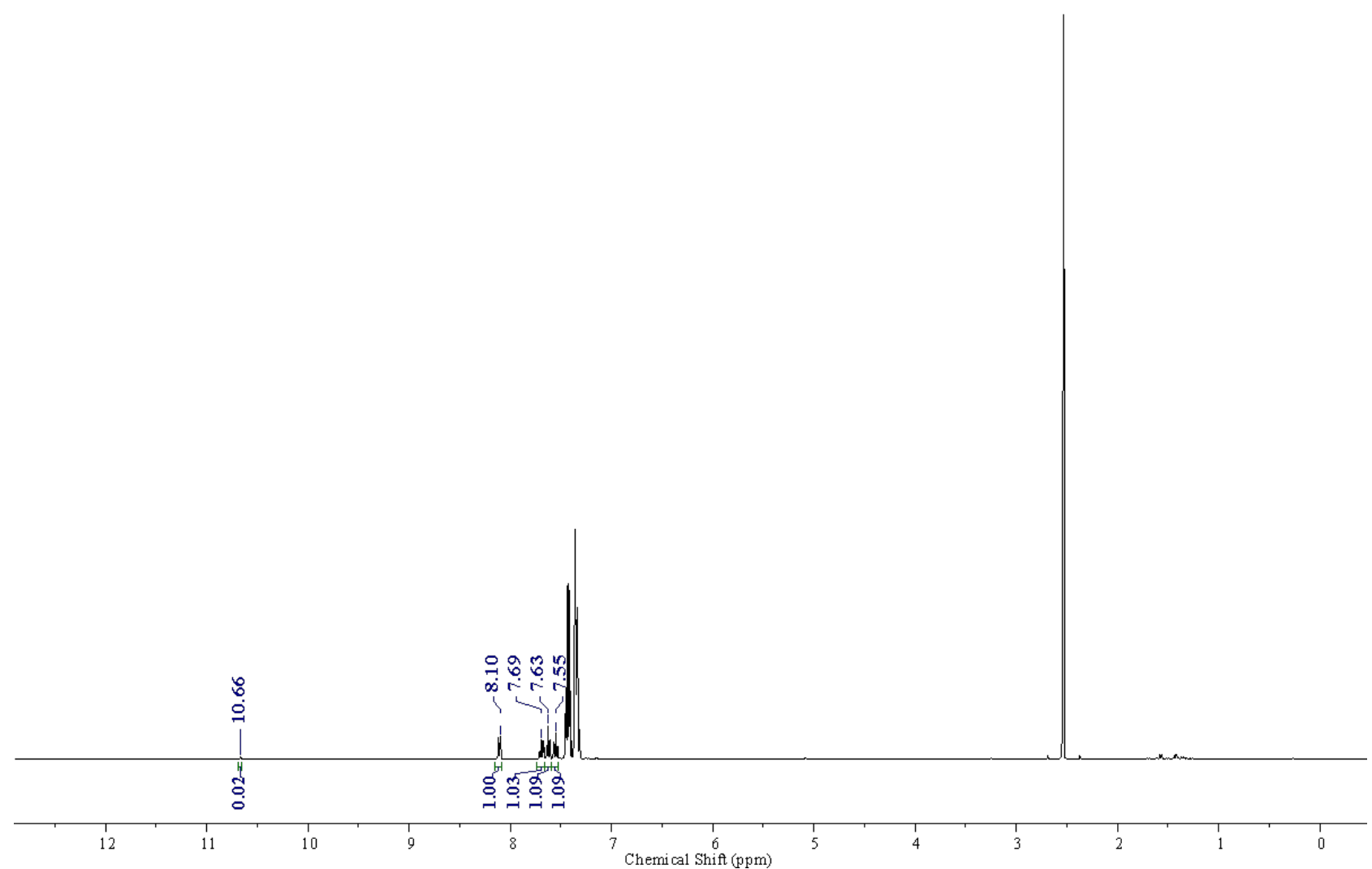

NMR after purification:

SCG-324-Distilled-D 1-50sec.1.fid

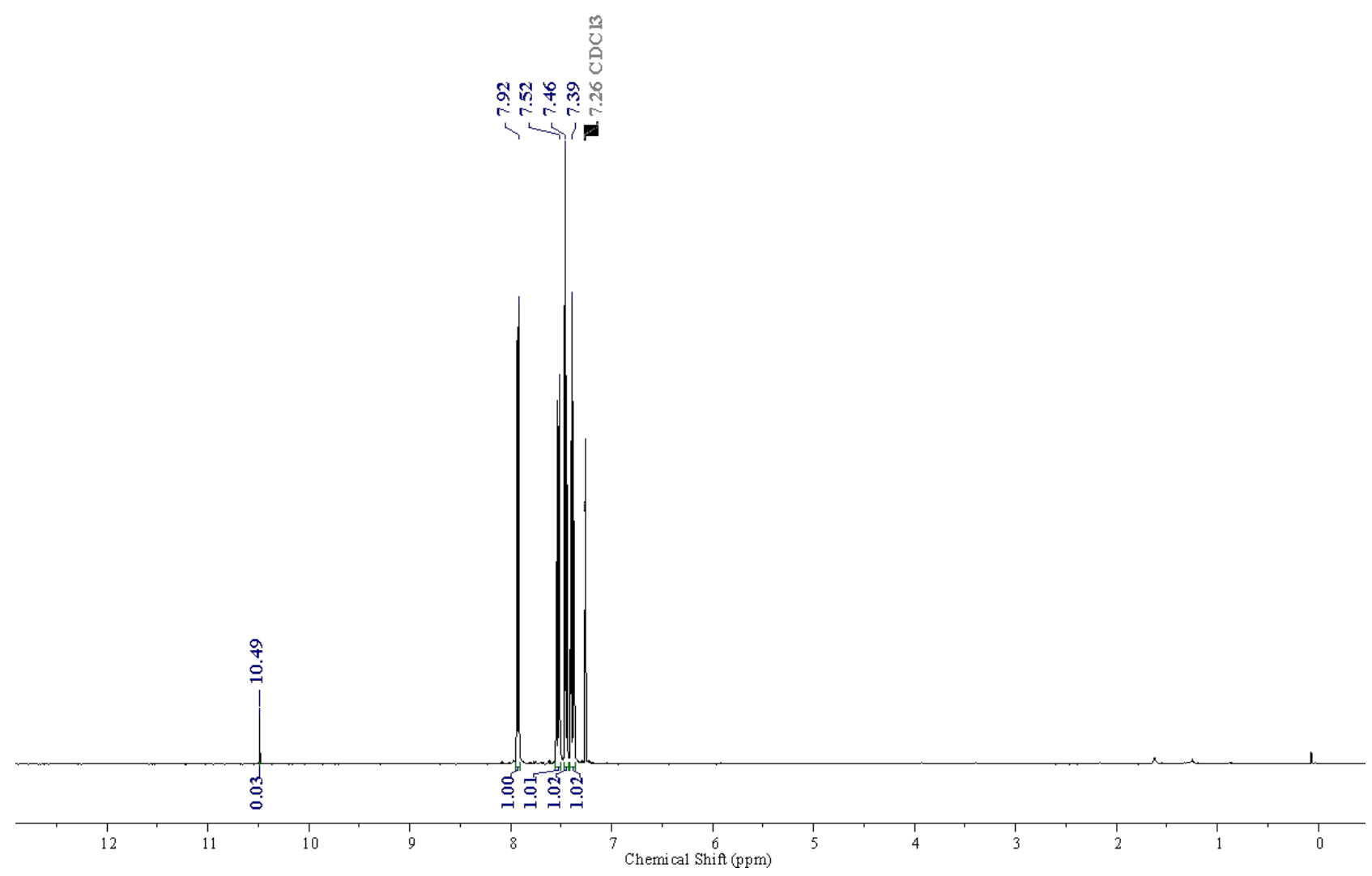


VDOS-587-A 1\&A2-pure 13C.1.fid

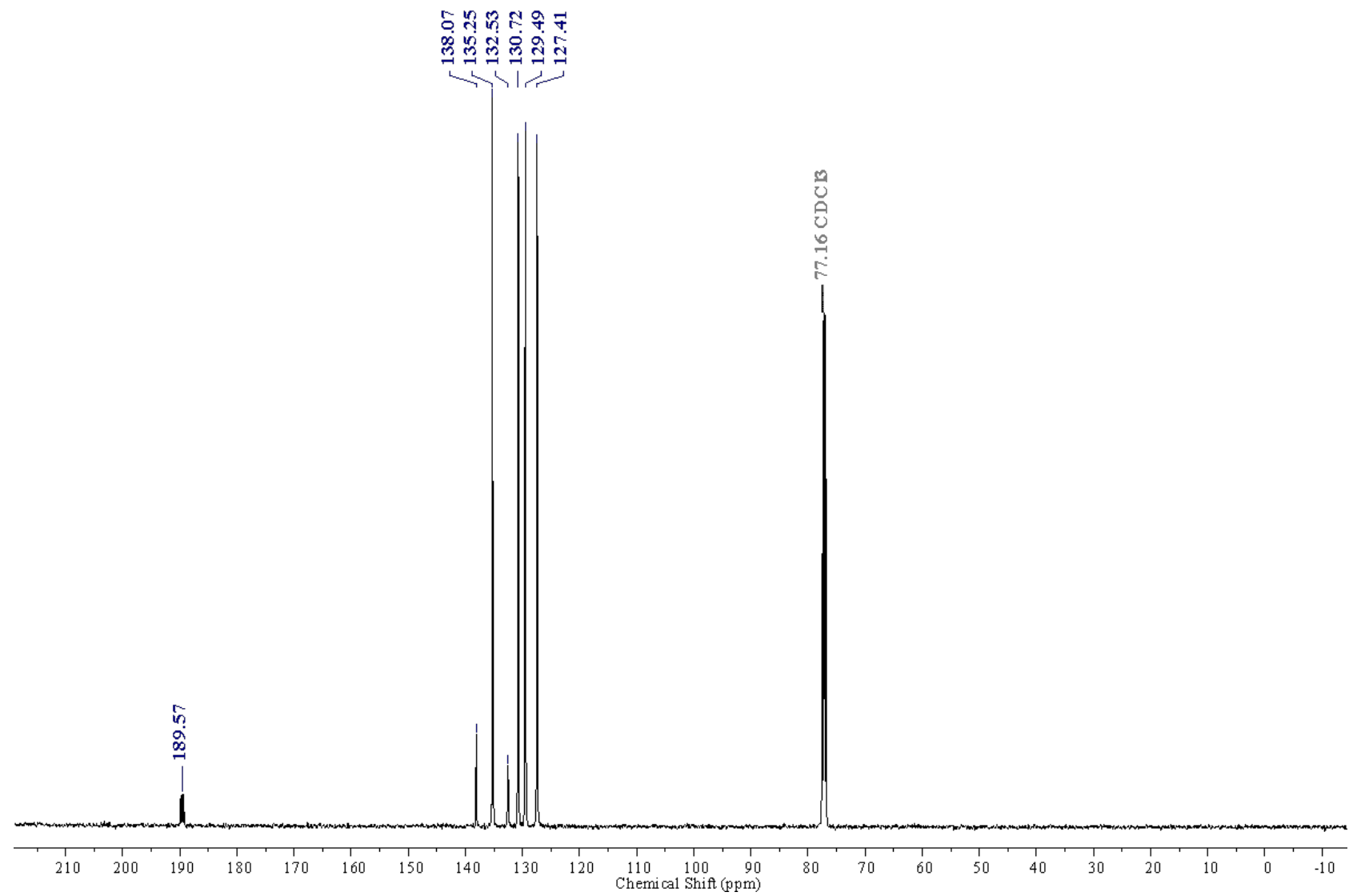


3. 2-Bromobenzaldehyde-d<smiles>[2H]C(=O)c1ccccc1Br</smiles>

Entry 1: NMR for Crude reaction mixture VDOS-590-A.2.2.fid

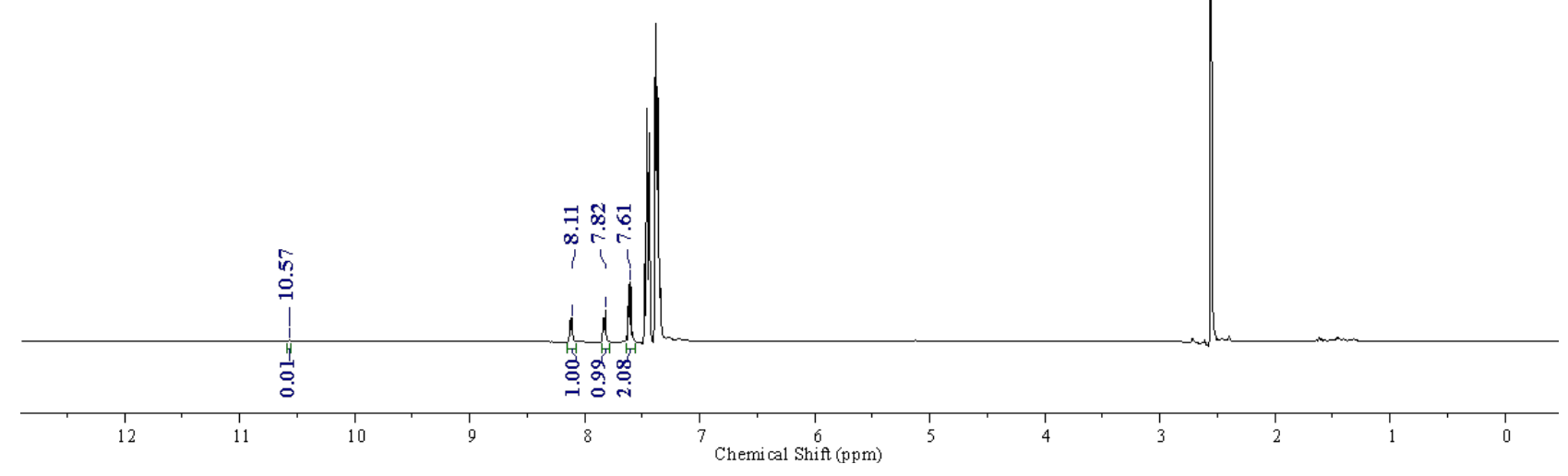


Entry 1': NMR for Crude reaction mixture, Duplicate

VDOS-590-A 1.1. fid

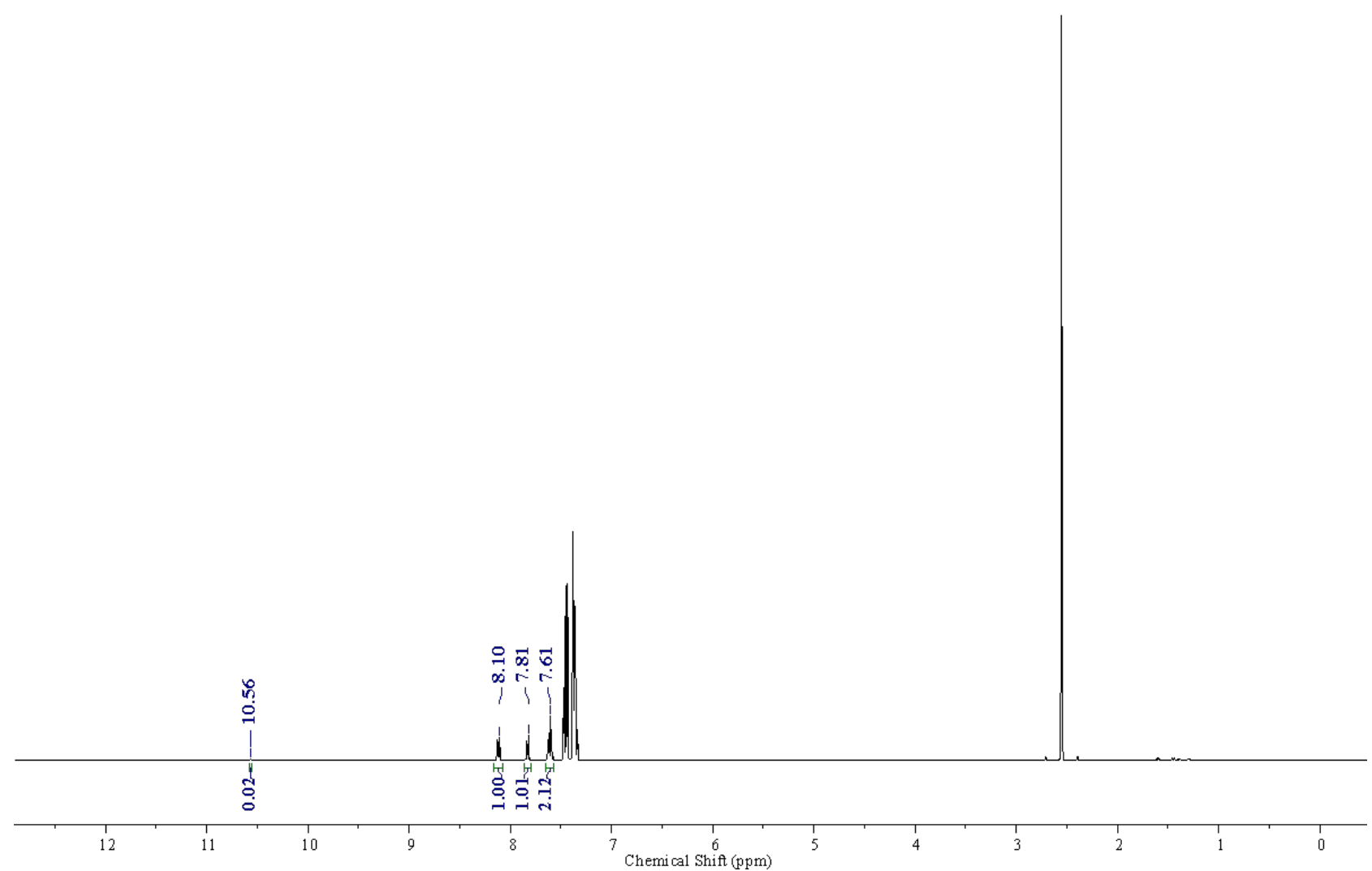

NMR after purification:

VDOS-590-A 1-A2-Pure.1.fid

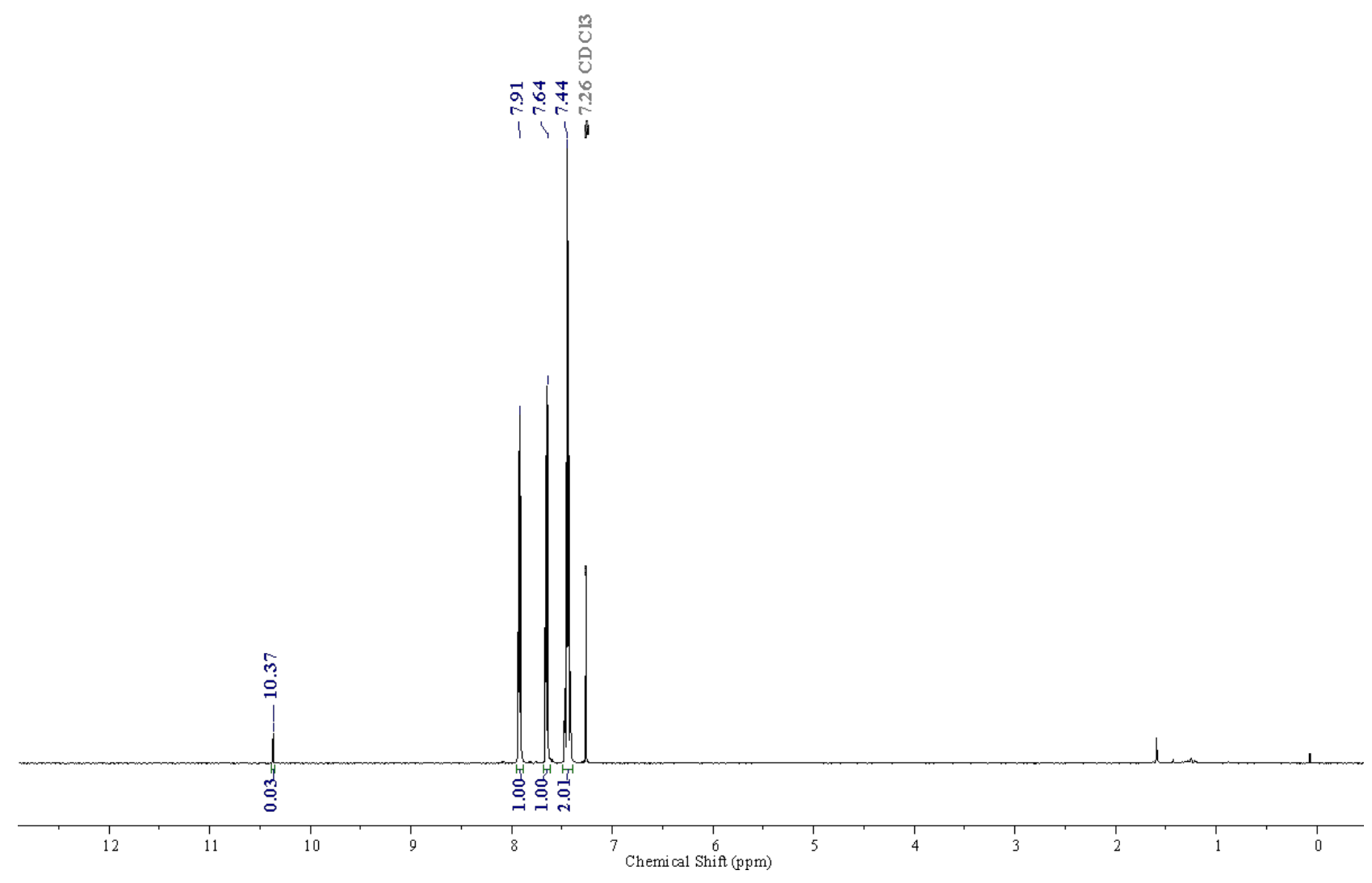




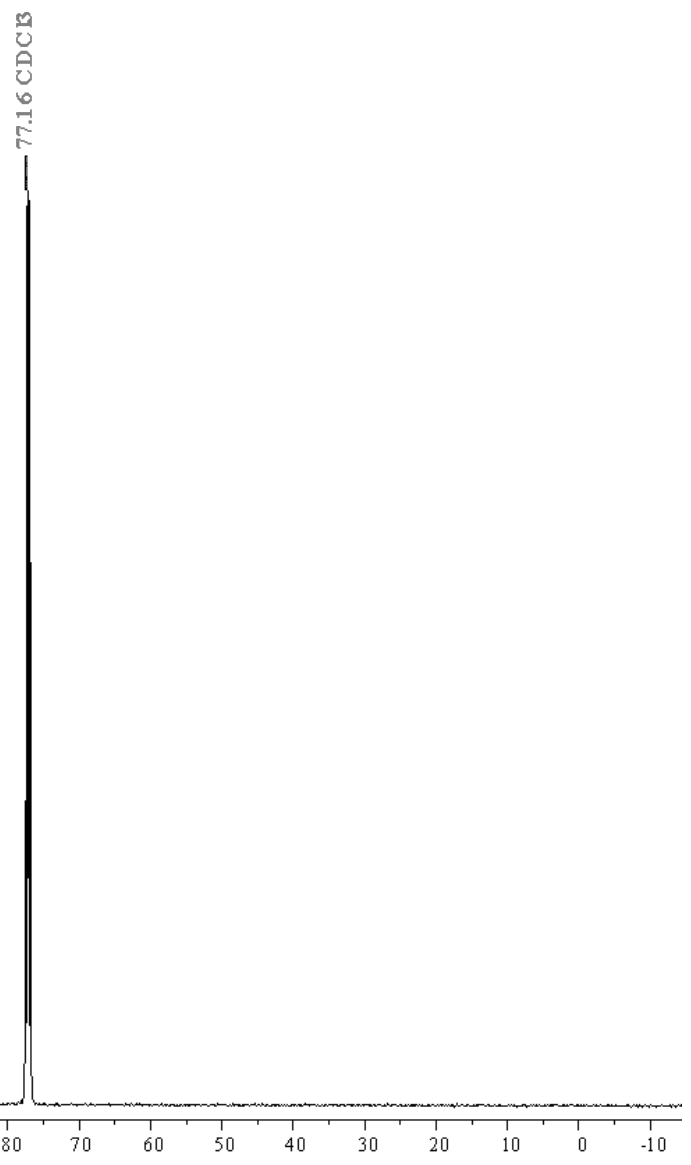

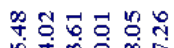

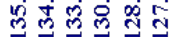

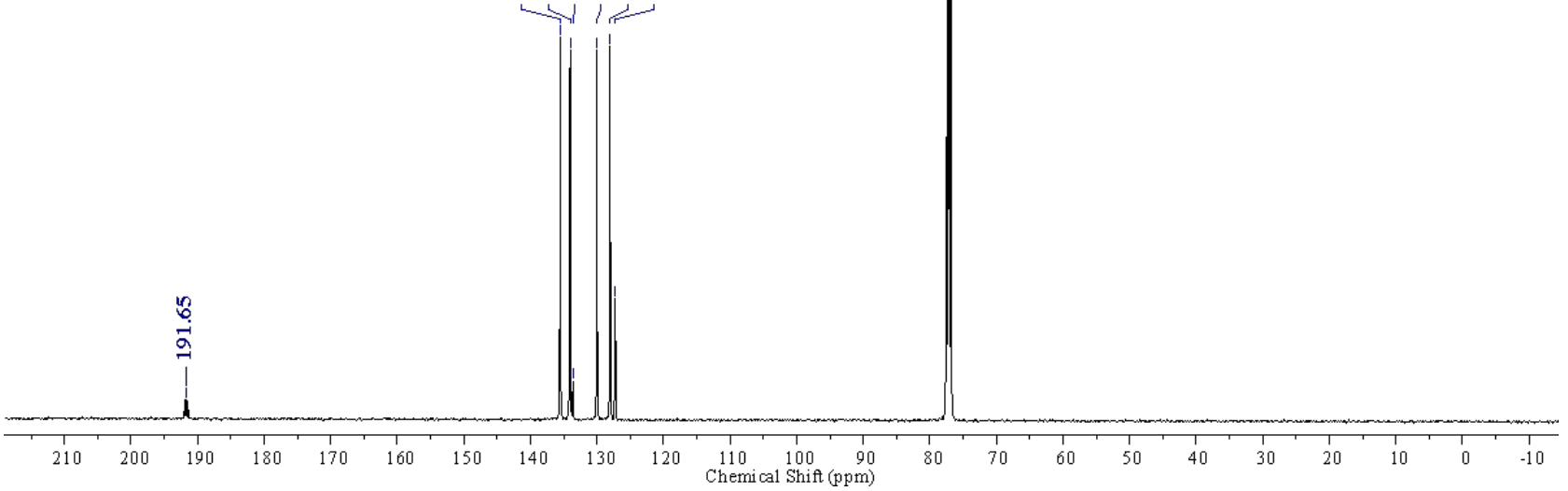


4. 3-Methoxybenzaldehyde-d<smiles>[2H]C(=O)c1cccc(OC)c1</smiles>

Entry 1: NMR for Crude reaction mixture SCG-312-U 1-16hr-D 1-50 sec

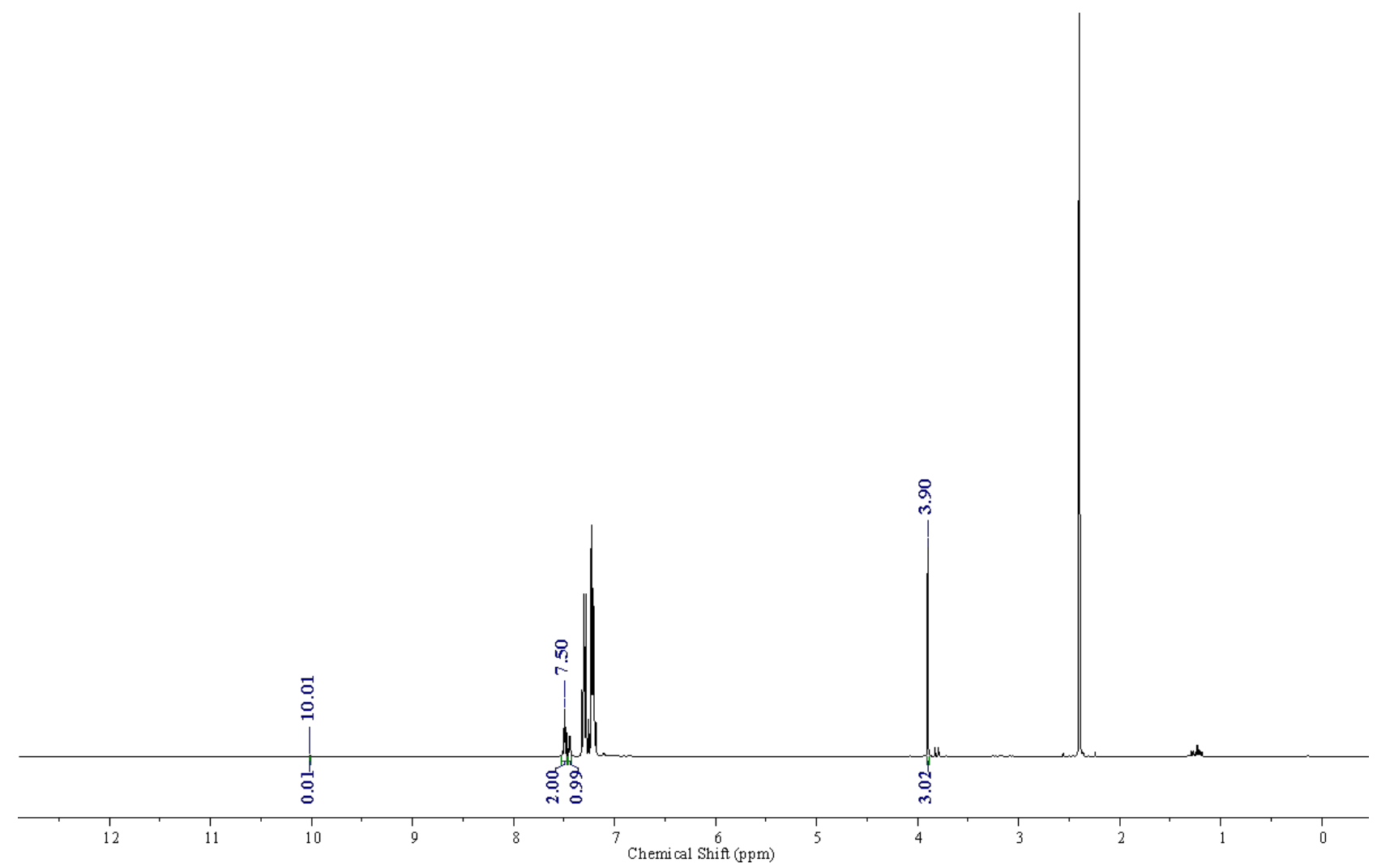


Entry 1': NMR for Crude reaction mixture, Duplicate

SCG-312-U2-16hr-D 1-50sec

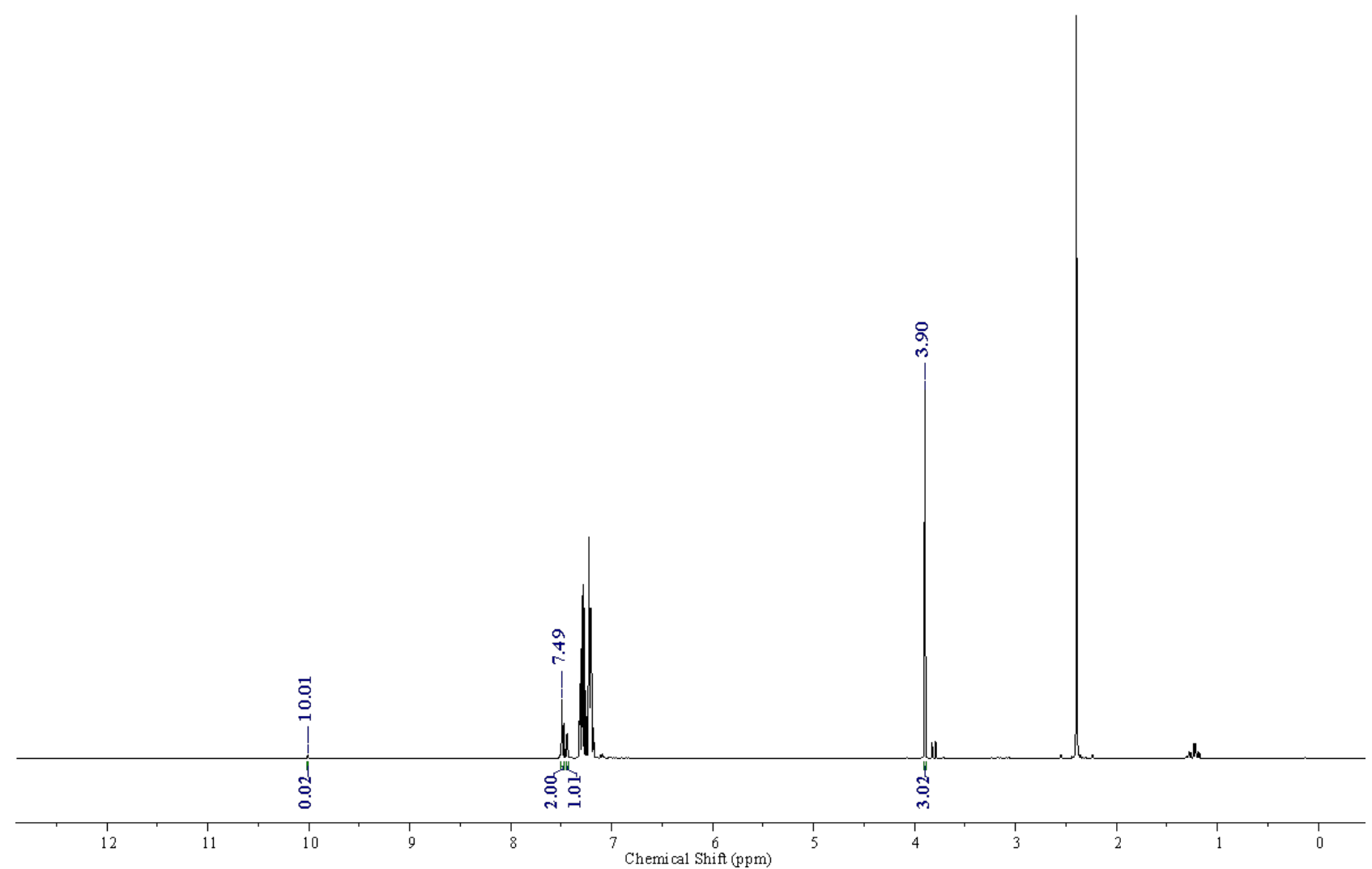

NMR after purification:

SCG-312-U 1-16hr-D 1-50sec-A C

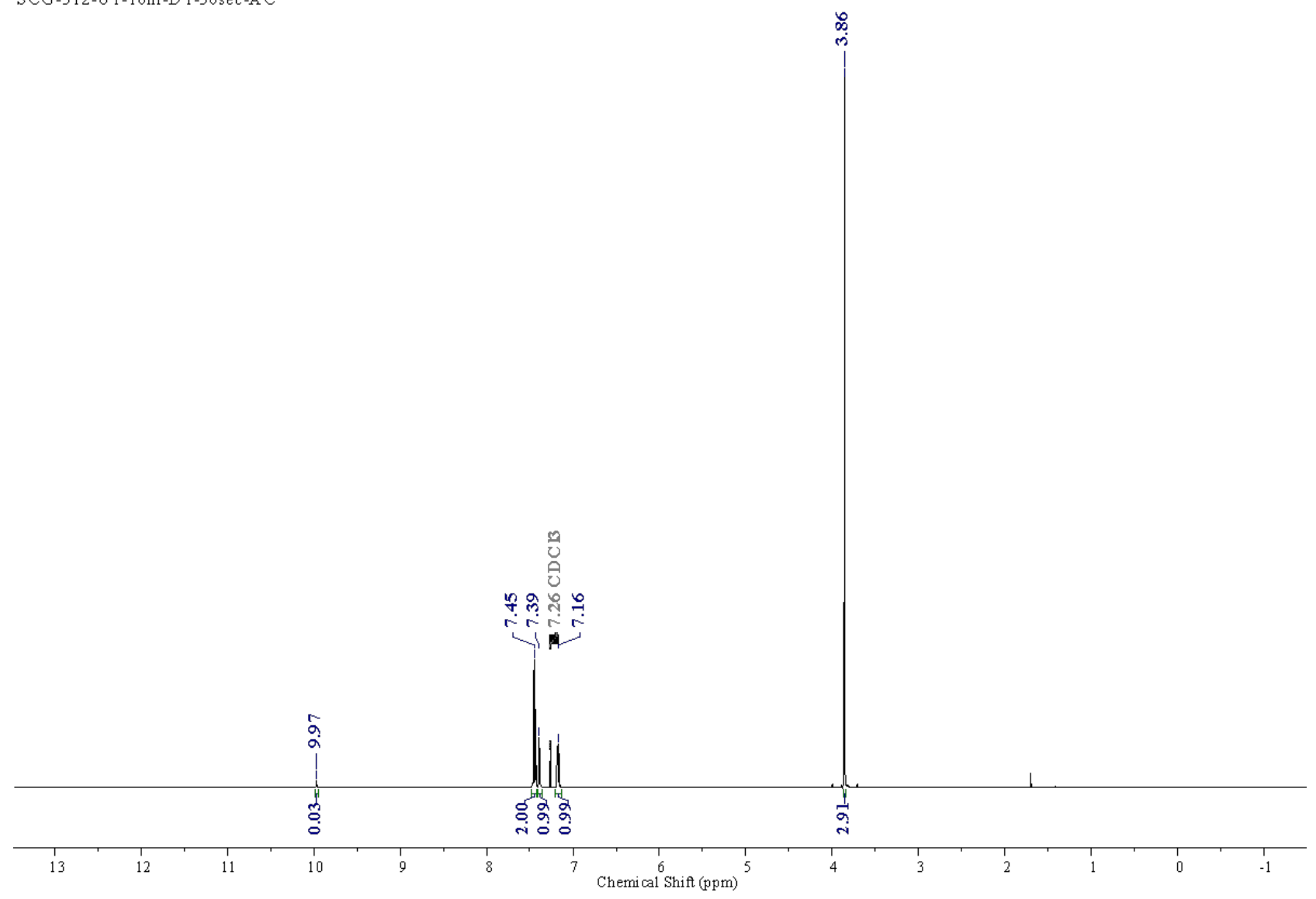


응ํำ

क्षं

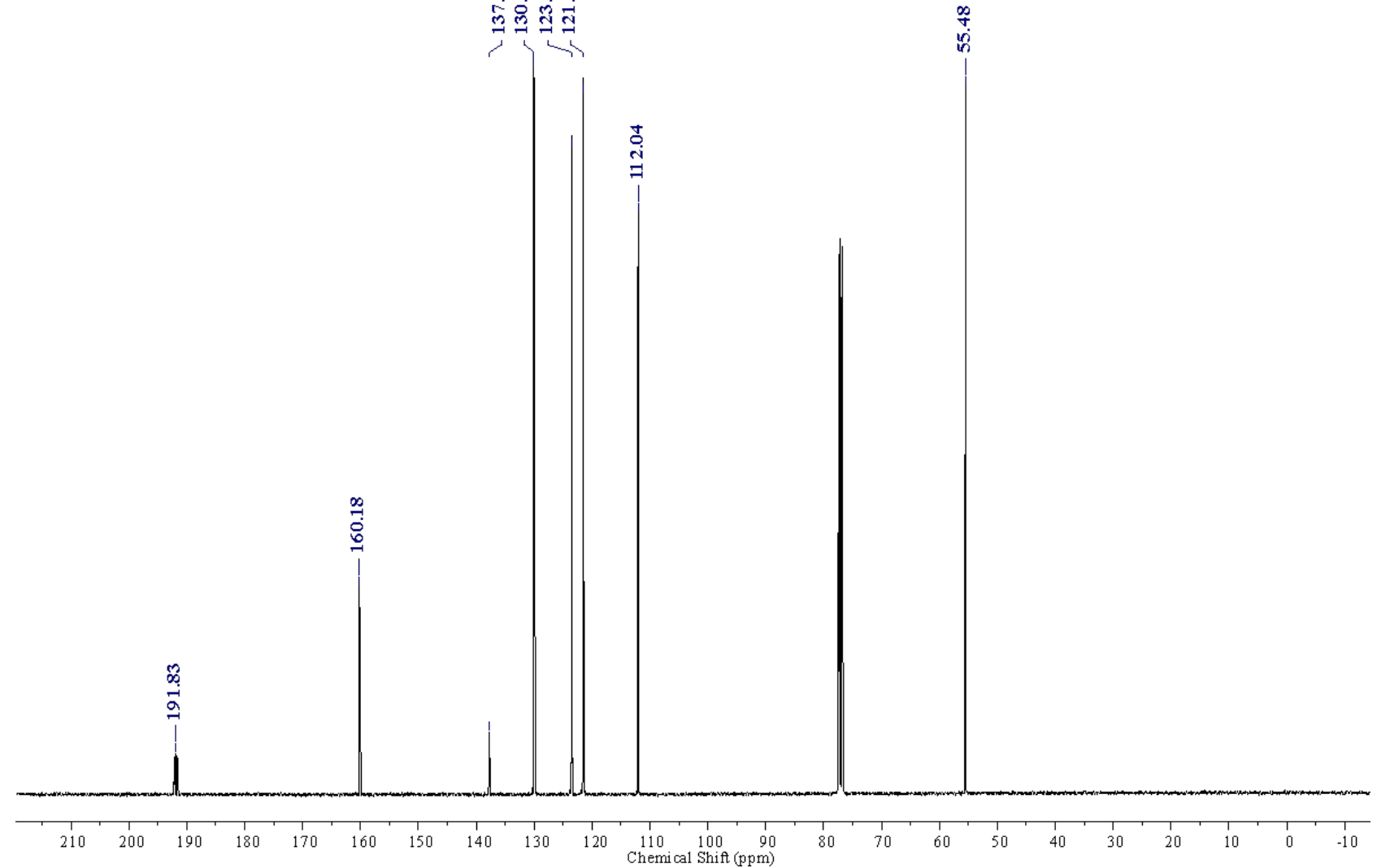


5. 4-Methoxybenzaldehyde-d

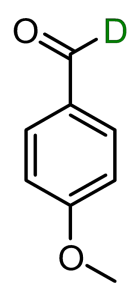

Entry 1: NMR for Crude reaction mixture

SCG-312-R 1-16hr-D 1-50sec. 1. fid

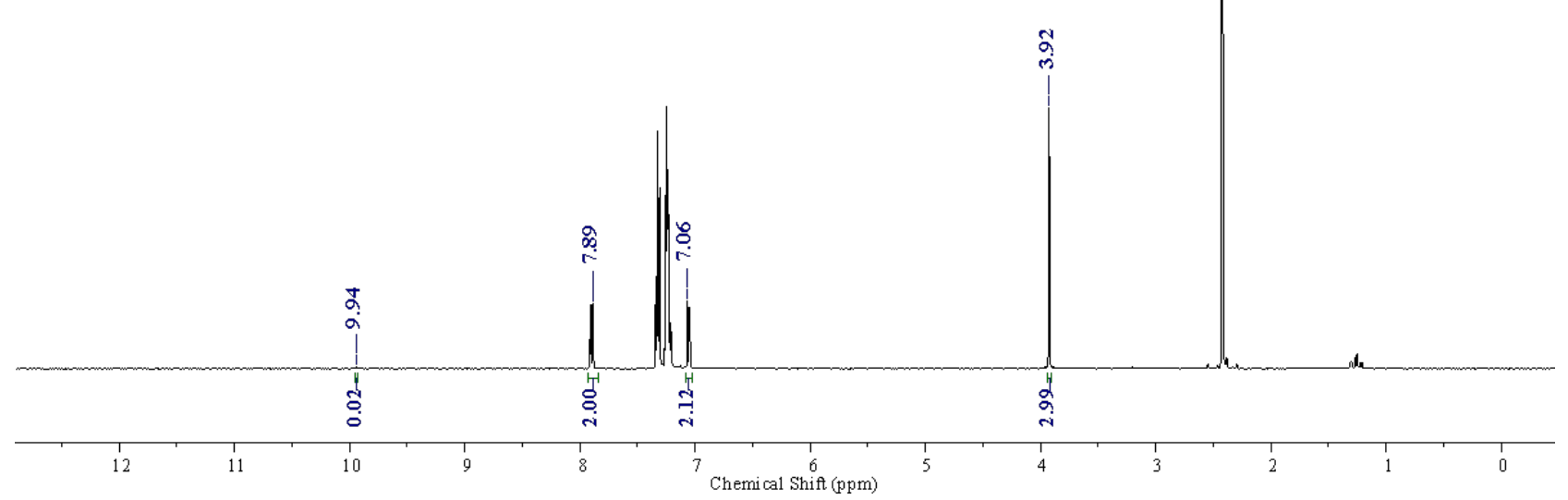

191 
Entry 1': NMR for Crude reaction mixture, Duplicate

SCG-312-R2-16hr-D 1-50sec.1. fid

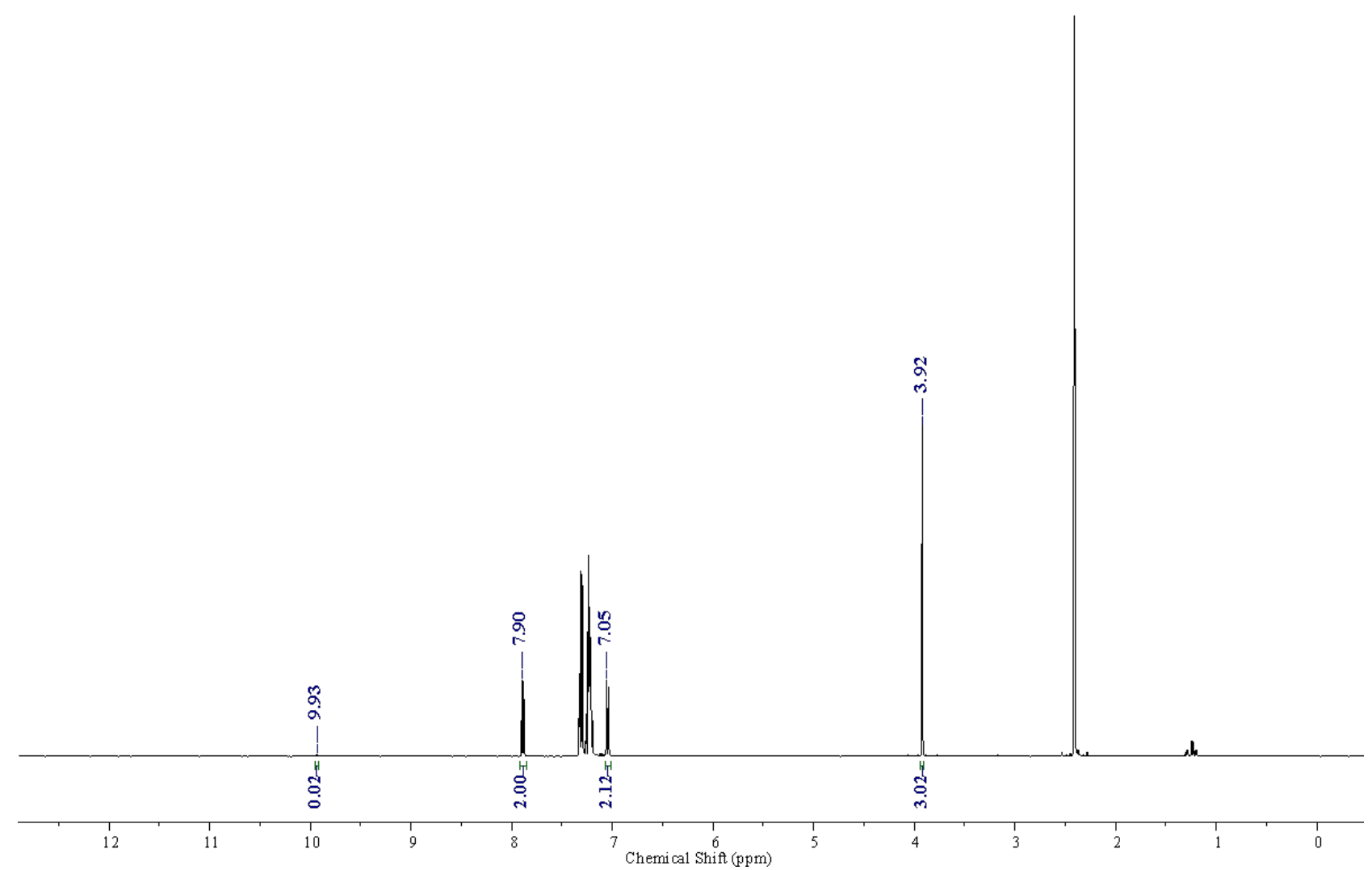

NMR after purification:

SCG-312-R 1-16hr-D 1-50sec-AC. 1 fid

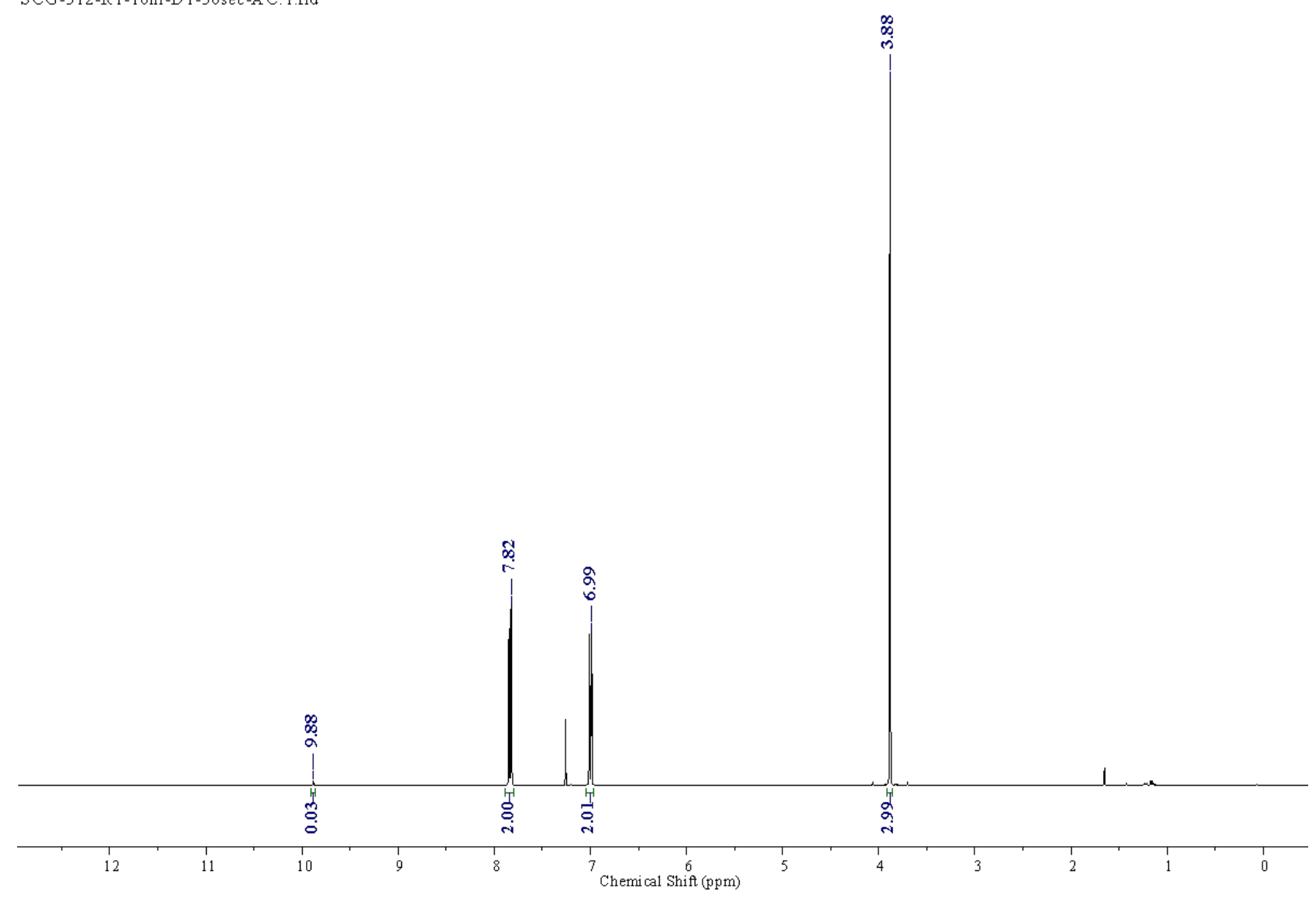


SCG-312-R 1-16hr-AC-13C.1.fid

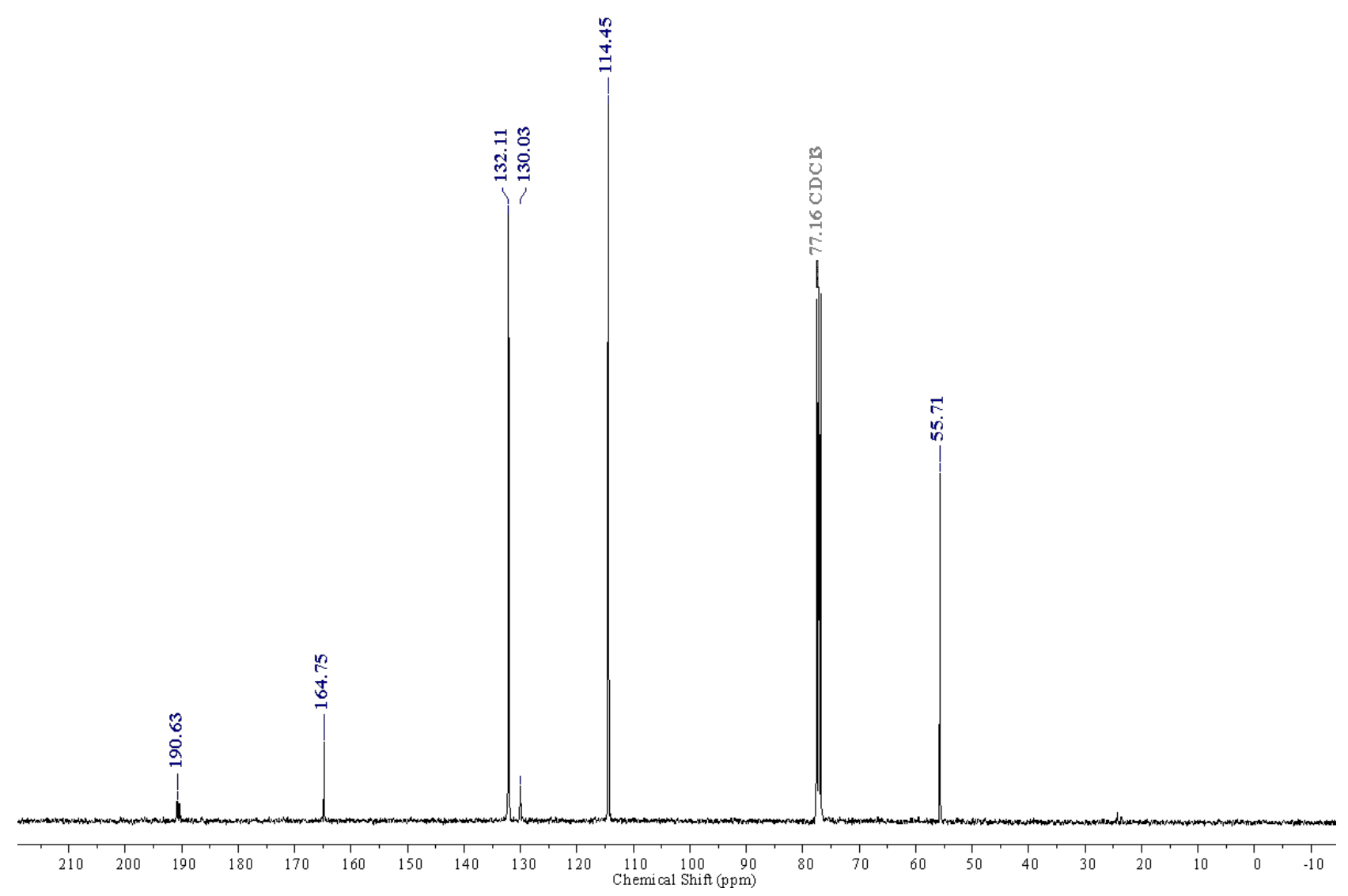

193 
6. Benzo $[d][1,3]$ dioxole-4-carbaldehyde- $d$<smiles>[2H]C(=O)c1cccc2c1OCO2</smiles>

Entry 1: NMR for Crude reaction mixture VDOS-608-H 1.4.fid

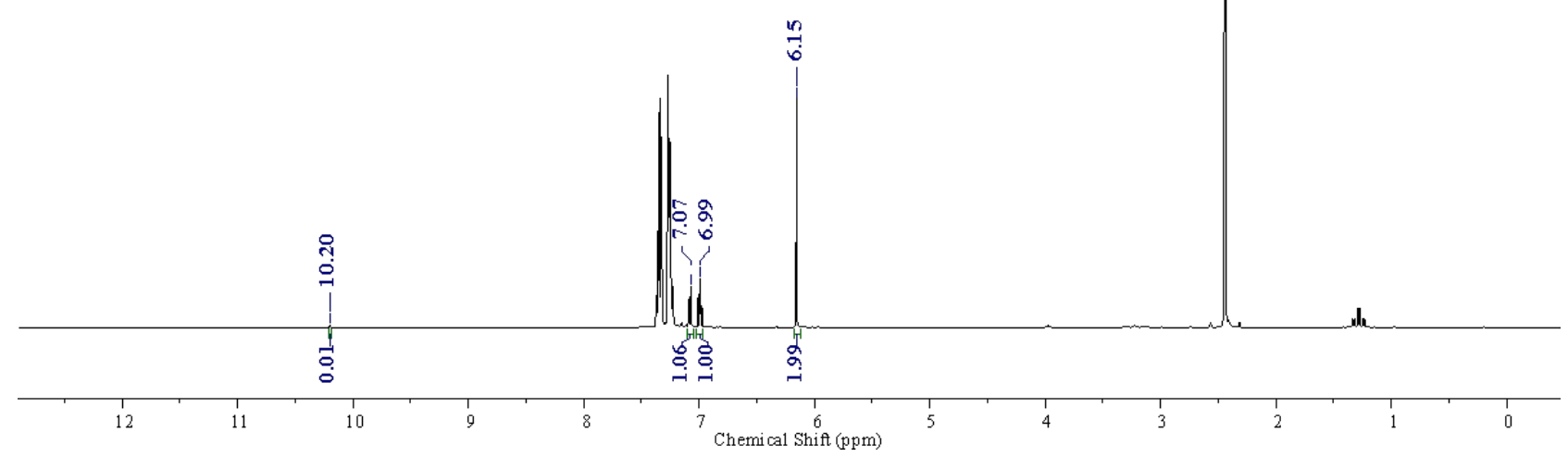


Entry 1': NMR for Crude reaction mixture, Duplicate

VDOS-608-H 3.6. fid

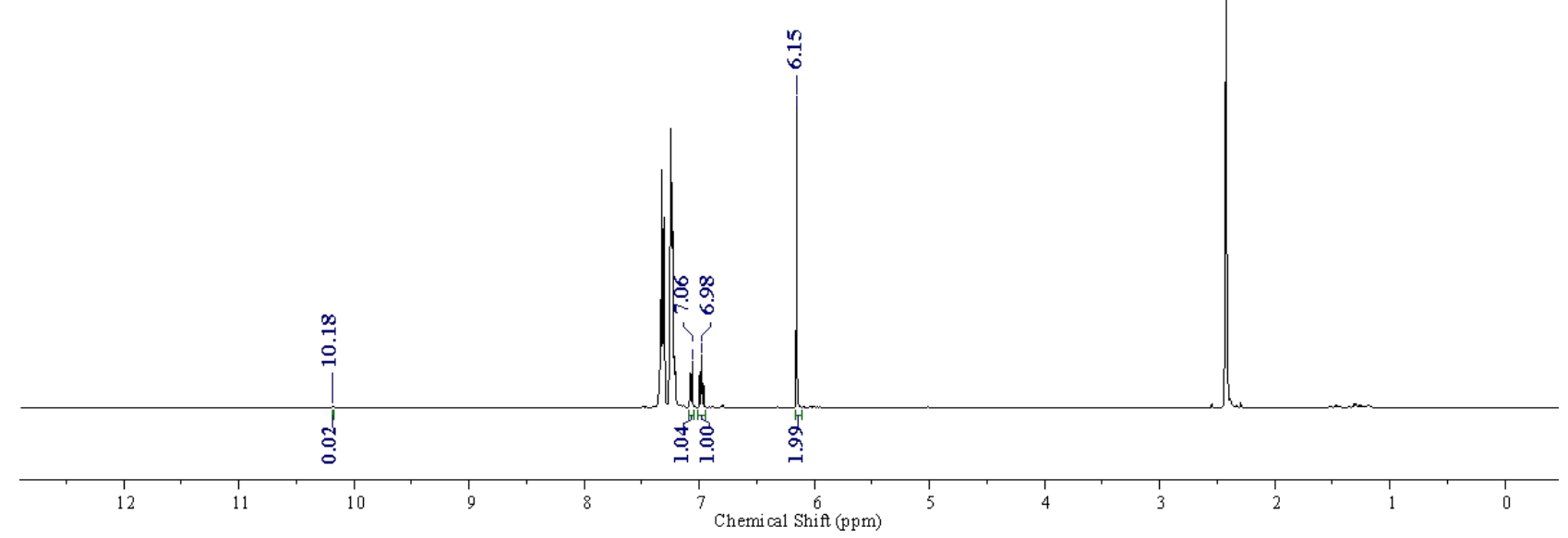

NMR after purification:

VDOS-608-H3 pure.7.fid

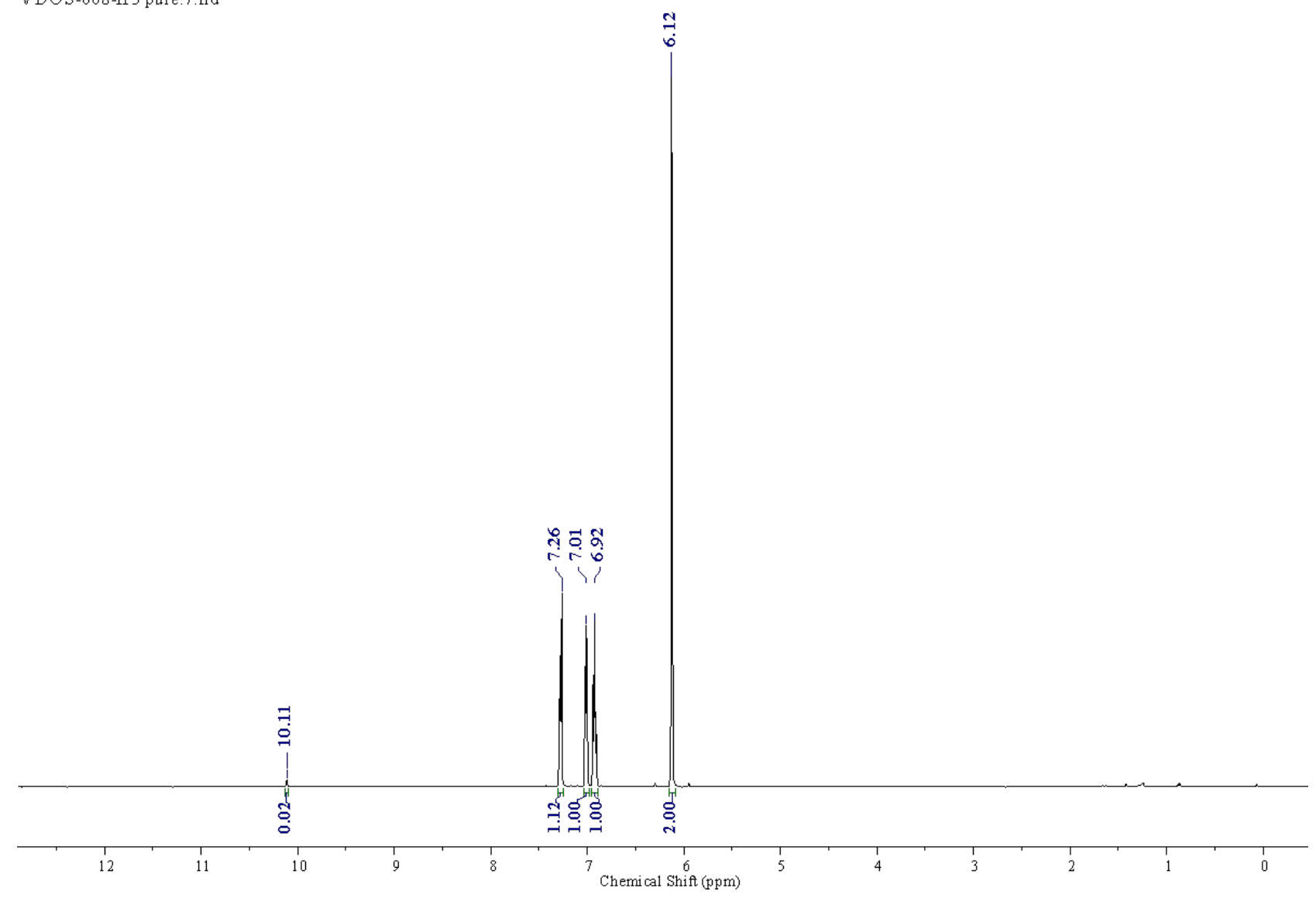




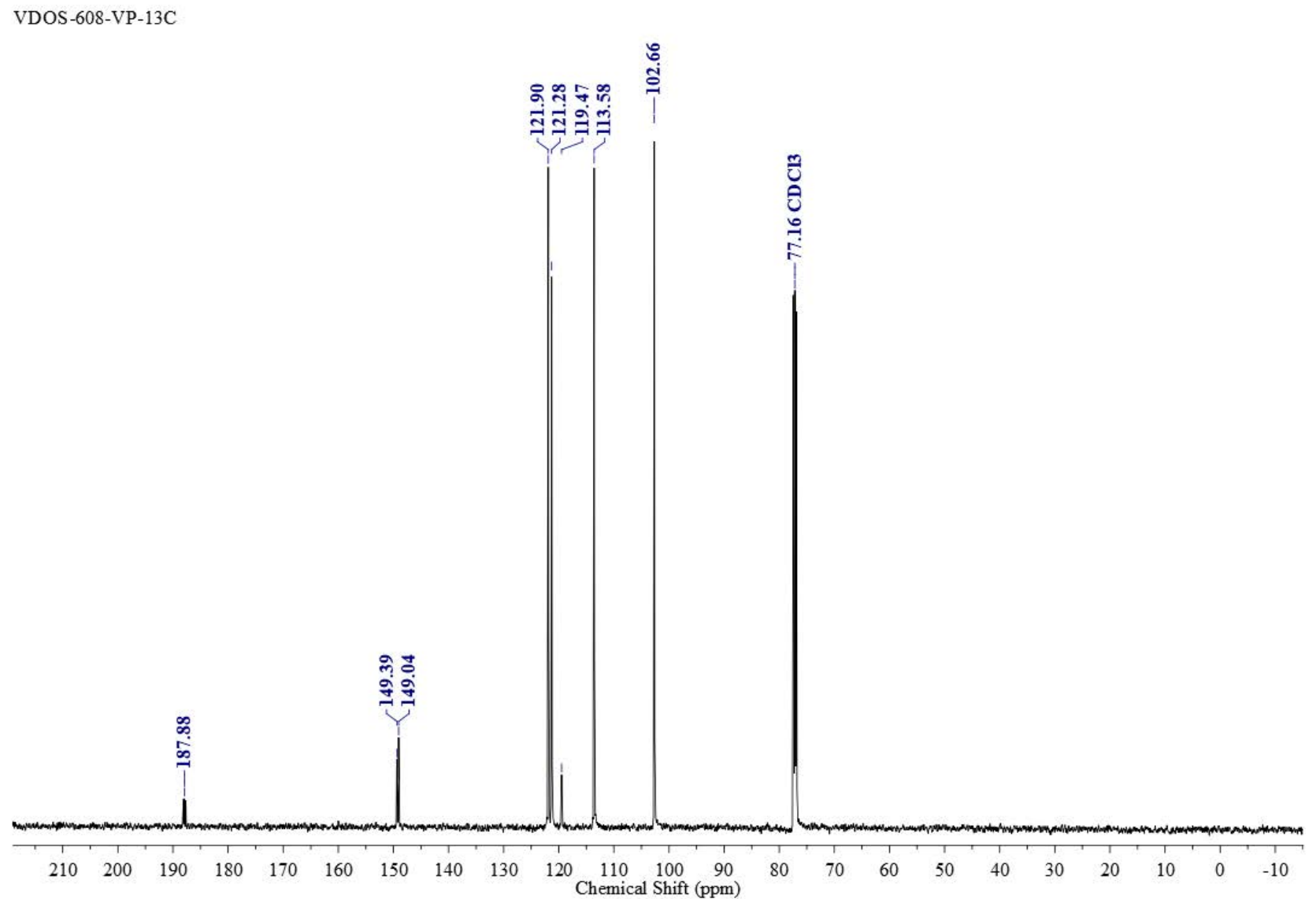


7. 1-Naphthaldehyde-d<smiles>[2H]C(=O)c1cccc2ccccc12</smiles>

Entry 1: NMR for Crude reaction mixture VDOS-616-A2-2.5.fid

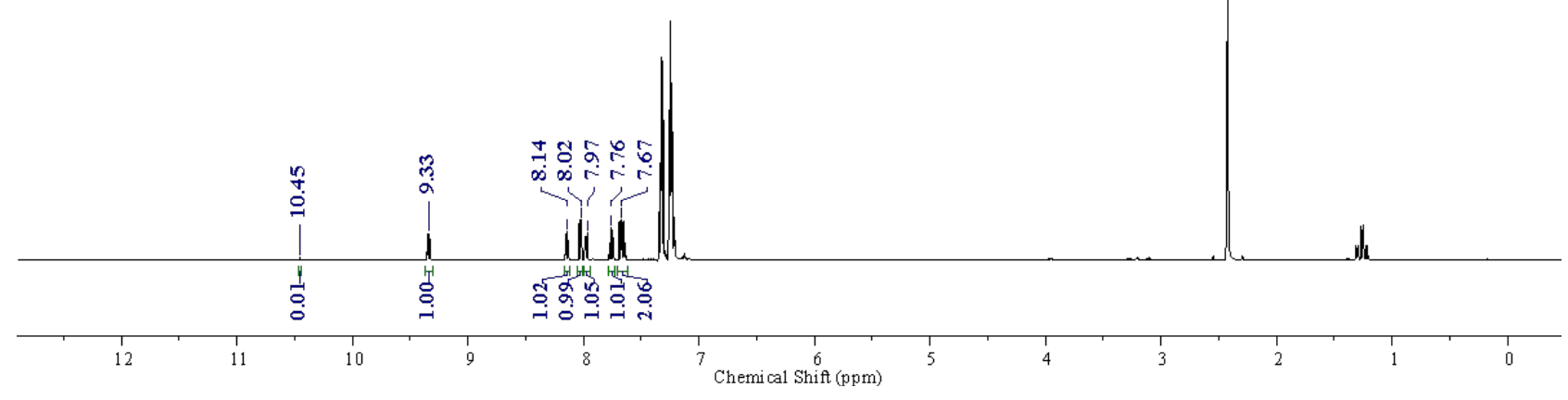


Entry 1': NMR for Crude reaction mixture, Duplicate

VDOS-616-A 1.3.fid

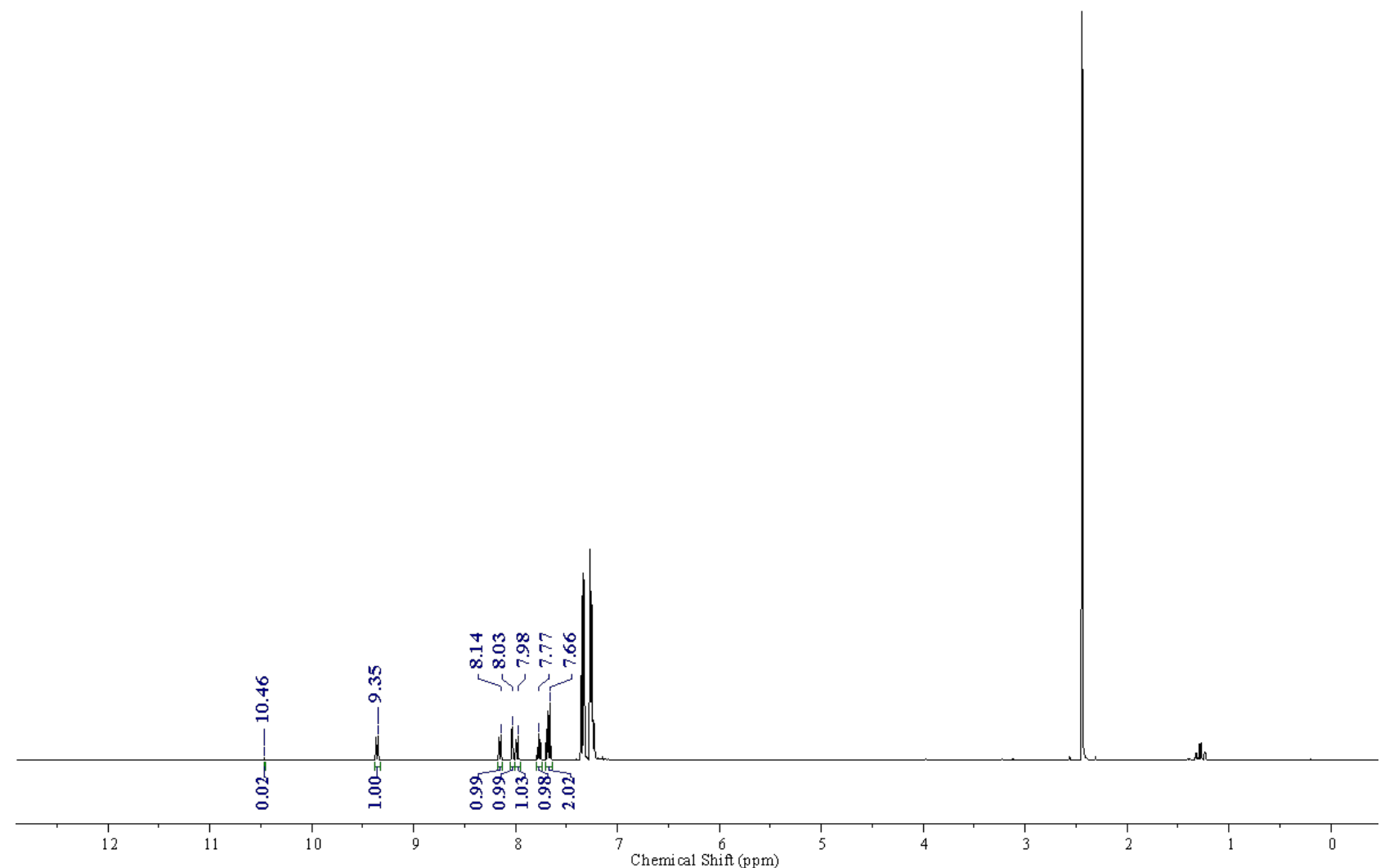

NMR after purification:

VDOS-616-A 2 pure.6.fid

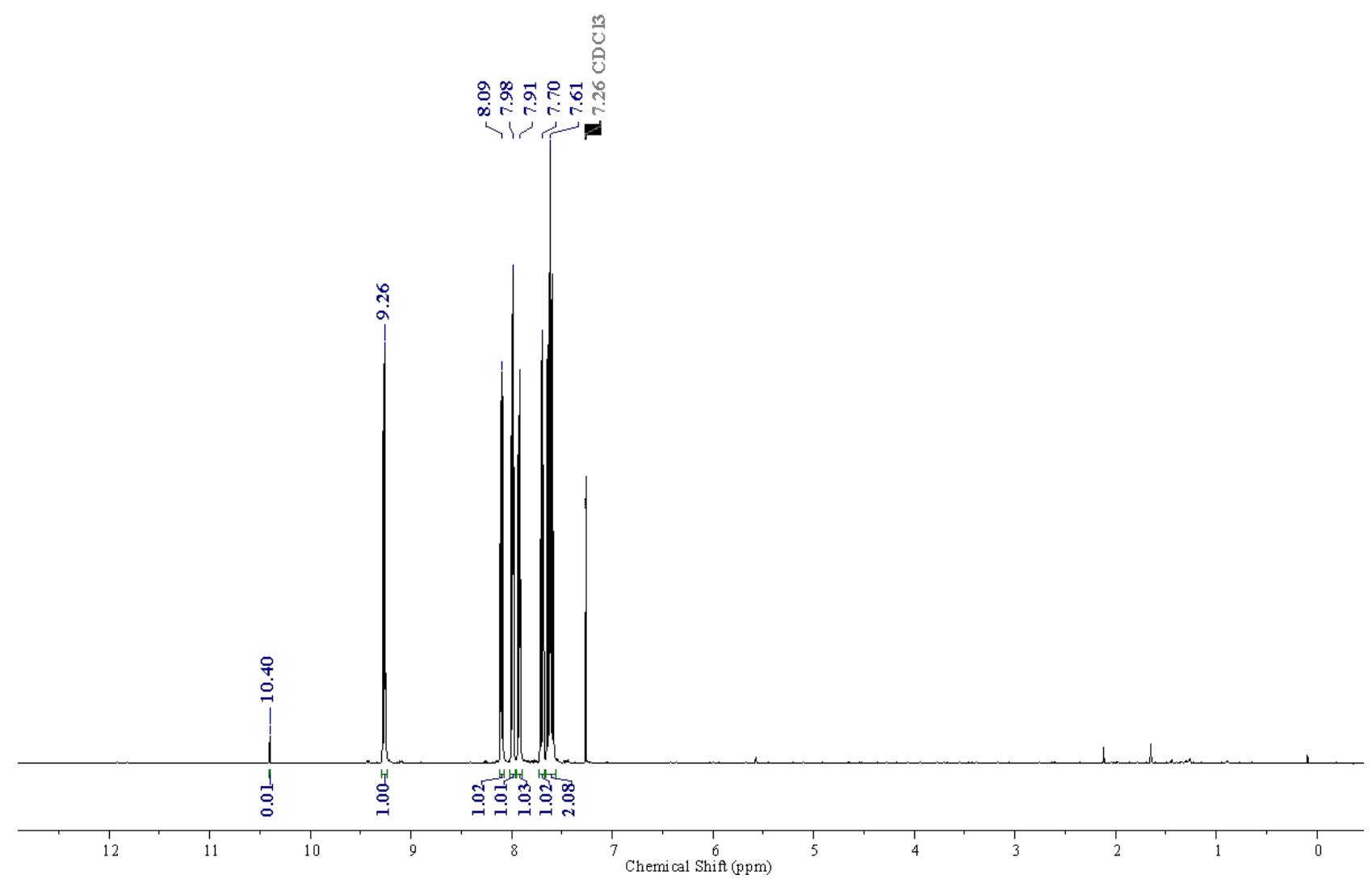




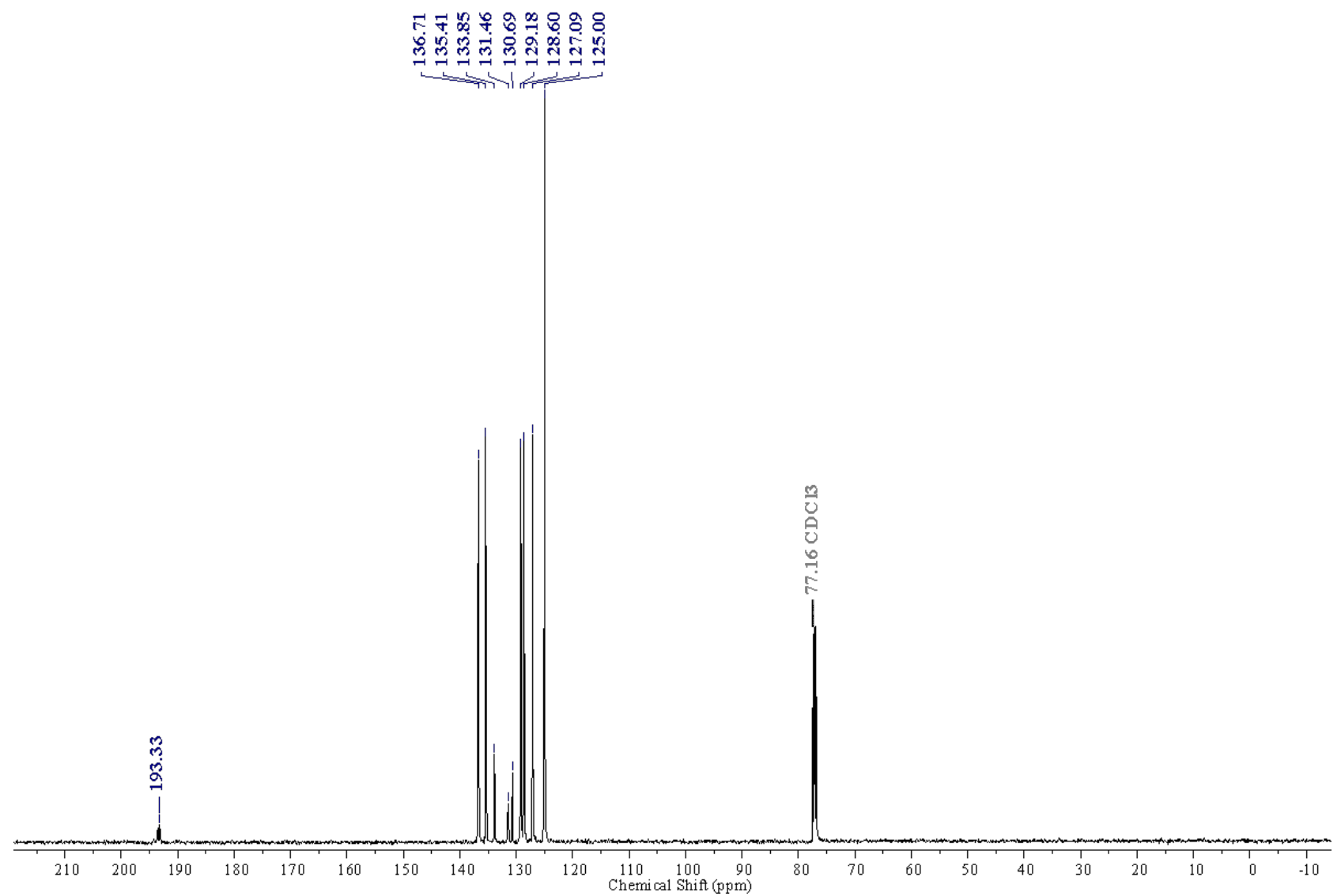




\section{2-(Trifluoromethoxy)benzaldehyde-d}<smiles>[2H]C(=O)c1ccccc1OC(F)(F)F</smiles>

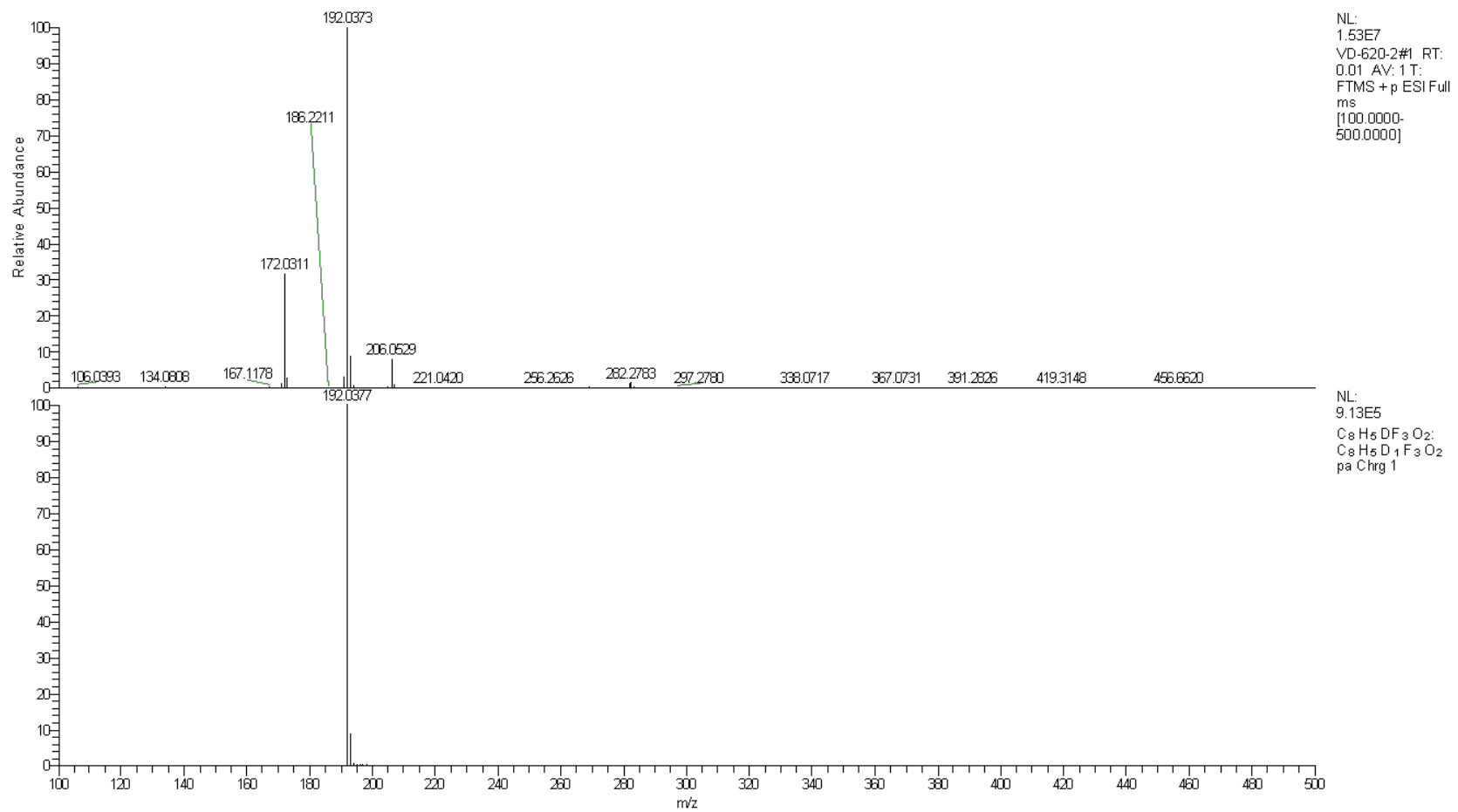

Entry 1: NMR for Crude reaction mixture VDOS-620-A 1.6.fid

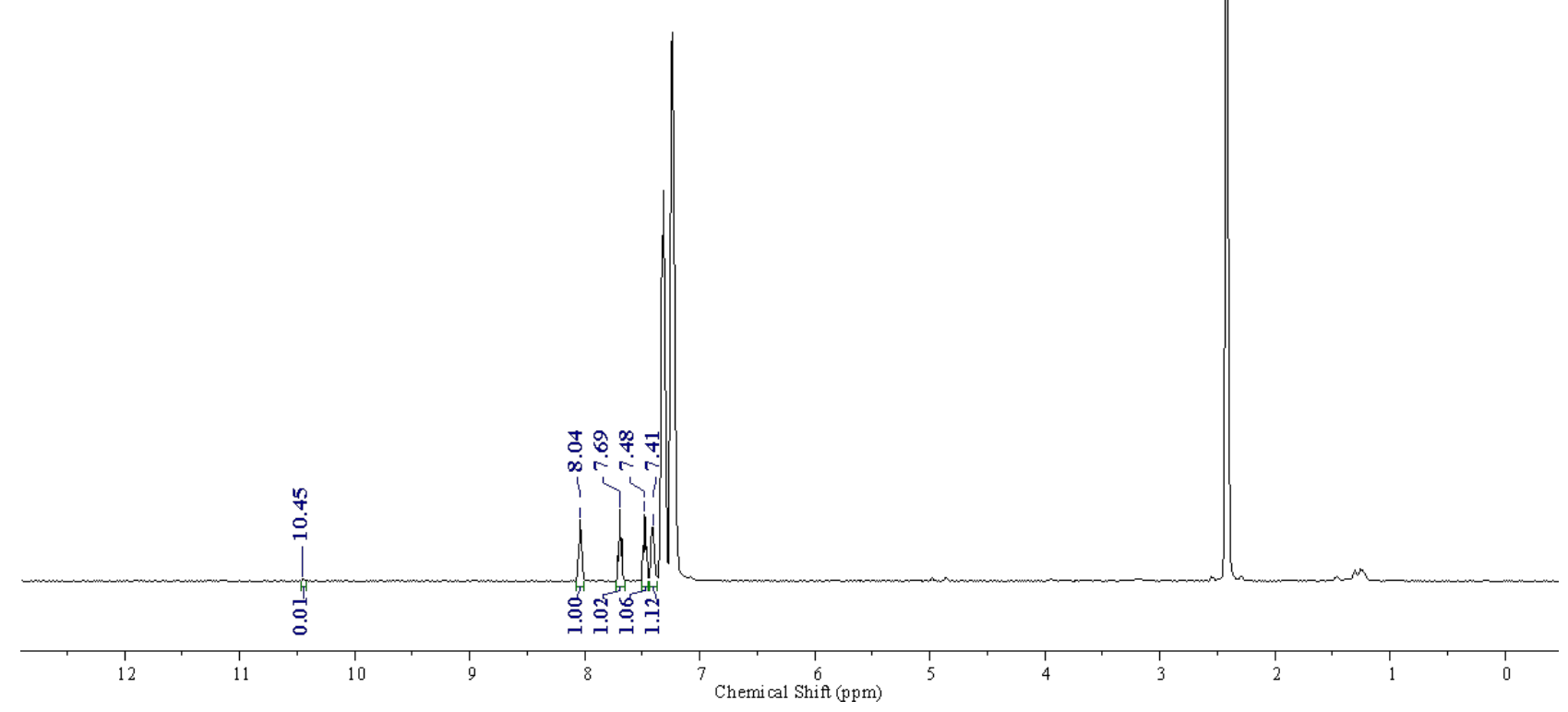

Entry 1': NMR for Crude reaction mixture, Duplicate 


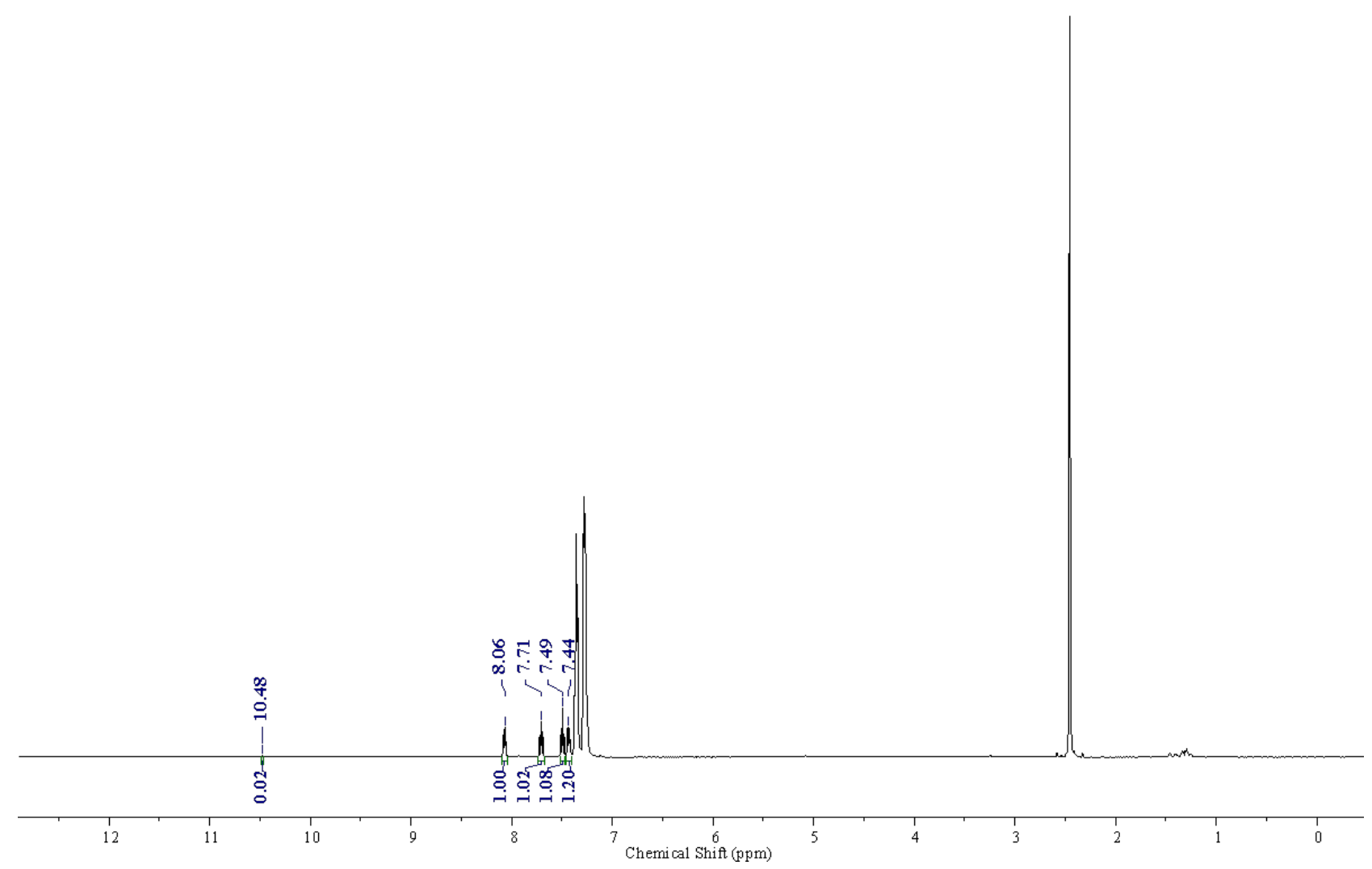

NMR after purification:

VDOS-620-A 2 pure.2.fid

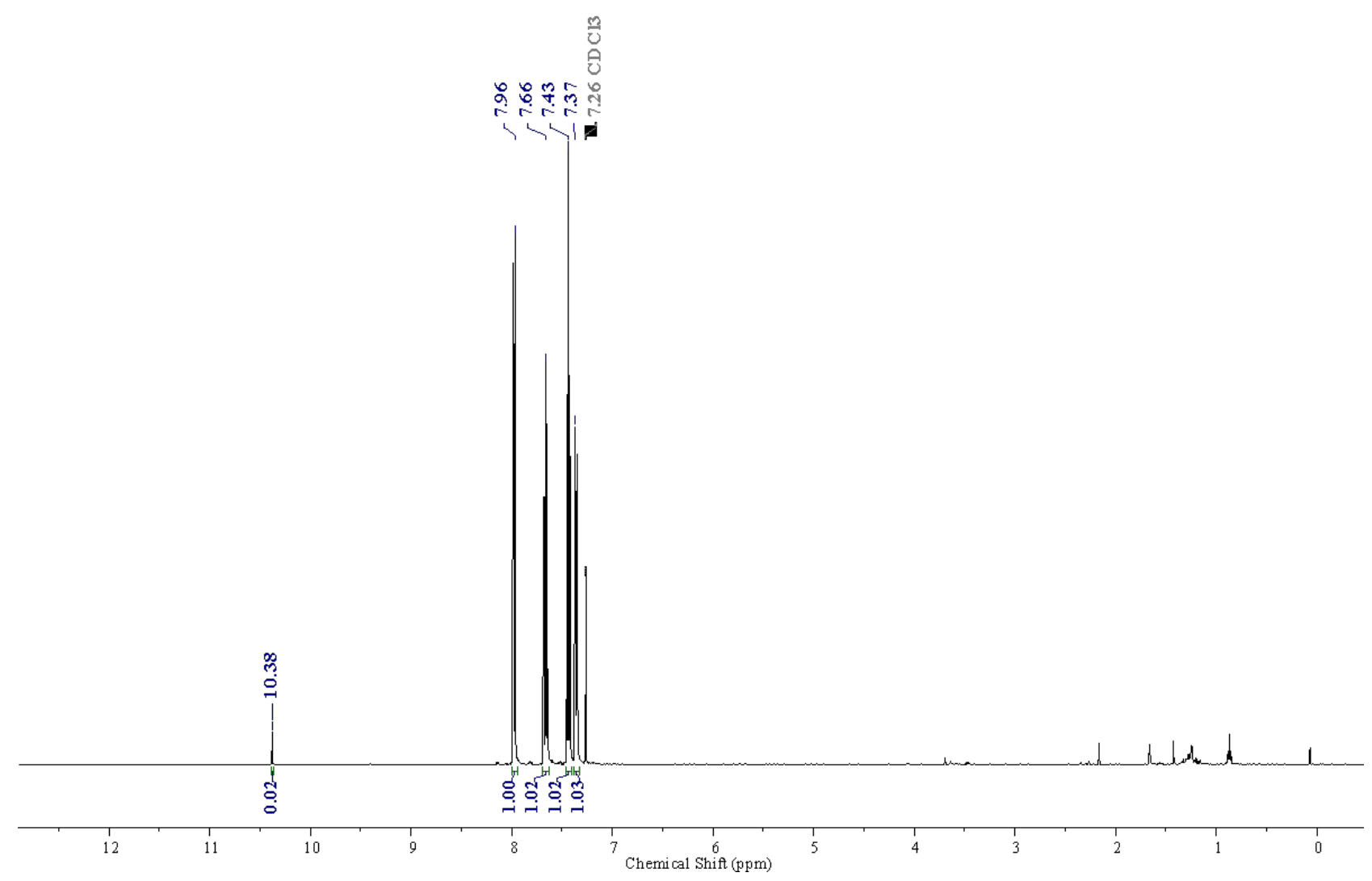



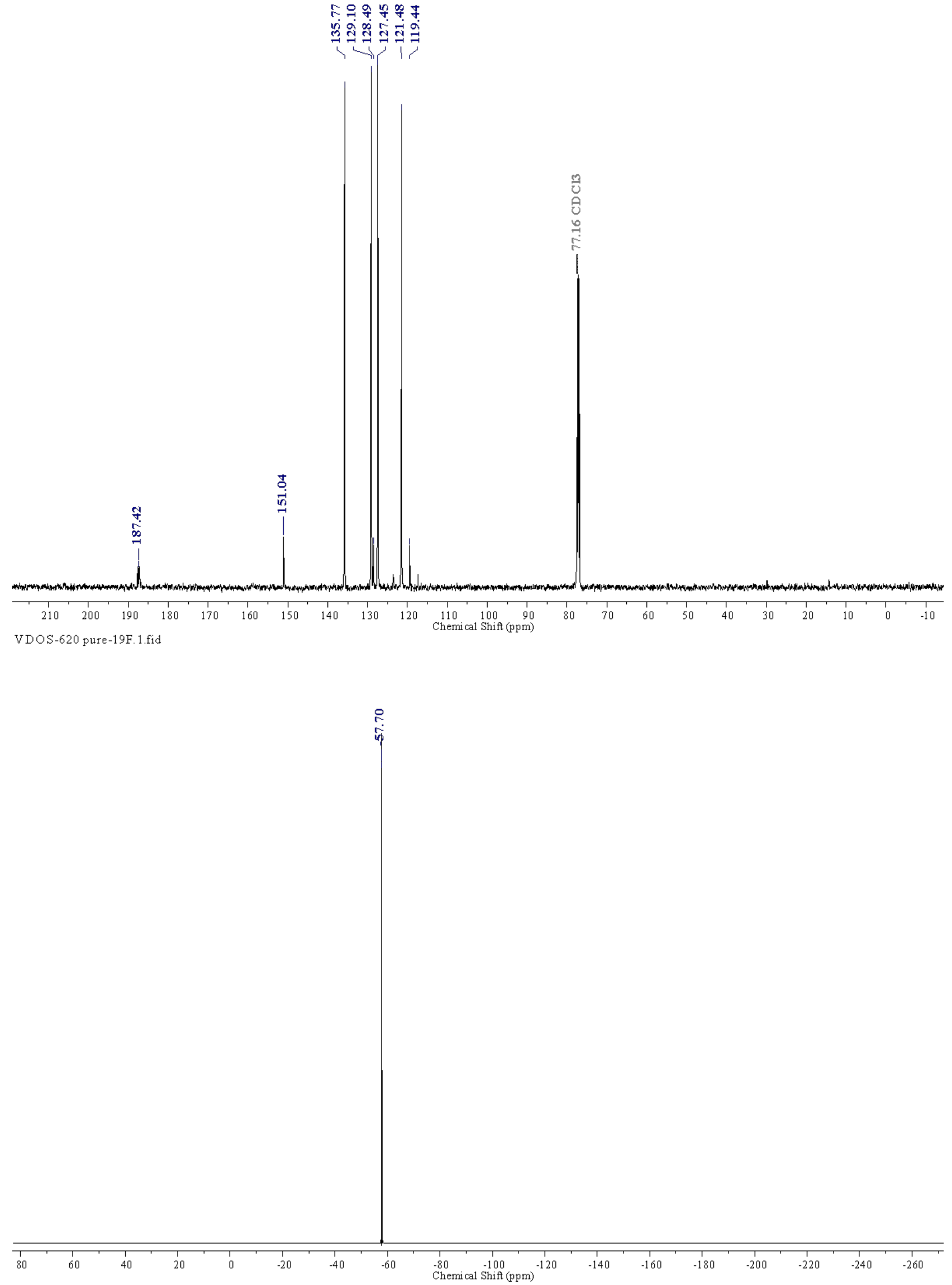


\section{2-Ethynylbenzaldehyde-d}

$95 \%$
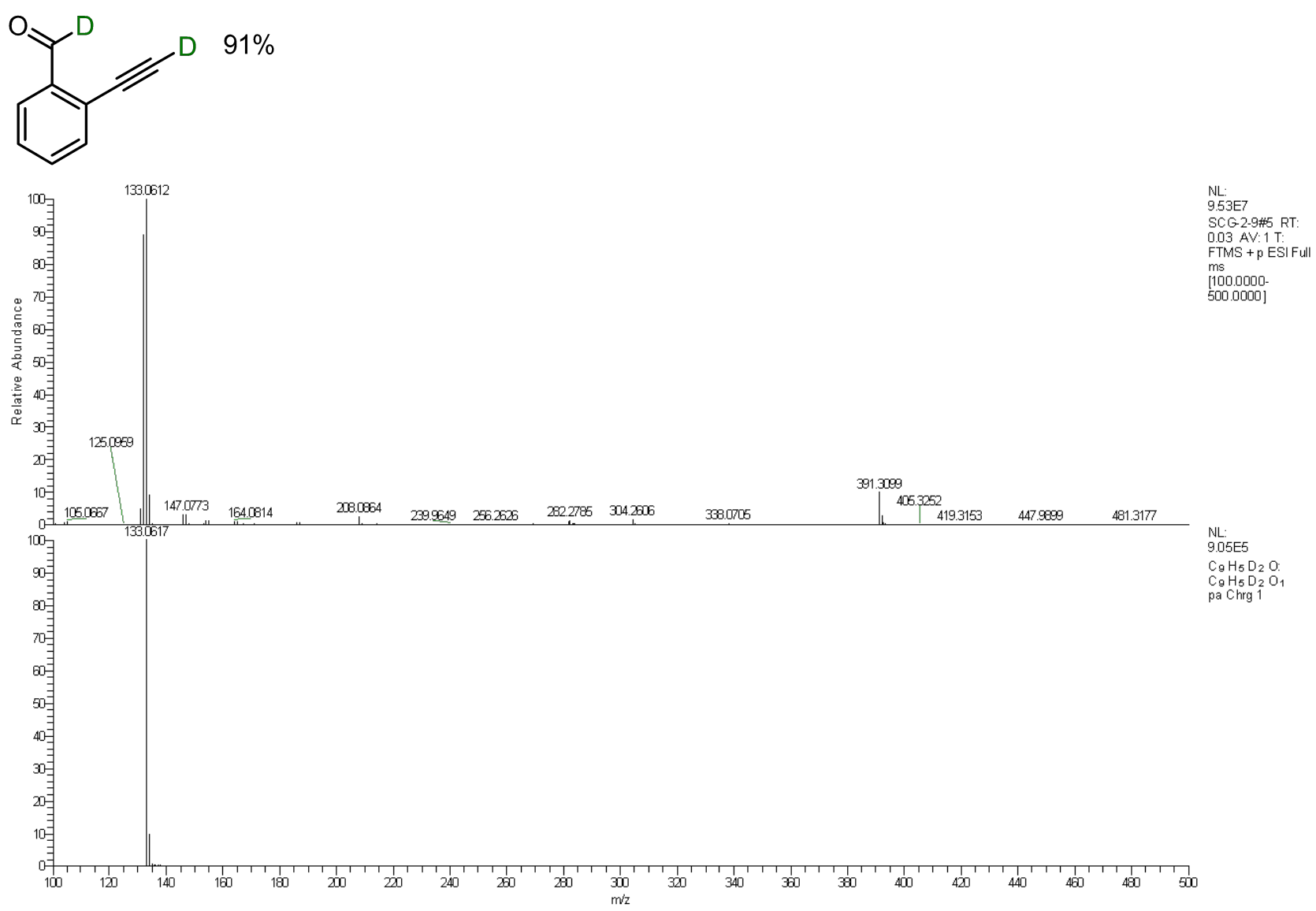

Entry 1: NMR for Crude reaction mixture VDOS-618-F1.7.fid

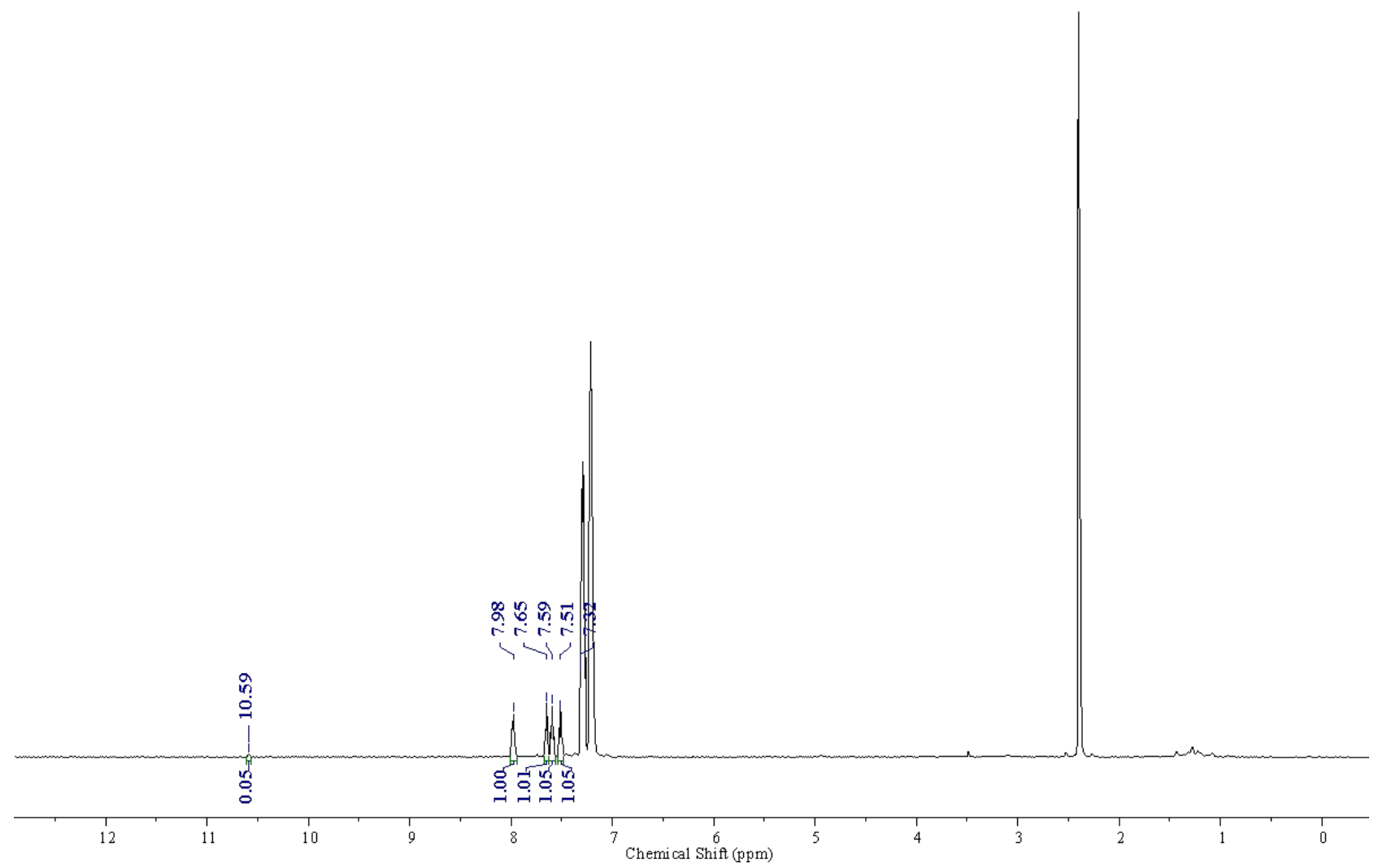


Entry 1': NMR for Crude reaction mixture, Duplicate

VDOS-618-A 1.2.fid

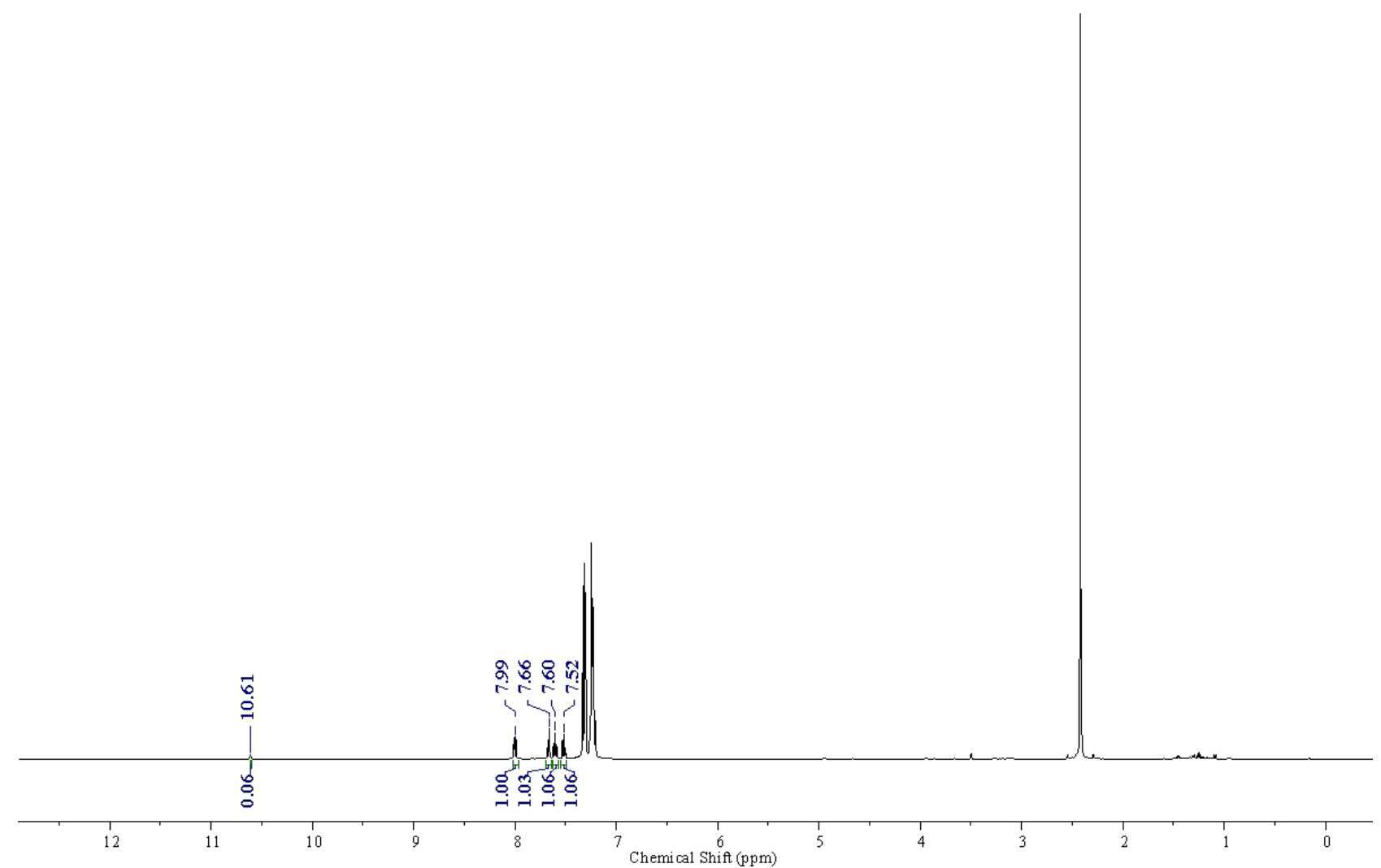

NMR after purification:

VDOS-618-A. 1 pure.2.fid

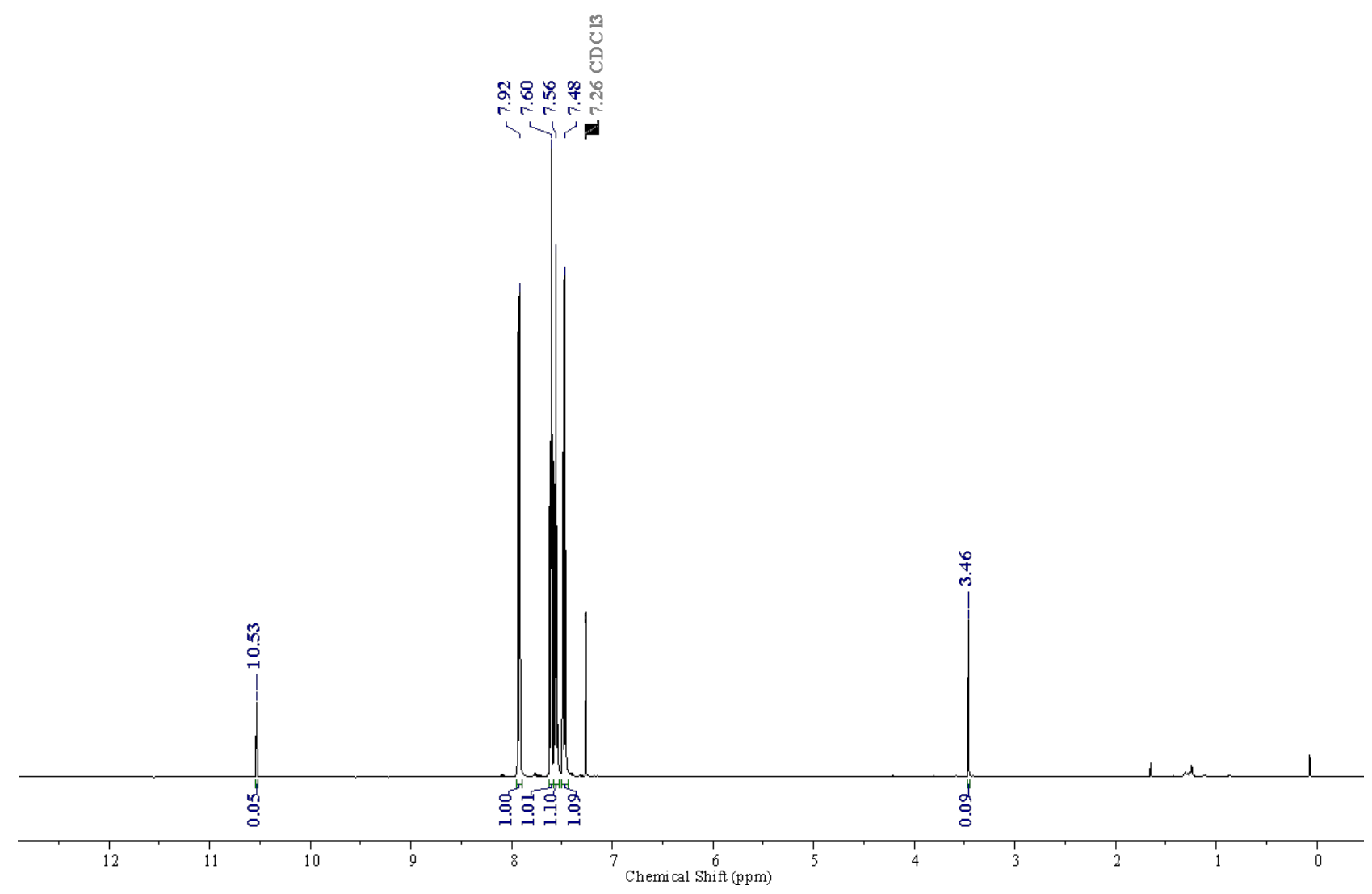




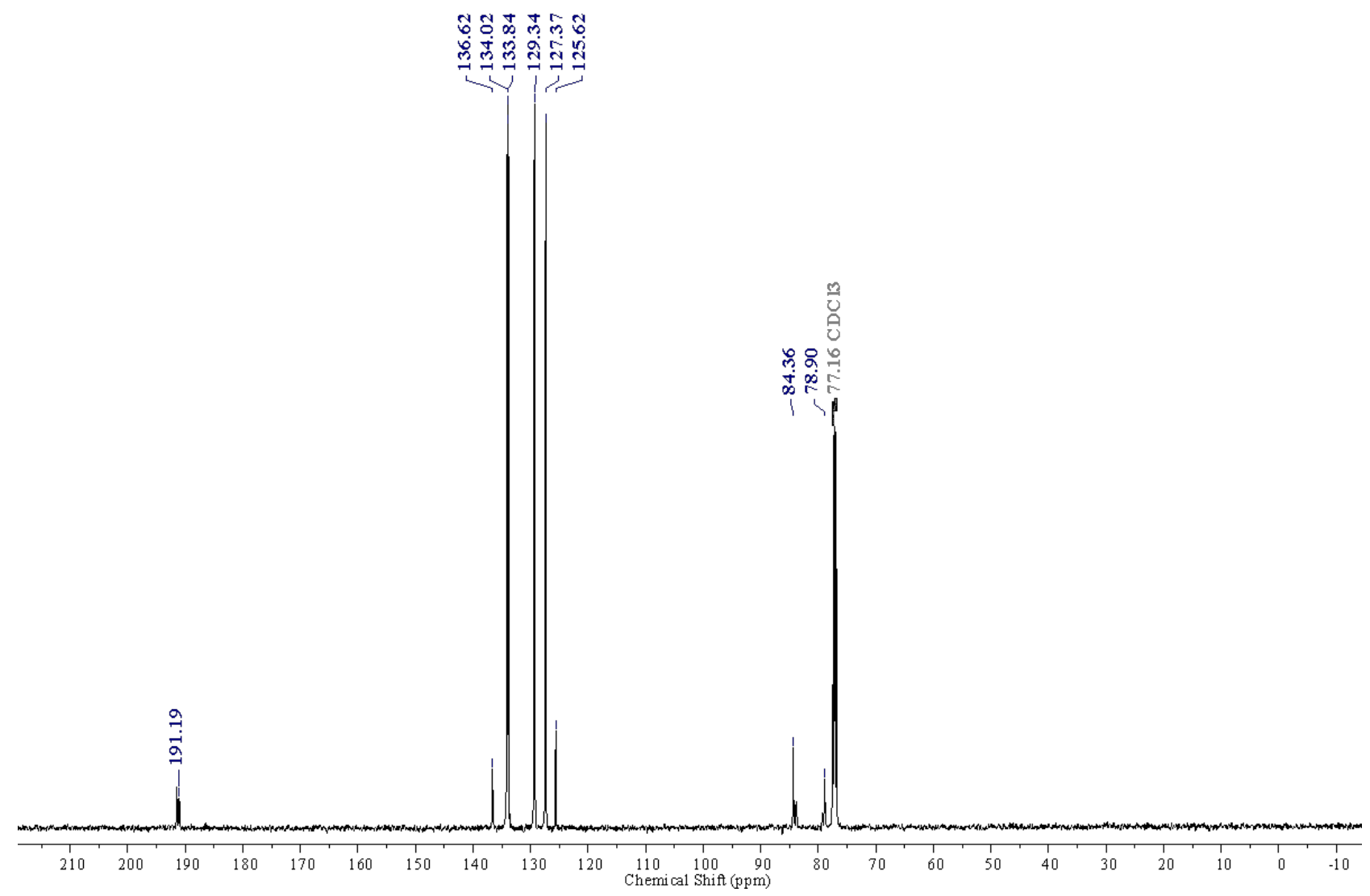


10. 2-Hydroxybenzaldehyde-d<smiles>[2H]C(=O)c1ccccc1O</smiles>

Entry 1: NMR for Crude reaction mixture

SCG-307-B1-16hr-D 1-50sec.1. fid

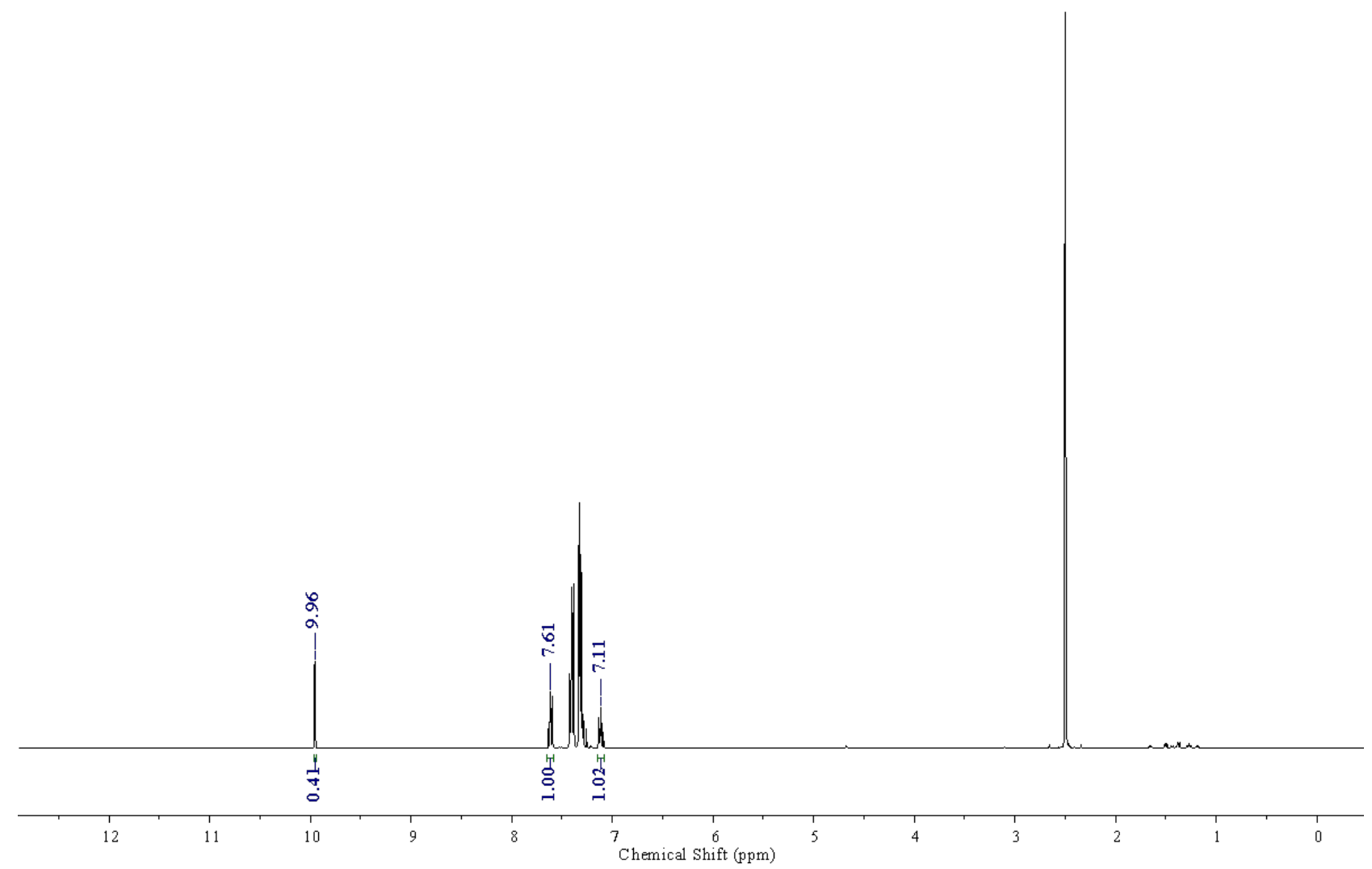


Entry 1': NMR for Crude reaction mixture, Duplicate

SCG-307-B2-16hr-D 1-50sec.1.fid

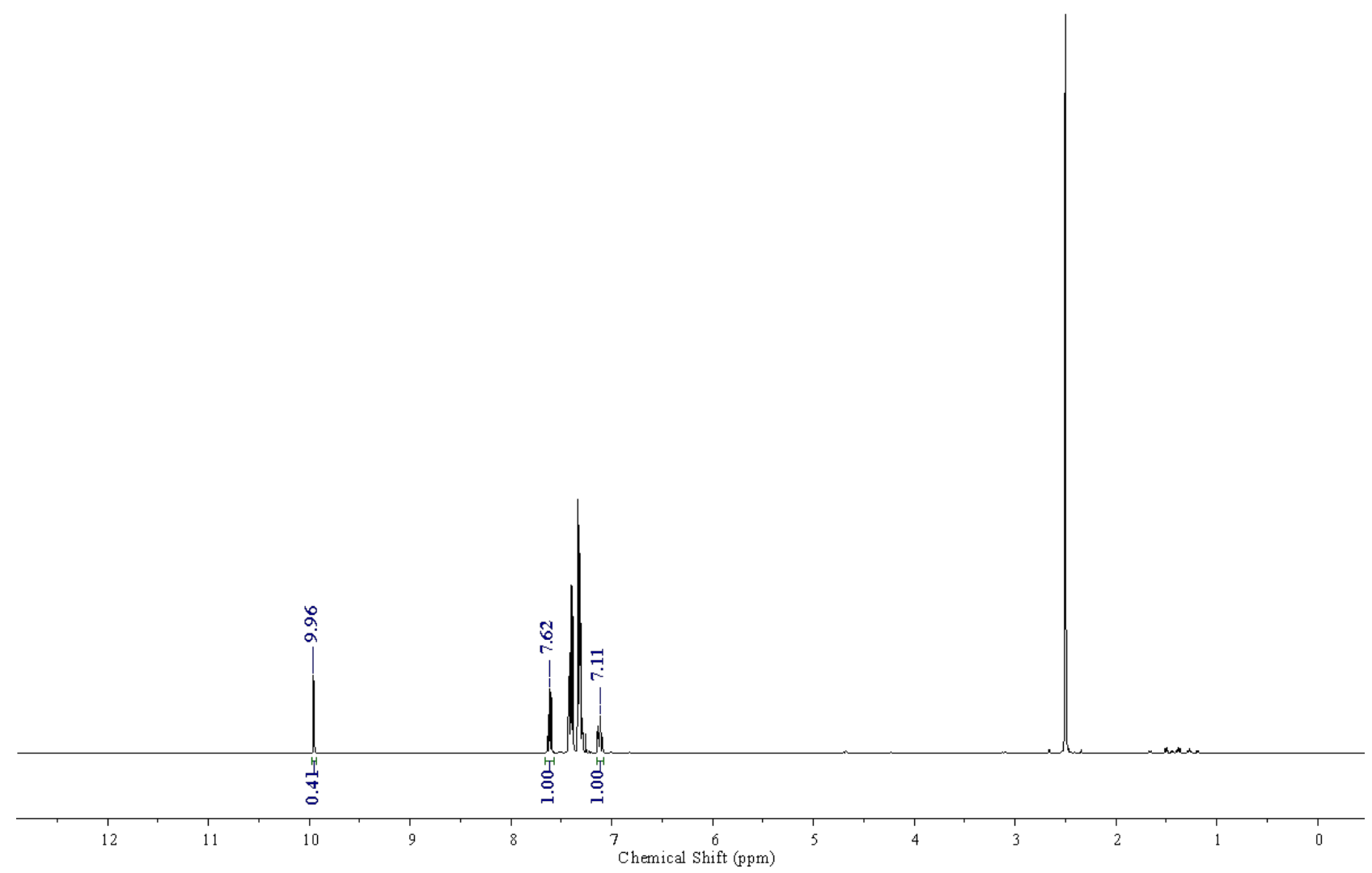

Entry 2: NMR for Crude reaction mixture

VDOS-594-B 1.7.fid

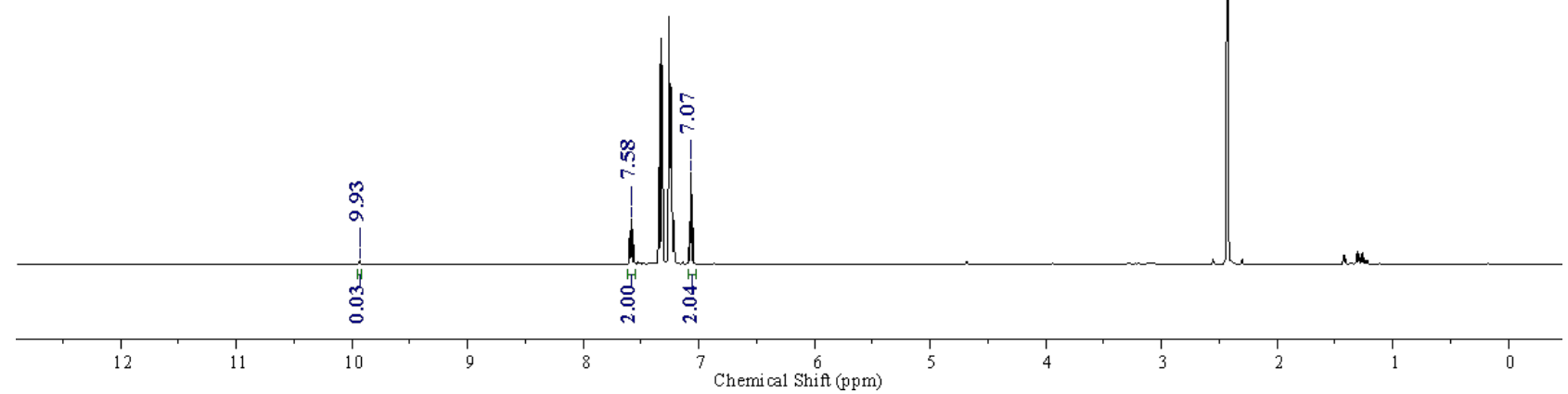

Entry 2': NMR for Crude reaction mixture, Duplicate 


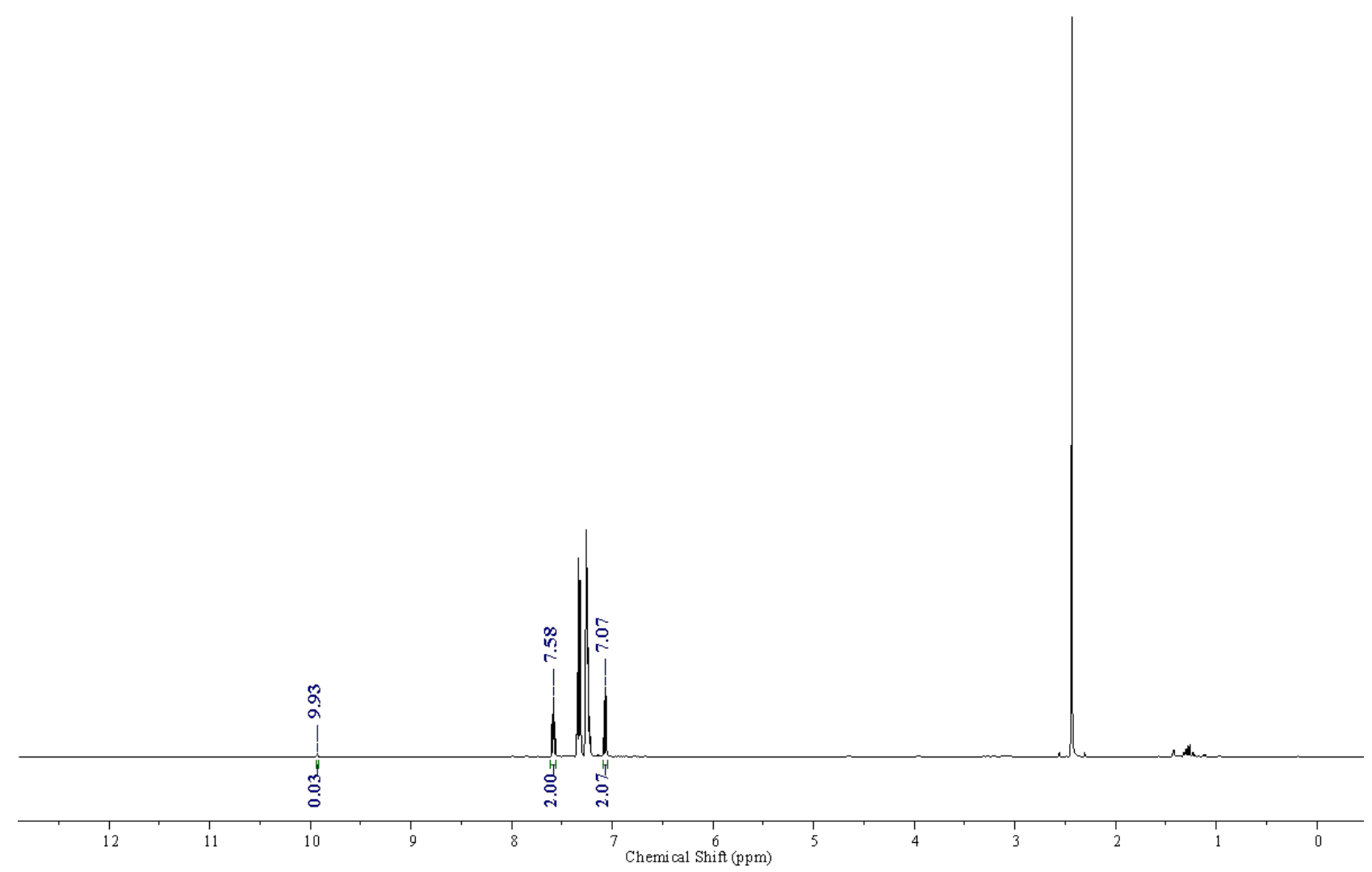

NMR after purification:

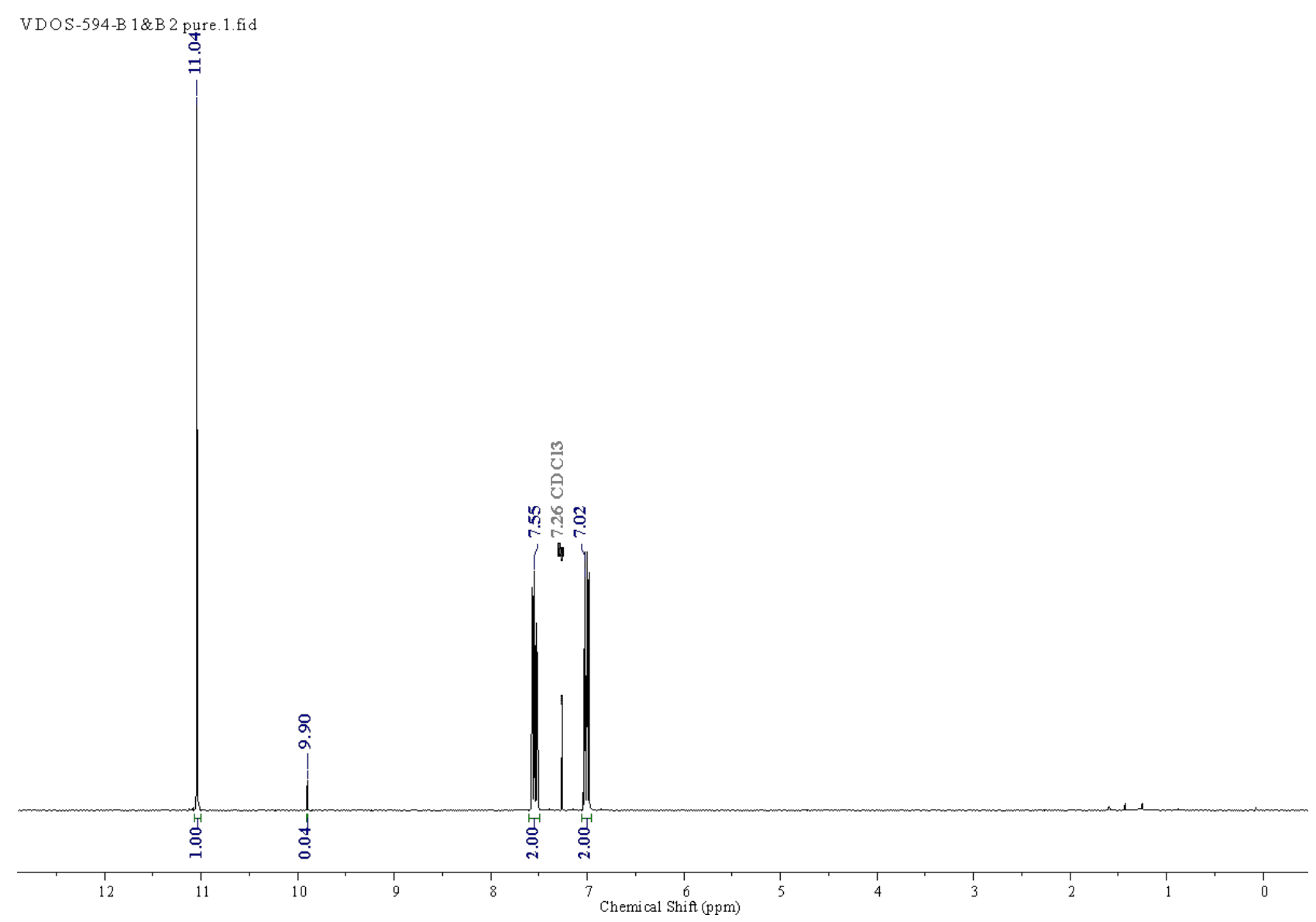




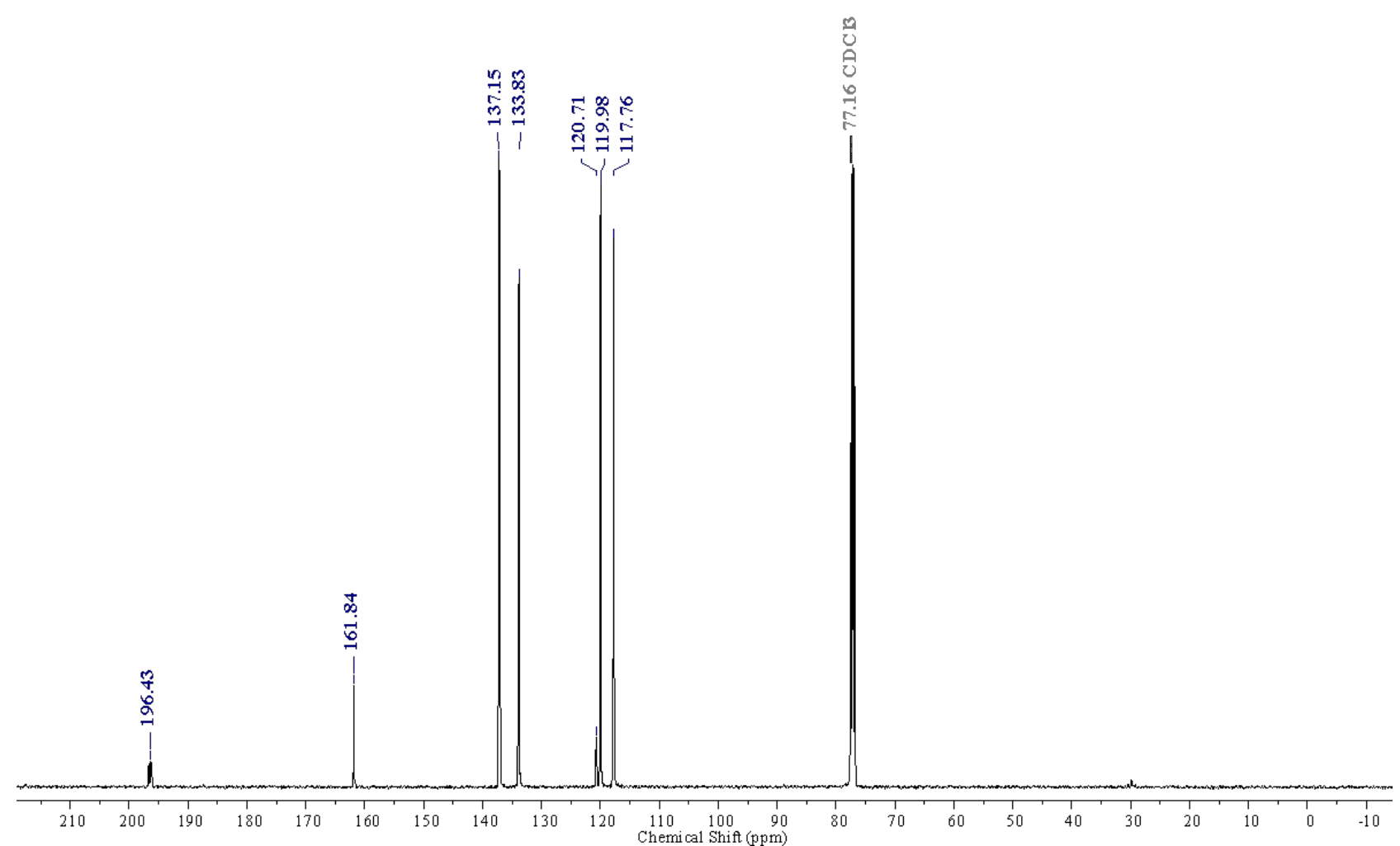


11. 3-Methylbenzaldehyde-d<smiles>[2H]C(=O)c1cccc(C)c1</smiles>

Entry 1: NMR for Crude reaction mixture SCG-312-I1-16hr-D 1-50sec

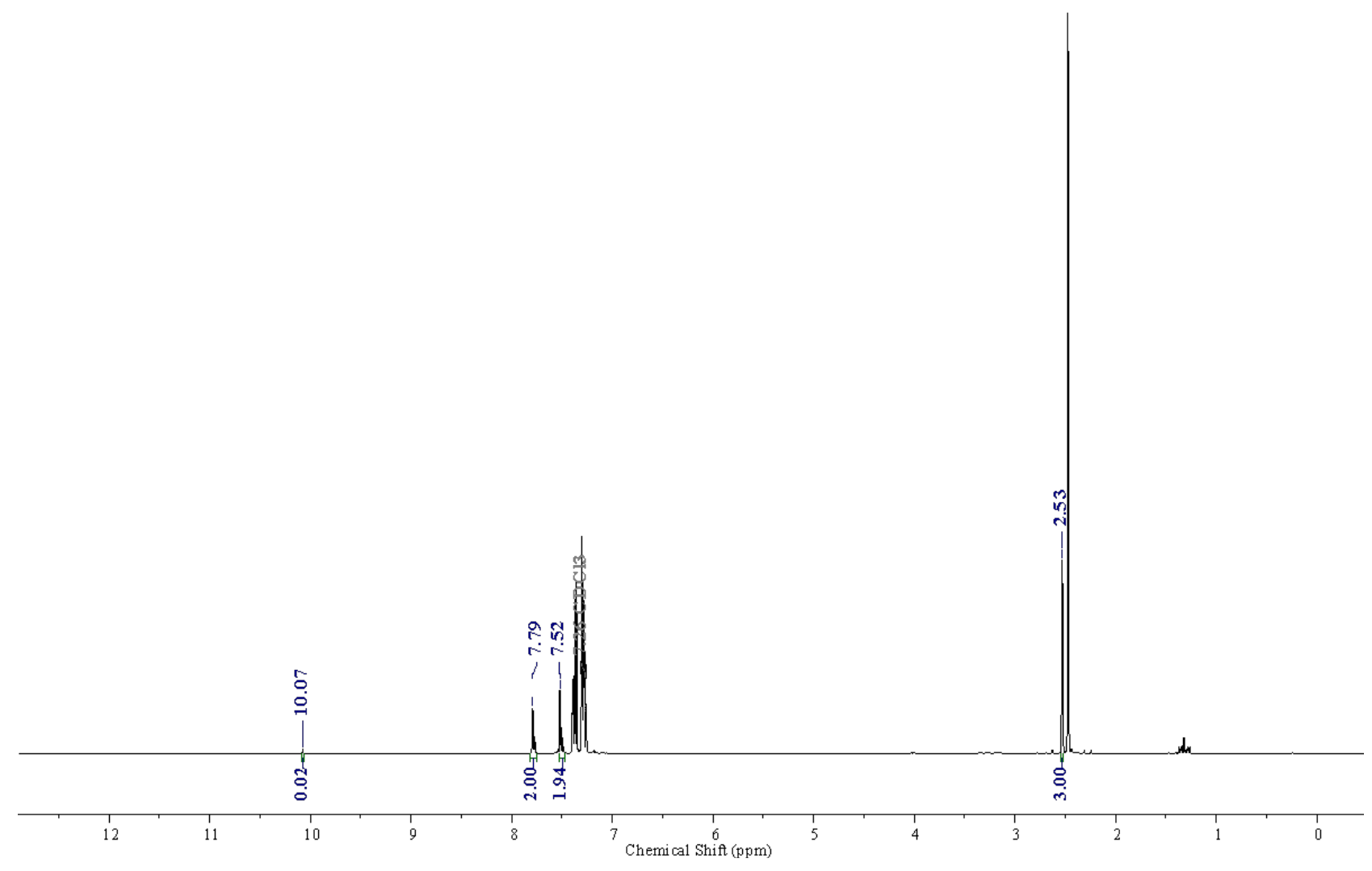


Entry 1': NMR for Crude reaction mixture, Duplicate

SCG-312-I2-16hr-D 1-50sec

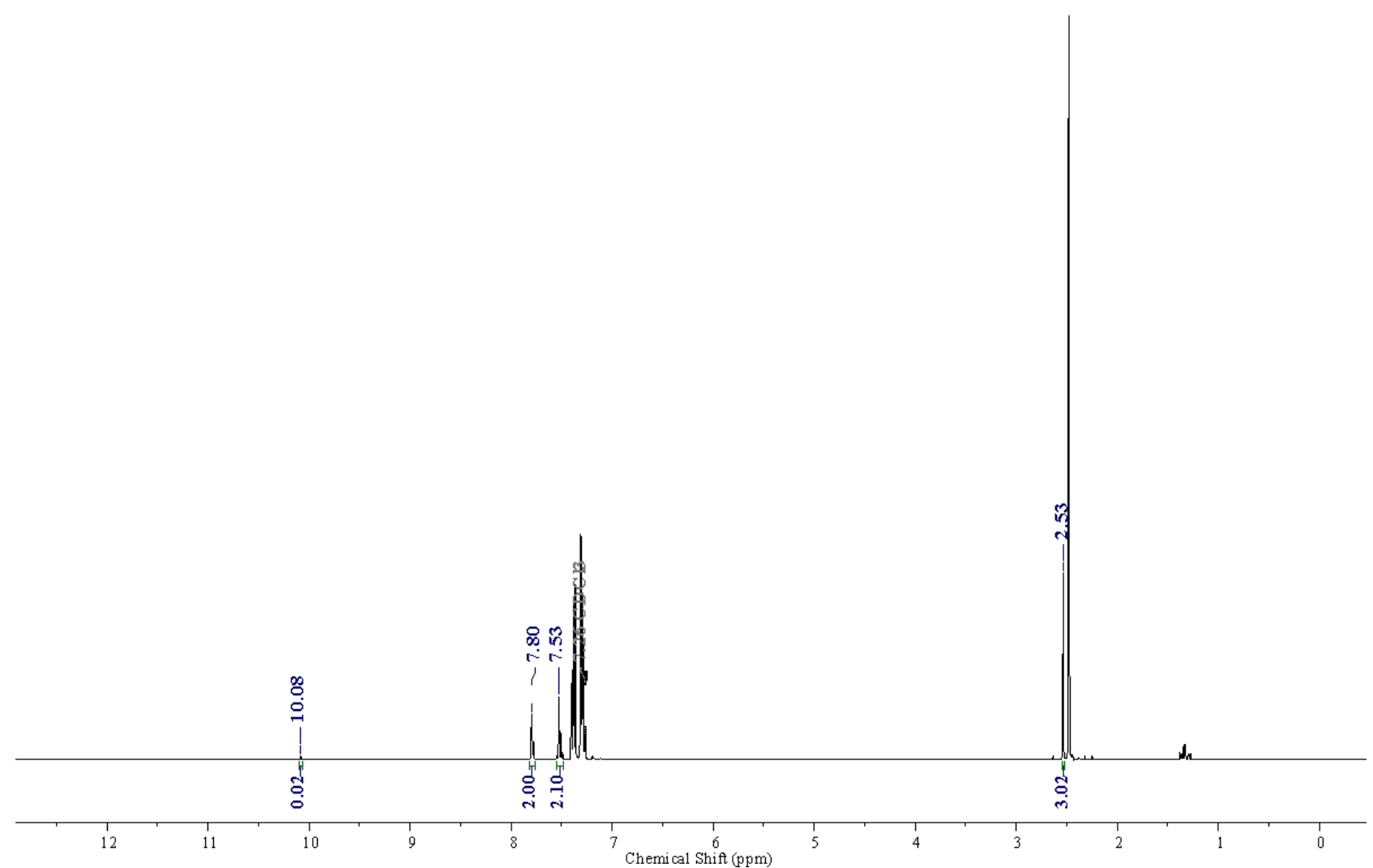

NMR after purification:

SCG-312-I5-16hr-D 1-50sec-AC

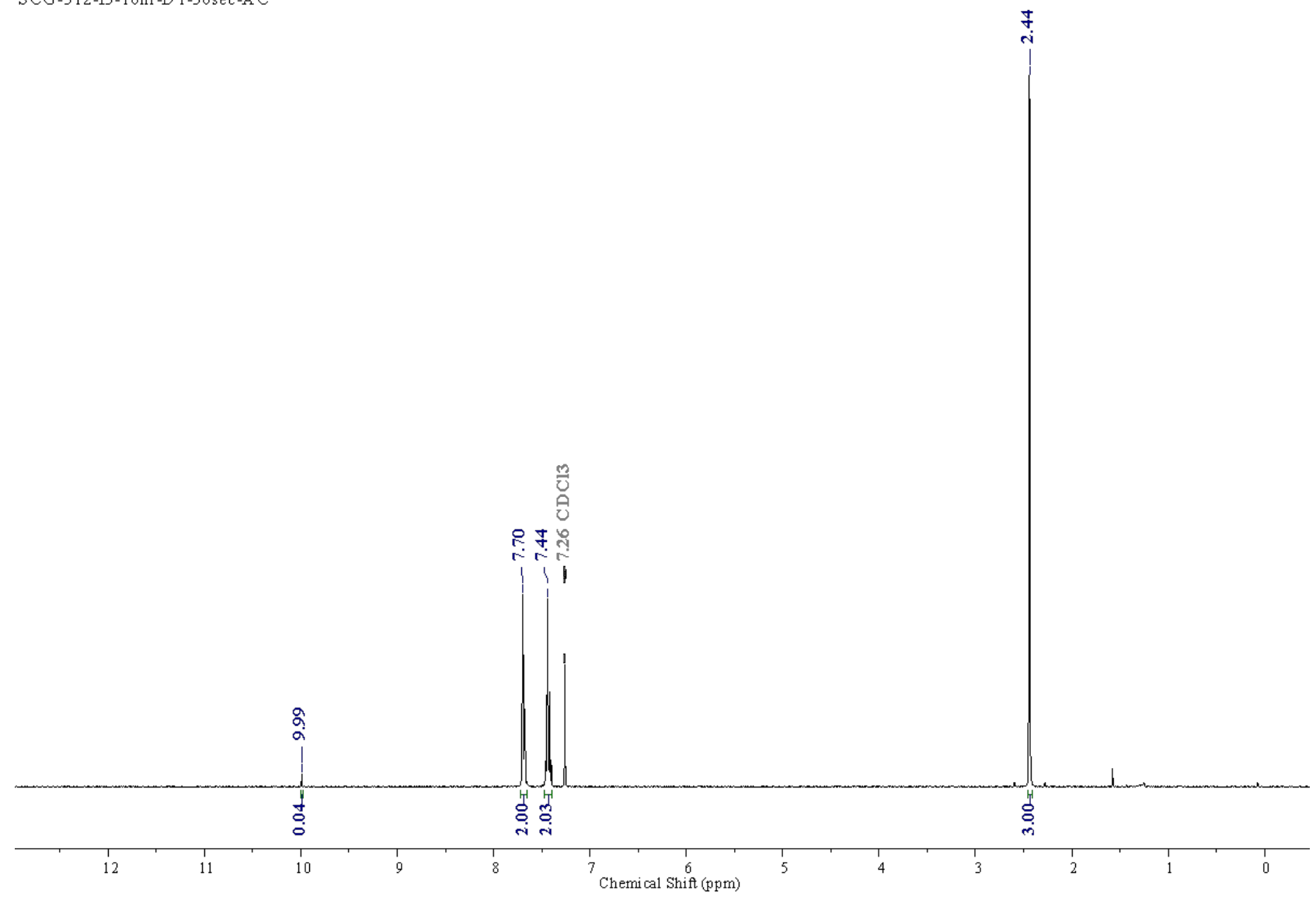




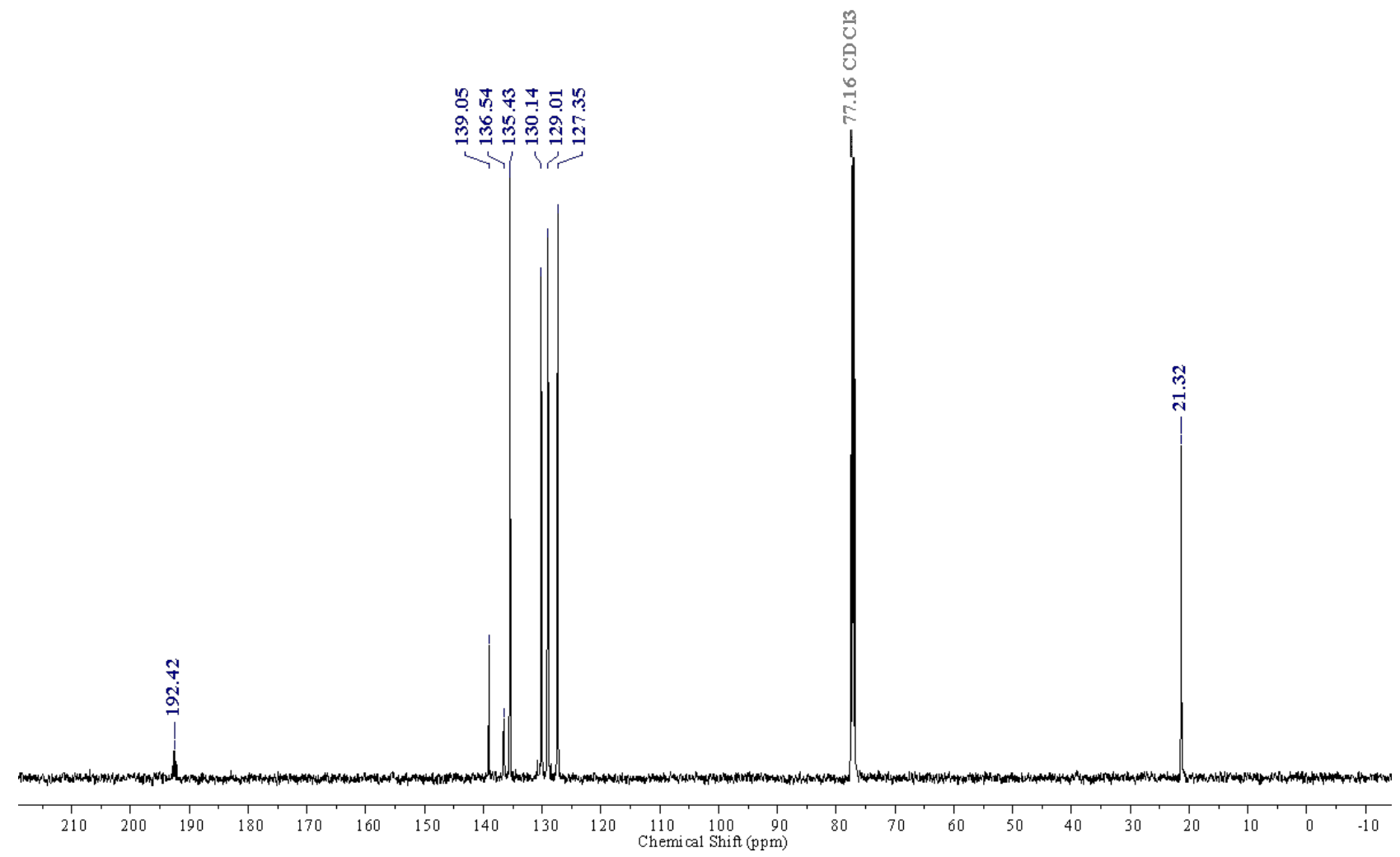


12. 4-(Diethoxymethyl)benzaldehyde-d

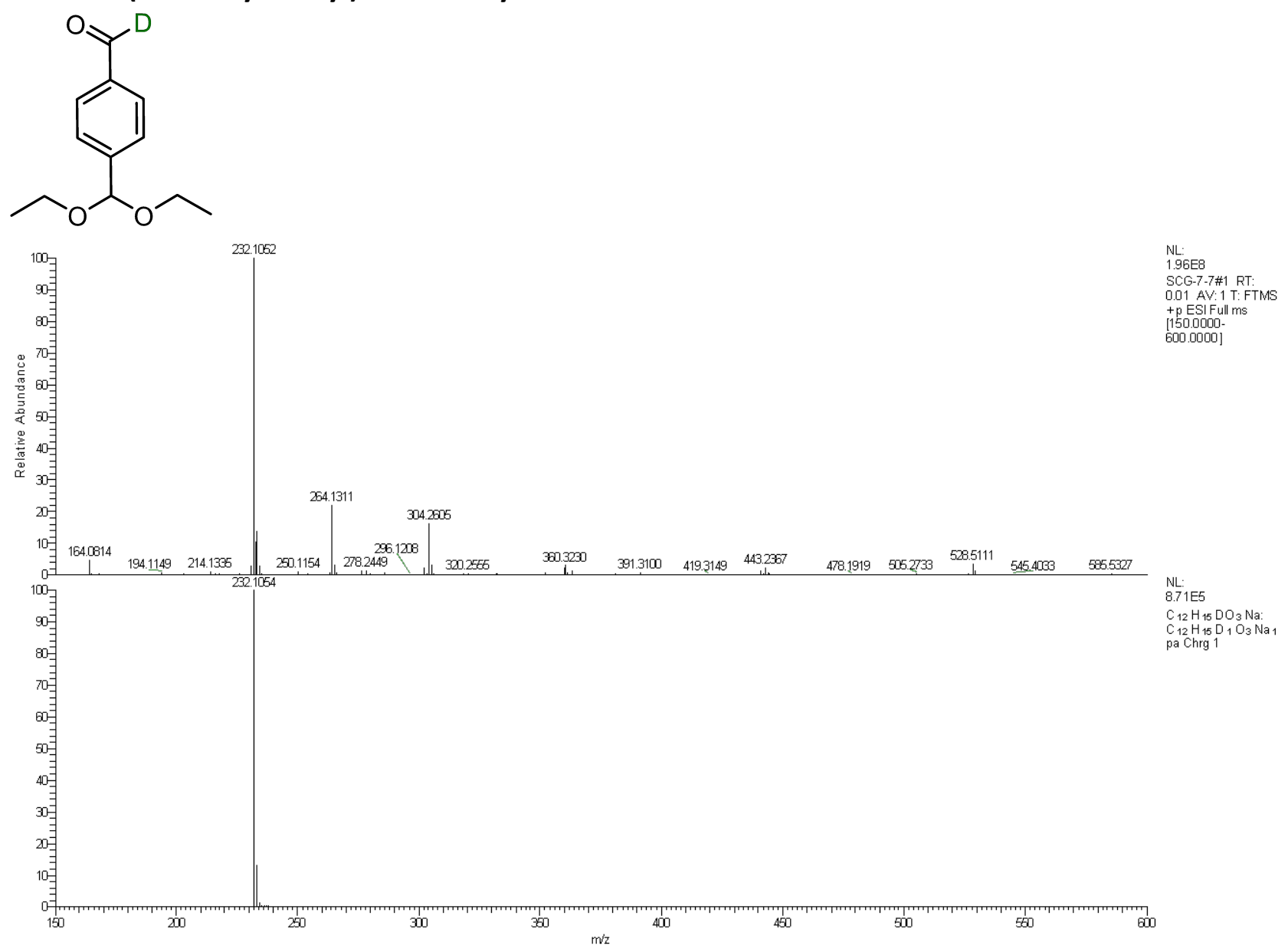


Entry 1: NMR for Crude reaction mixture

SCG-318-C1-16hr-D 1-50sec

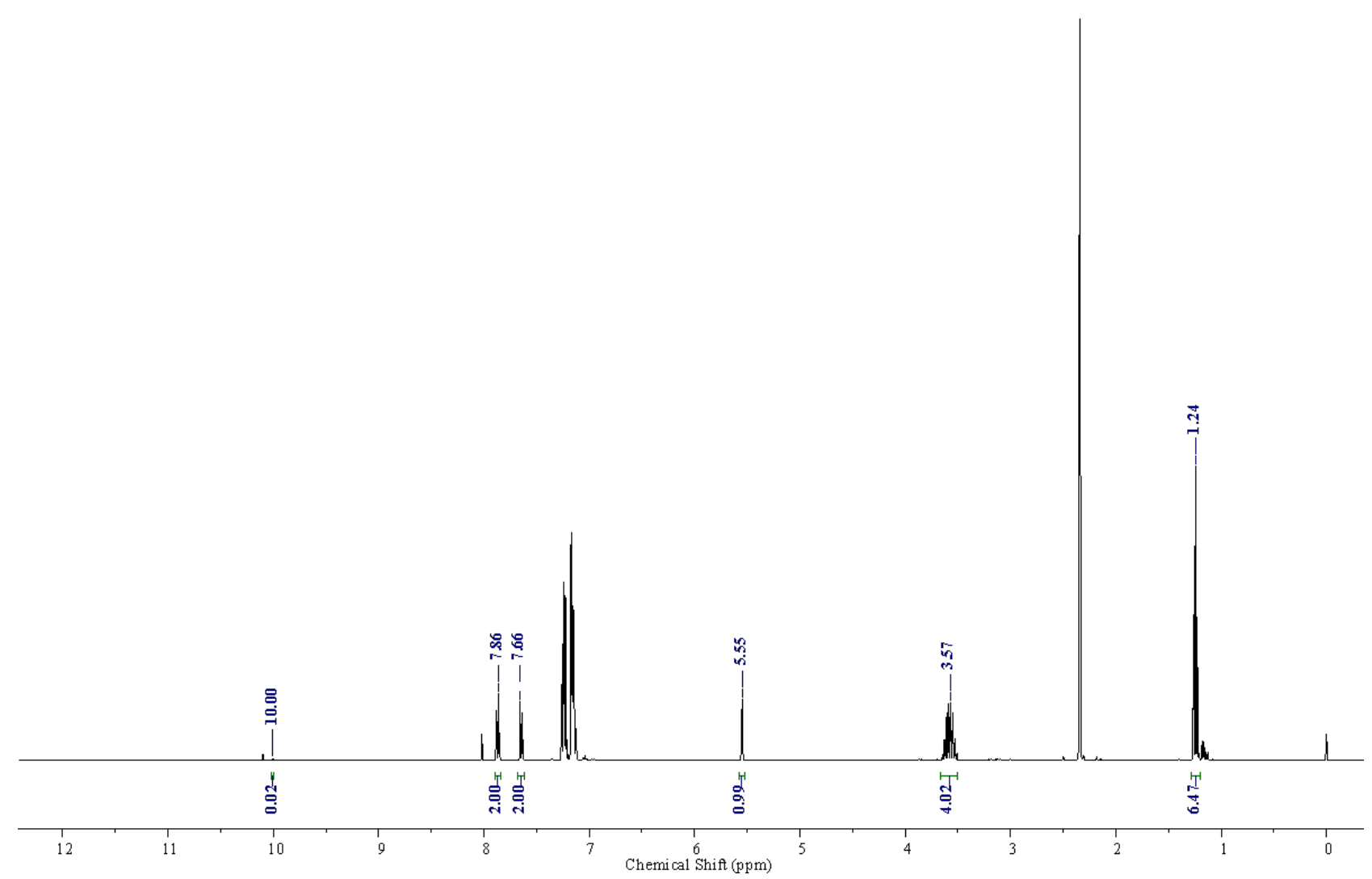

Entry 1': NMR for Crude reaction mixture, Duplicate

SCG-318-C2-16hr-D 1-50sec 
NMR after purification:

SCG-318-C1-16hr-D 1-50sec-AC
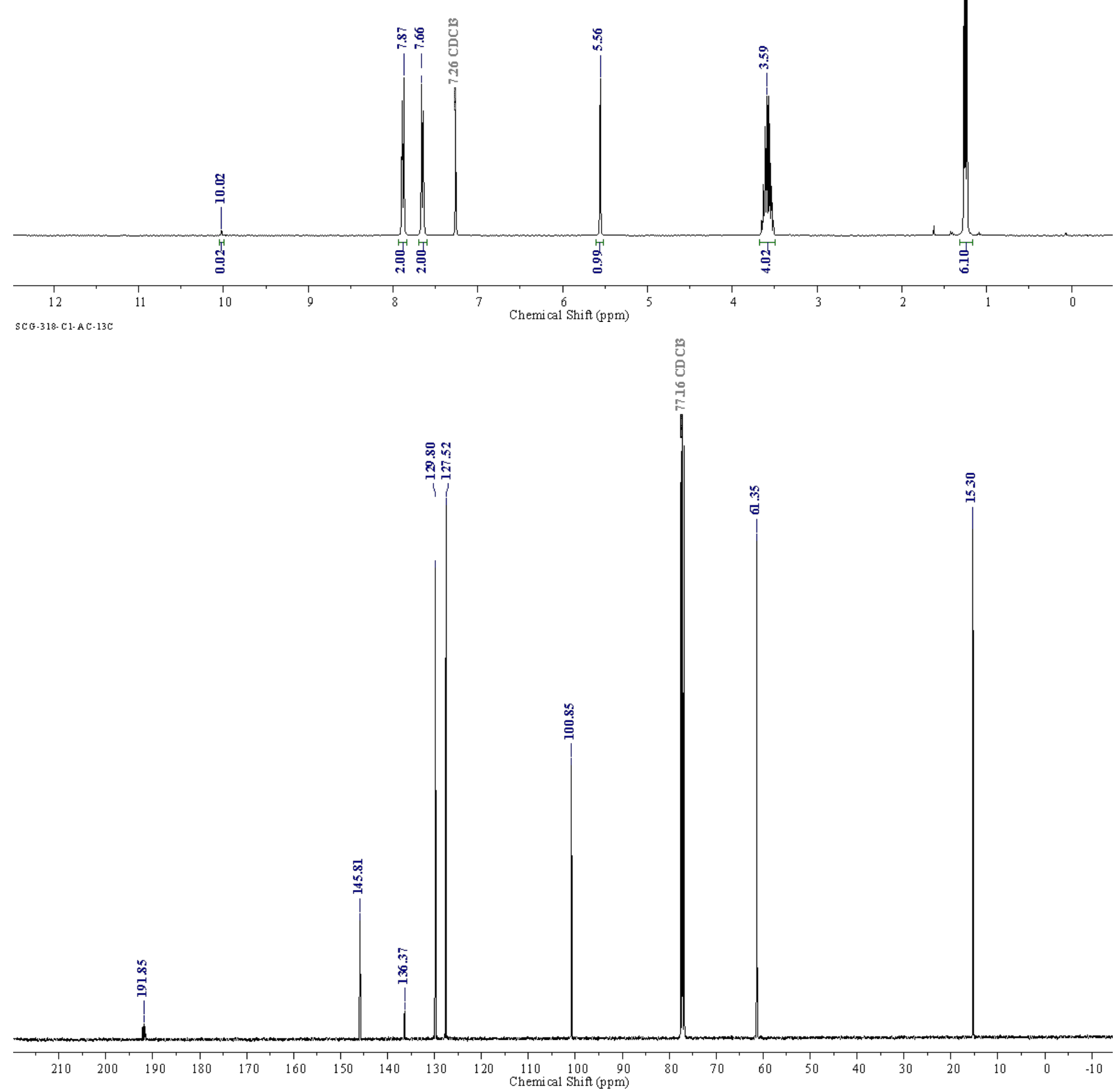


\section{3-Hydoxybenzaldehyde-d}<smiles>[2H]C(=O)c1cccc(O)c1</smiles>

2 \#1 RT: 0.01 AV 1 NL-1.63E8

T: FTMS + p ESI Full ms [50.0000-750.0000]

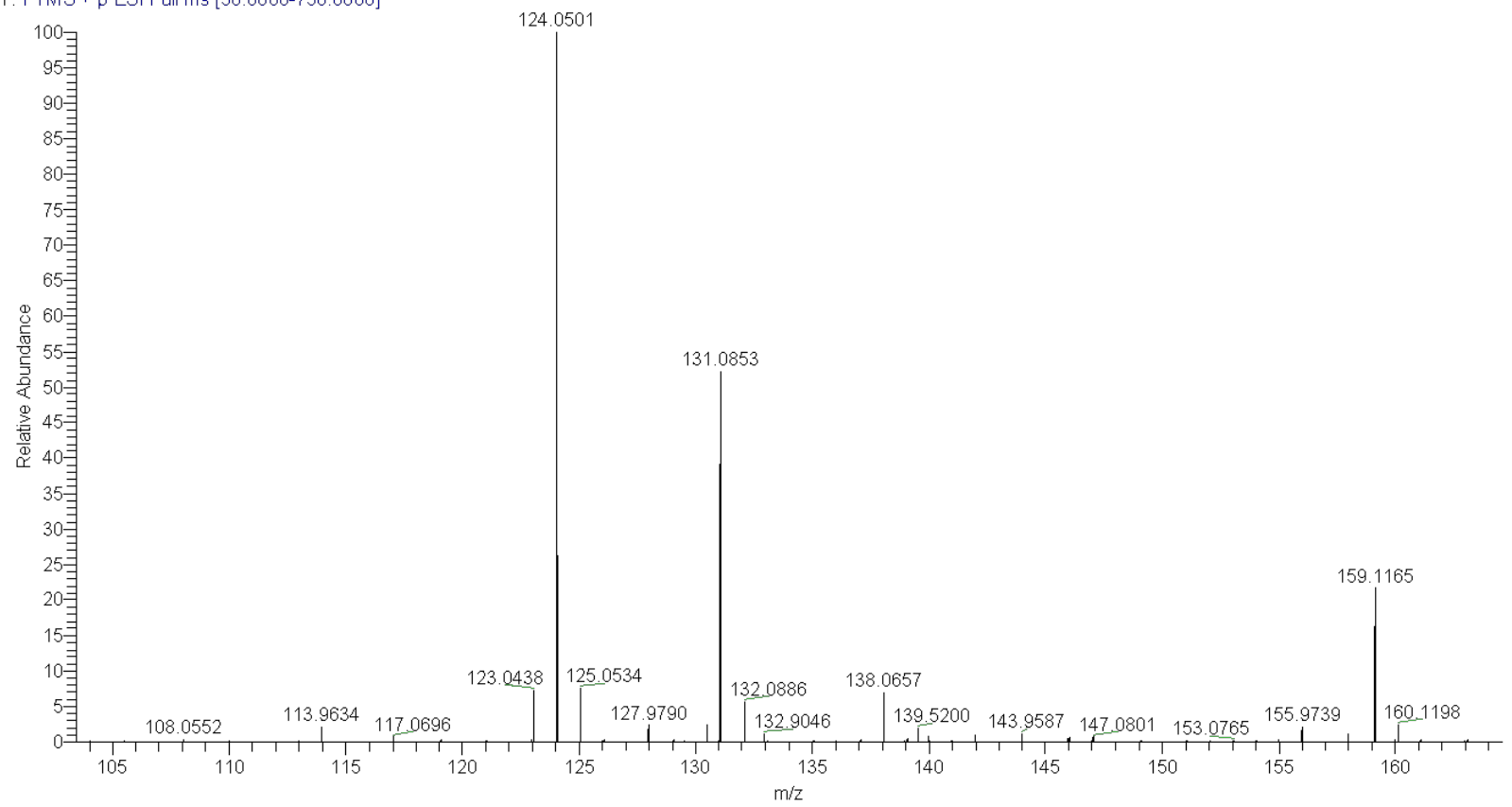

Entry 1: NMR for Crude reaction mixture vDOS-695-A

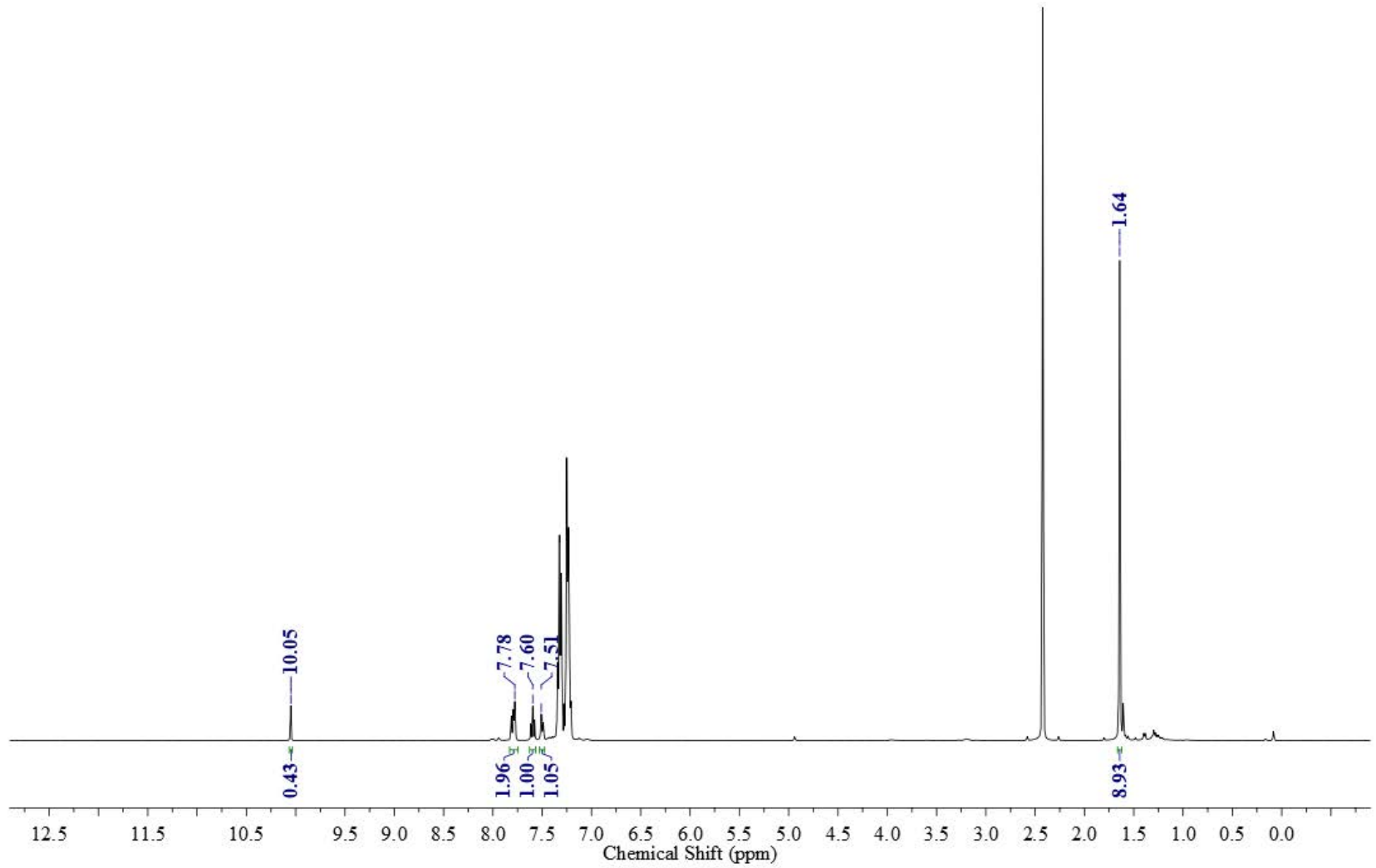


Entry 2: NMR for Crude reaction mixture

vDOS-695-B1 Crude

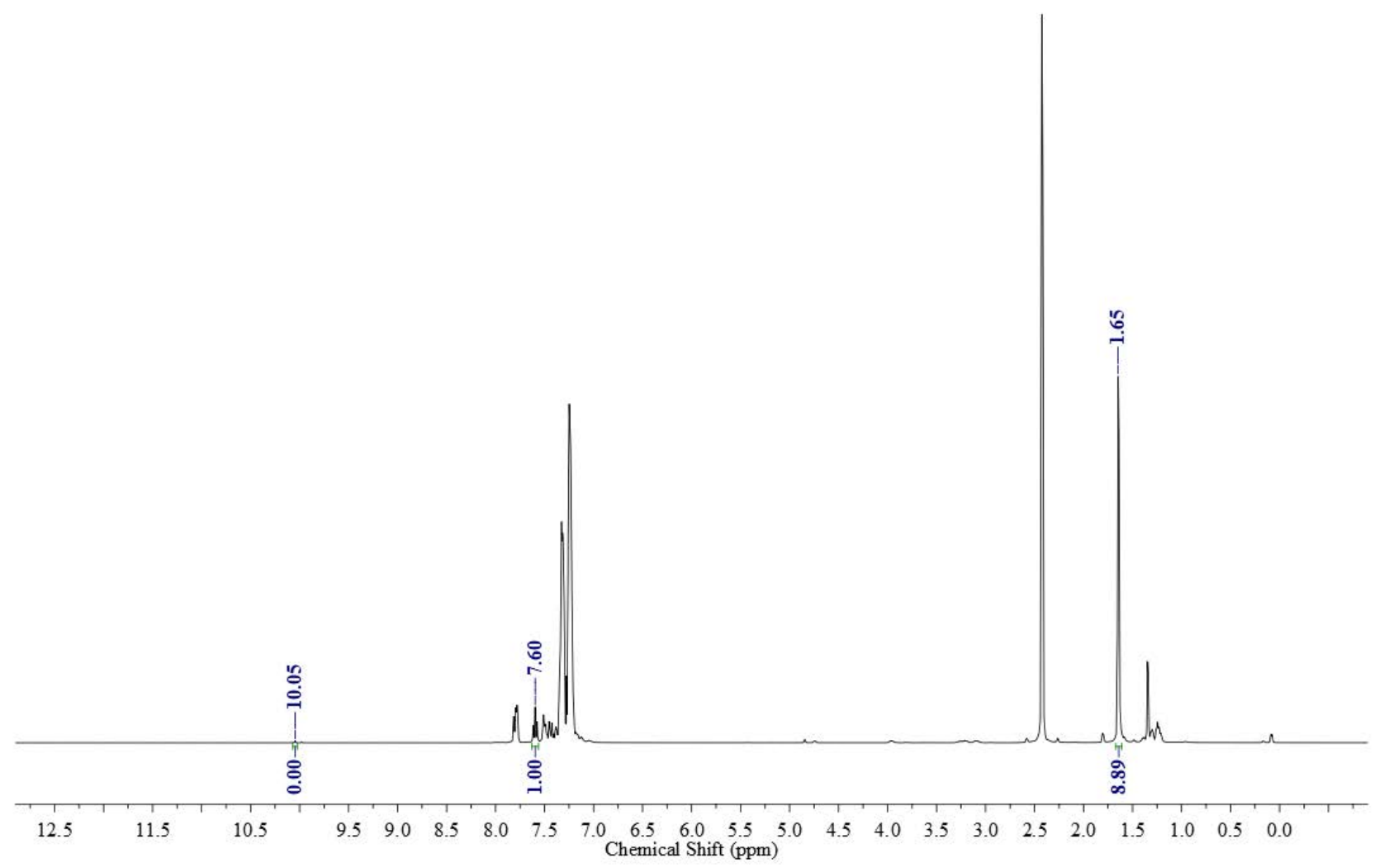

NMR after purification:

3-OBocbenzaldehyde- $d$

VDOS-695-B1-OBoc pure

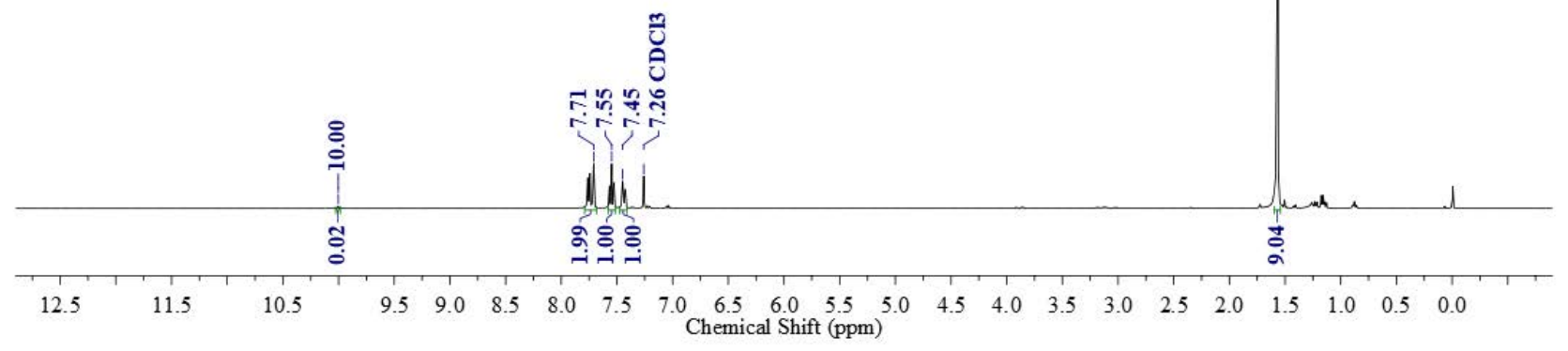




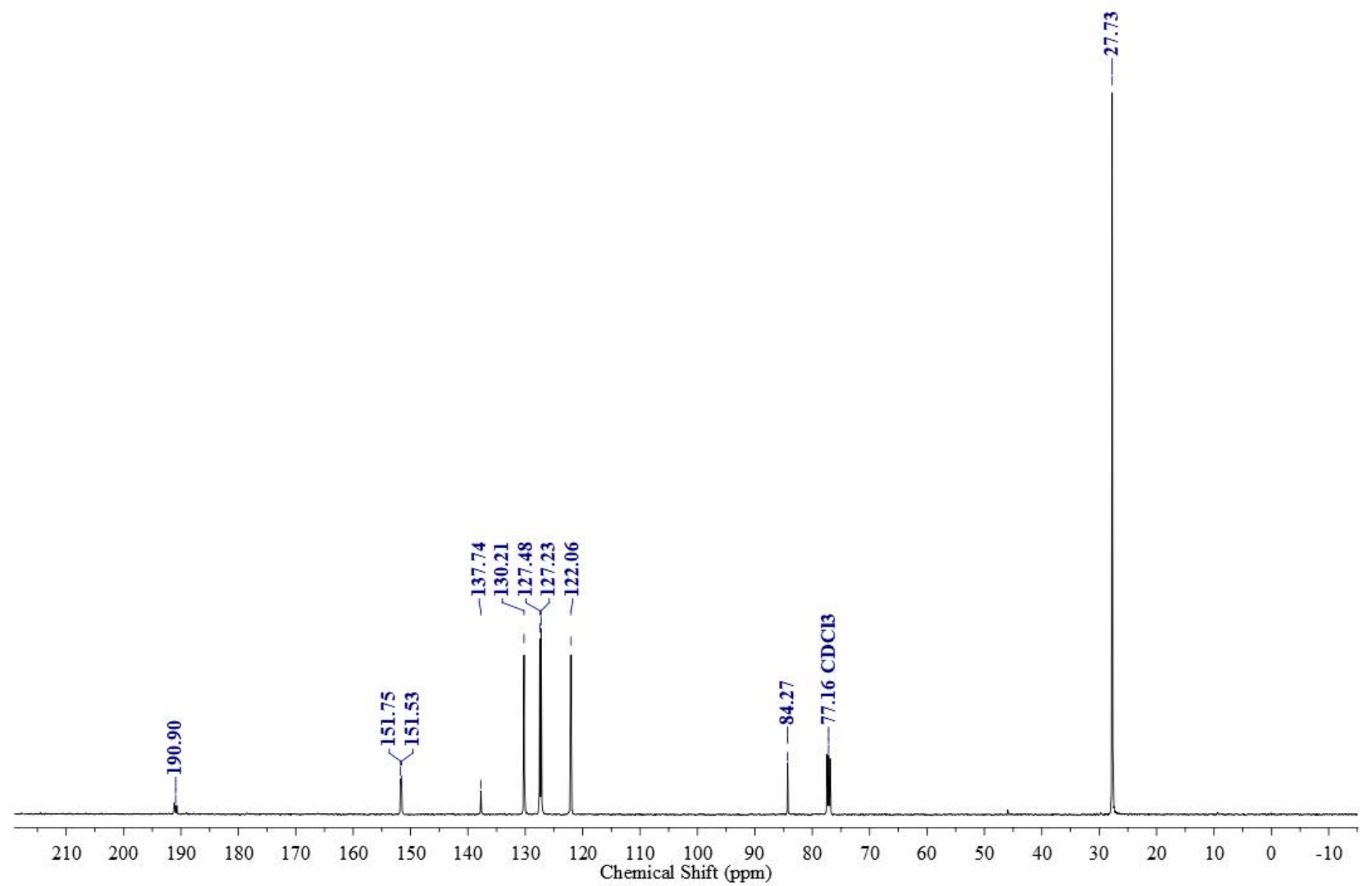

3-Hydoxybenzaldehyde-d

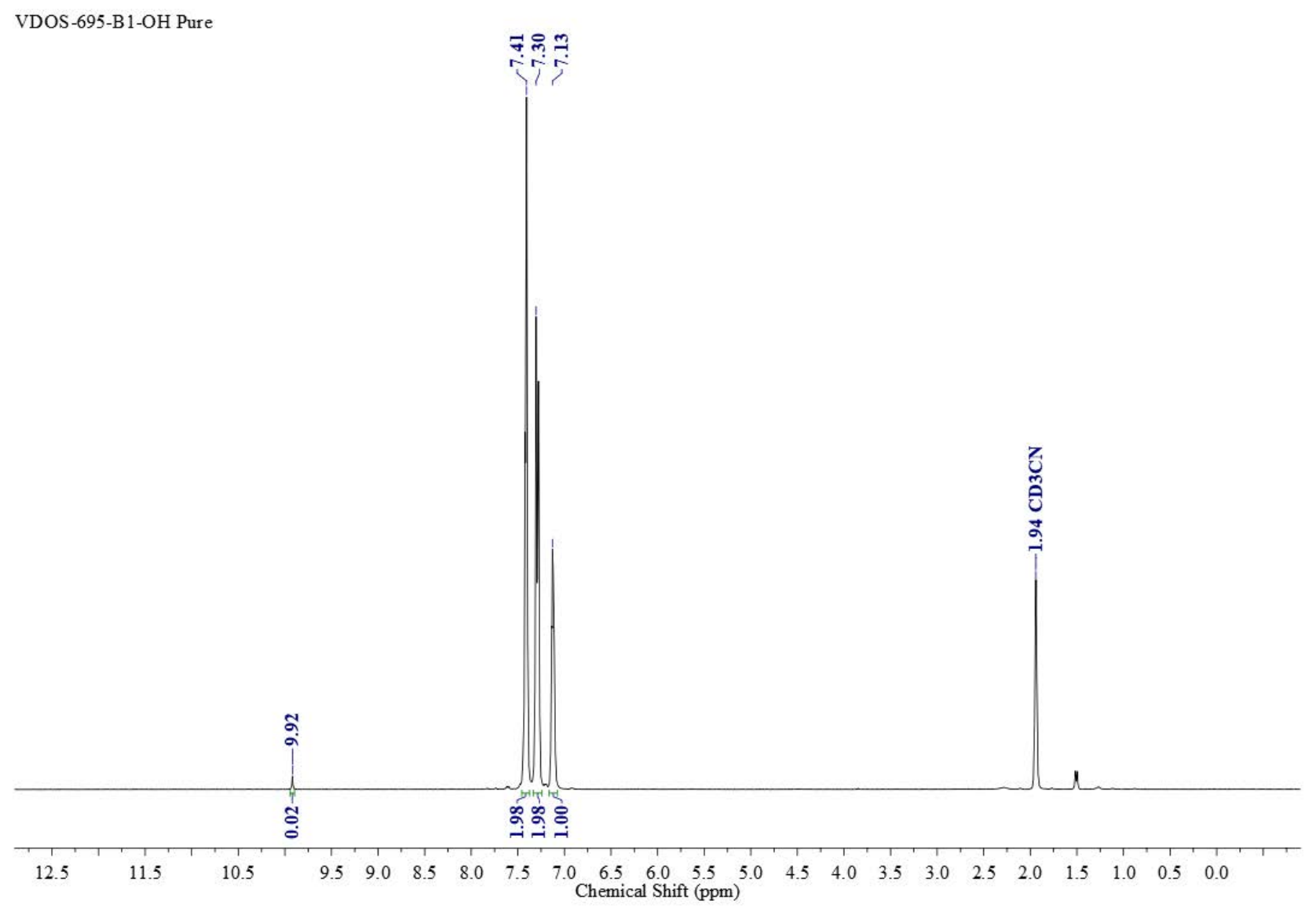


Entry 3: NMR for Crude reaction mixture

vDOS-695-B4 CD3CN-F2

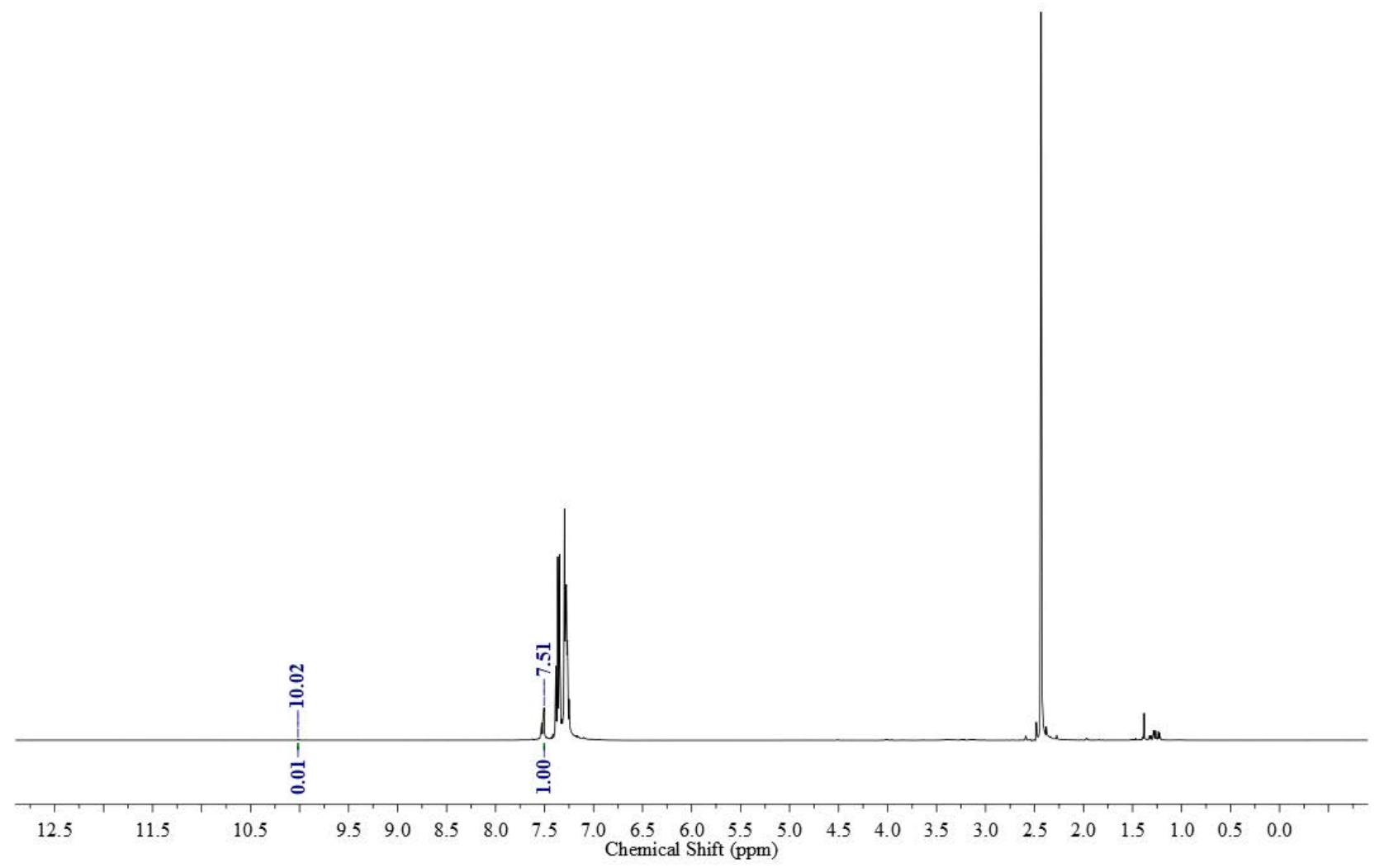

Entry 3': NMR for Crude reaction mixture, Duplicate

vDOS-695-B3 Crude

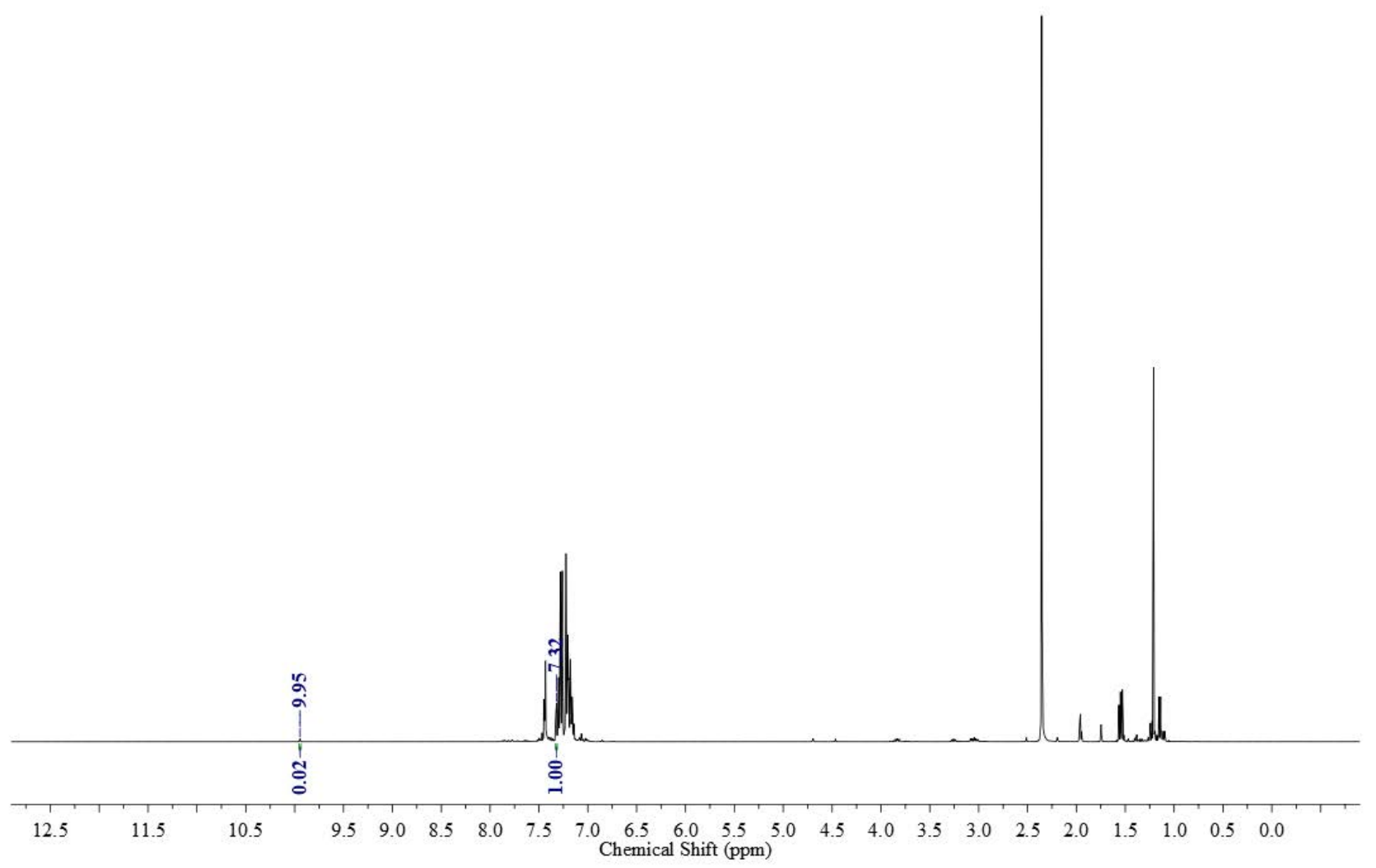


NMR after purification:

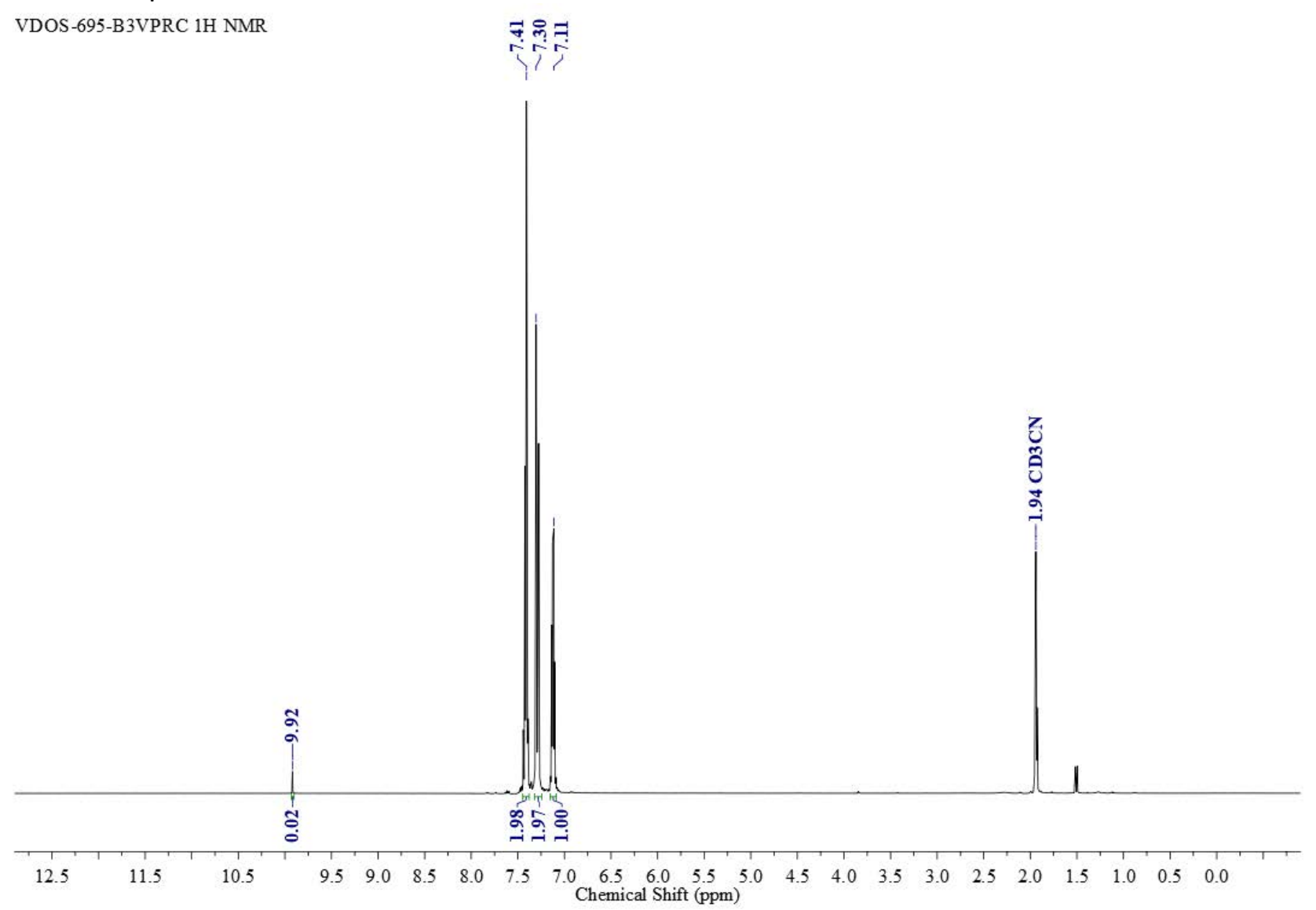

VDOS-695-B3VPRC -13CNMR

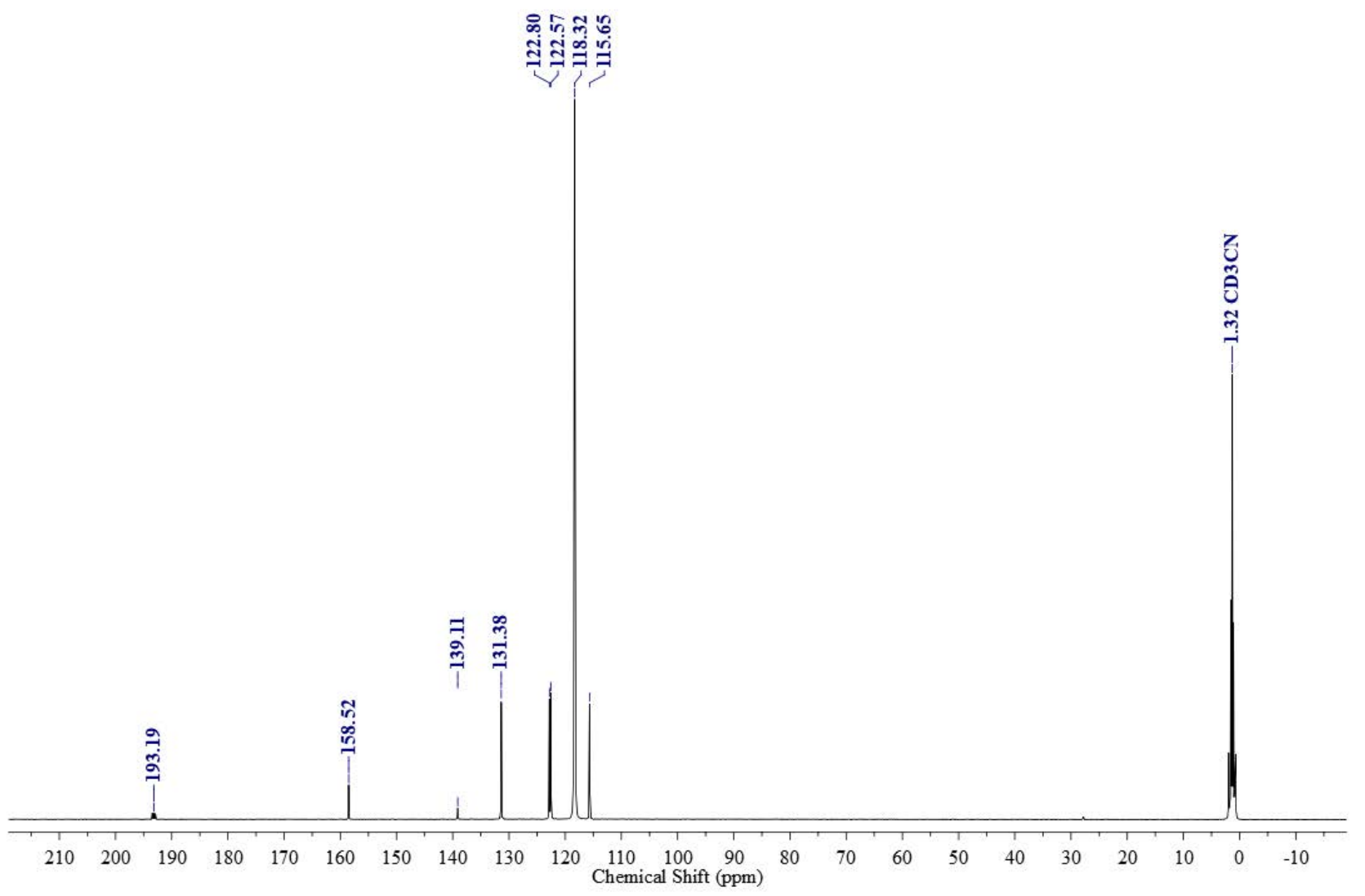


Entry 4: NMR for Crude reaction mixture vDOS-695-B5

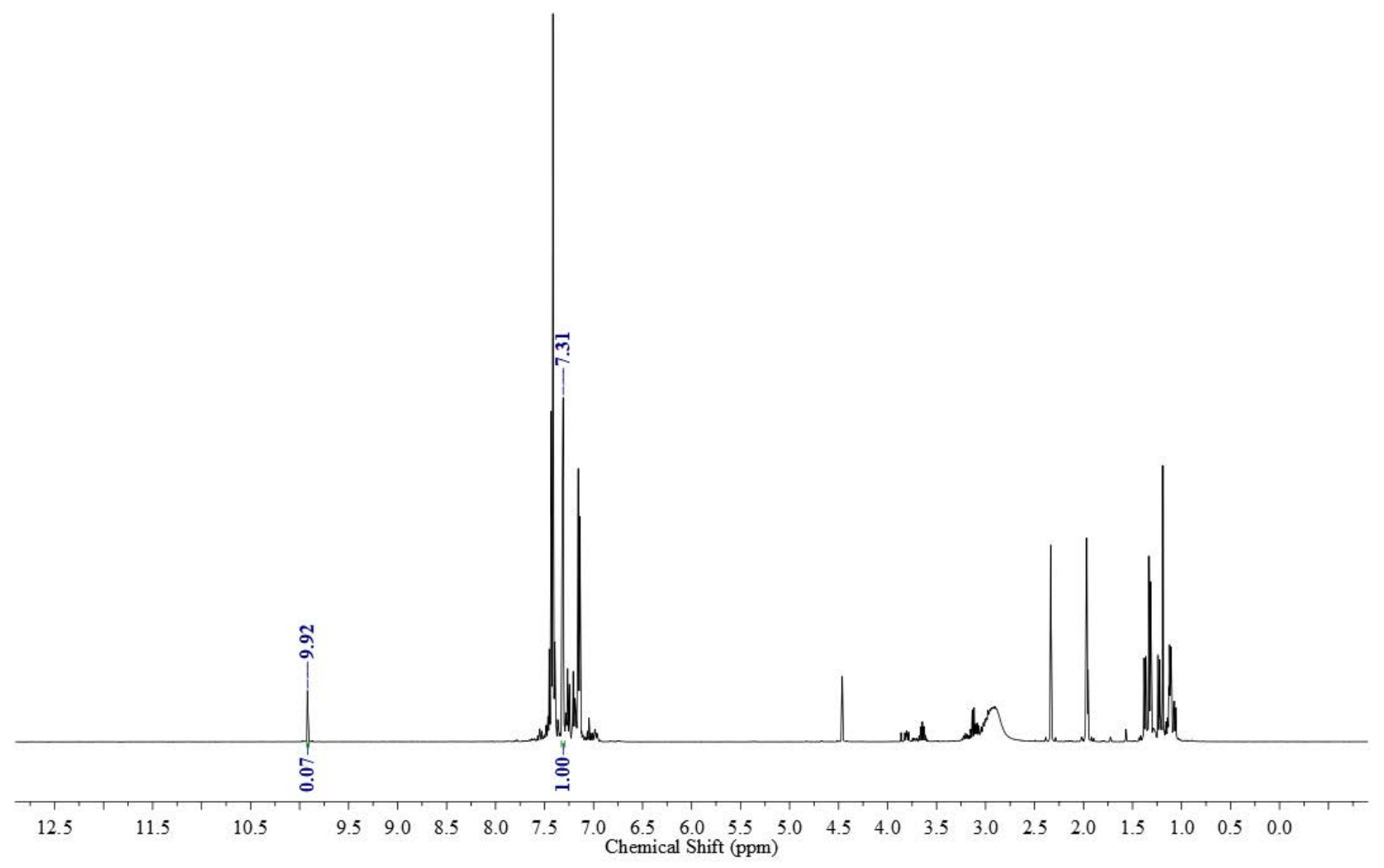

NMR after purification:

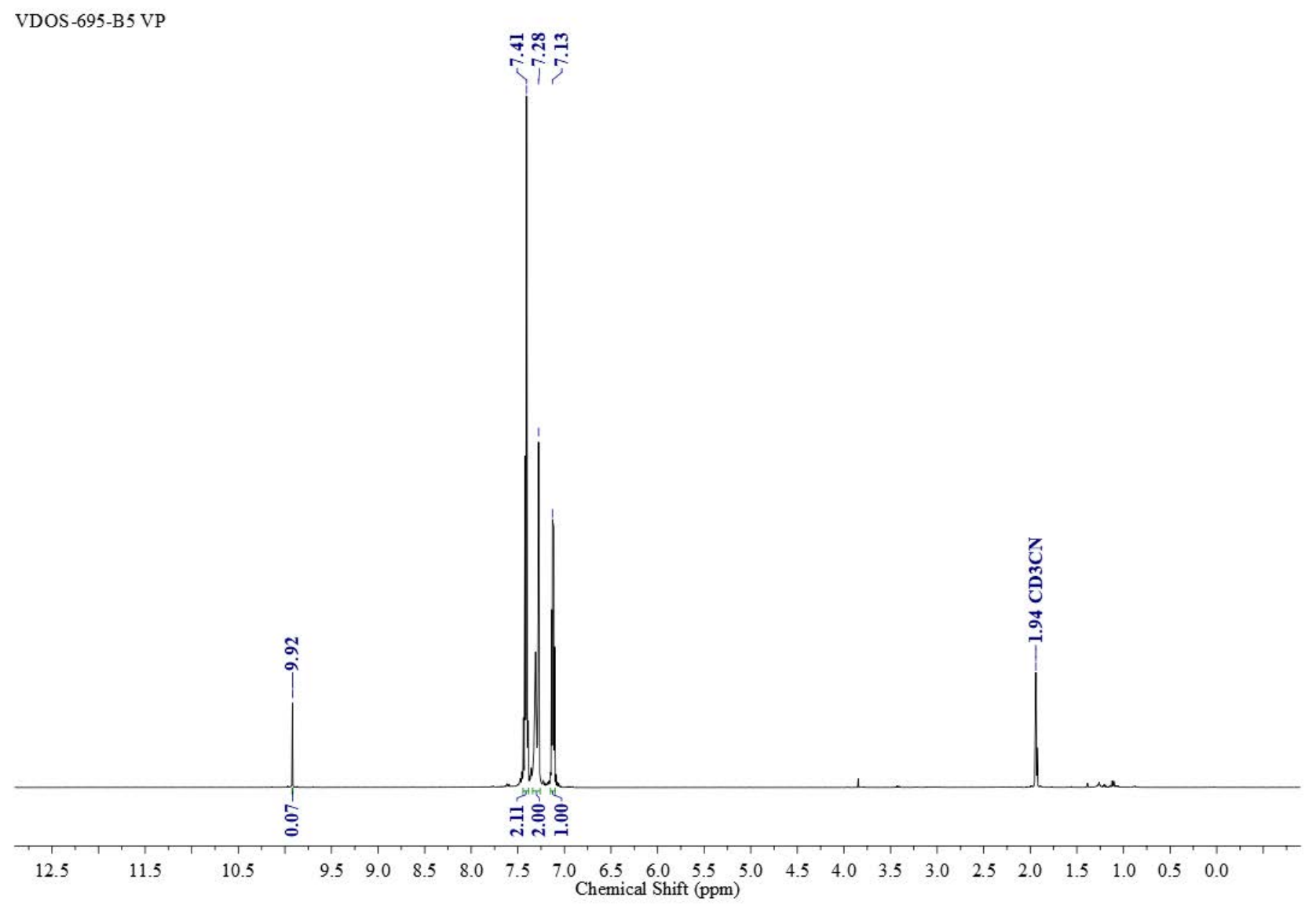


14. 2-(Trifluoromethyl)benzaldehyde-d<smiles>[2H]C(=O)c1ccccc1C(F)(F)F</smiles>

Entry 1: NMR for Crude reaction mixture SCG-309-K1-16hr-D1-50sec.1.fid

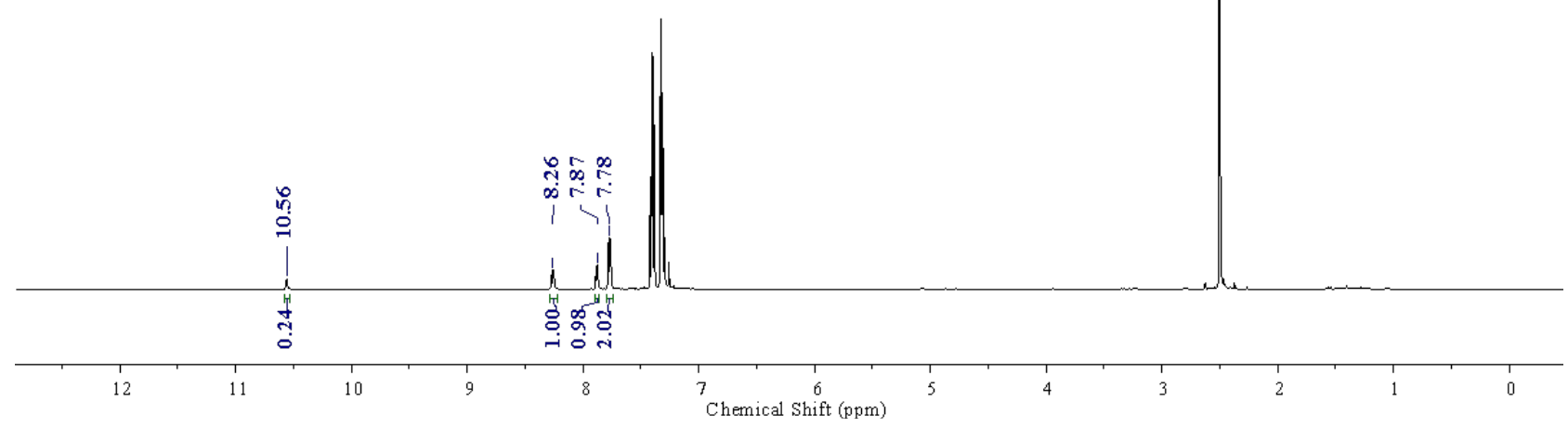


Entry 1': NMR for Crude reaction mixture, Duplicate

SCG-309-K2-16hr-D1-50sec.1. fid

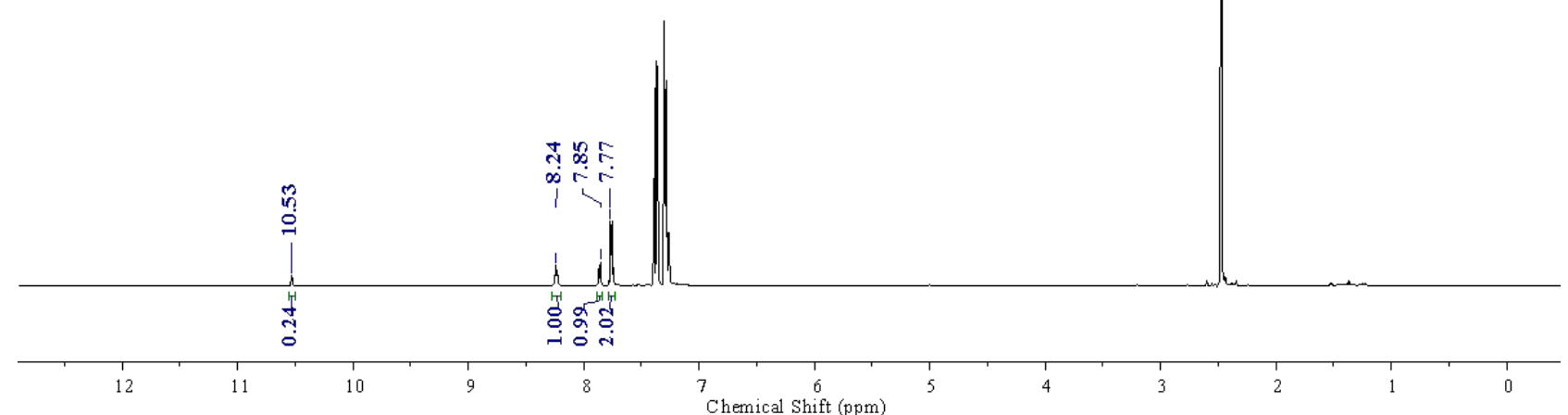

Entry 2: NMR for Crude reaction mixture

VDOS-595-B 2.2.fid

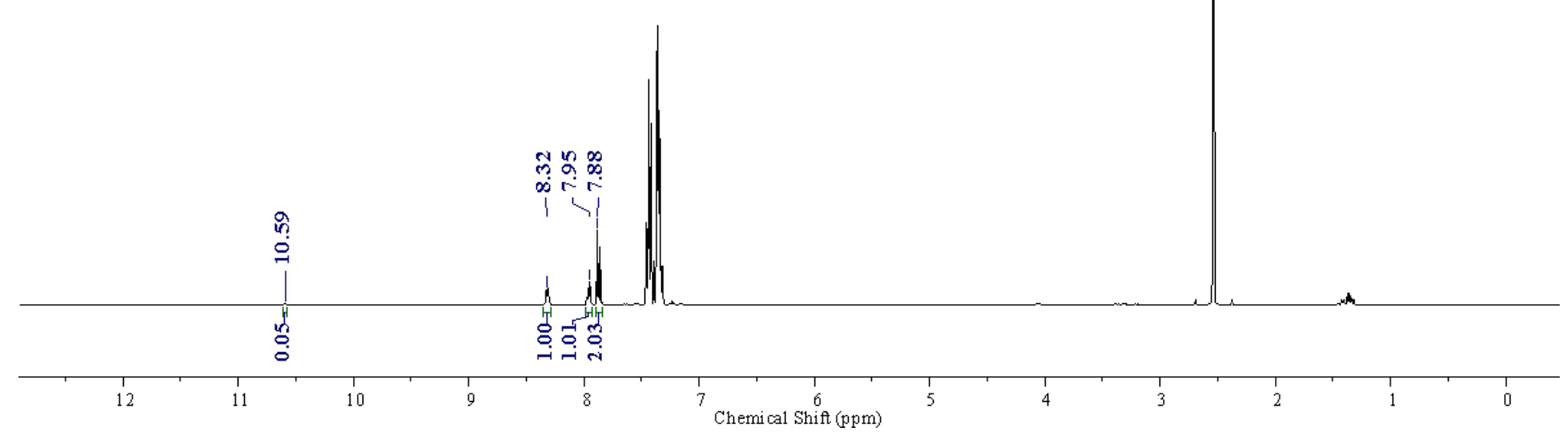


Entry 2': NMR for Crude reaction mixture, Duplicate VDOS-595-B 1.1. fid

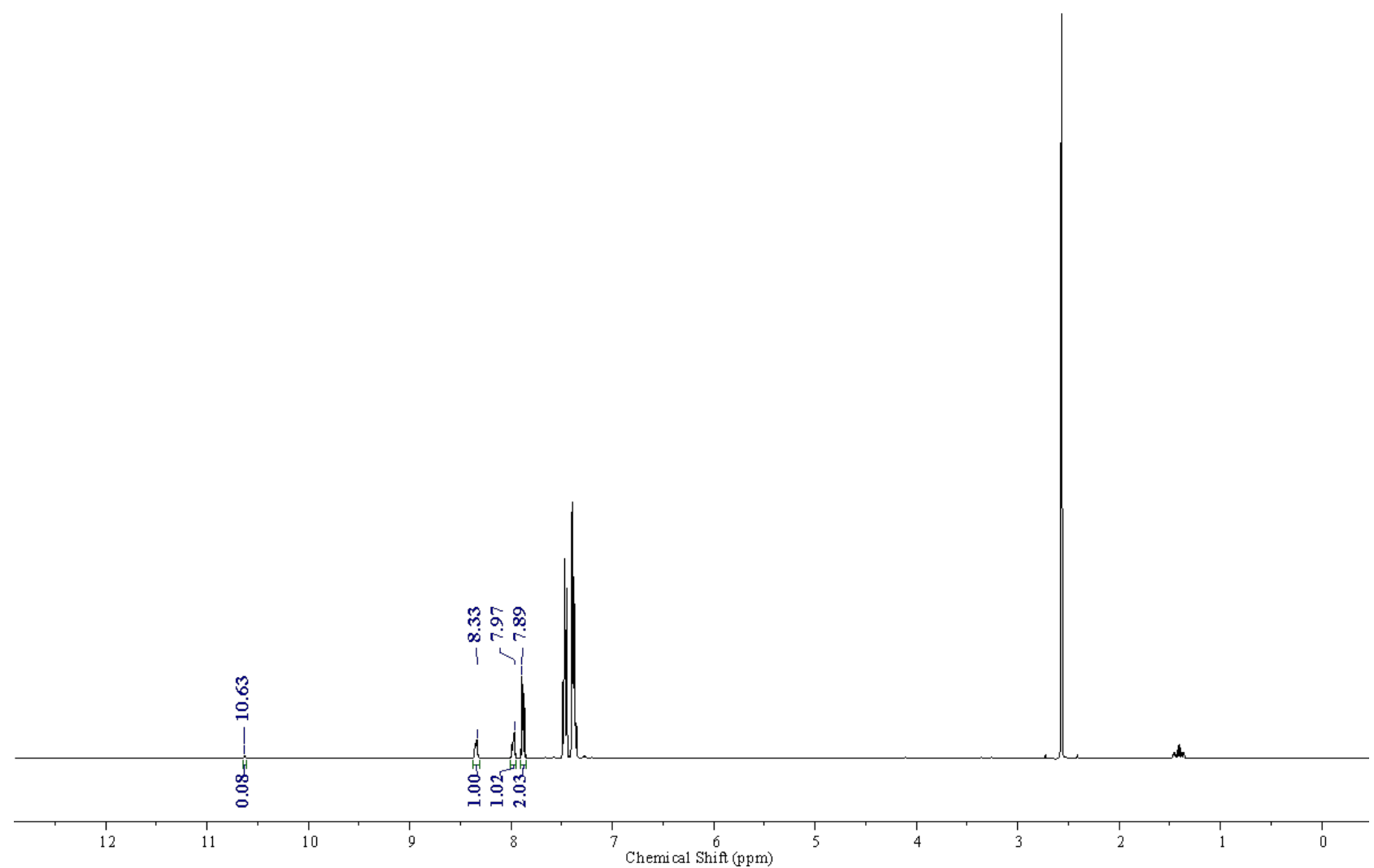

Entry 3: NMR for Crude reaction mixture

VDOS-595--F1.5.fid

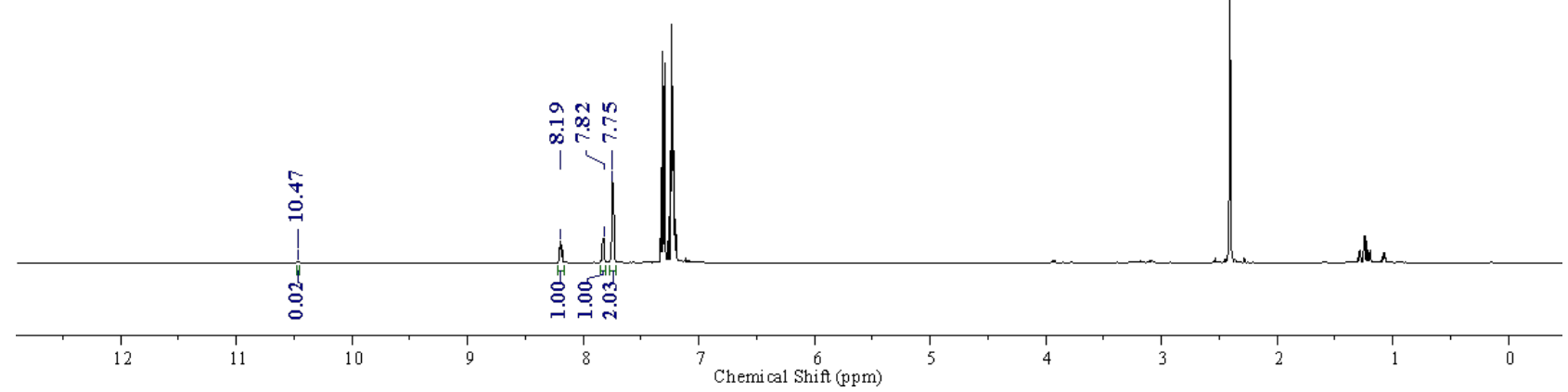


Entry 3': NMR for Crude reaction mixture, Duplicate

VDOS-595--F2.6.fid

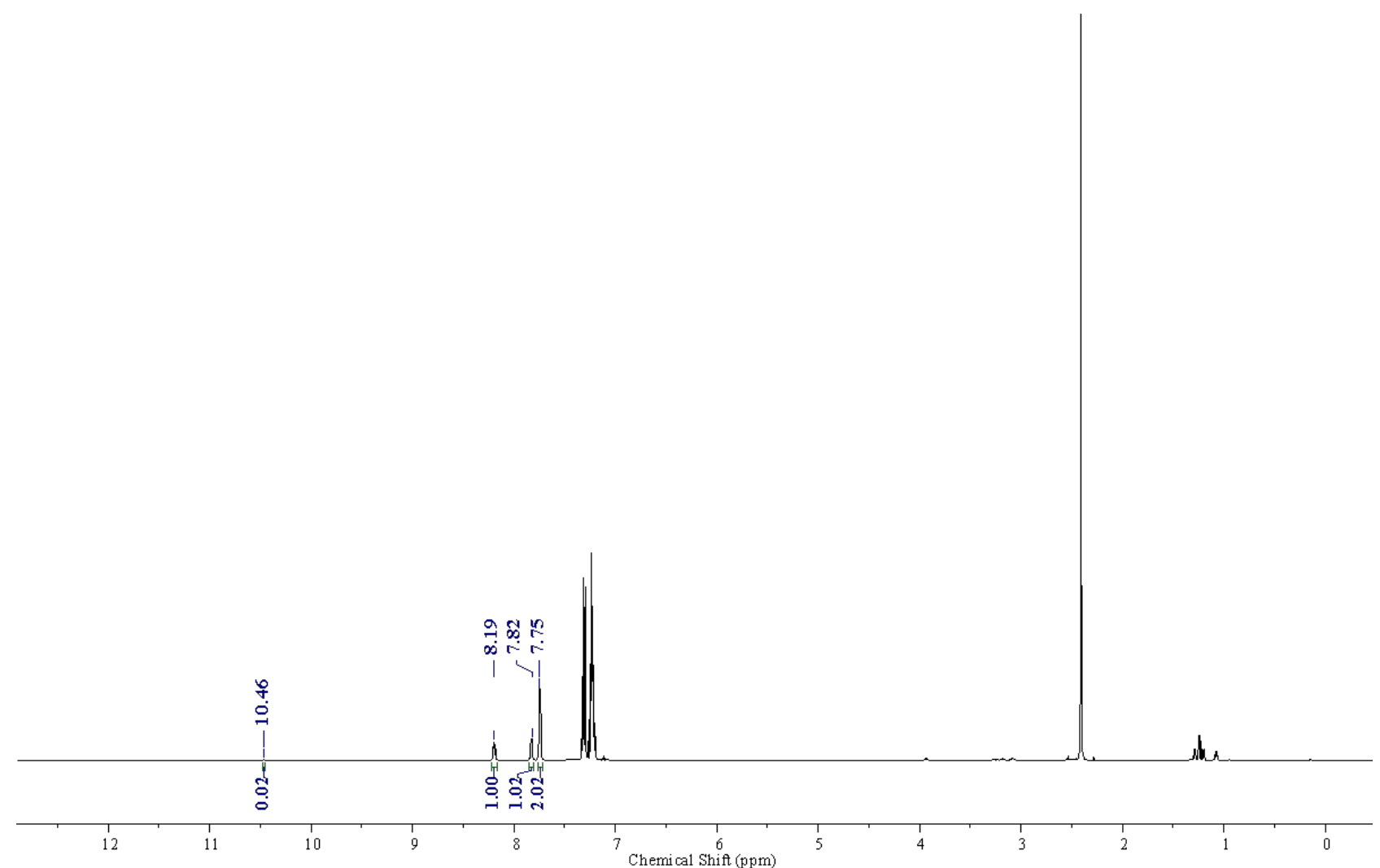

Entry 4: NMR for Crude reaction mixture

VDOS-595-D 2.2.fid

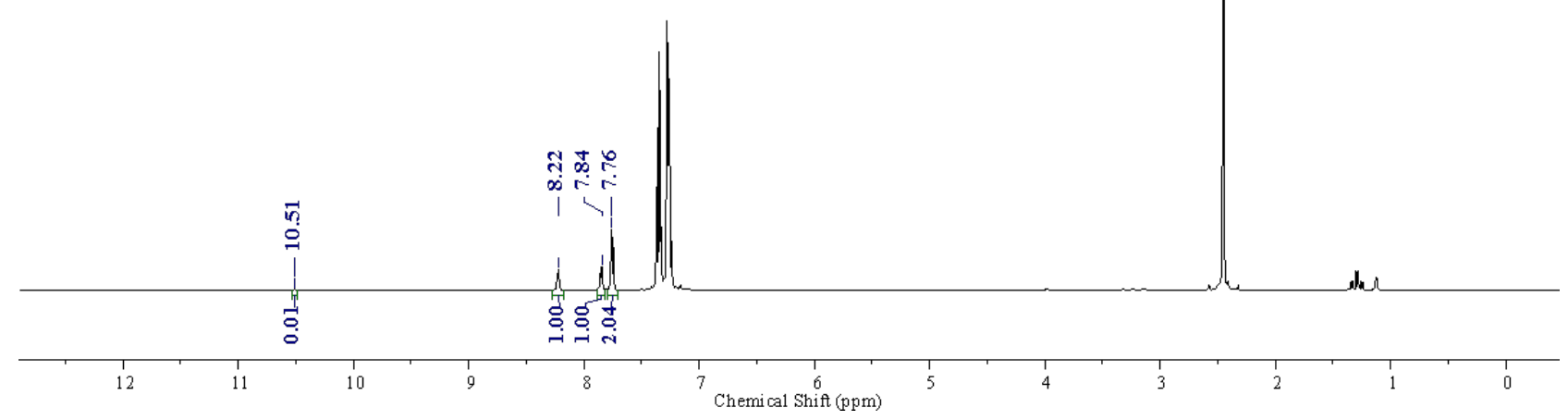


Entry 4': NMR for Crude reaction mixture, Duplicate

VDOS-595-D 1.3.fid

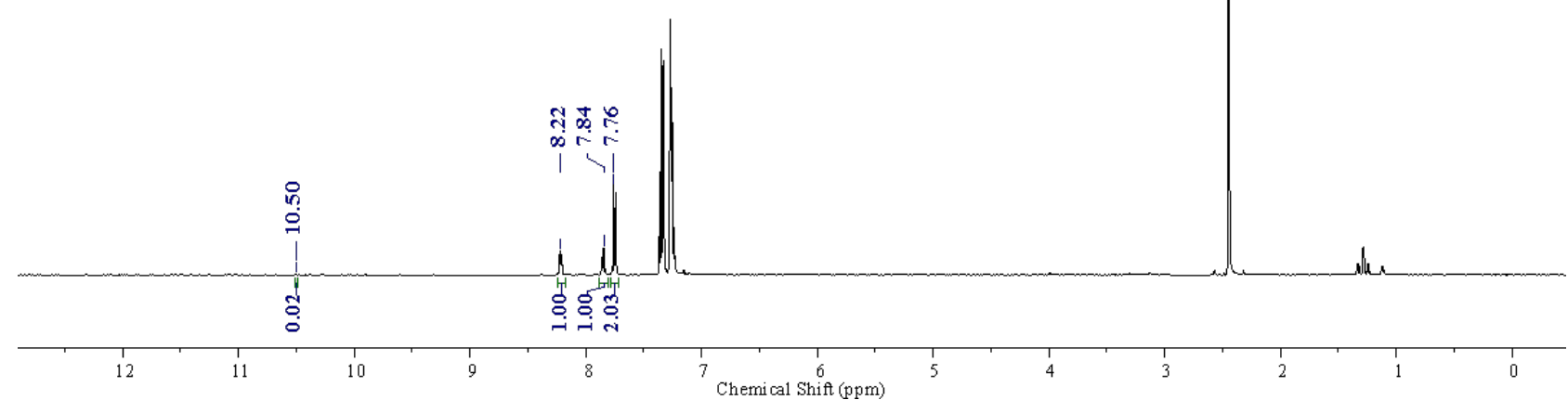

NMR after purification:

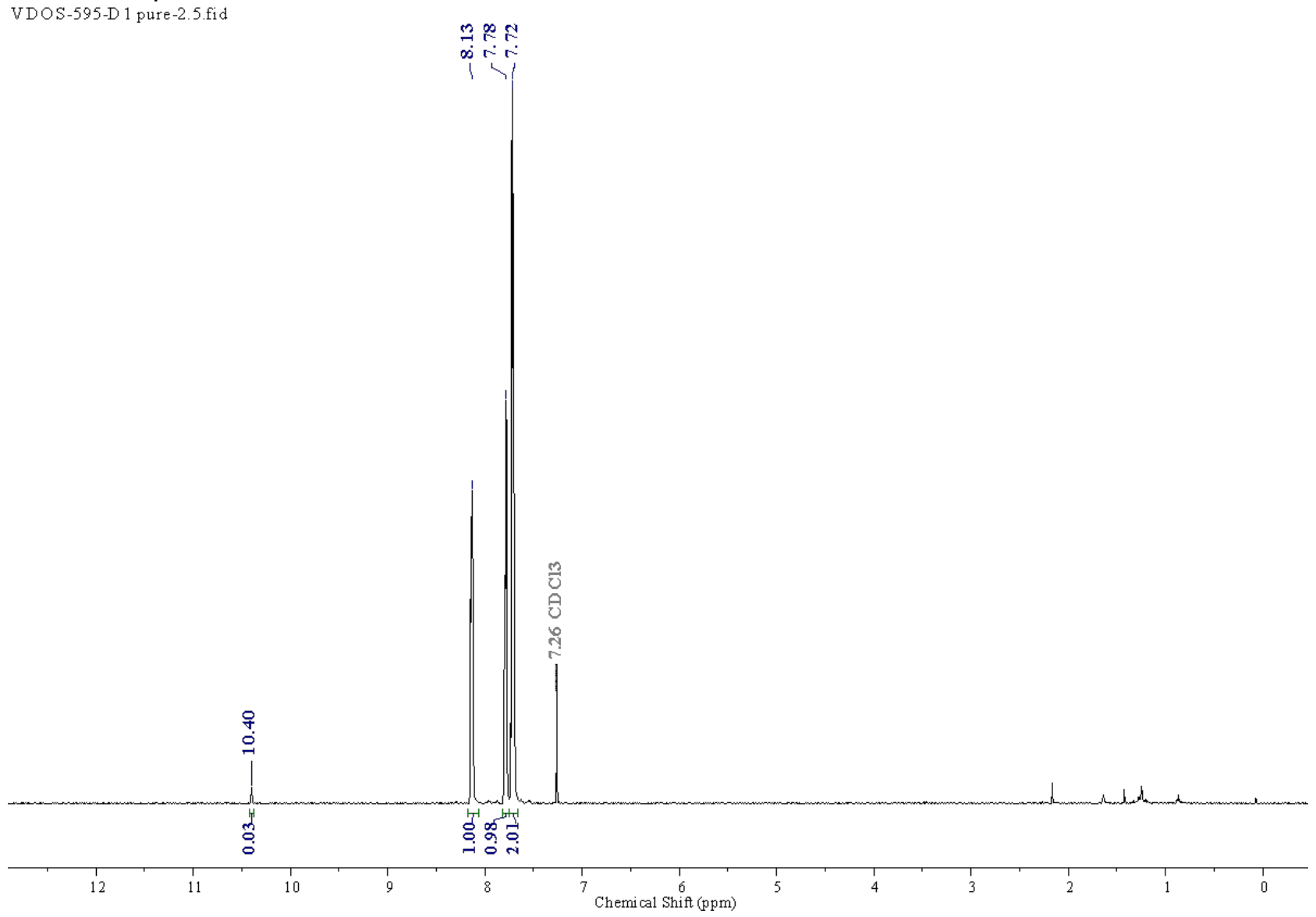




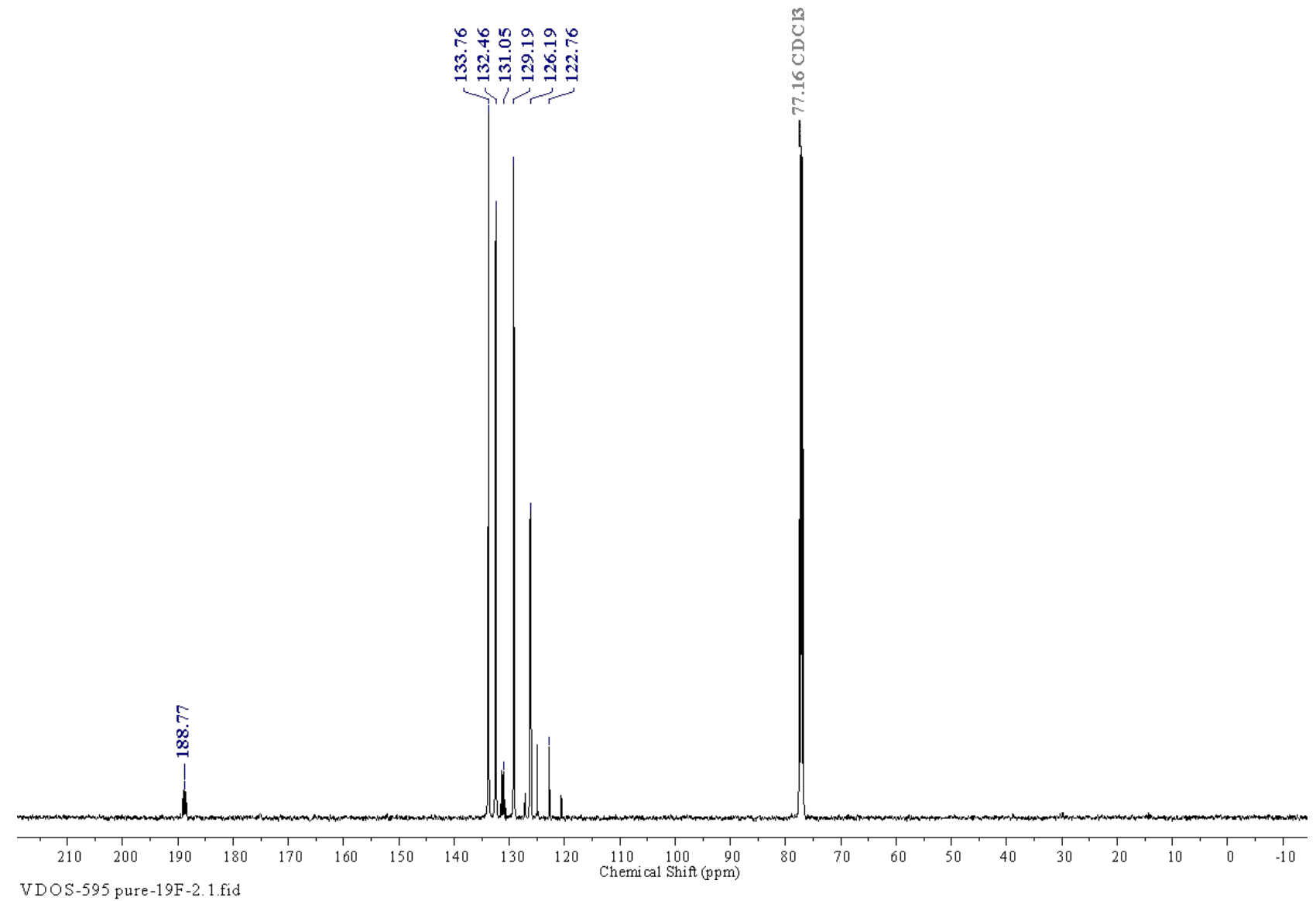

5

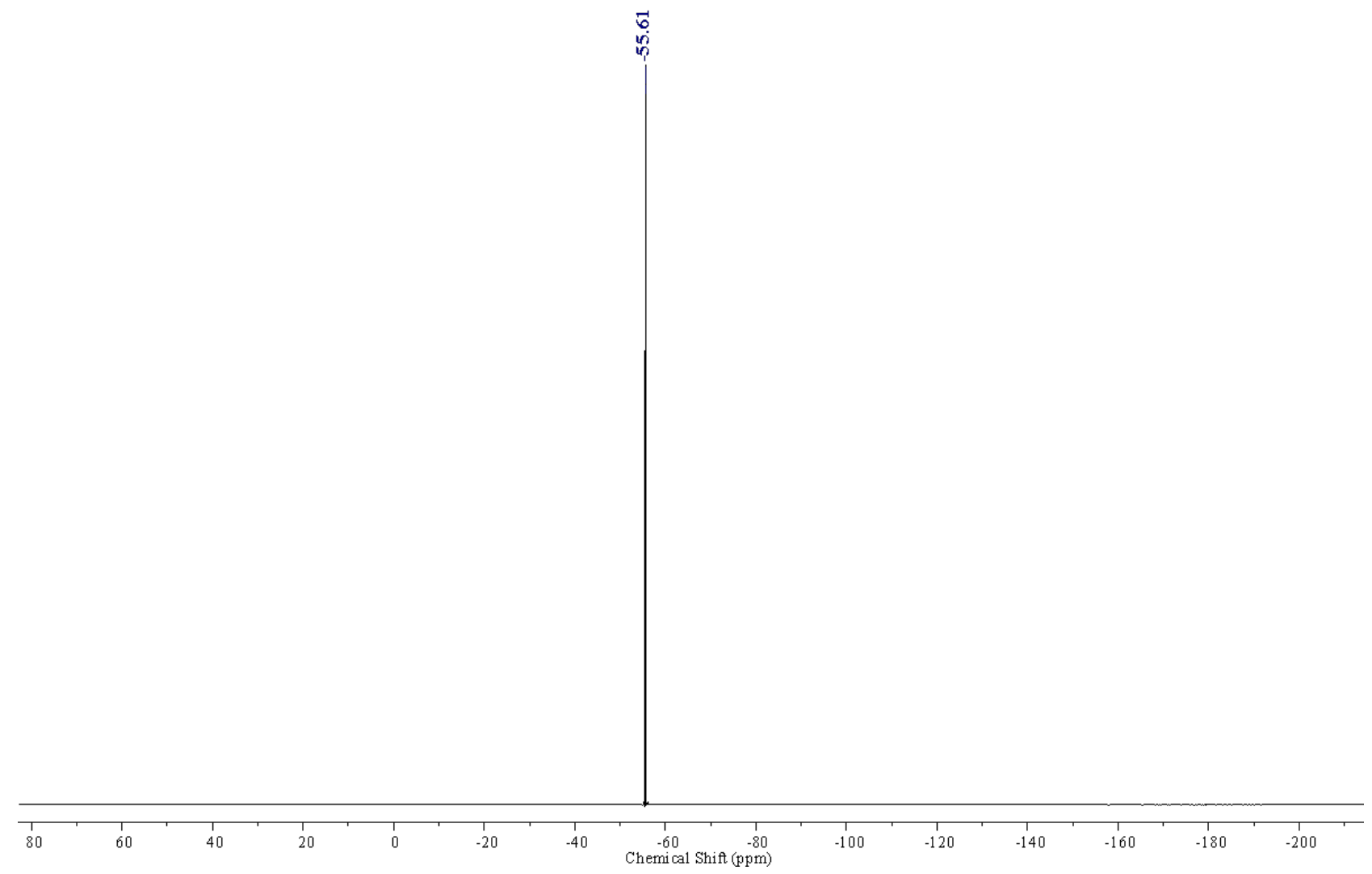


15. 2-Methoxybenzaldehyde-d<smiles>[2H]C(=O)c1ccccc1OC</smiles>

Entry 1: NMR for Crude reaction mixture SCG-307-A2-16hr-D1-50sec.1. fid

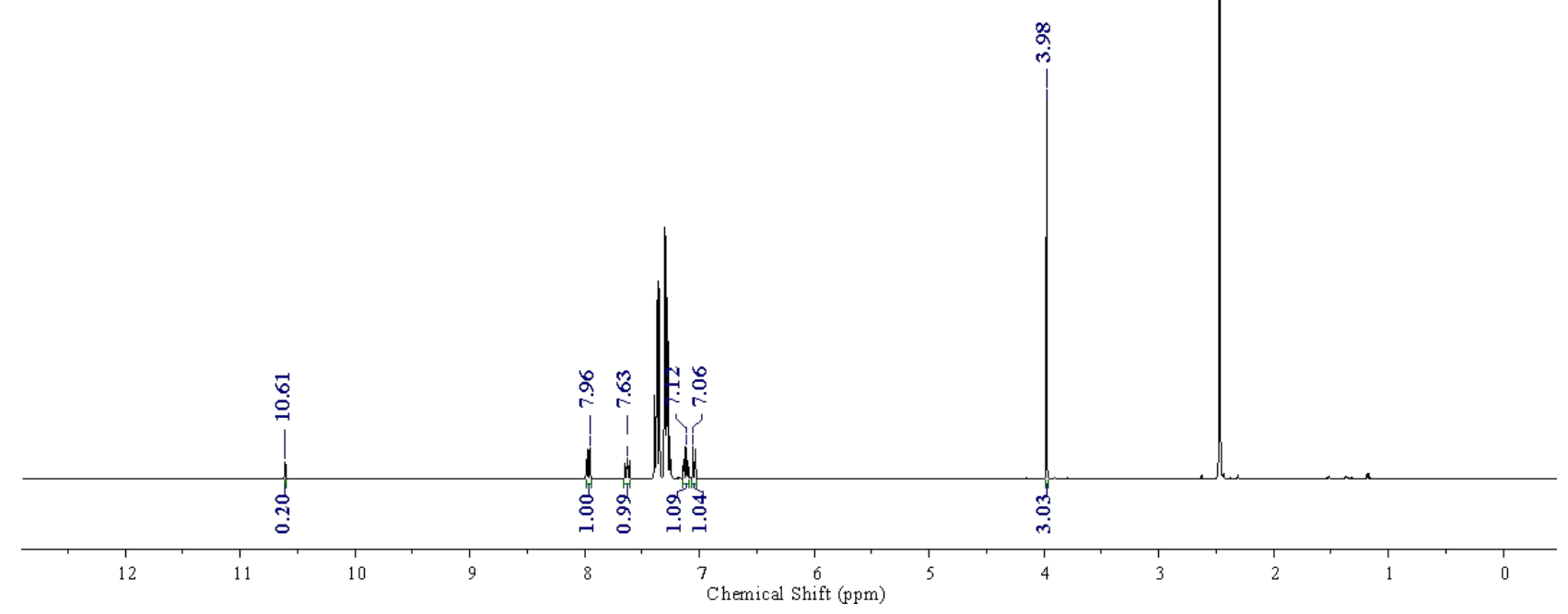


Entry 1': NMR for Crude reaction mixture, Duplicate

SCG-307-A3-16hr-D1-50sec.1. fid

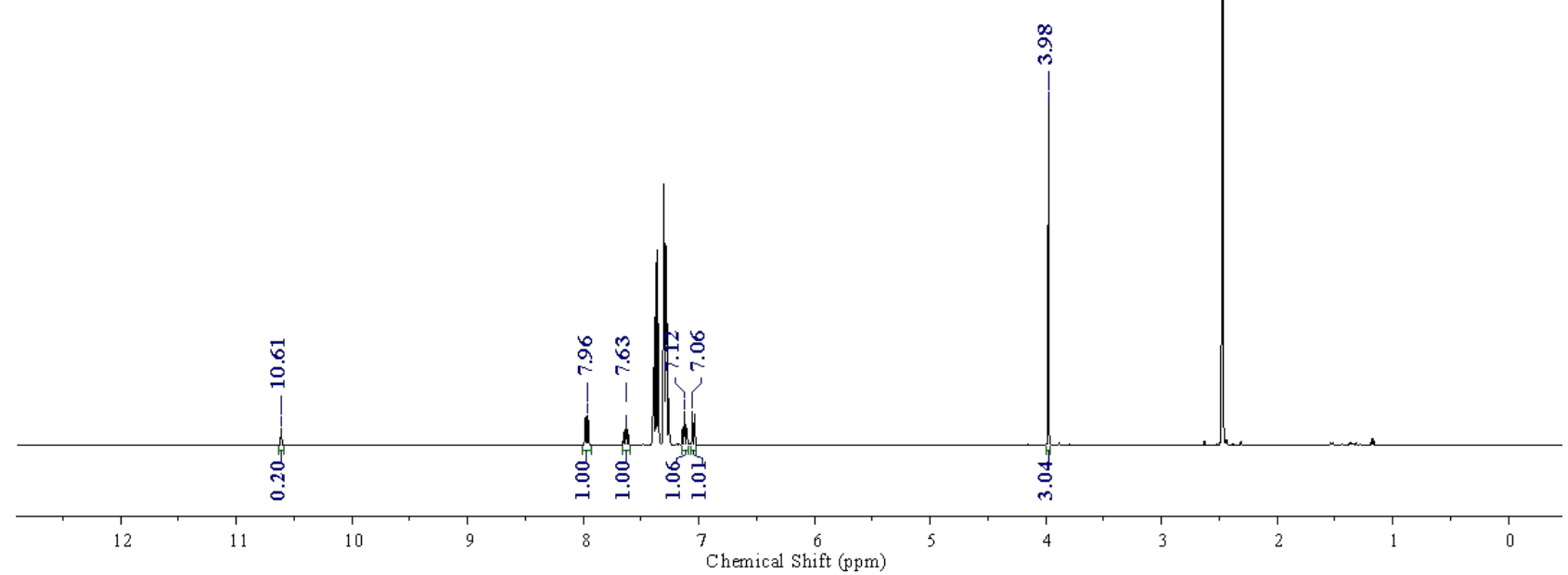

Entry 2: NMR for Crude reaction mixture

SCG-298-J-16hr-D1-50sec.1. fid

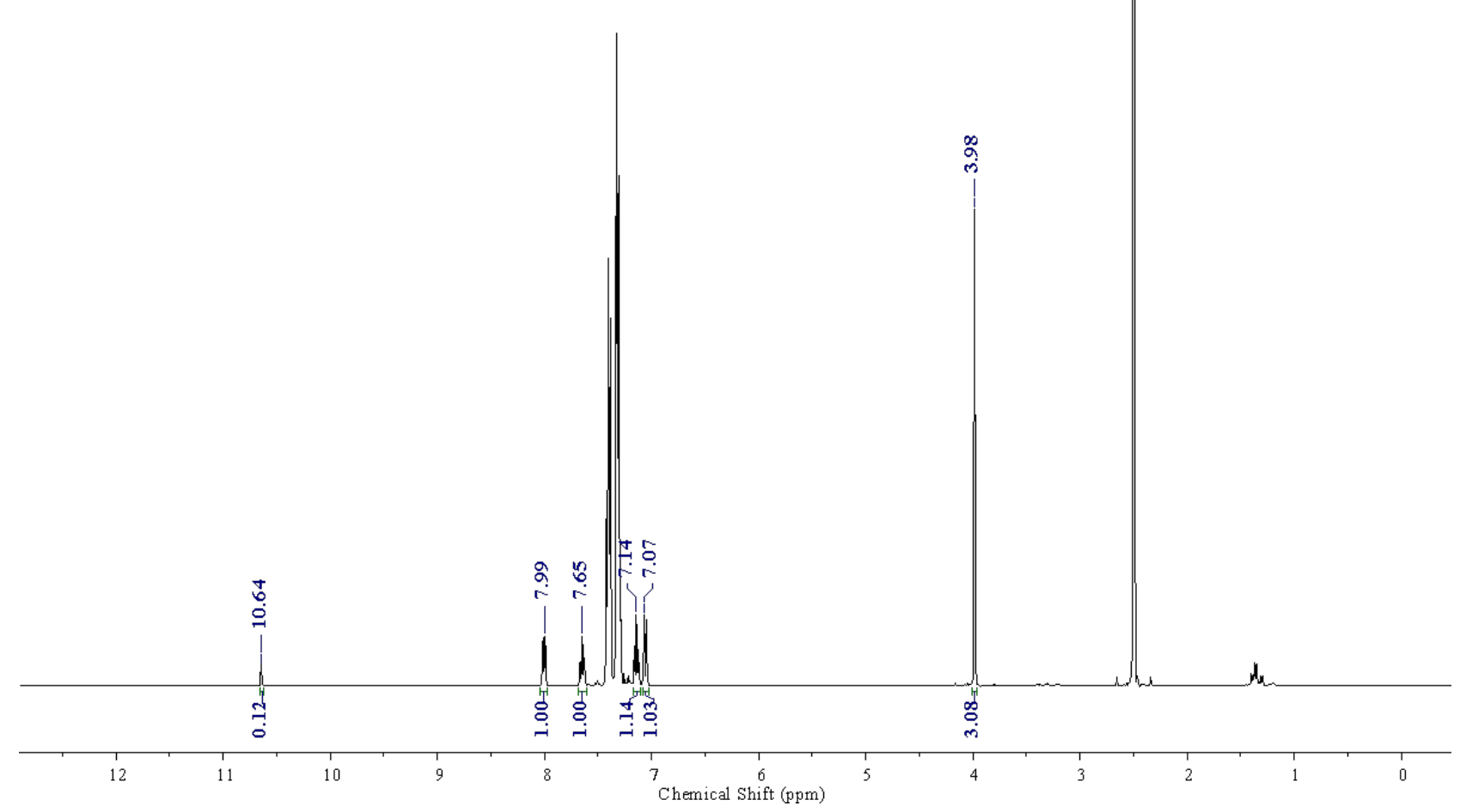


Entry 3: NMR for Crude reaction mixture

VDOS- 2OMe-A1.1.fid

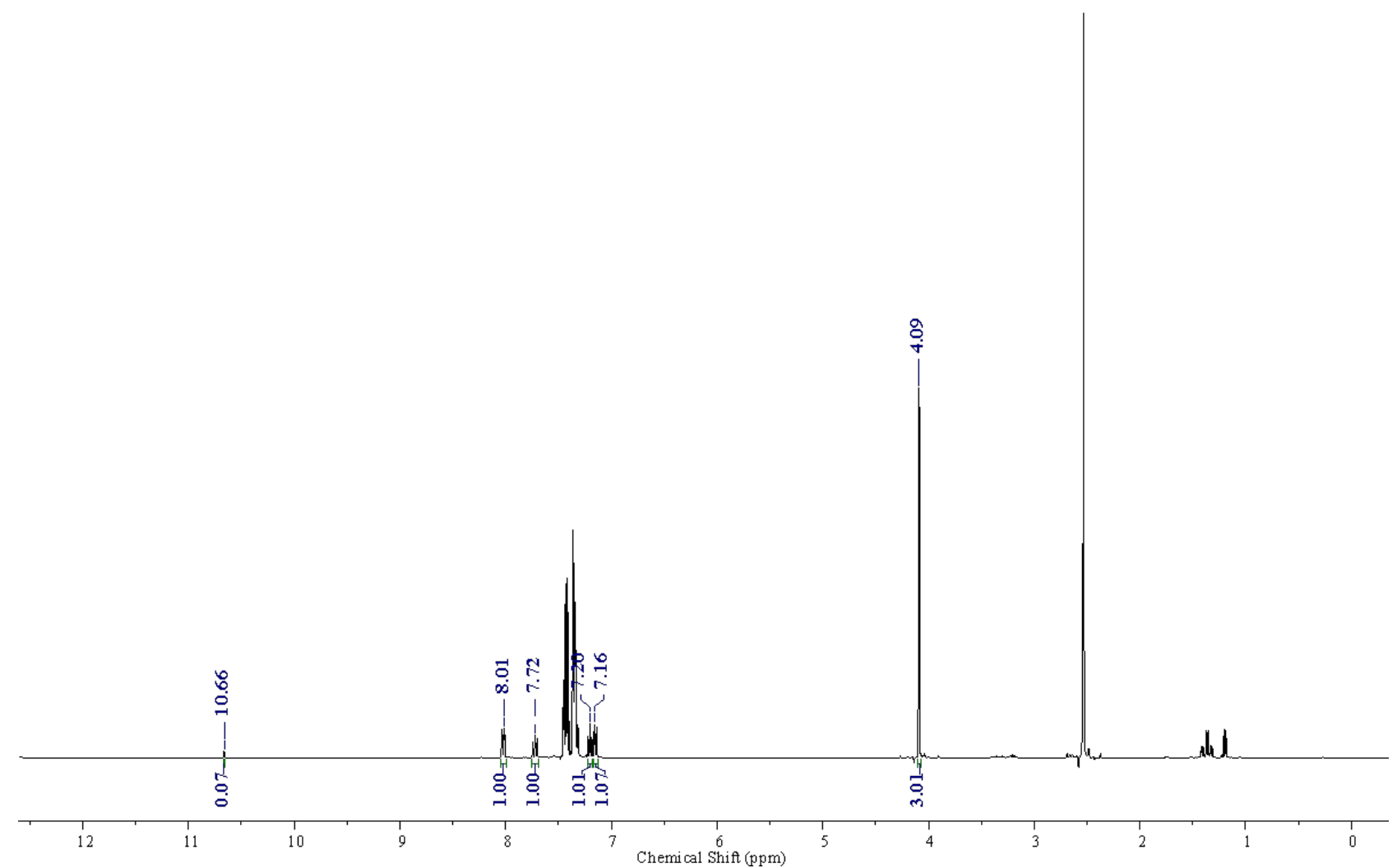

Entry 3': NMR for Crude reaction mixture, Duplicate

VDOS-2OMe-G2.1. fid

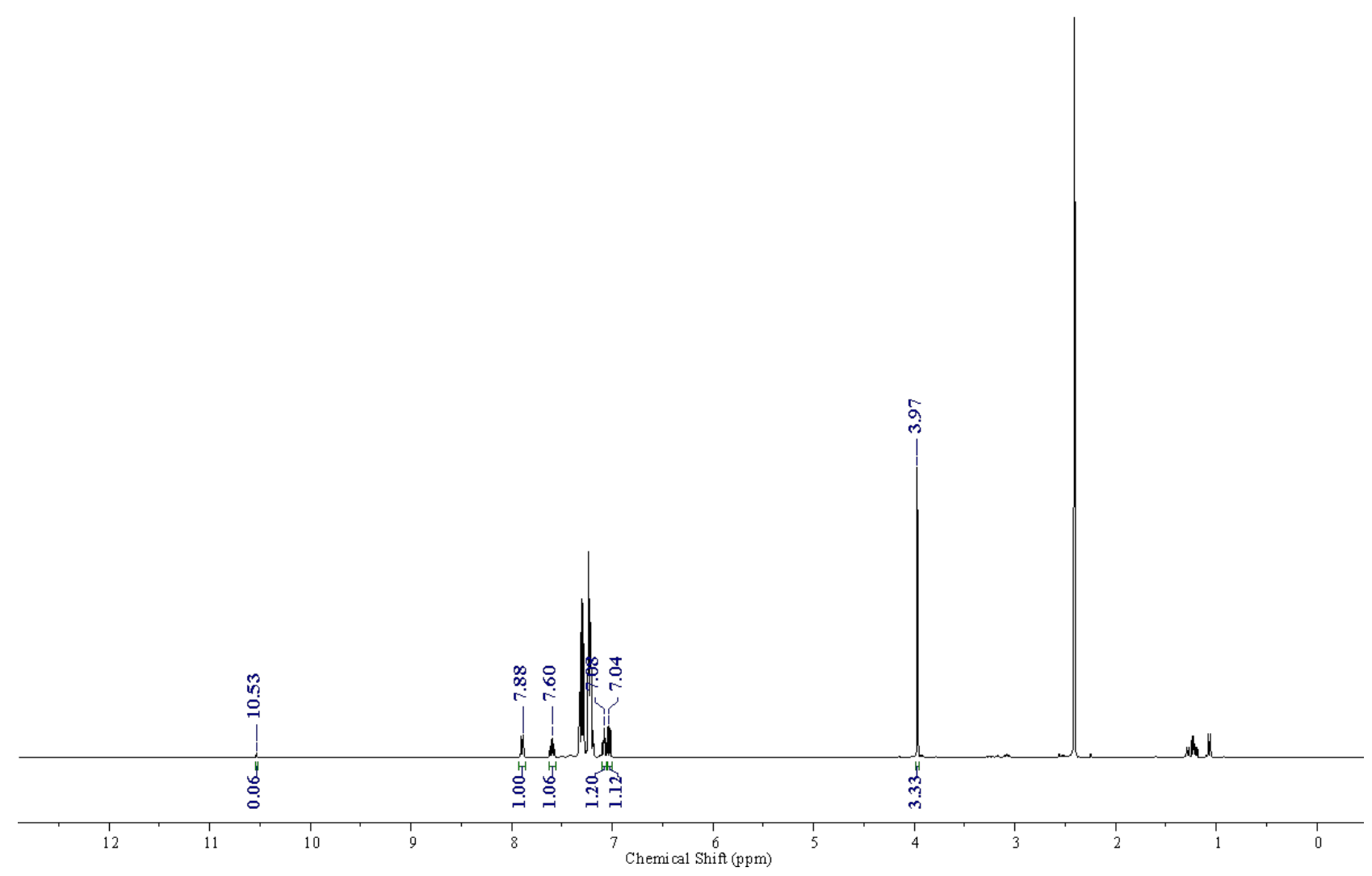


Entry 4: NMR for Crude reaction mixture

VDOS-589-E 1.2.fid

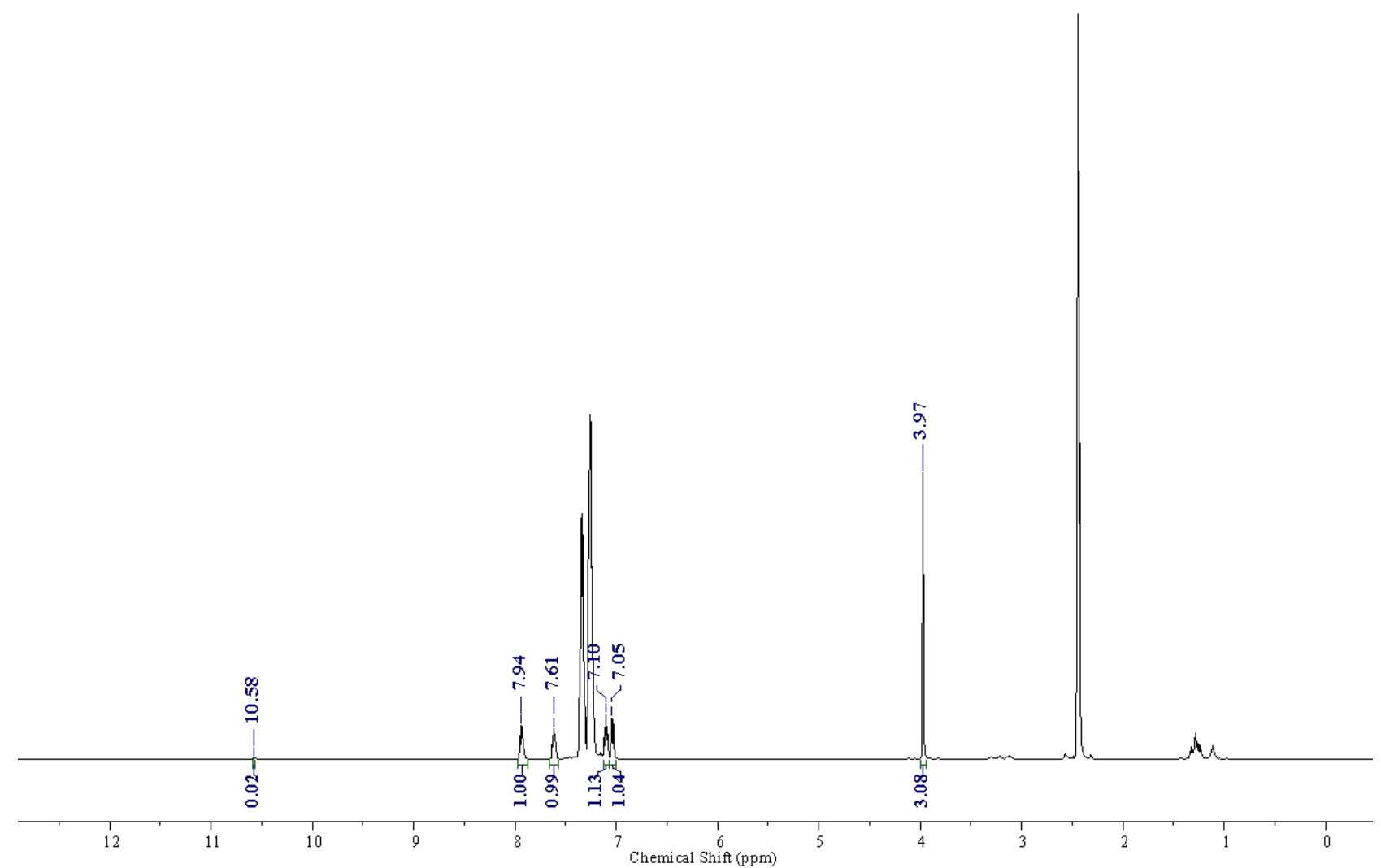

Entry 4': NMR for Crude reaction mixture, Duplicate

VDOS-589-E2.3.fid

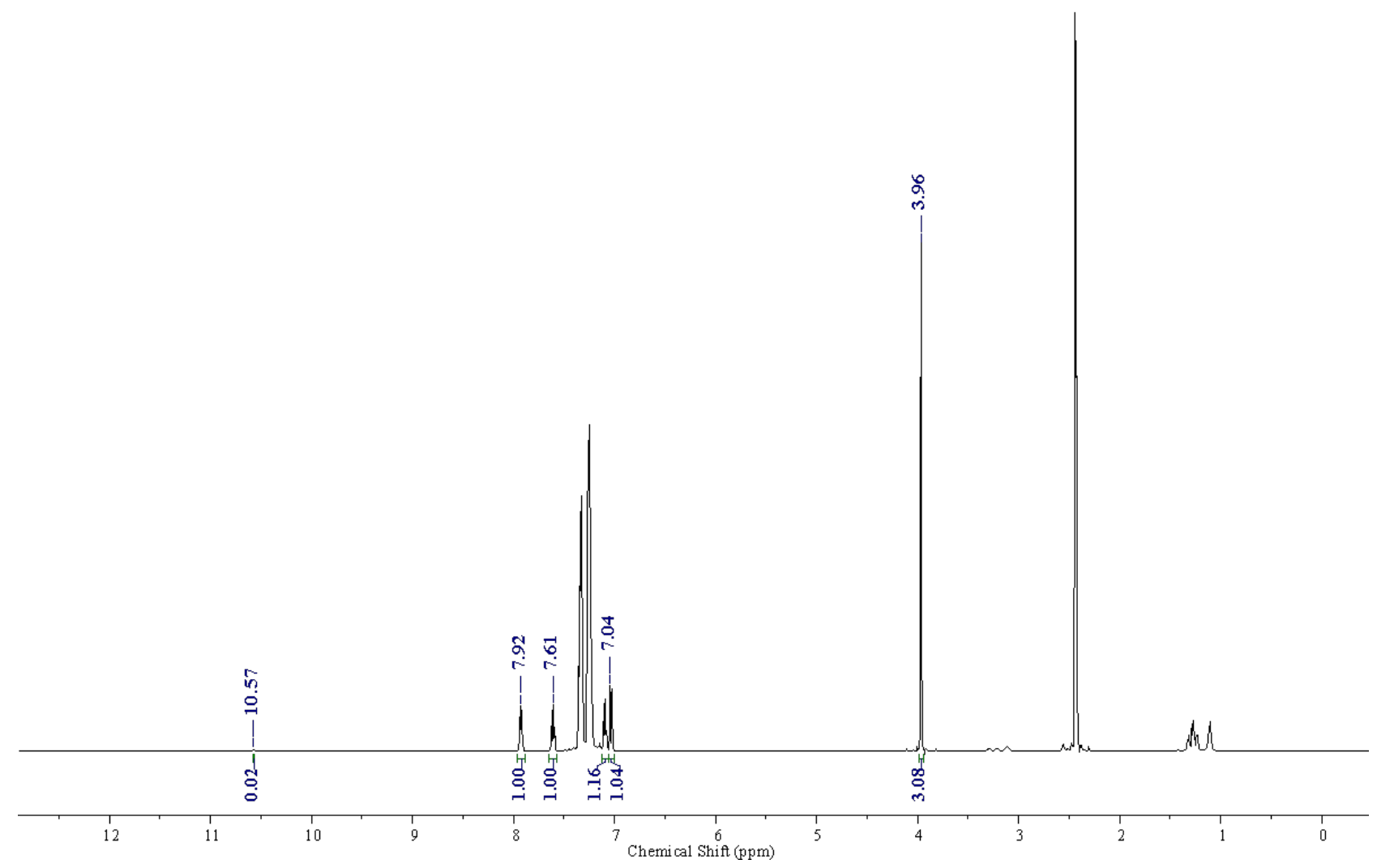


NMR after purification:

VDOS-589-C 1 and C2-Pure.2.fid
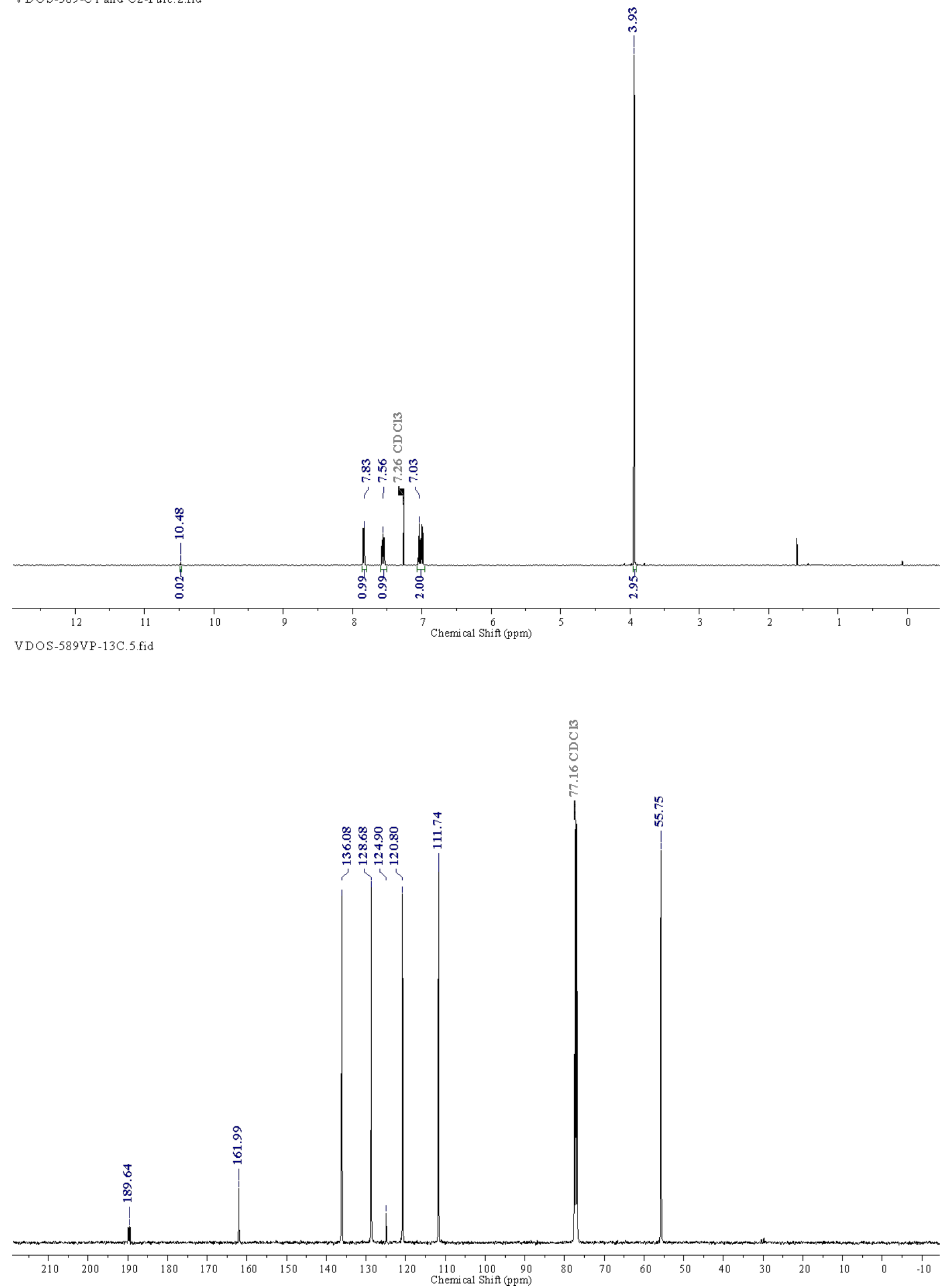
16. 4-Methylbenzaldehyde-d<smiles>[2H]C(=O)c1ccc(C)cc1</smiles>

Entry 1: NMR for Crude reaction mixture SCG-319-I1-16hr-D 1-50sec.1.fid

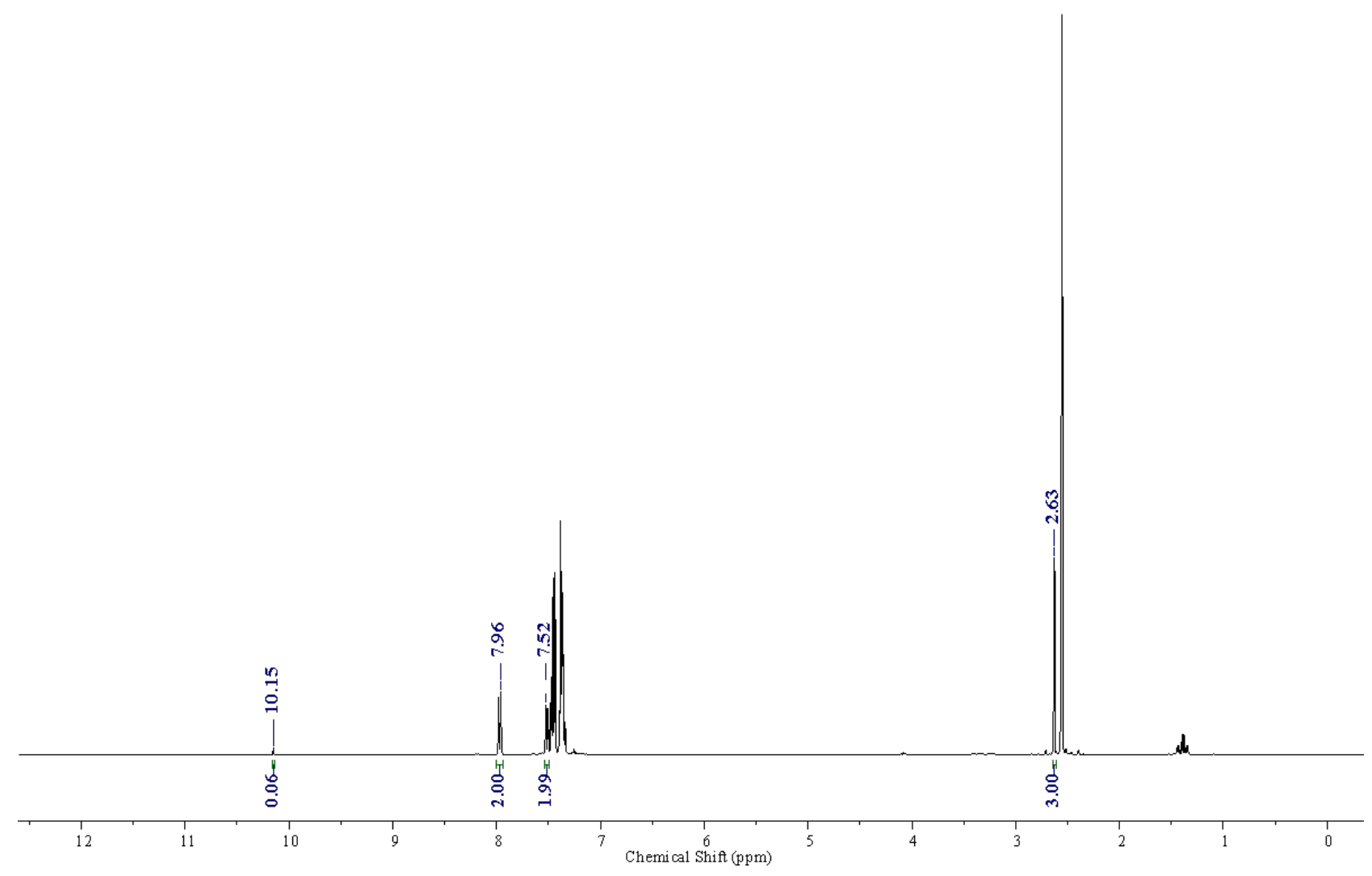


Entry 1': NMR for Crude reaction mixture, Duplicate

SCG-318-A 1-16hr-D 1-50sec.1. fid

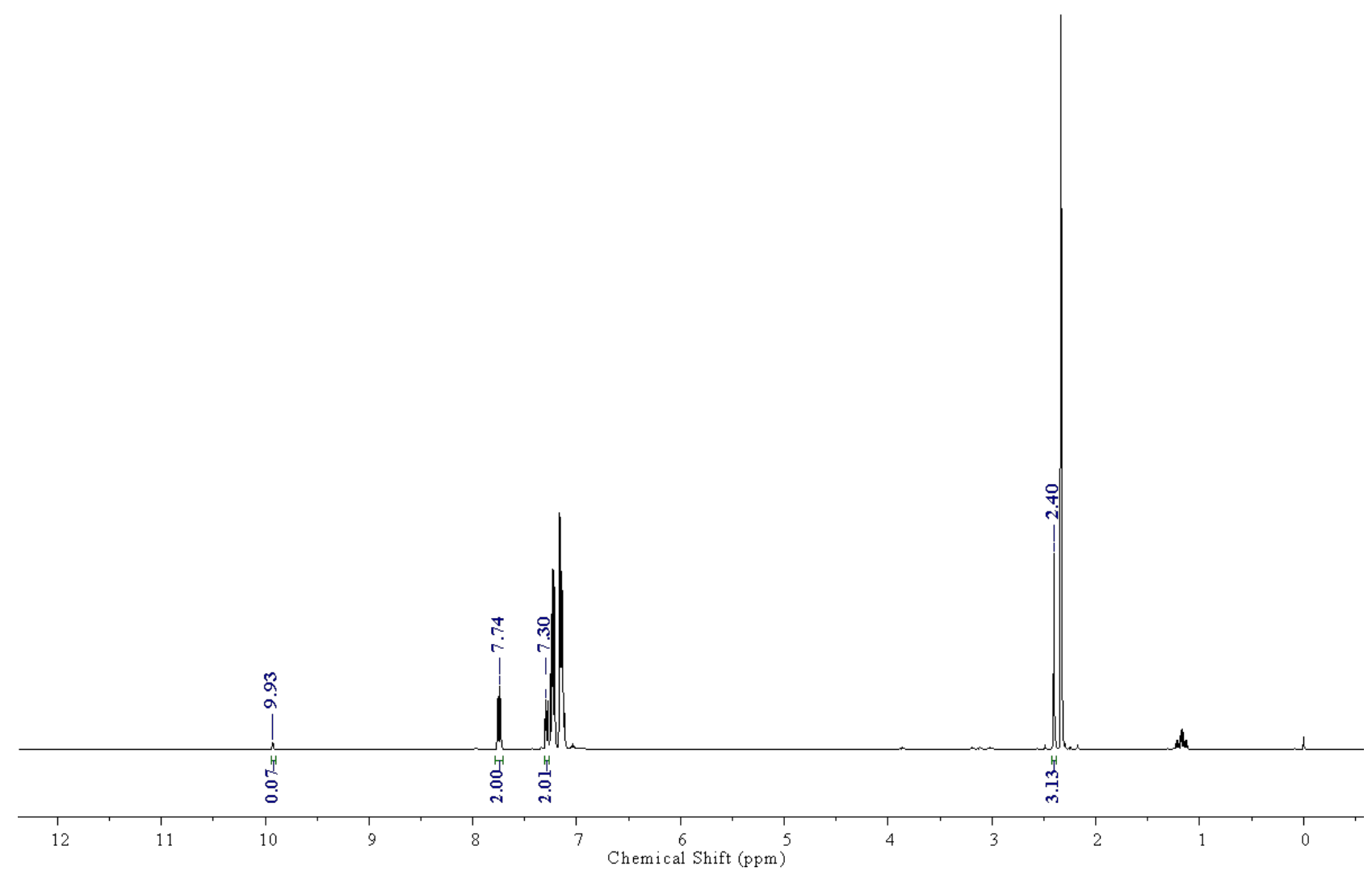

Entry 2: NMR for Crude reaction mixture

VDOS-637-A 1.1. fid

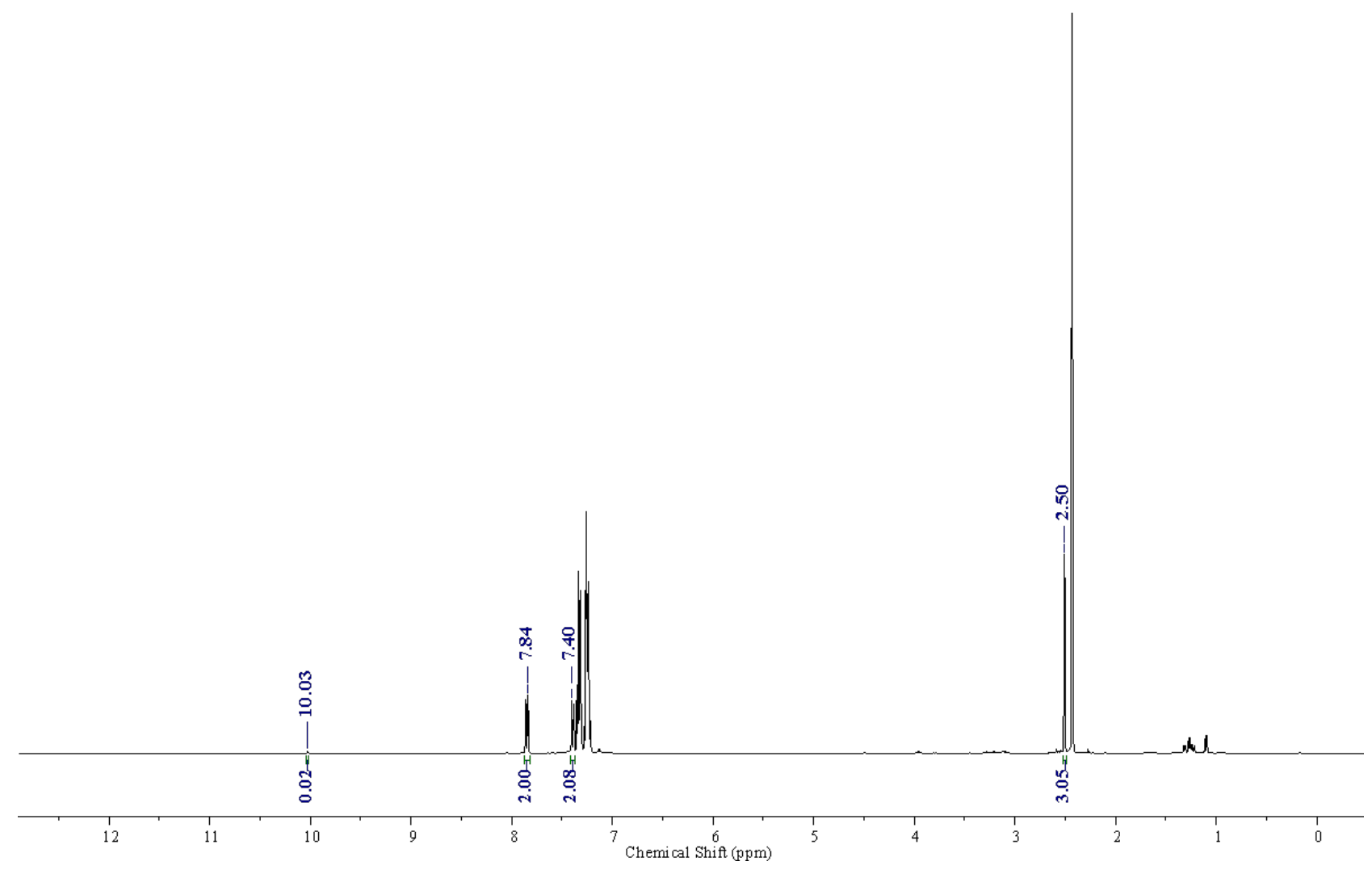


Entry 2: NMR for Crude reaction mixture, Duplicate

VDOS-637-A.2.1.fid

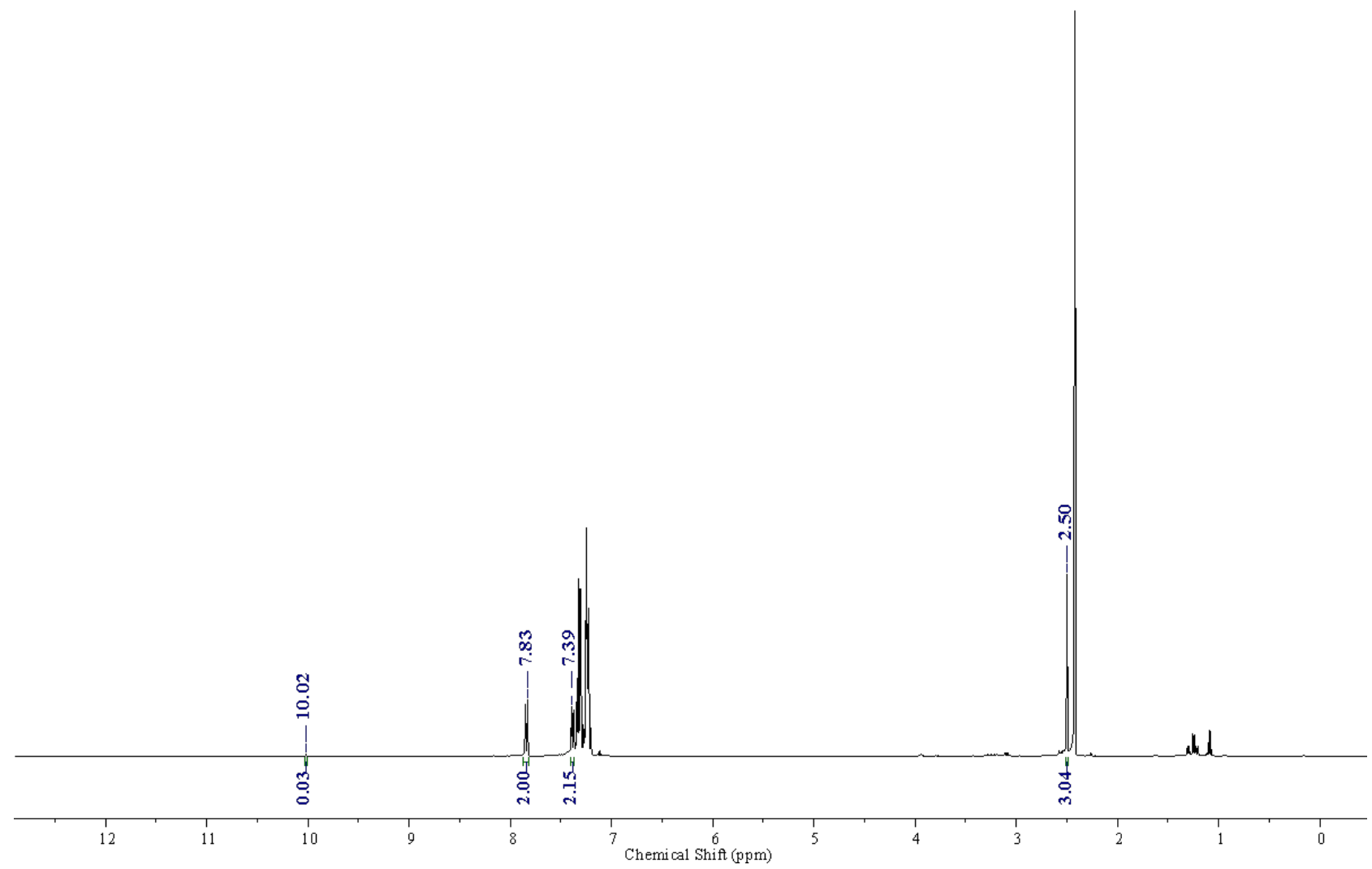

Entry 3: NMR for Crude reaction mixture

SCG-319-I2-16hr-D 1-50sec.1.fid

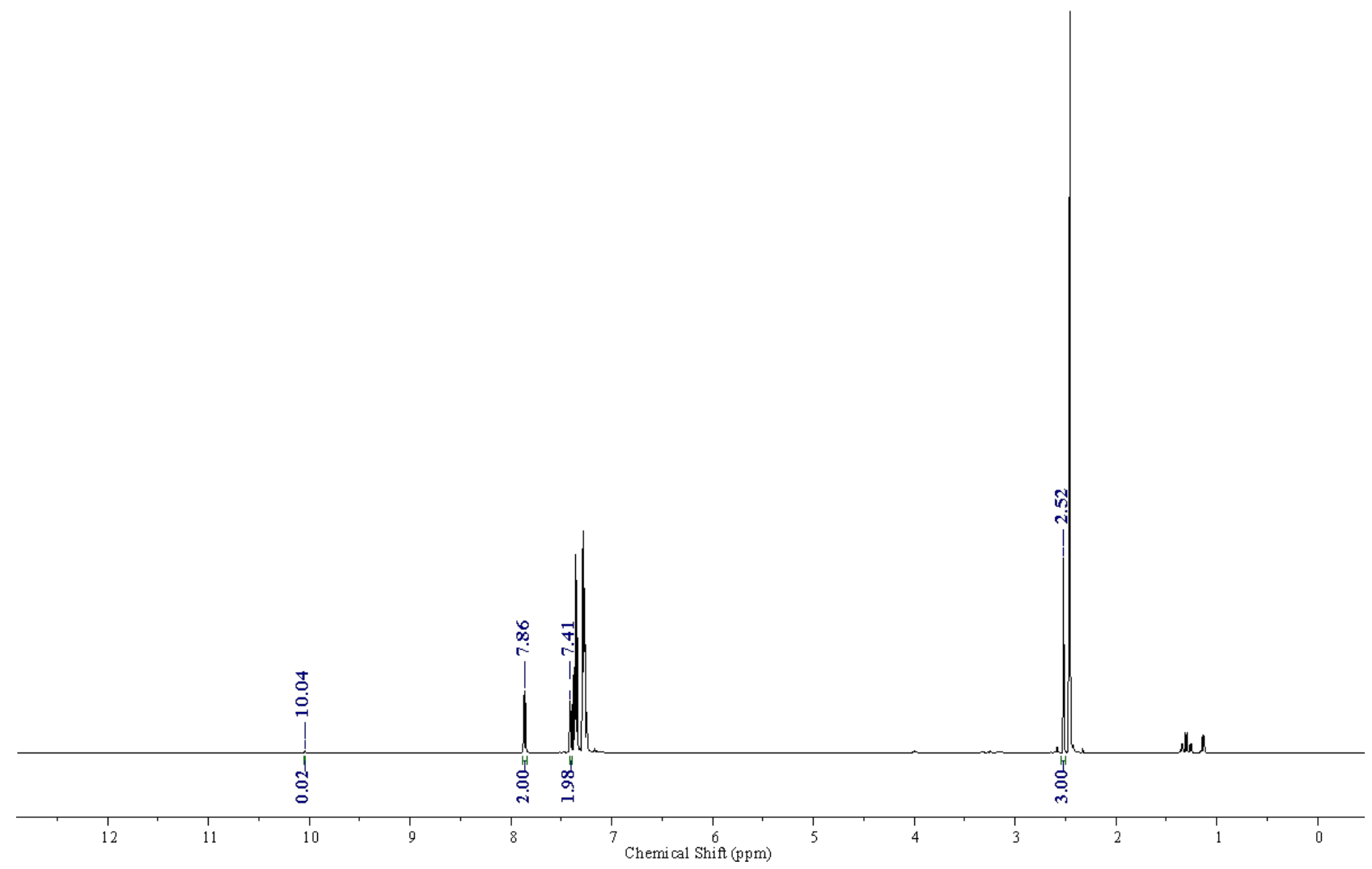


Entry 3': NMR for Crude reaction mixture, Duplicate

SCG-319-I3-16hr-D 1-50sec.1. fid

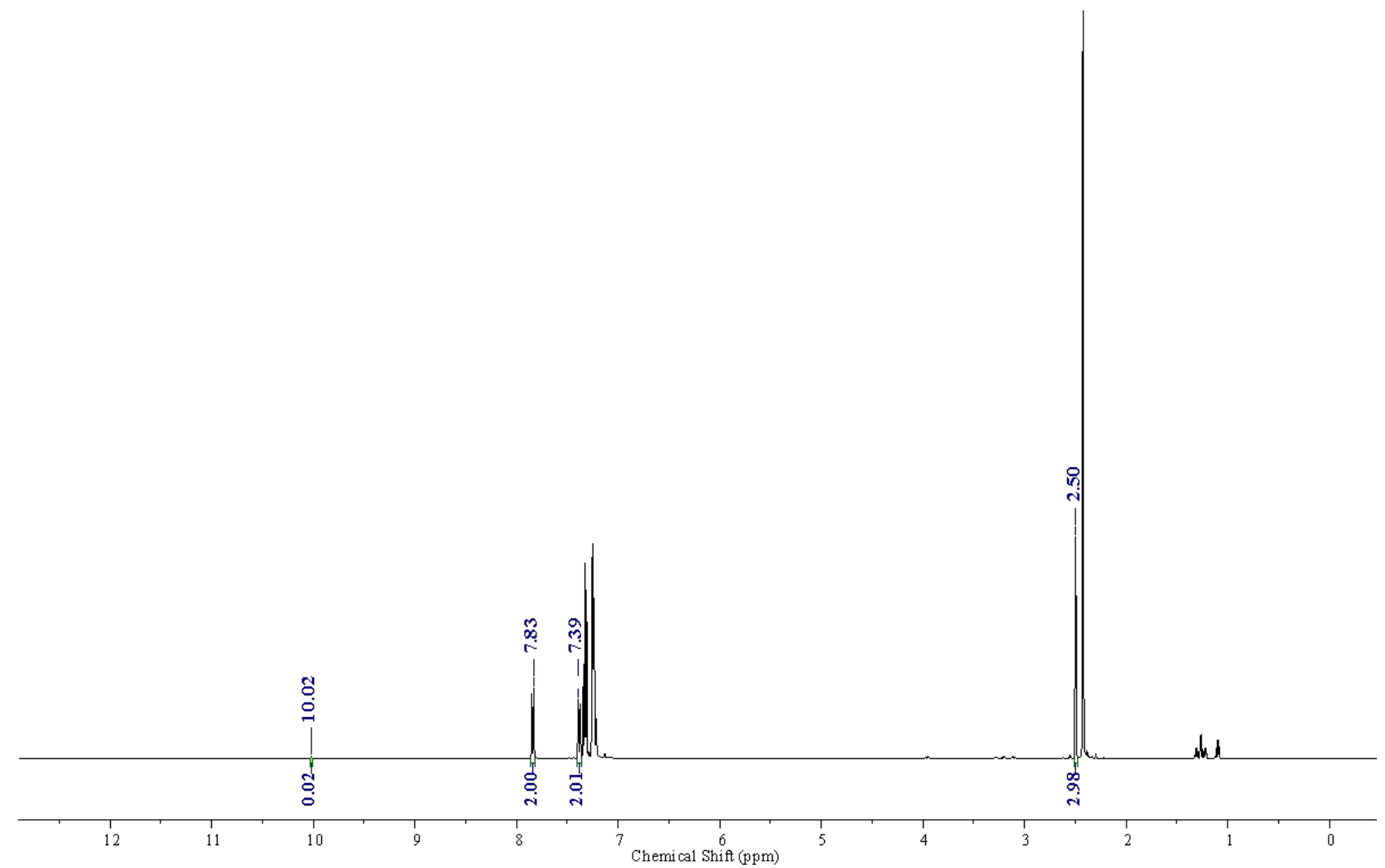

NMR after purification:

VDOS-637-A 2 pure.2.fid

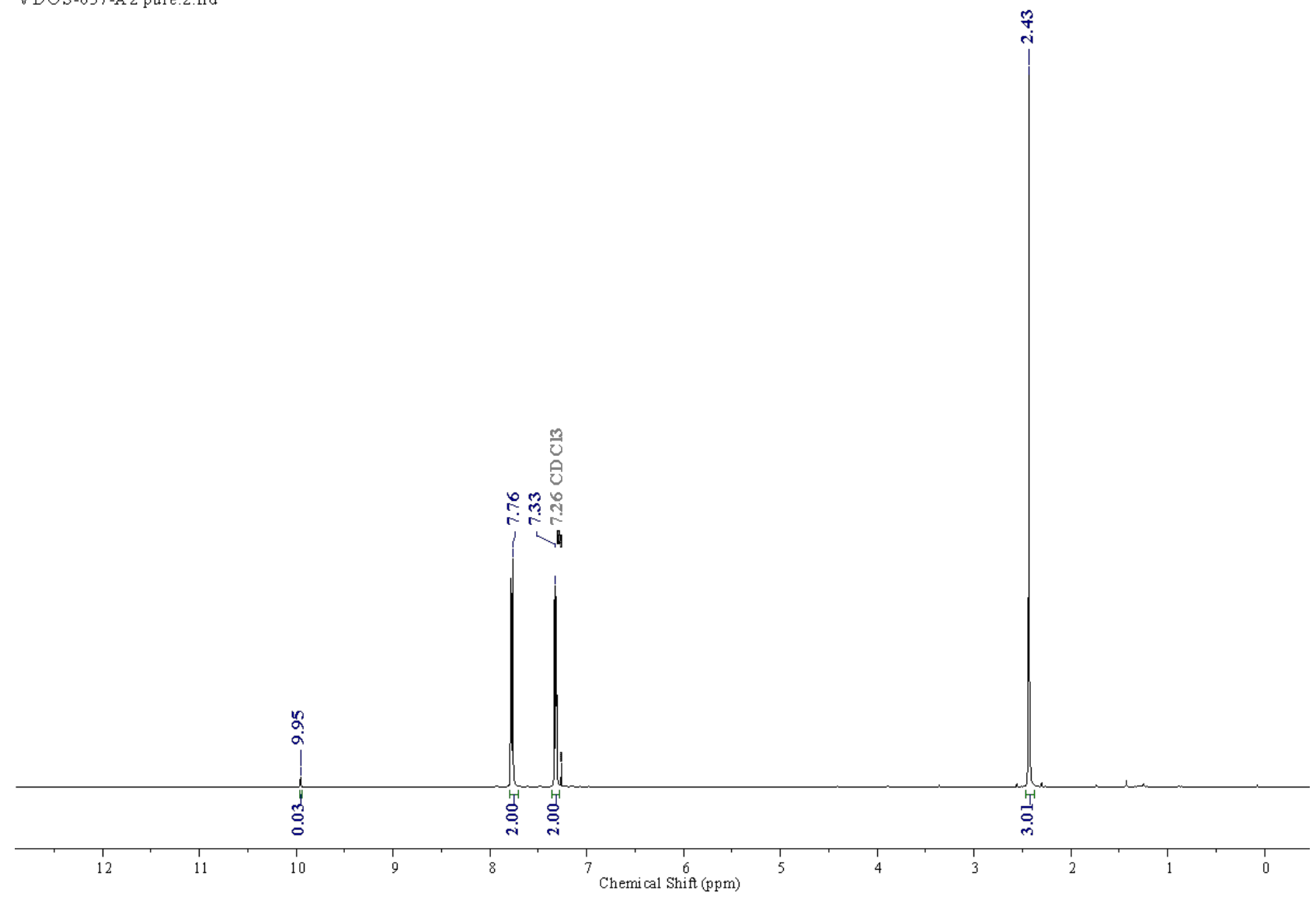




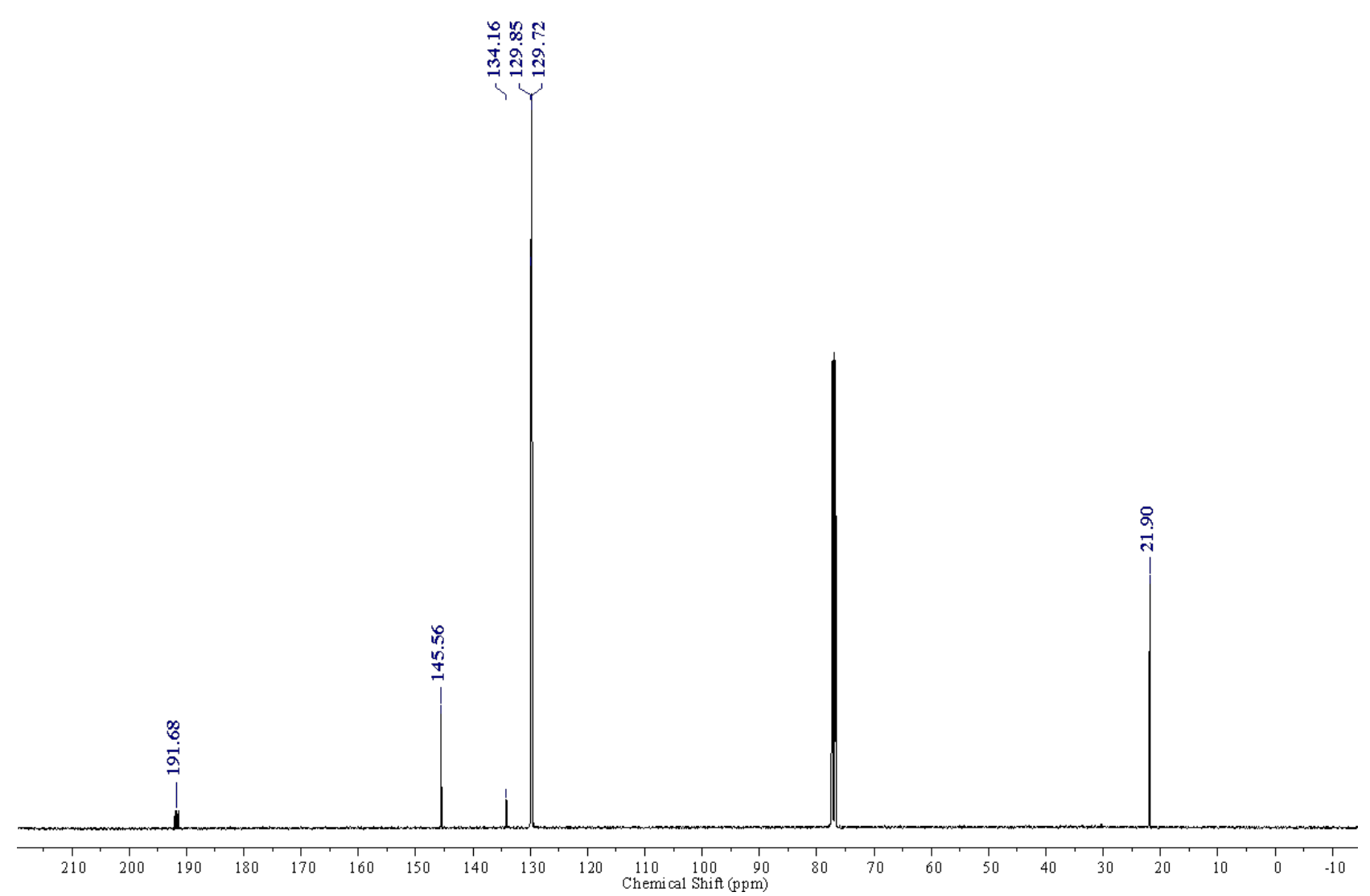


17. 4-Isopropylbenzaldehyde-d<smiles>[2H]C(=O)c1ccc(C(C)C)cc1</smiles>

Entry 1: NMR for Crude reaction mixture SCG-318-B2-16h-D 1-50sec.1.fid

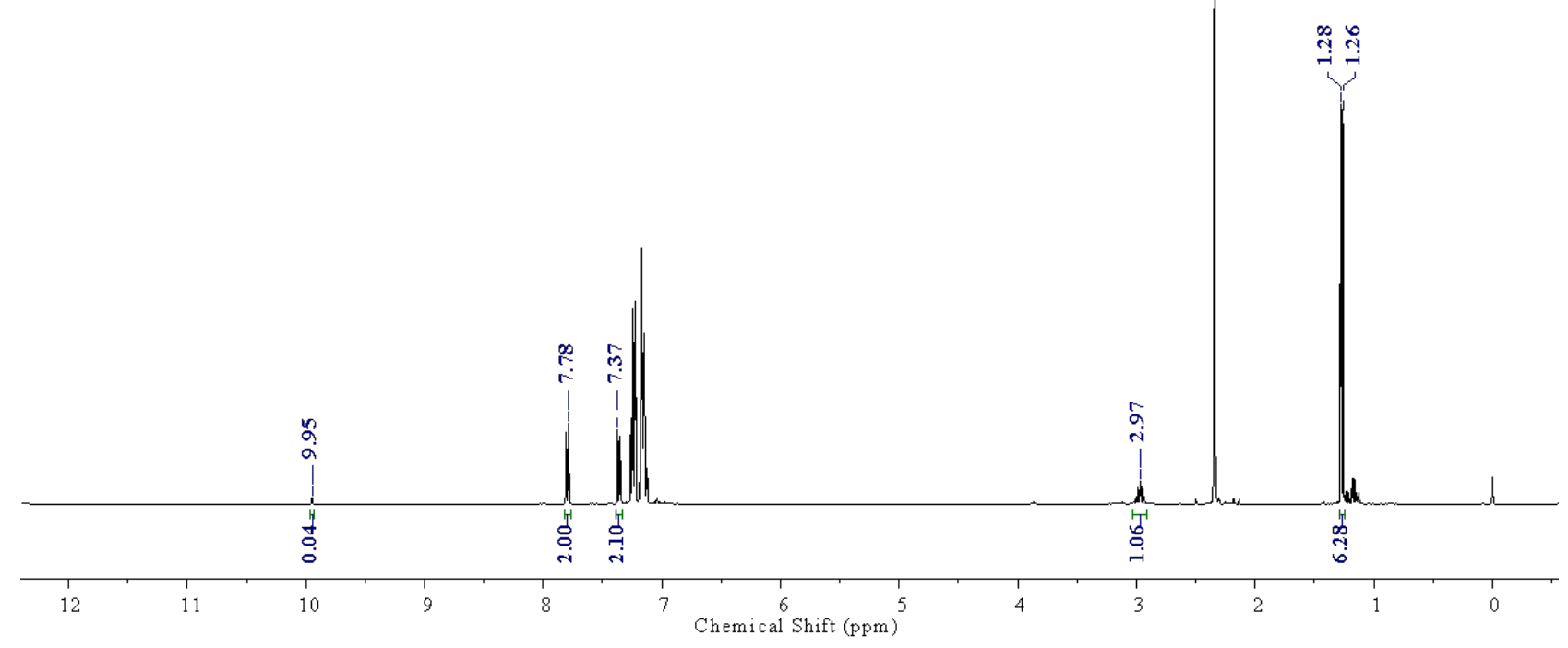


Entry 1': NMR for Crude reaction mixture, Duplicate

SCG-318-B 1-16hr-D 1-50sec.1. fid

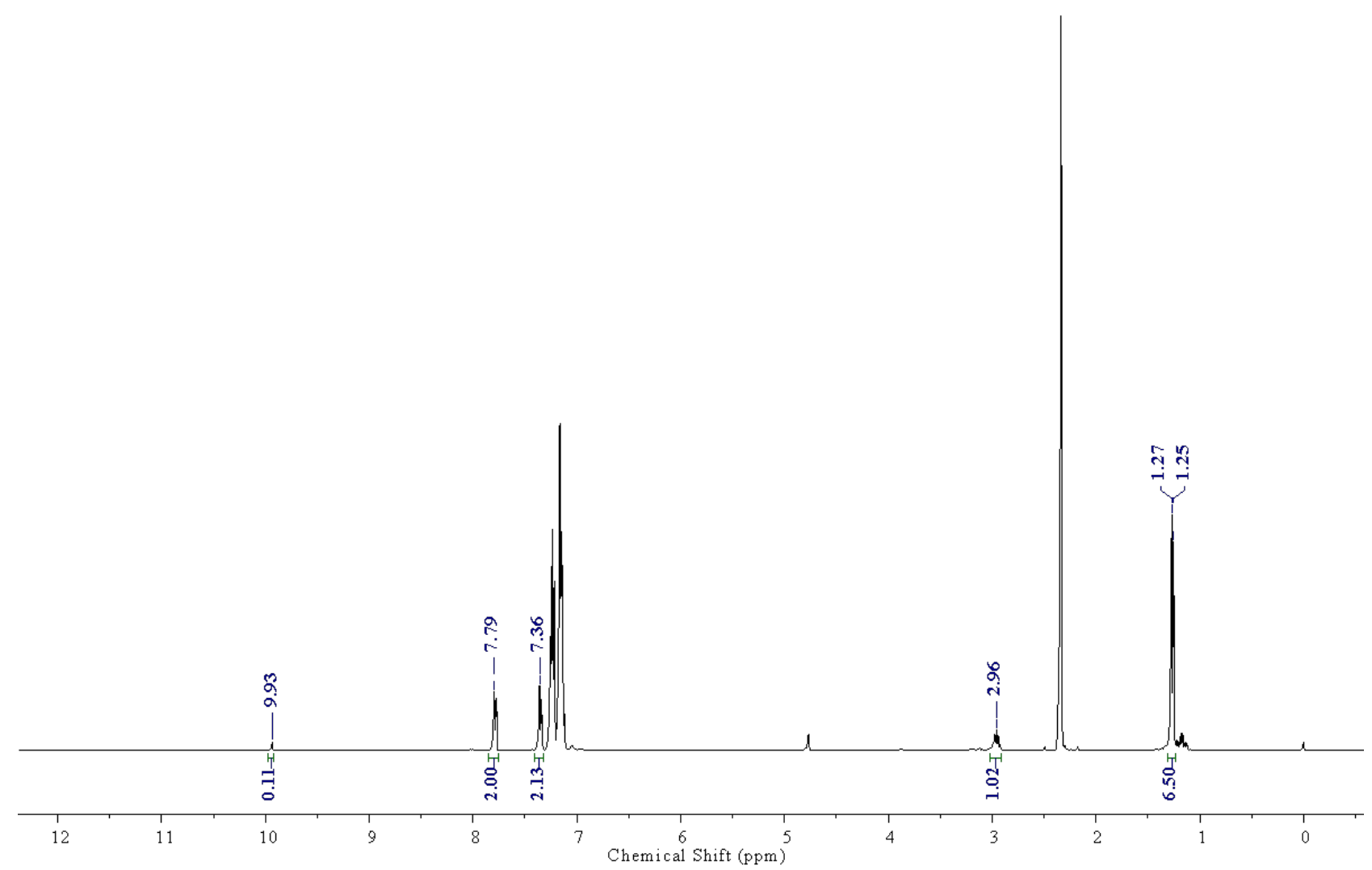

Entry 2: NMR for Crude reaction mixture

VDOS-636-B 1.7.fid

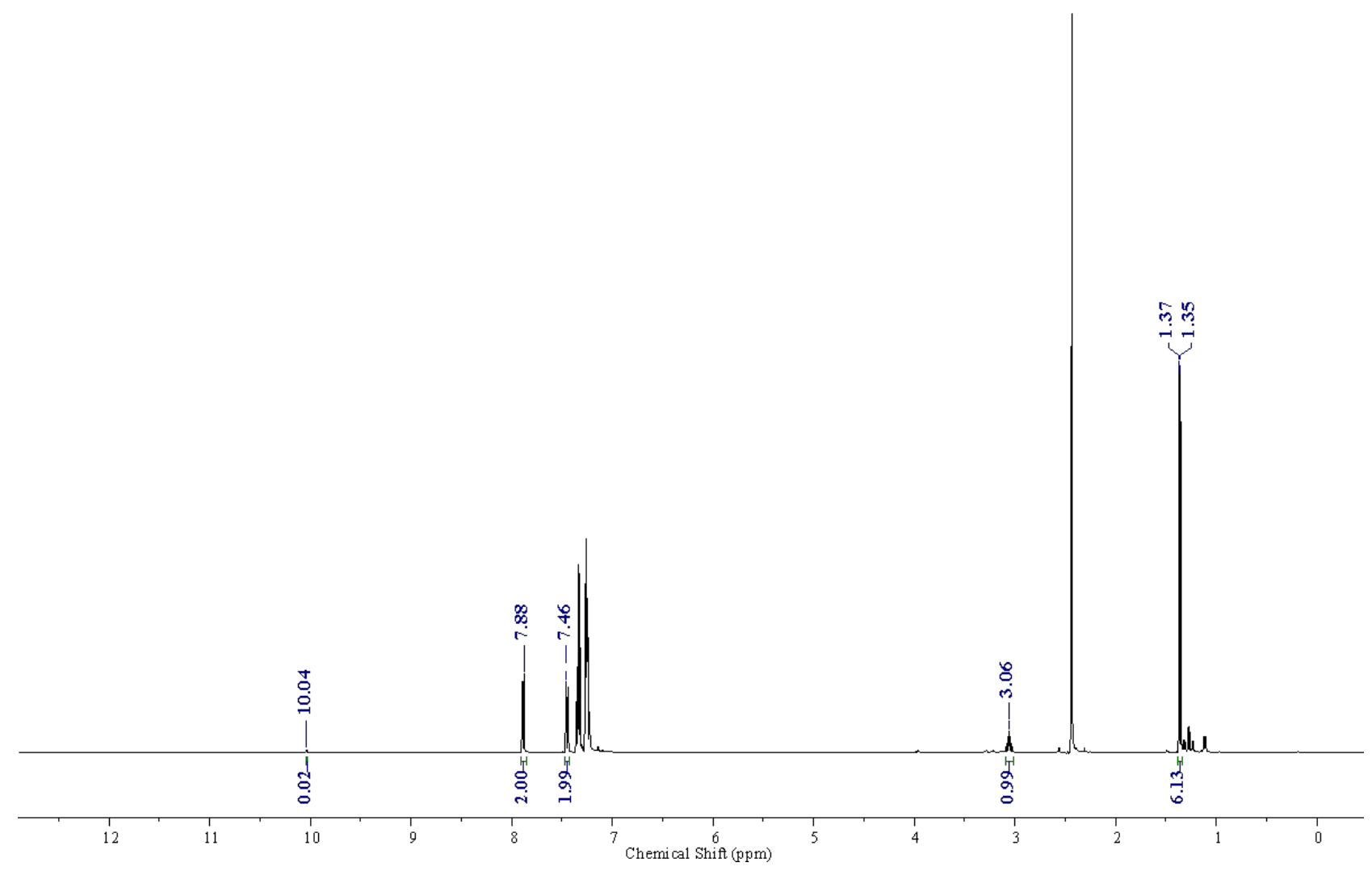


Entry 2': NMR for Crude reaction mixture, Duplicate VDOS-636-B 2.8.fid

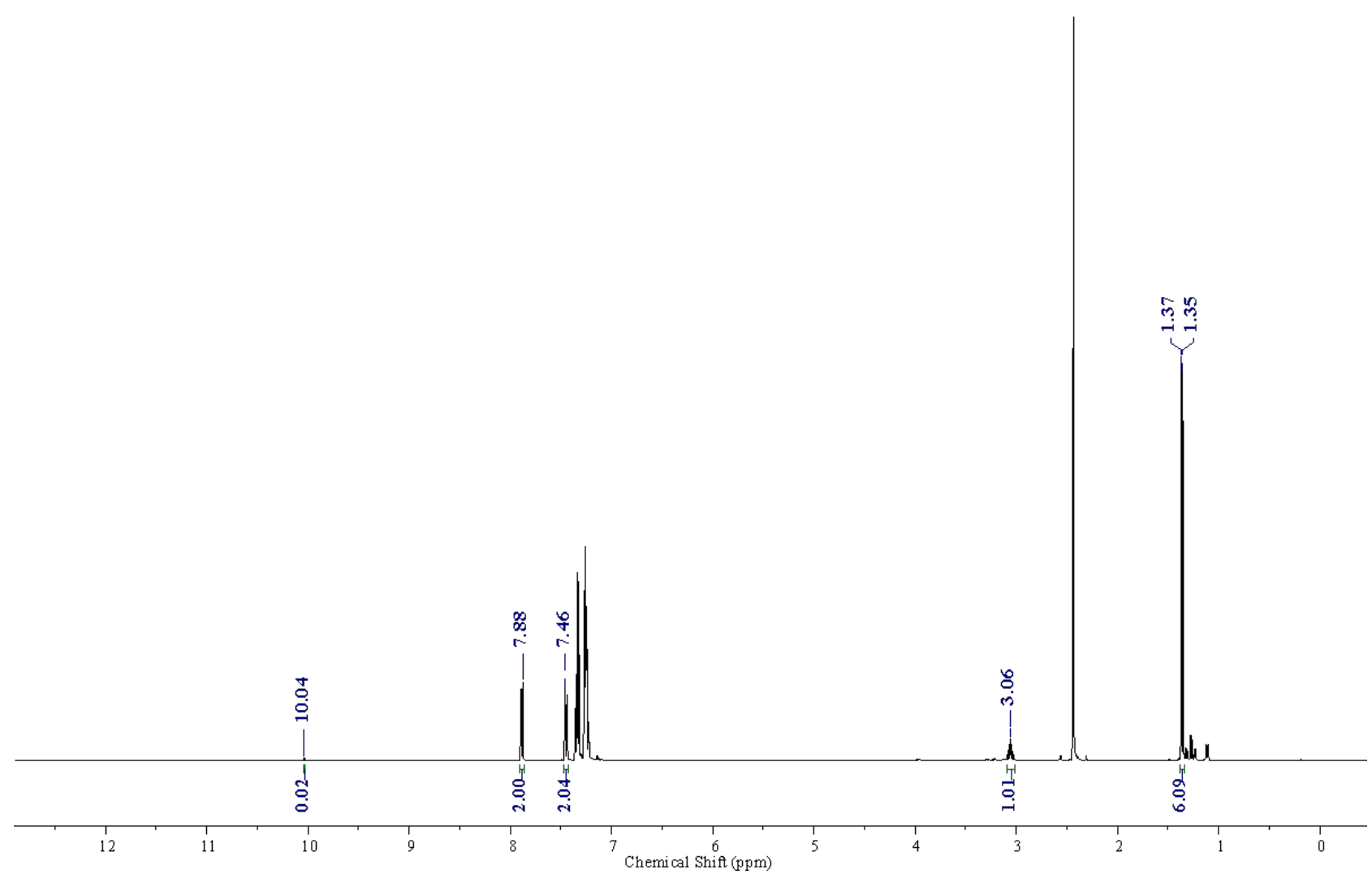

Entry 3: NMR for Crude reaction mixture

VDOS-636-C 1.3.fid

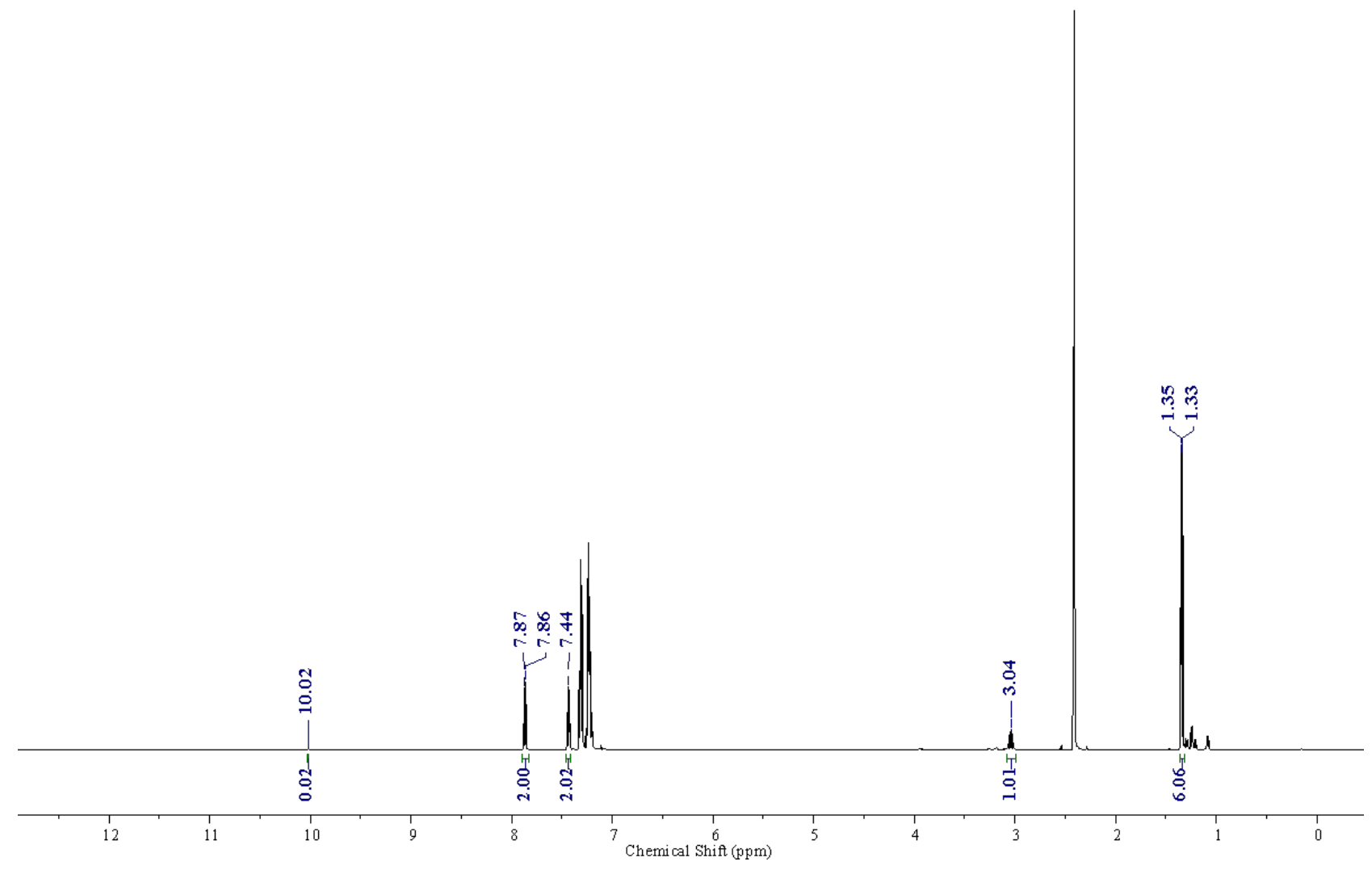


Entry 3': NMR for Crude reaction mixture, Duplicate

VDOS-636-C 2.4.fid

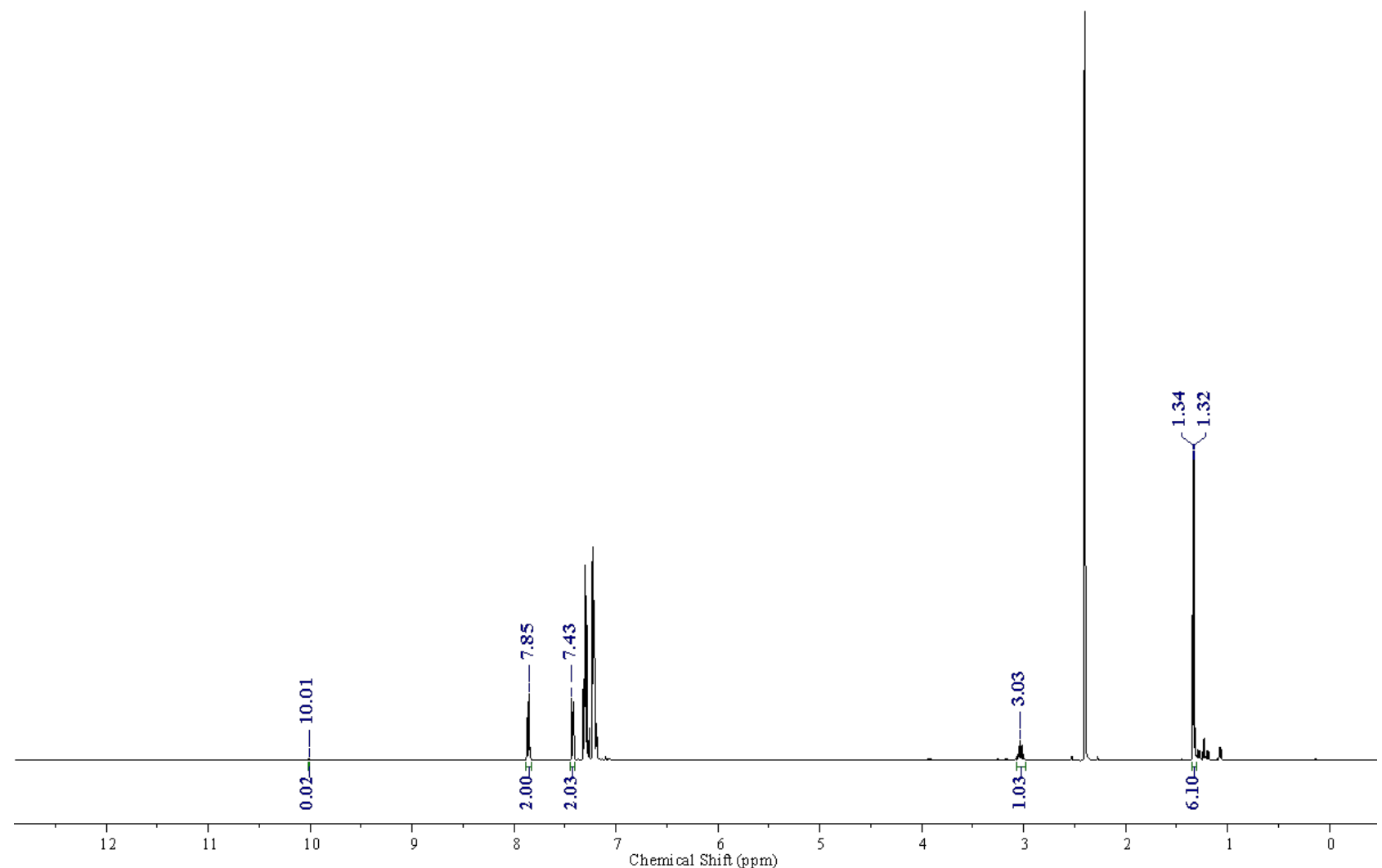

NMR after purification:

VDOS-636-C1 pure. 2fid

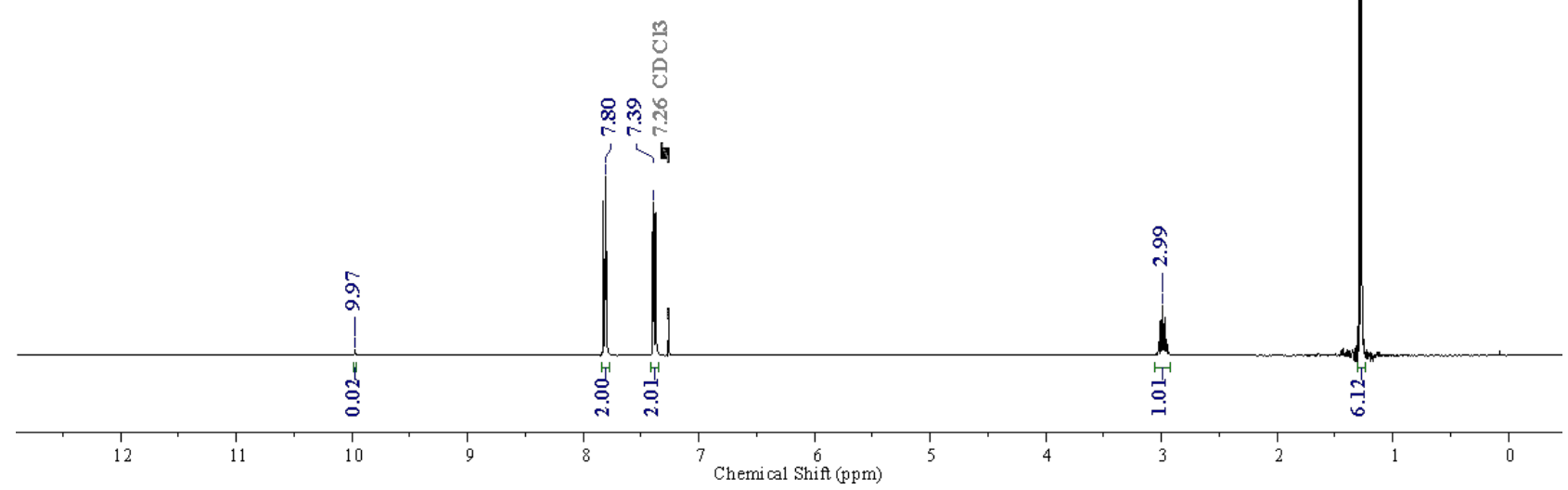




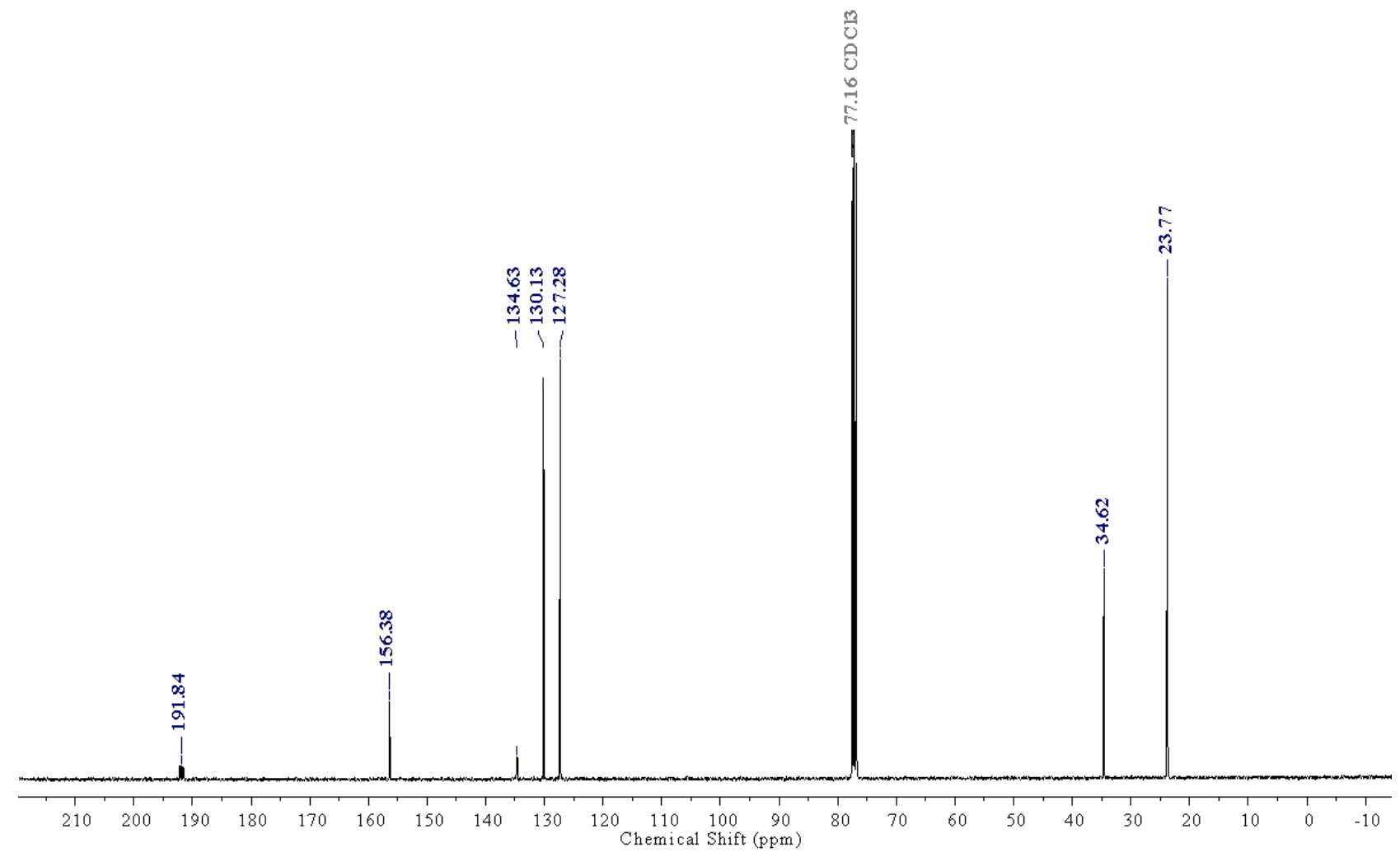


18. 3-Fluorobenzaldehyde-d

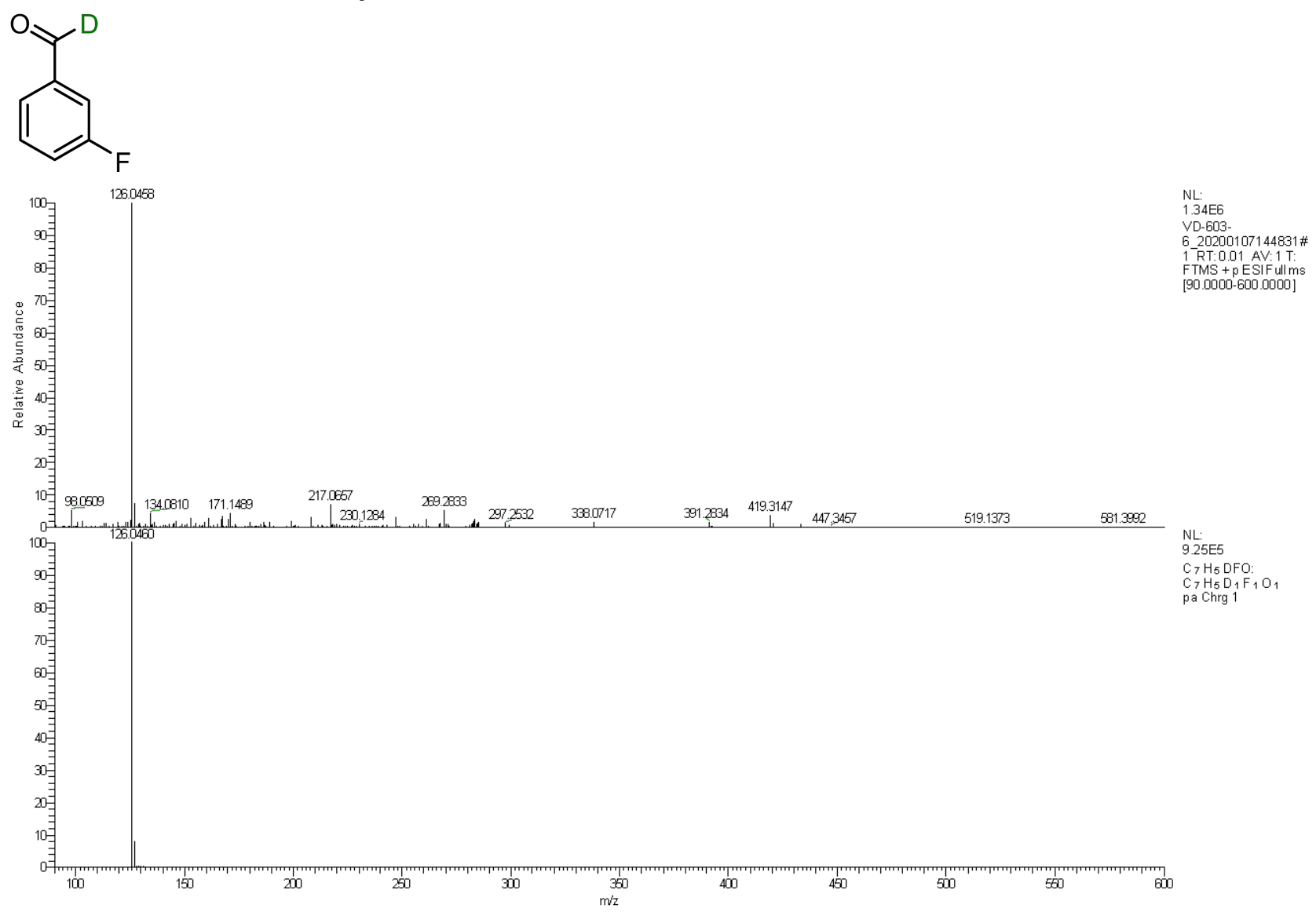

Entry 1: NMR for Crude reaction mixture SCG-312-01-16hr-D 1-50sec.1.fid

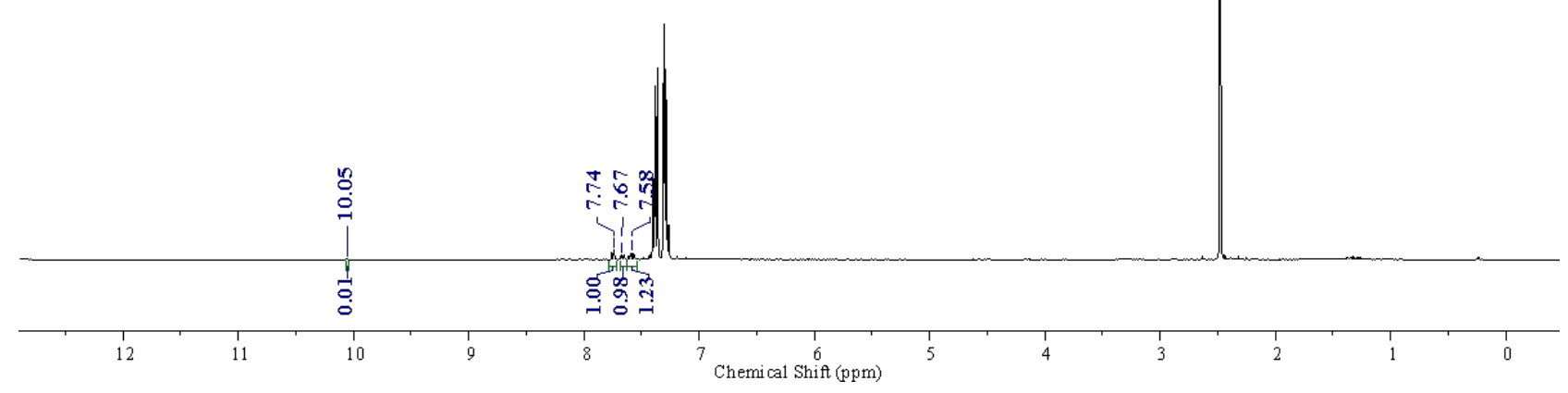


Entry 1': NMR for Crude reaction mixture, Duplicate

SCG-312-O2-16hr-D 1-50sec.2.fid

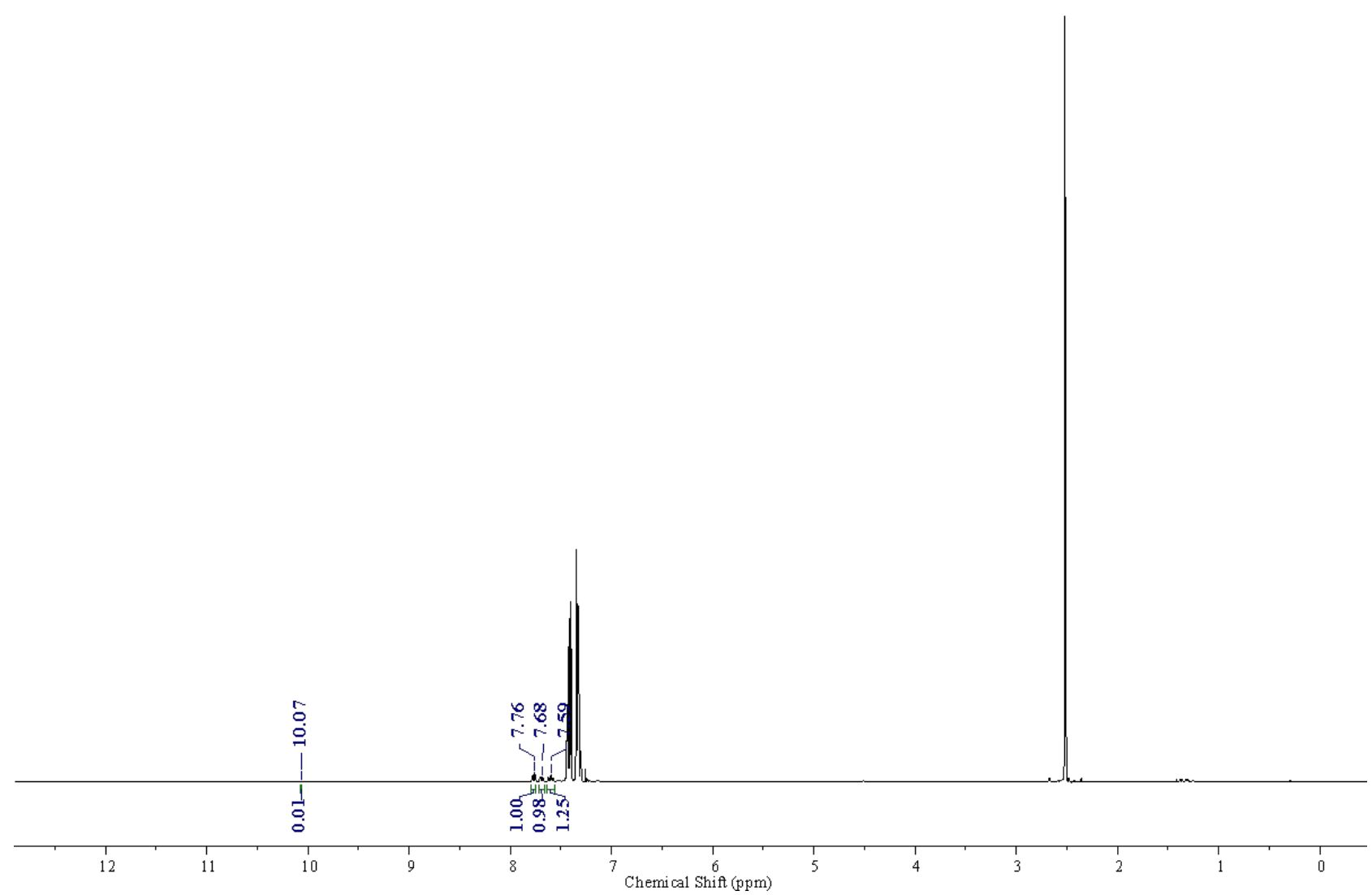

NMR after purification:

VDOS-603-VP-2.3.fid

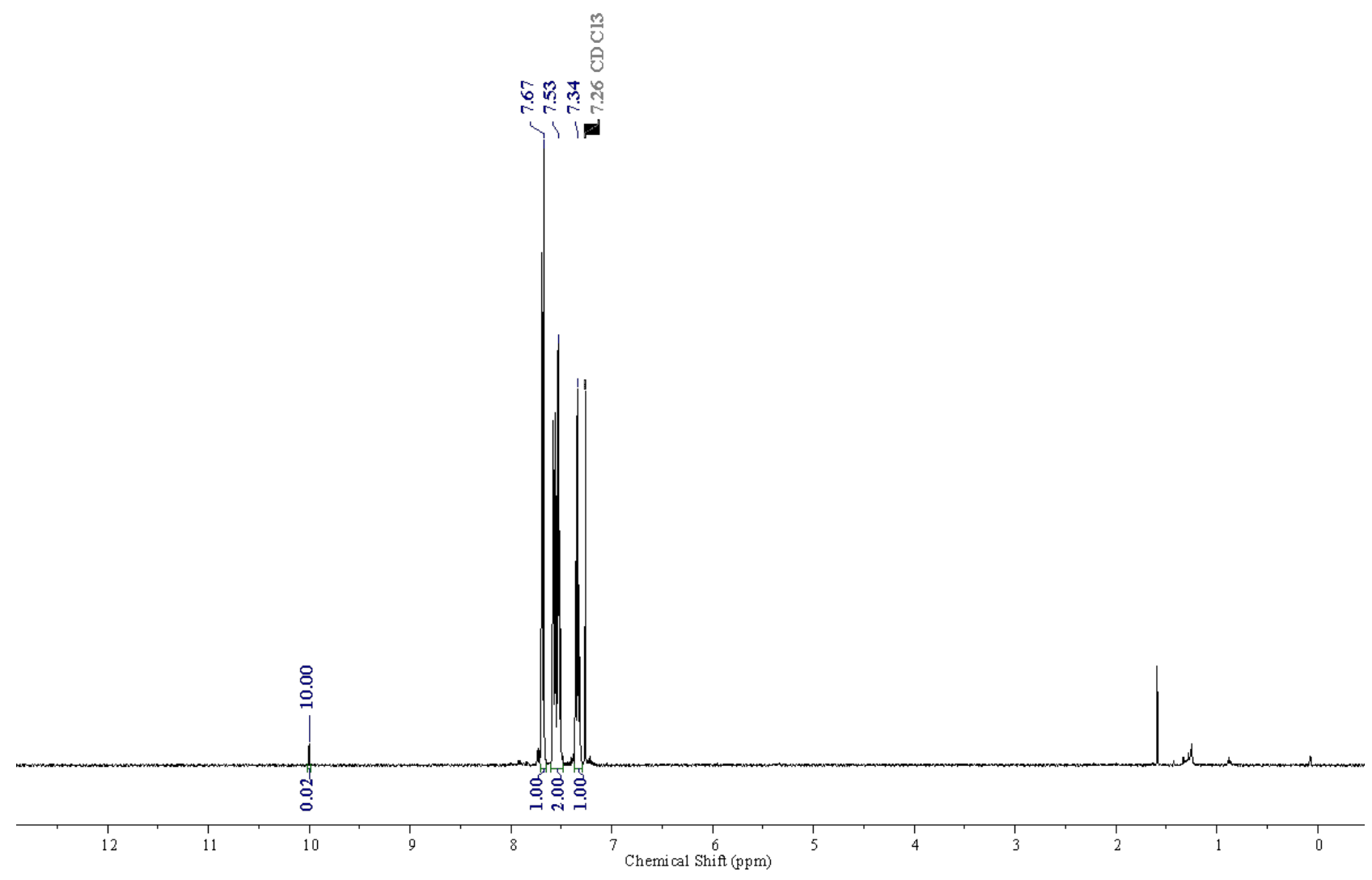




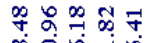

兽余突

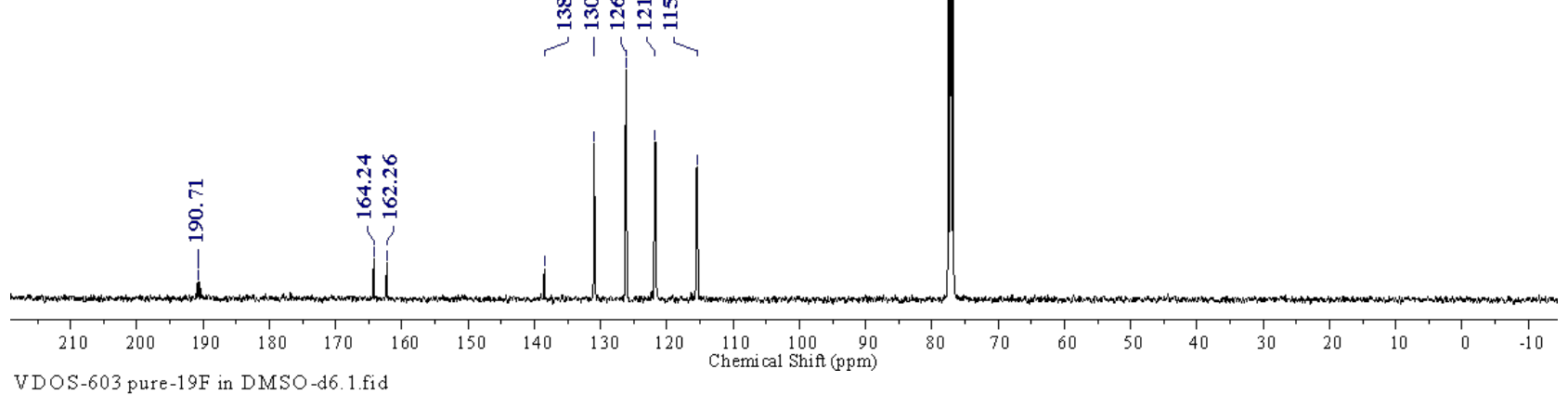
VDOS-603 pure-19F in DMSO-d6.1.fid

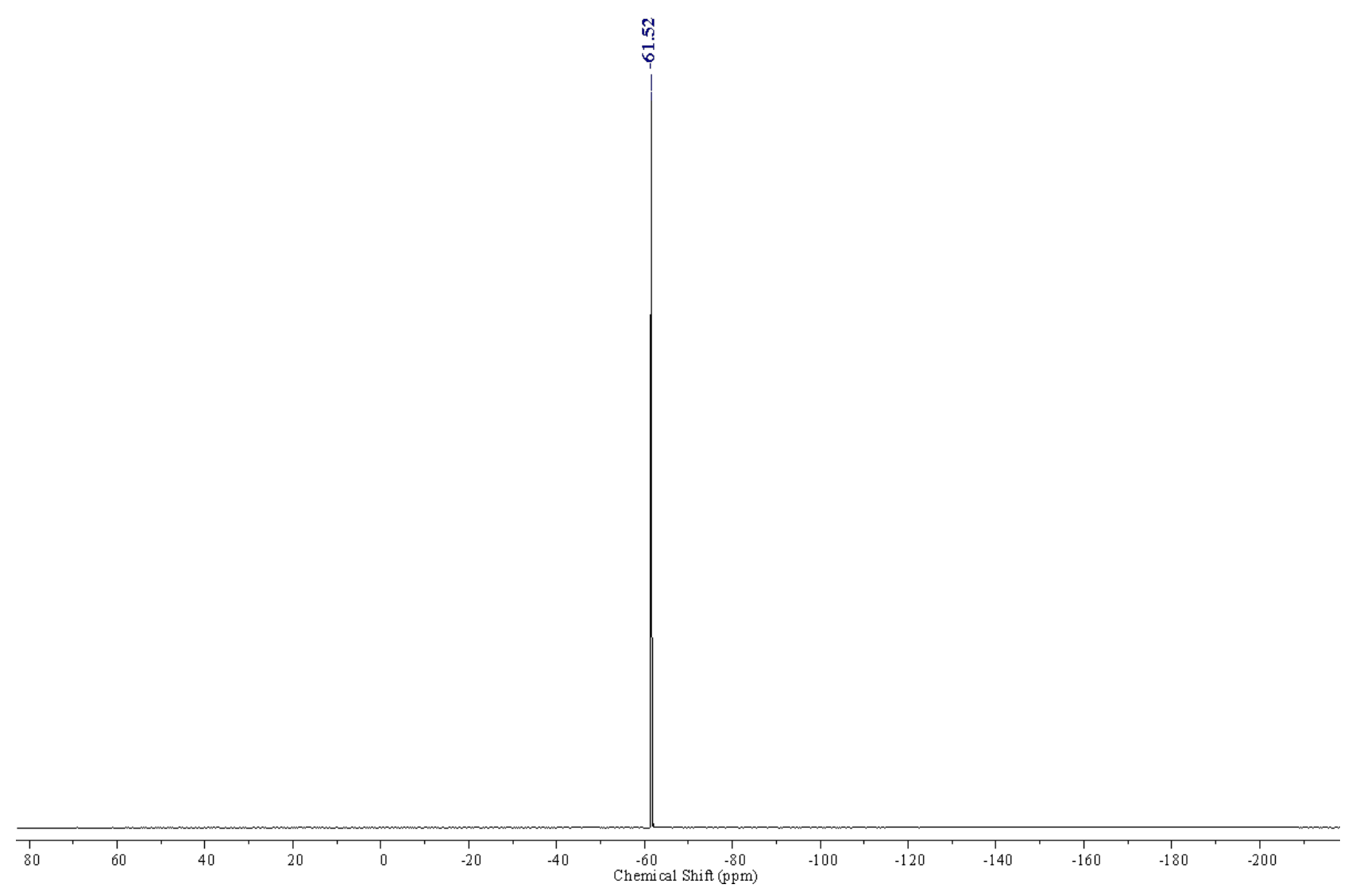


19. 3-Chlorobenzaldehyde-d<smiles>[2H]C(=O)c1cccc(Cl)c1</smiles>

Entry 1: NMR for Crude reaction mixture SCG-312-K1-16hr-D 1-50sec

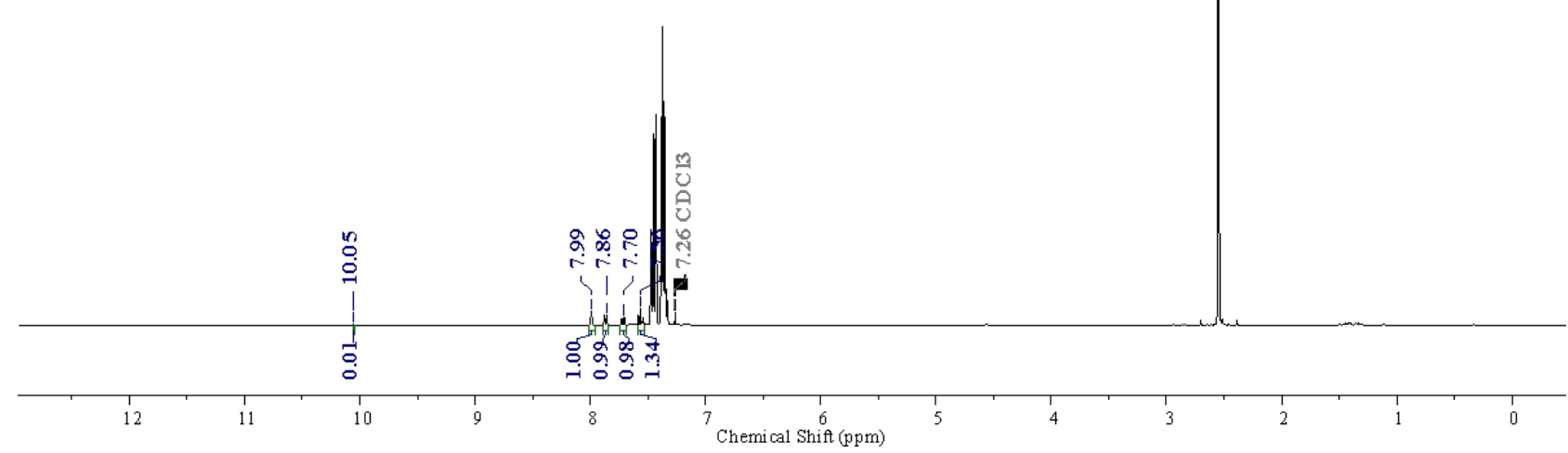


Entry 1': NMR for Crude reaction mixture, Duplicate

SCG-312-K2-16hr-D 1-50sec

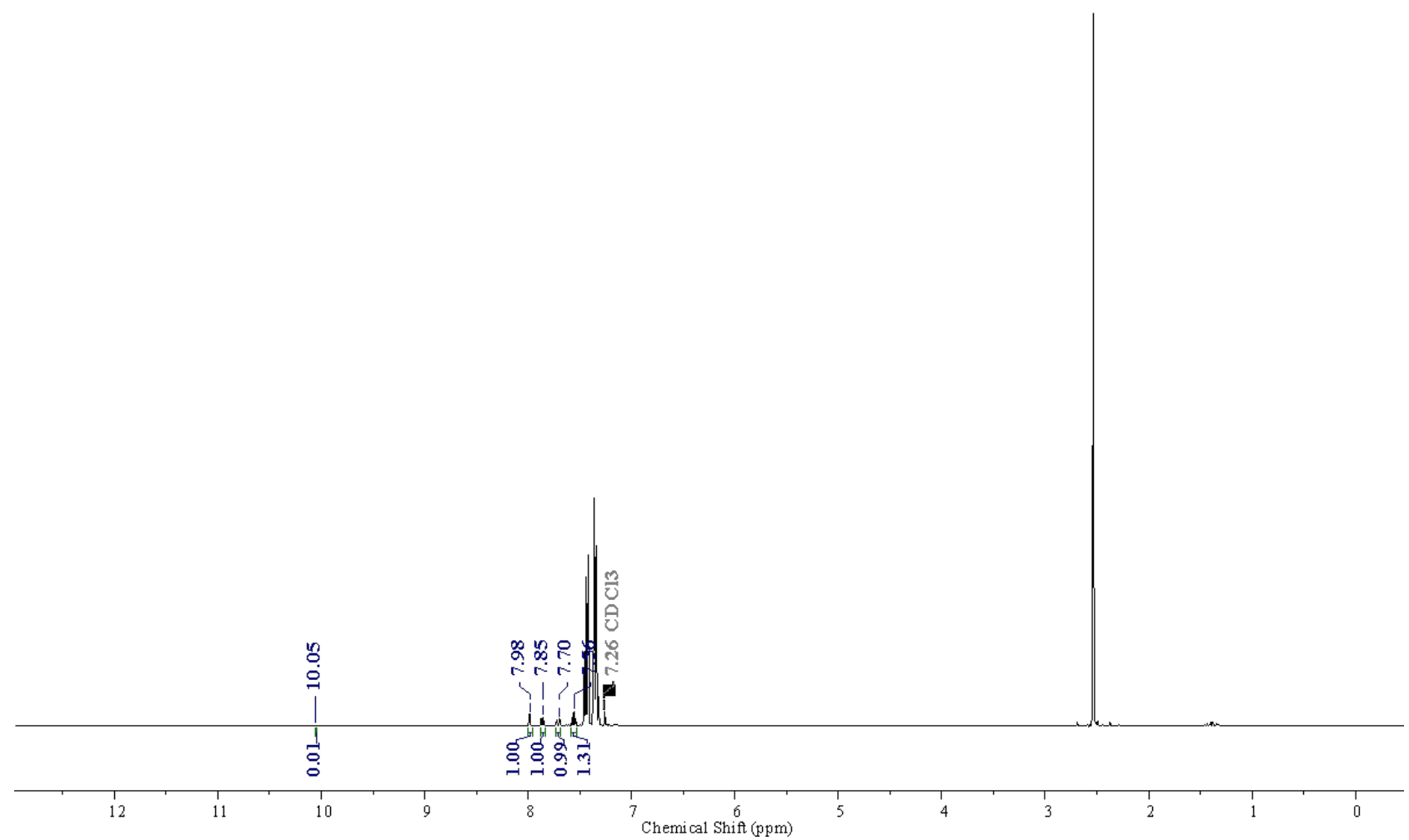

NMR after purification:

SCG-312-K3-16hr-D1-50sec-AC

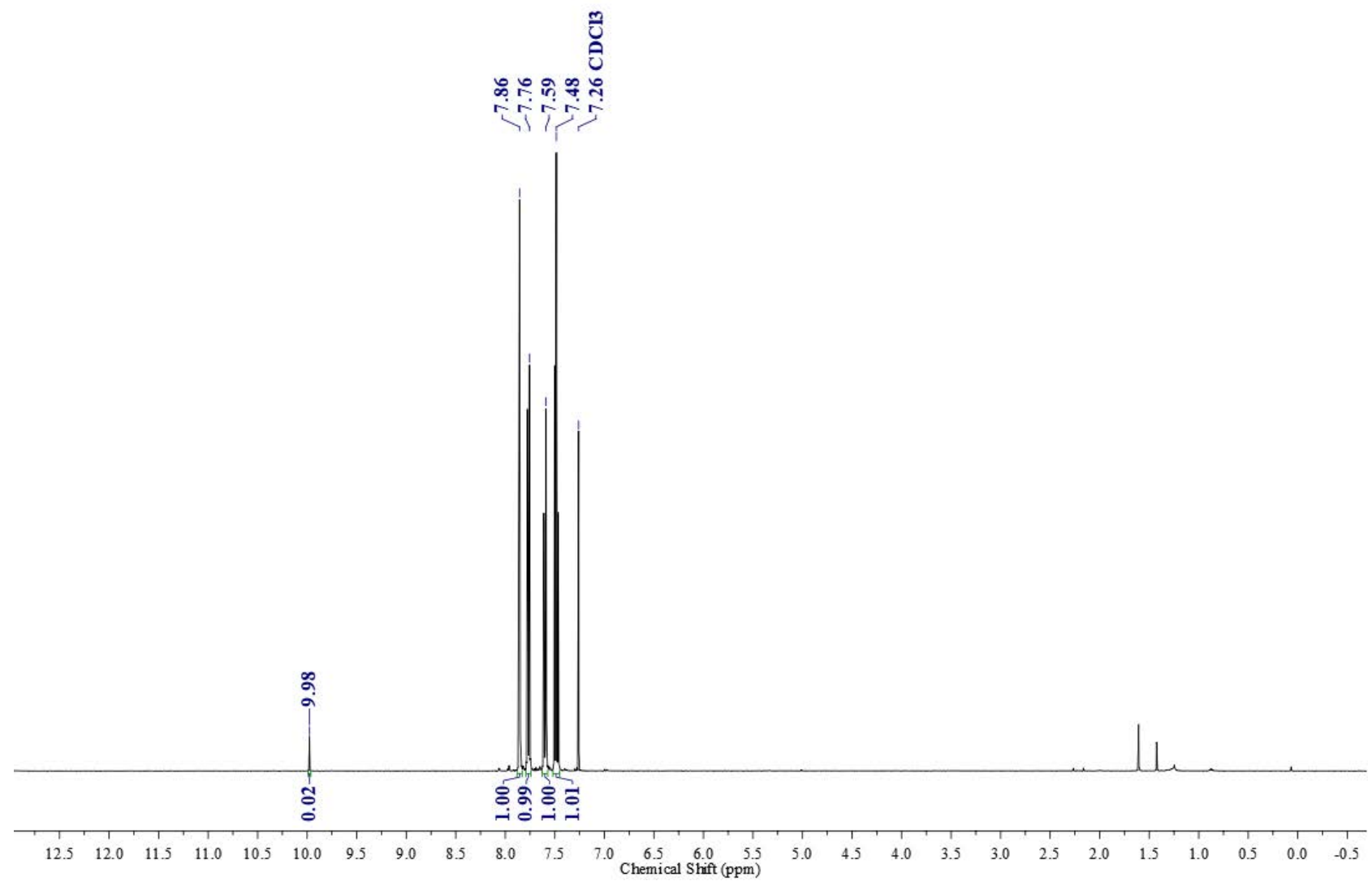




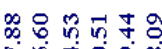

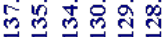

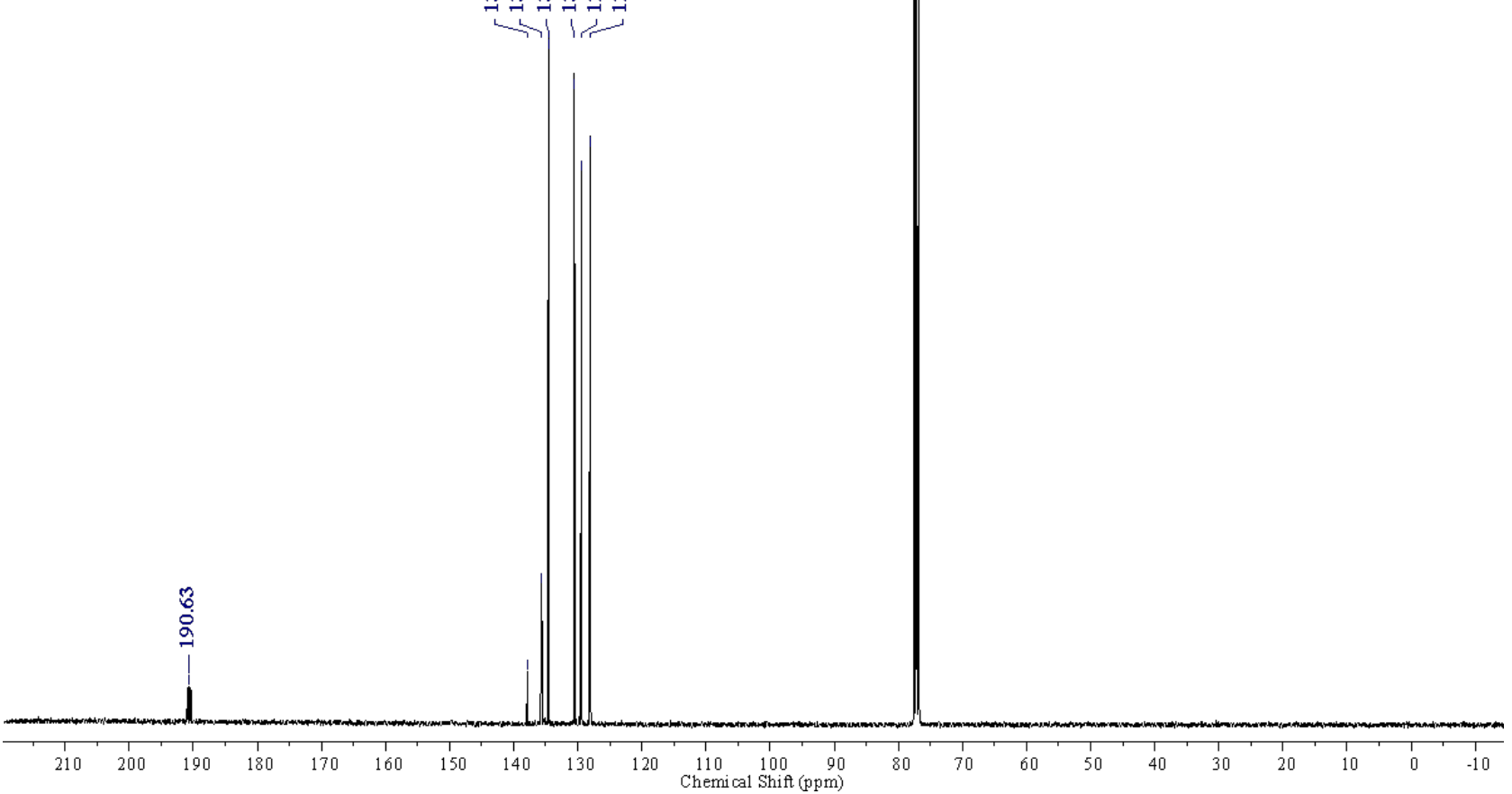


20. 4-Chlorobenzaldehyde-d<smiles>[2H]C(=O)c1ccc(Cl)cc1</smiles>

Entry 1: NMR for Crude reaction mixture SCG-312-J2-16hr-D 1-50sec

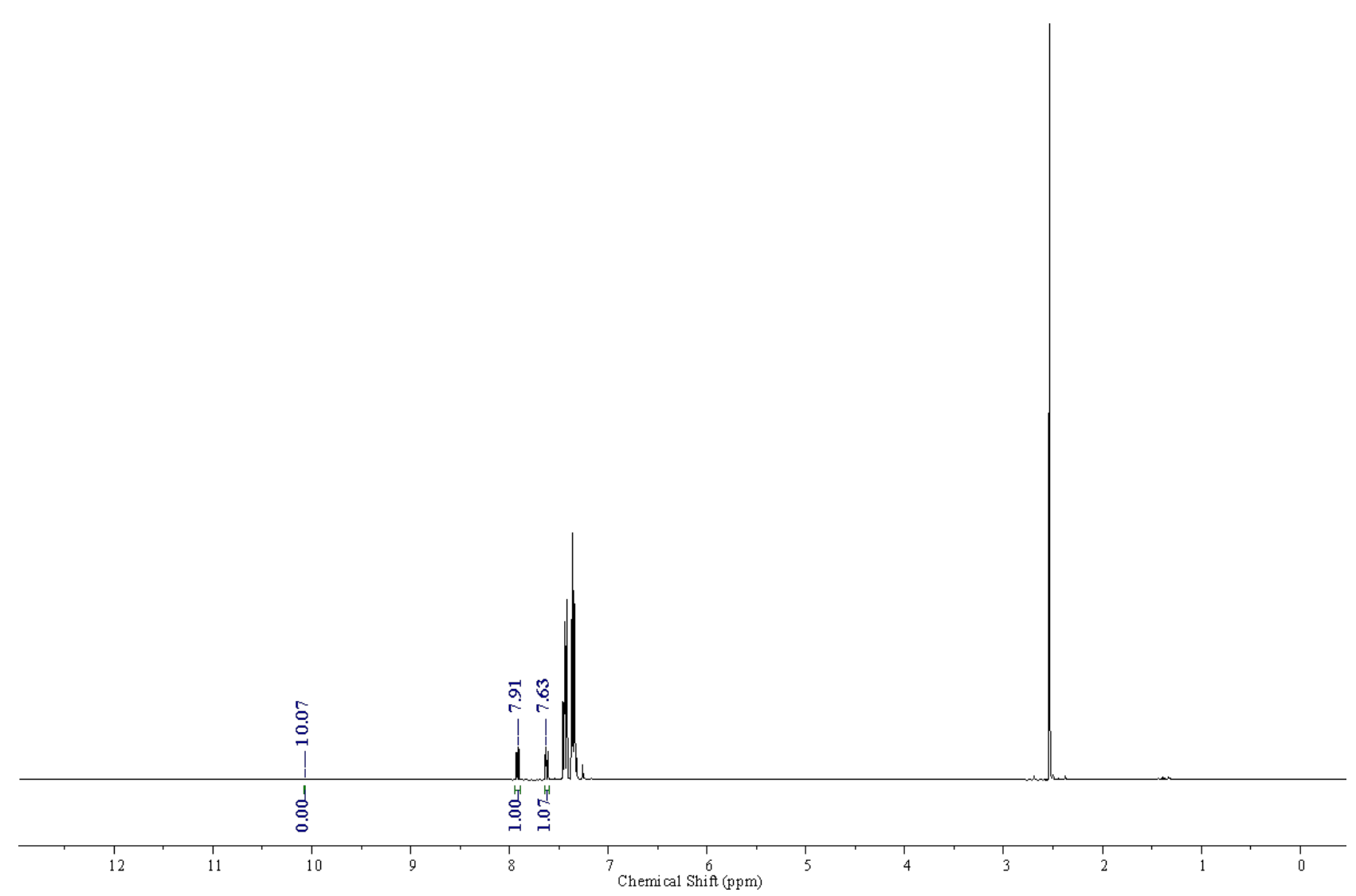


Entry 1': NMR for Crude reaction mixture, Duplicate SCG-312-J1-16hr-D 1-50sec

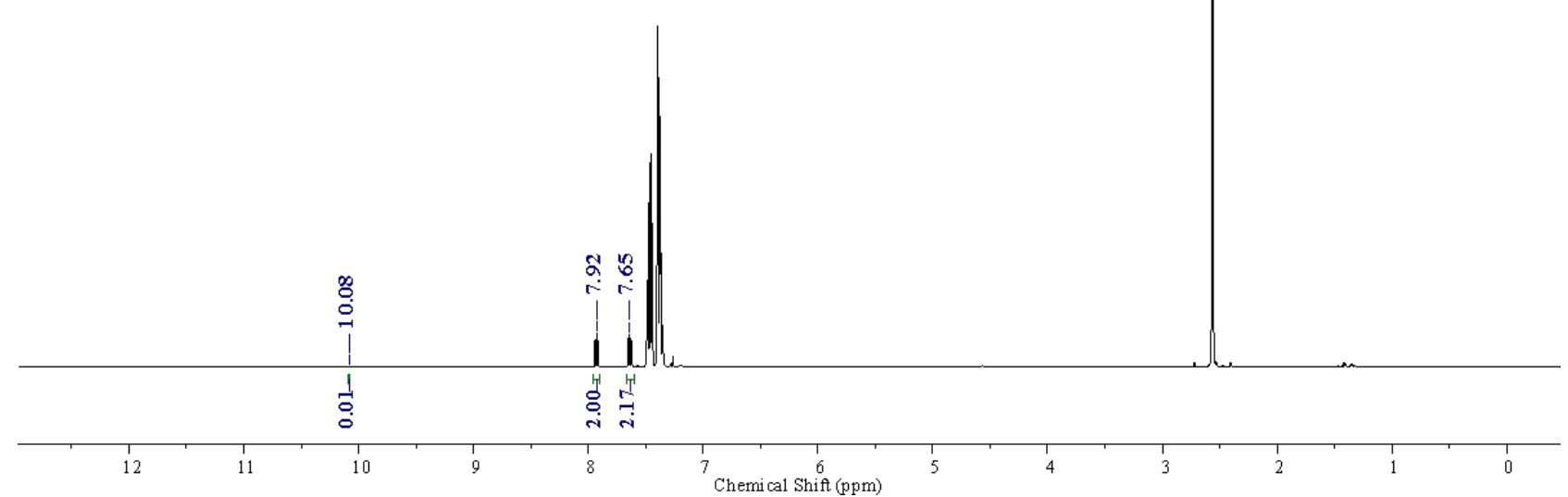

NMR after purification:

SCG-312-J1-16hr-D 1-50sec-AC

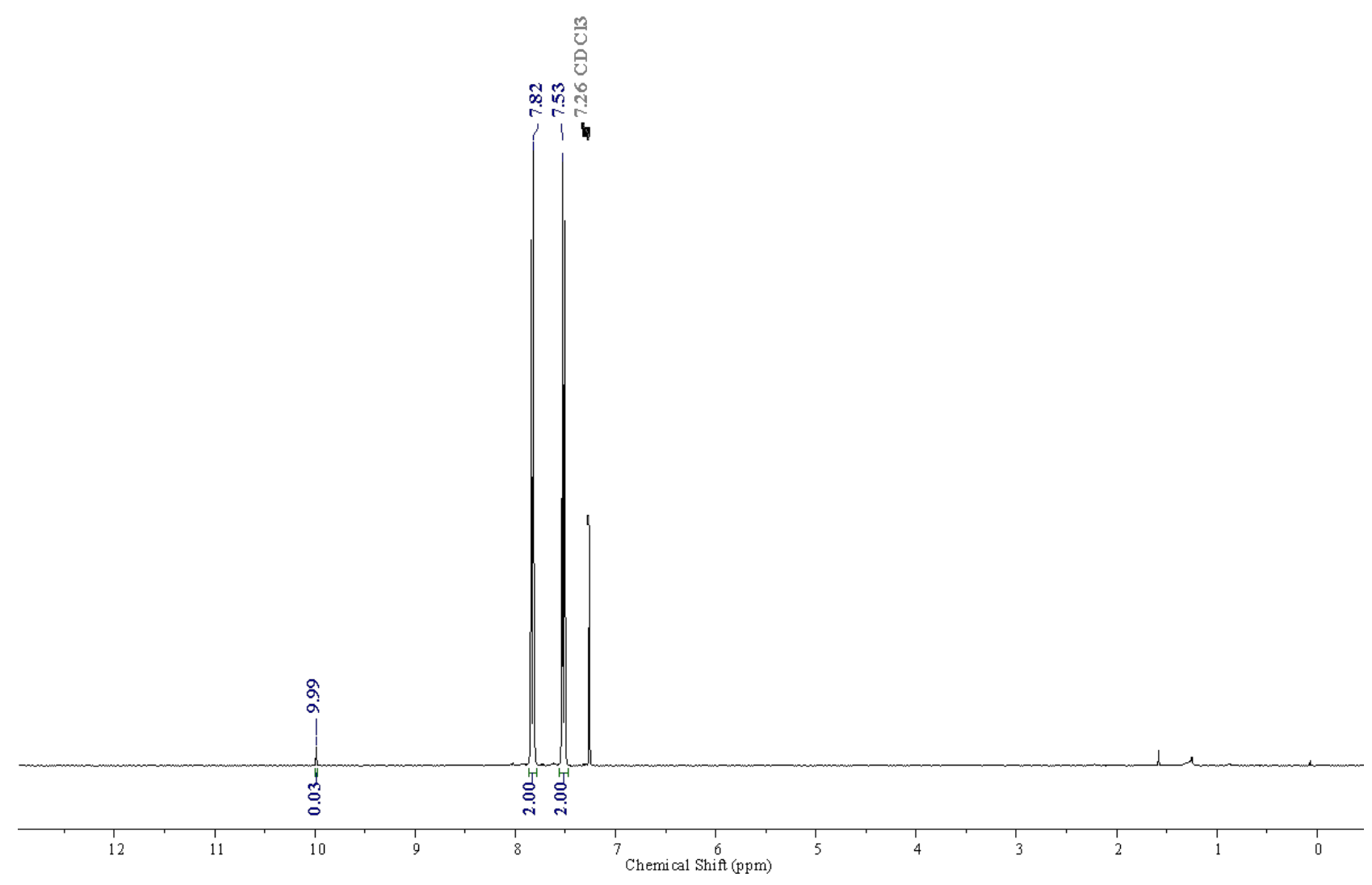




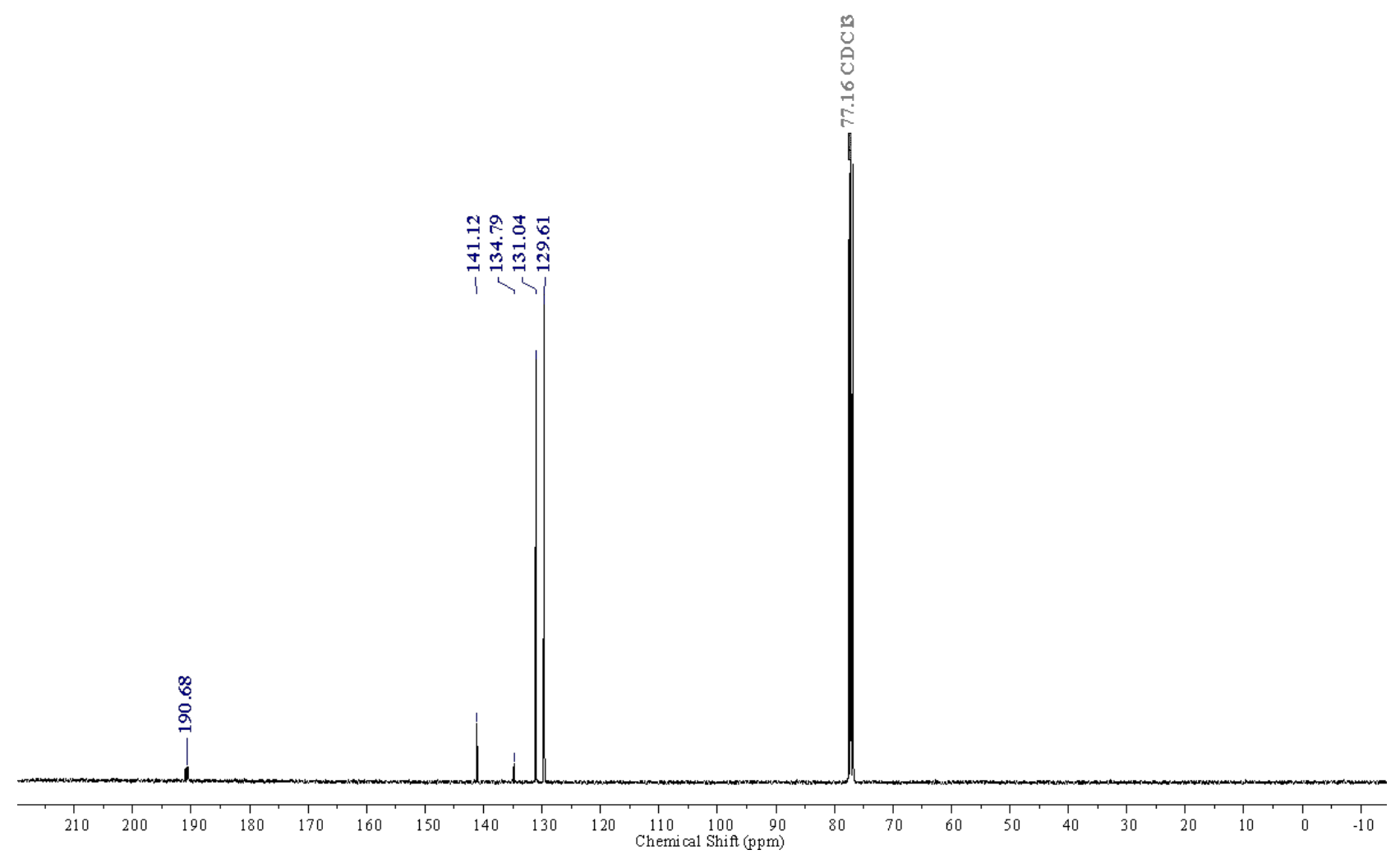




\section{3,5-Dichlorobenzaldehyde-d}<smiles>[2H]C(=O)c1cc(Cl)cc(Cl)c1</smiles>

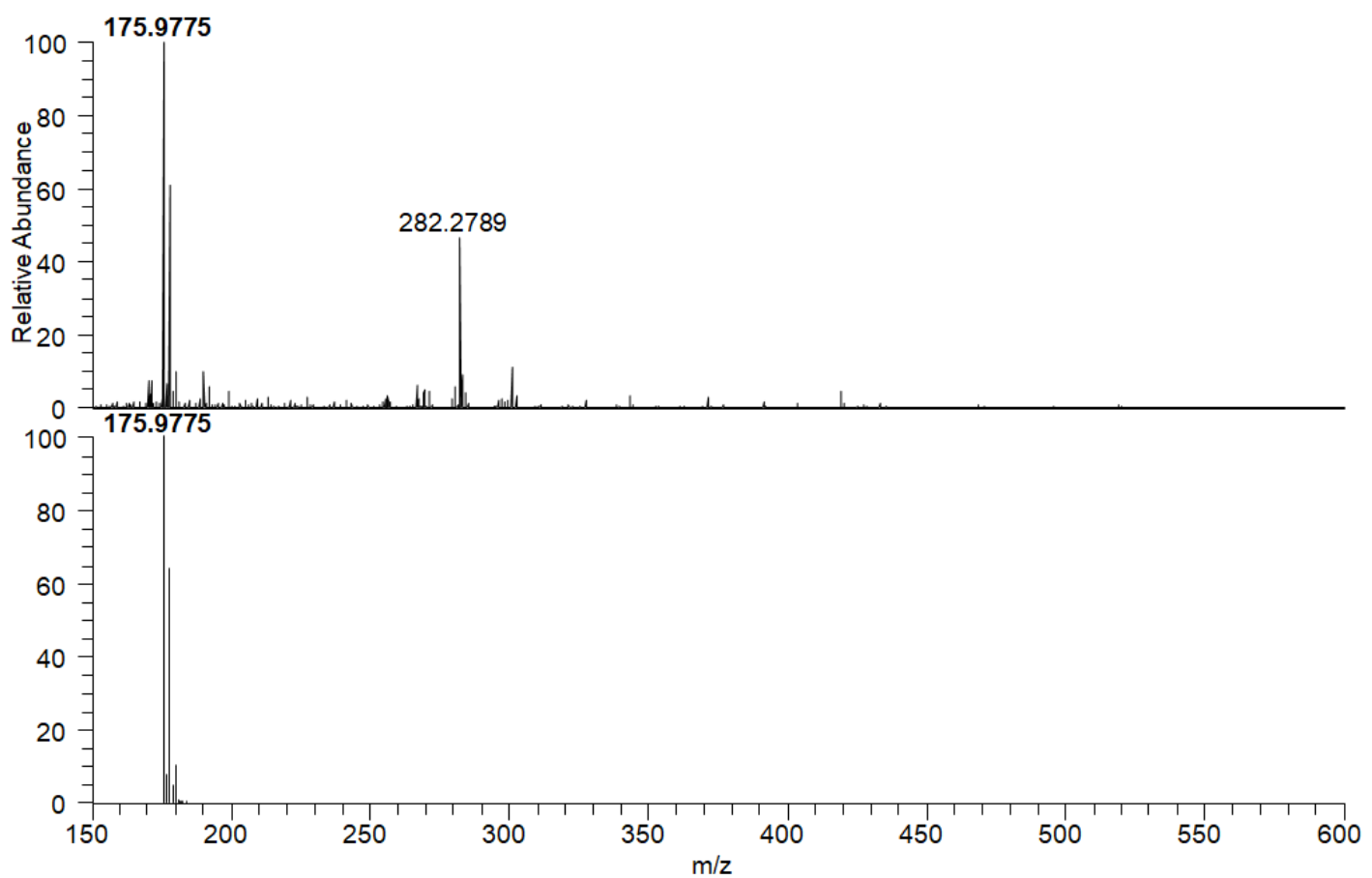

NL:

2.04E6

ilz-o1 \#30-2RT:

$0.01 \mathrm{AV}: 1 \mathrm{~T}$

FTMS + p ESI Full

lock ms

$-150.0000$

[600.0000
5.31E5

$\mathrm{C}_{7} \mathrm{H}_{4} \mathrm{DCl}_{2} \mathrm{O}$ :

$\mathrm{C}_{7} \mathrm{H}_{4} \mathrm{D}_{1} \mathrm{Cl}_{2} \mathrm{O}_{1}$ pa Chrg 1

Entry 1: NMR for Crude reaction mixture SCG-318-D 2-16hr-D 1-50sec

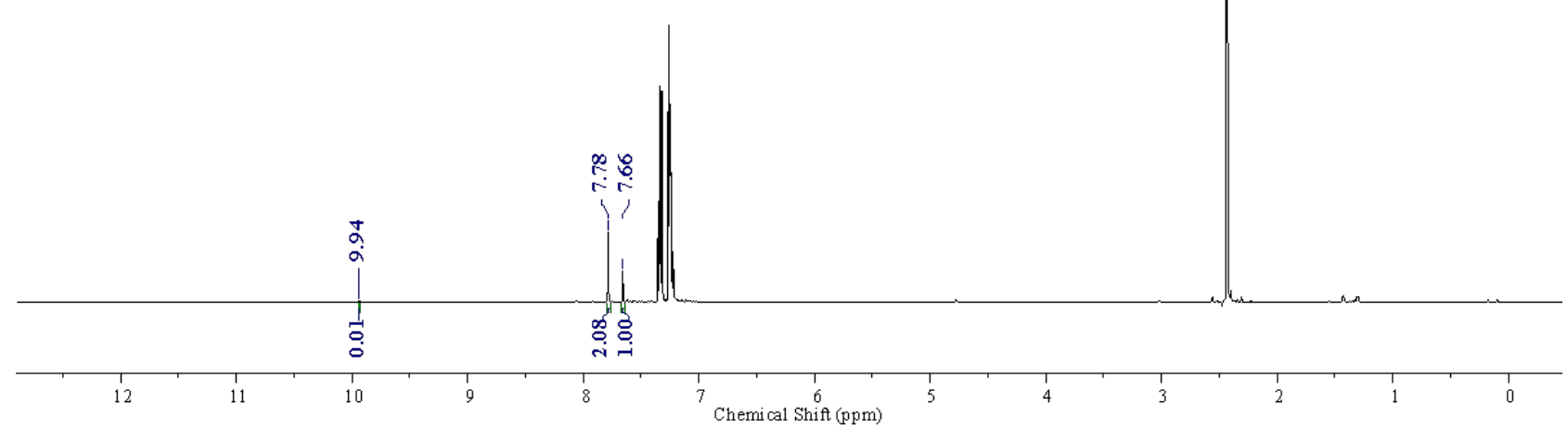


Entry 1': NMR for Crude reaction mixture, Duplicate

SCG-318-D 1-16hr-D 1-50sec

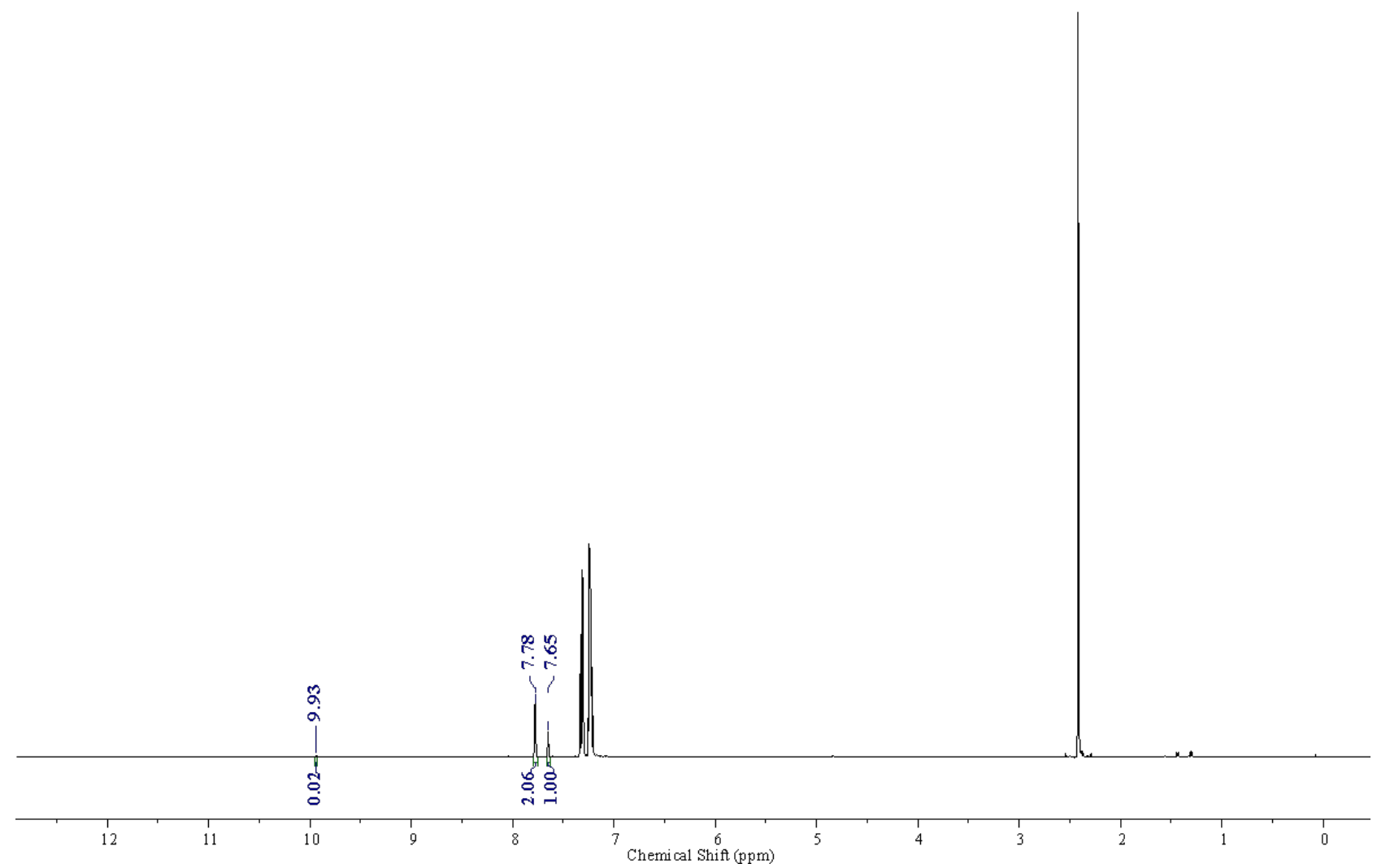

NMR after purification:

VDOS-601-VP

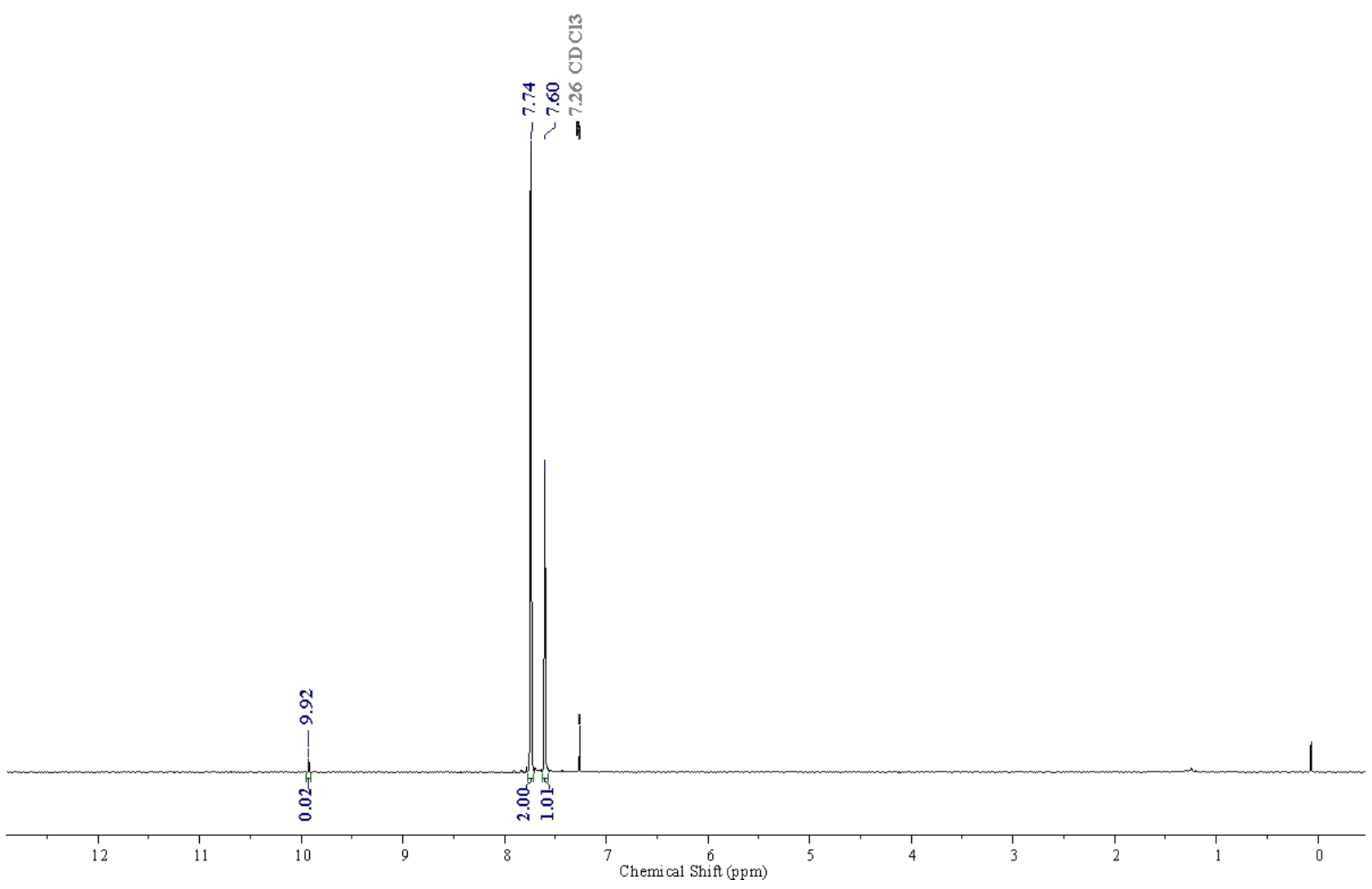


VDOS-601-VP 13C

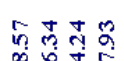

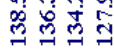

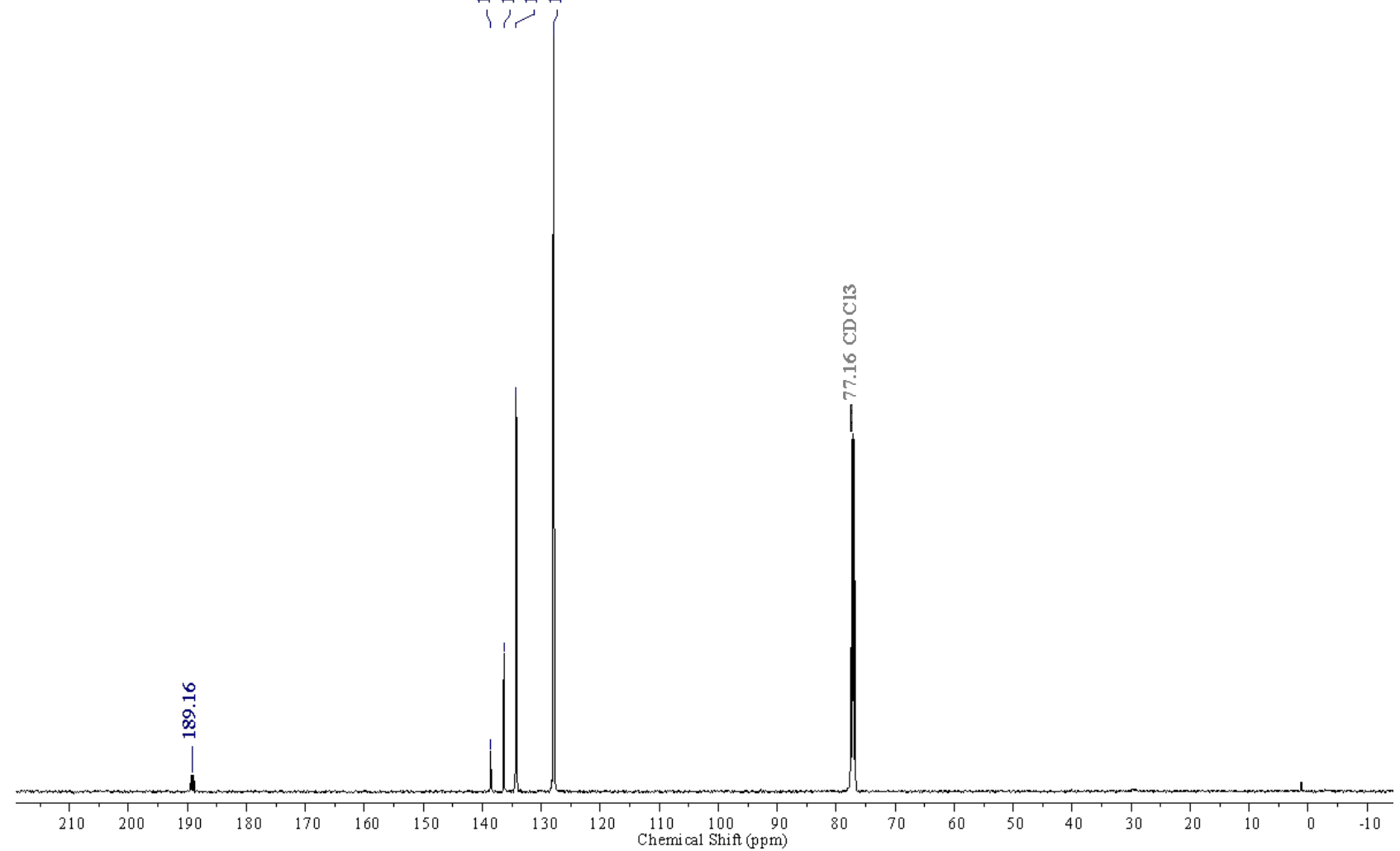


22. 3-Bromobenzaldehyde-d<smiles>[2H]C(=O)c1cccc(Br)c1</smiles>

Entry 1: NMR for Crude reaction mixture SCG-312-L 1-16hr-D 1-50sec

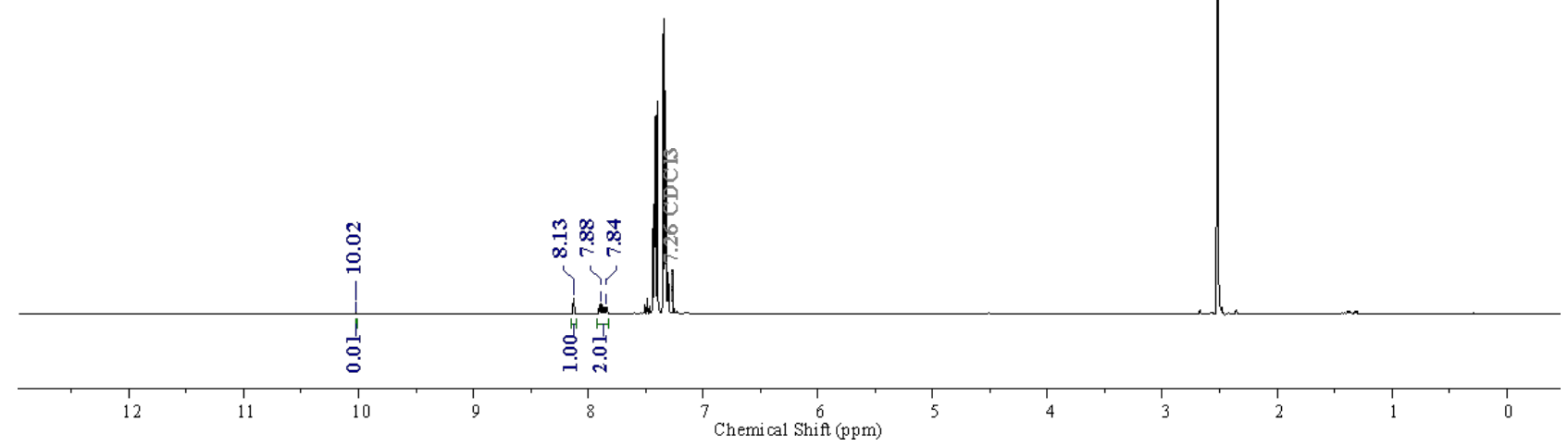


Entry 1': NMR for Crude reaction mixture, Duplicate

SCG-312-L2-16hr-D 1-50sec

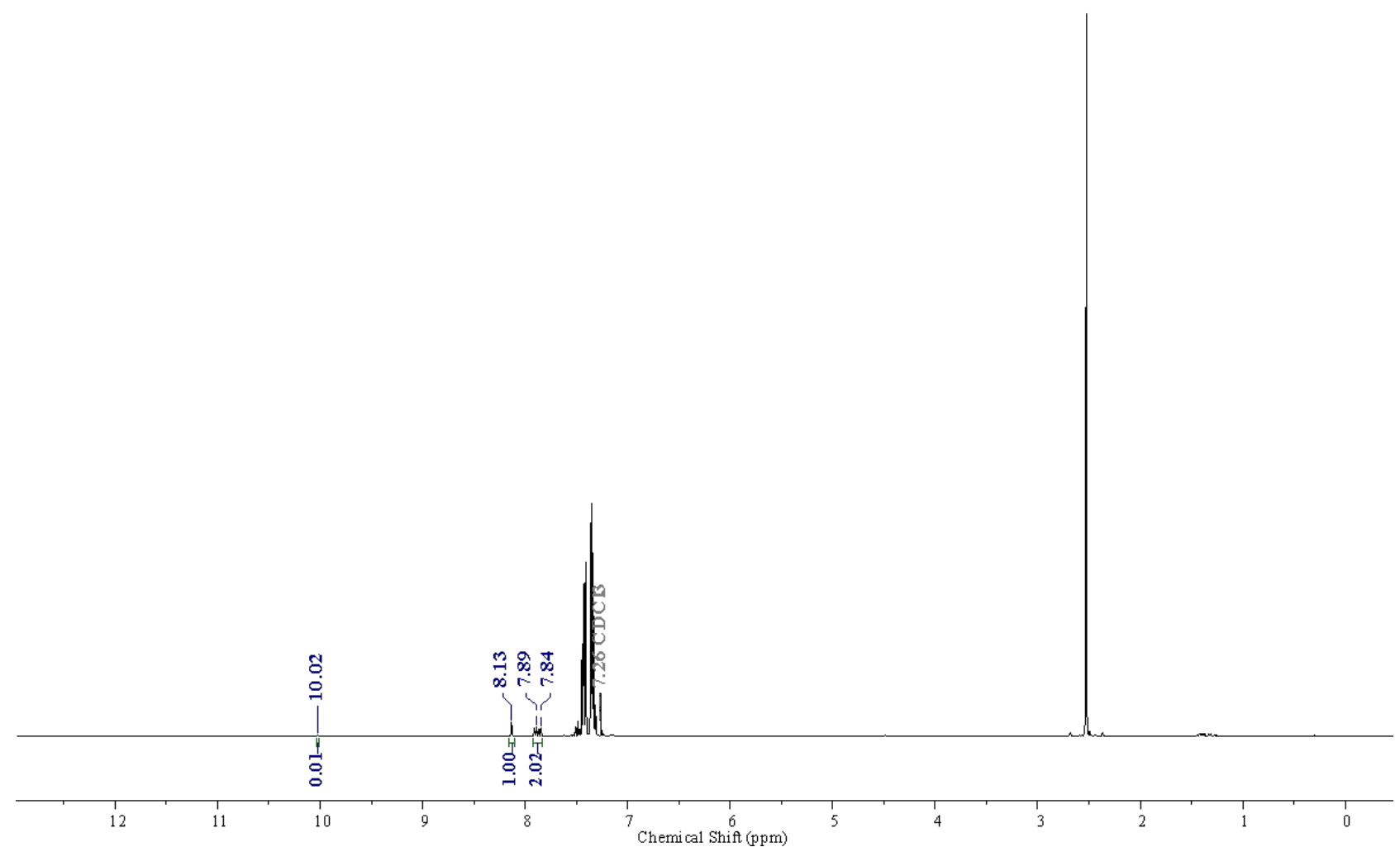

NMR after purification:

SCG-312-L1-16hr-D 1-50sec-A C-07052019

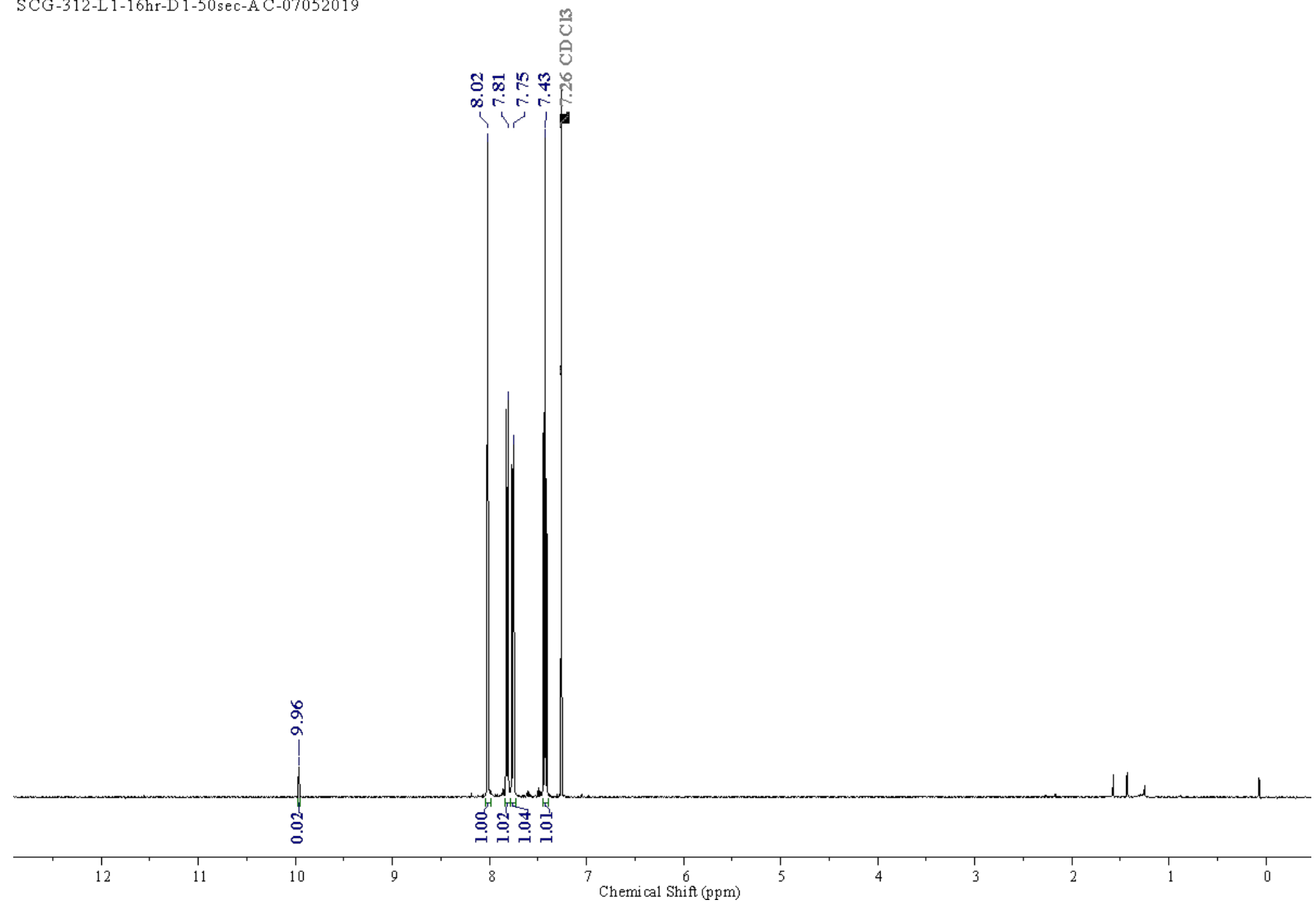




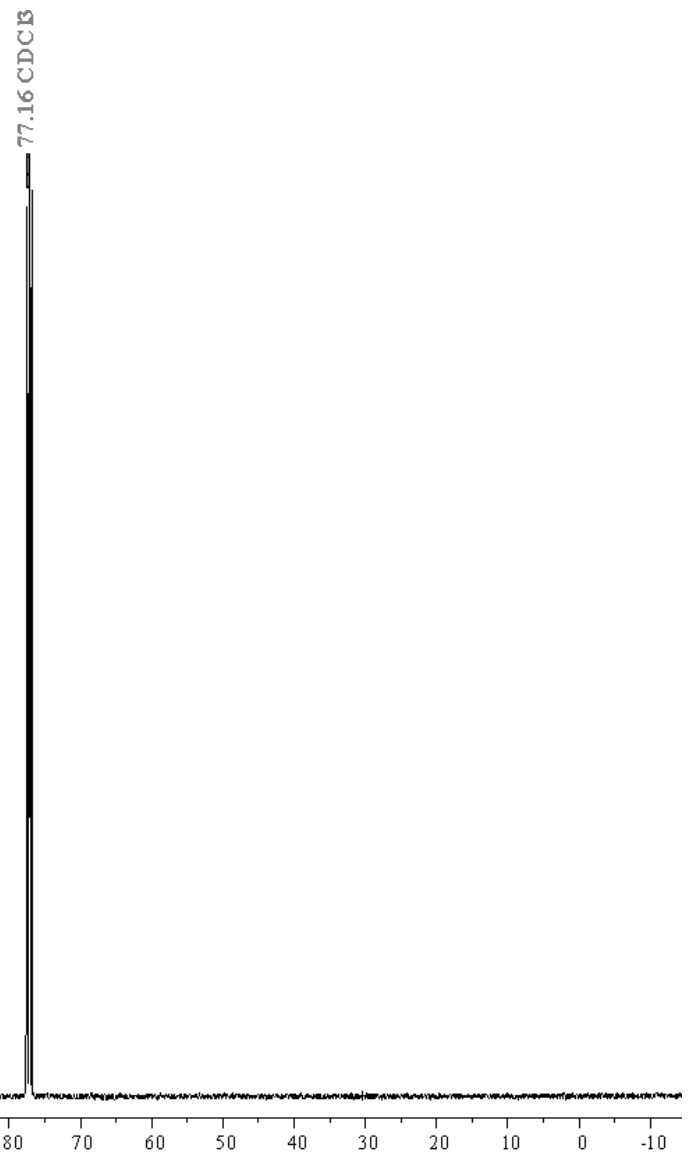

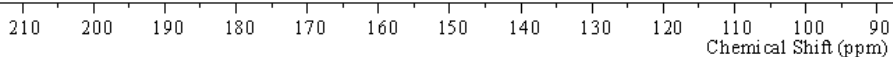


23. 4-Bromobenzaldehyde-d<smiles>[2H]C(=O)c1ccc(Br)cc1</smiles>

Entry 1: NMR for Crude reaction mixture SCG-312-B 1-16hr-D 1-50sec

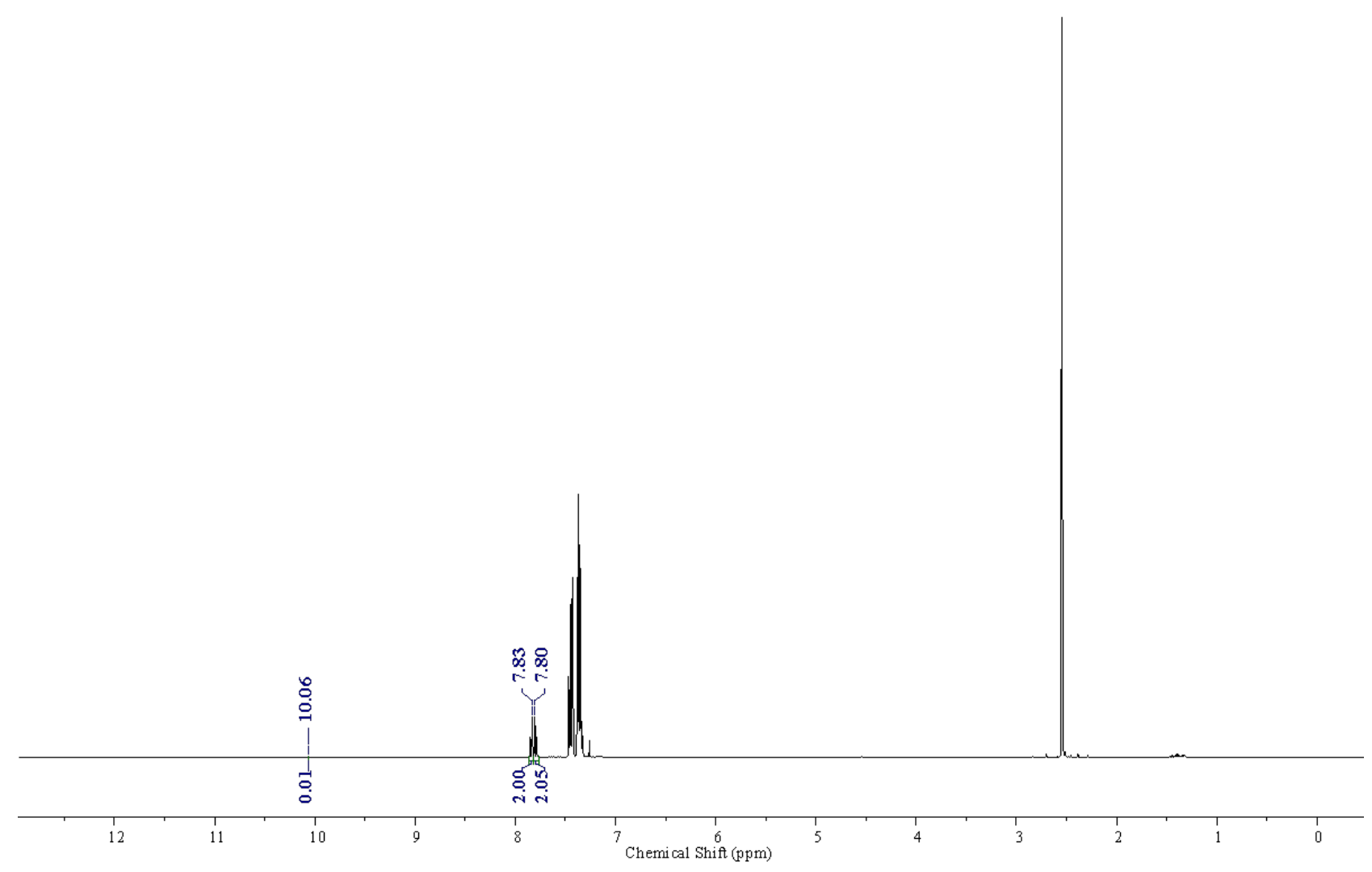


Entry 1': NMR for Crude reaction mixture, Duplicate

SCG-312-B 2-16hr-D 1-50sec

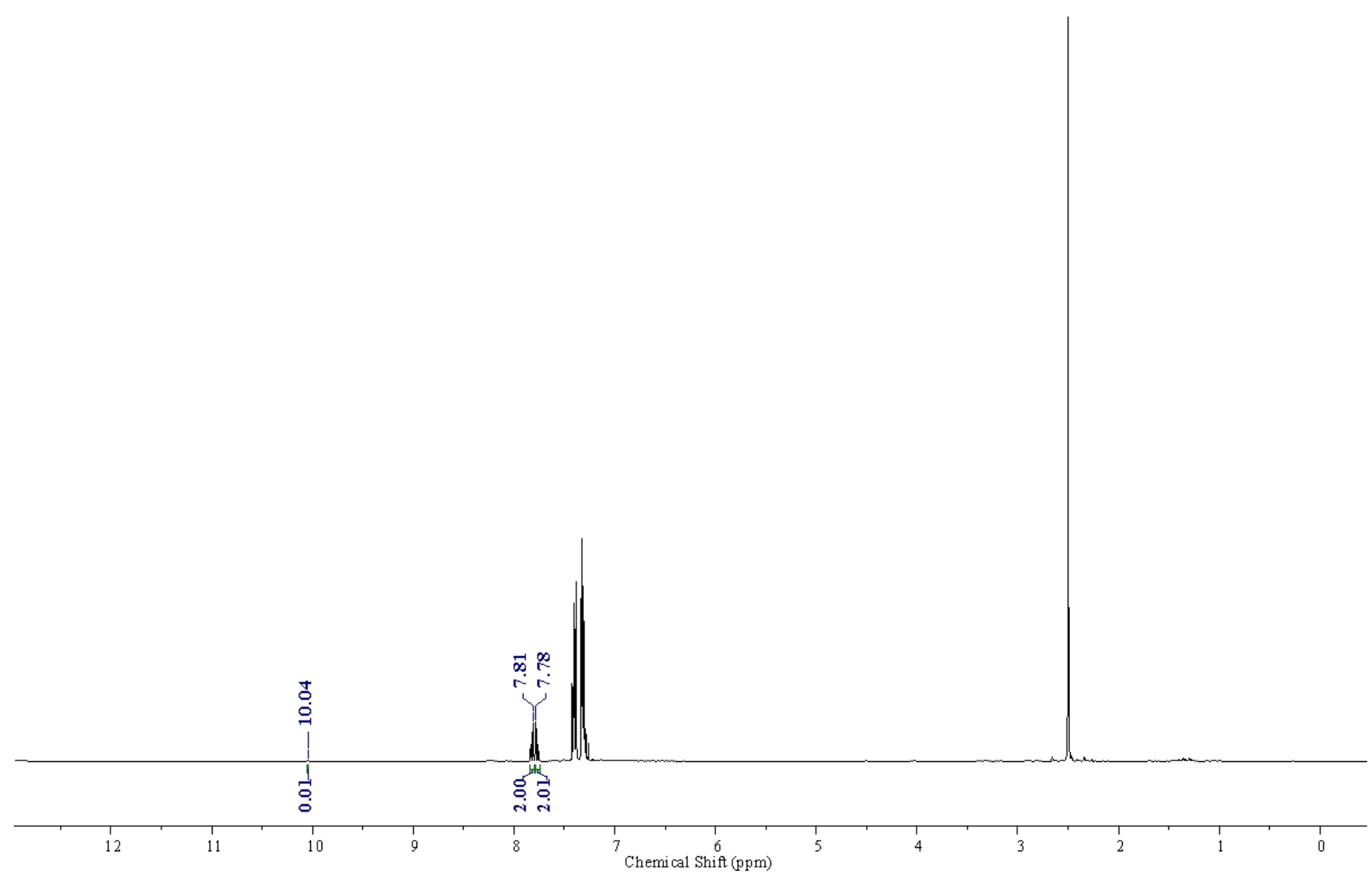

NMR after purification:

SCG-312-B 4-16hr-D 1-50sec-A C-2

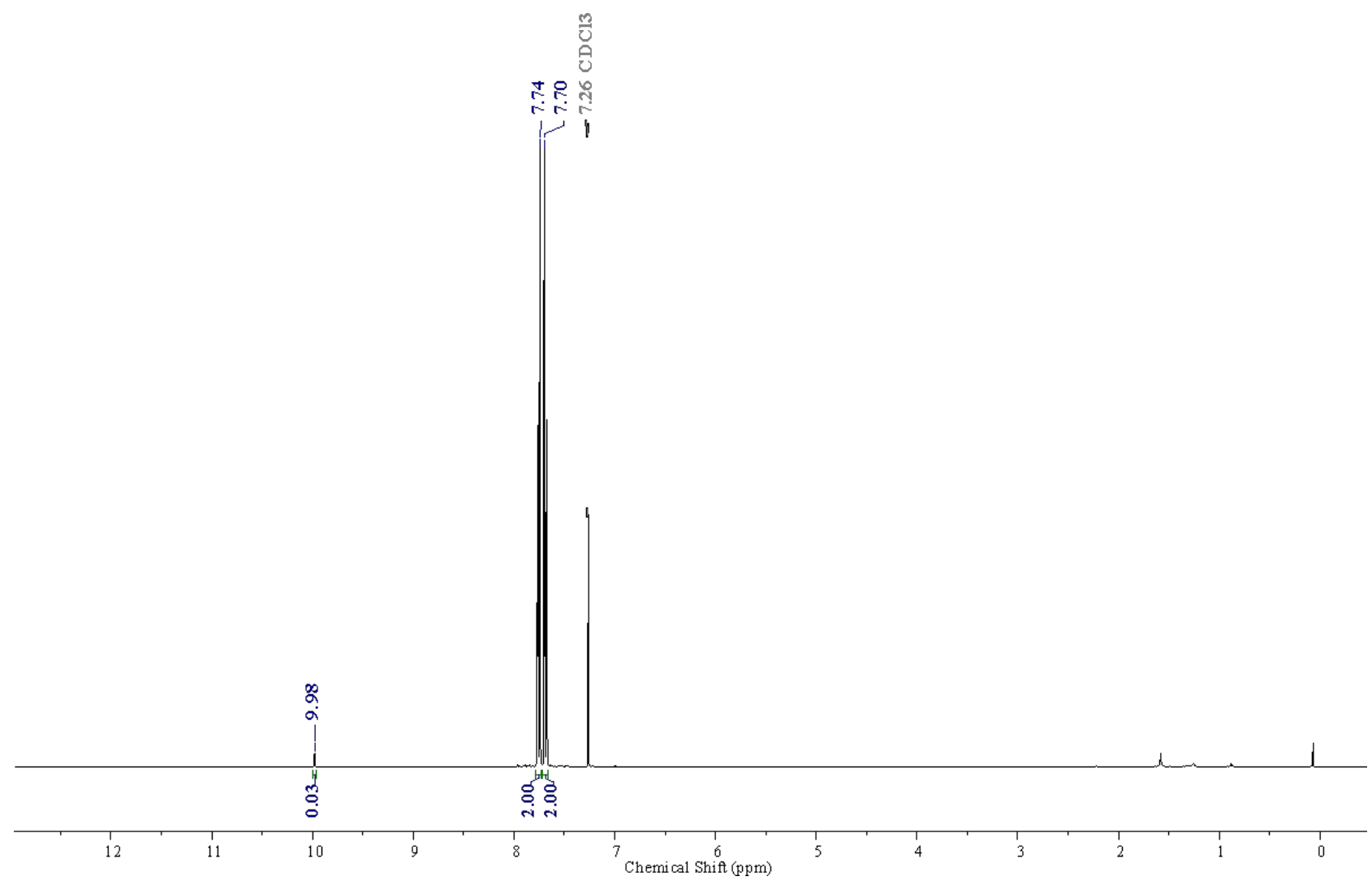




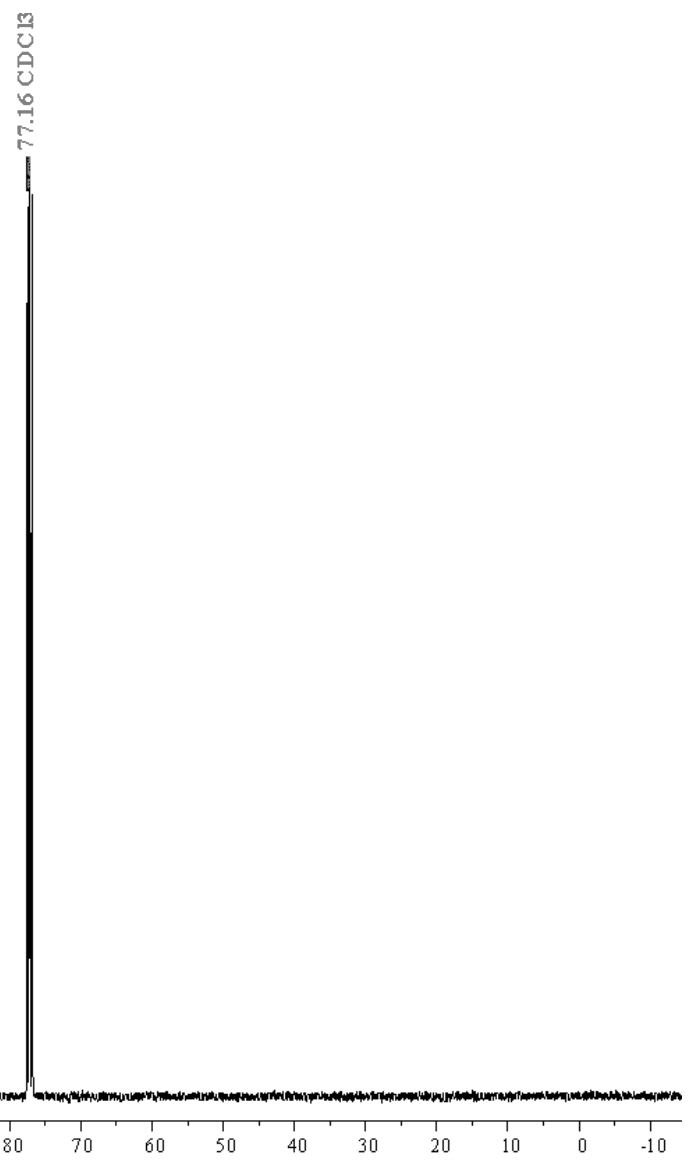

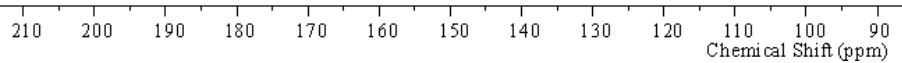




\section{3,5-Dibromobenzaldehyde-d}<smiles>[2H]C(=O)c1cc(Br)cc(Br)c1</smiles>

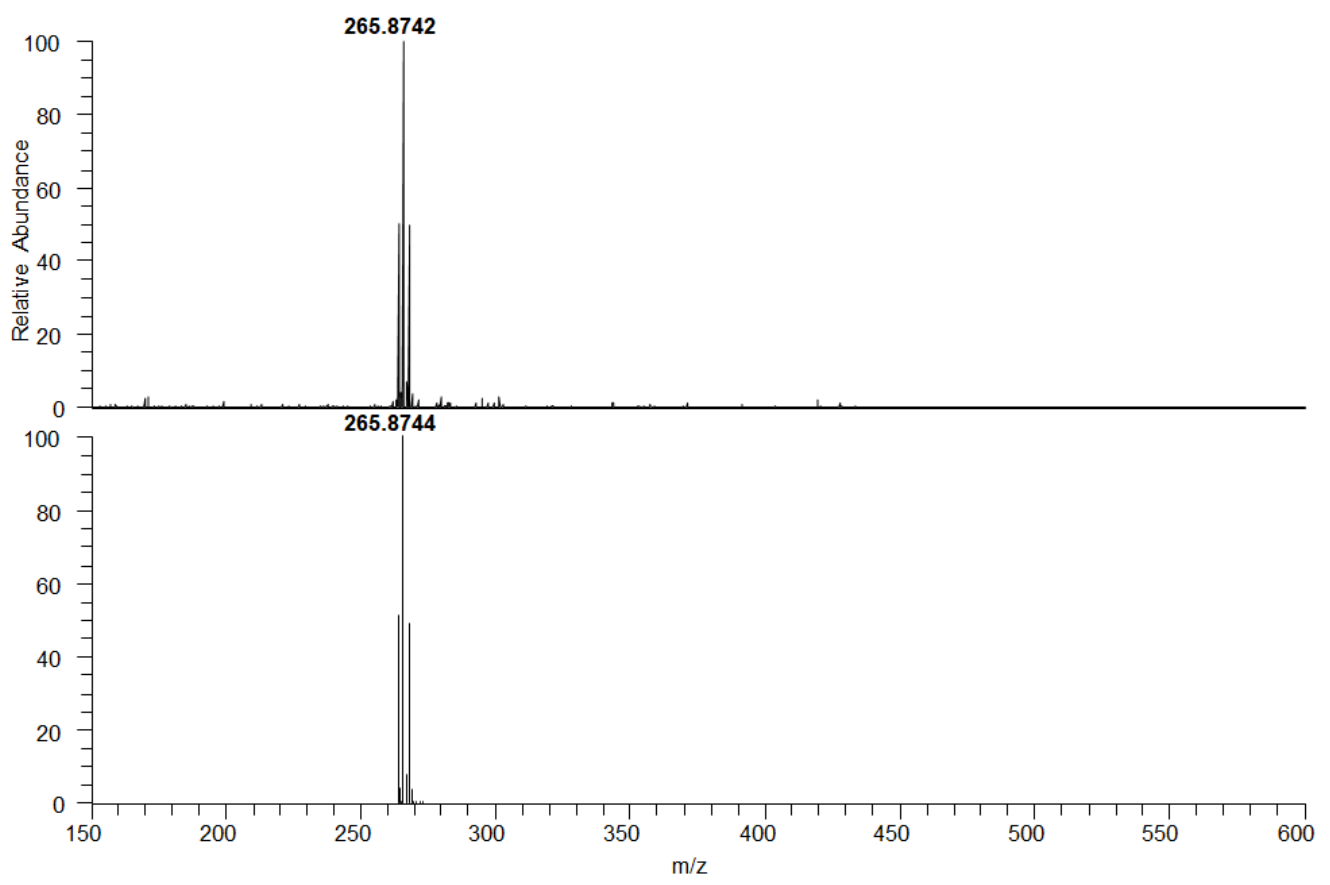

NL: 5.25E6

ilz-031

$1 \# 20200915145608$ RT: 0.01 AV: $1 \mathrm{~T}$ FTMS + p ESI Full lock

150.0000-600.0000]

$\mathrm{C}_{7} \mathrm{H}_{4} \mathrm{DBr} 2 \mathrm{O}$

$\mathrm{C}_{7} \mathrm{H}_{4} \mathrm{D}_{1} \mathrm{Br}_{2} \mathrm{O}_{1}$ pa Chrg 1

Entry 1: NMR for Crude reaction mixture SCG-318-E 1-16hr-D 1-50sec

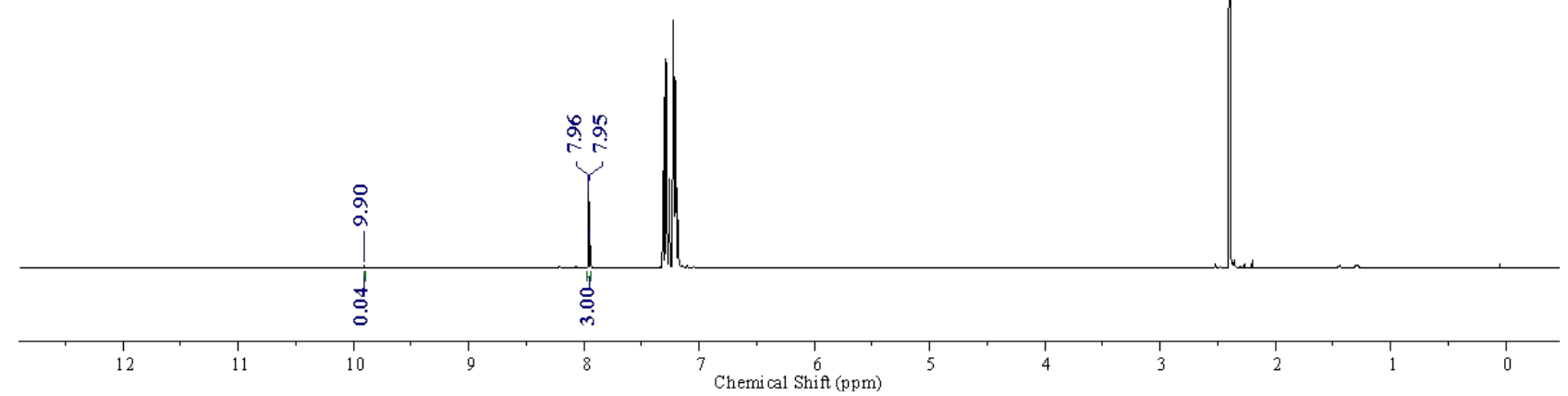


Entry 1': NMR for Crude reaction mixture, Duplicate

SCG-318-E2-16hr-D 1-50sec

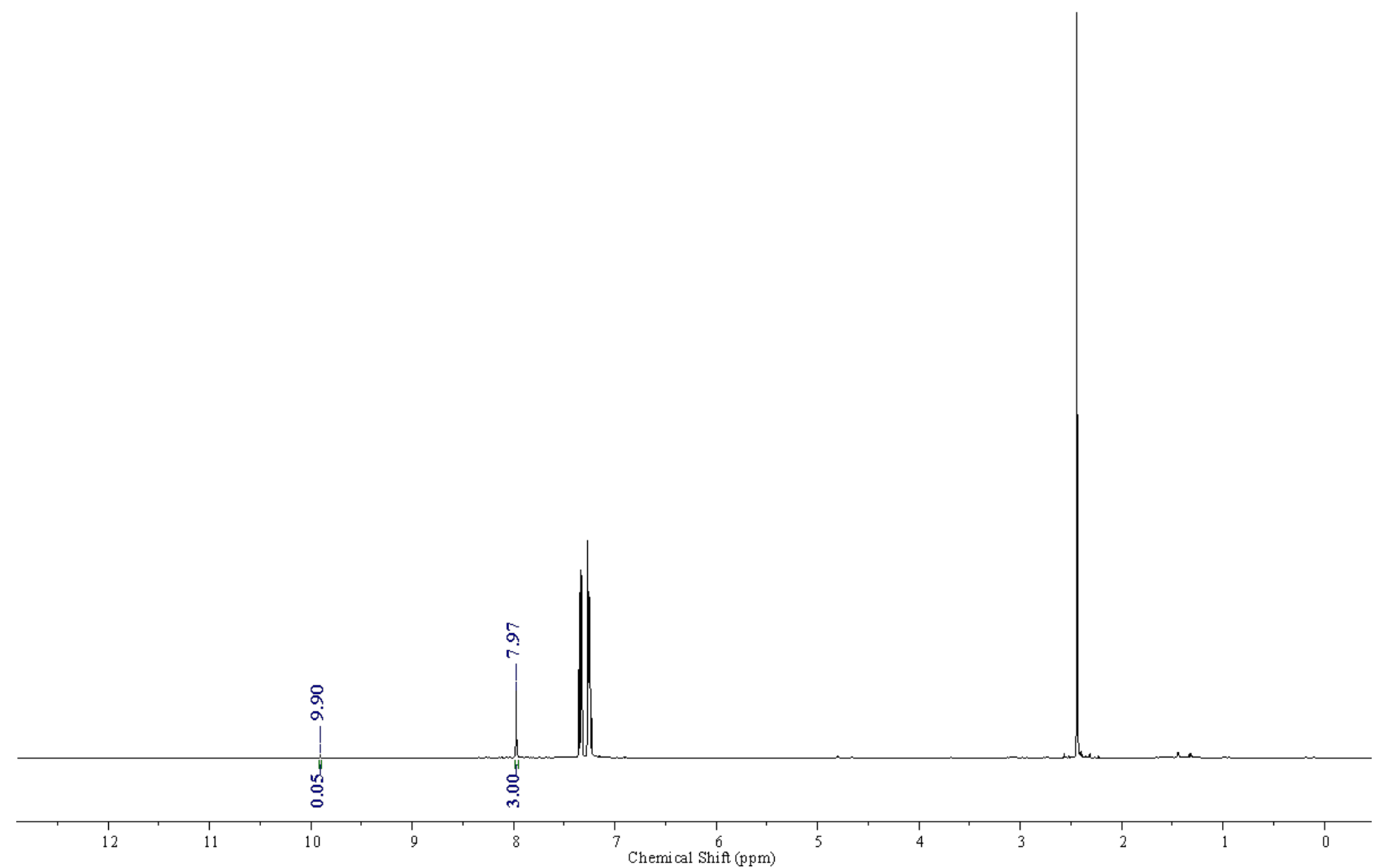

NMR after purification:

SCG-318-E1-16hr-D1-50sec-AC

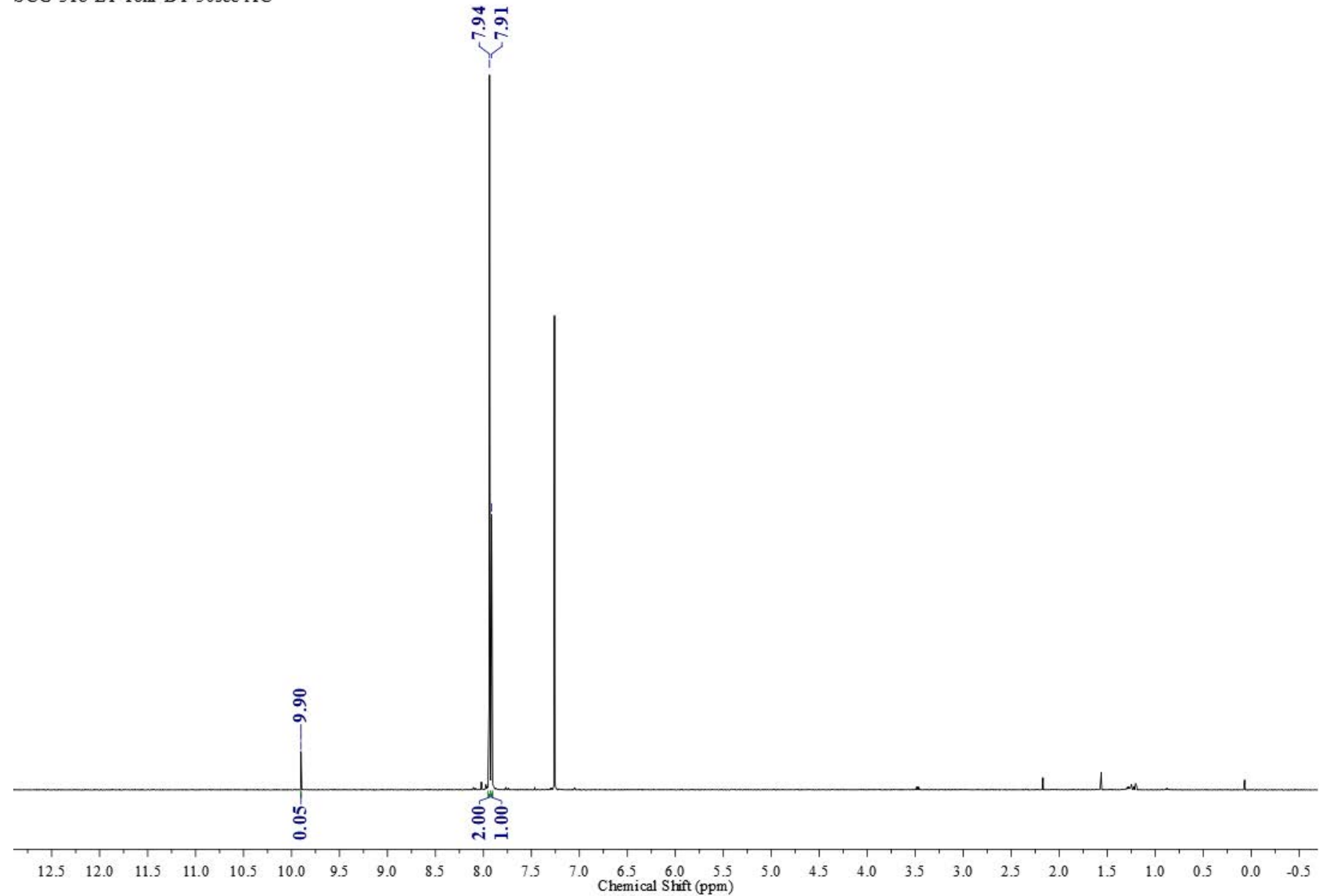




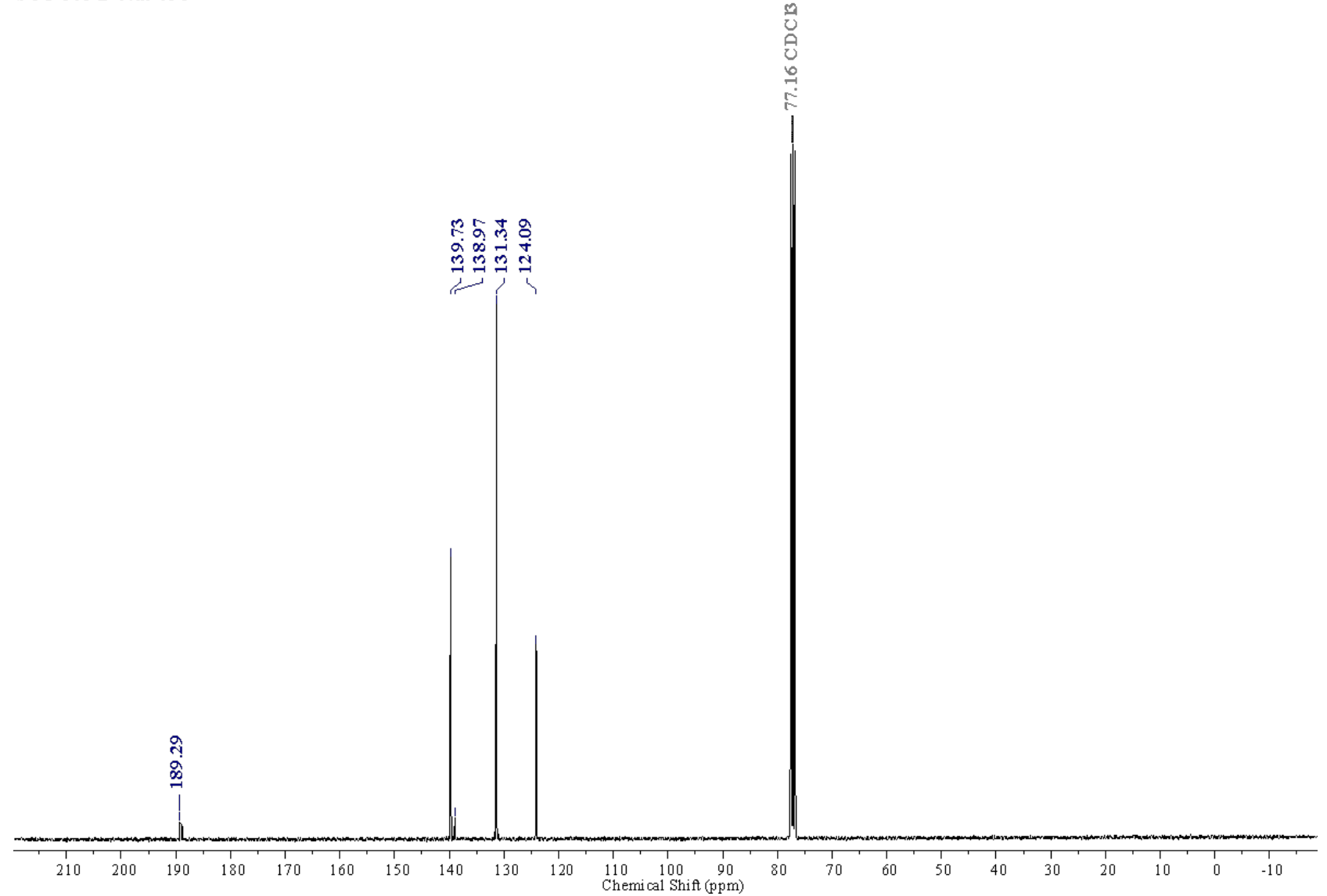


25. 3-lodobenzaldehyde-d<smiles>[2H]C(=O)c1cccc(I)c1</smiles>

Entry 1: NMR for Crude reaction mixture SCG-312-A1-16hr-D 1-50sec

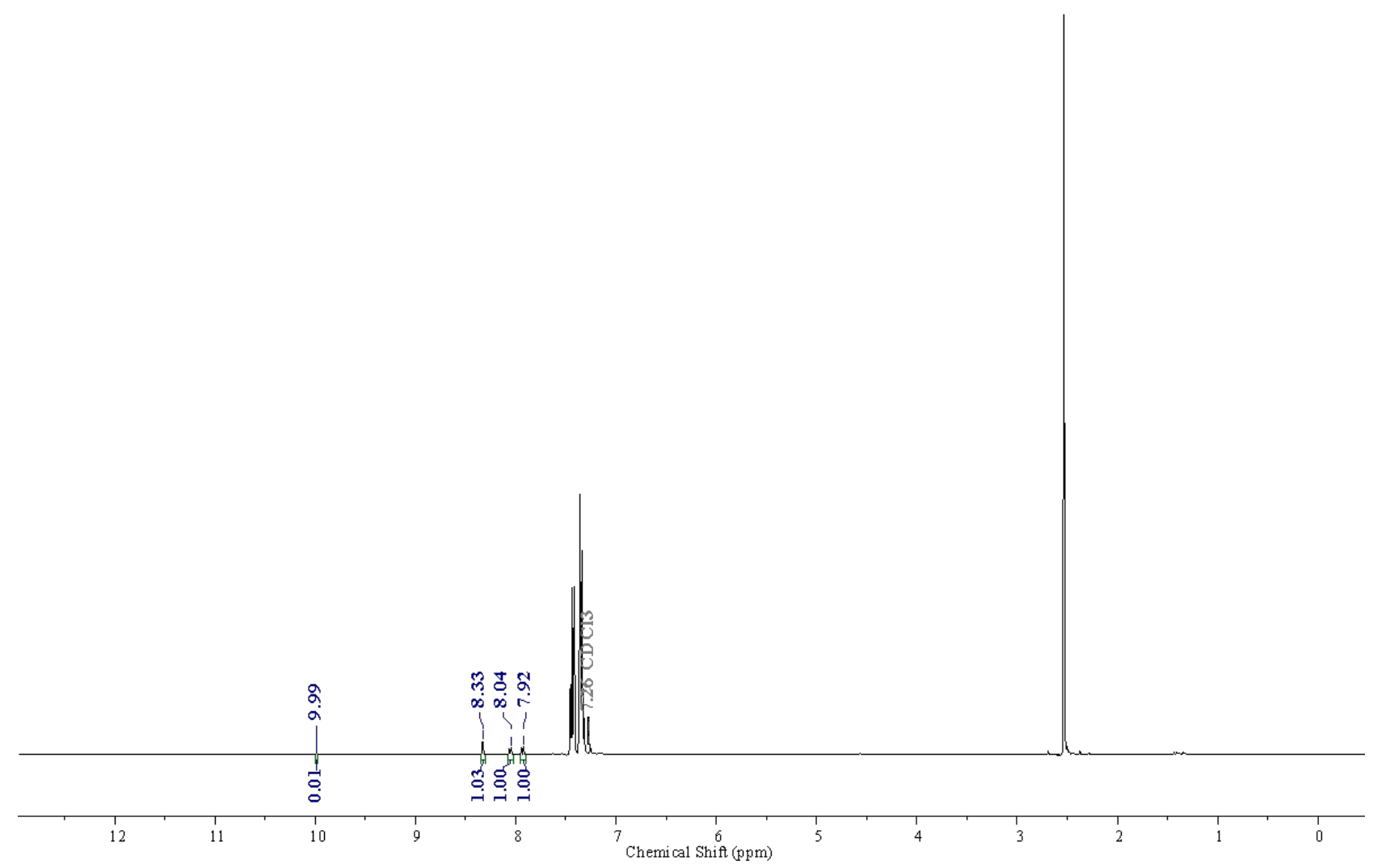


Entry 1': NMR for Crude reaction mixture, Duplicate

SCG-312-A2-16hr-D 1-50sec

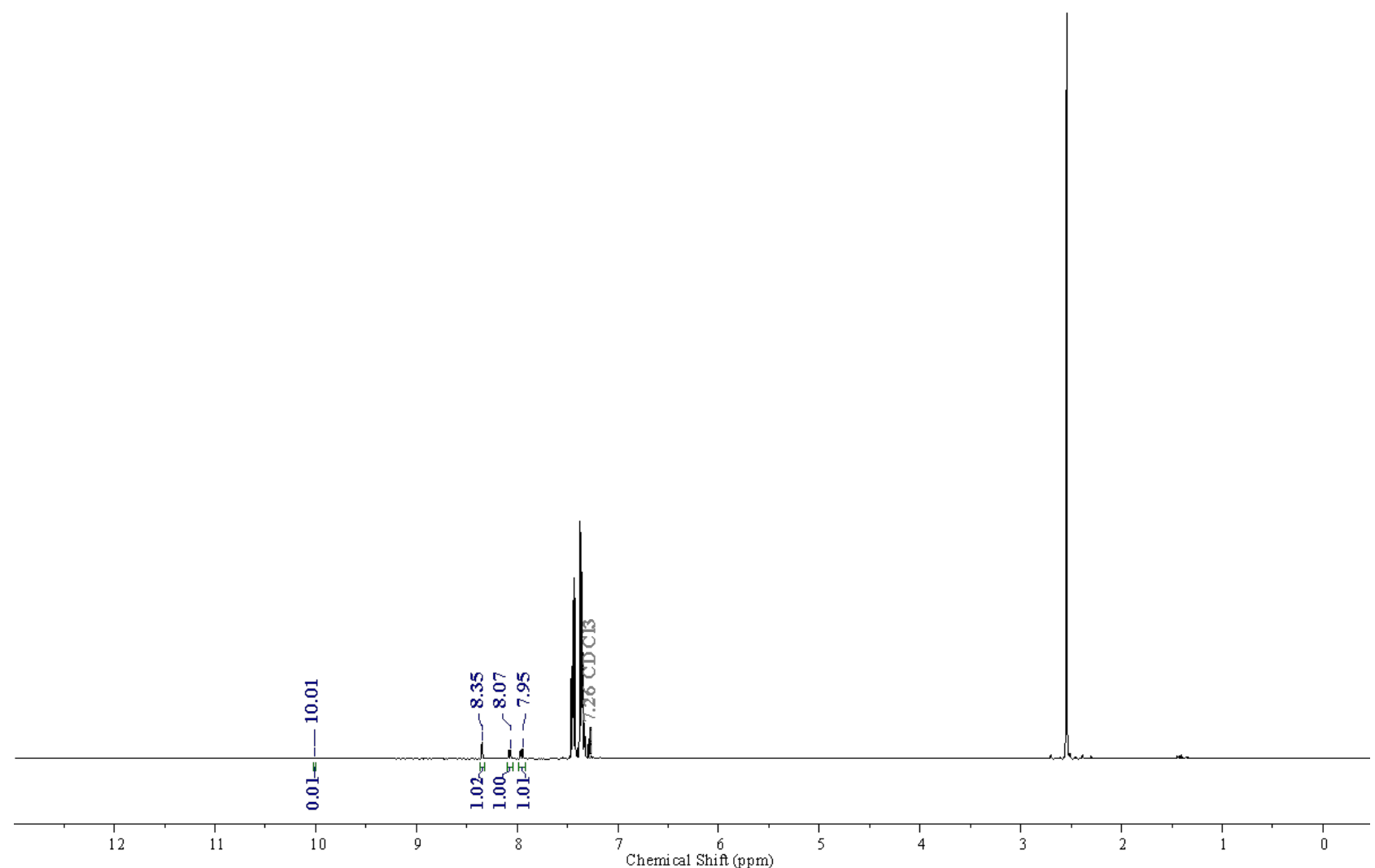

NMR after purification:

SCG-312-A4-16hr-D 1-50sec-A C-07052019

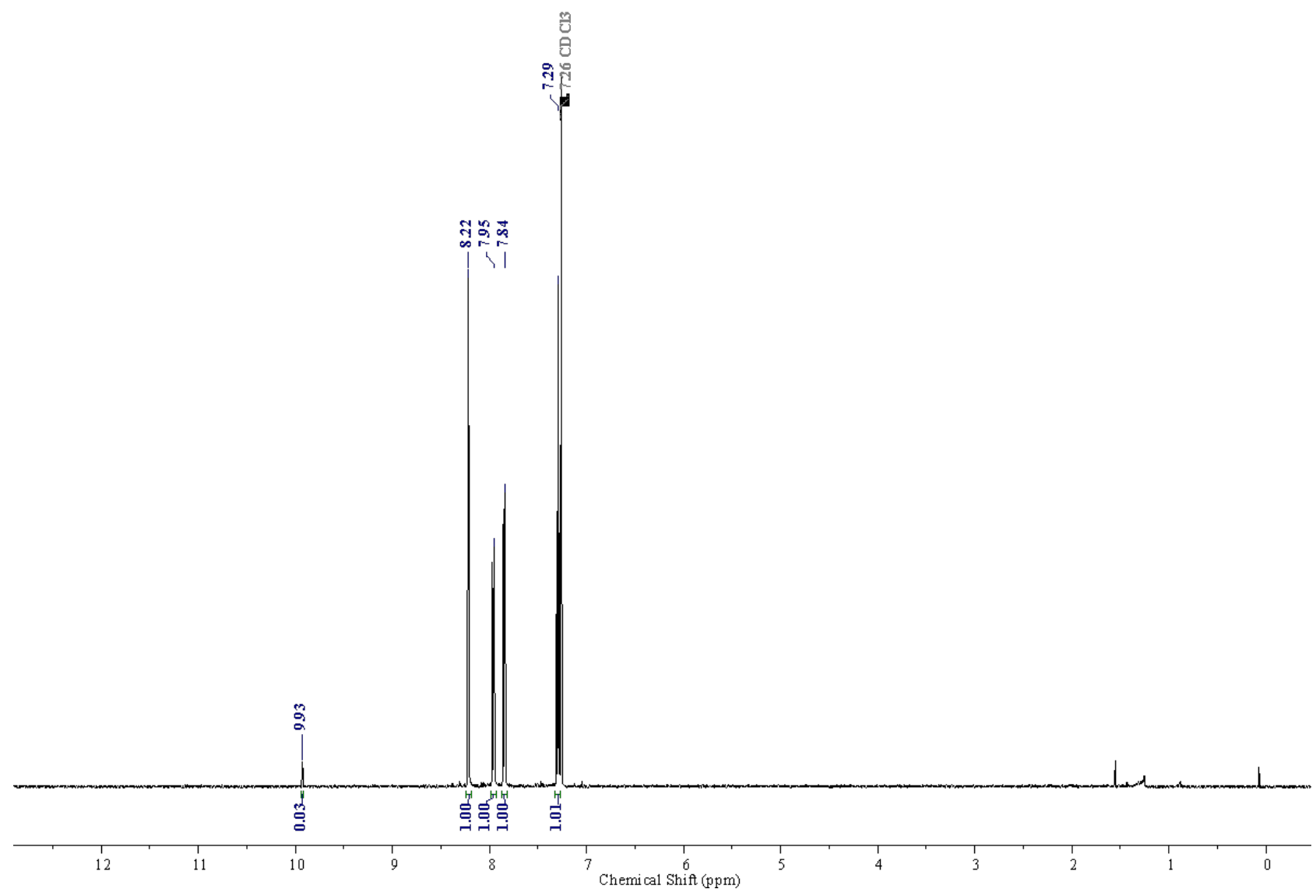




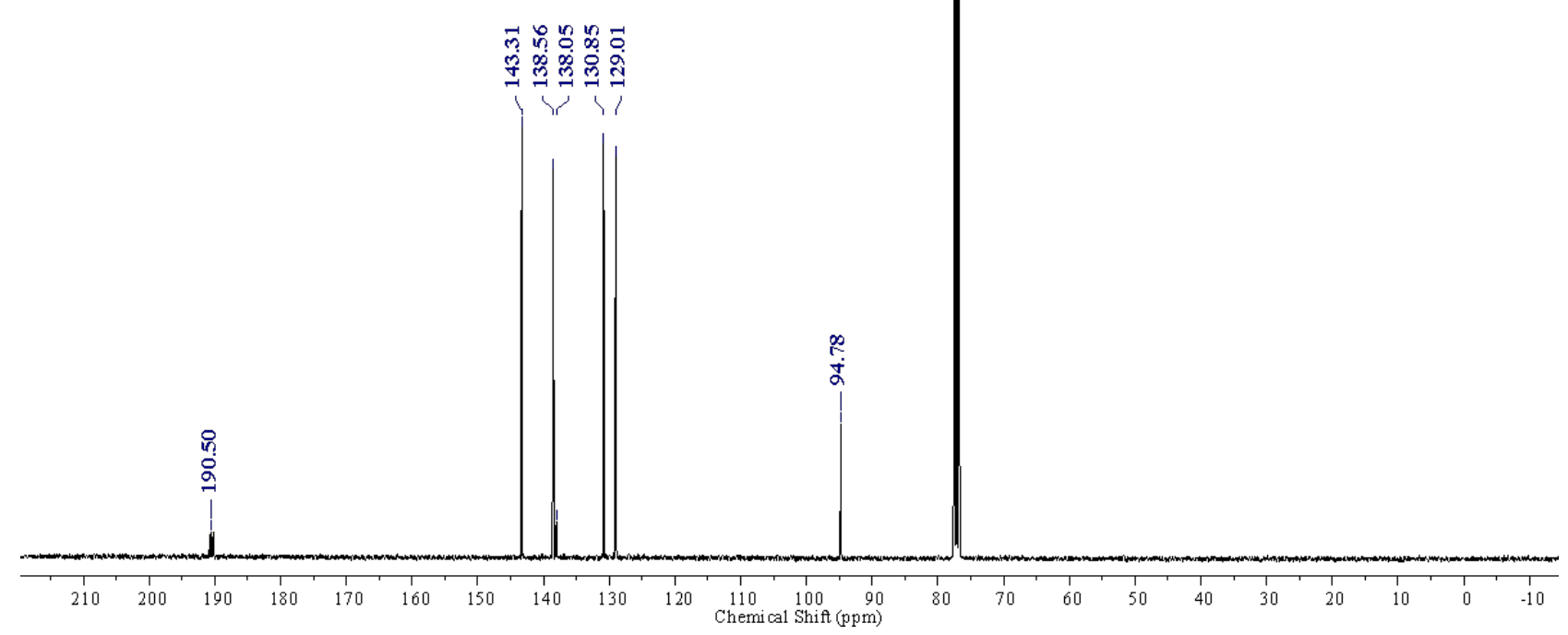


26. 4-lodobenzaldehyde-d<smiles>[2H]C(=O)c1ccc(I)cc1</smiles>

Entry 1: NMR for Crude reaction mixture SCG-312-M2-16hr-D 1-50sec.1. fid

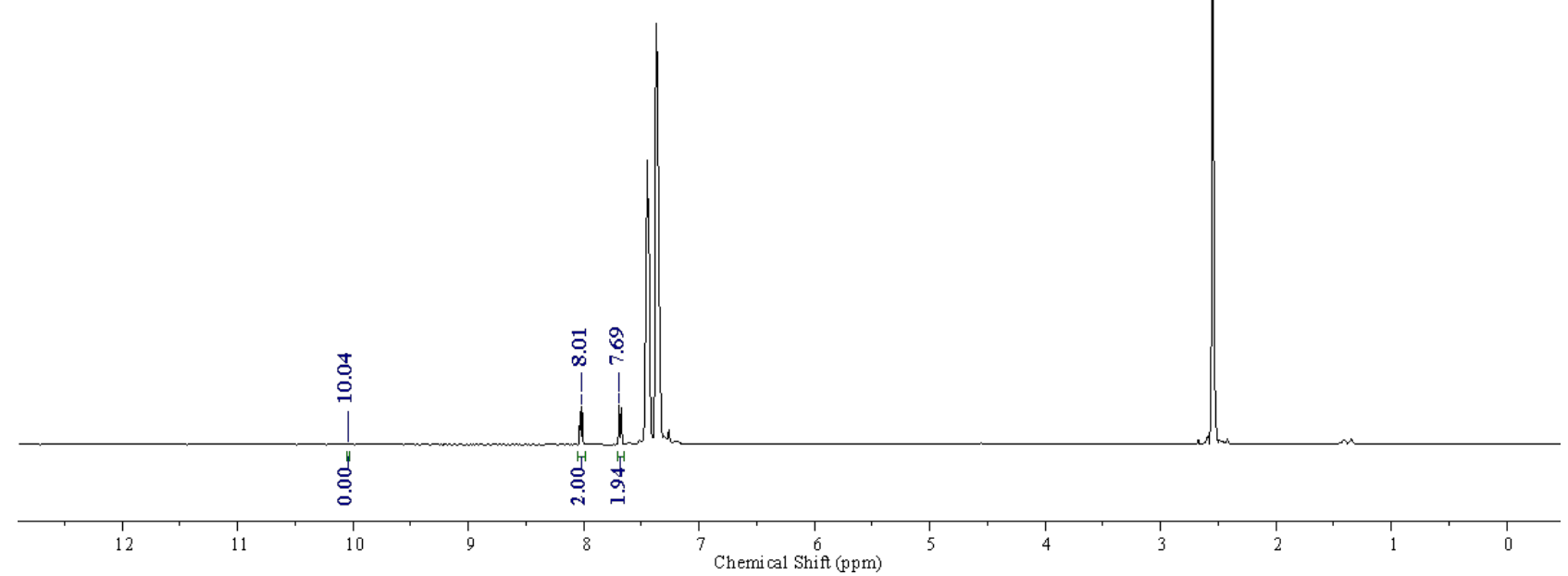


Entry 1': NMR for Crude reaction mixture, Duplicate

SCG-312-M1-16hr-D 1-50sec.1.fid

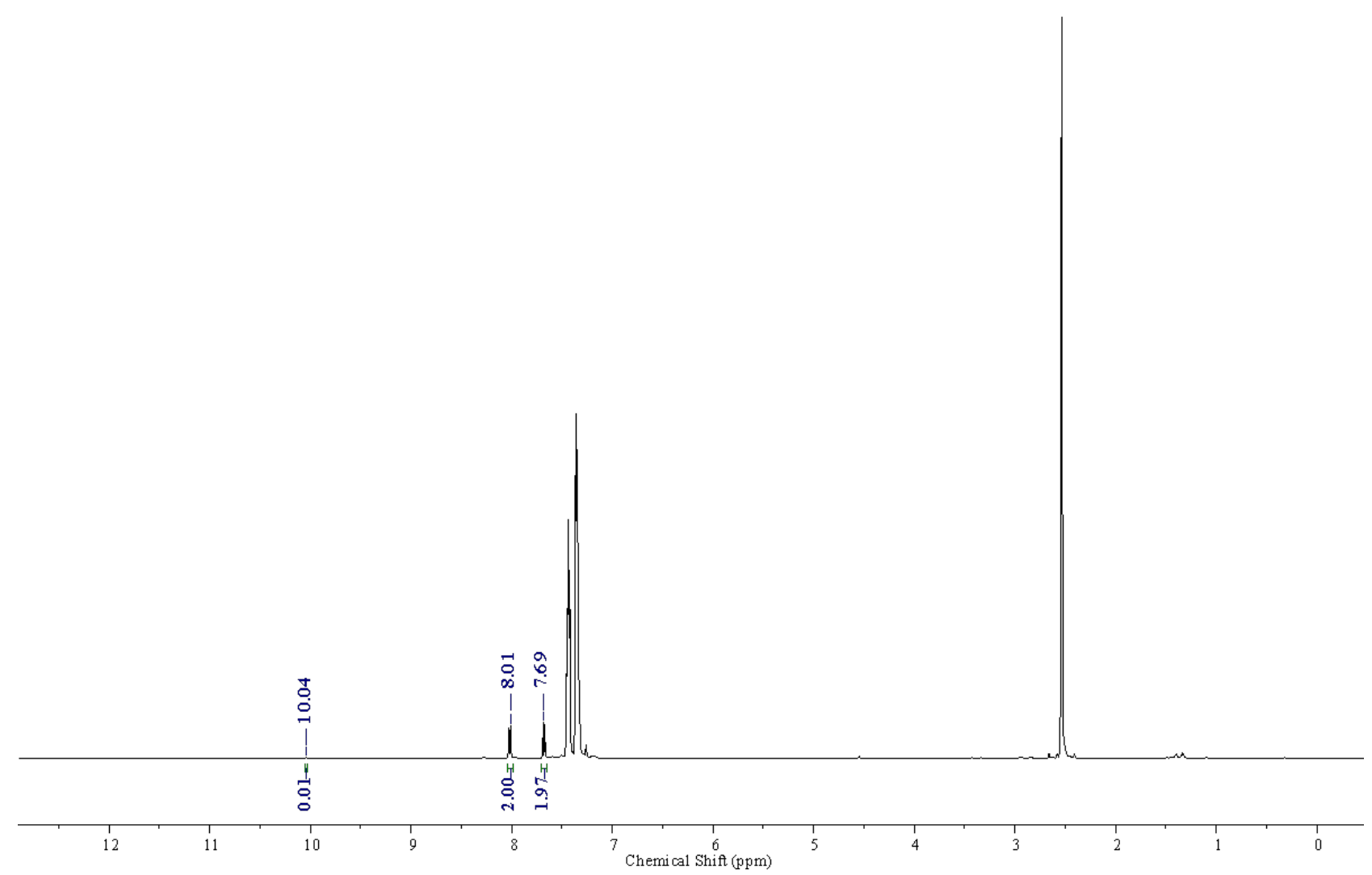

NMR after purification:

SCG-312-M1-16hr-D 1-50sec-AC.1.fid

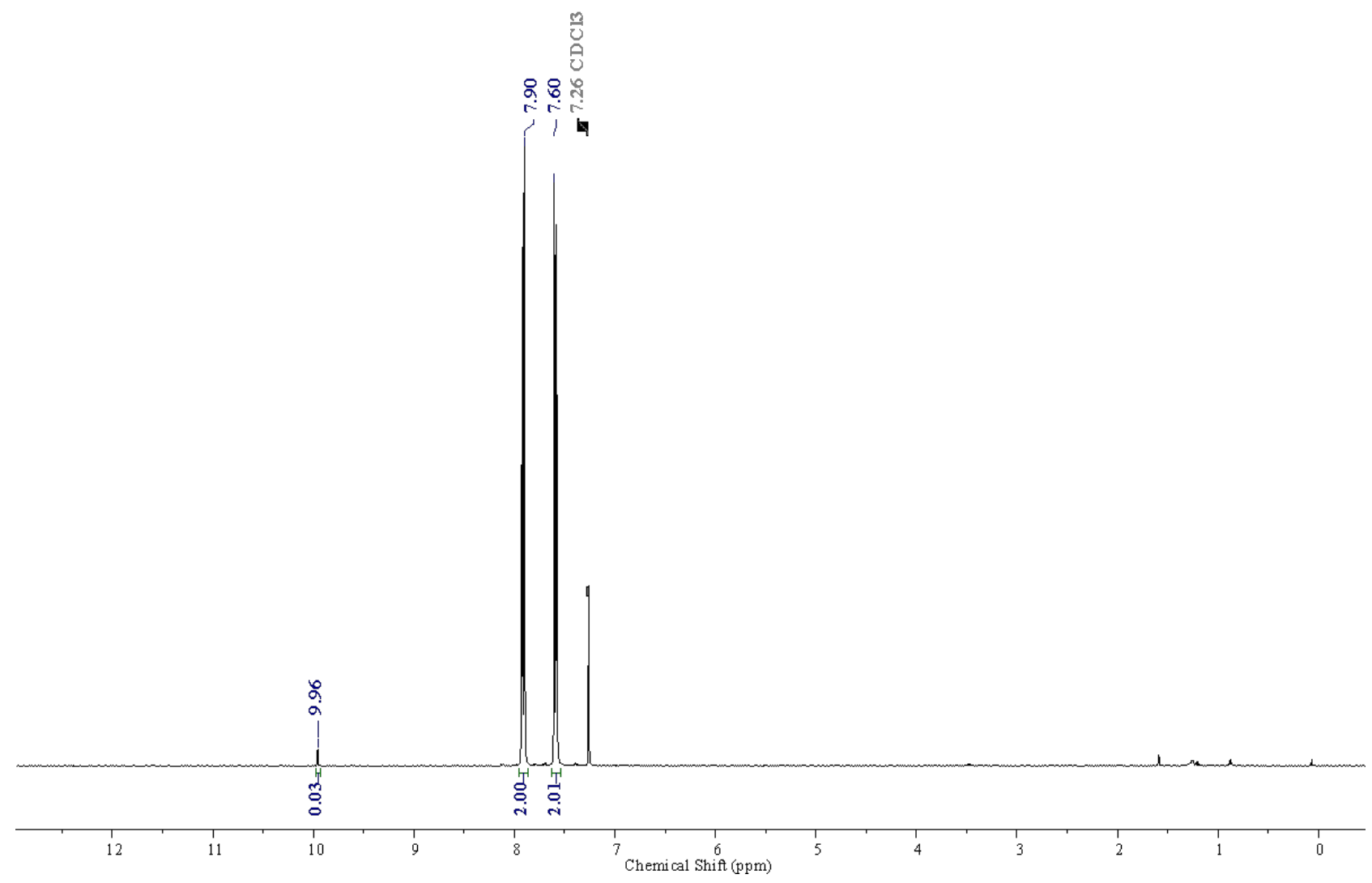


นึ:

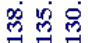

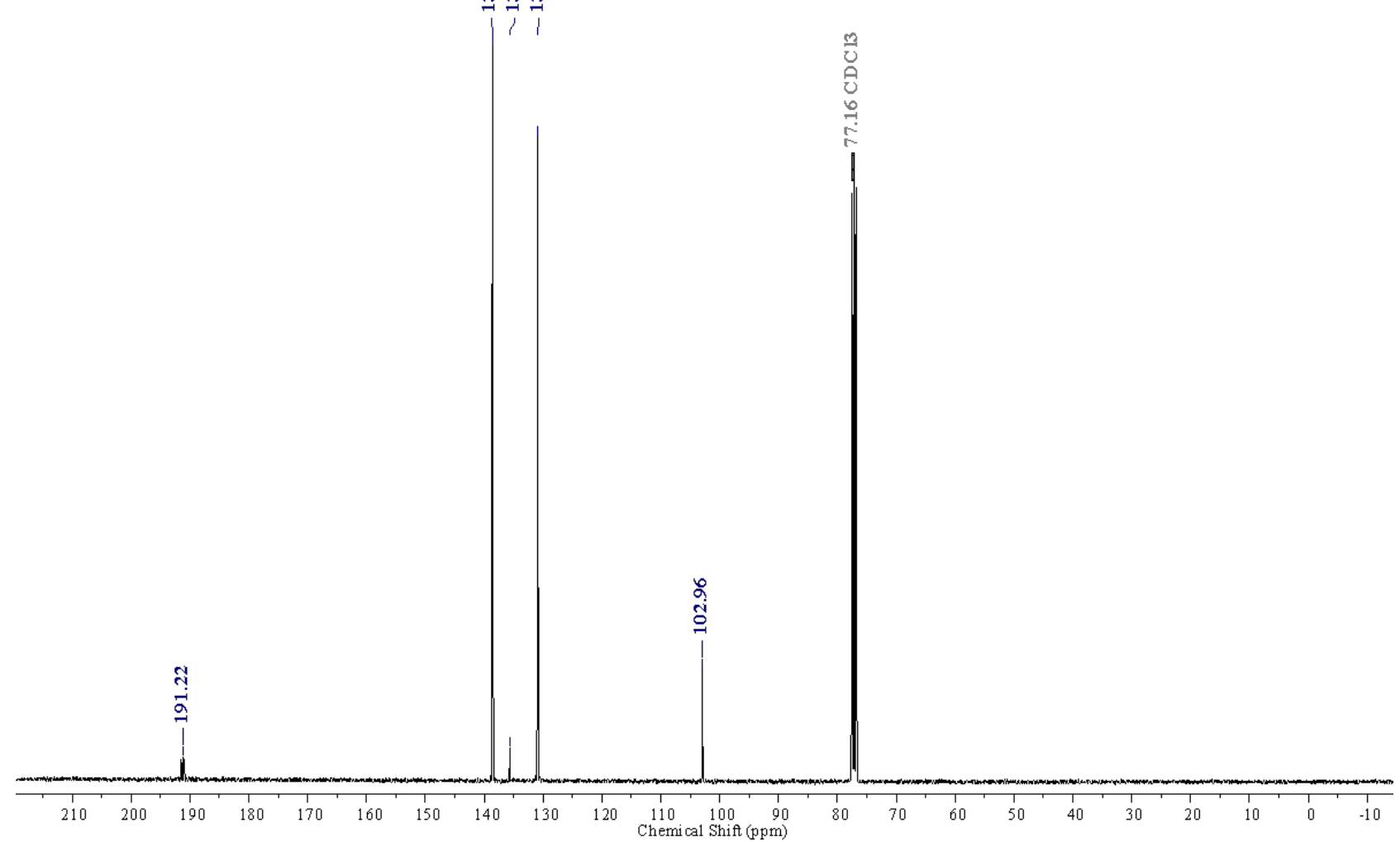


27. Methyl 3-formylbenzoate-d<smiles>[2H]C(=O)c1cccc(C(=O)OC)c1</smiles>

Entry 1: NMR for Crude reaction mixture SCG-312-C1-16hr-D 1-50sec

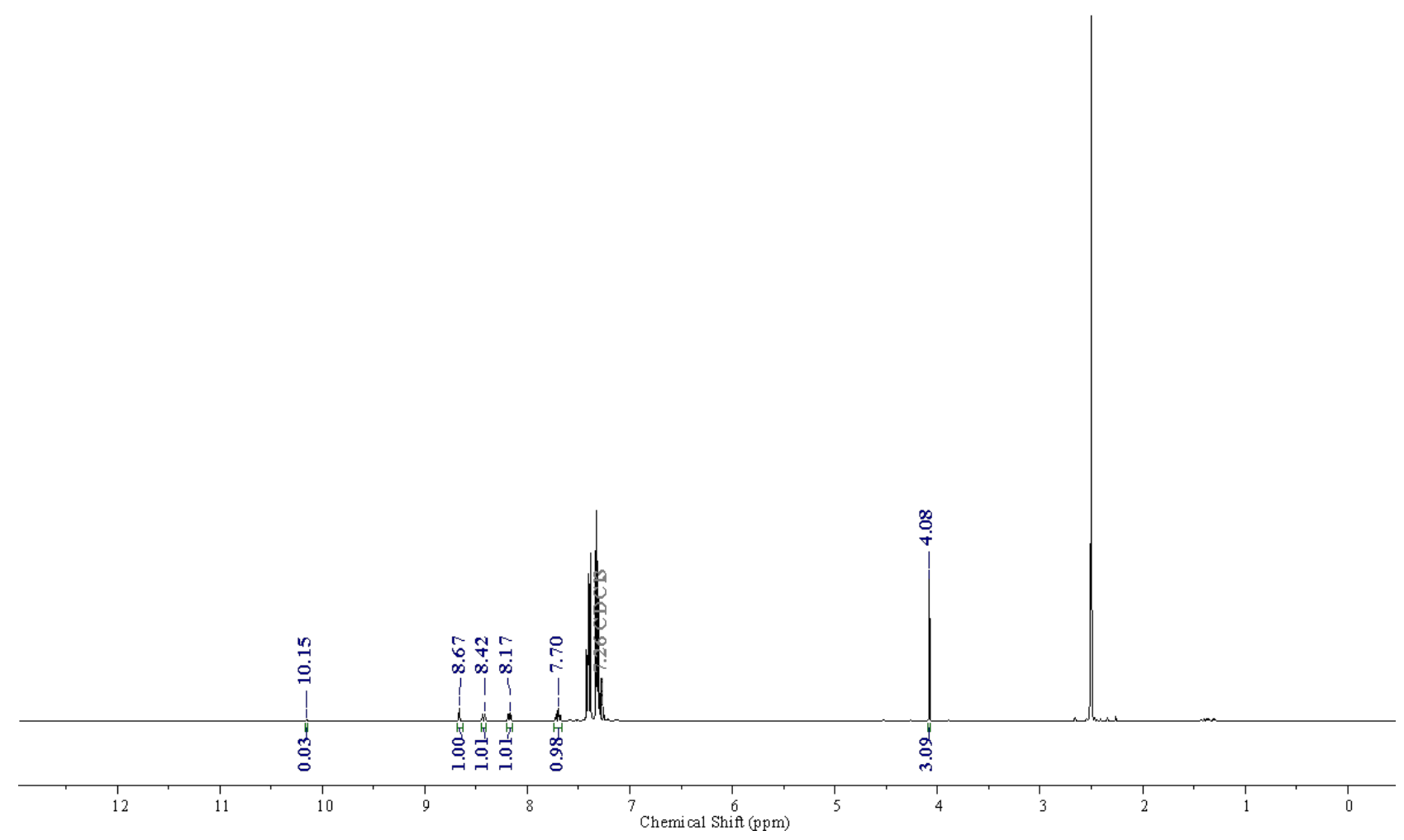


Entry 1': NMR for Crude reaction mixture, Duplicate

SCG-312-C2-16hr-D 1-50sec

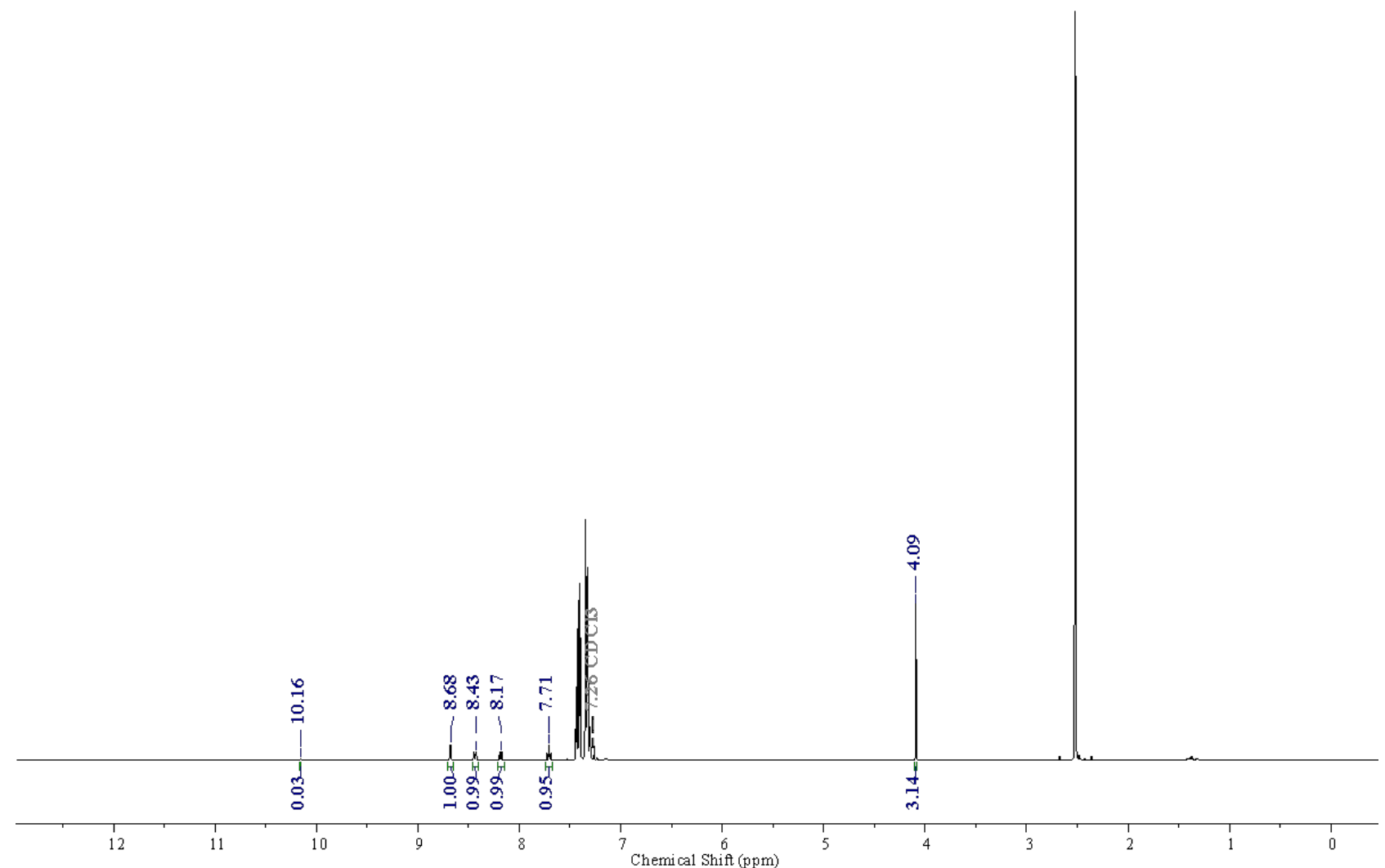

NMR after purification:

SCG-312-C4-16hr-D 1-50sec-AC

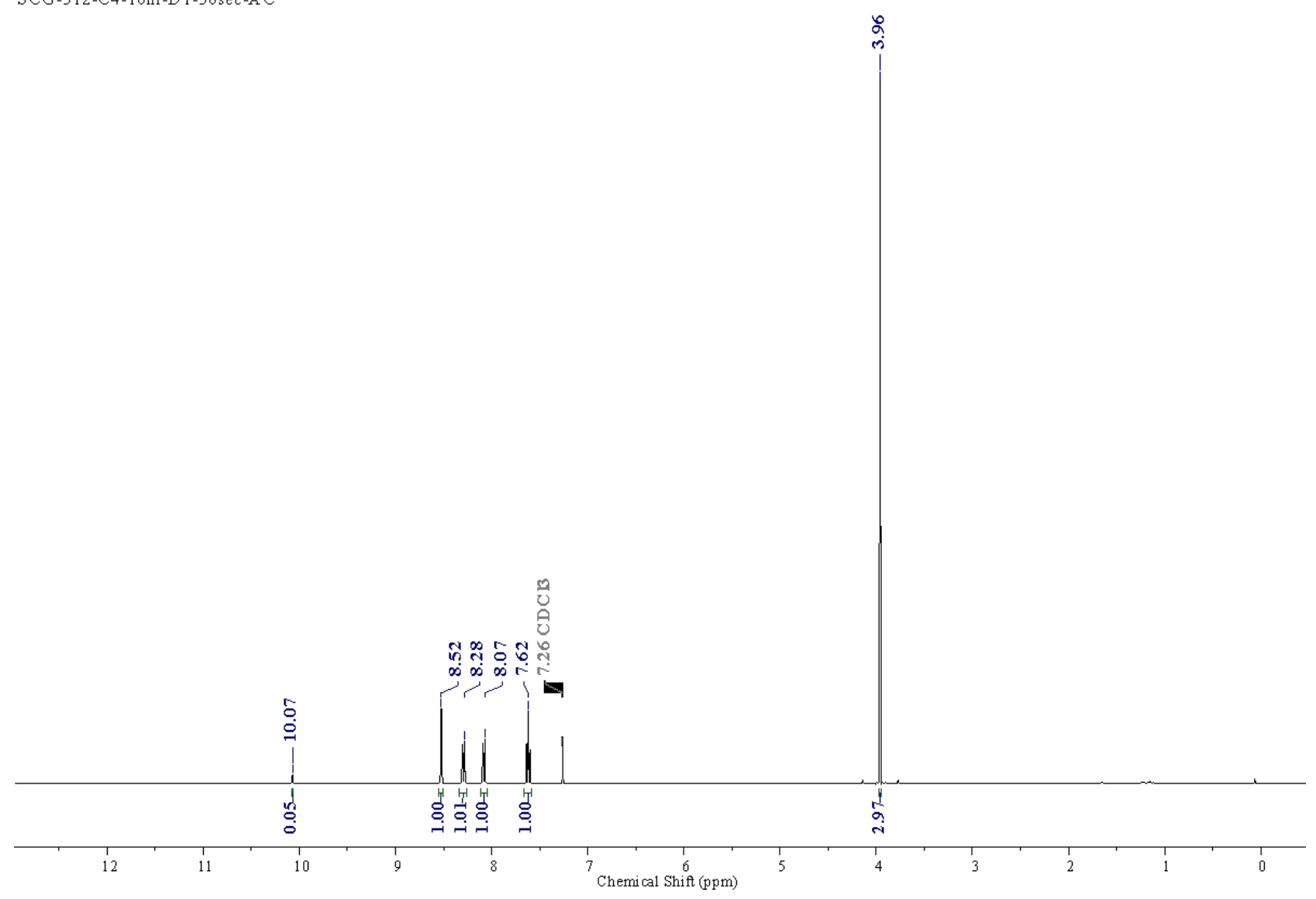




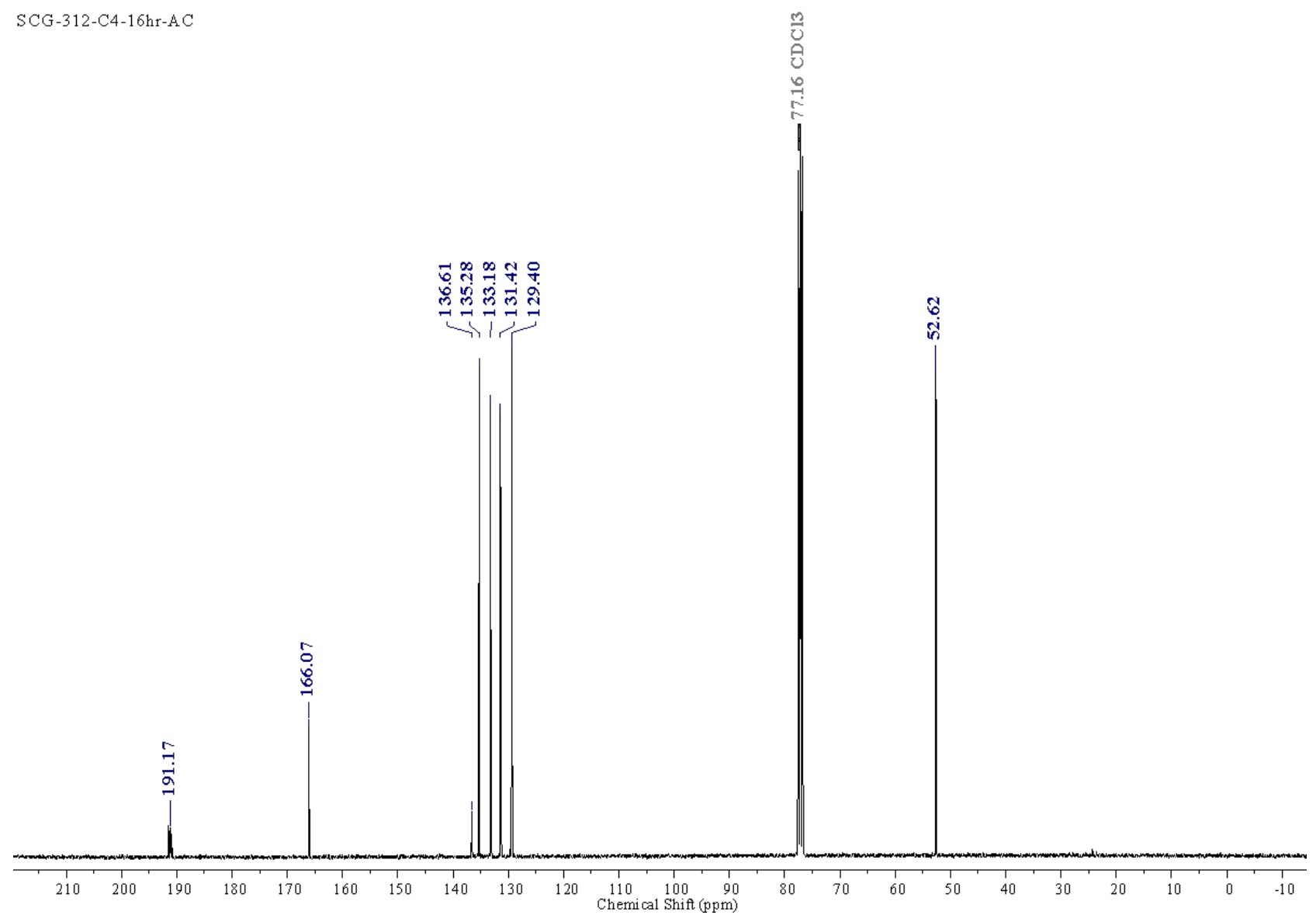


28. Methyl 4-formylbenzoate-d<smiles>[2H]C(=O)c1ccc(C(=O)OC)cc1</smiles>

Entry 1: NMR for Crude reaction mixture SCG-312-D 1-16hr-D 1-50sec.1.fid

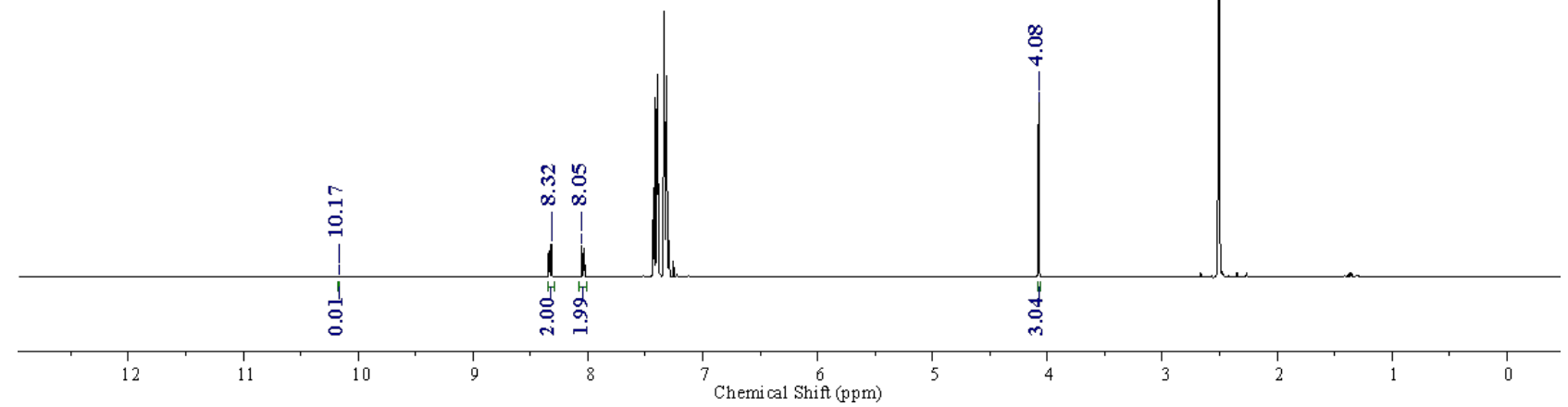


Entry 1': NMR for Crude reaction mixture, Duplicate

SCG-312-D 2-16hr-D 1-50sec.1. fid

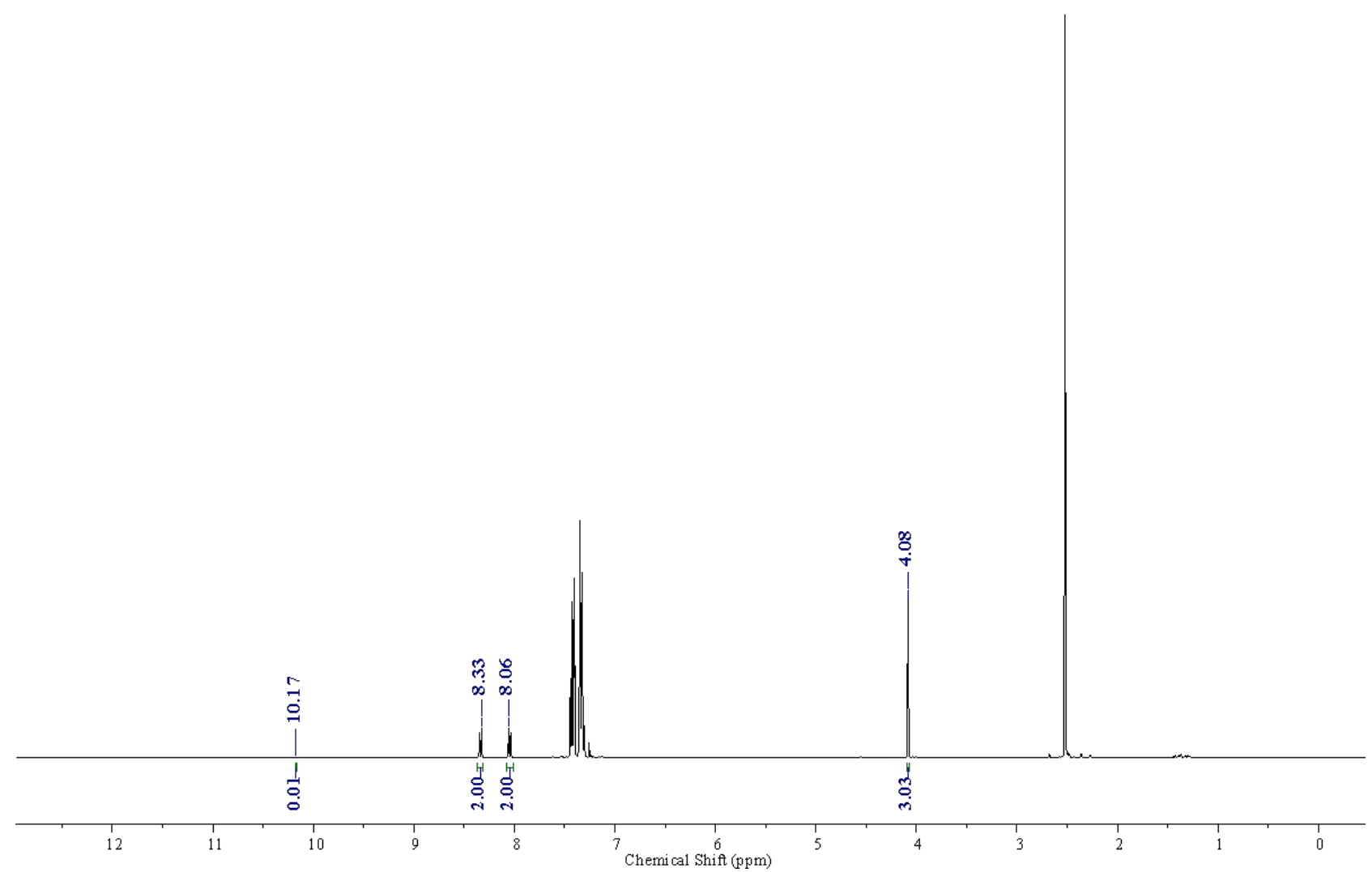

NMR after purification:

SCG-312-D 3-16ht- 1-50sec-AC 1 fid

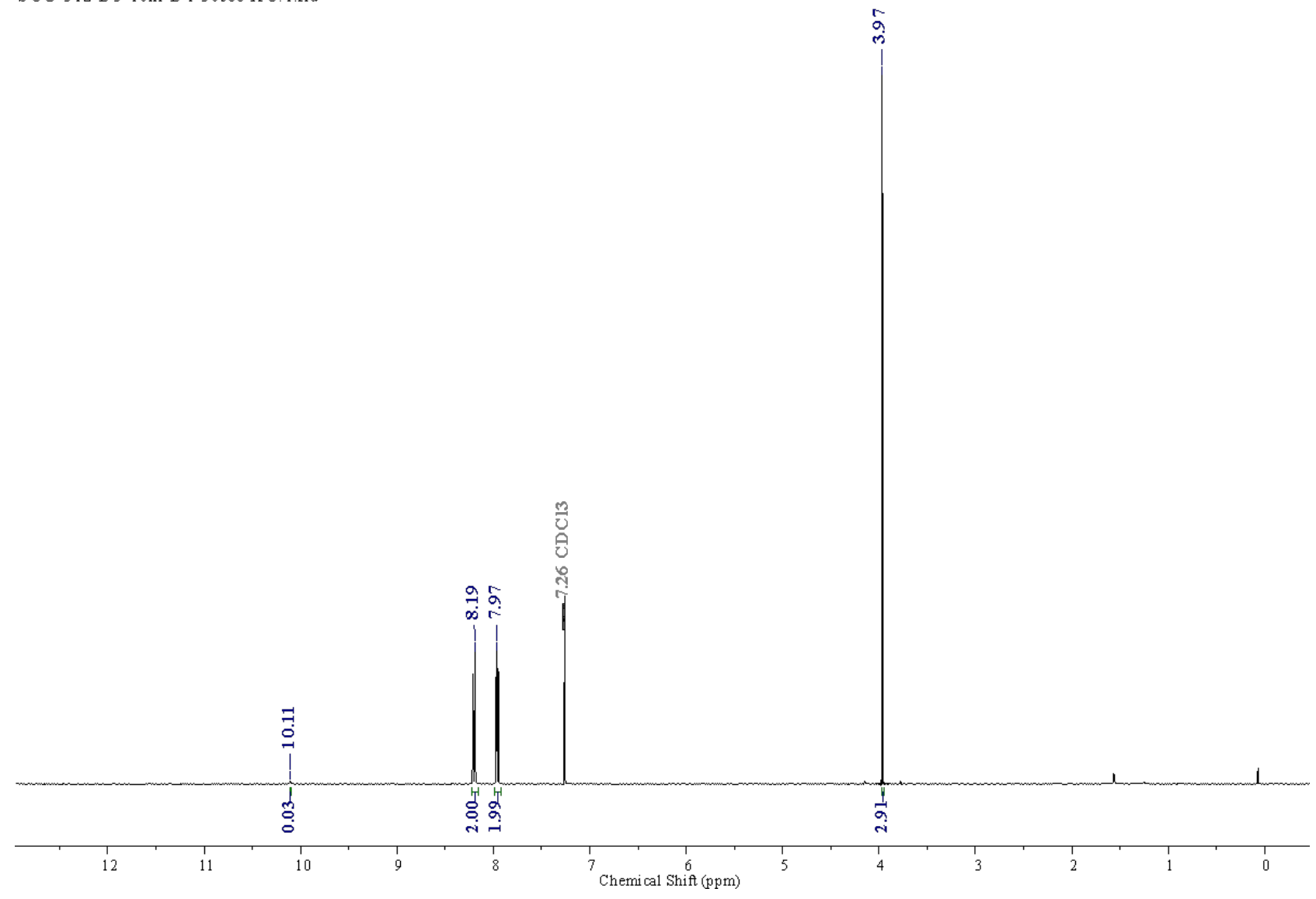




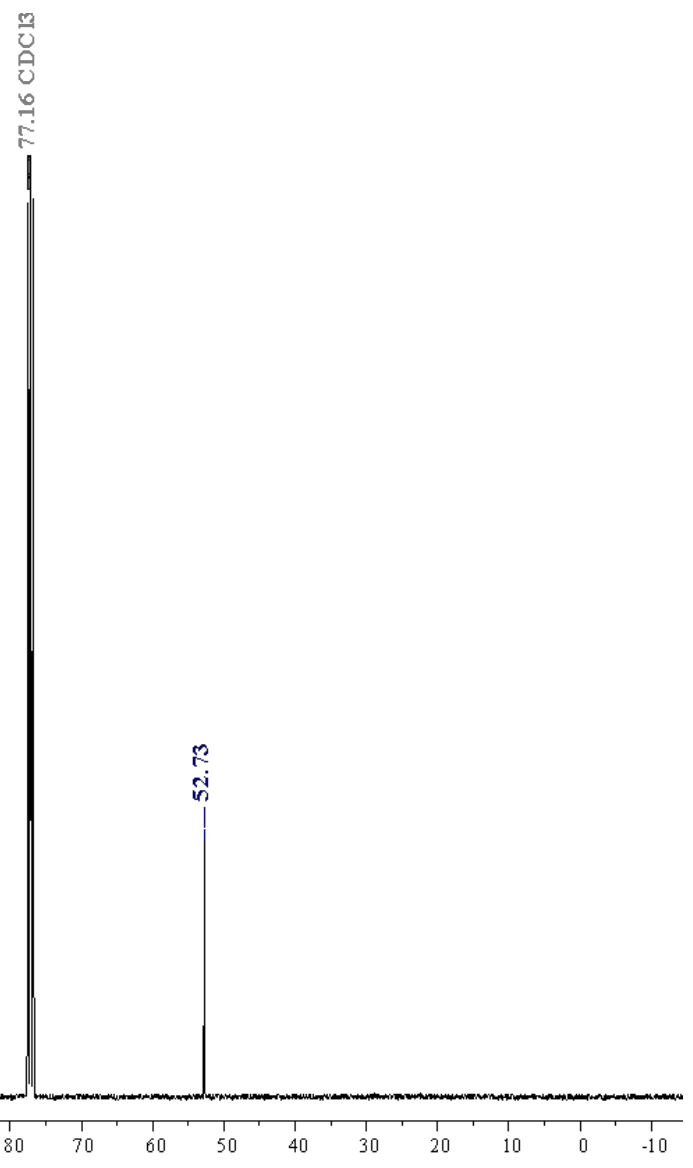


29. 3-(Trifluoromethyl)benzaldehyde-d<smiles>[2H]C(=O)c1cccc(C(F)(F)F)c1</smiles>

Entry 1: NMR for Crude reaction mixture SCG-319-B 3-16hr-D 1-50sec.1. fid

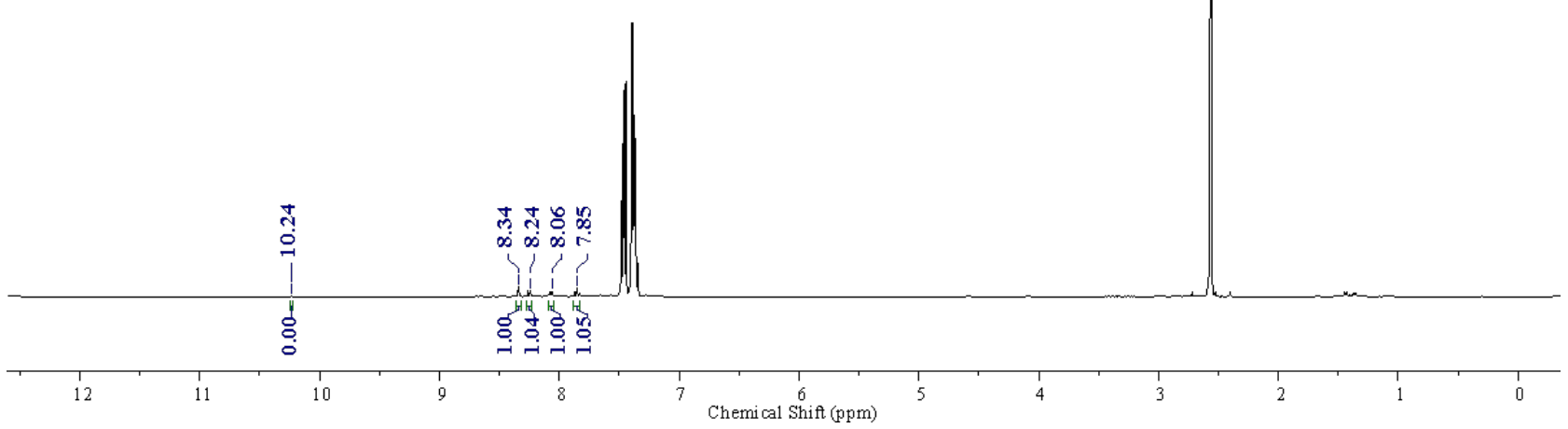


Entry 1': NMR for Crude reaction mixture, Duplicate

SCG-312-Q1-16hr-D 1-50sec.1.fid

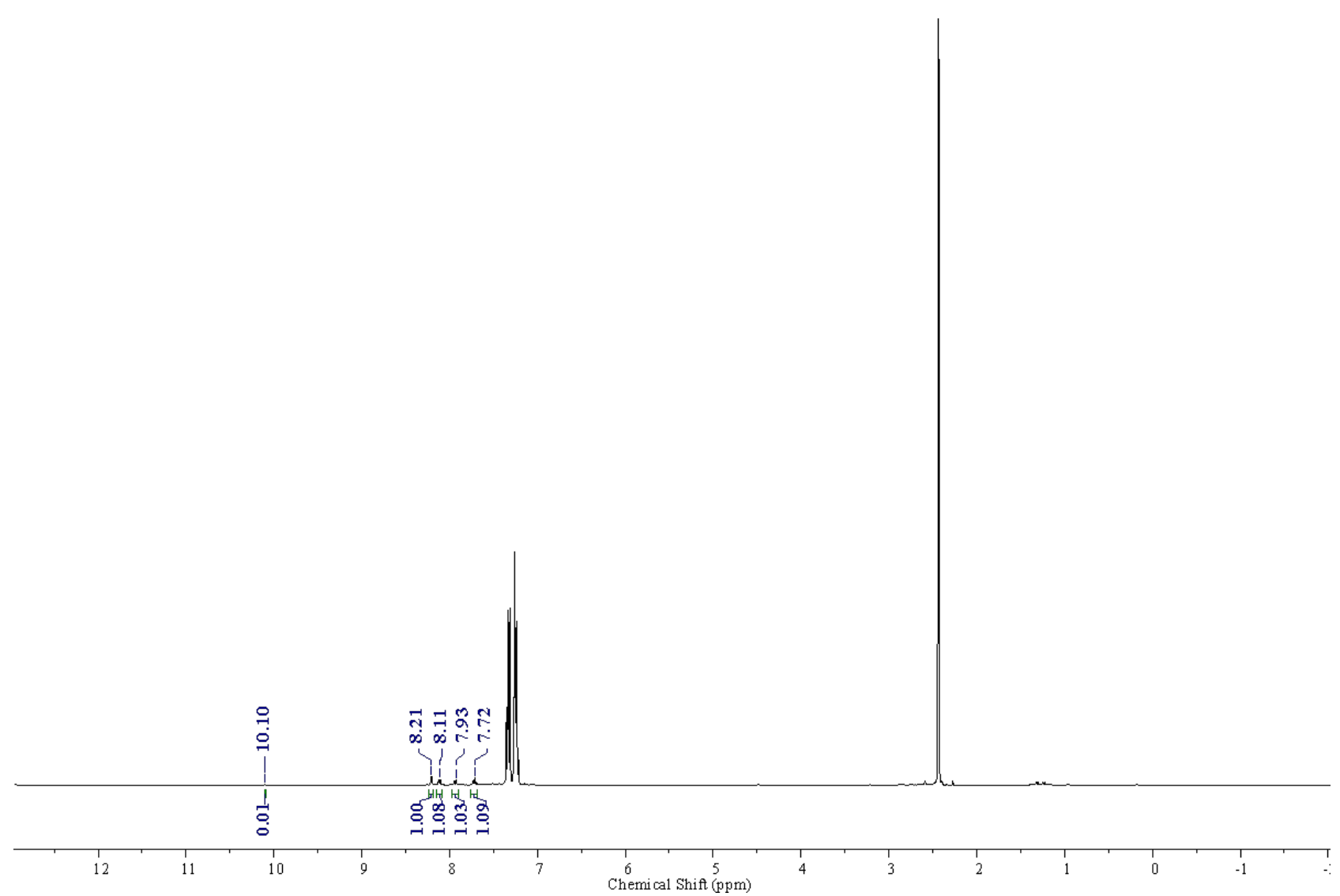

NMR after purification:

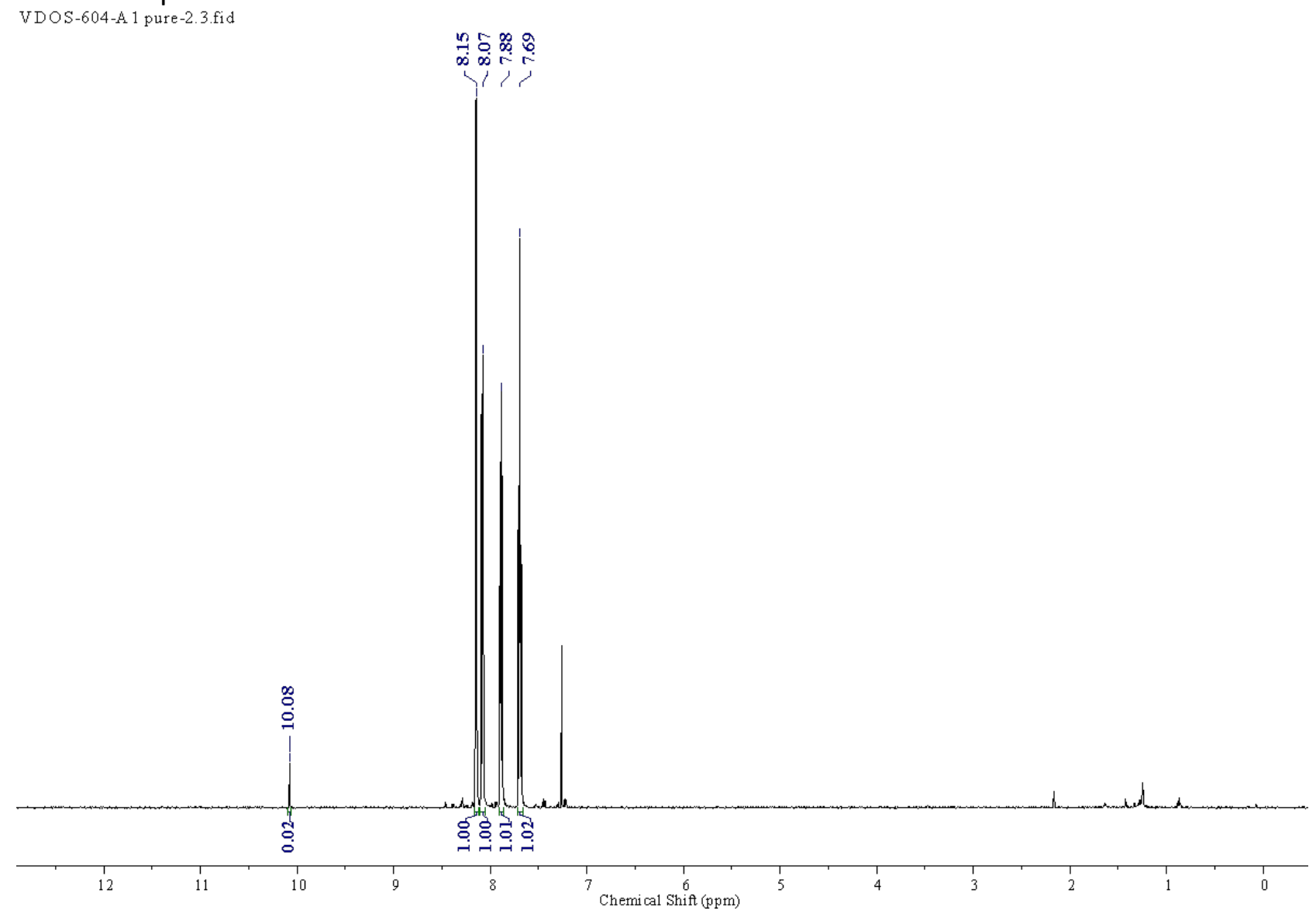



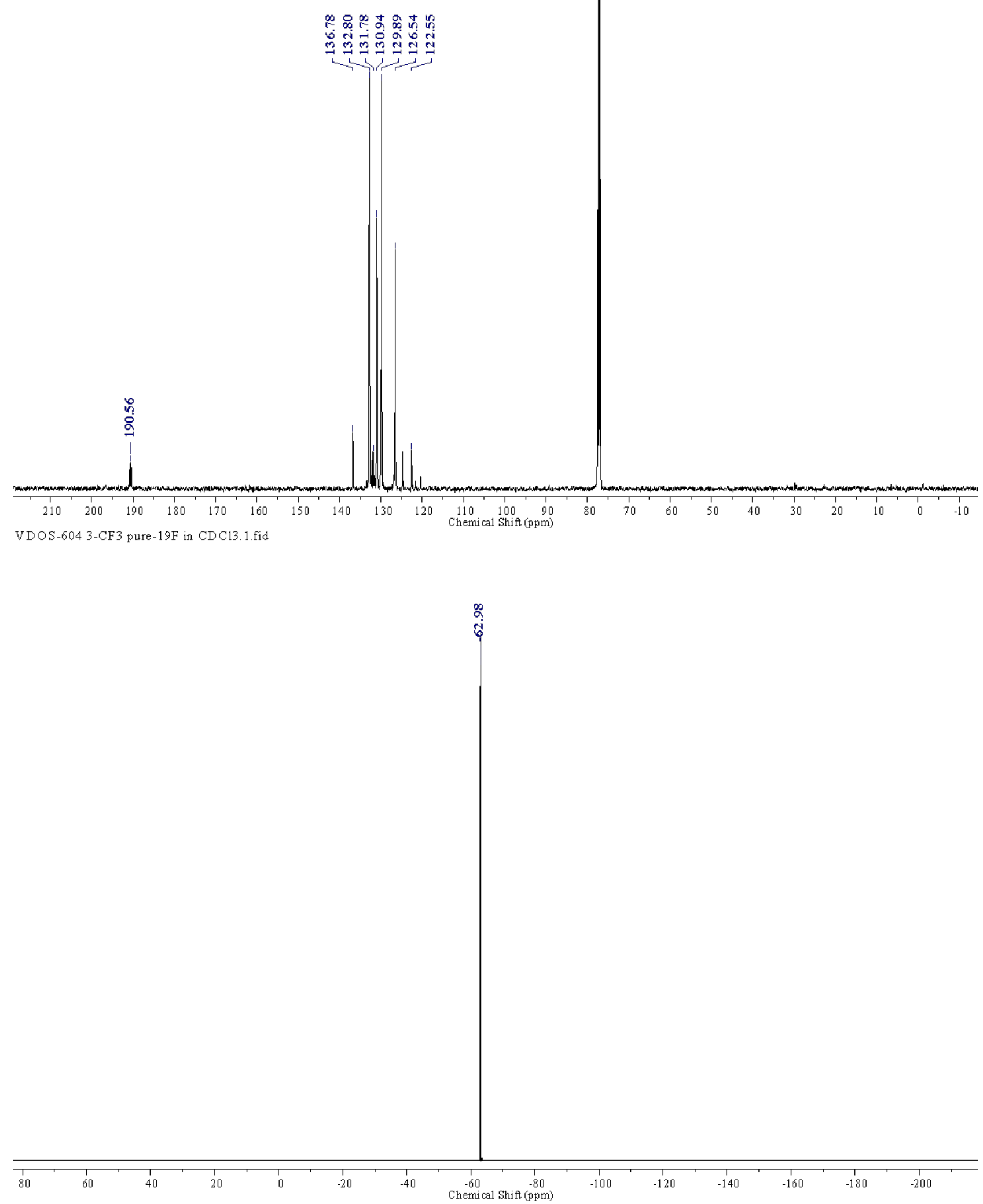
30. 4-(Trifluoromethyl)benzaldehyde-d<smiles>[2H]C(=O)c1ccc(C(F)(F)F)cc1</smiles>

Entry 1: NMR for Crude reaction mixture SCG-312-P1-16hr-D 1-50sec

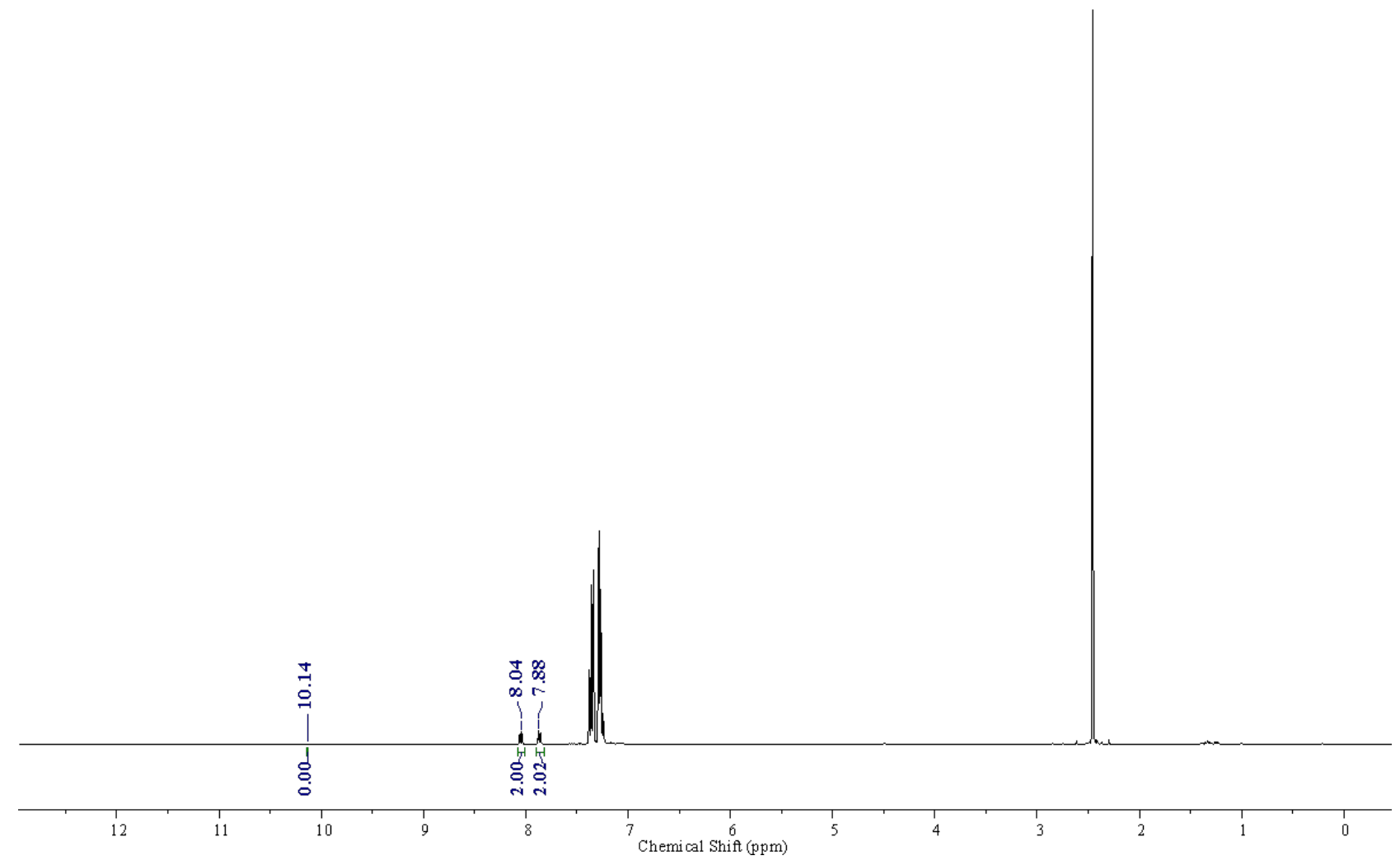


Entry 1': NMR for Crude reaction mixture, Duplicate

SCG-312-P2-16hr-D 1-50sec

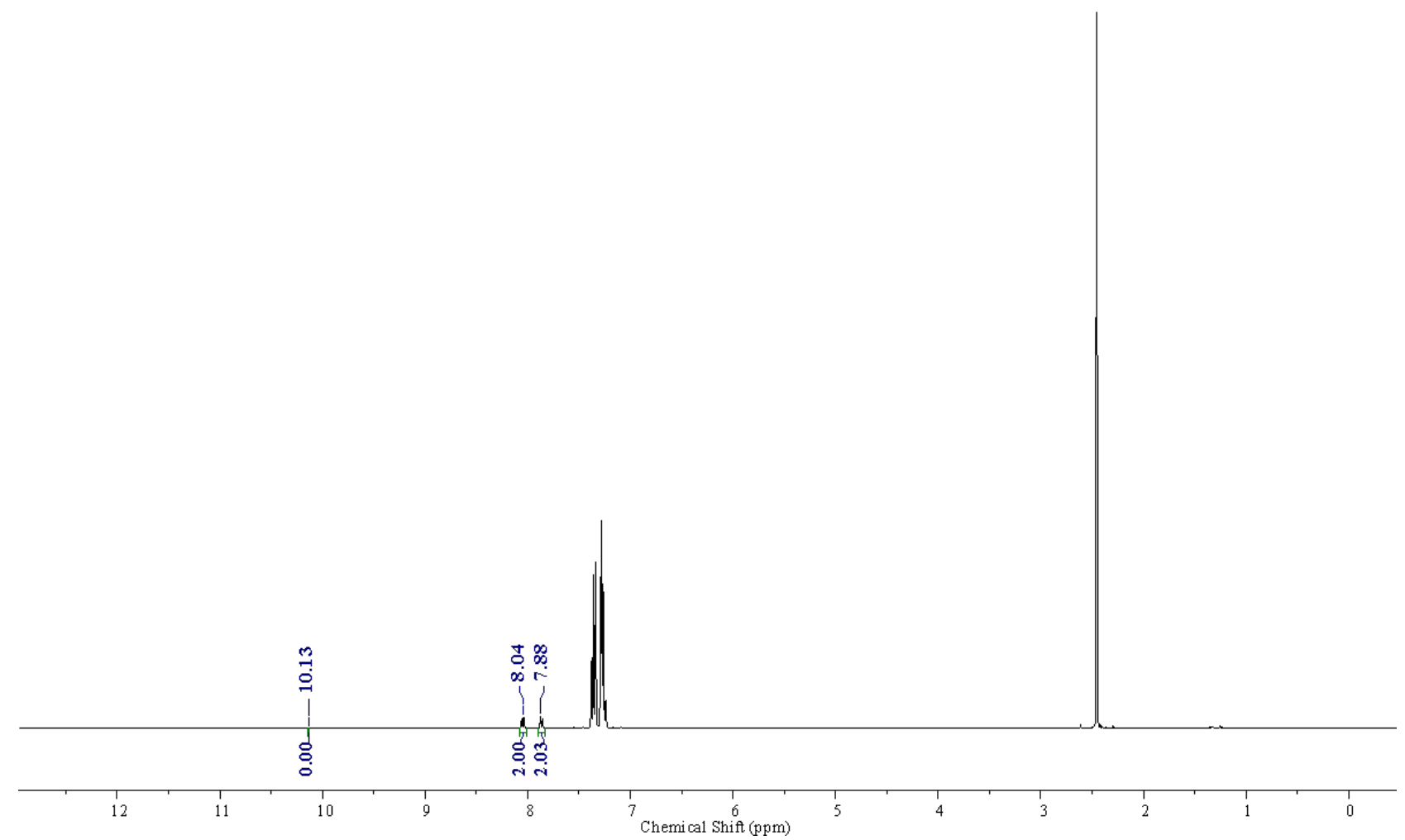

NMR after purification:

VDOS-605-A 1 pure-3.6.fid

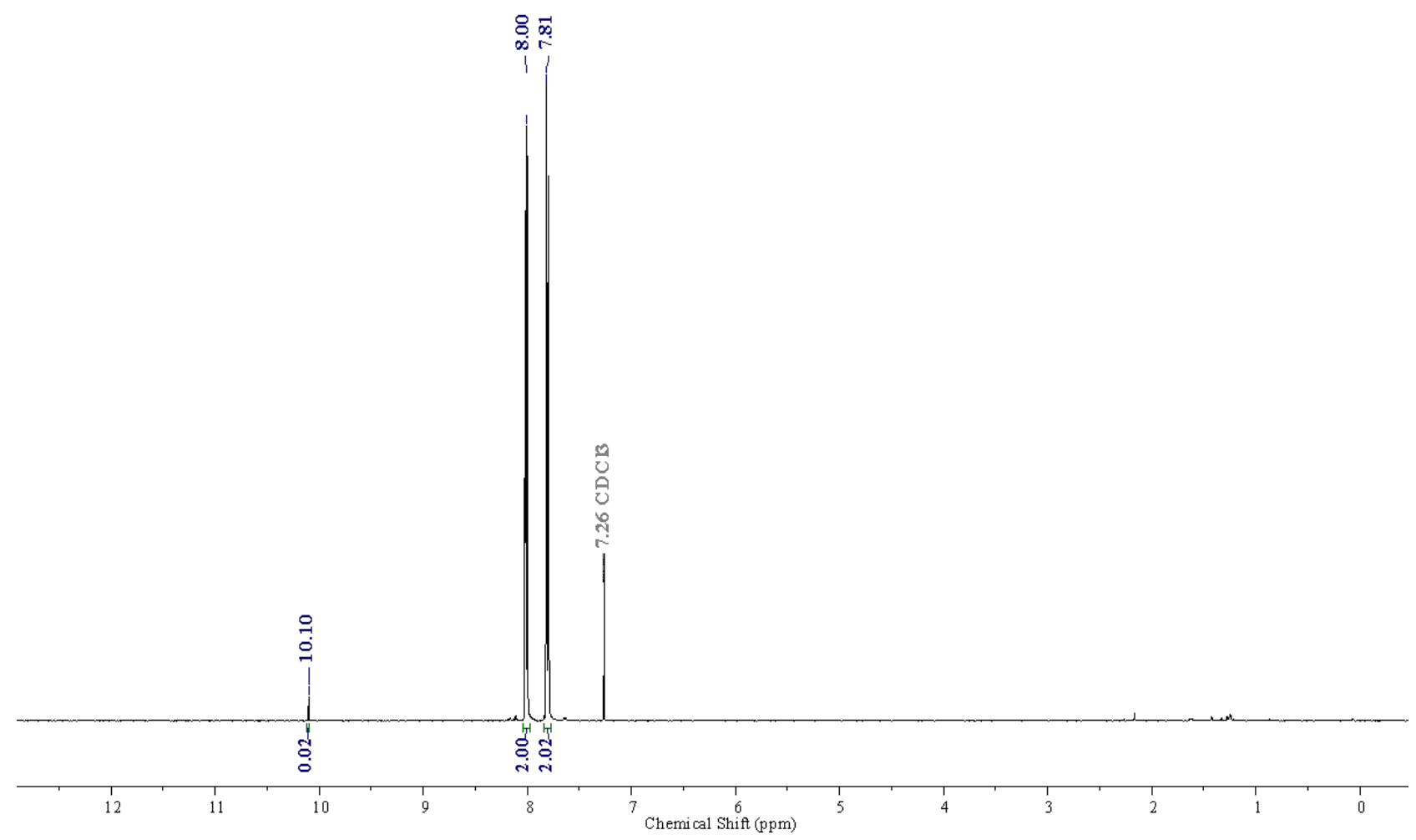



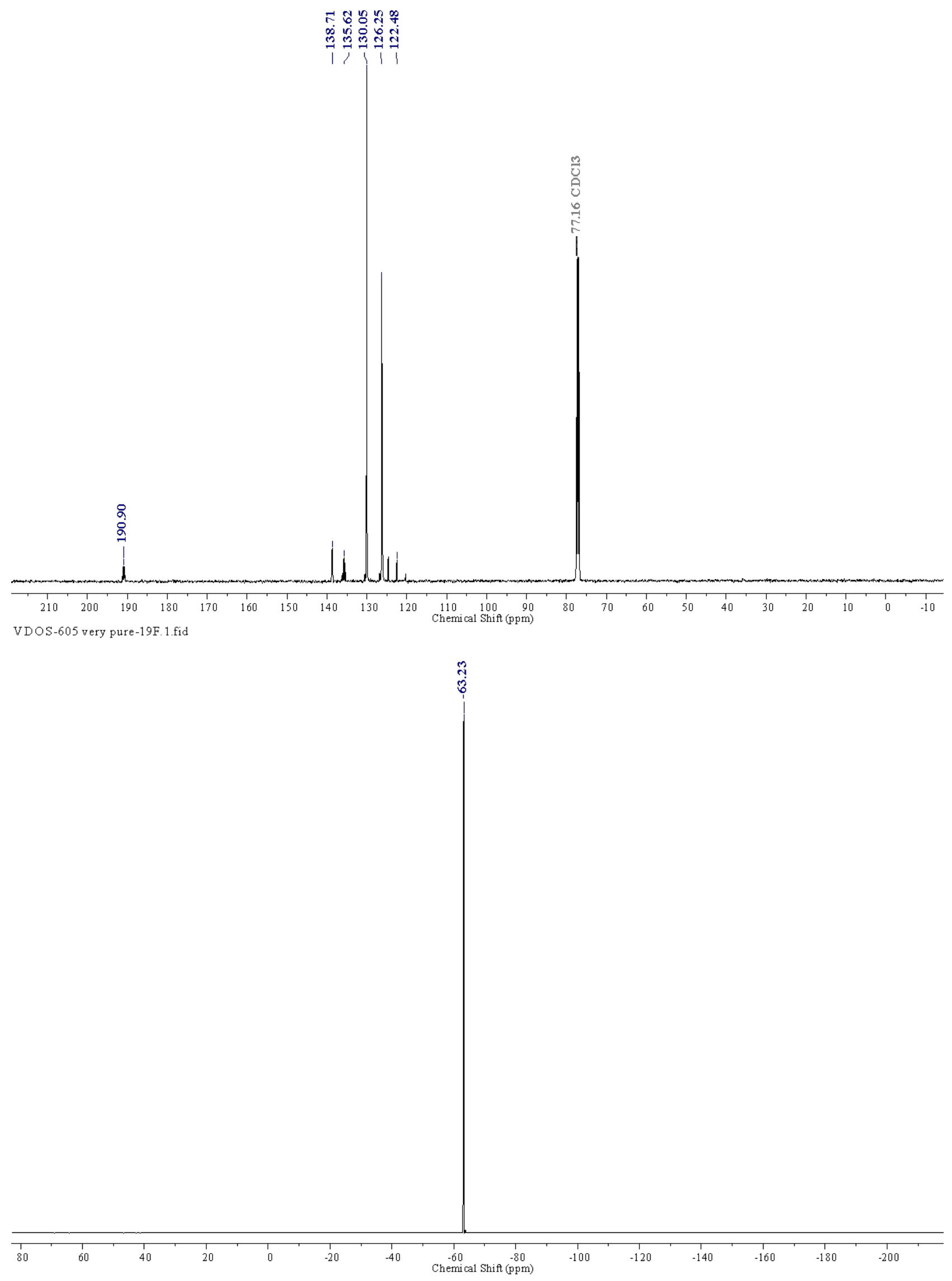
31. 4-Cyanobenzaldehyde-d<smiles>[2H]C(=O)c1ccc(C#N)cc1</smiles>

Entry 1: NMR for Crude reaction mixture SCG-312-F1-16hr-D 1-50sec.1.fid

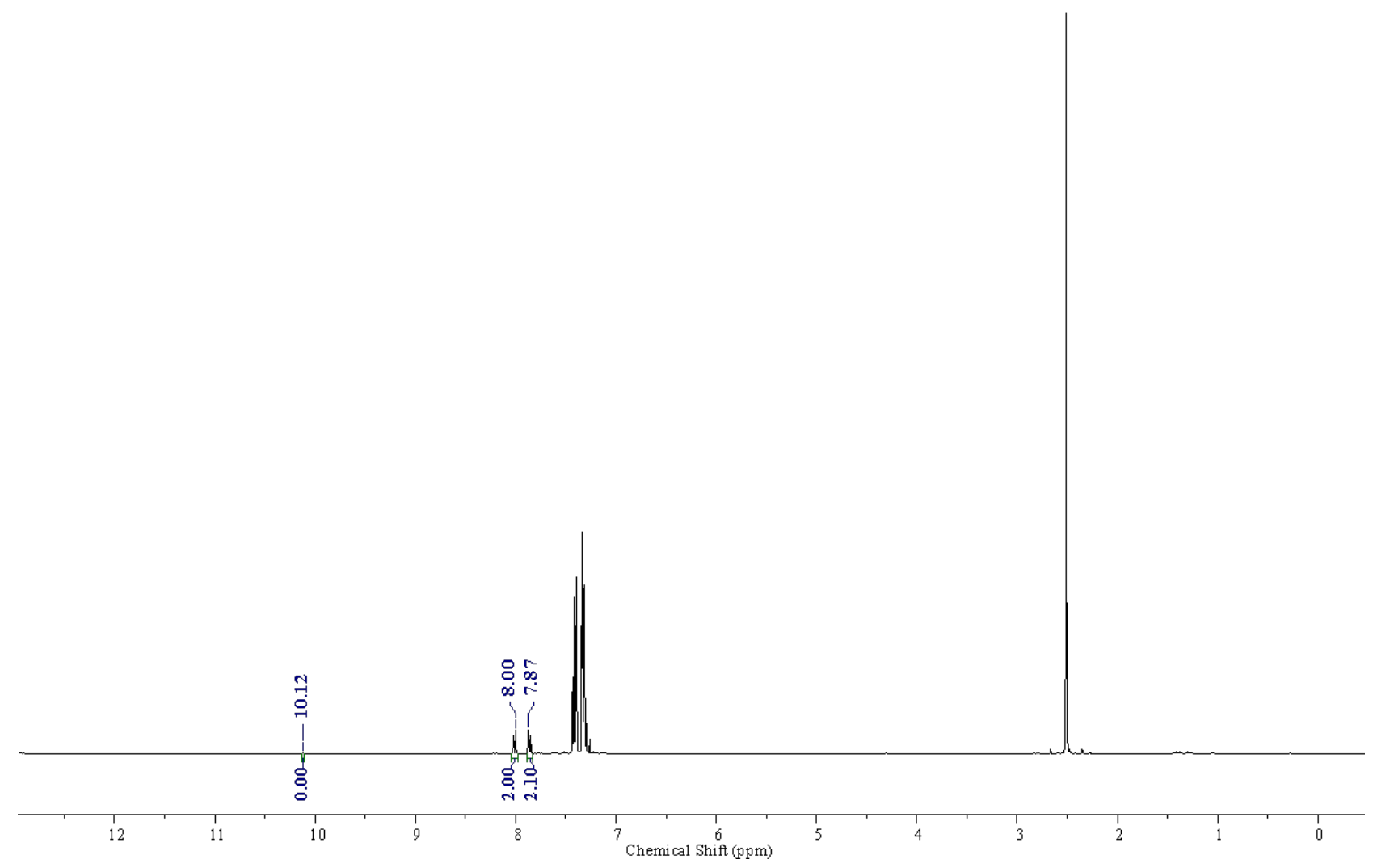


Entry 1': NMR for Crude reaction mixture, Duplicate

SCG-312-F2-16hr-D 1-50sec. 1.fid

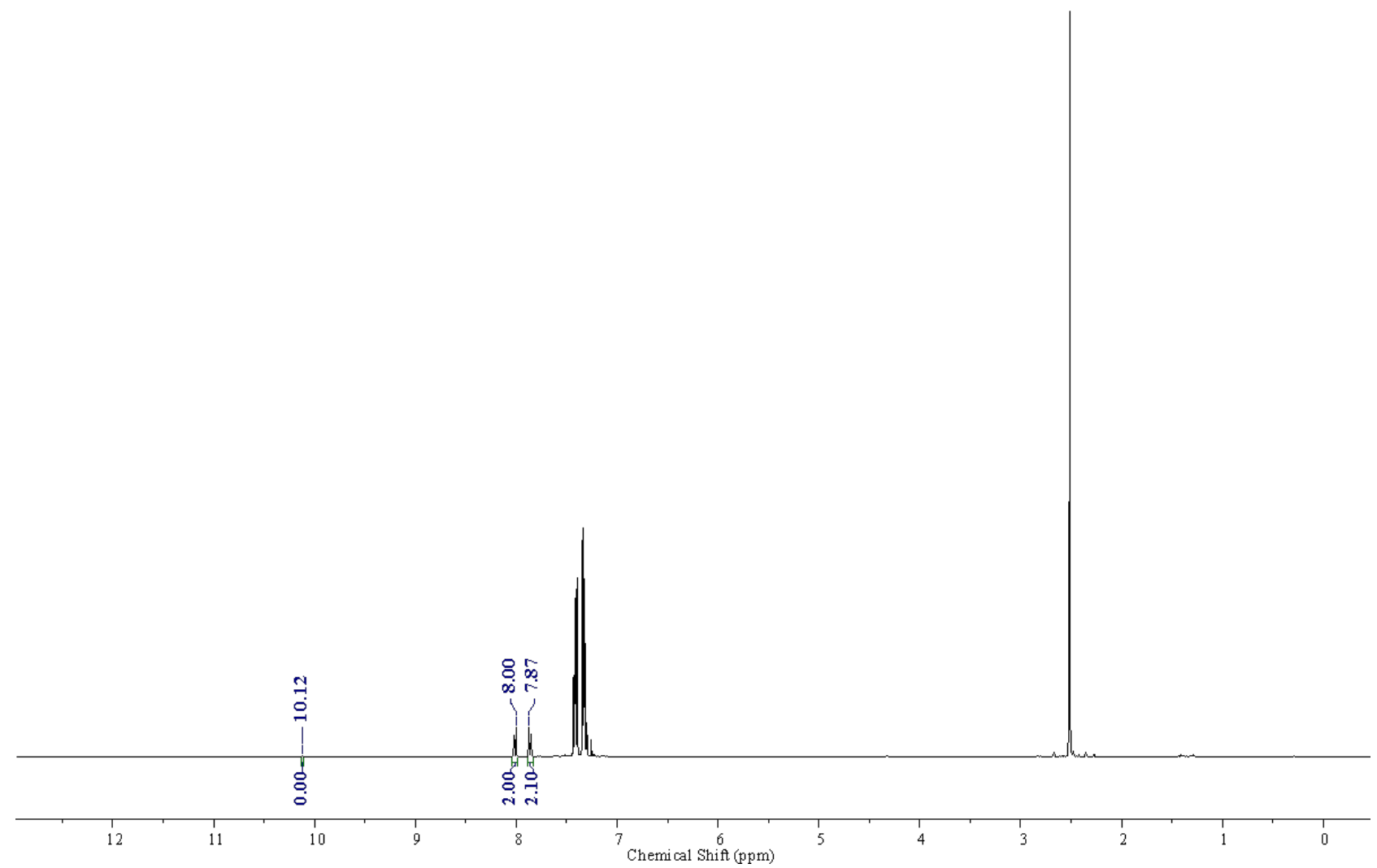

NMR after Purification:

SCG-312-F3-16hr-D 1-50sec-AC.1.fid

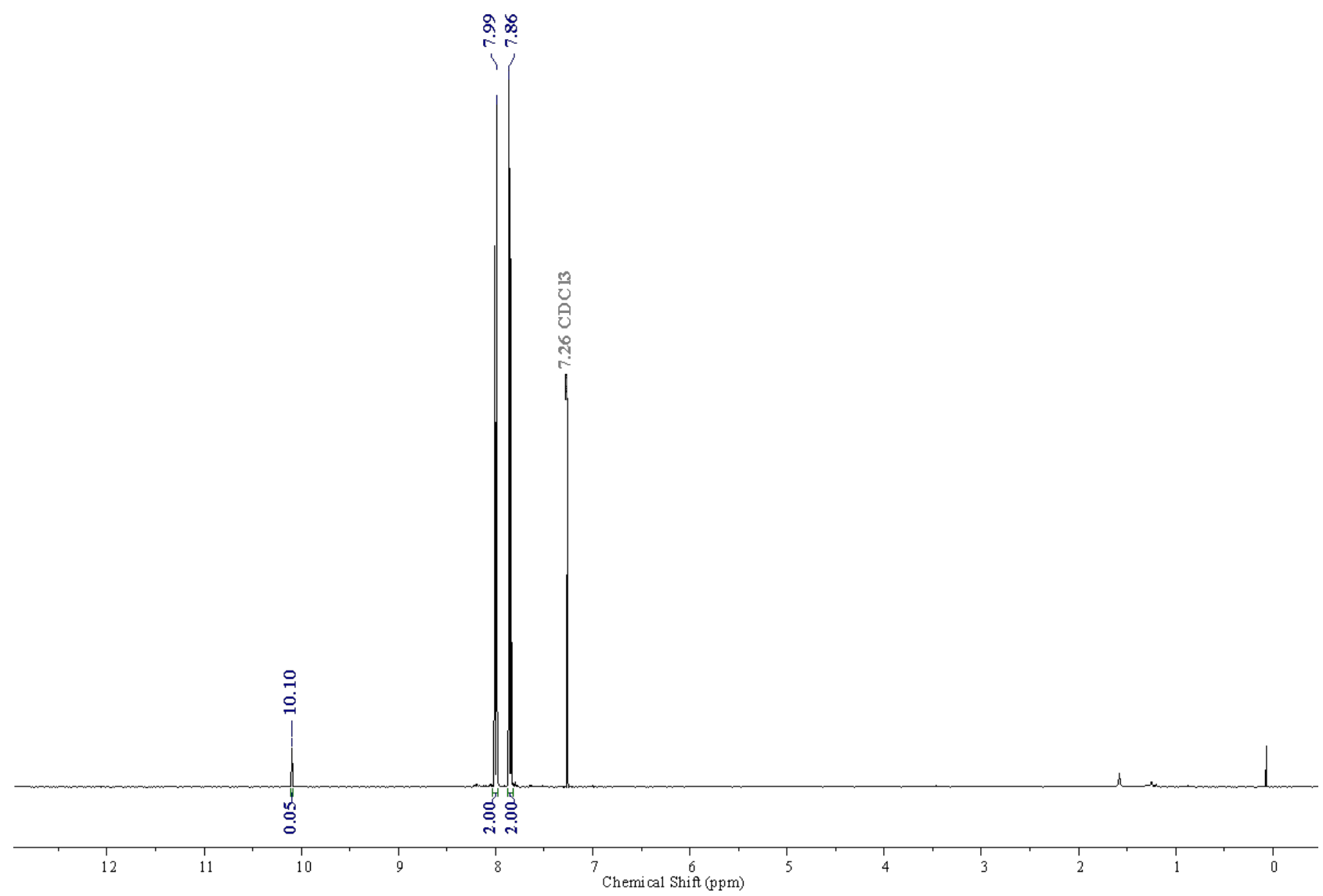




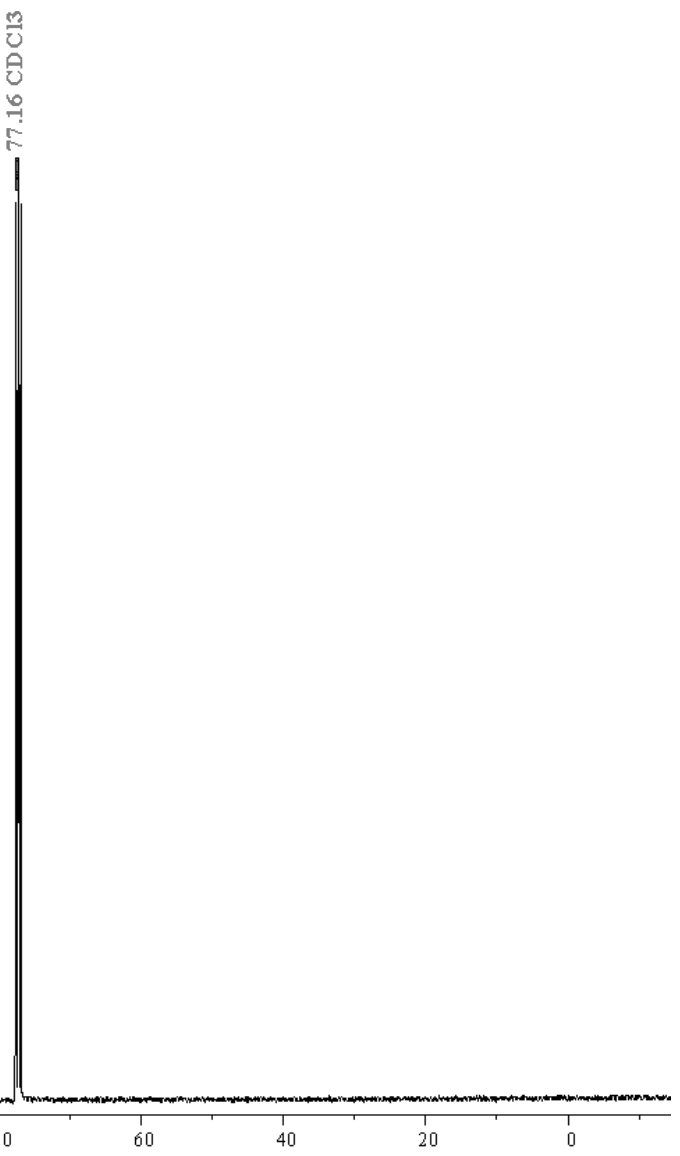


32. 3-Cyanobenzaldehyde-d<smiles>[2H]C(=O)c1cccc(C#N)c1</smiles>

Entry 1: NMR for Crude reaction mixture SCG-312-N1-16hr-D 1-50sec.1.fid

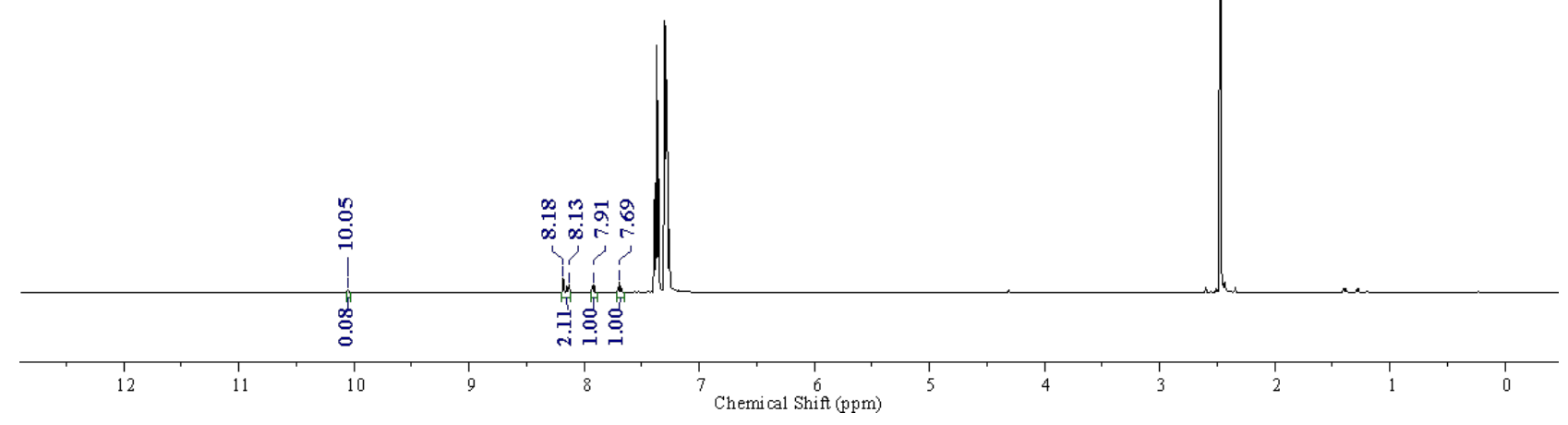


Entry 1': NMR for Crude reaction mixture, Duplicate

SCG-312-N2-16hr-D 1-50sec.2.fid

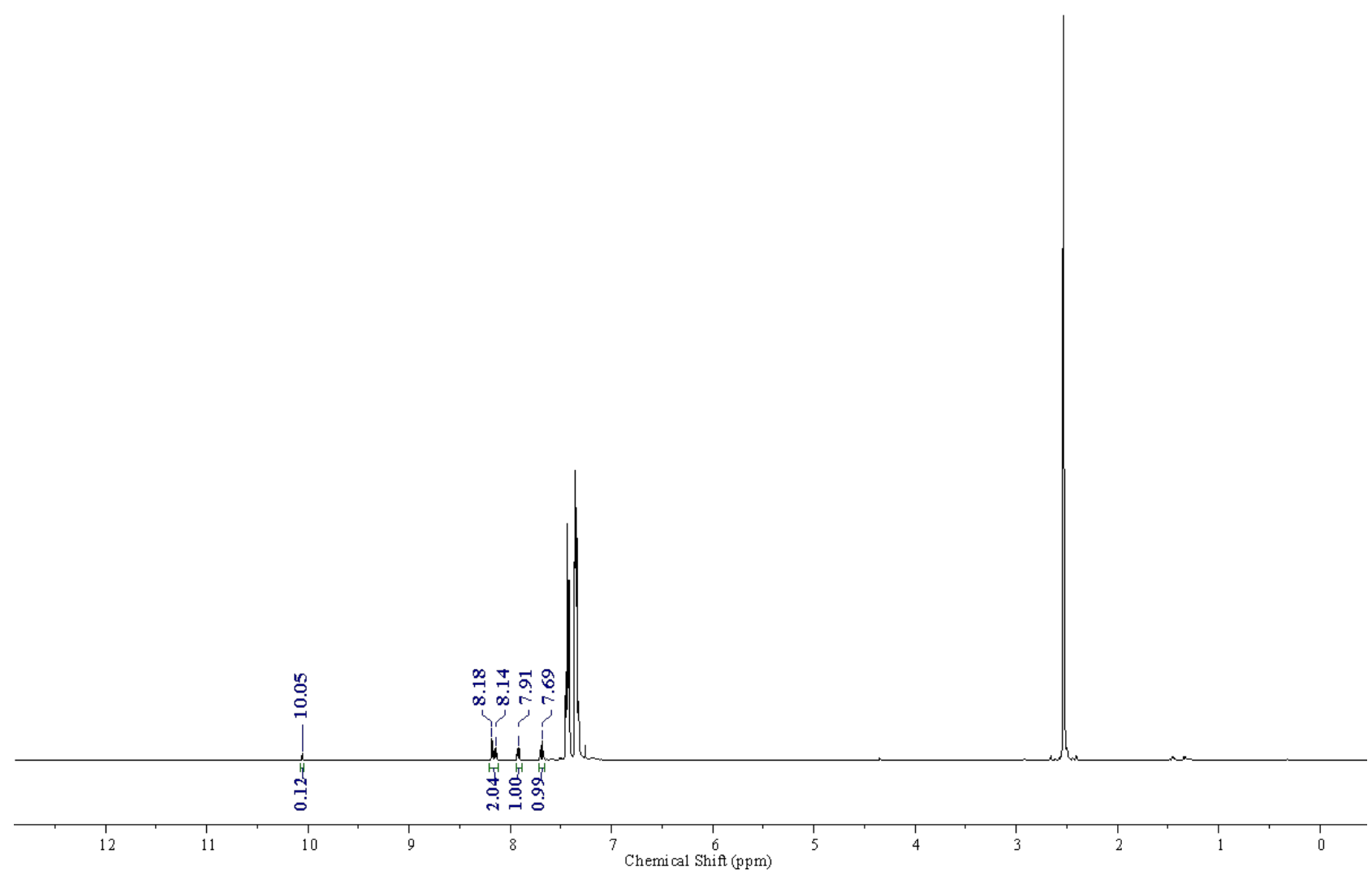

Entry 2: NMR for Crude reaction mixture

SCG-312-N4-16hr-D 1-50sec

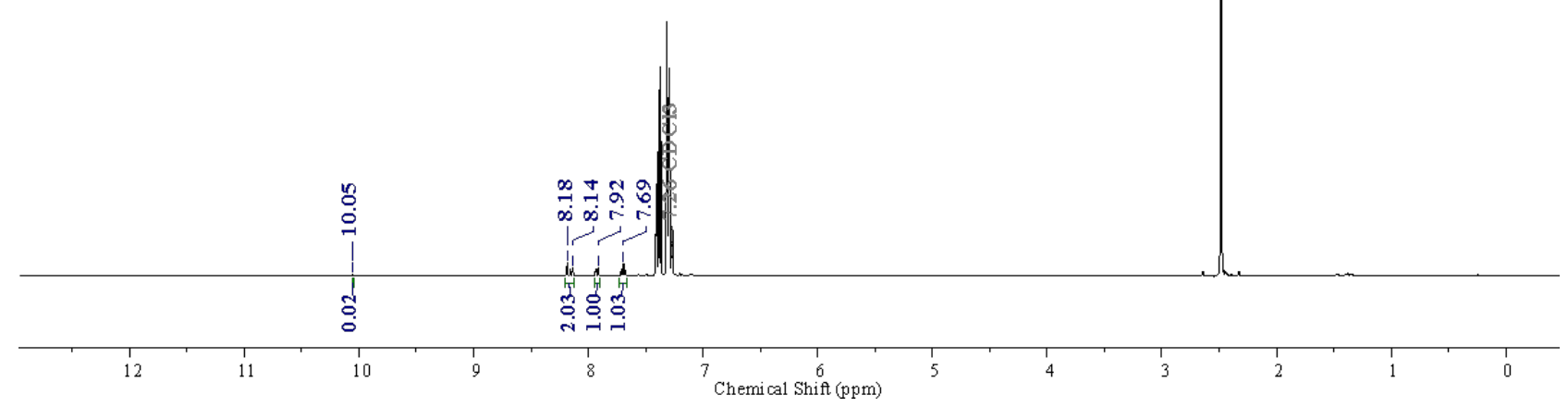


Entry 2': NMR for Crude reaction mixture, Duplicate SCG-312-N3-16hr-D 1-50sec

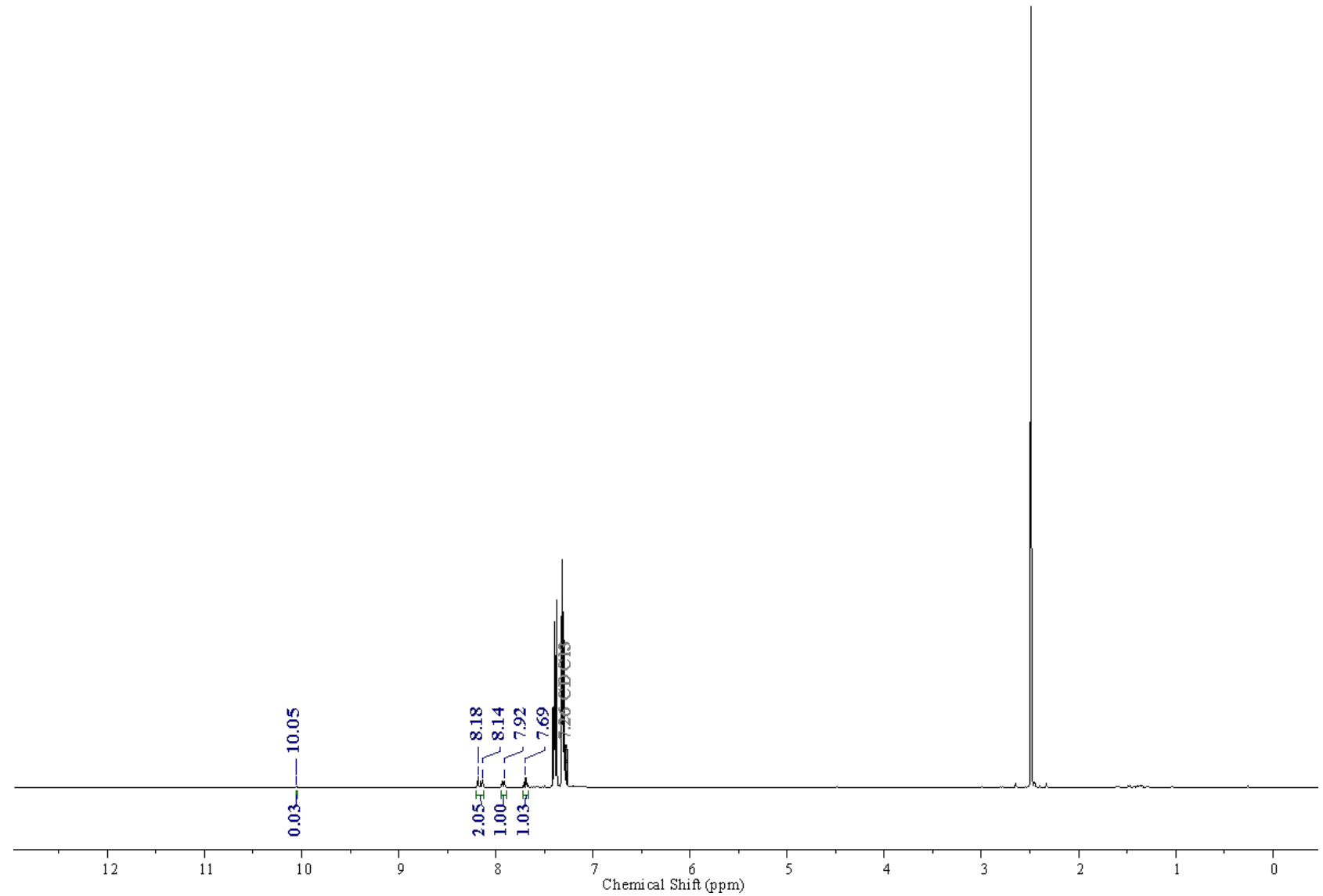

NMR after purification:

SCG-312-N7-16hr-D 1-50sec-AC

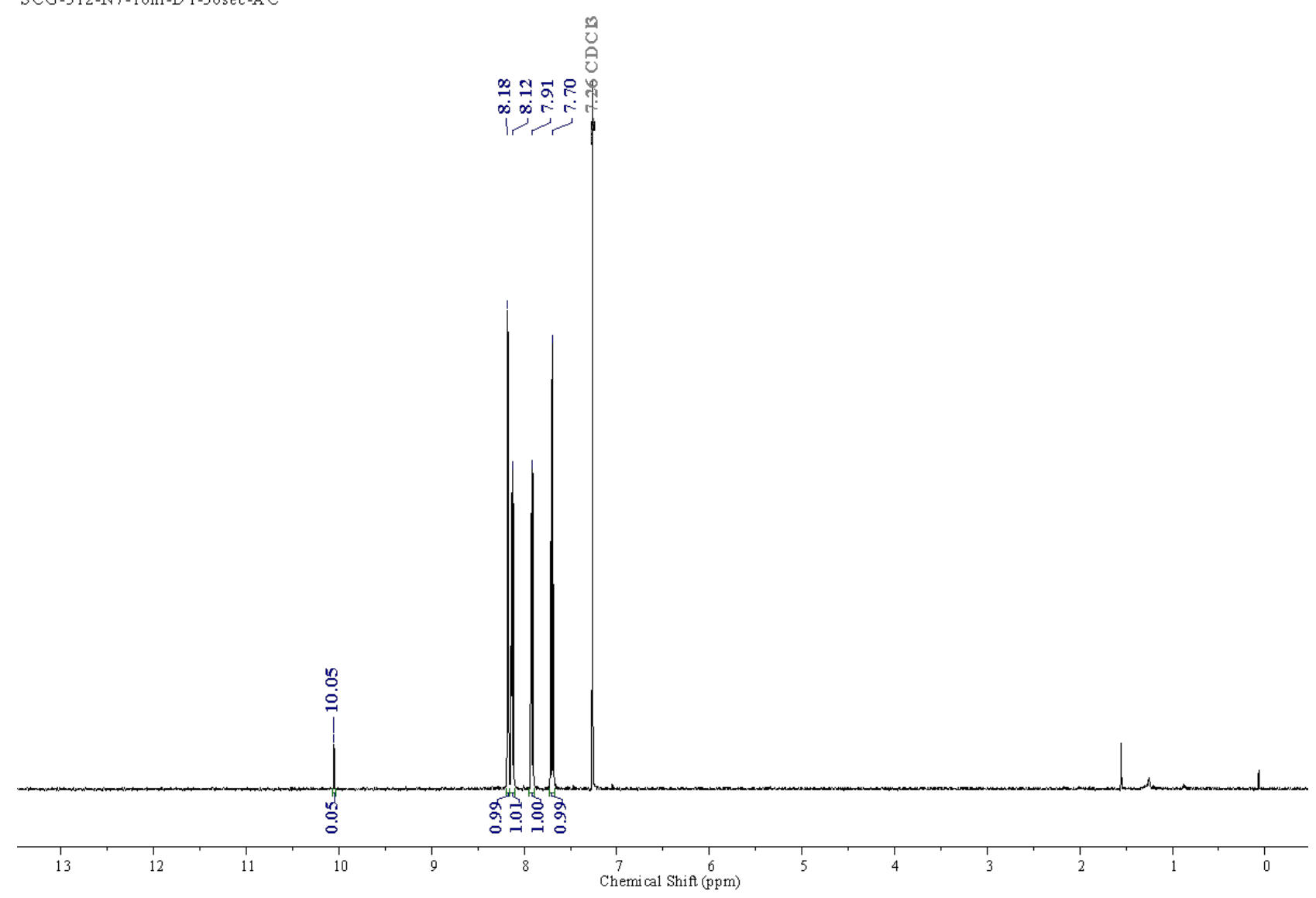




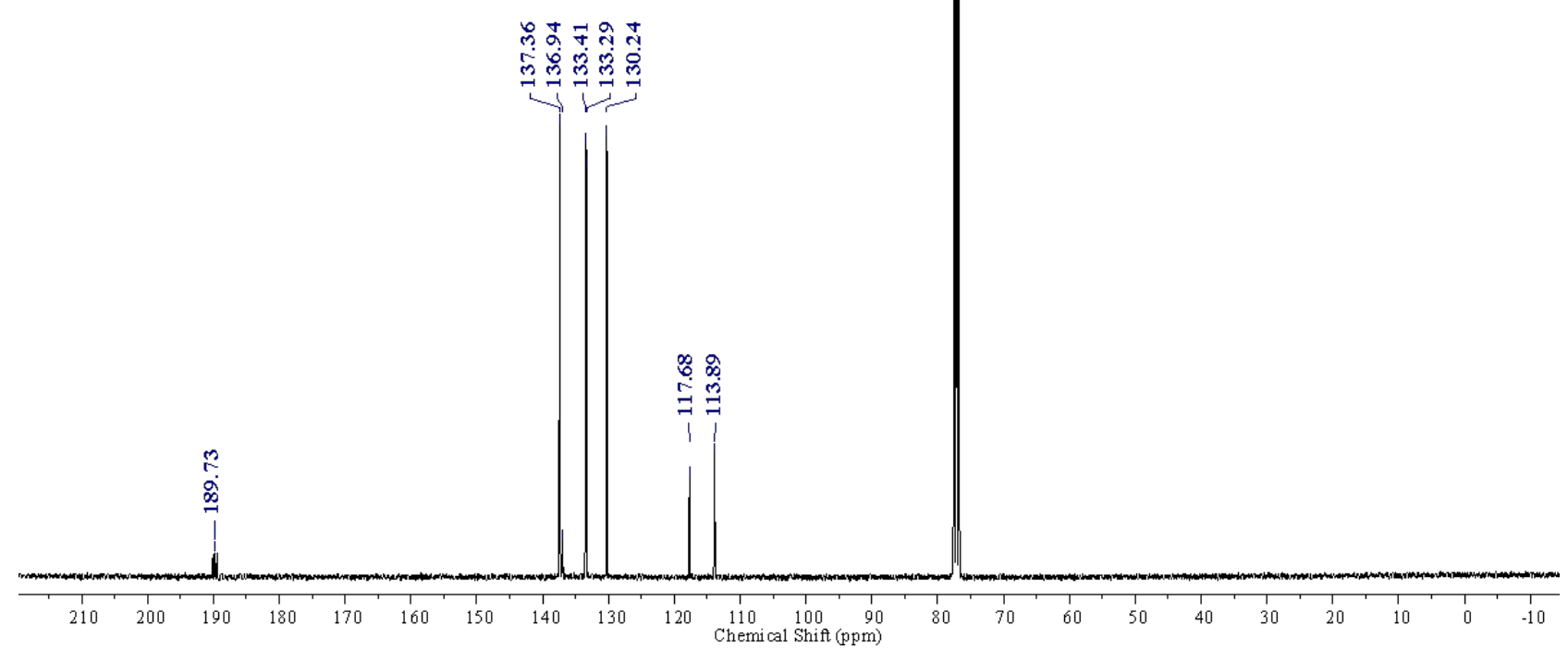


33. 4-Fluorobenzaldehyde-d<smiles>[2H]C(=O)c1ccc(F)cc1</smiles>

Entry 1: NMR for Crude reaction mixture SCG-312-E2-16hr-D 1-50sec. 1.fid

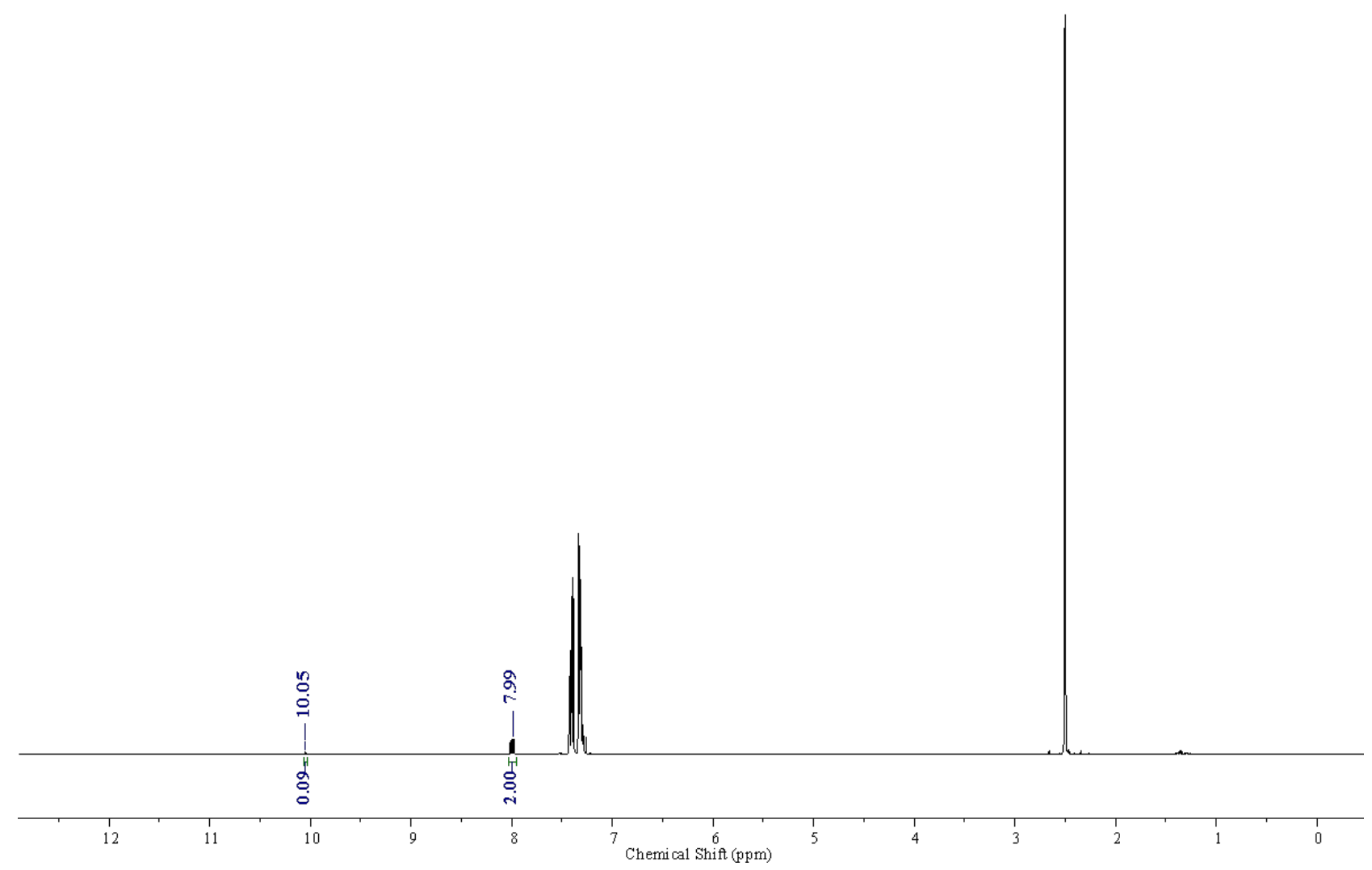


Entry 1': NMR for Crude reaction mixture, Duplicate SCG-312-E1-16hr-D 1-50sec.1.fid

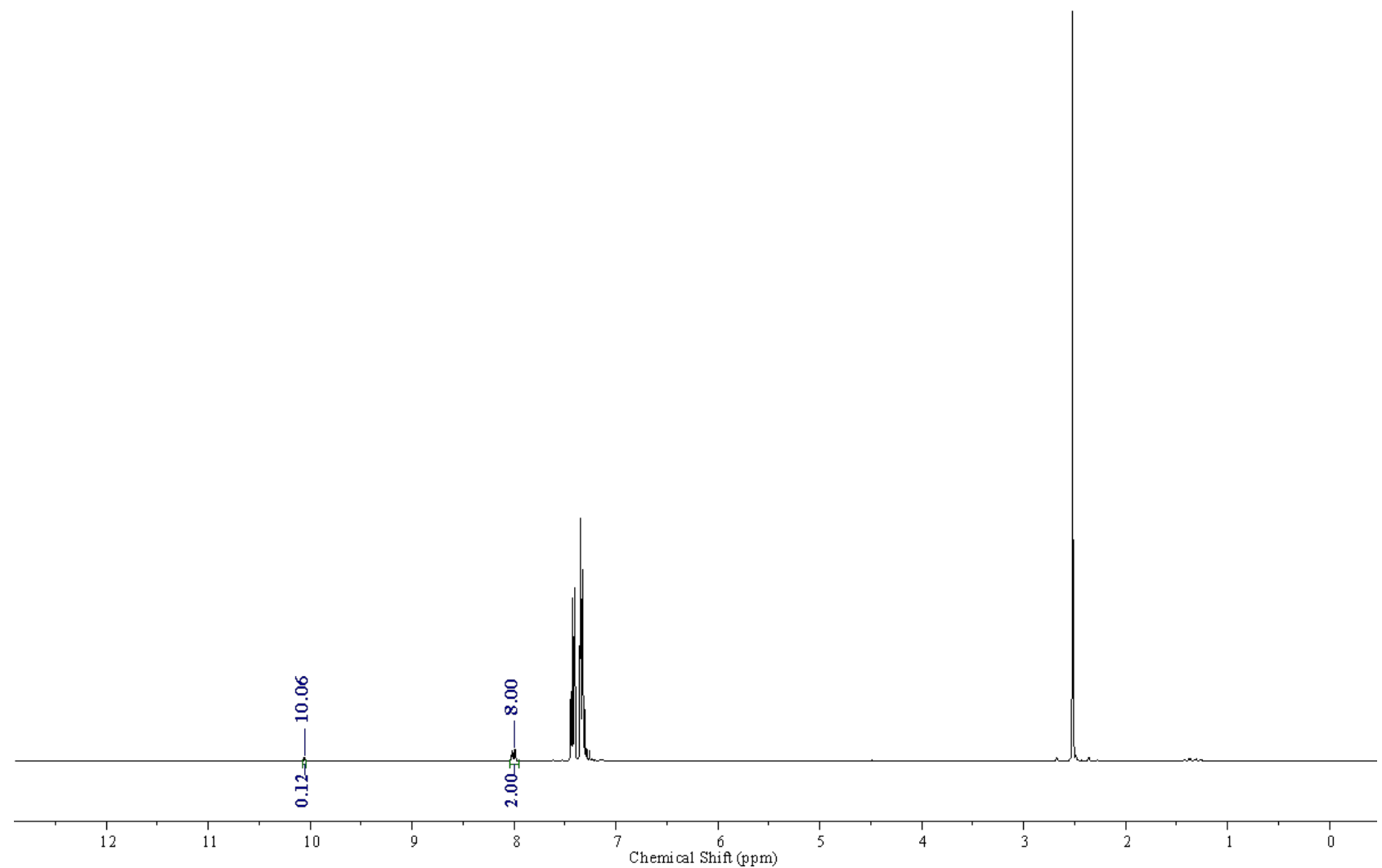

Entry 2: NMR for Crude reaction mixture

SCG-312-E3-16hr-D 1-50sec. 1.fid

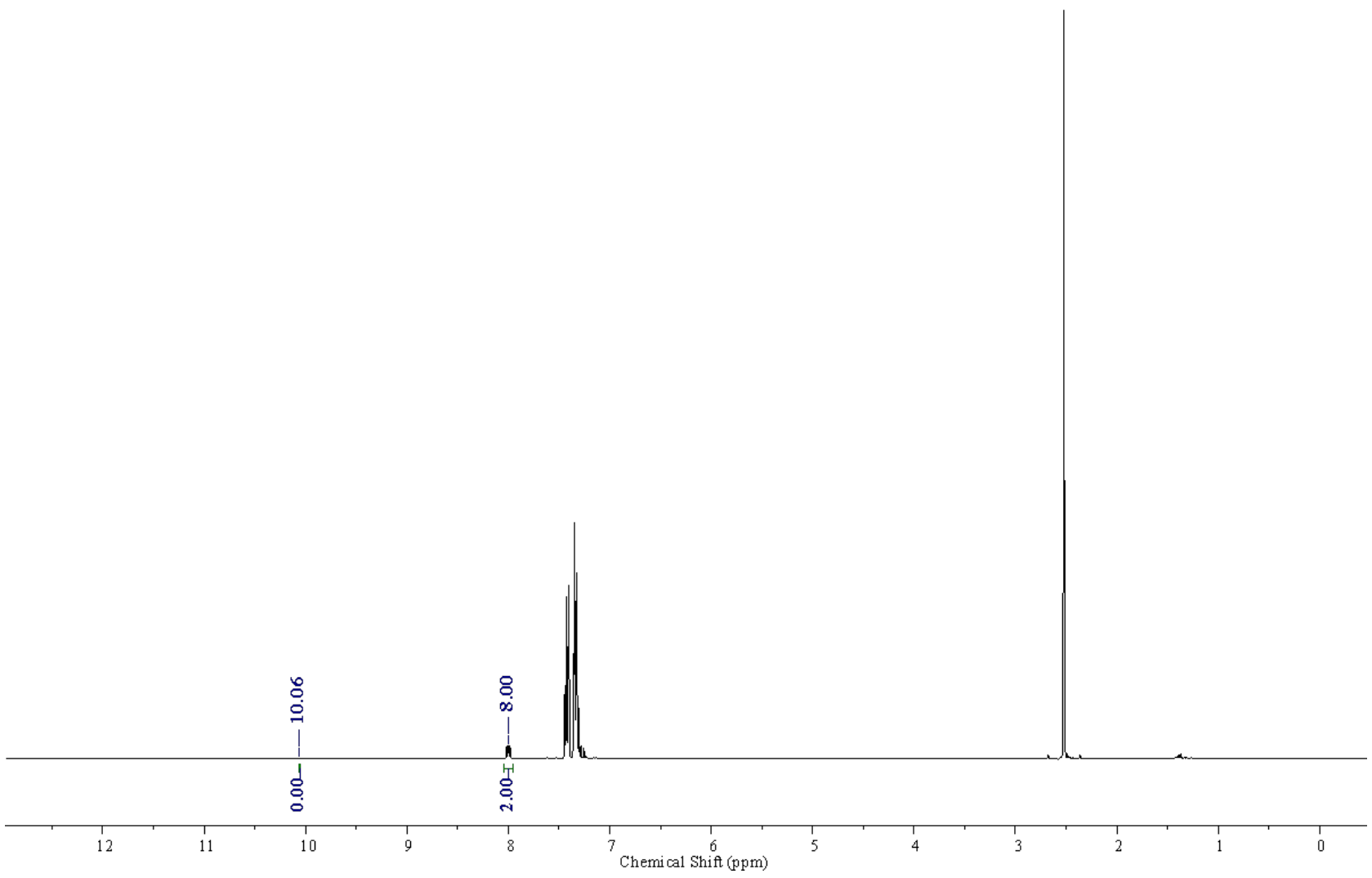


Entry 2': NMR for Crude reaction mixture, Duplicate SCG-319-D 1-16hr-D 1-50sec.1.fid

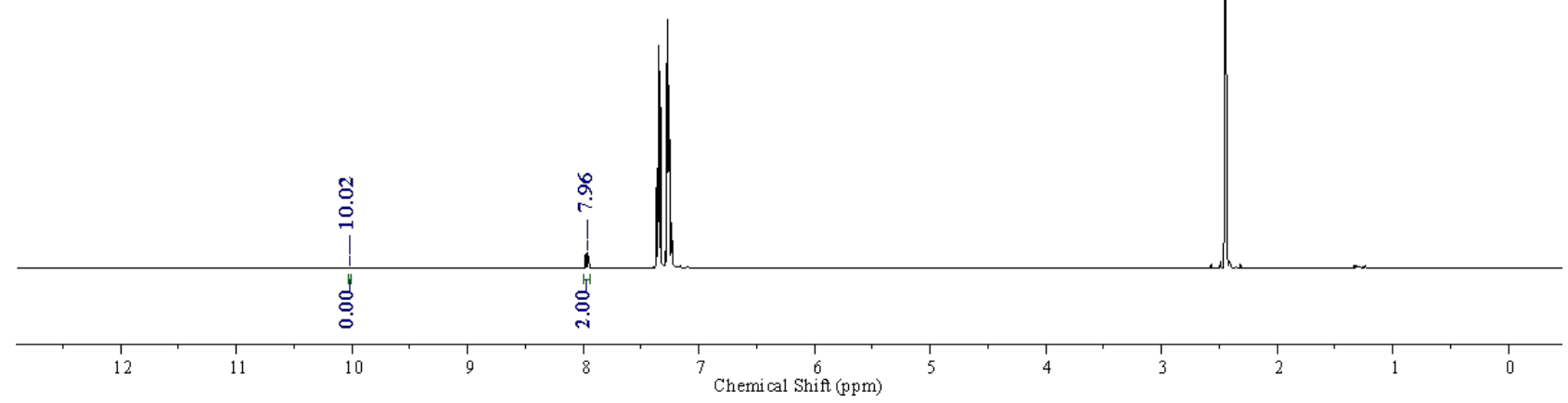


NMR after purification:

VD OS-602-A1pure.1.fid

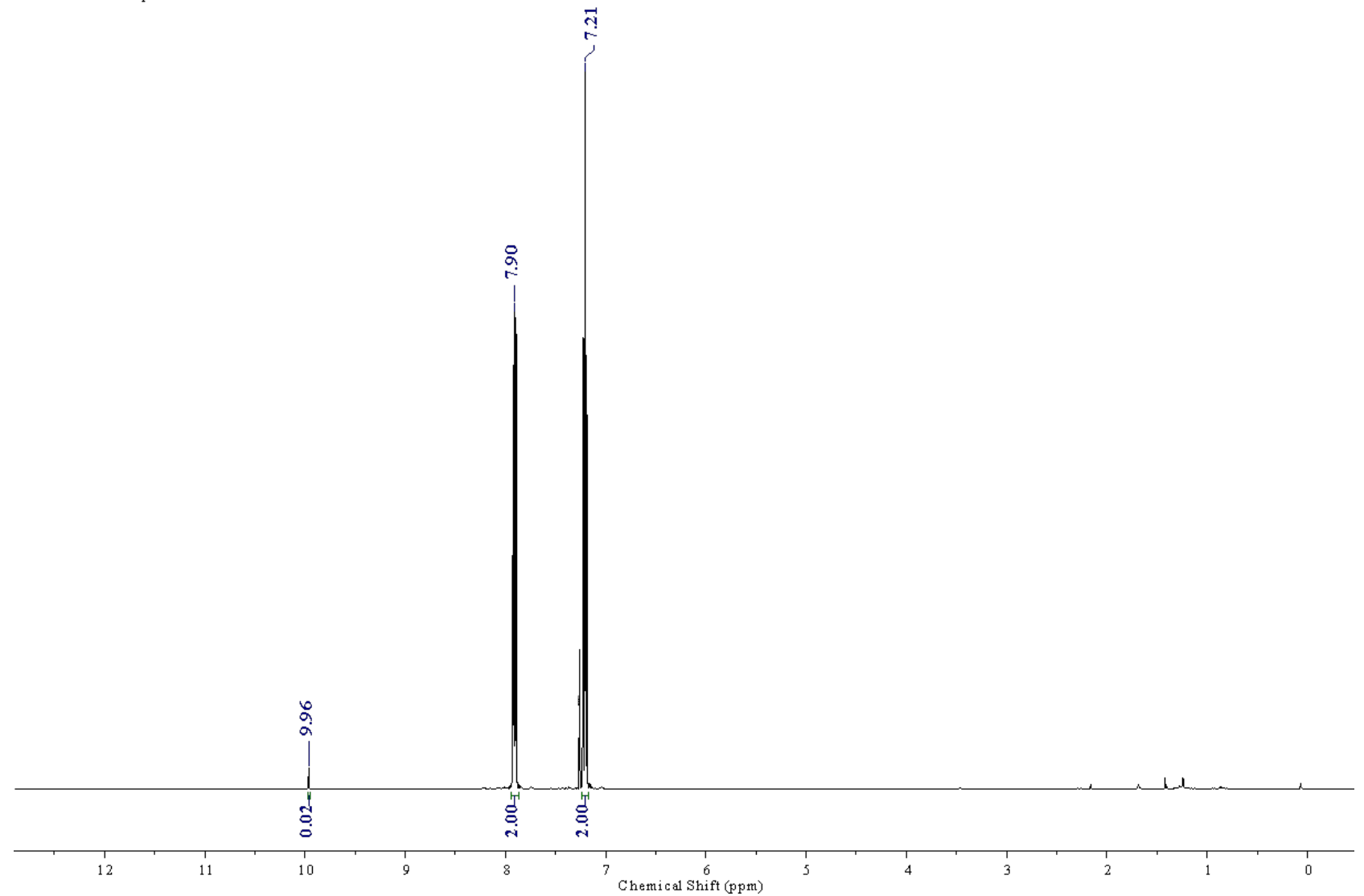

VDOS-602-A 1pure-VP 13C.4.fid

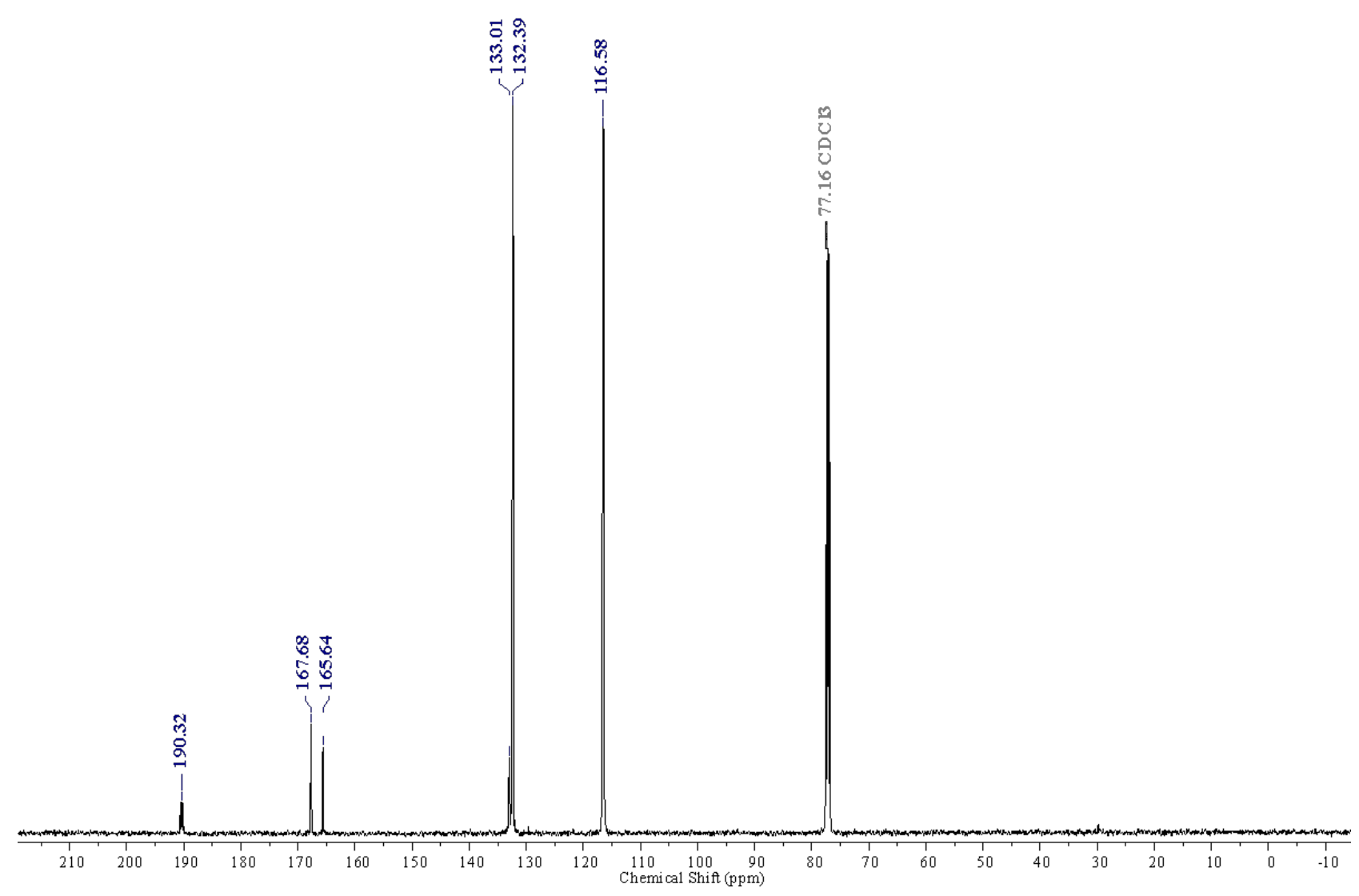




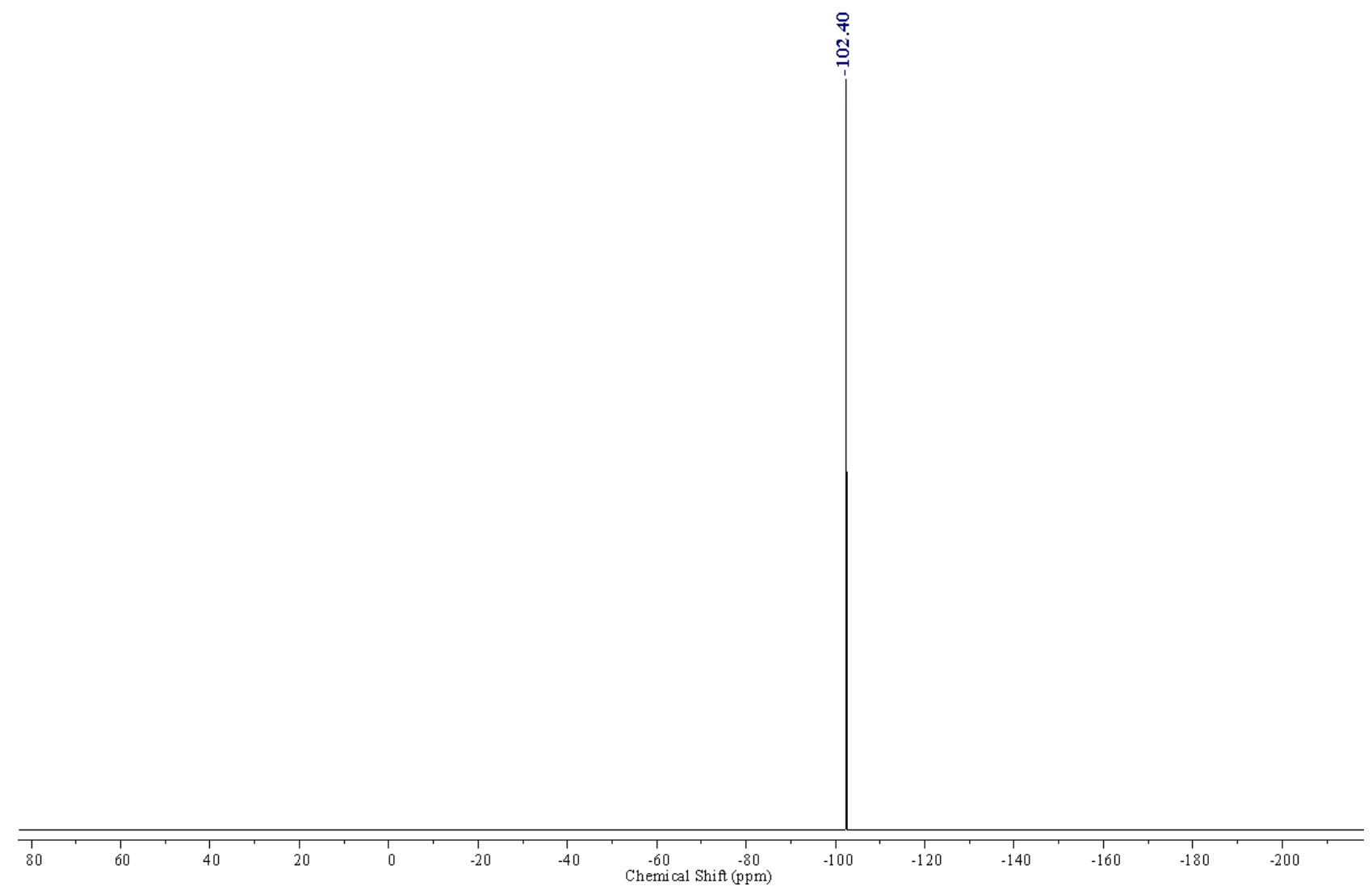


34. 2-Fluorobenzaldehyde-d<smiles>[2H]C(=O)c1ccccc1F</smiles>

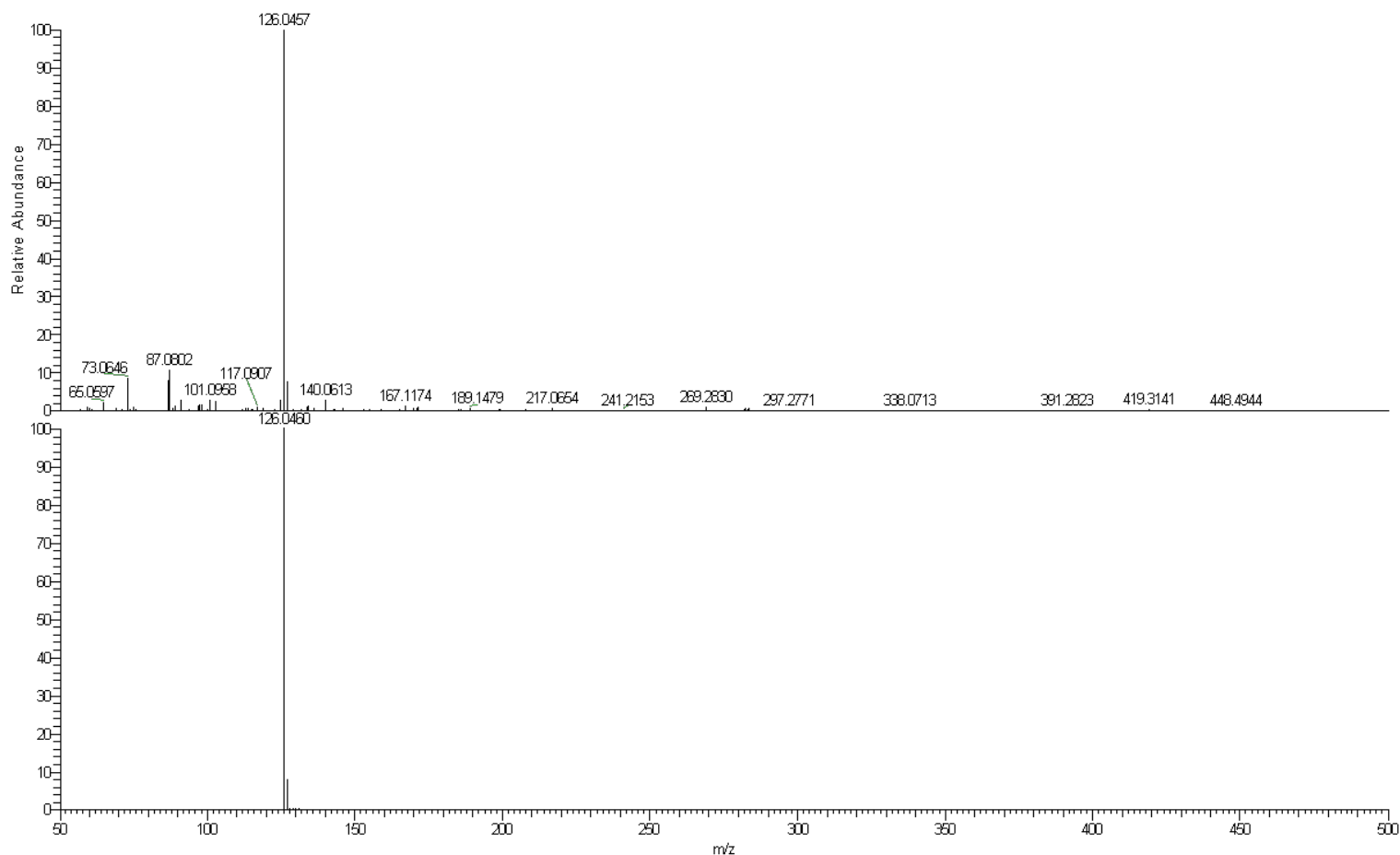

Entry 1: NMR for Crude reaction mixture SCG-309-A1-16hr-D1-50sec.1. fid

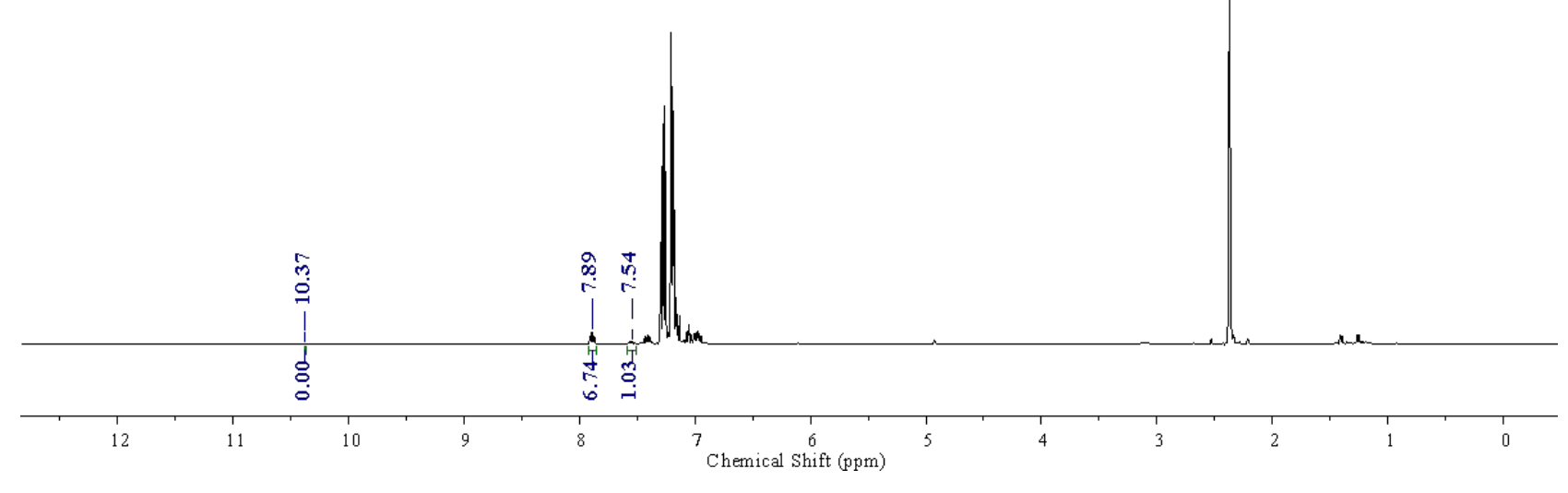


Entry 1': NMR for Crude reaction mixture, Duplicate

SCG-309-A2-16hr-D1-50sec.1. fid

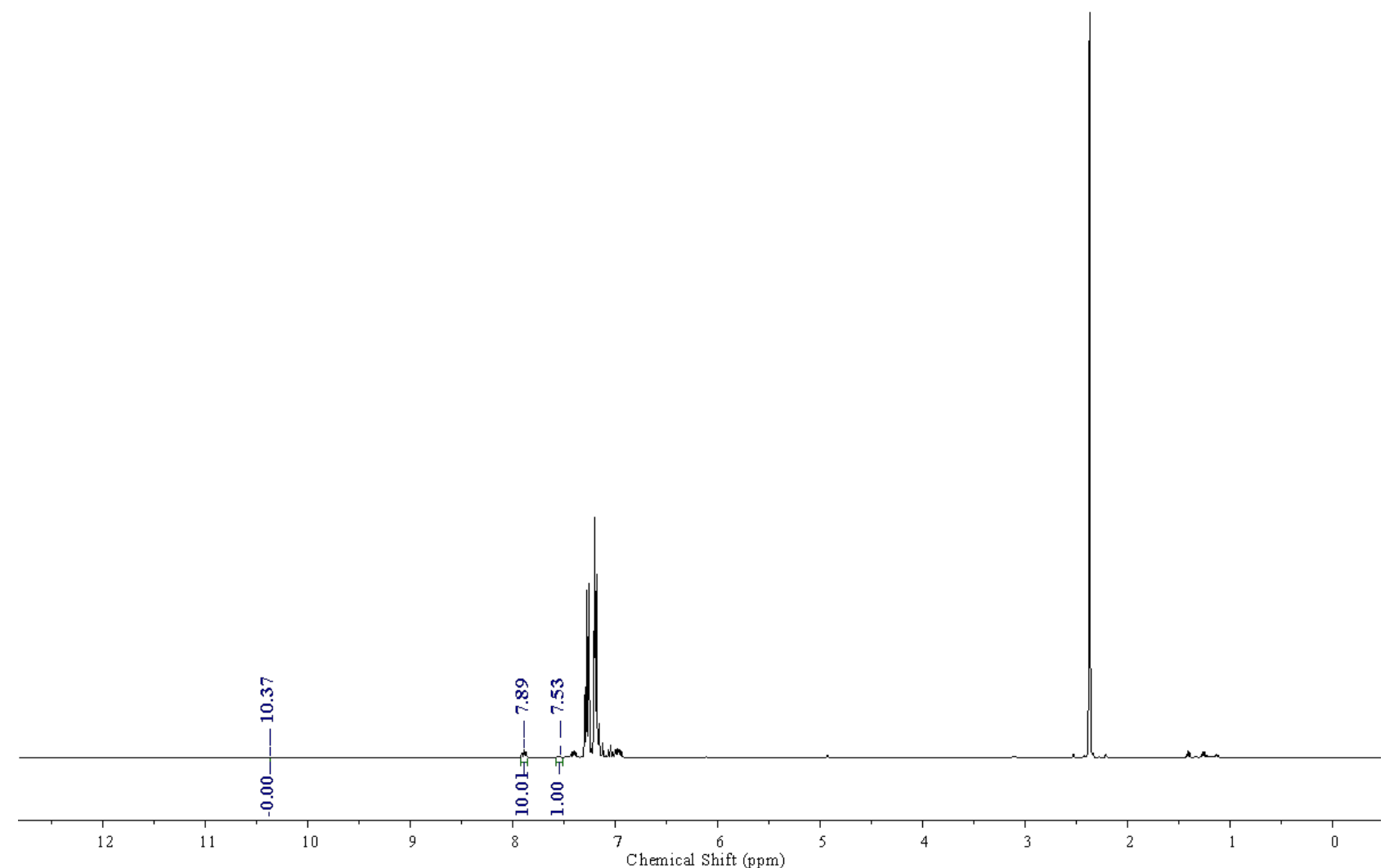

Entry 2: NMR for Crude reaction mixture

SCG-309-G2-16hr-D1-50sec.1. fid

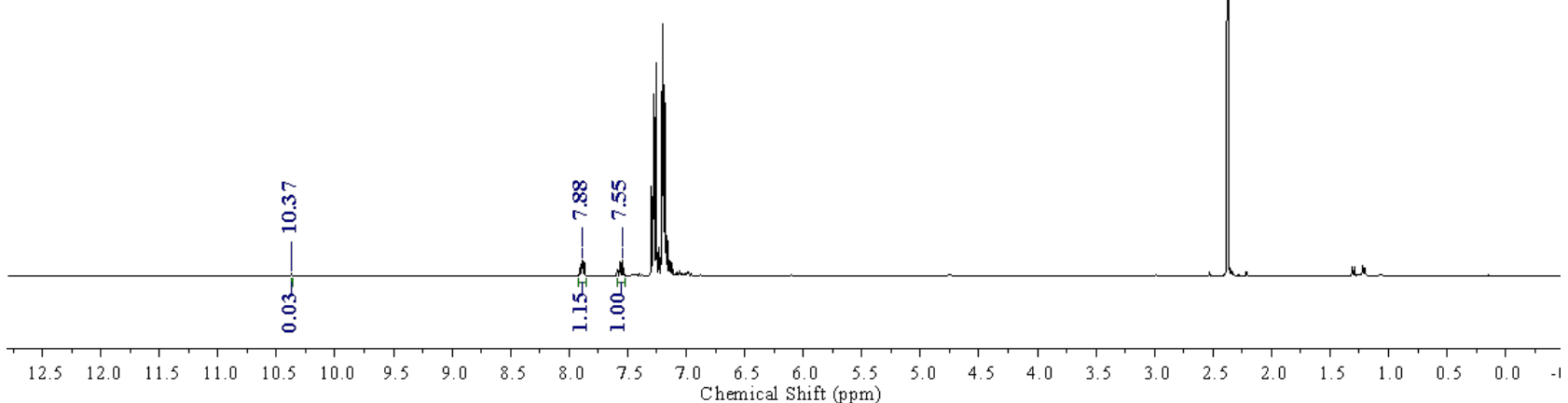


Entry 3: NMR for Crude reaction mixture

SCG-309-G1-16hr-D1-50sec.1. fid

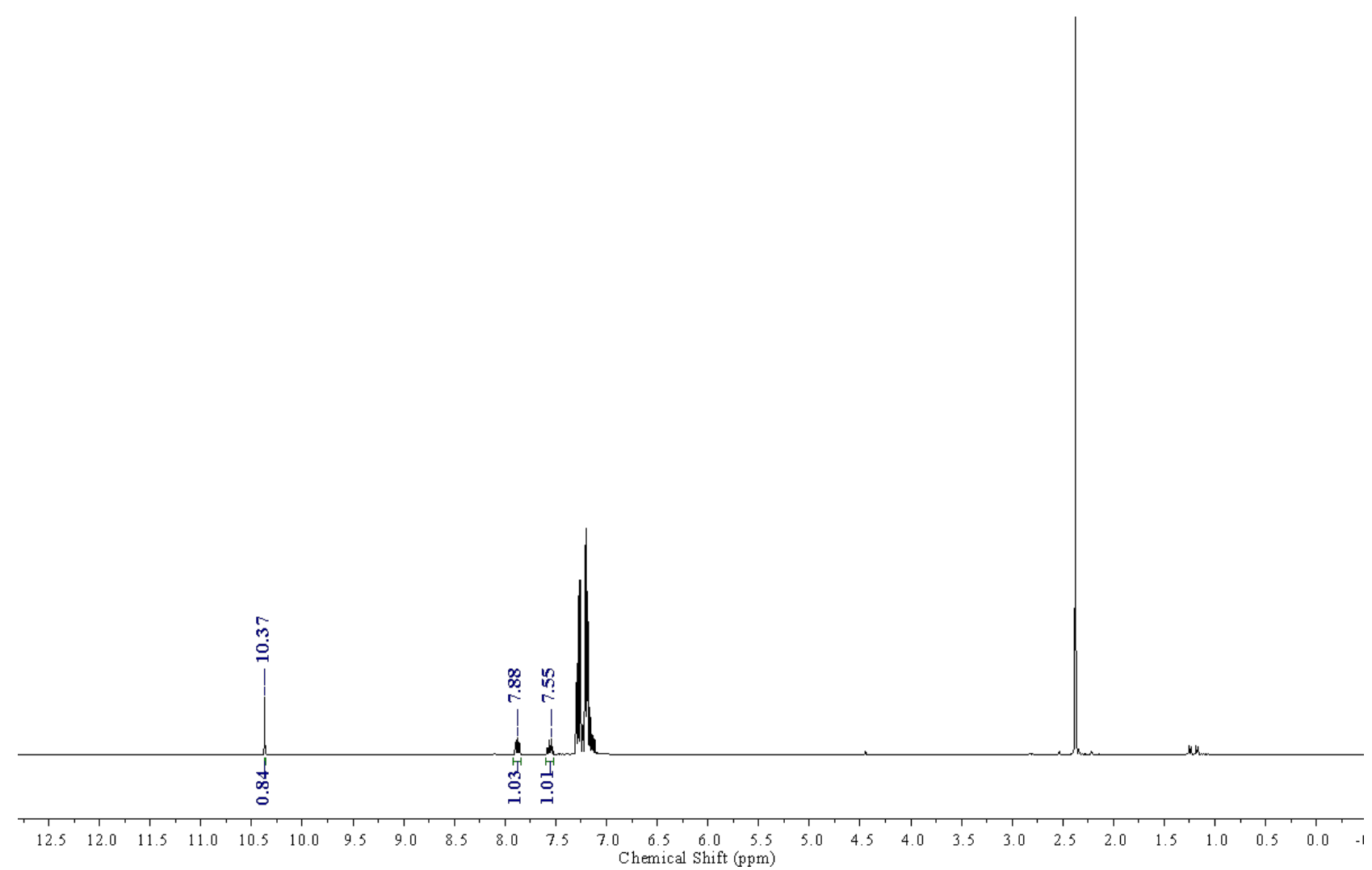

Entry 4: NMR for Crude reaction mixture

VDOS-625-B 1.2.fid

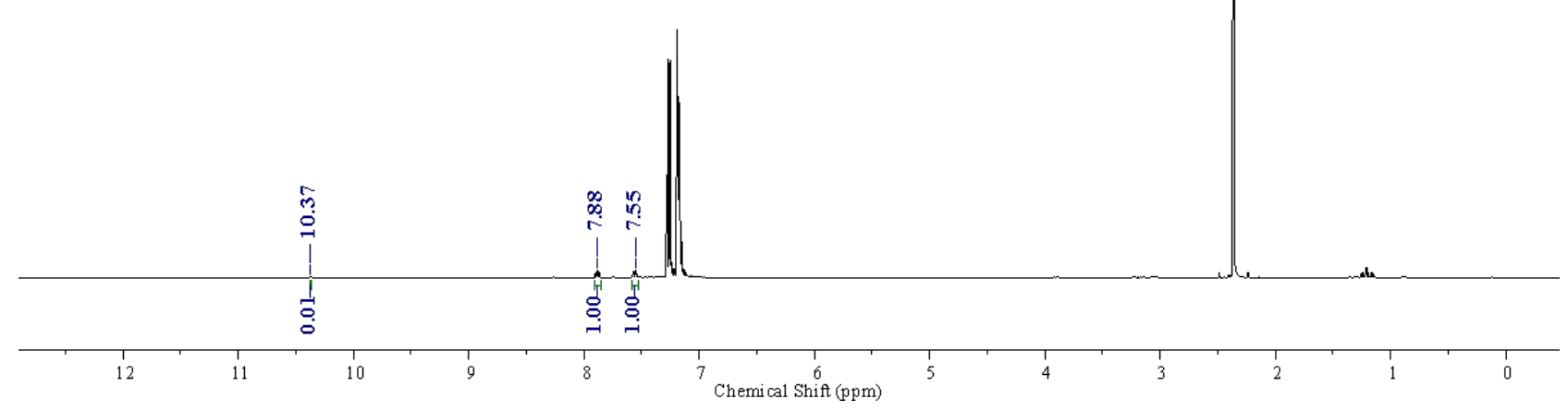


Entry 4': NMR for Crude reaction mixture, Duplicate VDOS-425-B 2-2.7.fid

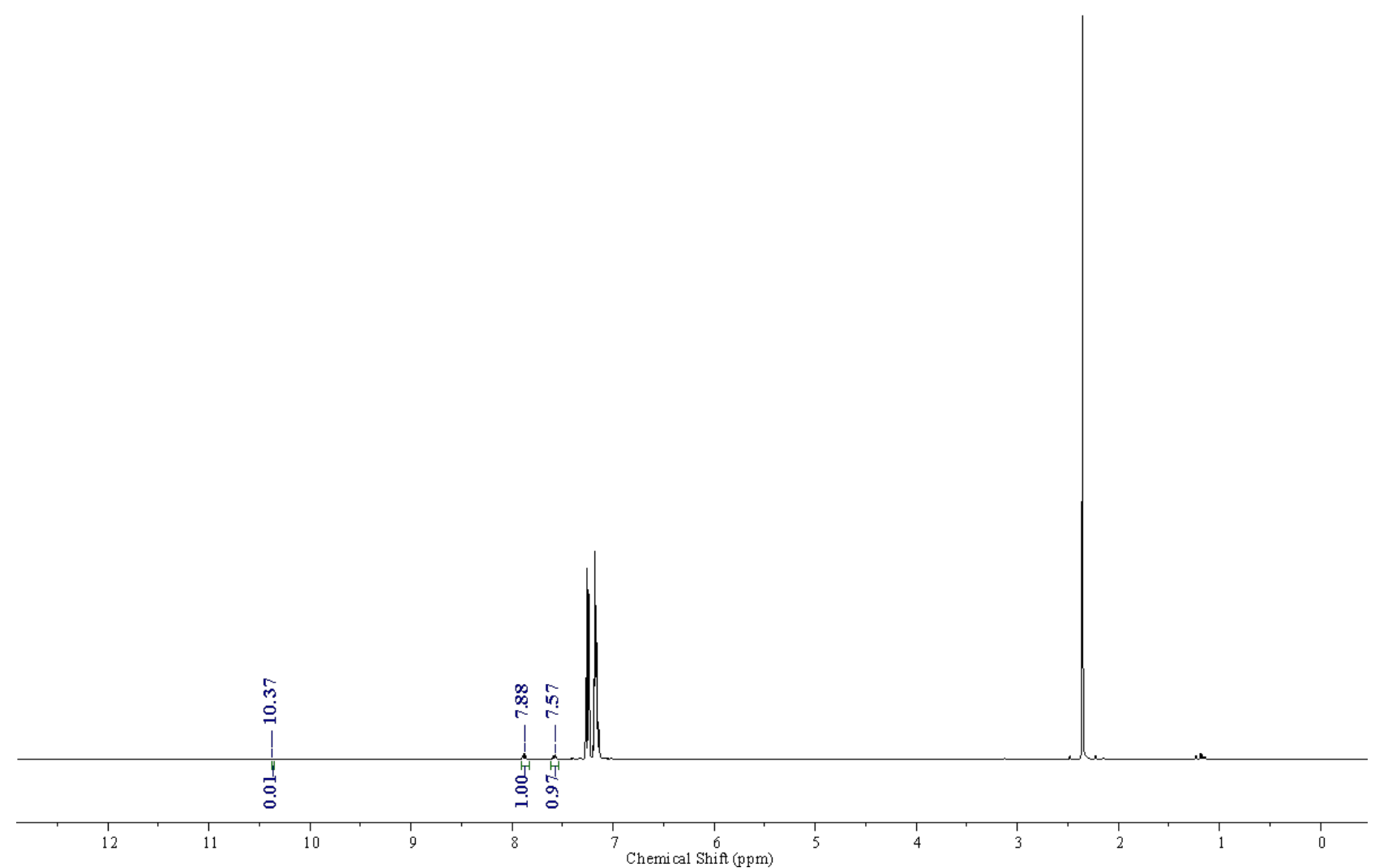

NMR after purification:

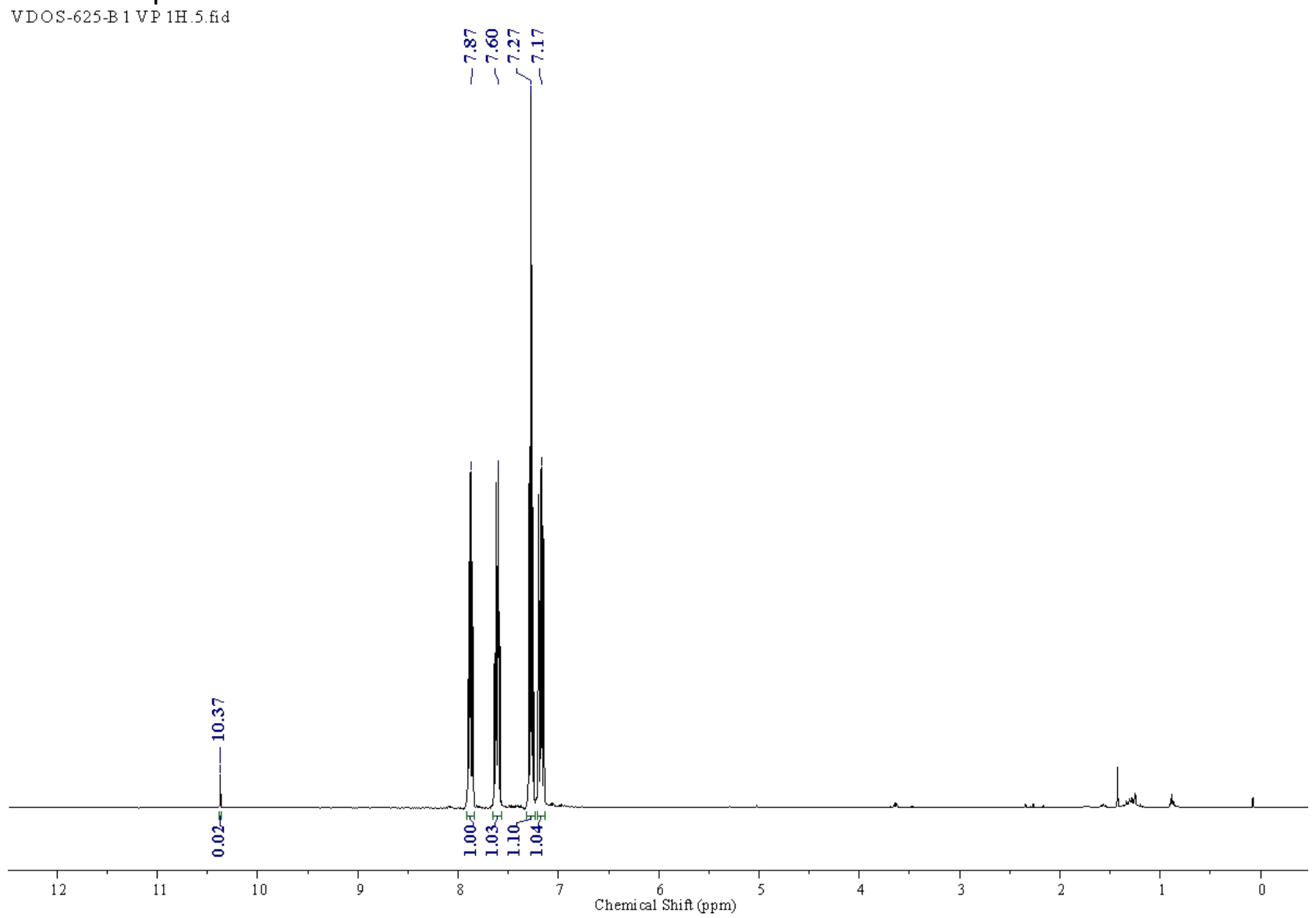




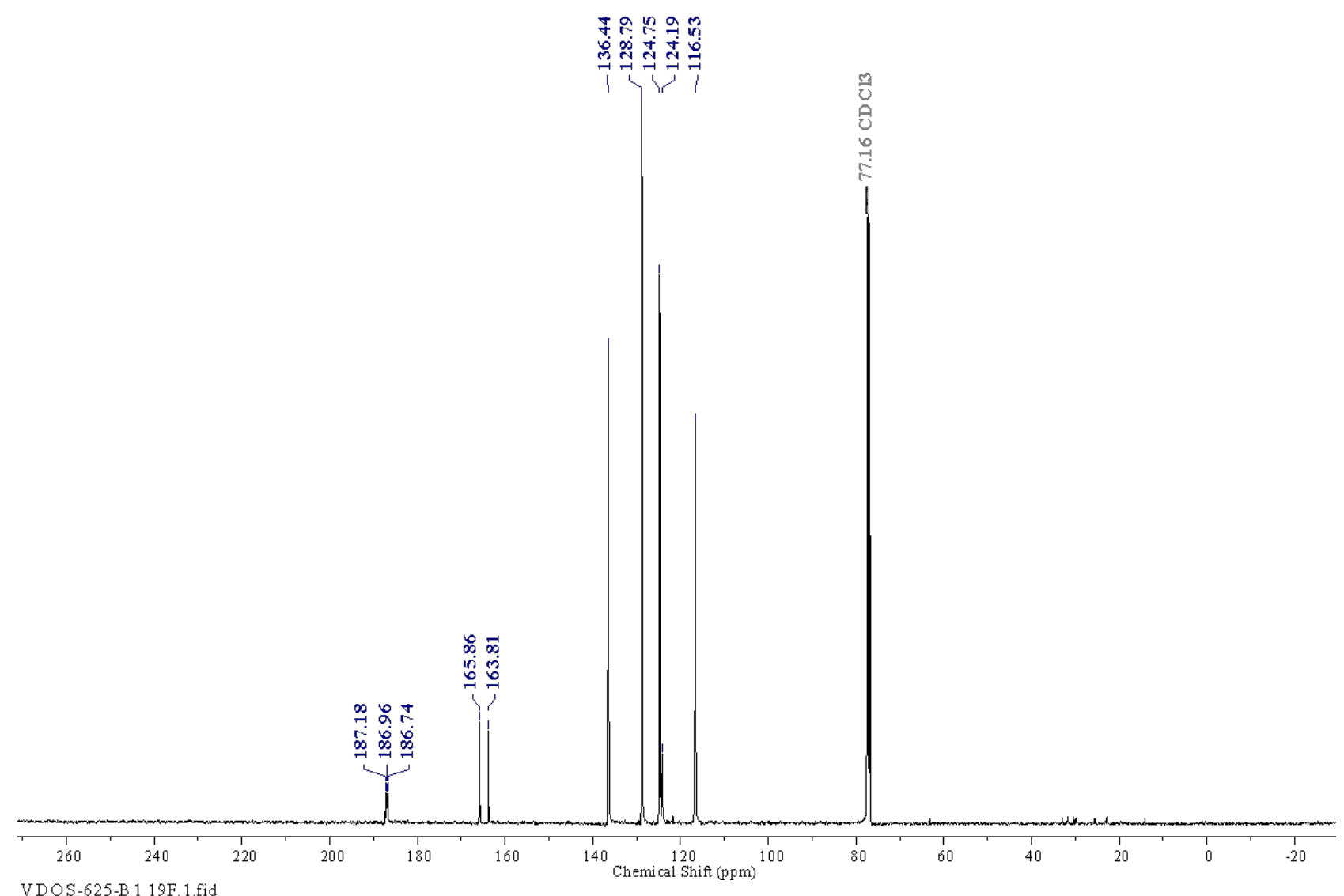

V DOS-625-B 1 19F. 1.fid

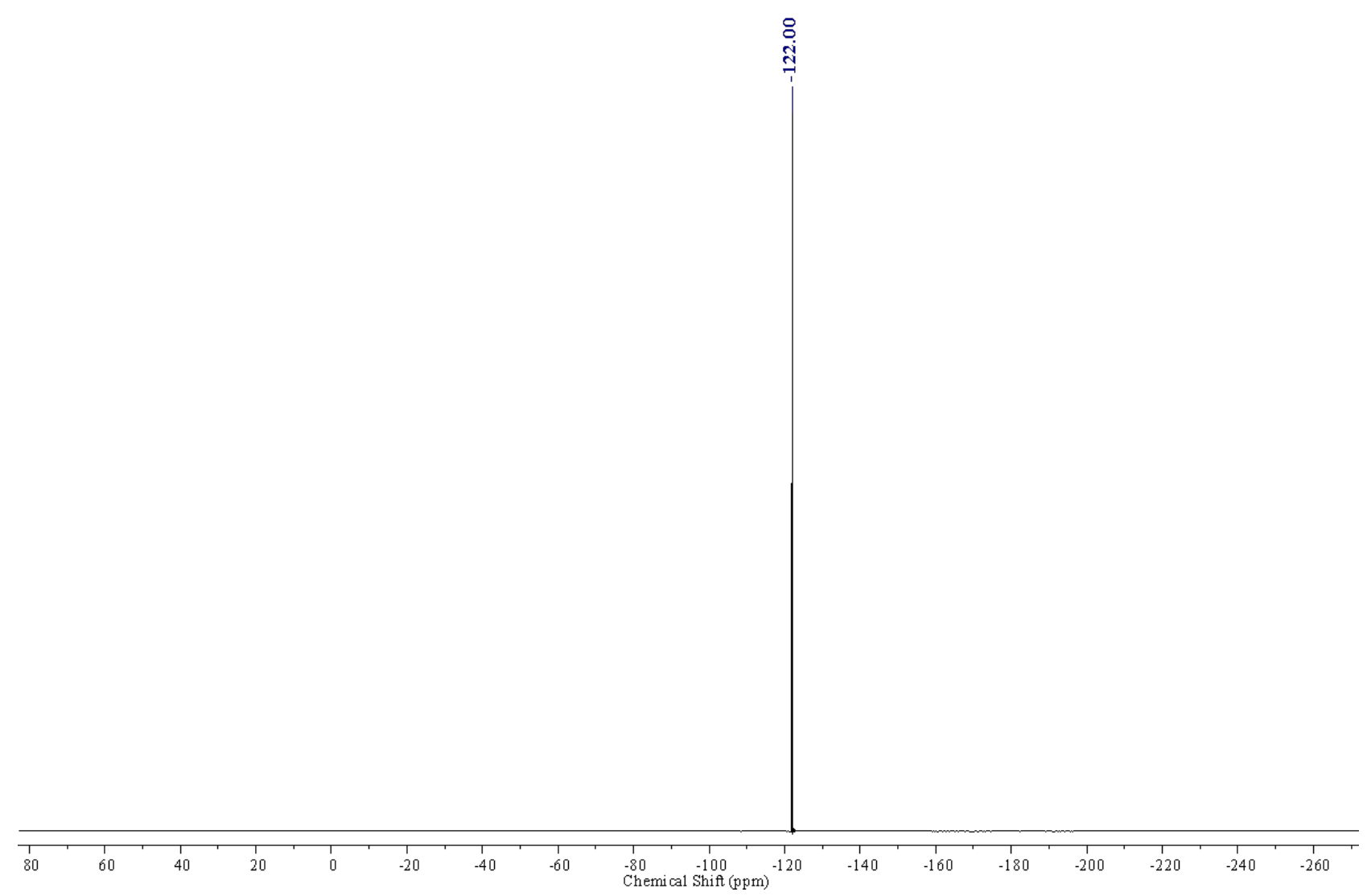


35. 2-Iodobenzaldehyde-d<smiles>[2H]C(=O)c1ccccc1I</smiles>

Entry 1: NMR for Crude reaction mixture SCG-307-M1-16ht-D1-50sec.1. fid

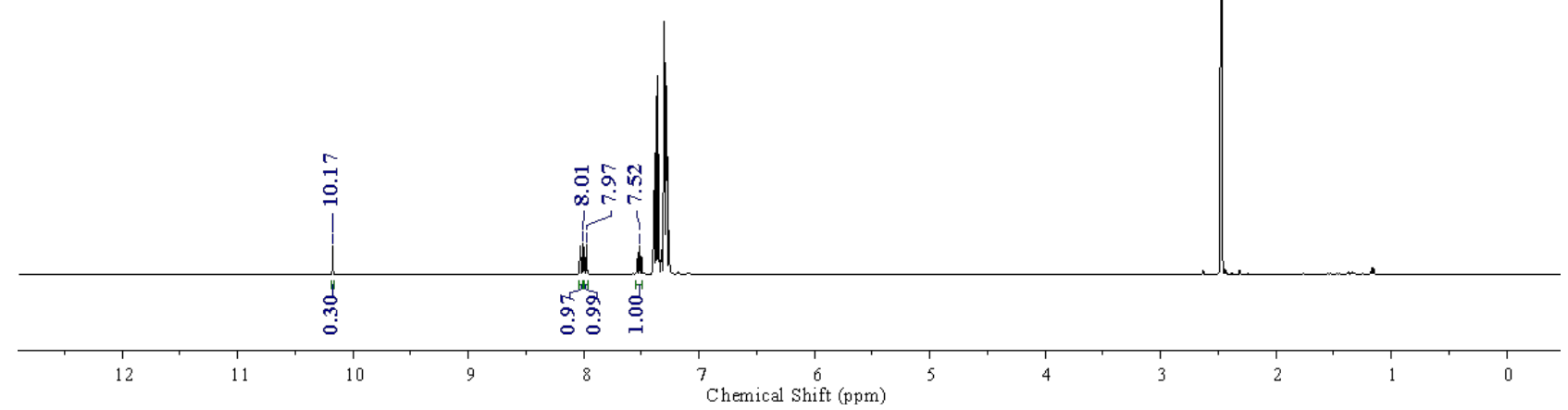


Entry 1': NMR for Crude reaction mixture, Duplicate

SCG-307-M2-16hr-D1-50sec.2. fid

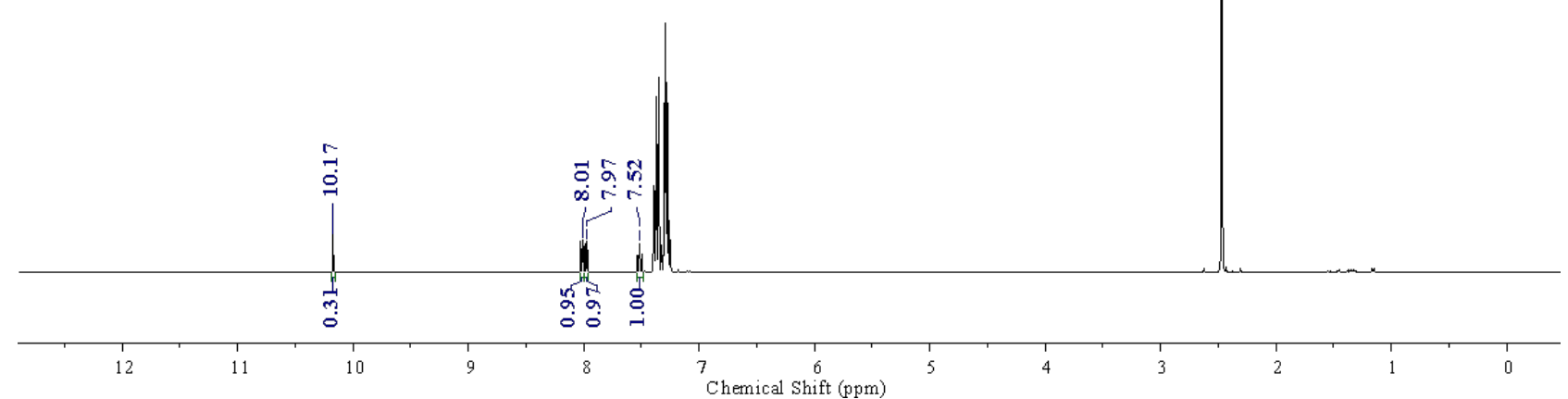

Entry 2: NMR for Crude reaction mixture

VDOS-591-B 1.1. fid

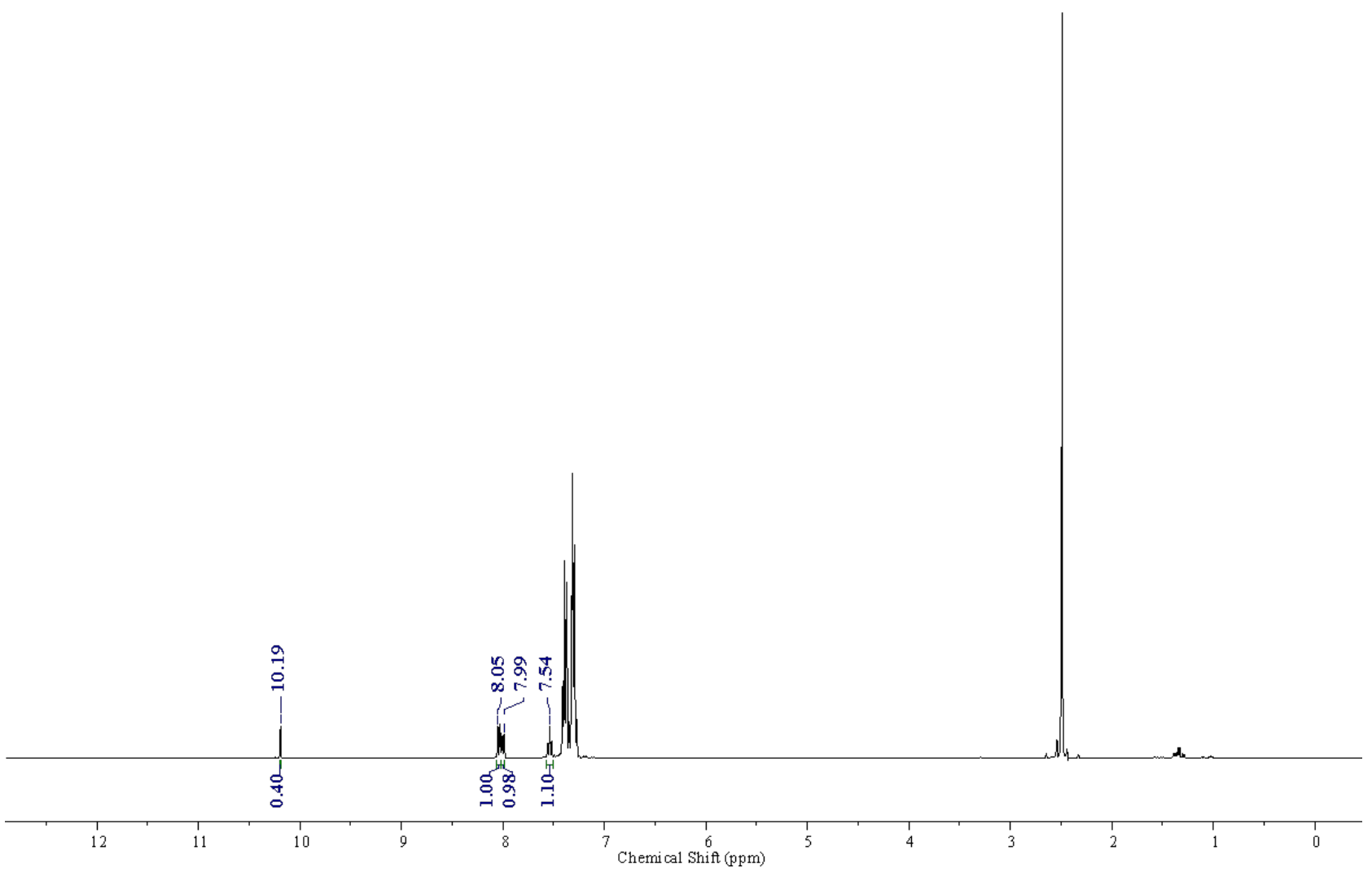


Entry 2': NMR for Crude reaction mixture, Duplicate VDOS-591-B 2.1.fid

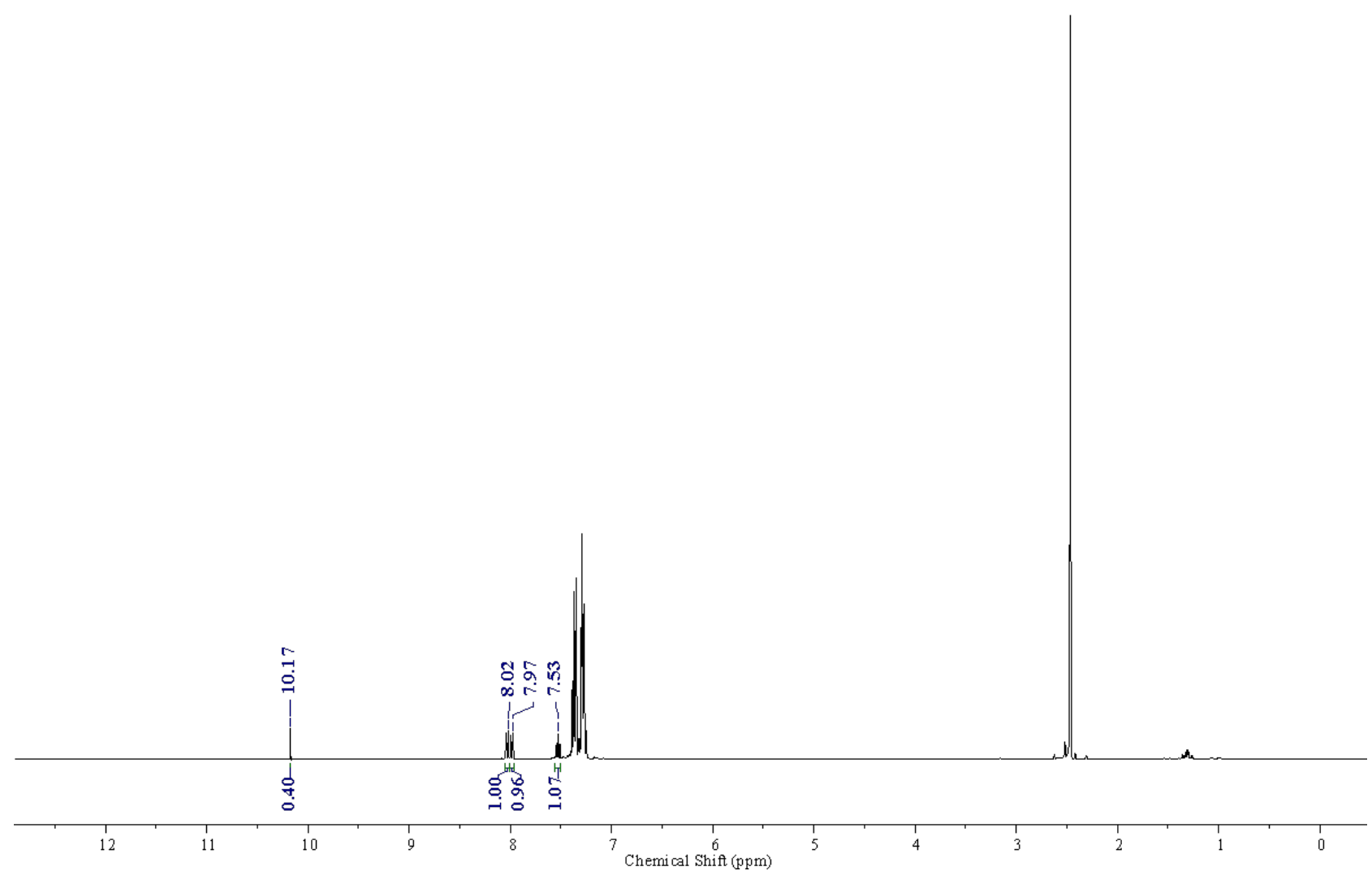

Entry 3: NMR for Crude reaction mixture

VDOS-591-A.2.3.fid

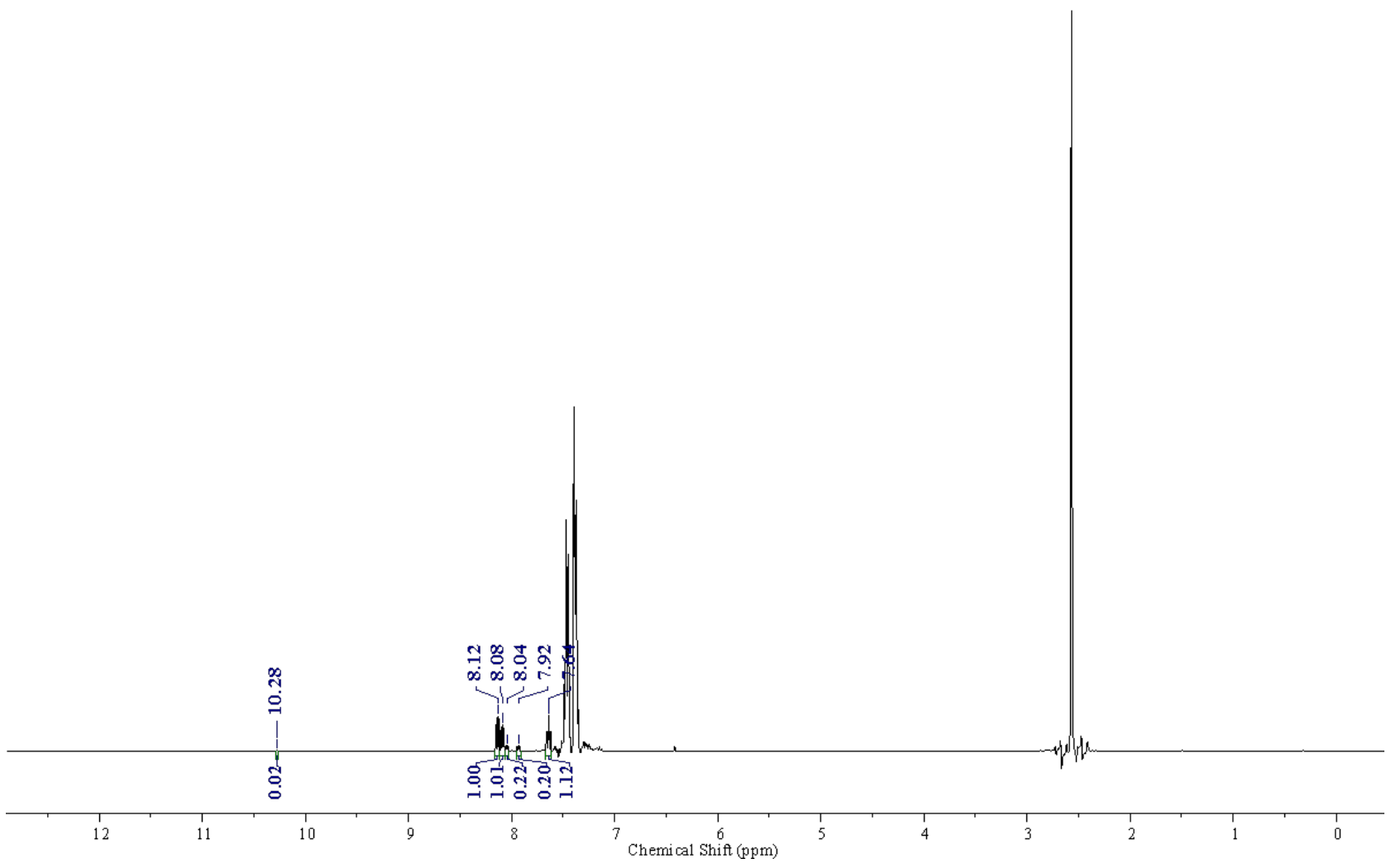


Entry 3': NMR for Crude reaction mixture, Duplicate

VDOS-591-A 1.2. fid

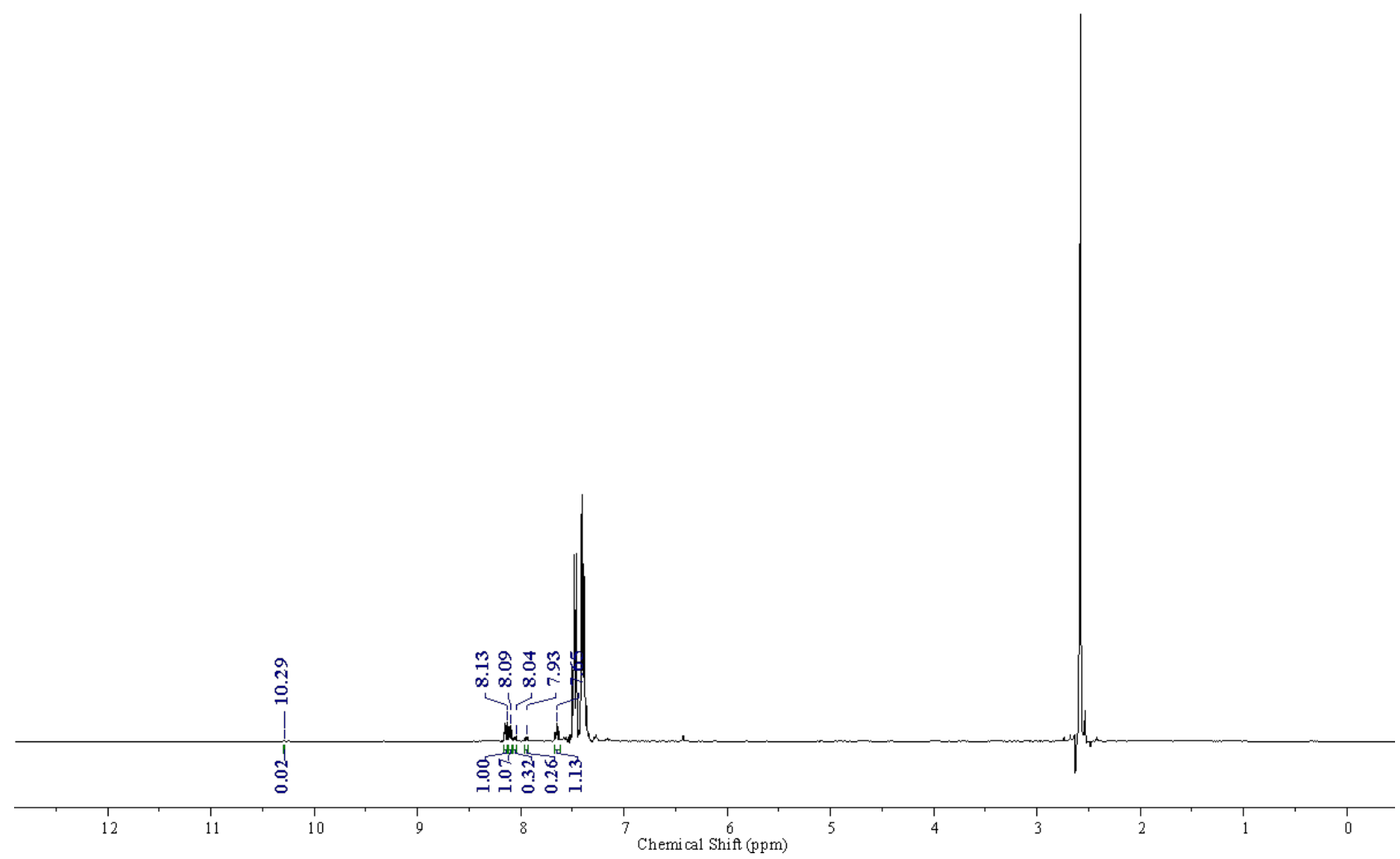

Entry 4: NMR for Crude reaction mixture

VDOS-591-C 1.4.fid

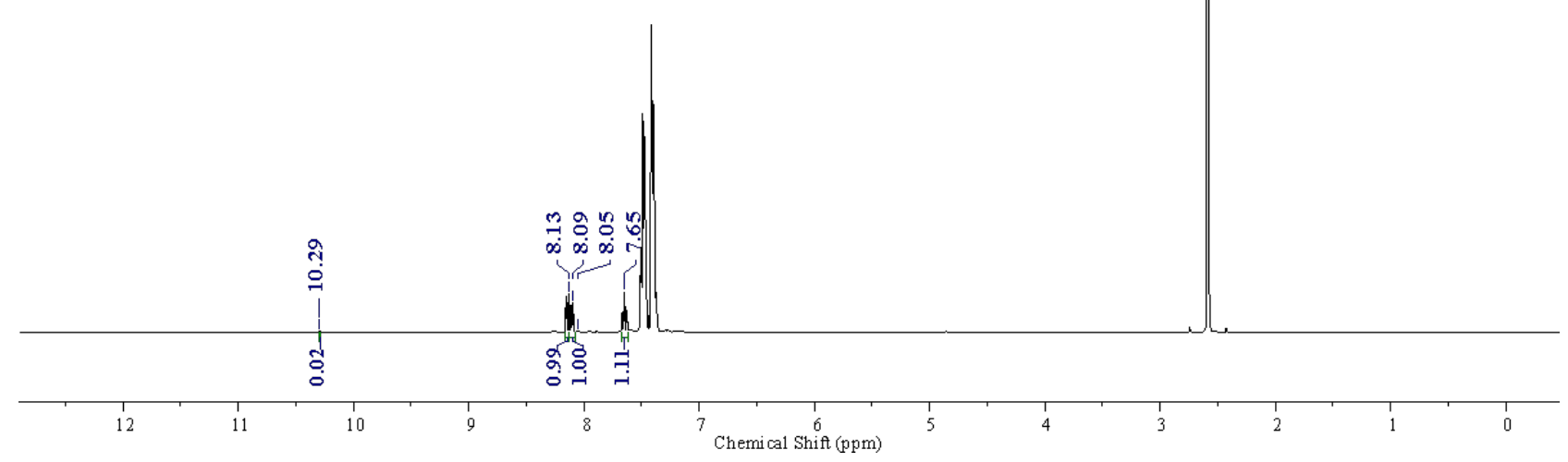


Entry 4': NMR for Crude reaction mixture, Duplicate

VDOS-591-C 2.5.fid

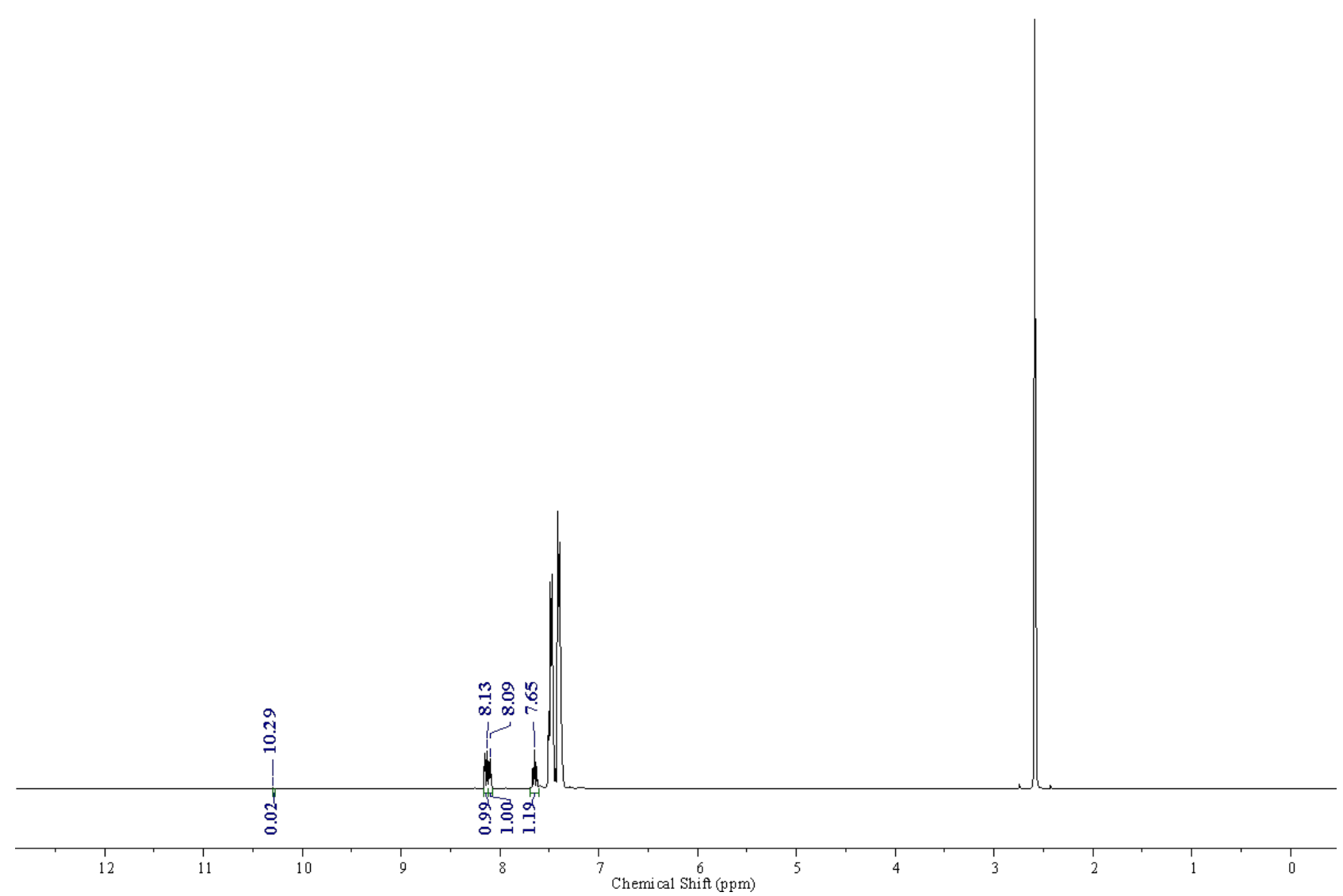

NMR after purification:

VDOS-591 $\mathrm{C} 1$ and $\mathrm{C} 2$ pure.1. fid

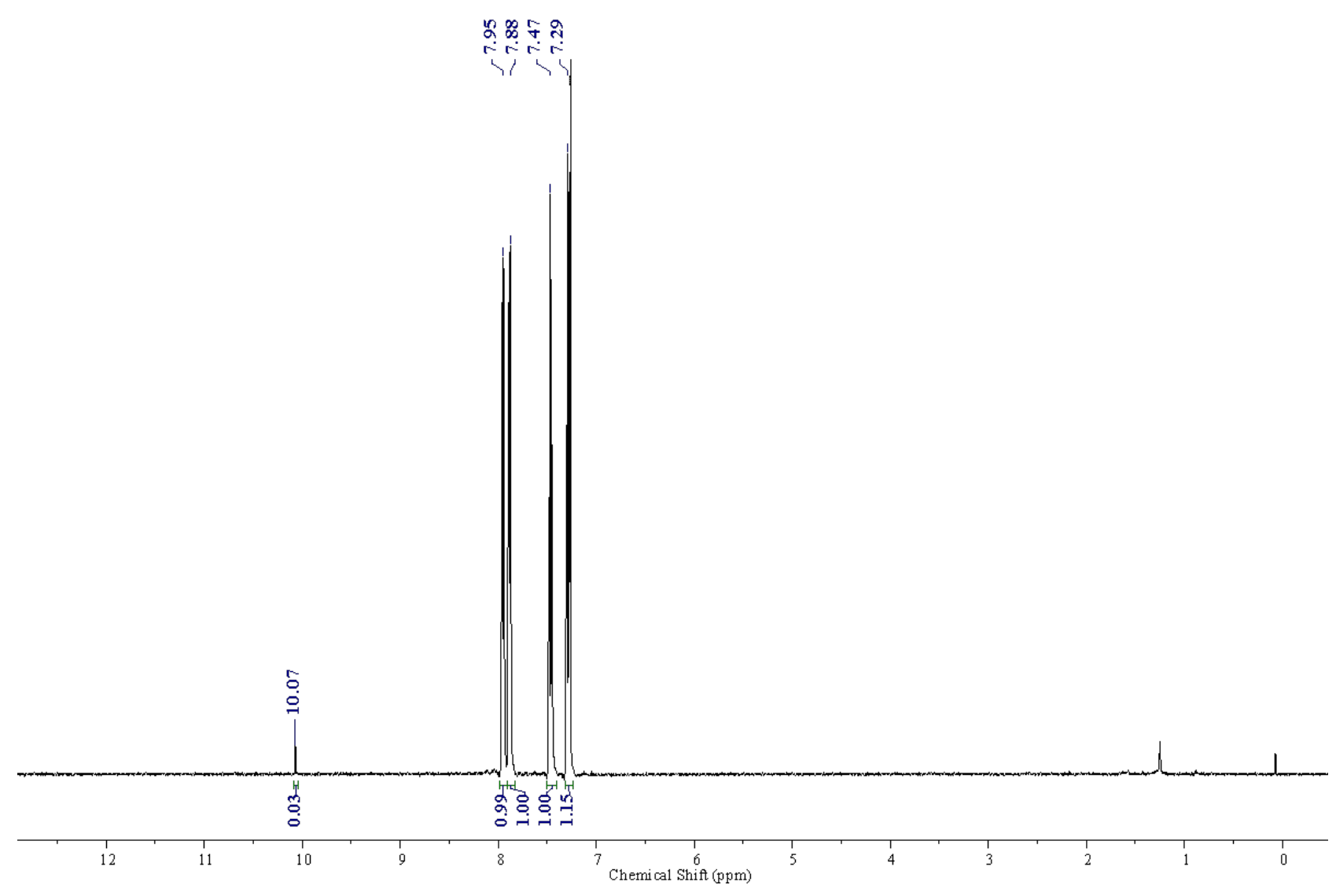


VDOS-591VP-A1-A2-Pure-13C.2.fid

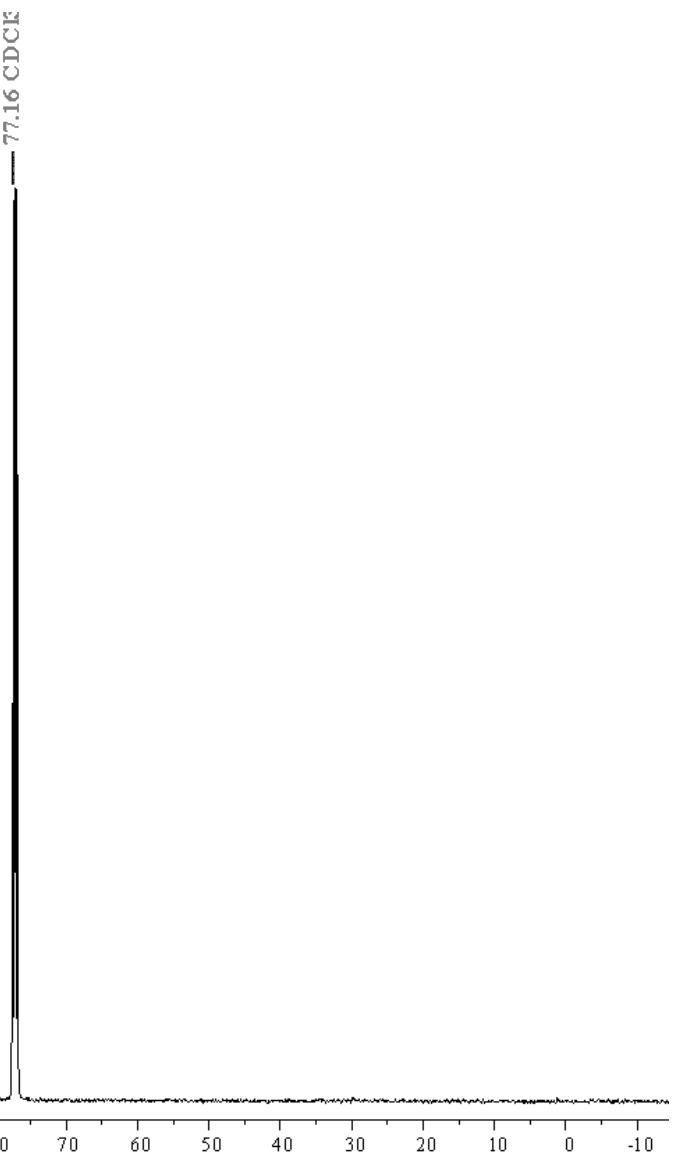

동하웅

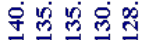

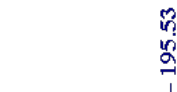

,

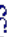

प ।

@

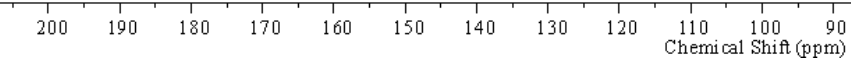


36. 2-" ${ }^{n}$ Butoxybenzaldehyde-d<smiles>[2H]C(=O)c1ccccc1OCCCC</smiles>

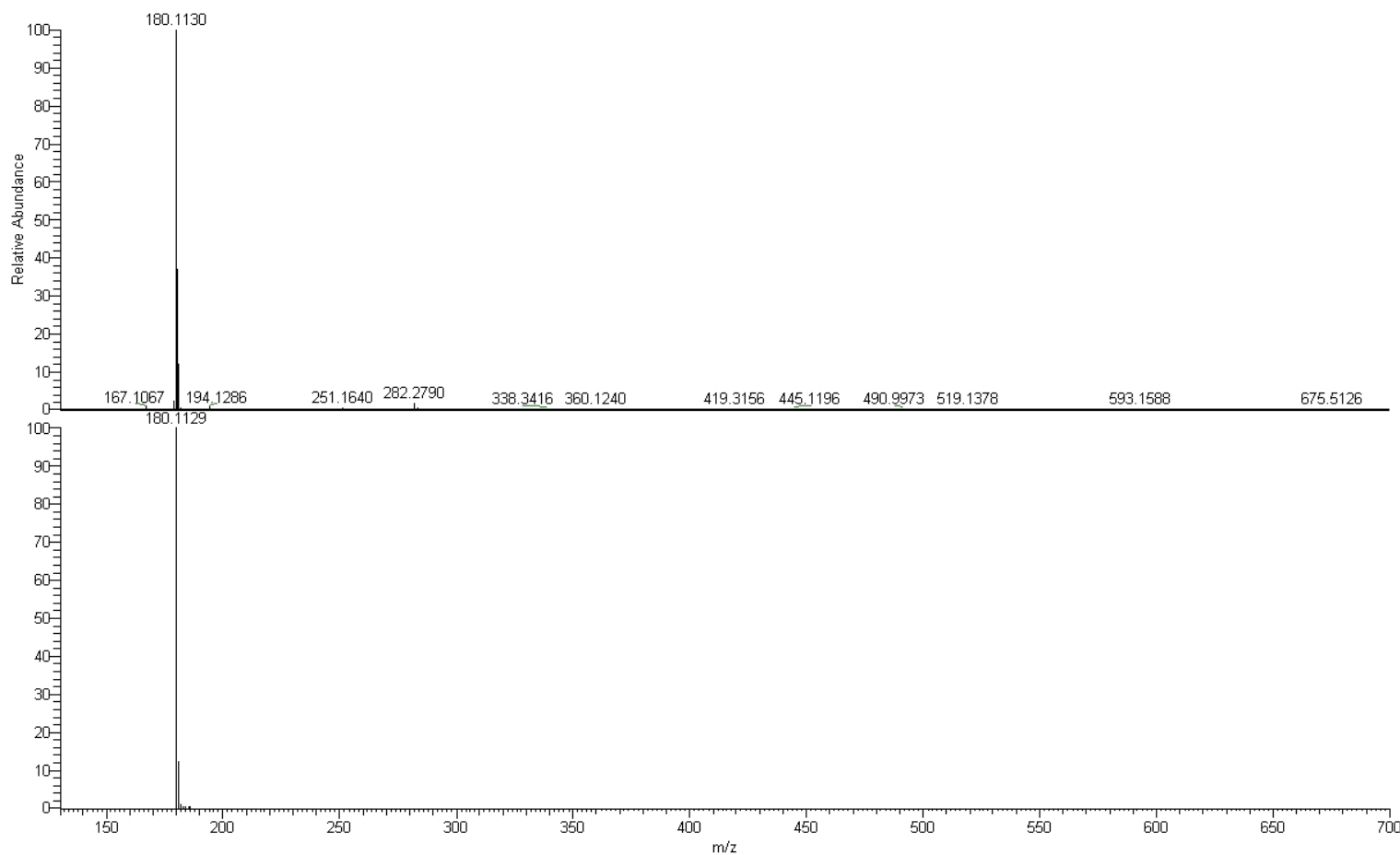

Entry 1: NMR for Crude reaction mixture SCG-348-G1-16hr-D 1-50sec.1.fid

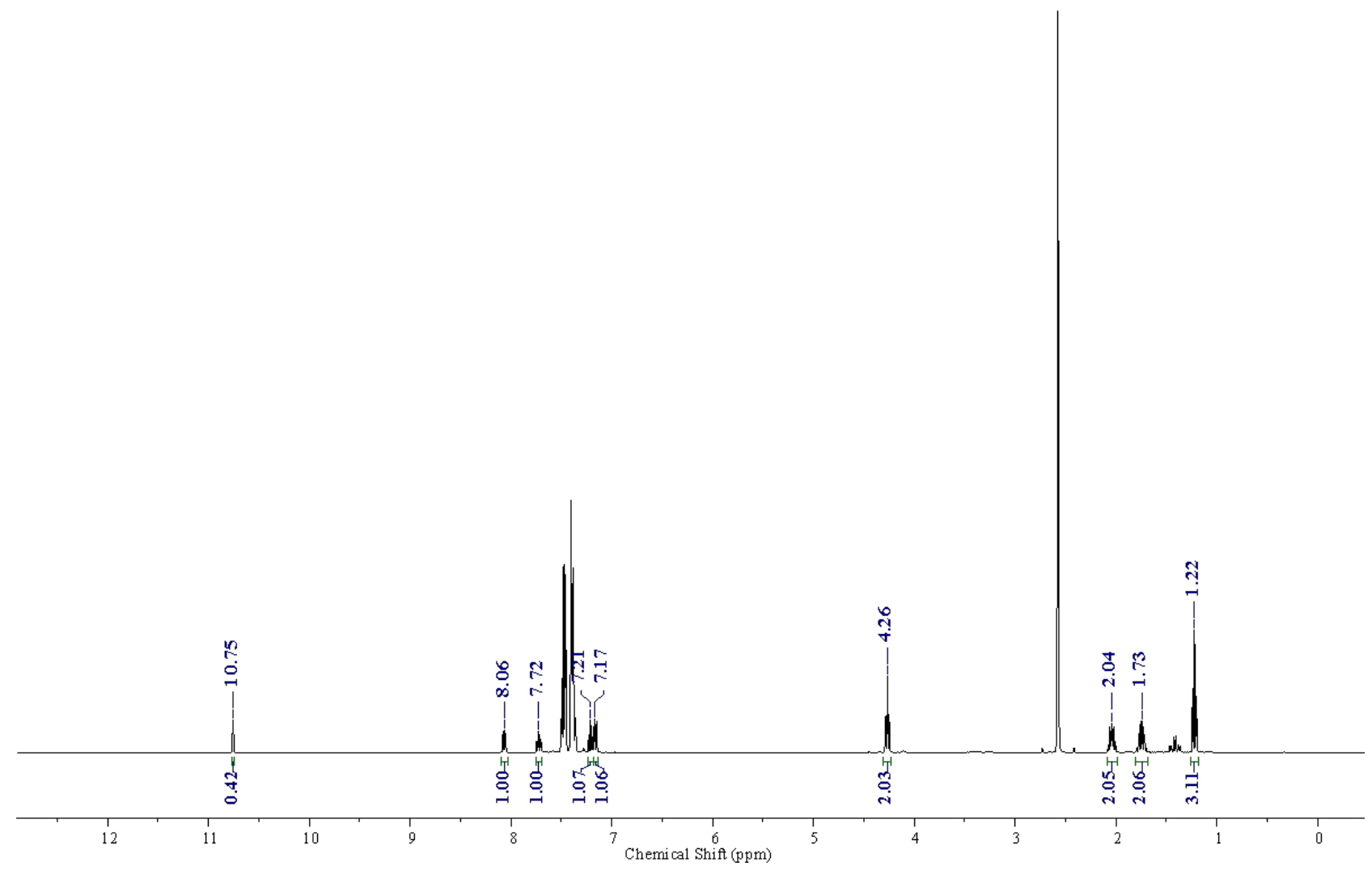


Entry 1': NMR for Crude reaction mixture, Duplicate

SCG-348-G2-16hr-D 1-50sec.1.fid

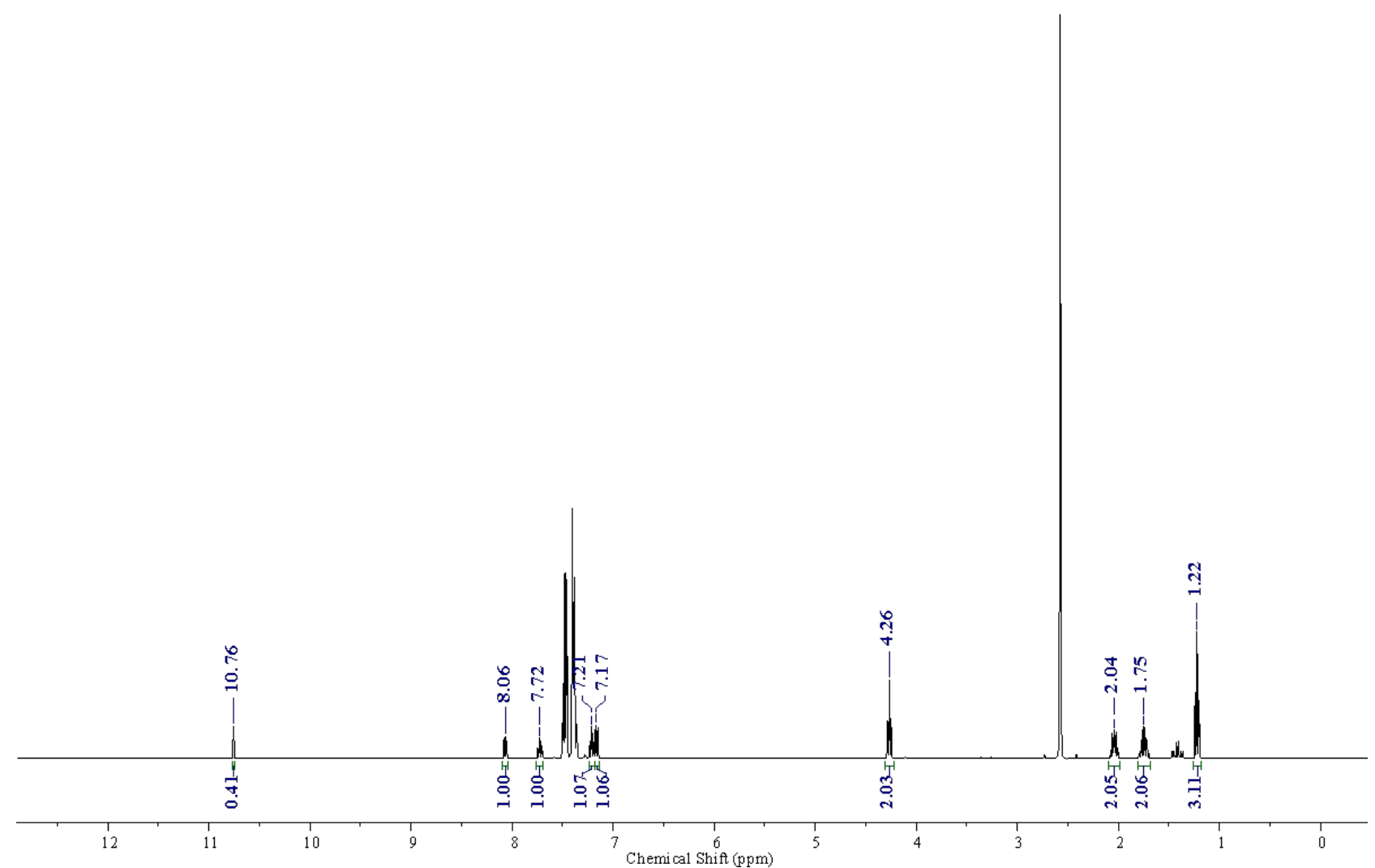

Entry 2: NMR for Crude reaction mixture

SCG-341-I2-16hr-D 1-50sec.1.fid

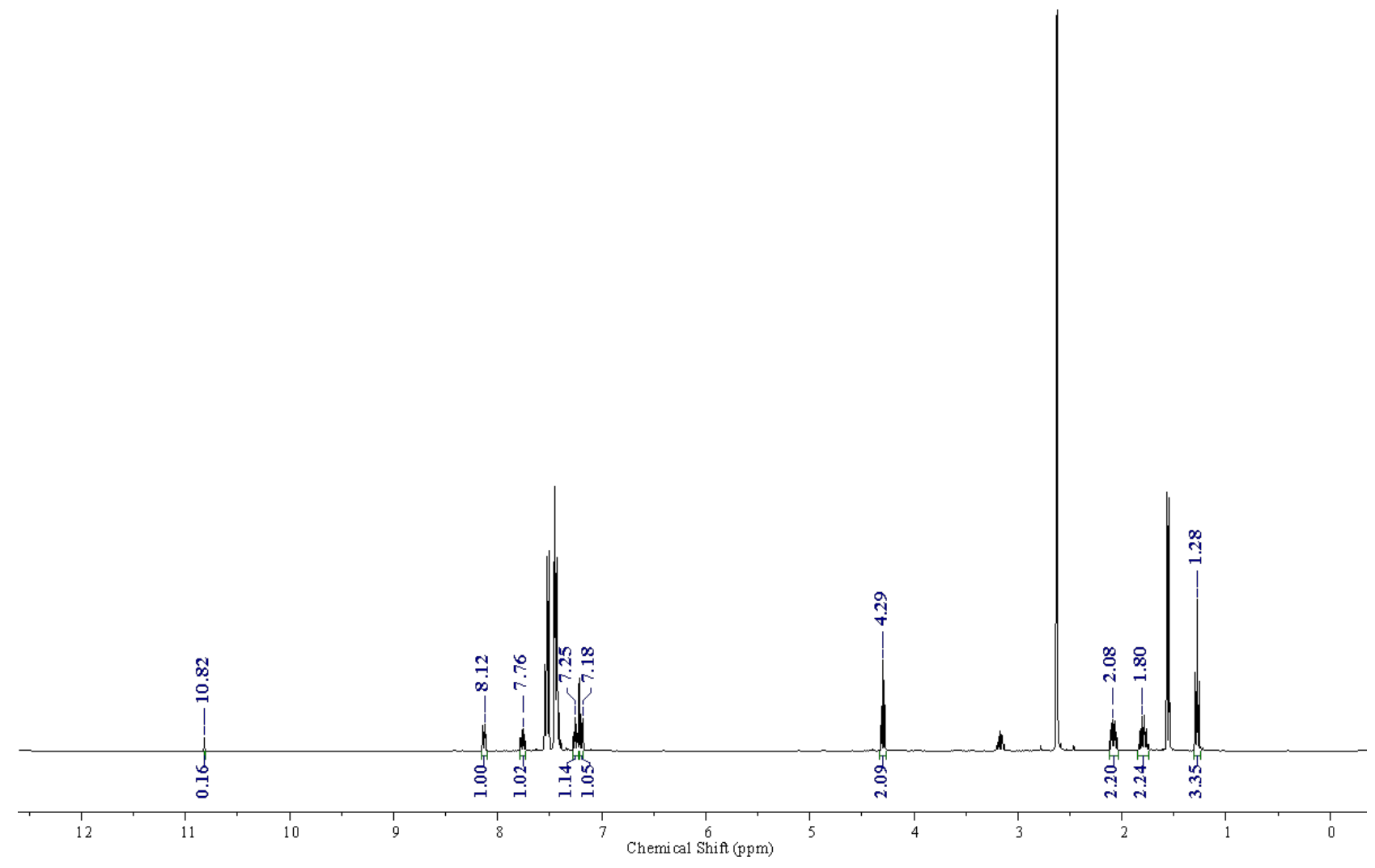


Entry 2': NMR for Crude reaction mixture, Duplicate

SCG-341-I1-16hr-D 1-50sec.1. fid

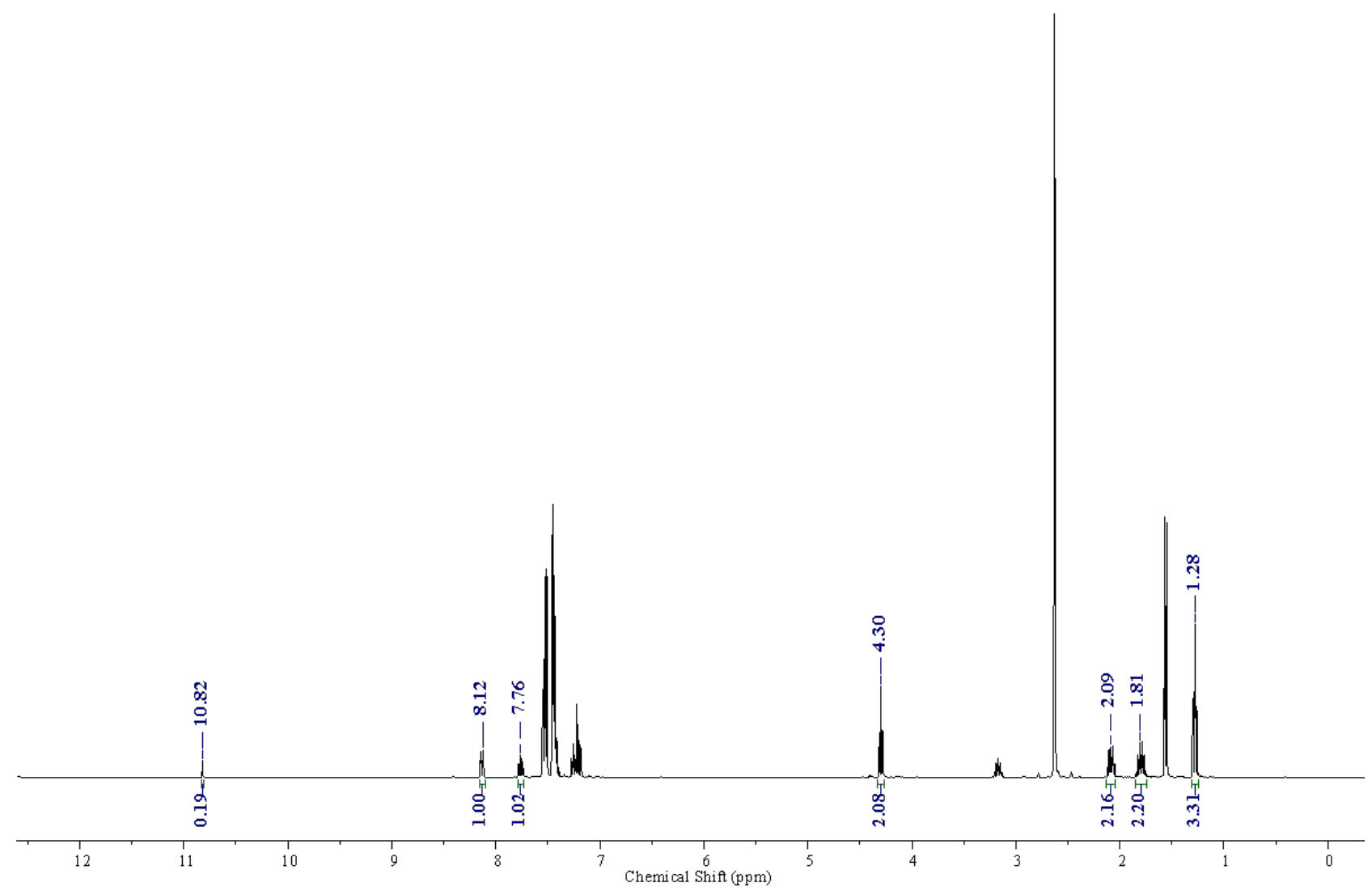

Entry 3: NMR for Crude reaction mixture

VDOS-598-A.1.1. fid

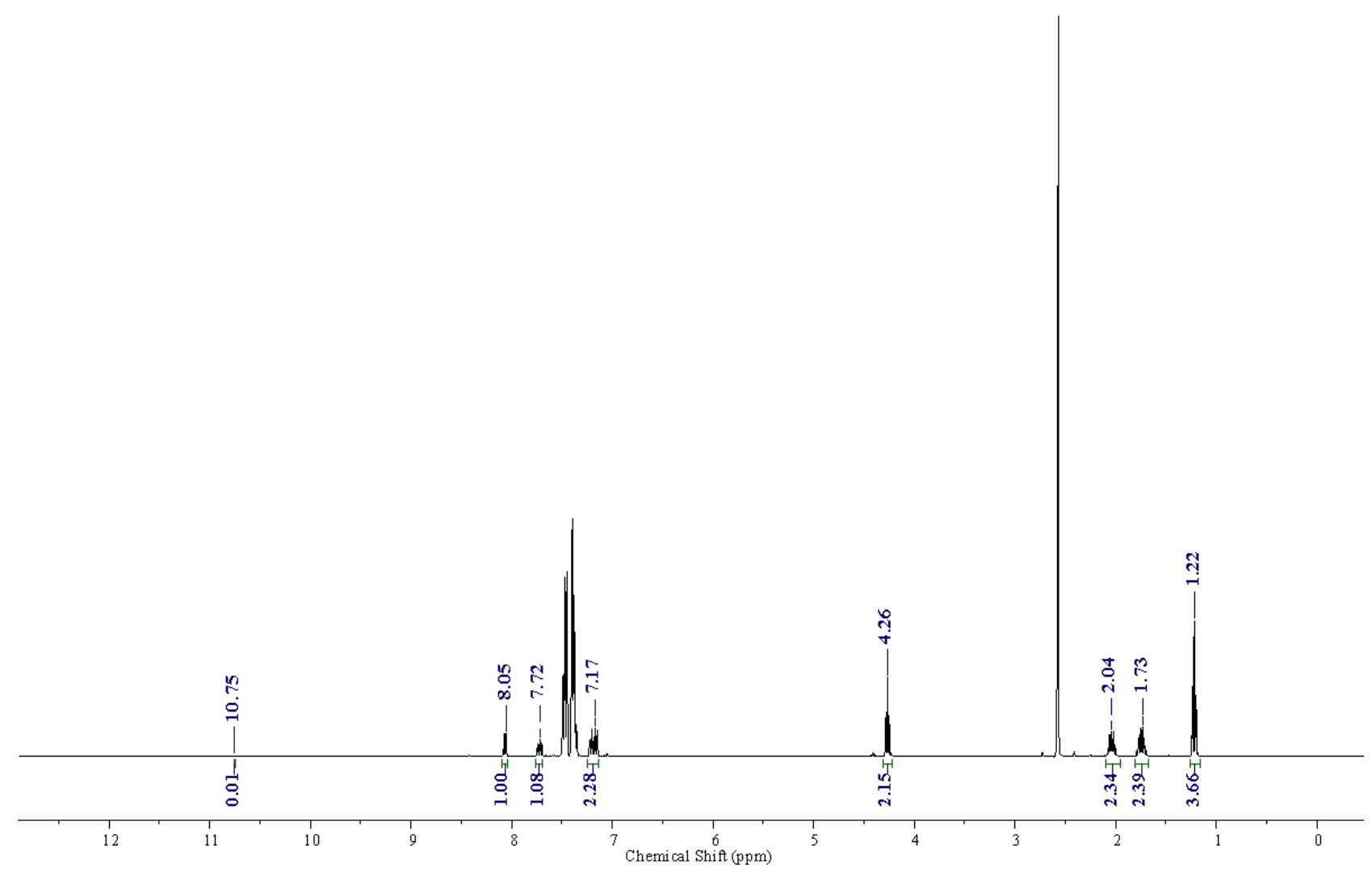


Entry 3': NMR for Crude reaction mixture, Duplicate

VDOS-598-A2.1.fid

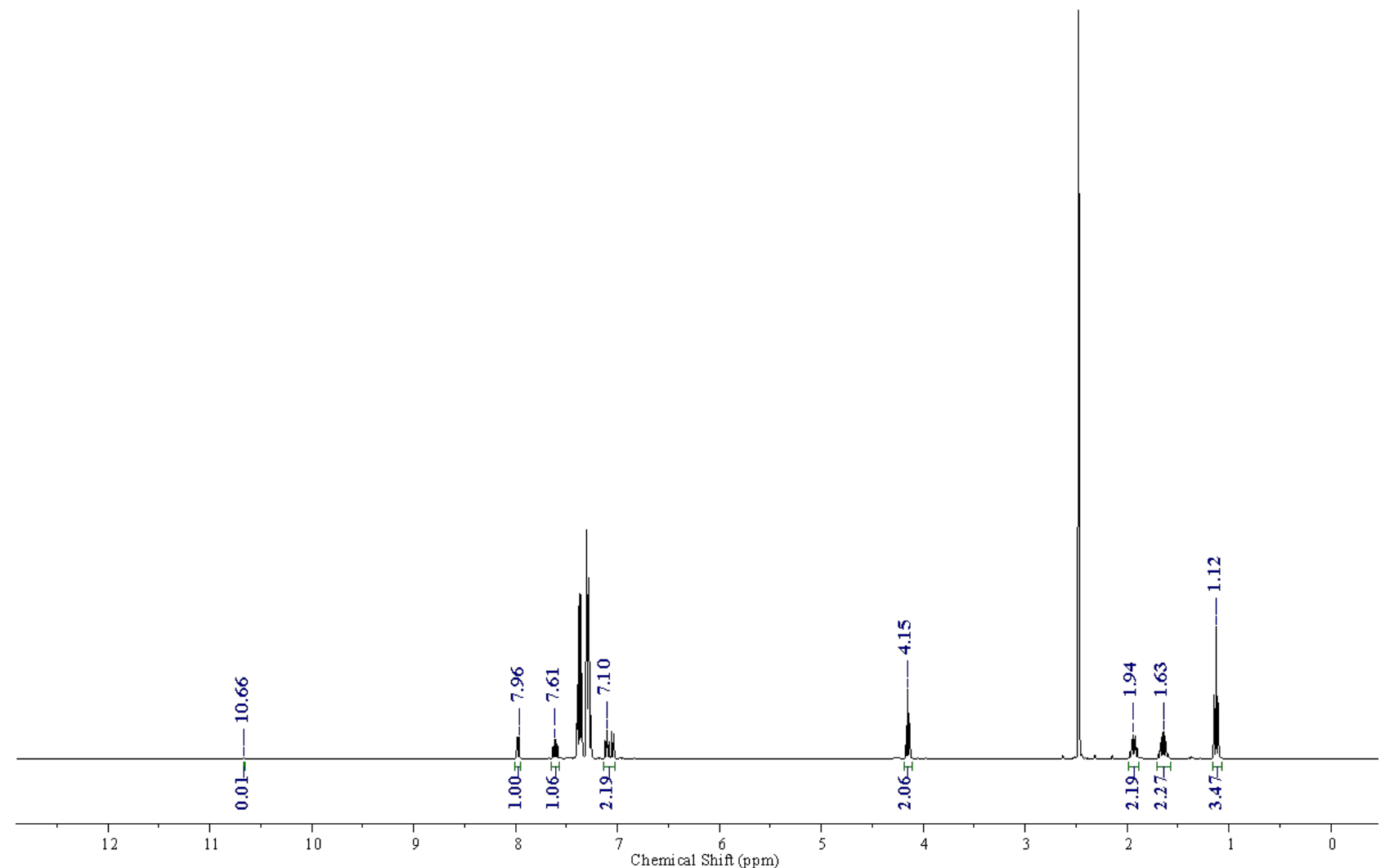

NMR after purification:

VDOS-598-A 3 pure. 4 fid

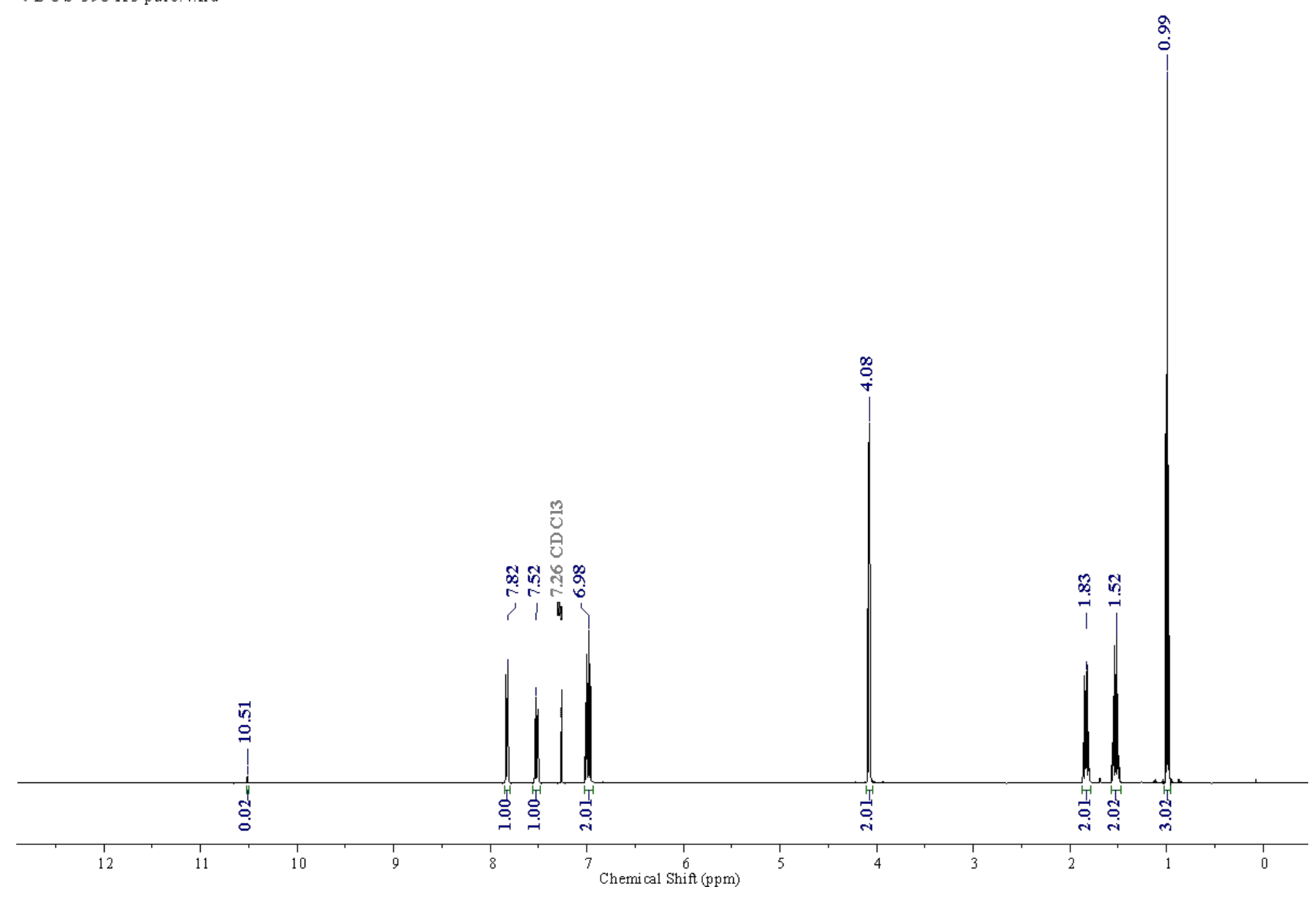


VDOS-598-VP 13C.2.fid

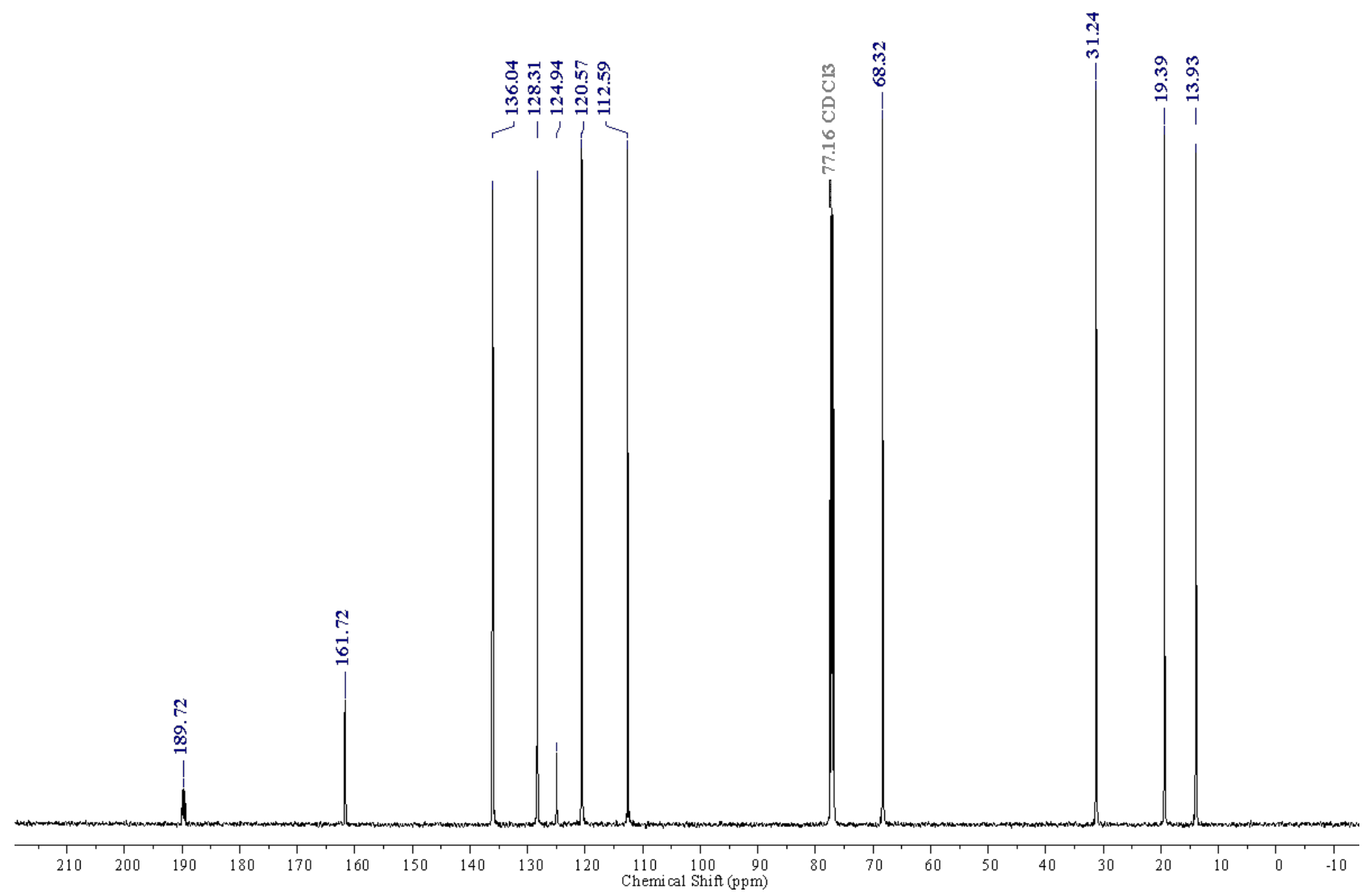

309 
37. 2-(-( ${ }^{\sec B u t o x y)}$ benzaldehyde-d<smiles>[2H]C(=O)c1ccccc1OC(C)CC</smiles>

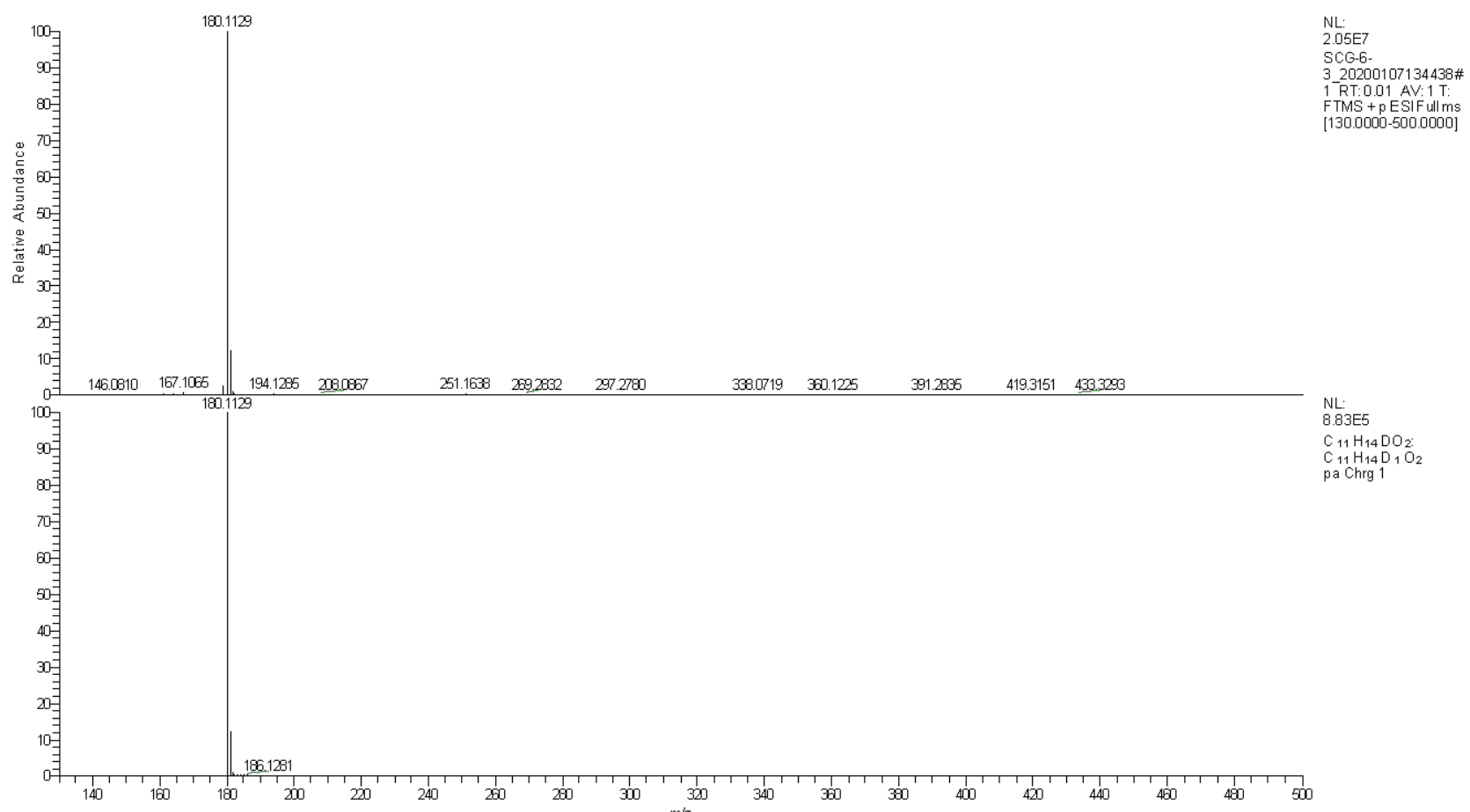

Entry 1: NMR for Crude reaction mixture SCG-348-H1-16hr-D 1-50sec.1.fid

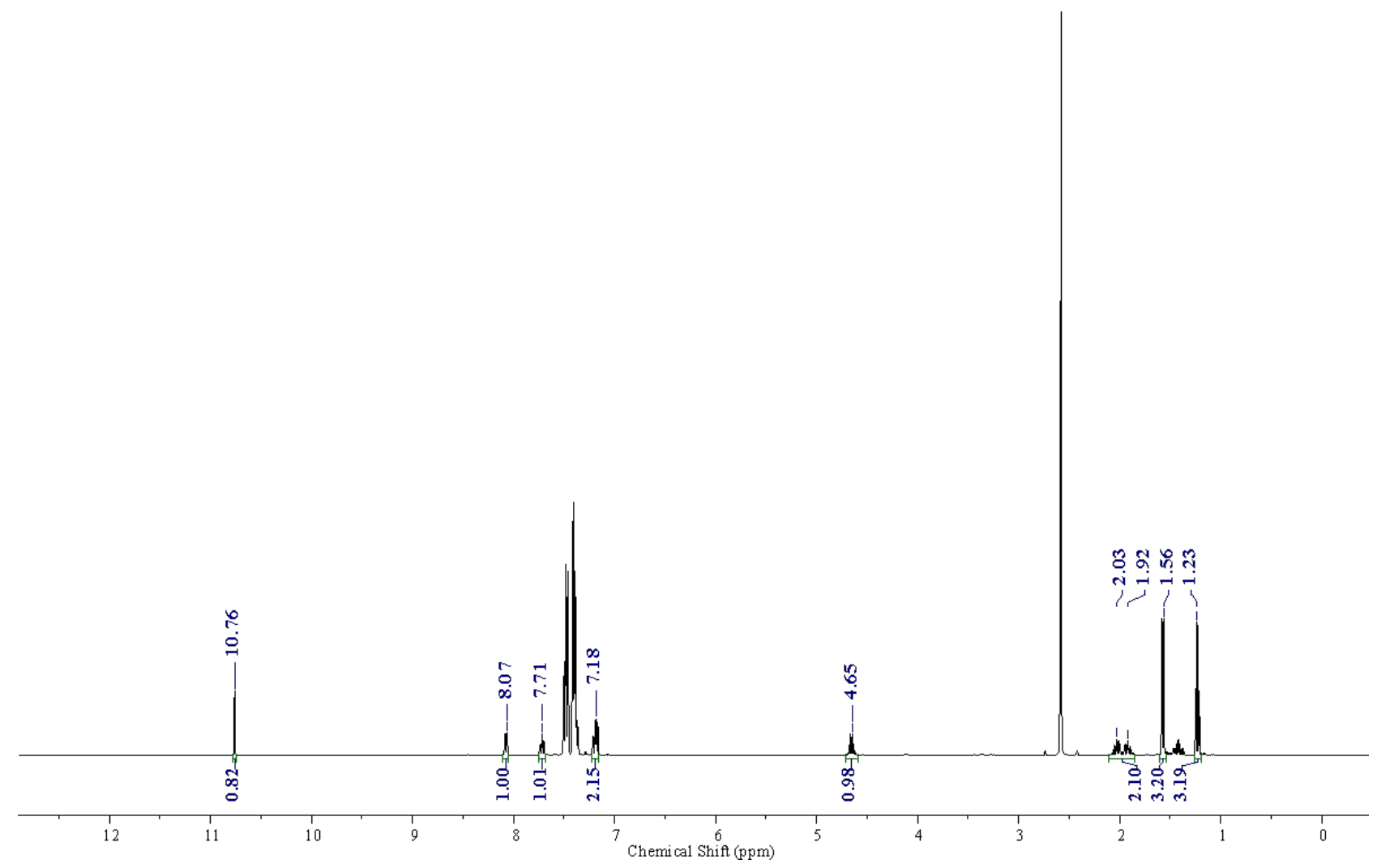


Entry 1': NMR for Crude reaction mixture, Duplicate

SCG-348-H2-16hr-D 1-50sec.1.fid

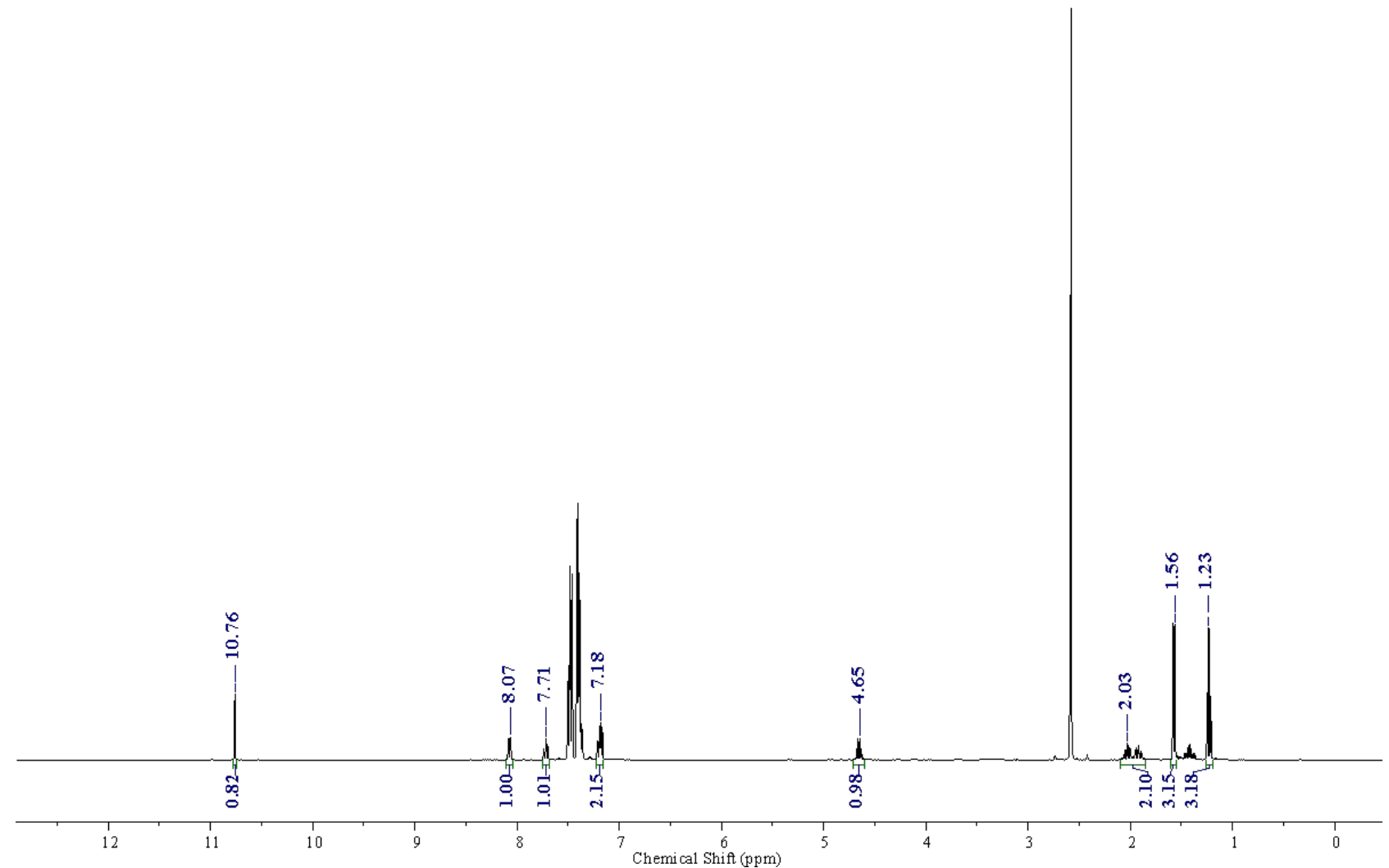

Entry 2: NMR for Crude reaction mixture

VDOS-599-A. 1.2.fid

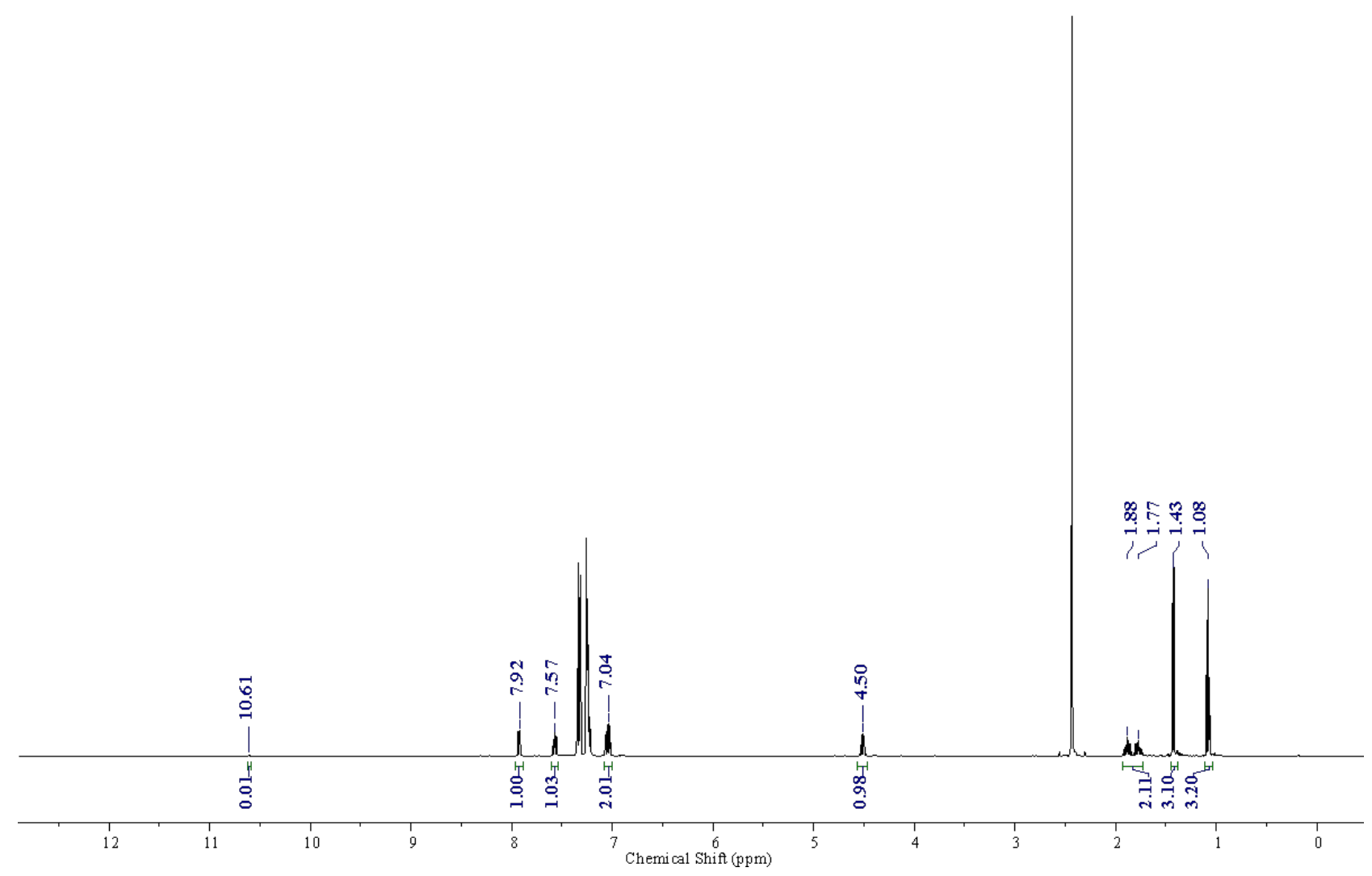


Entry 2': NMR for Crude reaction mixture, Duplicate

VDOS-599-A2.1.fid

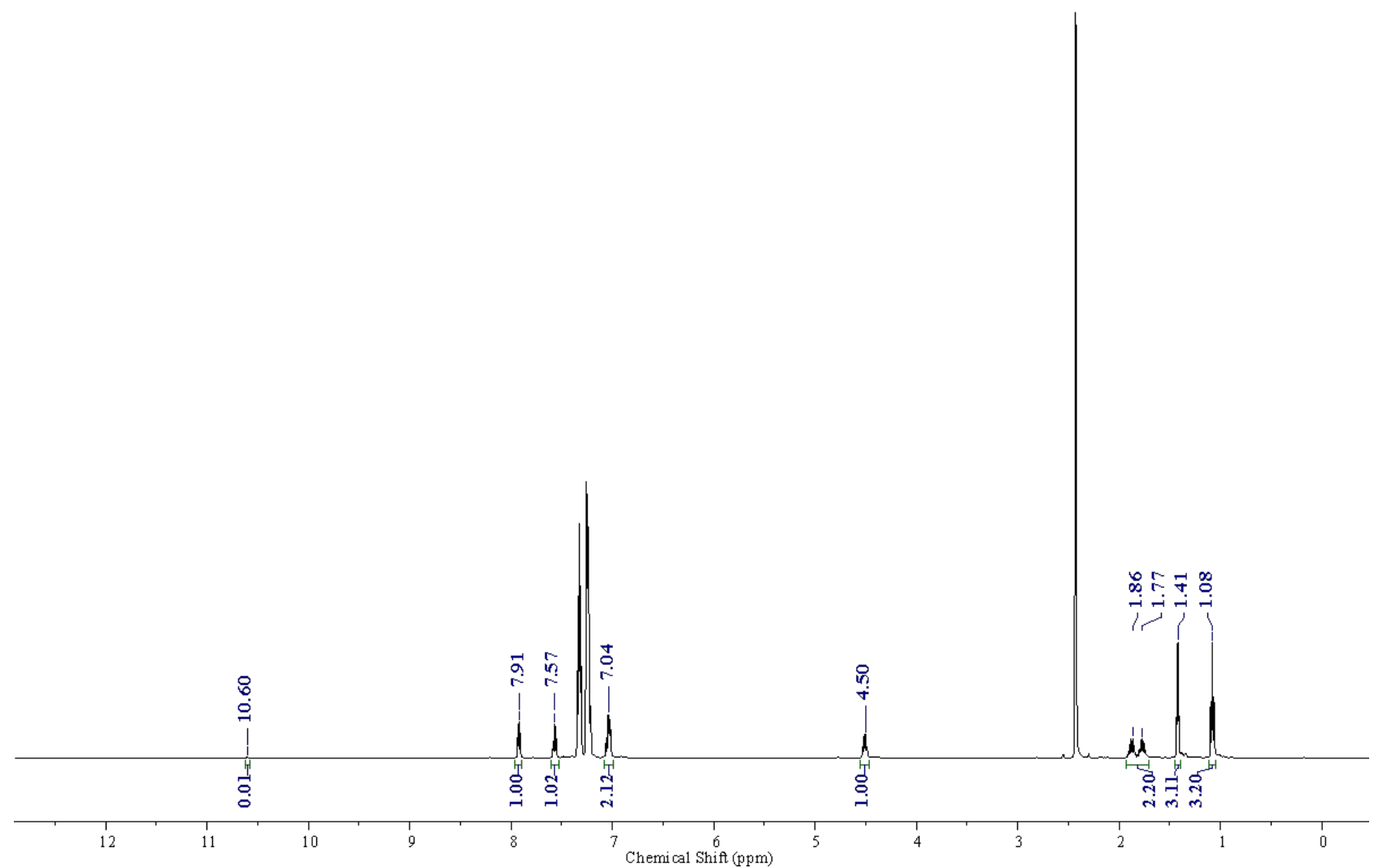

NMR after purification:

VDOS-599-A 1 and A2 pure. 3.fid

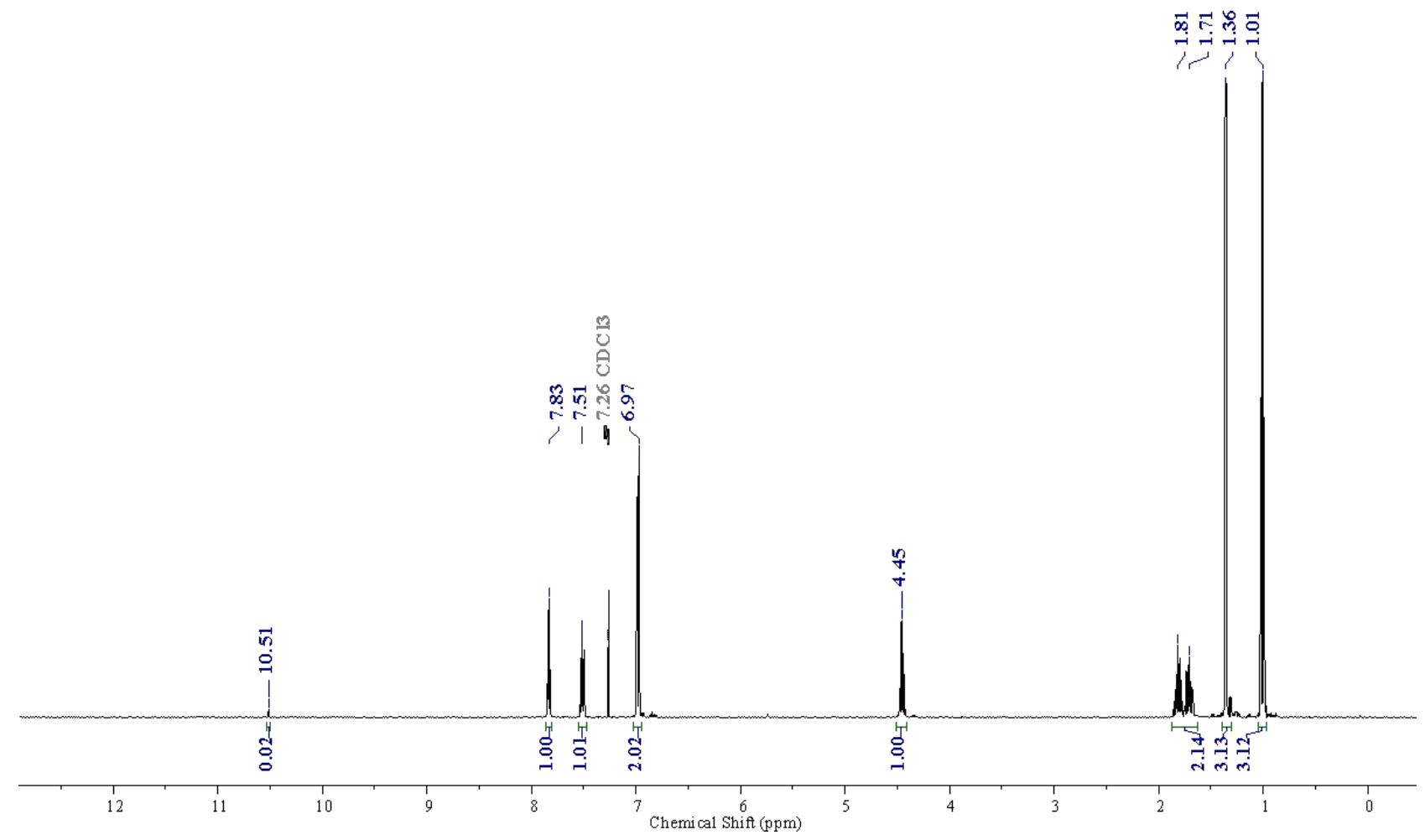




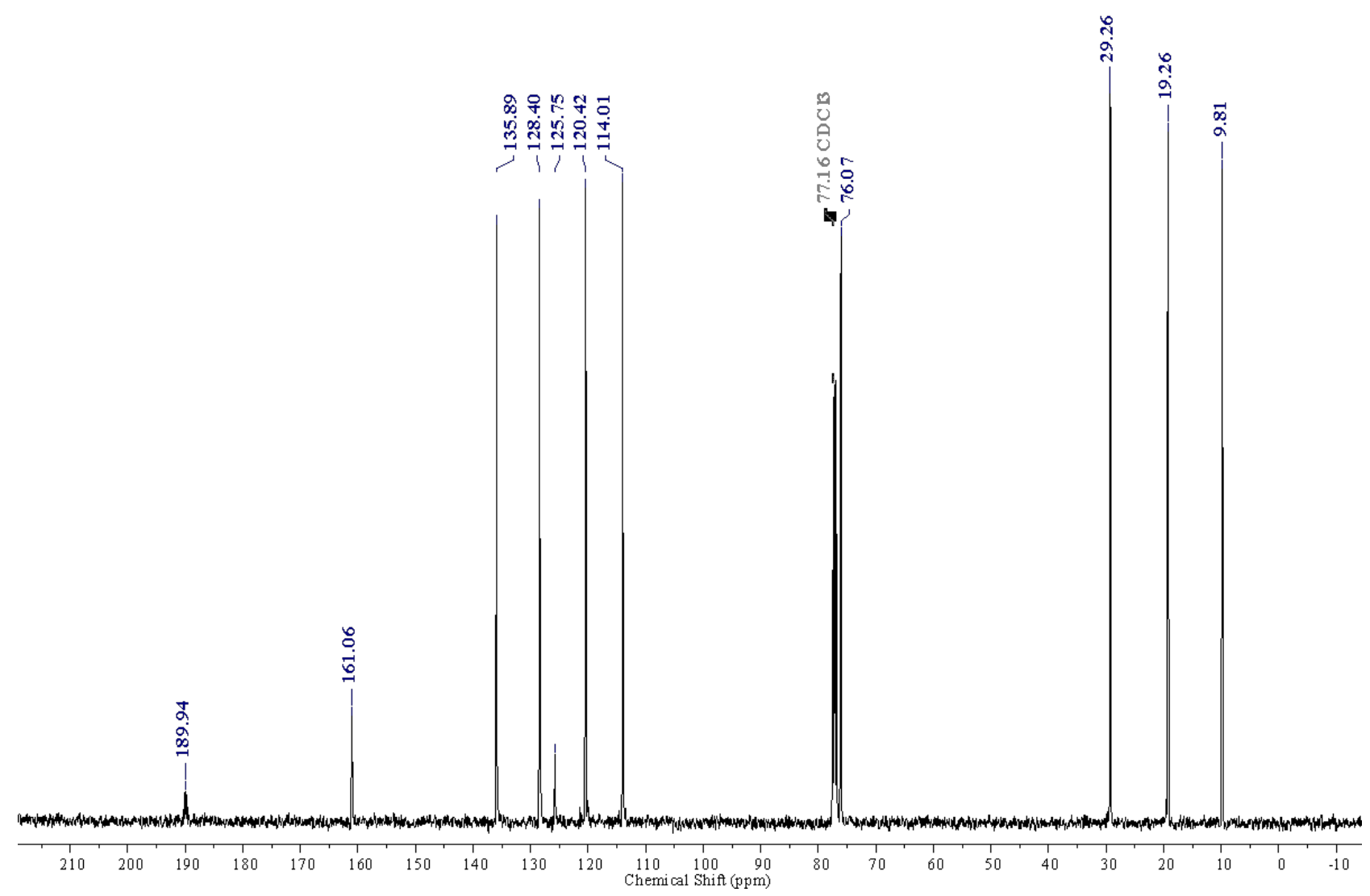


38. [1,1'-biphenyl]-2-carbaldehyde-d<smiles>[2H]C(=O)c1ccccc1-c1ccccc1</smiles>

Entry 1: NMR for Crude reaction mixture SCG-307-N1-16hr-D1-50sec.1.fid

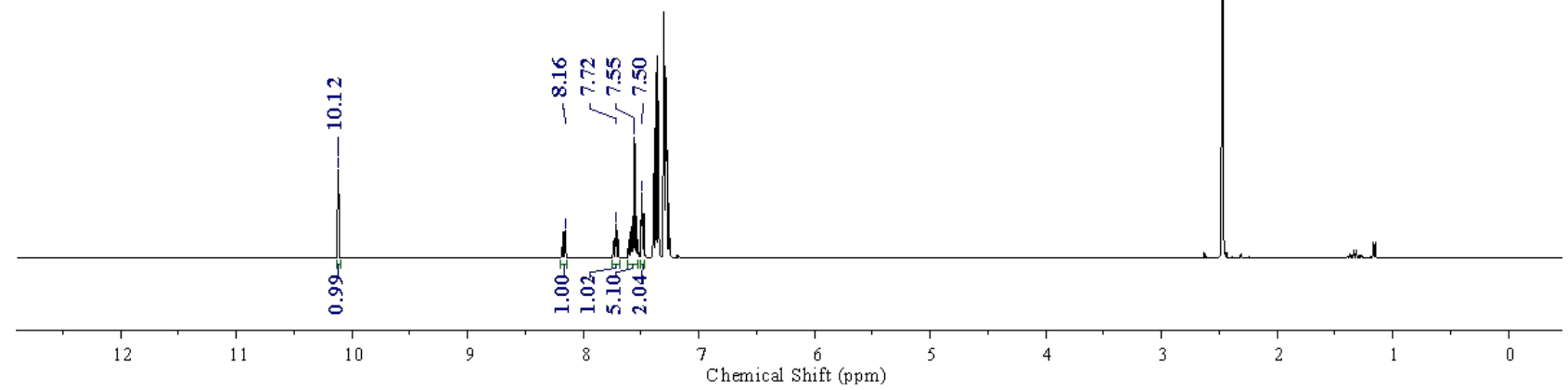


Entry 1': NMR for Crude reaction mixture, Duplicate

SCG-307-N2-16hr-D1-50sec.1. fid

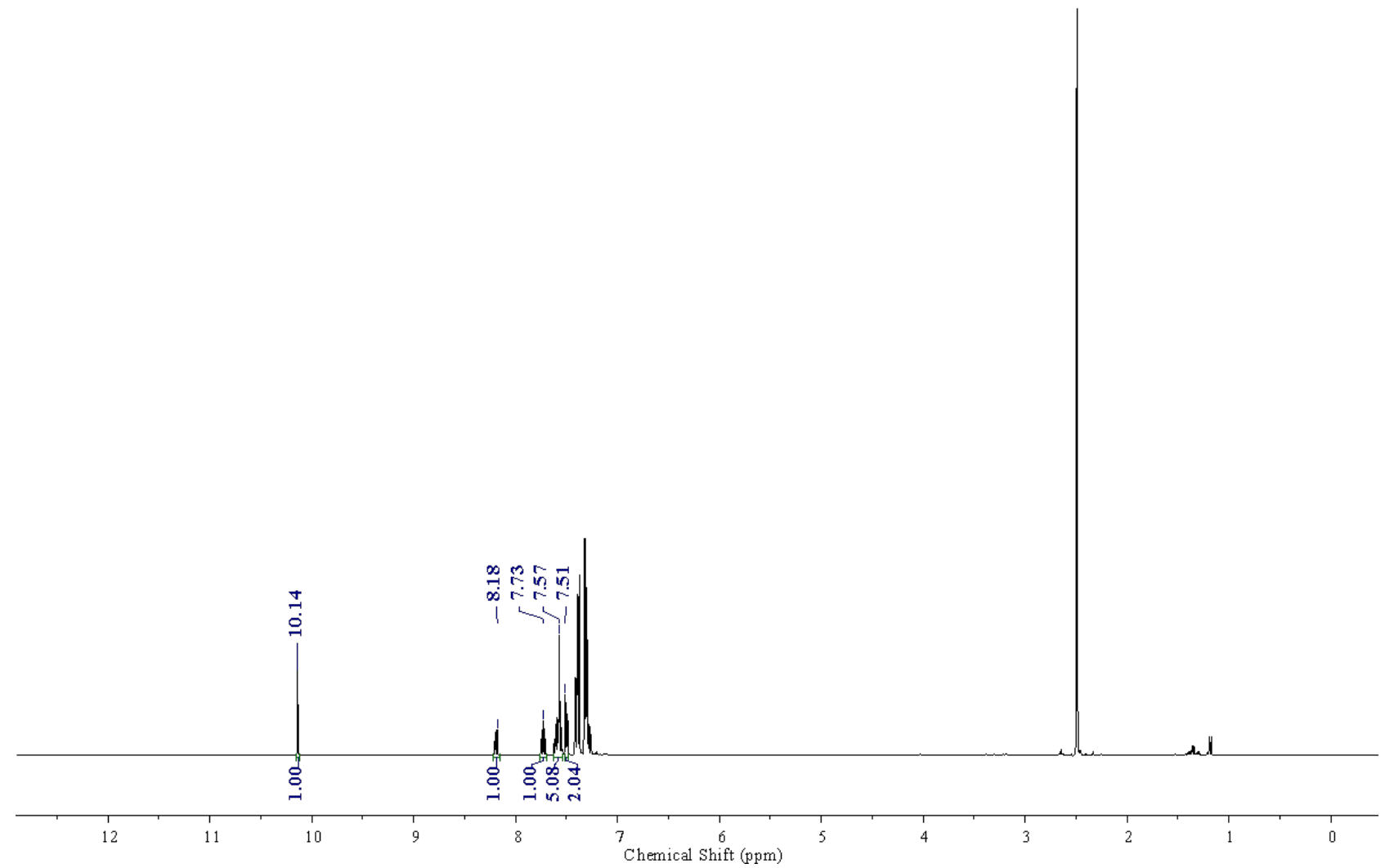

Entry 2: NMR for Crude reaction mixture

VDOS-593-A2.4.fid

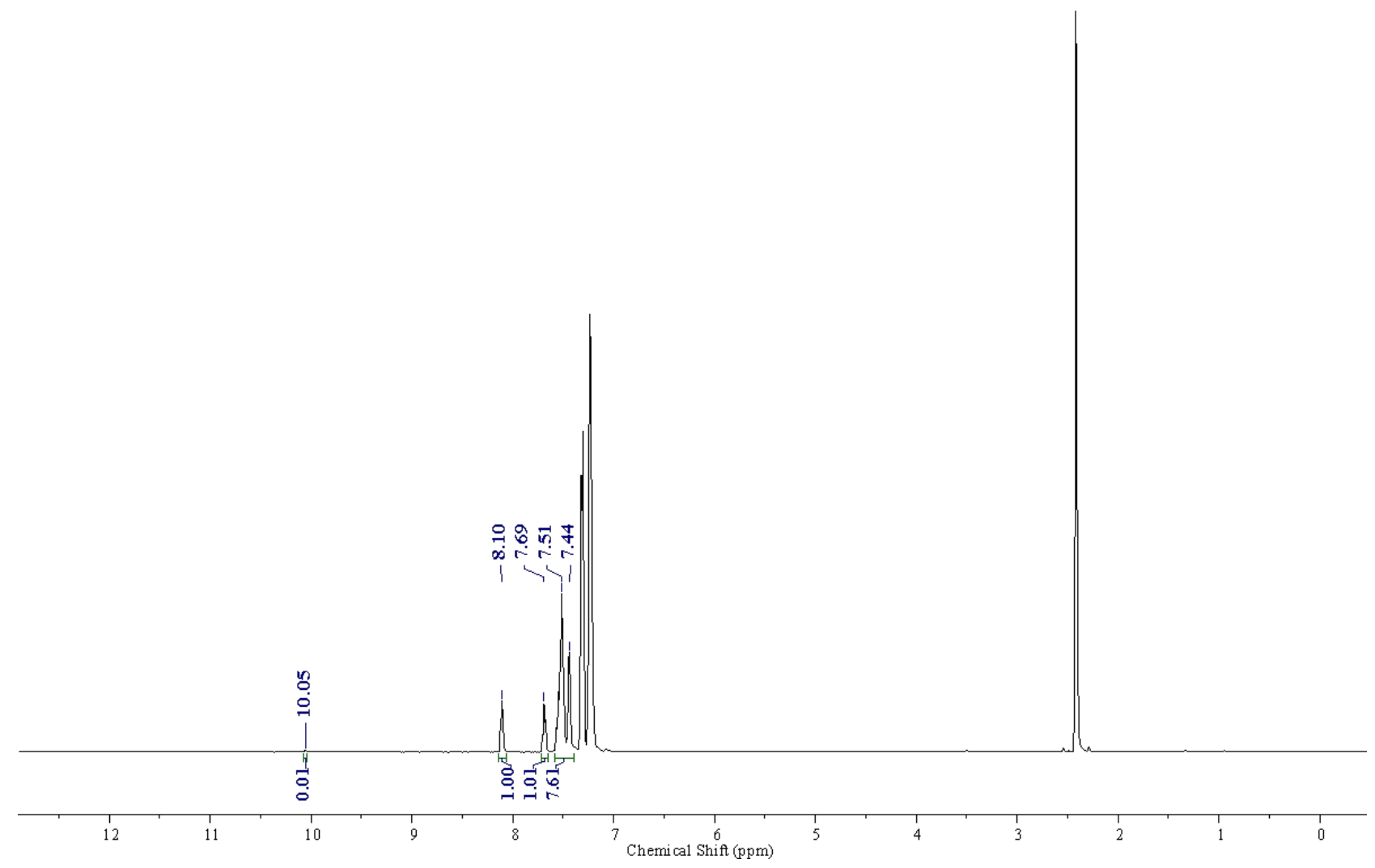


Entry 2': NMR for Crude reaction mixture, Duplicate

VDOS-593-A 1.3.fid

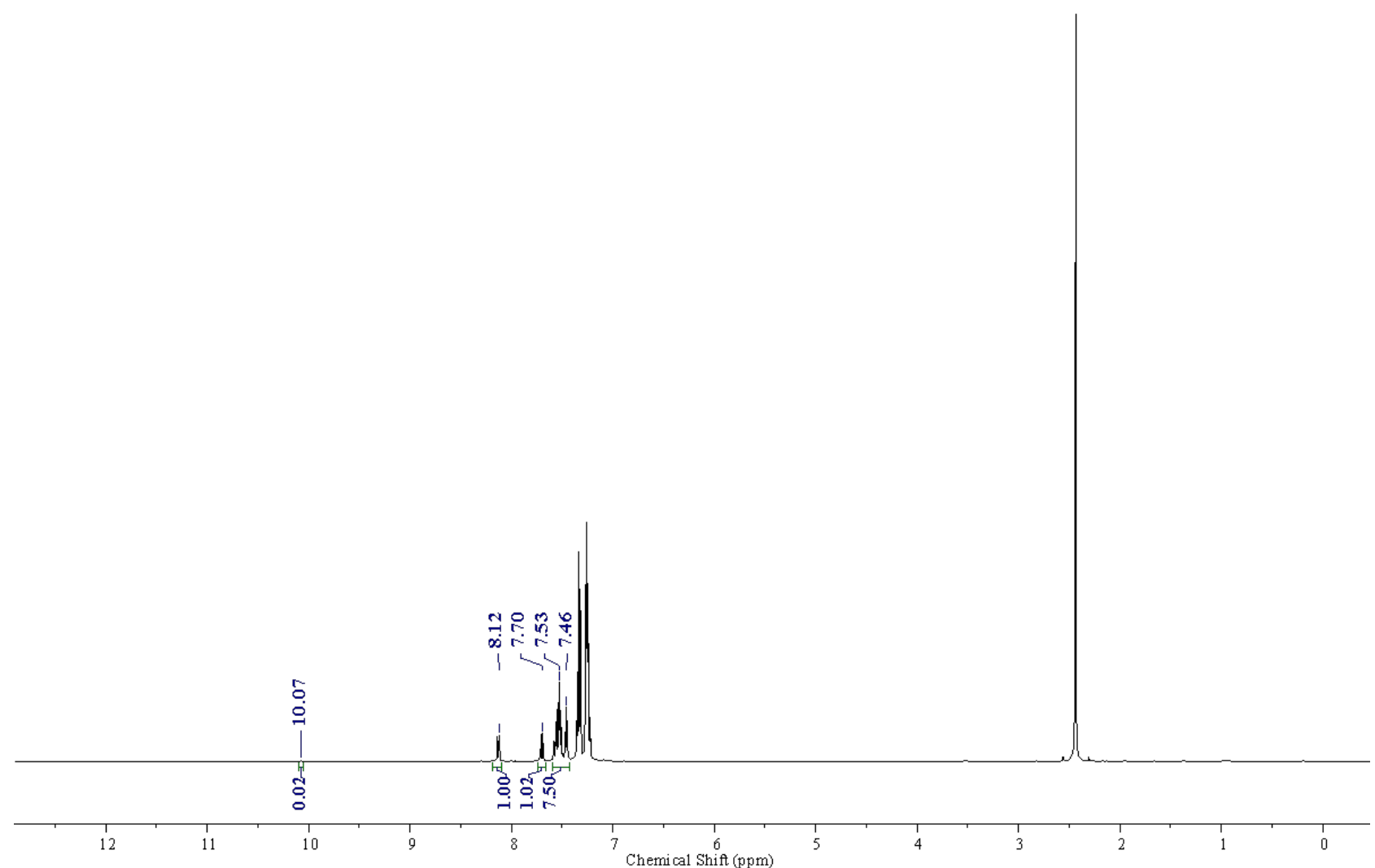

NMR after purification:

VDOS-593-A 1-A2-Pure.2.fid

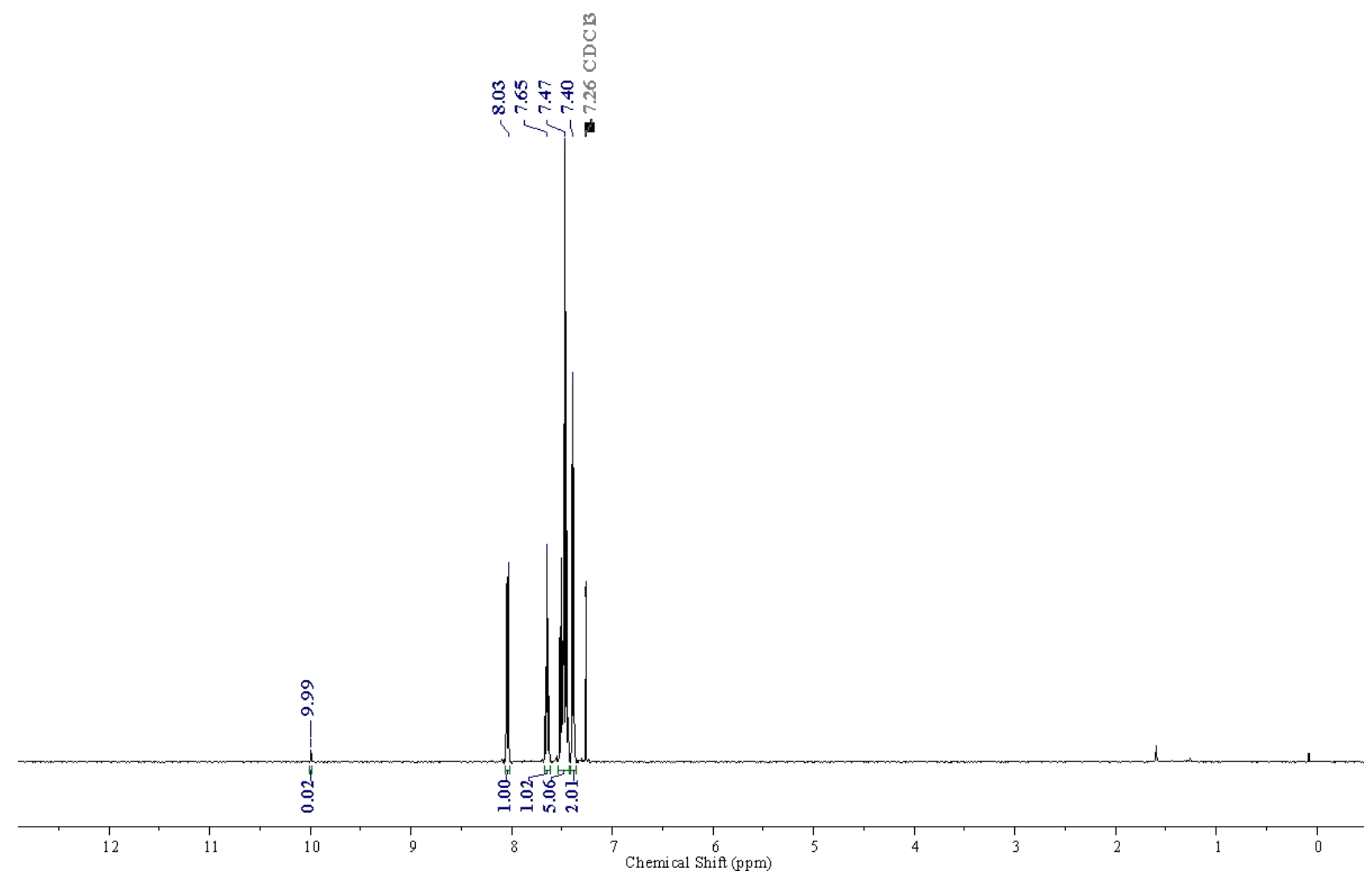




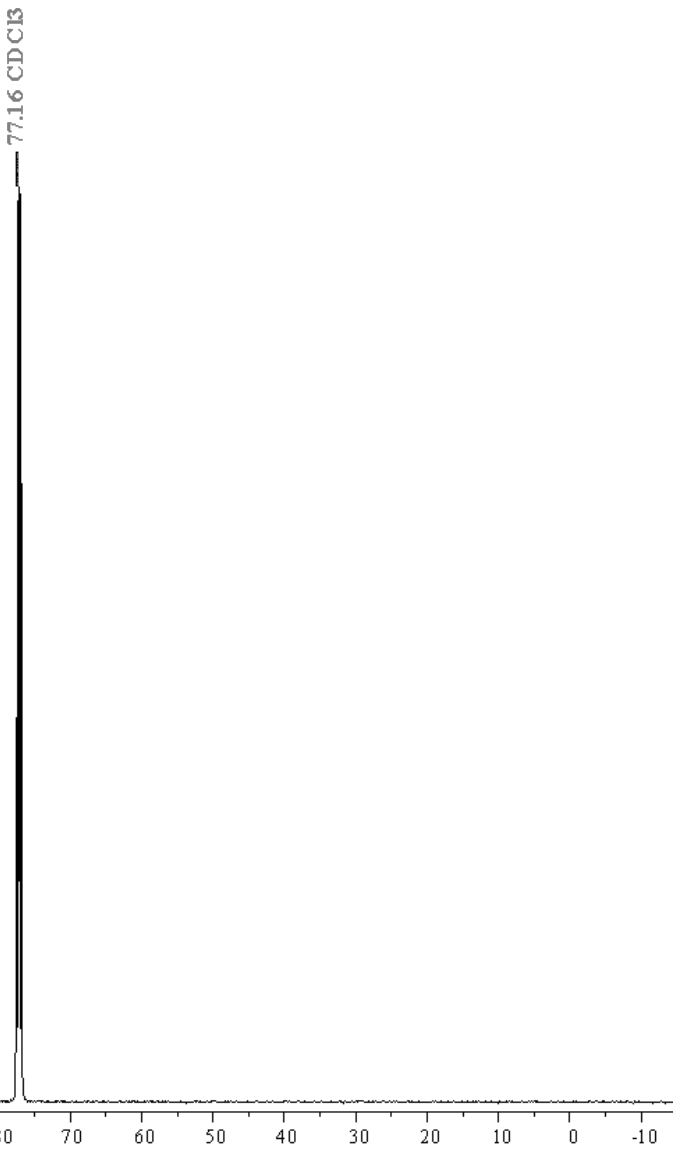


39. Phenanthrene-9-carbaldehyde-d

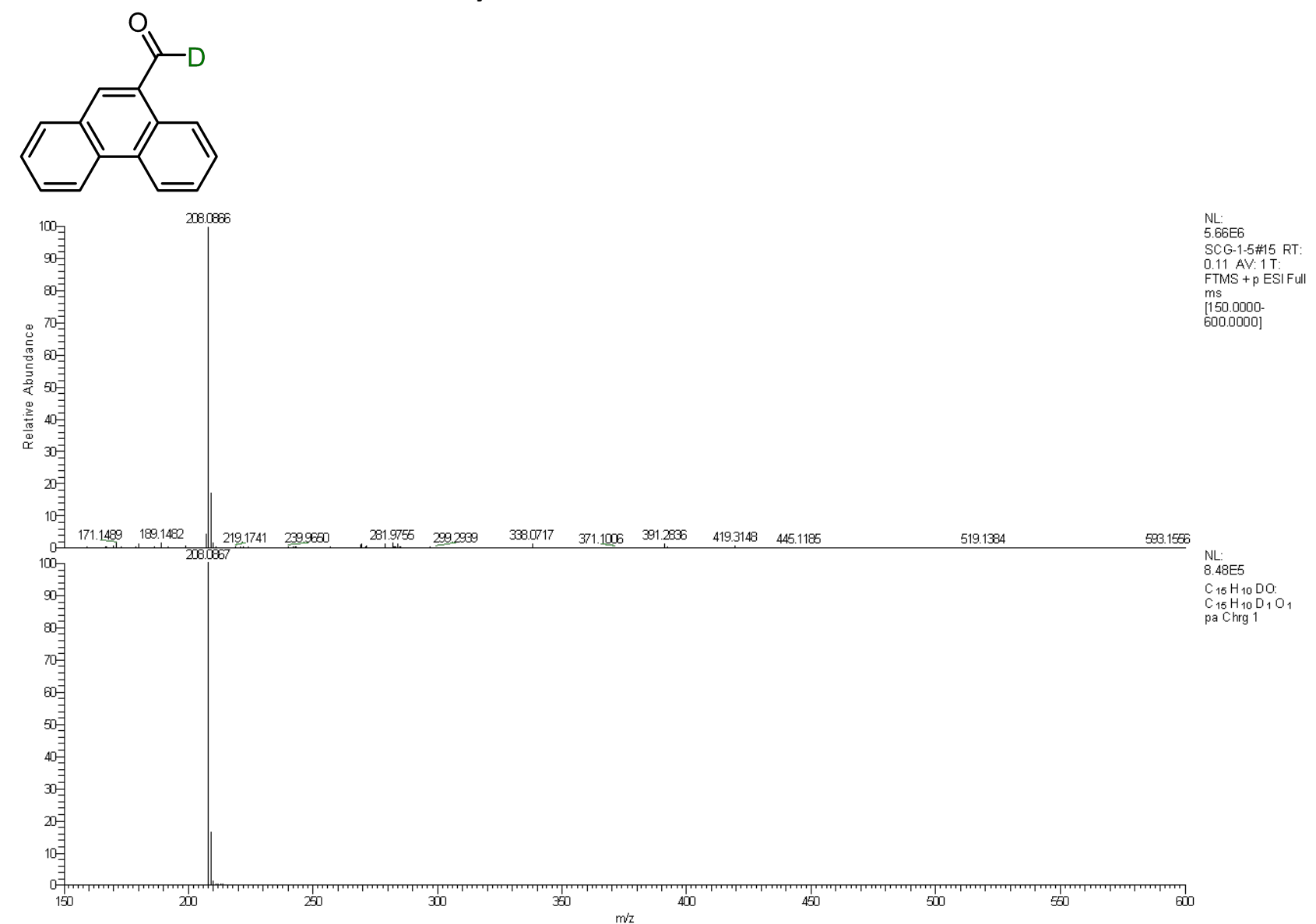

Entry 1: NMR for Crude reaction mixture SCG-309-L1-16hr-D1-50sec.1.fid

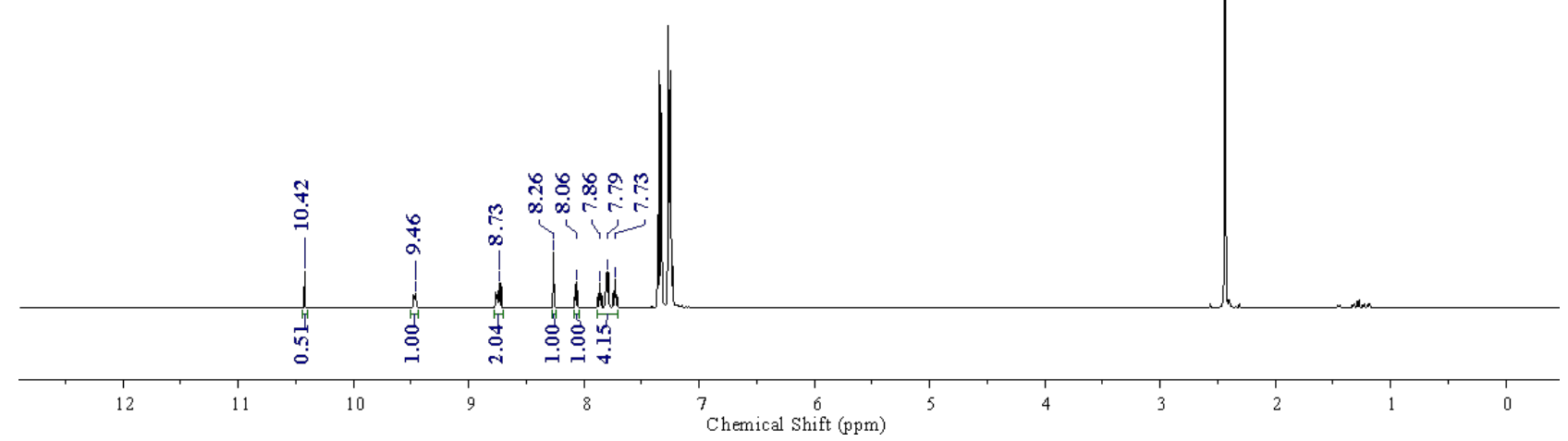

Entry 1': NMR for Crude reaction mixture, Duplicate 


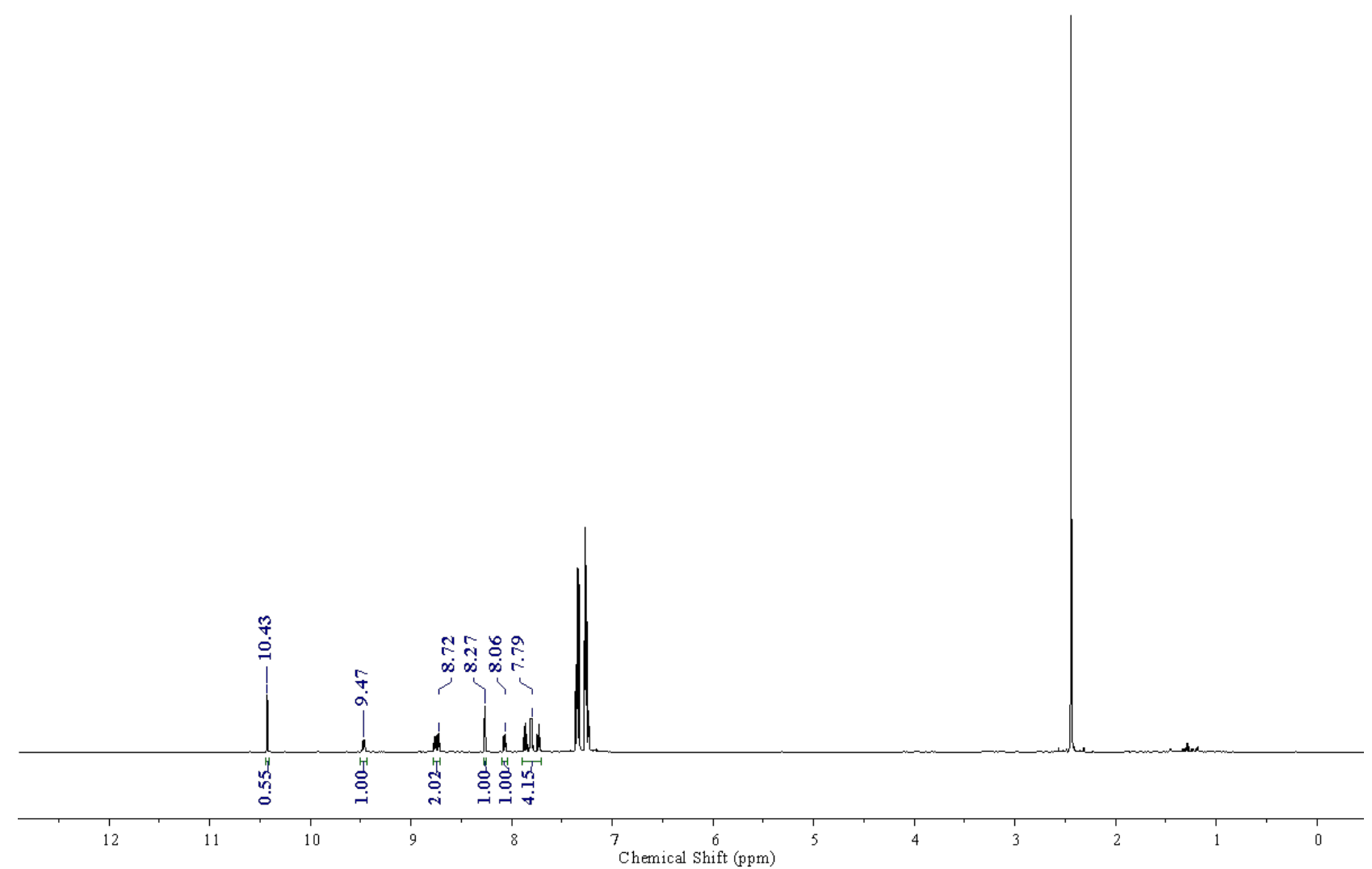

Entry 2: NMR for Crude reaction mixture VDOS-610-A 1.2.fid

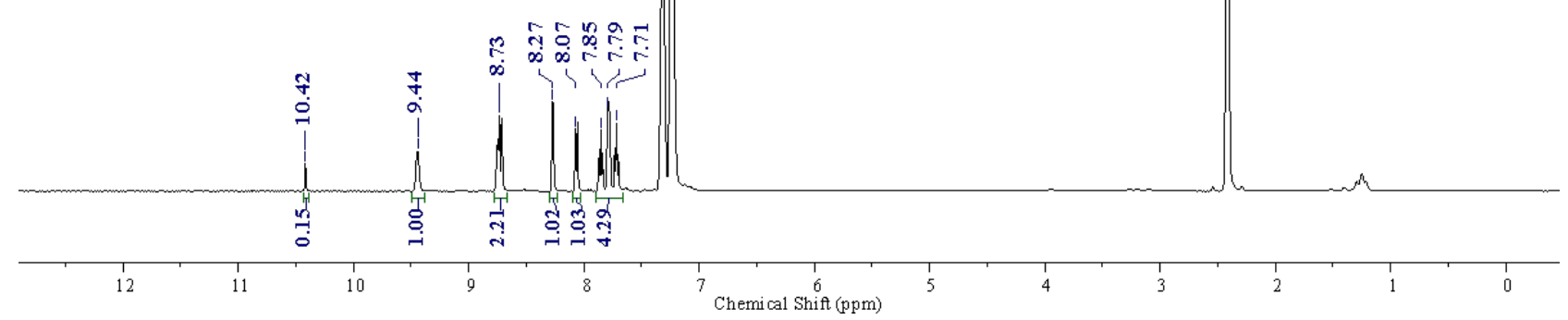


Entry 3: NMR for Crude reaction mixture

VDOS-610-A.2.4.fid

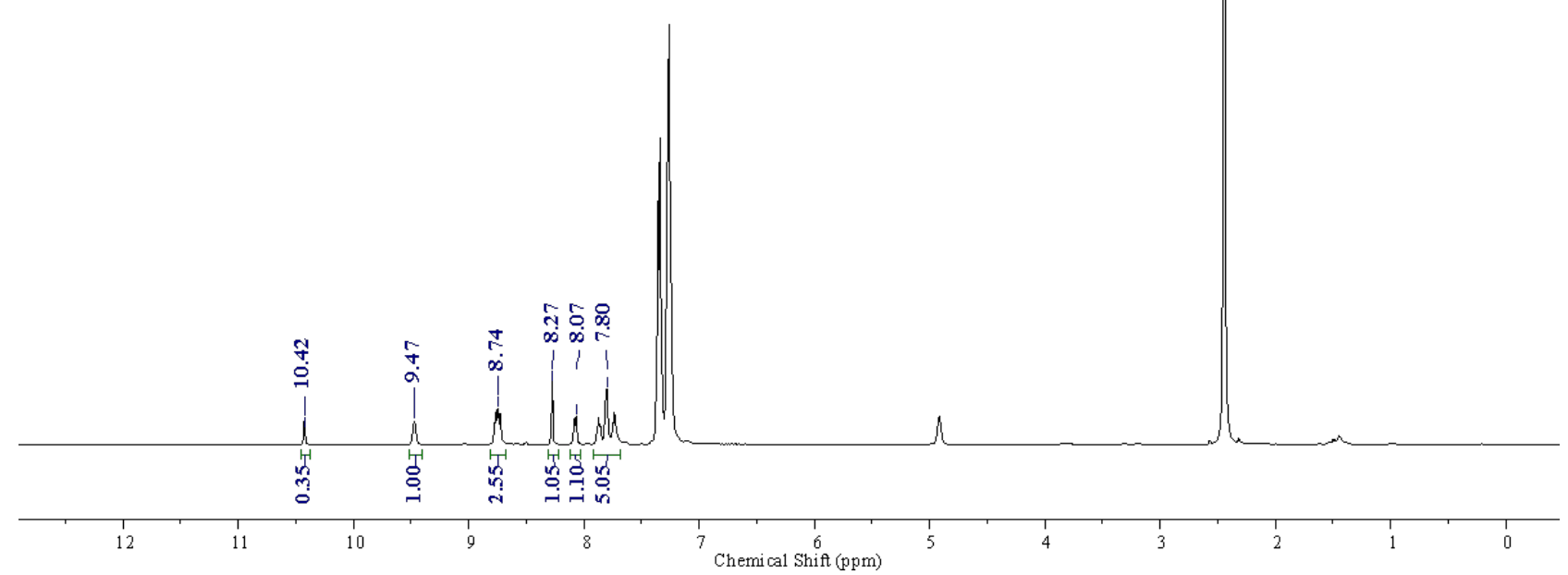

Entry 4: NMR for Crude reaction mixture

SCG-342-A4-16hr-D 1-50sec.1.fid

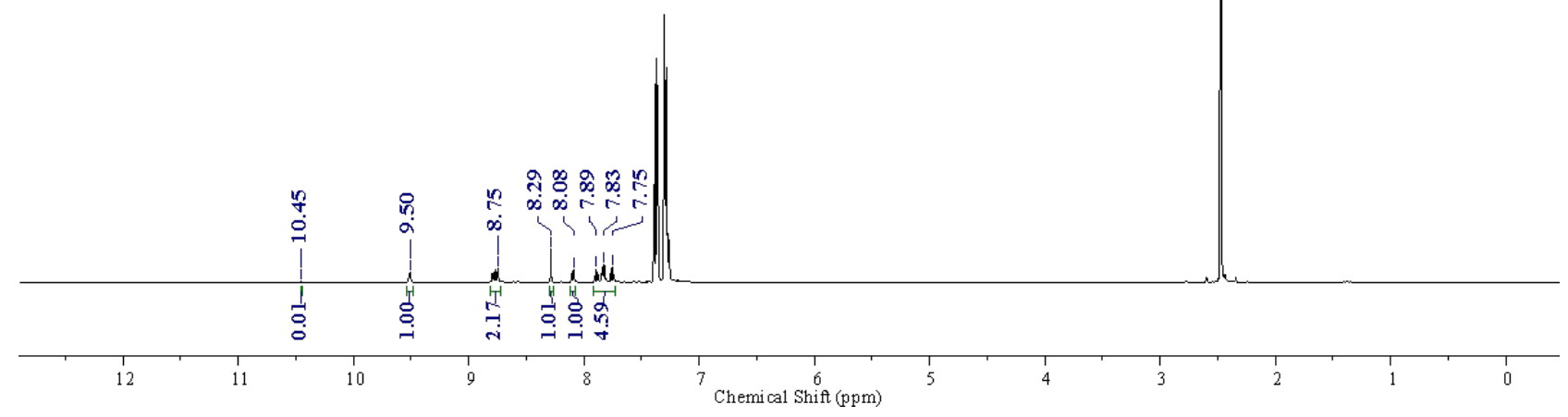


Entry 4': NMR for Crude reaction mixture, Duplicate

VDOS-610-A.3.3.fid

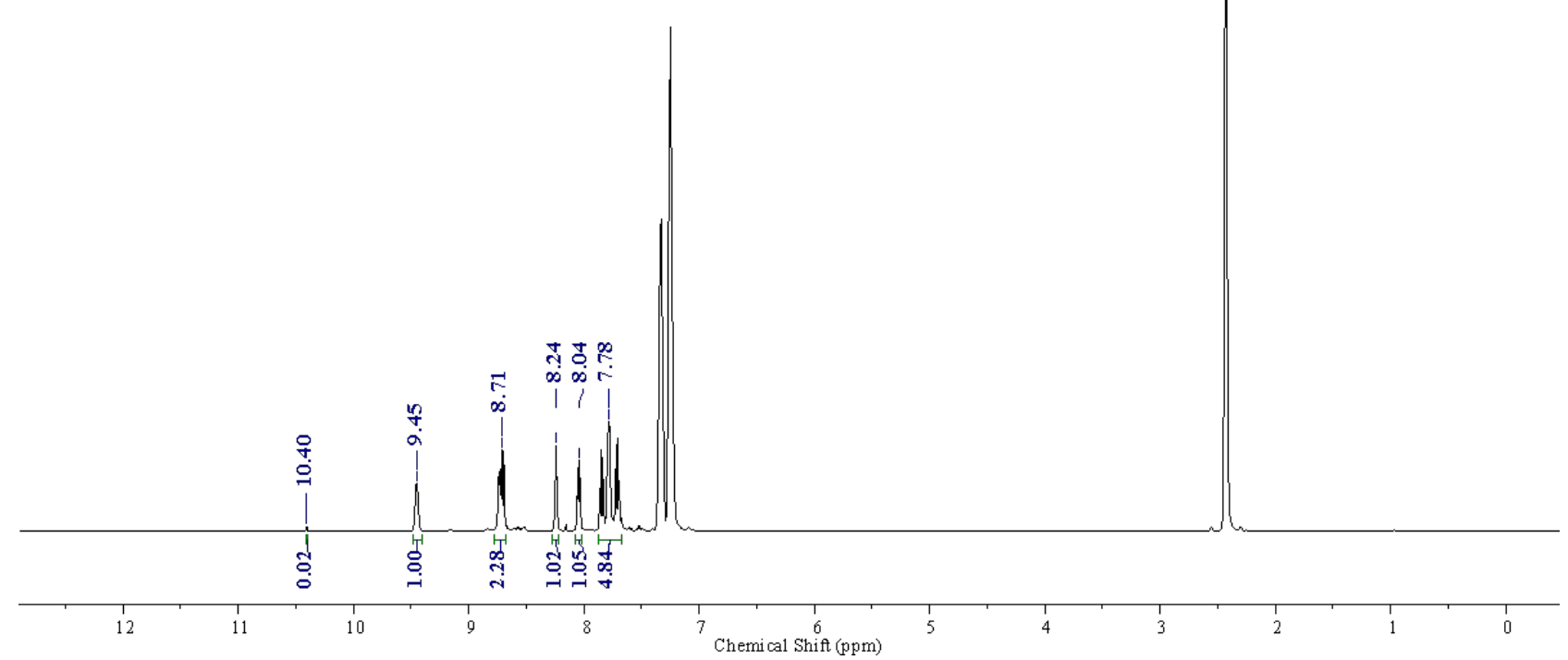

NMR after purification:

VDOS-610-A3 pure- 2.5 fid

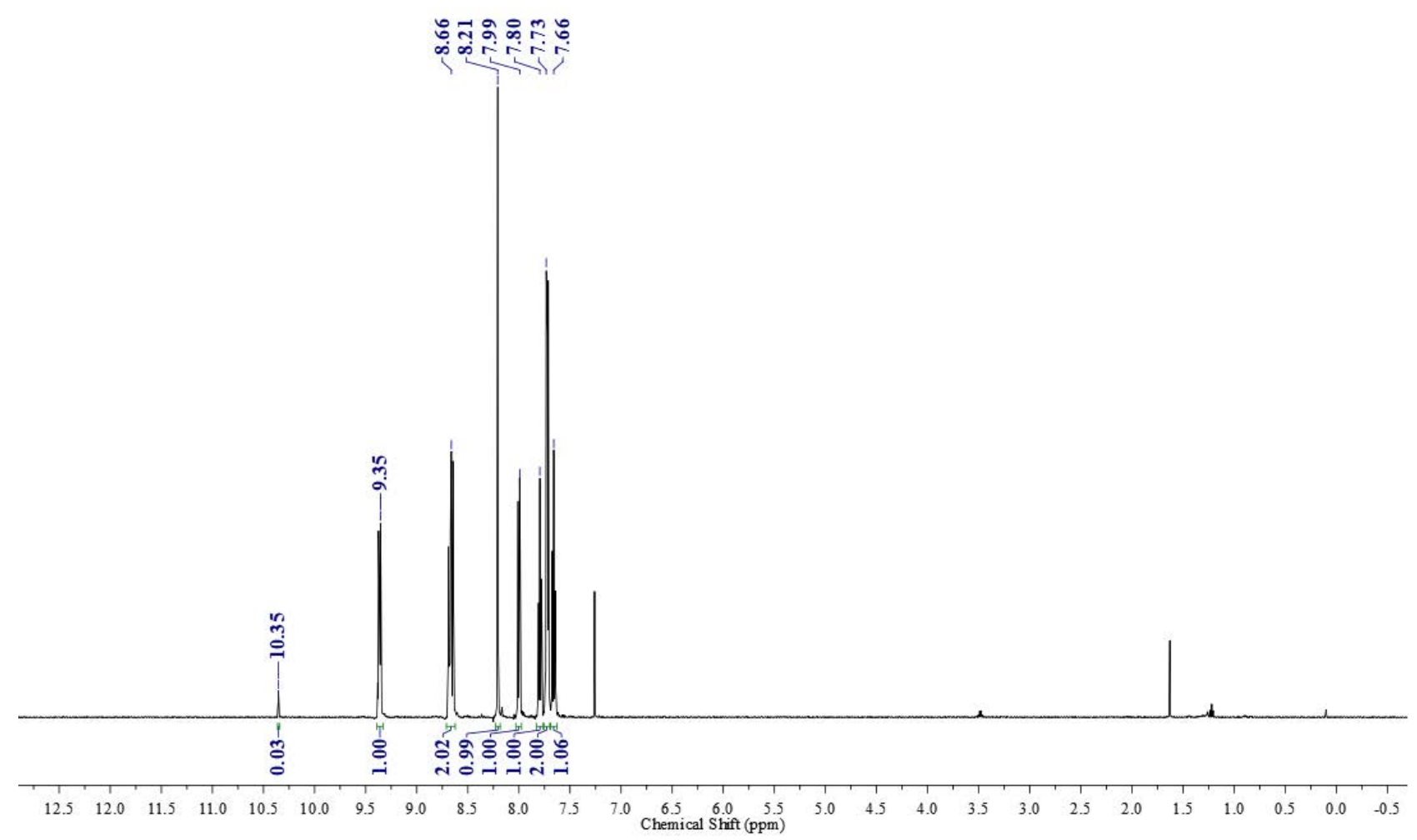


VDOS-610-VP-13C.3.fid

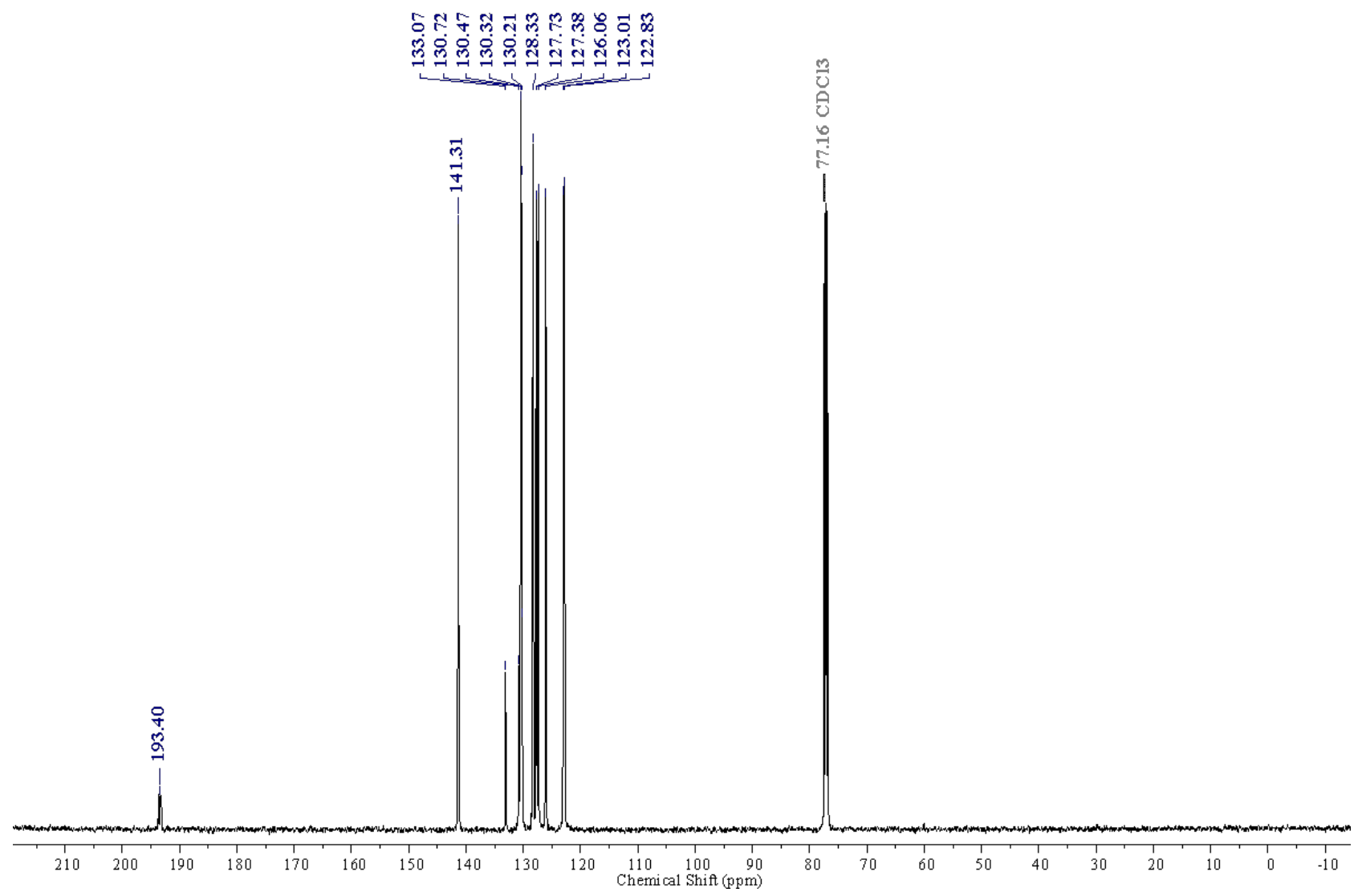

322 
40. 4-Hydoxybenzaldehyde-d<smiles>[2H]C(=O)c1ccc(O)cc1</smiles>

Entry 1: NMR for Crude reaction mixture SCG-312-T2-16hr-D 1-50sec.1.fid

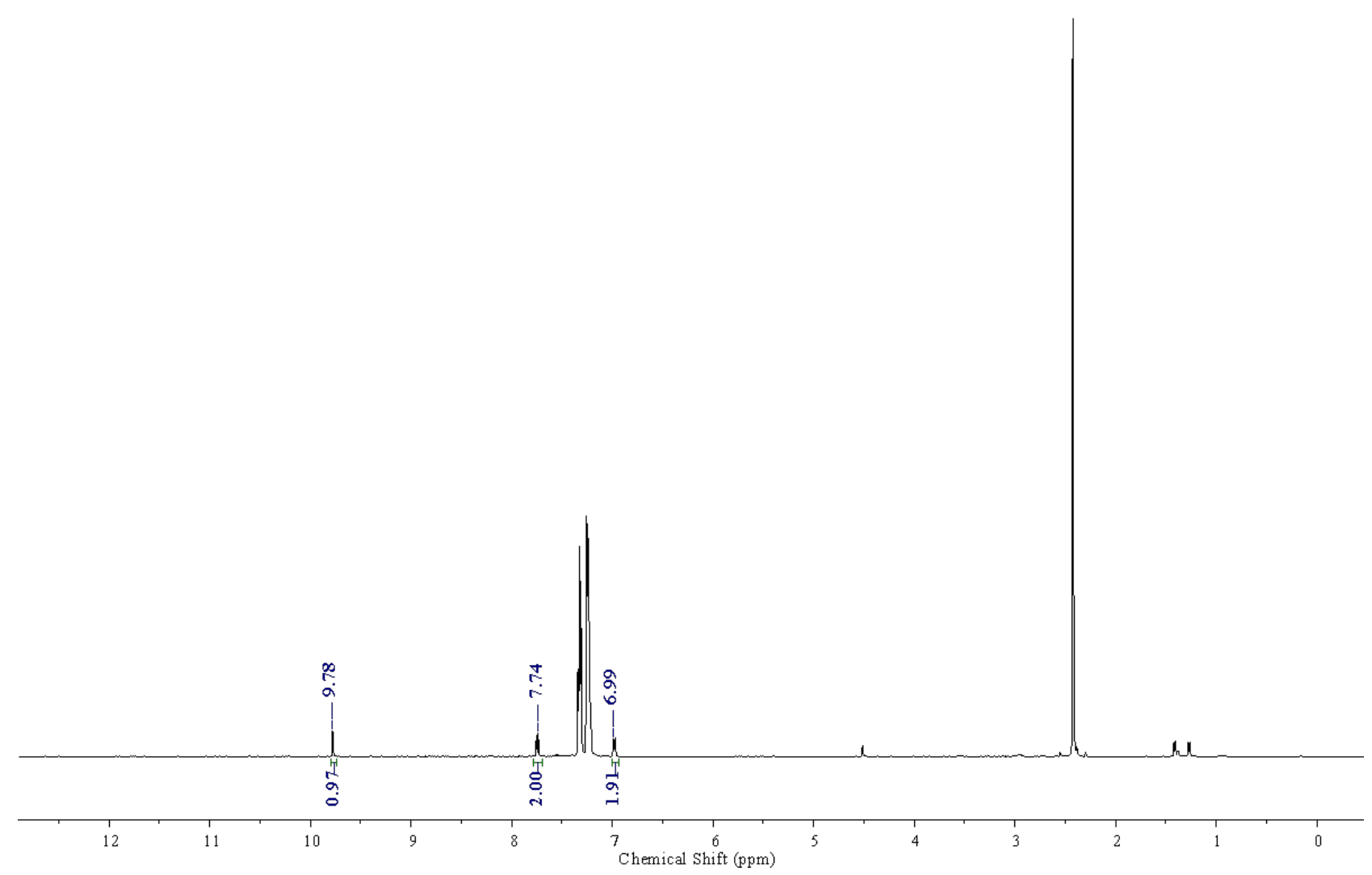


Entry 1': NMR for Crude reaction mixture, Duplicate

SCG-312-T1-16hr-D 1-50sec.1.fid

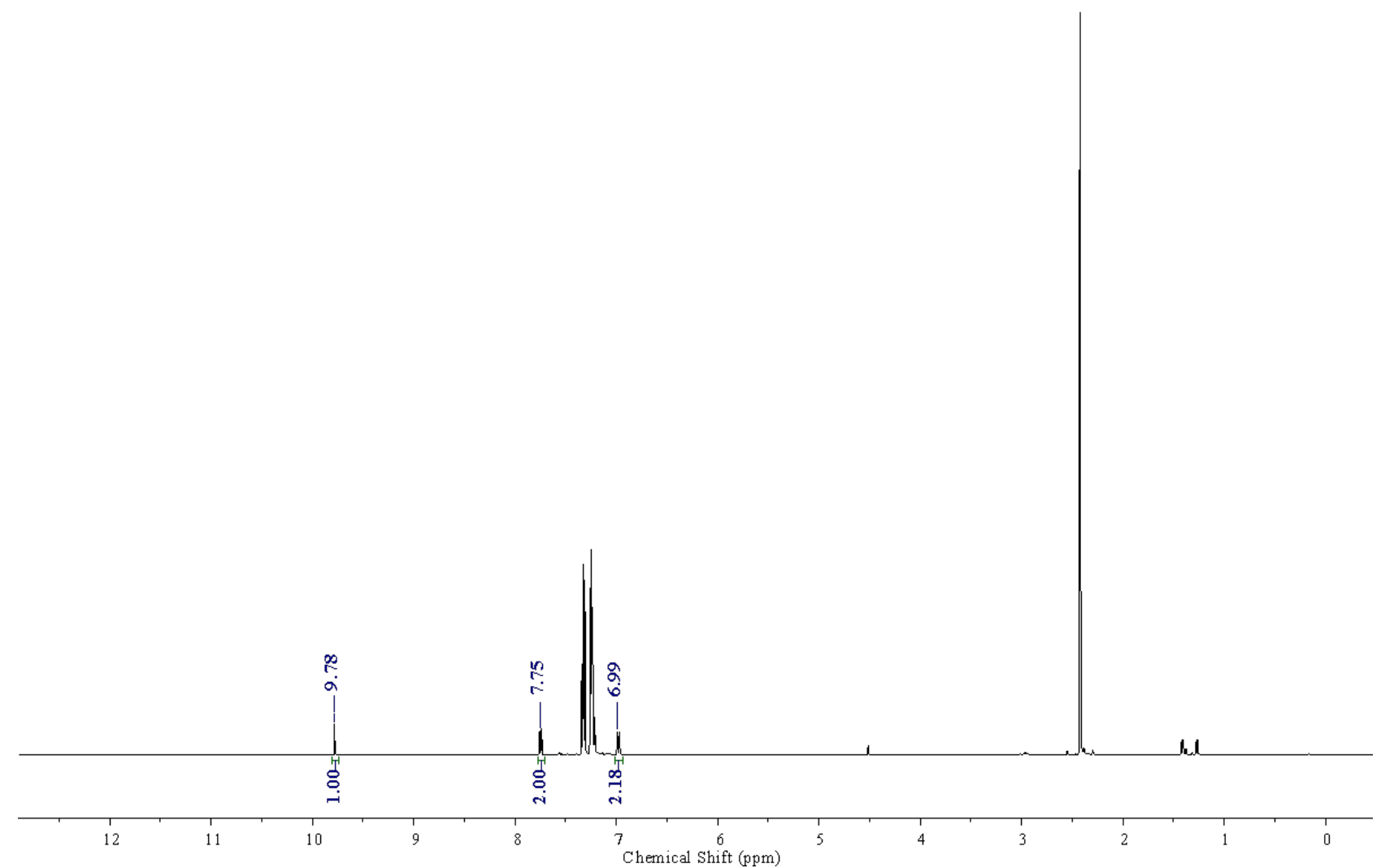

Entry 2: NMR for Crude reaction mixture

SCG-312-T3-16hr-D1-50sec 1 fid

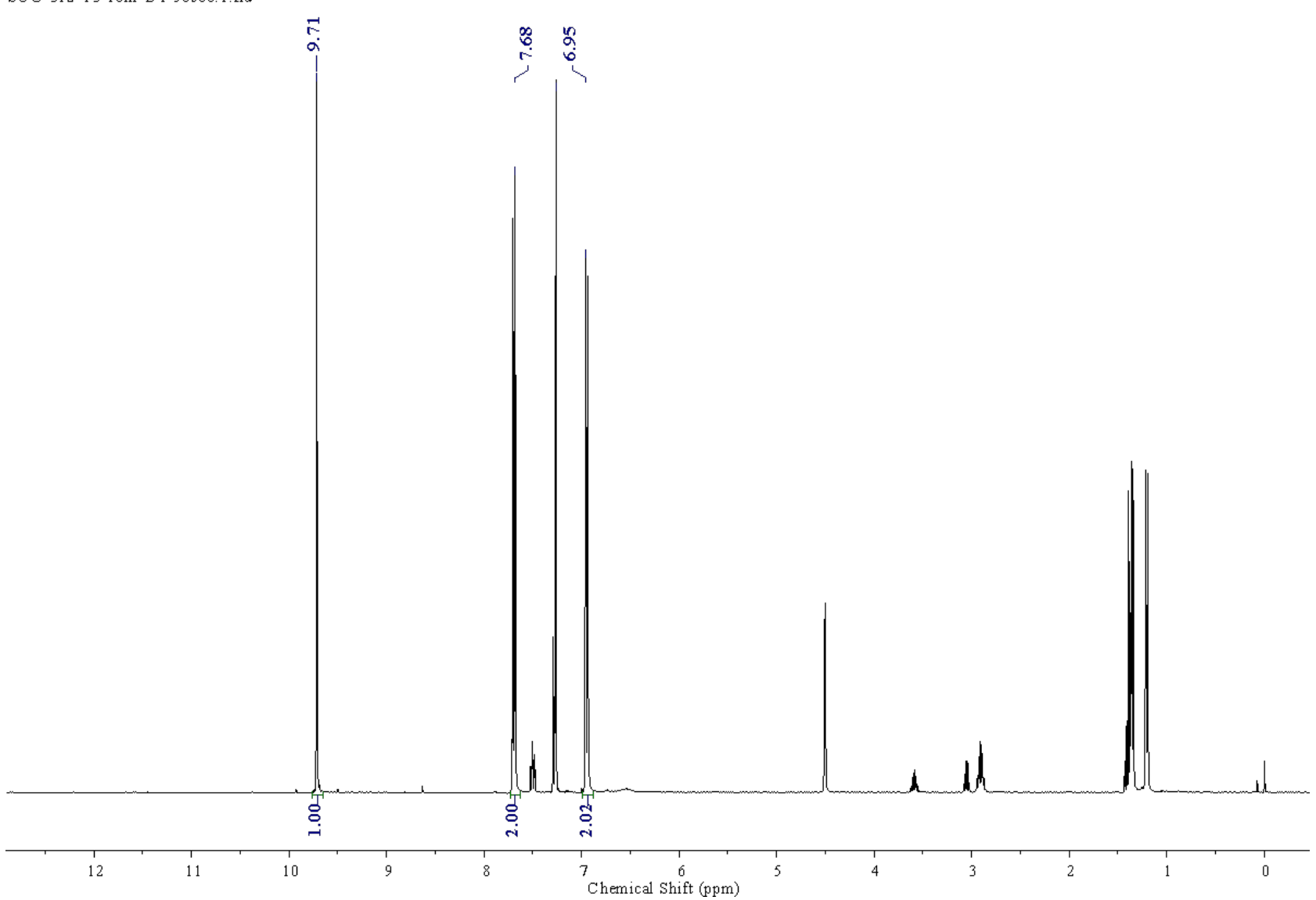


Entry 3: NMR for Crude reaction mixture

SCG-312-T5-16hr-D1-50sec 1 fid

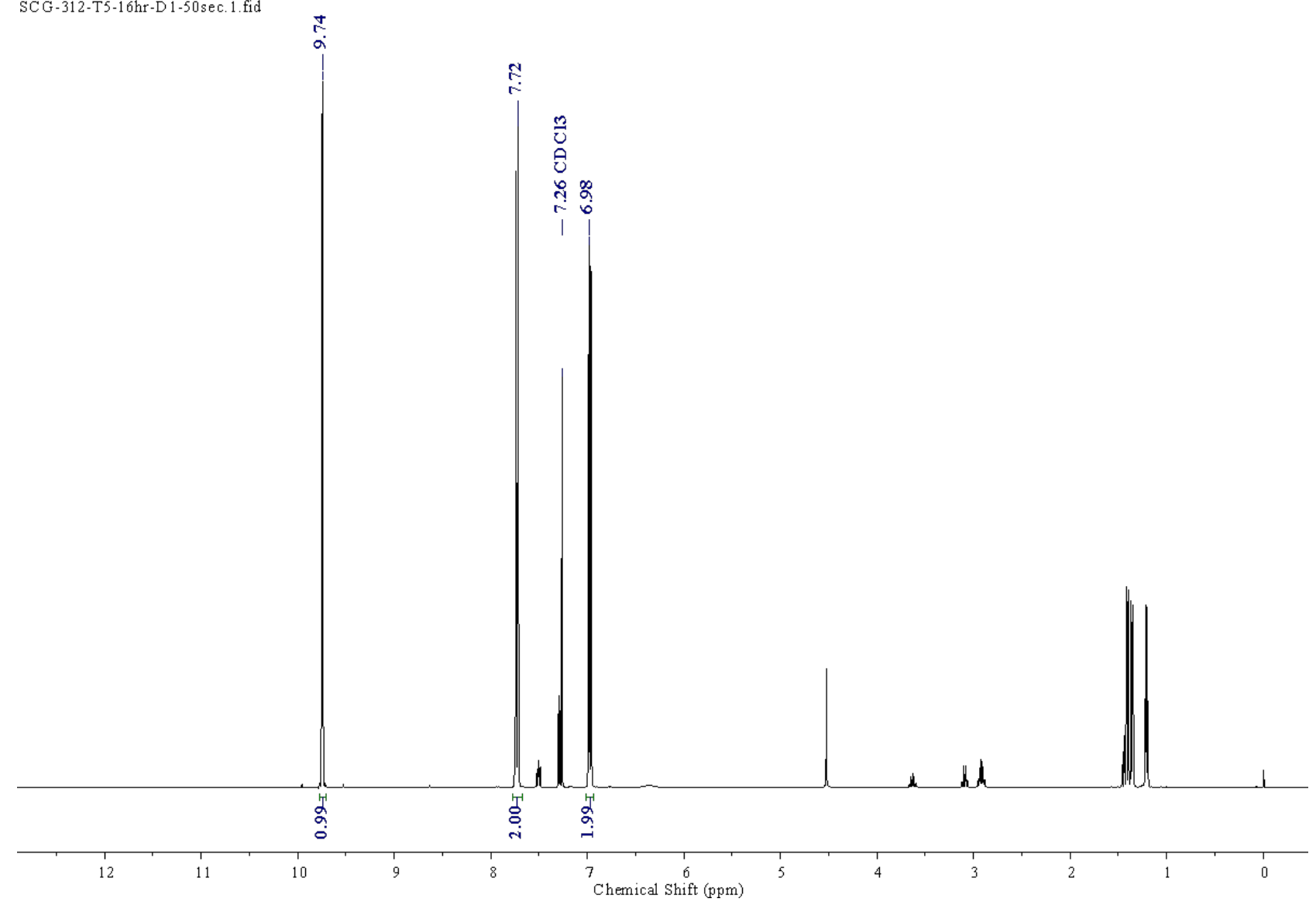

Entry 4: NMR for Crude reaction mixture

SCG-313-H5-16hr-D1-50sec.1.fid

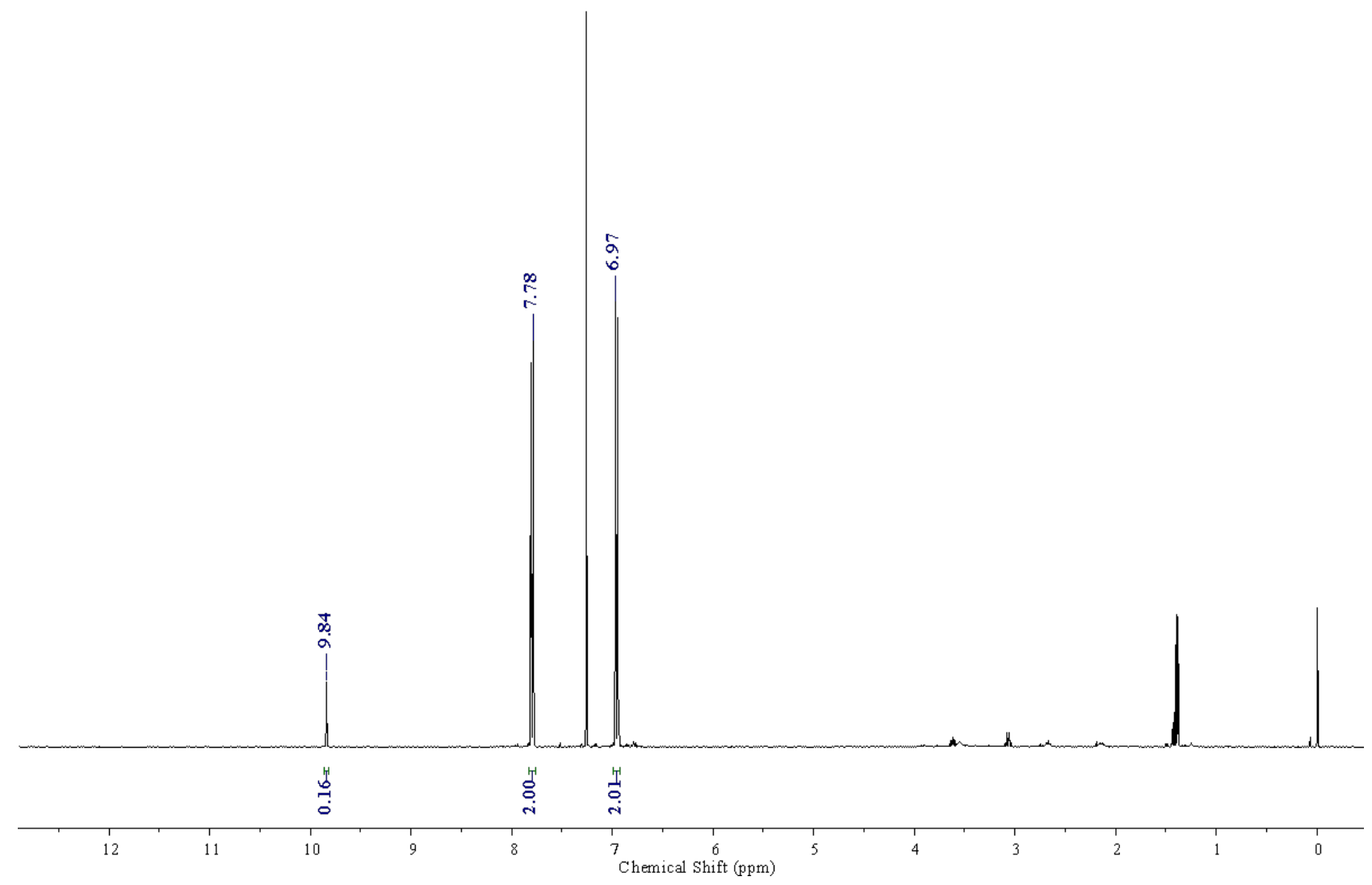


Entry 5: NMR for Crude reaction mixture

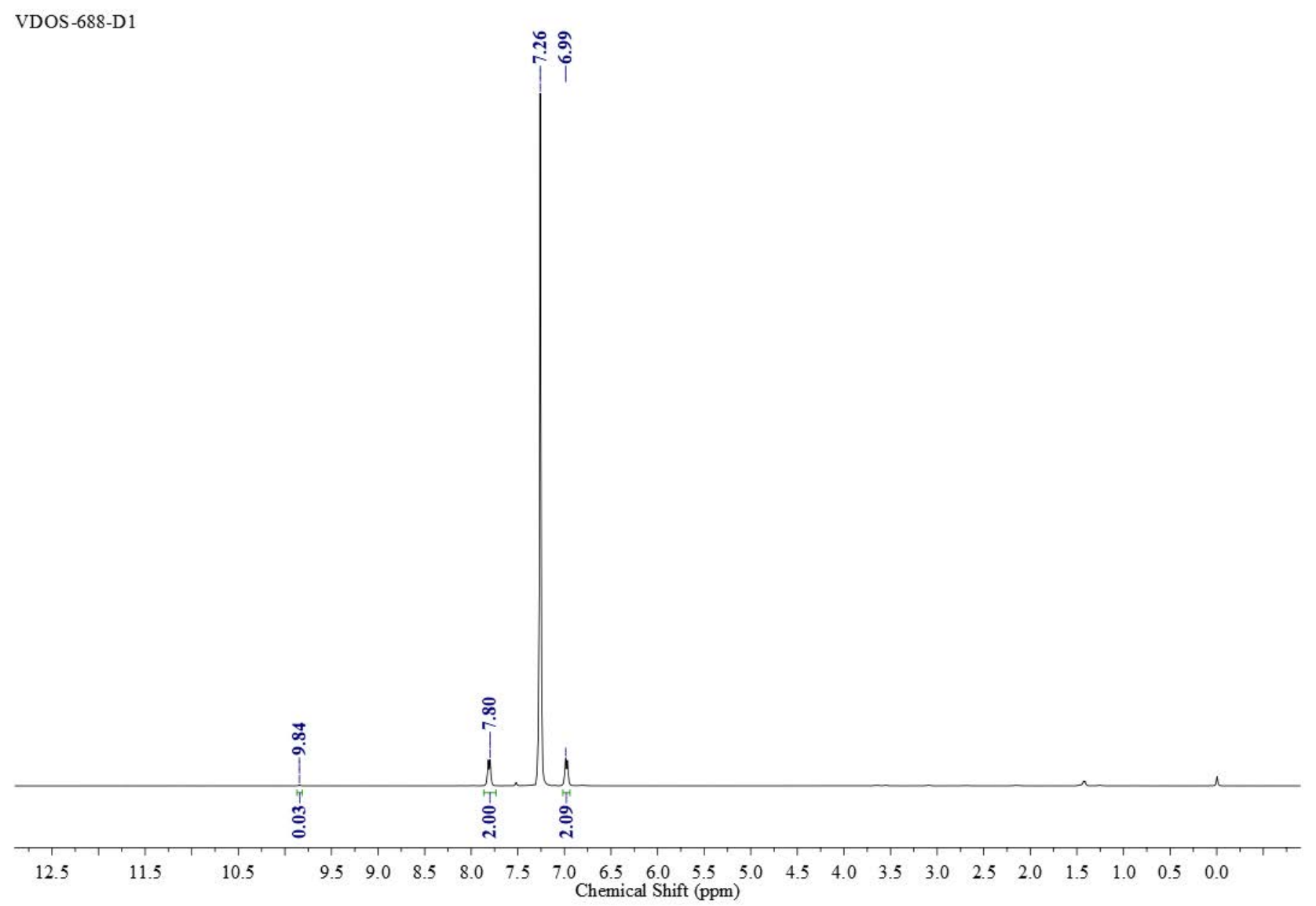

Entry 5': NMR for Crude reaction mixture, Duplicate VDOS-688-D2

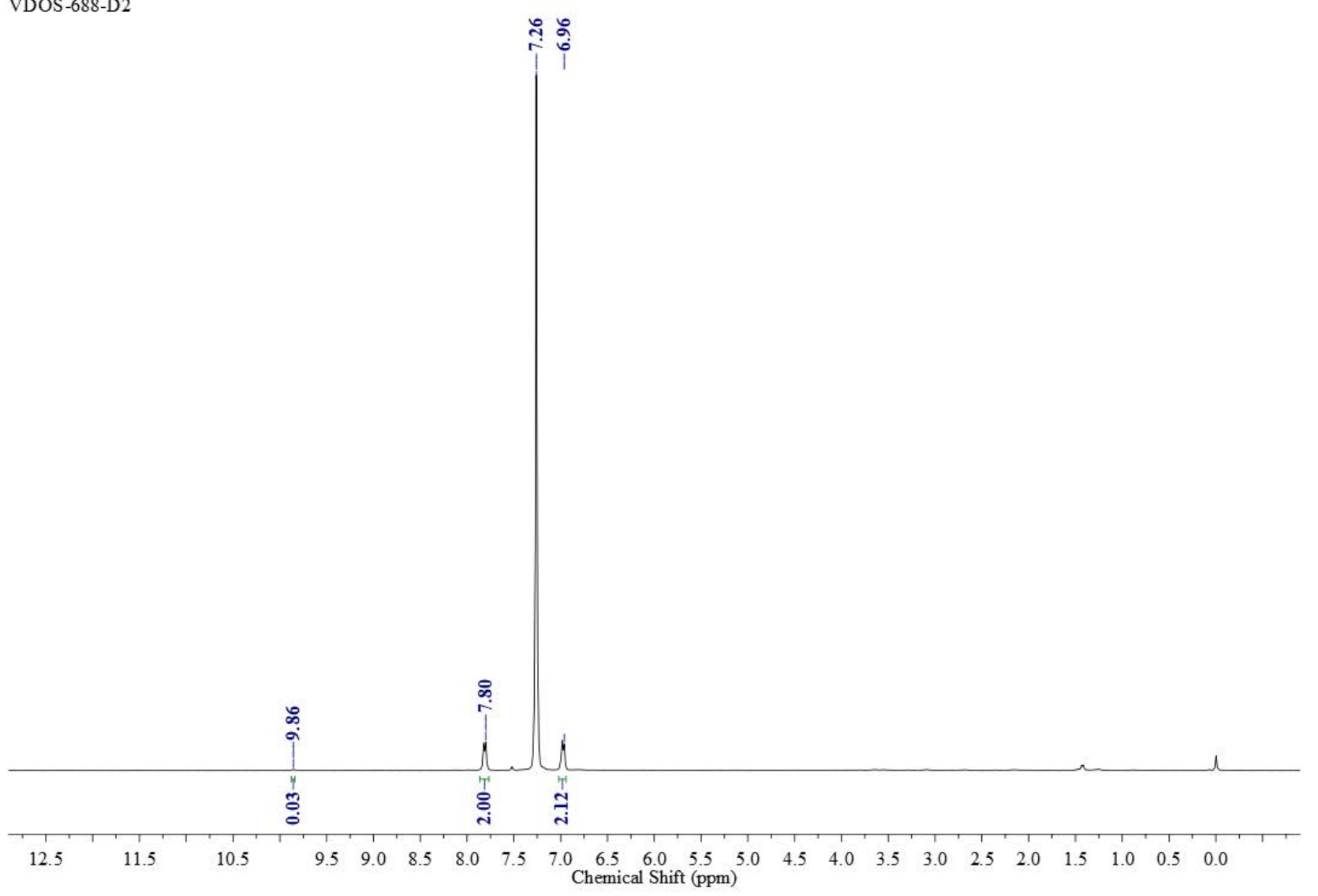


NMR after purification:

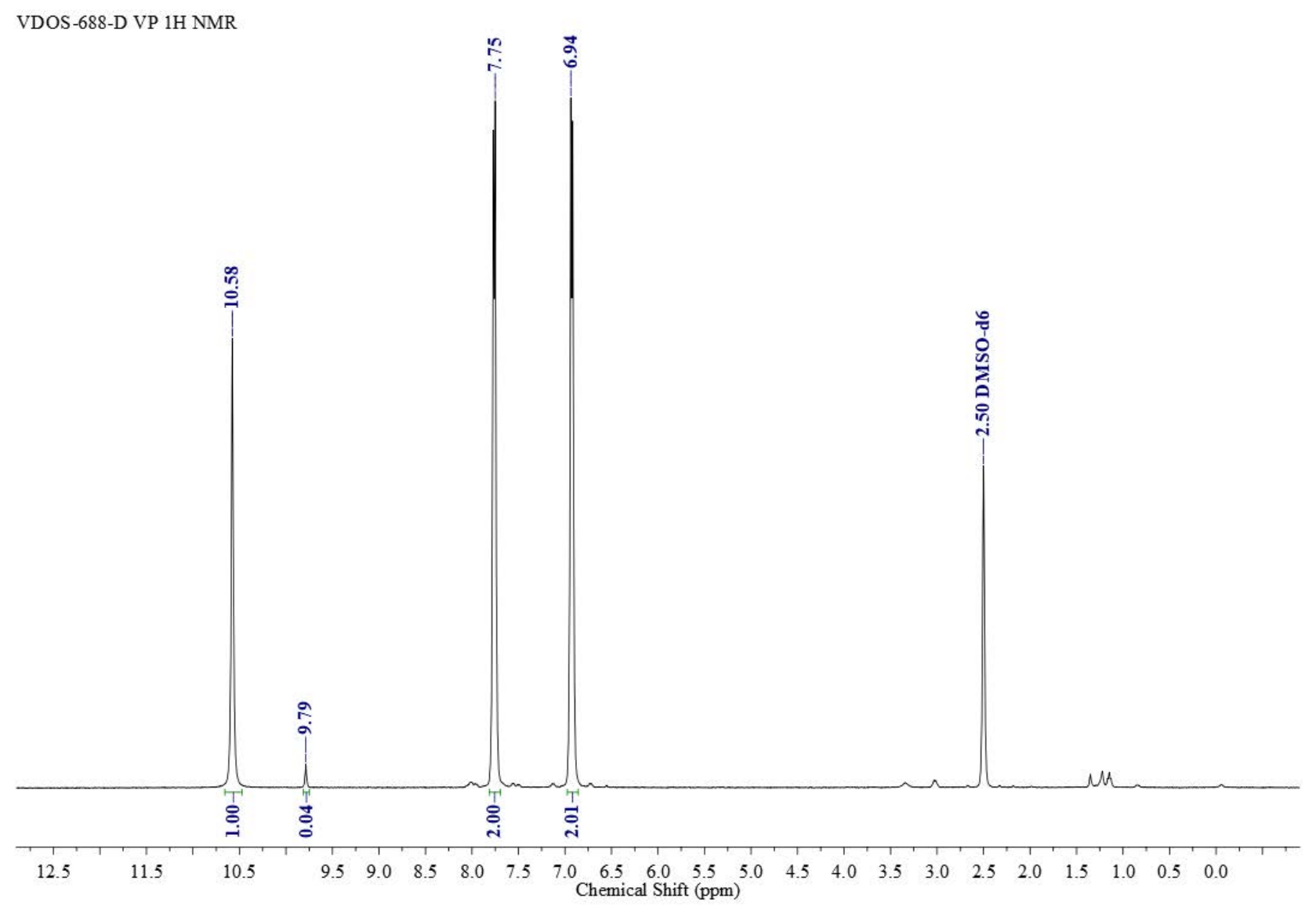

SCG-313-H6-16hr-A C.2.fid

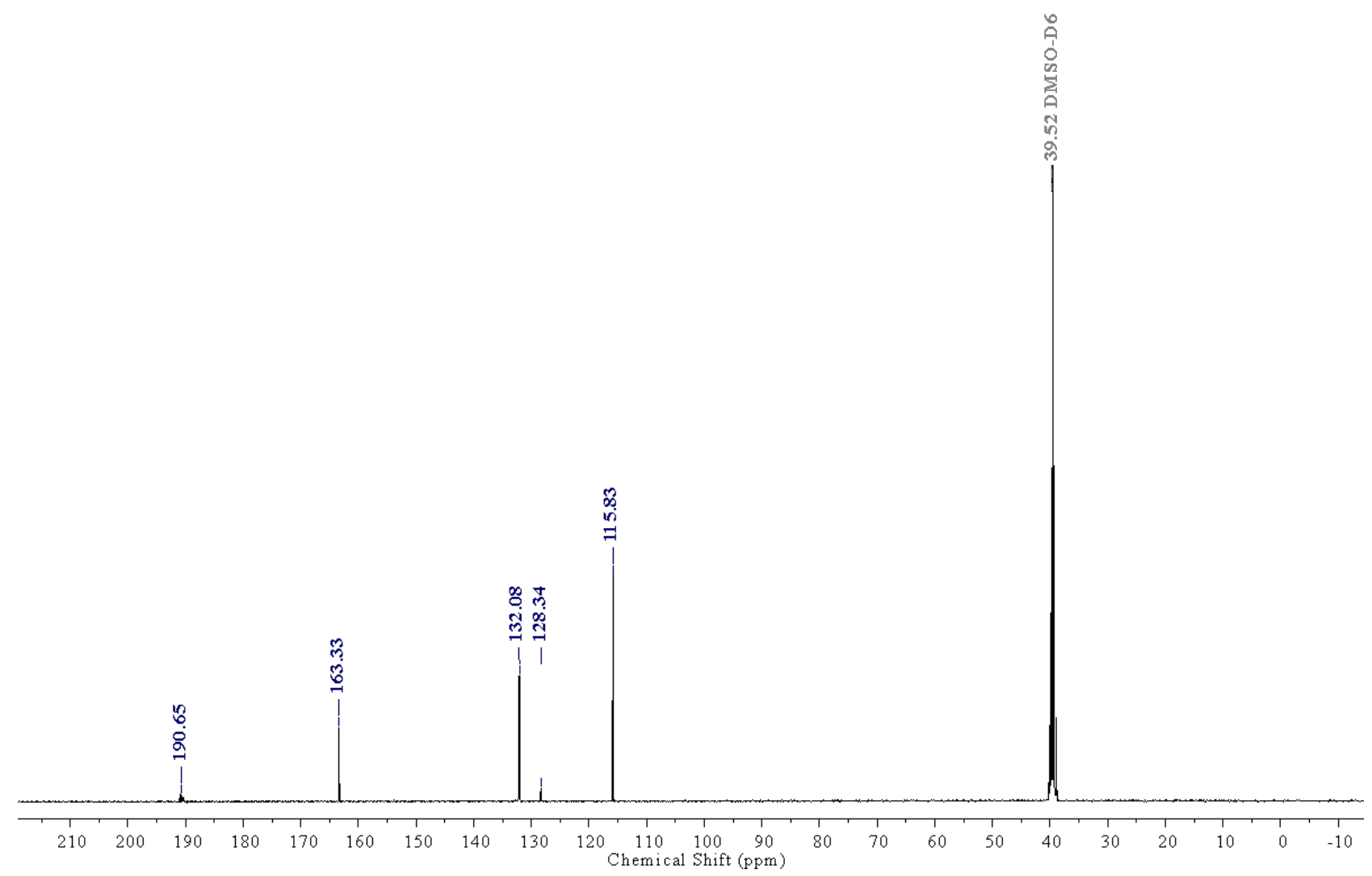


Substrate Scope with Heterocycles and Biologically Active Molecules:

41. Thiophene-2-carboxaldehyde-d<smiles>O=C(O)c1cccs1</smiles>

Entry 1: NMR for Crude reaction mixture SCG-309-11-16ht-D1-50sec.1. fid

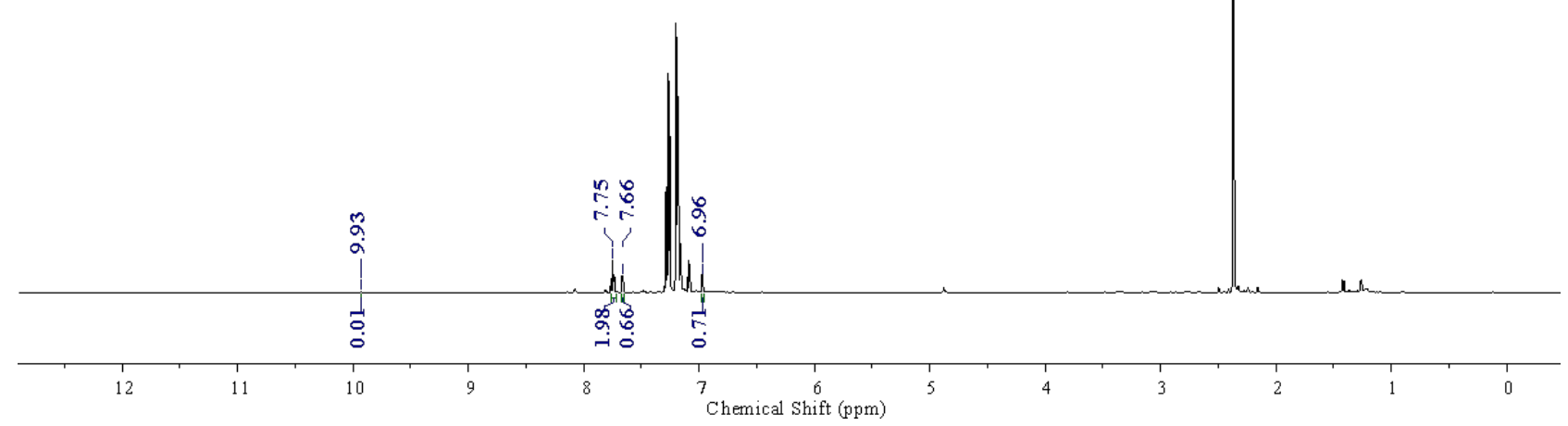


Entry 1': NMR for Crude reaction mixture, Duplicate

SCG-309-12-16hr-D1-50sec.1.fid

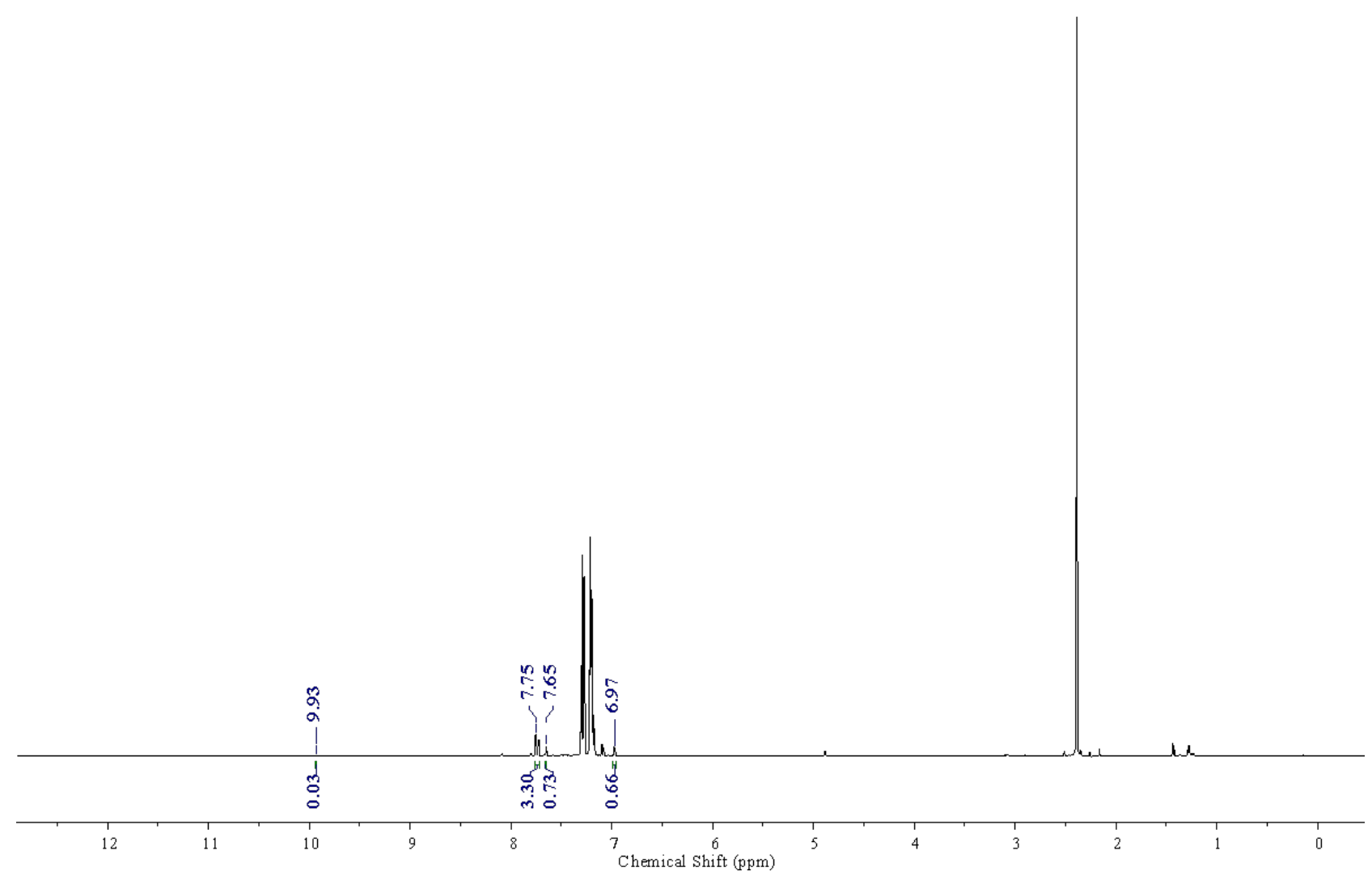

Entry 2: NMR for Crude reaction mixture

SCG-319-E2.2.fid

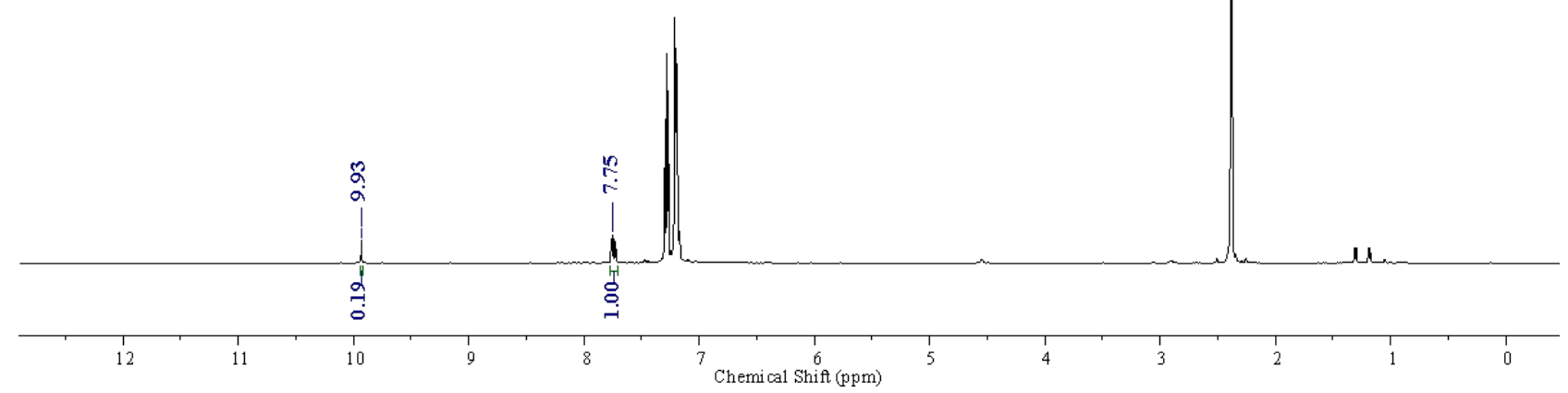


Entry 3: NMR for Crude reaction mixture

SCG-319-E1.1. fid

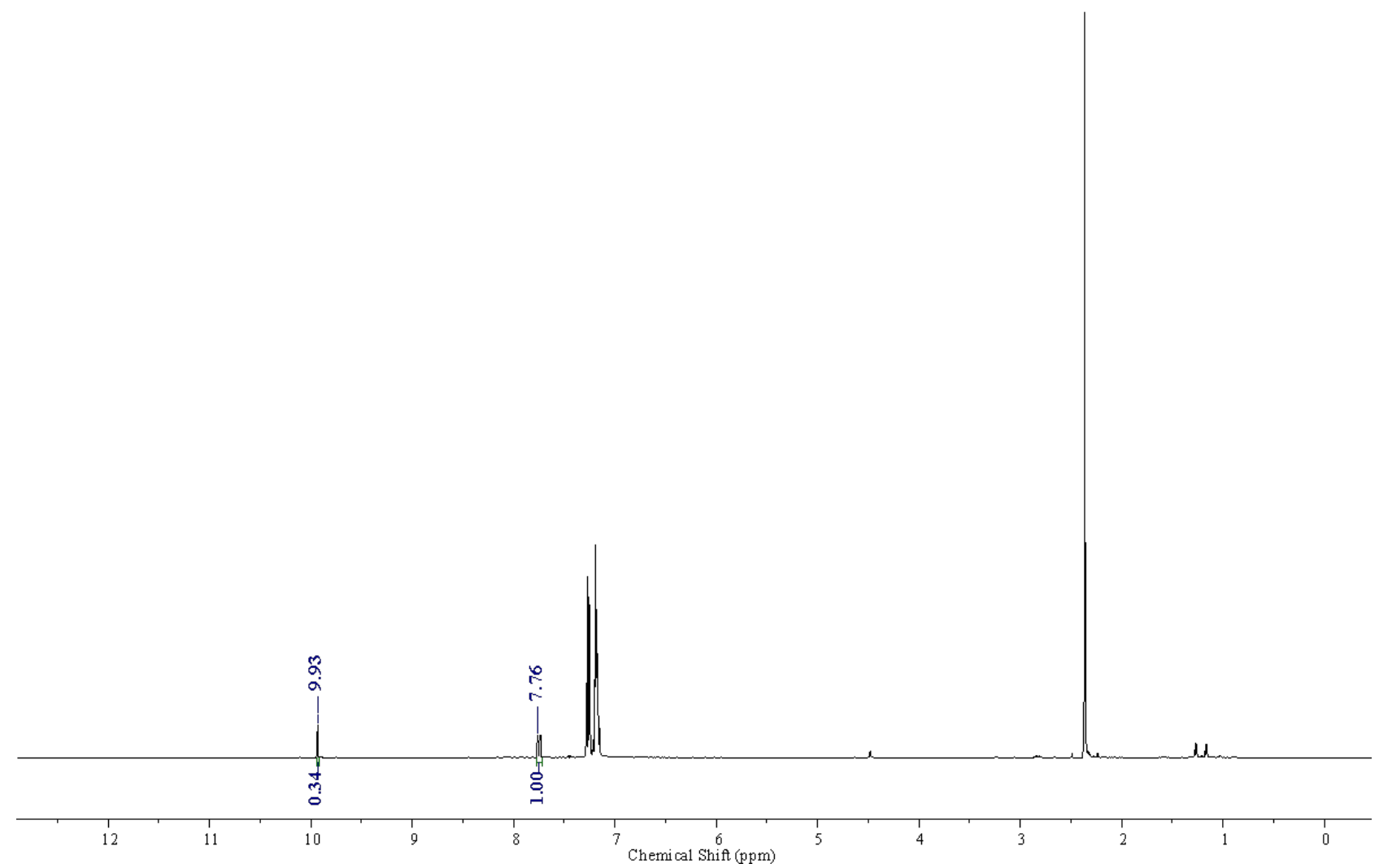

Entry 4: NMR for Crude reaction mixture

VDOS-606-E5.1.fid

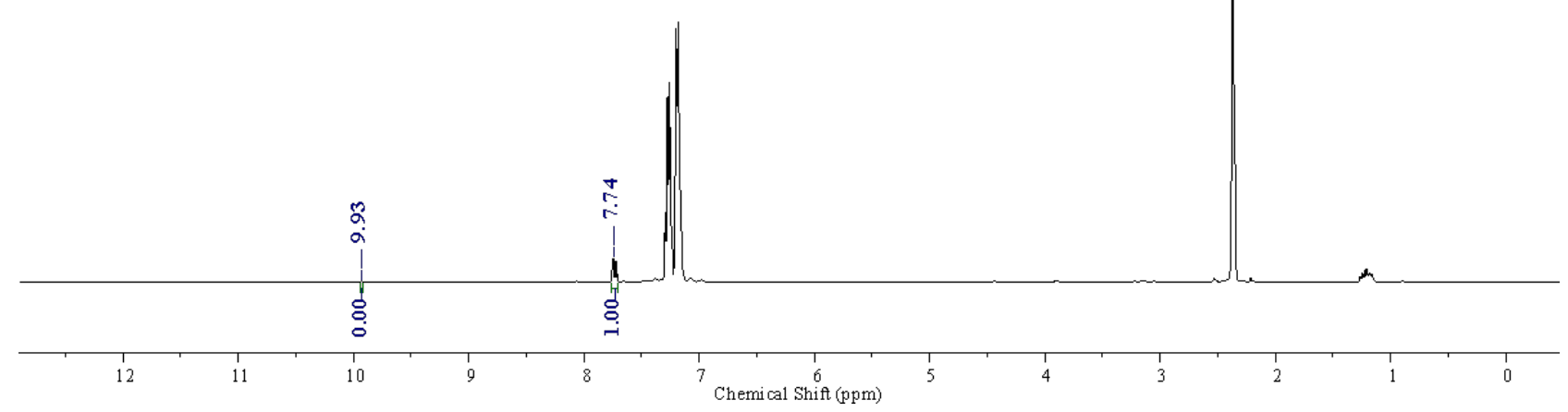


NMR after purification:

VDOS-606-VP final-2.8.fid

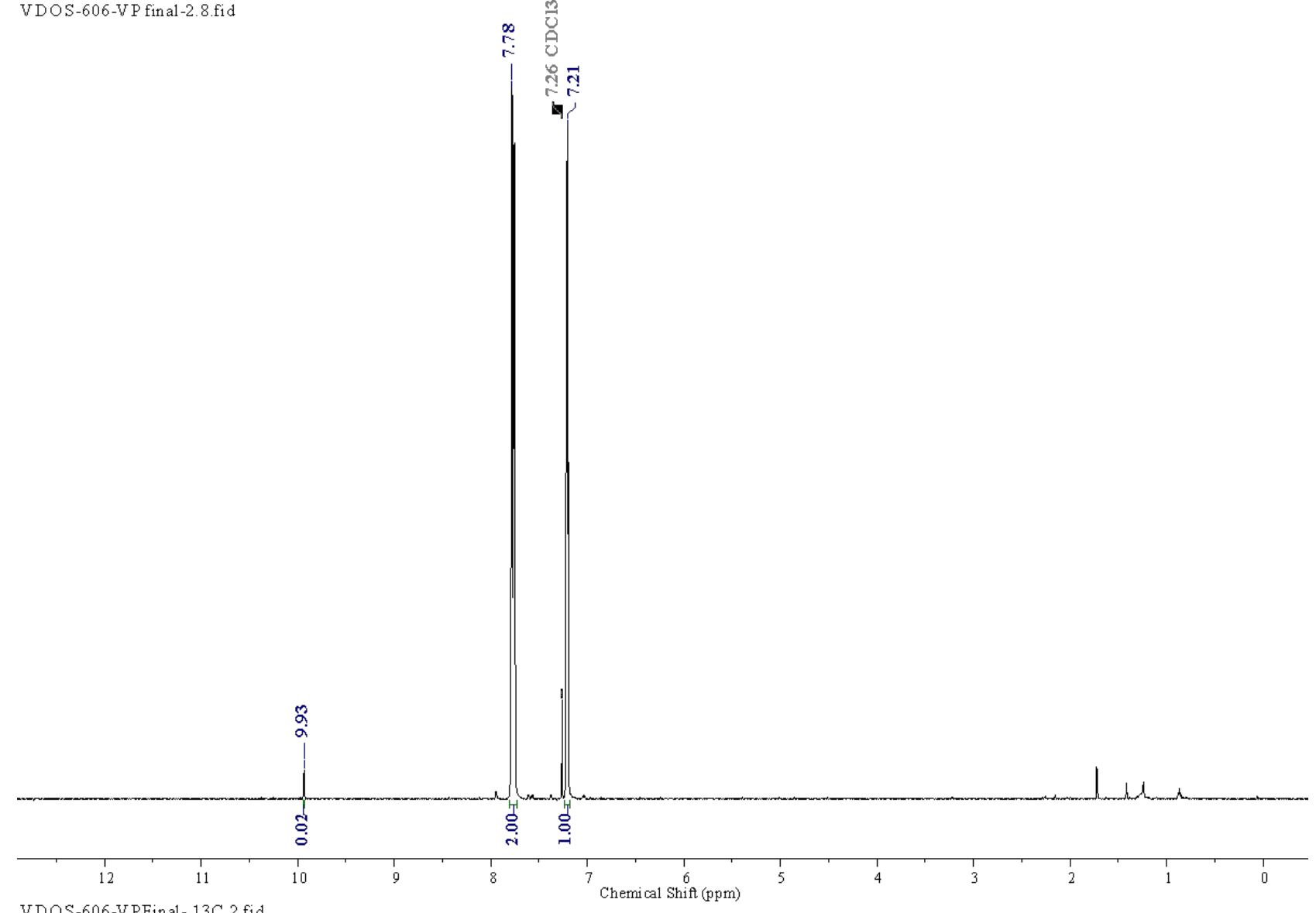

VDOS-606-VPFinal-13C.2.fid

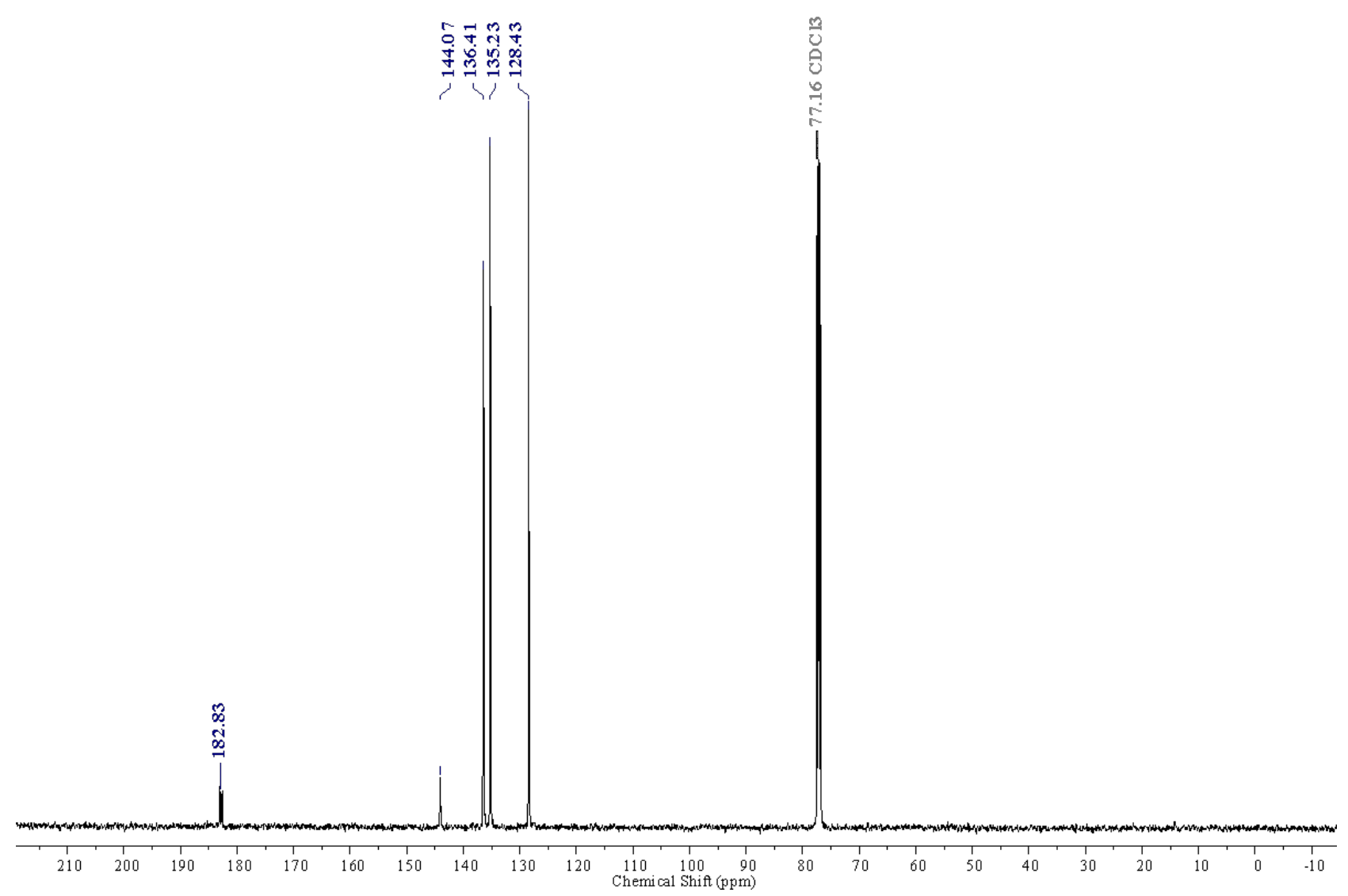


42. Indole-4-carboxaldehyde

45\%

Entry 1: NMR for Crude reaction mixture

VDOS-Indole-4 CHO-A1.2fid

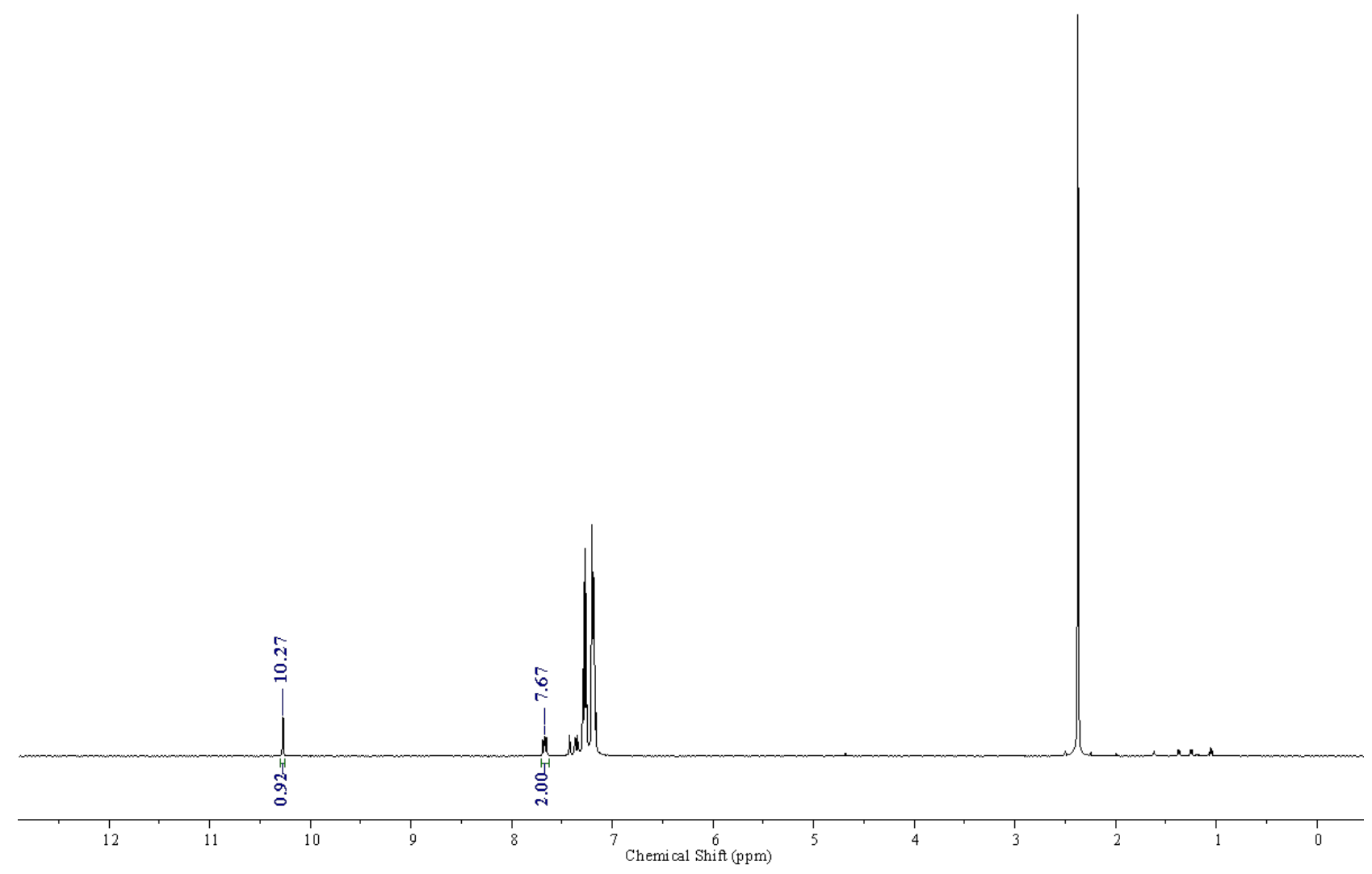


Entry 2: NMR for Crude reaction mixture

VDOS-Indole-4 $\mathrm{CHO}-\mathrm{B} 1.3$ fid

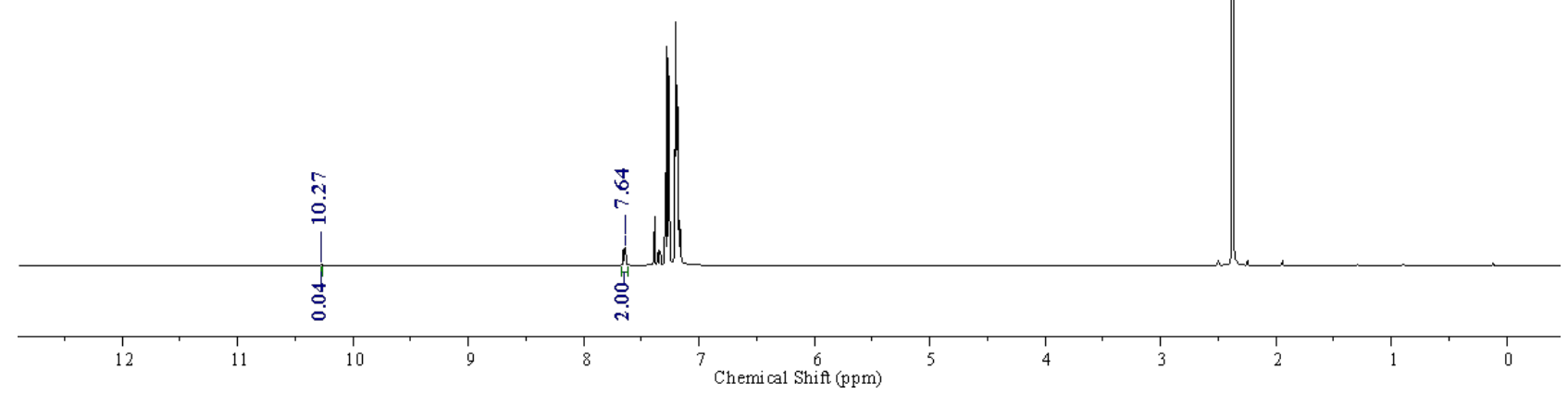

Entry 2': NMR for Crude reaction mixture, Duplicate

VDOS-633-B 2.6.fid

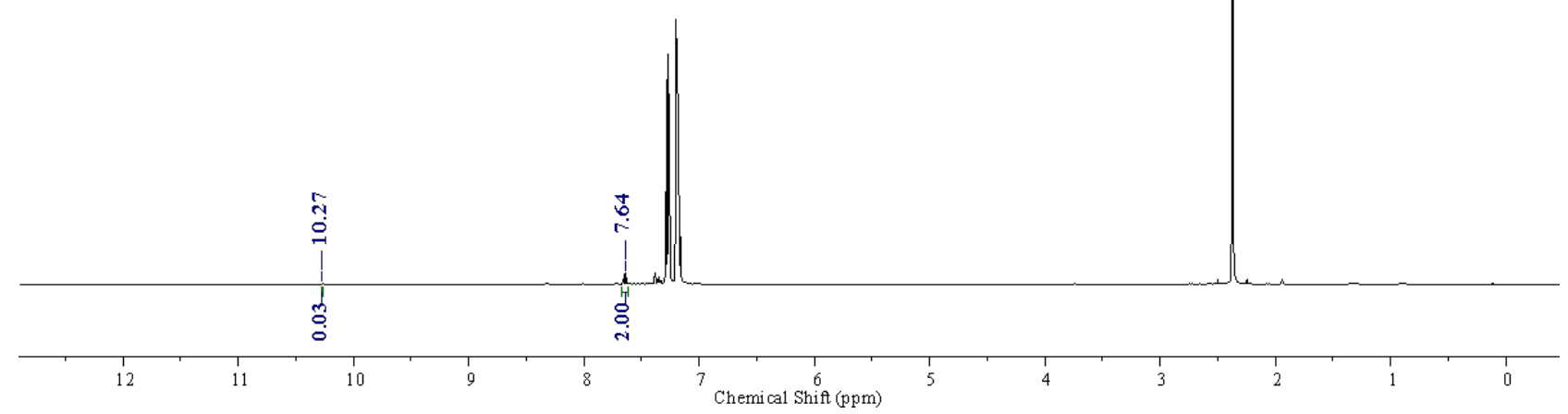


NMR after purification:

VD OS-633 VP.4.fid

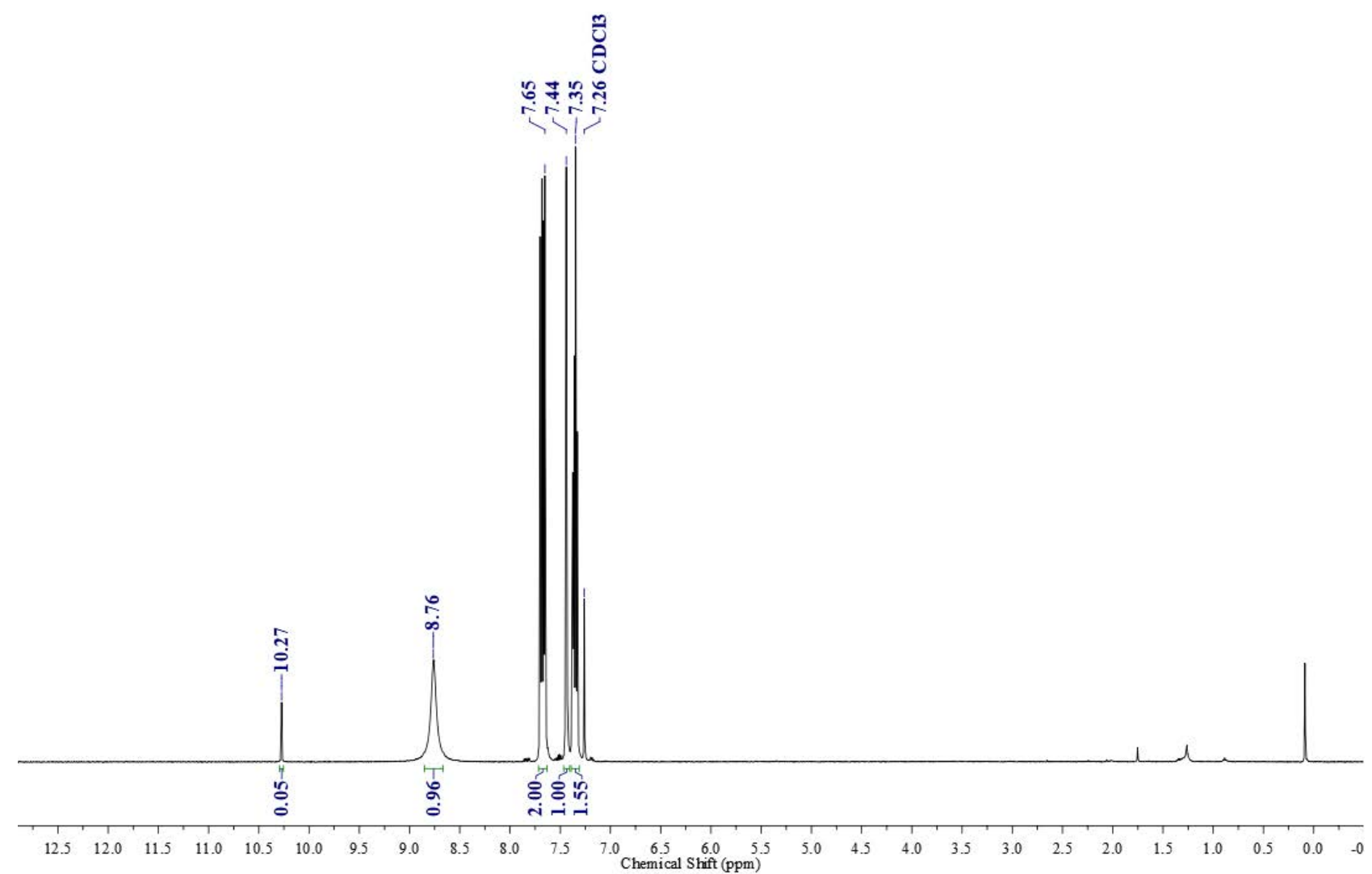
VDOS-633 VP 13C 3 fid

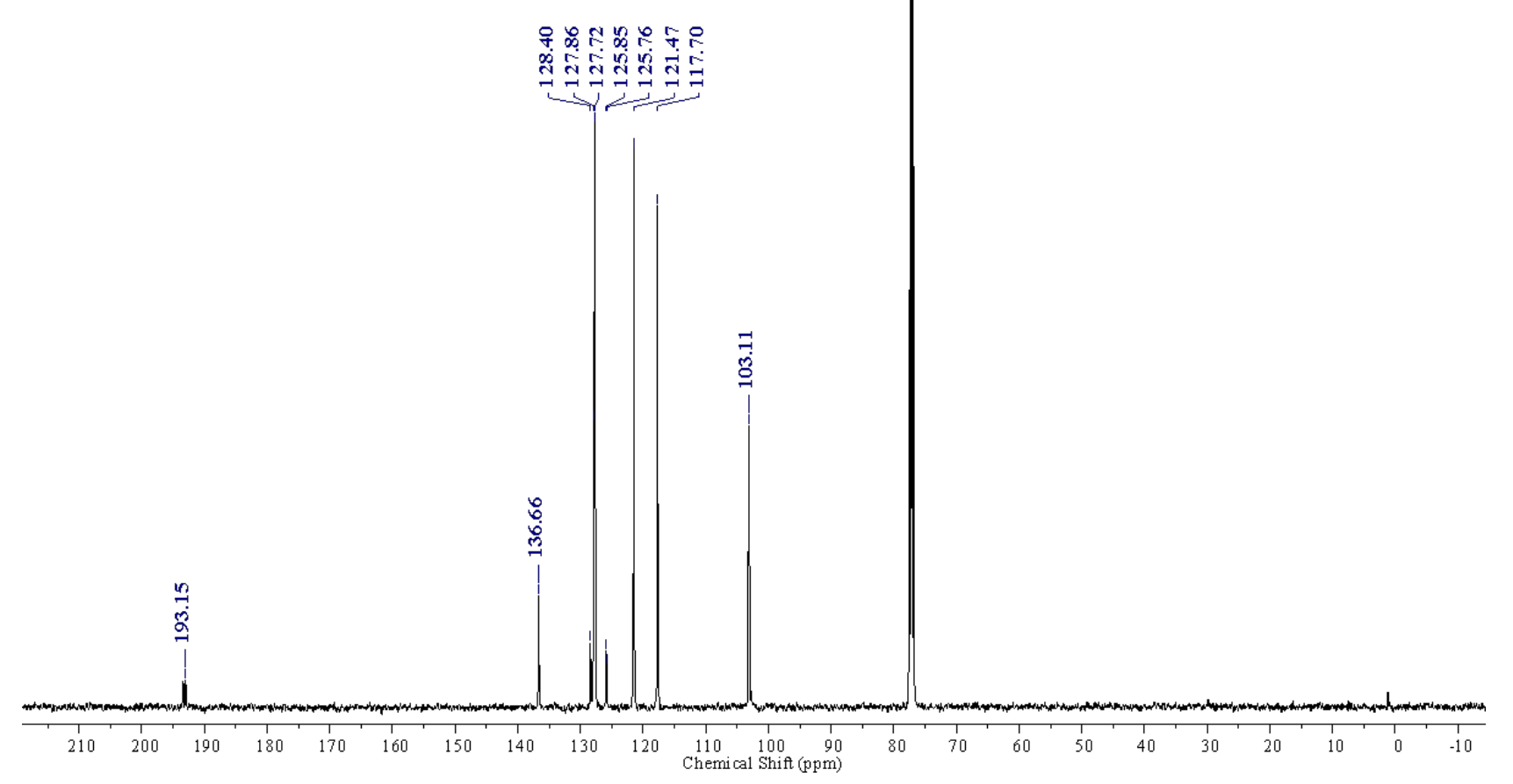


43. Quinoline-4-carbaldehyde-d<smiles>[2H]C(=O)c1ccnc2ccccc12</smiles>

Entry 1: NMR for Crude reaction mixture vDOS-617-A 1-2.3.fid

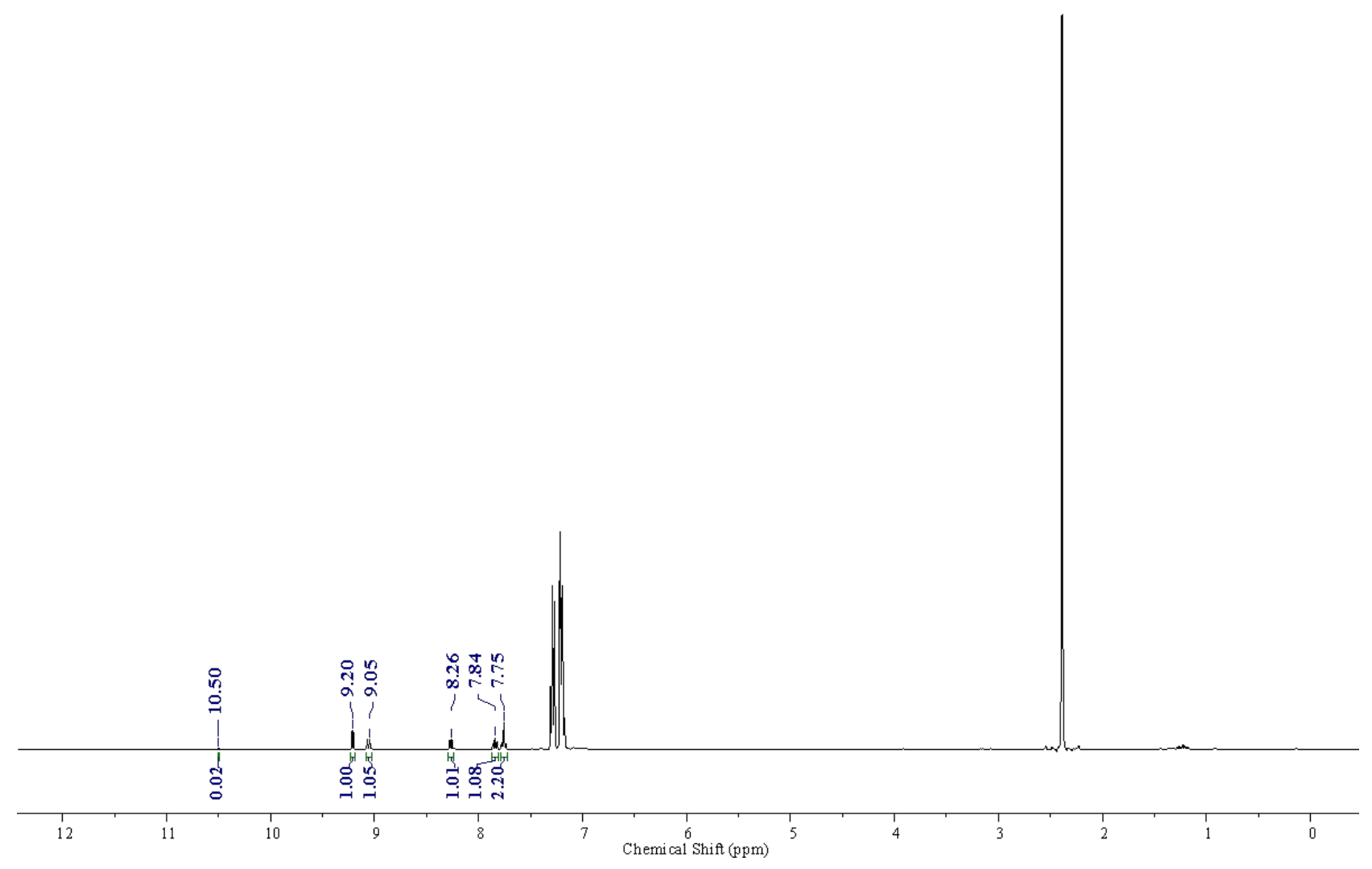


Entry 1': NMR for Crude reaction mixture, Duplicate VDOS-617-A2-2.2.fid

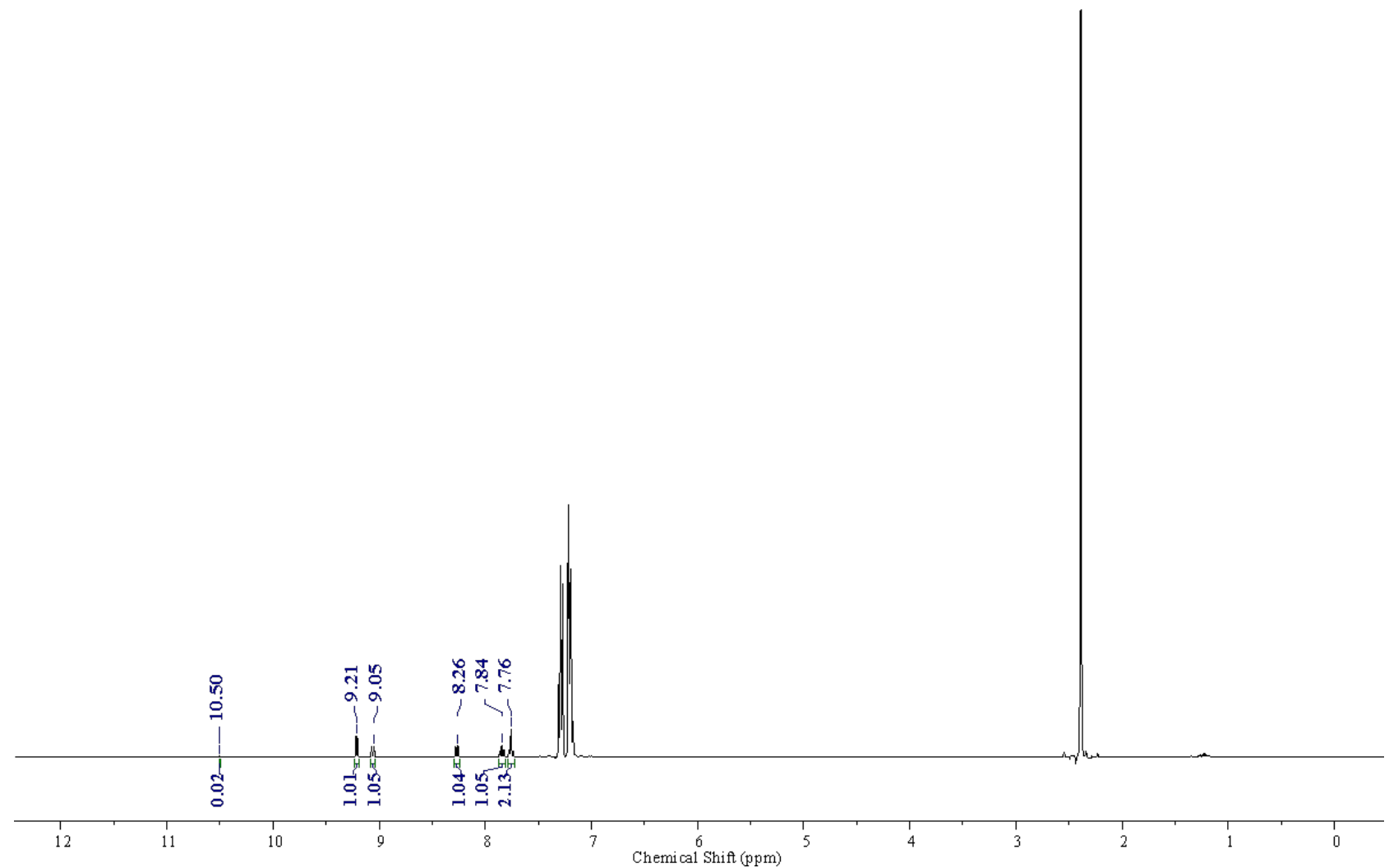

Entry 2': NMR for Crude reaction mixture

VDOS-617 2 MPB dup

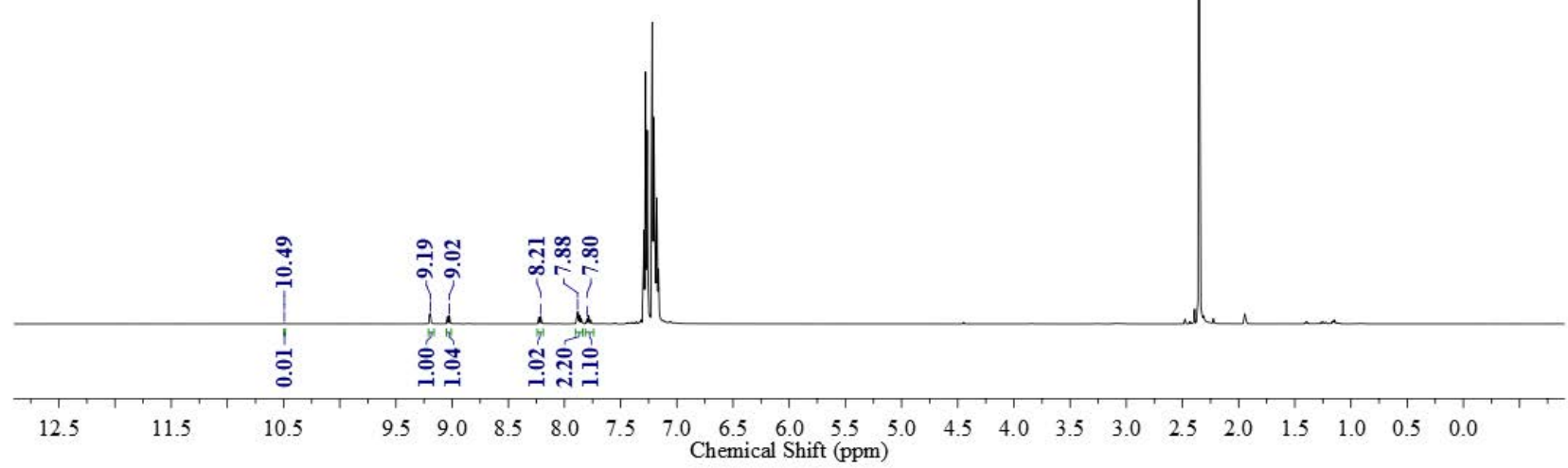


Entry 2': NMR for Crude reaction mixture, Duplicate

vDOS-617 2mol PB

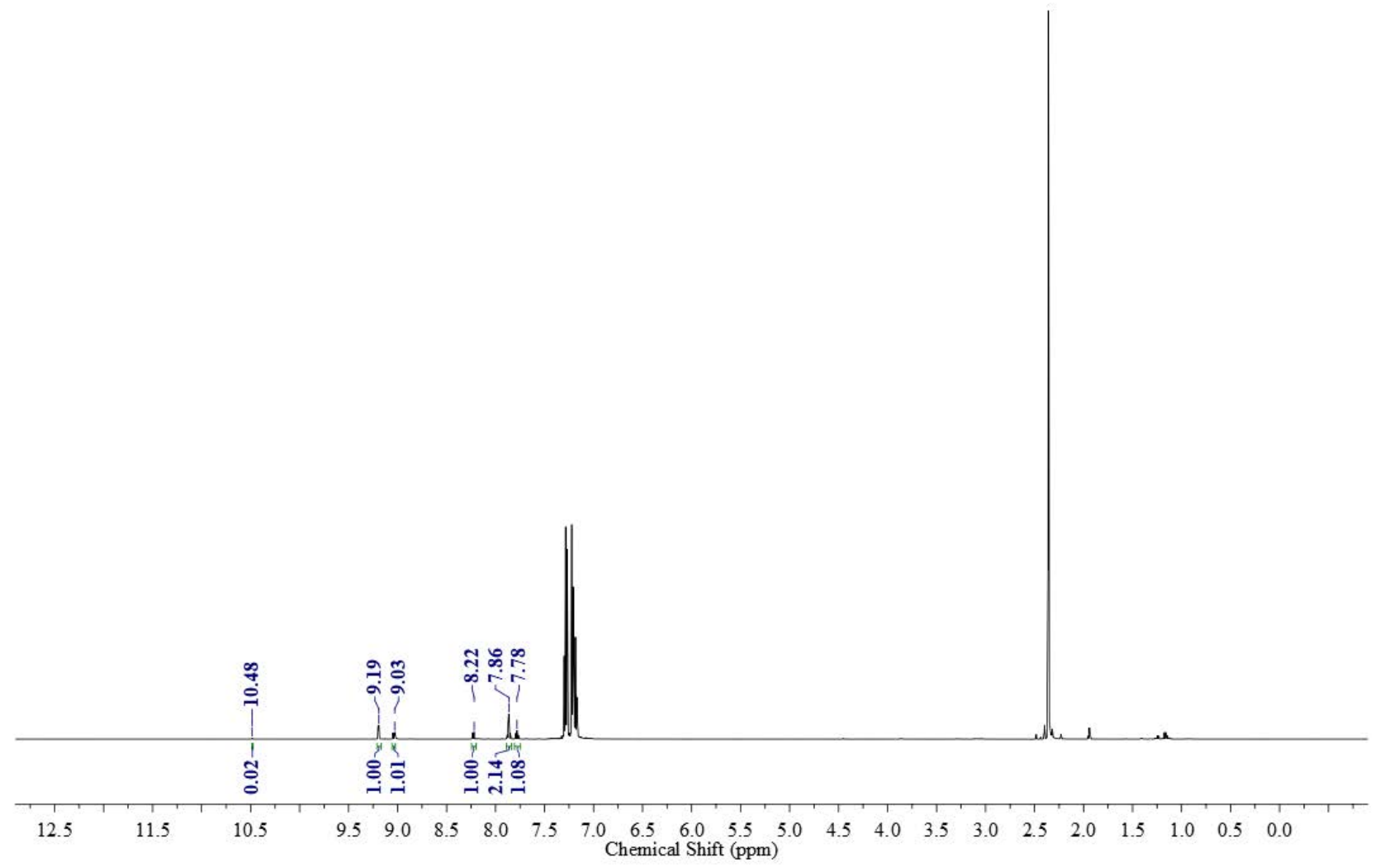

NMR after purification:

VDOS-617-A1 pure.2.fid

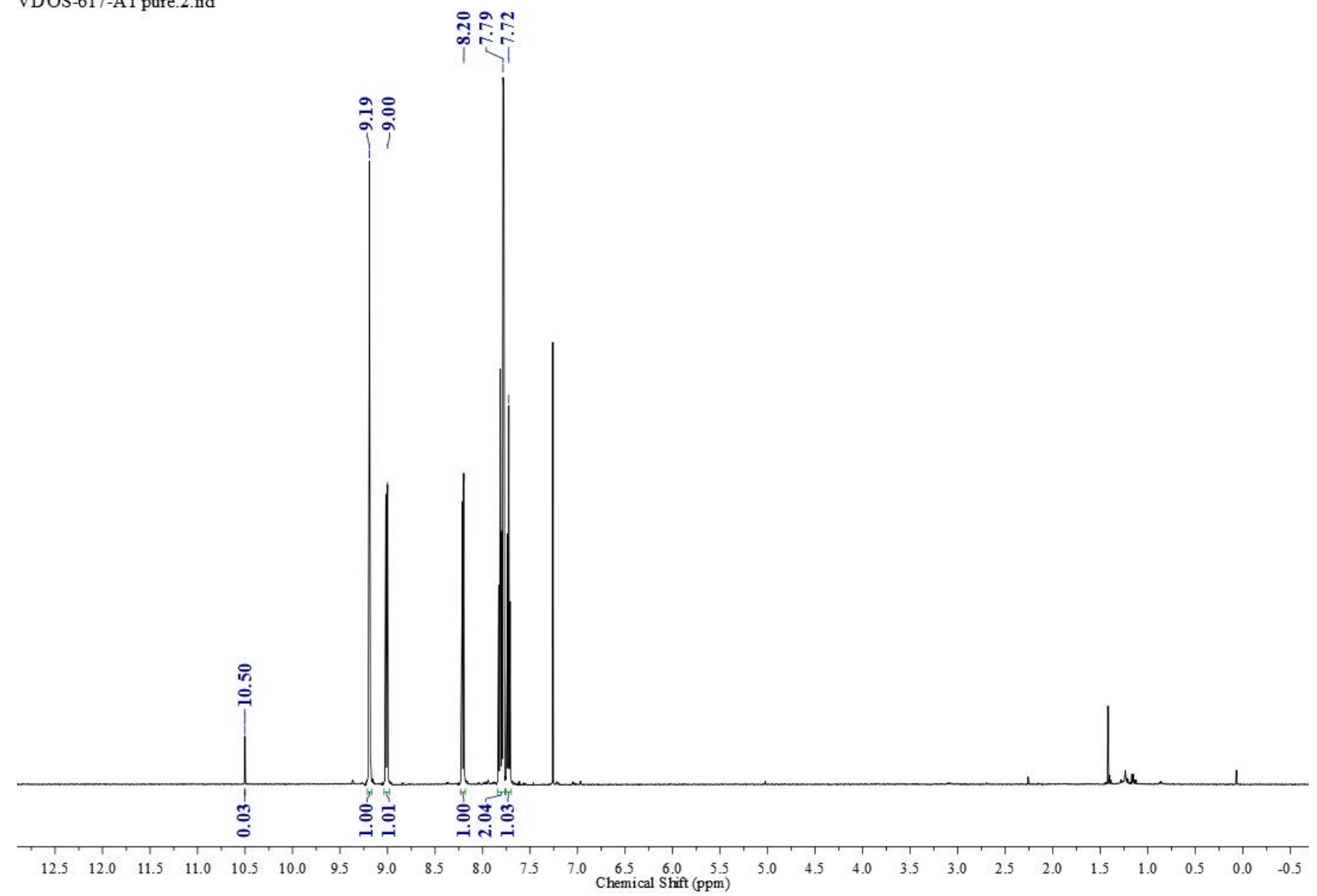


VDOS-617-A 1 pure 13C .4.fid

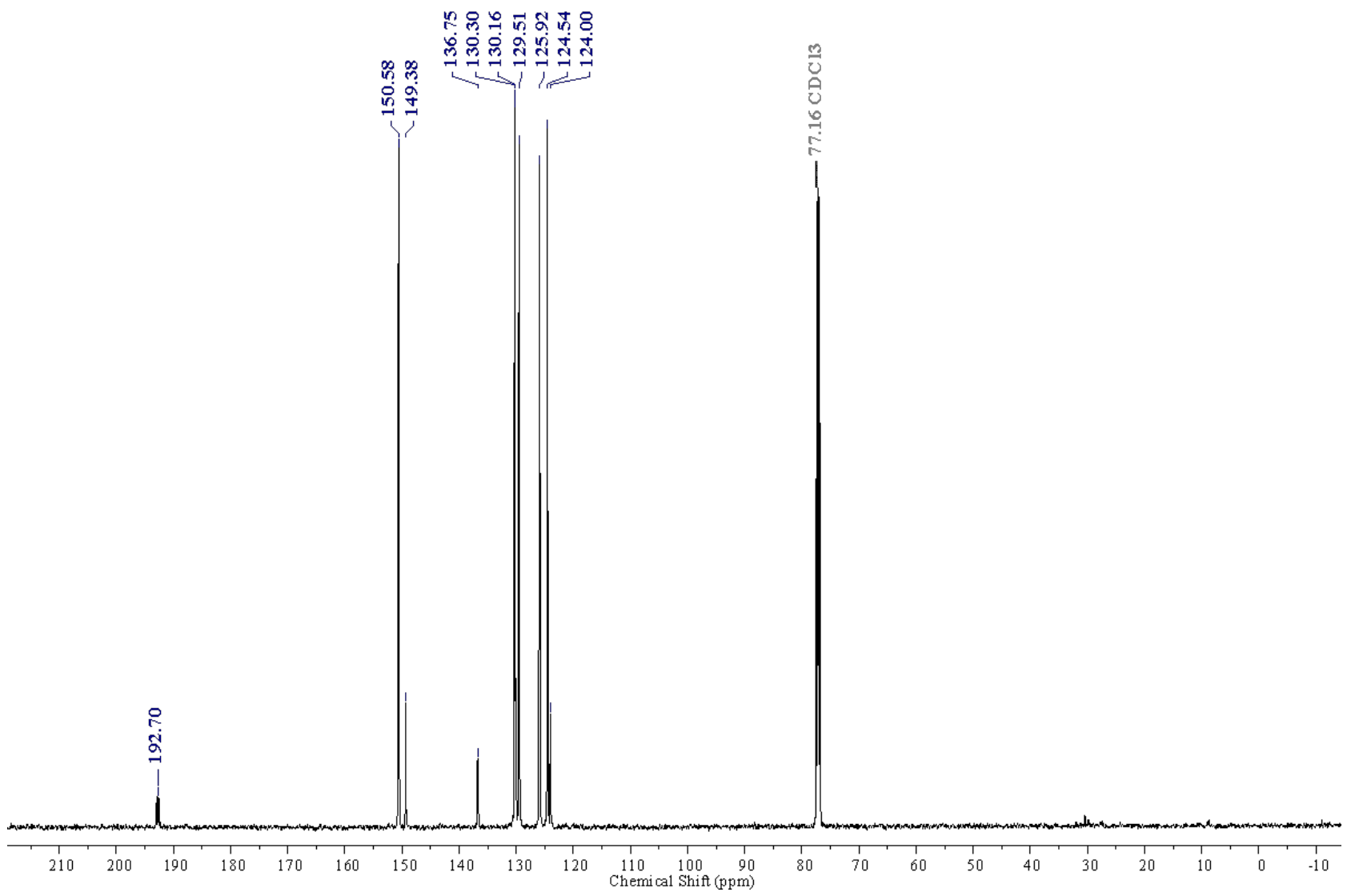

338 
44. Benzo[b]thiophene-2-carbaldehyde-d<smiles>[2H]C(=O)c1cc2ccccc2s1</smiles>

1,2-bis(benzo[b]thiophen-2-yl)-2-hydroxyethan-1-one:

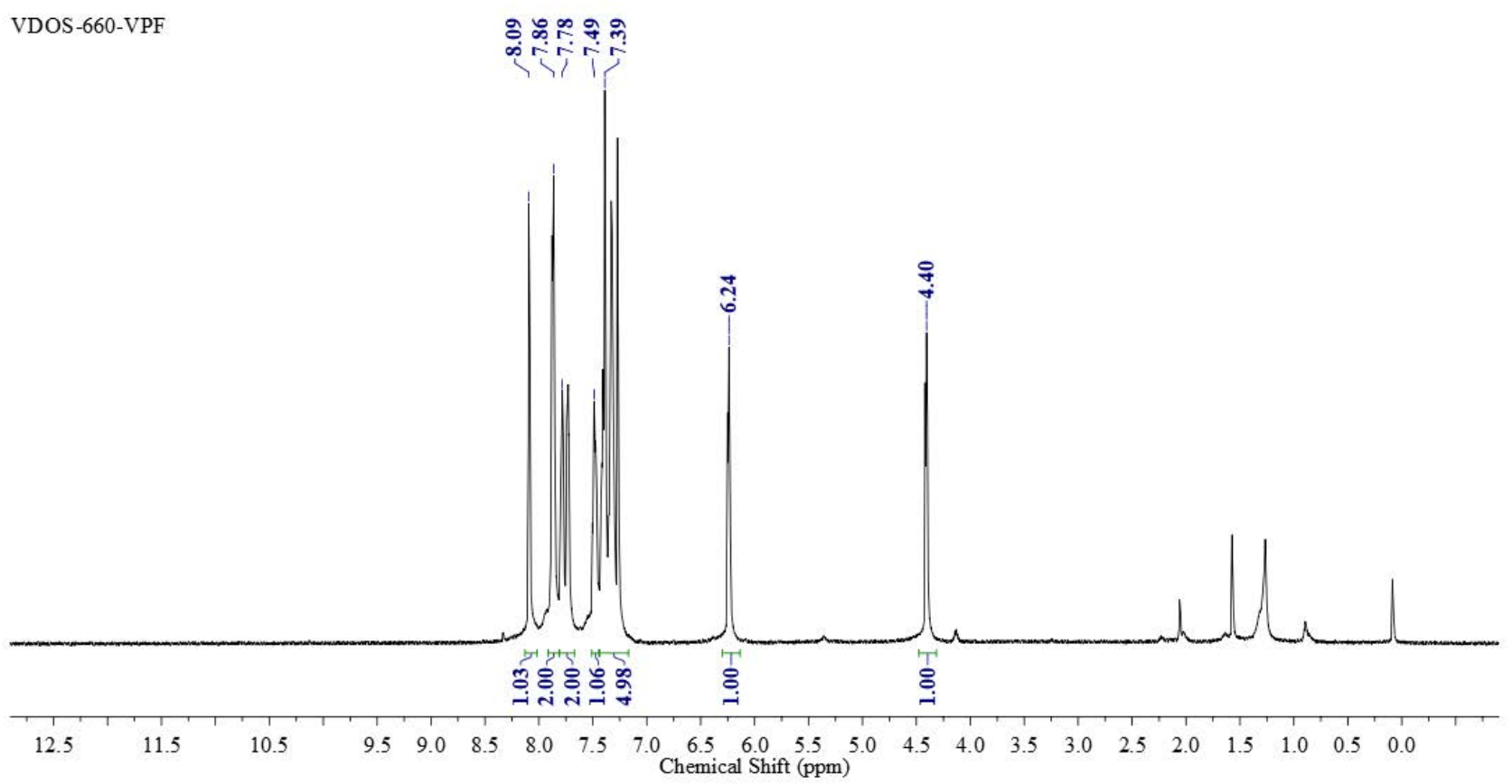

Entry 1: NMR for Crude reaction mixture vDOS-615-A1.5.fid

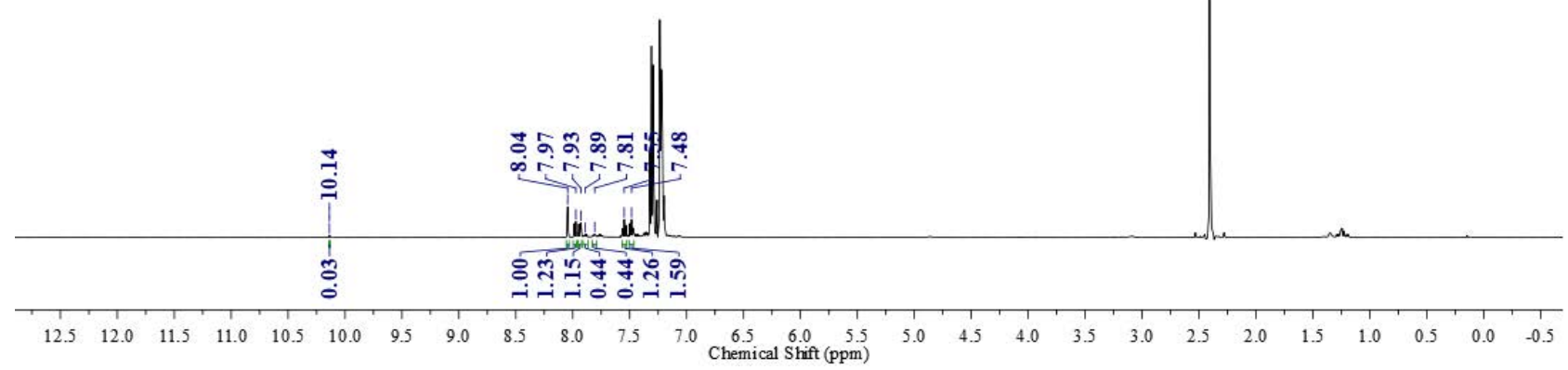


Entry 2: NMR for Crude reaction mixture

VDOS-615-C 1.5.fid

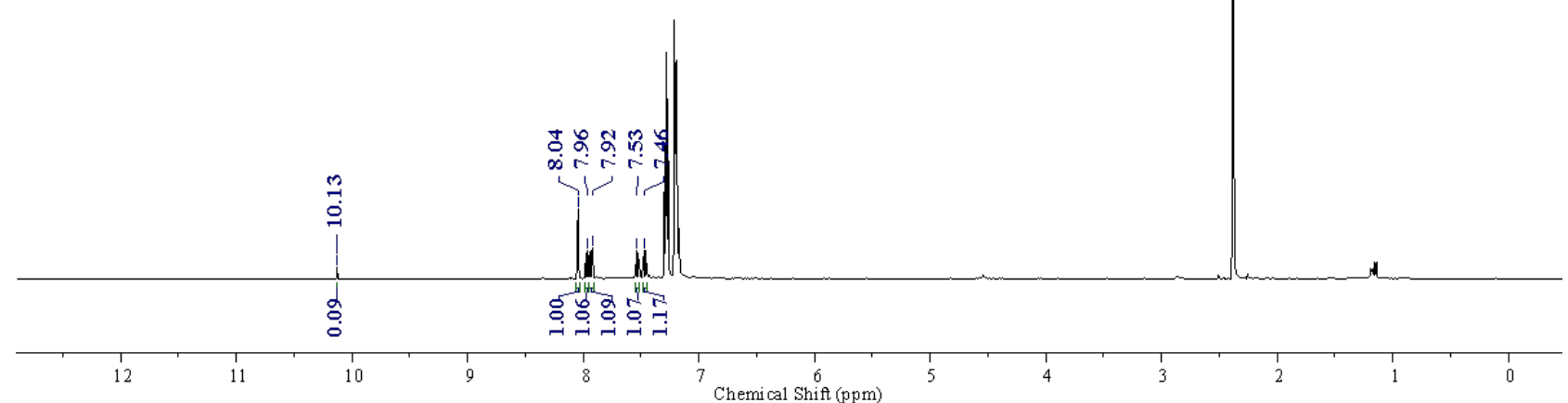

Entry 3: NMR for Crude reaction mixture

VDOS-615-B 1.6. fid

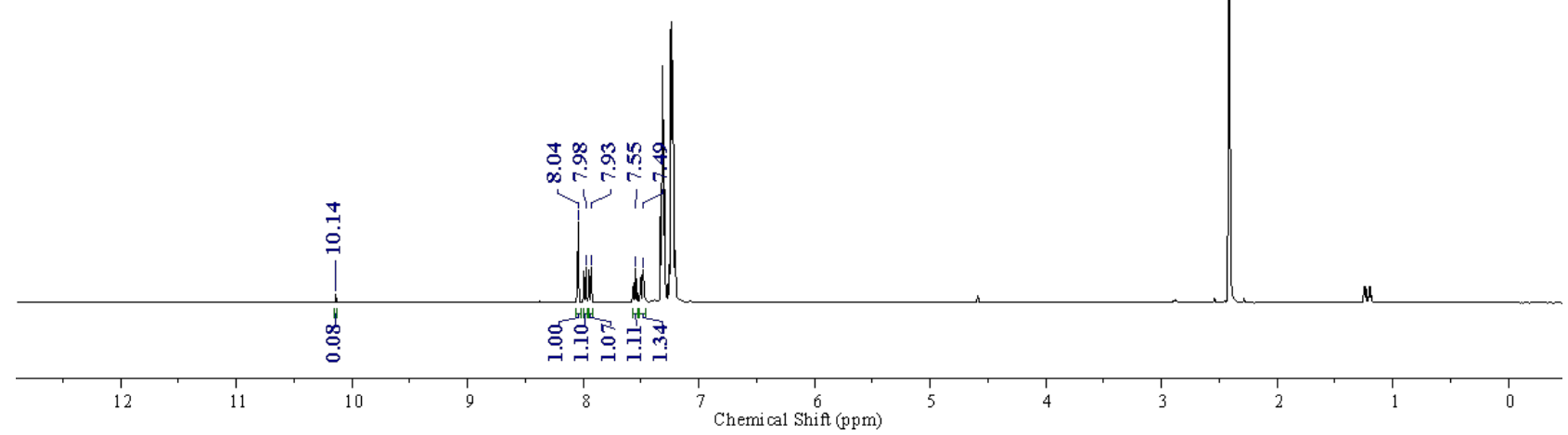


Entry 4: NMR for Crude reaction mixture

VDOS-615-D 1.6.fid

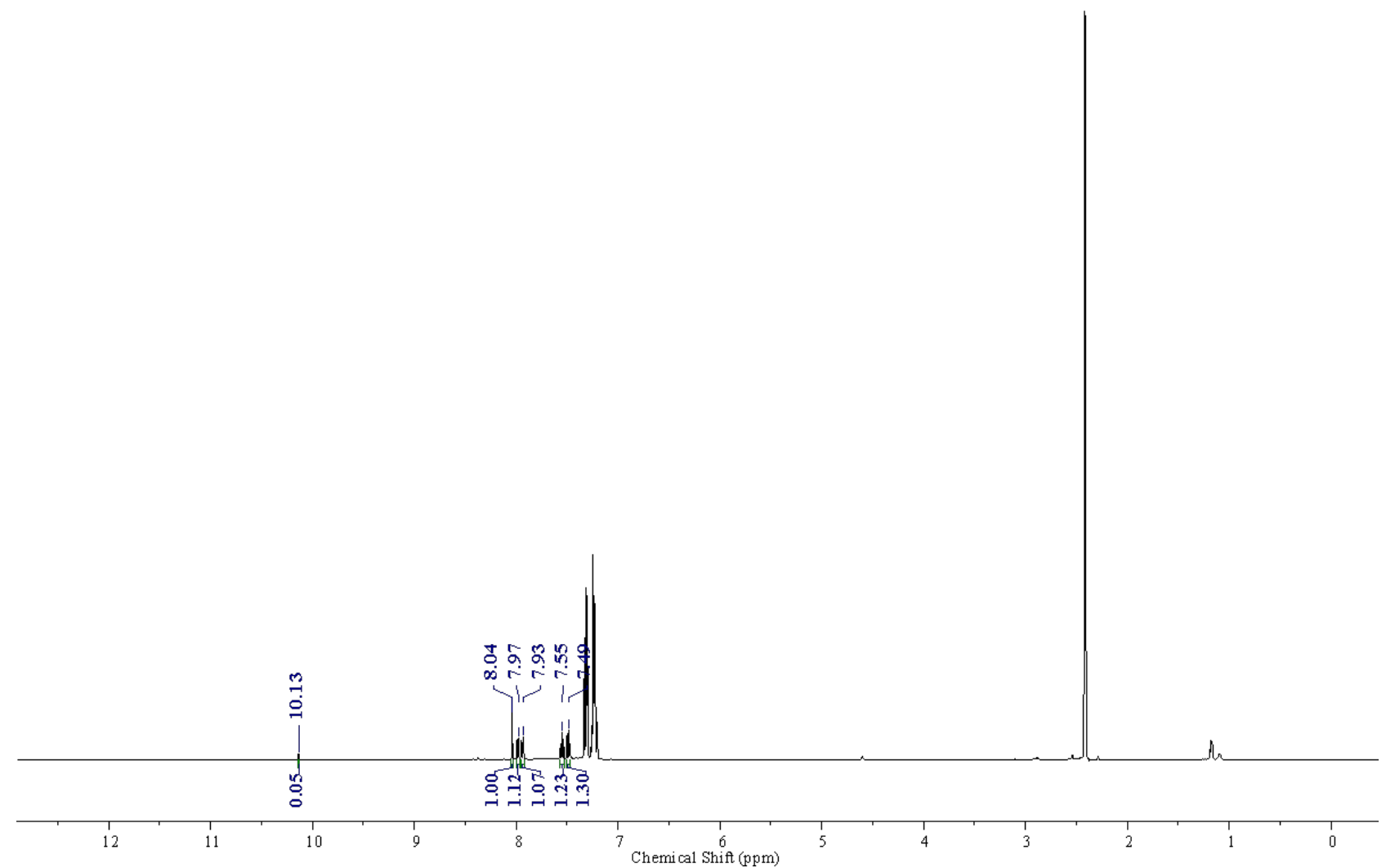

Entry 4': NMR for Crude reaction mixture, Duplicate

vDOS-615-F3.3fid

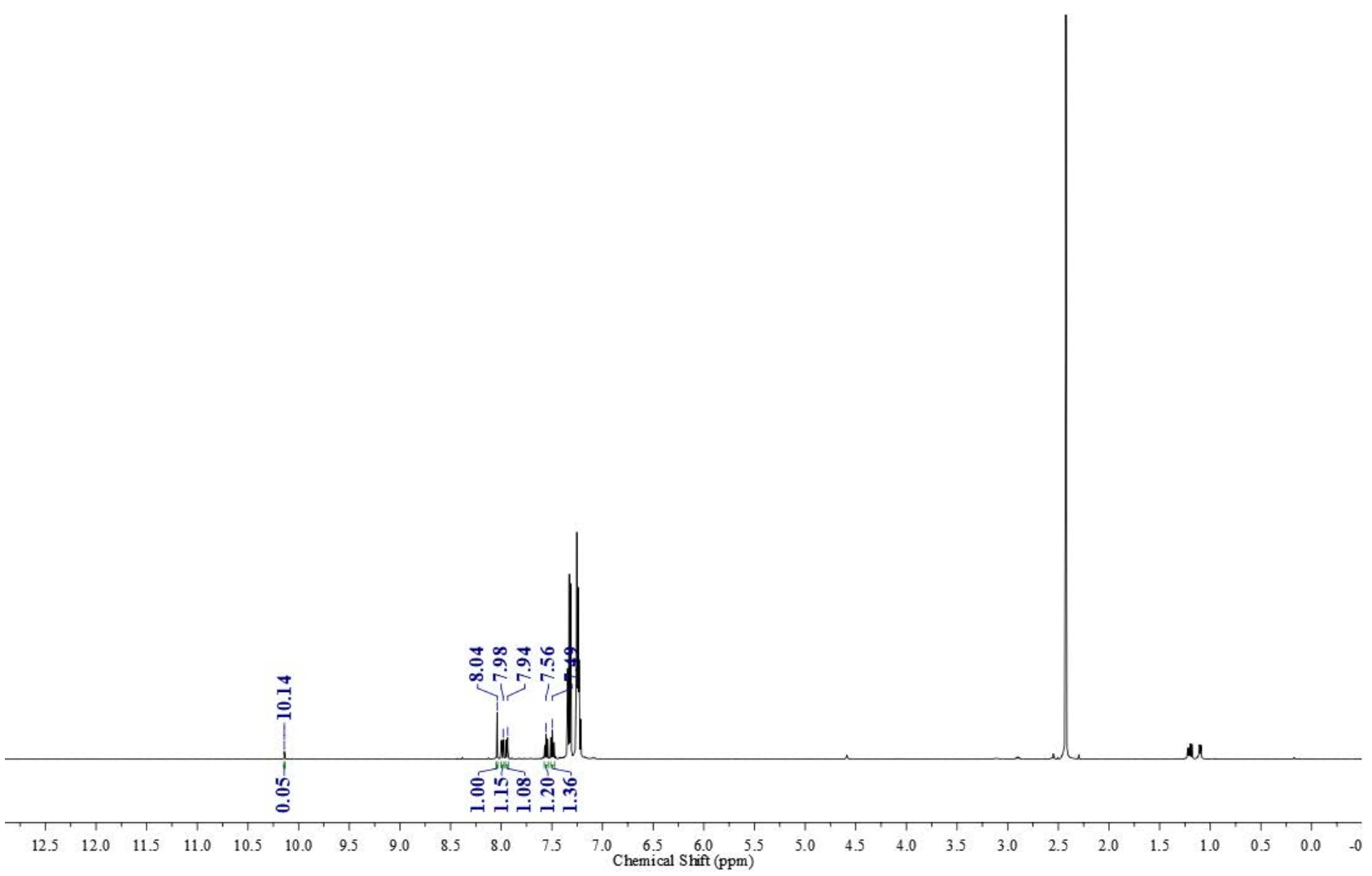


Entry 5: NMR for Crude reaction mixture

VDOS-615-G5.3.fid

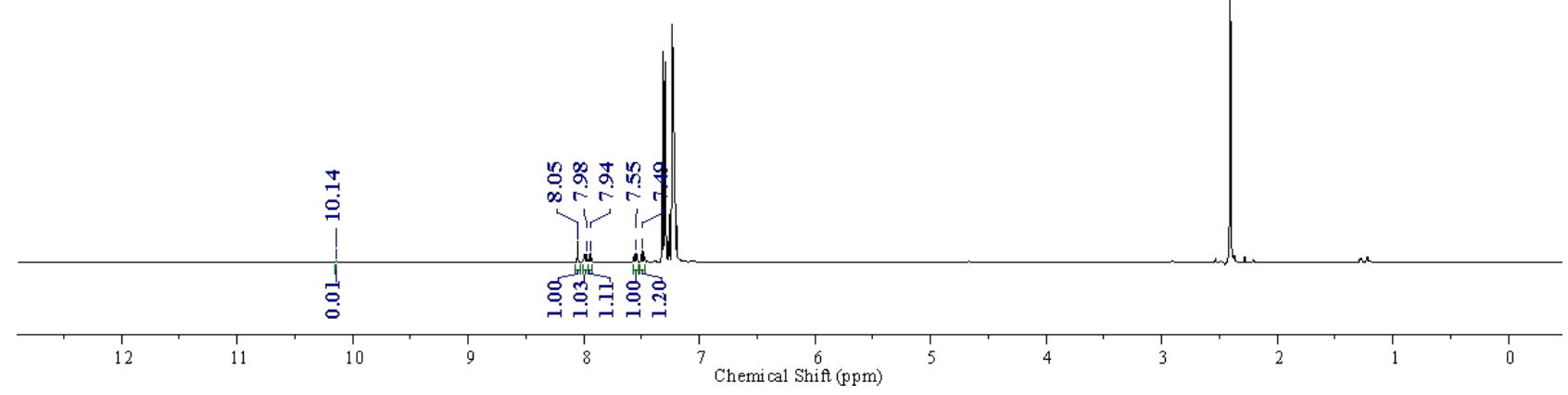

Entry 5': NMR for Crude reaction mixture, Duplicate

VDOS-615-G 1.3.fid

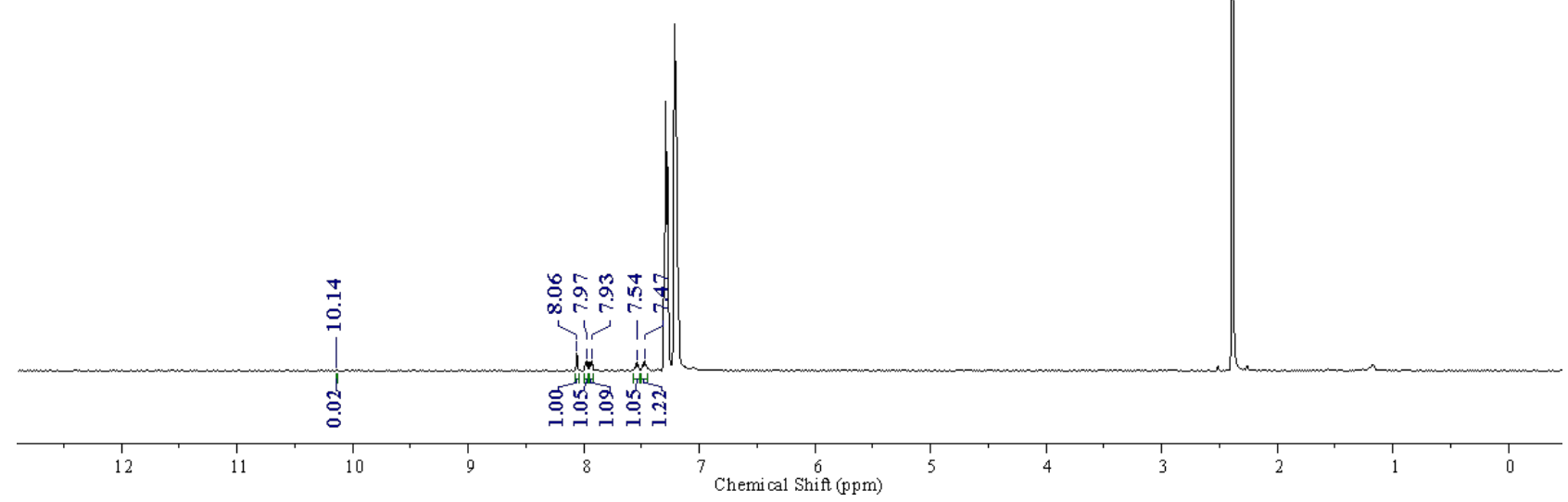


NMR after purification:

VDOS-615-G1 VP.5.fid

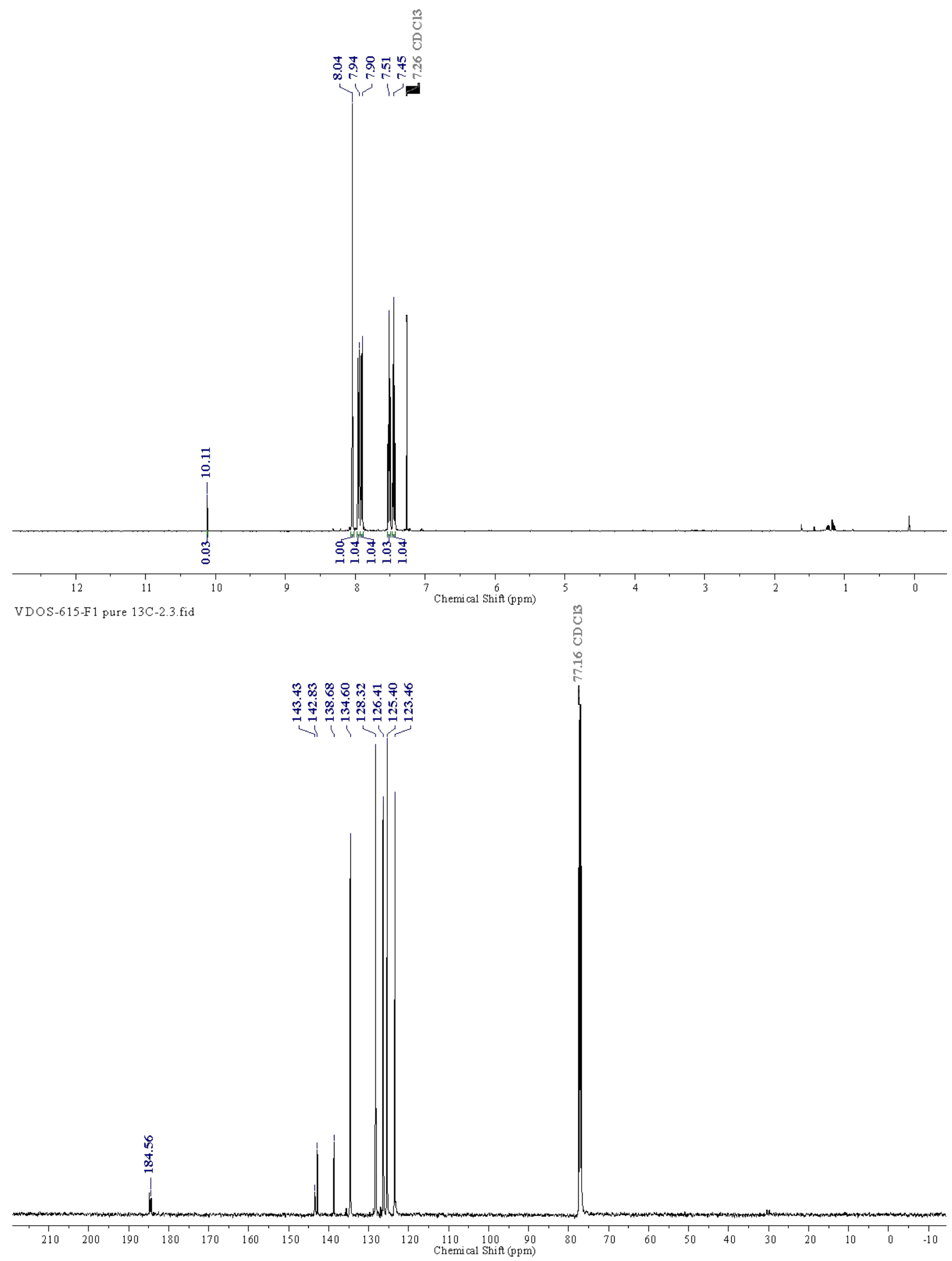


45. ${ }^{t}$ Butyl-3-formyl-indole-1-carboxylate- $d$<smiles>[2H]C(=O)c1cn(C(=O)OC(C)(C)C)c2ccccc12</smiles>

Entry 1: NMR for Crude reaction mixture VDOS-629-C 1.6.fid

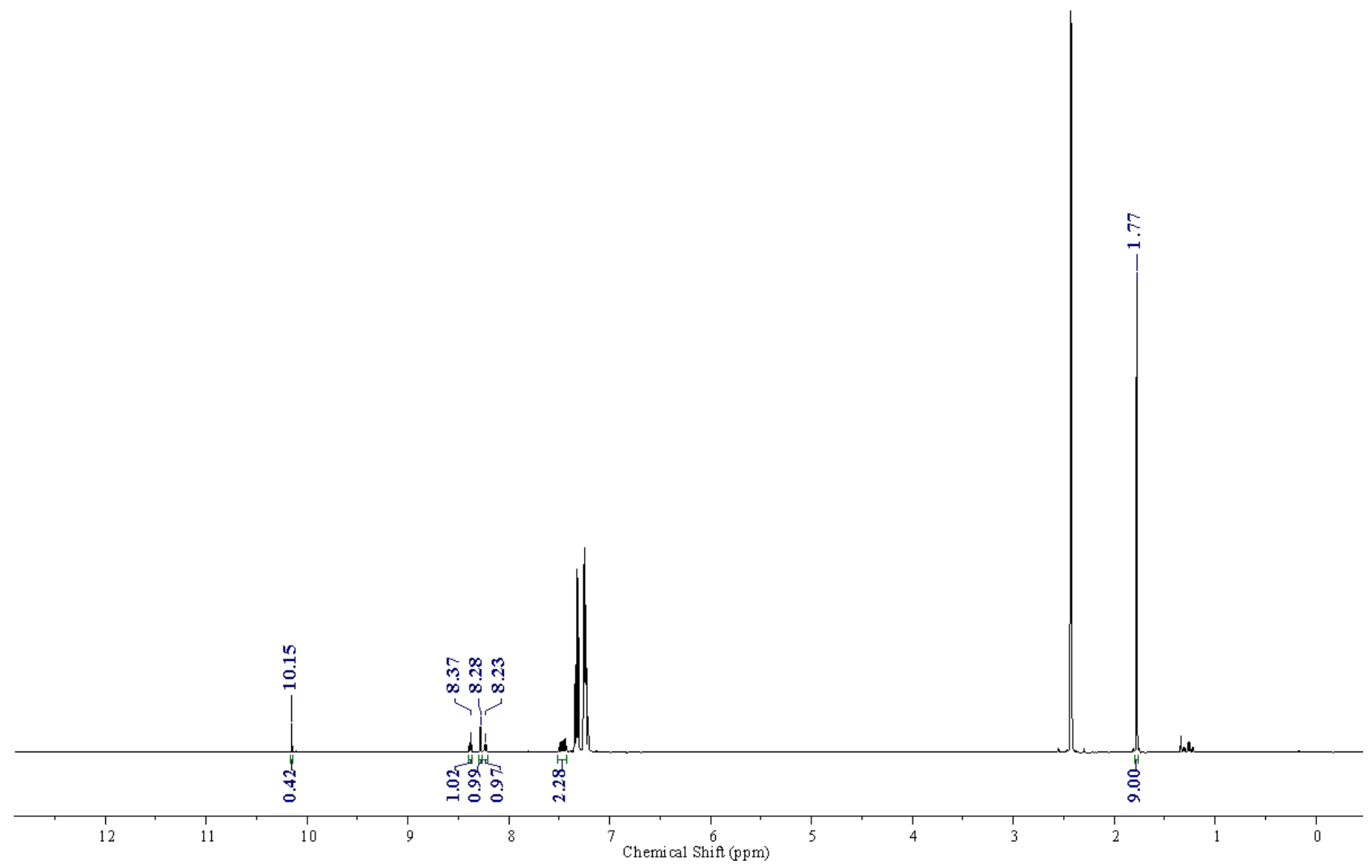


Entry 2: NMR for Crude reaction mixture

VDOS-629-D 1.7.fid

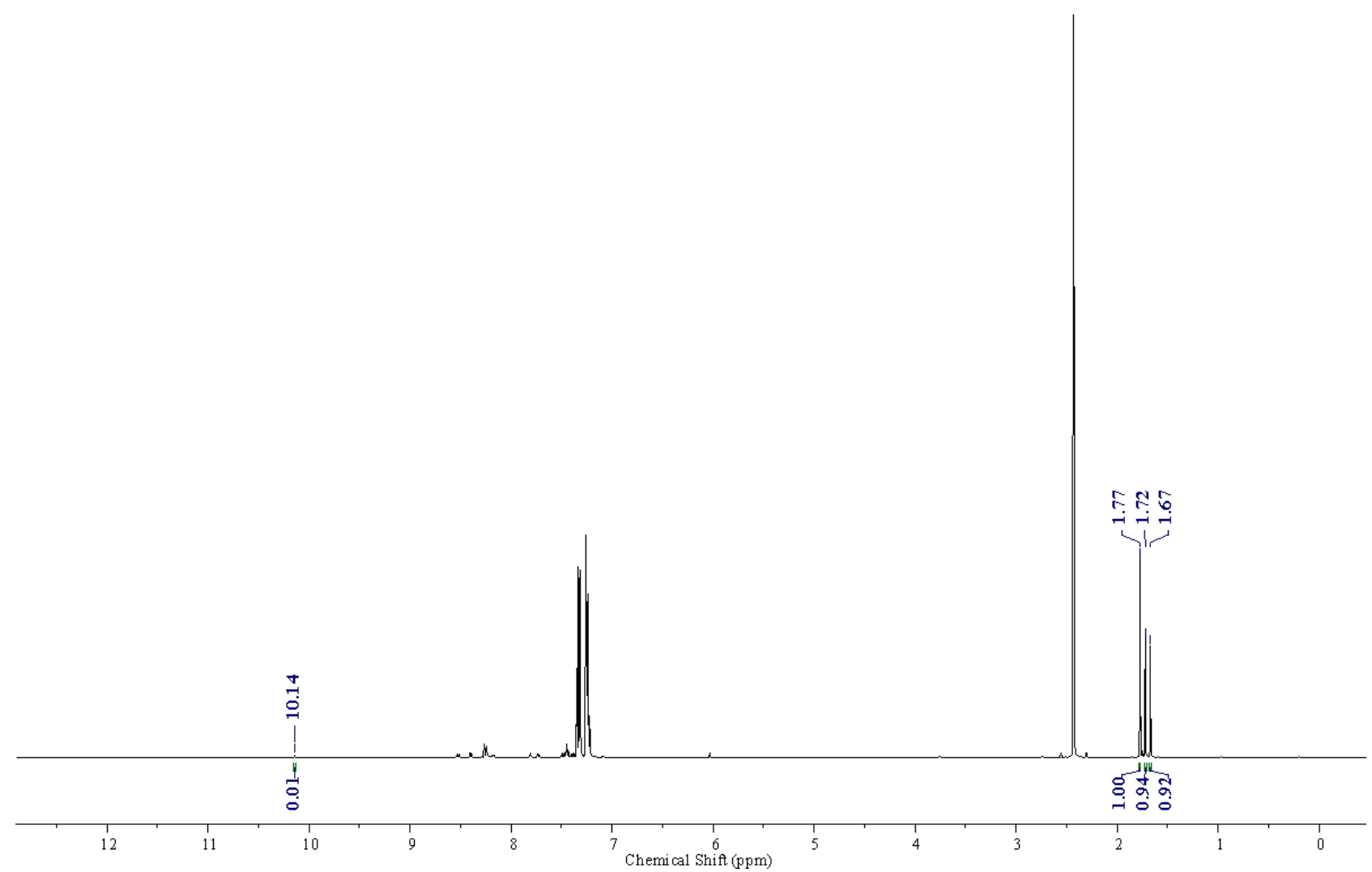

Entry 3: NMR for Crude reaction mixture

VDOS-629-E 1.8.fid

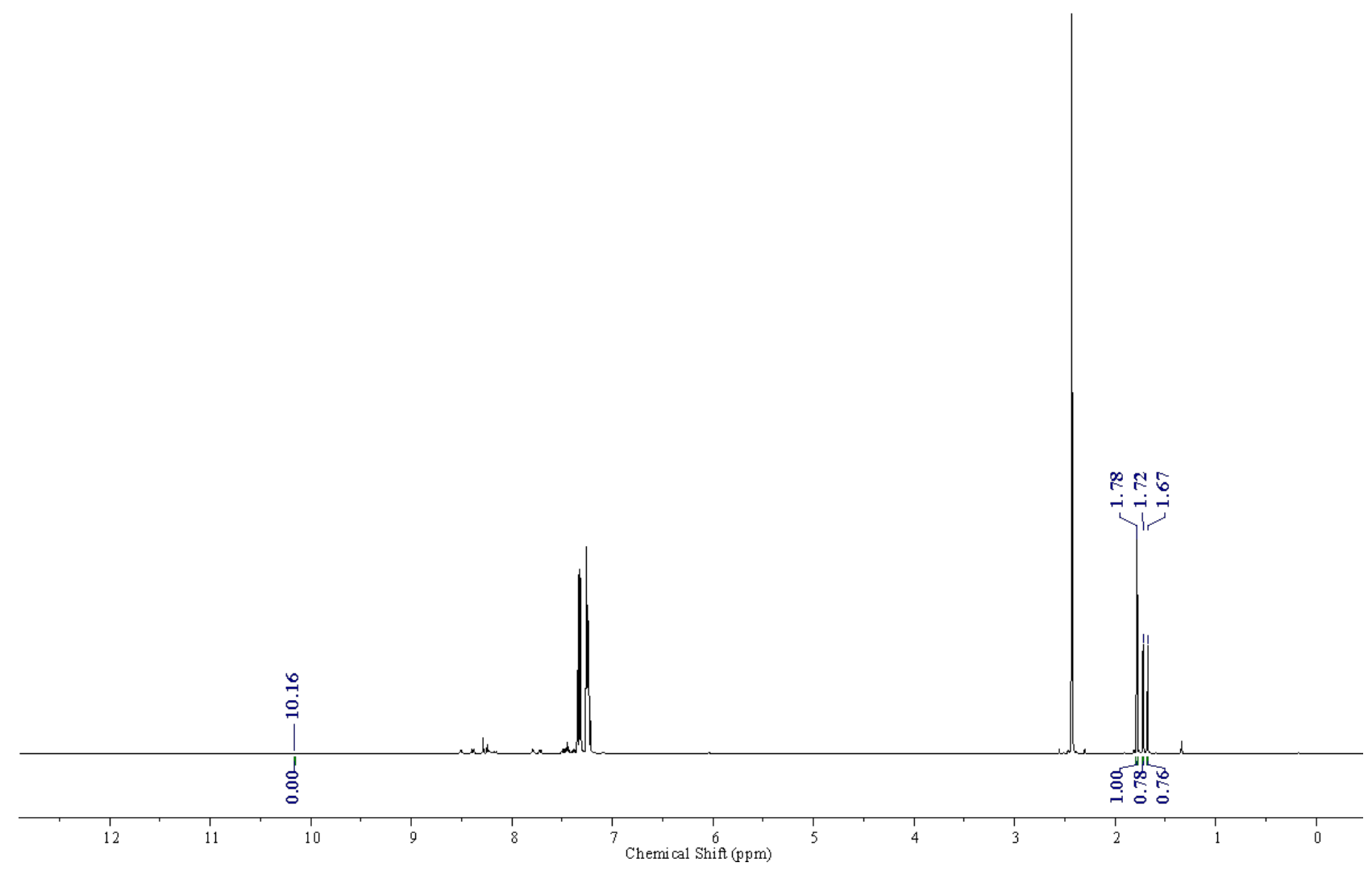


Entry 4: NMR for Crude reaction mixture

VDOS-629-F1.7.fid

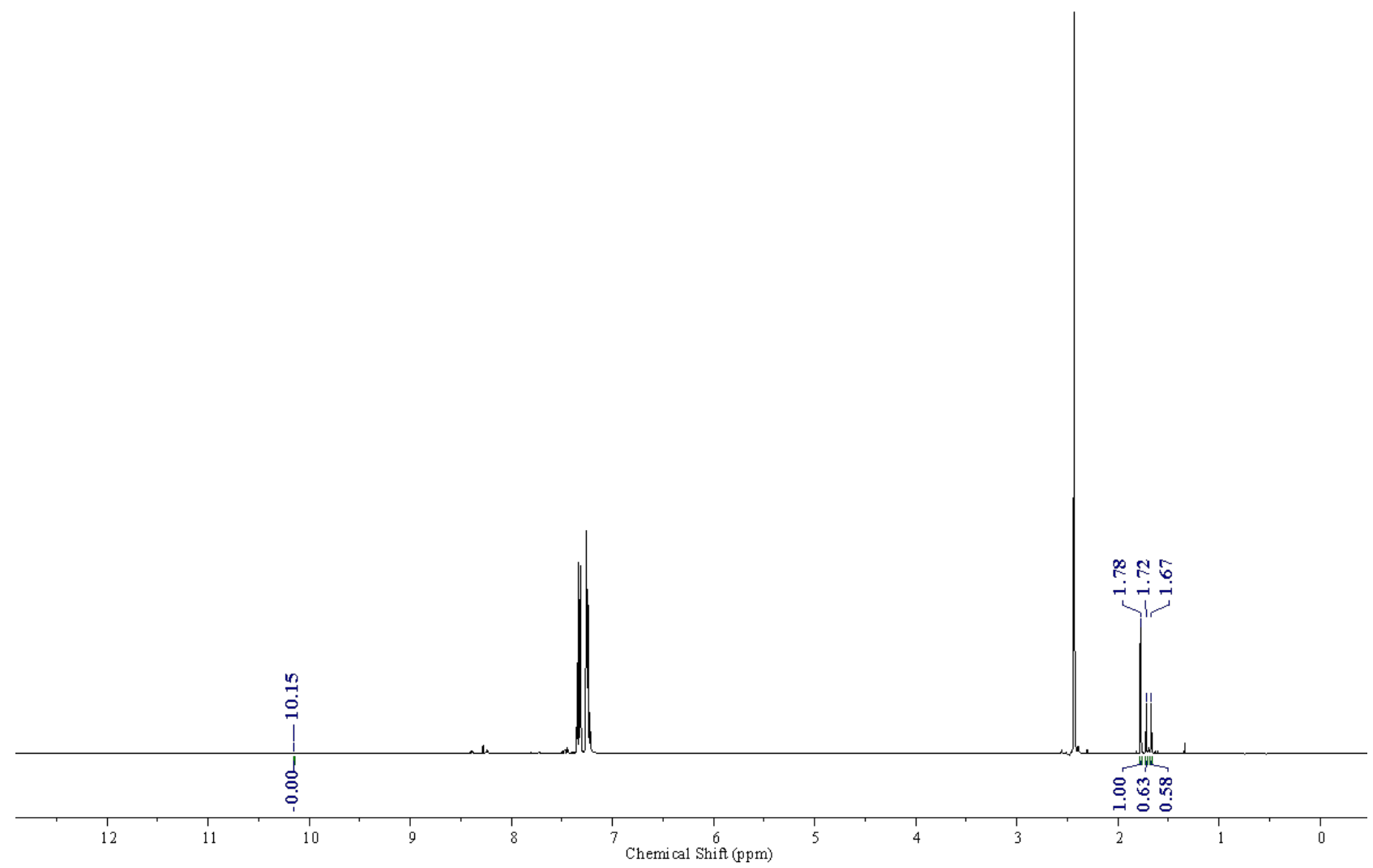

Entry 5: NMR for Crude reaction mixture

VDOS-629-10m o1\% PB.3.fid

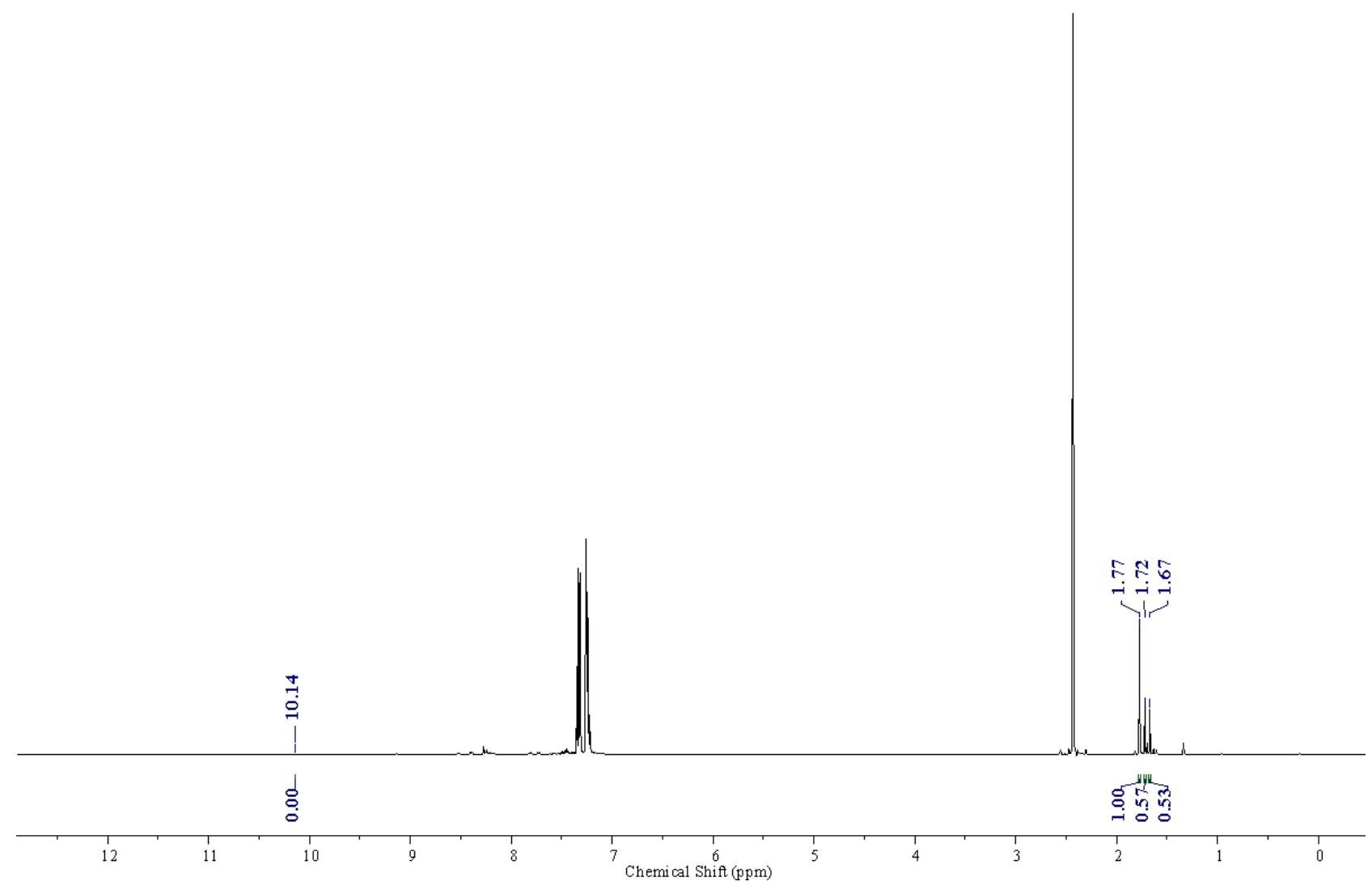


Entry 6: NMR for Crude reaction mixture

VDOS-629-30m ol\% PB.4.fid

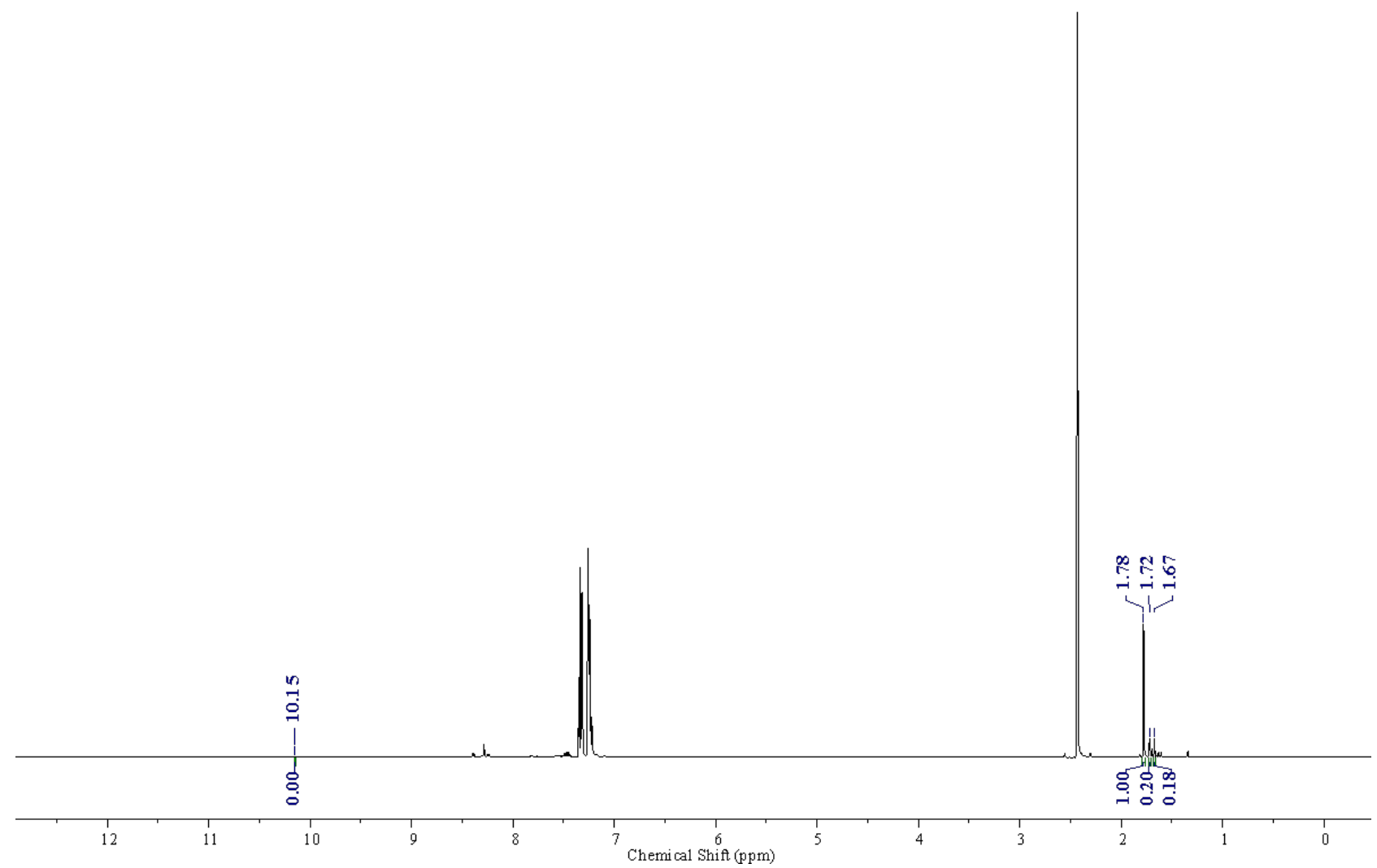

Entry 7: NMR for Crude reaction mixture

VDOS-629-50m o1\% PB. 5.fid

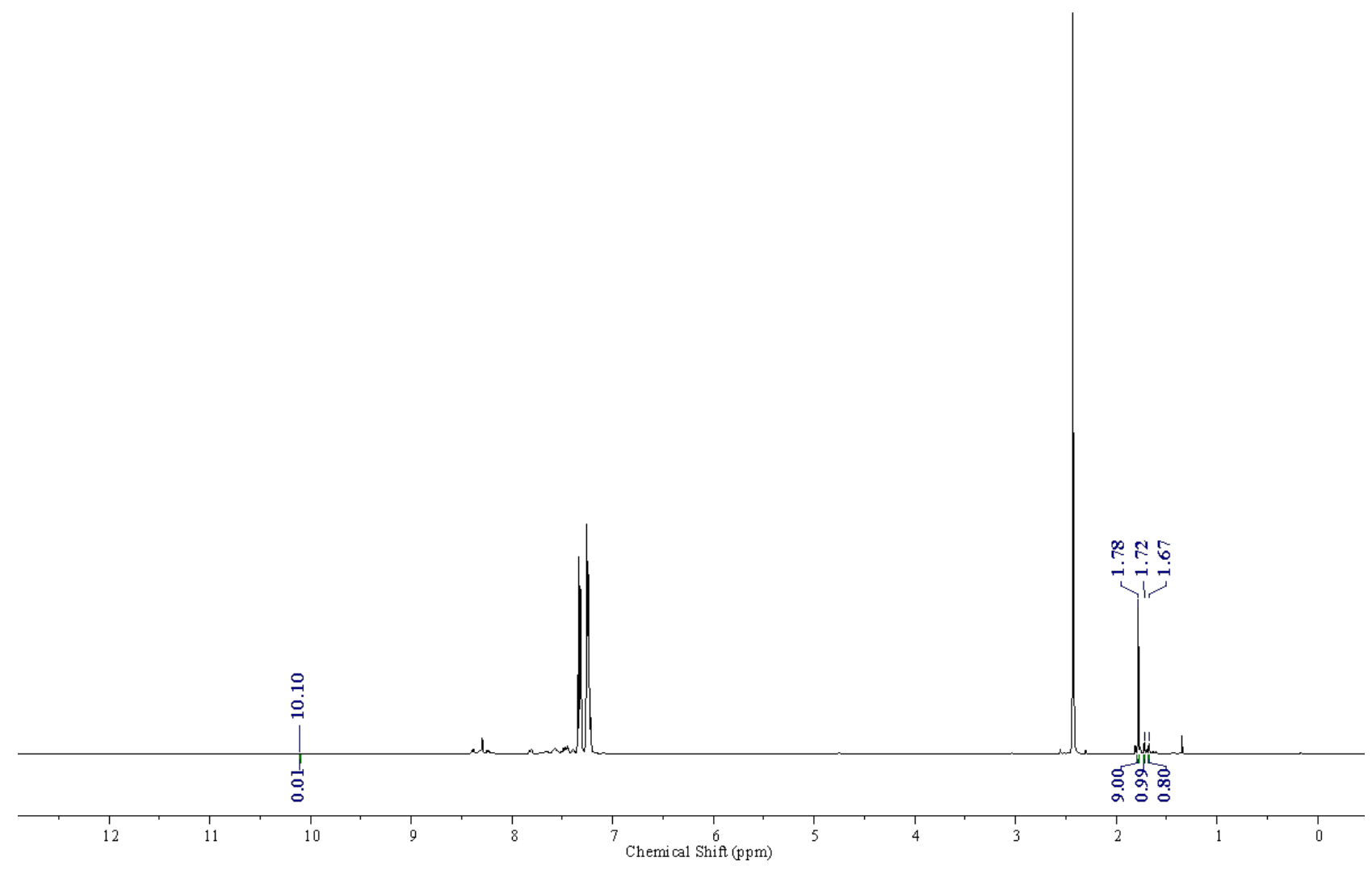


Entry 7': NMR for Crude reaction mixture, Duplicate

VDOS-629-A 2-2, $50 \mathrm{~m}$ ol\% PB .4. fid

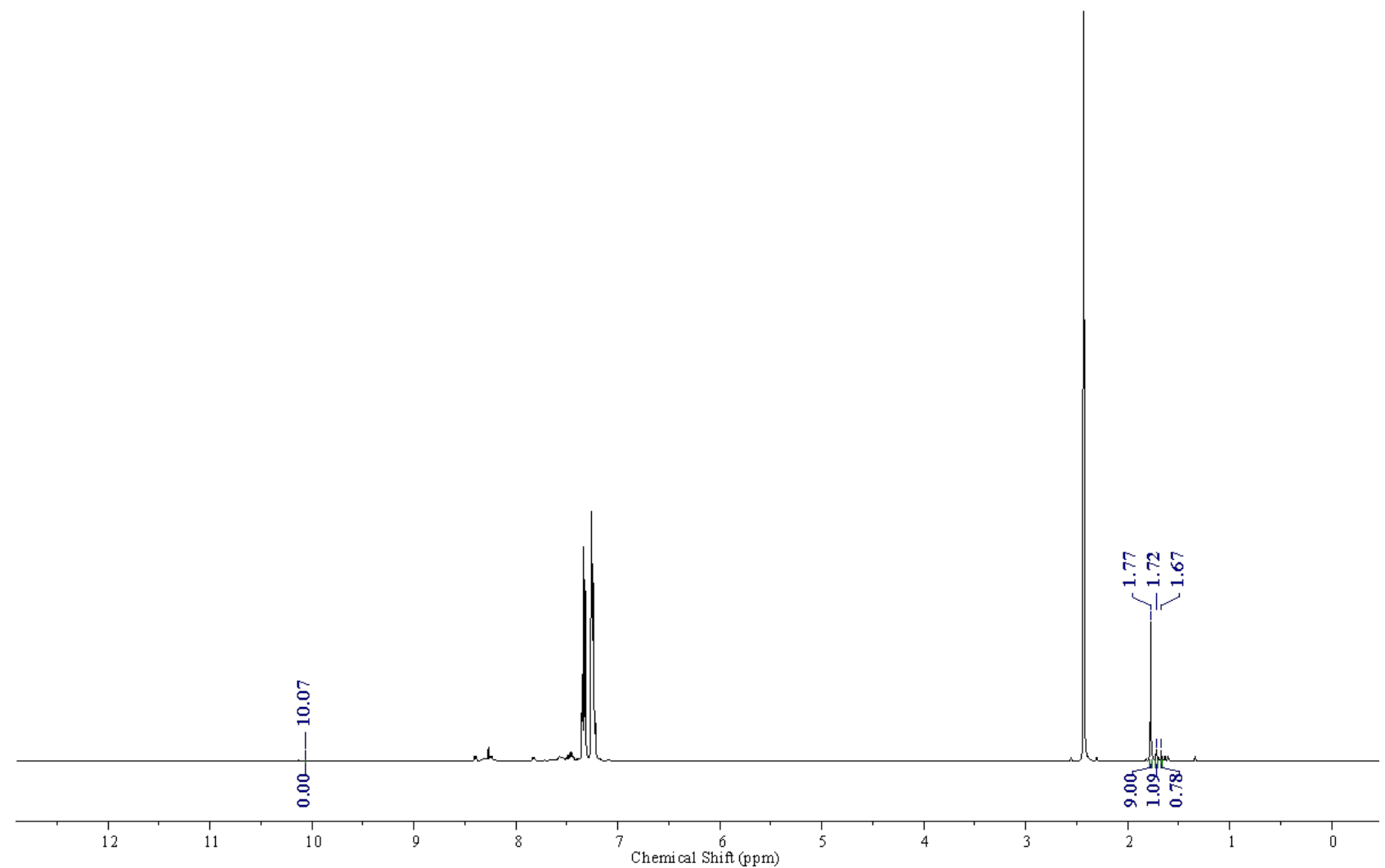

NMR after purification:

VDOS-629 VP in DMSO-d6.5.fid

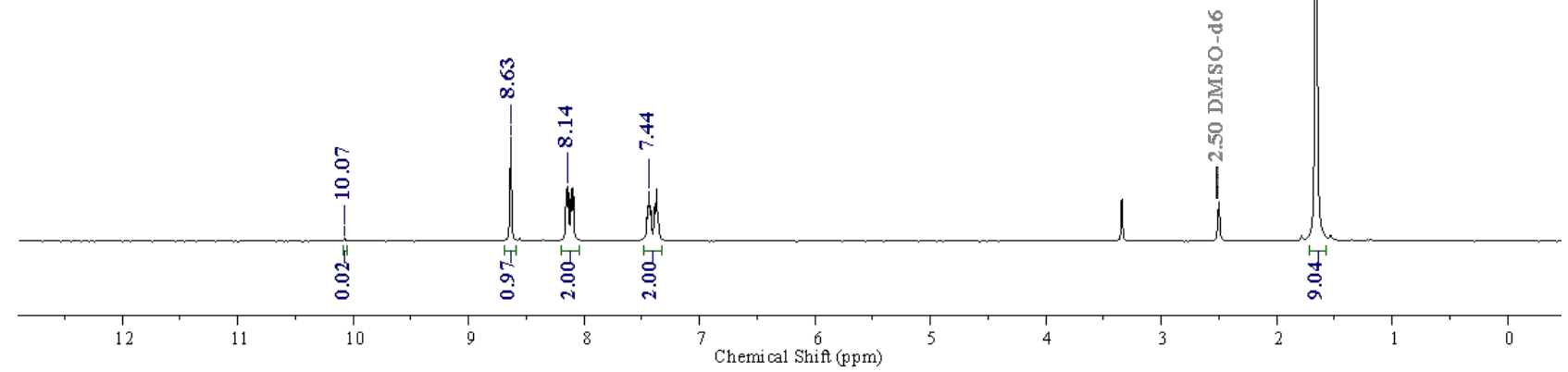




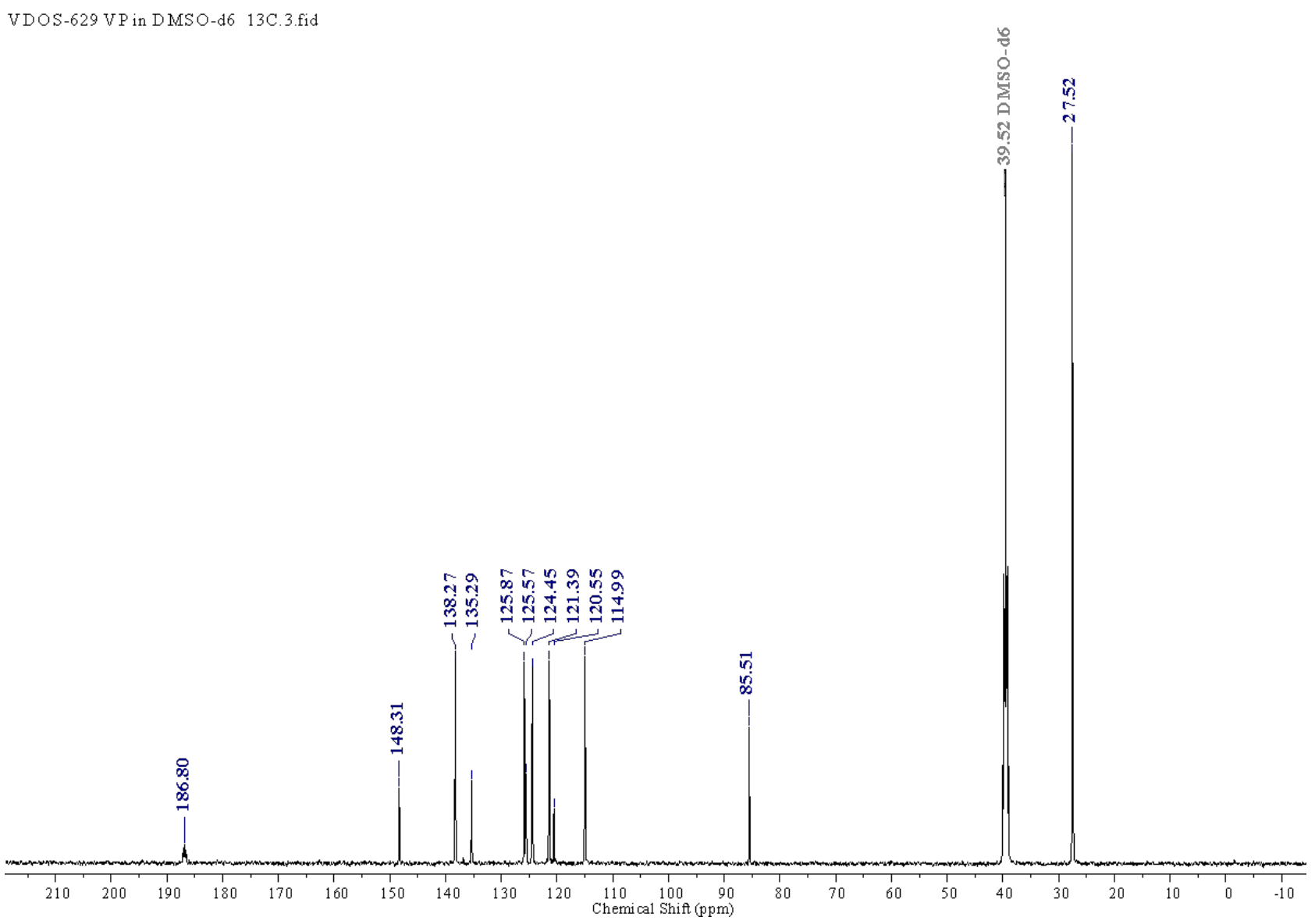


46. 1-Methylindole-3-carboxaldehyde-d<smiles>[2H]C(=O)c1cn(C)c2ccccc12</smiles>

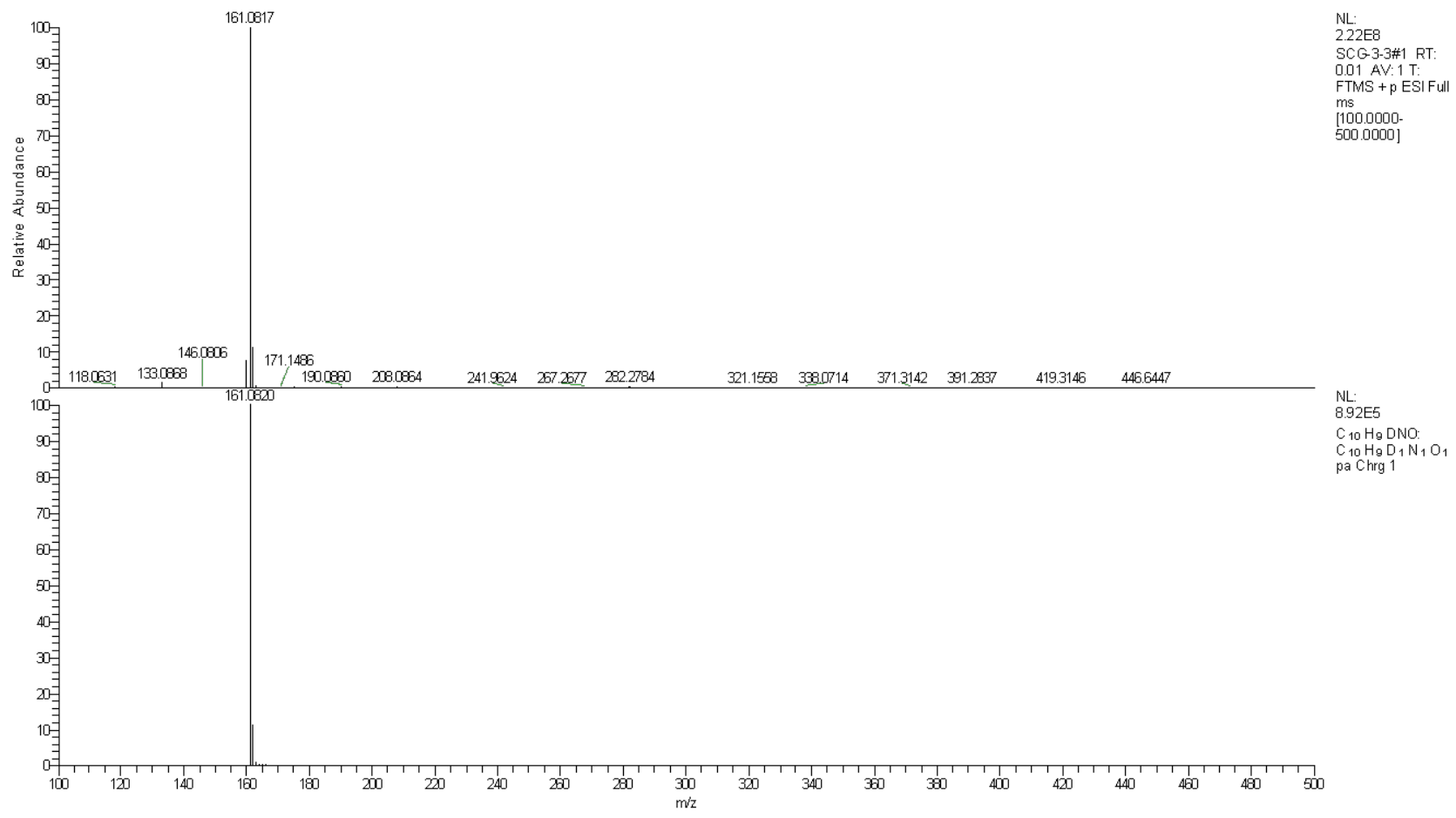


Entry 1: NMR for Crude reaction mixture

VDOS-612-A 1.1.fid

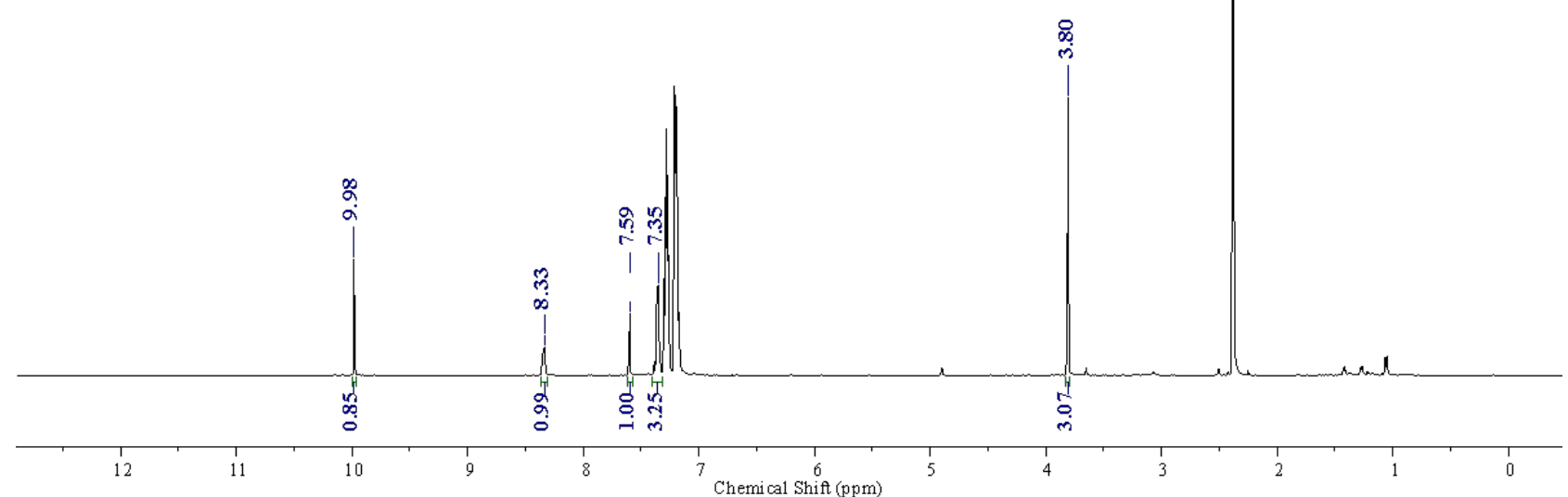

Entry 2: NMR for Crude reaction mixture

VDOS-612-B 1.2.fid

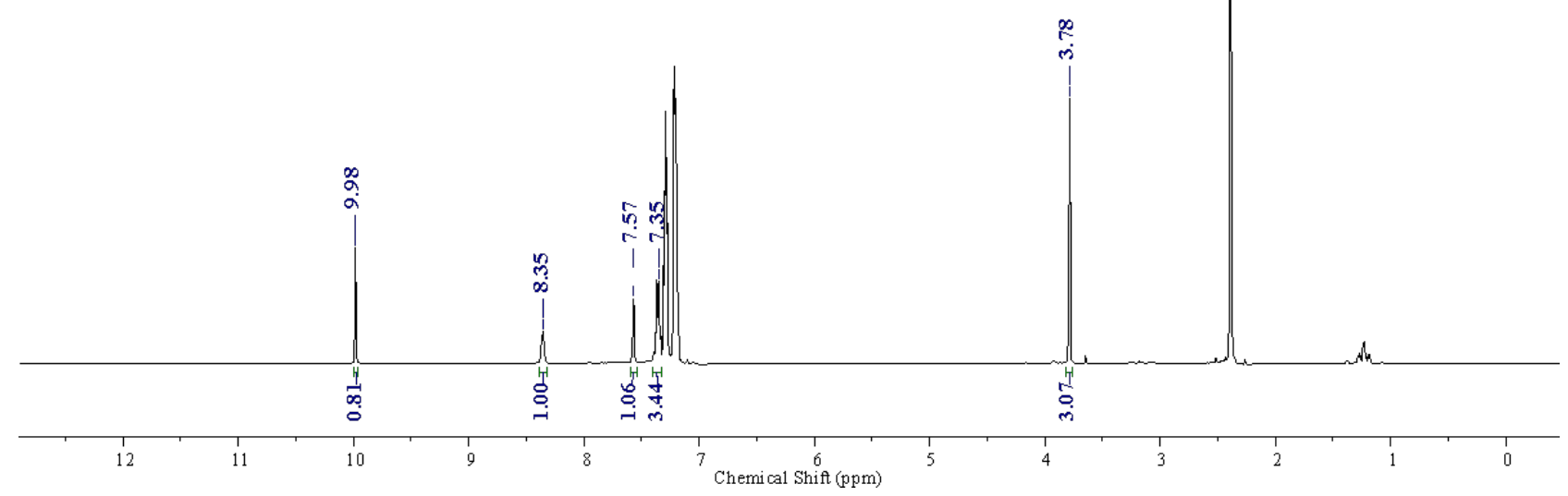


Entry 3: NMR for Crude reaction mixture

VDOS-612-C 1.3.fid

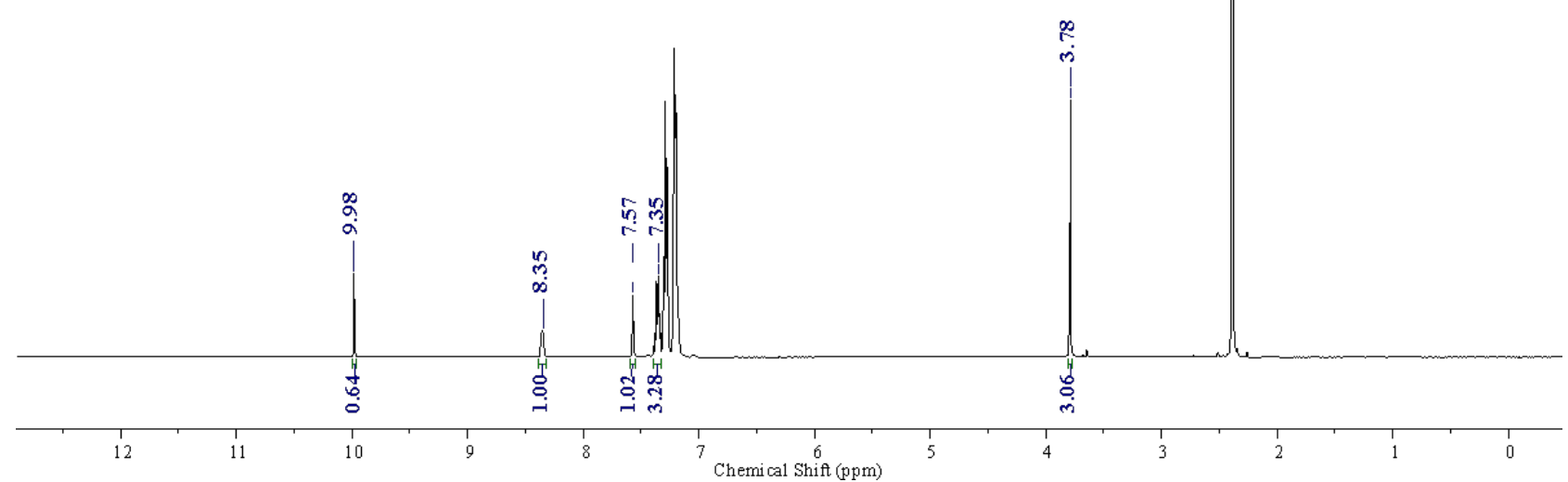

Entry 4: NMR for Crude reaction mixture

VDOS-612-D 1.4.fid

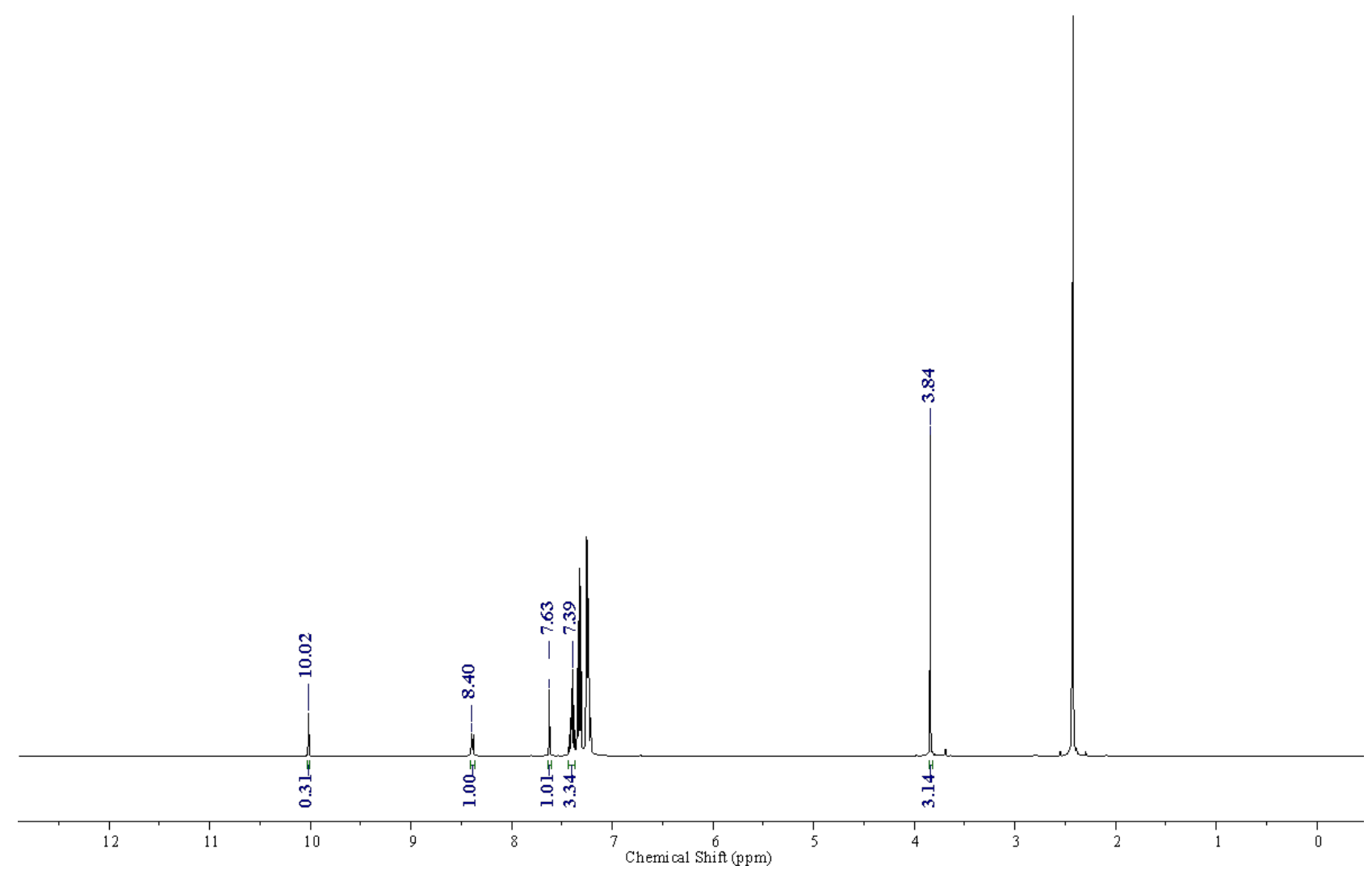


Entry 5: NMR for Crude reaction mixture

VDOS-612-F1, d1 50sec.5.fid

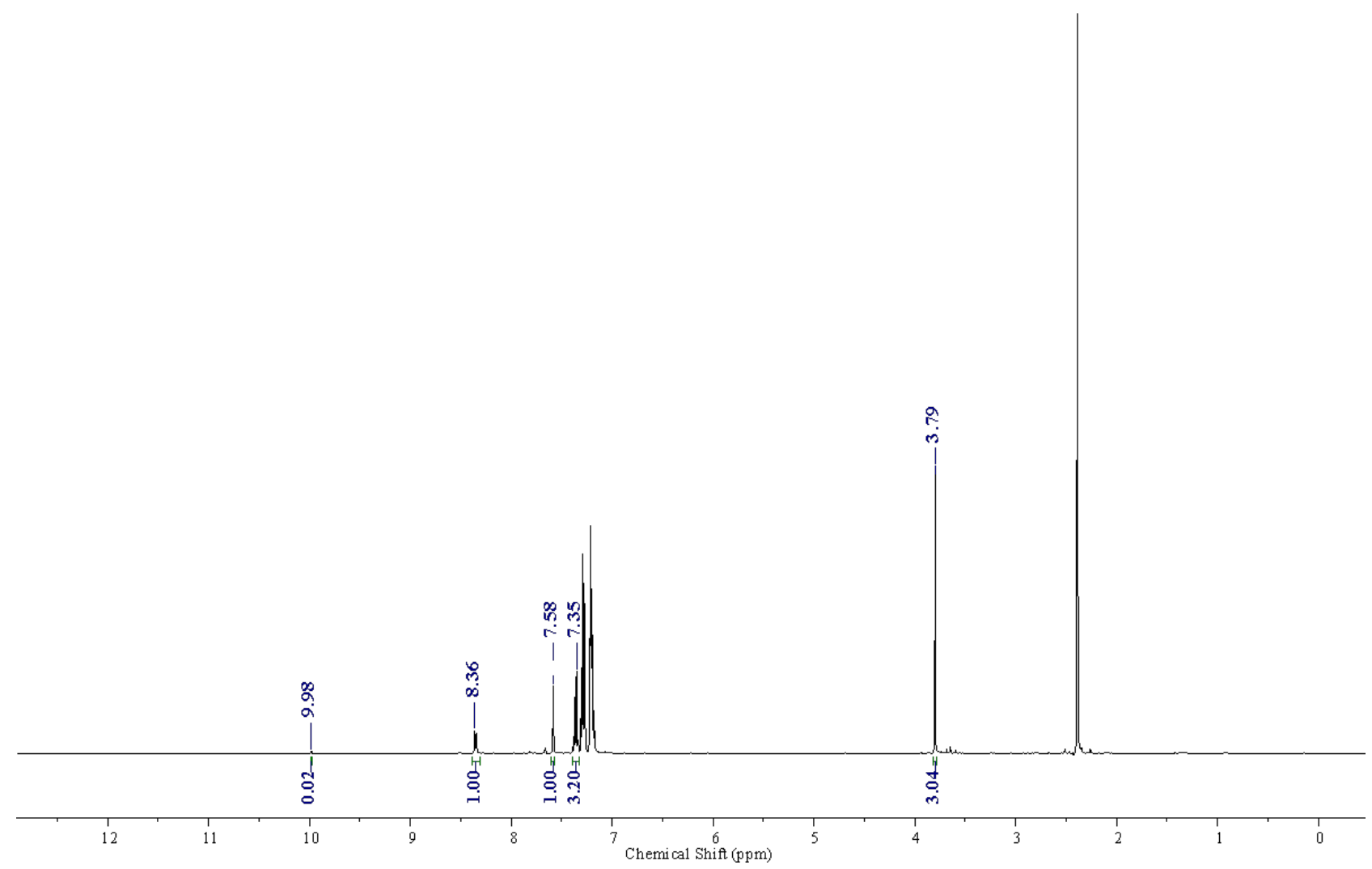

Entry 5': NMR for Crude reaction mixture, Duplicate

VDOS-612-E 1 , d1 $50 \mathrm{sec} .4$. fid

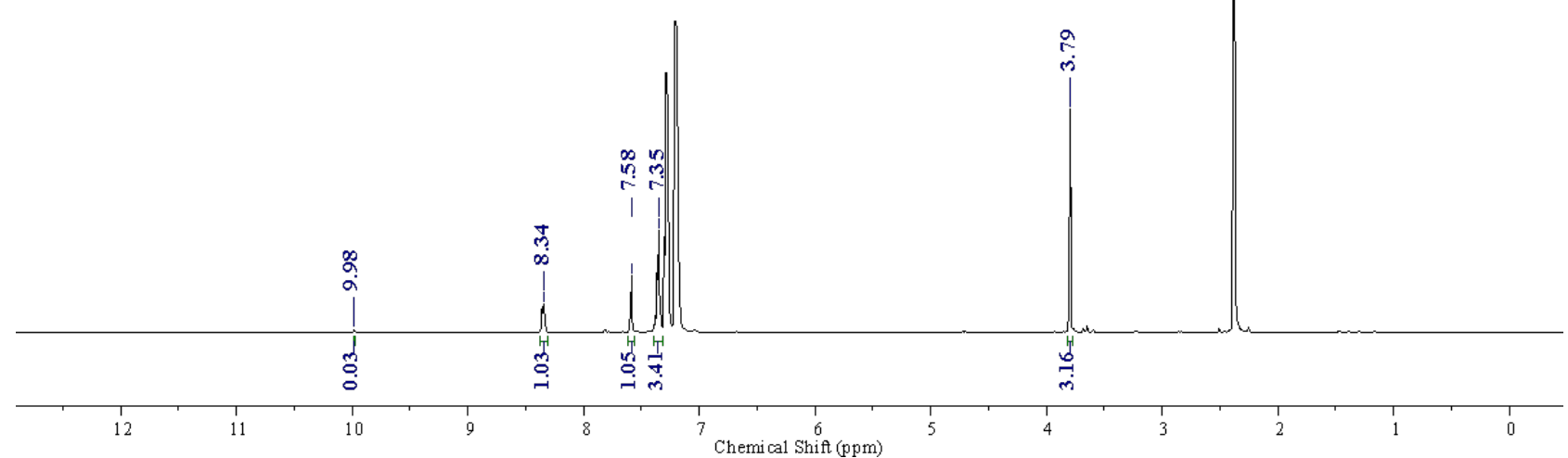


NMR after purification:

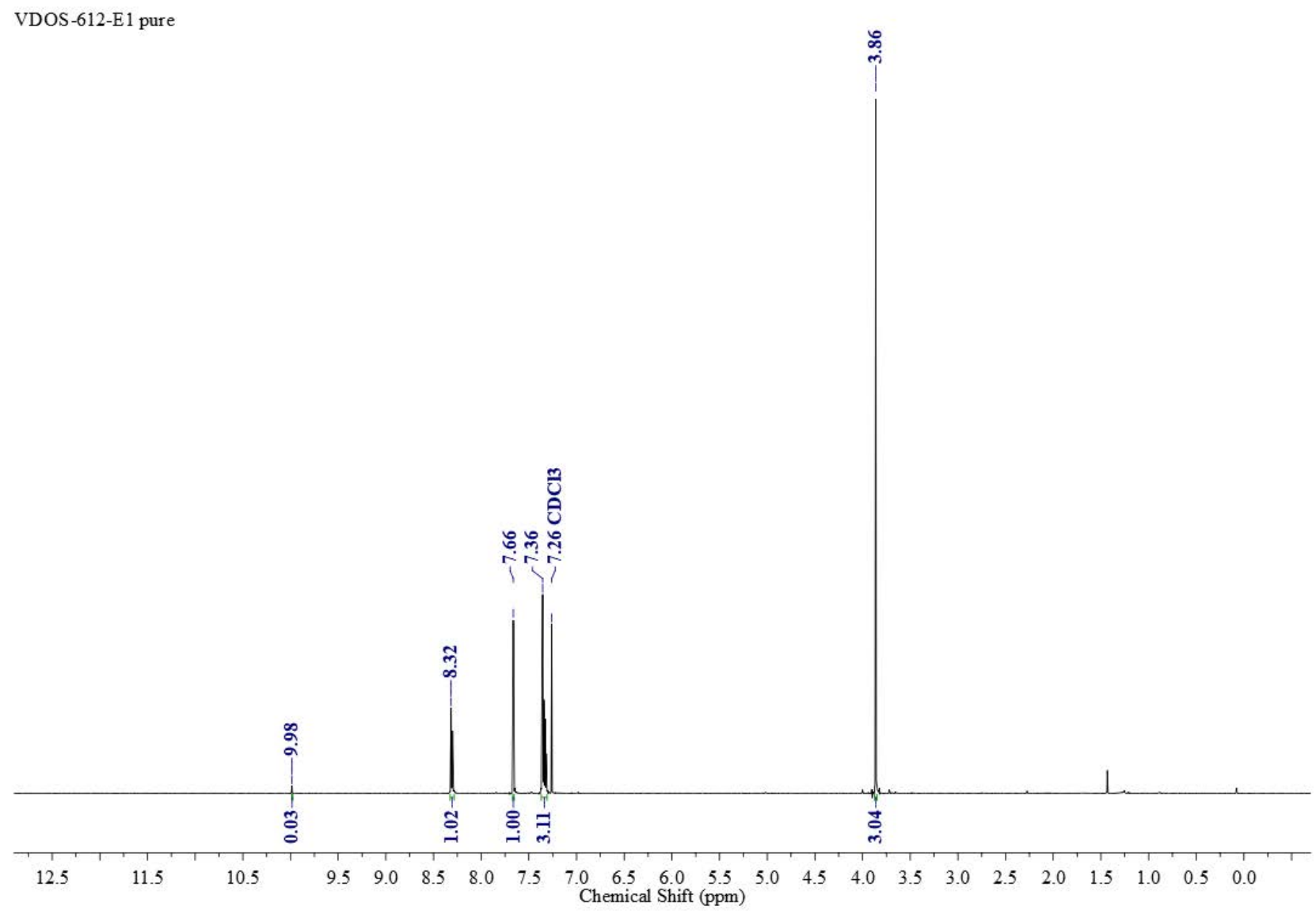

VDOS-612-E 1 pure-13C.6.fid

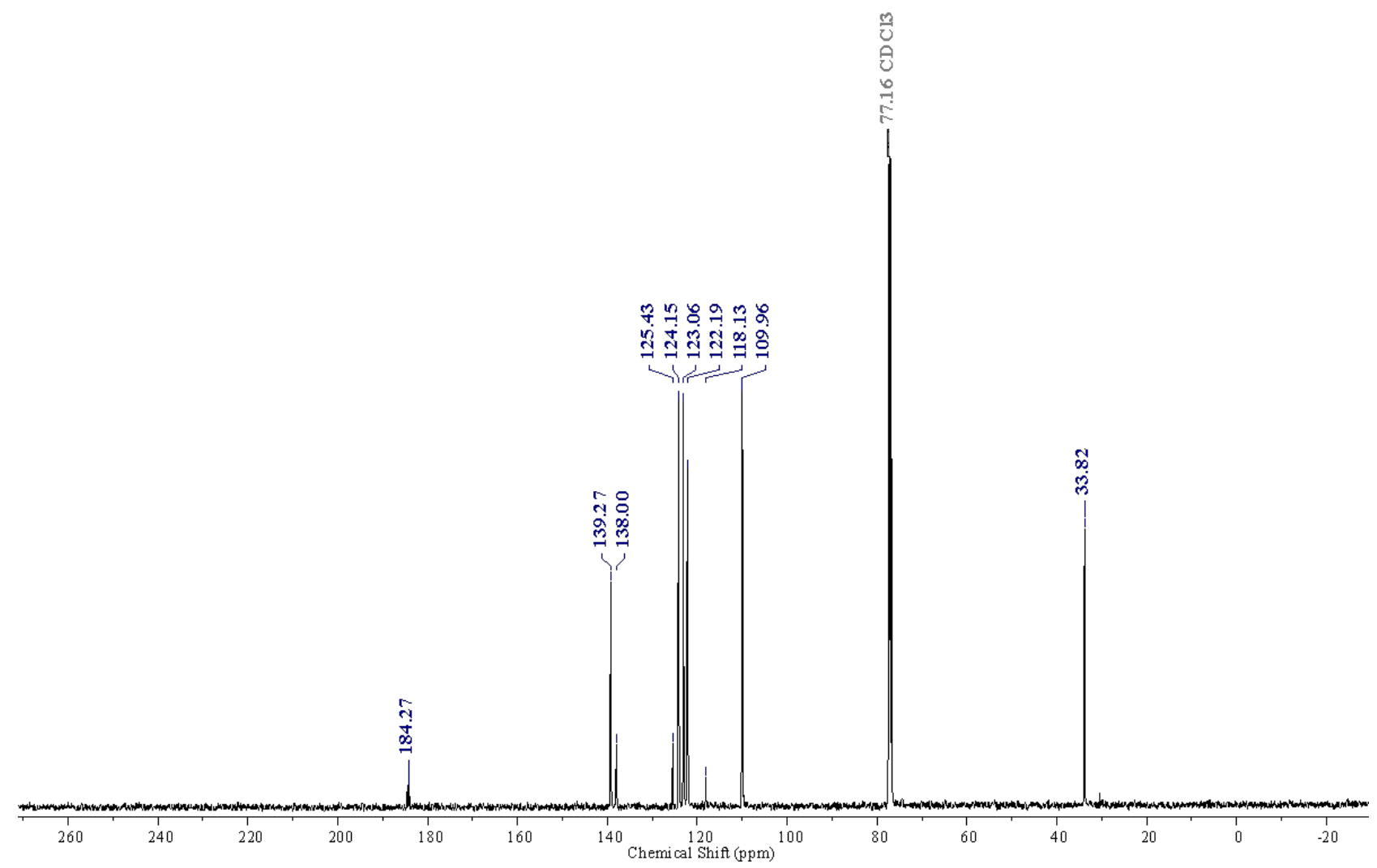


47. 9-Ethyl-3-carbazolecarboxaldehyde-d

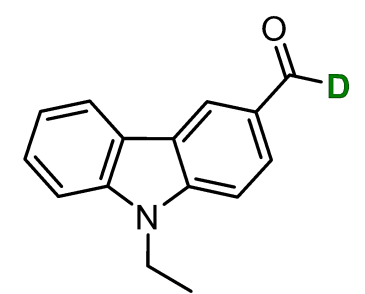

Entry 1: NMR for Crude reaction mixture VDOS-621-A 1.1.fid

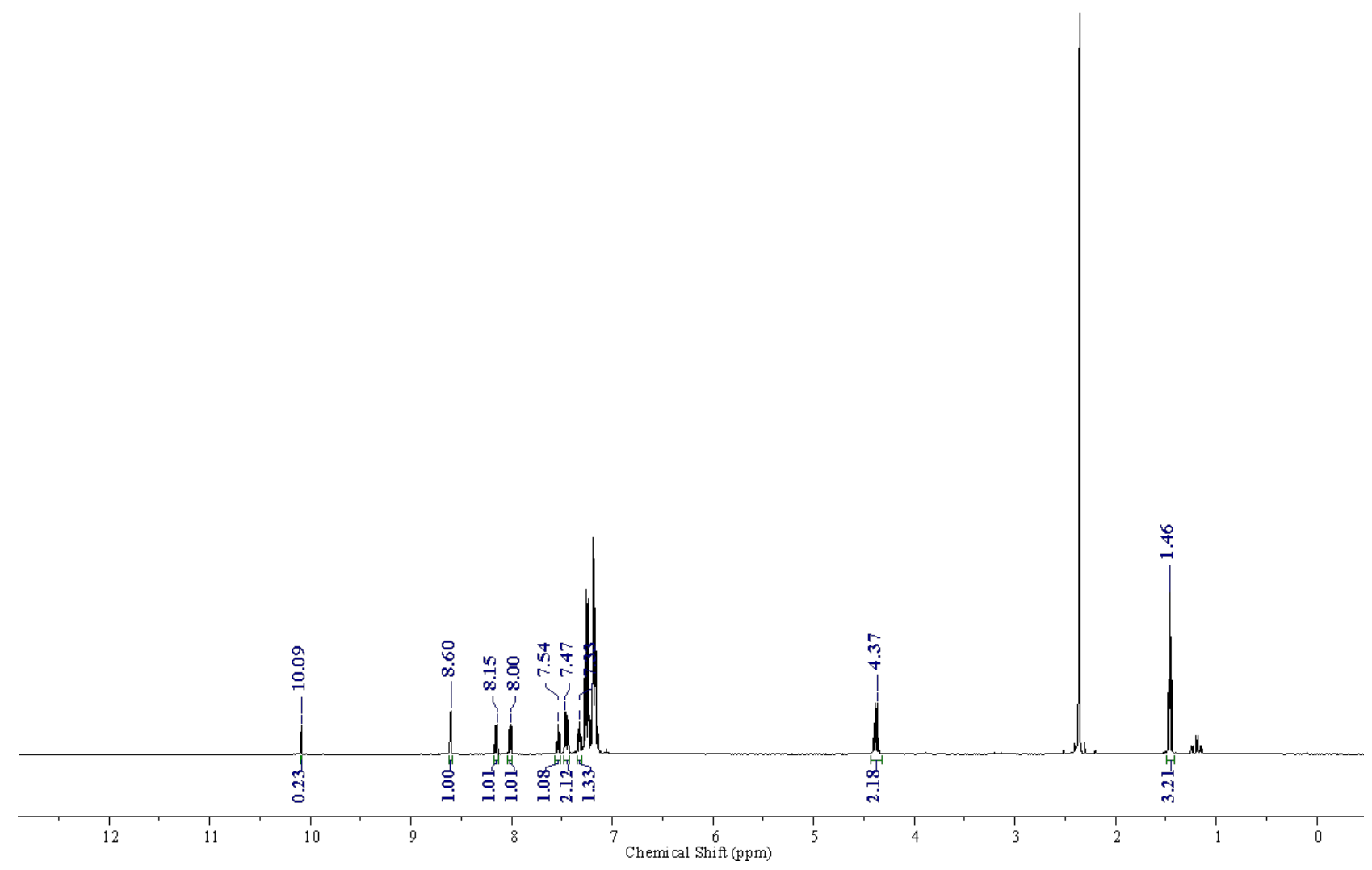


Entry 2: NMR for Crude reaction mixture

VDOS-621-B1.1.fid

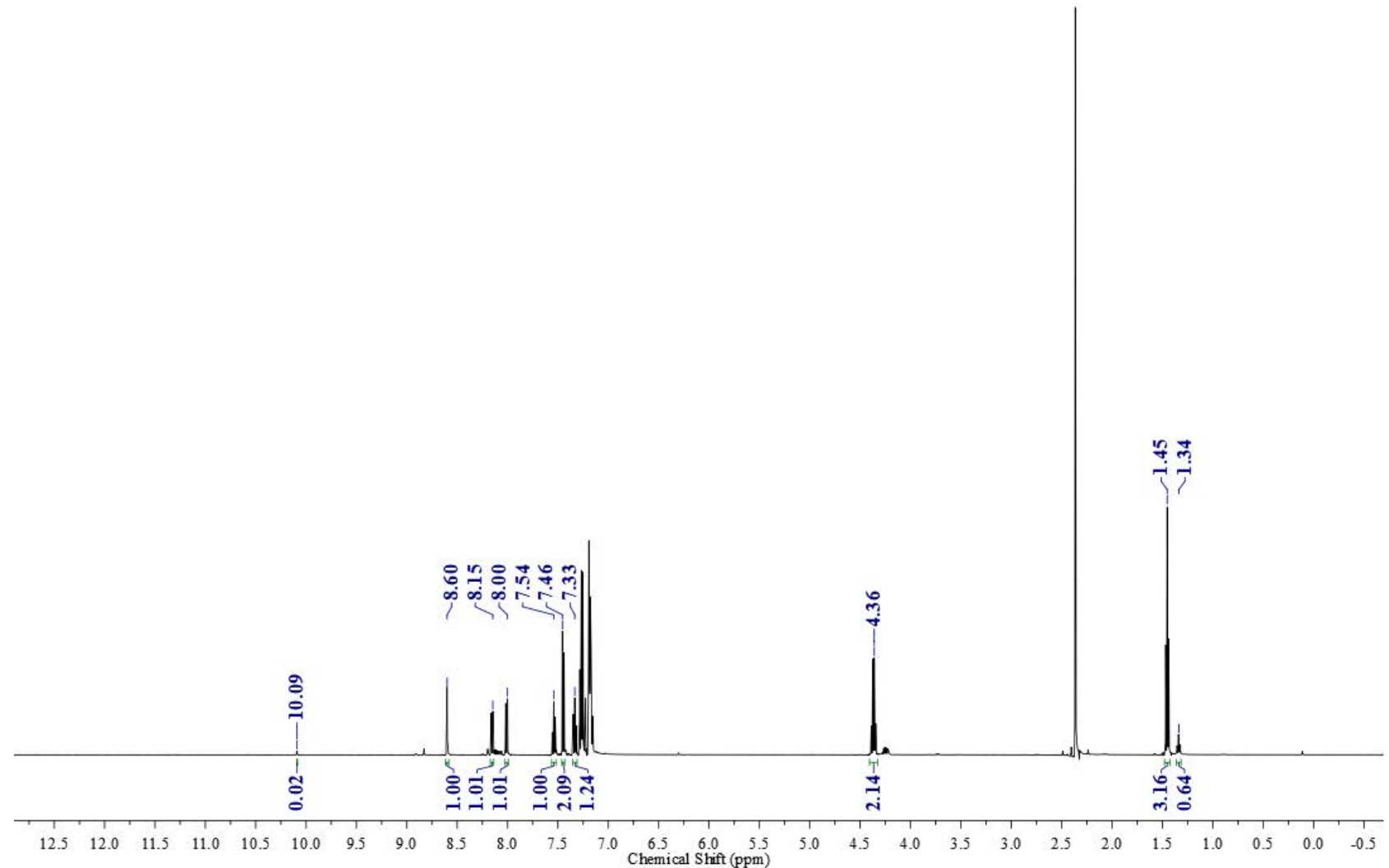

Entry 3: NMR for Crude reaction mixture

VDOS-621-C1.2fid

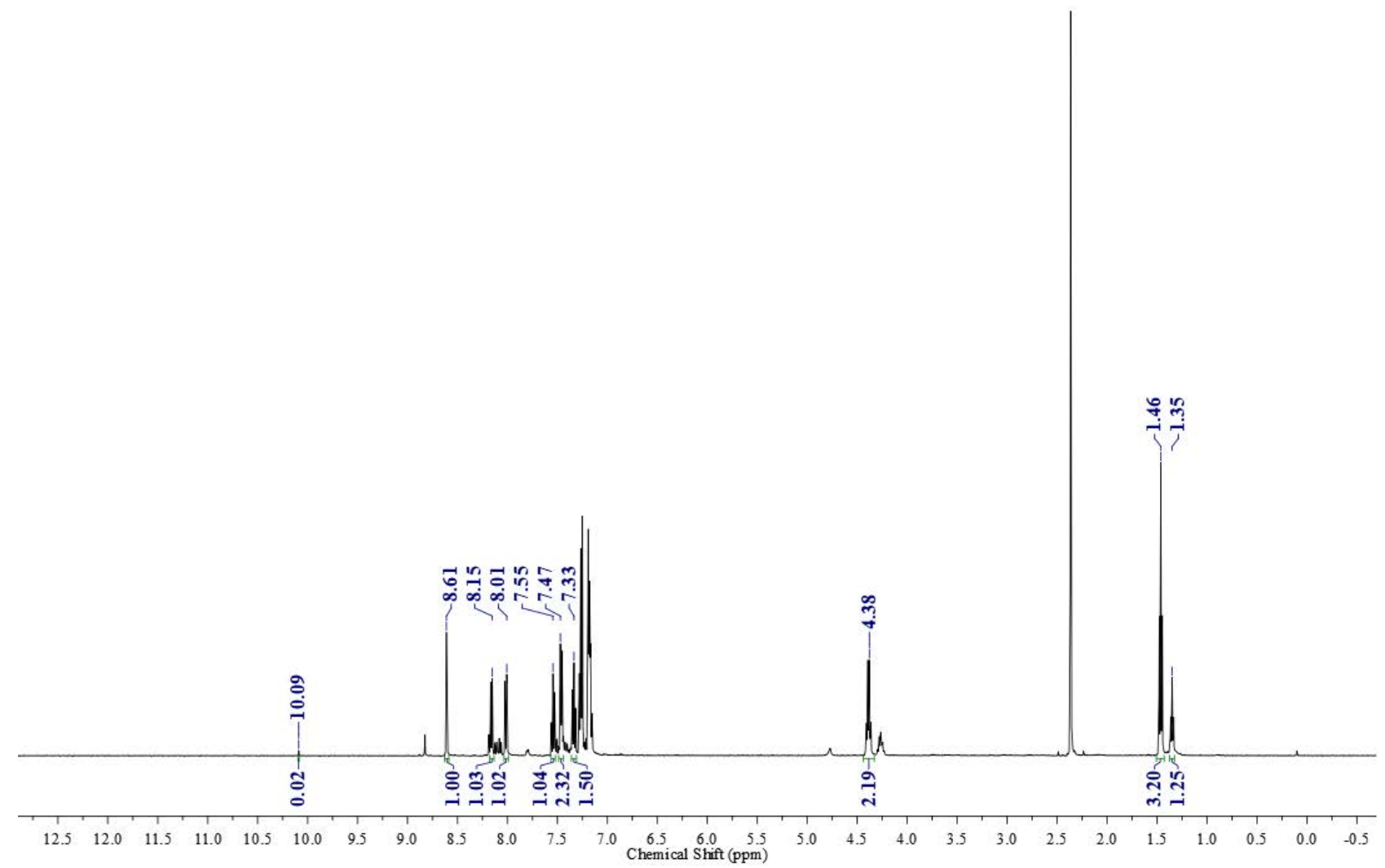


Entry 4: NMR for Crude reaction mixture

vDOS-621-D1.4fid

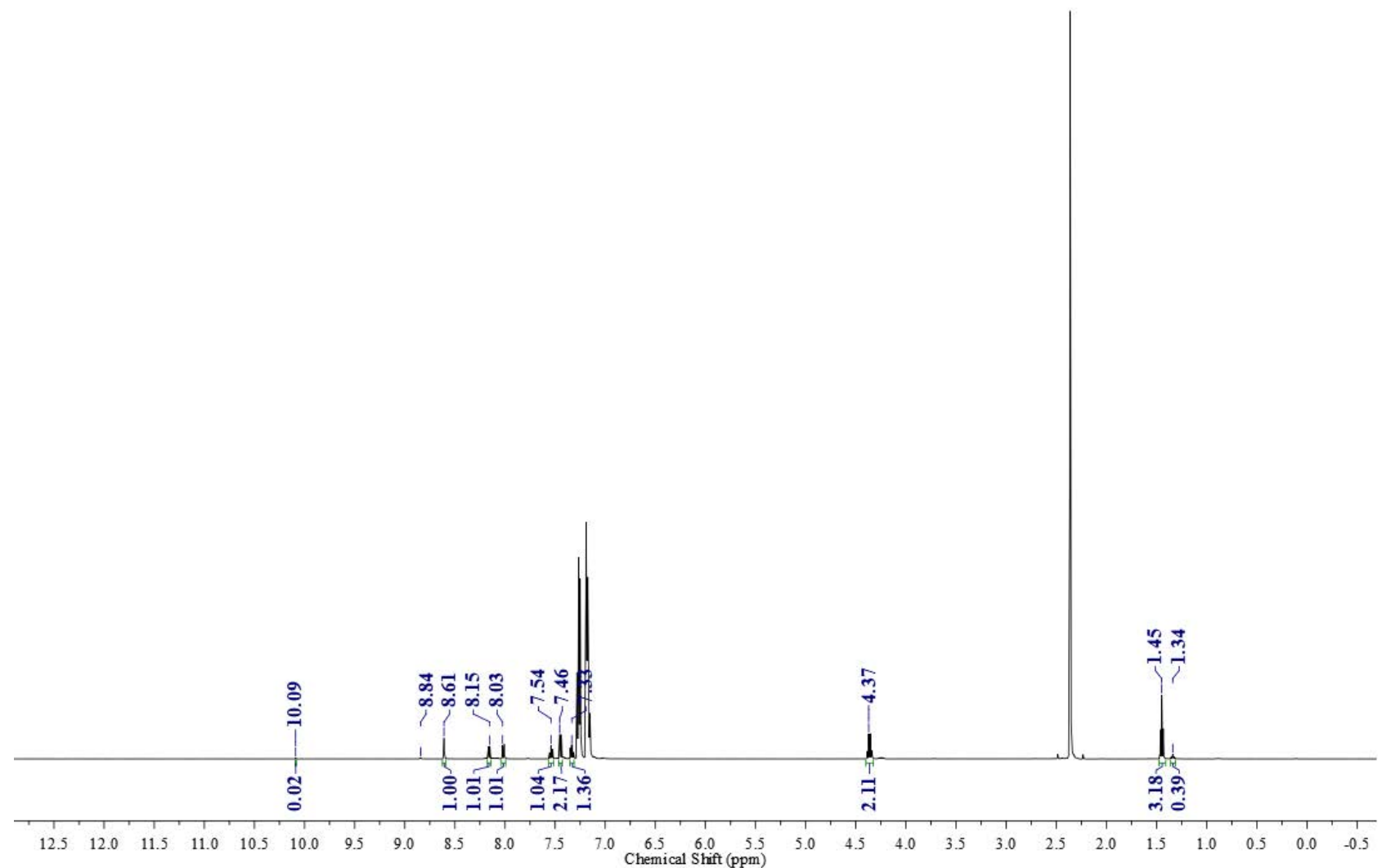

Entry 4': NMR for Crude reaction mixture, Duplicate VDOS-621-D2.8.fid

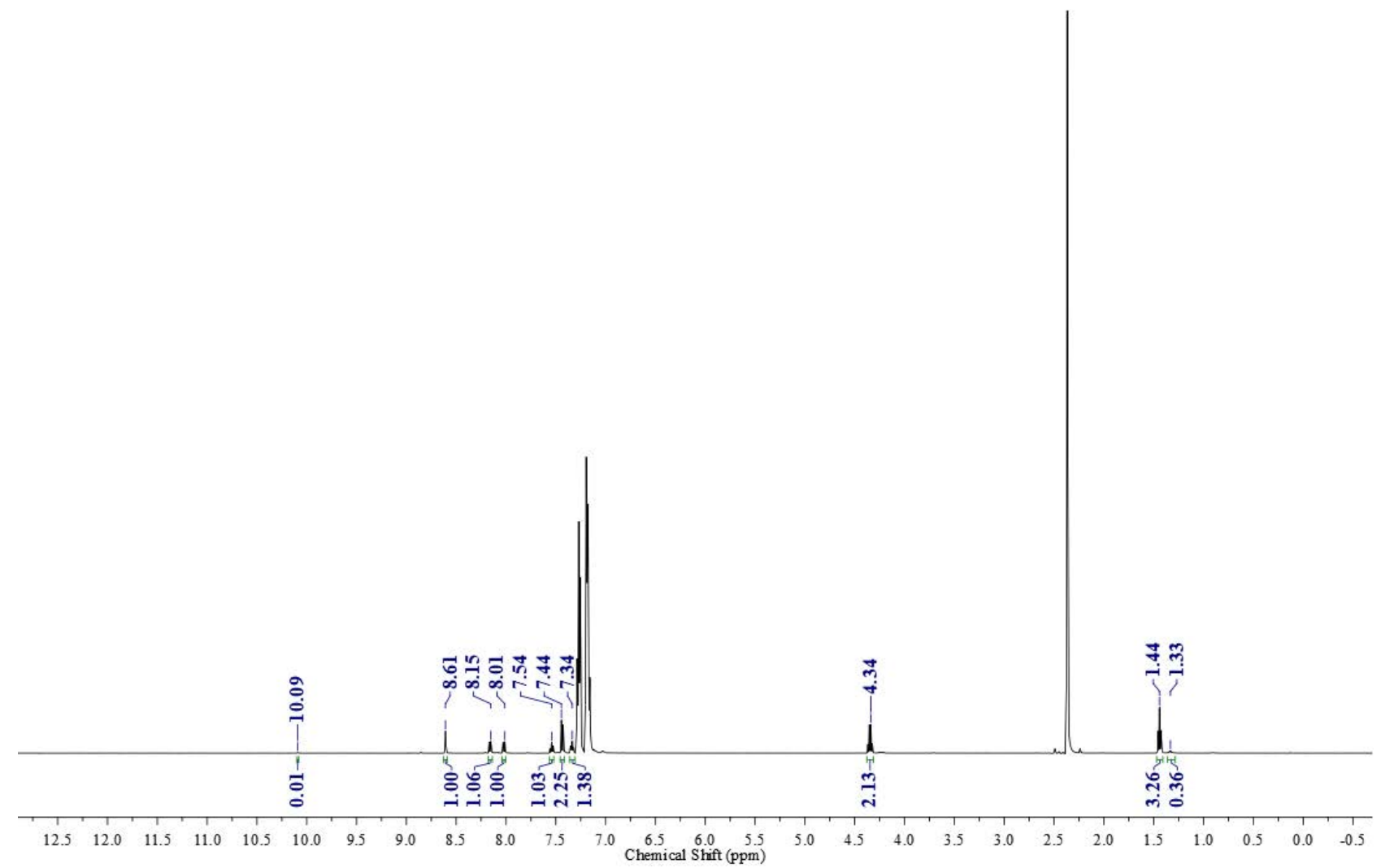


NMR after purification:

VDOS-621-B 1 pure $1 \mathrm{H}-2.3$ fid

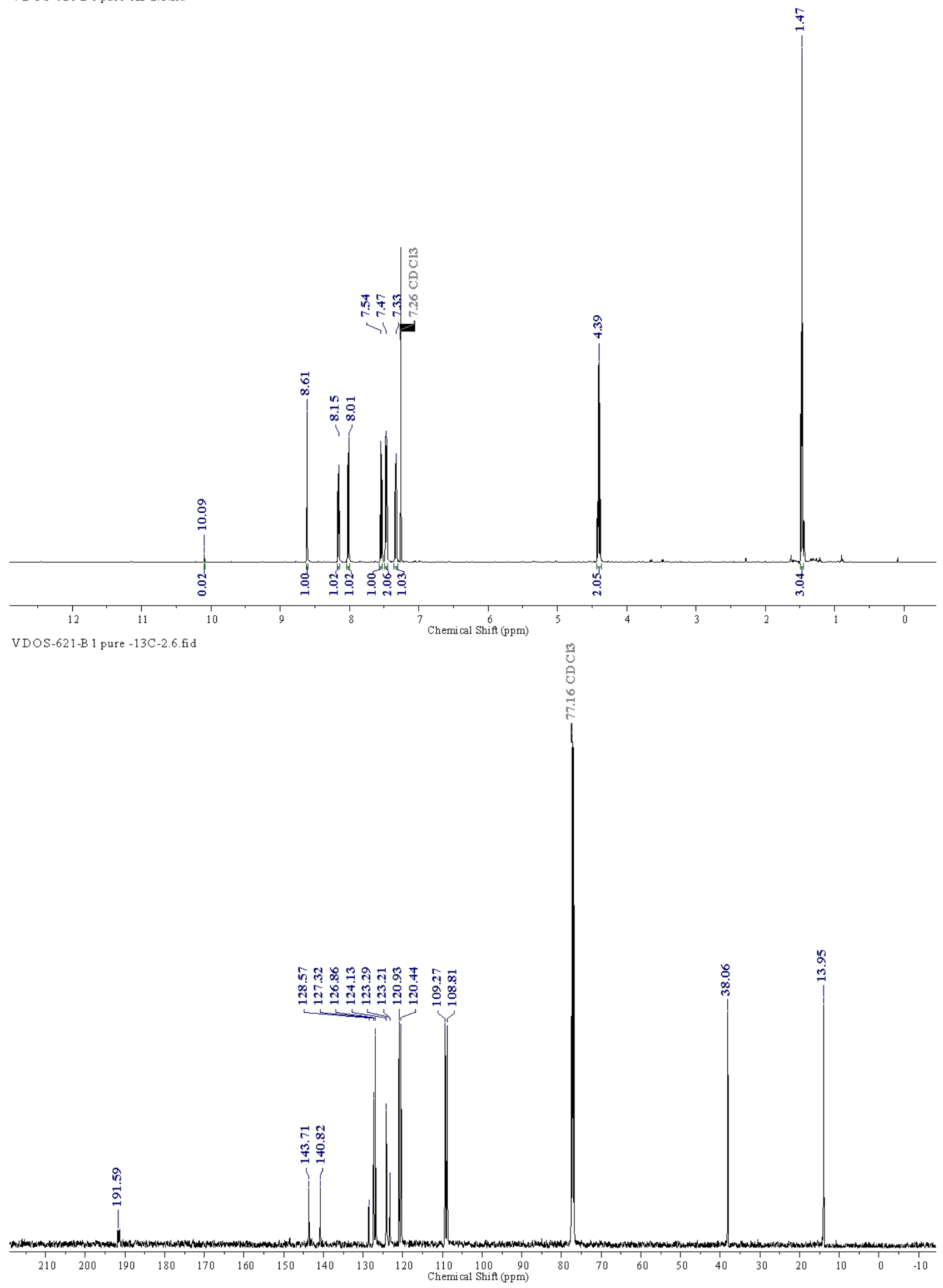


48. Pyrrole-2-carboxaldehyde-d

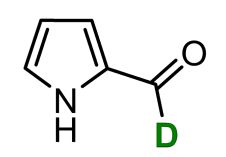

Entry 1: NMR for Crude reaction mixture SCG-307-J1-16hr-D1-50sec. 1. fid

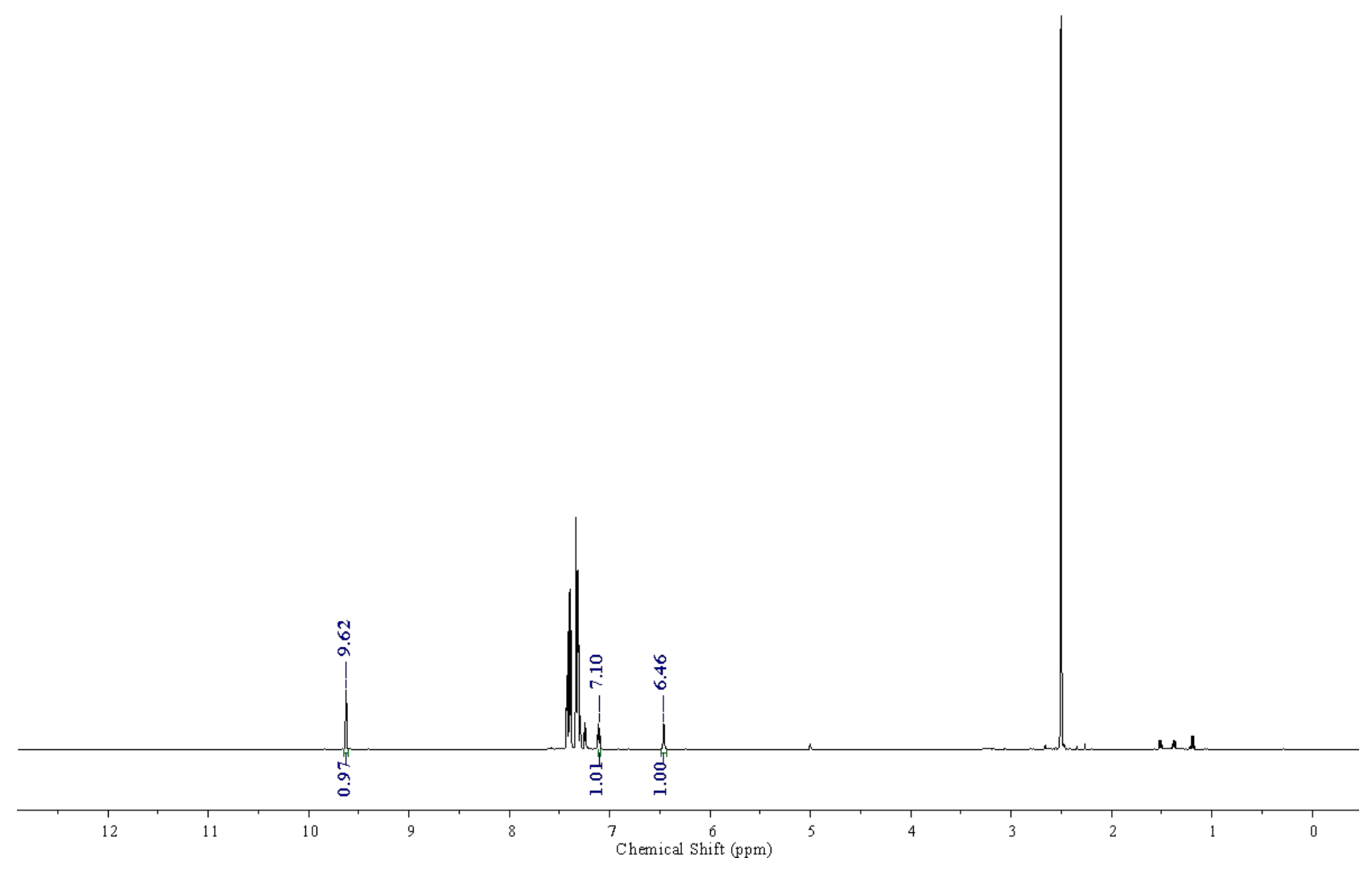


Entry 1': NMR for Crude reaction mixture, Duplicate

SCG-307-J2-16hr-D1-50sec.1. fid

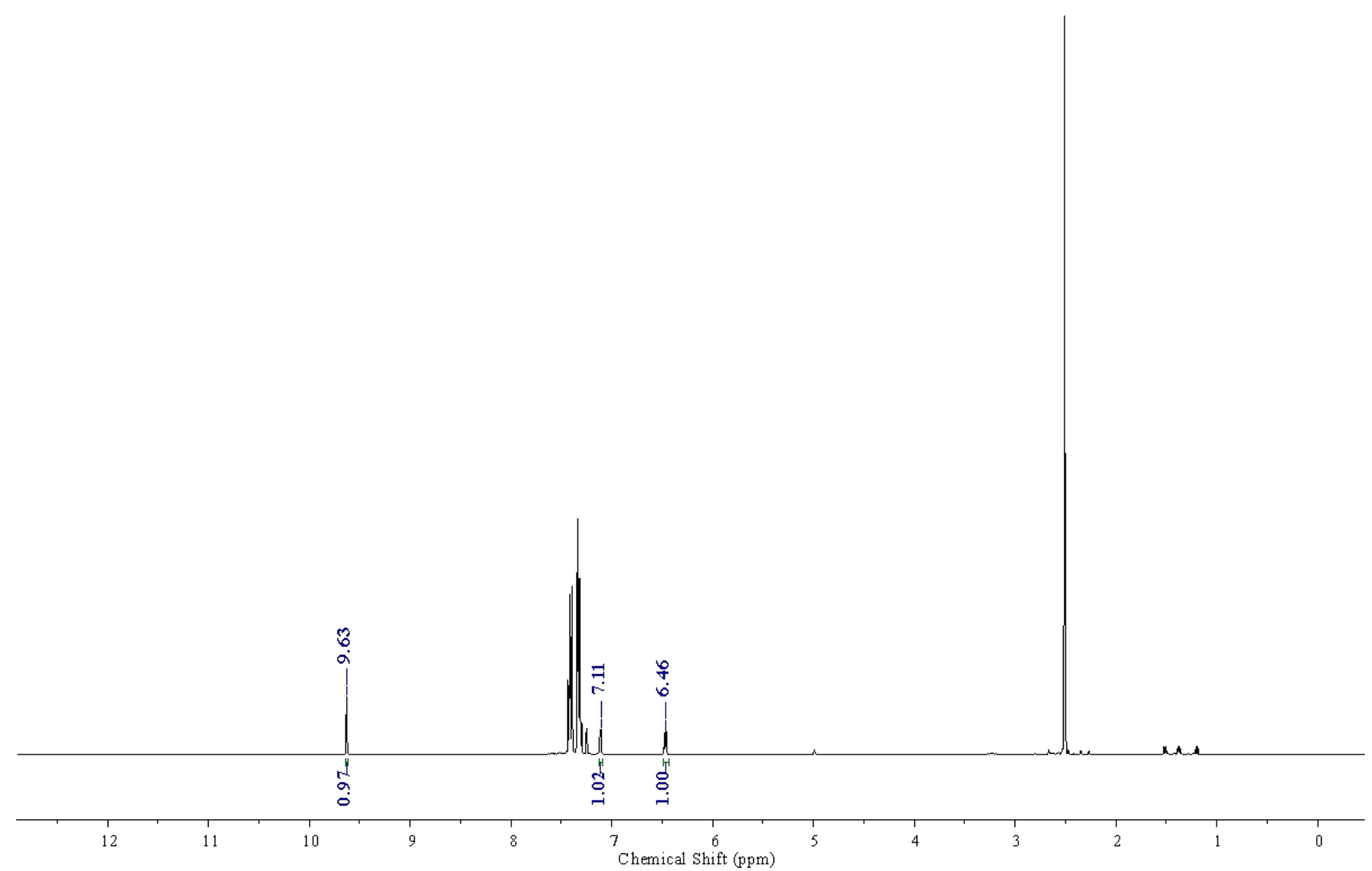

Entry 2: NMR for Crude reaction mixture

VDOS-614-B.2.fid

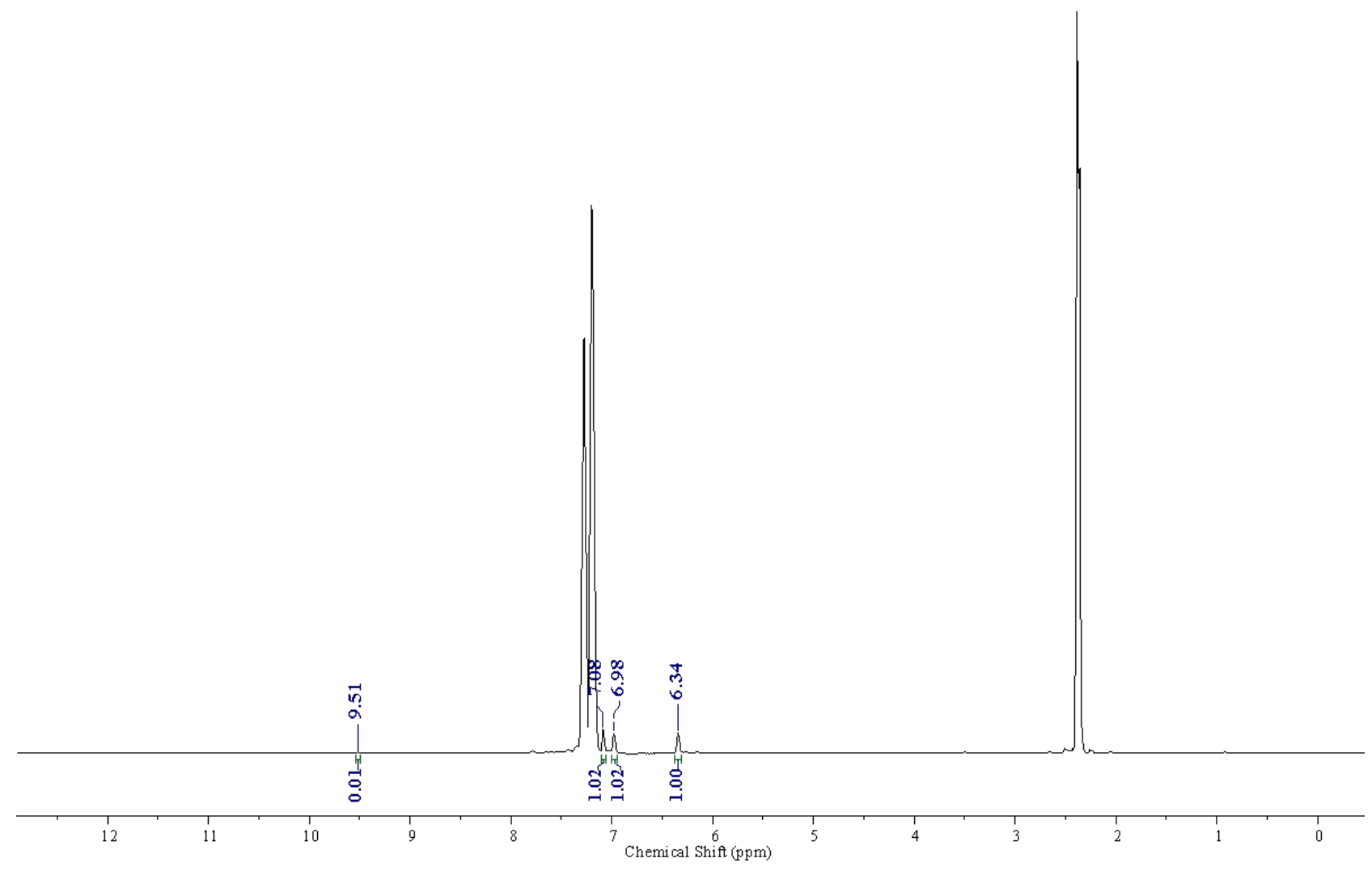


Entry 2': NMR for Crude reaction mixture, Duplicate VDOS-614-B 2.1.fid

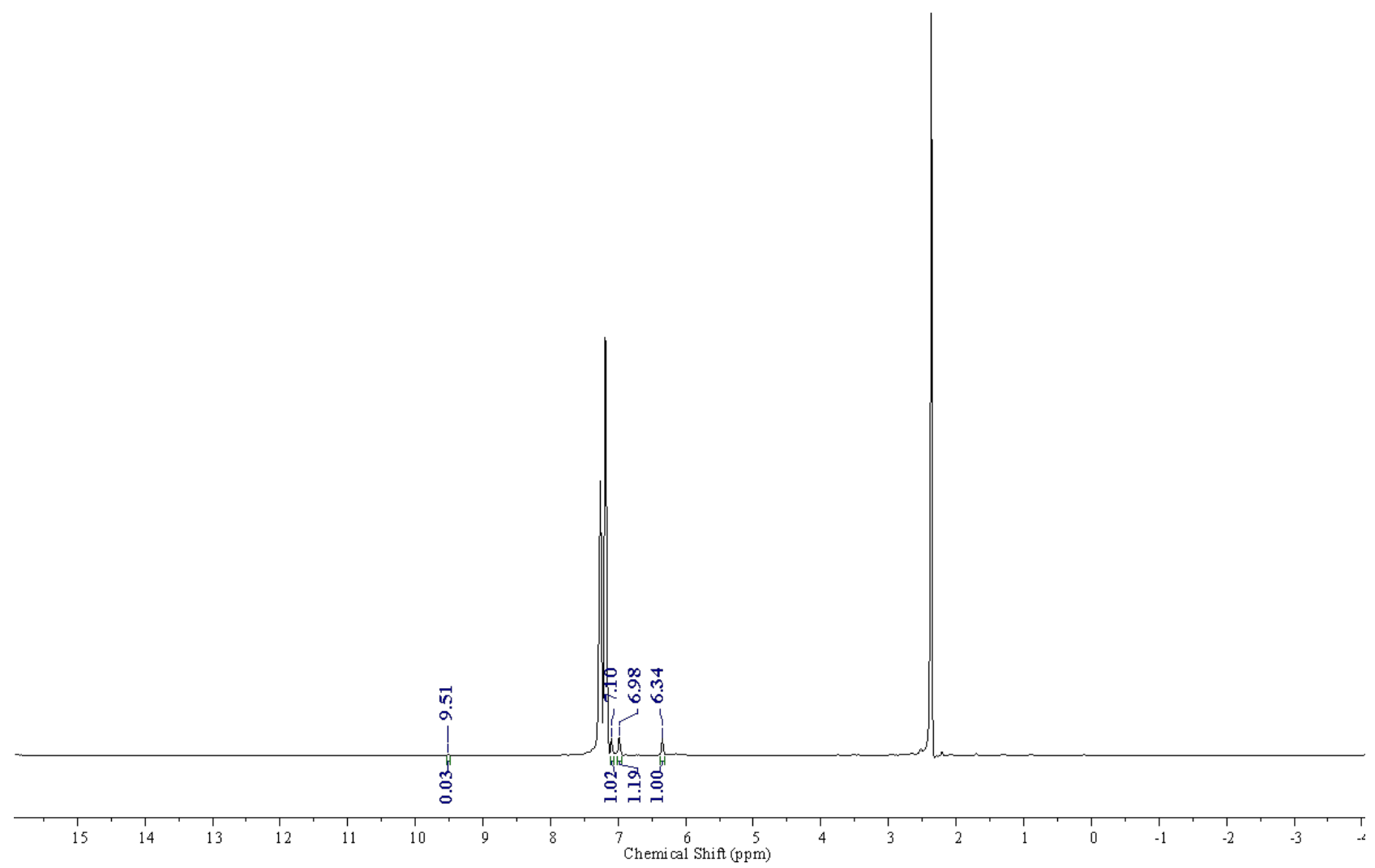

NMR after purification:

VDOS-614-B2 pure.1.fid

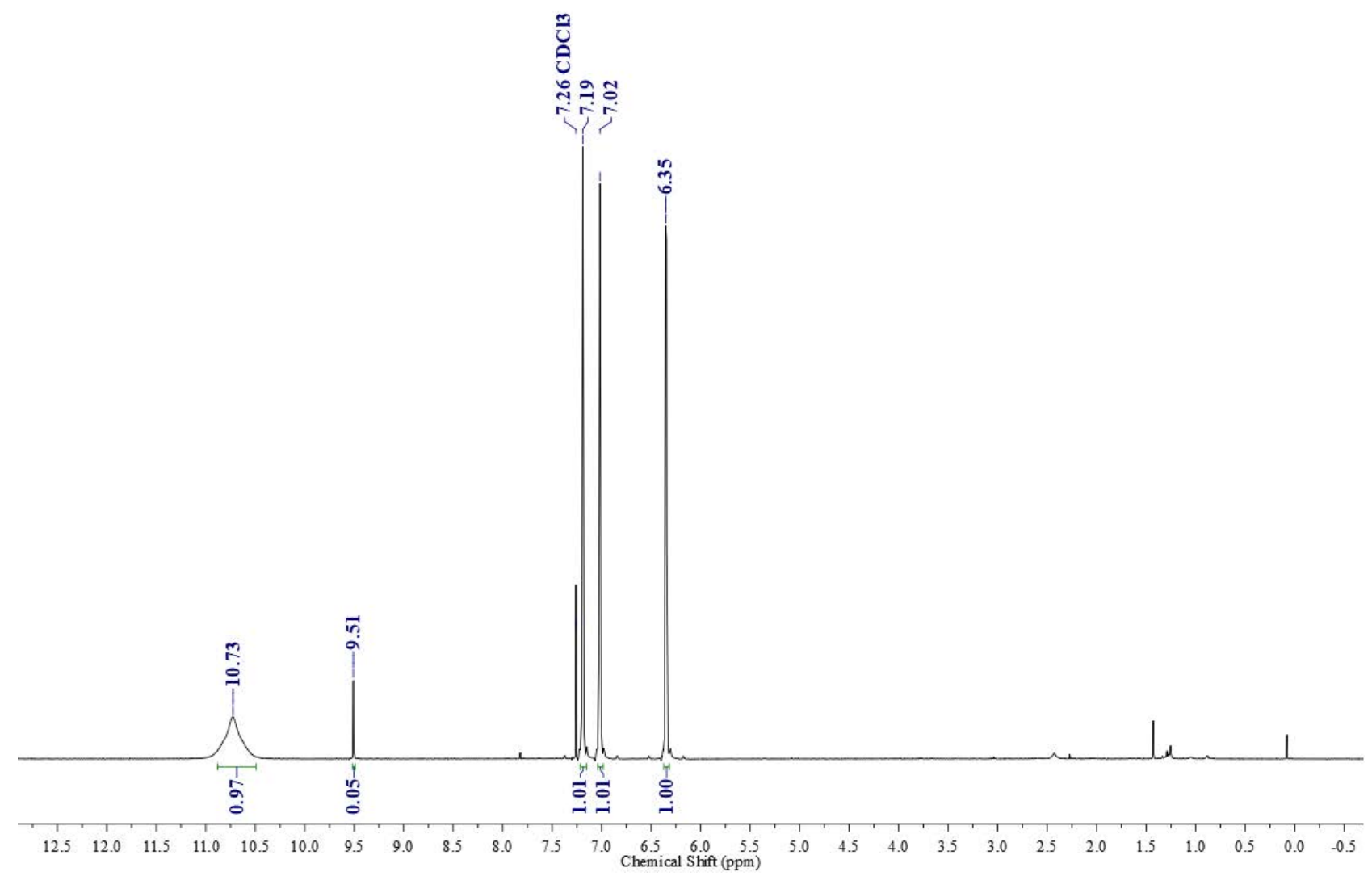




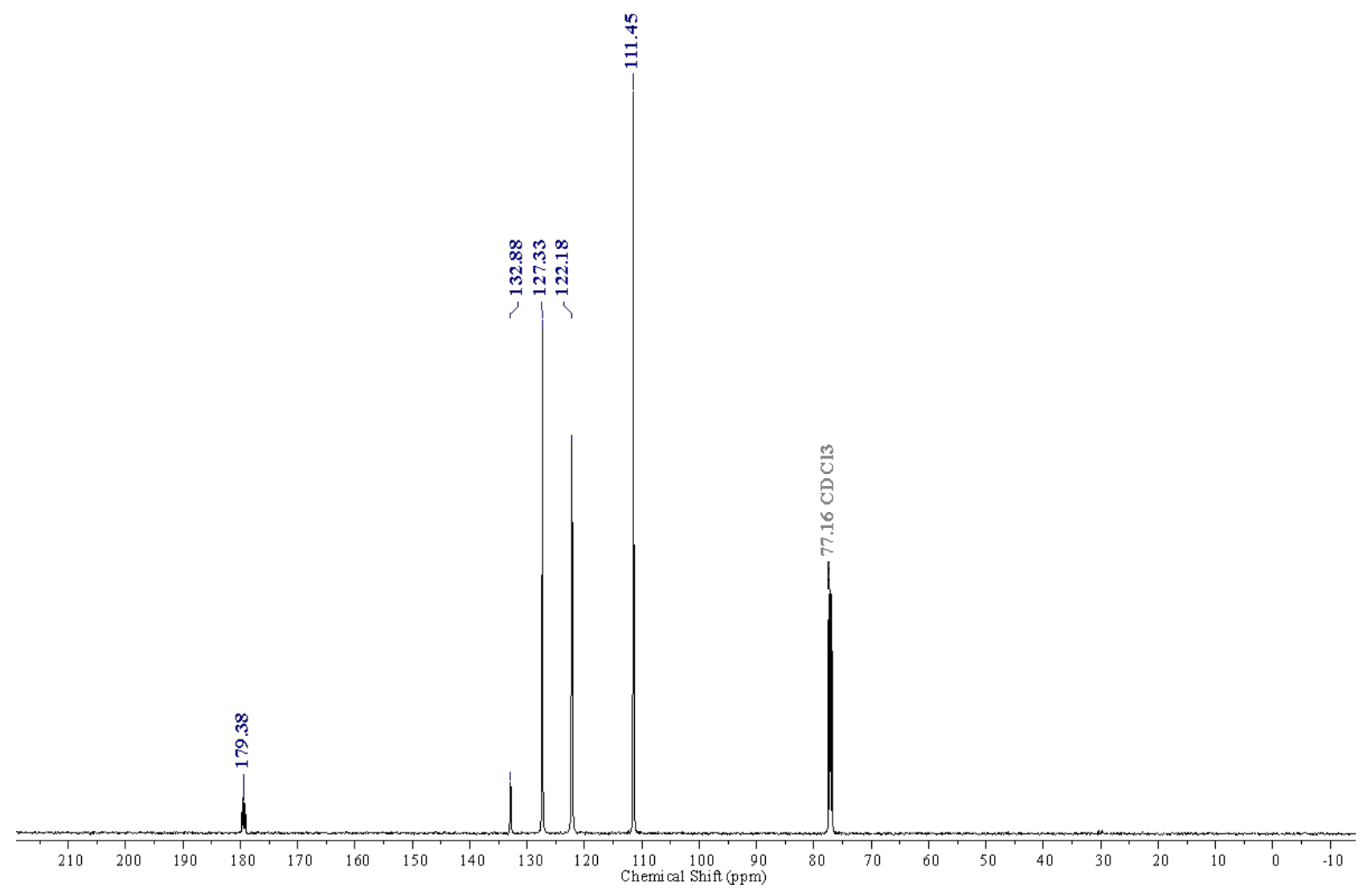


49. 1-(phenylsulfonyl)-pyrrole-2-carbaldehyde-d

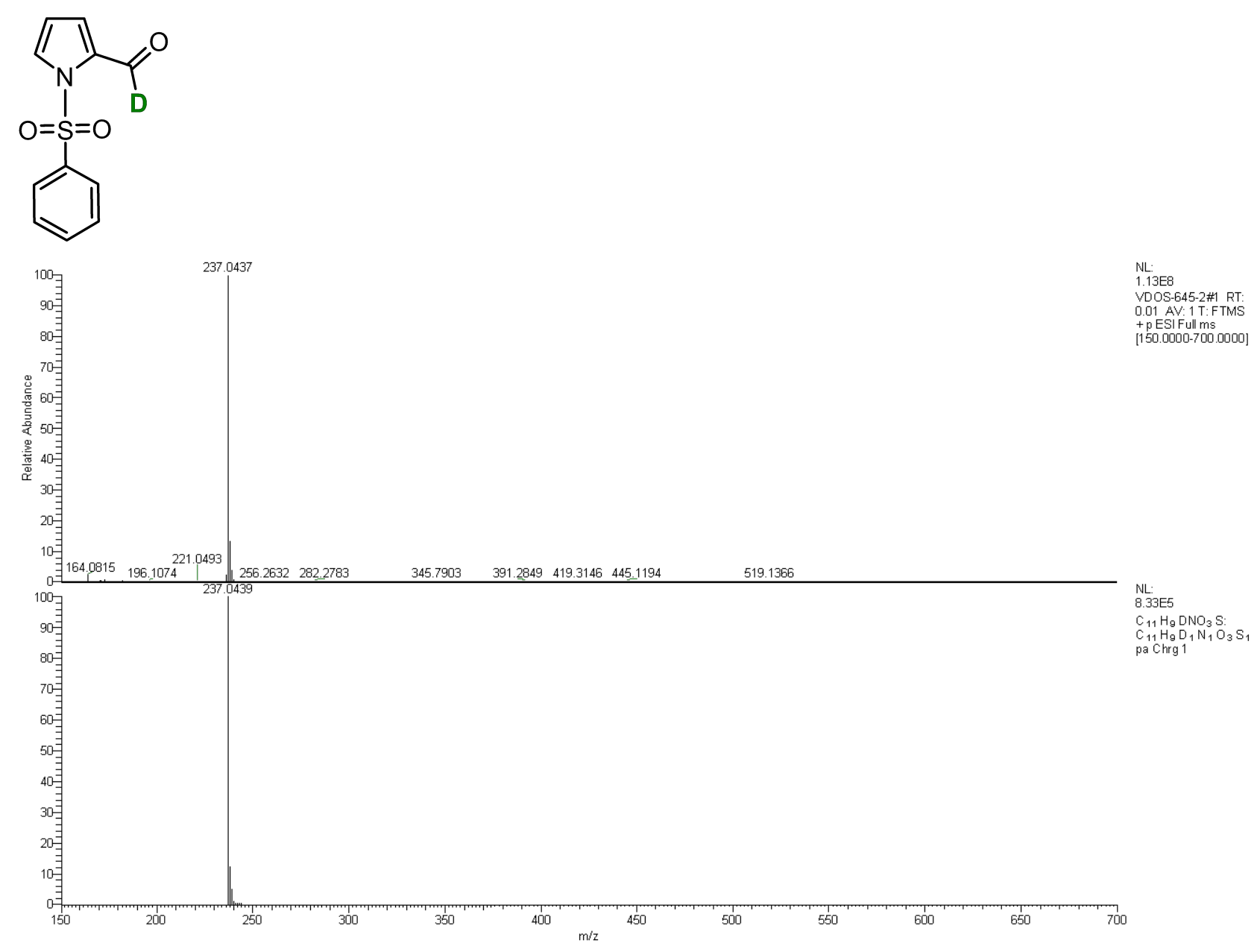


Entry 1: NMR for Crude reaction mixture

vDOS-645-A1

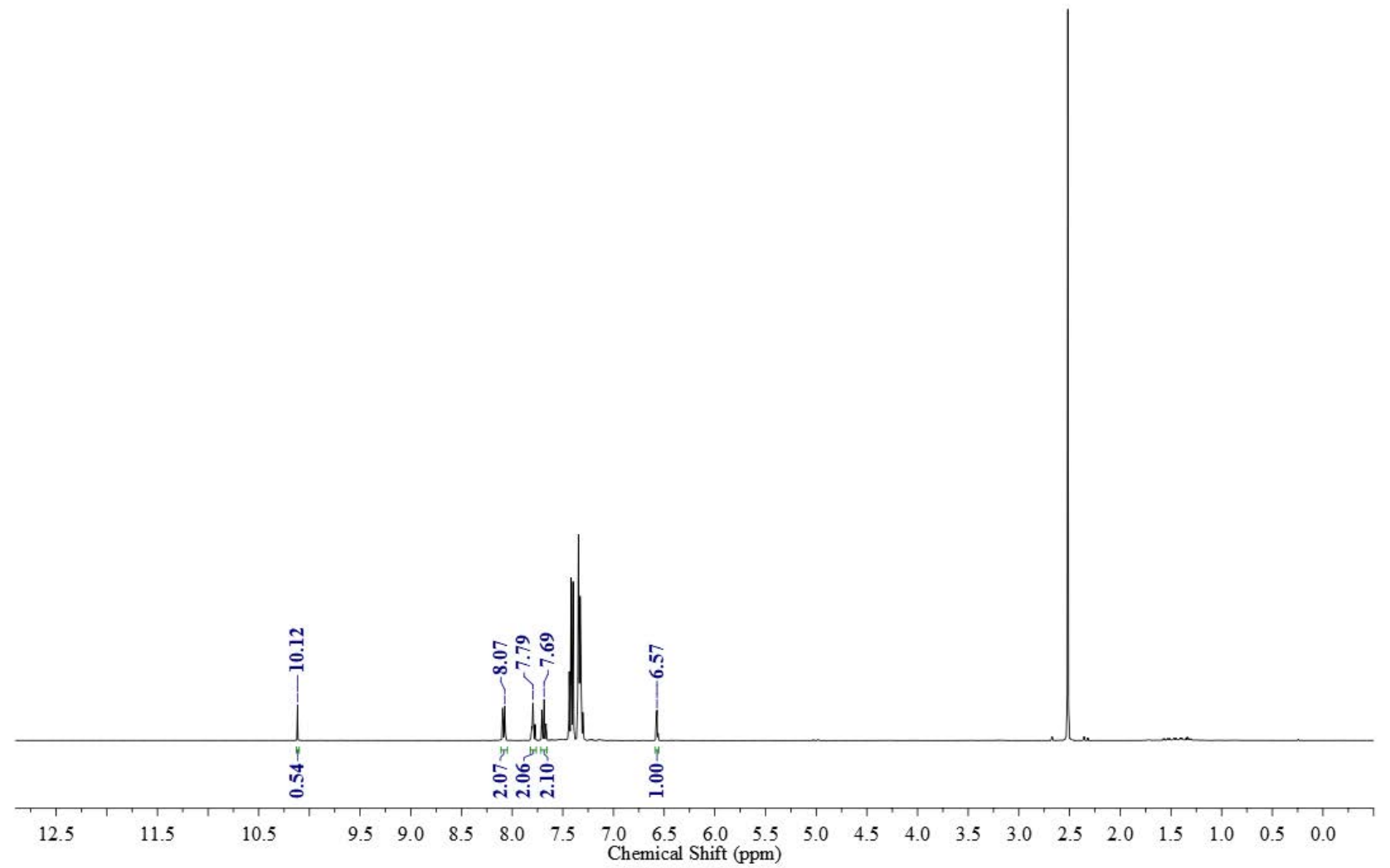

Entry 2: NMR for Crude reaction mixture

vDOS-645-B1

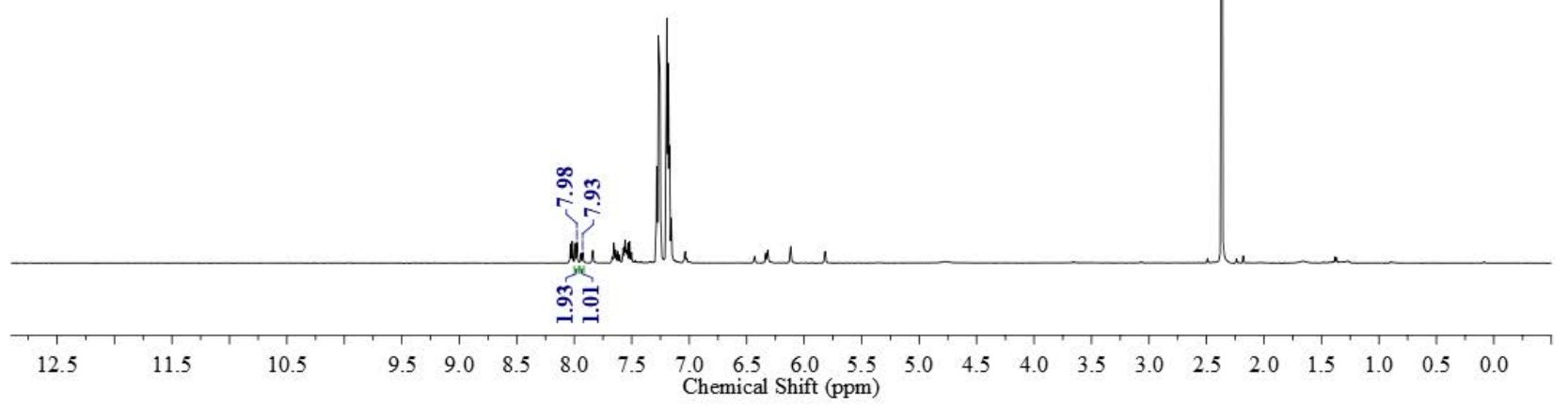


Entry 3: NMR for Crude reaction mixture

vDOS-645-C1

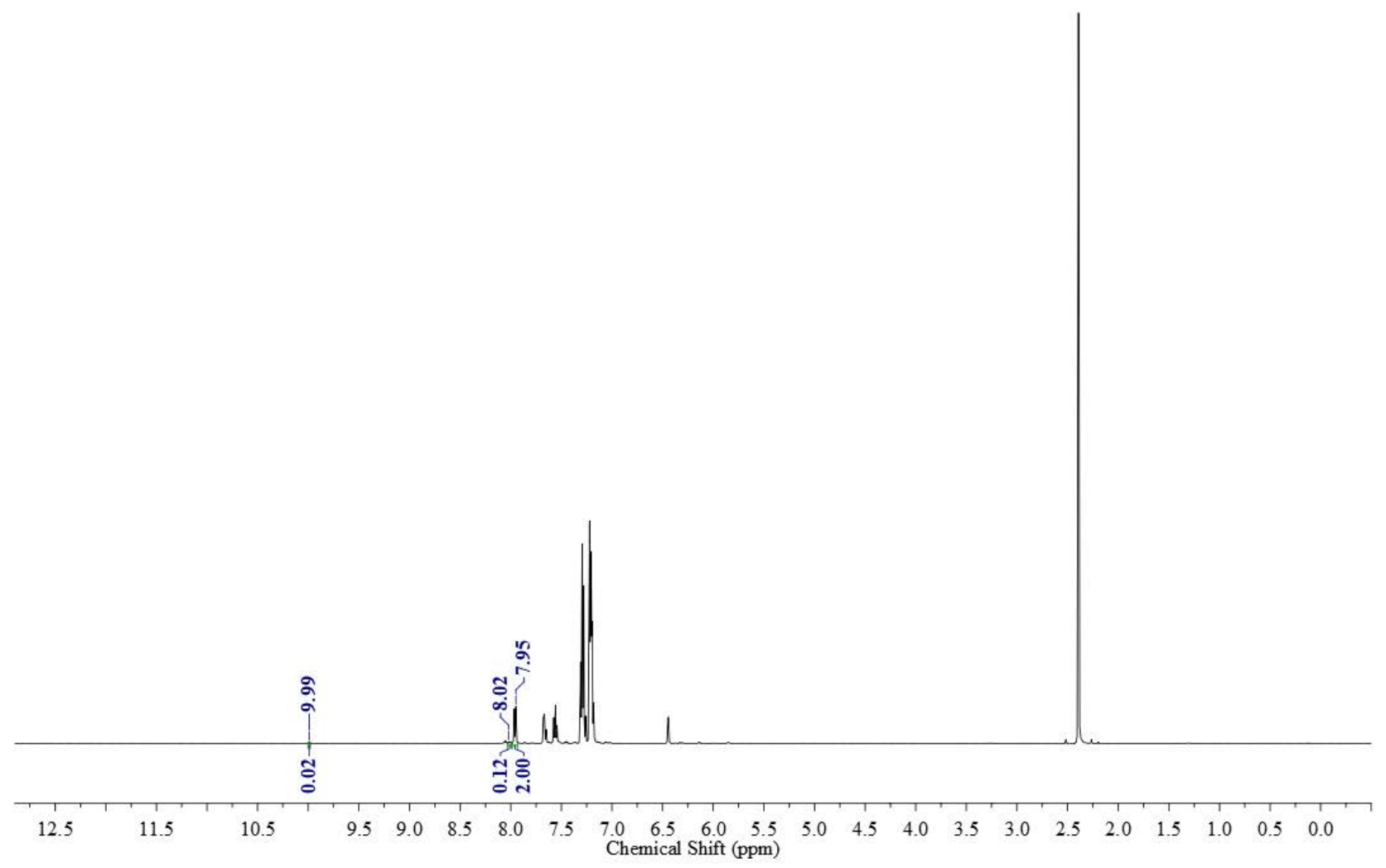

Entry 3': NMR for Crude reaction mixture, Duplicate vDOS-645-C2

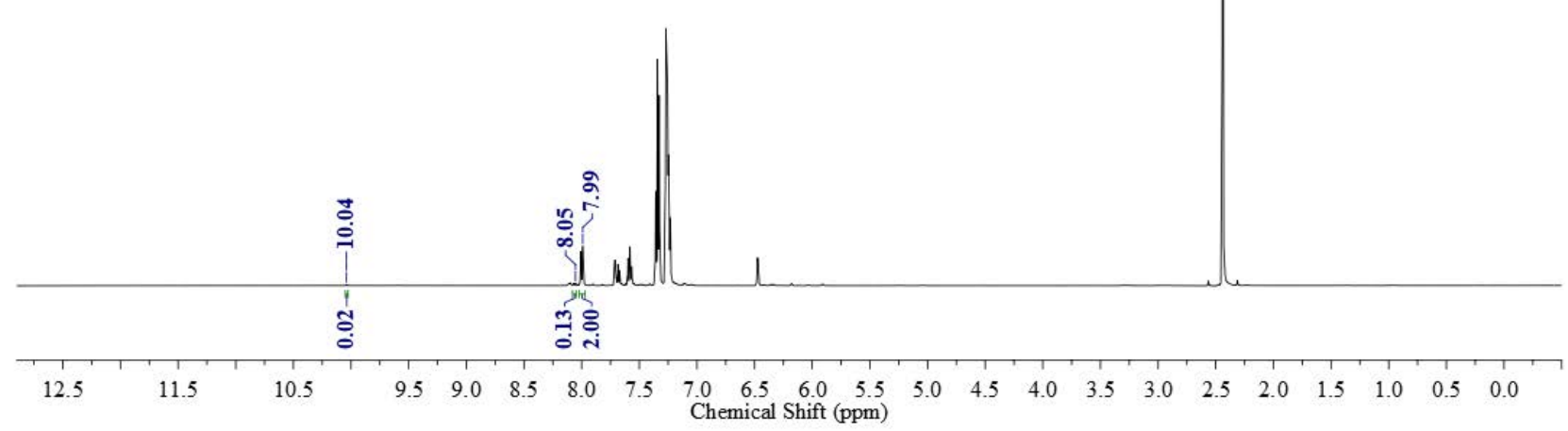

NMR after purification: 


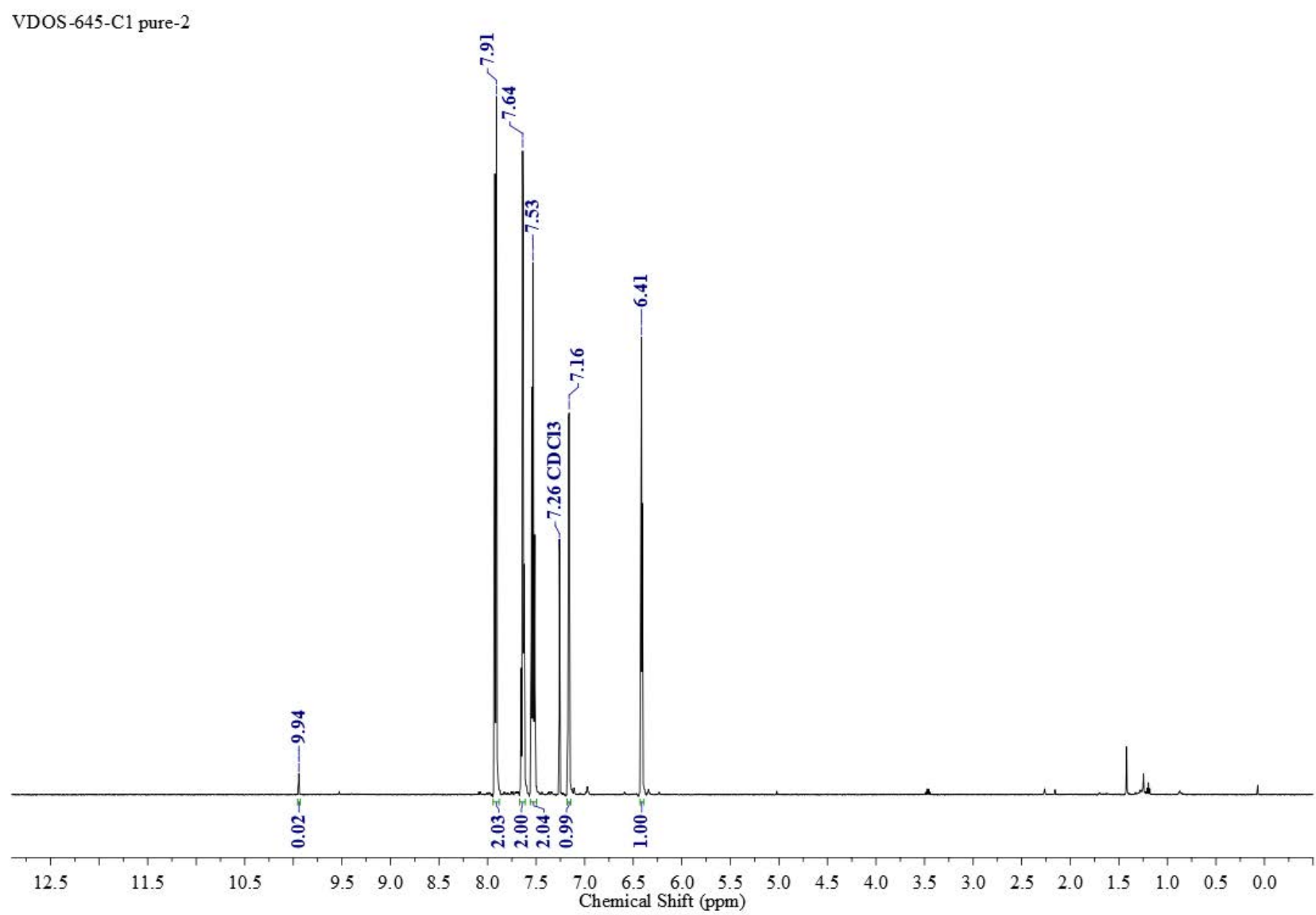

VDOS-645-C1 pure 13C

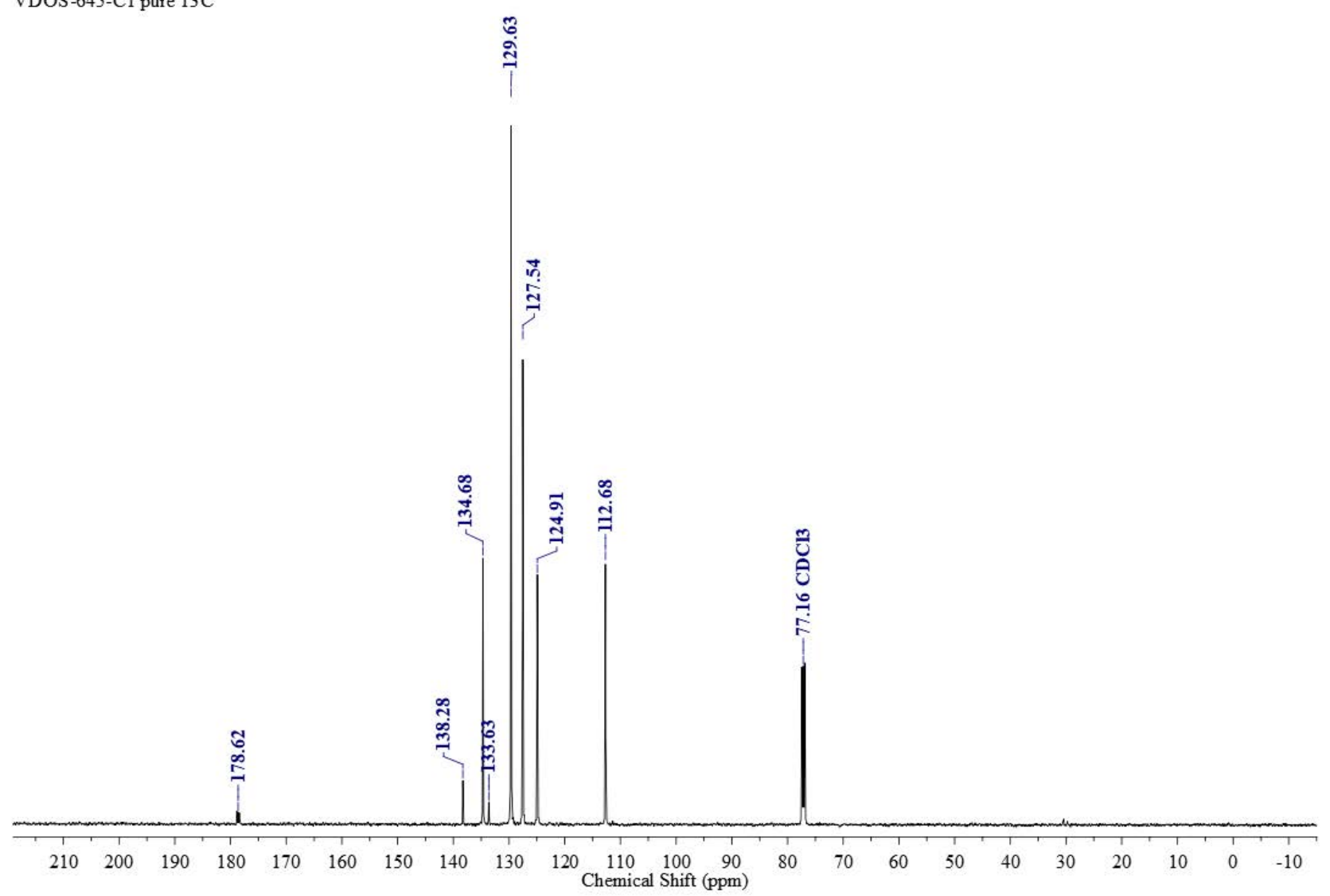

50. Dibenzo[b,d]furan-1-carbaldehyde-d 

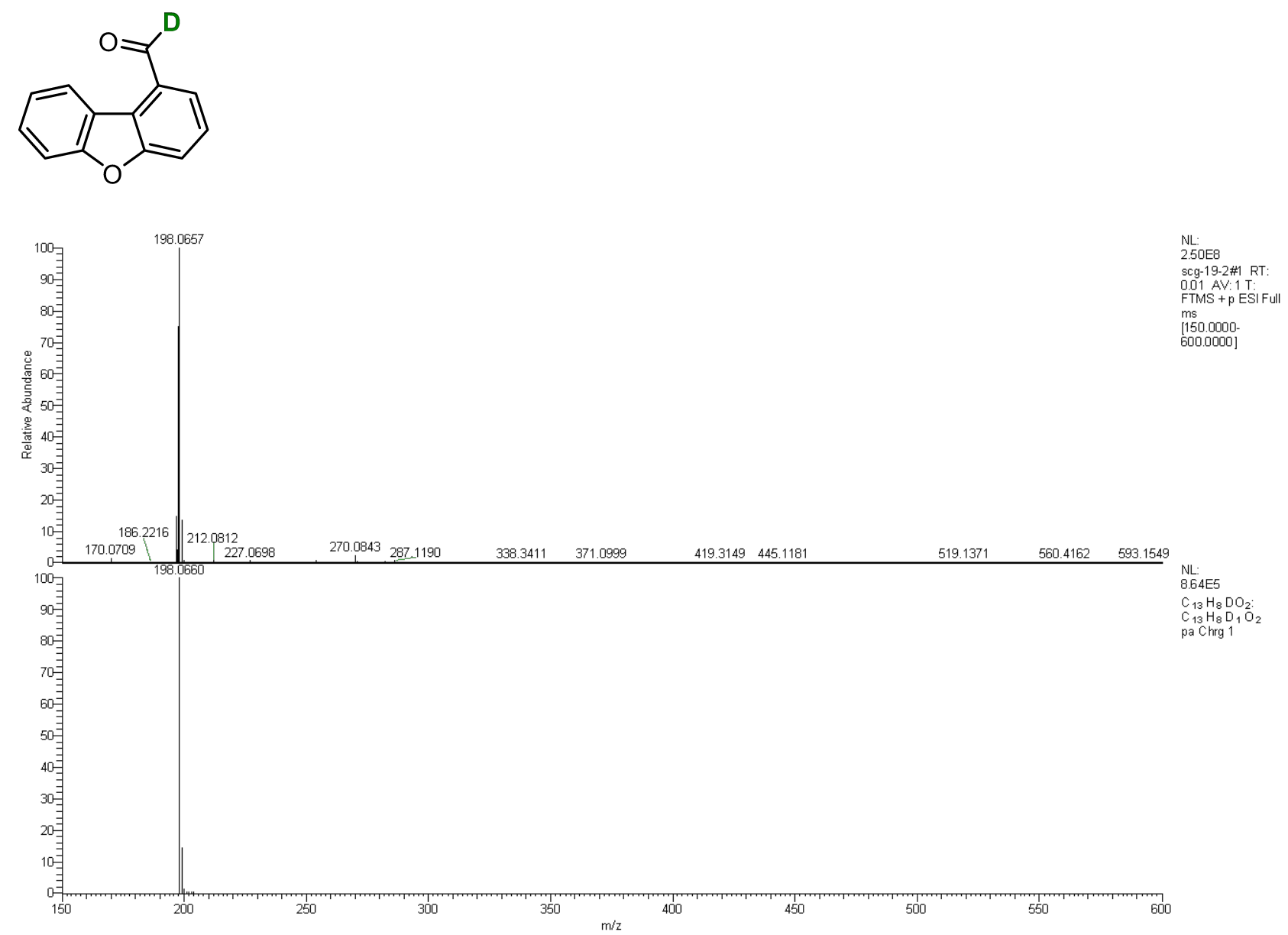
Entry 1: NMR for Crude reaction mixture

VDOS-640-A 1.6. fid

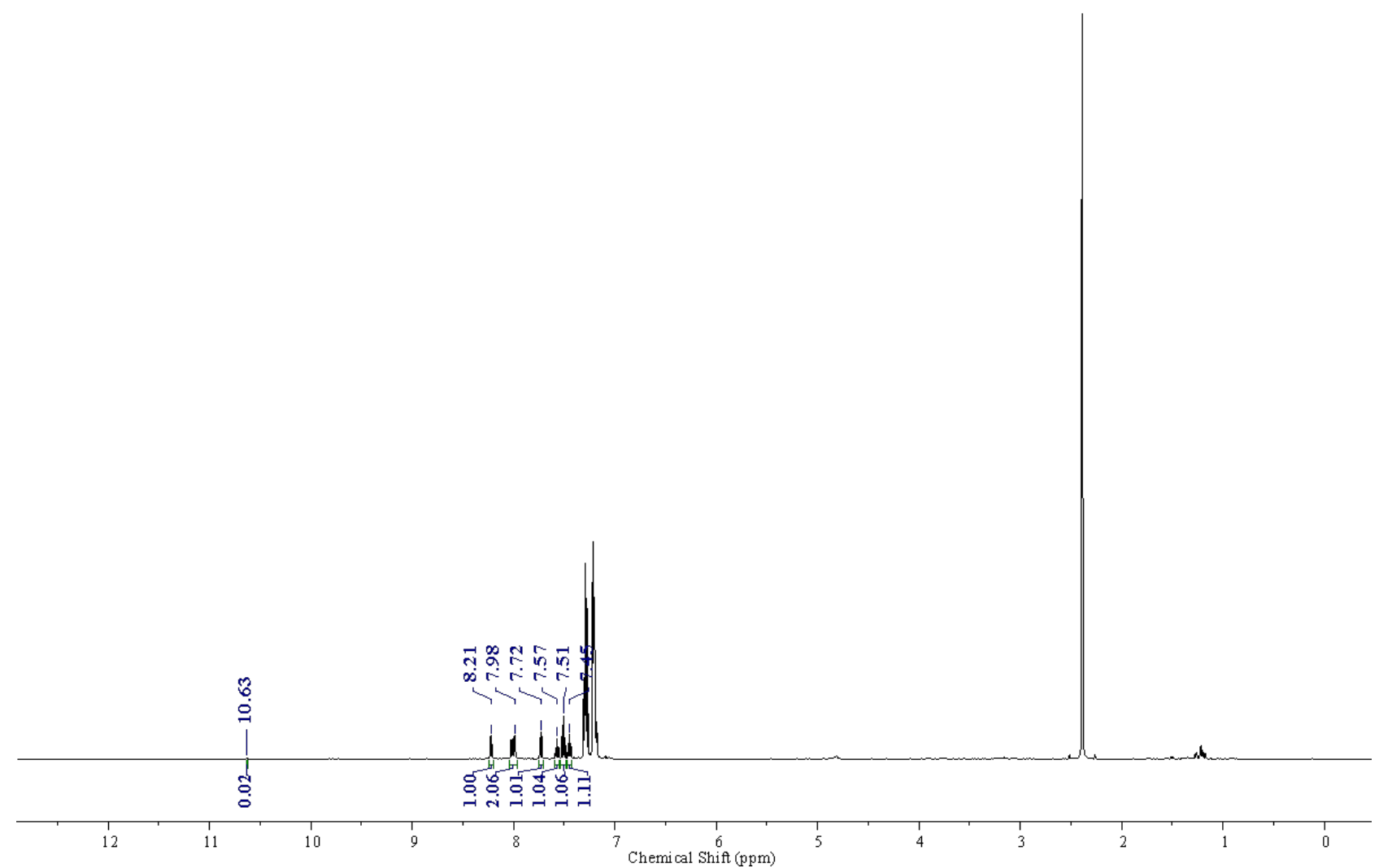

Entry 1': NMR for Crude reaction mixture, Duplicate

VDOS-640-A 2.1. fid

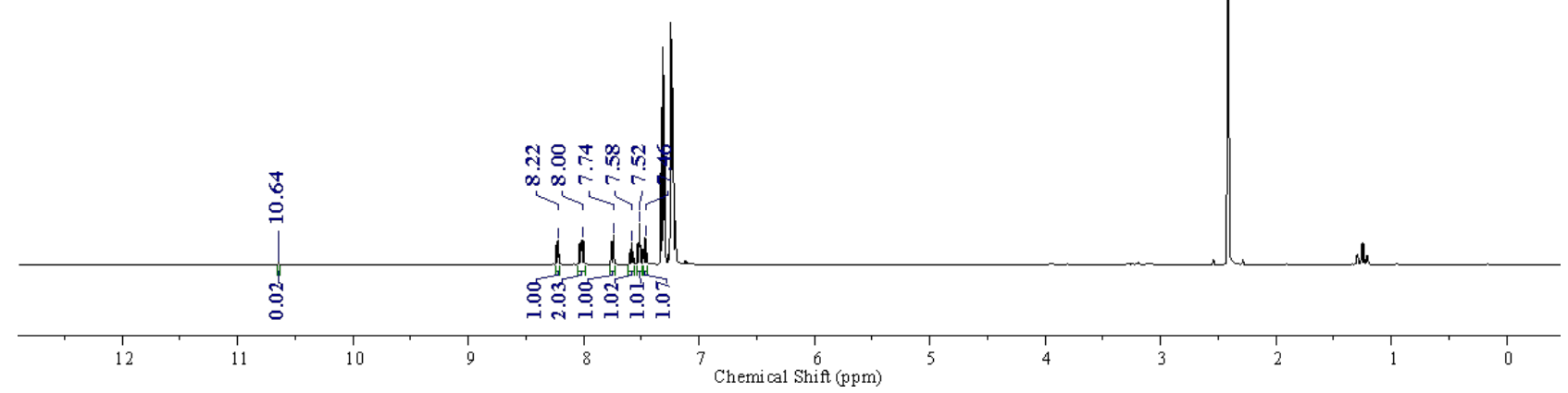


NMR after purification:

VDOS-639-A 1 pure.2.fid
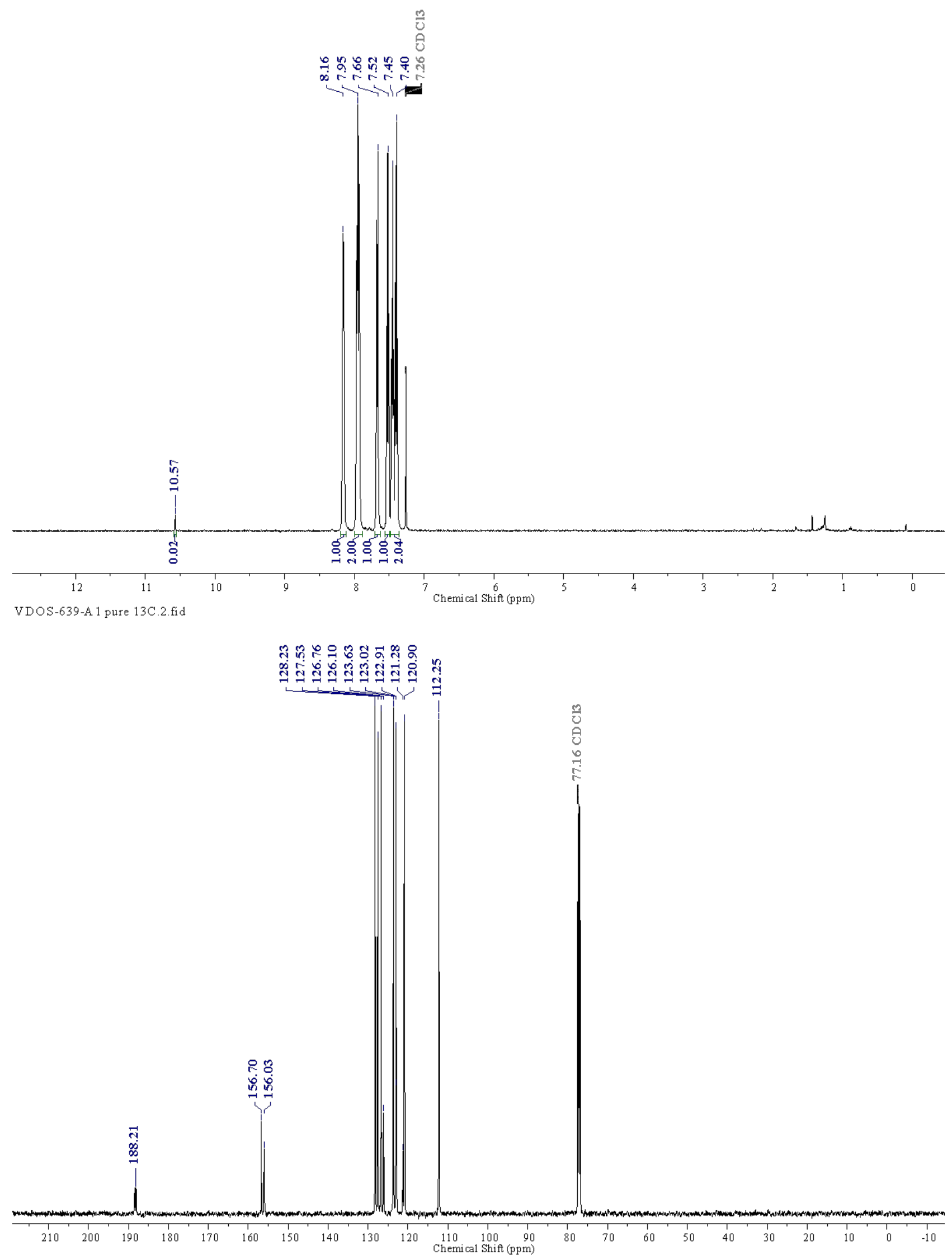


\section{Ibuprofen Derivative-d}<smiles>[2H]C(=O)c1cccc(OC(=O)[C@H](C)c2ccc(CC(C)C)cc2)c1</smiles>

Entry 1: NMR for Crude reaction mixture vDOS-632-A 3.5.fid

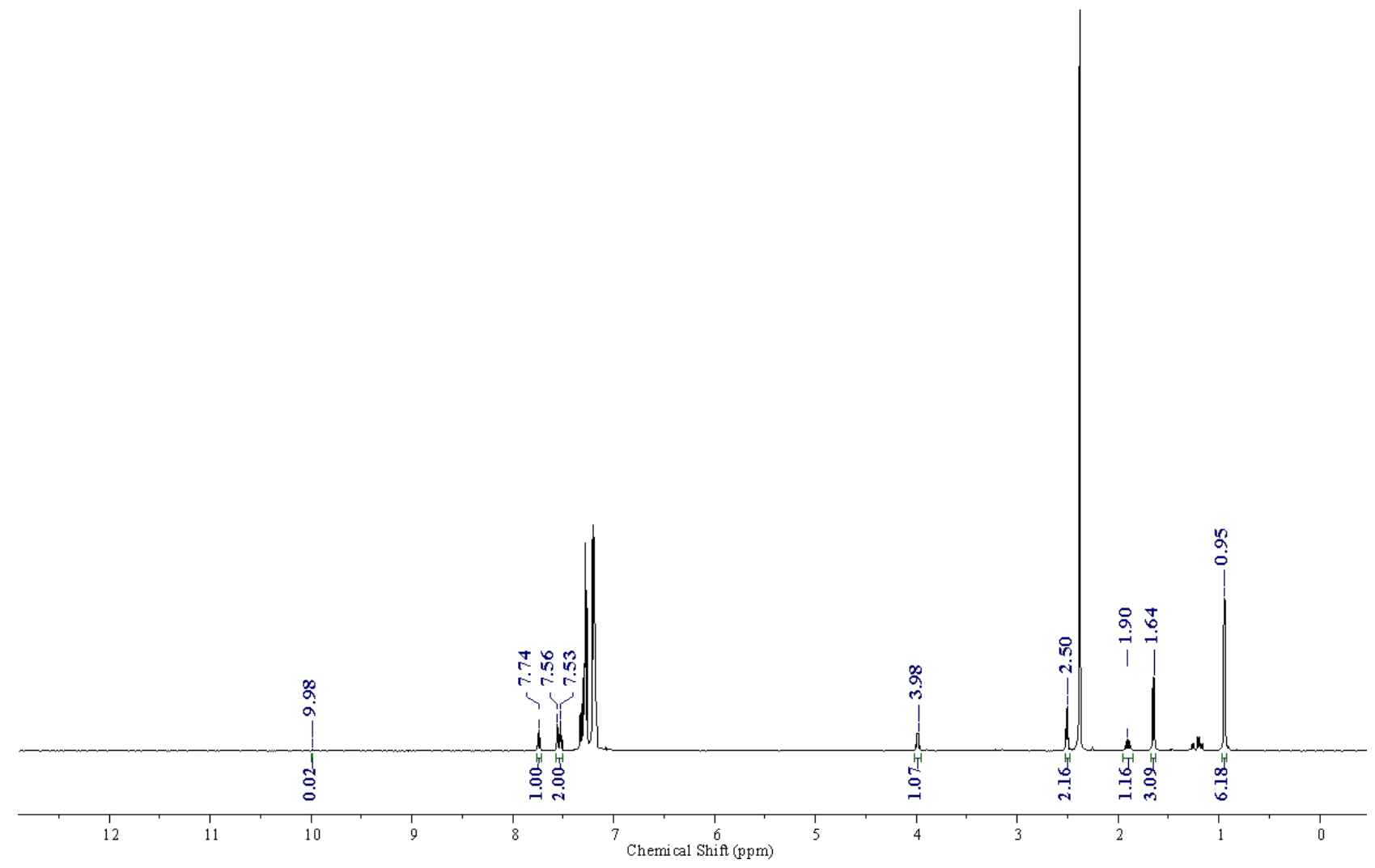


Entry 1': NMR for Crude reaction mixture, Duplicate

VDOS-Ibupropen-A 2.1. fid

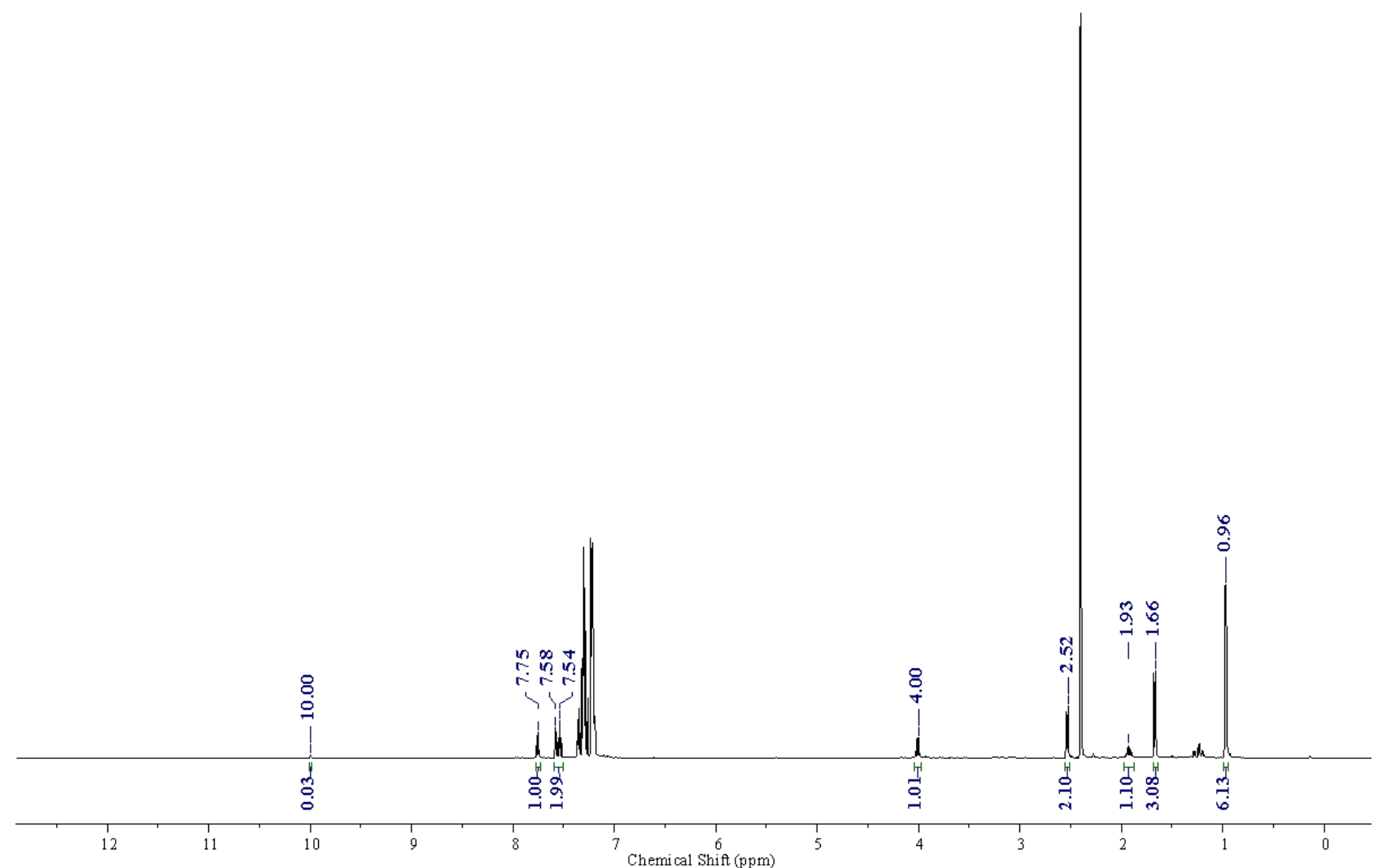

NMR after purification:

VDOS-632-A 2 VP Final.4.fid

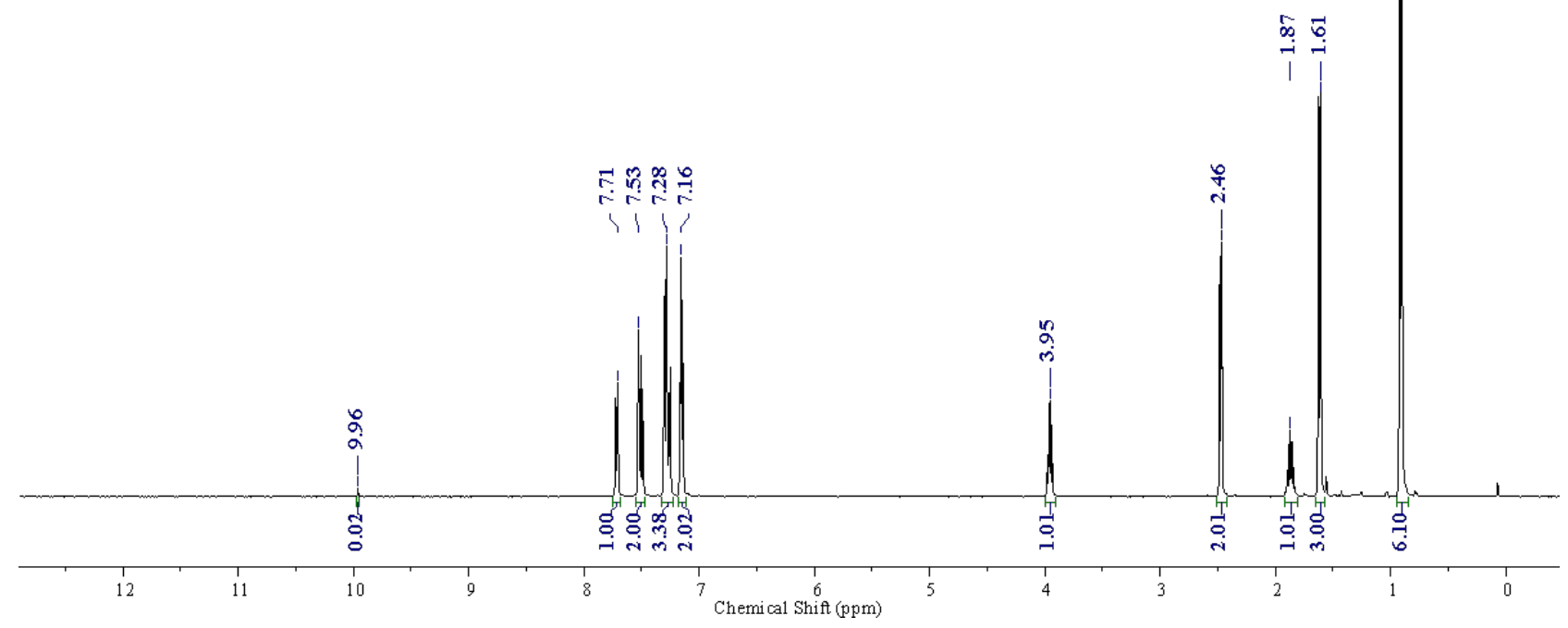




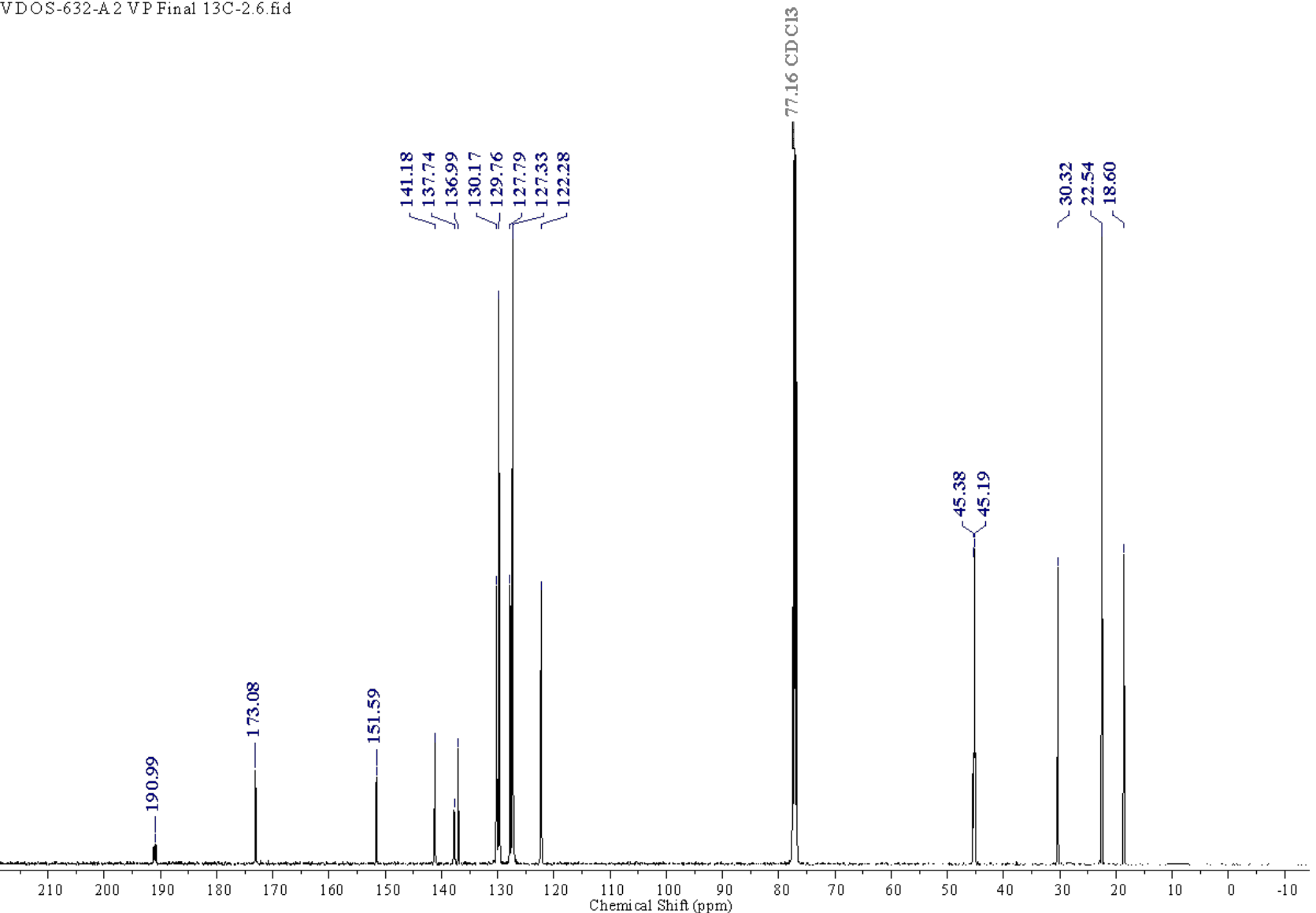




\section{HPLC Traces:}

HPLC Conditions: Chiralpak, IC, $1 \mathrm{~mL} / \mathrm{min}^{-1}$, 99.5/0.5 (hexane-IPA), RT: 34 and 37 minutes.

Racemic product of Ibuprofen Derivative<smiles>CC(C)Cc1ccc(C(C)C(=O)Oc2cccc(C=O)c2)cc1</smiles>

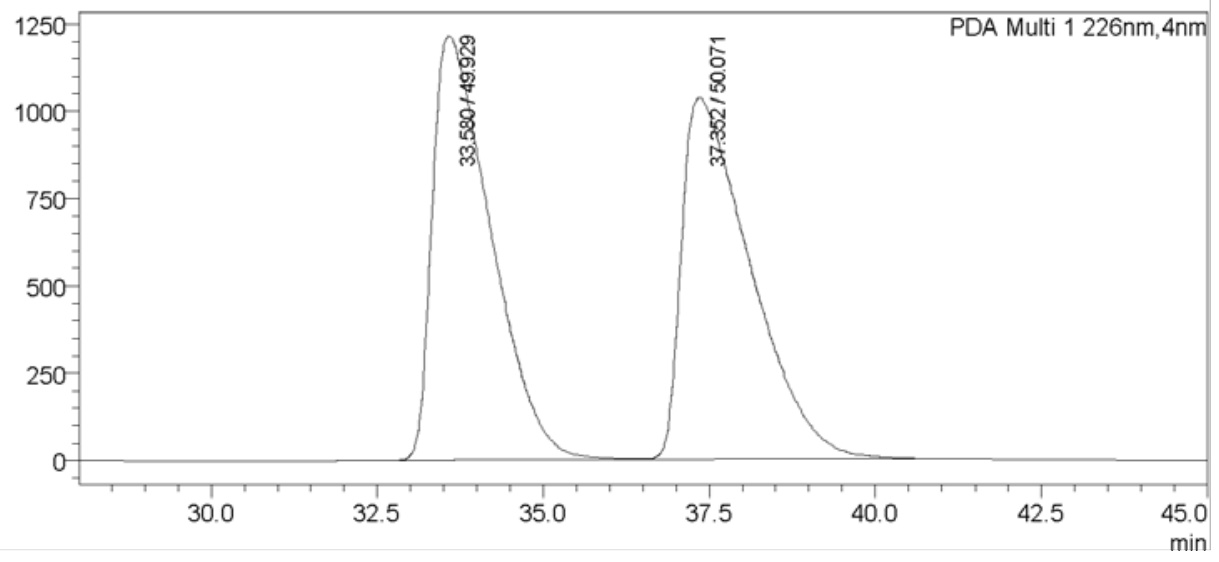

Racemic product of Ibuprofen Derivative- $d$<smiles>[2H]C(=O)c1cccc(OC(=O)C(C)c2ccc(CC(C)C)cc2)c1</smiles>

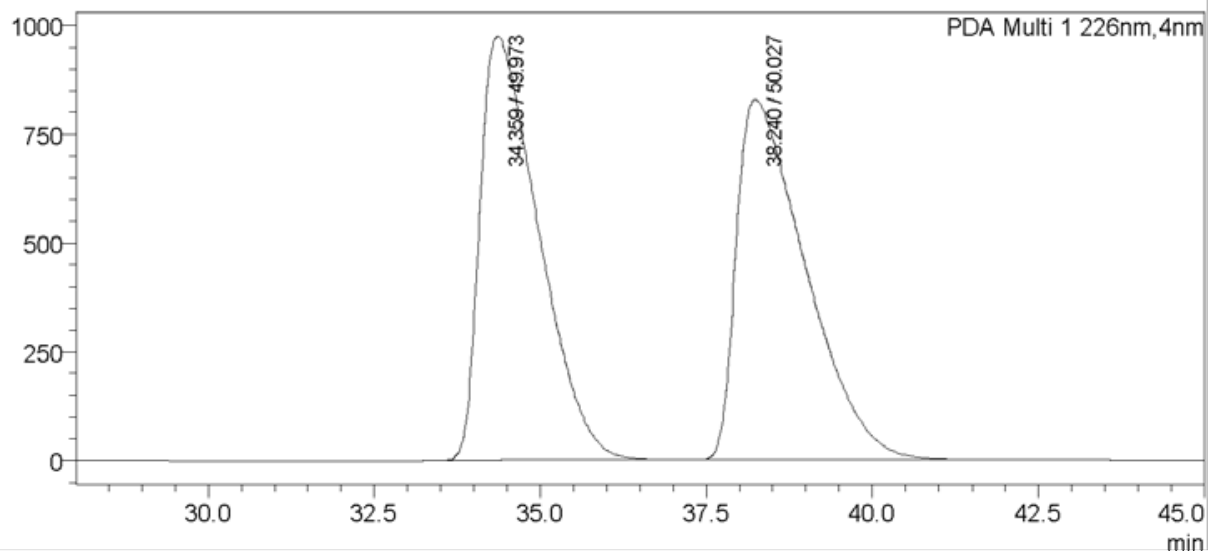


Starting Material, $S$-Ibuprofen Derivative (er 95.8:4.2)<smiles>CC(C)Cc1ccc([C@H](C)C(=O)Oc2cccc(C=O)c2)cc1</smiles>

\section{$S, 92 \%$ ee}

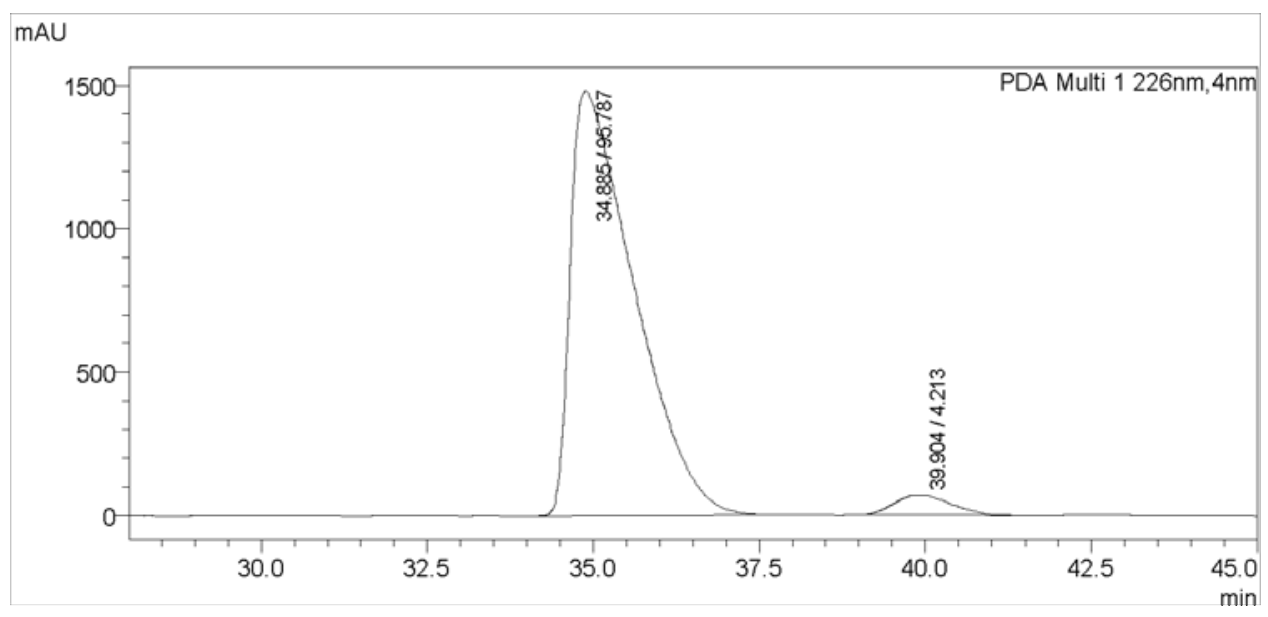

$S$-Ibuprofen Derivative- $d$ (er 95.4:4.6)<smiles>[2H]C(=O)c1cccc(OC(=O)[C@H](C)c2ccc(CC(C)C)cc2)c1</smiles>

\section{$S, 91 \%$ ee}

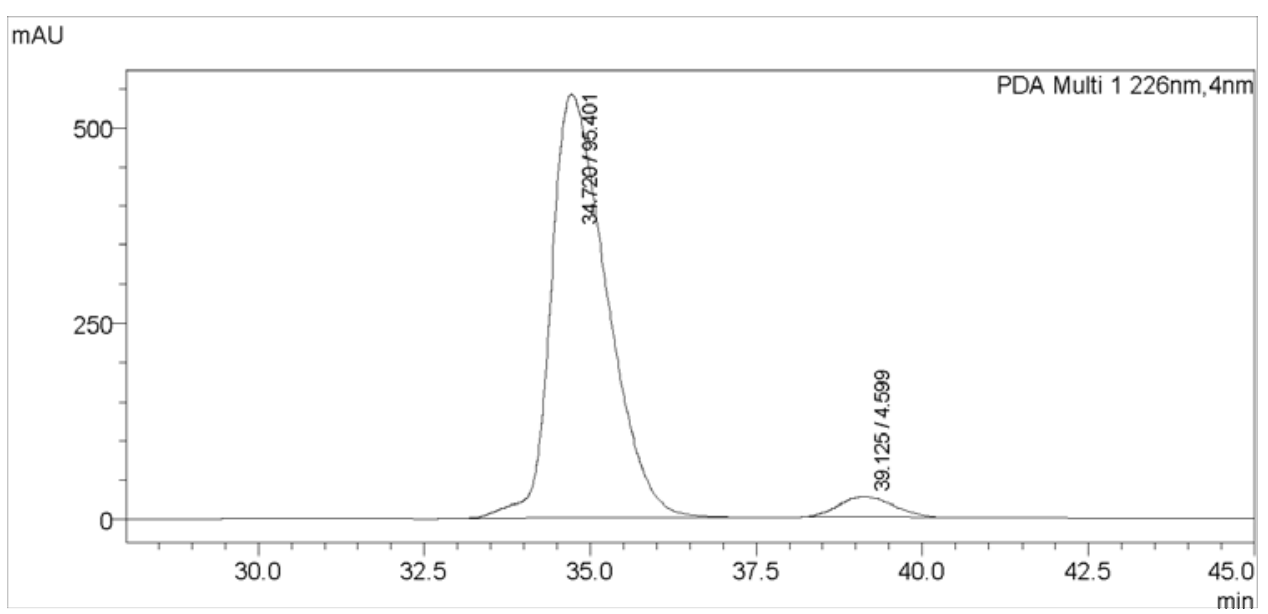


52. Menthol Derivative-d<smiles>[2H]C(=O)c1cccc(C(=O)OC2C[C@@H](C)CC[C@H]2C(C)C)c1</smiles>

Entry 1: NMR for Crude reaction mixture vDOS-638-B 1.1.fid

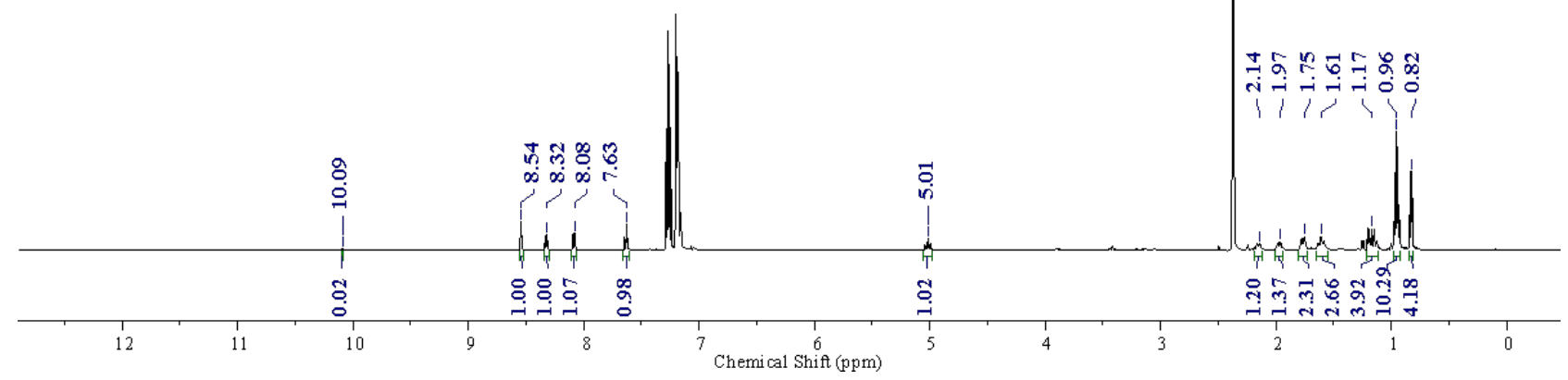


Entry 1': NMR for Crude reaction mixture, Duplicate

VDOS-638-B 2.5.fid

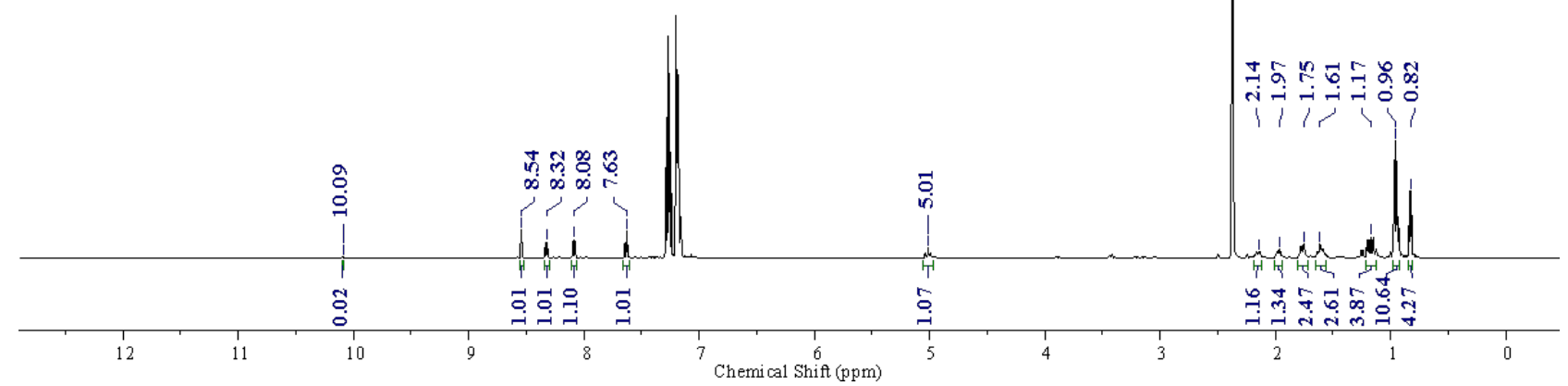

NMR after purification:

VDOS-638-B 1 -very pure.2.fid

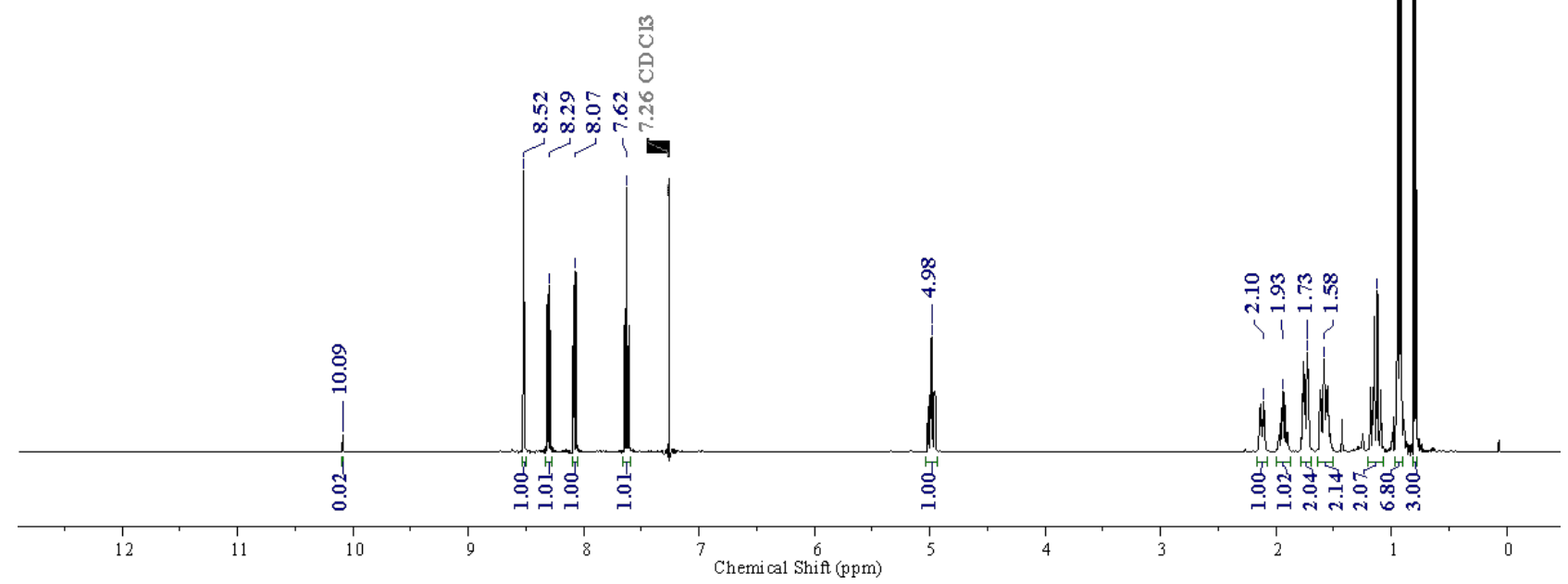




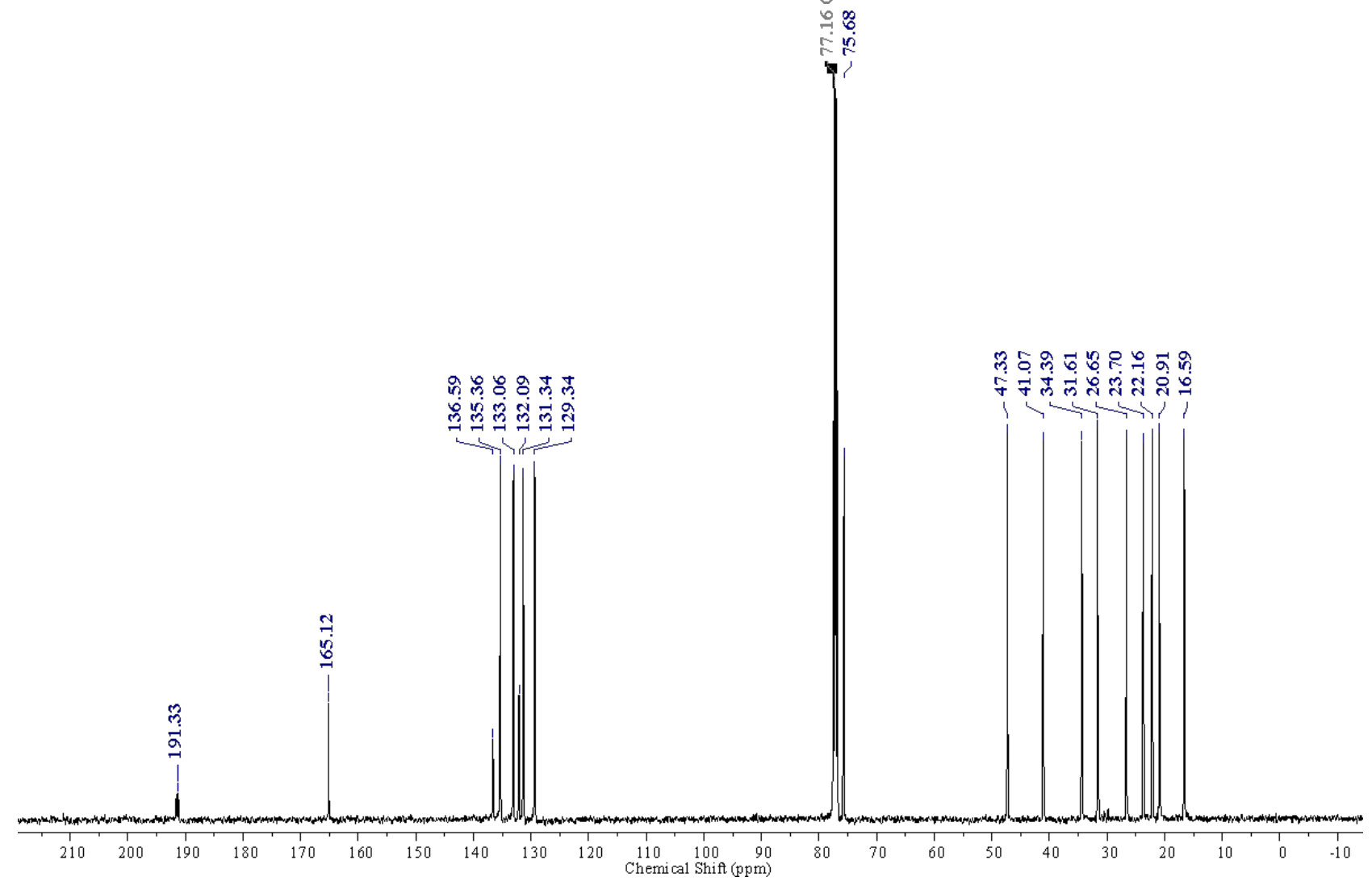




\section{HPLC Traces:}

HPLC Conditions: Chiralpak, IA, $0.5 \mathrm{~mL} / \mathrm{min}^{-1}, 99.5 / 0.5$ (hexane-IPA), RT: 17 and 24 minutes.

Racemic product of Menthol Derivative<smiles>CC1CCC(C(C)C)C(OC(=O)c2cccc(C=O)c2)C1</smiles>

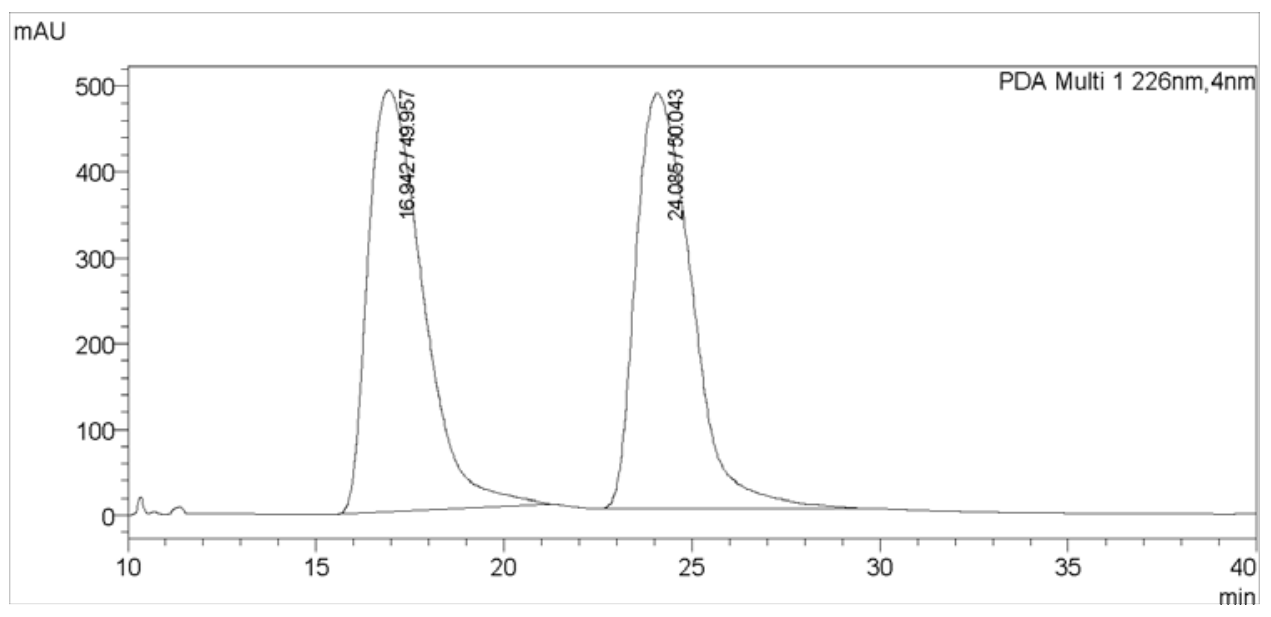

Racemic product of Menthol Derivative- $d$<smiles>[2H]C(=O)c1cccc(C(=O)OC2CC(C)CCC2C(C)C)c1</smiles>

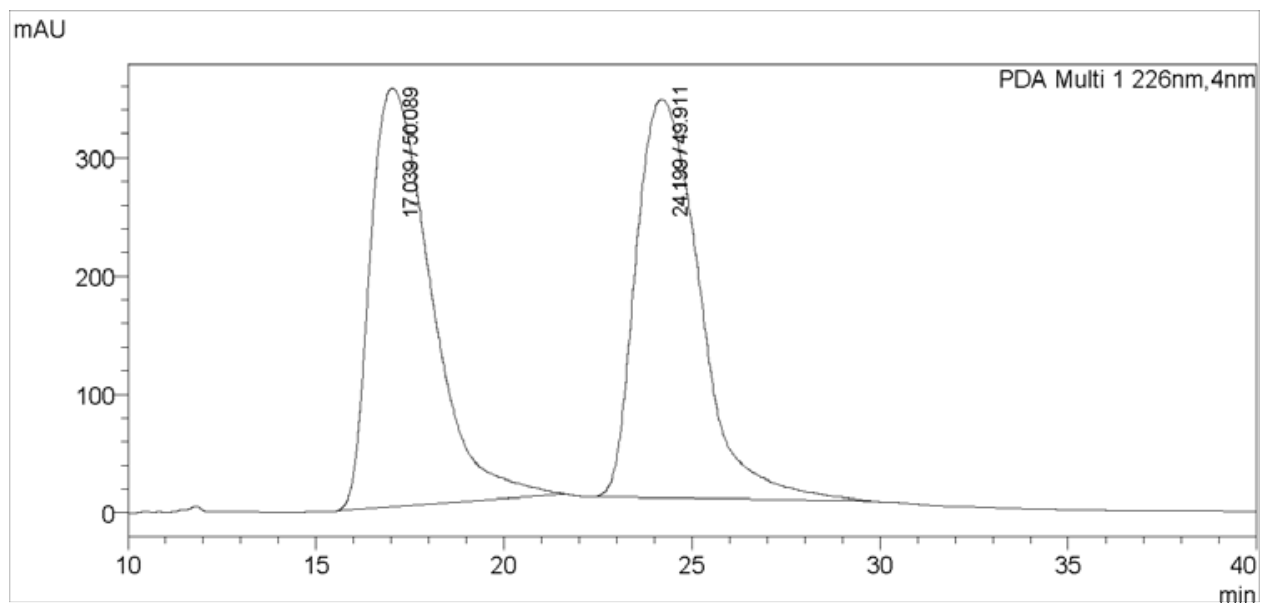


Starting Material, L-Menthol Derivative (er 0.3:99.7)<smiles>CC1CC[C@H](C(C)C)C(OC(=O)c2cccc(C=O)c2)C1</smiles>

$99 \%$ ee

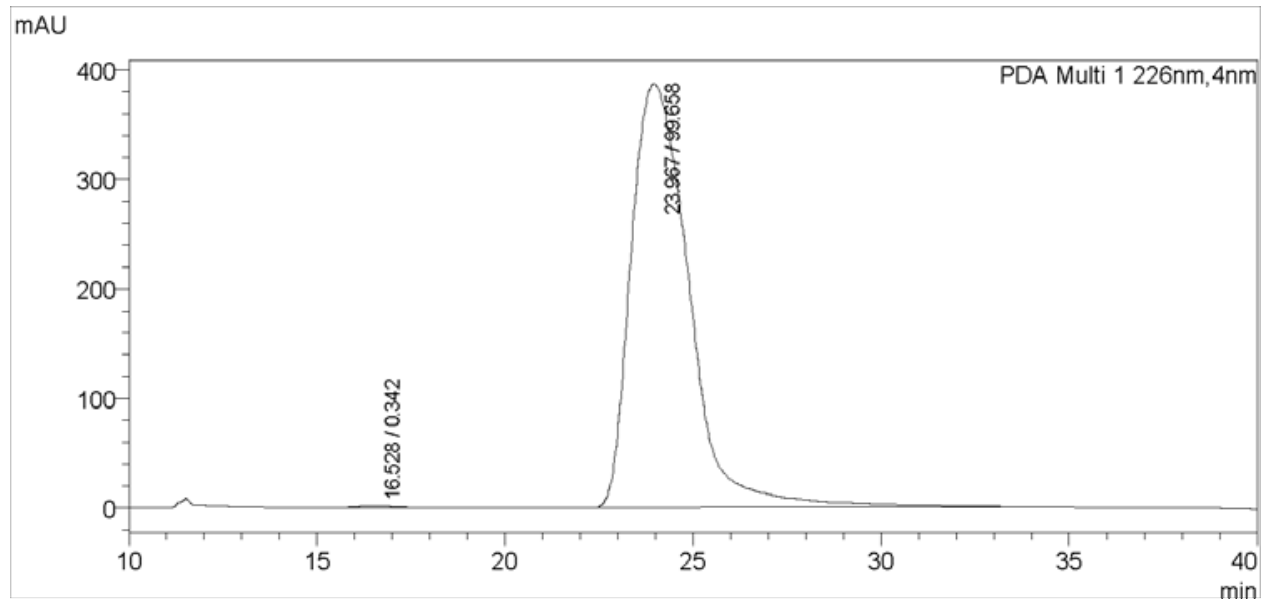

L-Menthol Derivative- $d$ (er 0.4:99.6)<smiles>[2H]C(=O)c1cccc(C(=O)OC2CC(C)CC[C@@H]2C(C)C)c1</smiles>

$99 \%$ ee

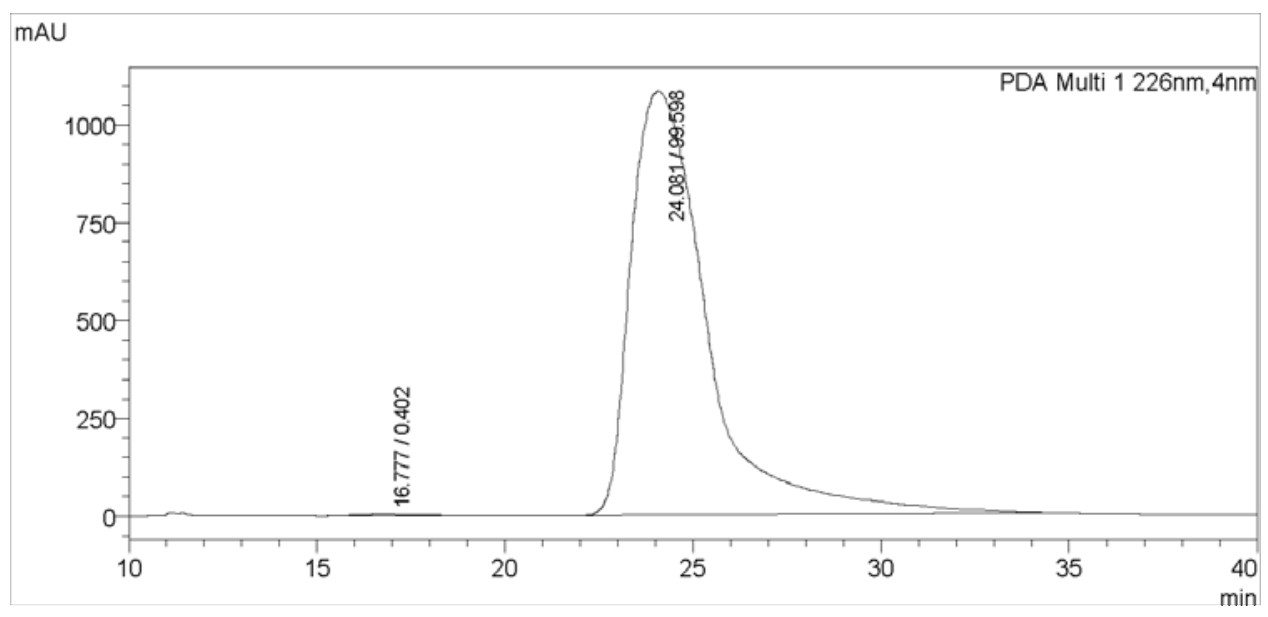


53. Naproxen Derivative- $d$

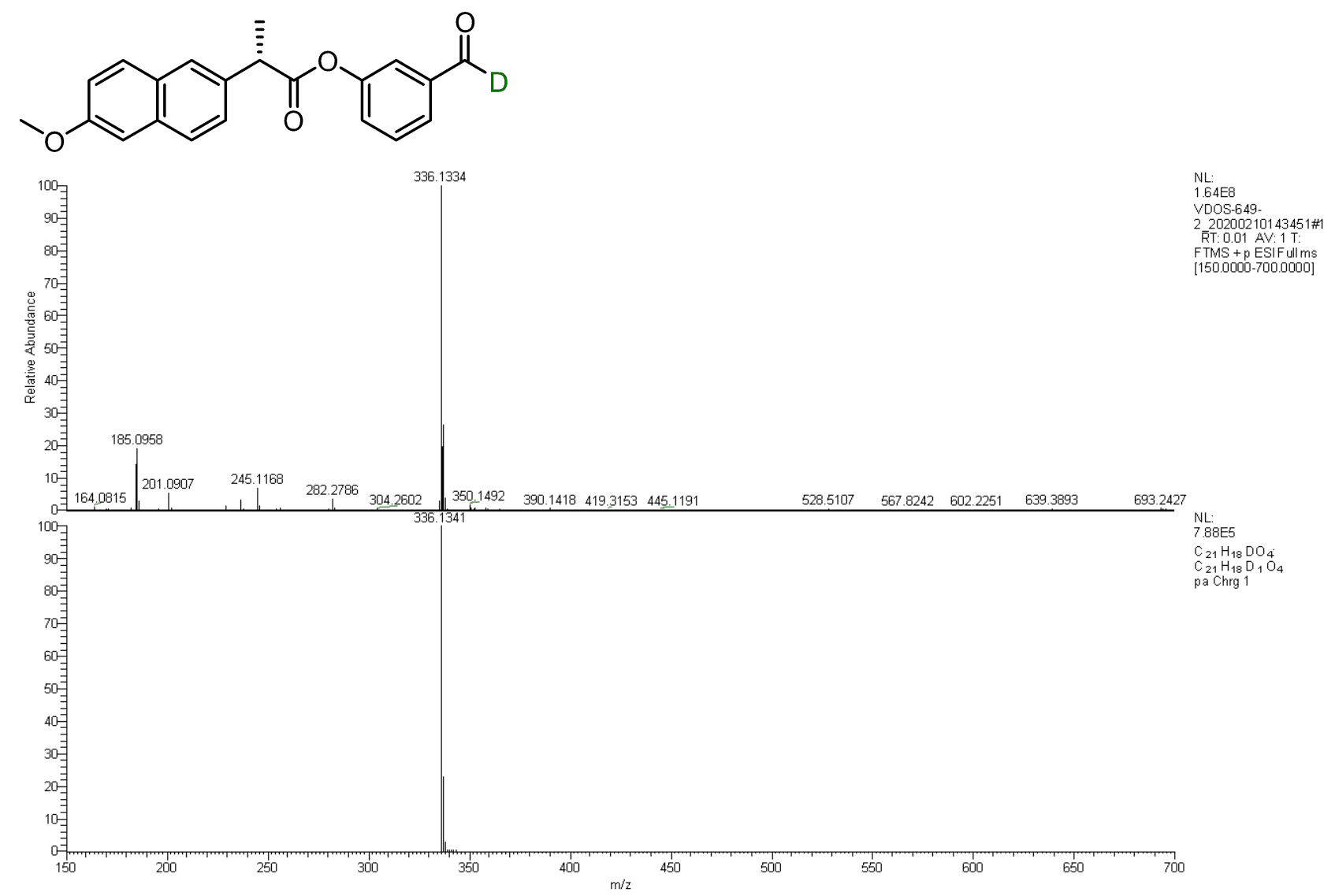

Entry 1: NMR for Crude reaction mixture

VDOS-649-A1

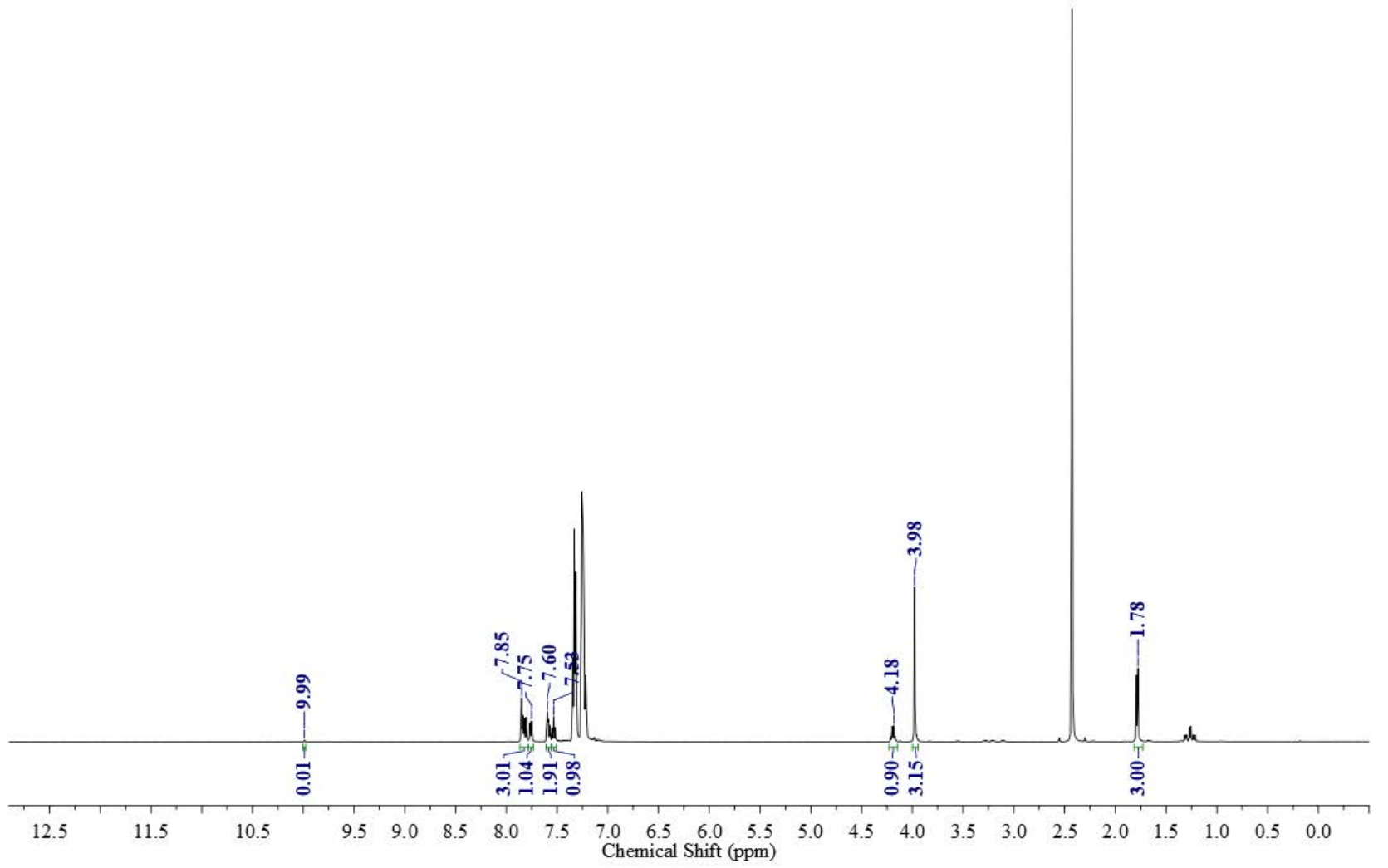


Entry 1': NMR for Crude reaction mixture, Duplicate

VDOS-649-A2-2

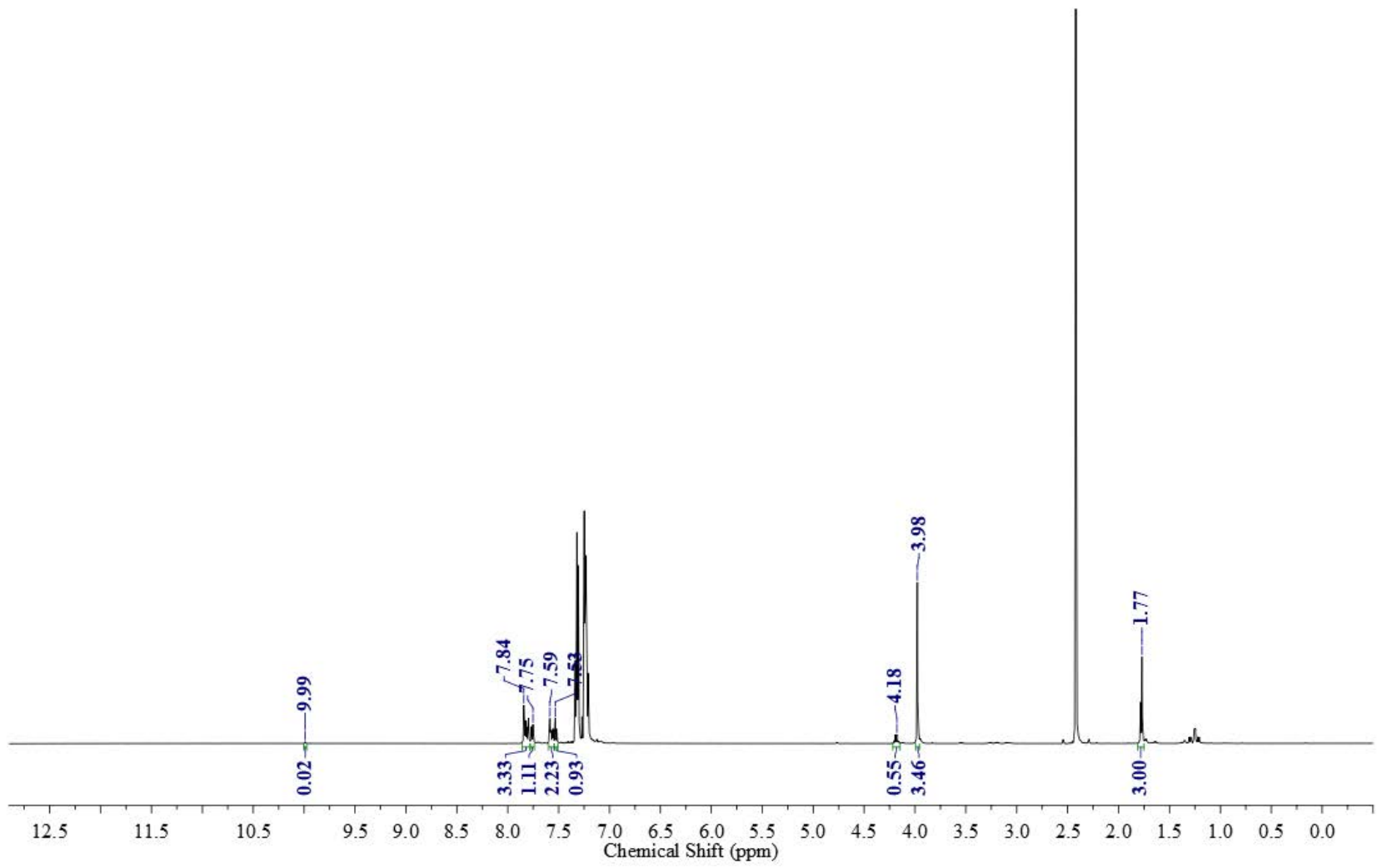

NMR after purification:

VDOS-649-A1 RC pure

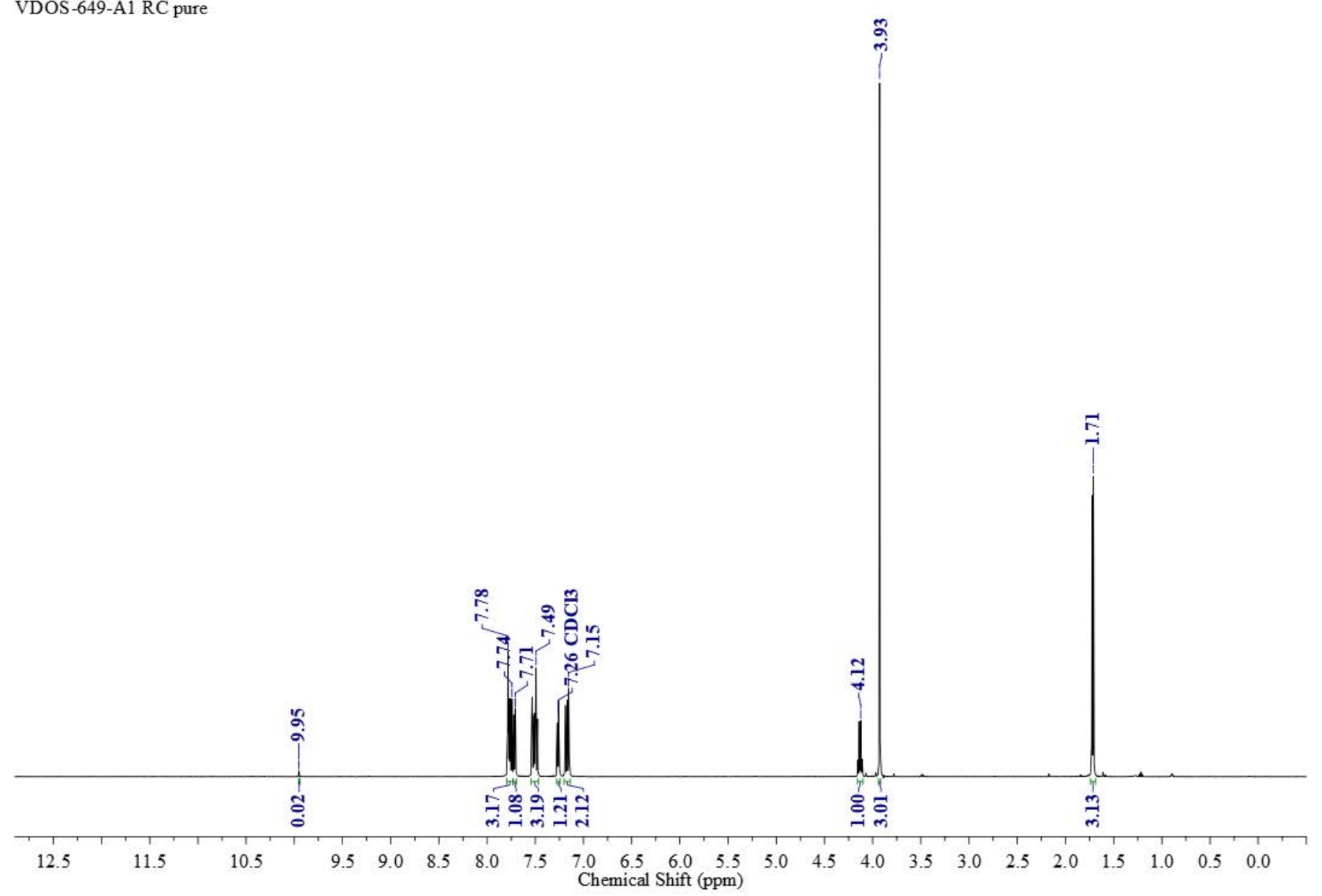


VDOS-649-A1 RC 13C

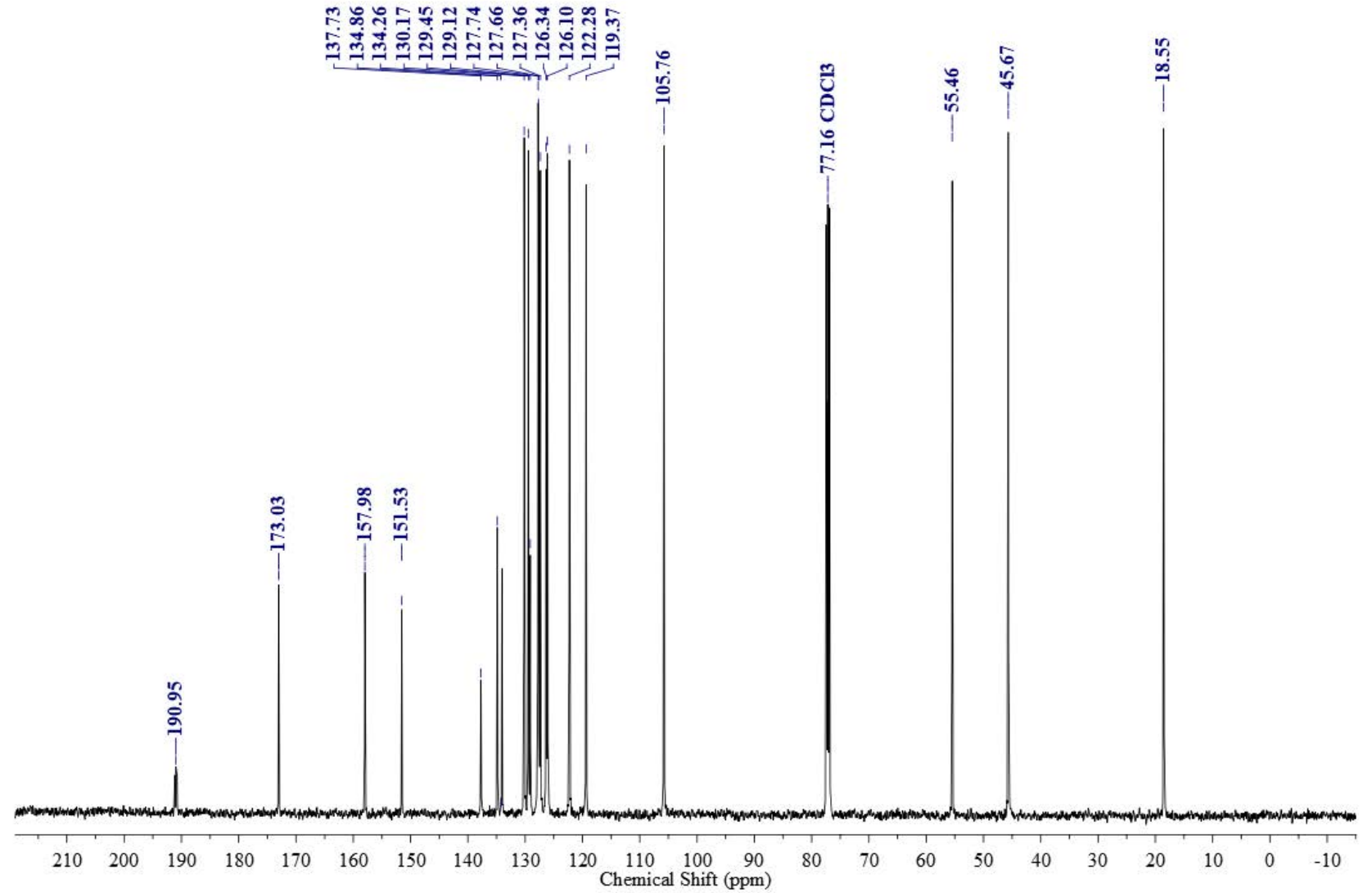


54. Epiandrosterone Derivative-d

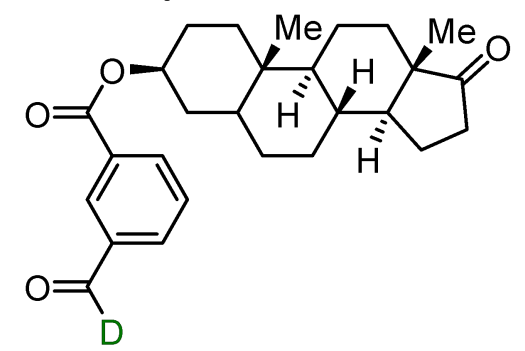

Entry 1: NMR for Crude reaction mixture vDOS-678-A1

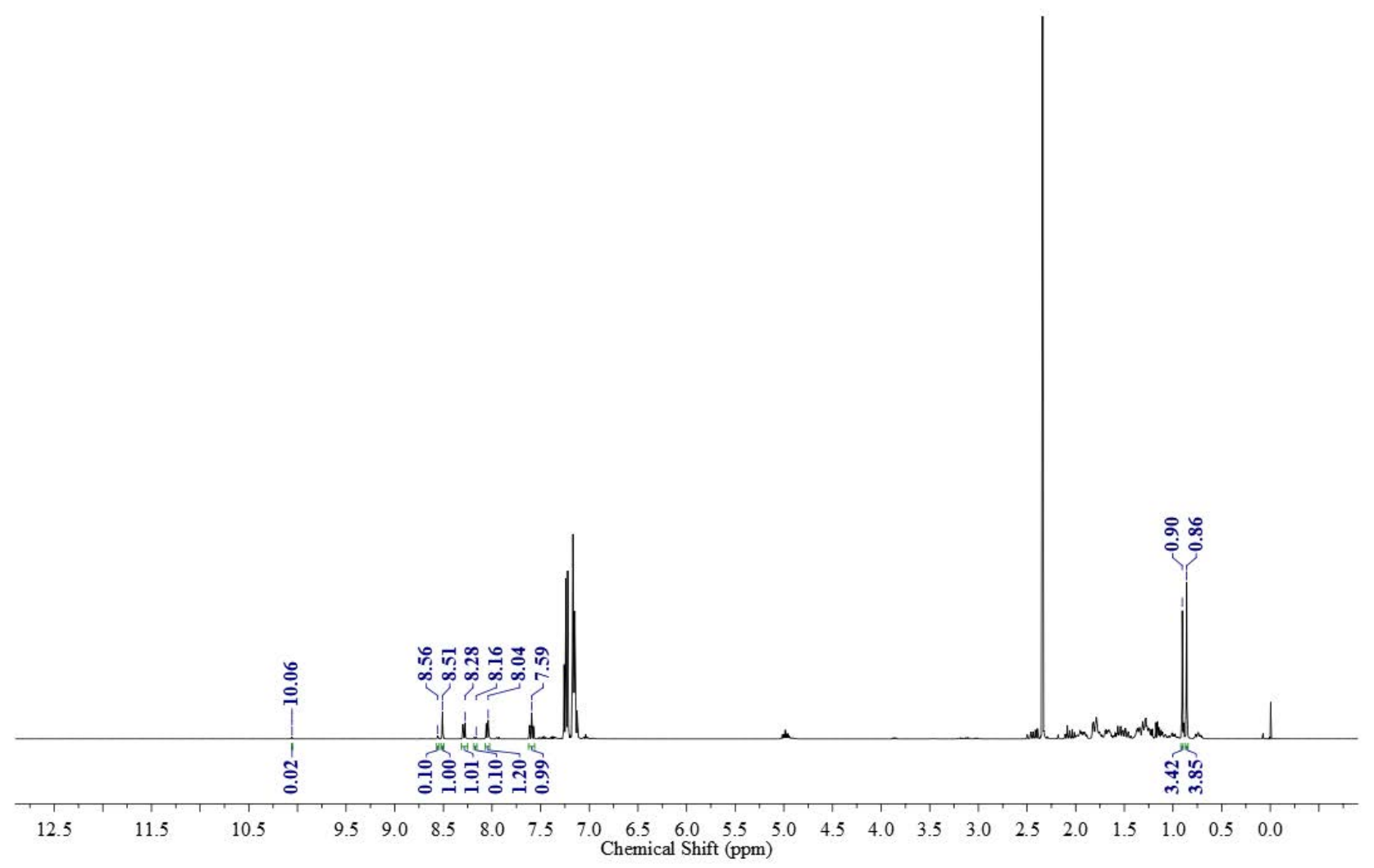


Entry 1': NMR for Crude reaction mixture, Duplicate

VDOS-678-A2

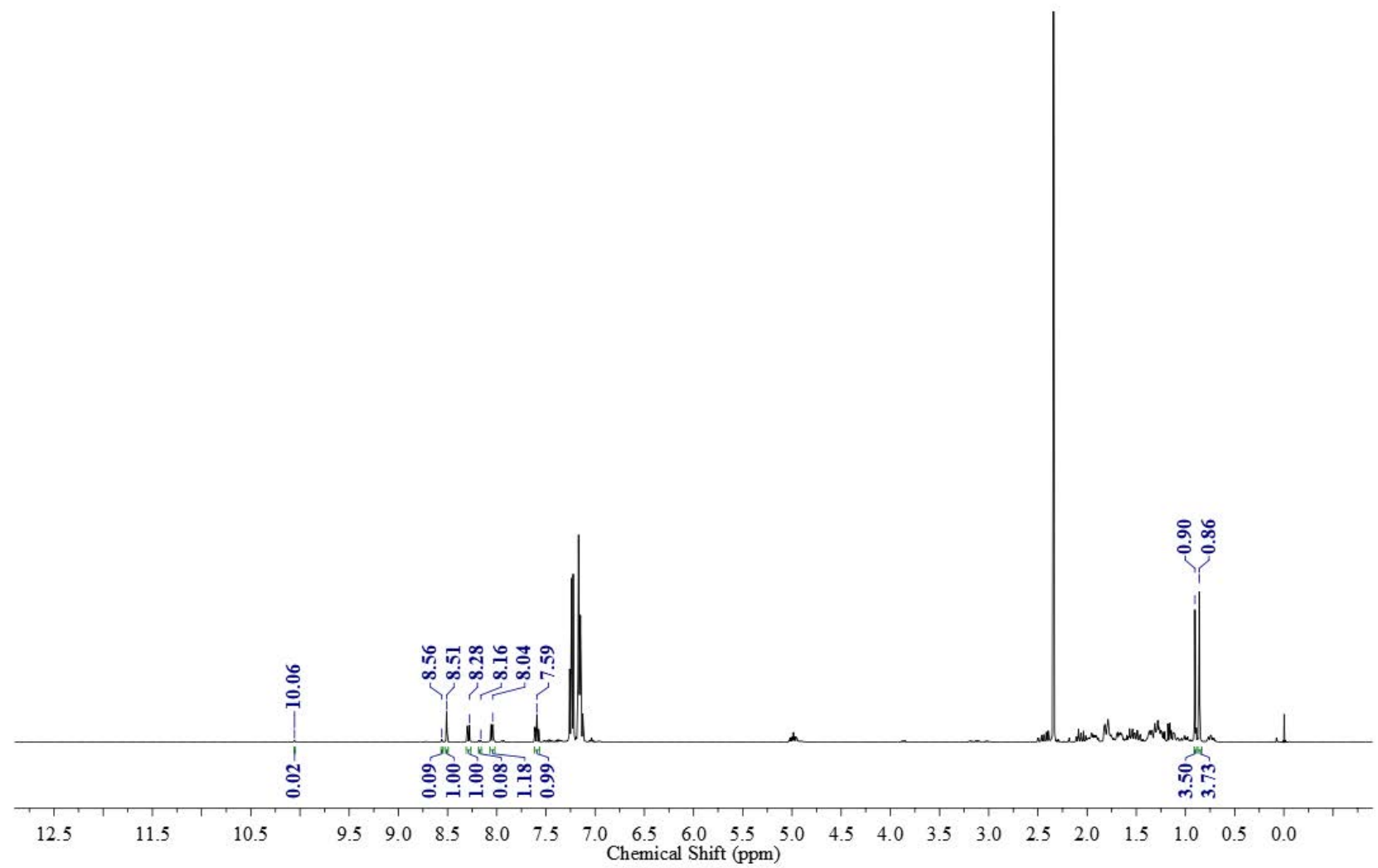

Entry 2: NMR for Crude reaction mixture

vDOS-678-B1

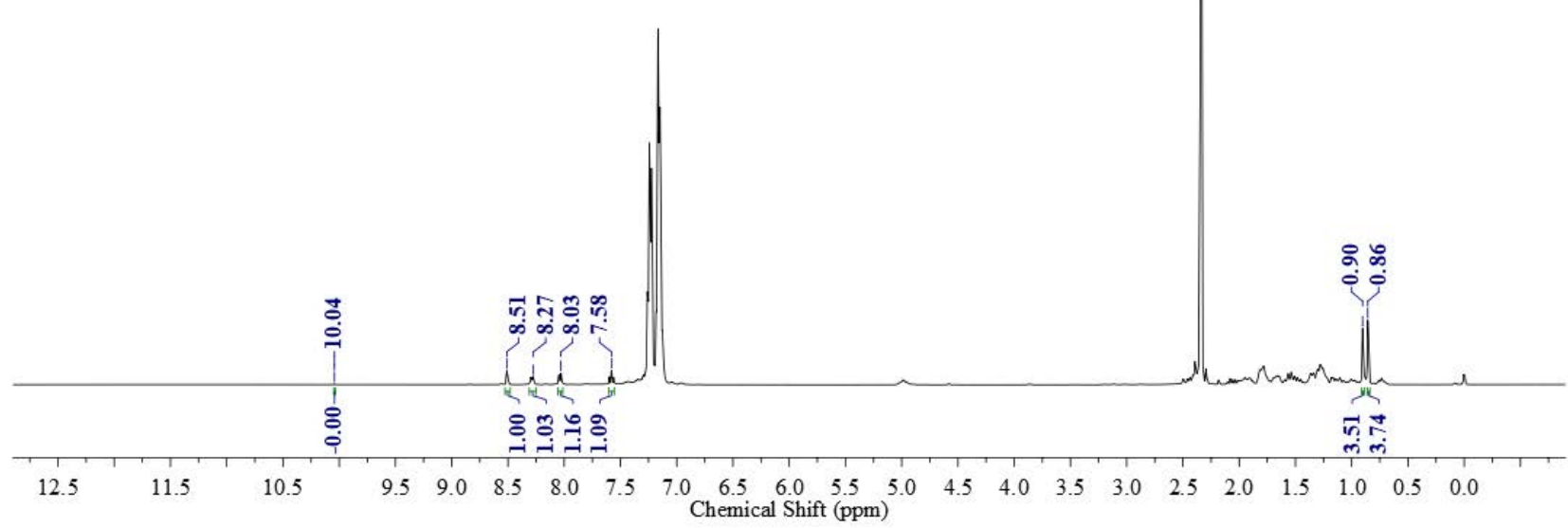


Entry 2': NMR for Crude reaction mixture, Duplicate vDOS-678-B2

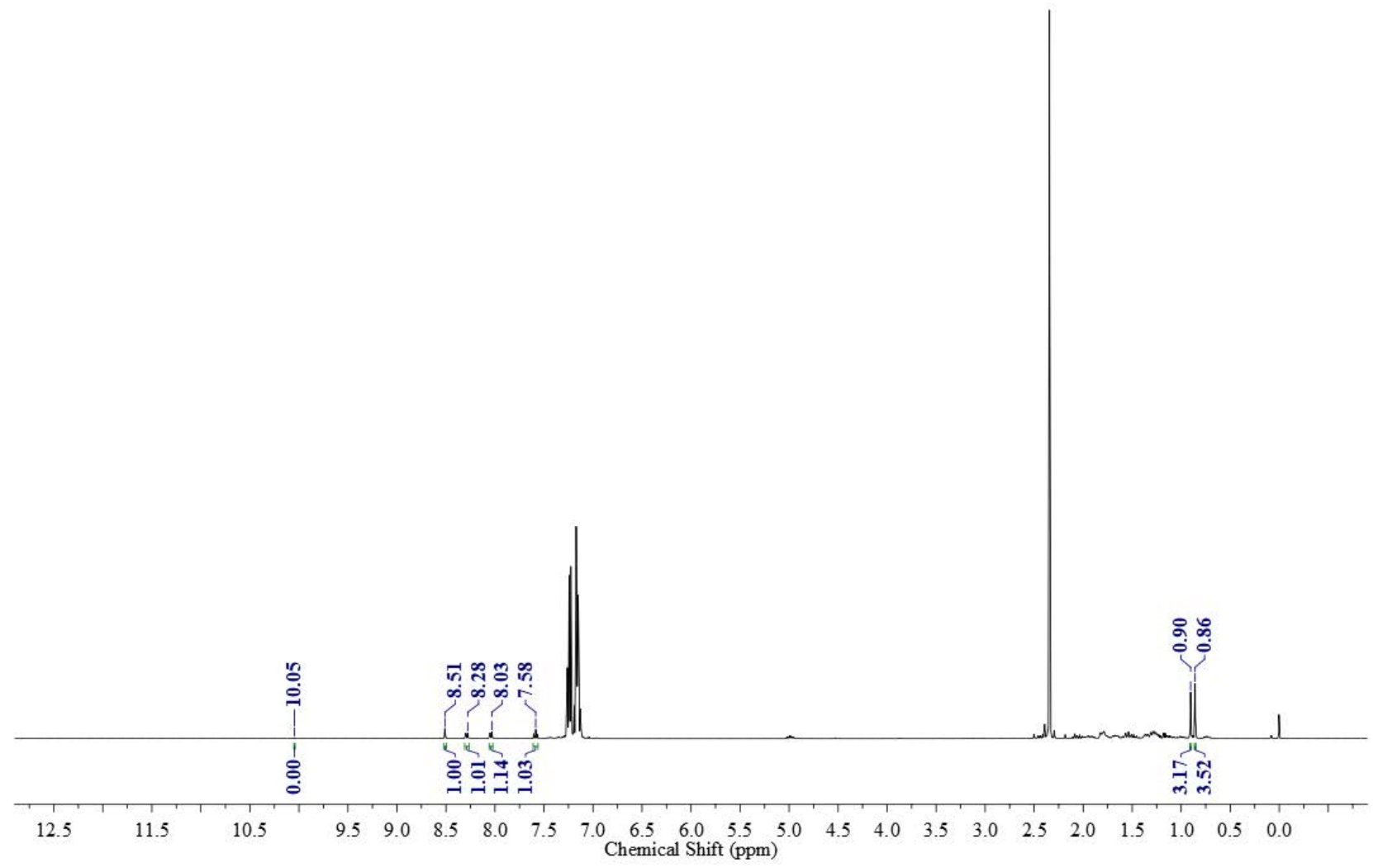

NMR after purification:

VDOS-678VP-1H NMR

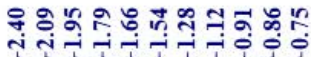
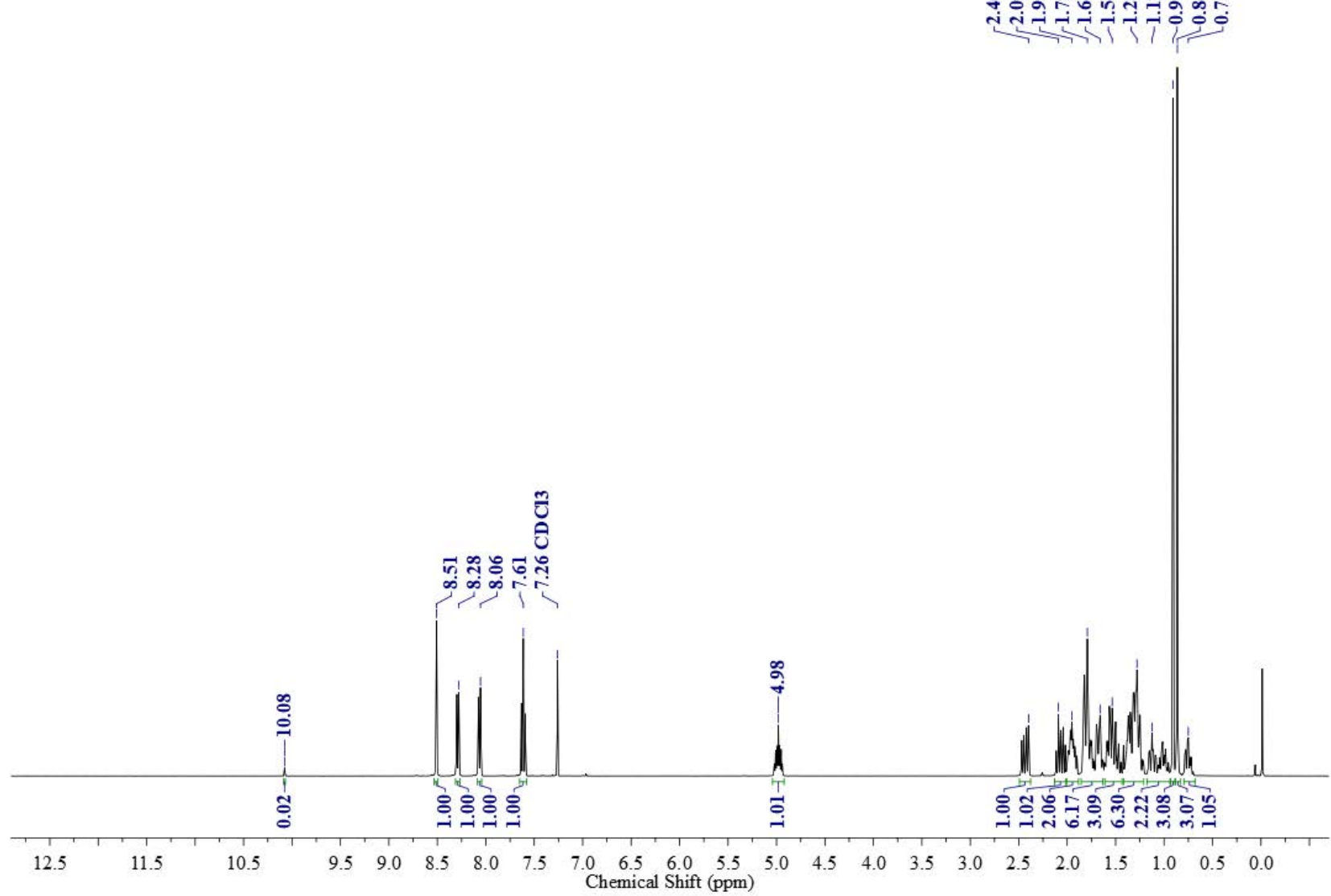


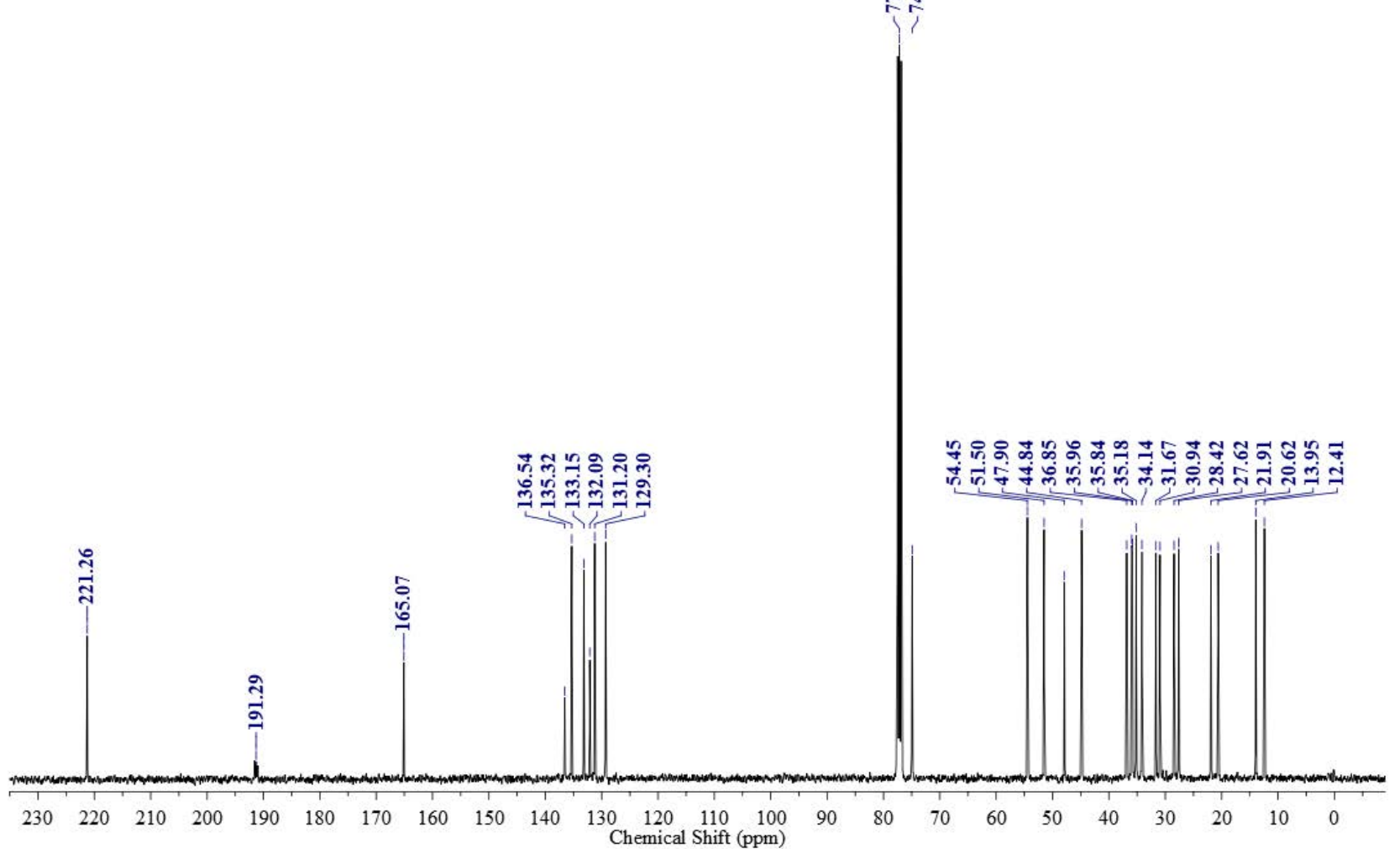


55. Pregnenolone Derivative-d

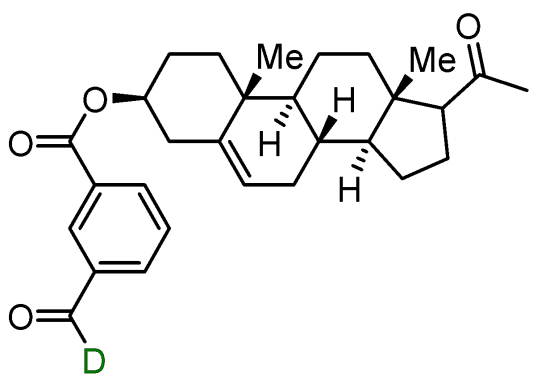

Entry 1: NMR for Crude reaction mixture VDOS-679-A1

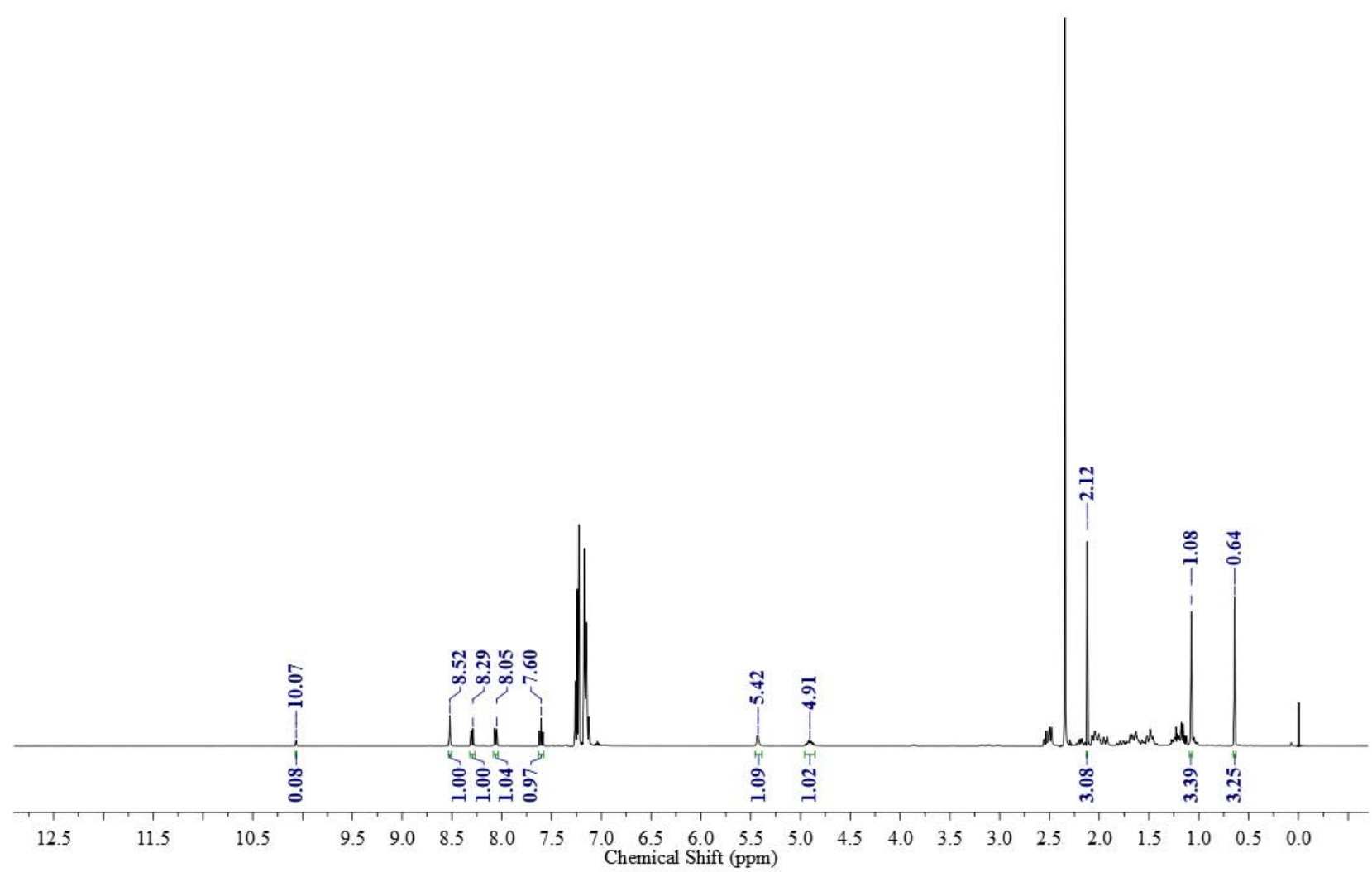


Entry 1': NMR for Crude reaction mixture, Duplicate

vDOS-679-A2

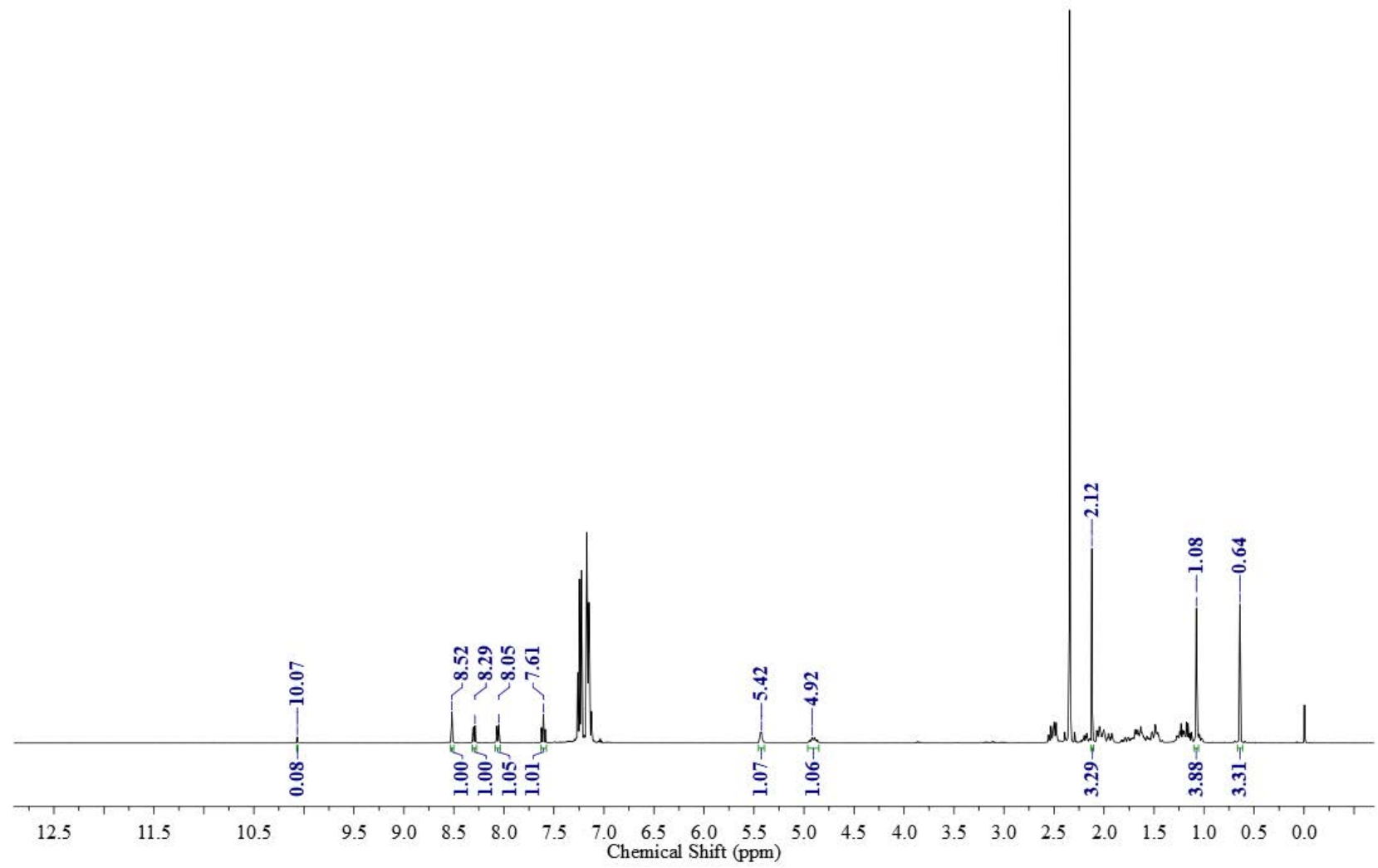

Entry 2: NMR for Crude reaction mixture

vDOS-679-B1

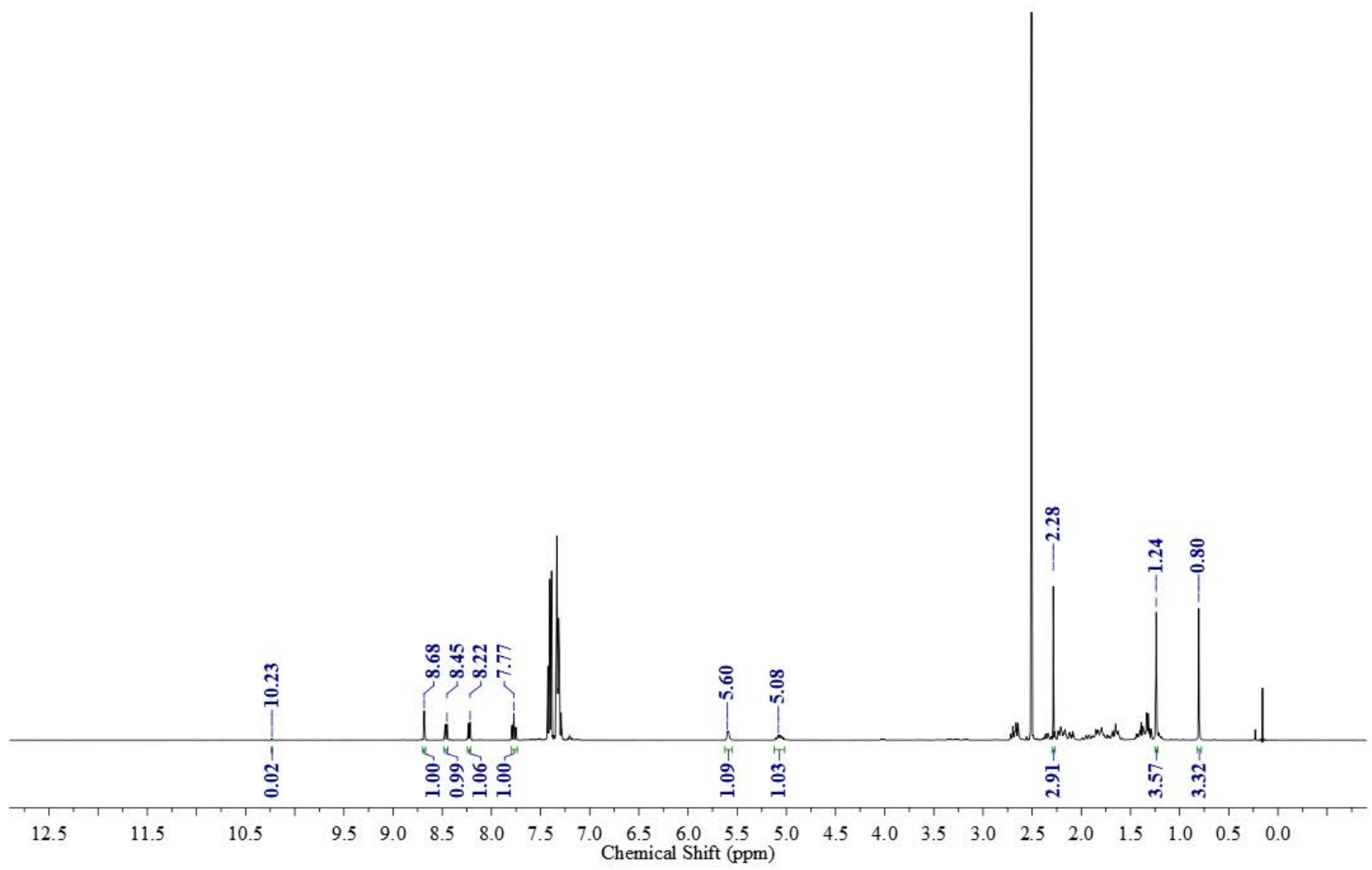


Entry 2': NMR for Crude reaction mixture, Duplicate

vDOS-679-B2

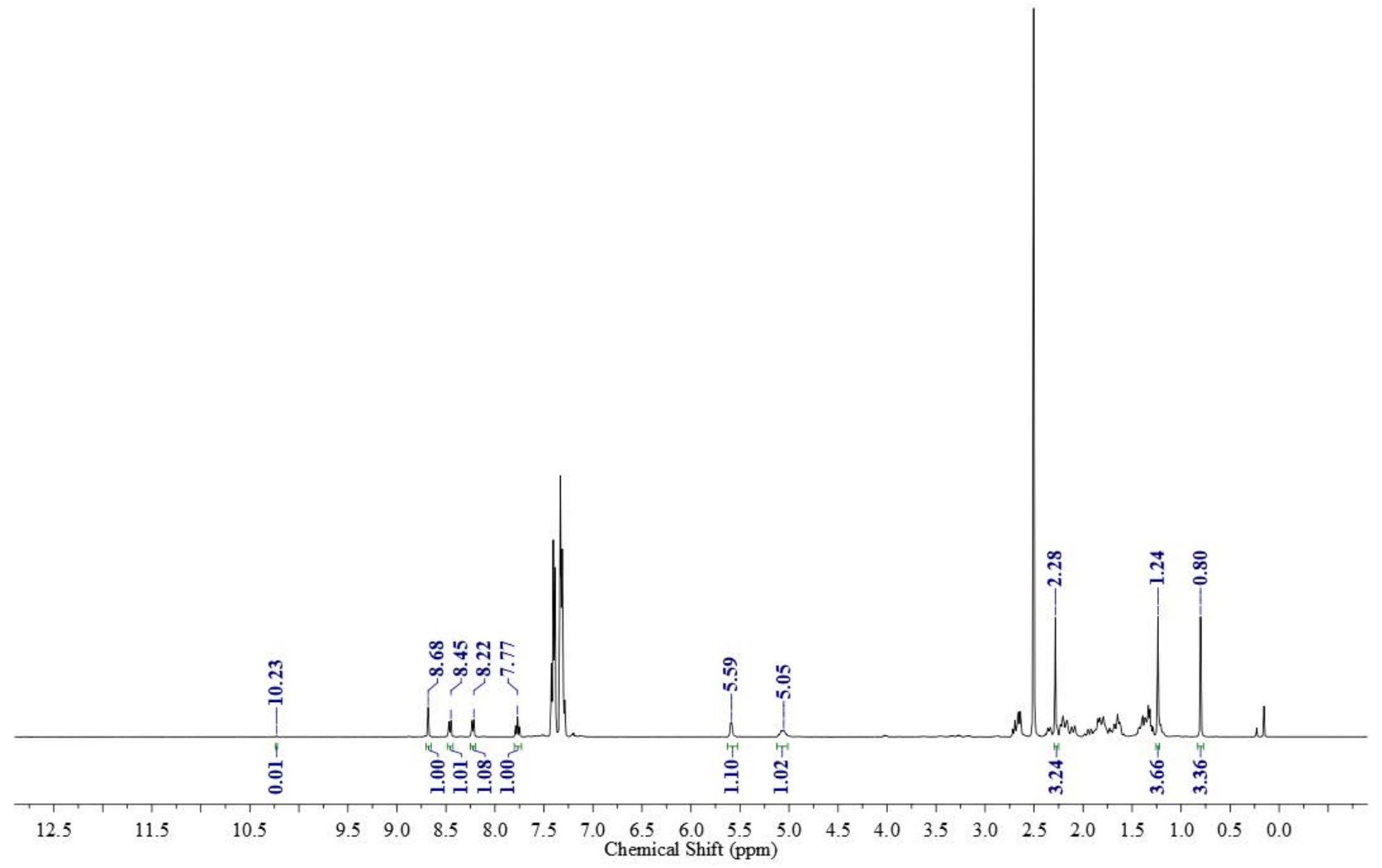

NMR after purification:

vDOS-679B-VP 1H NMR

\section{กู่ สิส}

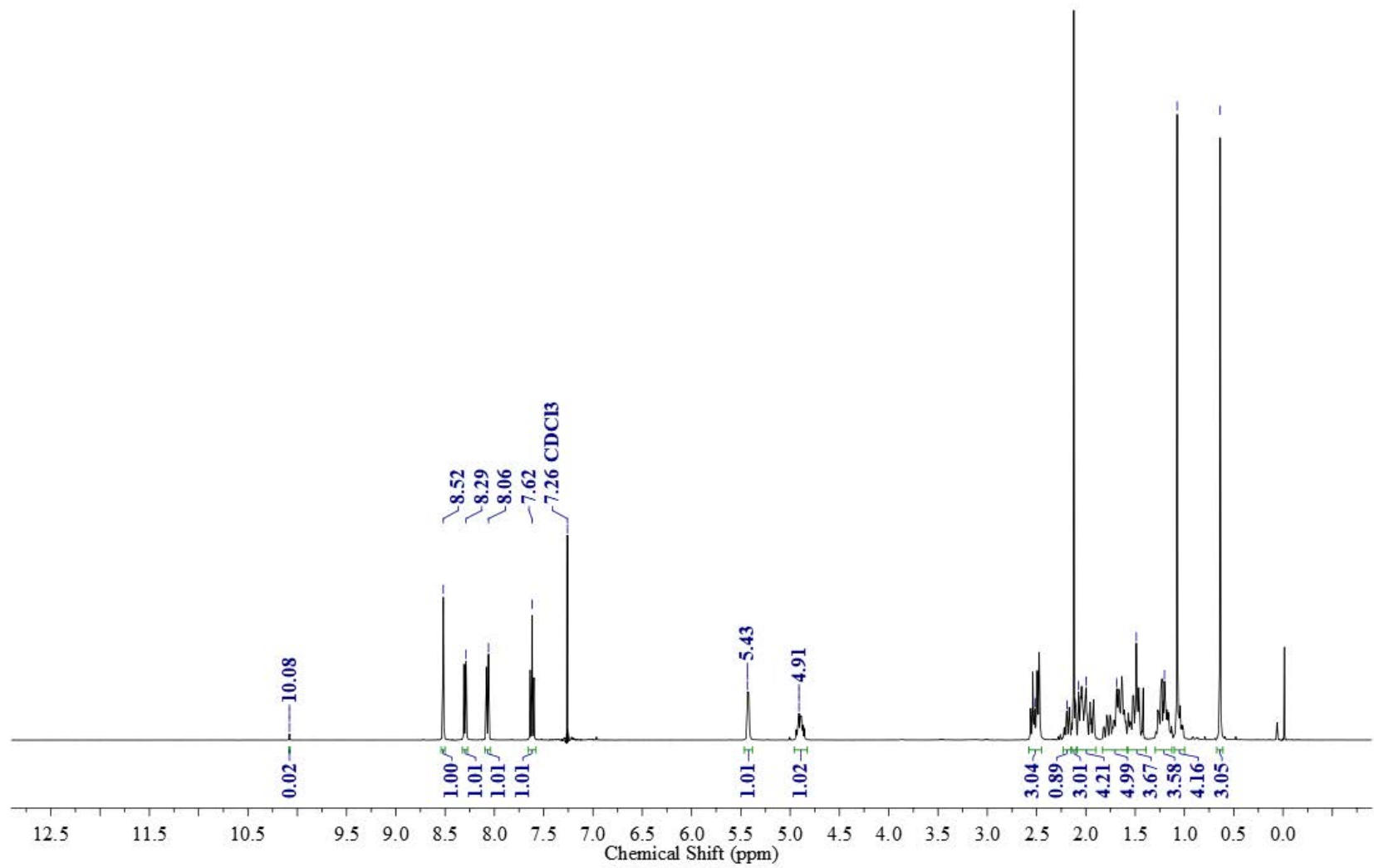




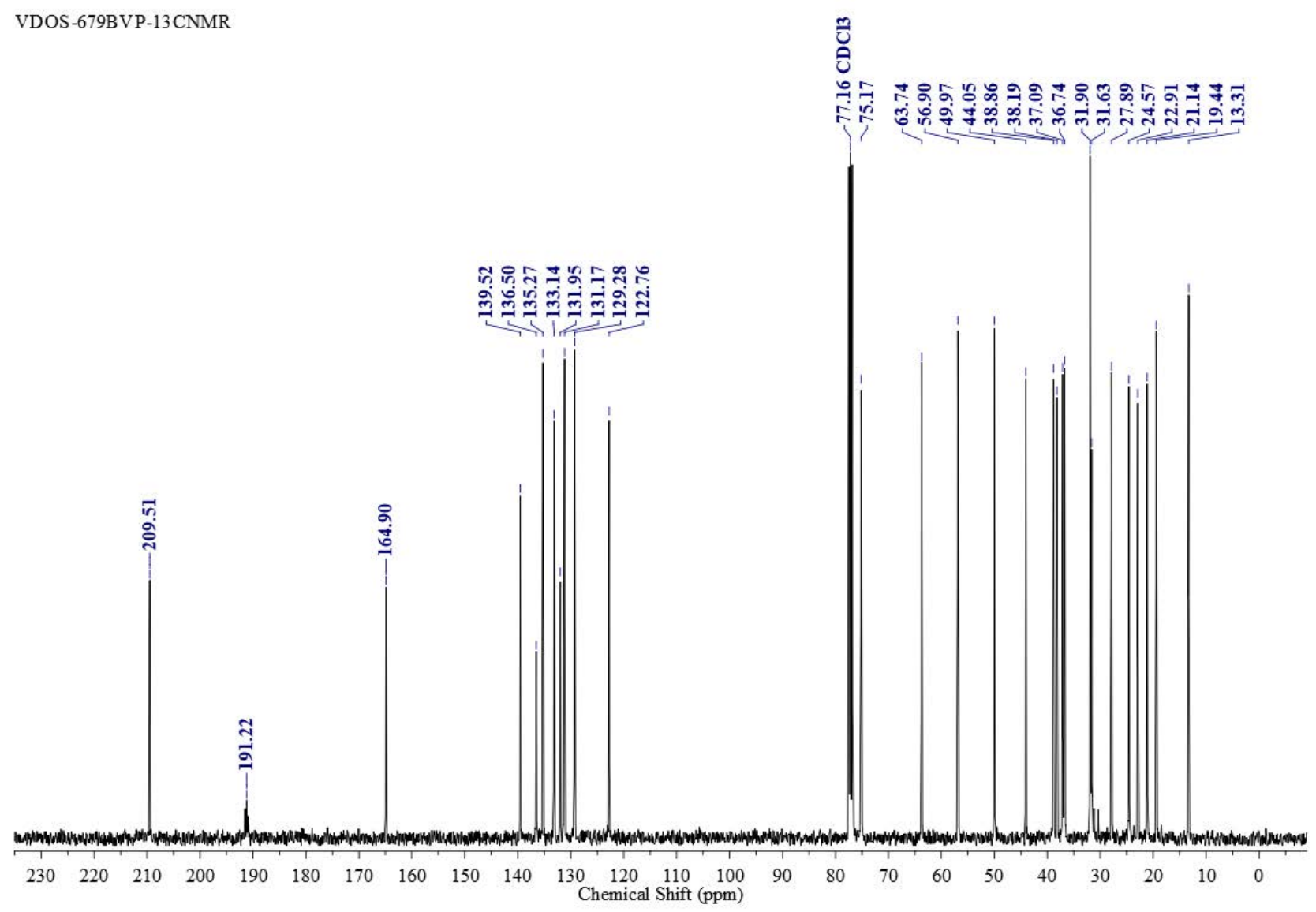

390 
56. Diacetone-glucose Derivative-d<smiles>[2H]C(=O)c1cccc(C(=O)O[C@H]2C3OC(C)(C)O[C@@H]3O[C@H]2C2COC(C)(C)O2)c1</smiles>

Entry 1: NMR for Crude reaction mixture vDOS-683-A1

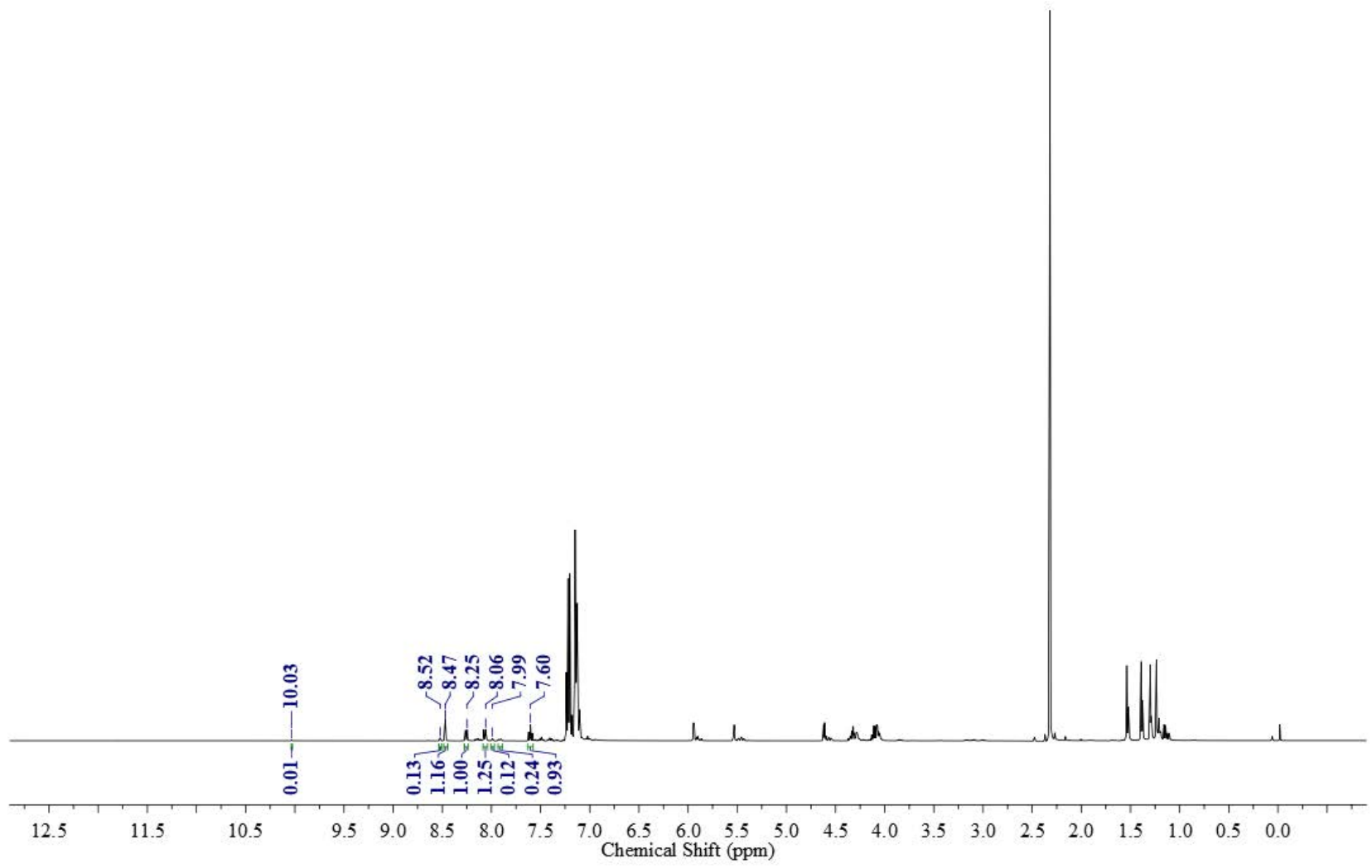


Entry 1': NMR for Crude reaction mixture, Duplicate VDOS-683-A2

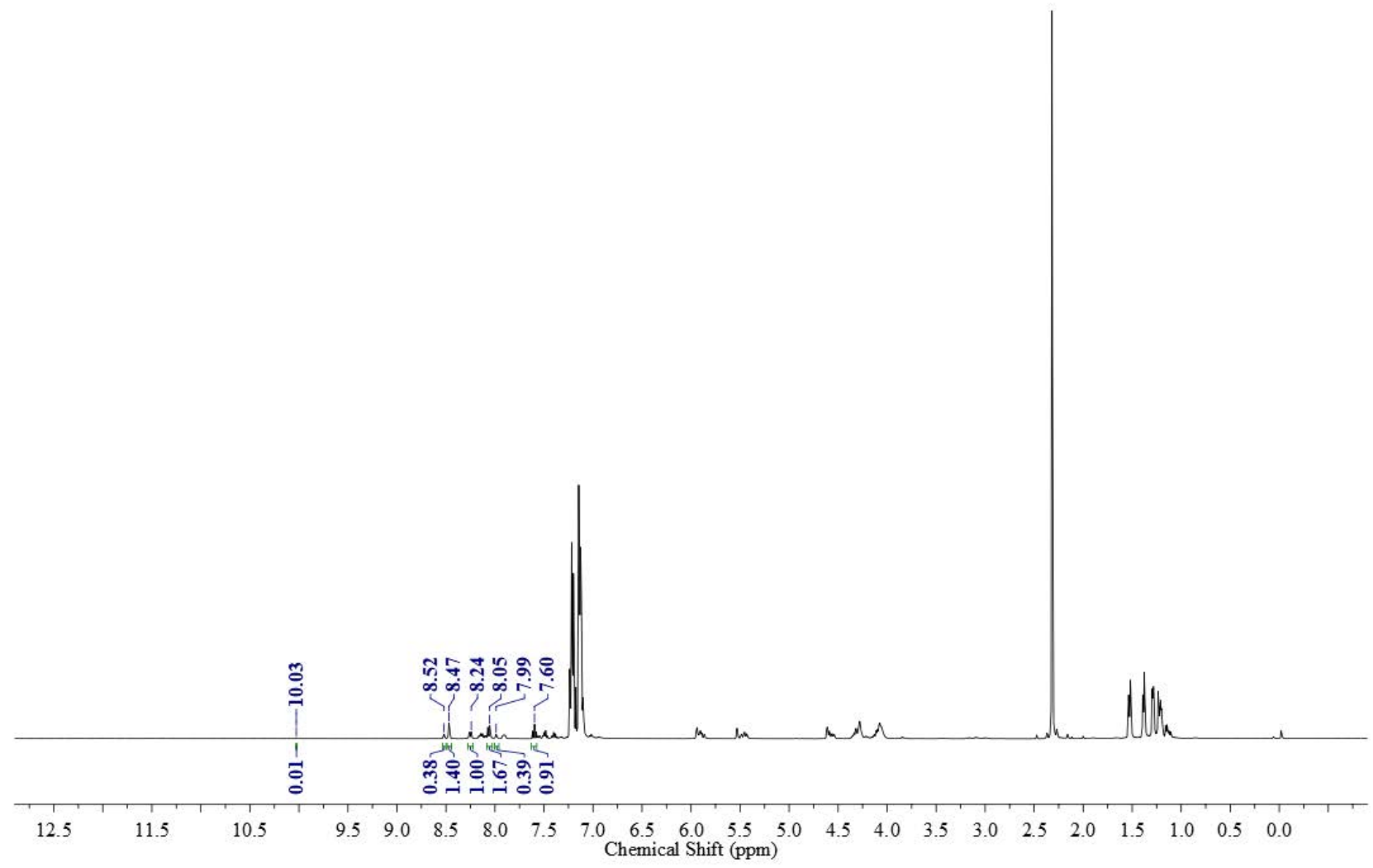

Entry 2: NMR for Crude reaction mixture vDOS-683-B1

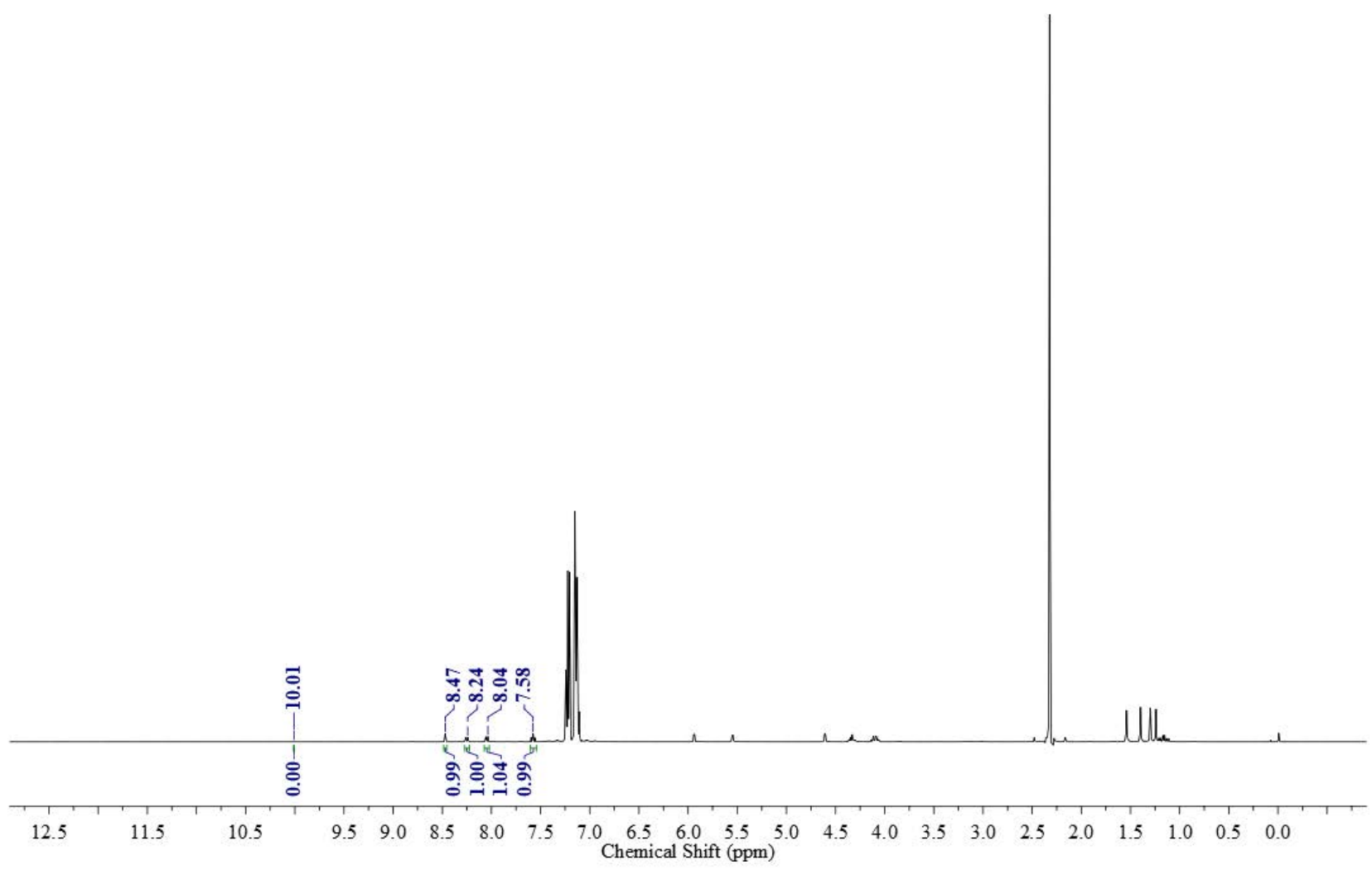


Entry 2': NMR for Crude reaction mixture, Duplicate

VDOS-683-B2

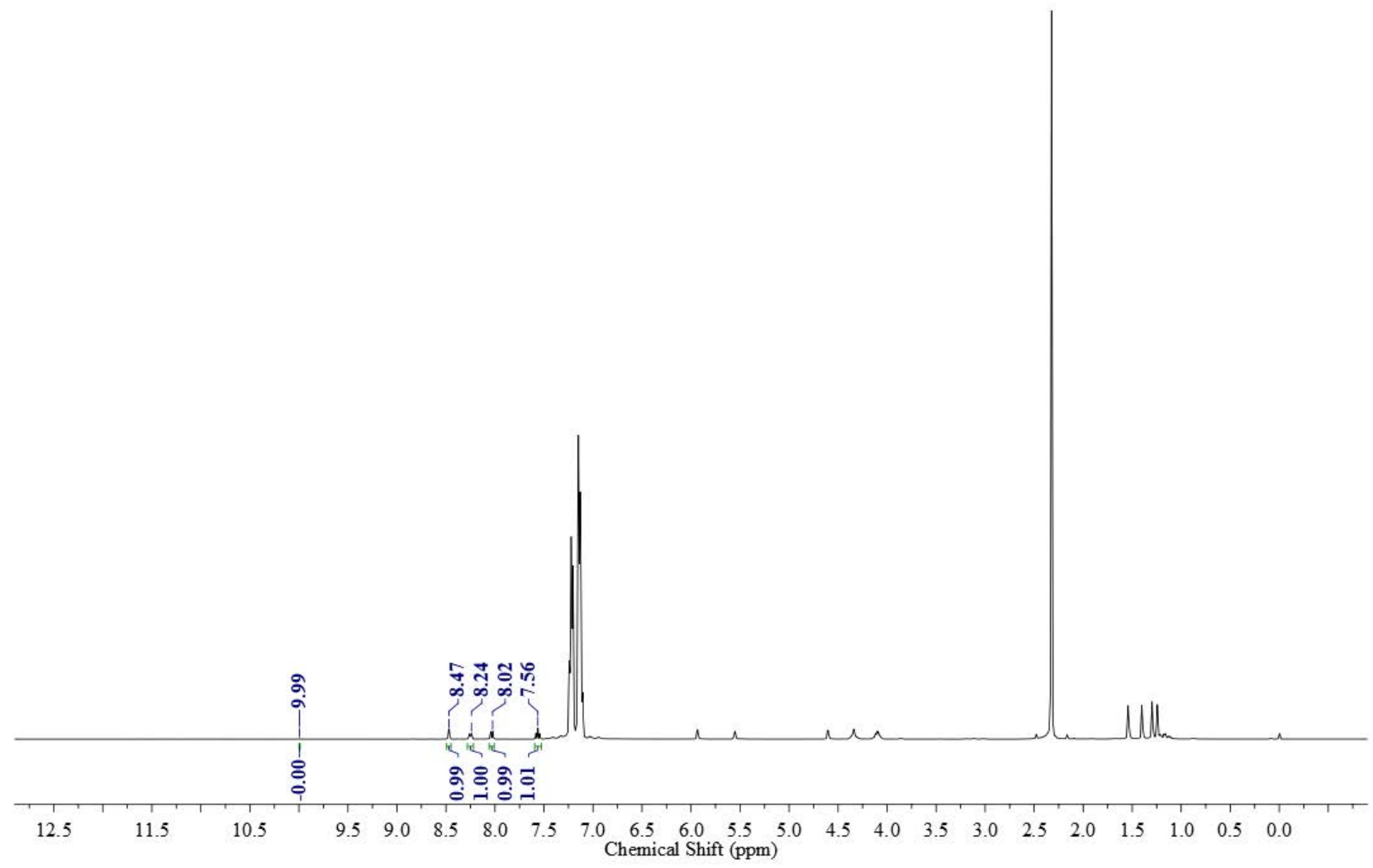

NMR after purification:

VDOS-683BVP-1H NMR in CD3CN-2

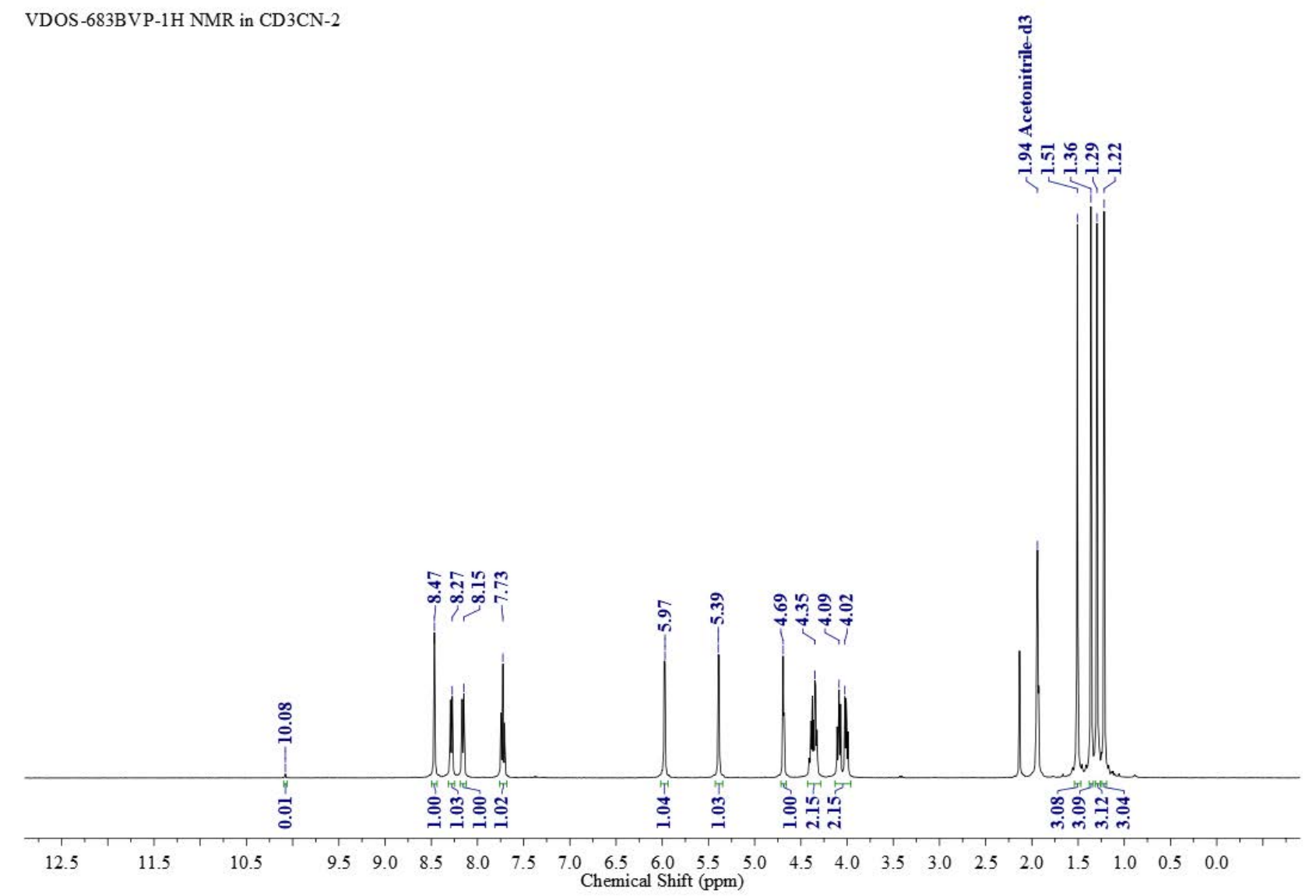




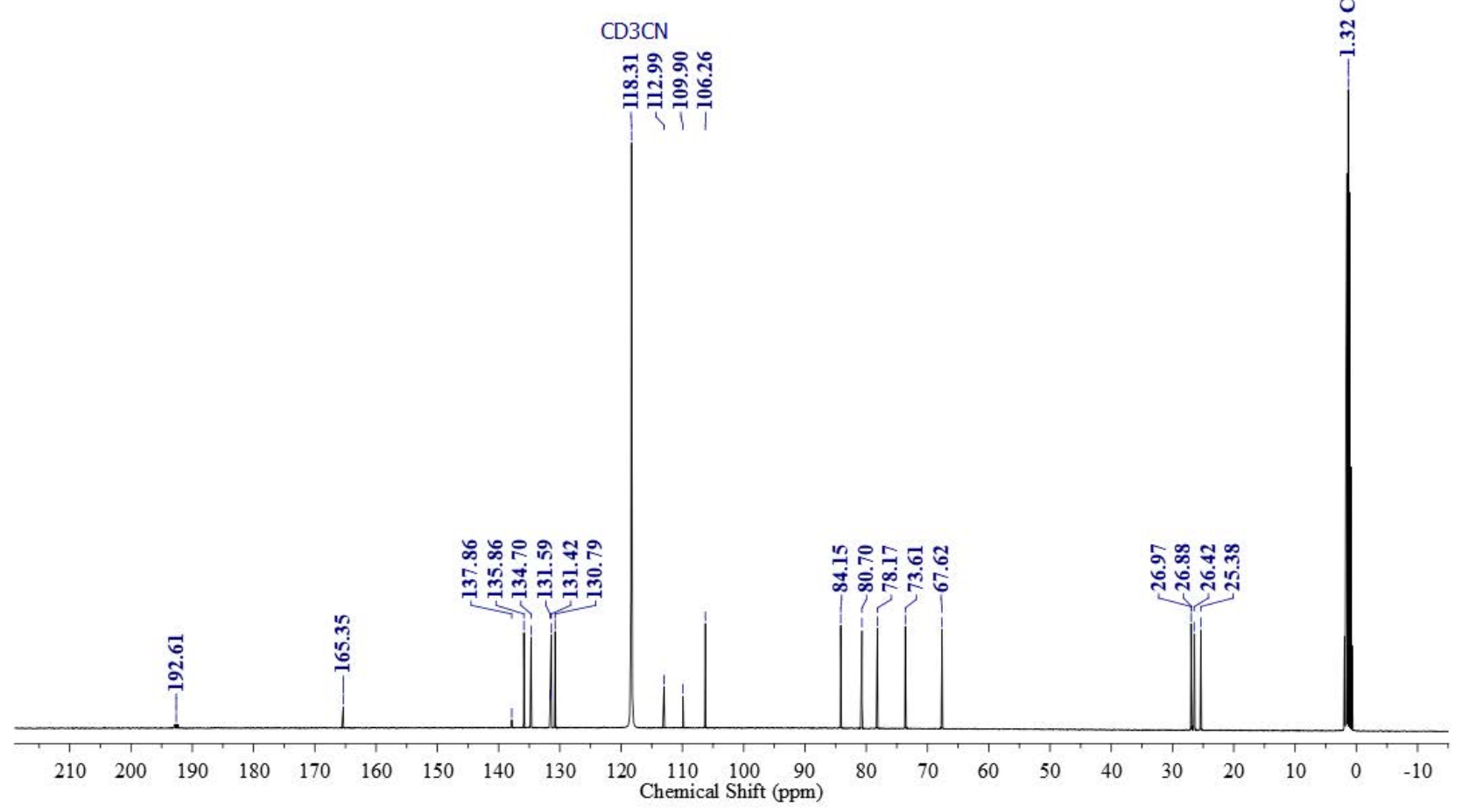


Initial Reaction Rates:

Initial Reaction Rate for 2-Fluorobenzaldehyde:

Entry 1: 1 minute, Set A

SCG-317-E8-1min-D 1-50sec.1. fid

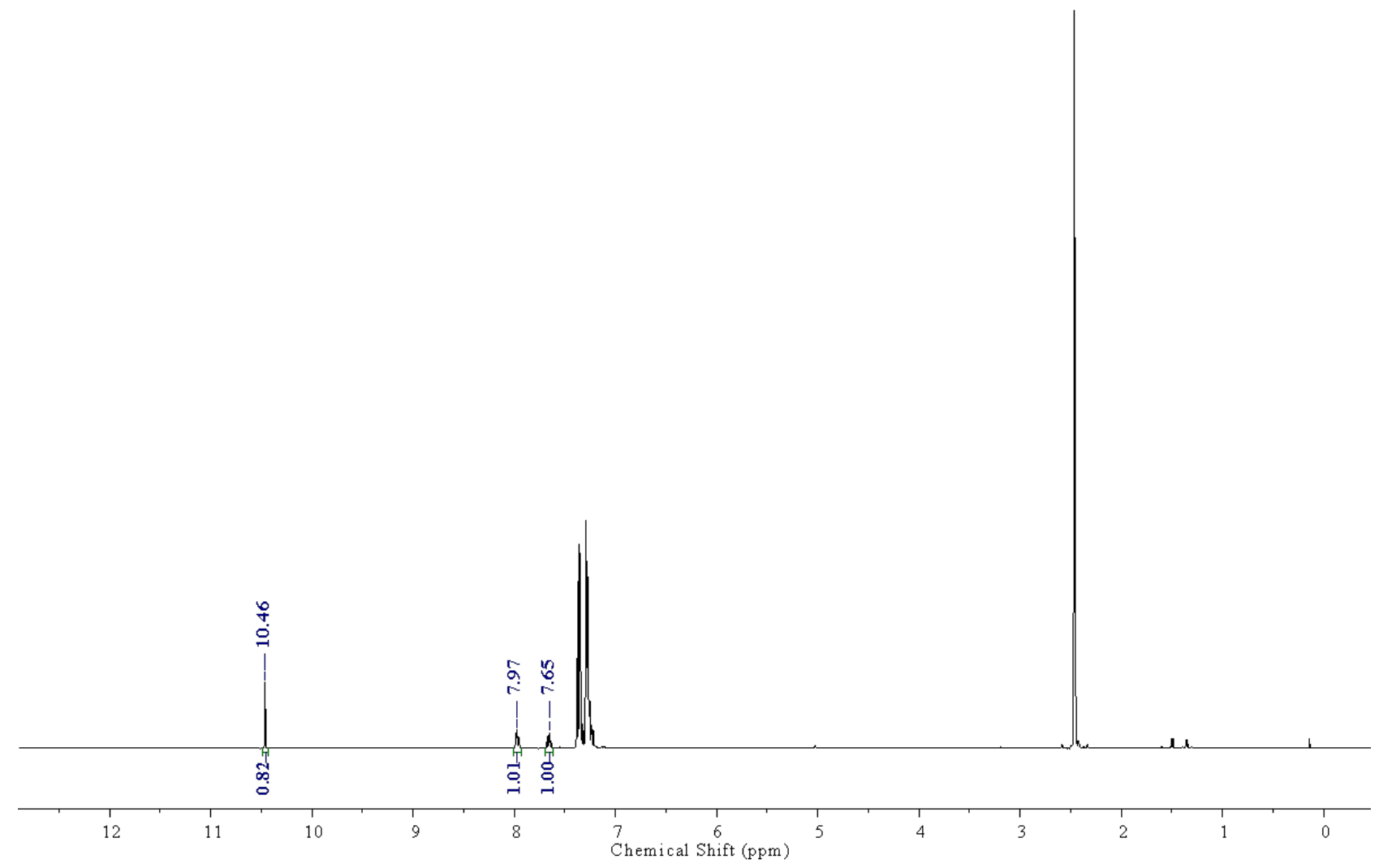


Entry 2: 2 minute, Set A

SCG-317-E9-2min-D 1-50sec.1. fid

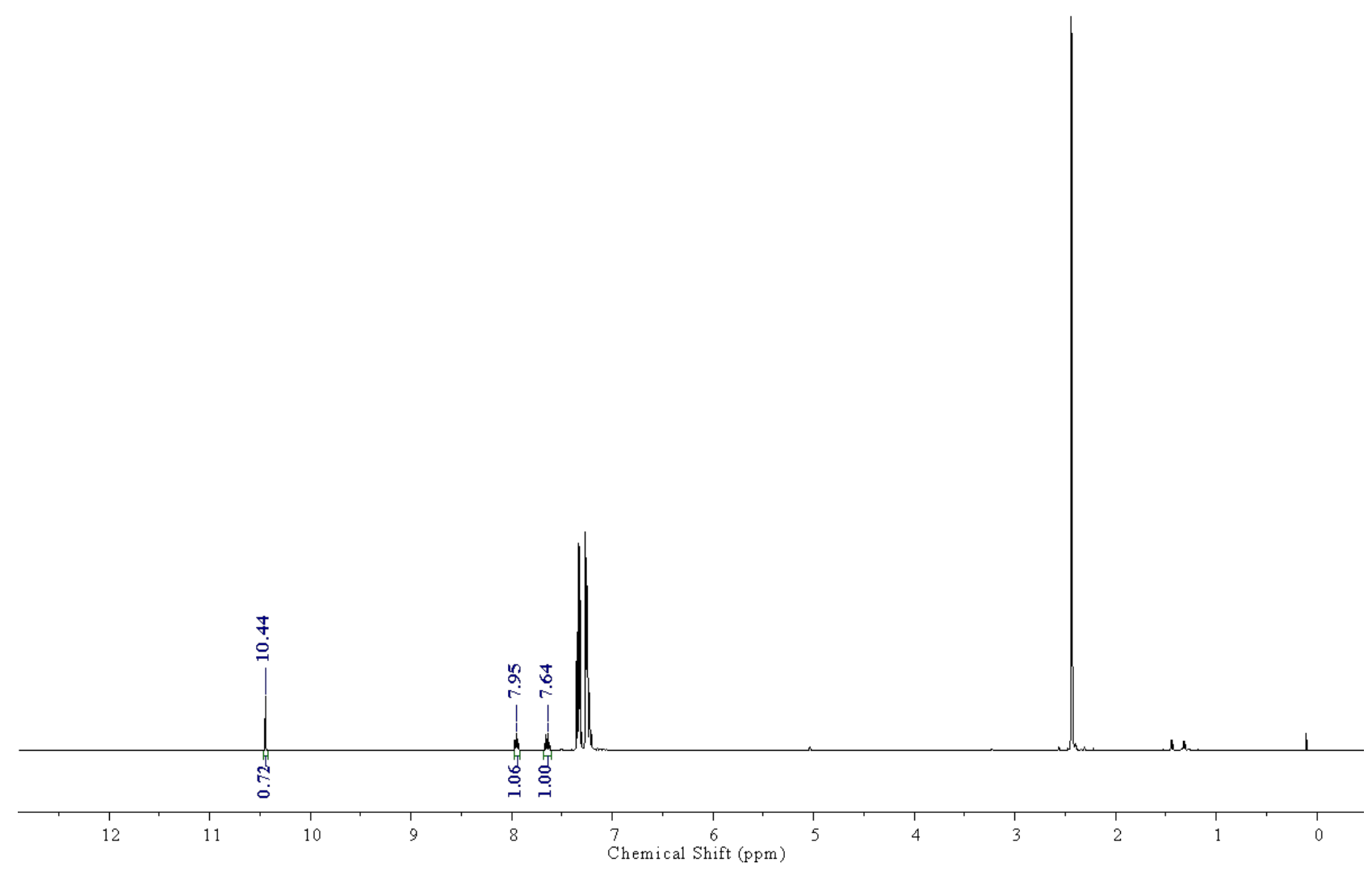

Entry 3: 3 minute, Set A

SCG-317-E 10-3min-D 1-50sec.1. fid

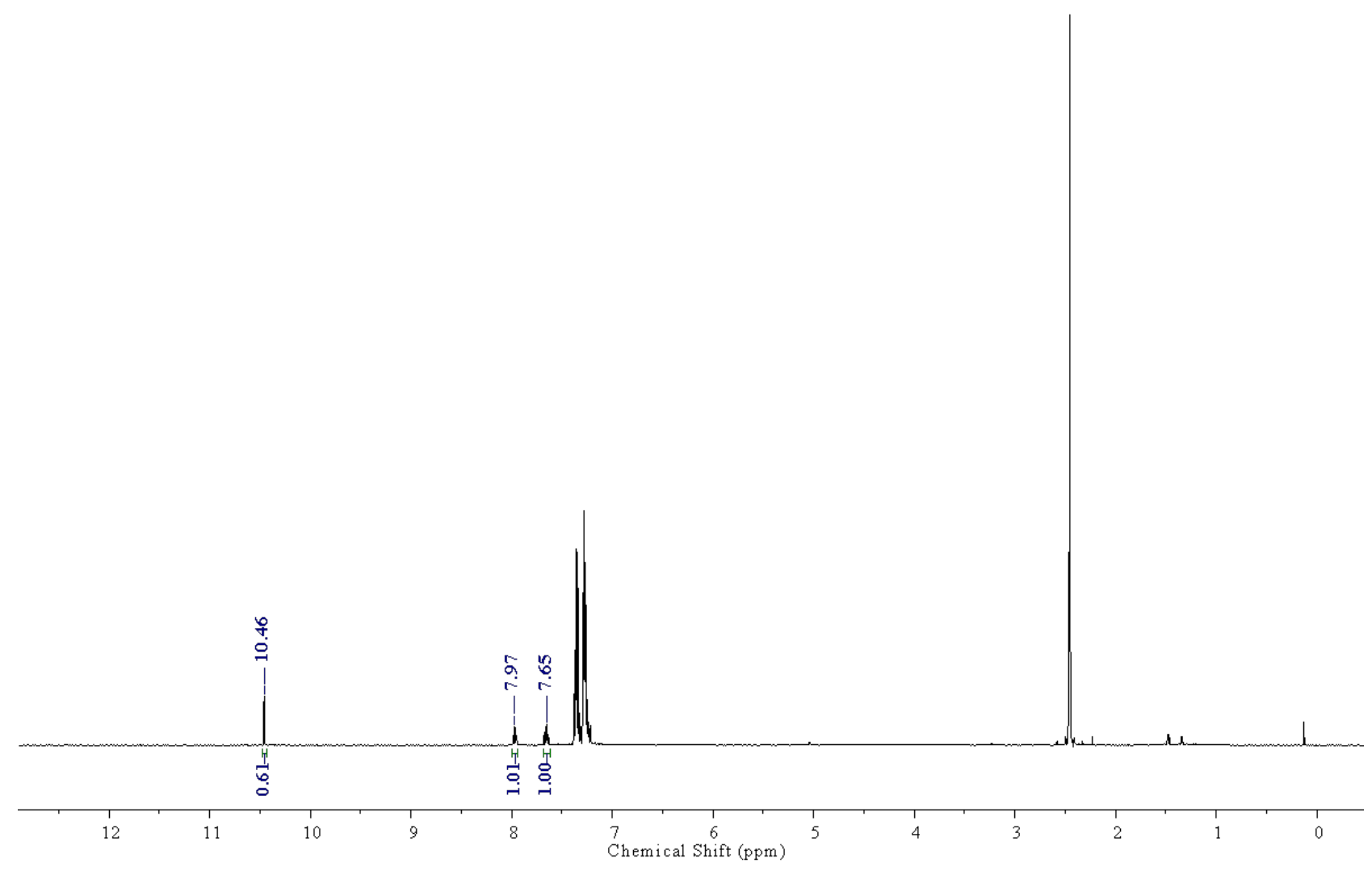


Entry 4: 4 minute, Set A

SCG-317-E11-4m in-D 1-50sec.1. fid

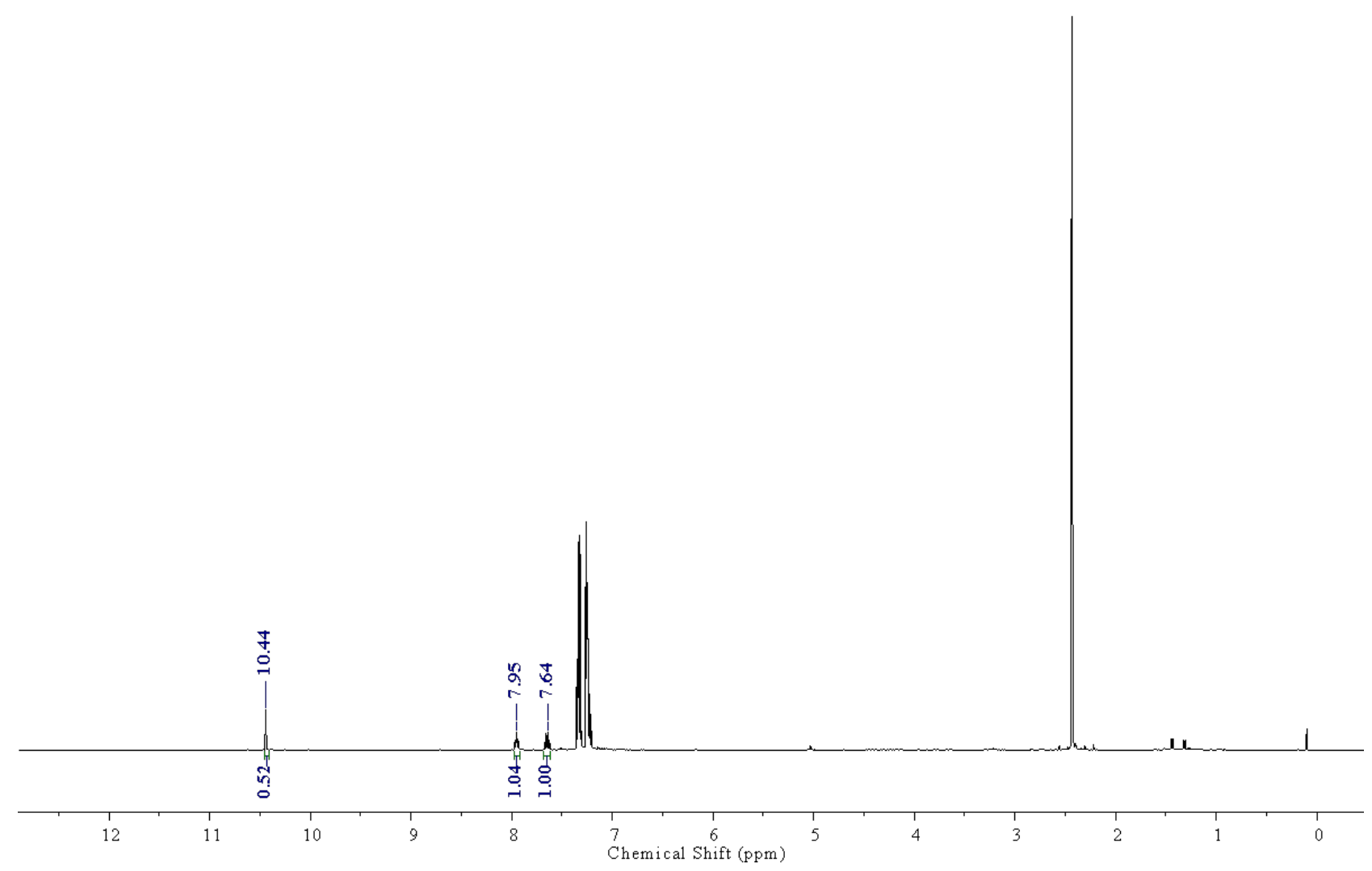

Entry 5: 5 minute, Set A

SCG-317-E 1-5min-D 1-50sec.1. fid

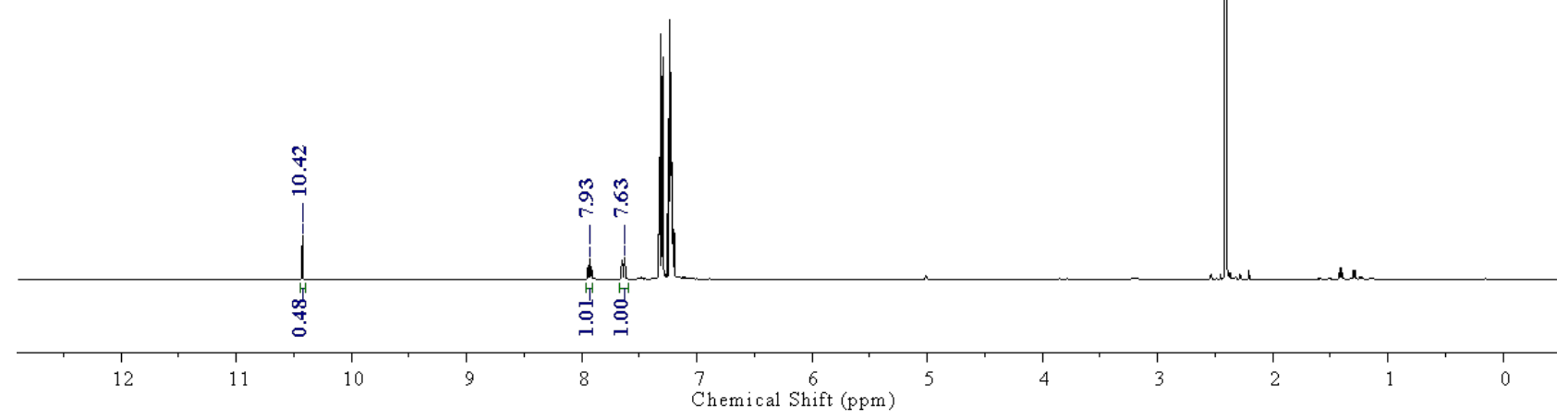


Initial Reaction Rate for 2-Chlorobenzaldehyde:

Entry 1: 5 minute, Set A

SCG-317-A6-5min-D 1-50sec. 1.fid

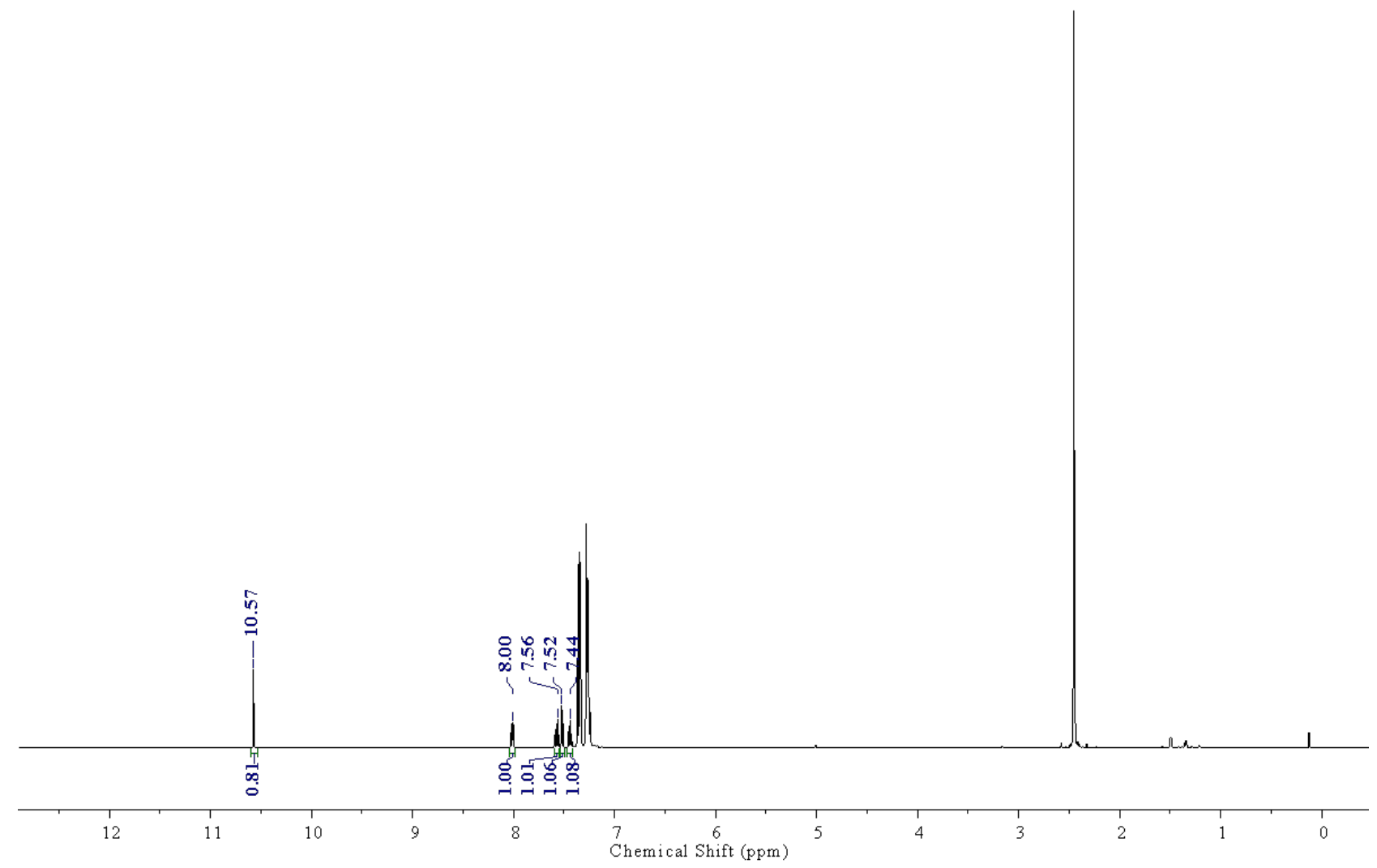

Entry 2: 10 minute, Set A

SCG-317-A1-10min-D 1-50sec. 1. fid

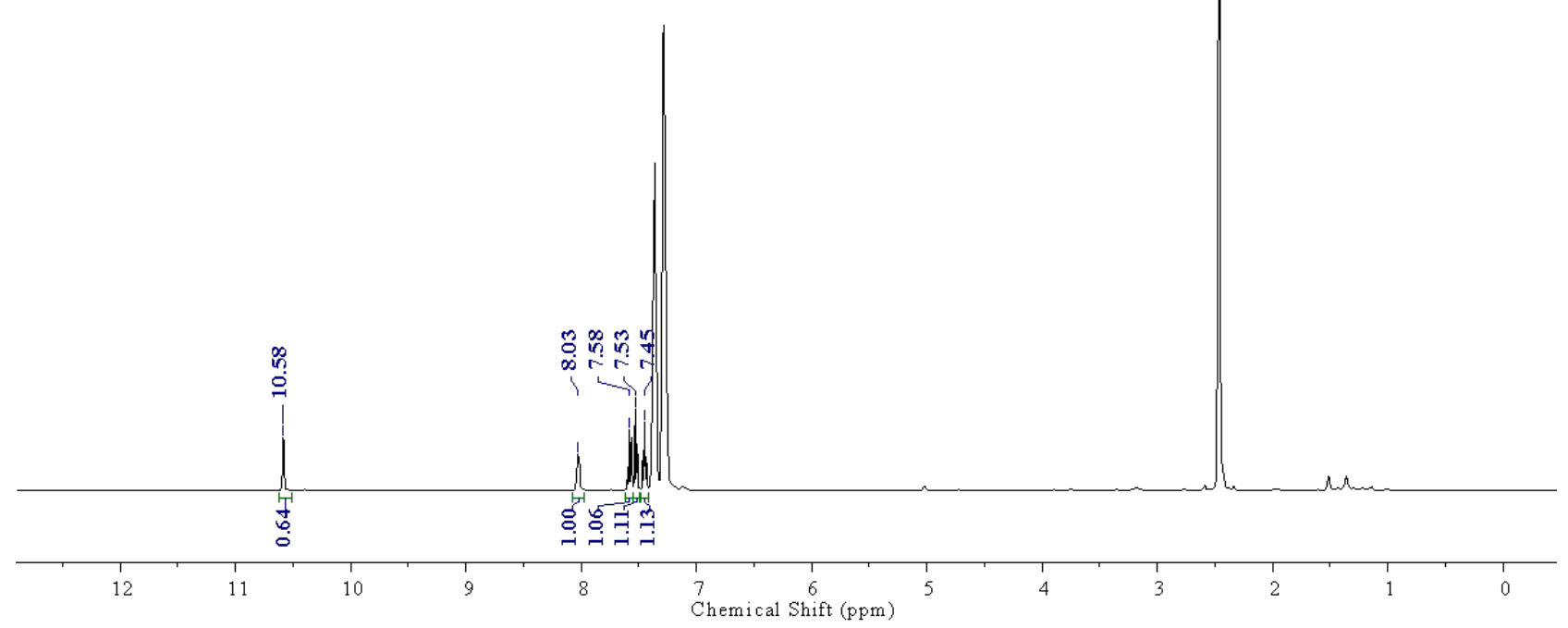


Entry 3: 15 minute, Set A

SCG-317-A 7-R-15min-D 1-50sec. 1.fid

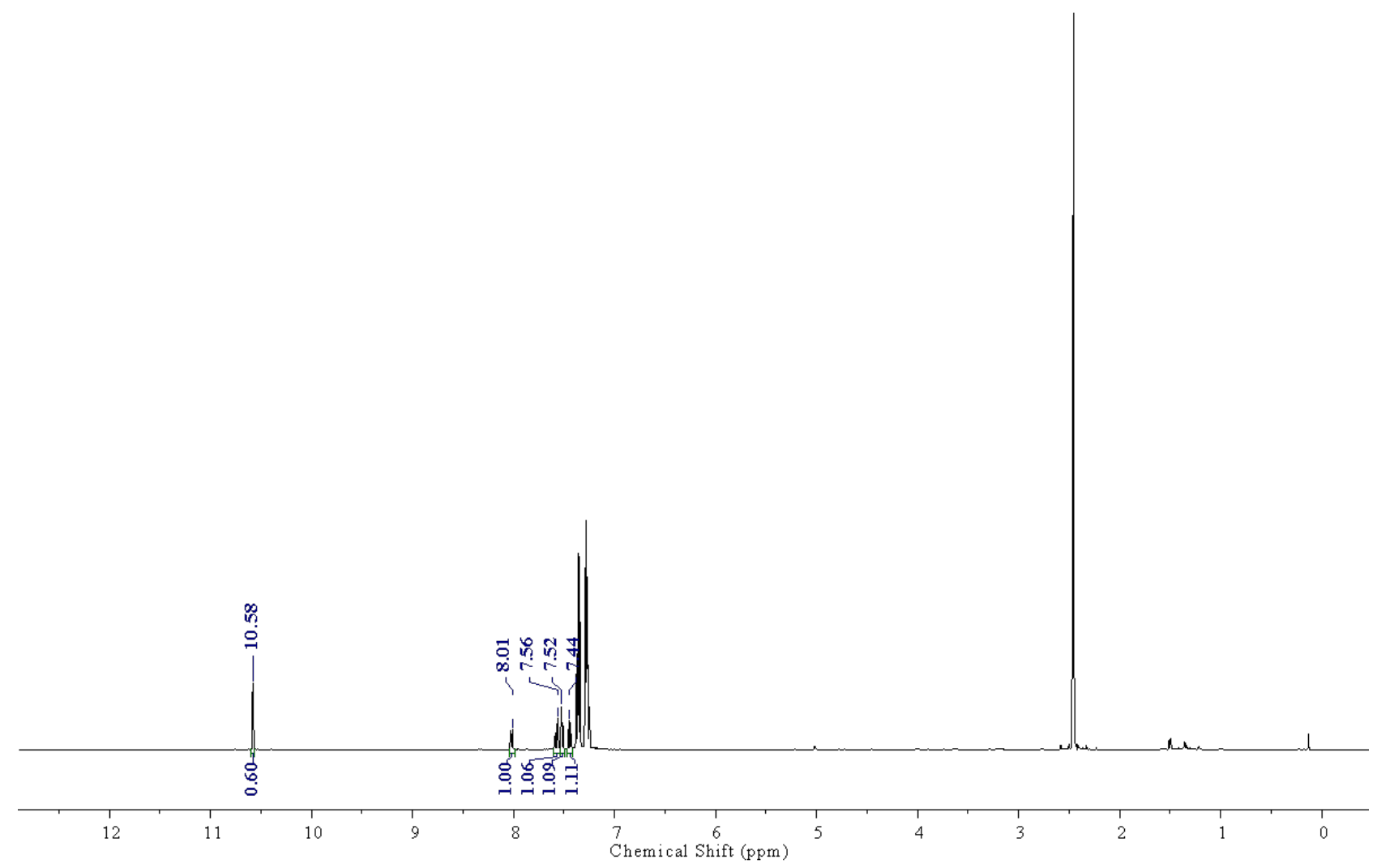

Entry 4: 20 minute, Set A

SCG-317-A2-20min-D 1-50sec. 1 fid

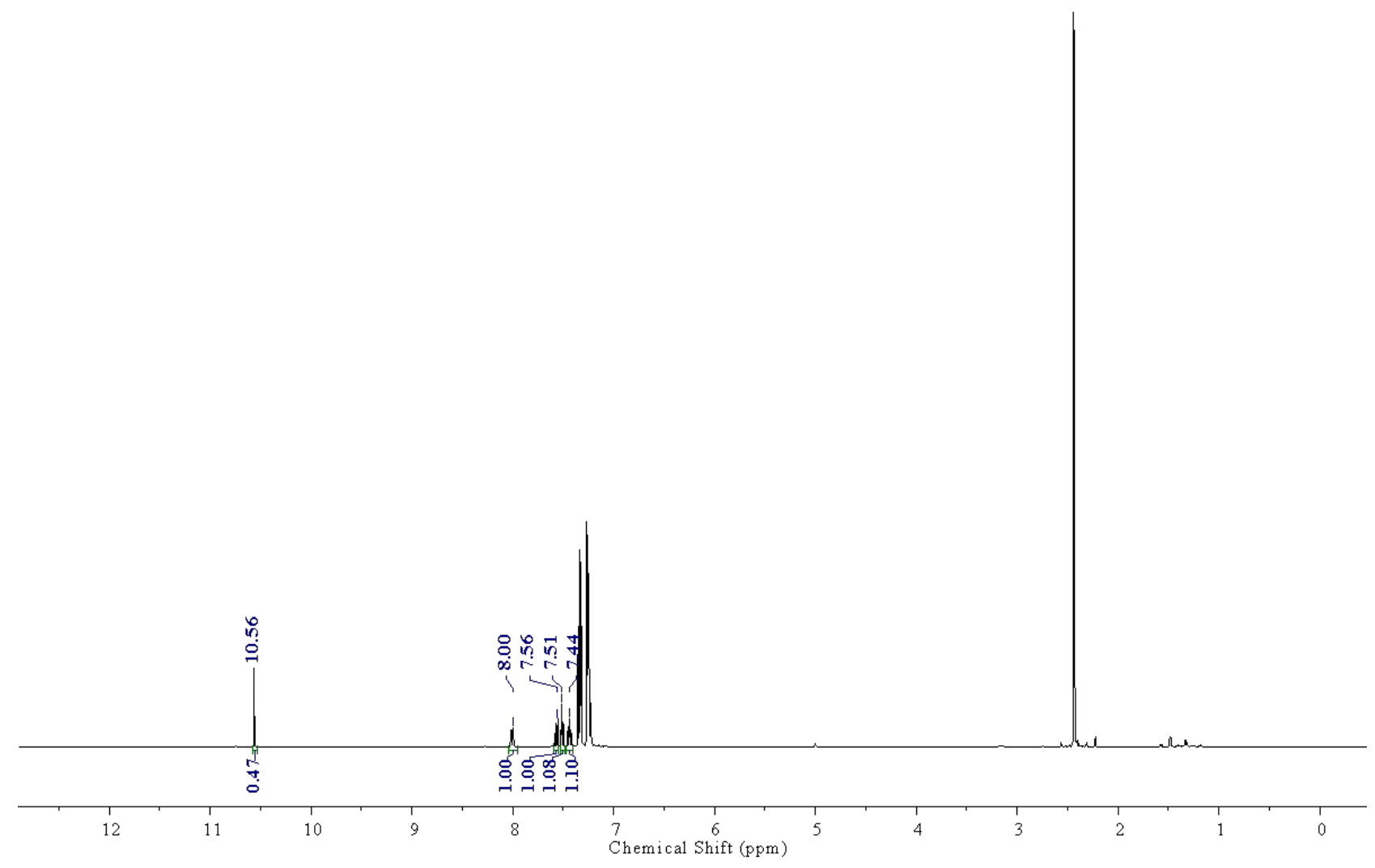


Entry 5: 25 minute, Set A

SCG-317-A8-25min-D 1-50sec. 1. fid

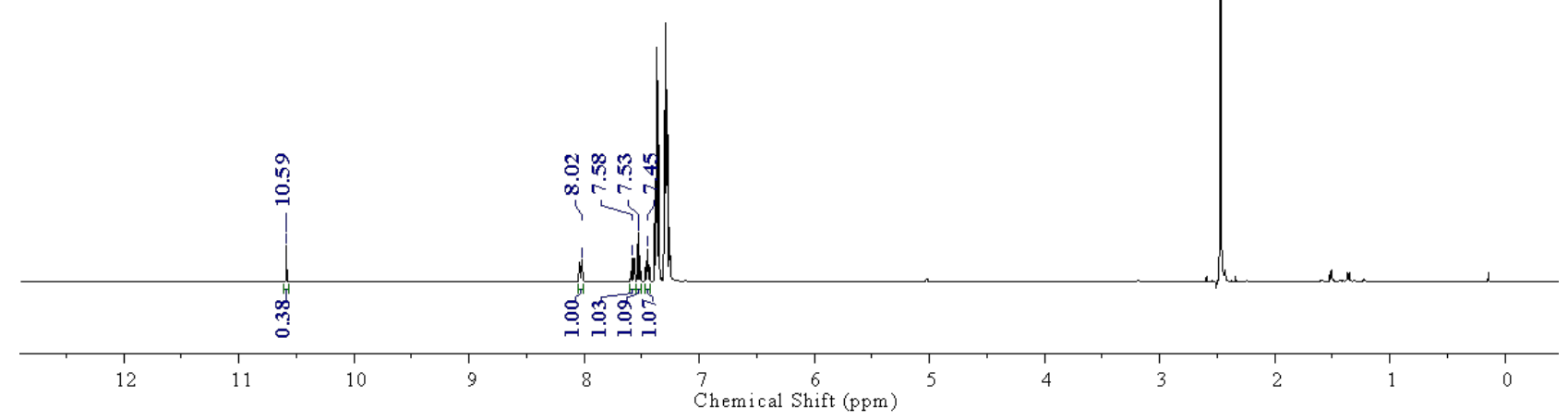


Initial Reaction Rate for 2-Bromobenzaldehyde:

Entry 1: 5 minute, Set A

SCG-317-C6-5min-D 1-50sec. 1.fid

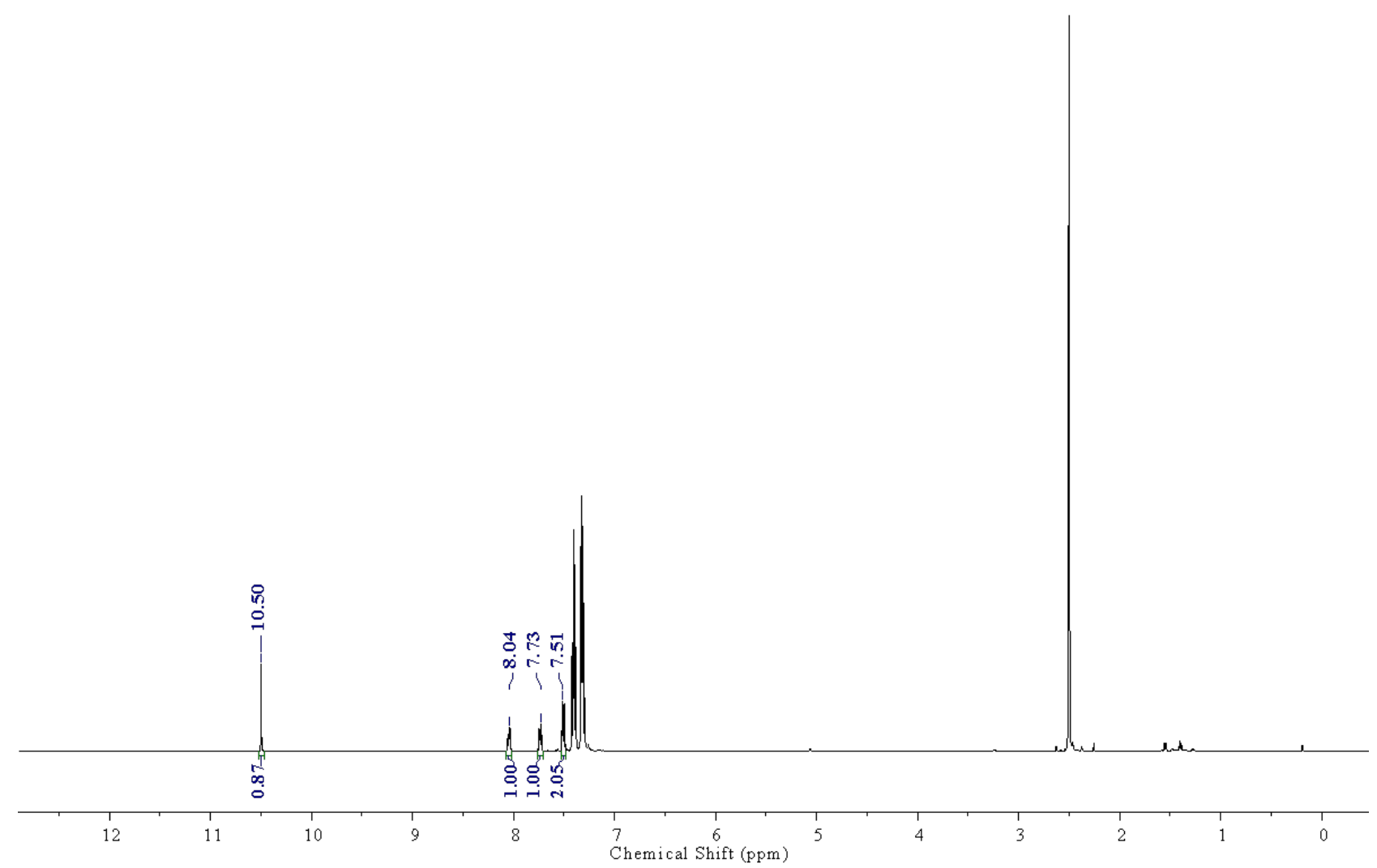

Entry 2: 10 minute, Set A

SCG-317-C1-10min-D 1-50sec. 1. fid

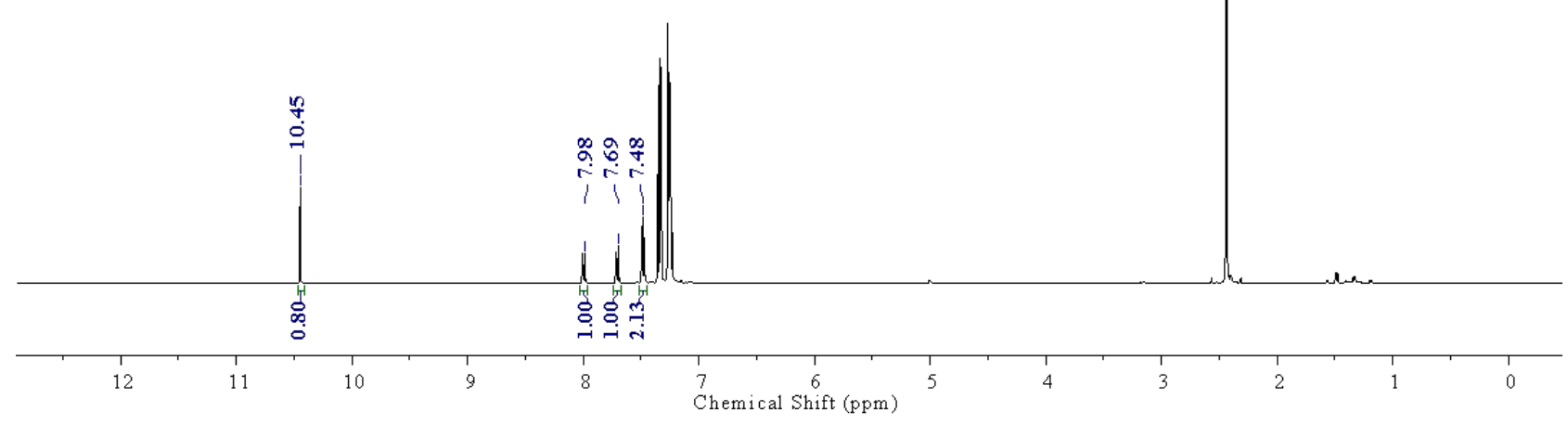


Entry 3: 15 minute, Set A

SCG-317-C7-15min-D 1-50sec. 1.fid

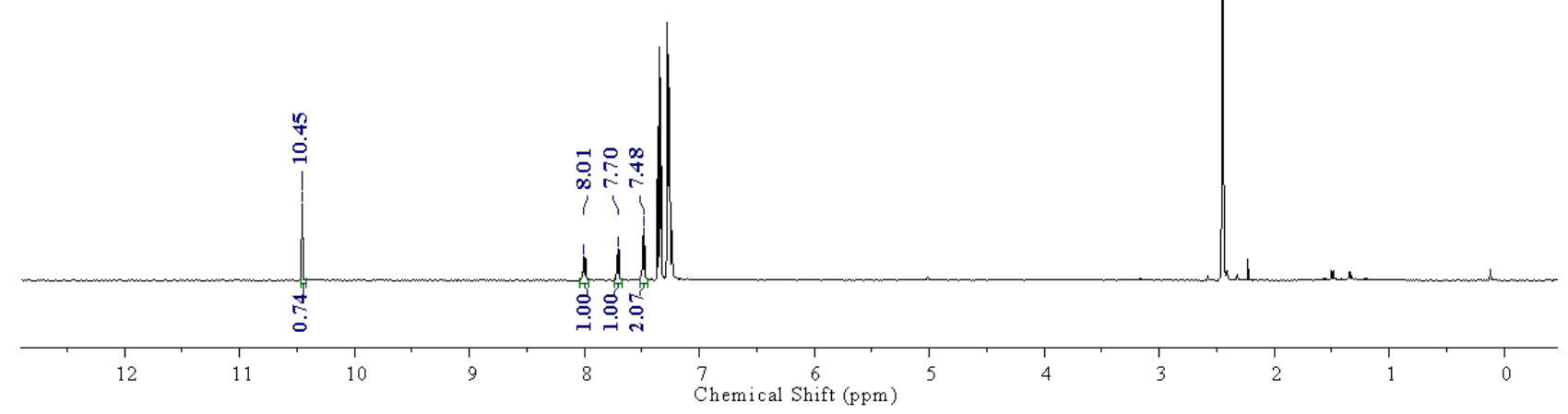

Entry 4: 20 minute, Set A

SCG-317-C2-20min-D 1-50sec. 1.fid

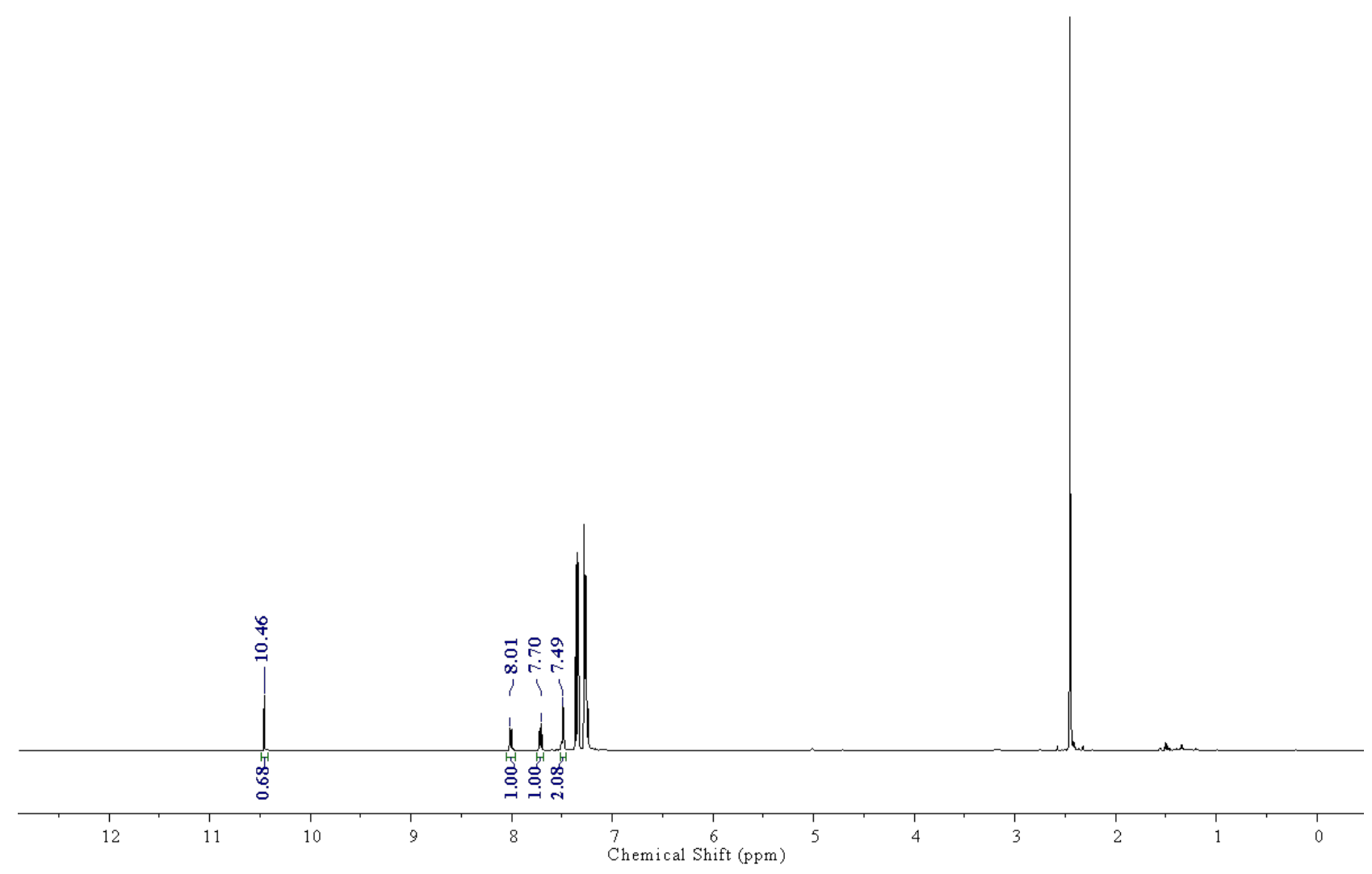


Entry 5: 25 minute, Set A

SCG-317-C8-25m in-D 1-50sec. 1 fid

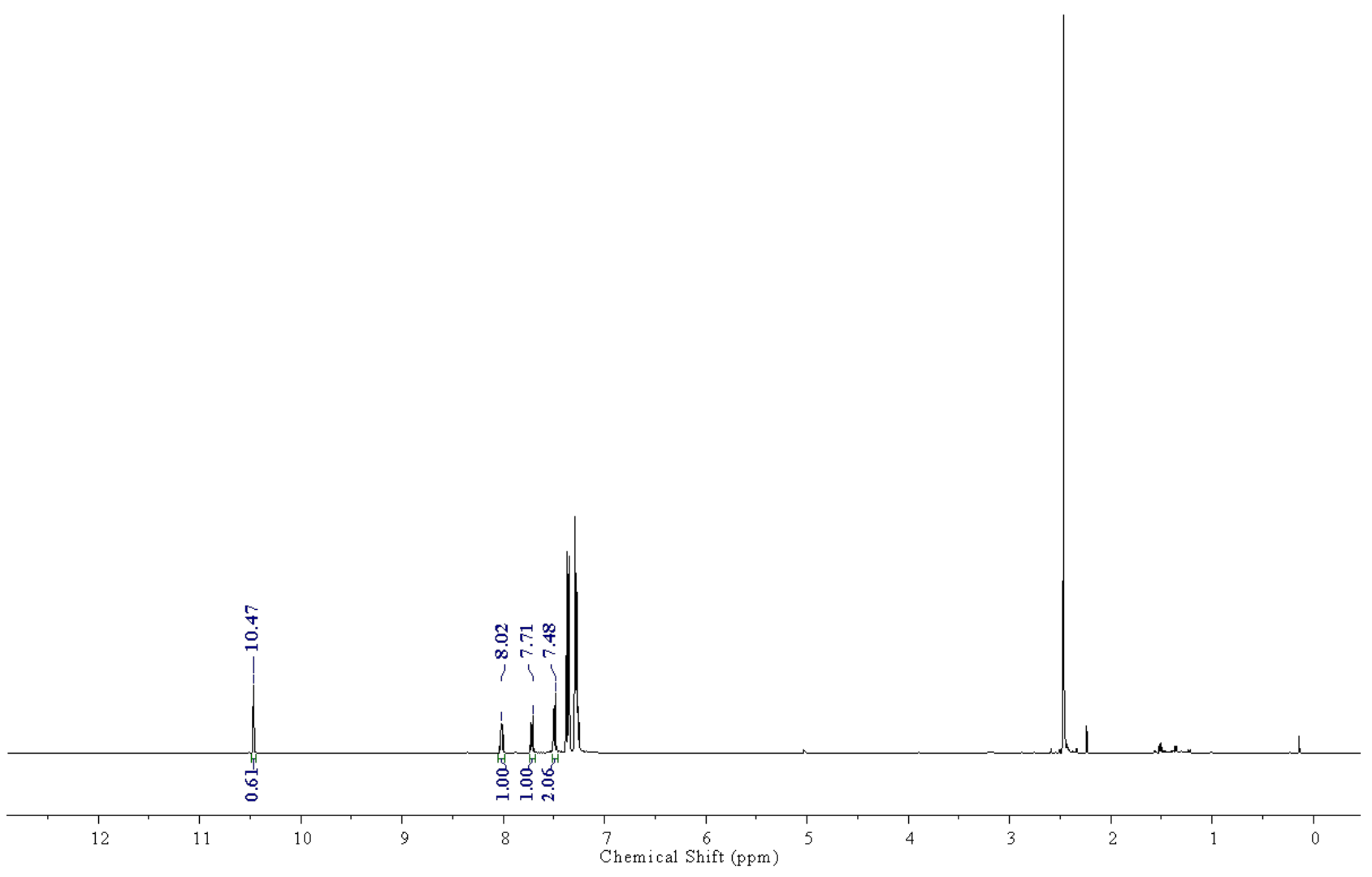


Initial Reaction Rate for 2-Methylbenzaldehyde:

Entry 1: 5 minute, Set A

ILZ-016-A 1.1. fid

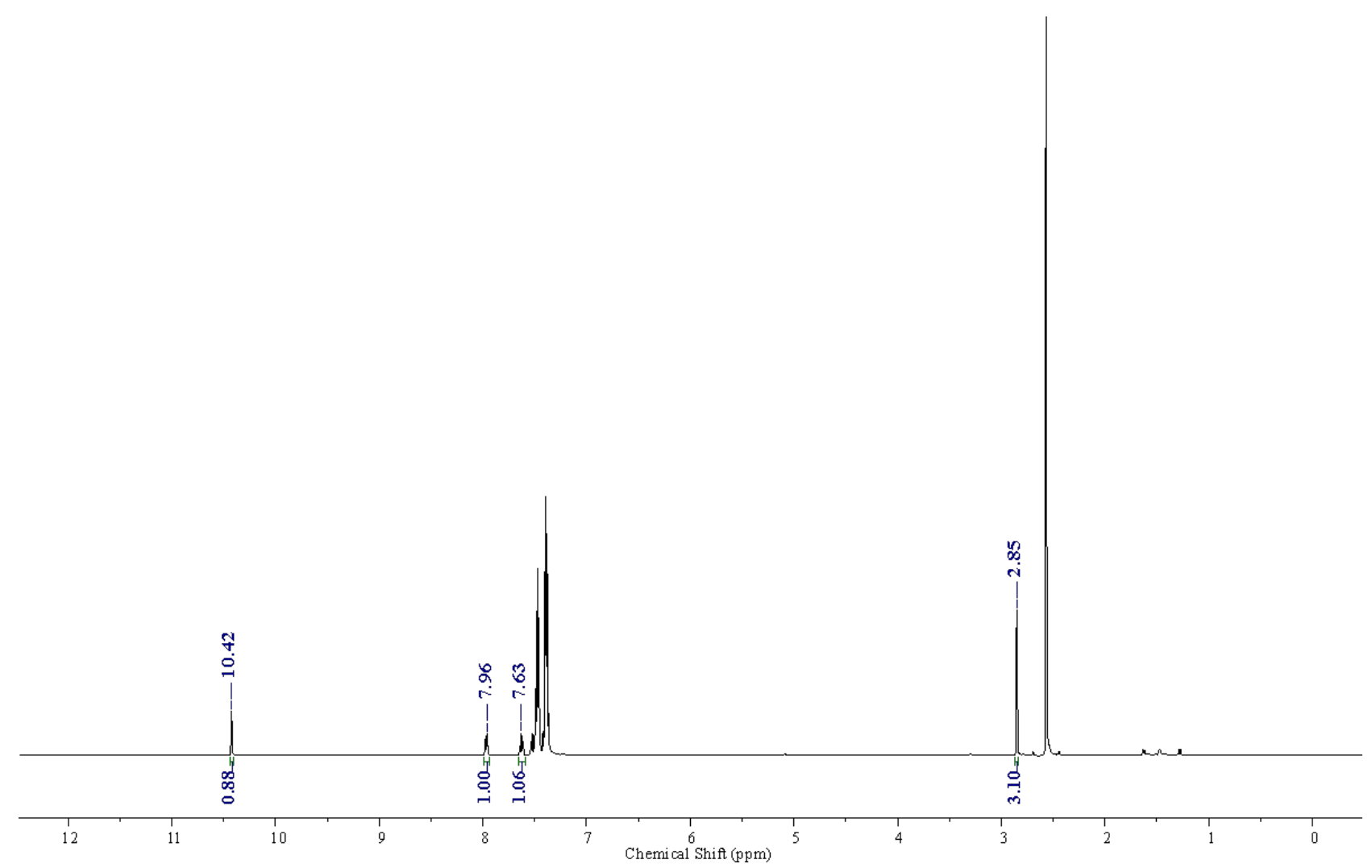

Entry 2: 10 minute, Set A

ILZ-016-A2.1.fid

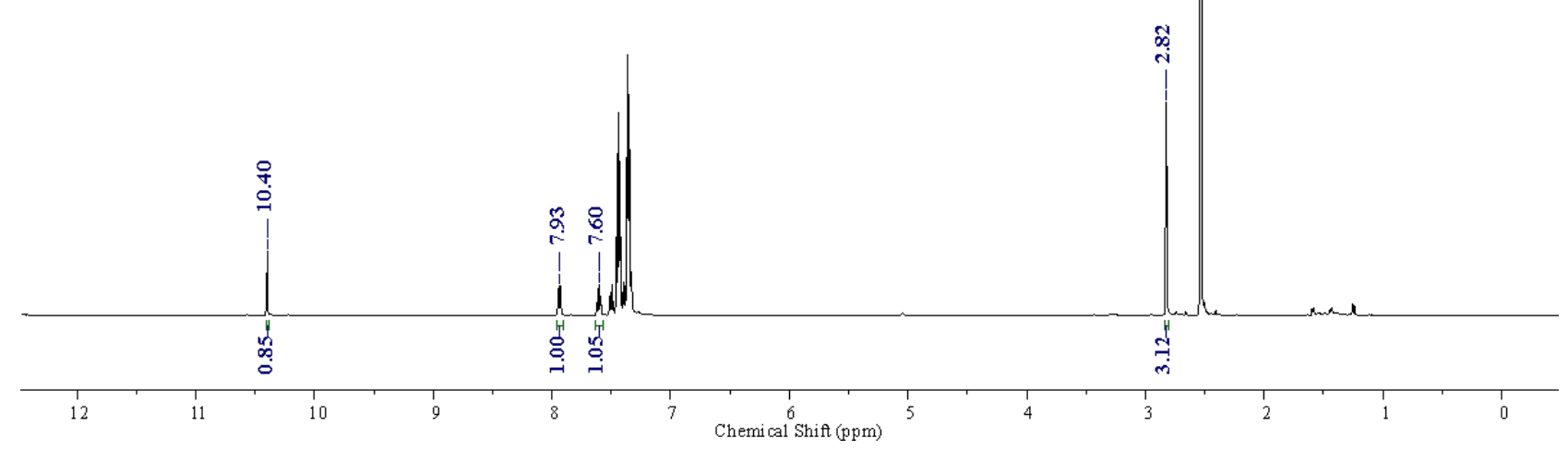


Entry 3: 15 minute, Set A

ILZ-016-A3.1.fid

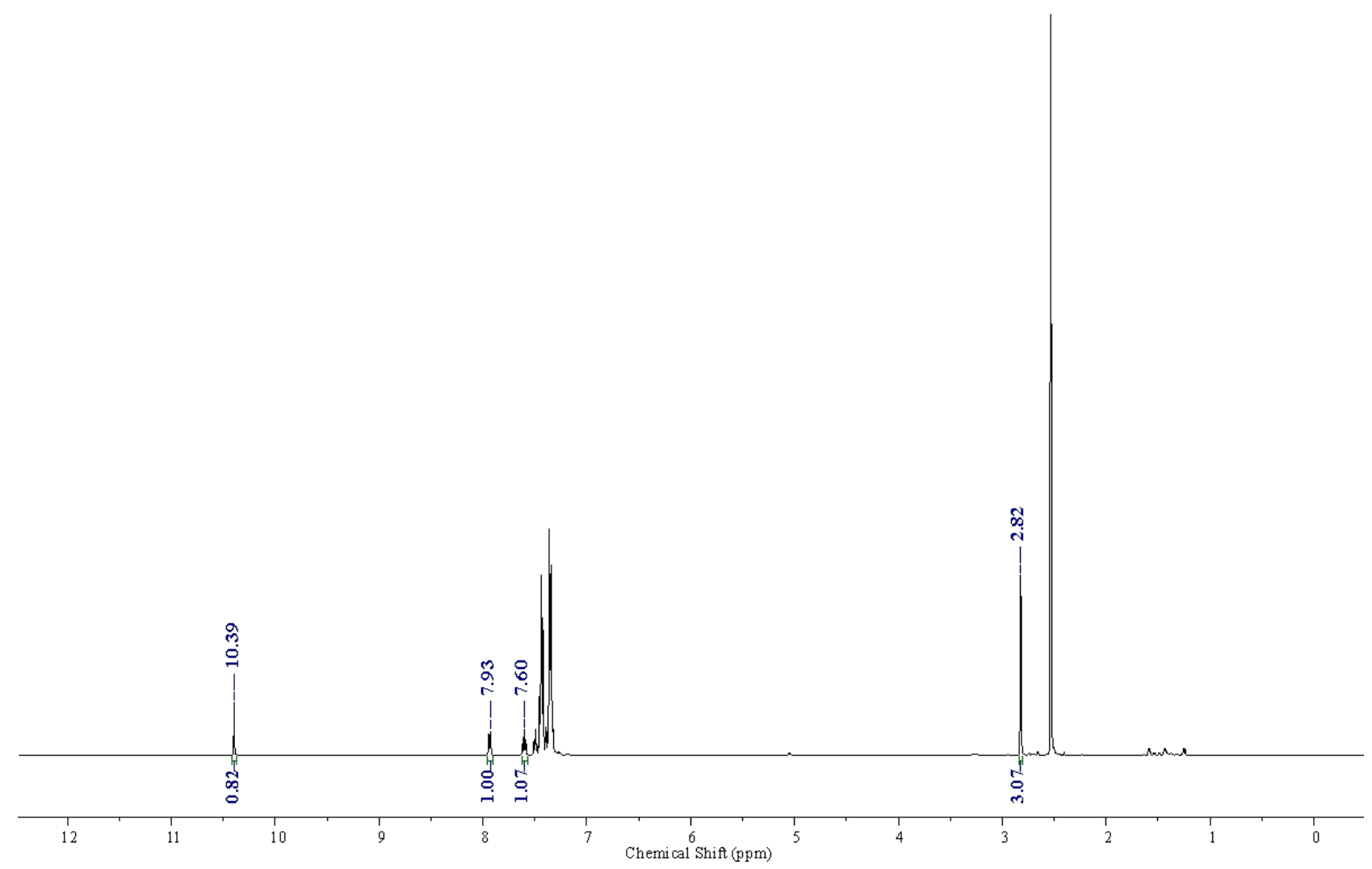

Entry 4: 20 minute, Set A

ILZ-016-A. 1. fid

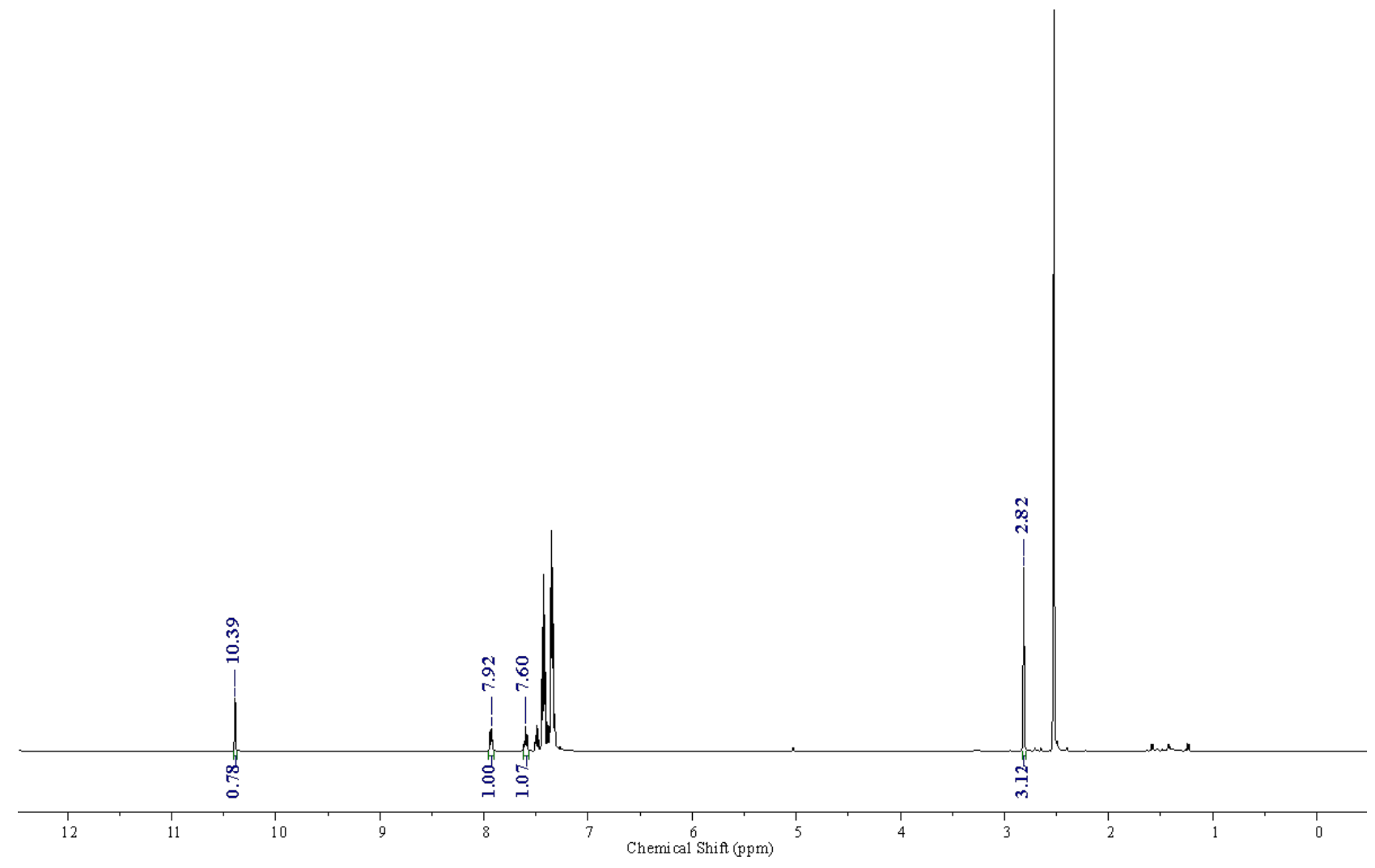


Entry 5: 25 minute, Set A

ILZ-016-A5.1.fid

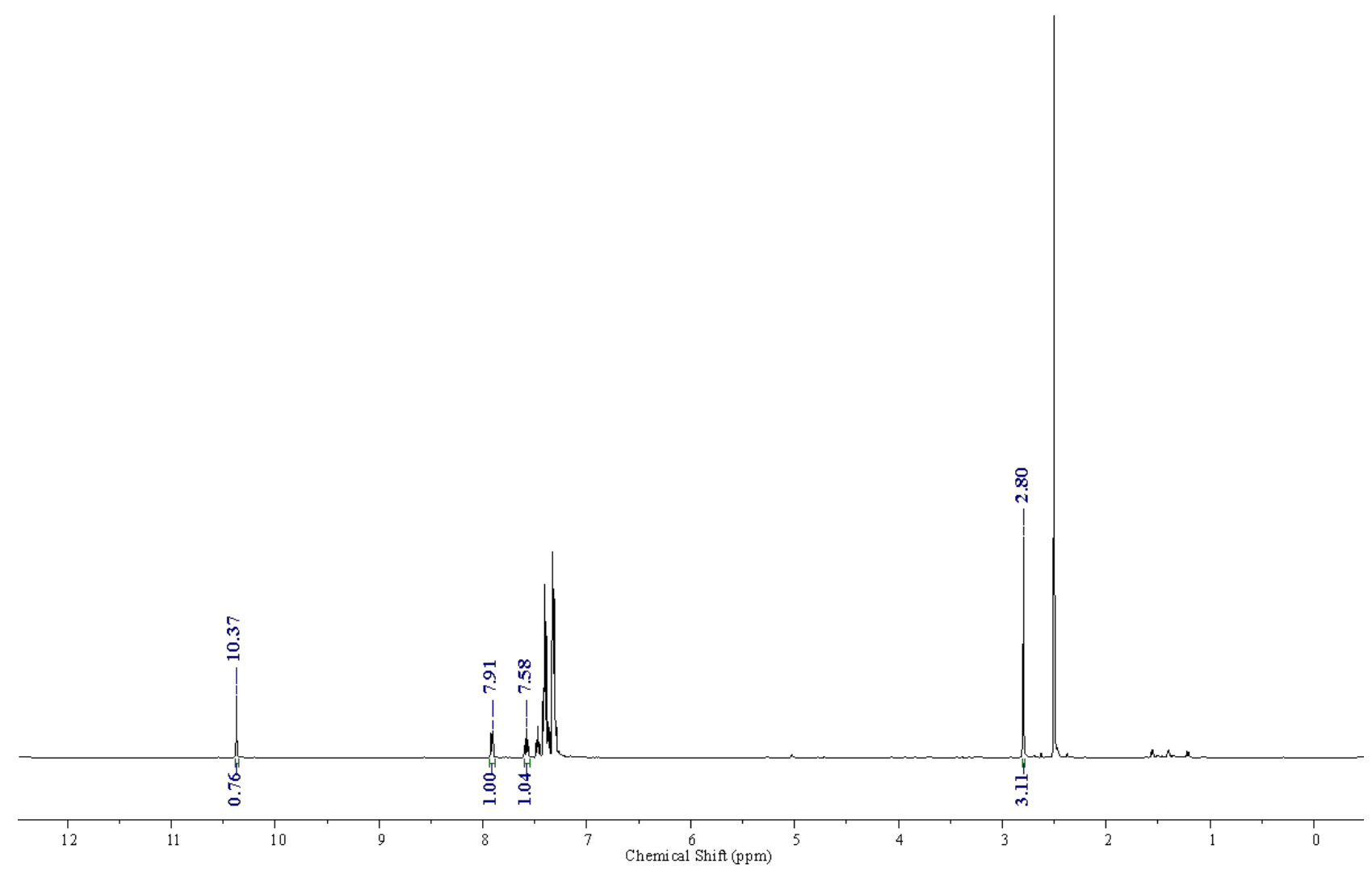


Initial Reaction Rate for 2-Methoxybenzaldehyde:

Entry 1: 30 minute, Set A

SCG-320-E1-30min-D 1-50sec.1. fid

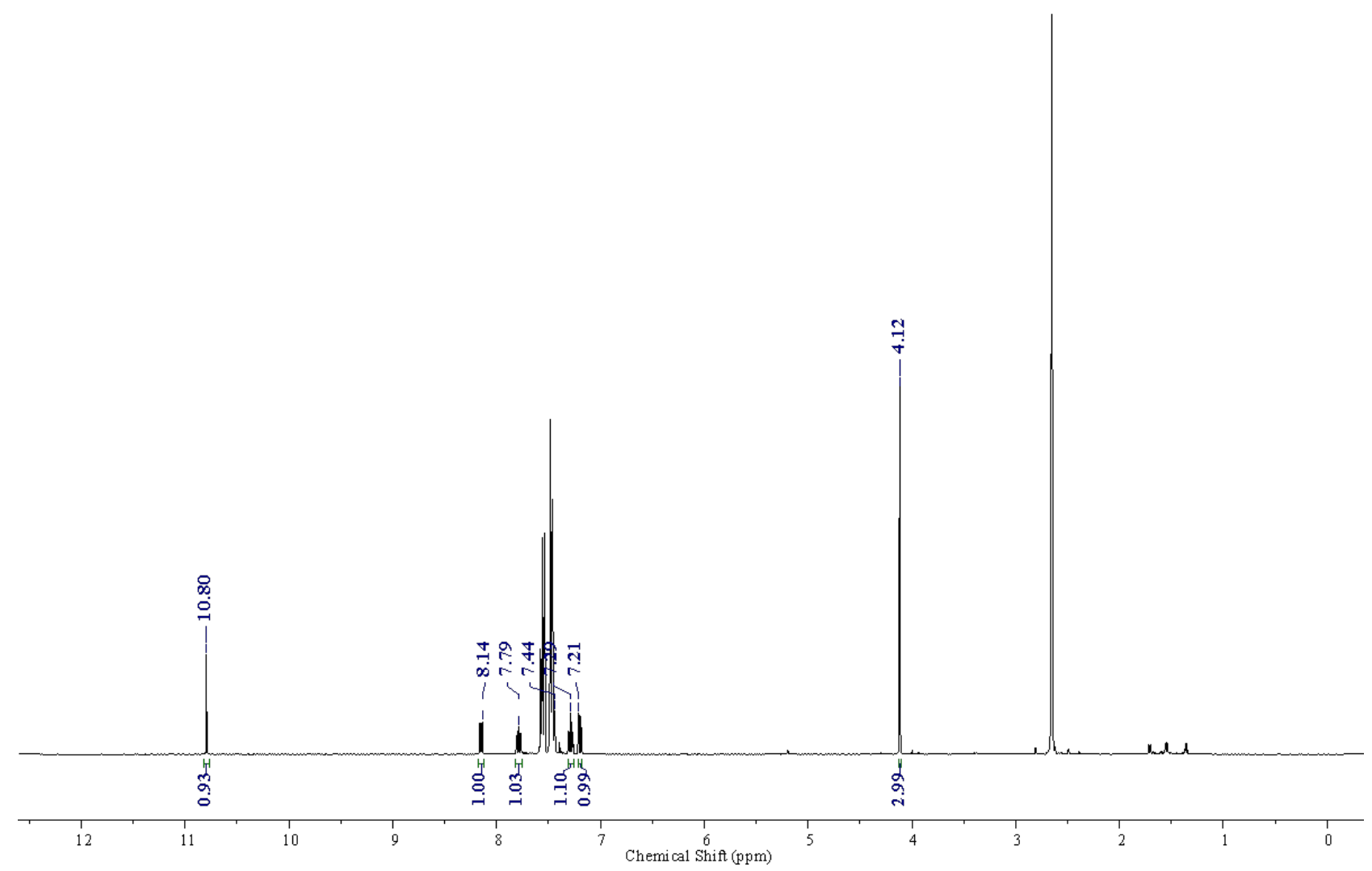

Entry 2: 60 minute, Set A

SCG-320-E2-1hr-D 1-50sec.1.fid

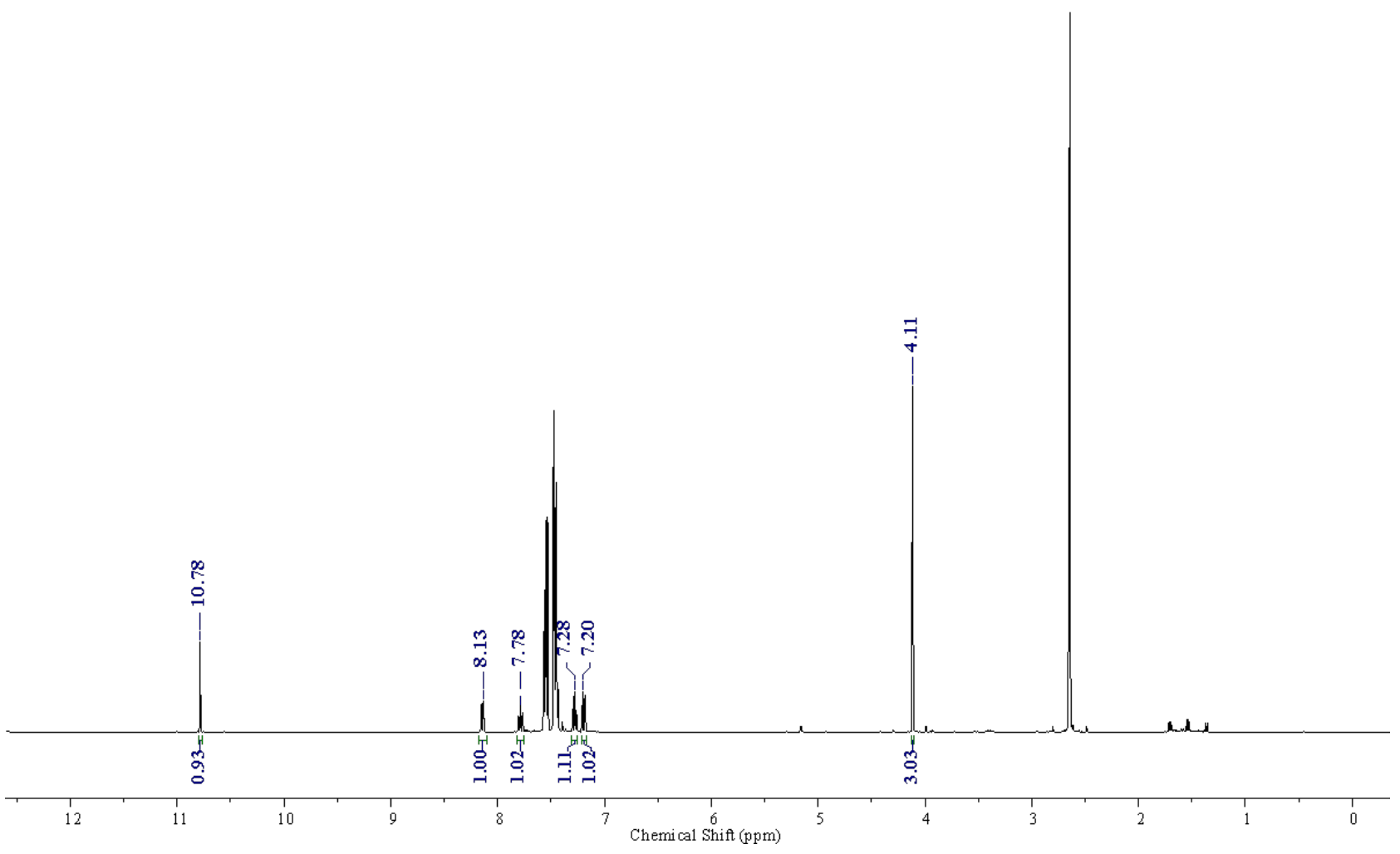


Entry 3: 90 minute, Set A

SCG-320-E3-1.5hr-D 1-50sec.1. fid

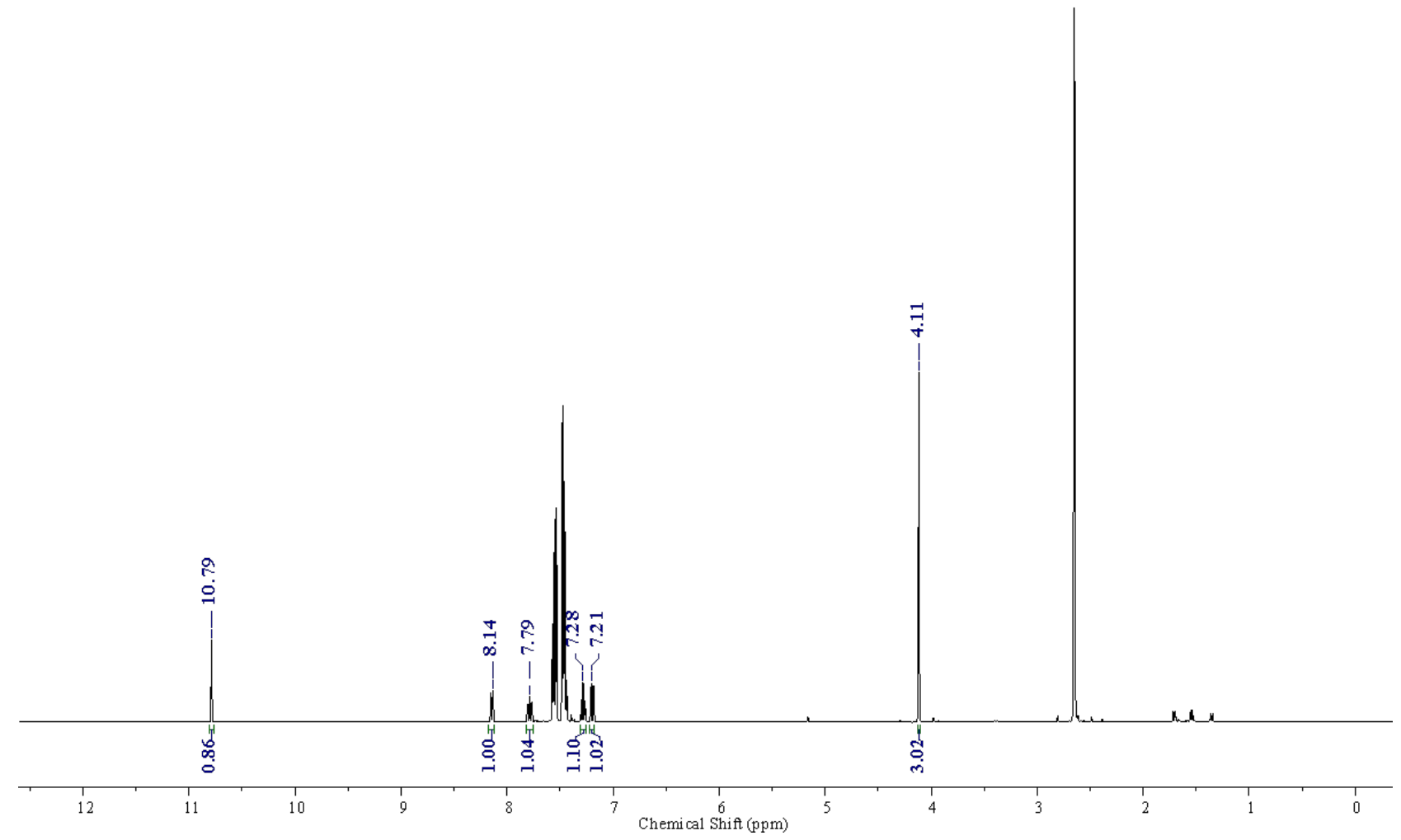

Entry 4: 120 minute, Set A

SCG-320-E4-2hr-D 1-50sec. 1.fid

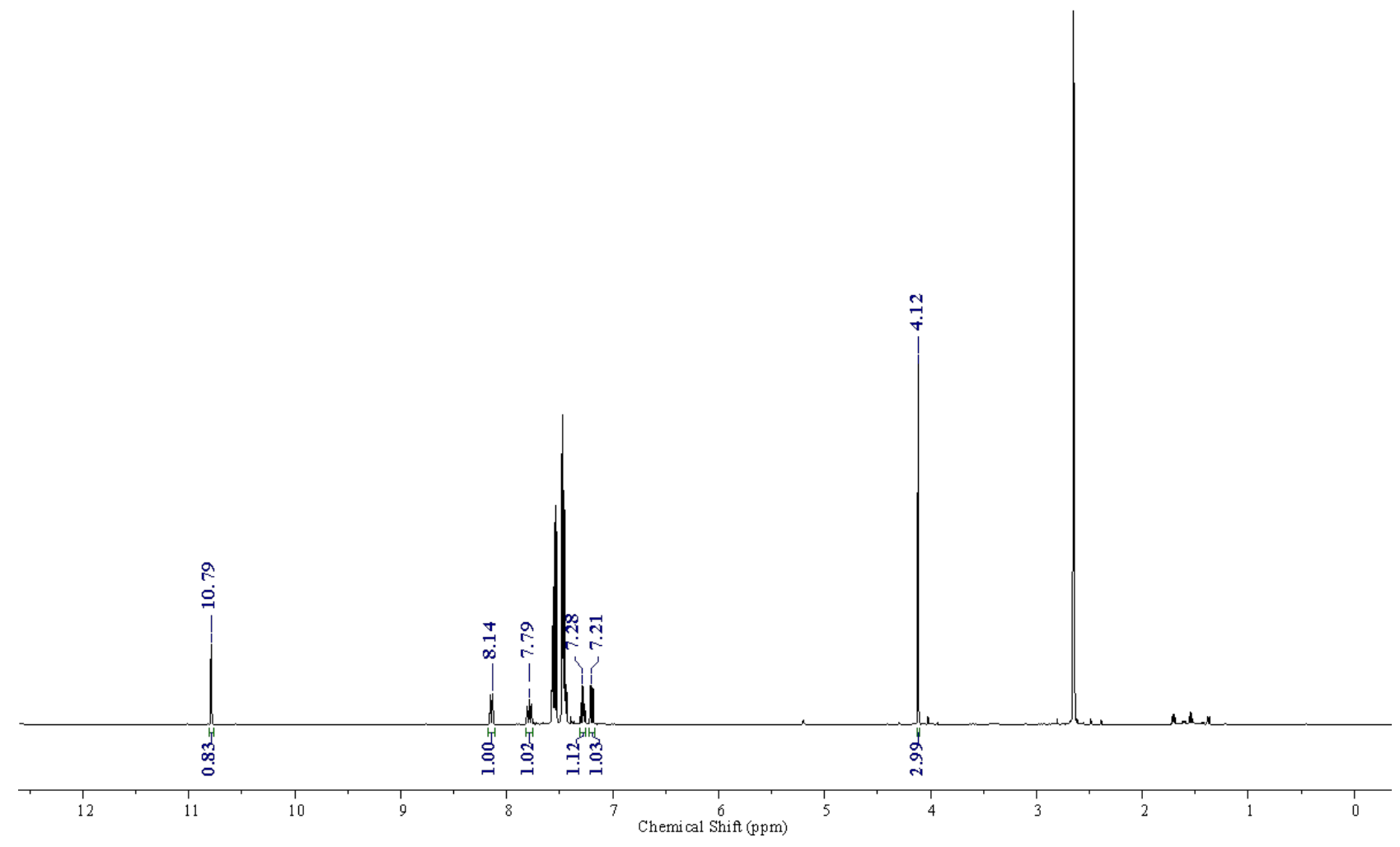


Entry 5: 150 minute, Set A

SCG-320-E5-2.5hr-D 1-50sec.1. fid

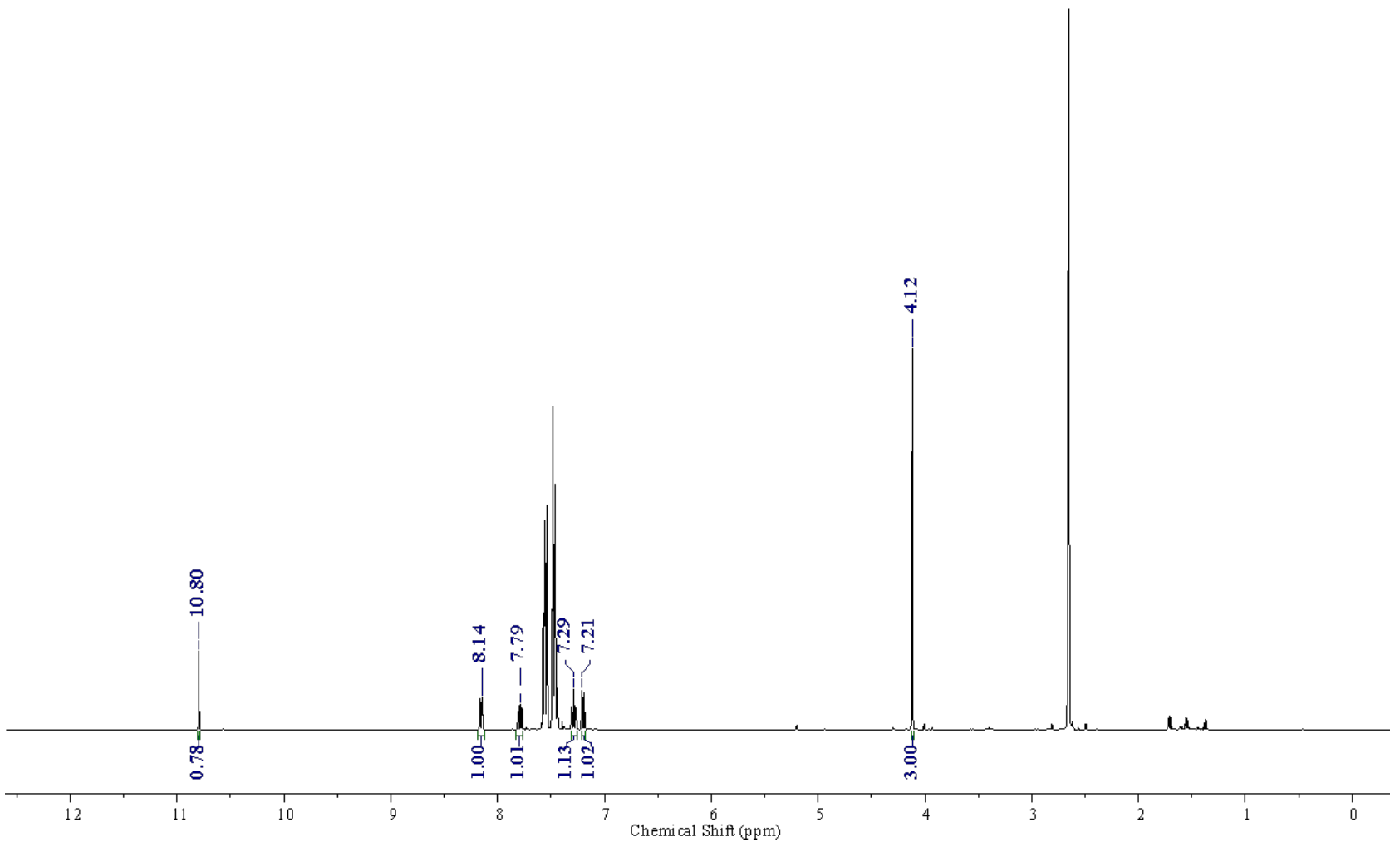


Initial Reaction Rate for 1-Naphthaldehyde:

Entry 1: 5 minute, Set A

ILZ-022-A1.1.fid

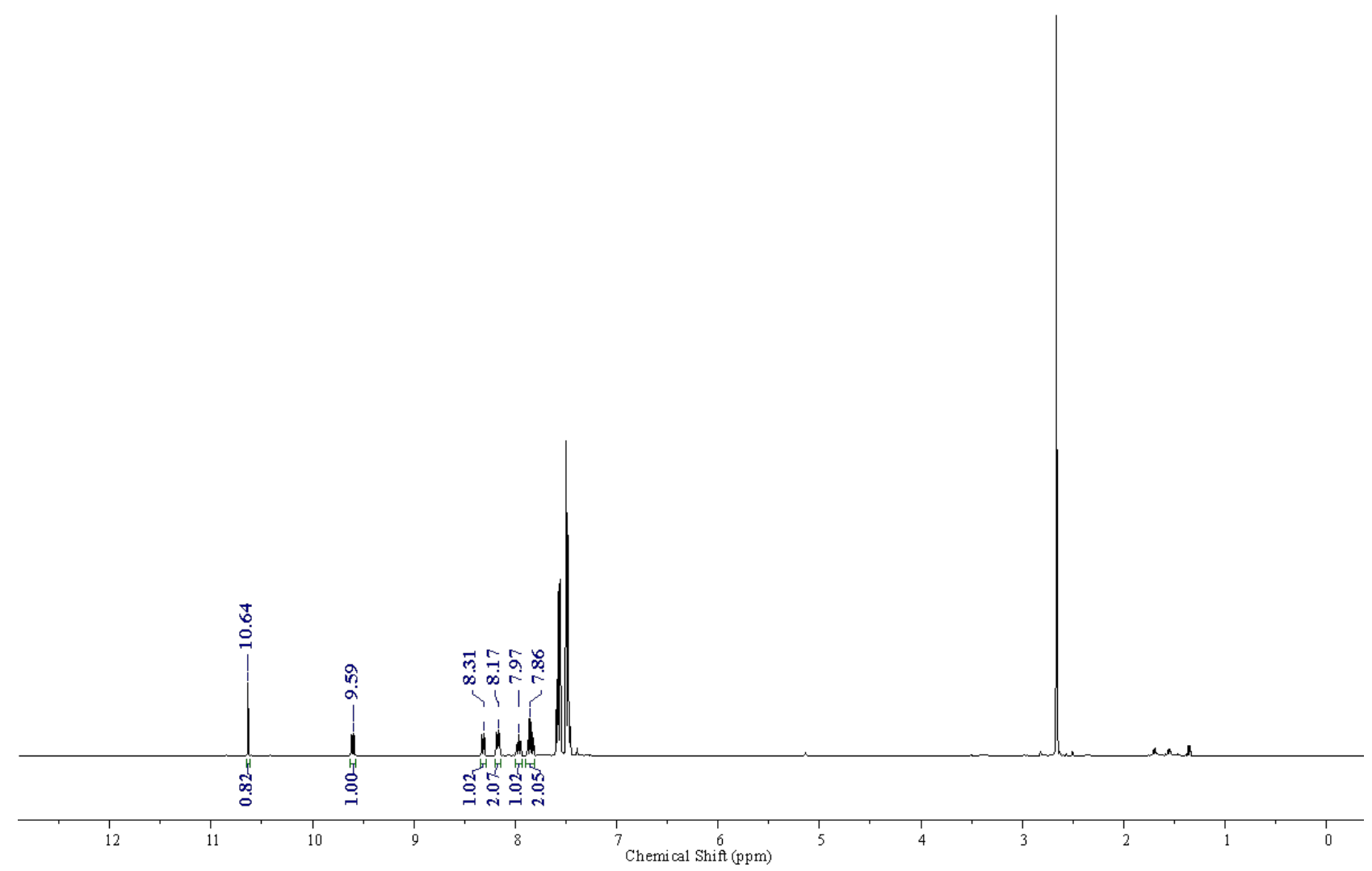

Entry 2: 10 minute, Set A

ILZ-022-A2. 1.fid

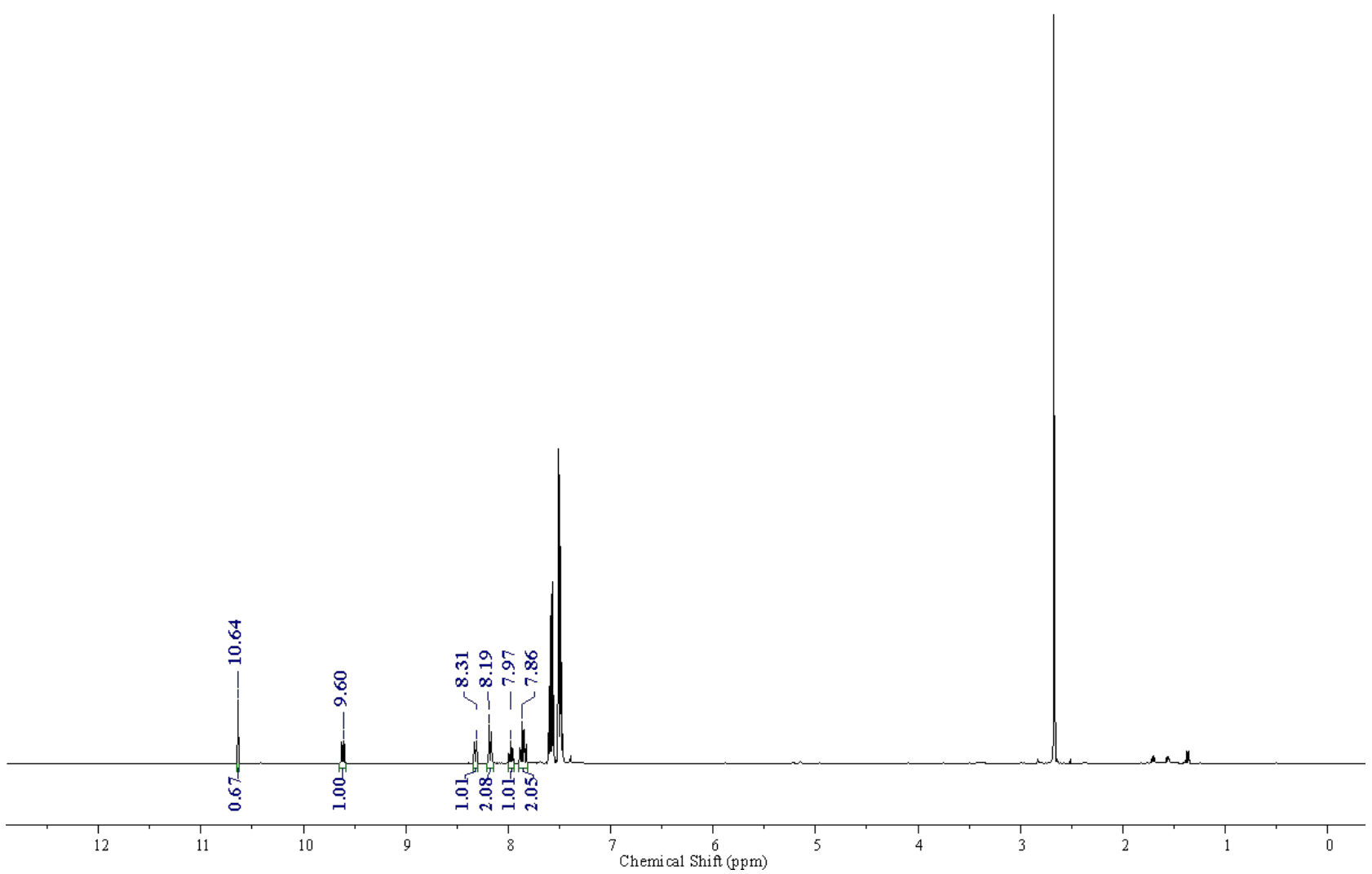


Entry 3: 15 minute, Set A

ILZ-022-A3.1. fid

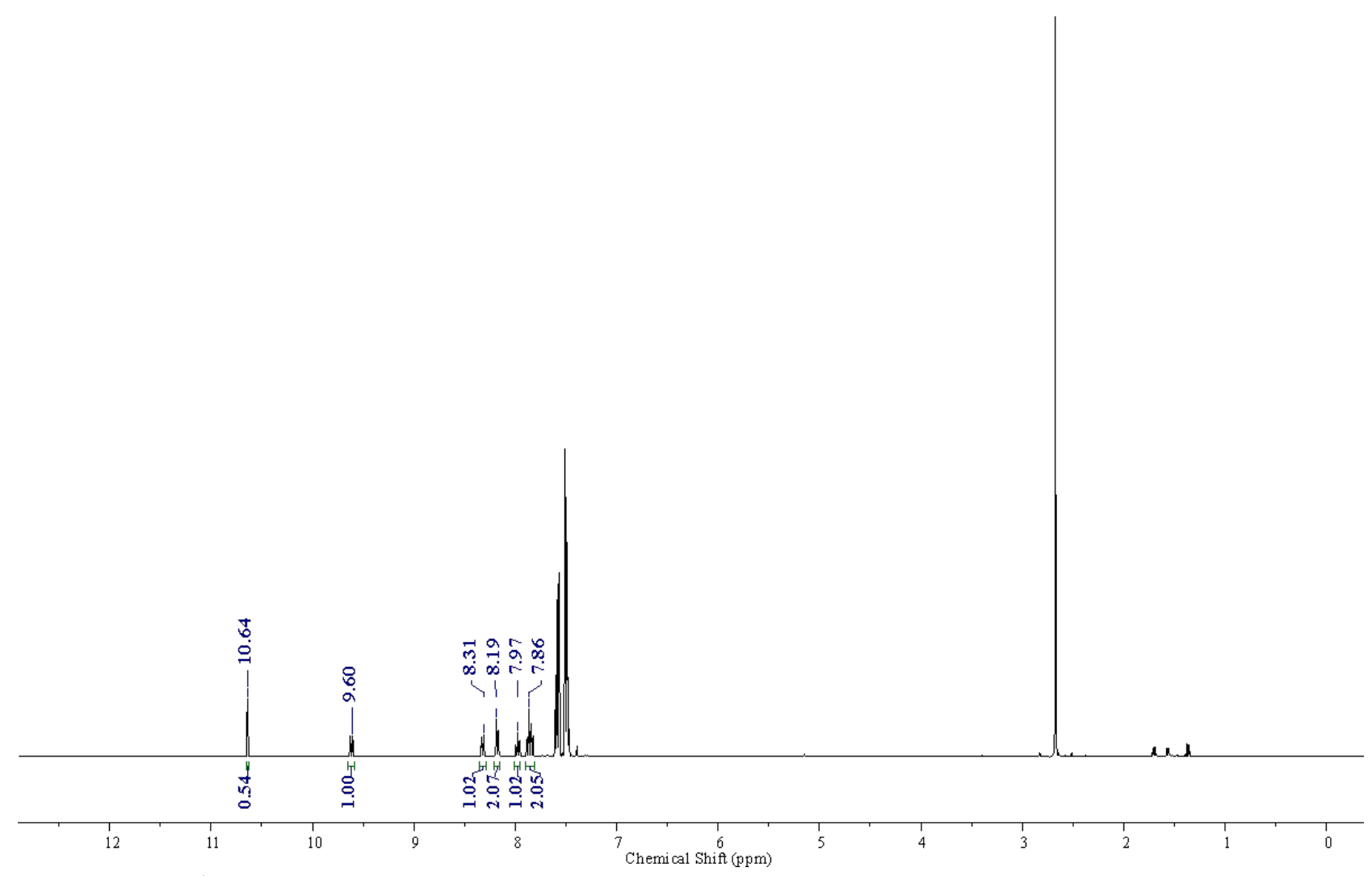

Entry 4: 20 minute, Set A

ILZ-022-A4. 1.fid

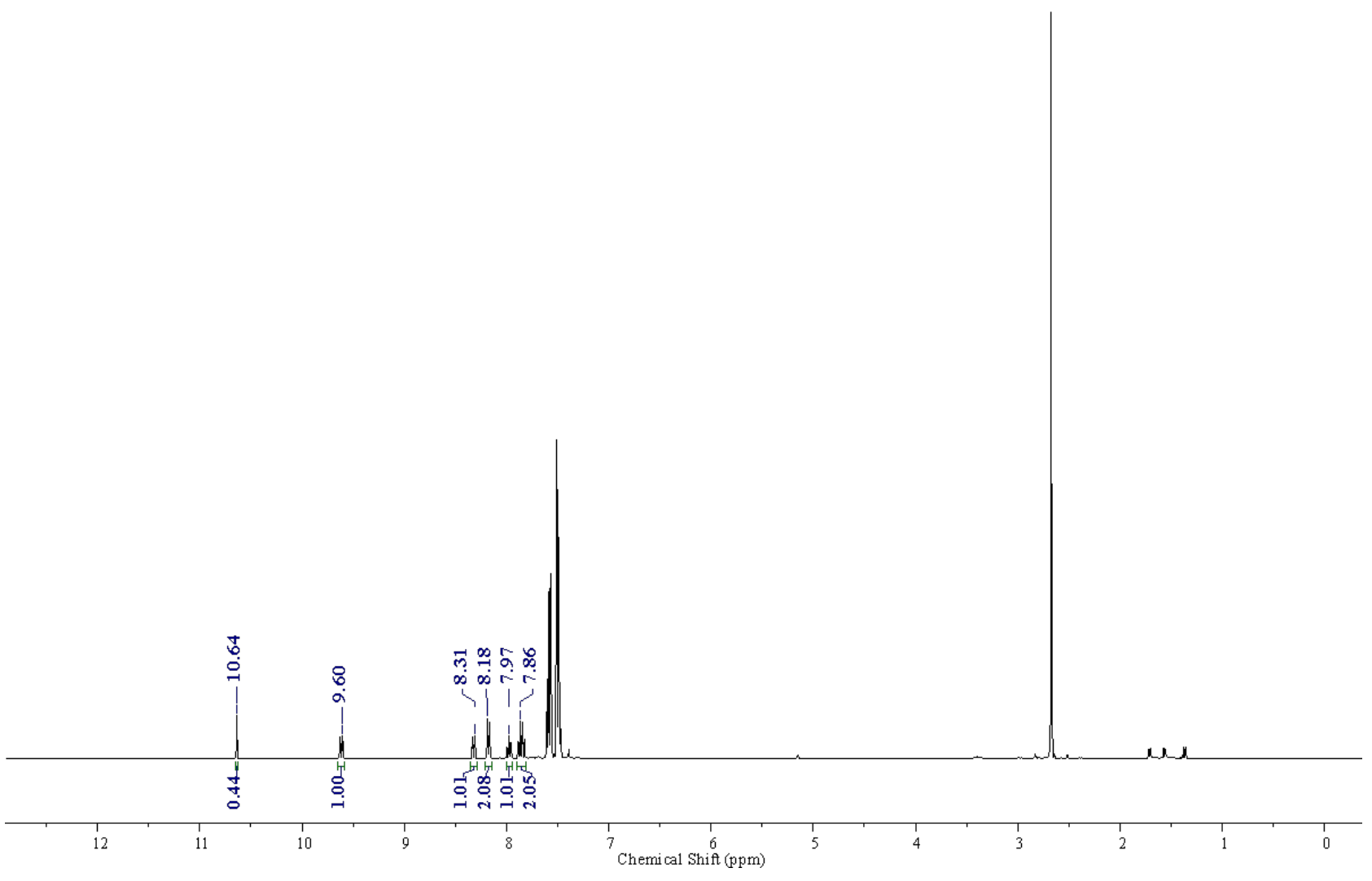


Entry 5: 25 minute, Set A

ILZ-022-A 5. 1. fid

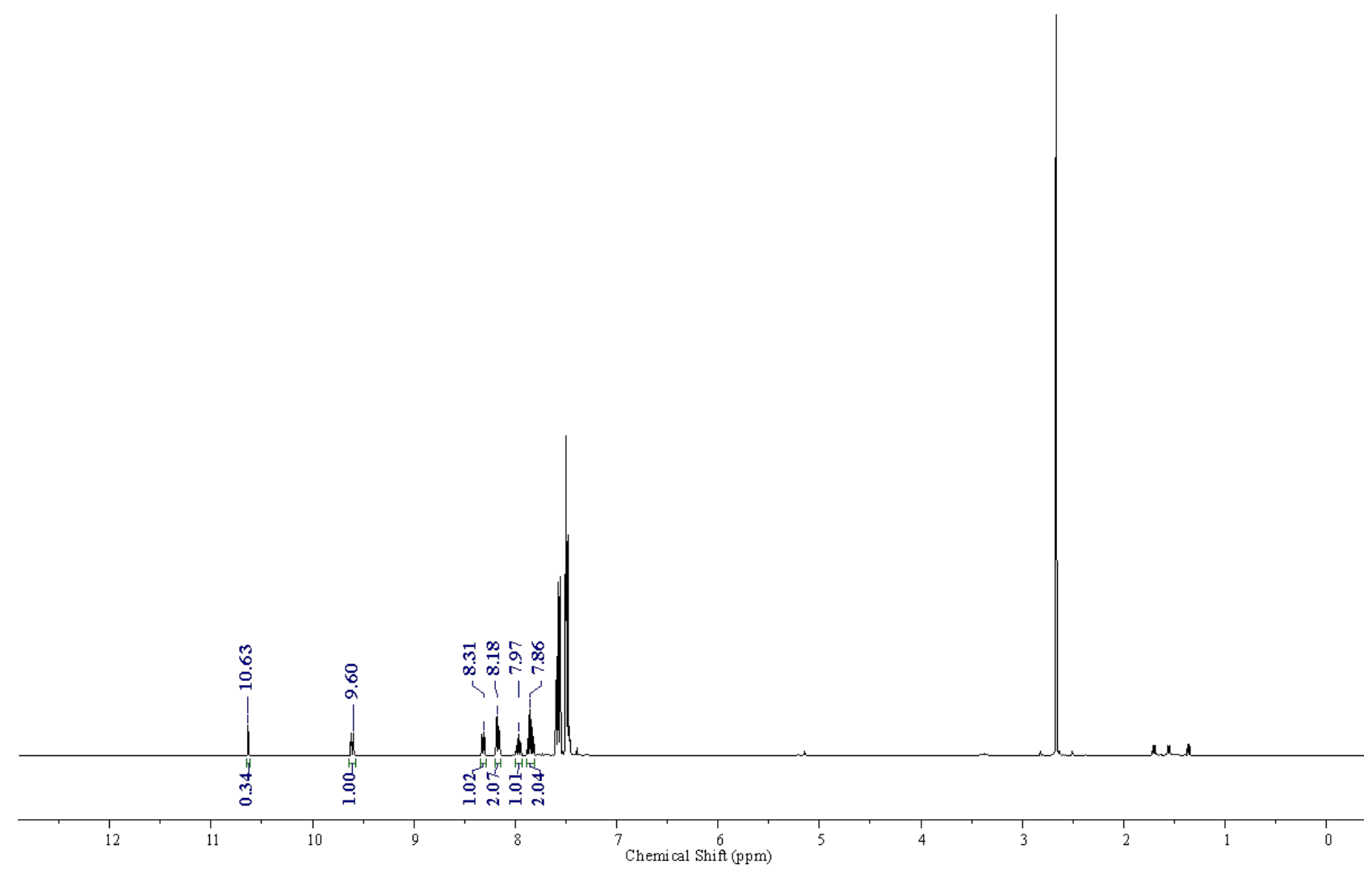


Initial Reaction Rate for Thiophene-2-carboxaldehyde:

Entry 1: 2.5 minute, Set A

ILZ-019-A6.1.fid

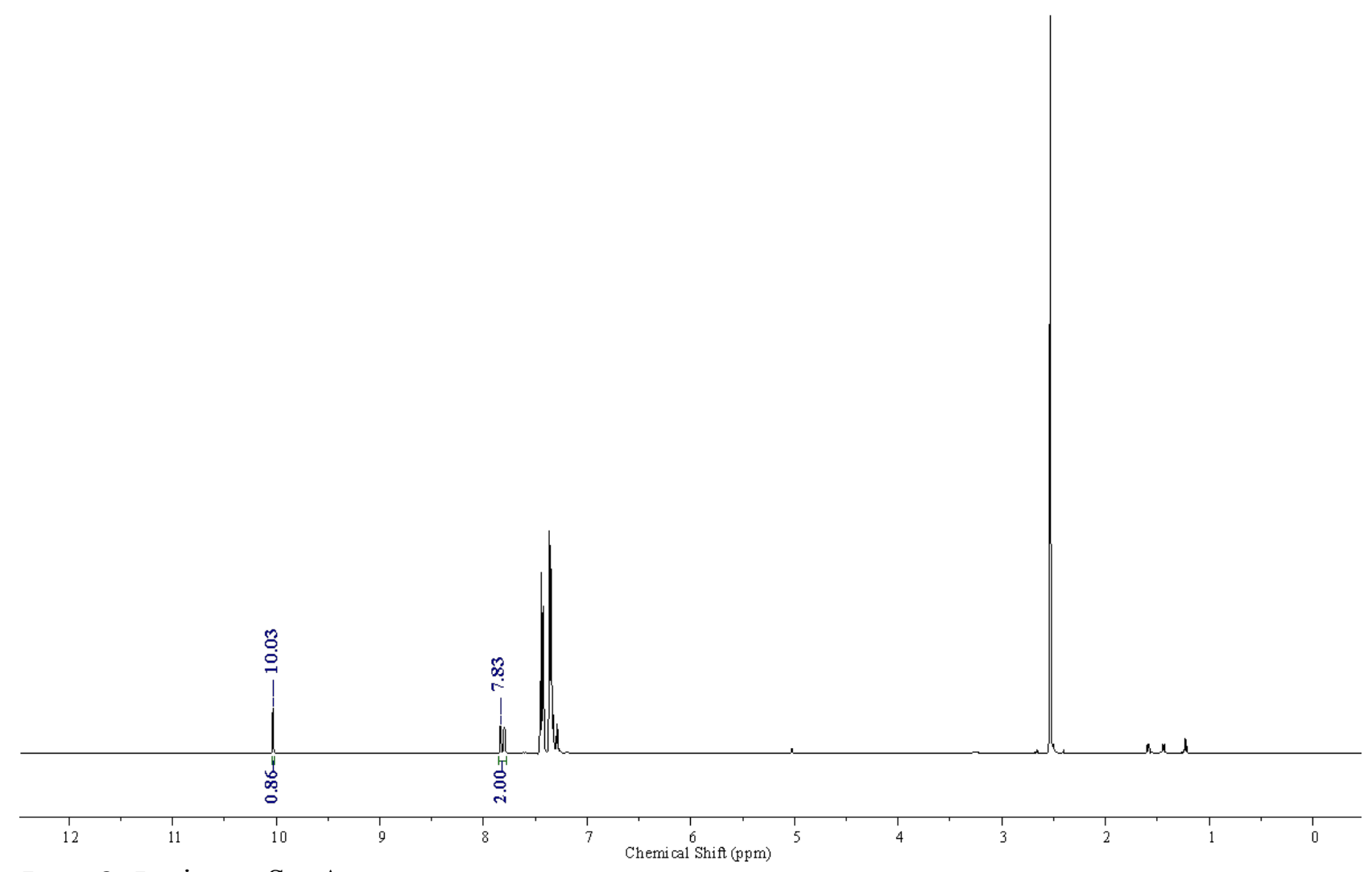

Entry 2: 5 minute, Set A

ILZ-019-A1.1.fid

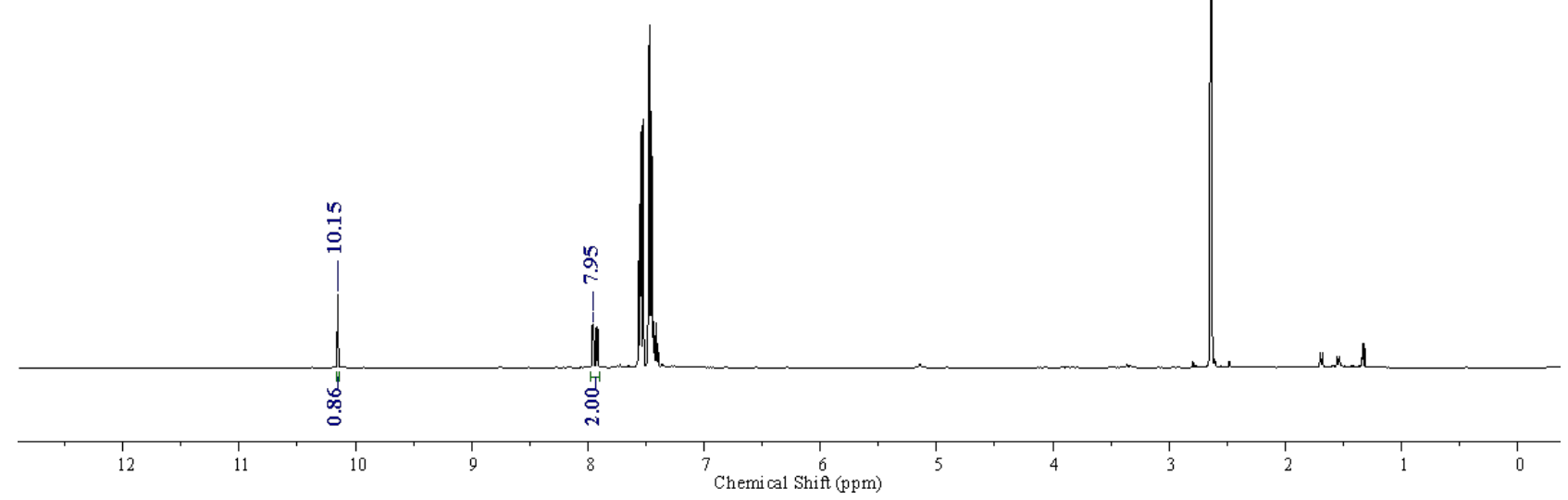


Entry 3: 7.5 minute, Set A

ILZ-019-A.7.1.fid

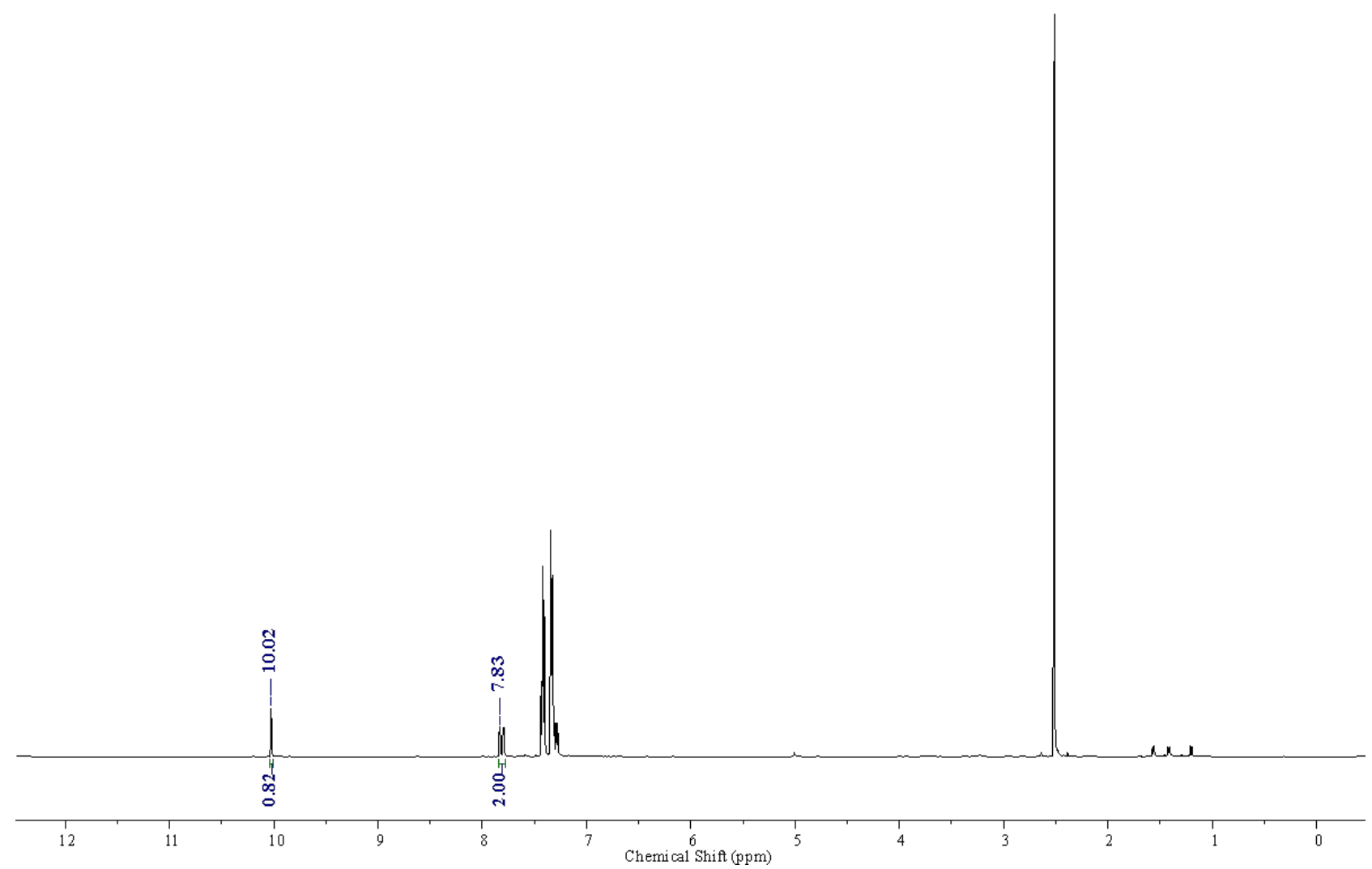

Entry 4: 10 minute, Set A

ILZ-019-A2.1. fid

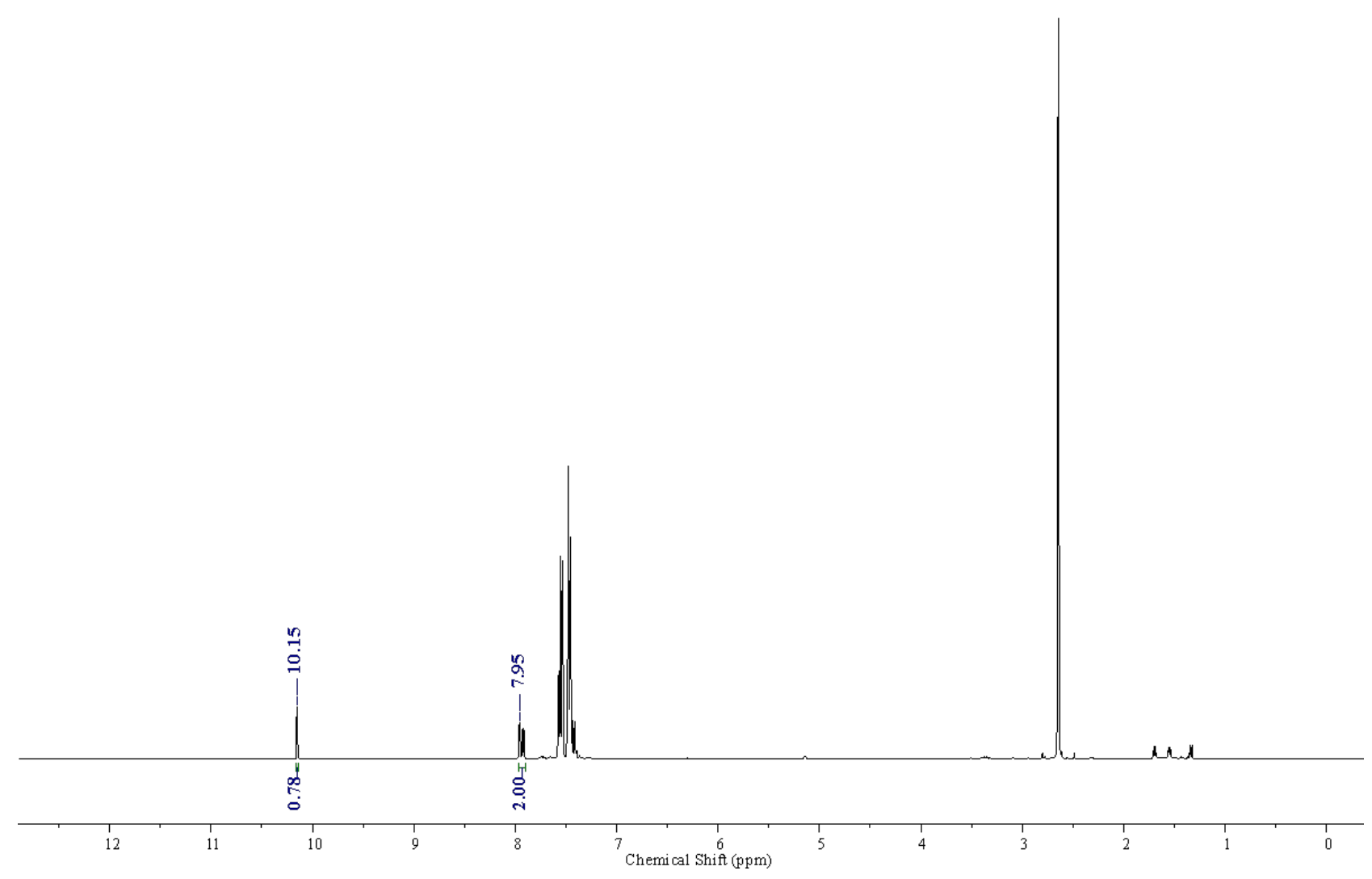


Entry 5: 12.5 minute, Set A

ILZ-019-A8.1.fid

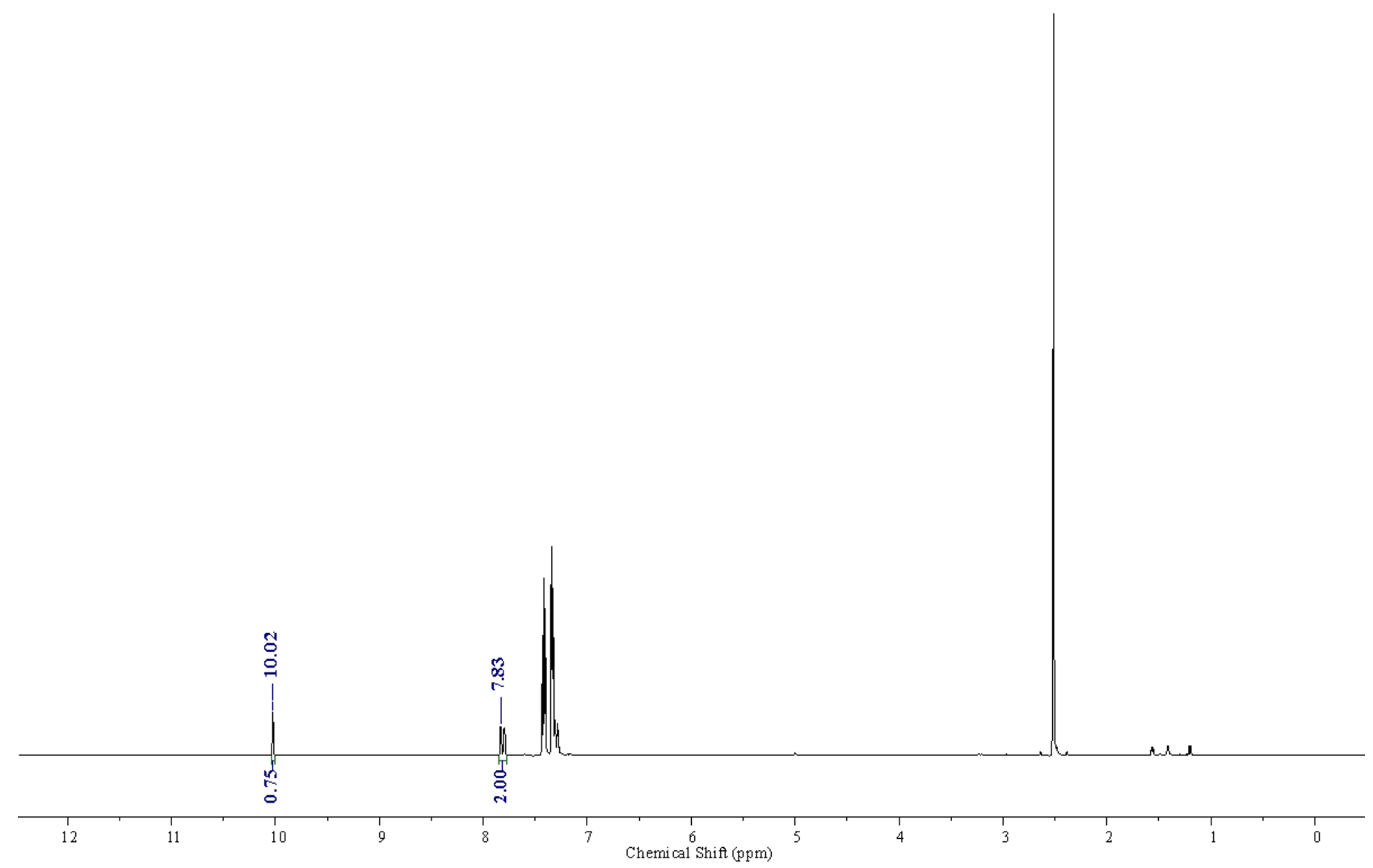


Initial Reaction Rate for $2{ }^{n}$ Butoxybenzaldehyde:

Entry 1: 60 minute, Set A

SCG-348-D 1-1hr-D 1-50sec.1.fid

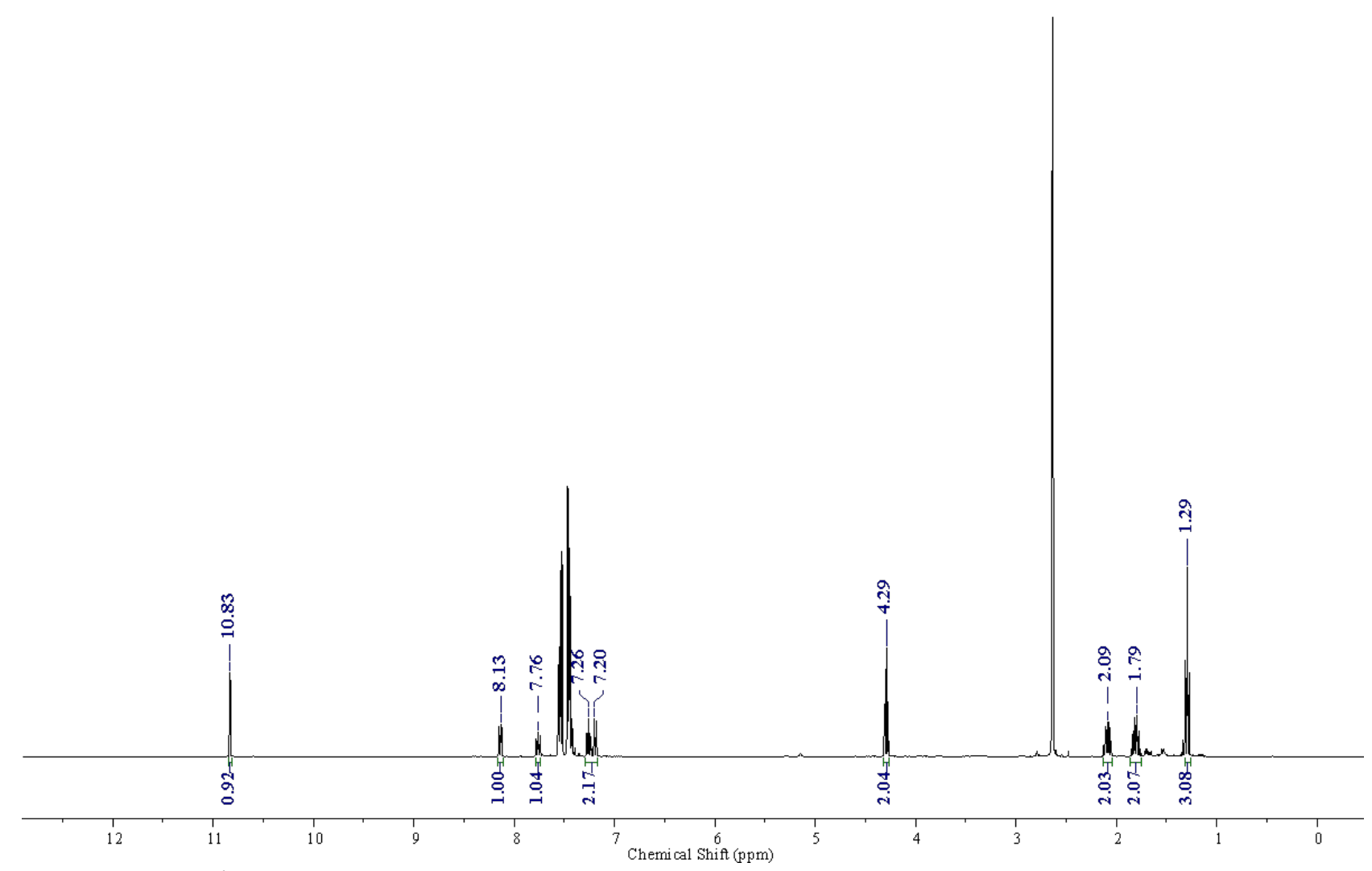

Entry 2: 120 minute, Set A

SCG-348-D 3-2hr-D 1-50sec.1.fid

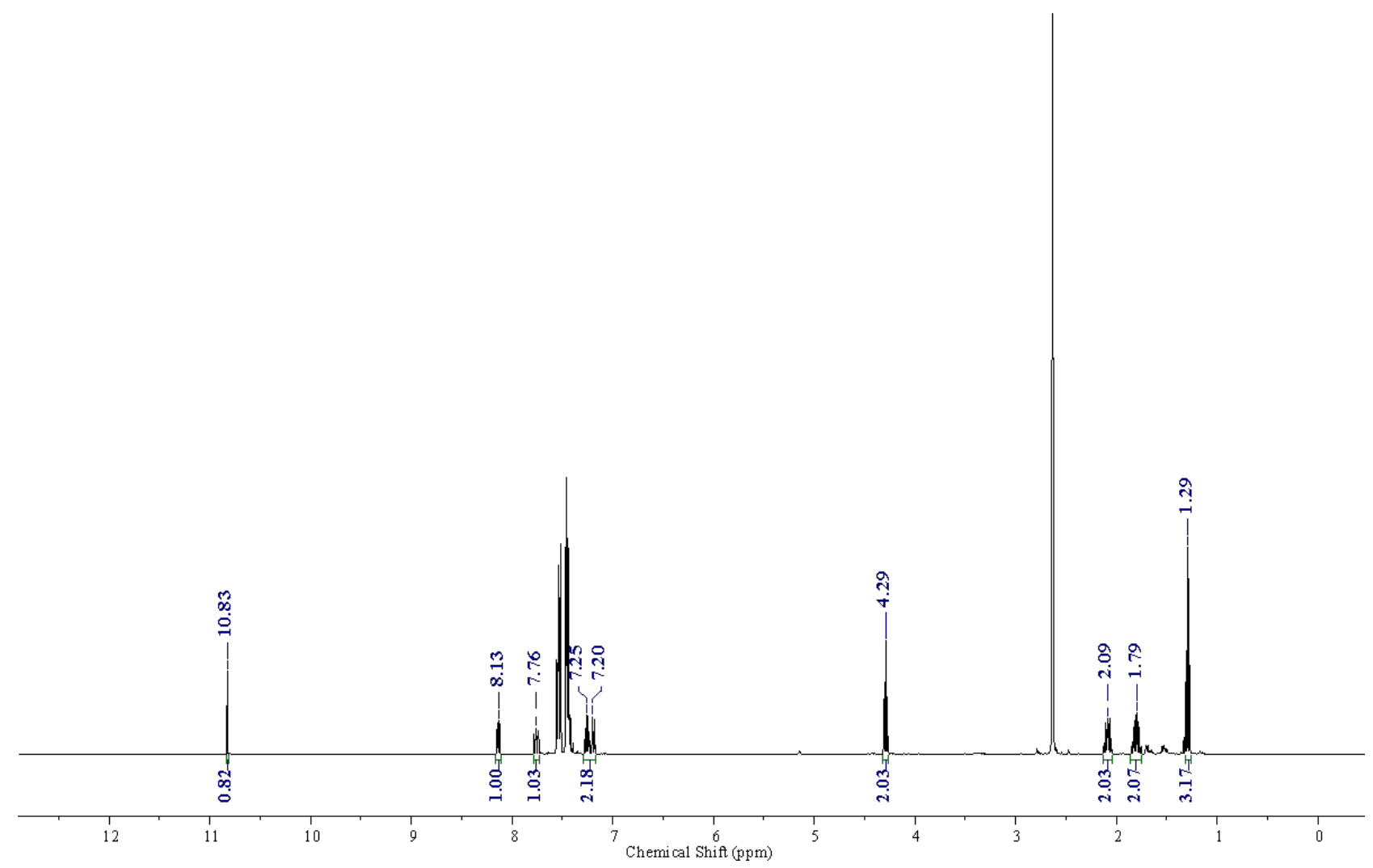


Entry 3: 180 minute, Set A

SCG-348-D 5-3hr-D 1-50sec.1. fid

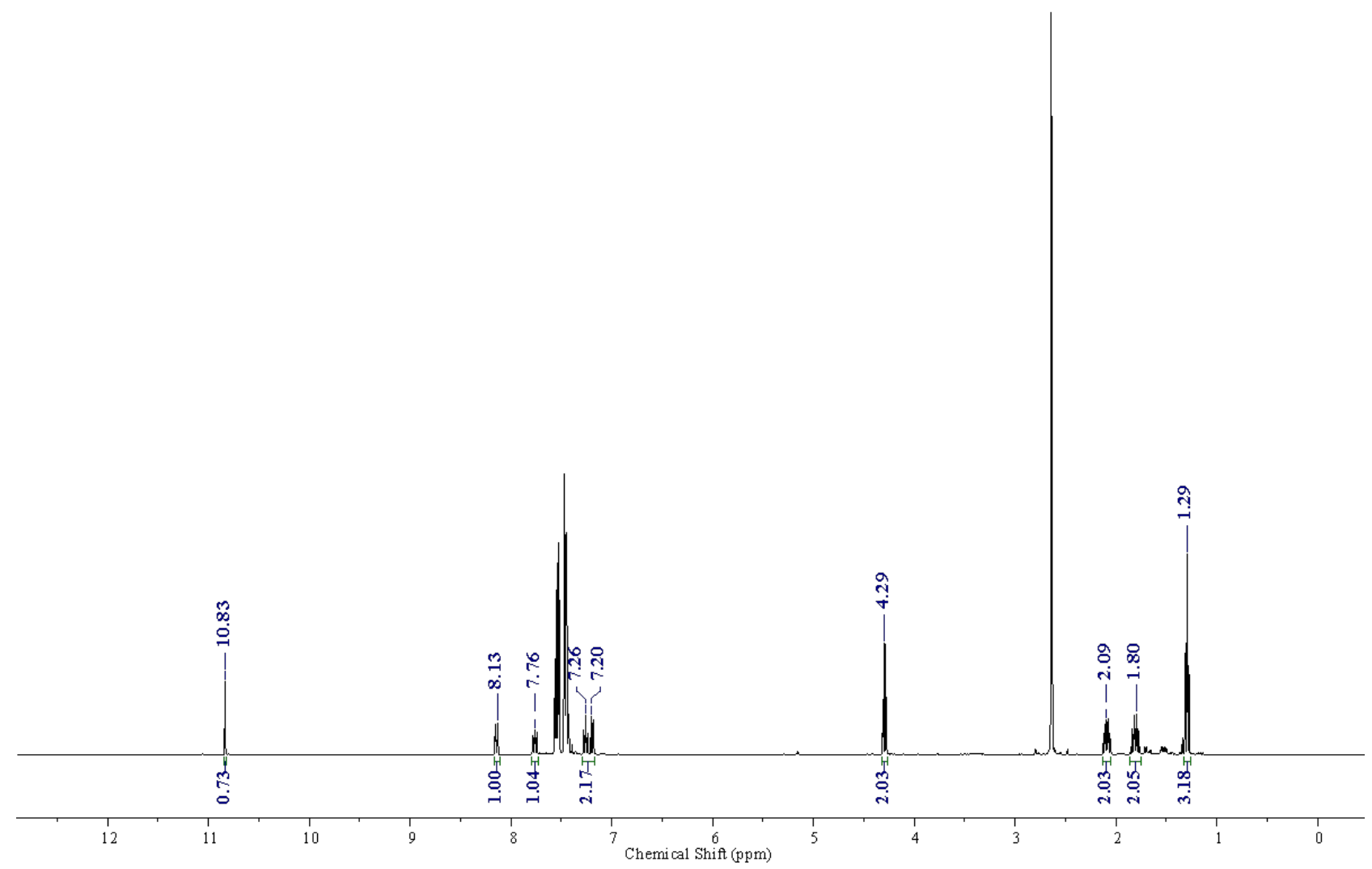

Entry 4: 240 minute, Set A

SCG-348-D 7-4hr-D 1-50 sec.1.fid

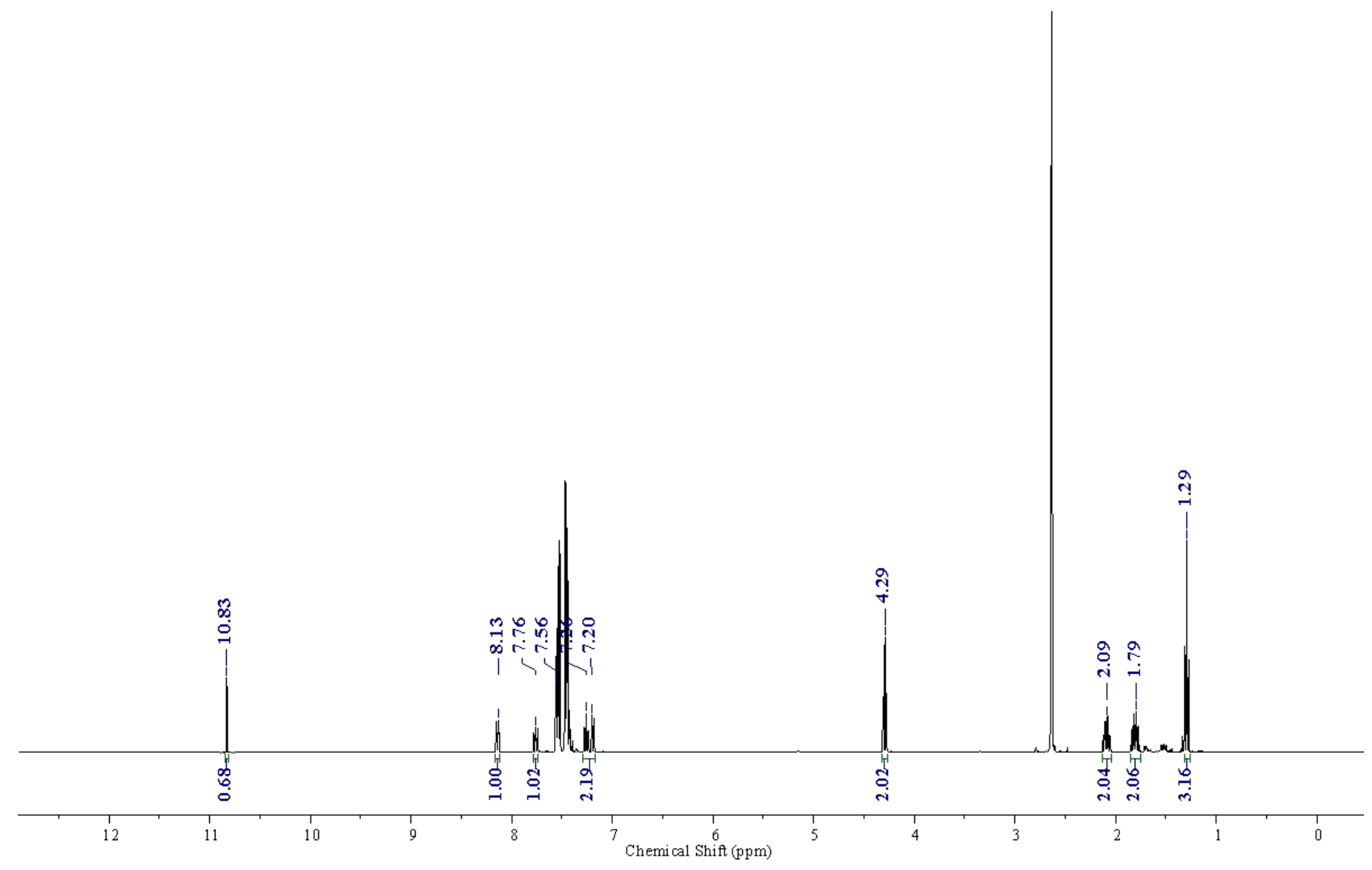


Entry 5: 300 minute, Set A

SCG-348-D 9-5hr-D 1-50sec.1. fid

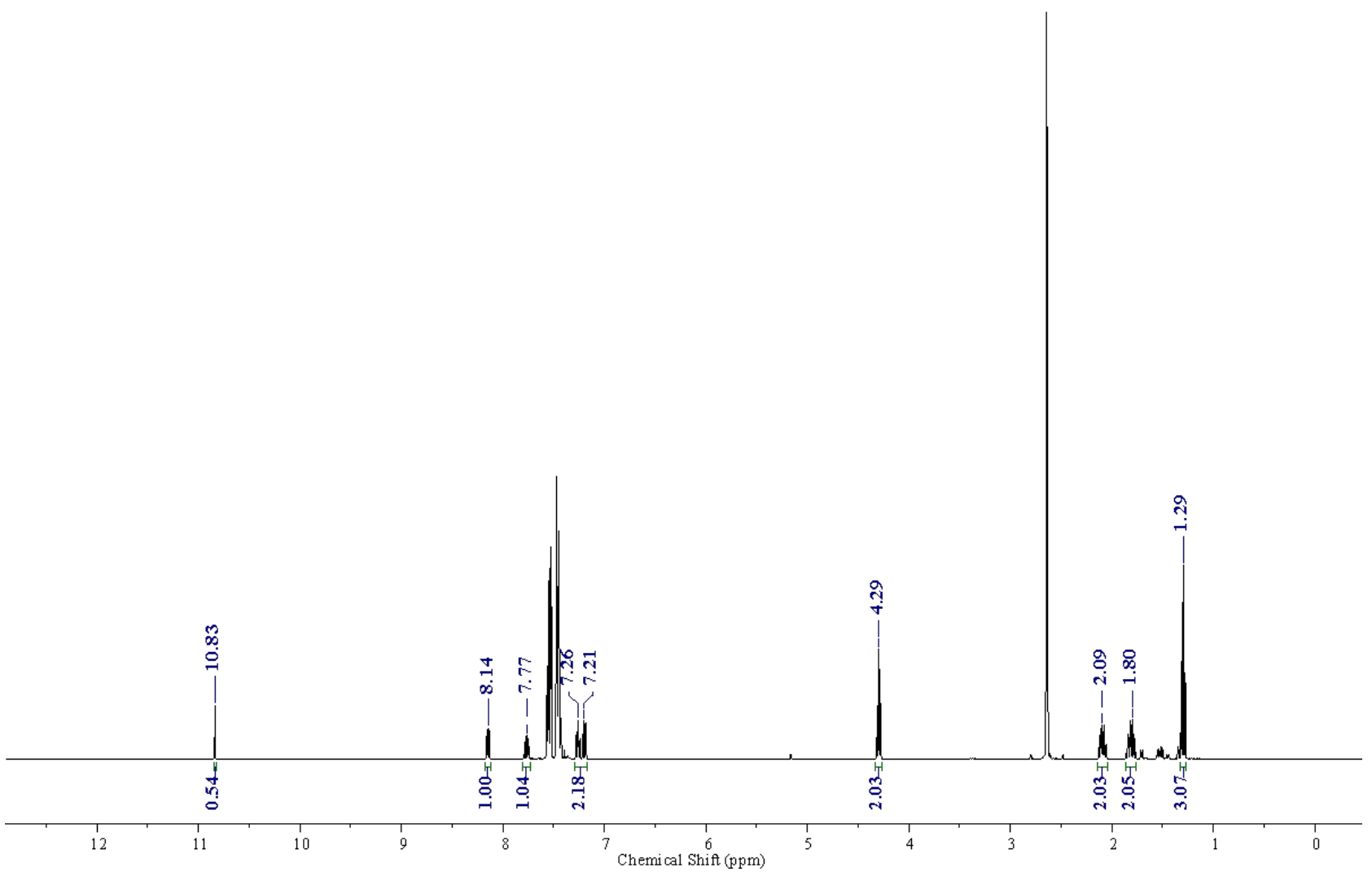


Initial Reaction Rate for 2-( $\left.{ }^{\left({ }^{e c} B u t o x y\right.}\right)$ benzaldehyde:

Entry 1: 60 minute, Set A

SCG-348-E1-1hr-D 1-50sec. 1.fid

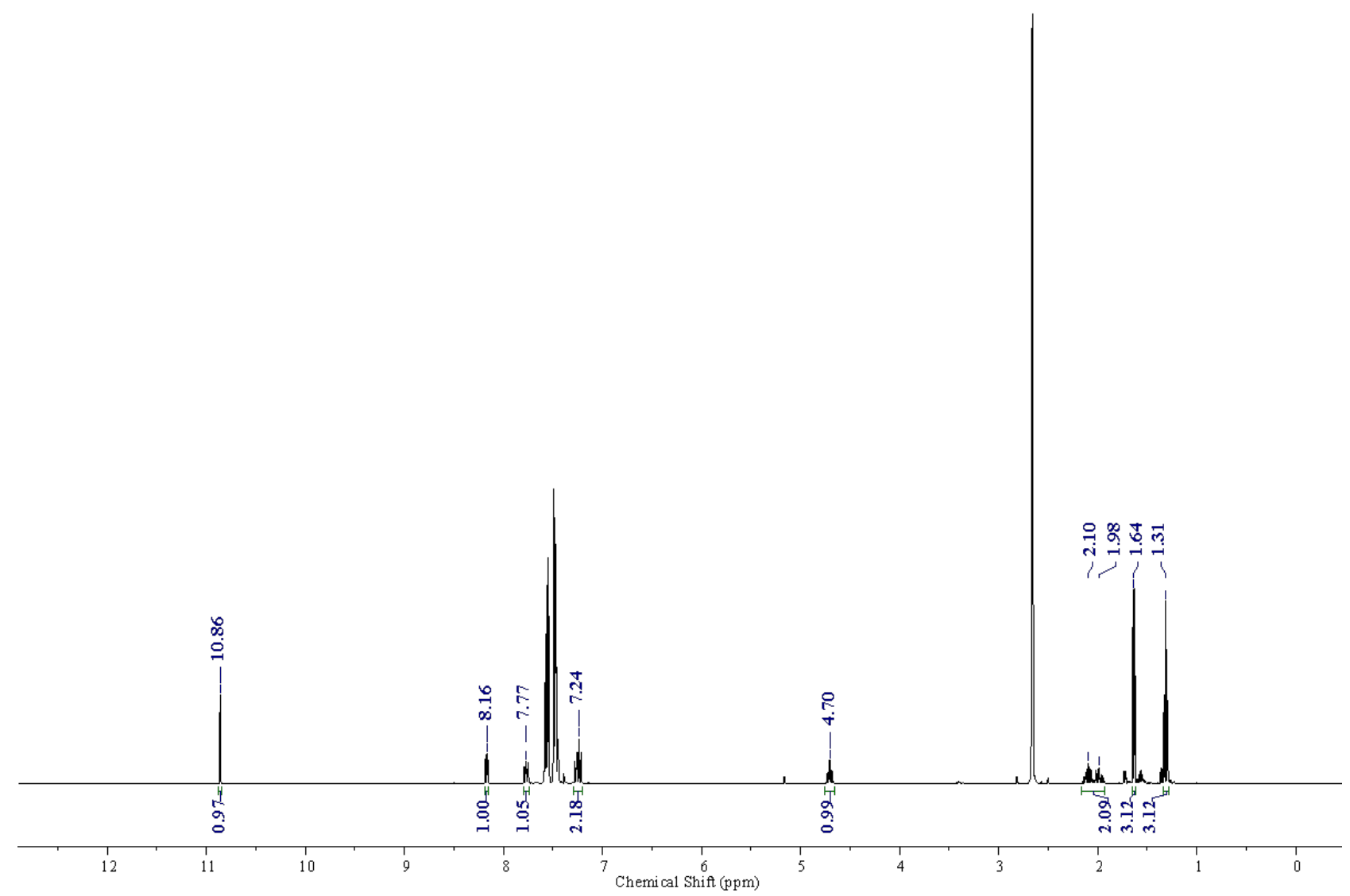

Entry 2: 120 minute, Set A

SCG-348-E3-2hr-D 1-50sec. 1.fid

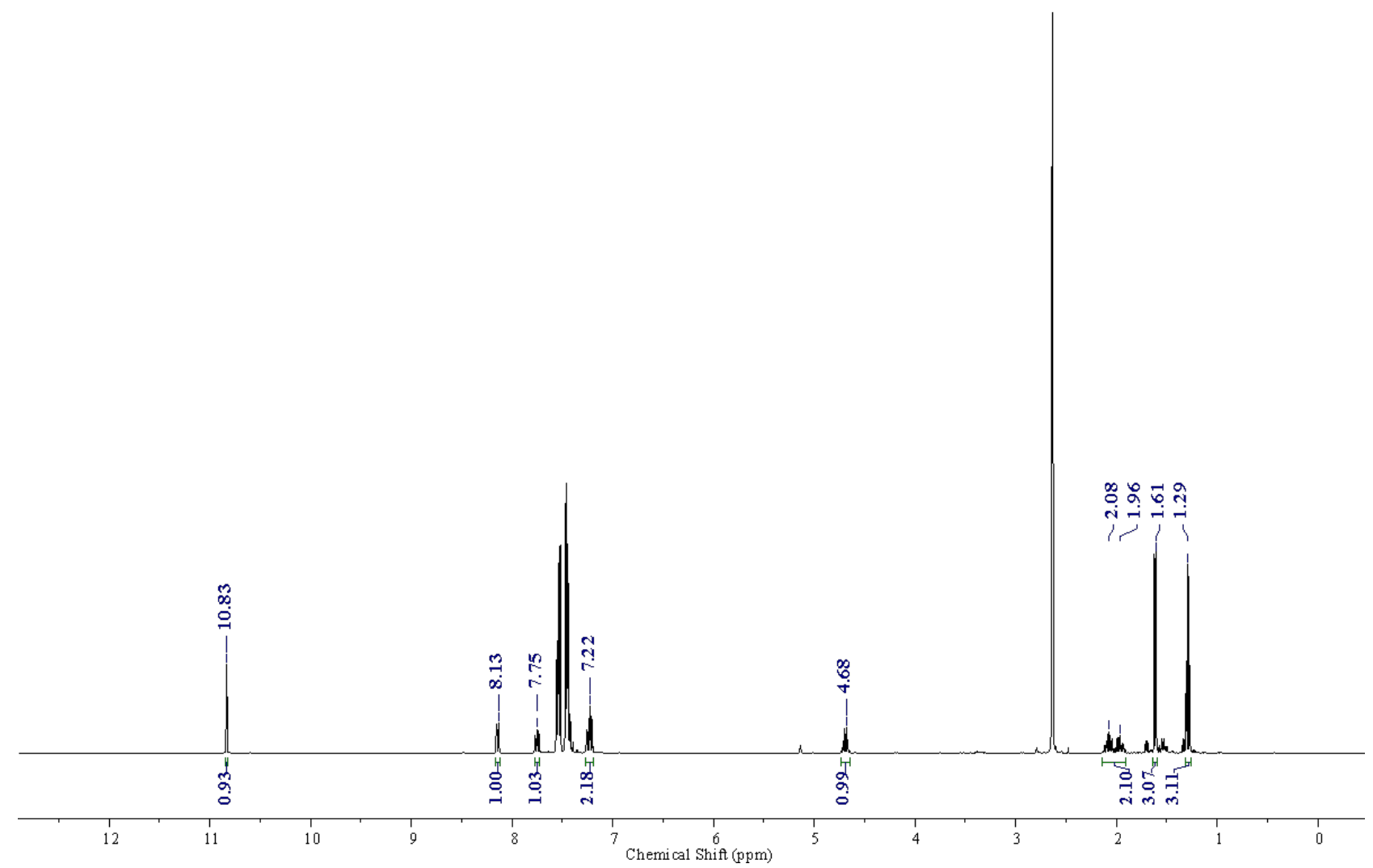


Entry 3: 180 minute, Set A

SCG-348-E5-3hr-D 1-50sec. 1.fid

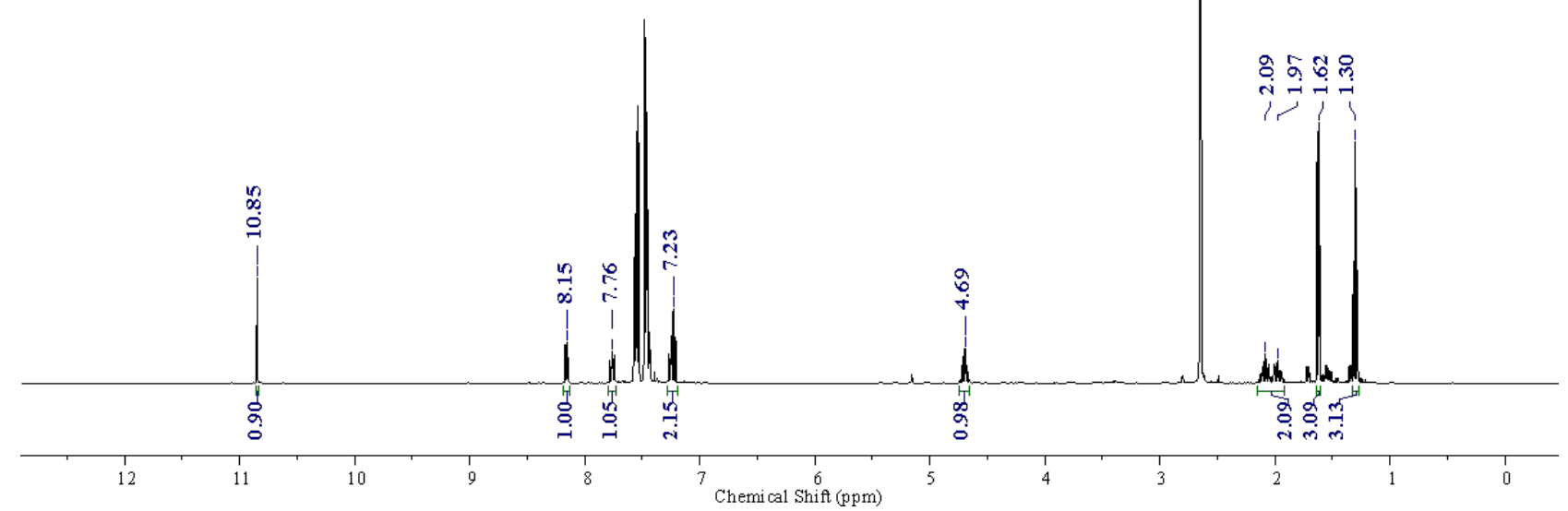

Entry 4: 240 minute, Set A

SCG-348-E7-4hr-D 1-50sec. 1.fid

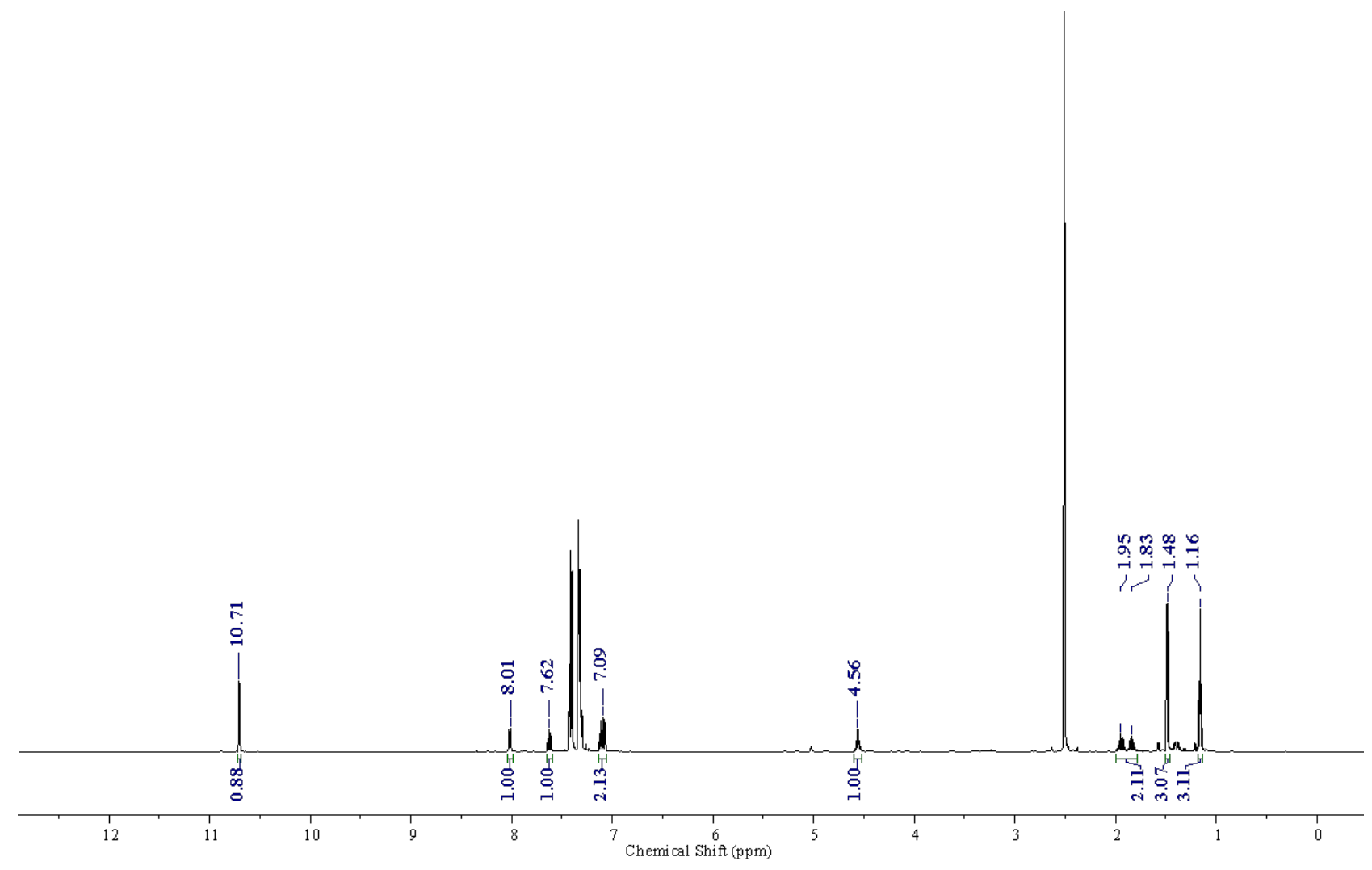


Entry 5: 300 minute, Set A

SCG-348-E9-5hr-D 1-50sec. 1.fid

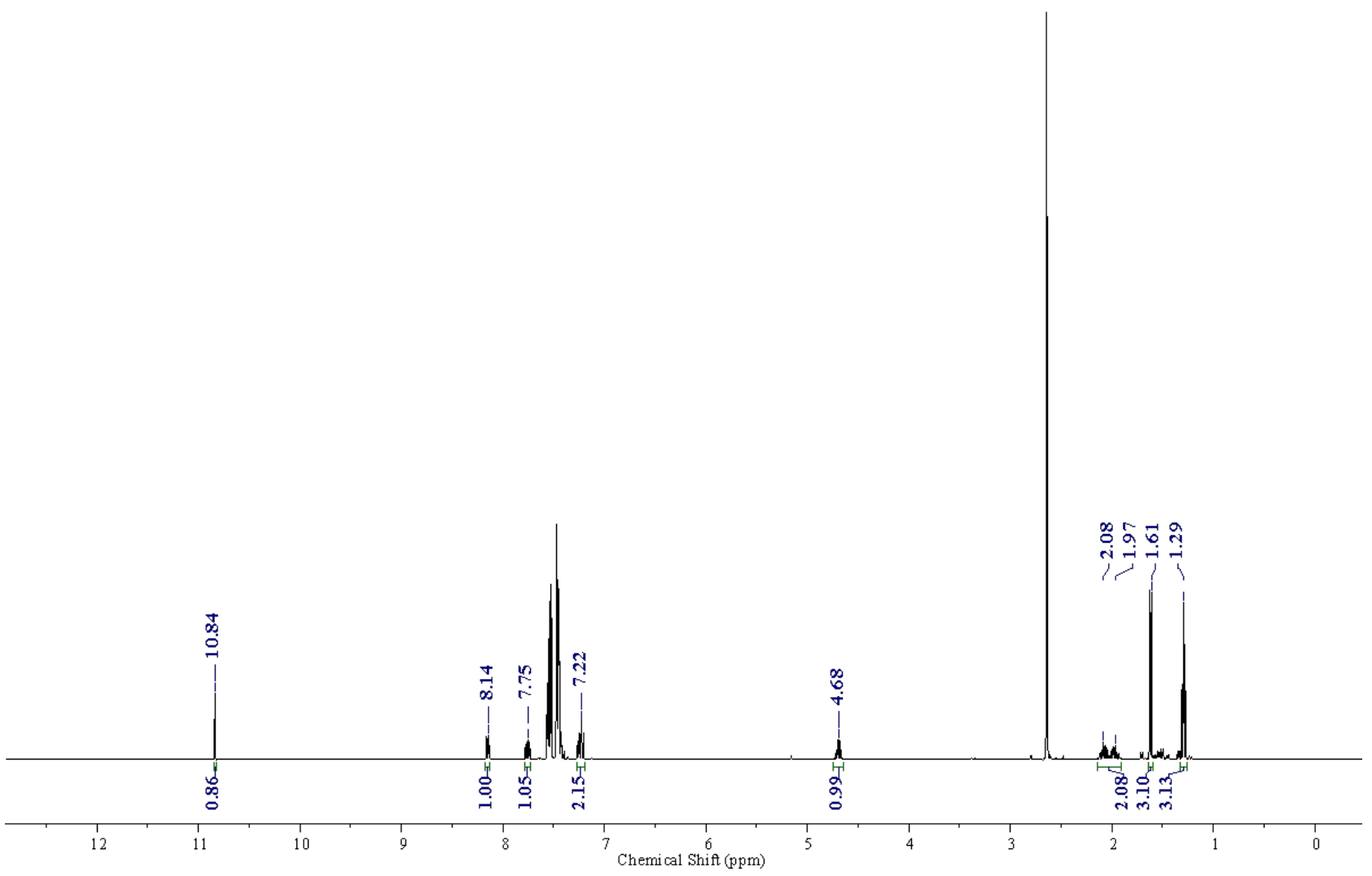


Initial Reaction Rate for 2-Ethynylbenzaldehyde:

Entry 1: 30 minute, Set A

ILZ-024-B 1.1. fid

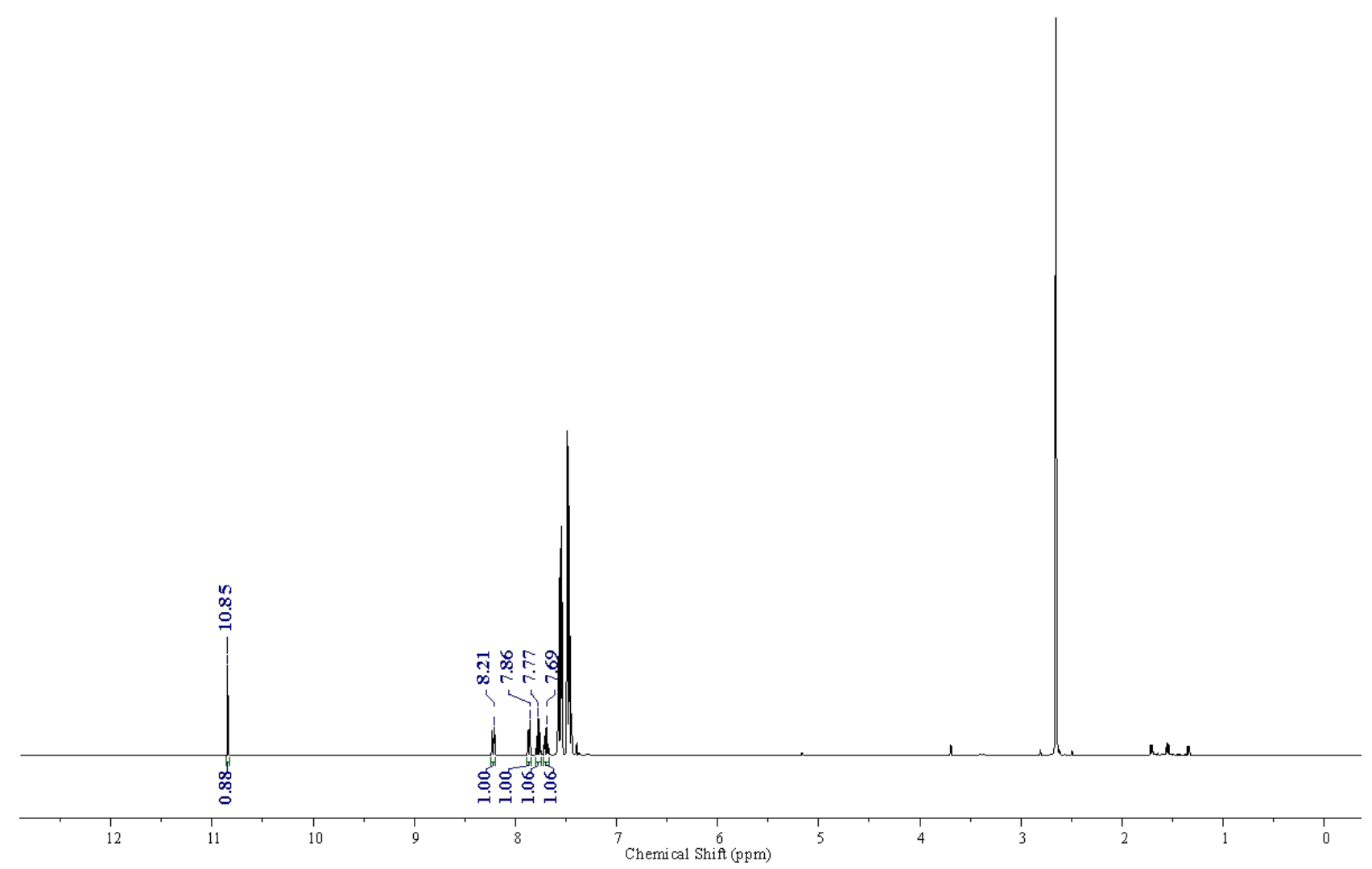

Entry 2: 60 minute, Set A

ILZ-024-B 2.1. fid

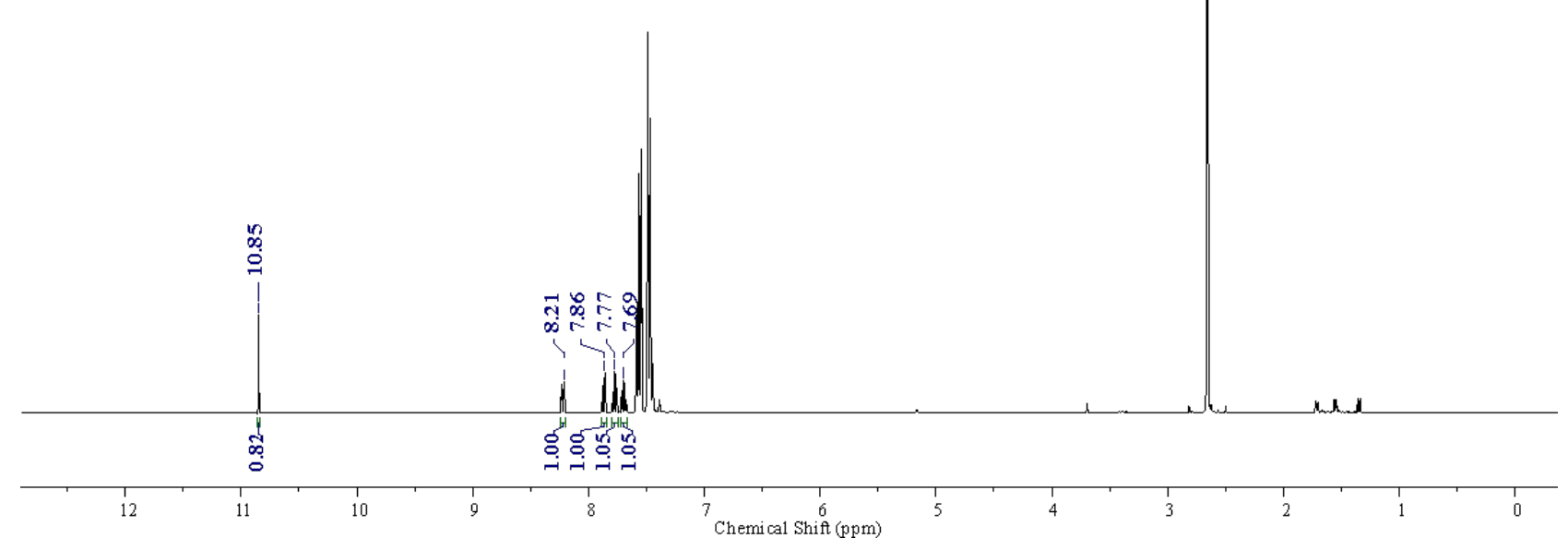


Entry 3: 90 minute, Set A

ILZ-024-B 3.1. fid

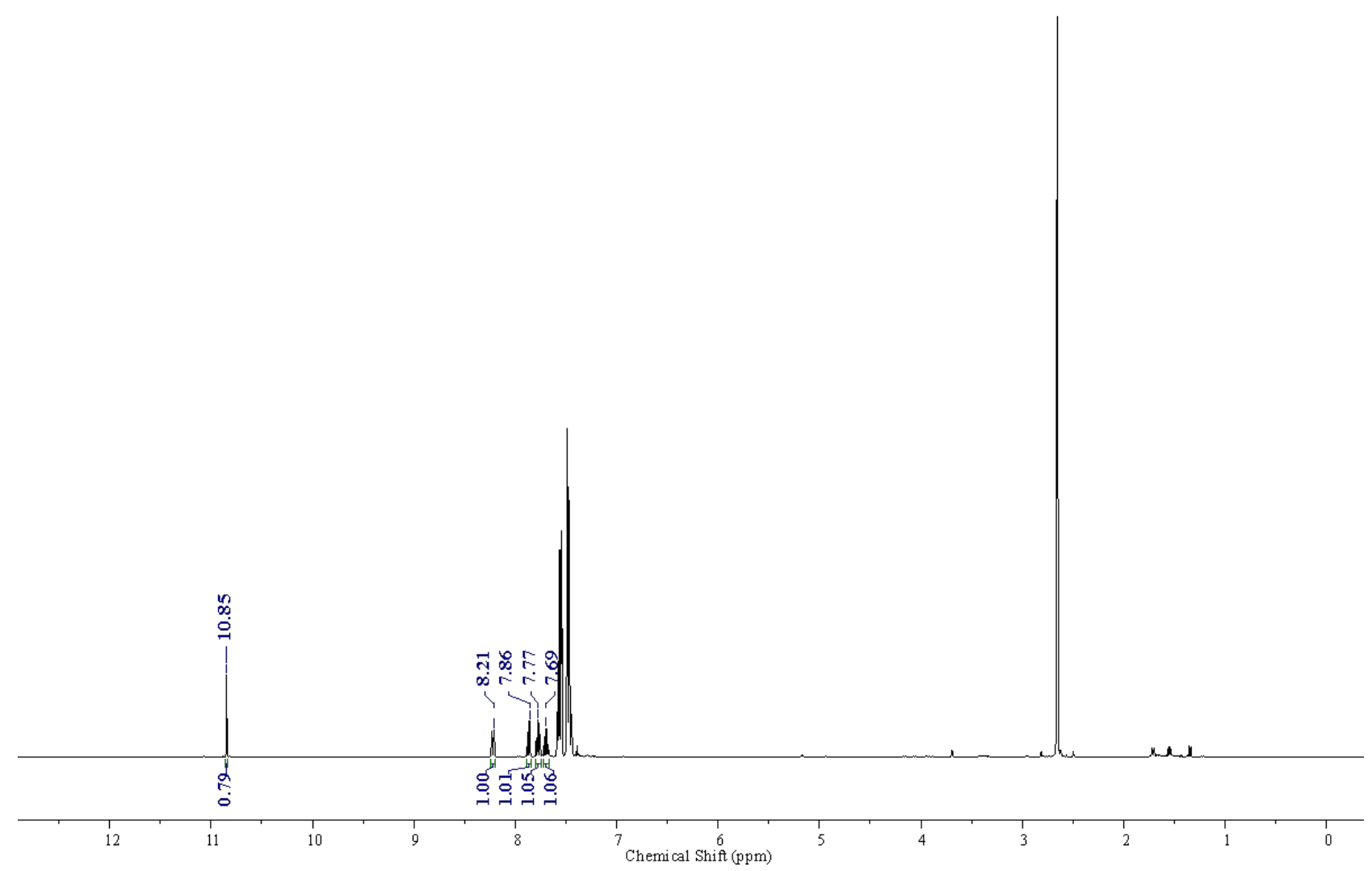

Entry 4: 120 minute, Set A

ILZ-024-B4.1.fid

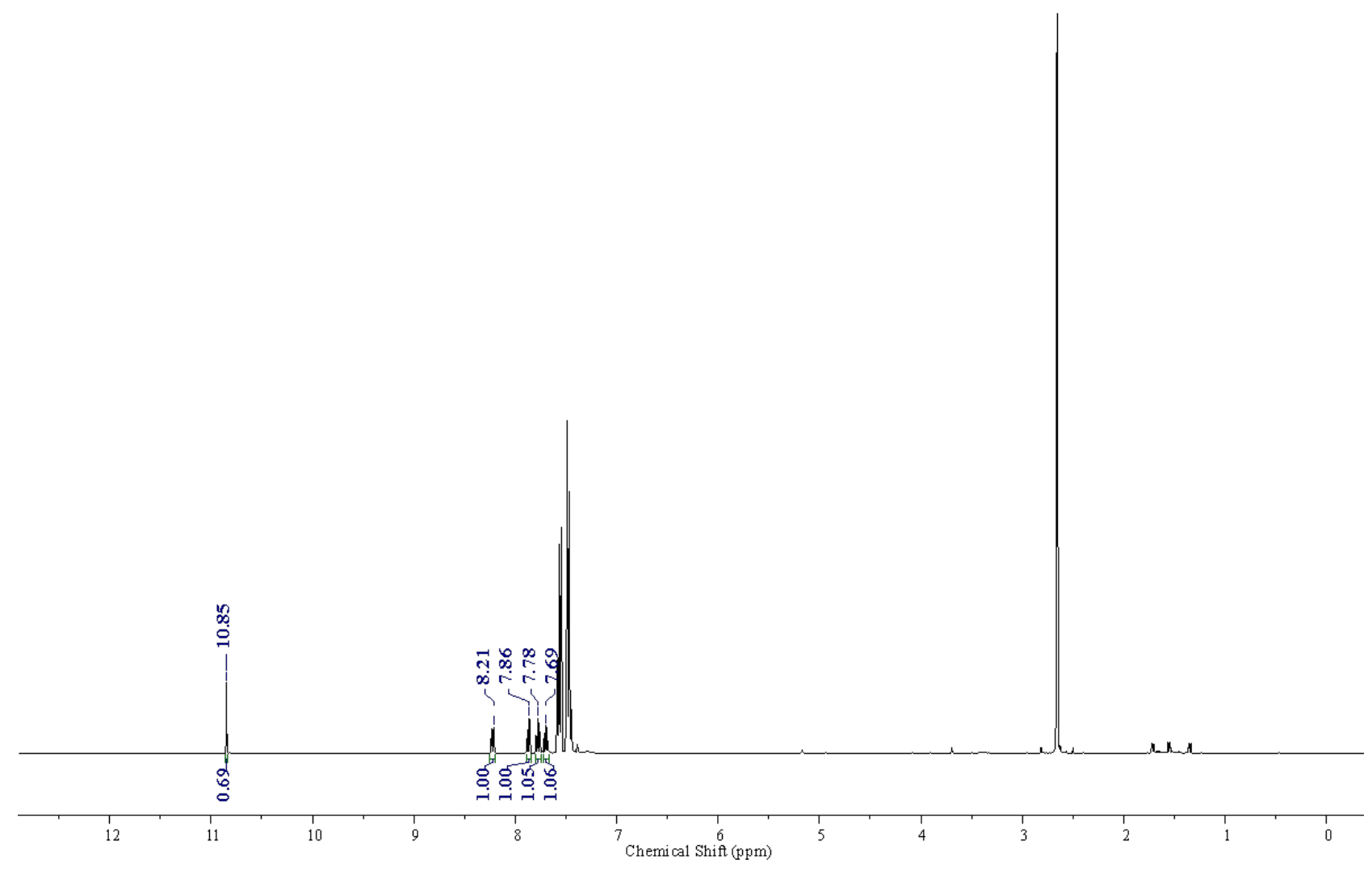


Entry 5: 150 minute, Set A IL Z-024-B 5. 1. fid

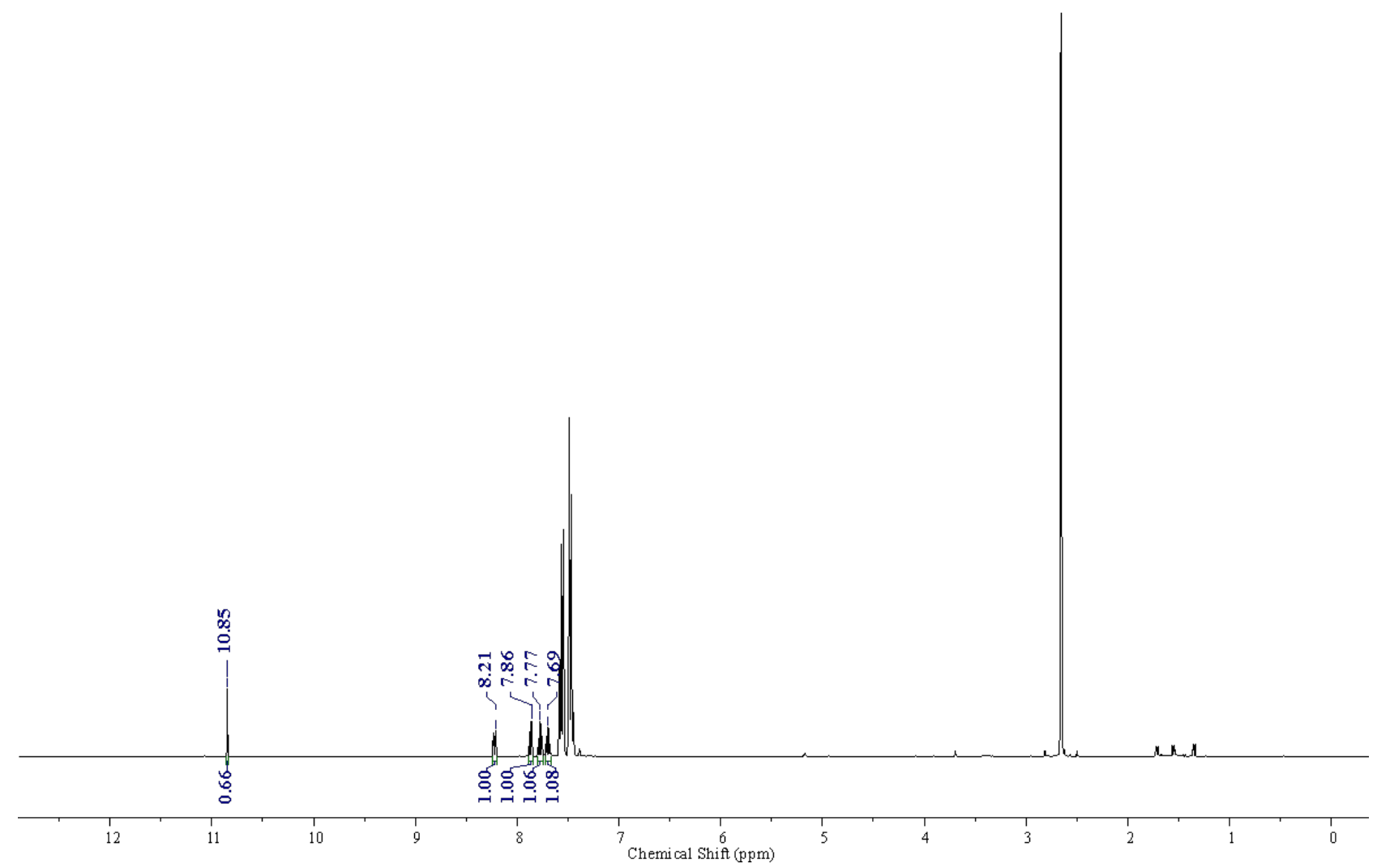


Initial Reaction Rate for 2-(Trifluoromethyl)benzaldehyde:

Entry 1: 5 minute, Set A

ILZ-017-E1.1. fid

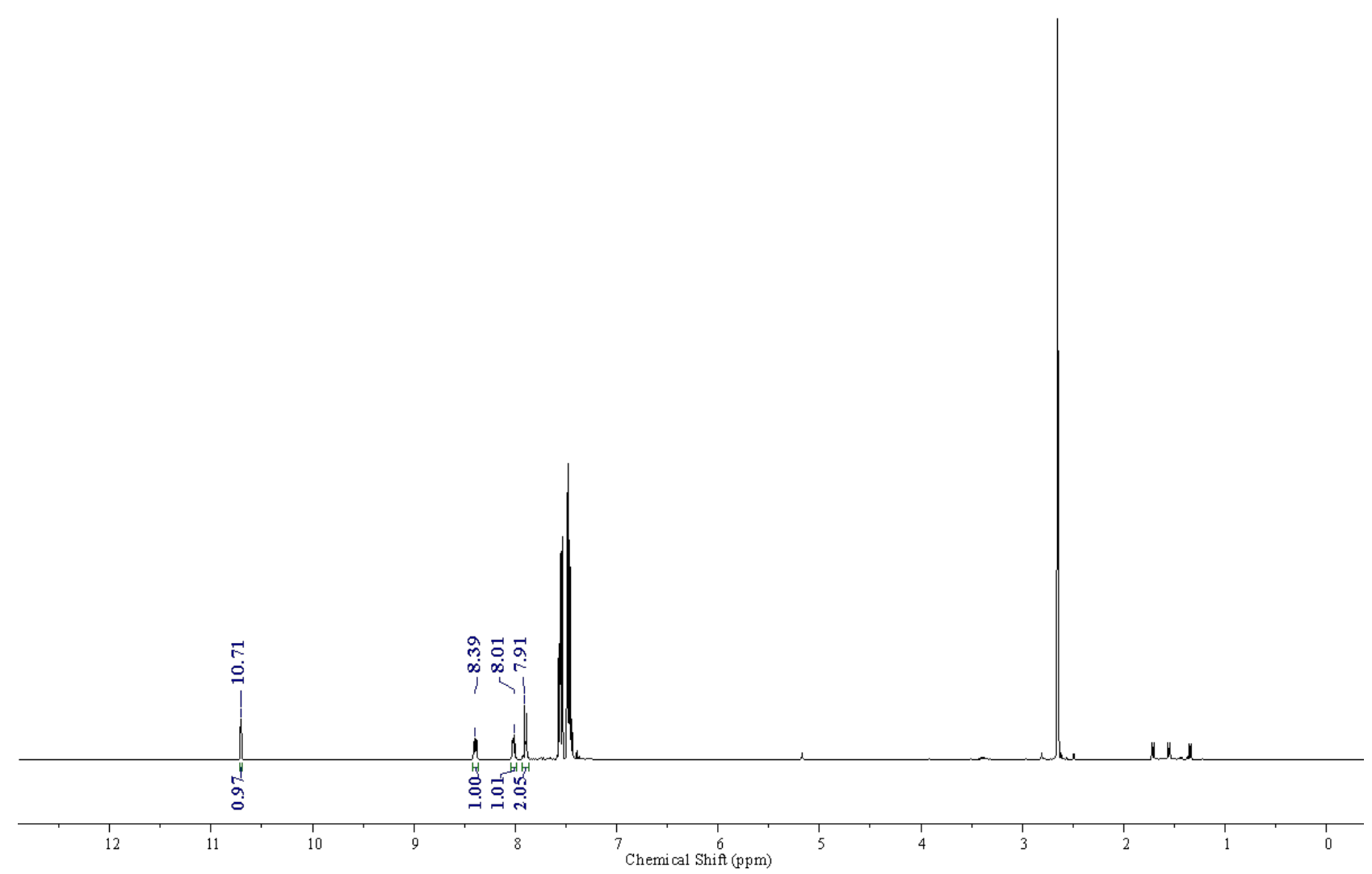

Entry 2: 10 minute, Set A

ILZ-017-E2.1.fid

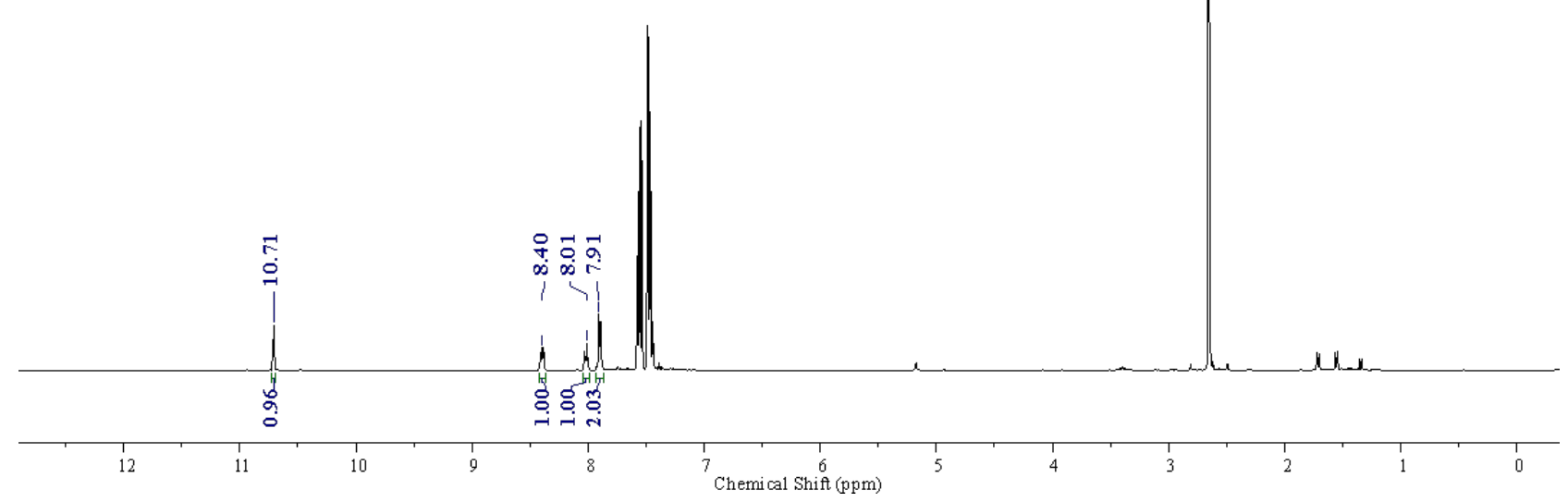


Entry 3: 15 minute, Set A

ILZ-017-E3.1.fid

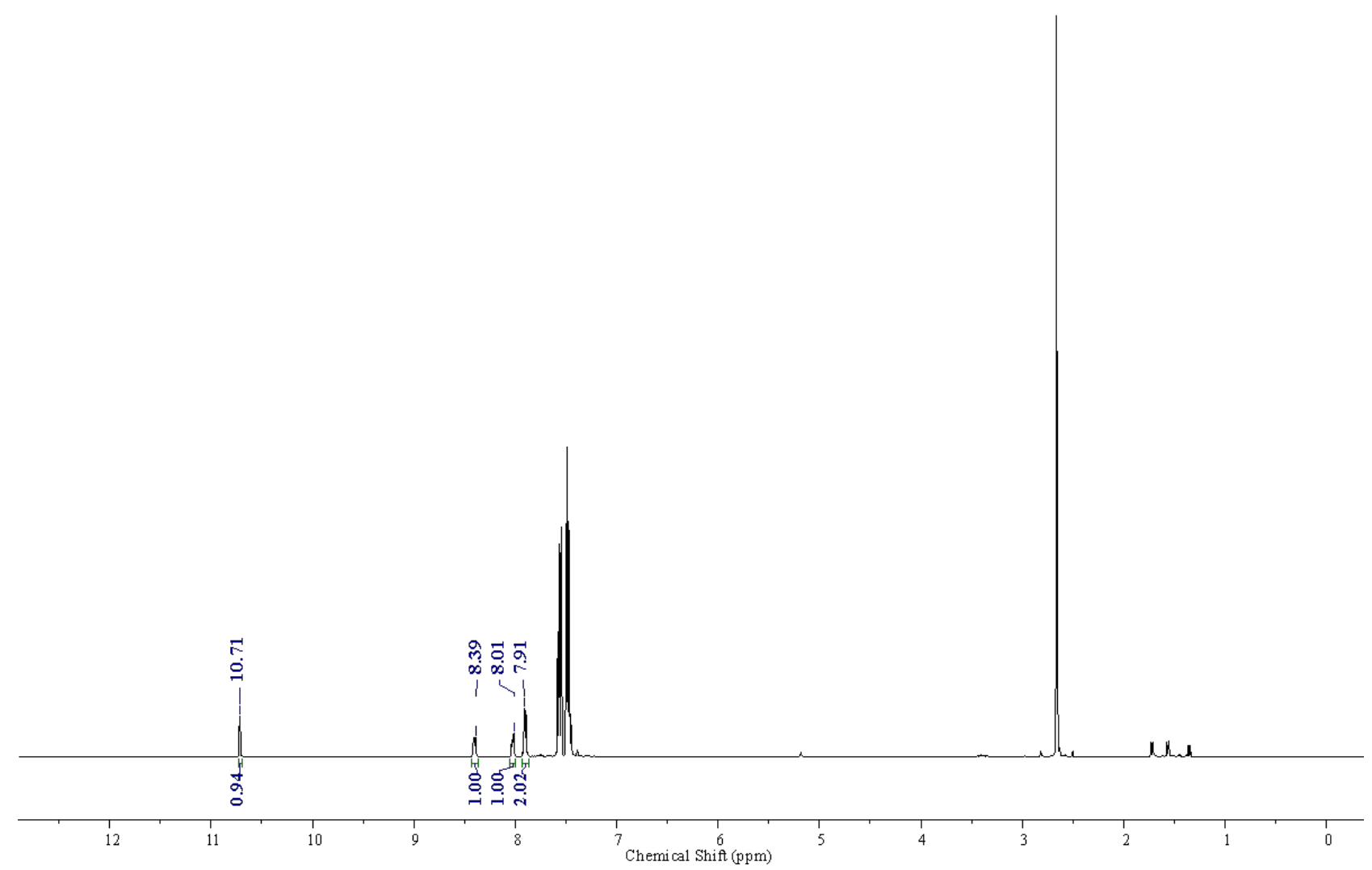

Entry 4: 20 minute, Set A

ILZ-017-E4.1. fid

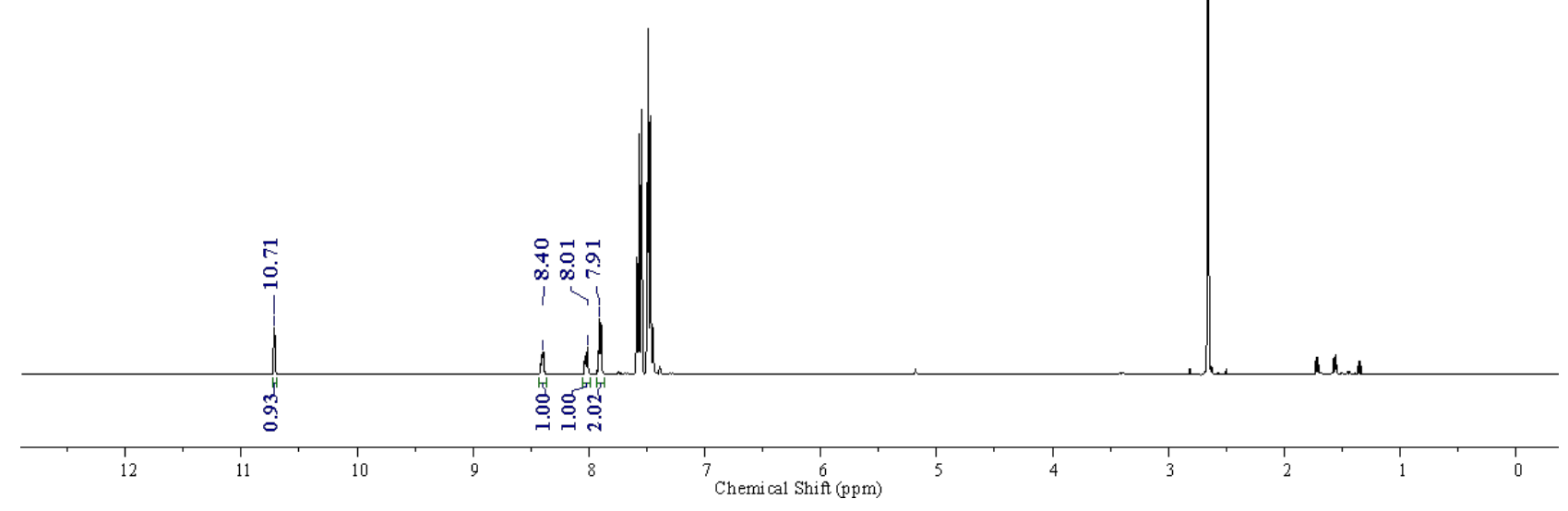


Entry 5: 25 minute, Set A ILZ-017-E5.1.fid

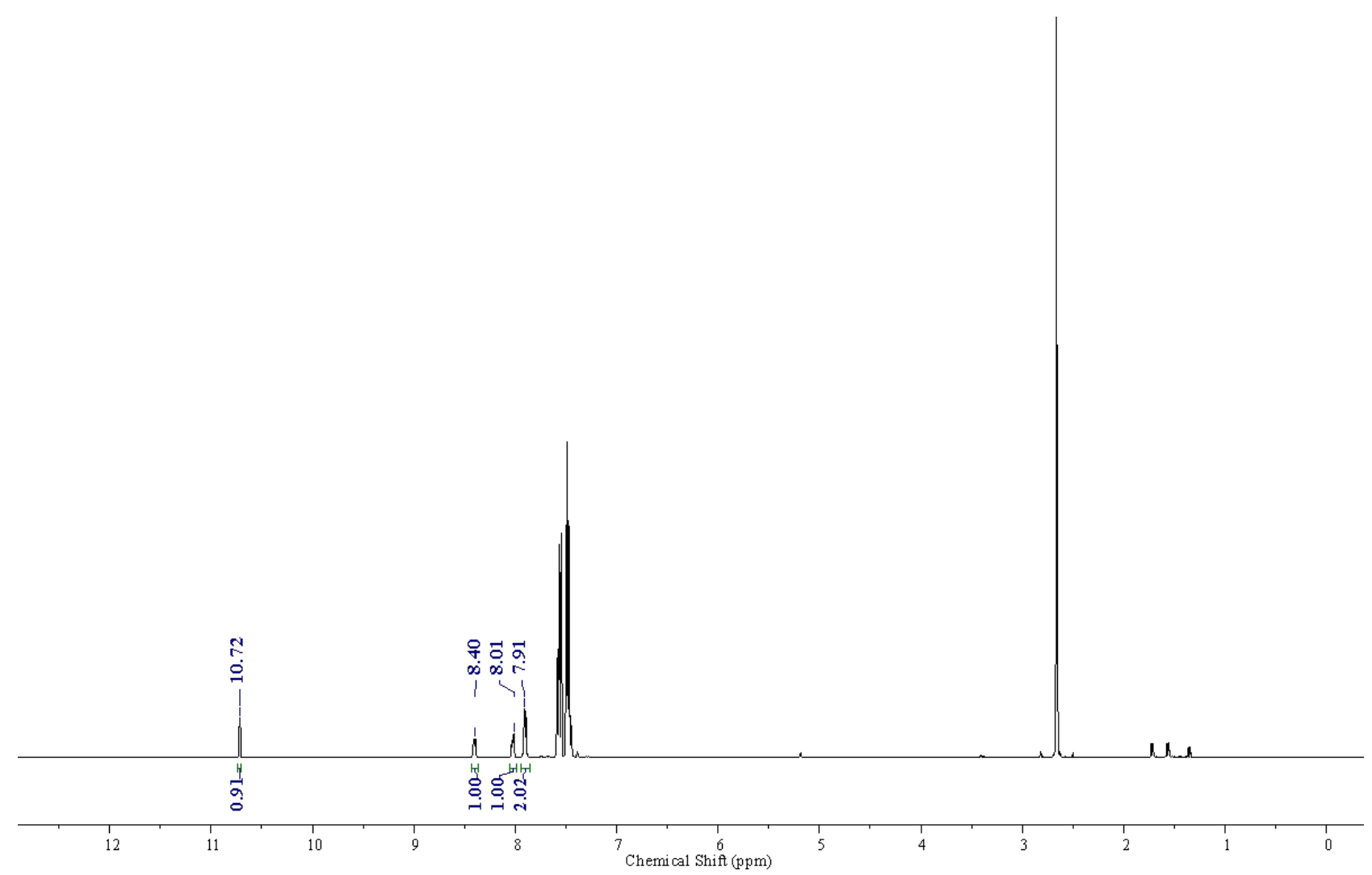


Initial Reaction Rate for 2-Chlorobenzaldehyde-d

Entry 1: 5 minute, Set A

SCG-325-A1-5min-D 1-50sec. 1.fid

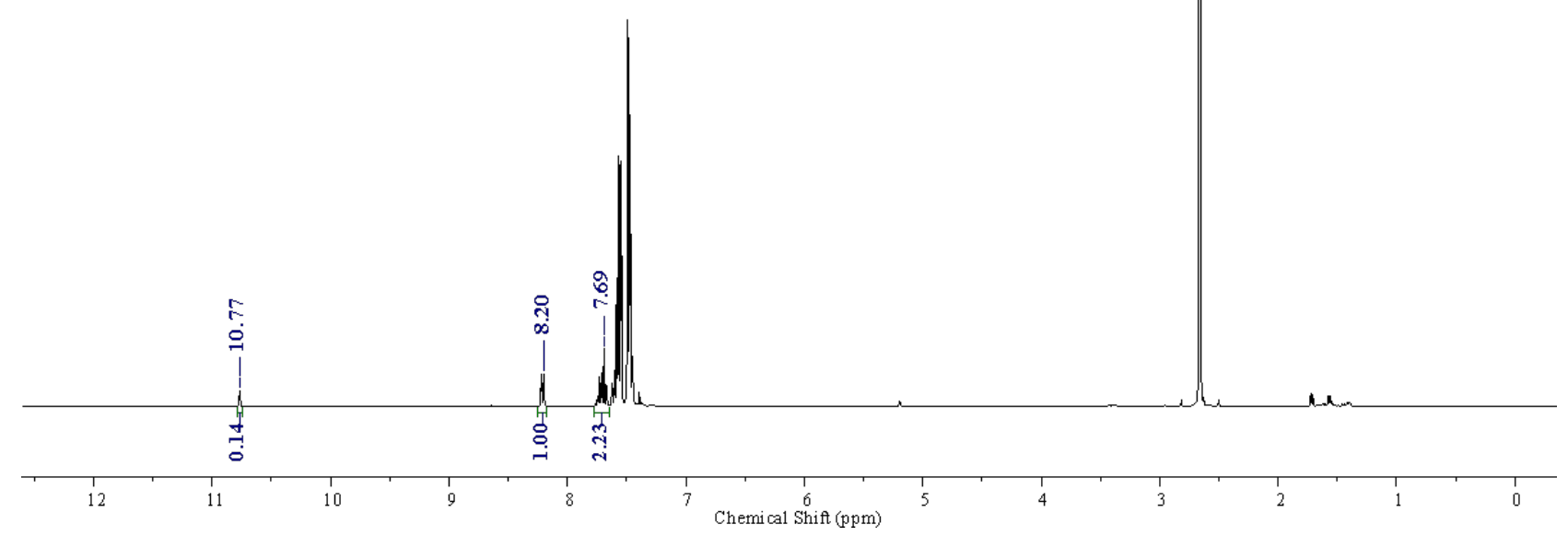

Entry 2: 10 minute, Set A

SCG-325-A2-10min-D 1-50sec. 1.fid

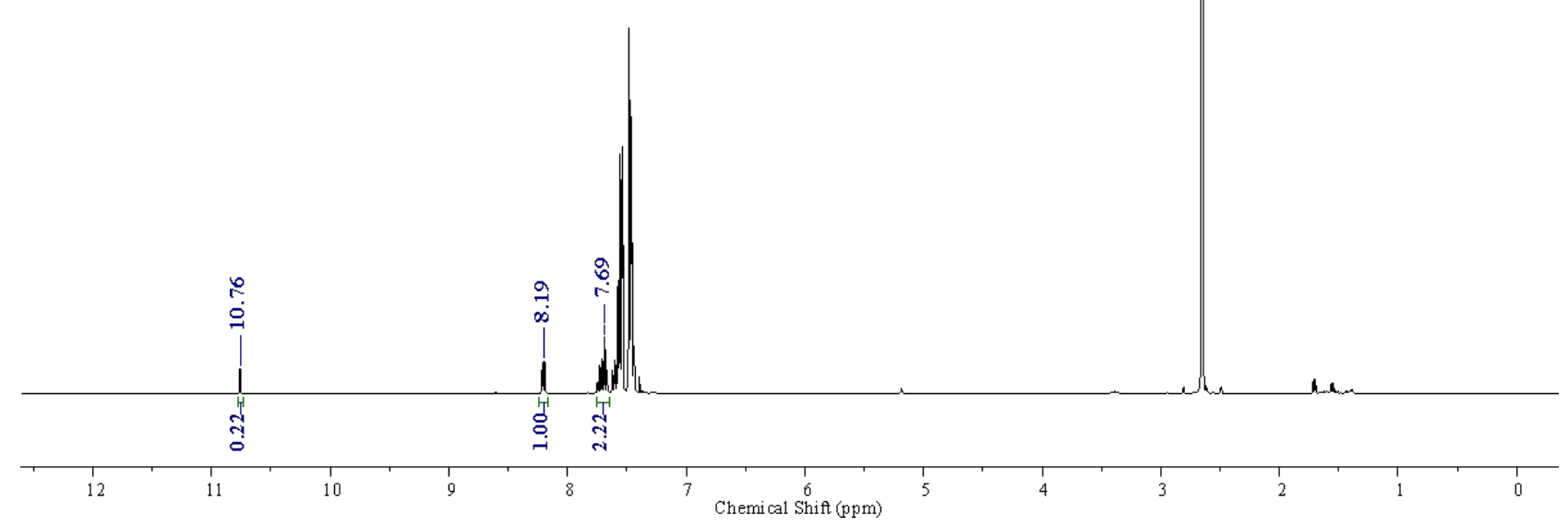


Entry 3: 15 minute, Set A

SCG-325-A3-15min-D 1-50sec. 1. fid

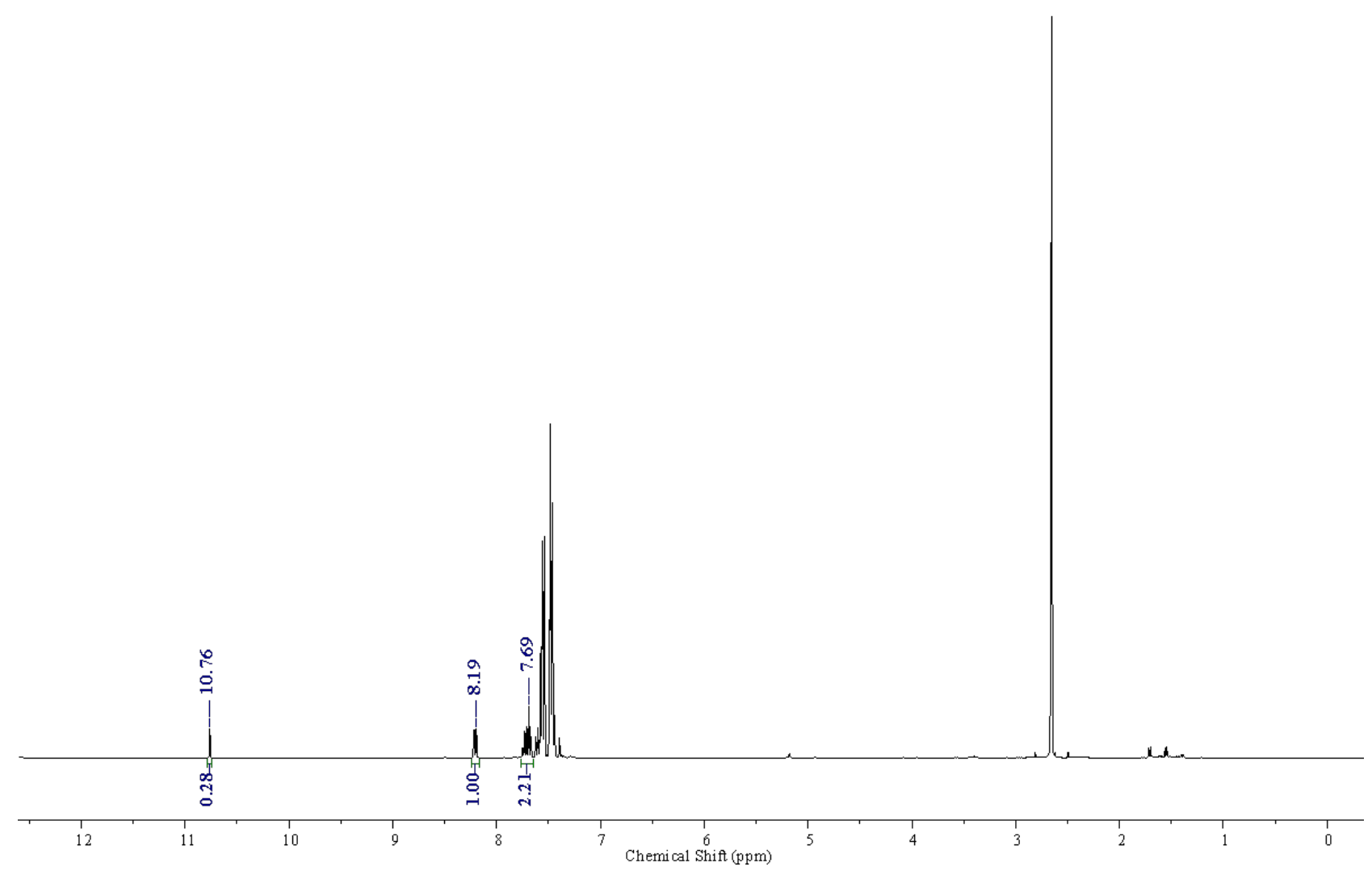

Entry 4: 20 minute, Set A

SCG-325-A.4-20min-D 1-50sec. 1. fid

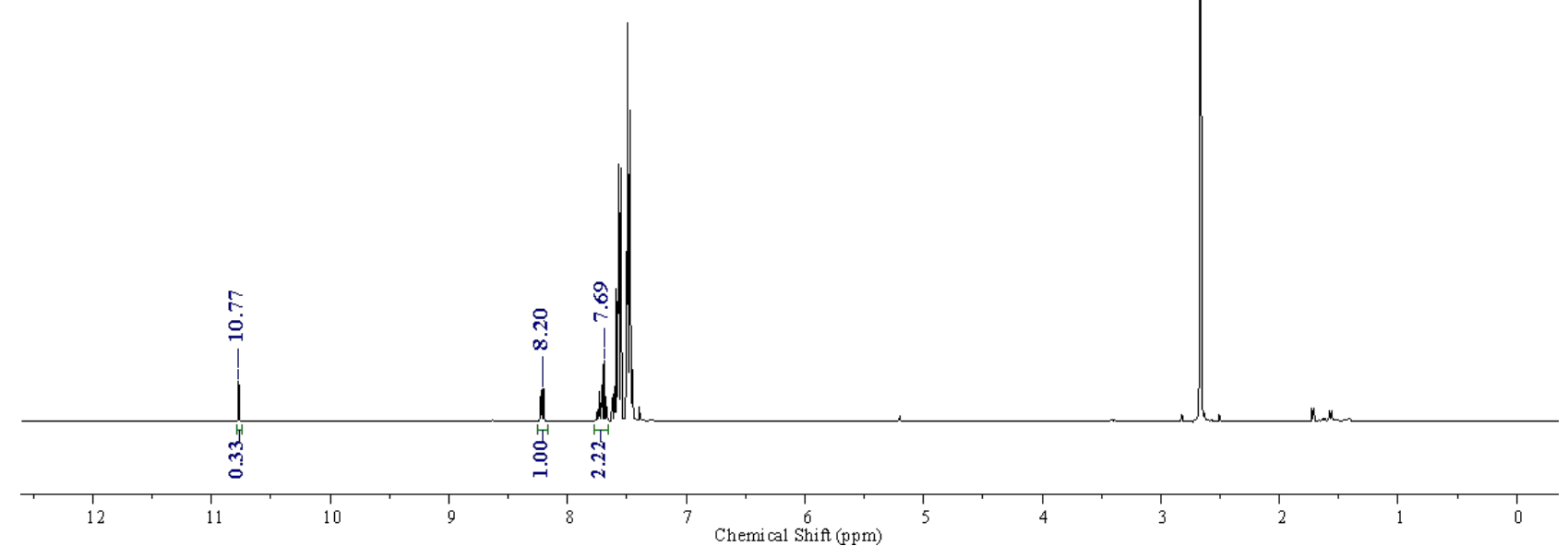


Entry 5: 25 minute, Set A

SCG-325-A 5-25min-D 1-50sec. 1. fid

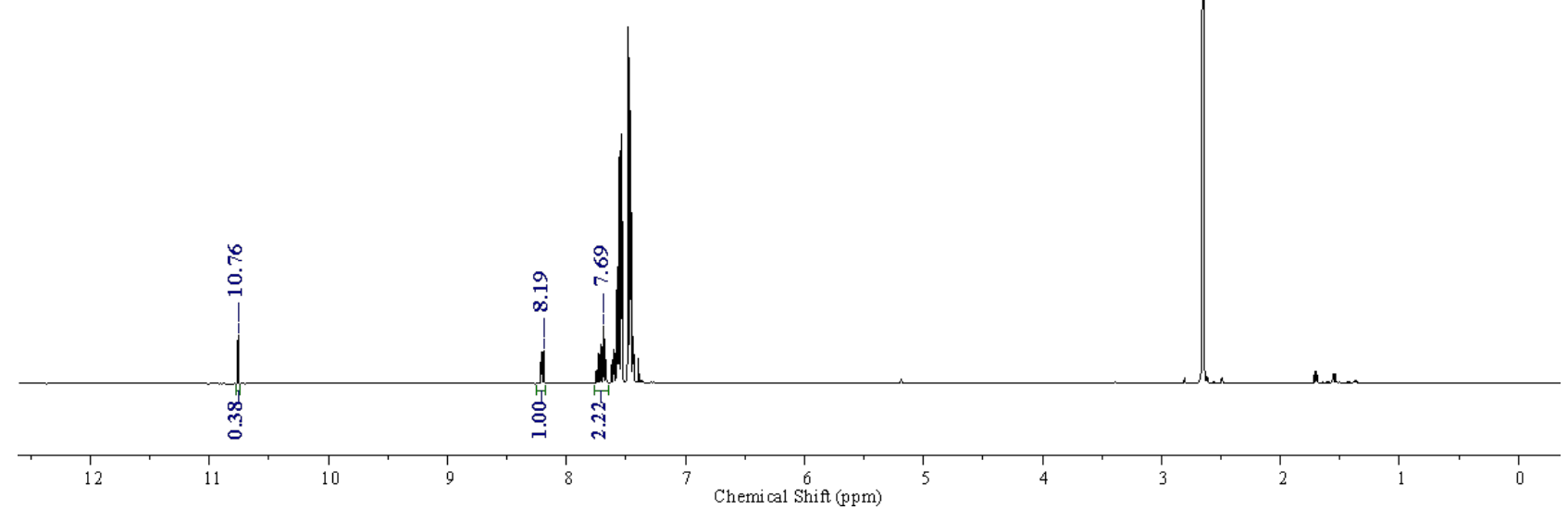


Reactions with different triazolium NHCs:

Entry 1: with 2,4,6-Triboromo-Phenyl-NHC (4a)

SCG-341-B 1-16hr-D 1-50sec.1.fid

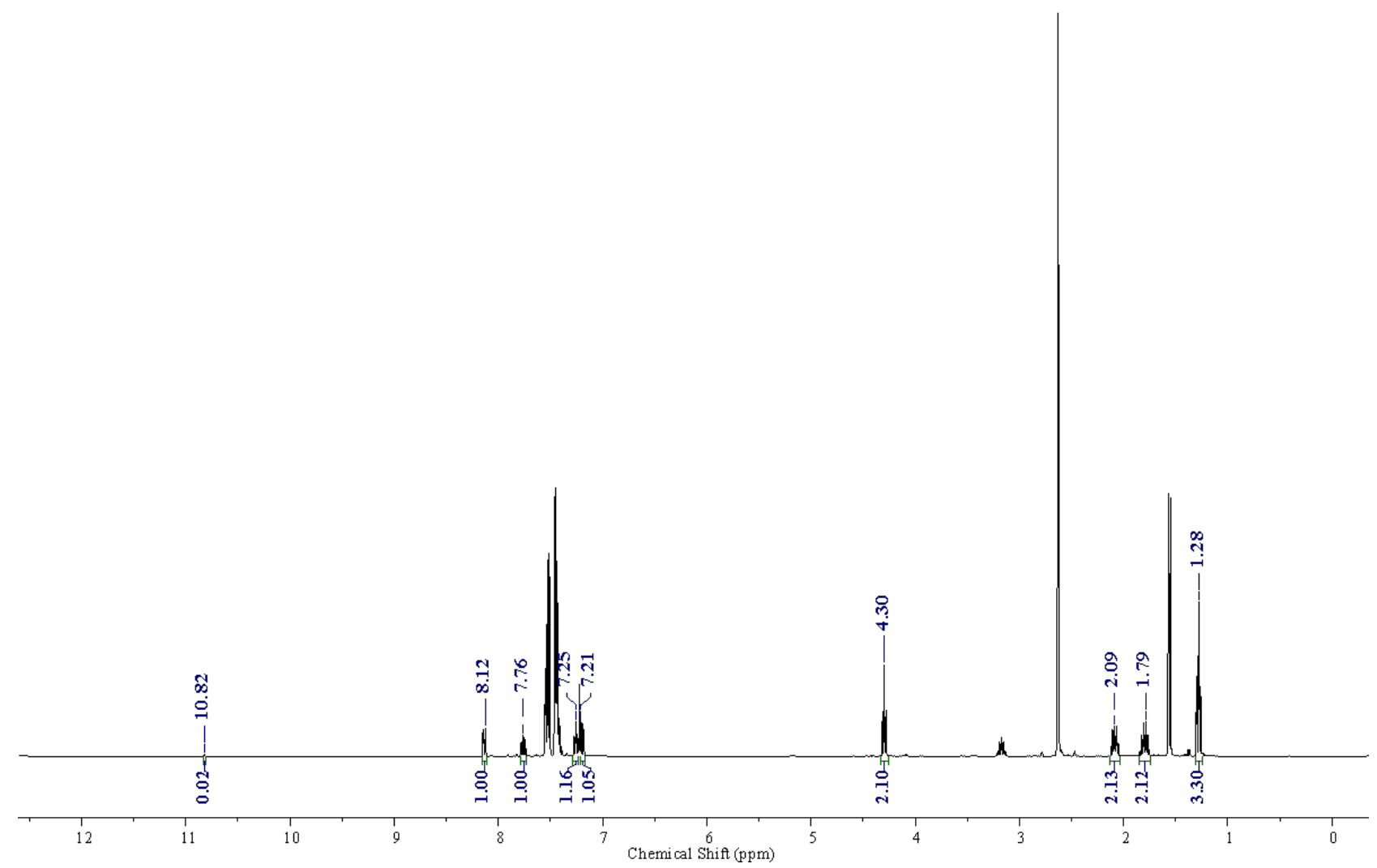

Entry 1': with 2,4,6-Triboromo-Phenyl-NHC (4a), Duplicate SCG-341-B 2-16hr-D 1-50sec.1. fid

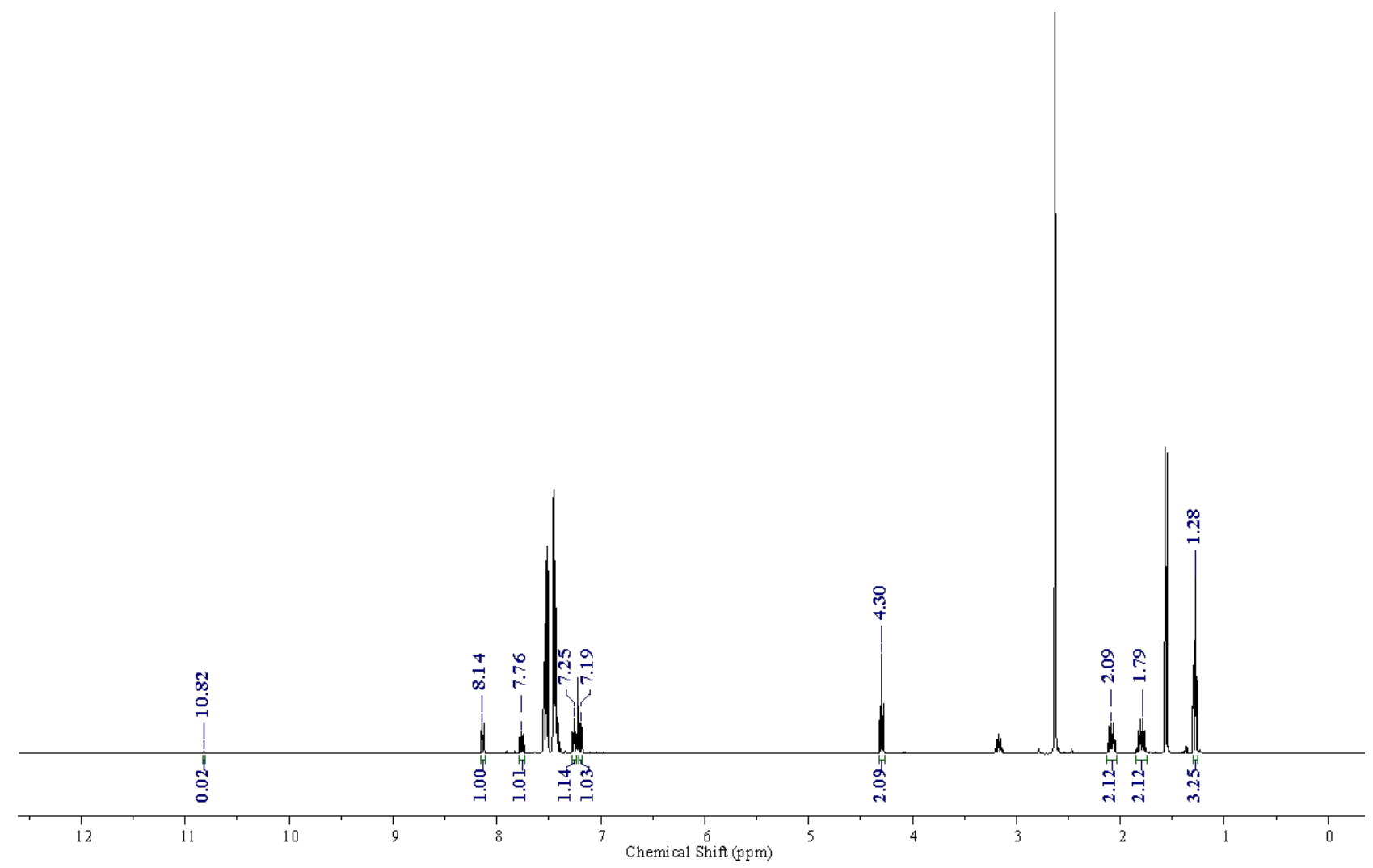


Entry 2: with 2,4,6-Trichloro-Phenyl-NHC (4b)

SCG-341-J1-16hr-D 1-50sec.1. fid

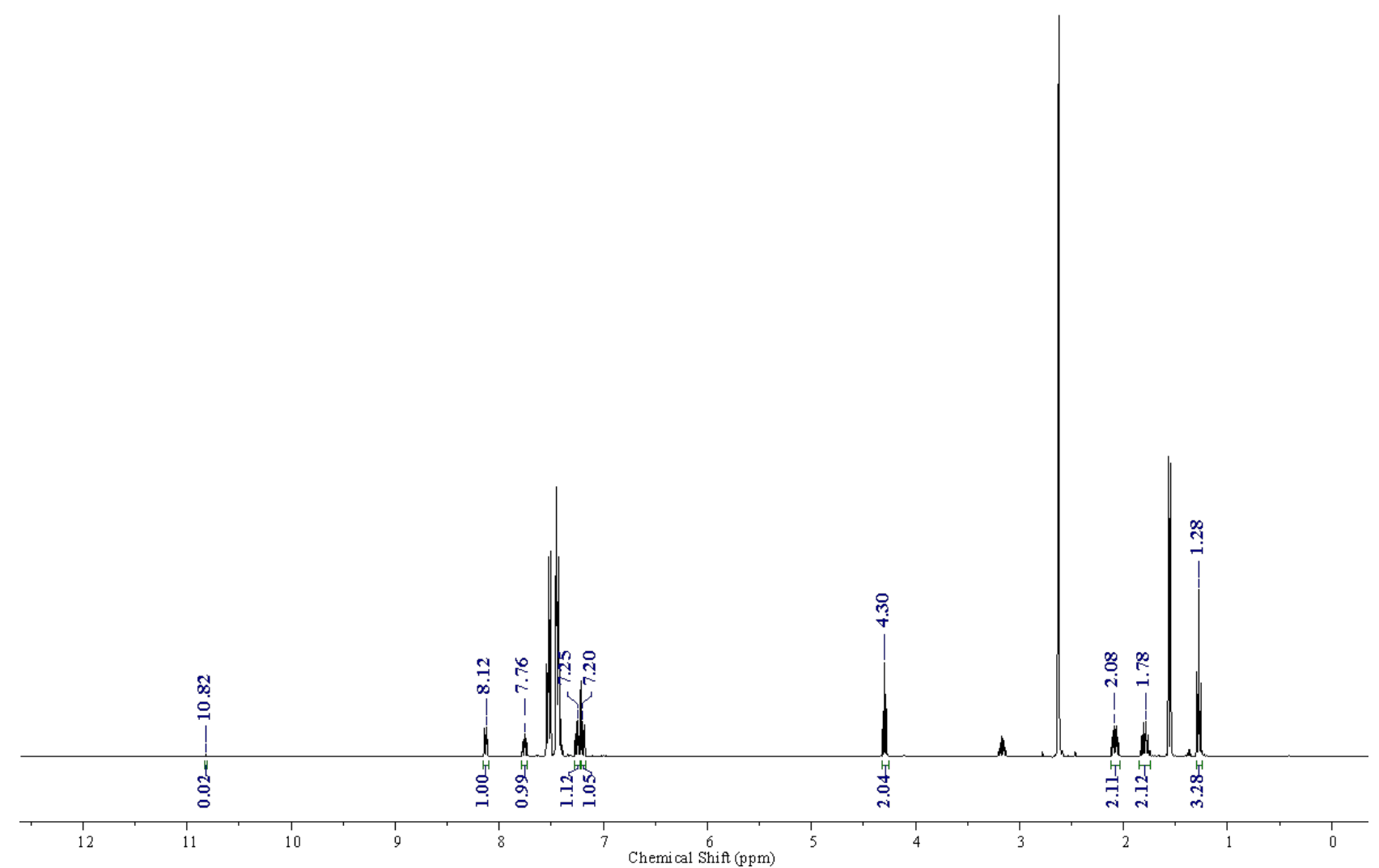

Entry 2': with 2,4,6- Trichloro-Phenyl-NHC (4b), Duplicate

SCG-341-J2-16hr-D 1-50sec.1.fid

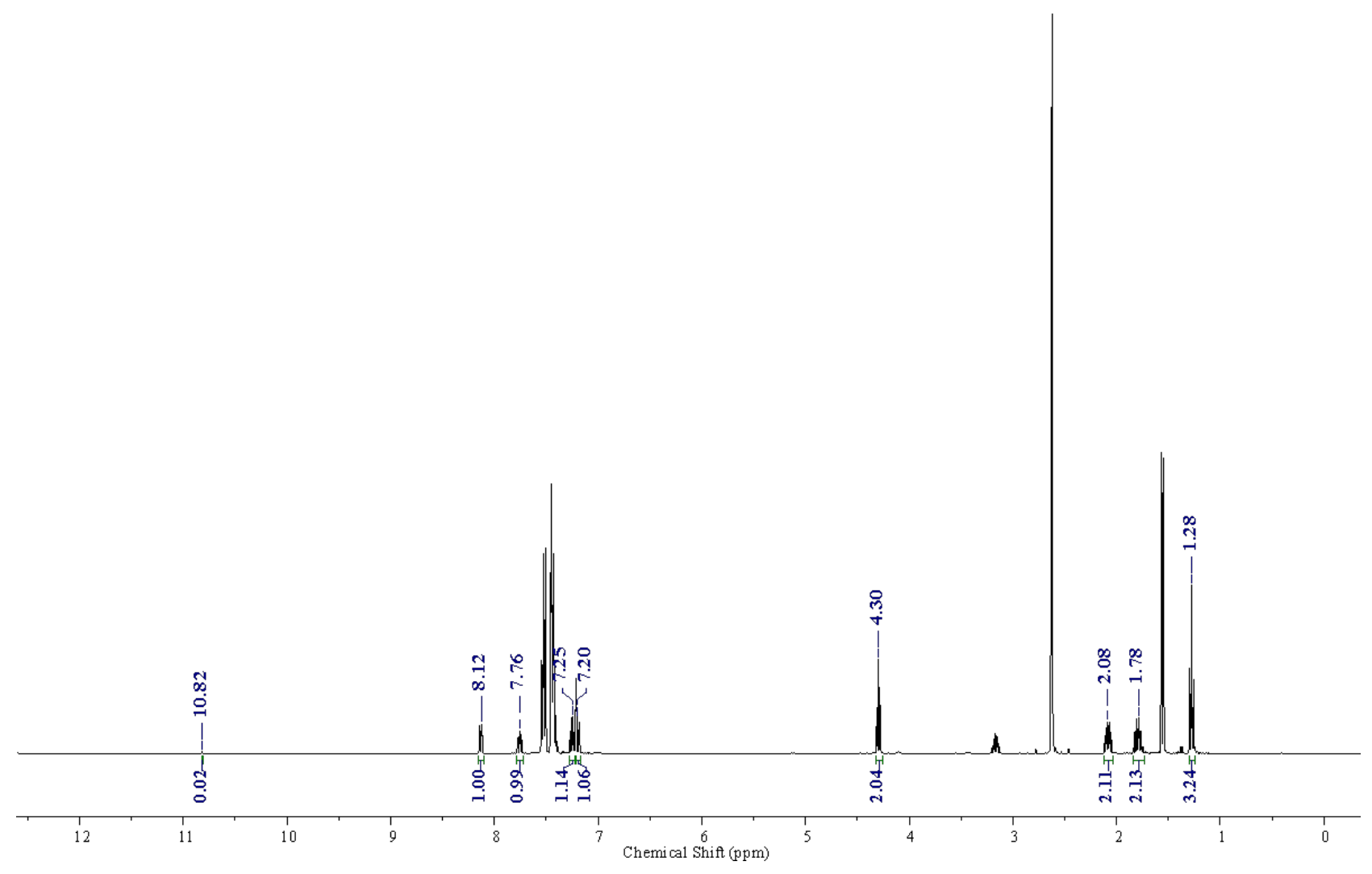


Entry 3: with 2,6-Dimethyl-Phenyl-NHC (4c)

SCG-341-F1-16hr-D 1-50sec. 1.fid

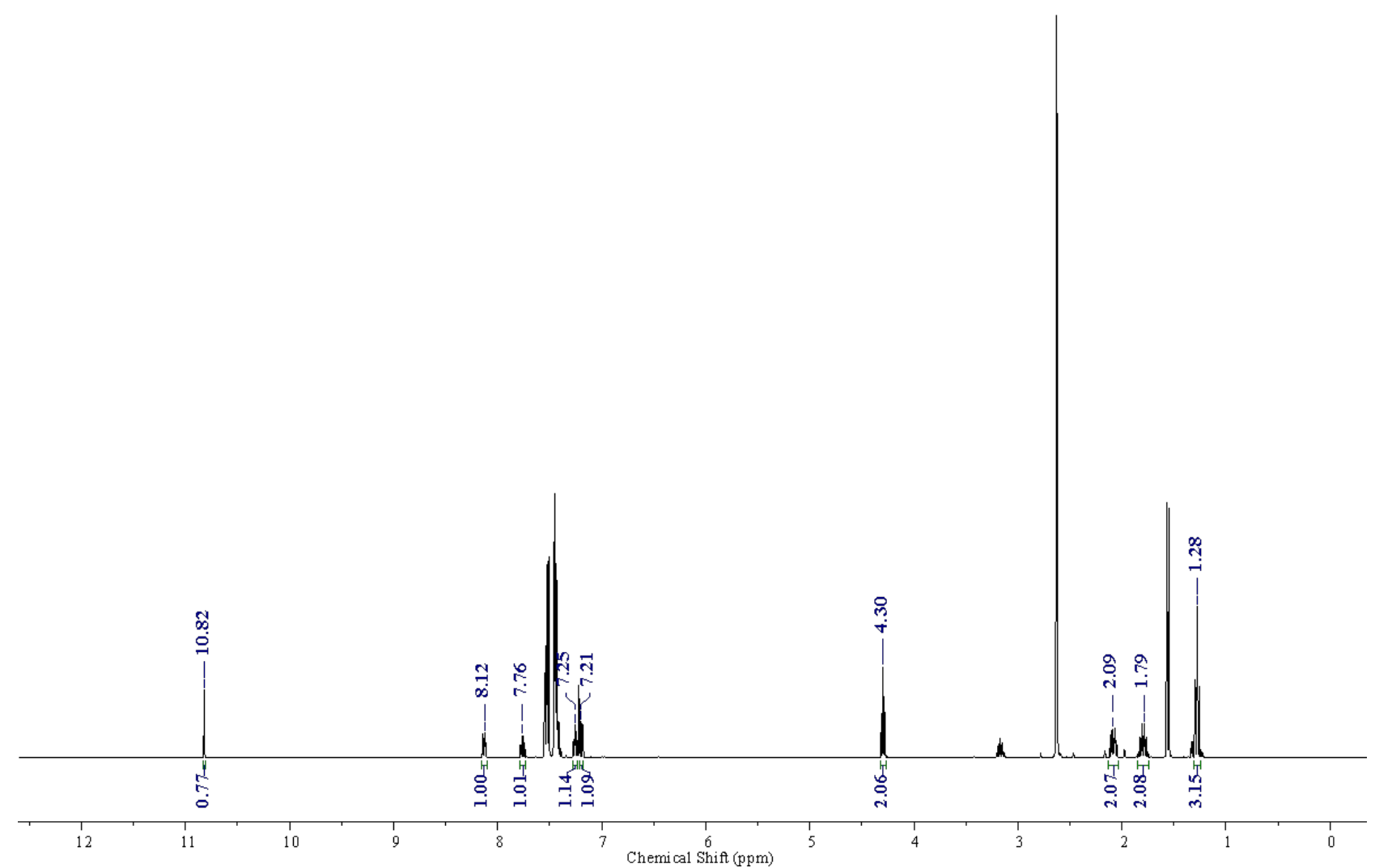

Entry 3': with 2,6-Dimethyl-Phenyl-NHC (4c), Duplicate

SCG-341-F2-16hr-D 1-50sec. 1.fid

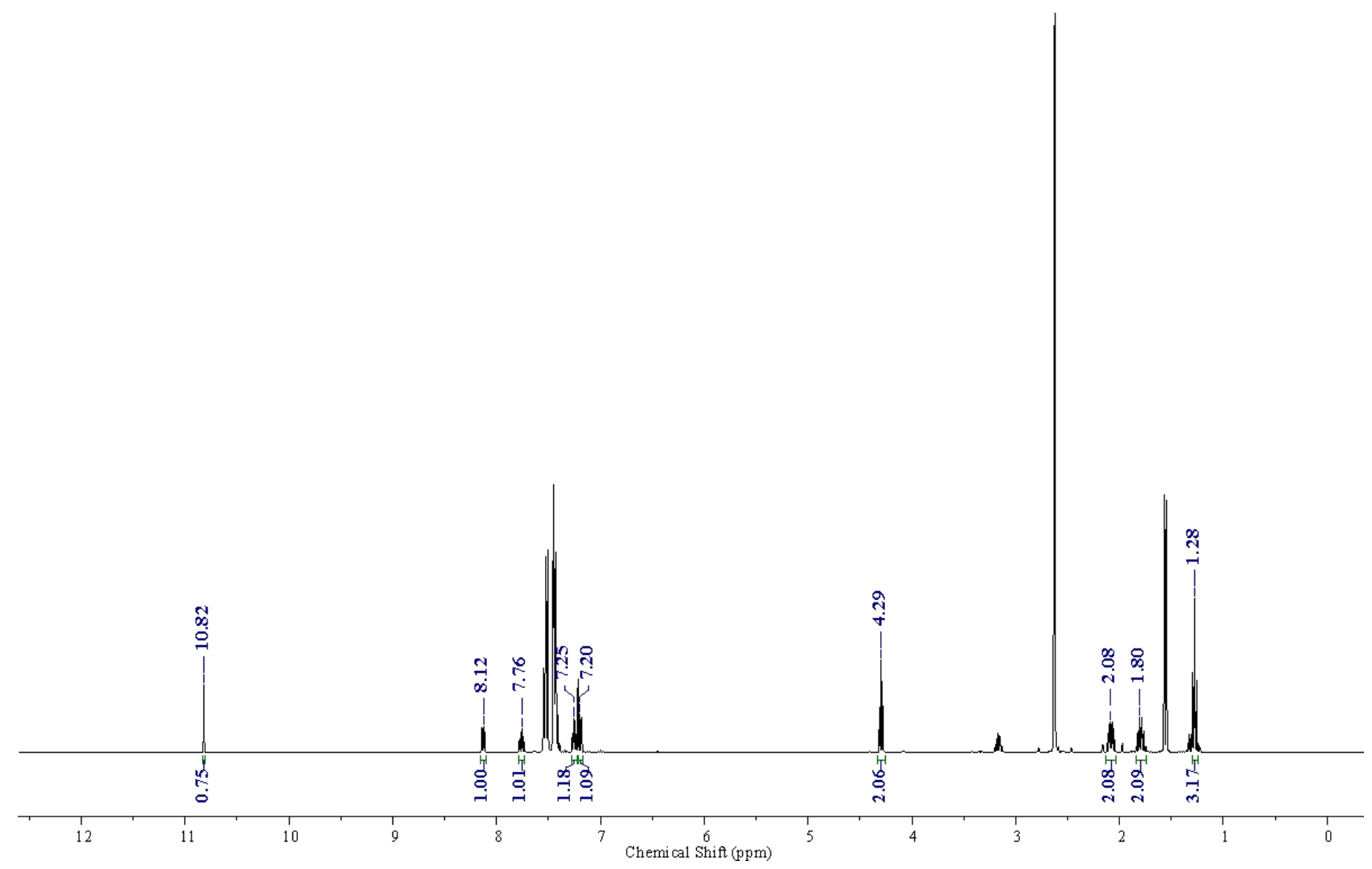


Entry 4: with 2-Methoxy-Phenyl-NHC (4d)

SCG-341-G1-16hr-D 1-50sec.1.fid

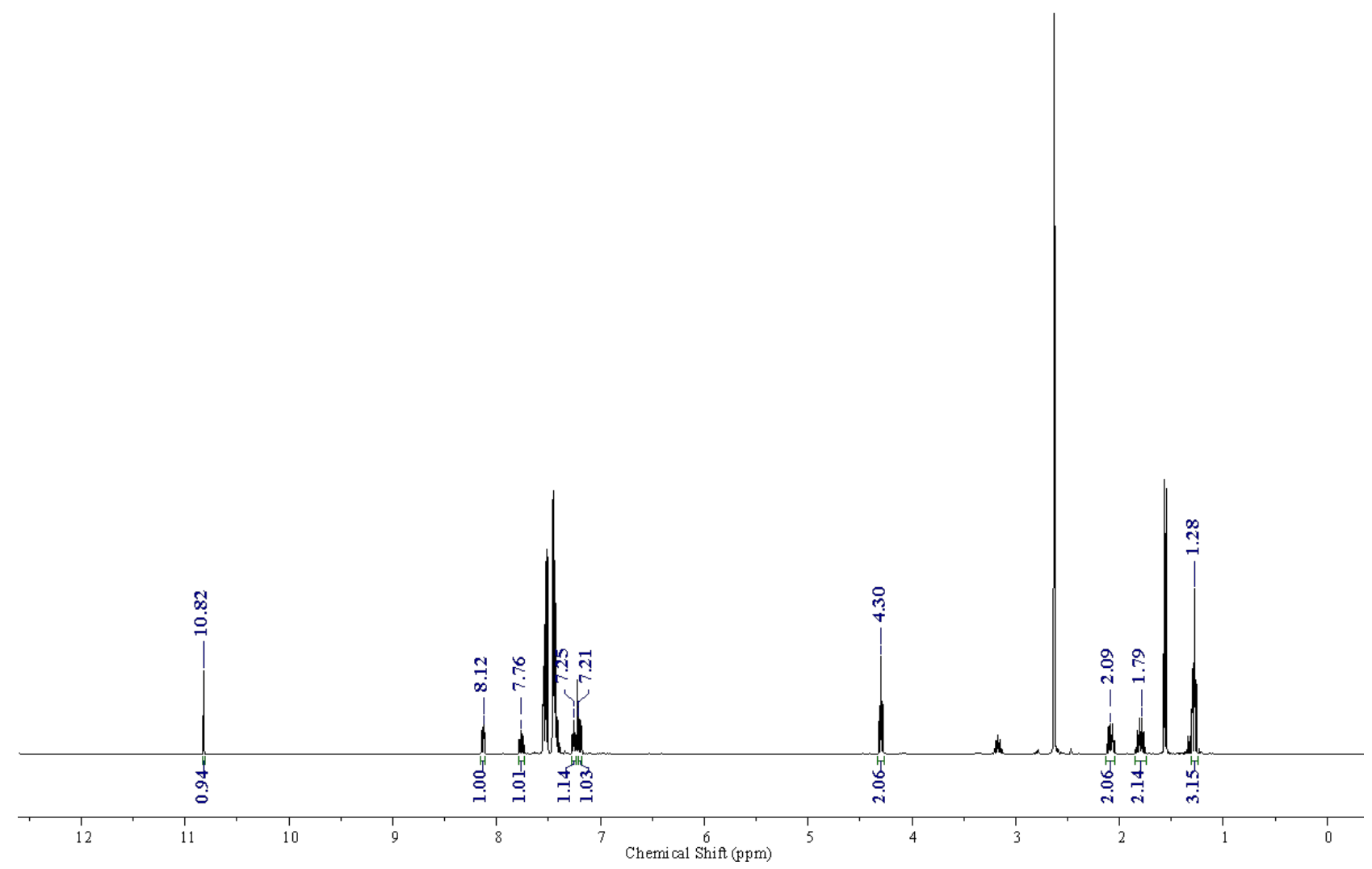

Entry 4': with 2-Methoxy-Phenyl-NHC (4d), Duplicate

SCG-341-G2-16hr-D 1-50sec.1. fid

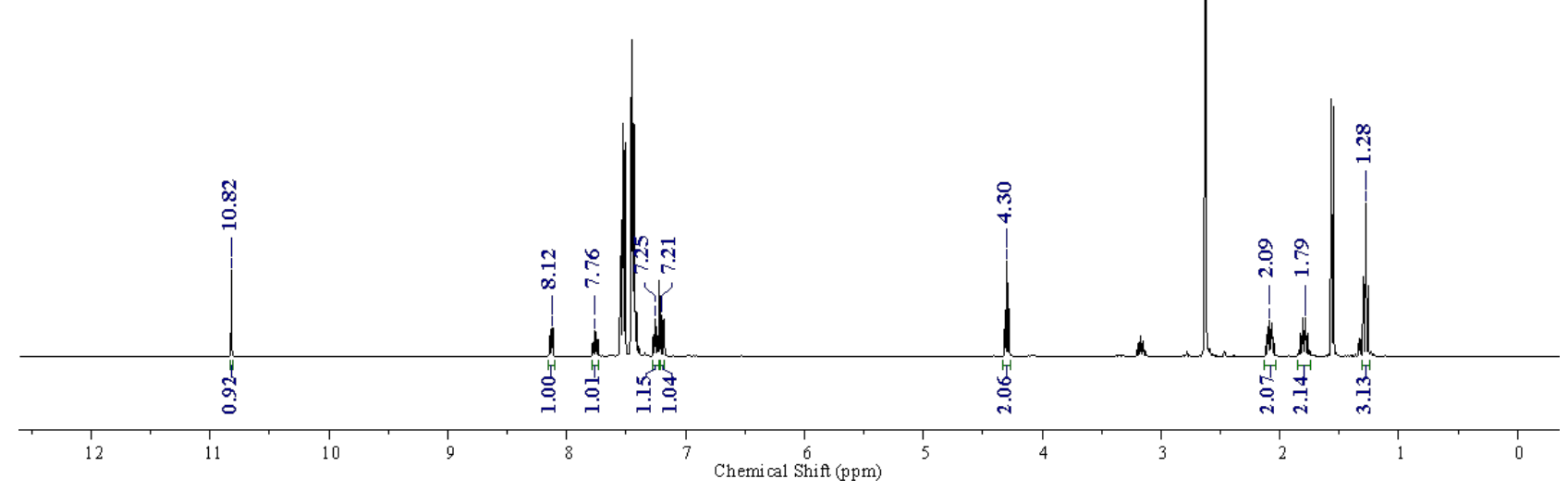


Entry 5: with 3,5-Ditrifluoromethyl-Phenyl-NHC (4e)

SCG-341-L1-16hr-D 1-50sec.1.fid

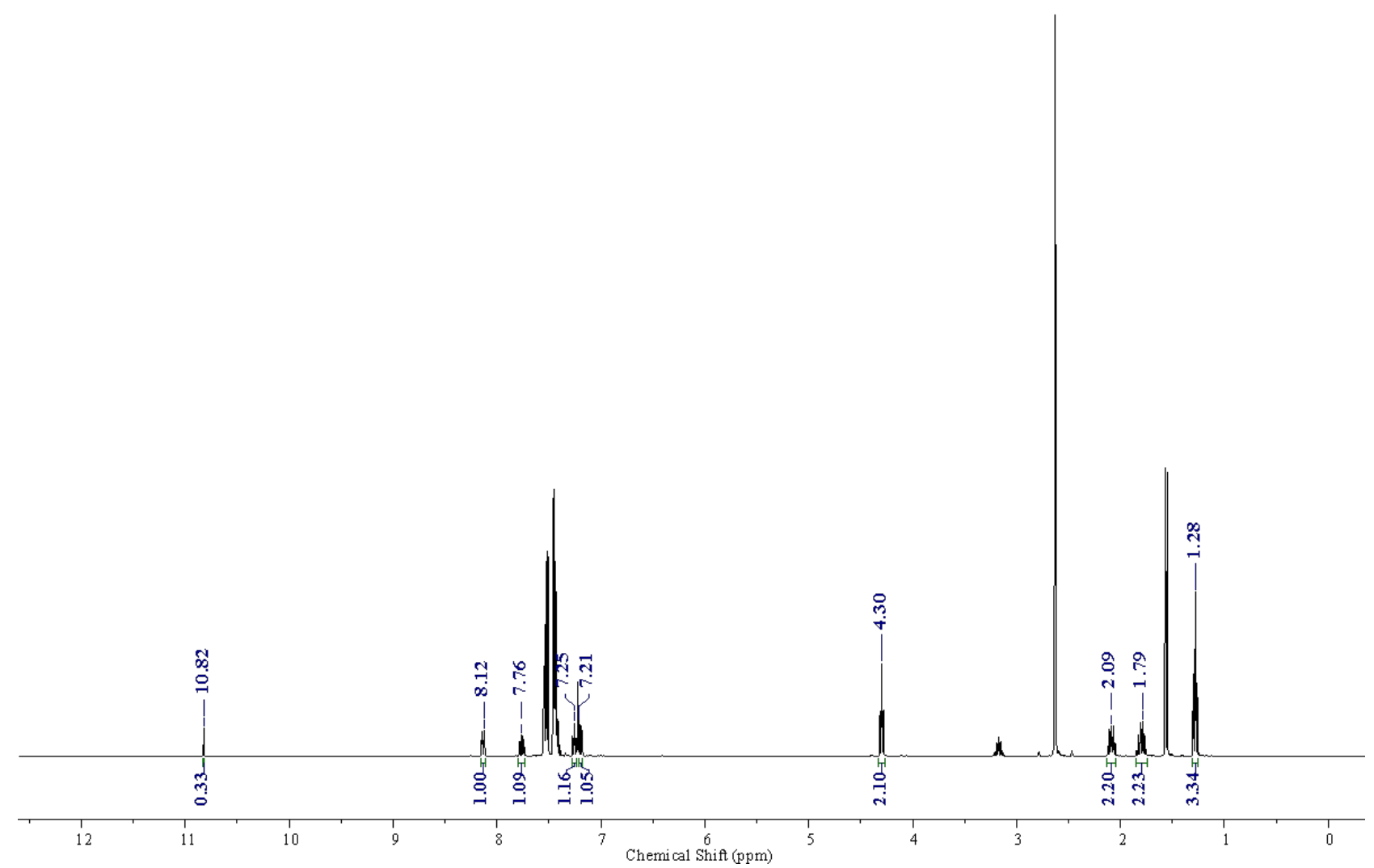

Entry 5: with 3,5-Ditrifluoromethyl-Phenyl-NHC (4e), Duplicate

SCG-341-L2-16hr-D 1-50sec.1.fid

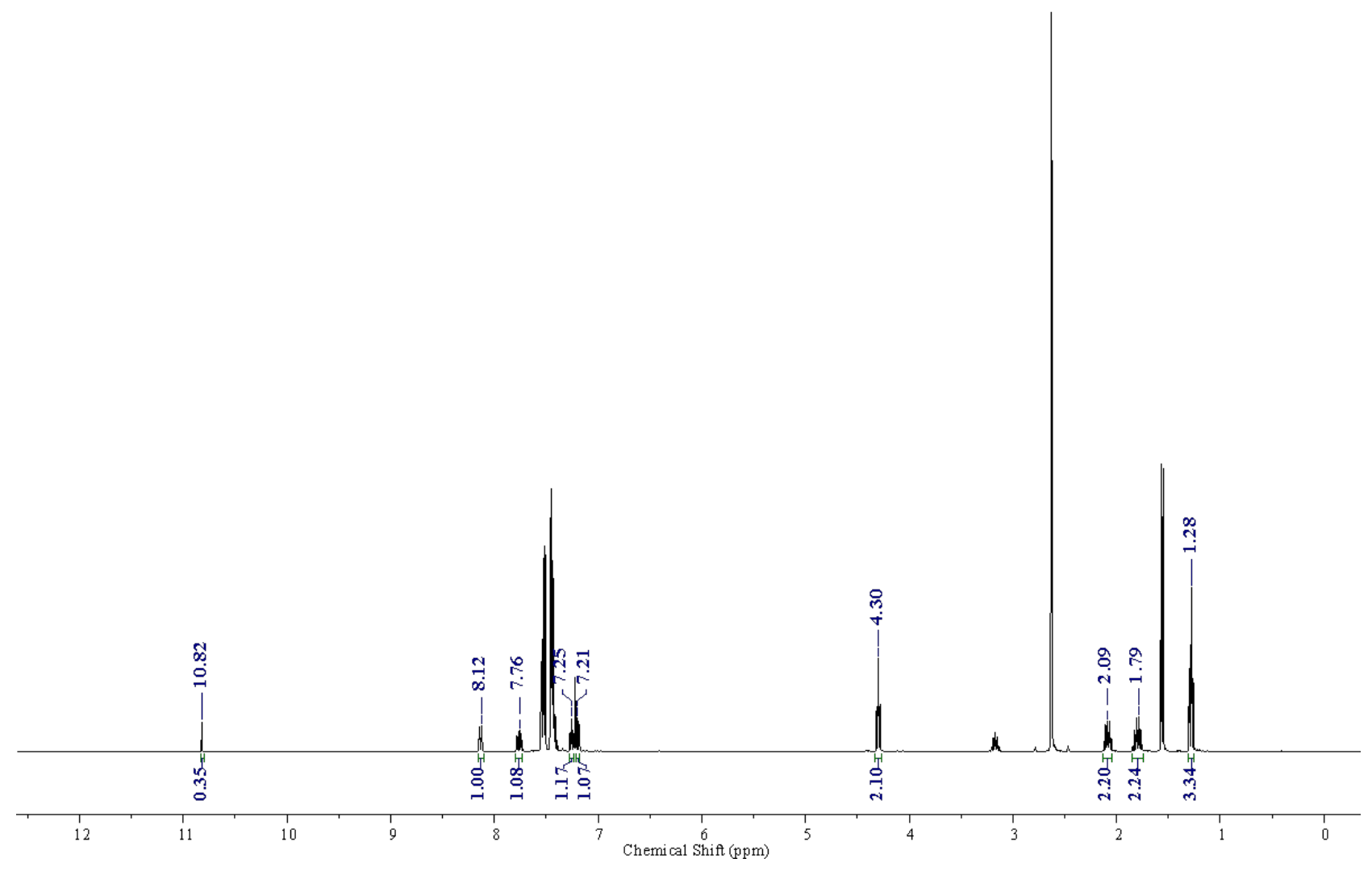


Entry 6: with 3,5-Dimethyl-Phenyl-NHC (4f)

SCG-341-D 1-16hr-D 1-50sec.1. fid

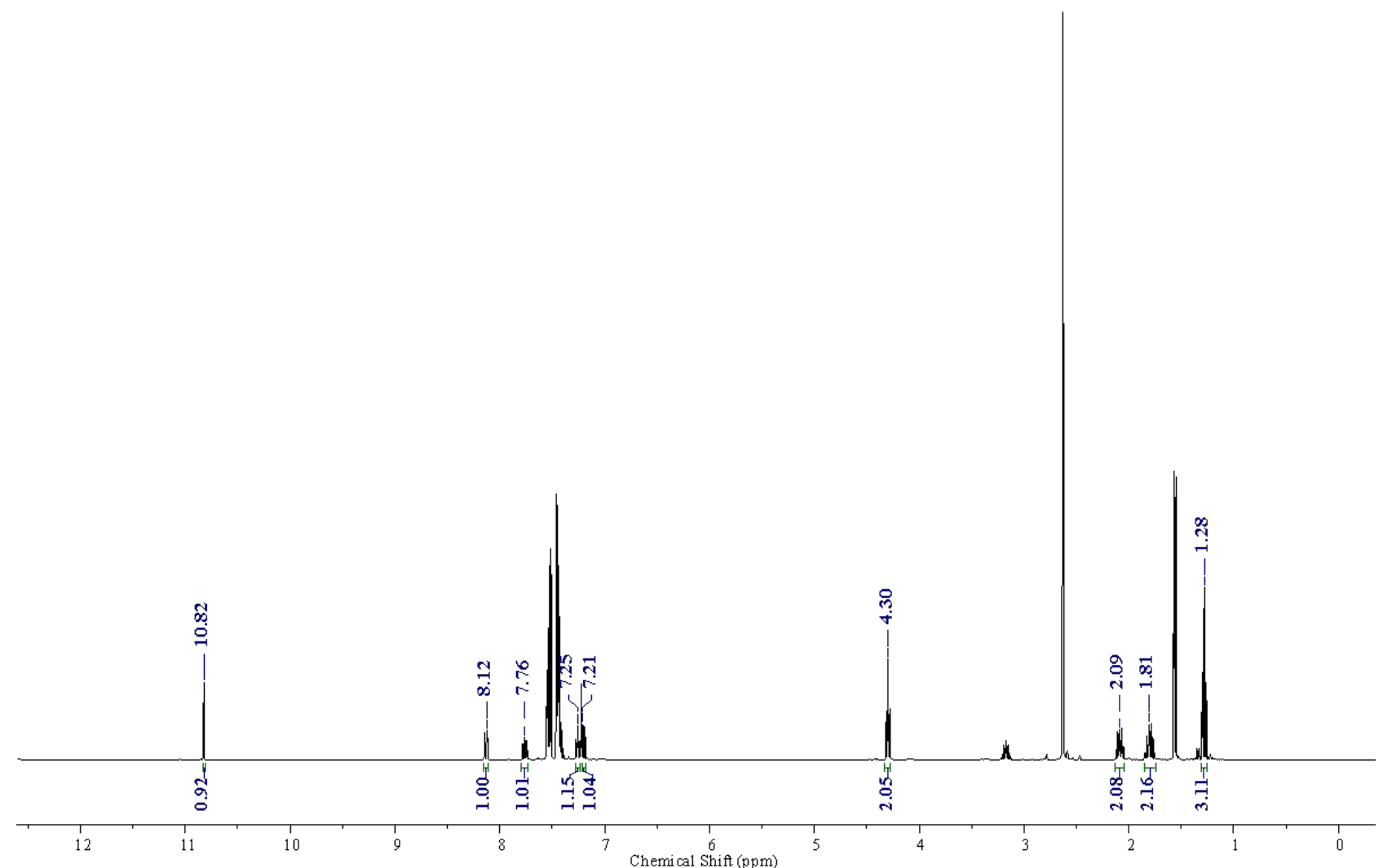

Entry 6': with 3,5-Dimethyl-Phenyl-NHC (4f), Duplicate SCG-341-D2-16hr-D 1-50sec.2.fid

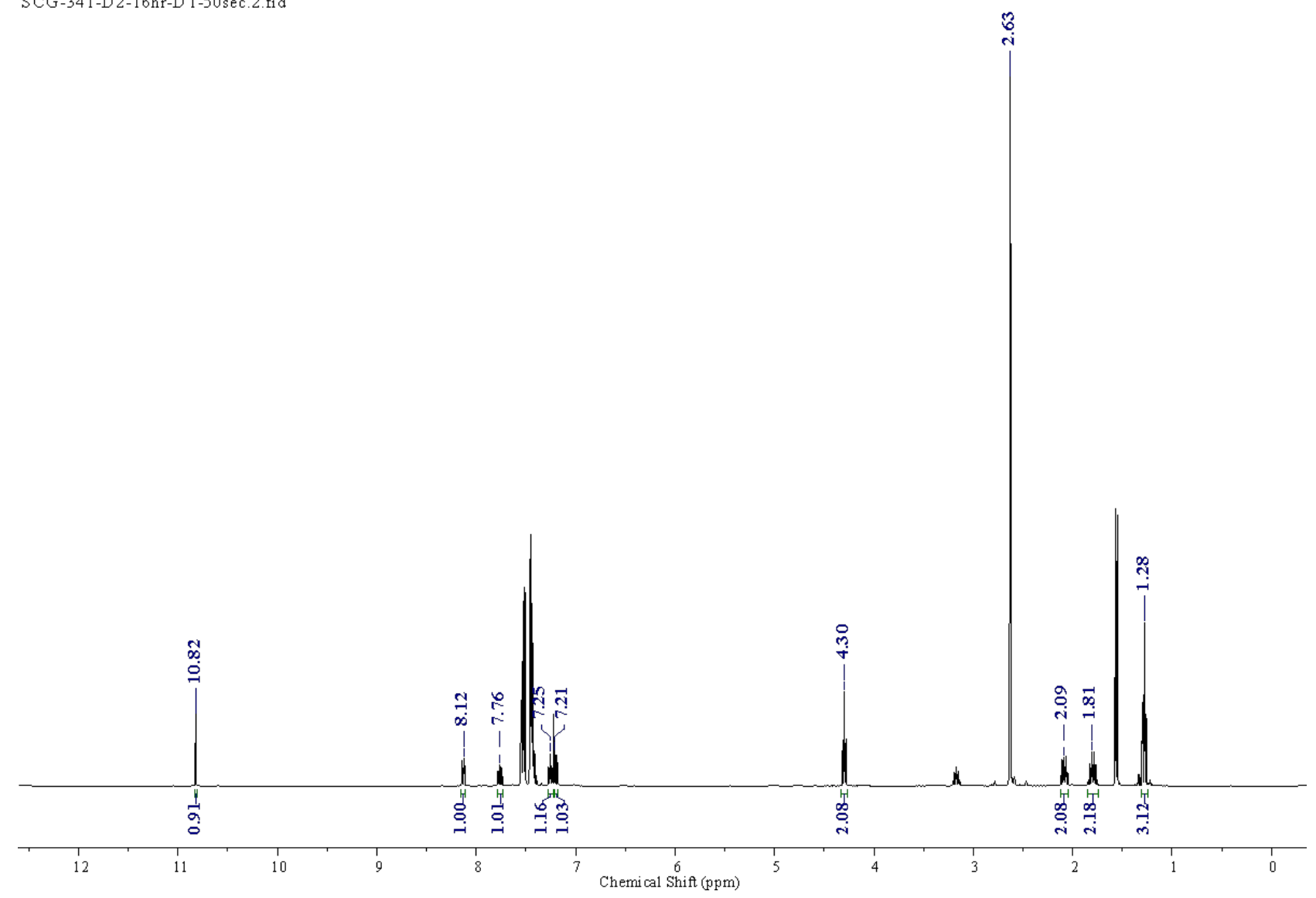


Entry 7: with 4-Trifluoromethyl-Phenyl-NHC (4g)

vDOS-598-A1 4CF4 NHC

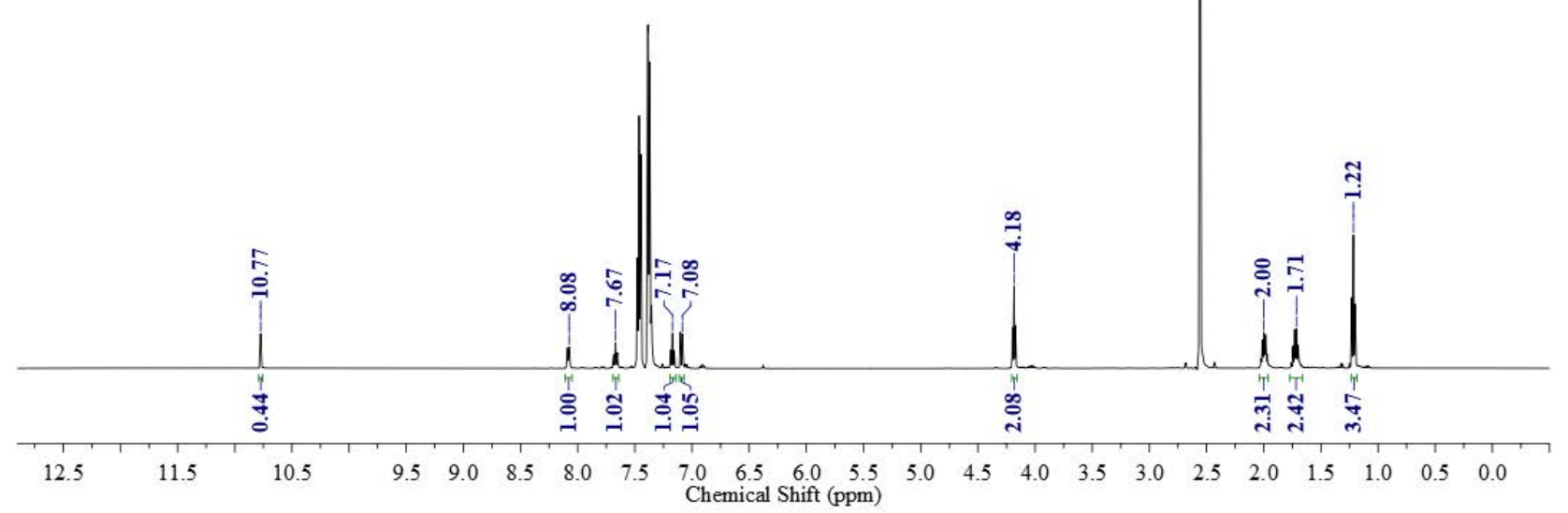

Entry 7': with 4-Trifluoromethyl-Phenyl-NHC (4g), Duplicate

vDOS-598-A2 4CF4 NHC

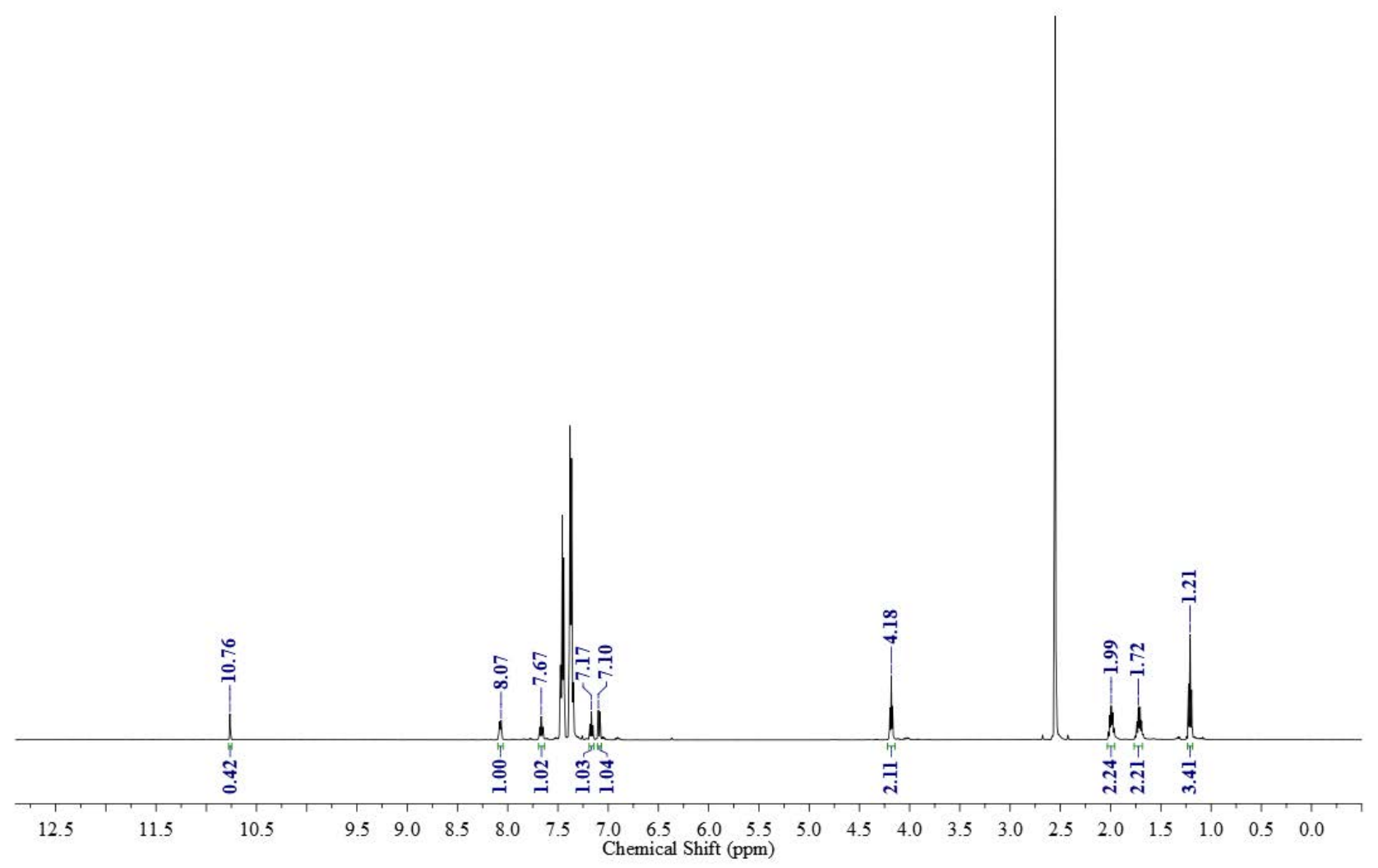


Entry 8: with 4-Methoxy-Phenyl-NHC (4h)

SCG-341-A 1-16hr-D 1-50sec.1.fid

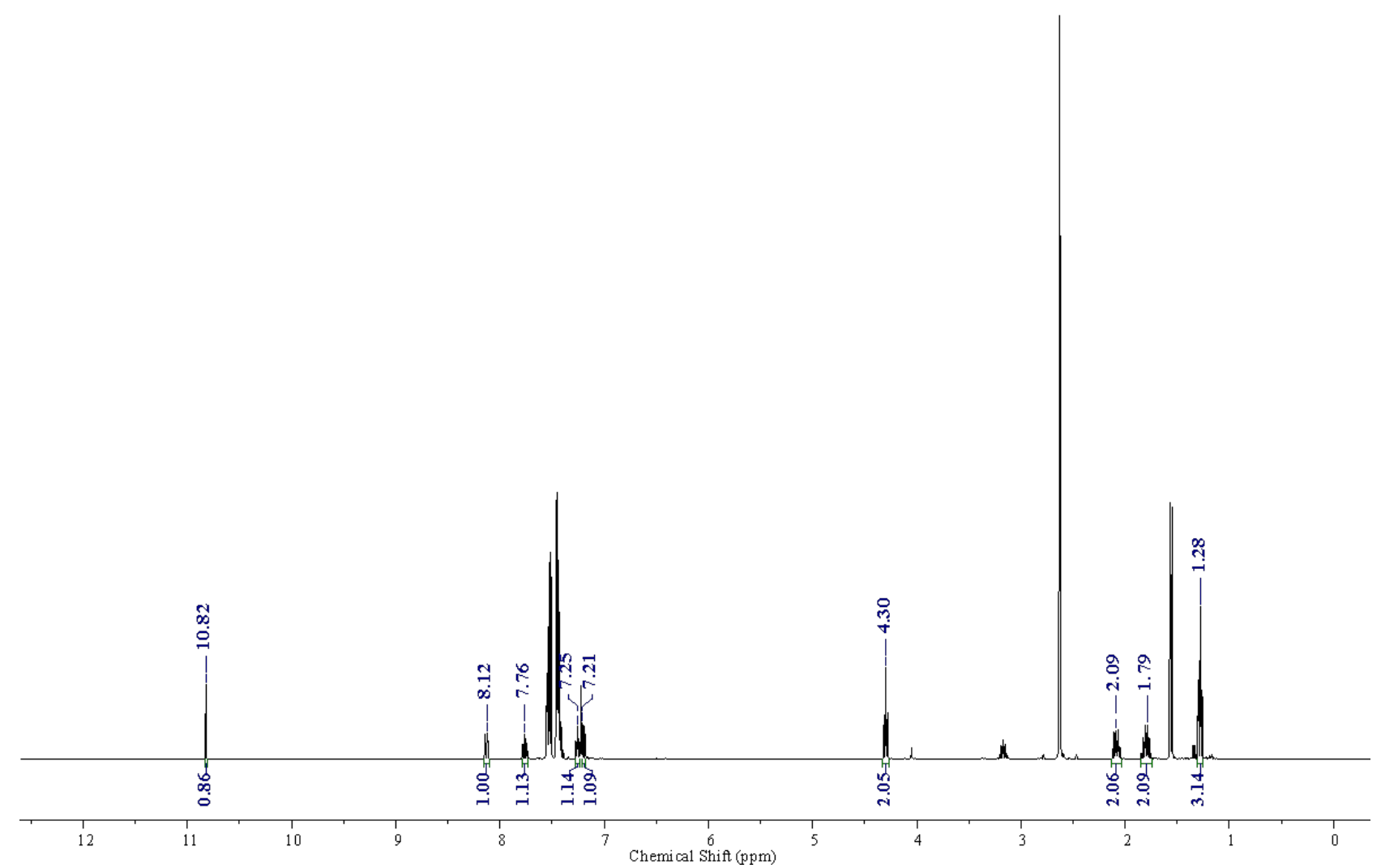

Entry 8': with 4-Methoxy-Phenyl-NHC (4h), Duplicate

SCG-341-A2-16hr-D 1-50sec.3.fid

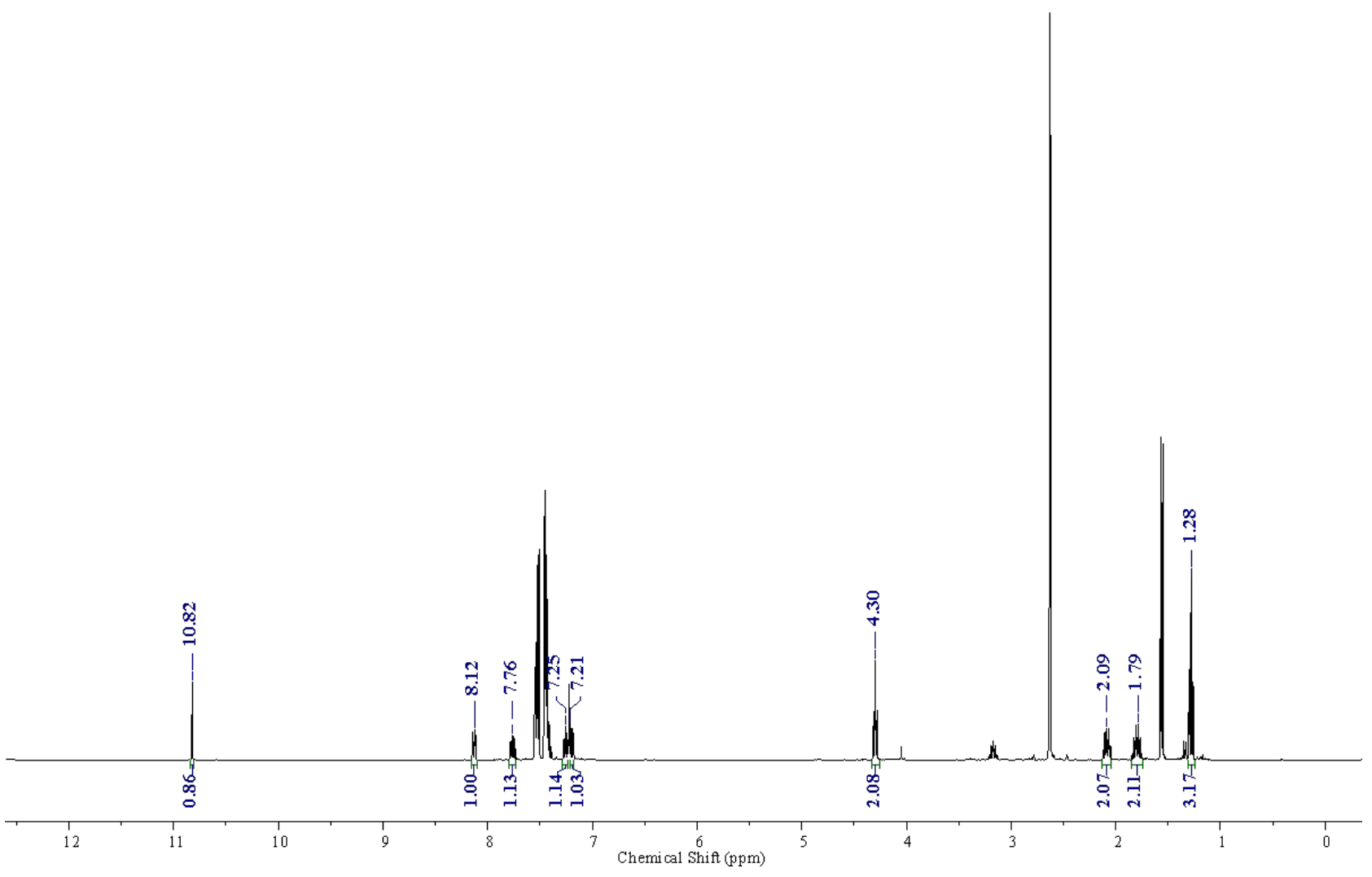


Entry 9: with Pentafluoro-Phenyl-NHC (4i)

SCG-341-I1-16hr-D 1-50sec.1. fid

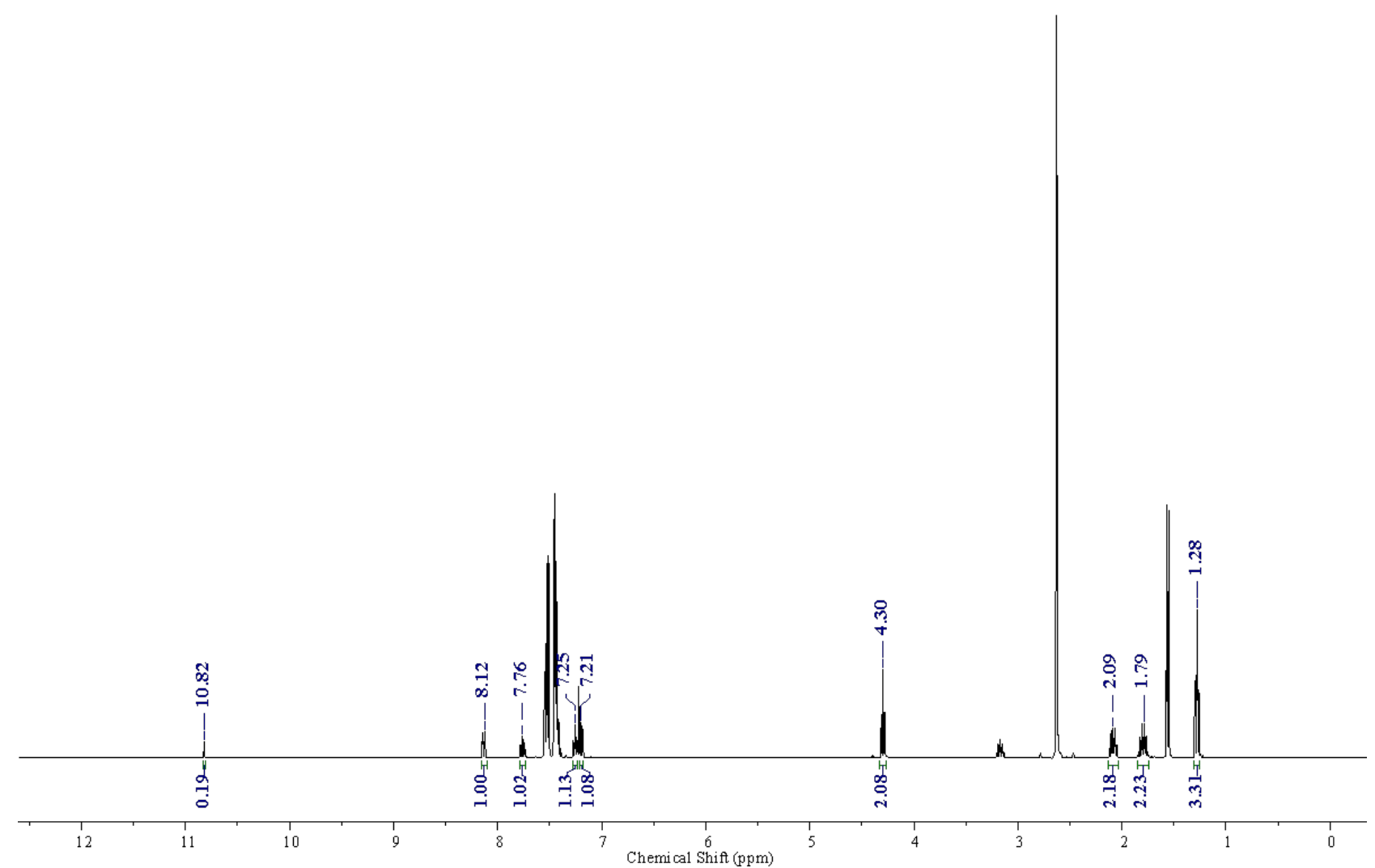

Entry 9': with Pentafluoro-Phenyl-NHC (4i), Duplicate

SCG-341-I2-16hr-D 1-50sec.1.fid

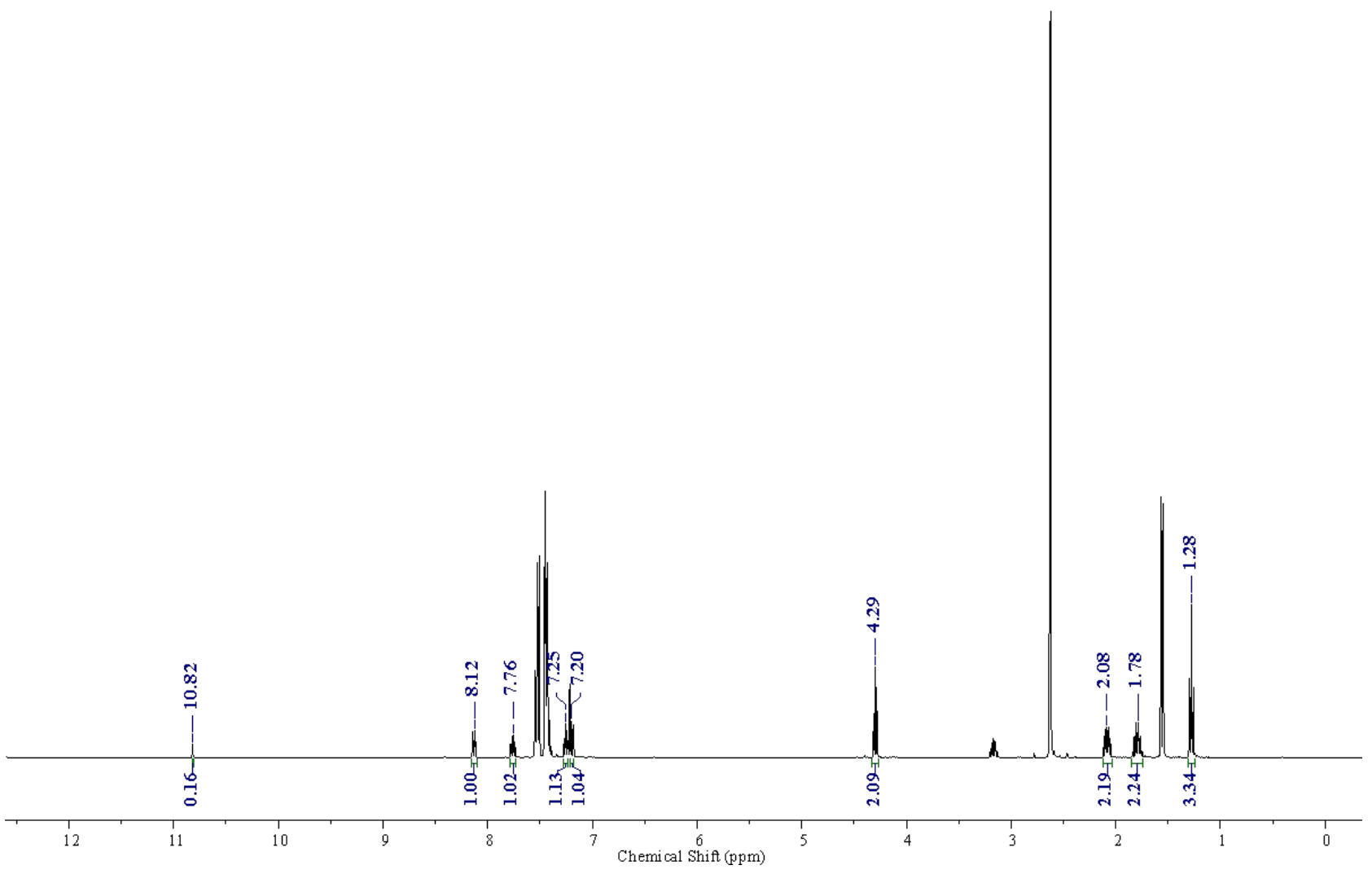


Entry 10: with Mesityl-Phenyl-NHC (4j)

SCG-341-C3-16hr-D 1-50sec.1.fid

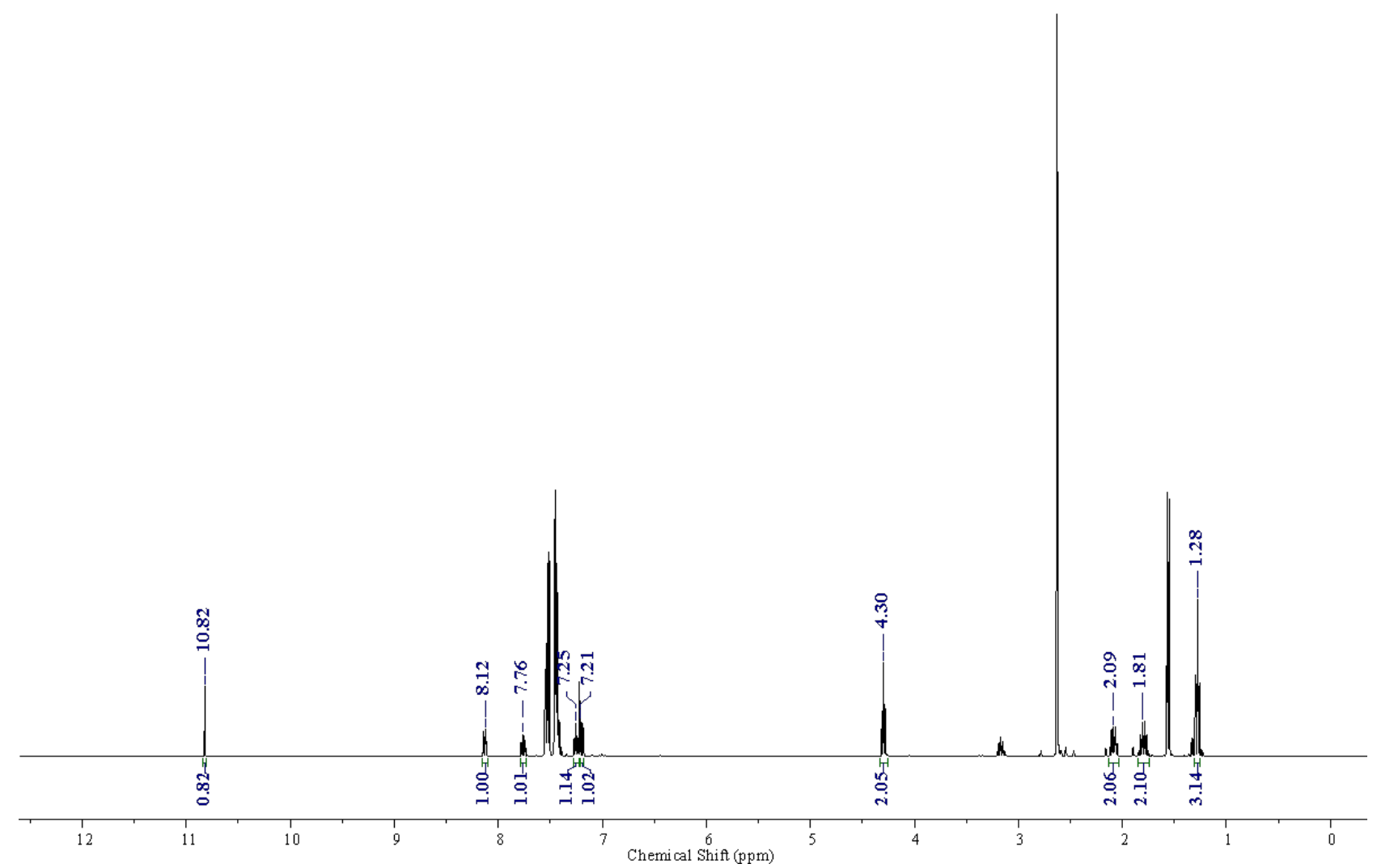

Entry 10': with Mesityl-Phenyl-NHC (4j), Duplicate

SCG-341-C4-16hr-D 1-50sec.1.fid

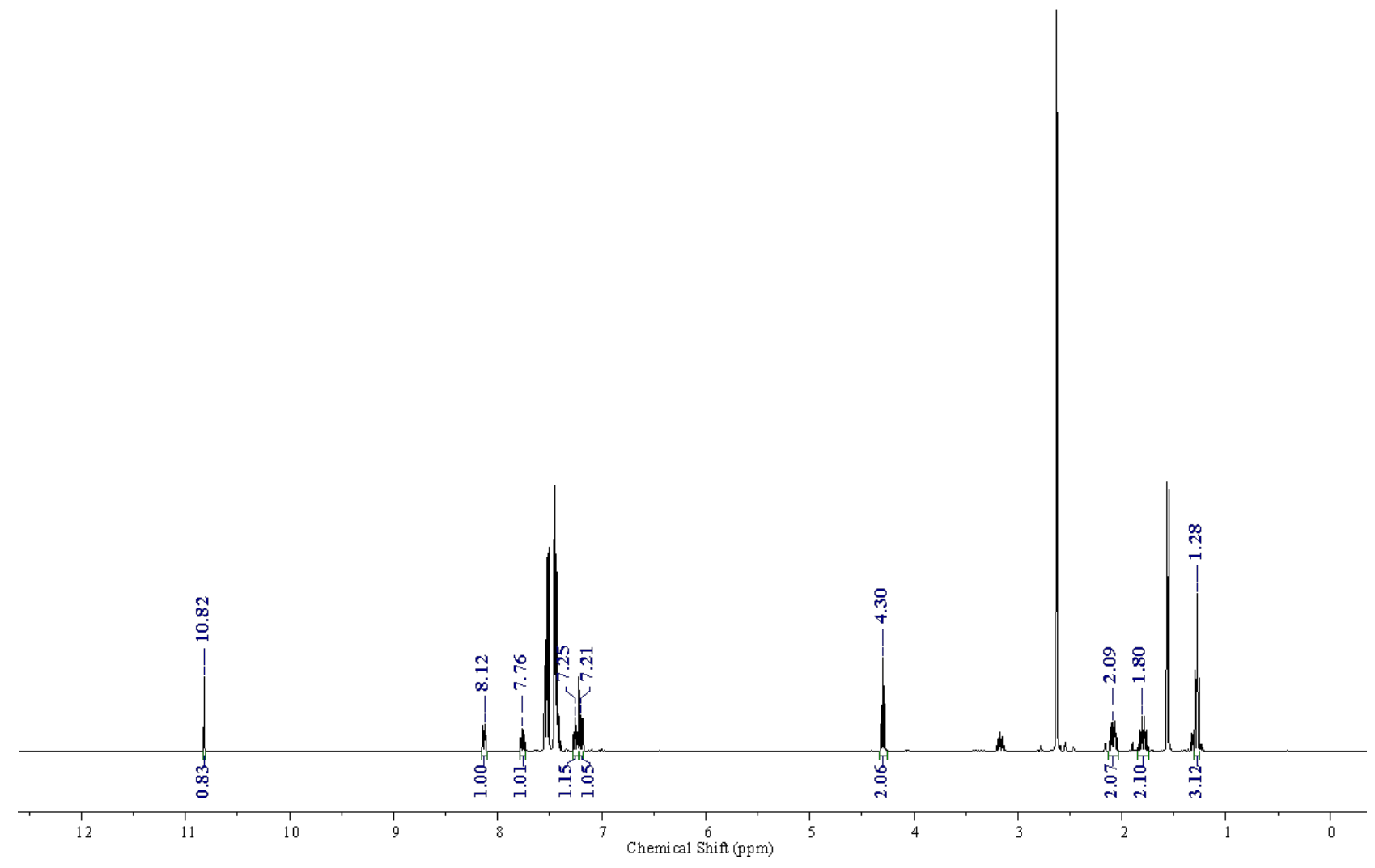


Entry 11: with Phenyl-NHC (4k)

SCG-341-H1-16hr-D 1-50sec.1.fid

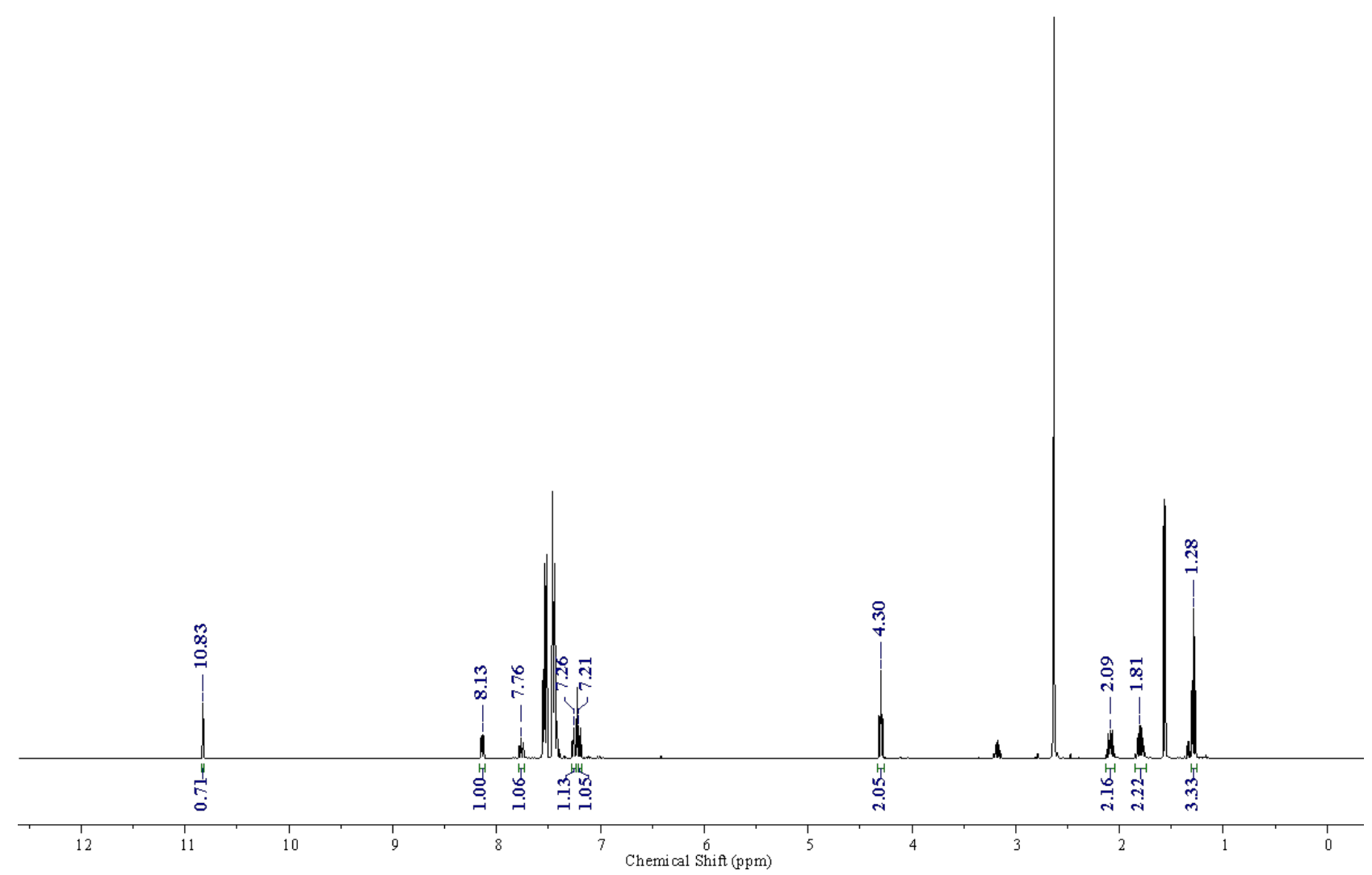

Entry 11': with Phenyl-NHC (4k), Duplicate

SCG-341-H2-16hr-D 1-50sec.1. fid

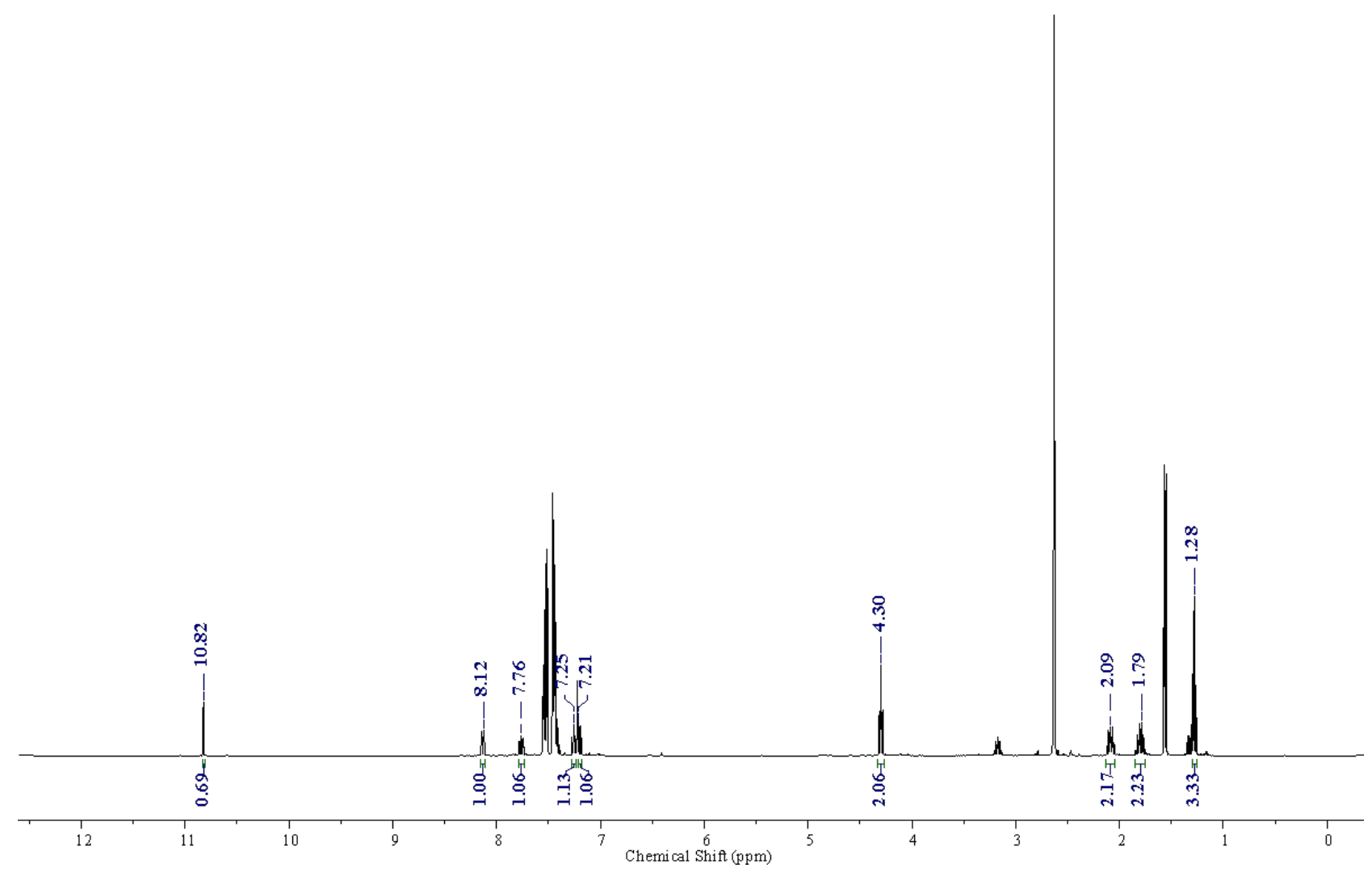


Entry 12: with 2-Chloro-Phenyl-NHC (4I)

SCG-341-M1-16hr-D 1-50sec.1.fid

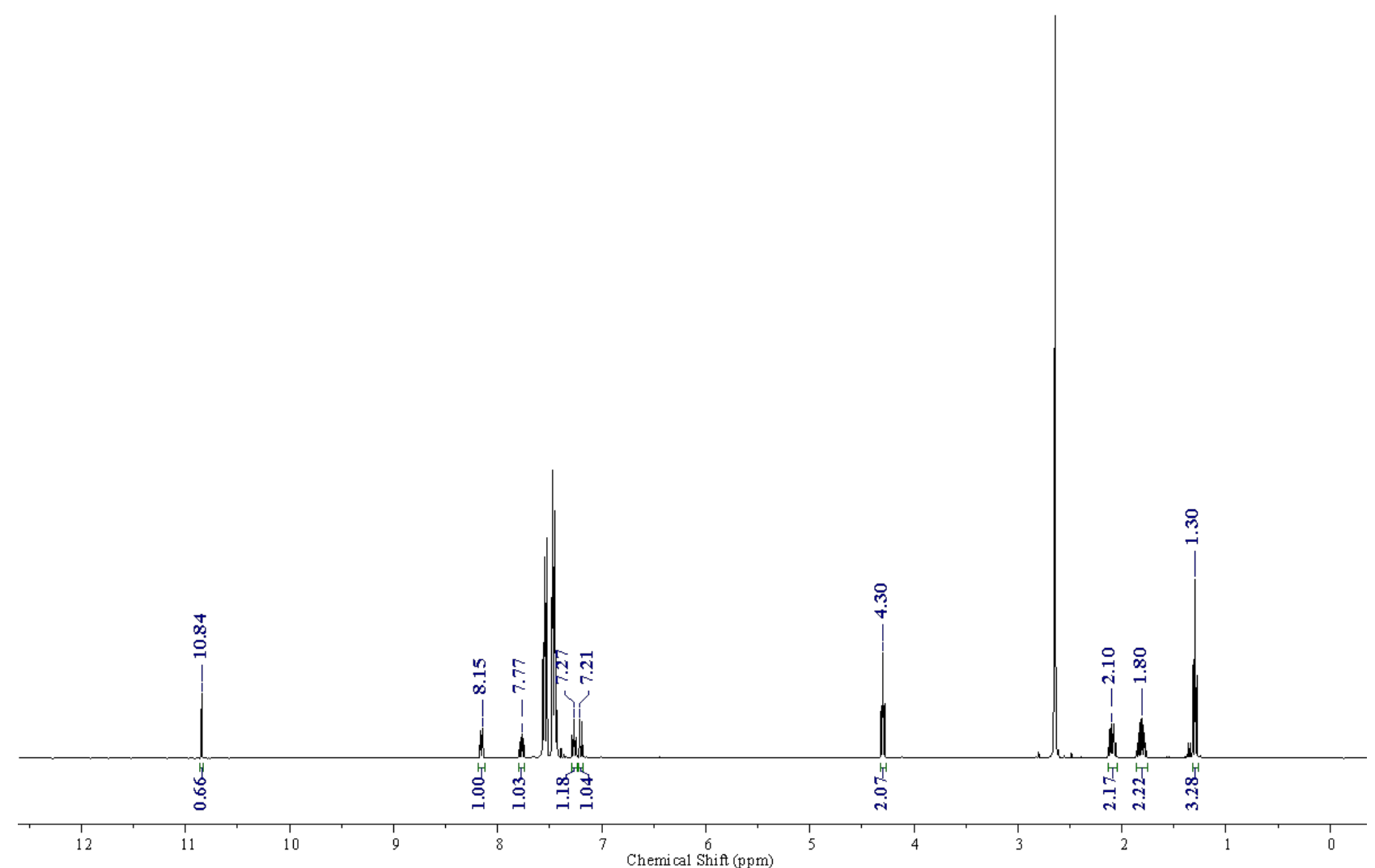

Entry 12': with 2-Chloro-Phenyl-NHC (4I), Duplicate

SCG-341-M2-16hr-D 1-50sec.1. fid

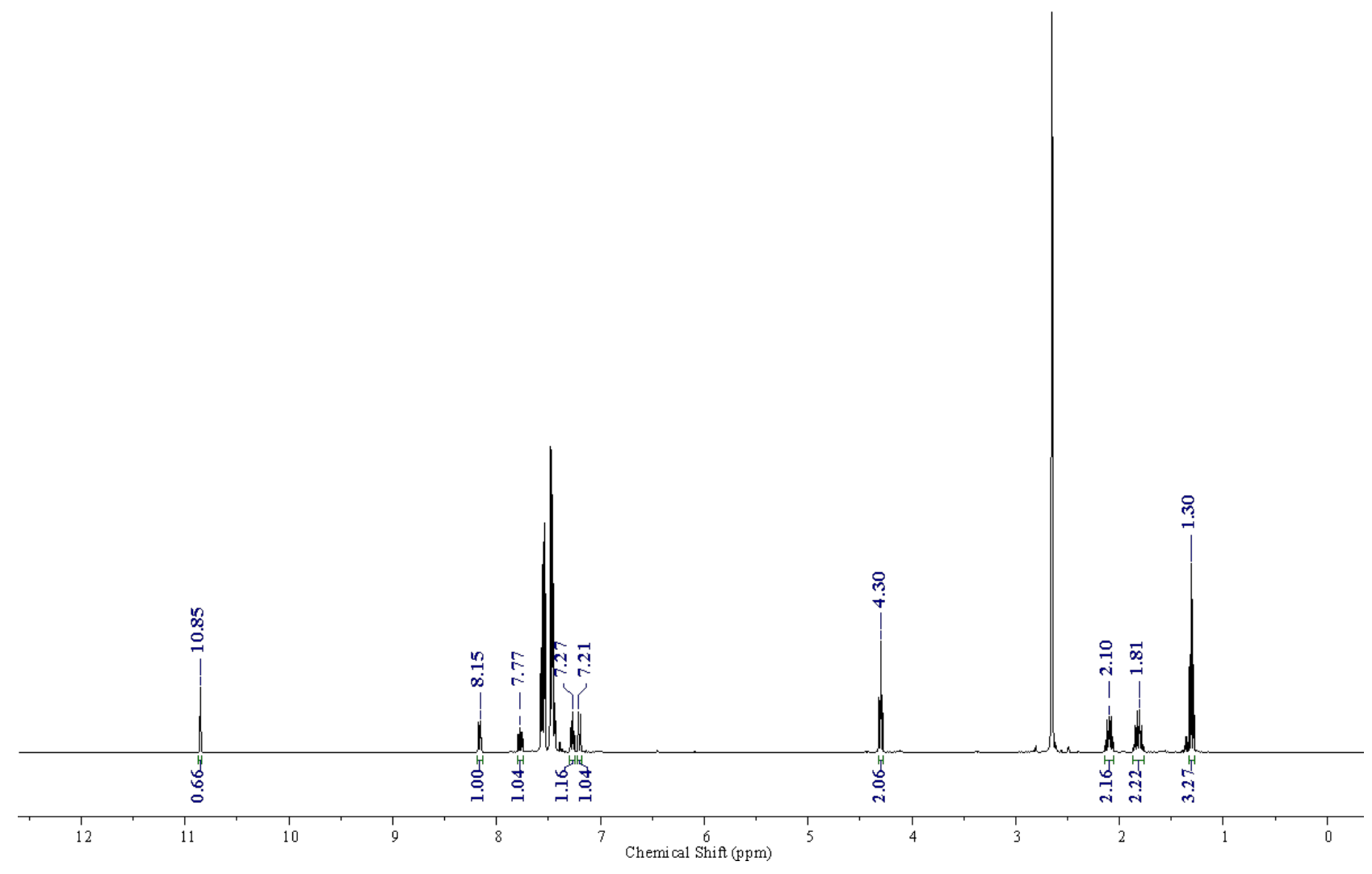


Entry 13: with 2-Trifluoromethyl-Phenyl-NHC (4m)

SCG-341-N1-16hr-D 1-50sec.1. fid

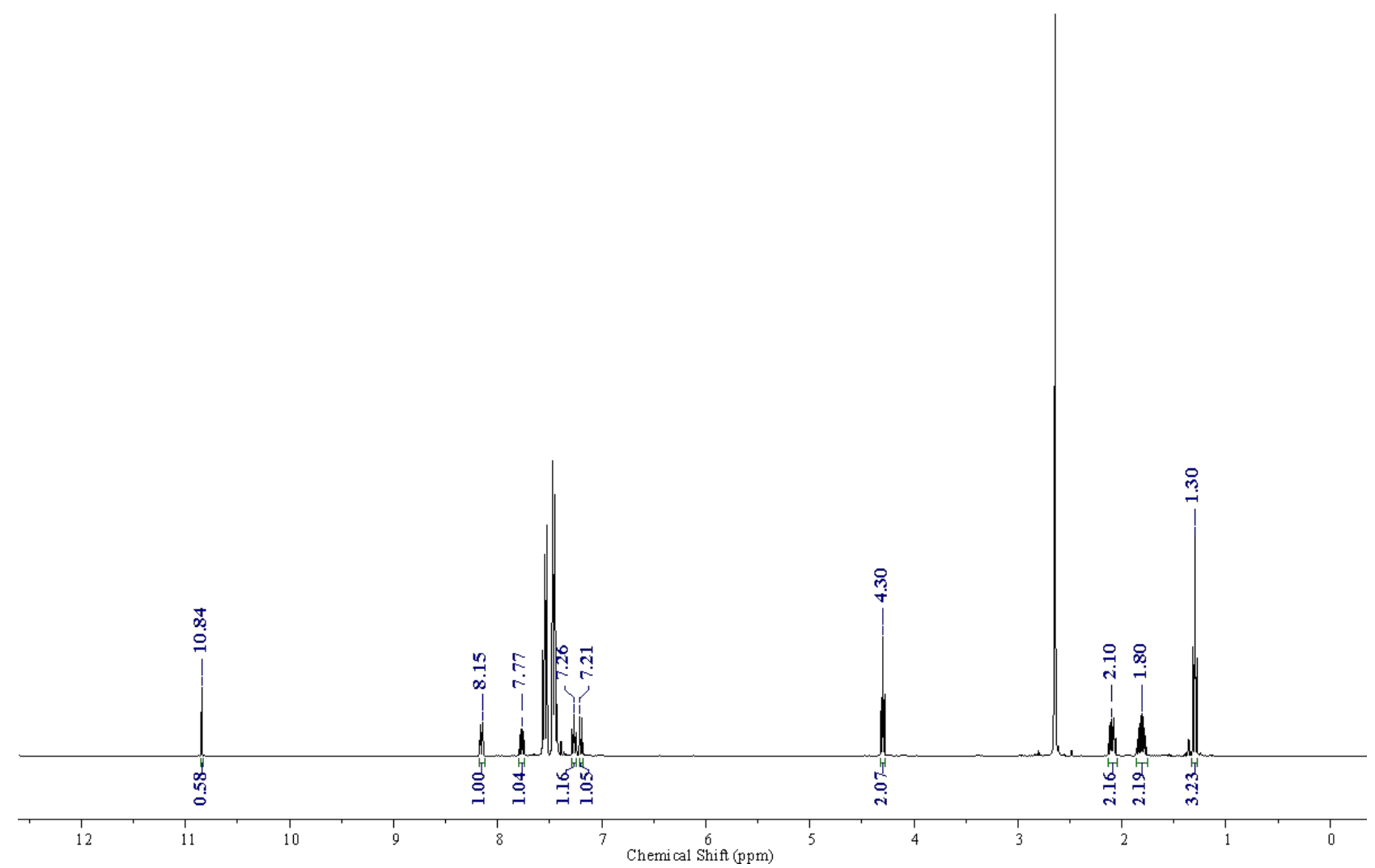

Entry 13': with 2-Trifluoromethyl-Phenyl-NHC (4m), Duplicate

SCG-341-N2-16hr-D 1-50sec.1. fid

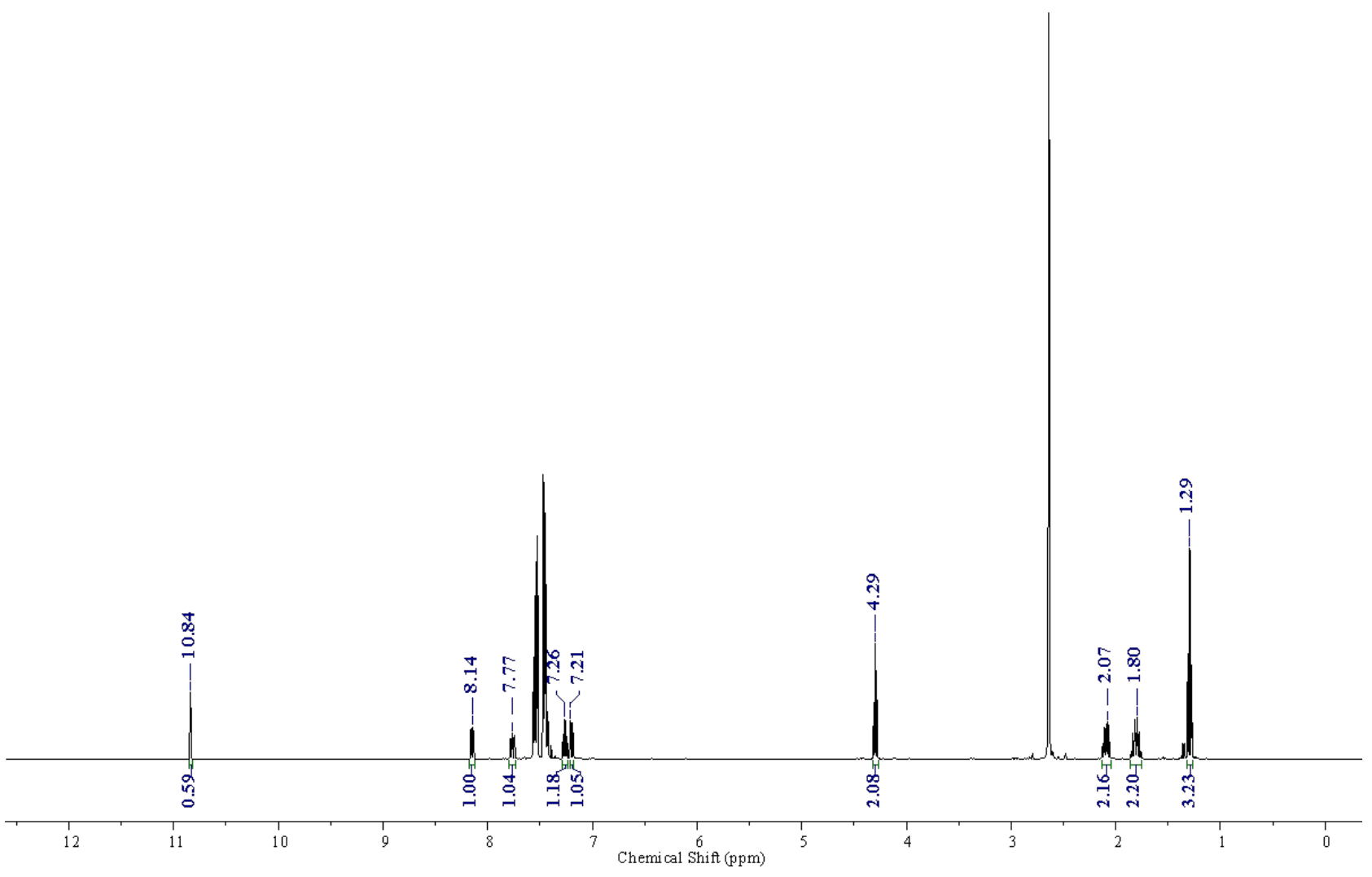


Entry 14: with 3-fluoro-Phenyl-NHC (4n)

SCG-341-K3-16hr-D 1-50sec.2.fid

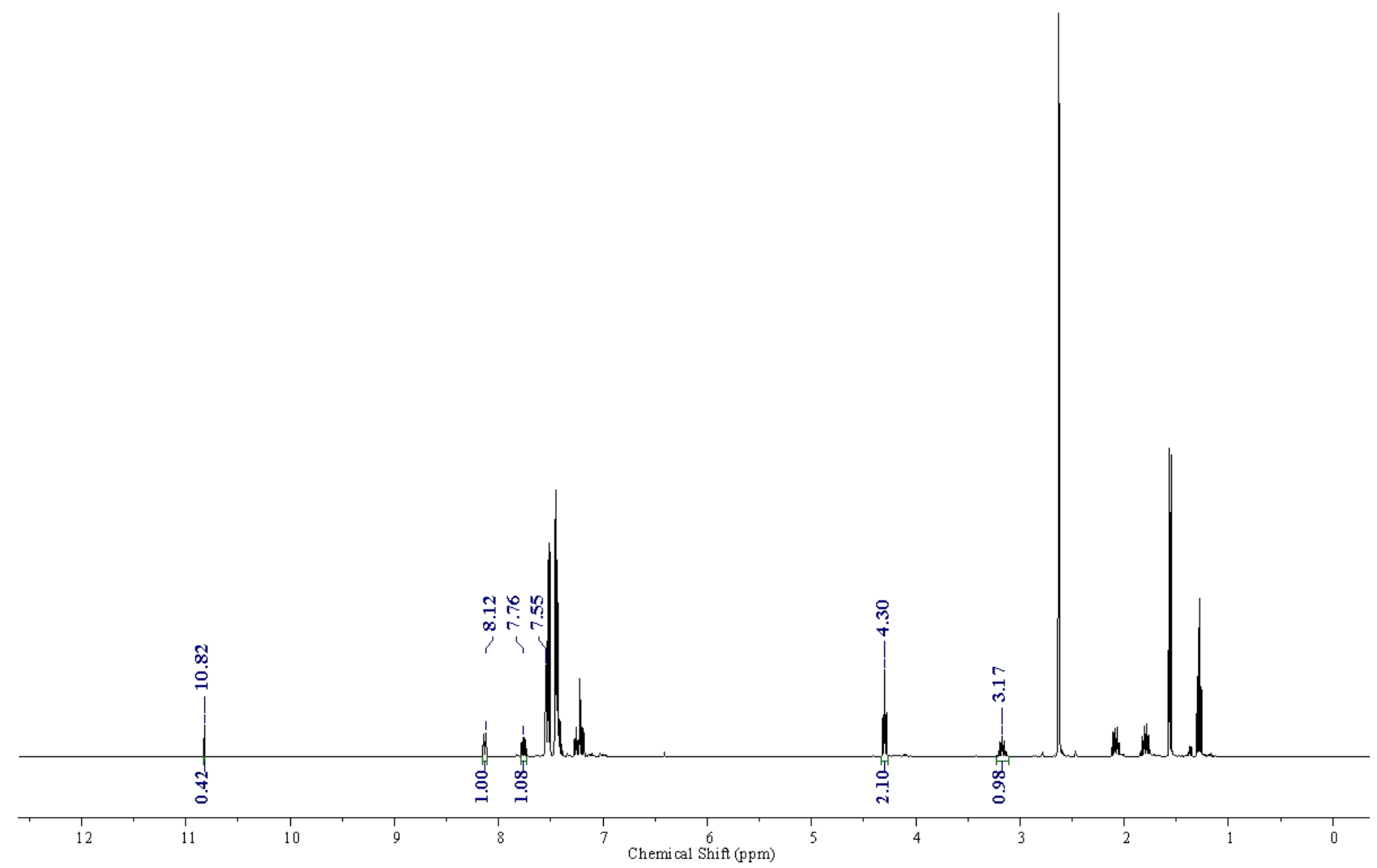

Entry 14': with 3-fluoro-Phenyl-NHC (4n), Duplicate

SCG-341-K4-16hr-D 1-50sec.1.fid

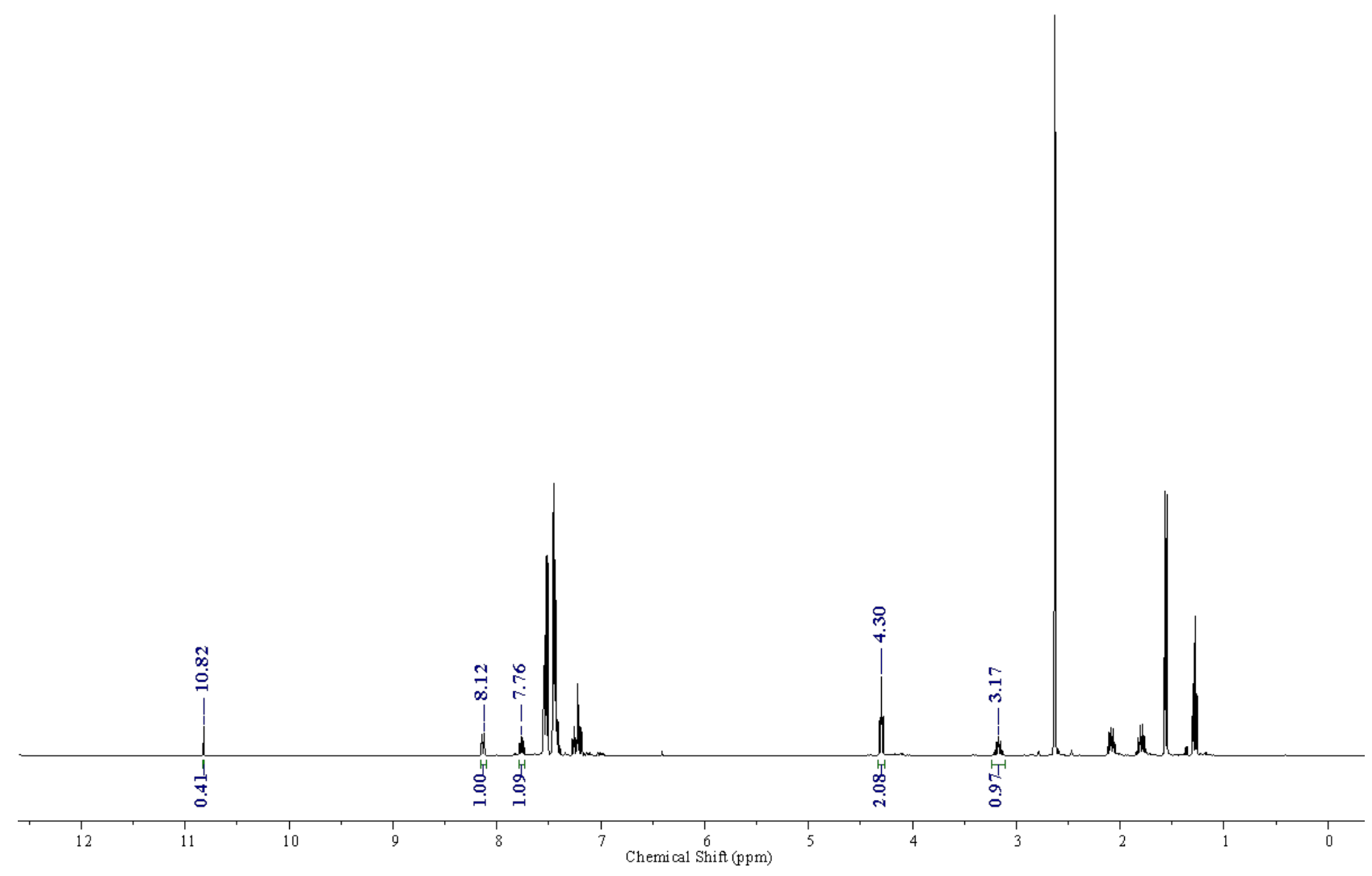




\section{Experiments with Alcohols:}

8.1 Reactions with 3a and R-OH (5 eq.)

Entry 1: With Water (1.85 mmol), 1 hour SCG-334-A1-60min-D 1-50sec.2.fid

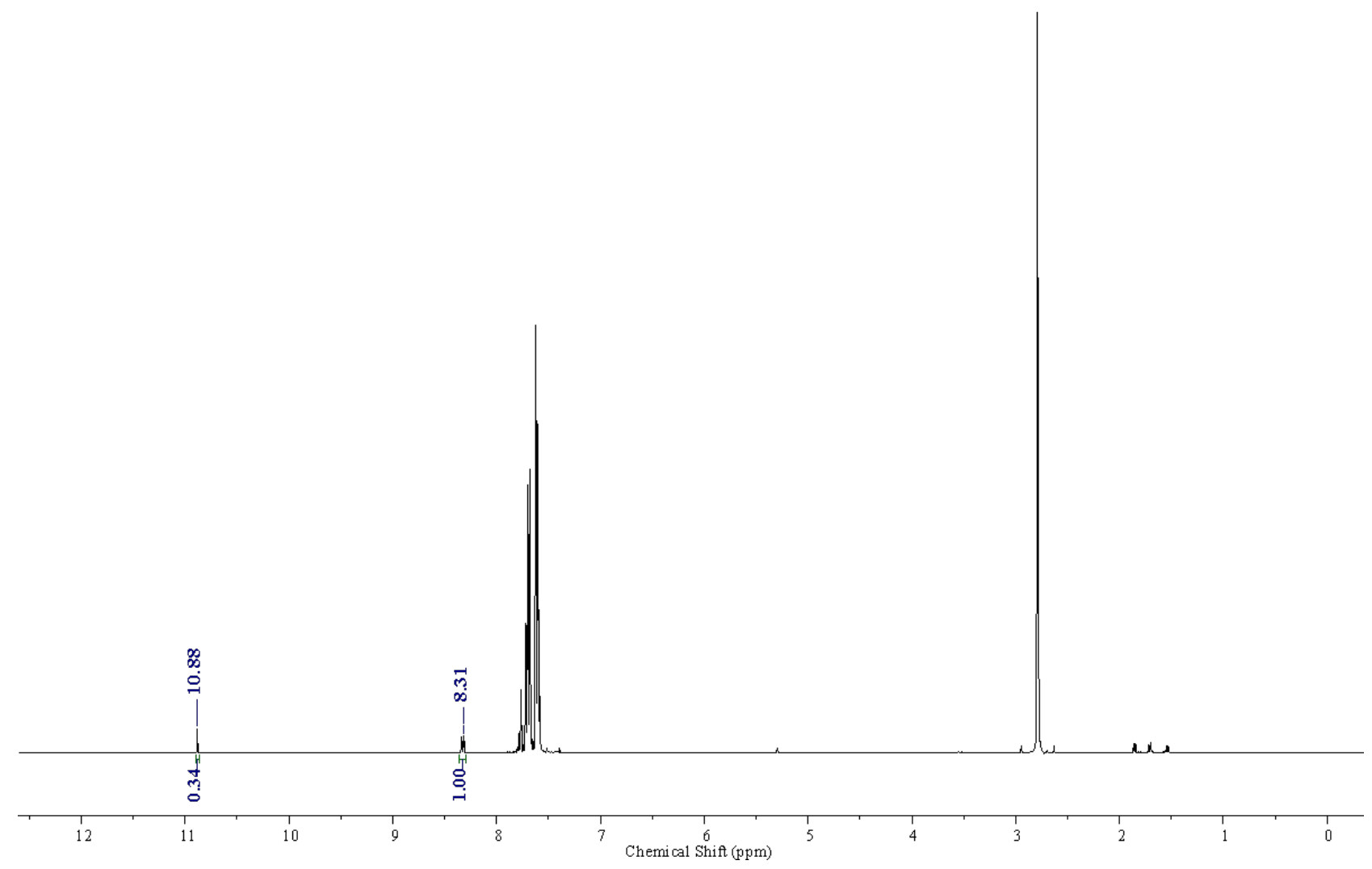


Entry 2: With Methanol (1.85 mmol), 1 hour

SCG-332-F1-60m in-D 1-50sec.1. fid

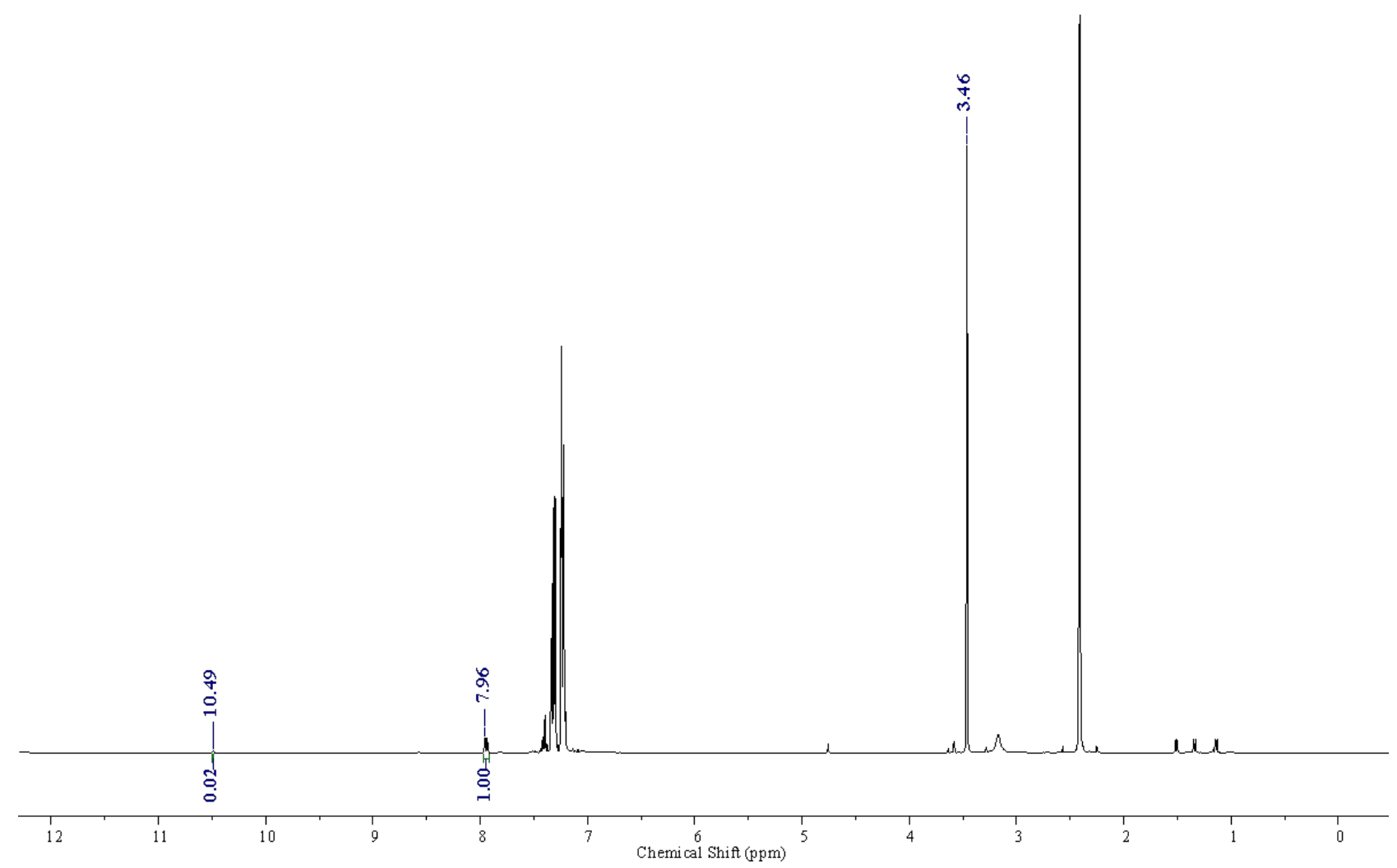

Entry 3: With Ethanol (1.85 mmol), 1 hour

SCG-332-A 1-60min-D 1-50sec. 1.fid

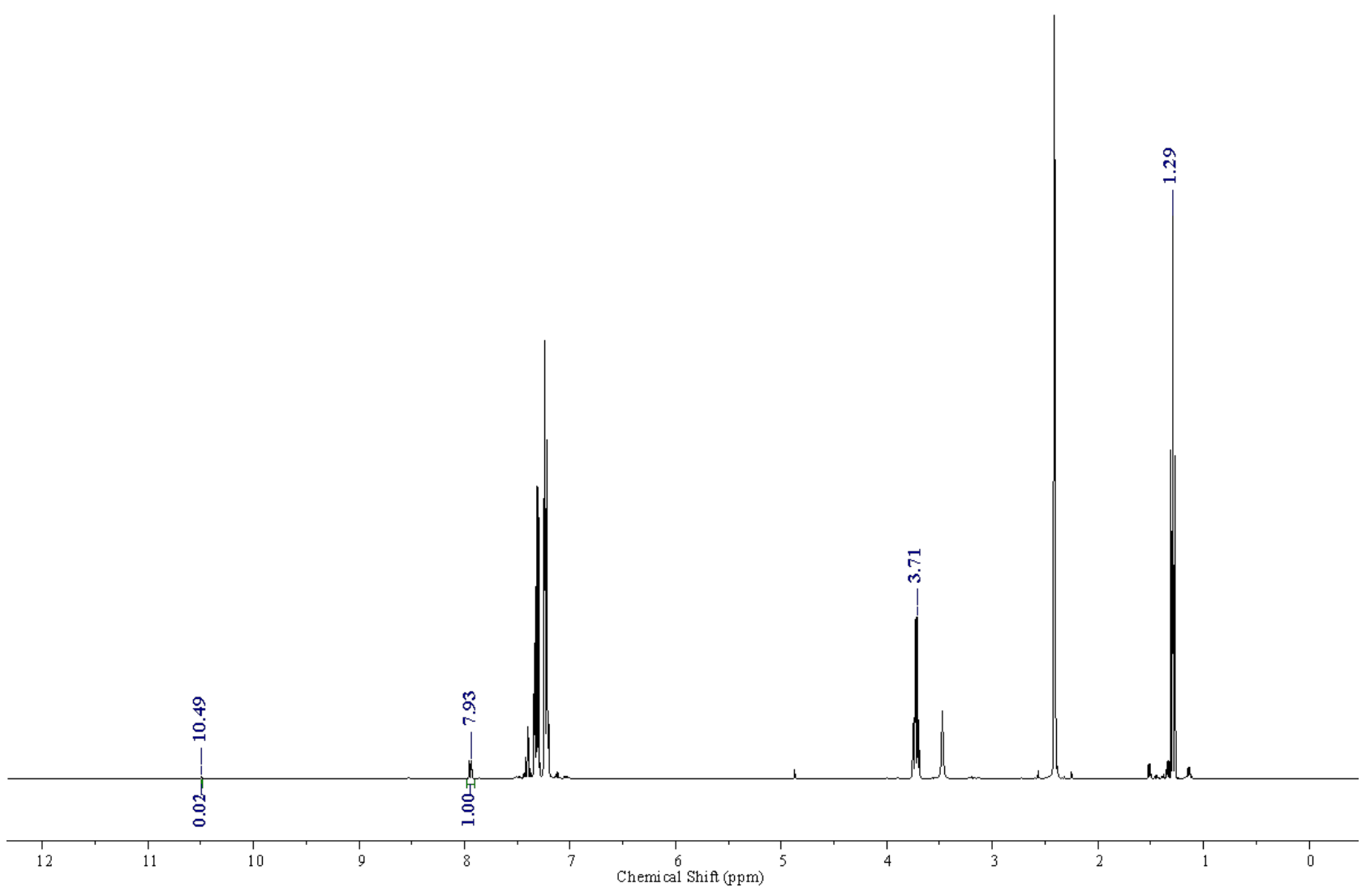


Entry 4: With Phenol (1.85 mmol), 1 hour

SCG-332-B 1-60m in -D 1-50sec. 1. fid

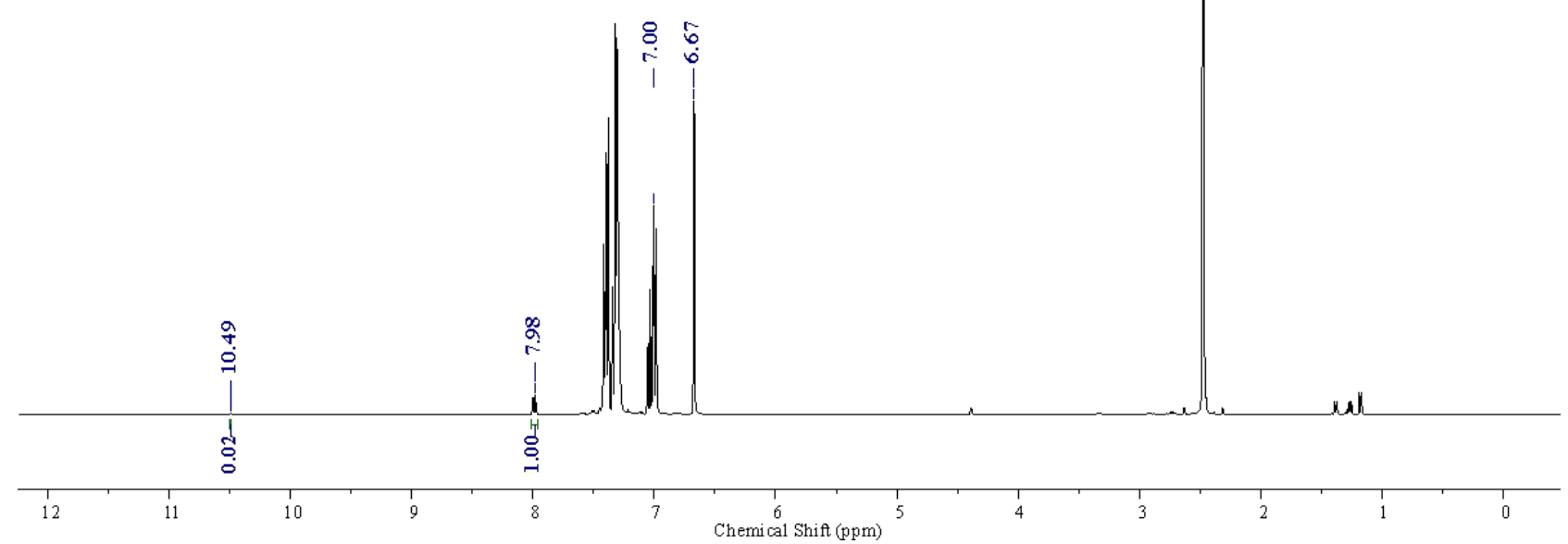

Entry 5: With 4-Methylphenol (1.85 mmol), 1 hour

SCG-332-C1-60min-D 1-50sec. 1. fid

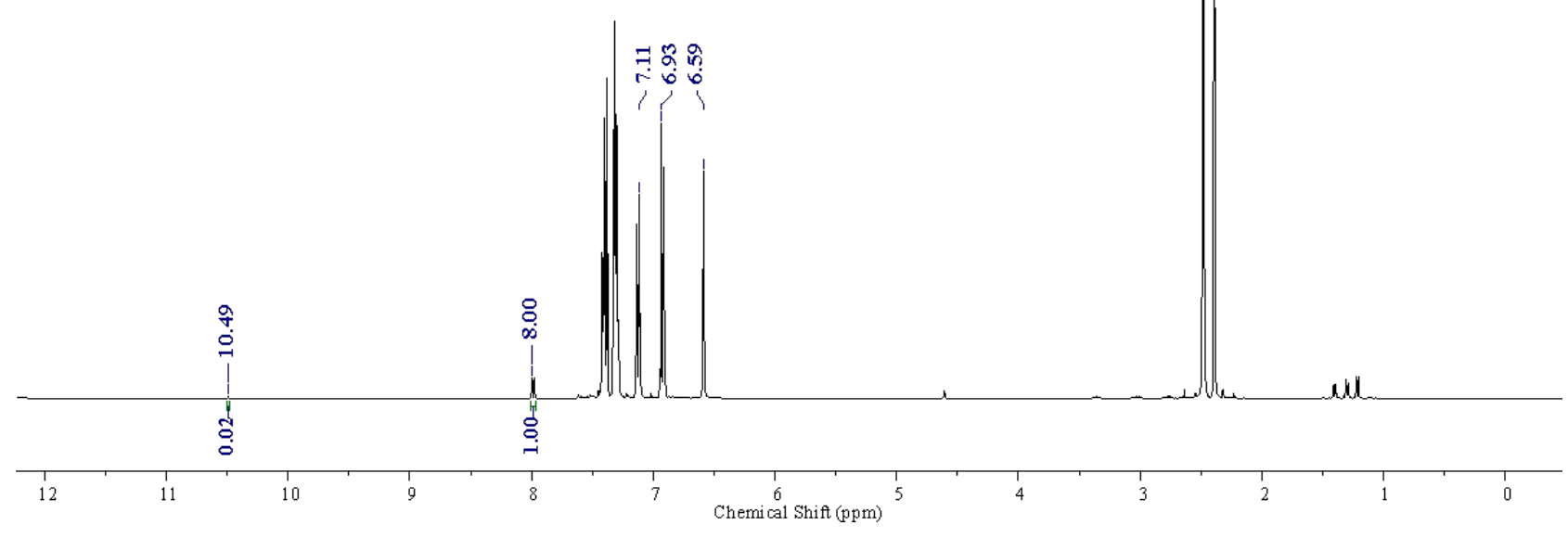


Entry 6: With Pentafluorophenol (1.85 mmol), 1 hour

SCG-332-D 1-60min-D 1-50sec. 1.fid

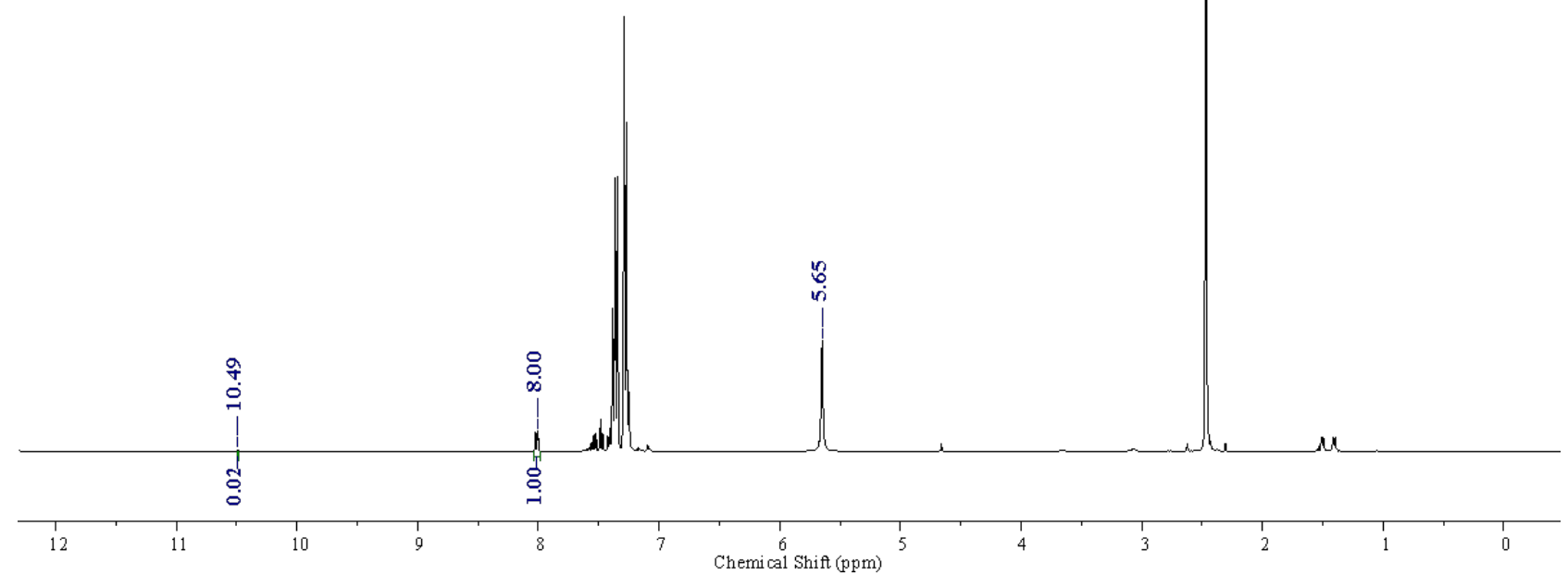

Entry 7: With 4-Fluorophenol (1.85 mmol), 1 hour

SCG-332-E 1-60min-D 1-50sec.1. fid

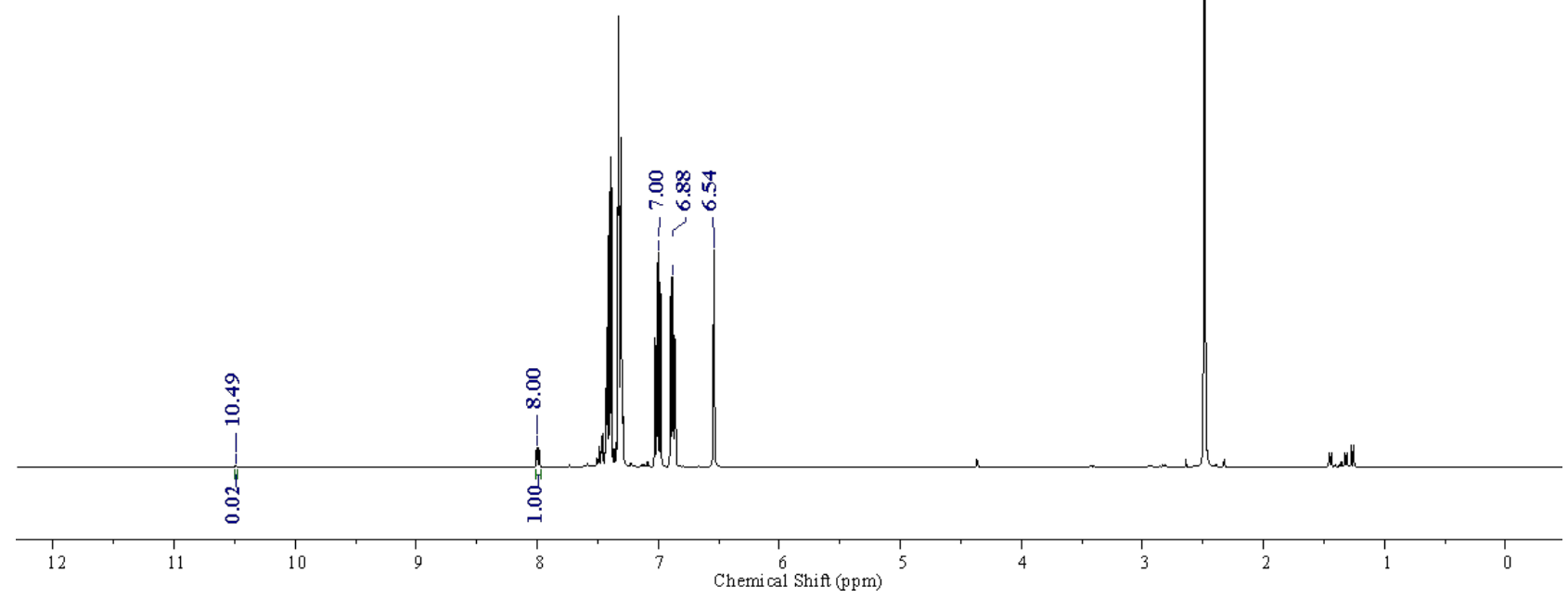


Entry 1: With Water (1.85 mmol), 17 hour

SCG-334-B 1-17hr-D 1-50sec.1. fid

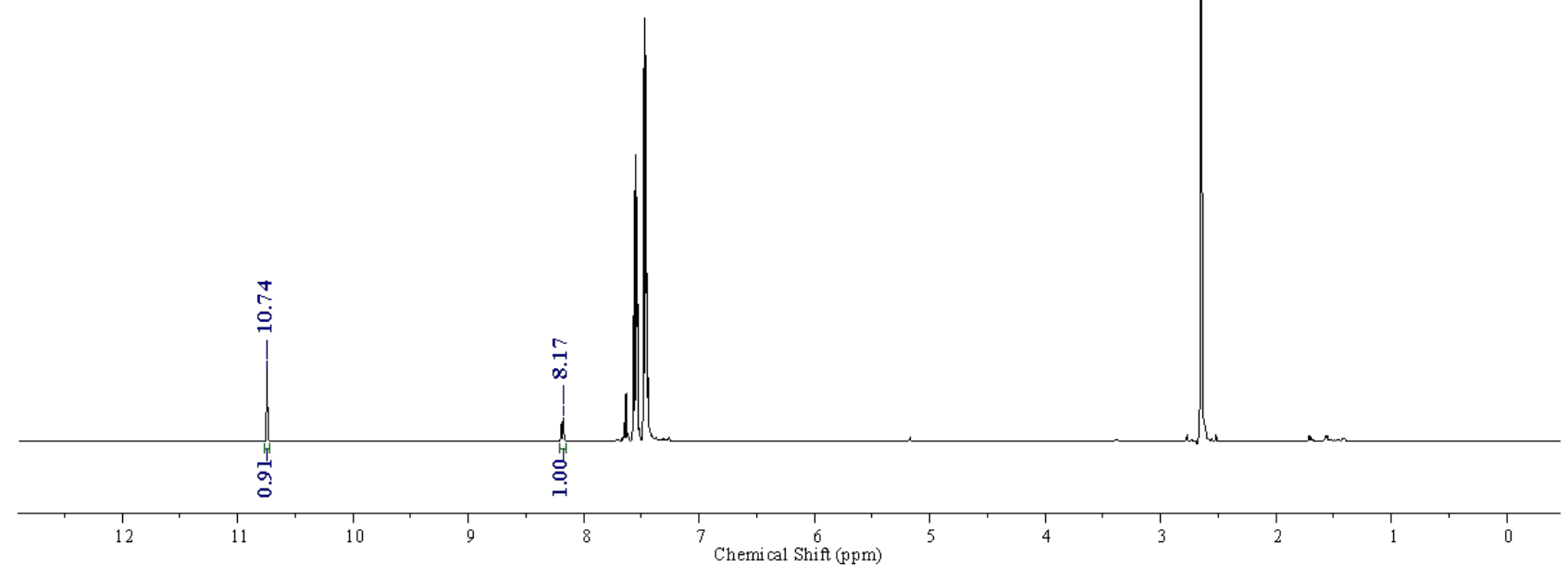

Entry 2: With Methanol (1.85 mmol), 17 hour

SCG-332-F3-17hr-D 1-50sec. 1.fid

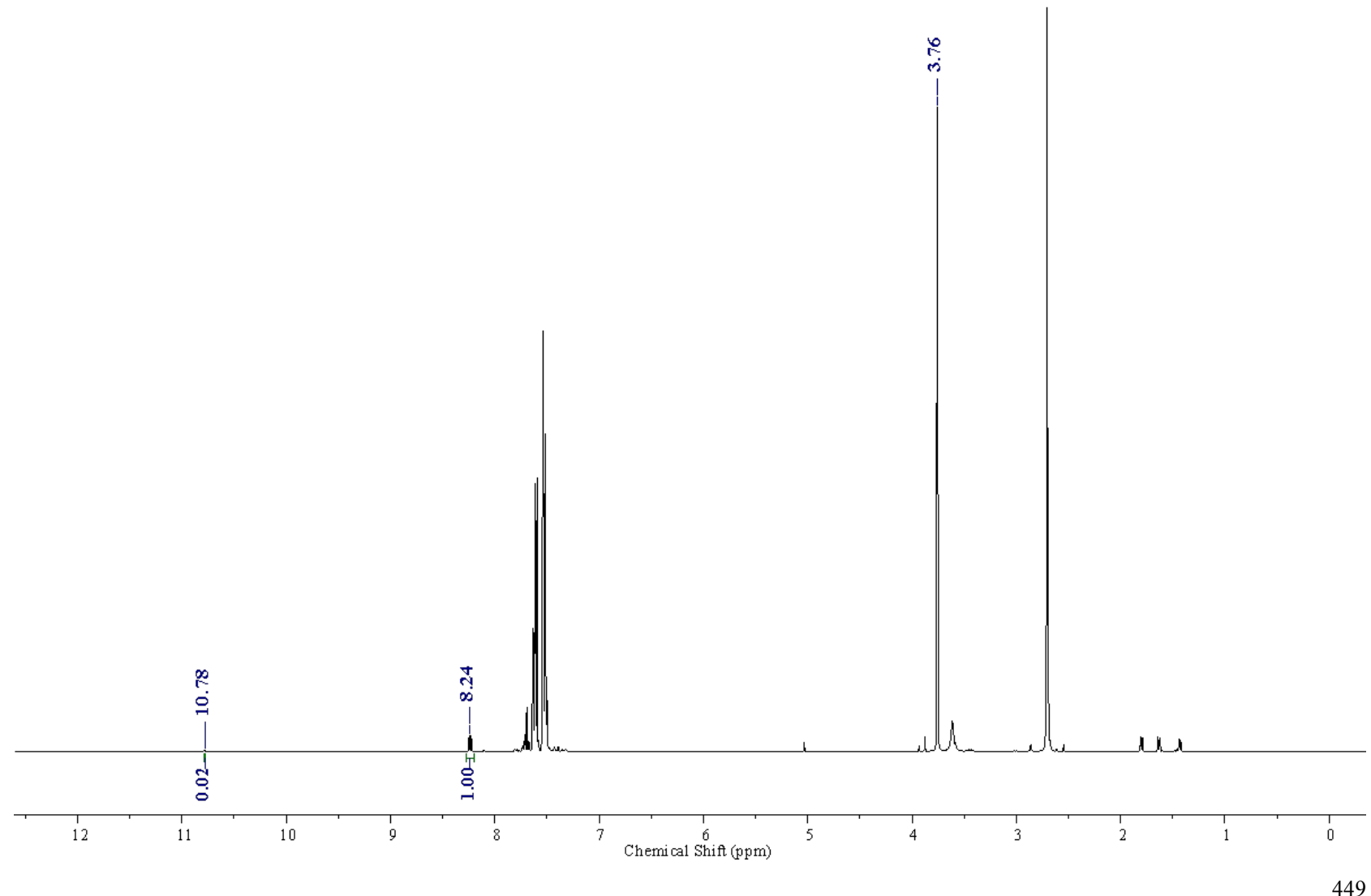


Entry 3: With Ethanol (1.85 mmol), 17 hour

SCG-332-A3-17hr-D 1-50sec. 1.fid

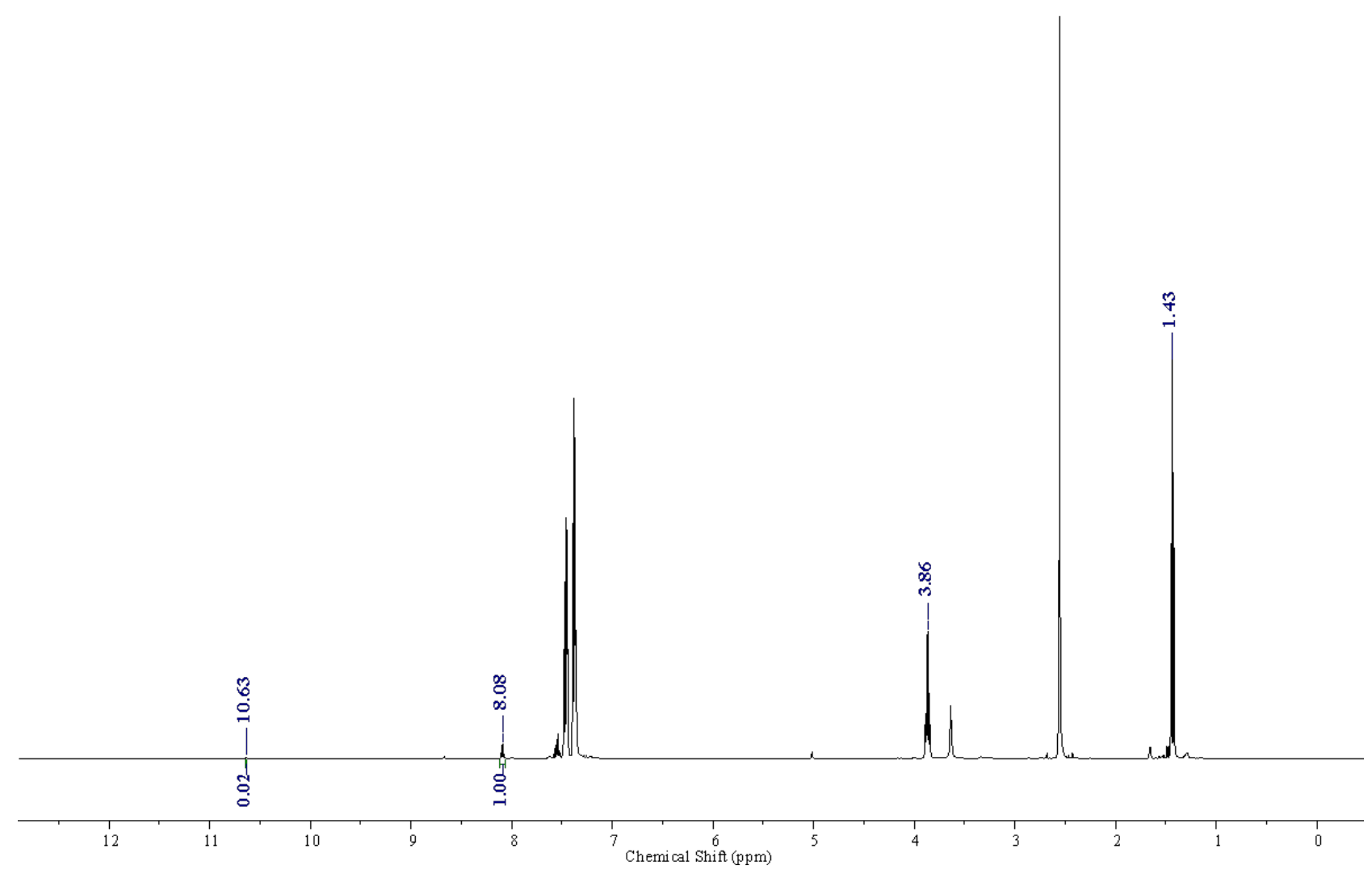

Entry 4: With Phenol (1.85 mmol), 17 hour

SCG-332-B 3-17hr-D 1-50sec. 1. fid

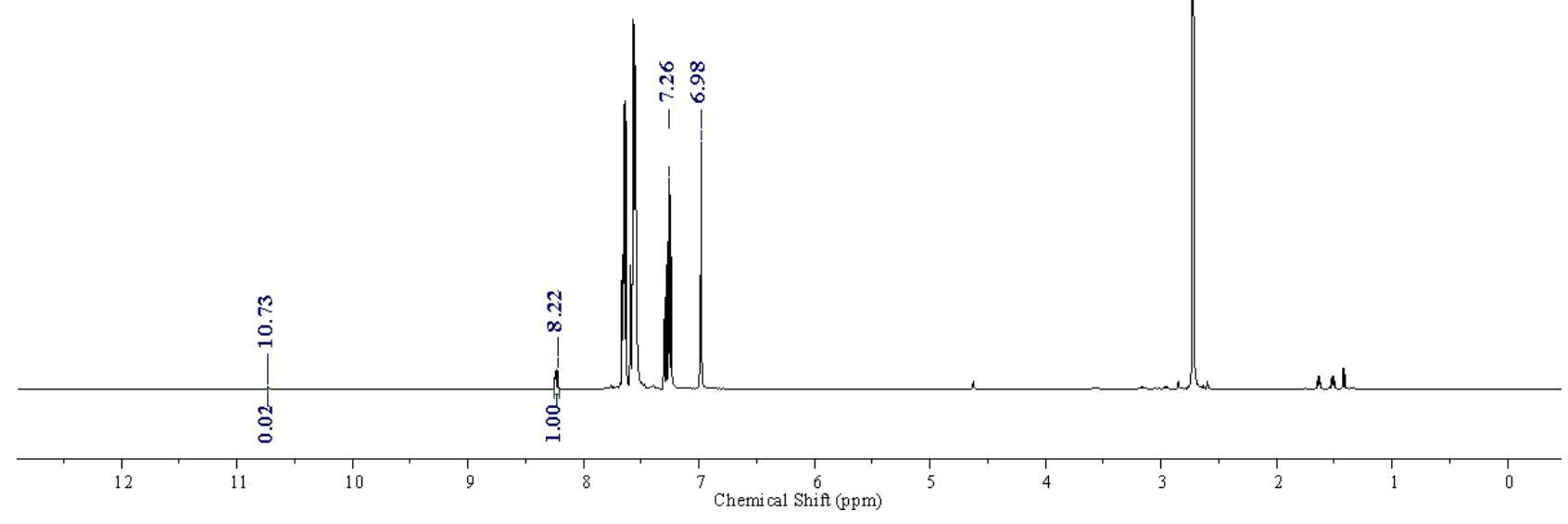


Entry 5: With 4-Methylphenol (1.85 mmol), 17 hour

SCG-332-C3-17hr-D 1-50sec.1. fid

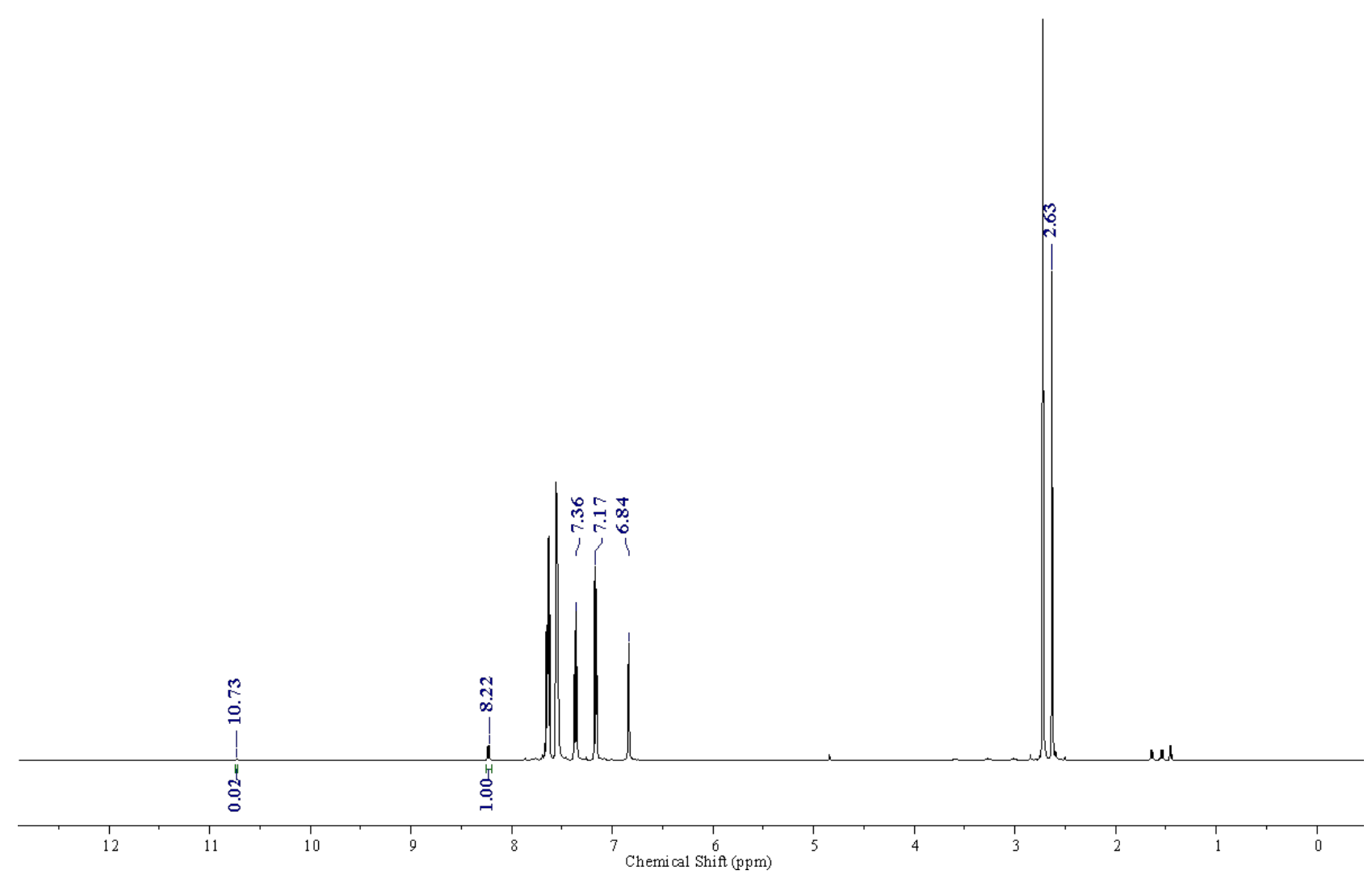

Entry 6: With Pentafluorophenol (1.85 mmol), 17 hour

SCG-332-D 3-17hr-D 1-50sec. 1. fid

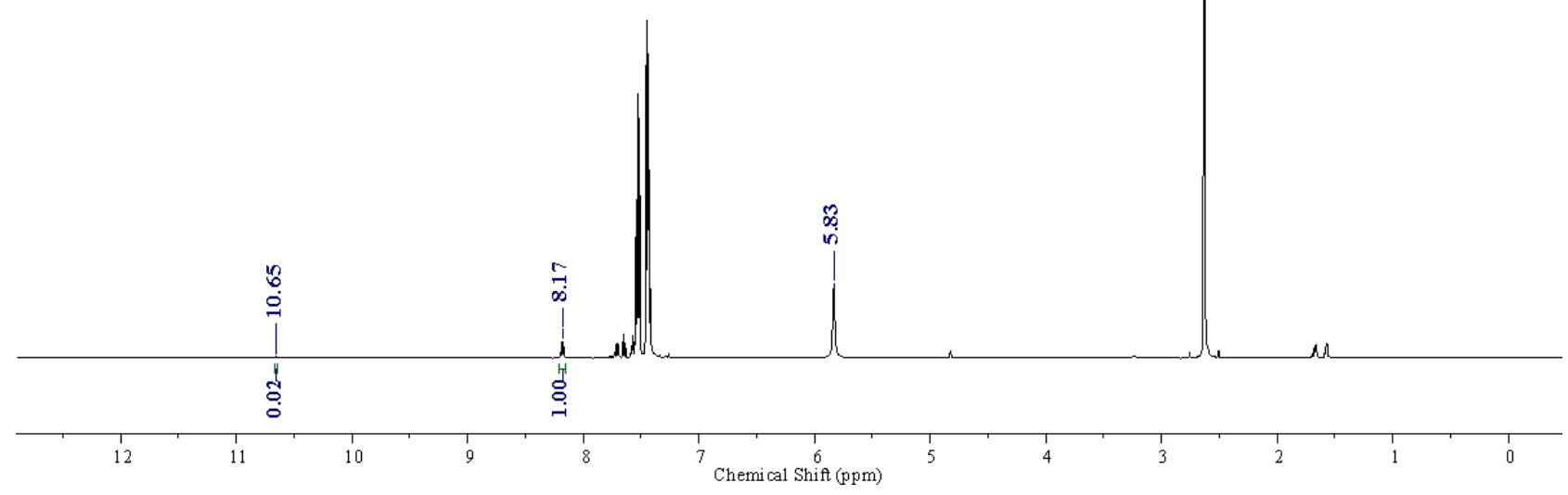


Entry 7: With 4-Fluorophenol (1.85 mmol), 17 hour

SCG-332-E3-17hr-D 1-50sec. 1.fid

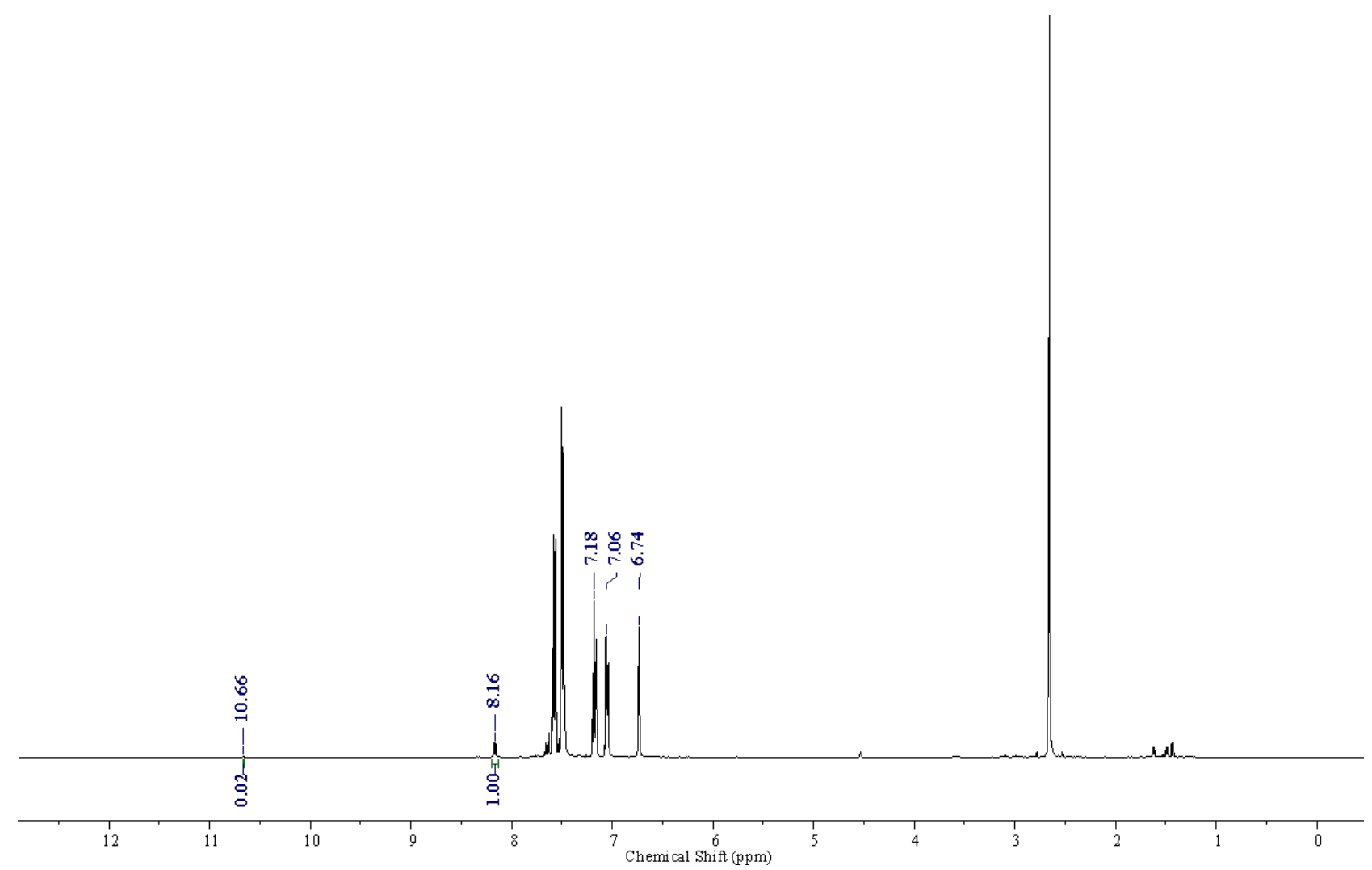


8.2 Reactions with $3 b$ and $\mathrm{R}-\mathrm{OH}$ (5 eq.)

Entry 1: With Methanol (1.85 $\mathrm{mmol}), 1$ hour

SCG-339-A 1-60min-D 1-50sec. 1. fid

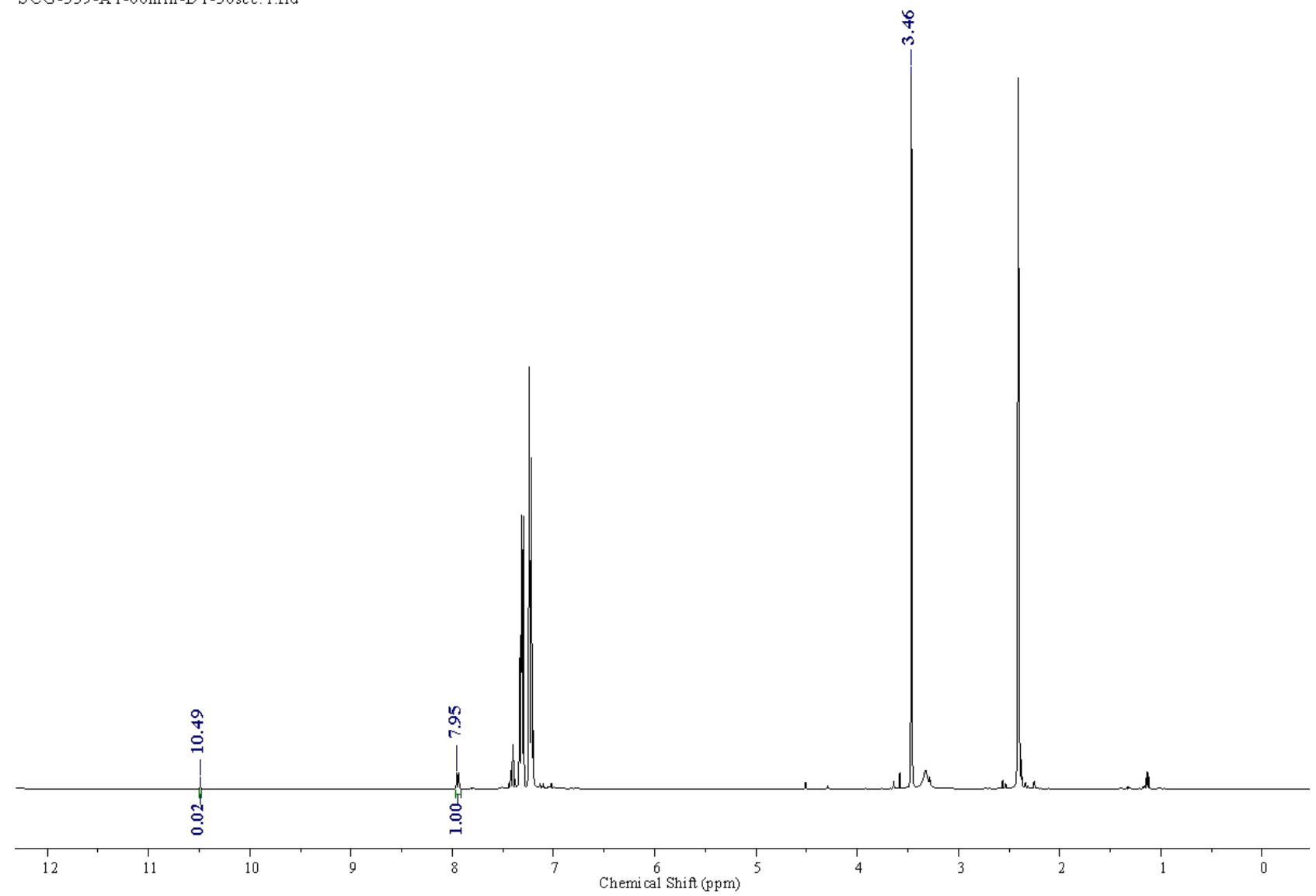

Entry 2: With Ethanol (1.85 mmol), 1 hour SCG-339-B 1-60min-D 1-50sec. 1. fid

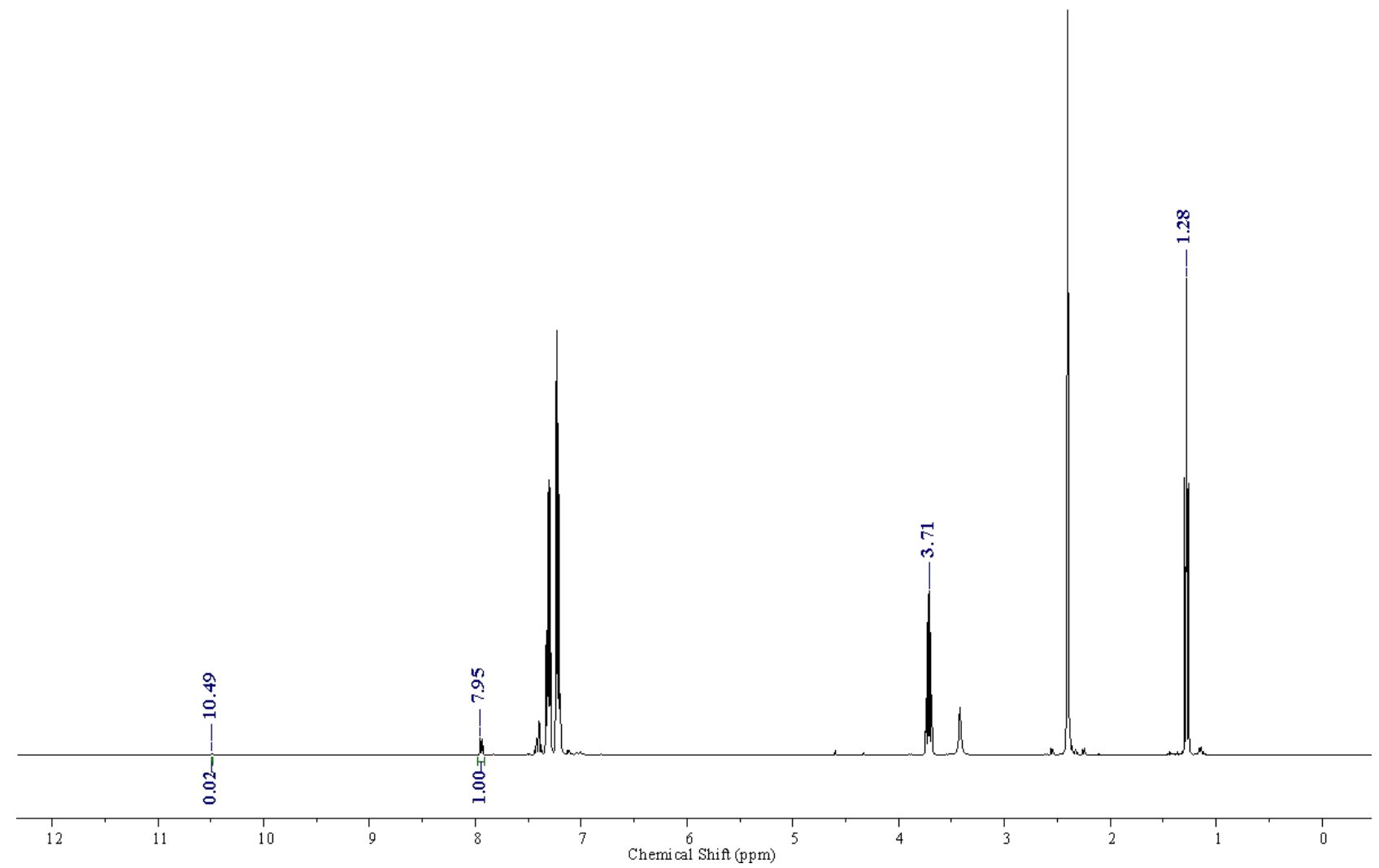


Entry 3: With Phenol (1.85 mmol), 1 hour

SCG-339-C1-60min-D 1-50sec. 1. fid

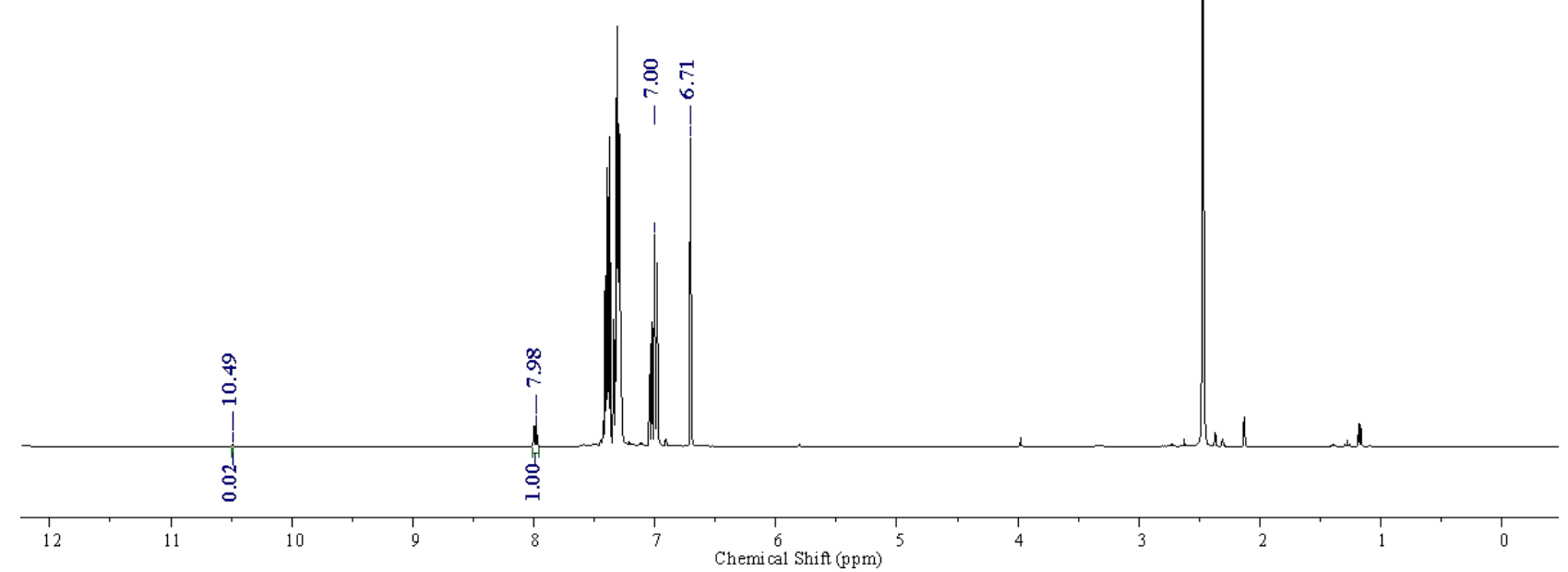

Entry 4: With 4-Methylphenol (1.85 mmol), 1 hour

SCG-339-D 1-60m in-D 1-50sec. 1. fid

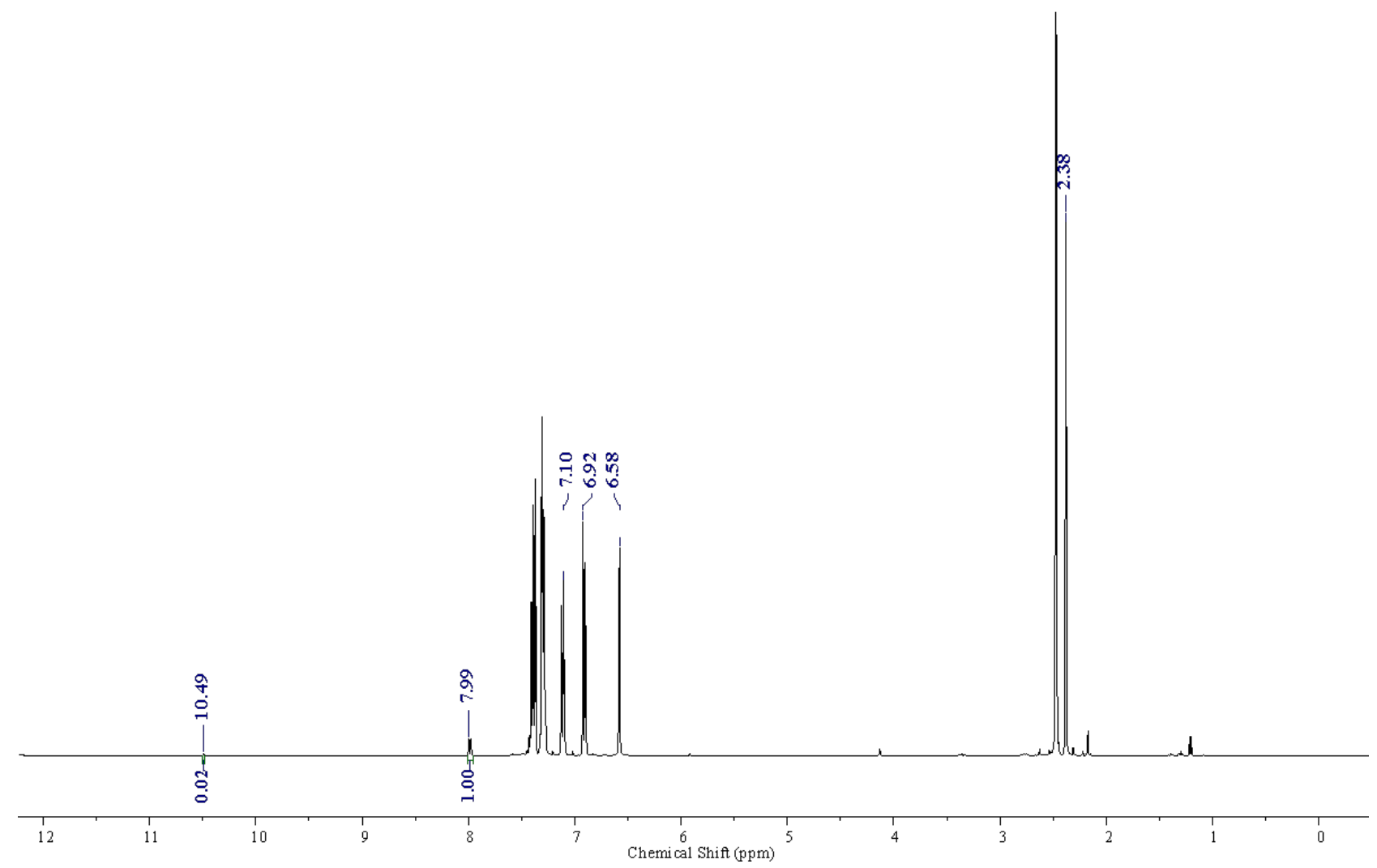


Entry 5: With Pentafluorophenol (1.85 mmol), 1 hour

SCG-339-E 1-60m in-D 1-50sec. 1. fid

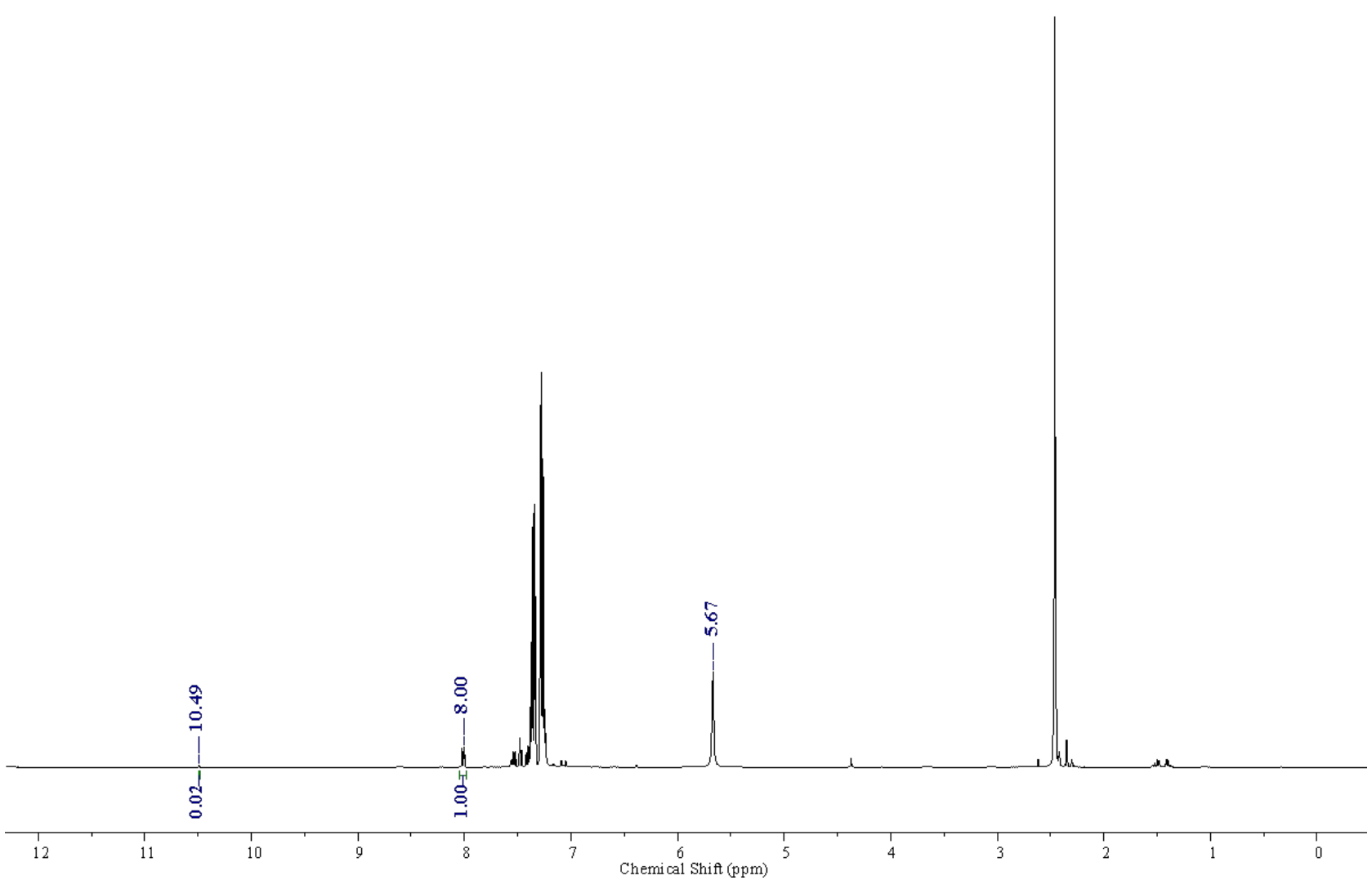

Entry 1: With Water (1.85 mmol), 17 hour

VDOS-651-2-C1

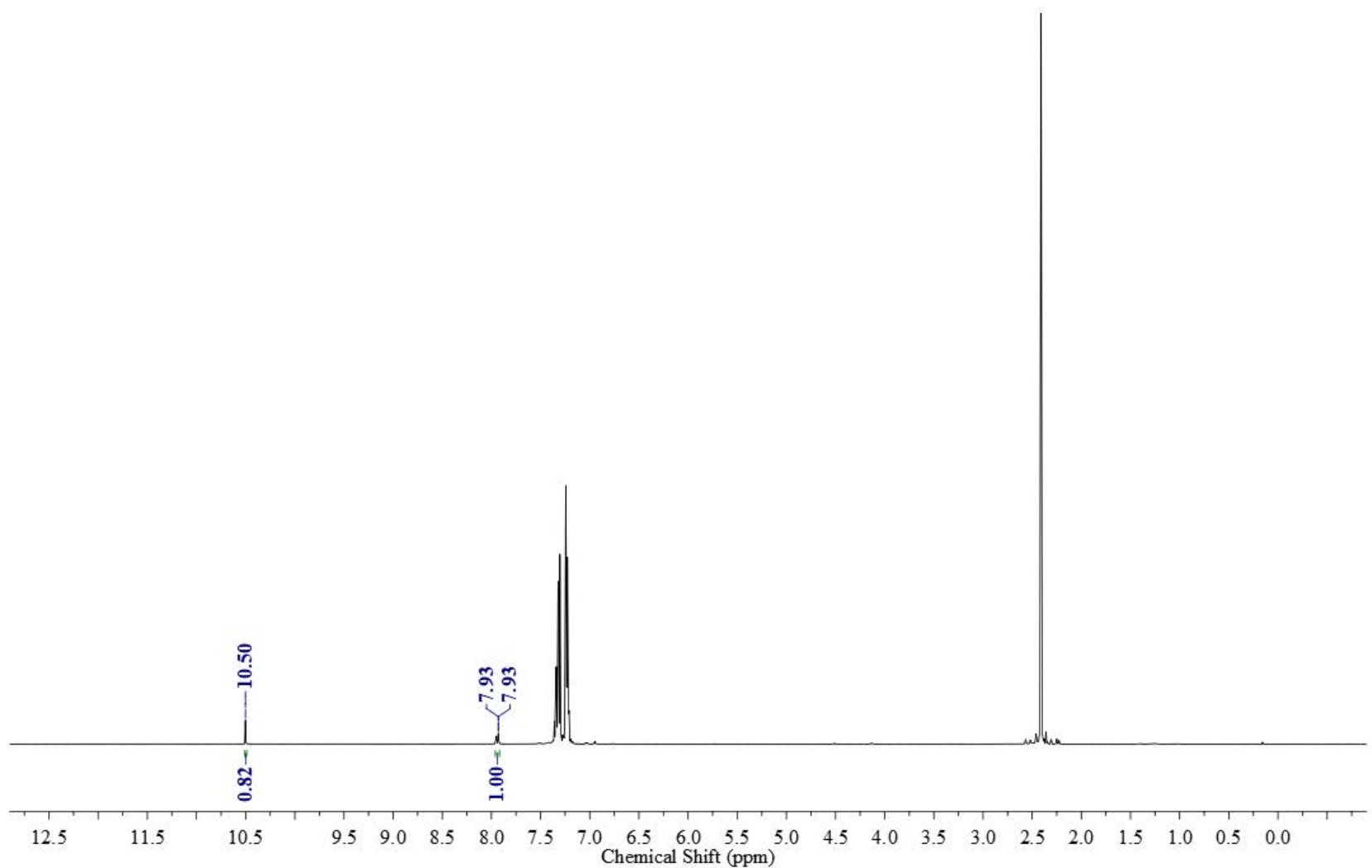


Entry 2: With Methanol (1.85 mmol), 17 hour

SCG-339-A 3-16hr-D 1-50sec.2.fid

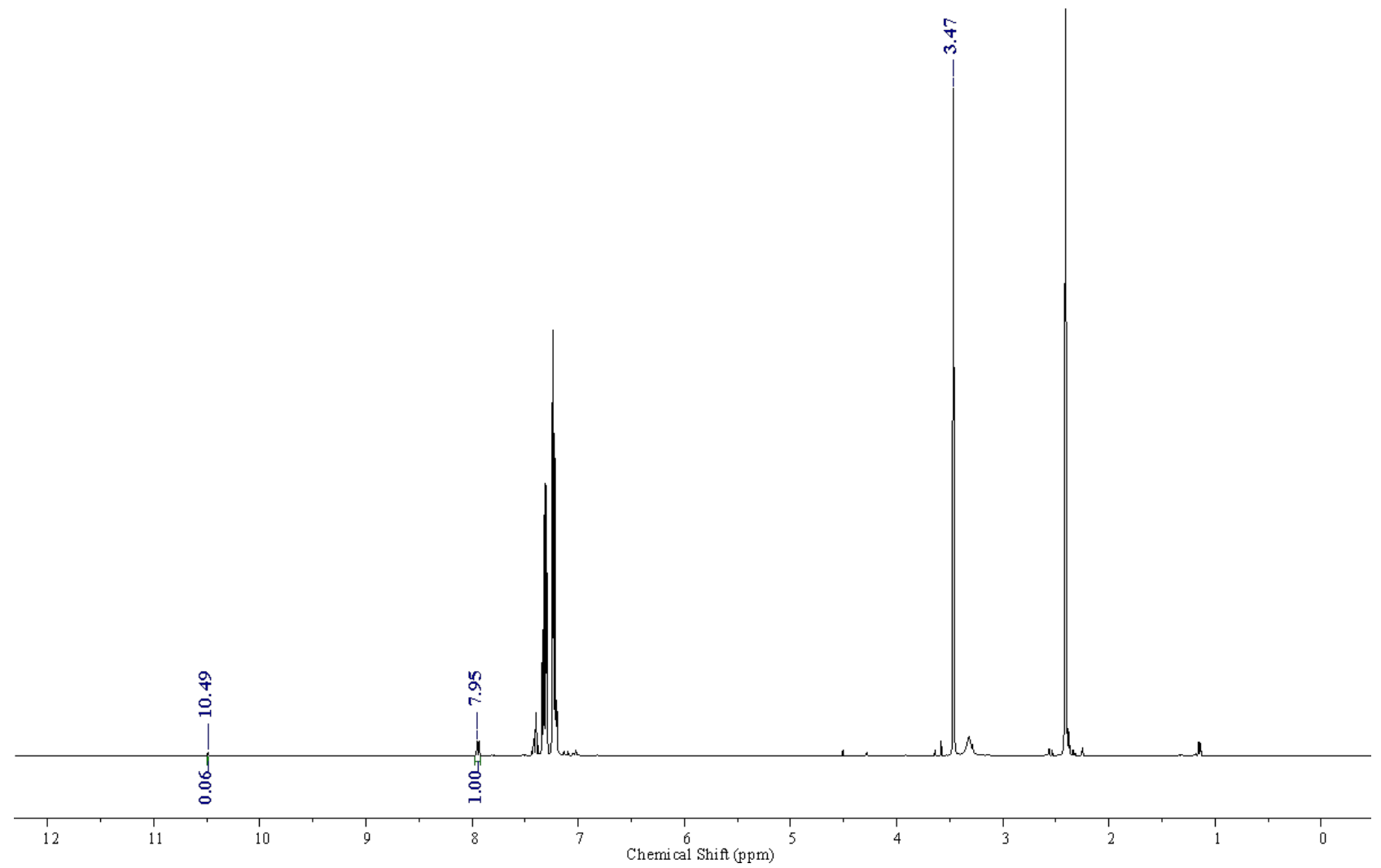

Entry 3: With Ethanol (1.85 mmol), 17 hour

SCG-339-B 3-16hr-D 1-50sec. 1. fid

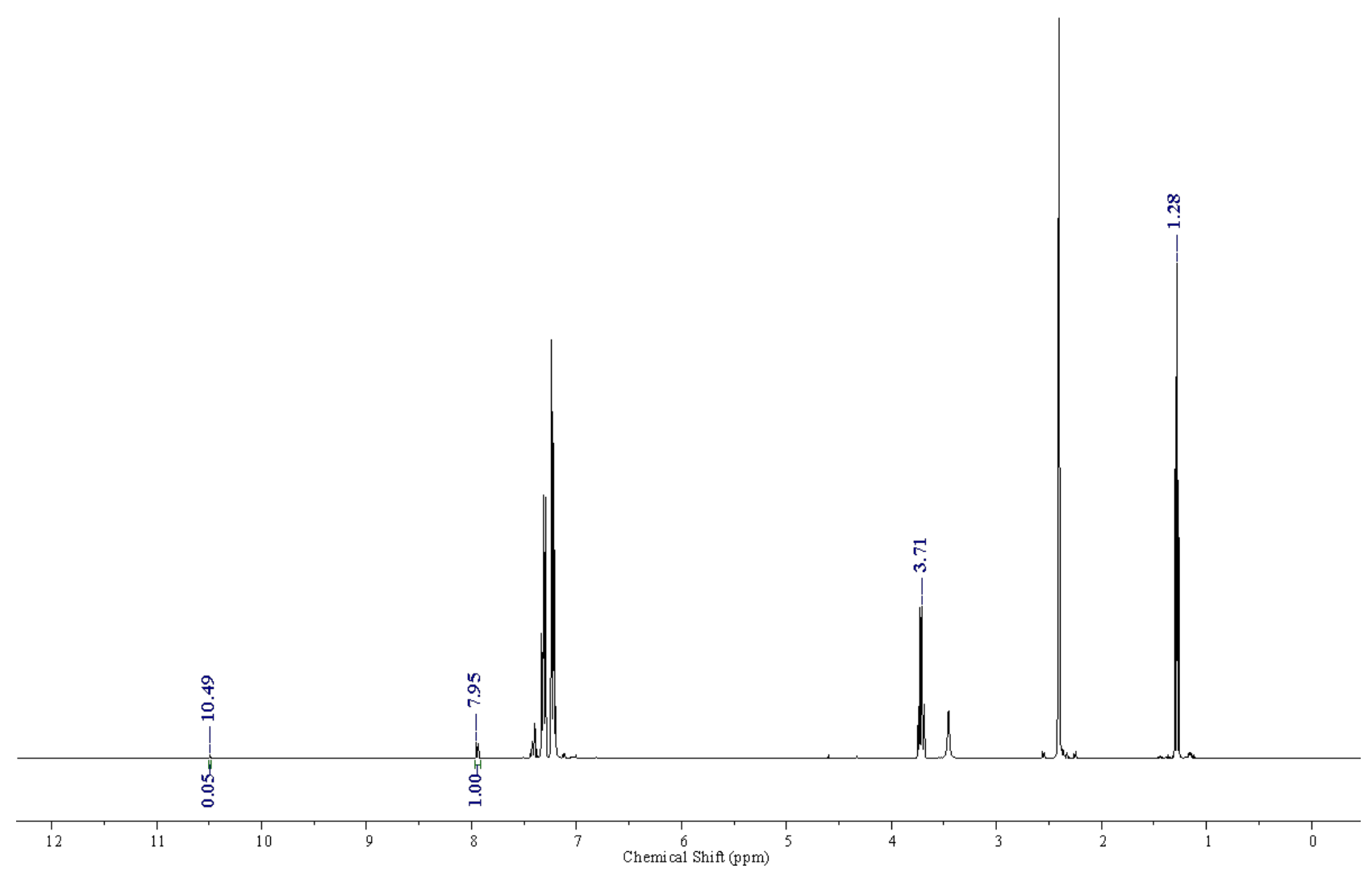


Entry 4: With Trifluoroethanol (1.85 mmol), 17 hour

VDOS-651-2-B1

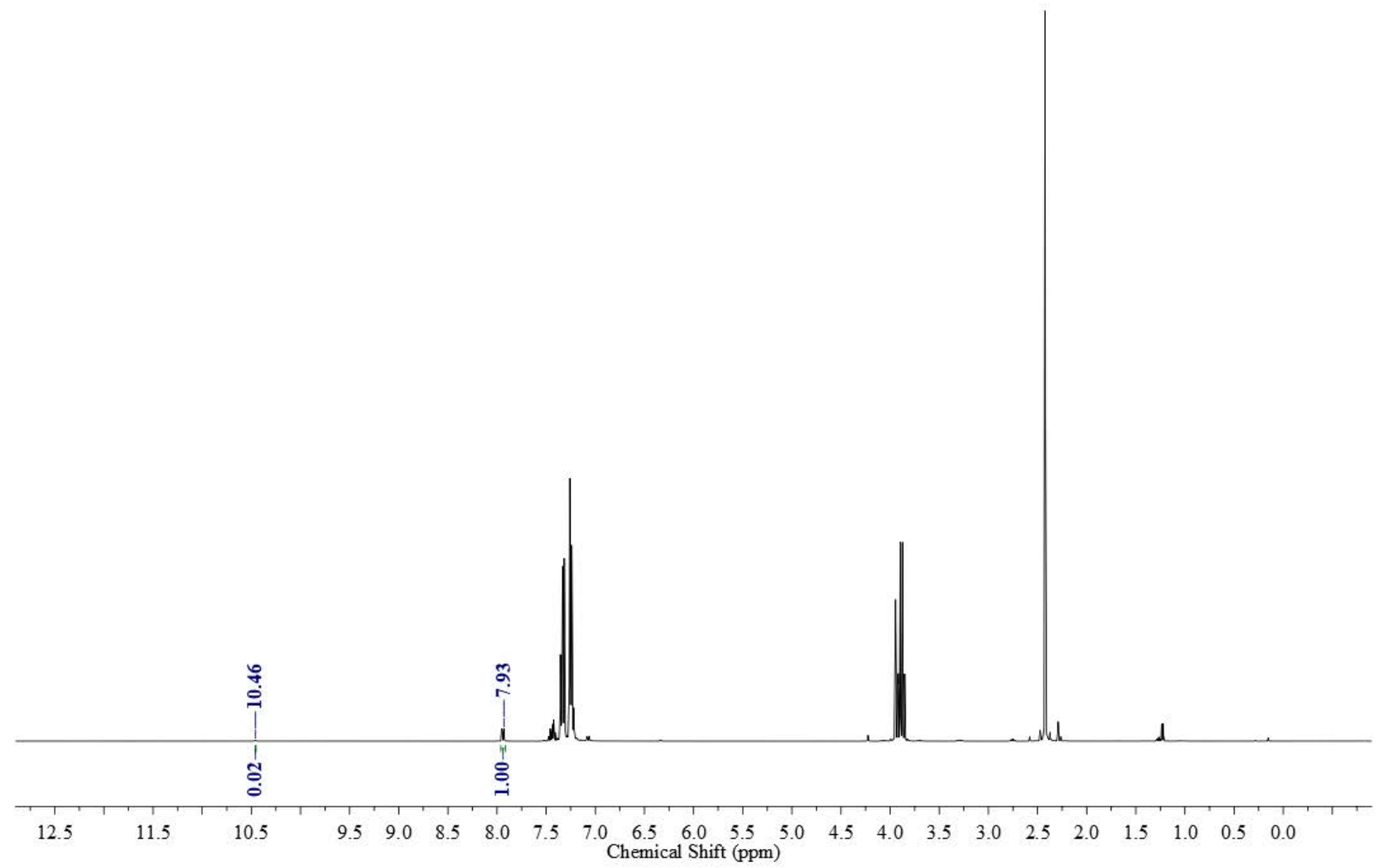

Entry 5: With Phenol (1.85 mmol), 17 hour

SCG-339-C3-16hr-D 1-50sec.1.fid

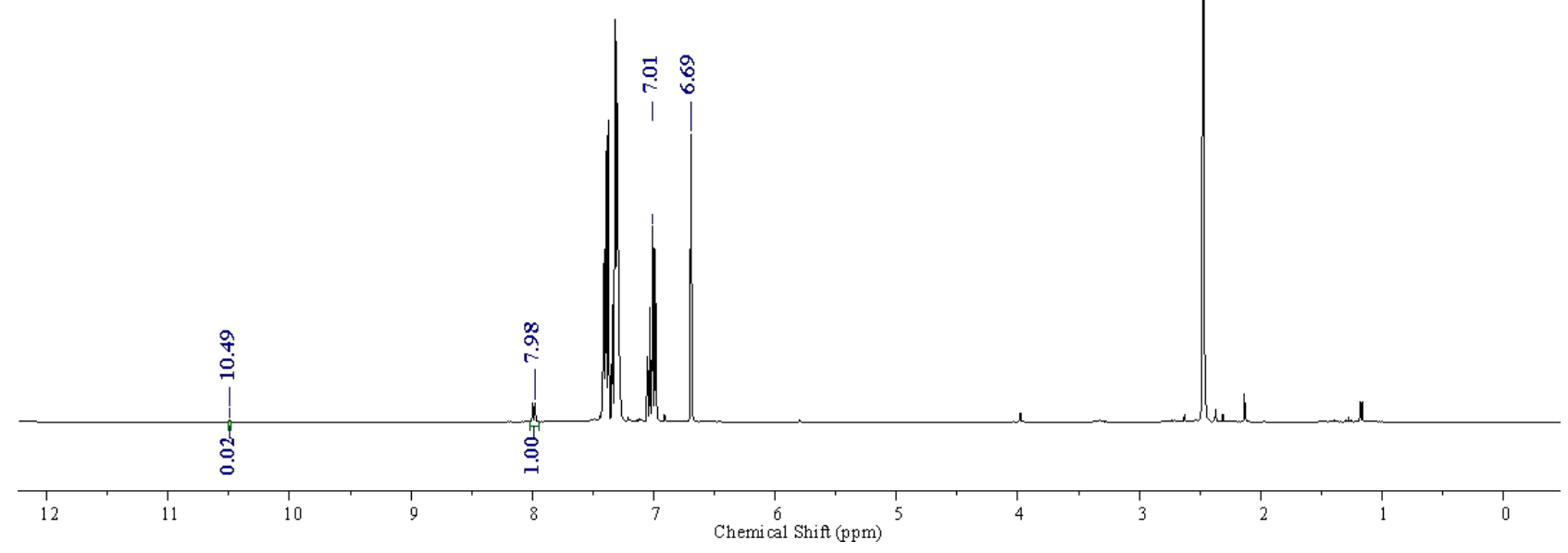


Entry 6: With 4-Methylphenol (1.85 mmol), 17 hour

SCG-339-D 3-16hr-D 1-50sec.1.fid

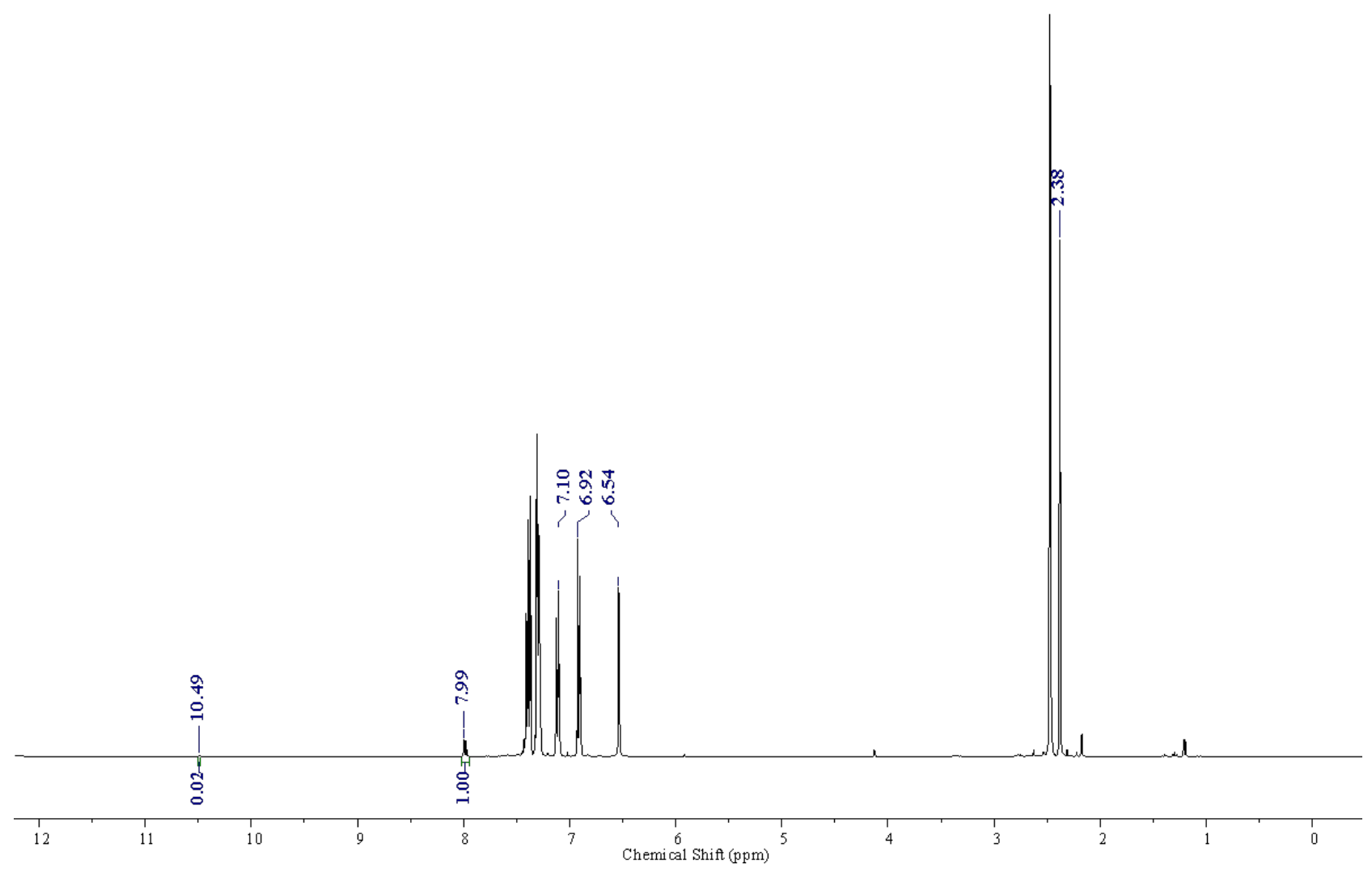

Entry 7: With Pentafluorophenol (1.85 mmol), 17 hour

SCG-339-E3-16hr-D 1-50sec. 1. fid

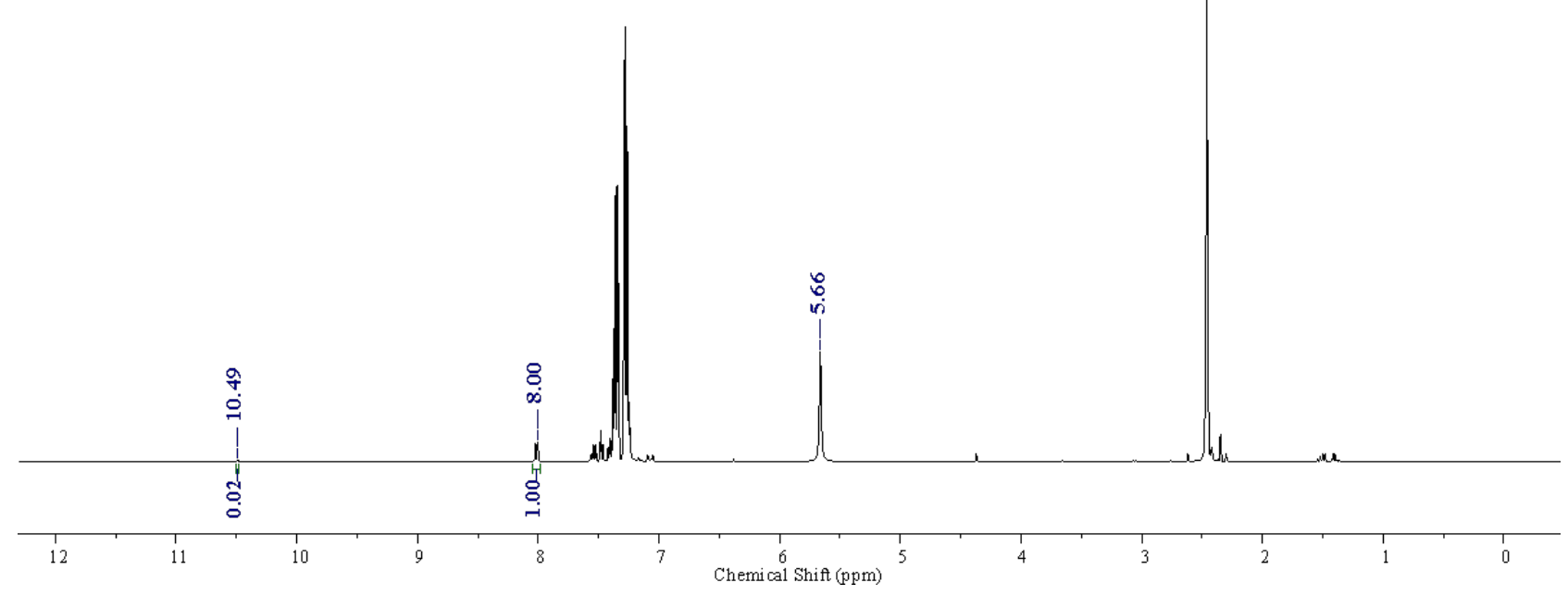


Entry 8: With 4-Fluorophenol (1.85 mmol), 17 hour vDOS-651-2-A1

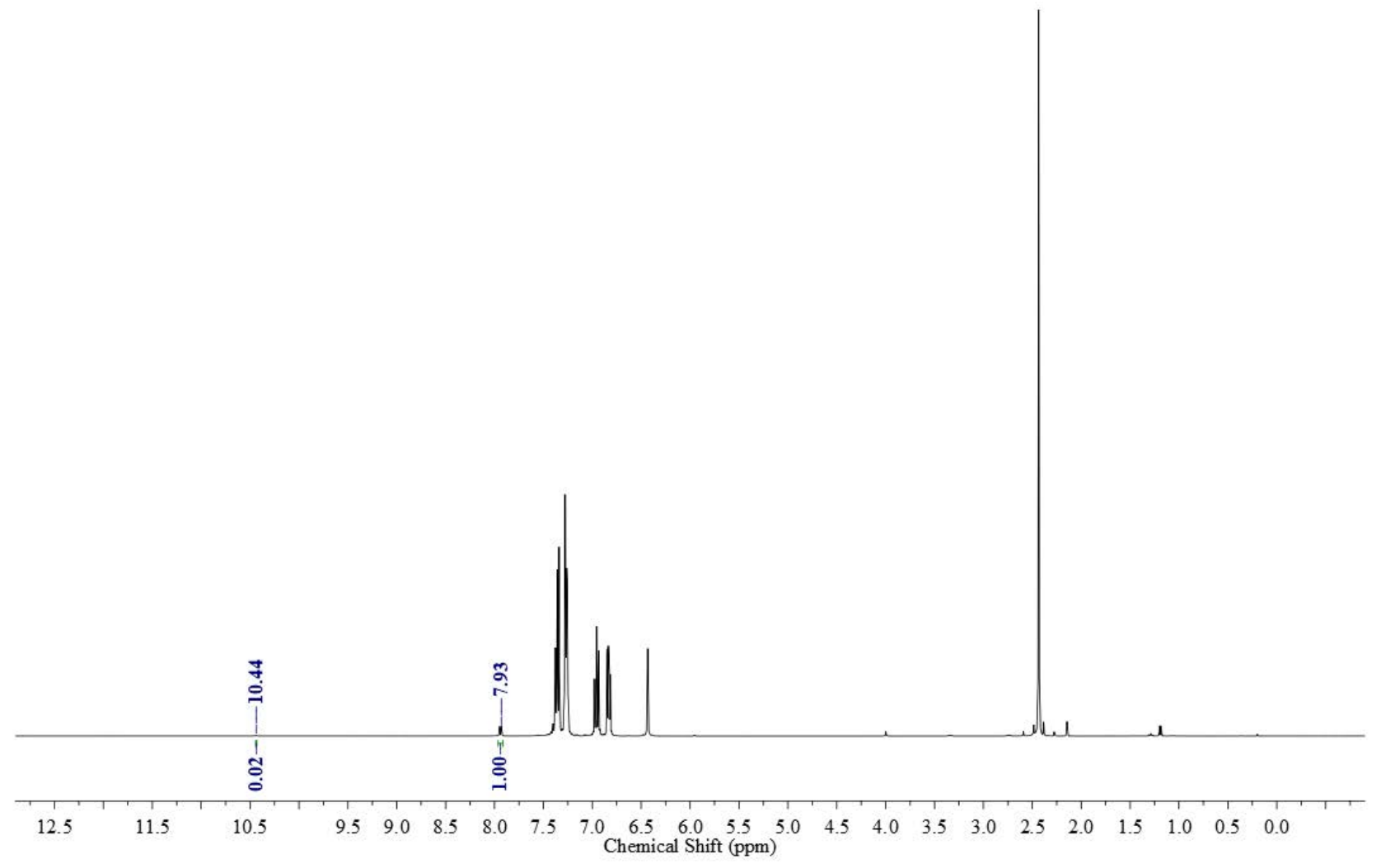


8.3 Reactions with $\mathbf{3 b}$ and Methanol (Different Concentrations):

Entry 1: With $0.00999 \mathrm{mmol}$ Methanol $(0.027 \mathrm{M}), 17$ hour

SCG-343-A 5-17hr-D 1-50sec.1.fid

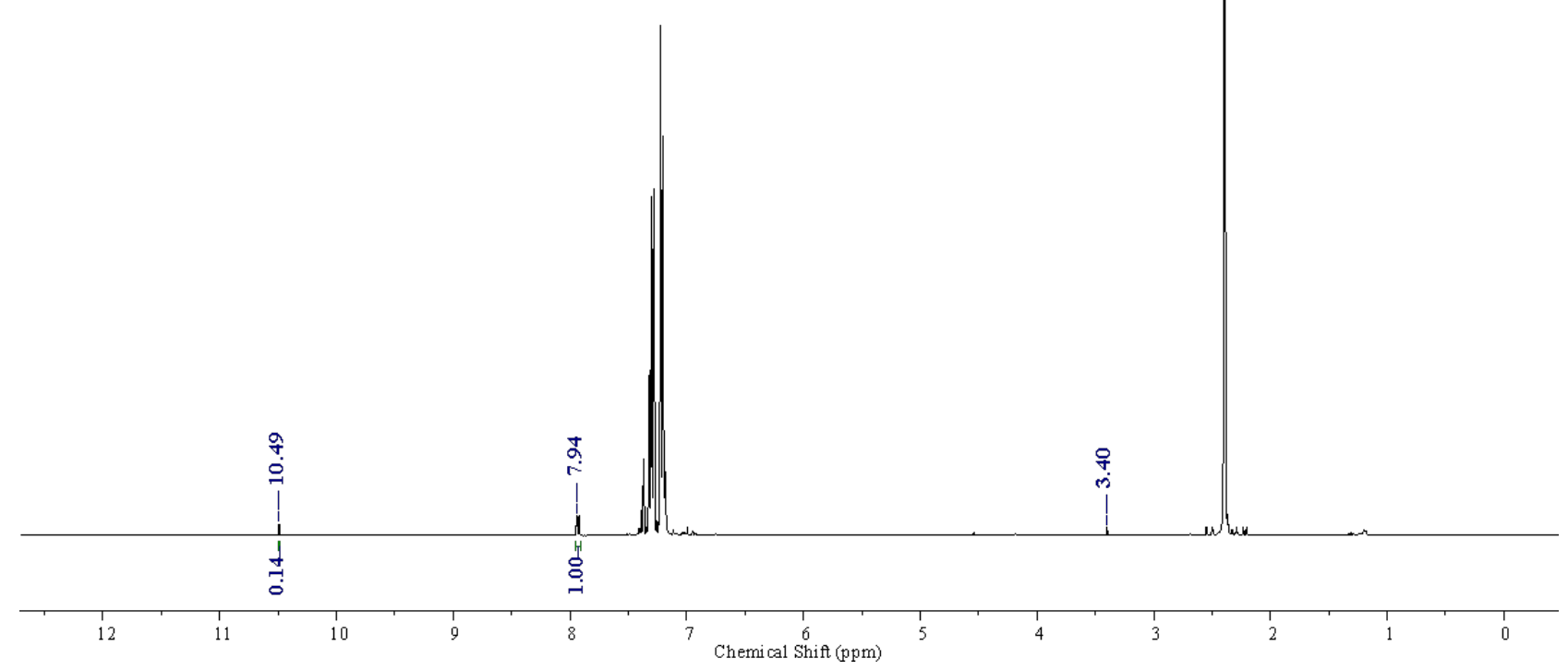

Entry 2: With $0.037 \mathrm{mmol}$ Methanol $(0.100 \mathrm{M}), 17$ hour SCG-343-B 5-17hr-D 1-50sec.2.fid

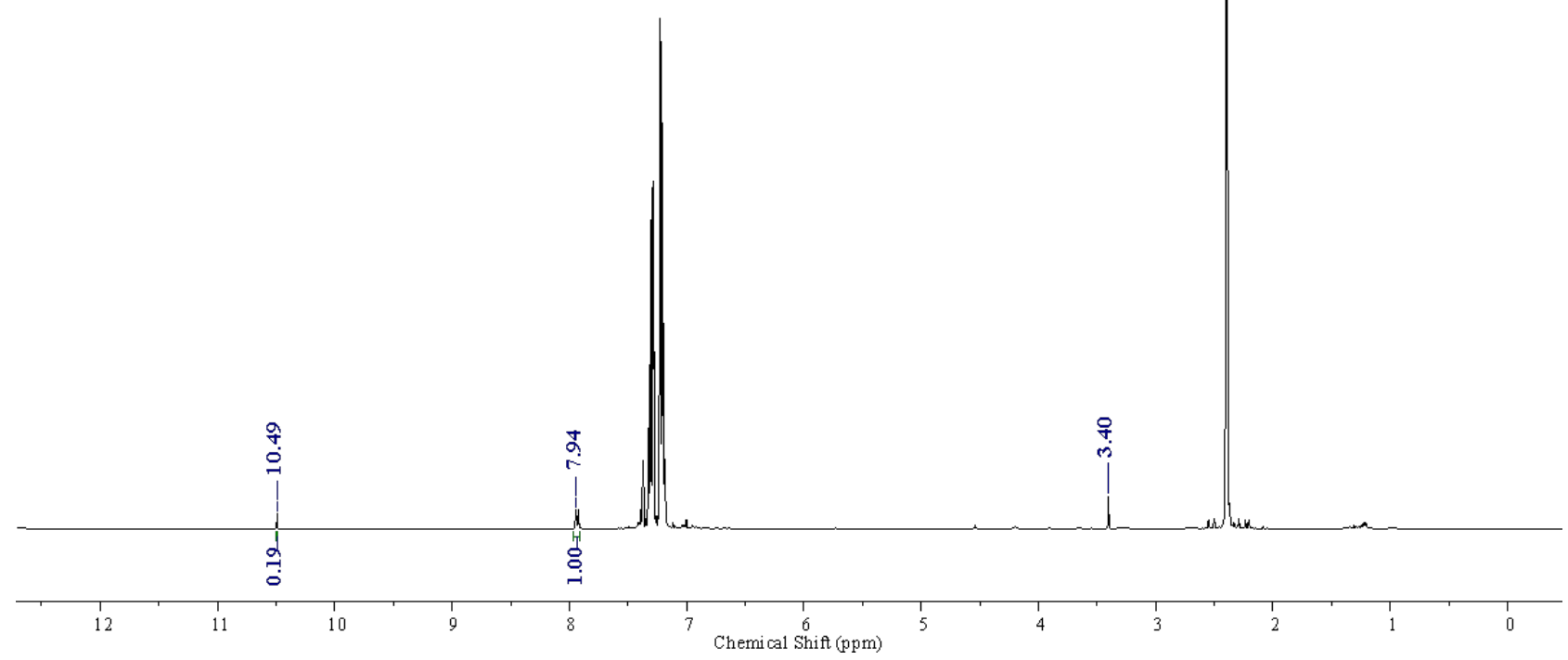


Entry 3: With $0.06 \mathrm{mmol}$ Methanol $(0.162 \mathrm{M}), 17$ hour SCG-343-E3-17hr-D 1-50sec. 1.fid

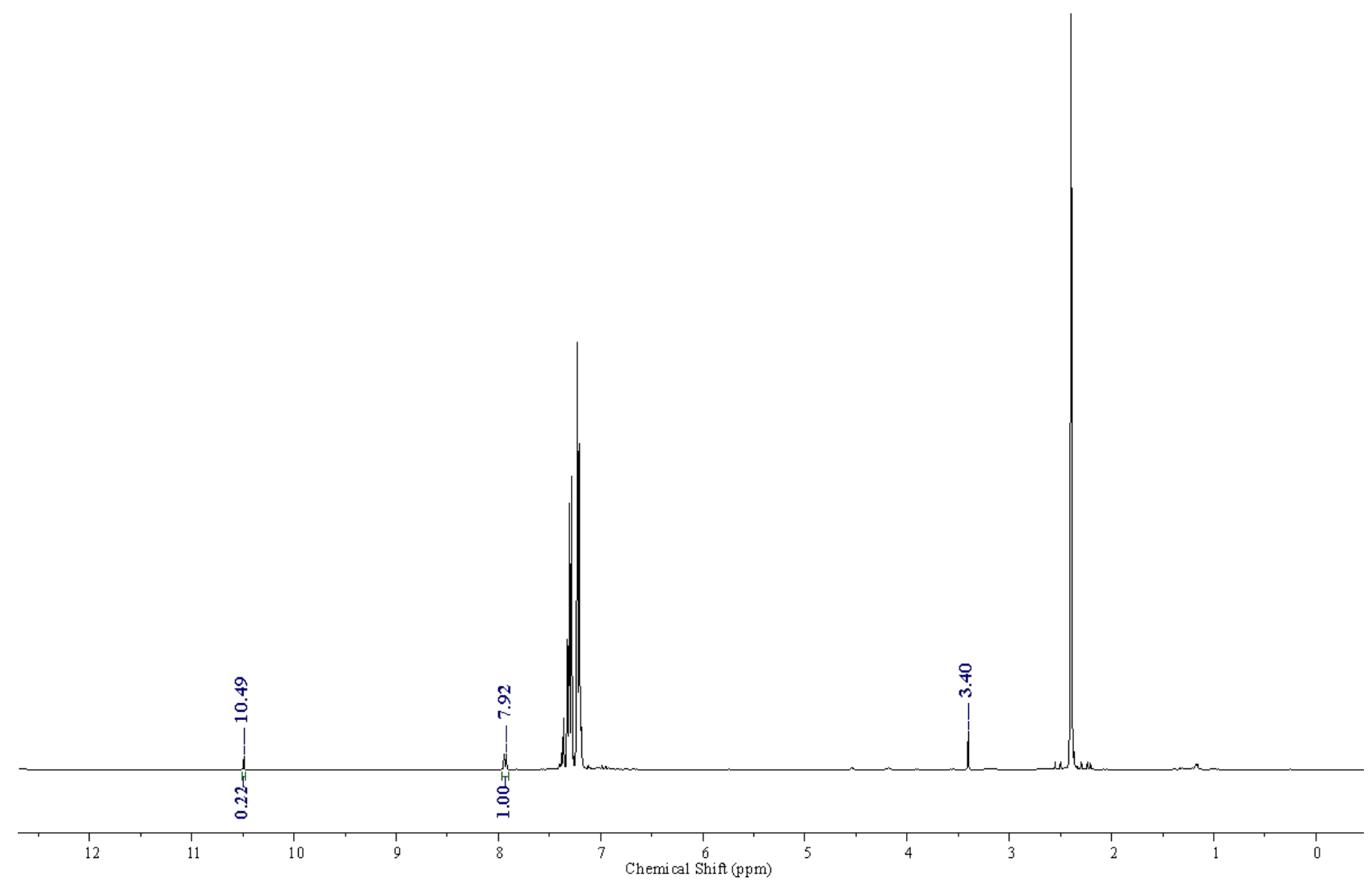

Entry 4: With $0.08 \mathrm{mmol}$ Methanol $(0.216 \mathrm{M}), 17$ hour

SCG-343-C3-17hr-D 1-50sec.1.fid

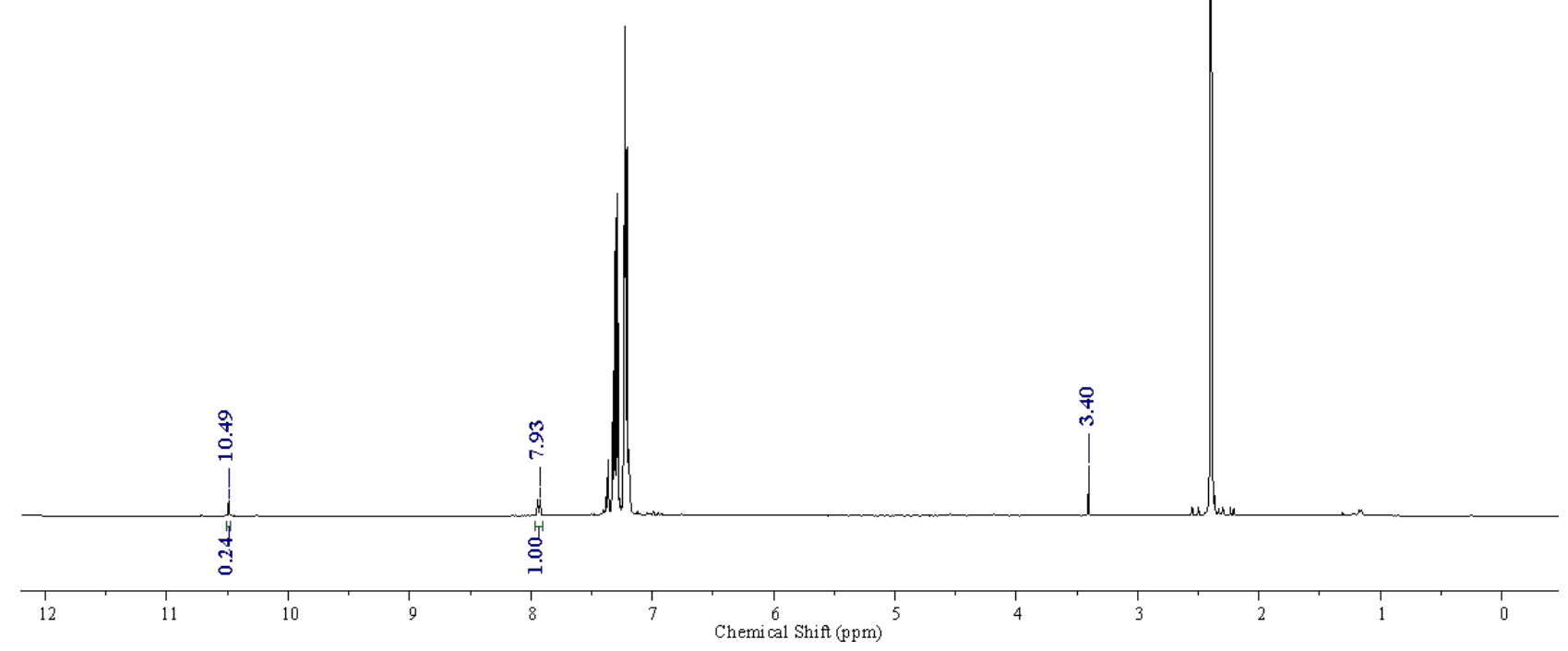


Entry 5: With $0.10 \mathrm{mmol}$ Methanol $(0.270 \mathrm{M}), 17$ hour SCG-343-F3-17hr-D 1-50sec. 1.fid

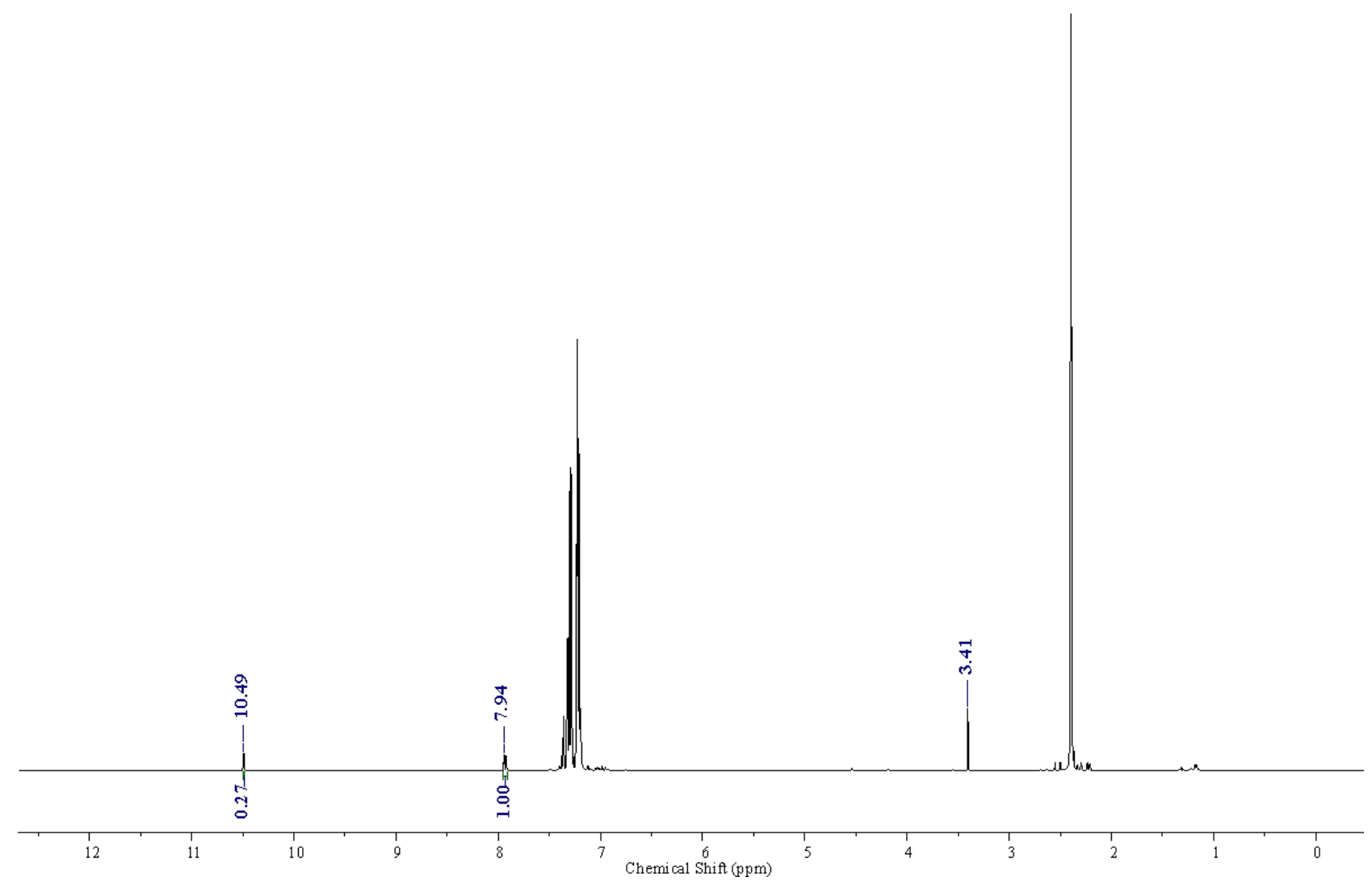

Entry 6: With $0.12 \mathrm{mmol}$ Methanol $(0.324 \mathrm{M}), 17$ hour

SCG-343-O1-17hr-D 1-50sec.1.fid

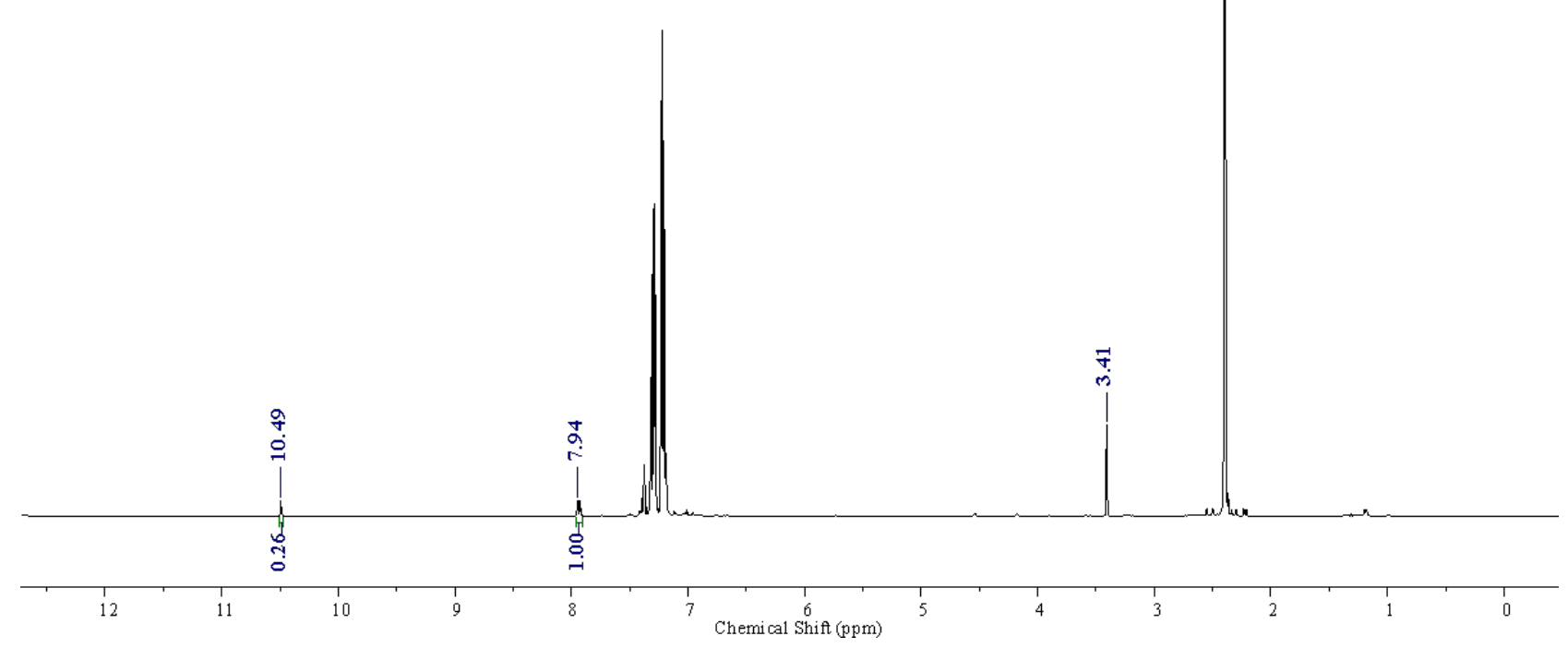


Entry 7: With $0.14 \mathrm{mmol}$ Methanol $(0.378 \mathrm{M}), 17$ hour SCG-343-N1-17hr-D 1-50sec.1. fid

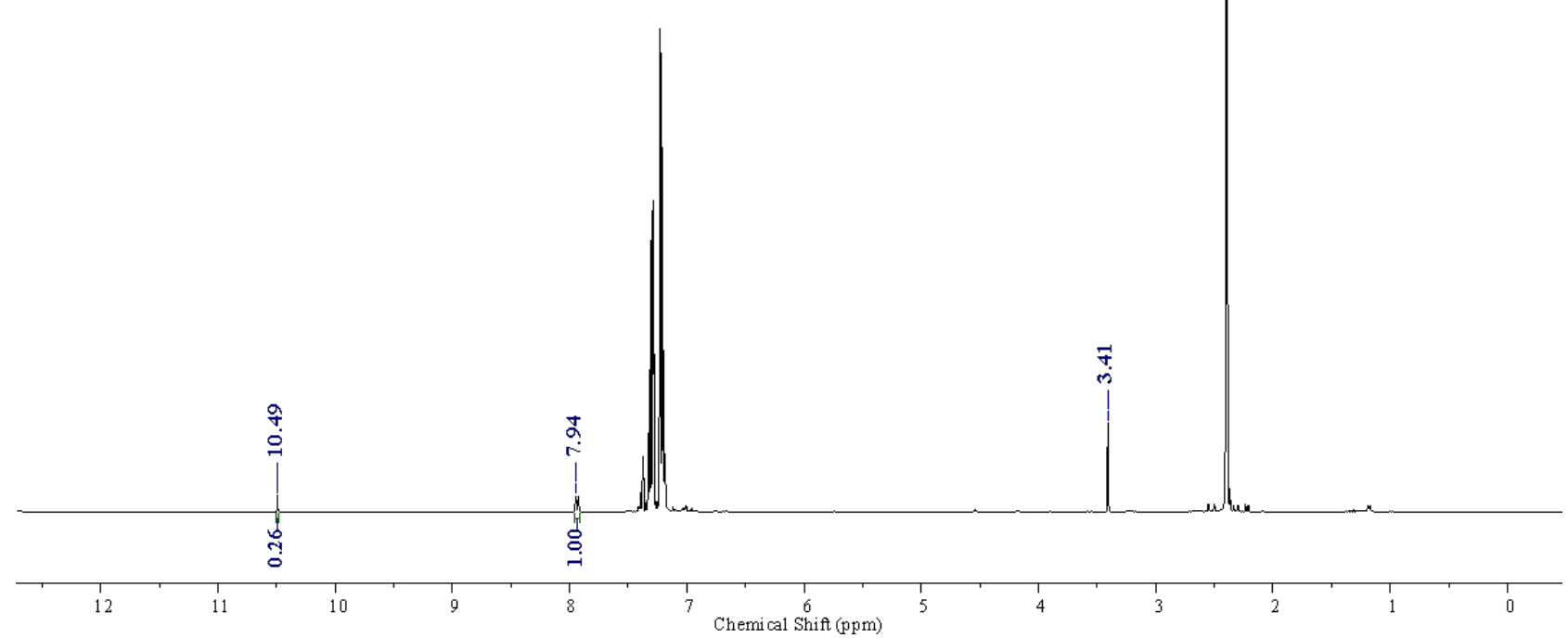

Entry 8: With $0.16 \mathrm{mmol}$ Methanol $(0.432 \mathrm{M}), 17$ hour

SCG-343-P1-17hr-D 1-50sec.1.fid

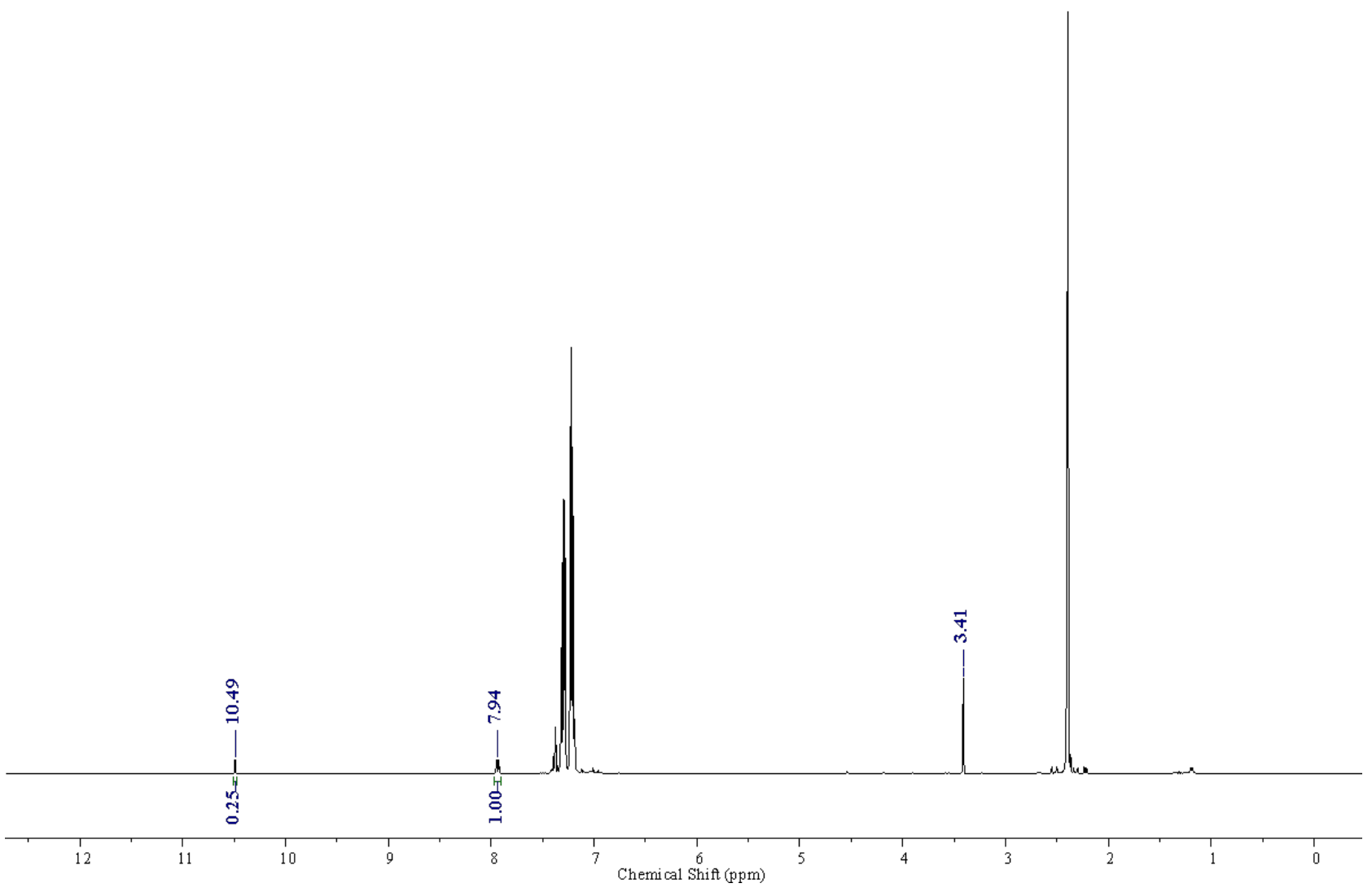


Entry 9: With $0.20 \mathrm{mmol}$ Methanol (0.541 M), 17 hour SCG-343-K1-17hr-D 1-50sec.1.fid

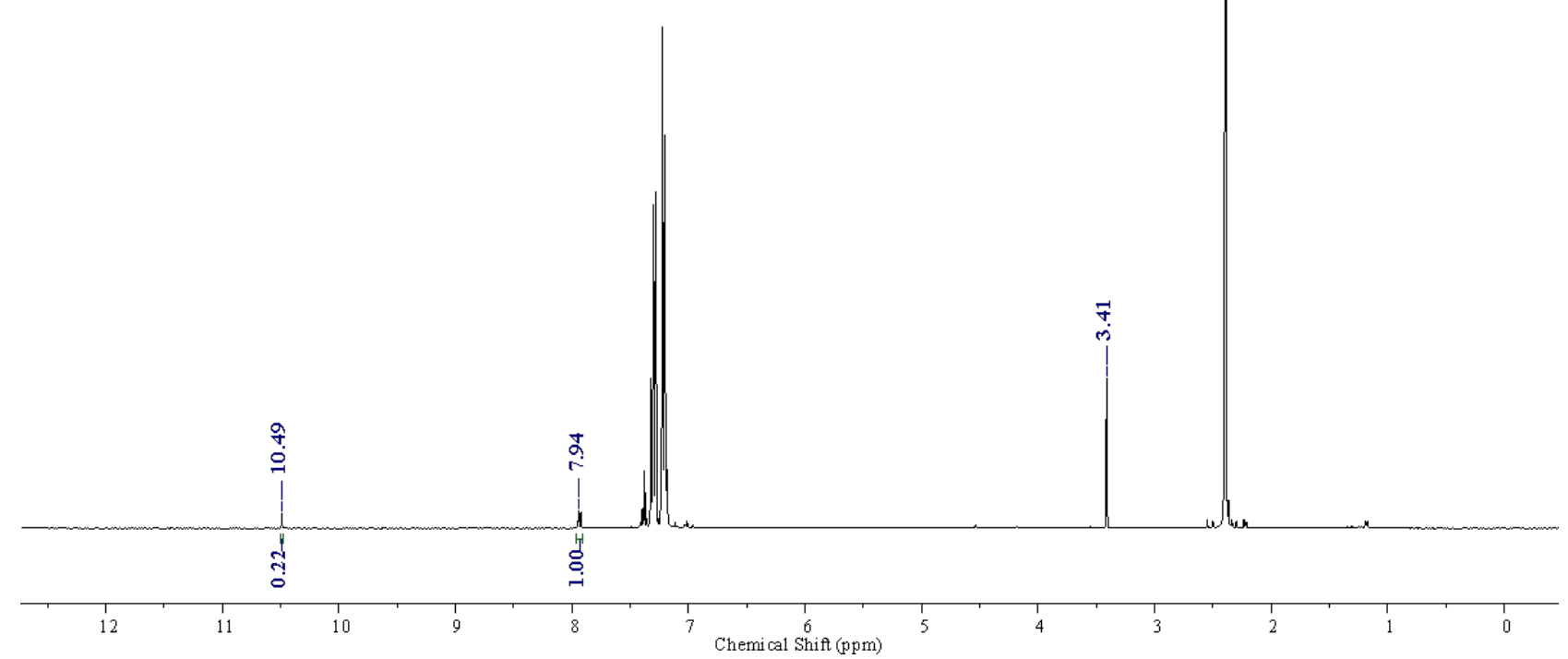

Entry 10: With $0.30 \mathrm{mmol}$ Methanol $(0.811 \mathrm{M}), 17$ hour

SCG-343-L1-17hr-D 1-50sec.1.fid

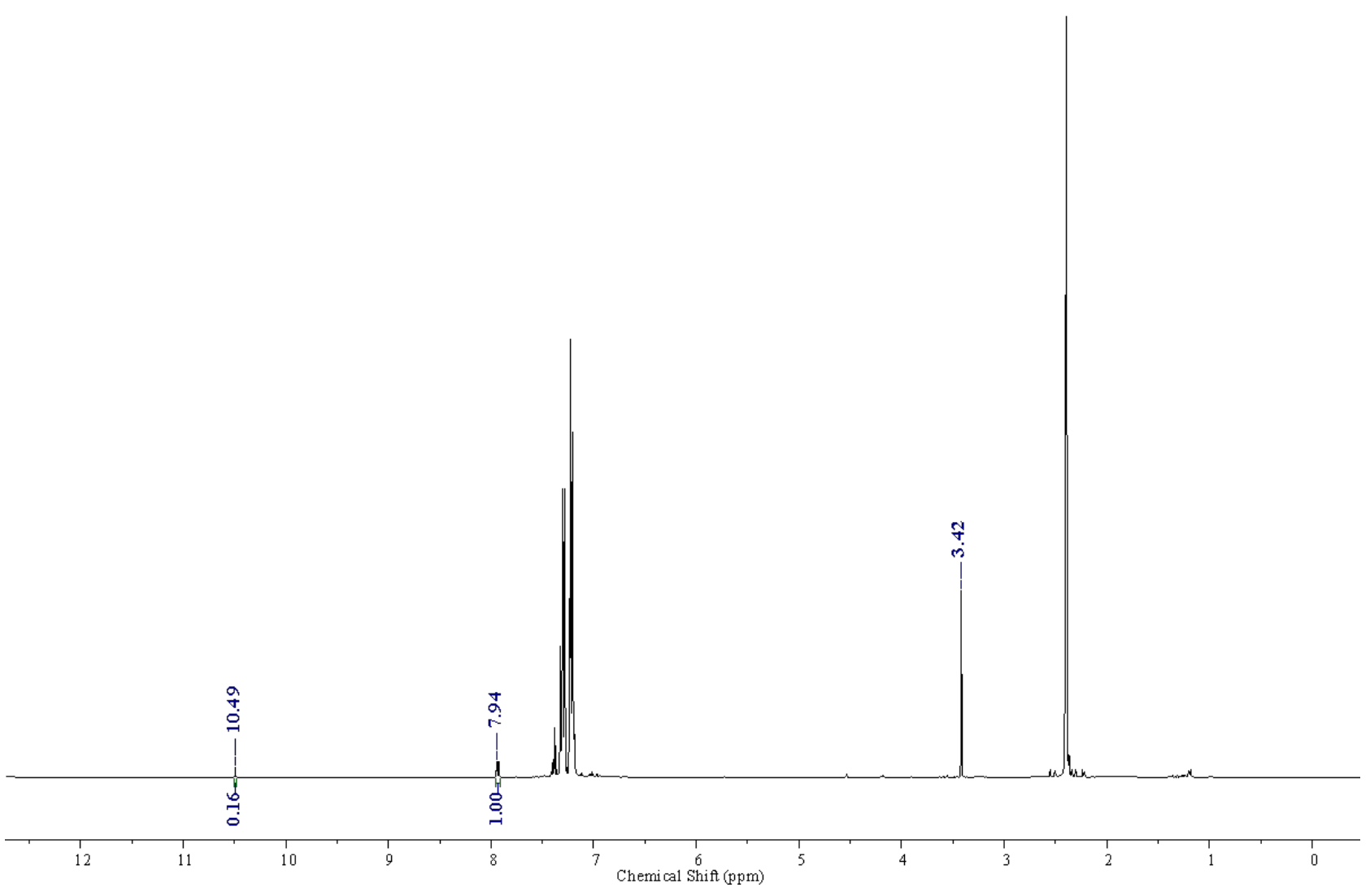


Entry 11: With $0.40 \mathrm{mmol}$ Methanol (1.081 M), 17 hour SCG-343-M1-17hr-D 1-50sec.1.fid

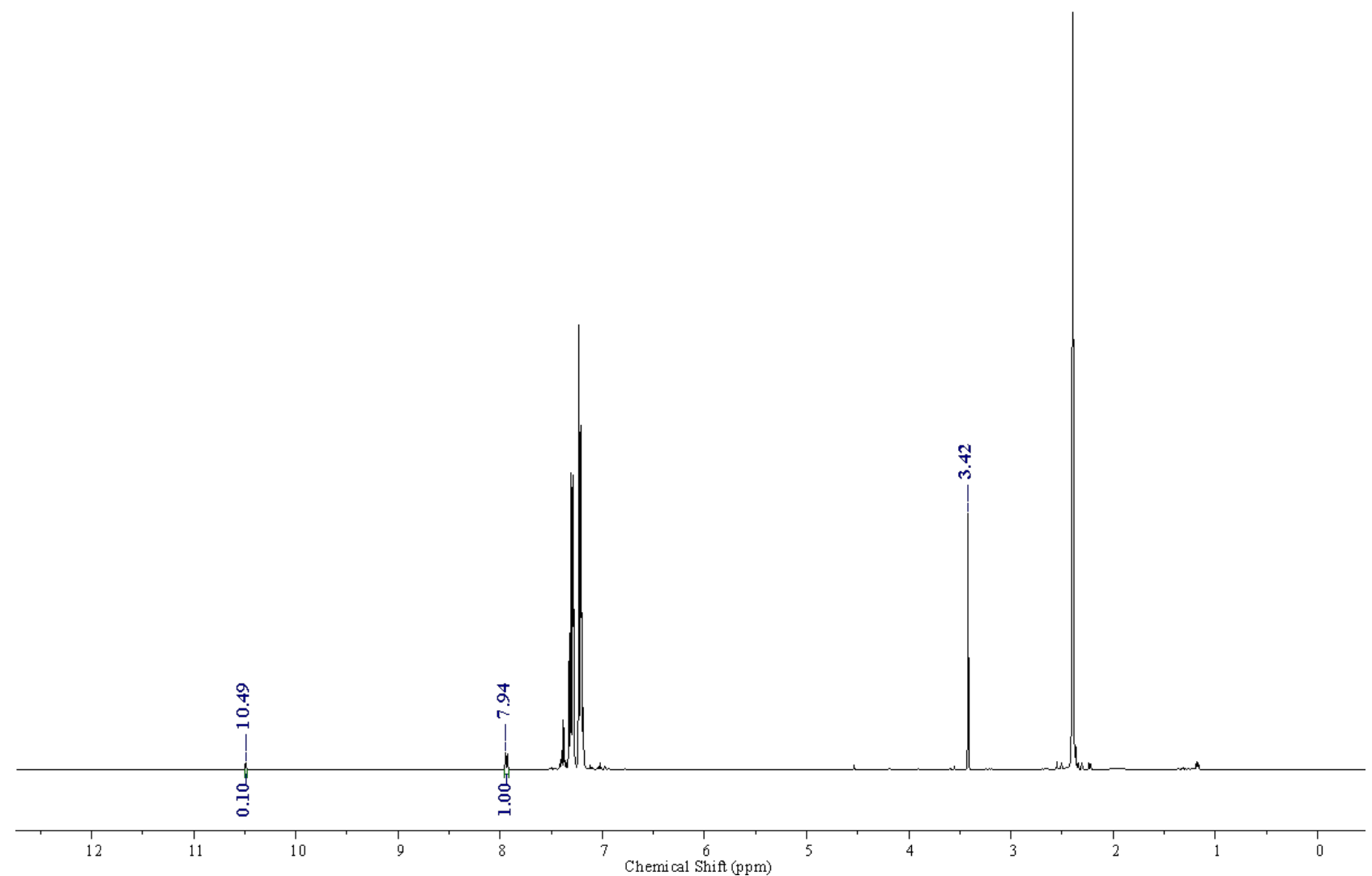

Entry 12: With $0.60 \mathrm{mmol}$ Methanol $(1.622 \mathrm{M}), 17$ hour SCG-343-G5-17hr-D 1-50sec.1. fid

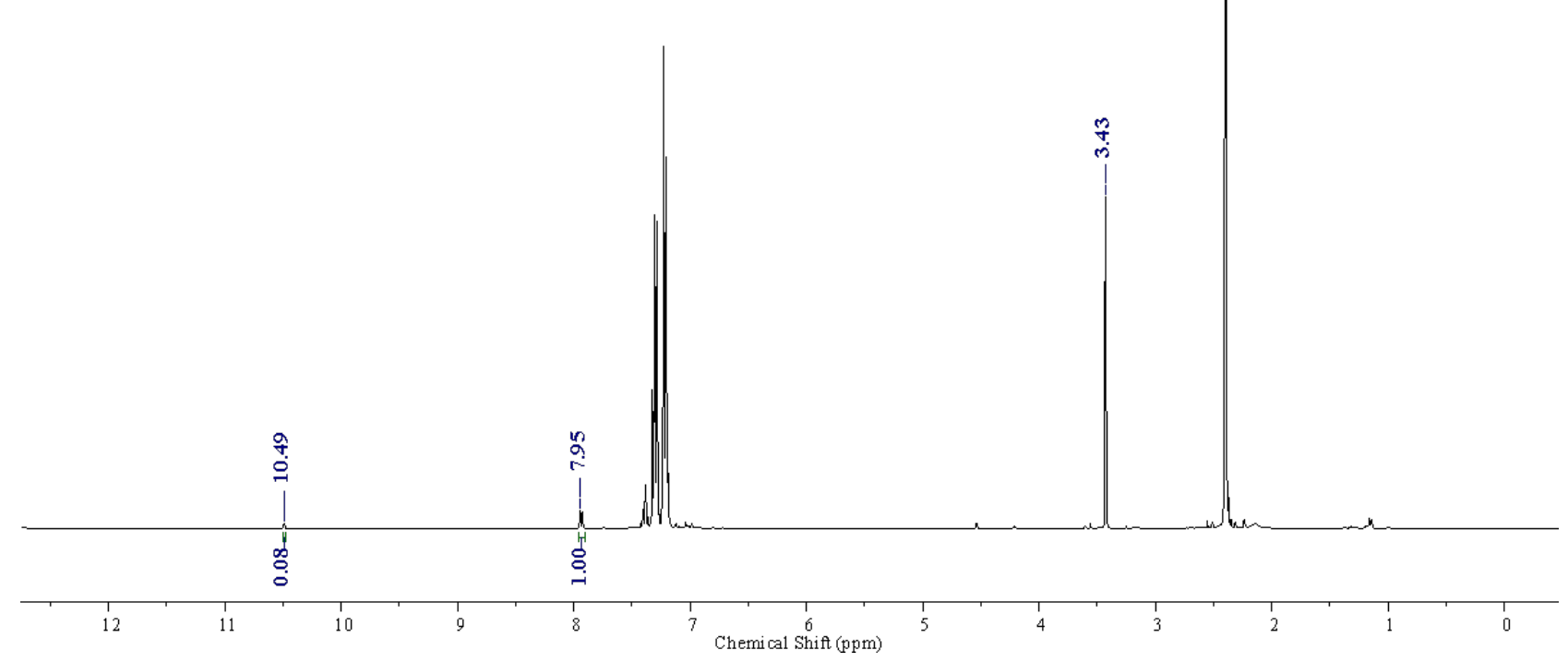


Entry 13: With $0.90 \mathrm{mmol}$ Methanol (2.432 M), 17 hour SCG-343-I3-17hr-D 1-50sec.1. fid

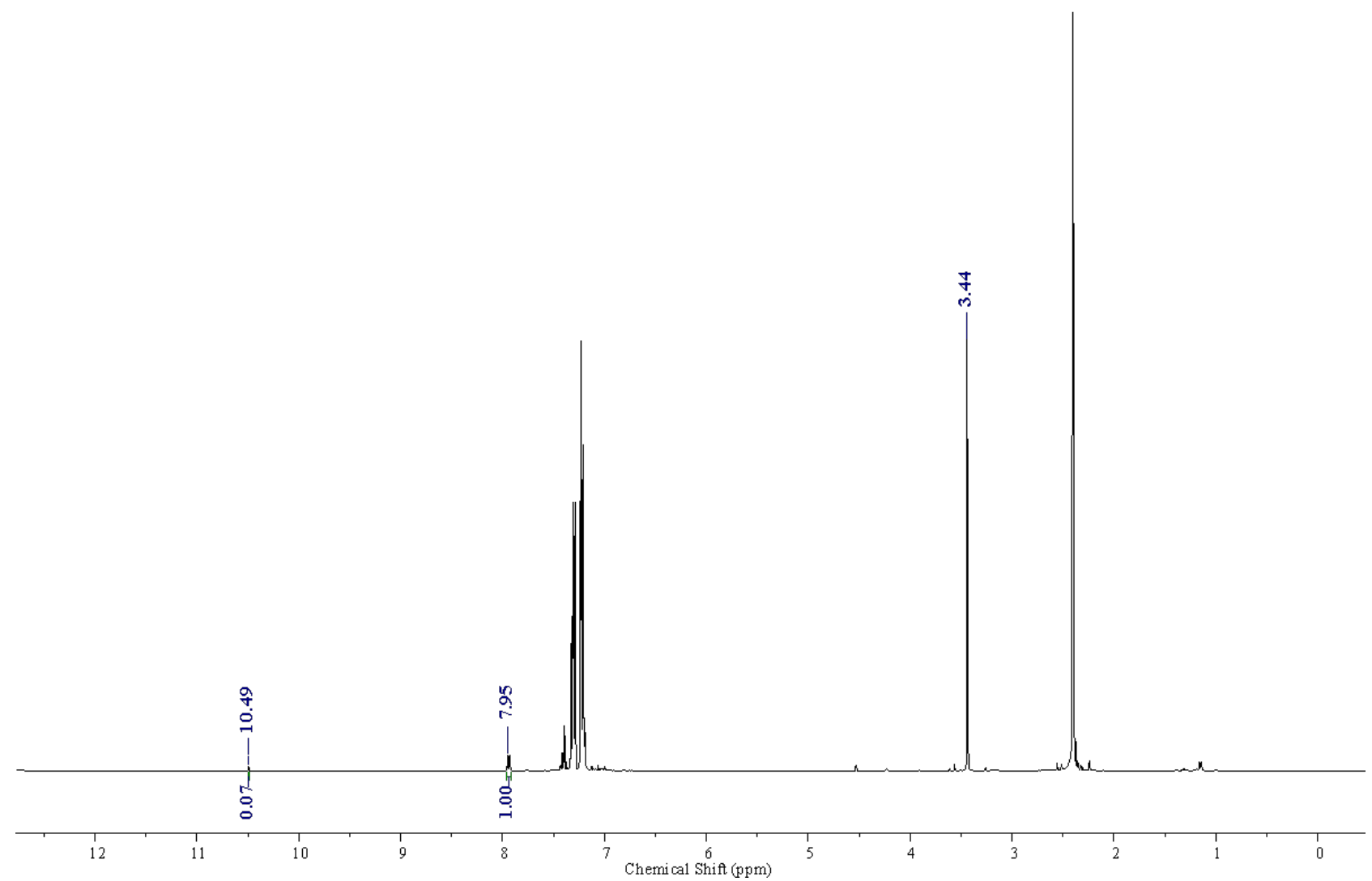

Entry 14: With $1.20 \mathrm{mmol}$ Methanol (3.243 M), 17 hour

SCG-343-H3-17hr-D 1-50sec.1.fid

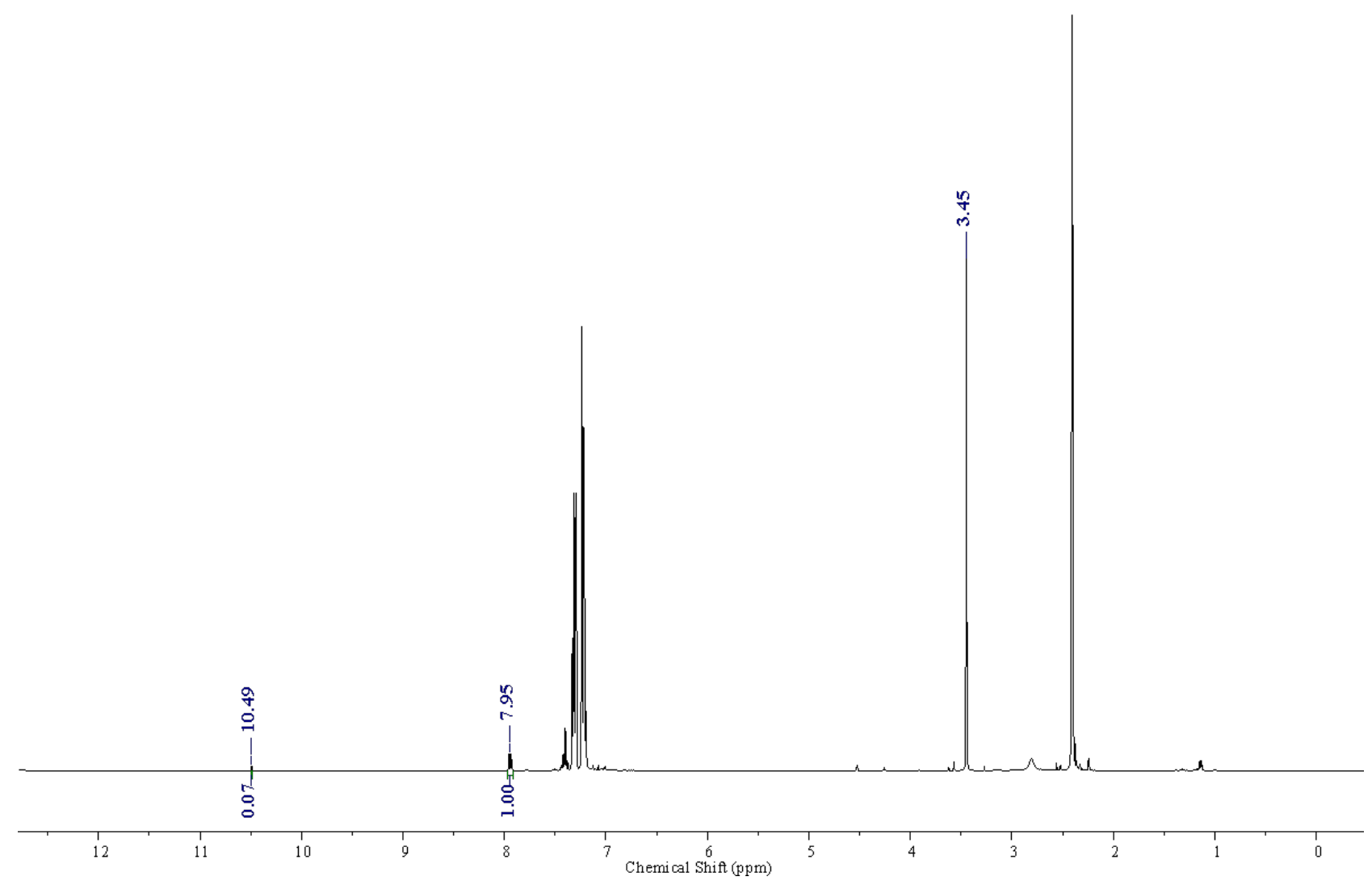


Entry 15: With $1.60 \mathrm{mmol}$ Methanol (4.324 M), 17 hour SCG-343-J3-17hr-D 1-50sec.1.fid

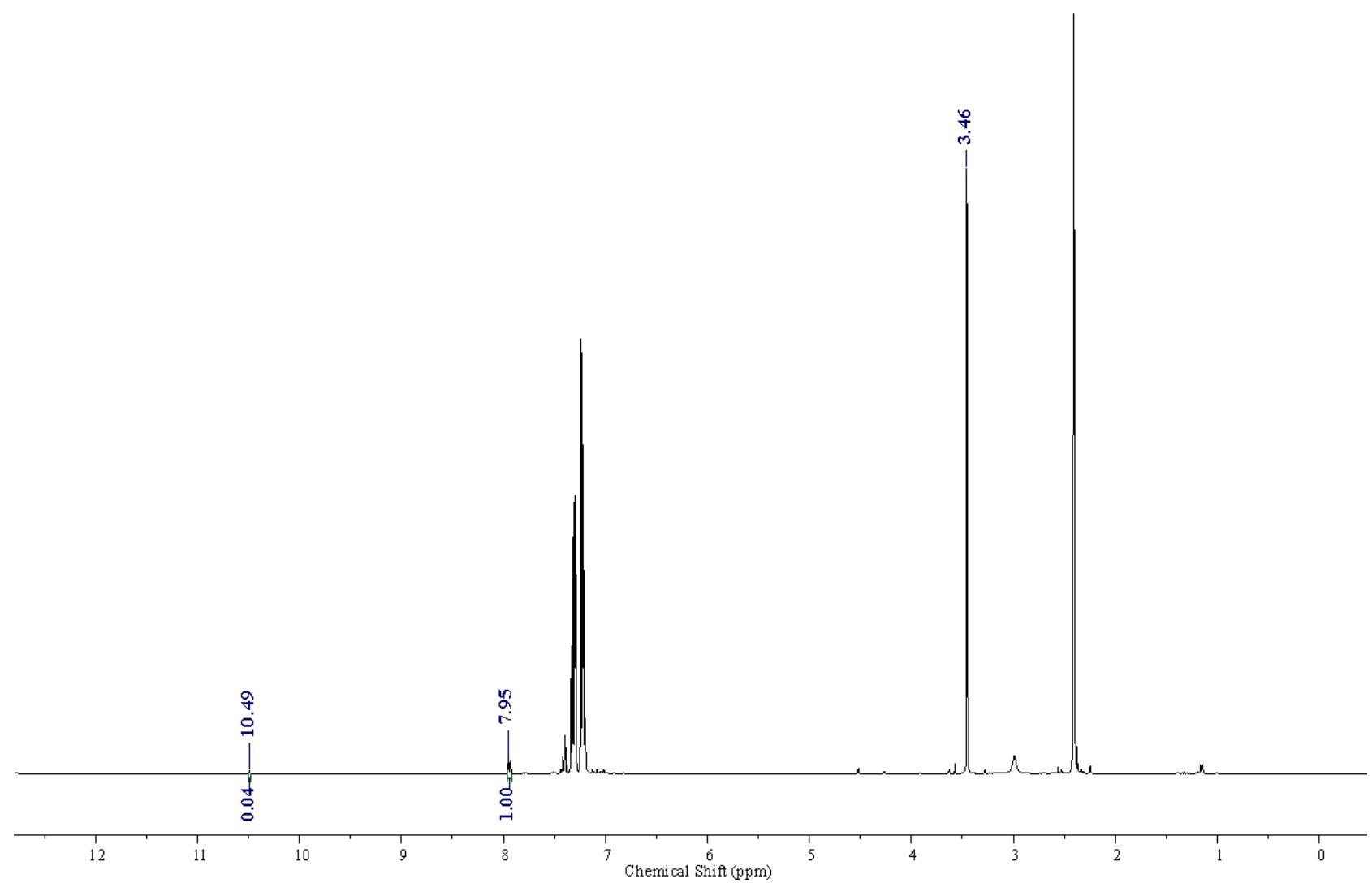

Entry 16: With $1.85 \mathrm{mmol}$ Methanol (5.000 M), 17 hour

SCG-339-A3-16hr-D 1-50sec.2.fid

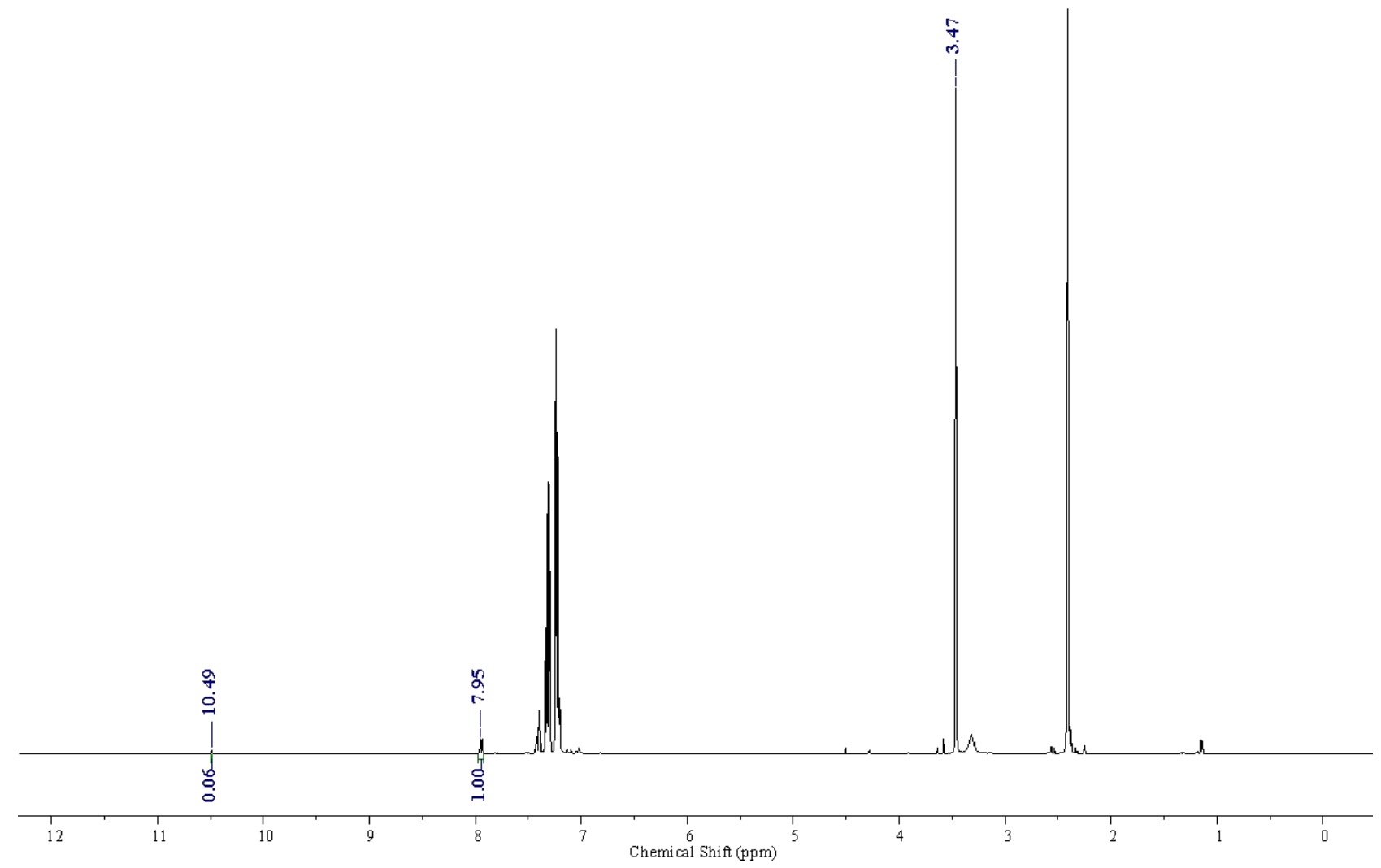


8.4 Reactions with $3 \mathrm{~b}$ (SIMes) and $\mathrm{R}-\mathrm{OH}$ and Water $(0.10 \mathrm{mmol})$ :

Entry 1: With $0.10 \mathrm{mmol}$ Water, 17 hour

VDOS-651-2-D1

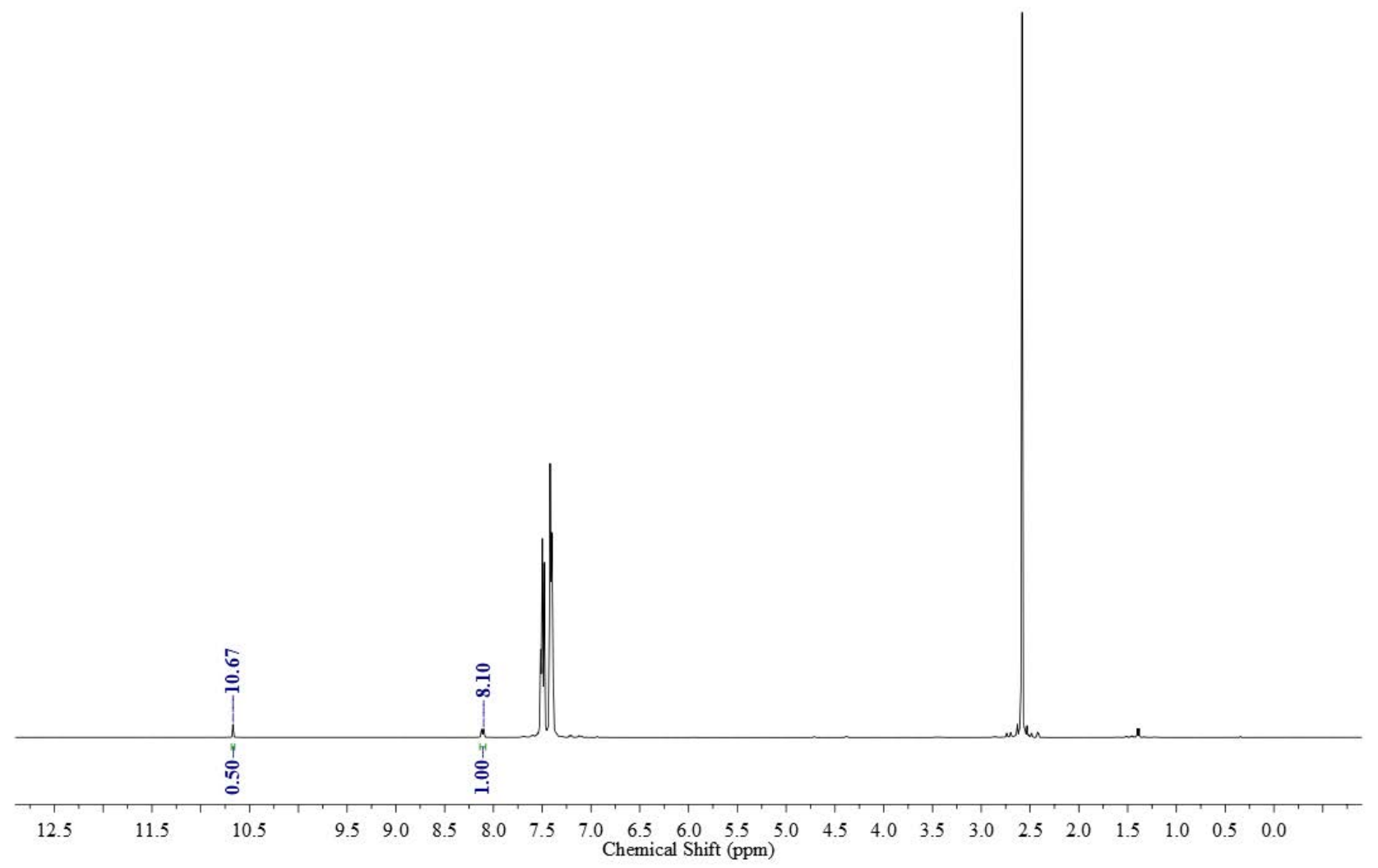

Entry 2: With 0.10 mmol Ethanol, 17 hour SCG-343-Q1-17hr-D 1-50sec.2.fid

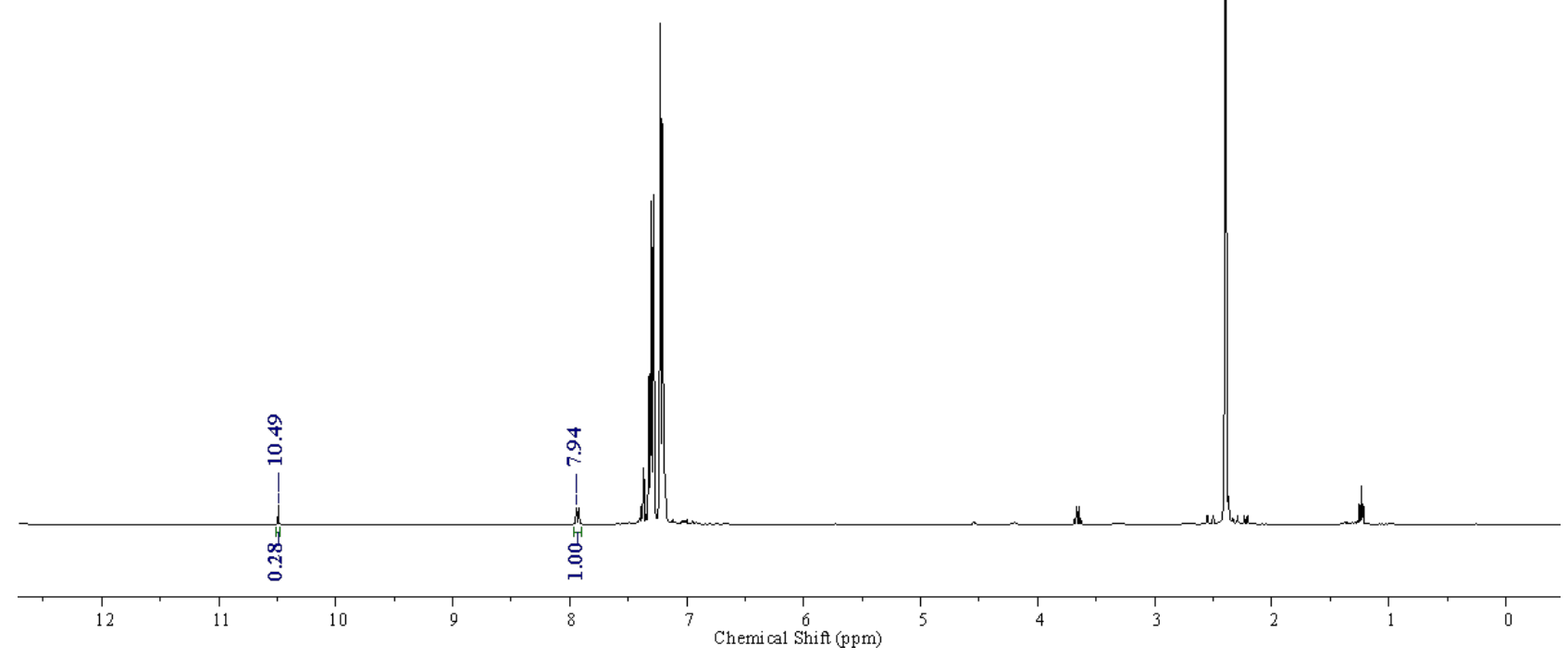


Entry 3: With $0.10 \mathrm{mmol}$ Trifluoroethanol, 17 hour

SCG-343-T1-17hr-D 1-50sec.1.fid

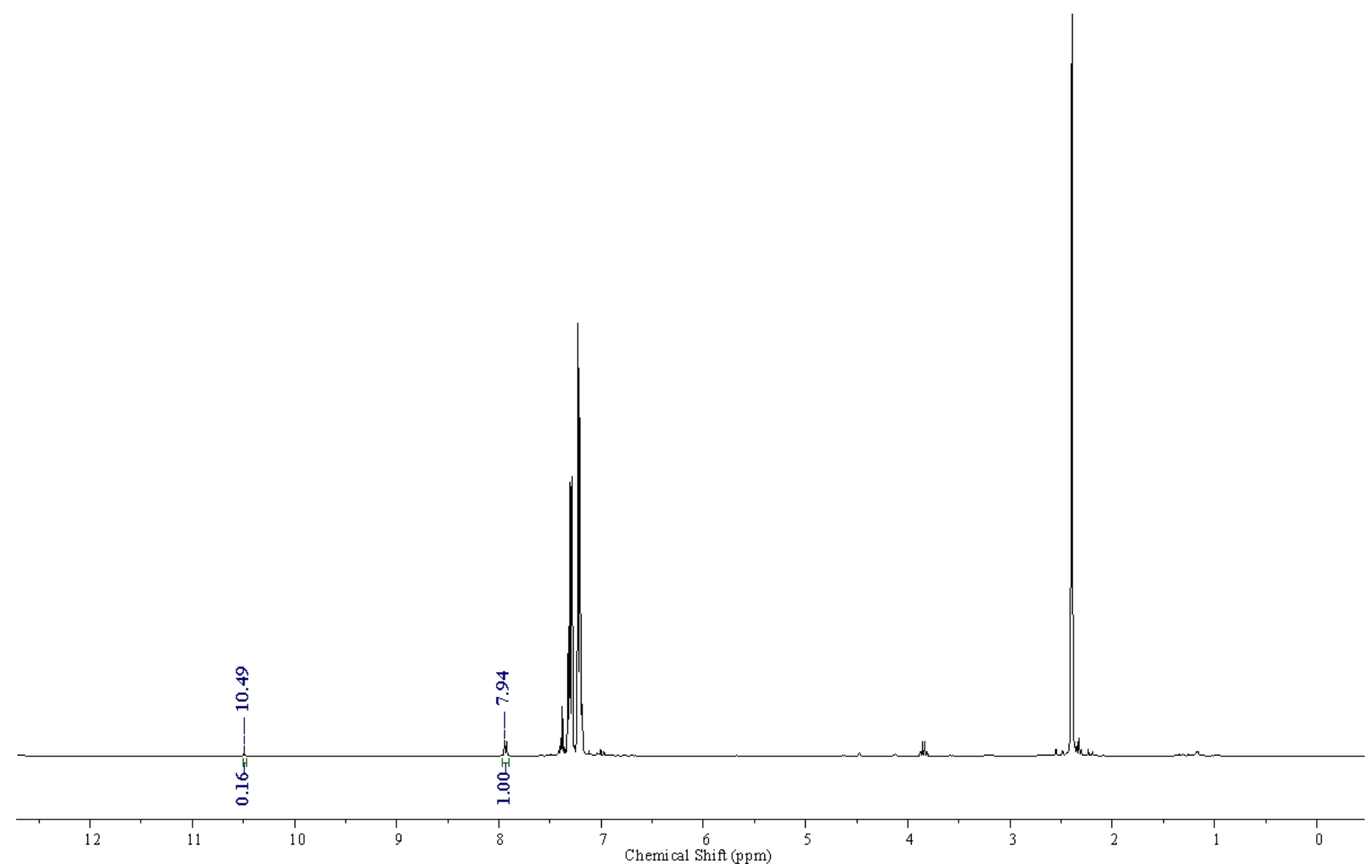

Entry 4: With $0.10 \mathrm{mmol}$ Phenol, 17 hour

SCG-343-R 1-17hr-D 1-50sec.2.fid

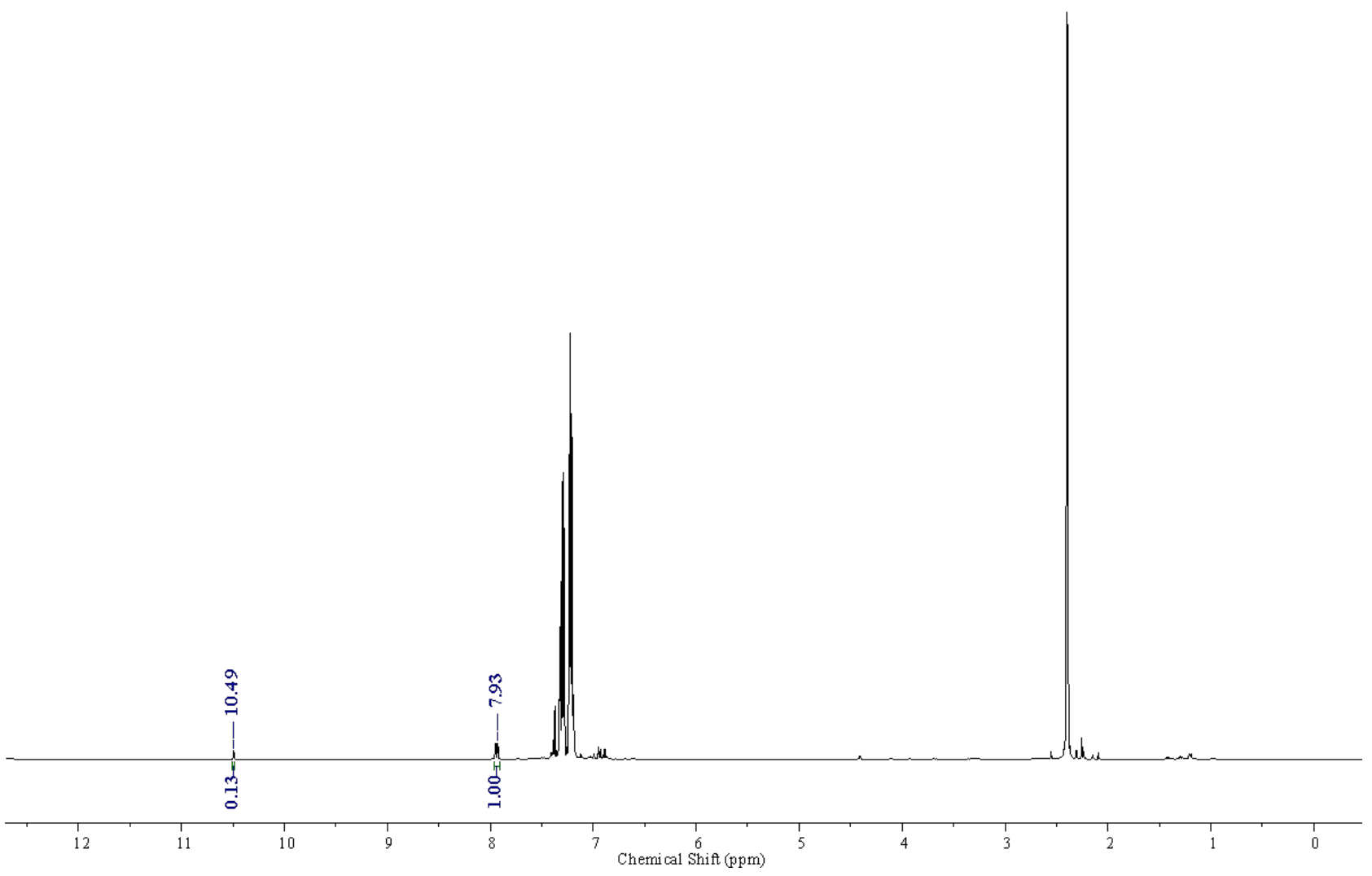


Entry 5: With $0.10 \mathrm{mmol}$ 4-Methylphenol, 17 hour

SCG-343-V 1-17hr-D 1-50sec.1.fid

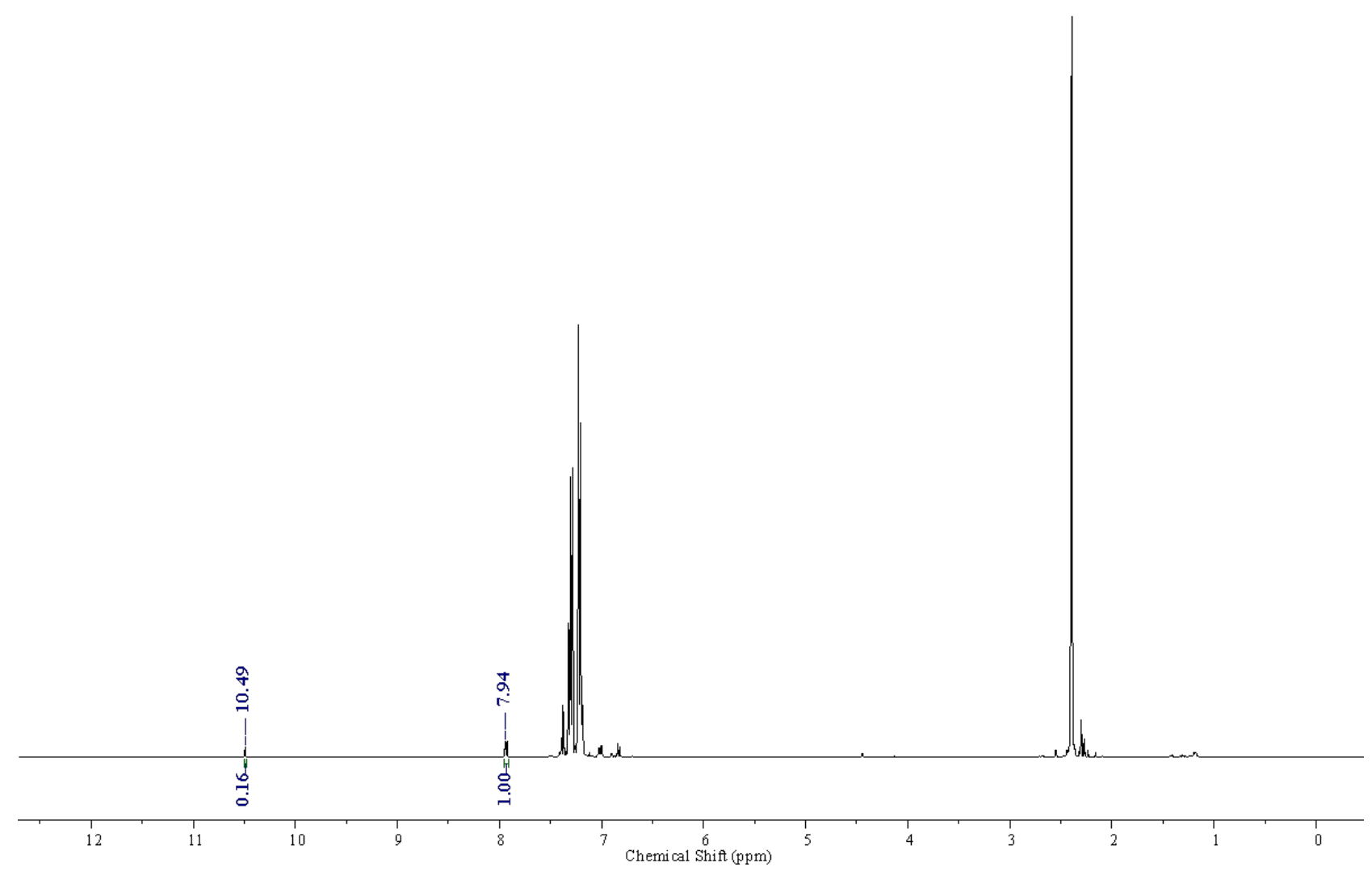

Entry 6: With $0.10 \mathrm{mmol}$ Pentafluorophenol, 17 hour

SCG-343-U 1-17hr-D 1-50sec.1. fid

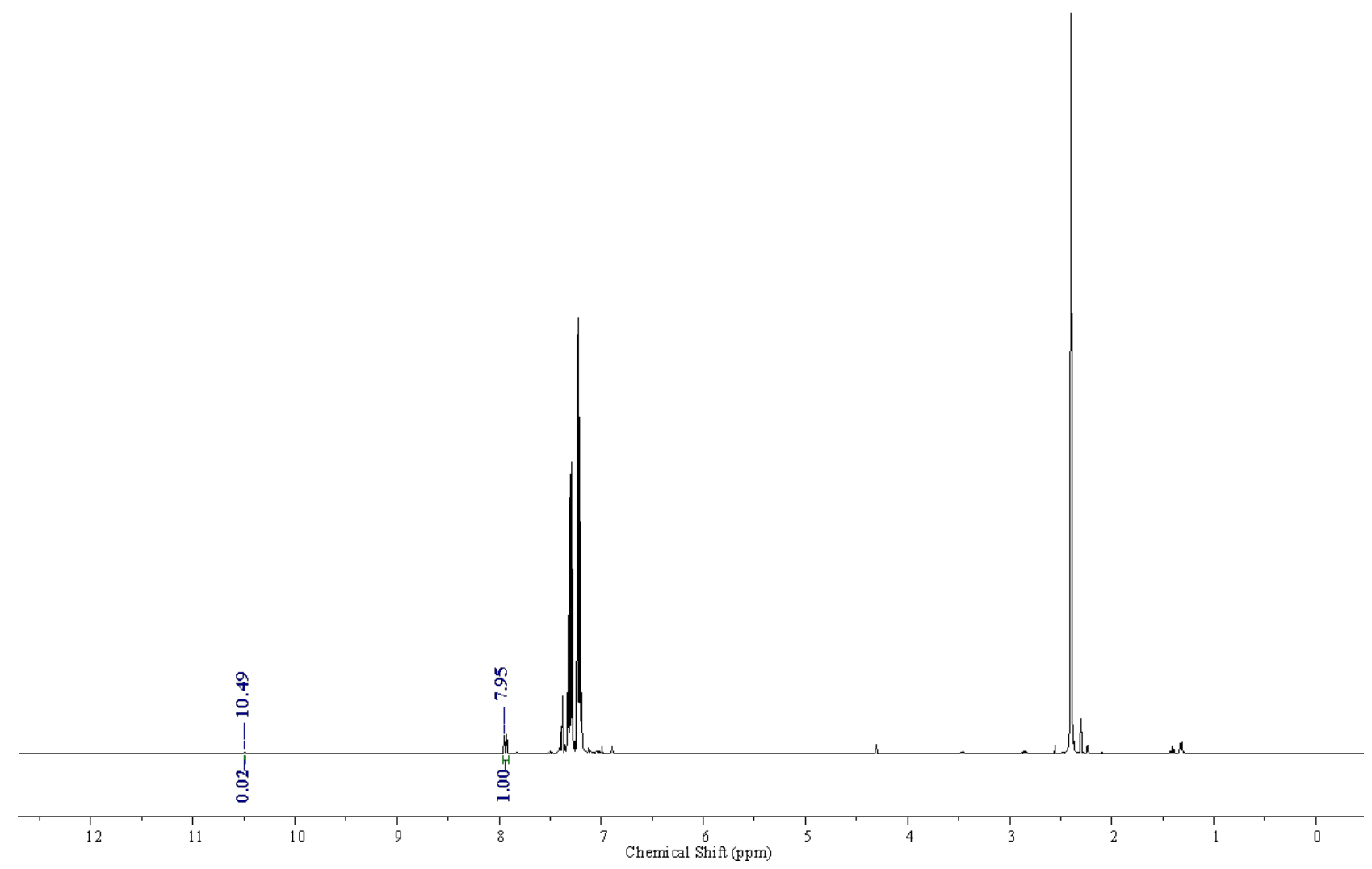


Entry 7: With $0.10 \mathrm{mmol}$ 4-Fluorophenol, 17 hour SCG-343-S1-17hr-D 1-50sec.1.fid

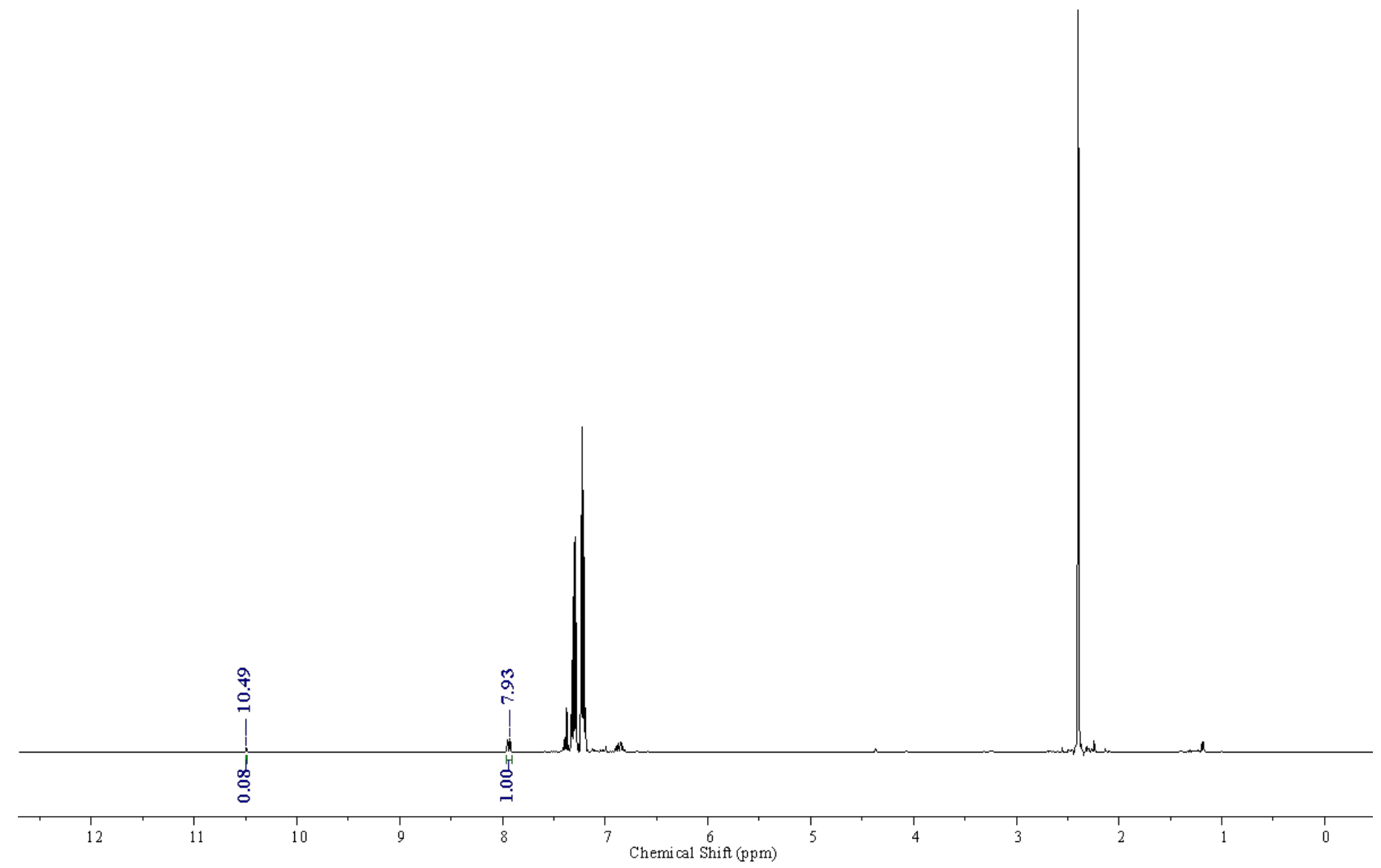


Reactions with 3-Chlorobenzaldehyde with $3 a$ and $D_{2} \mathrm{O}$ (5 eq.):

Entry 1: Reaction with $\mathrm{D}_{2} \mathrm{O}$ (5 eq.), 1 hour

SCG-334-C1-60min-D 1-50 sec.1.fid

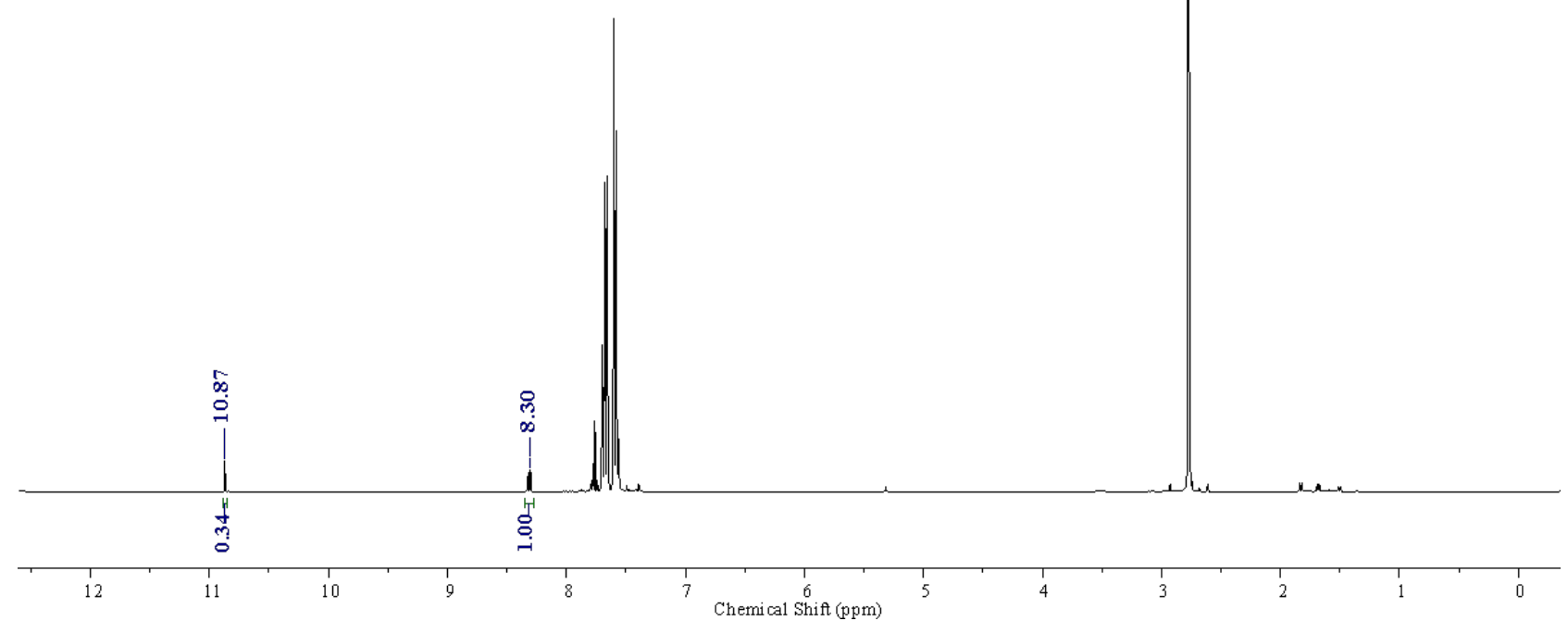

Entry 2: Reaction with $\mathrm{D}_{2} \mathrm{O}$ (5 eq.), 17 hour SCG-334-D 1-17hr-D 1-50sec.1.fid

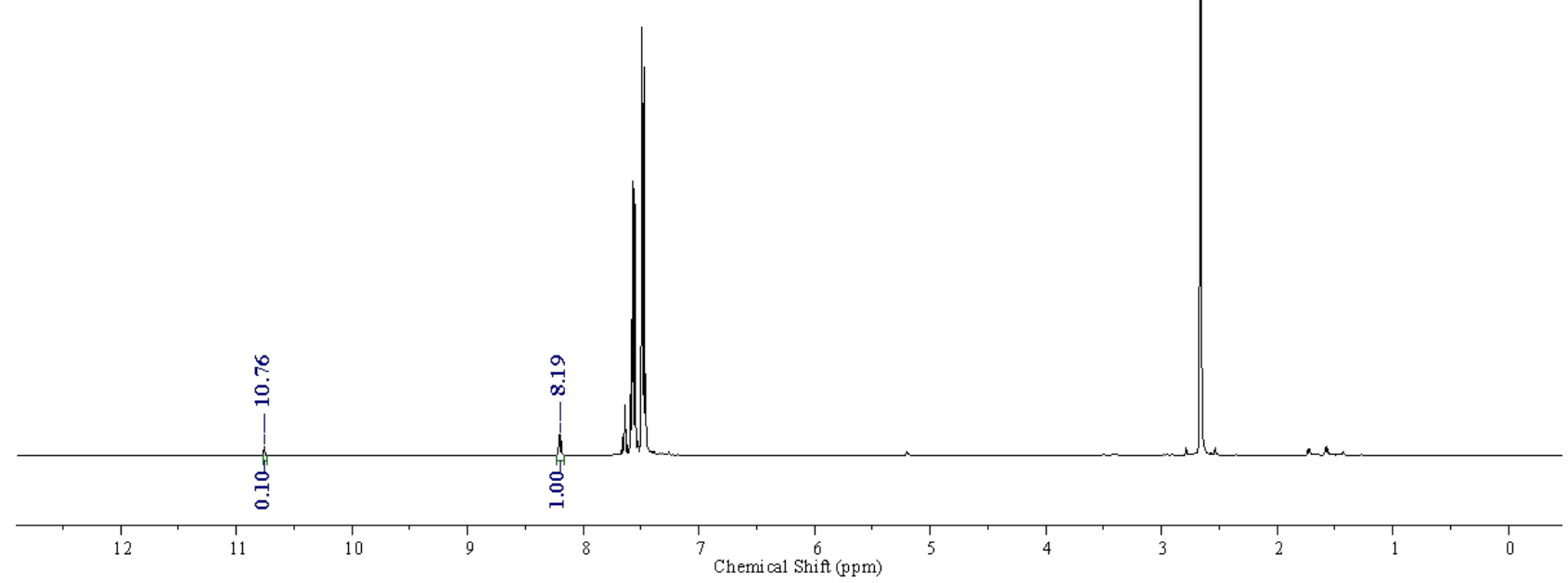




\section{Large Scale Reactions}

Entry 1:

SCG-345-A1-16hr-D1-50sec

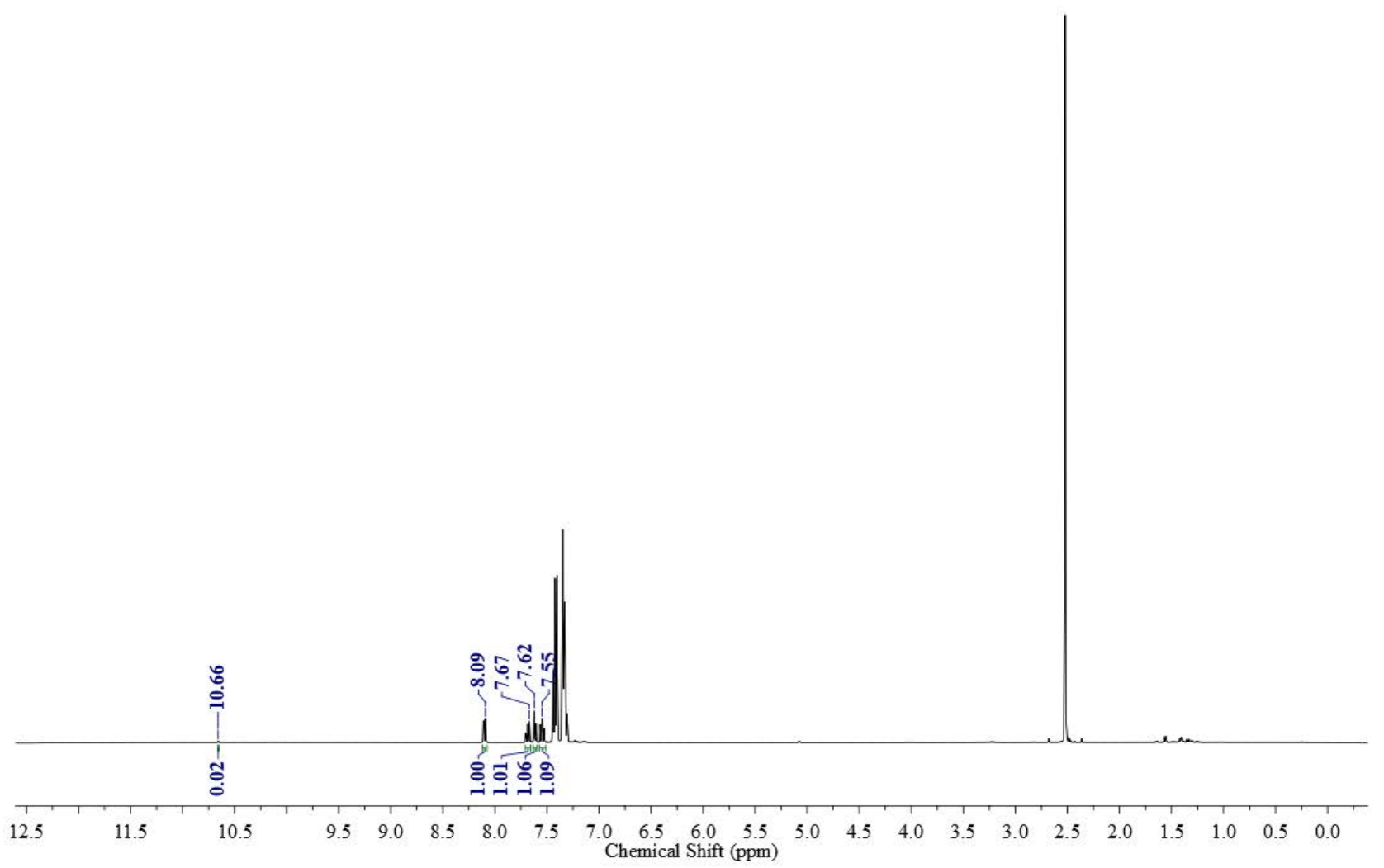




\section{After Purification:}

SCG-345-A1-A2-16hr-D1-50sec-AC-Distilled

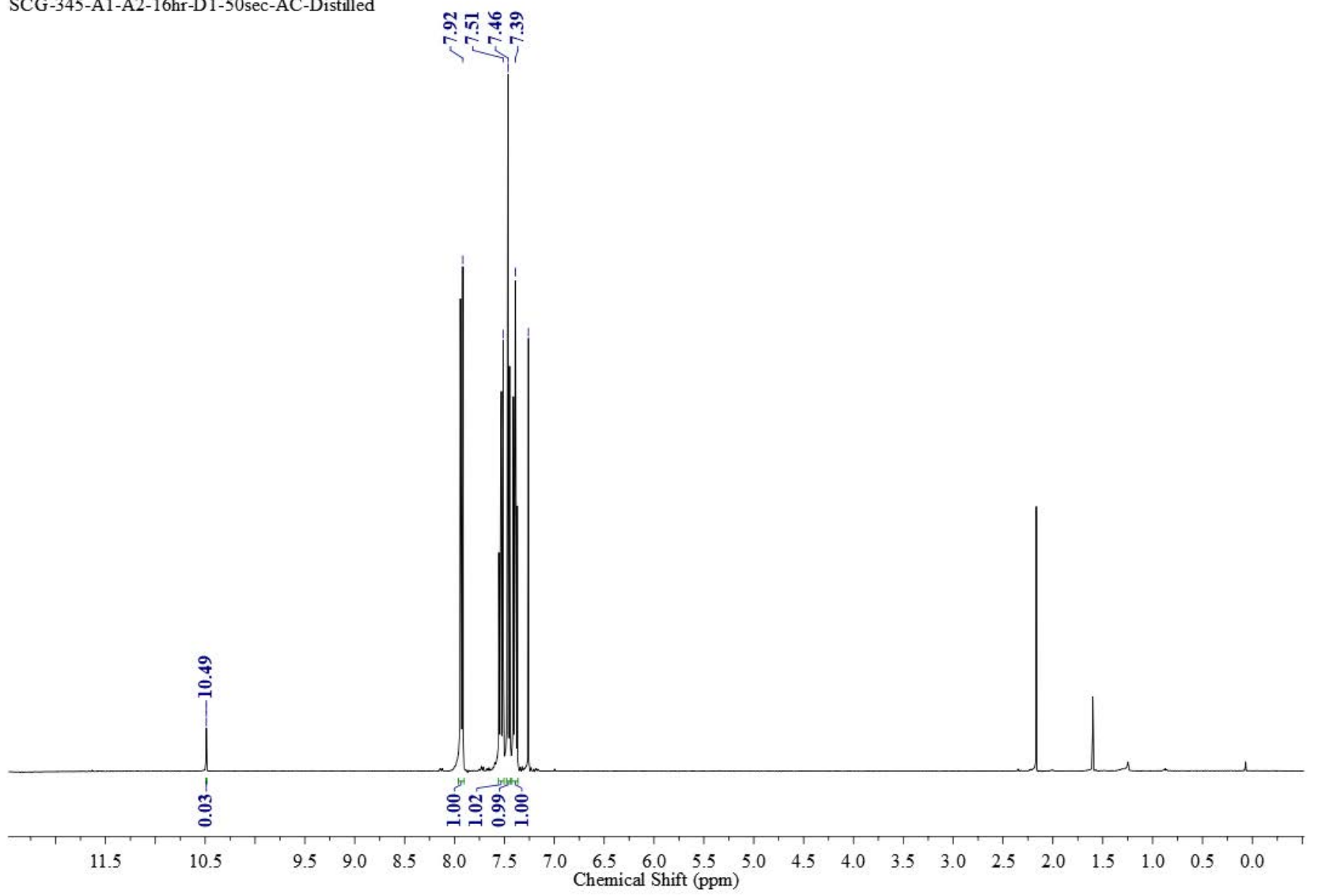

Entry 2:

VDOS -587-11 mmol-A1

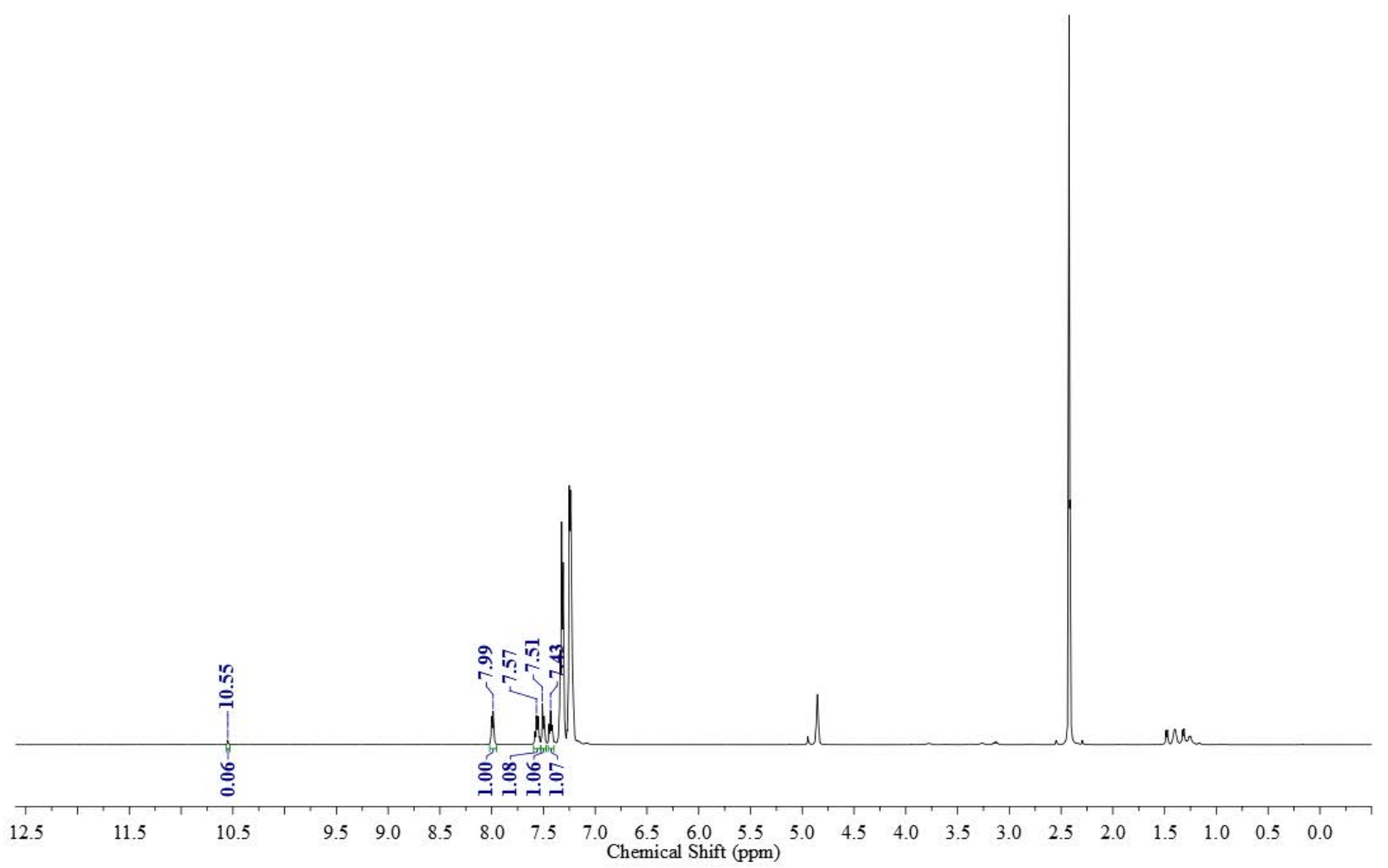


Entry 3:

VDOS $-587-11 \mathrm{mmol}, \mathrm{B} 2$

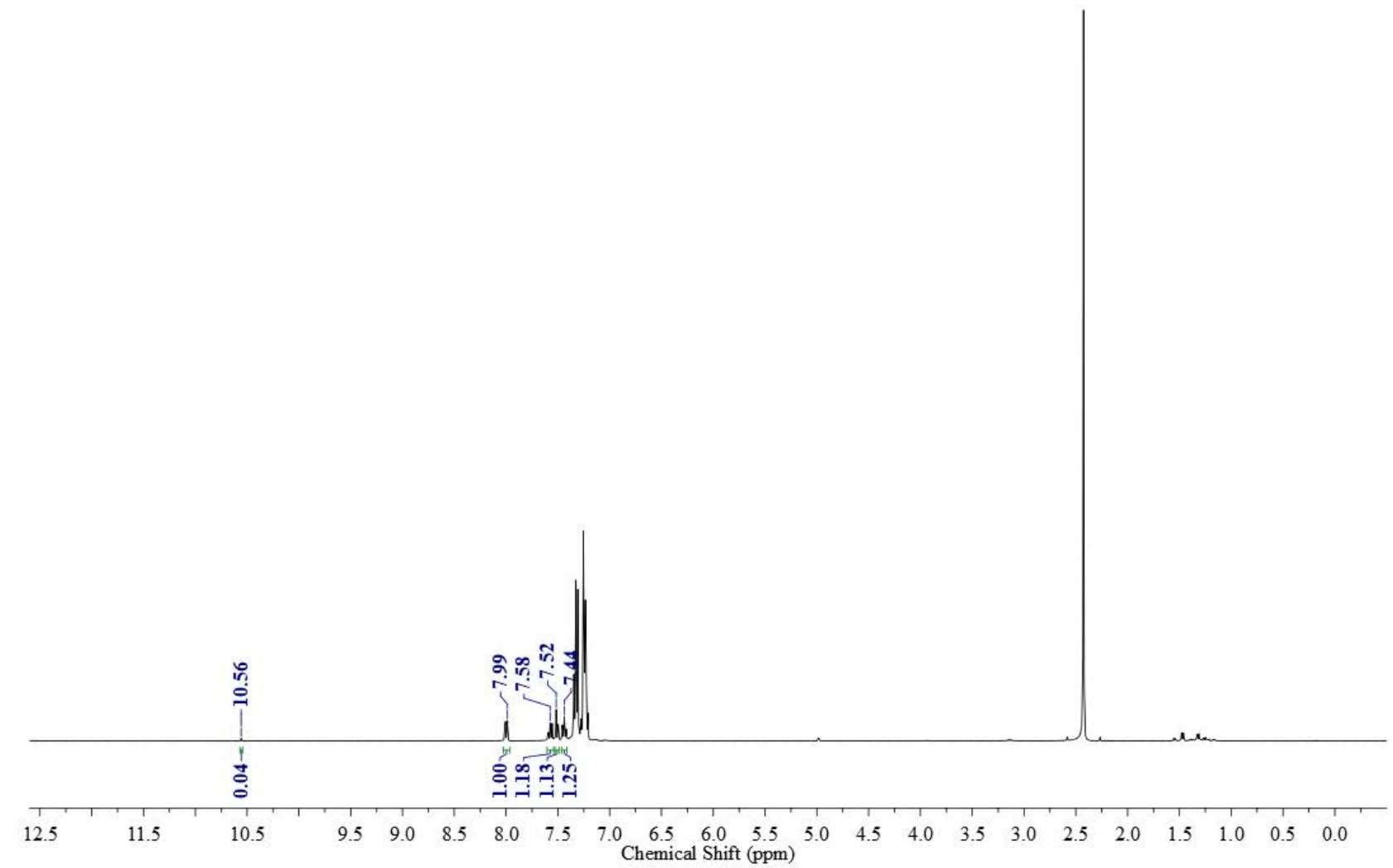

After Purification:

VDOS-587-B1 pure

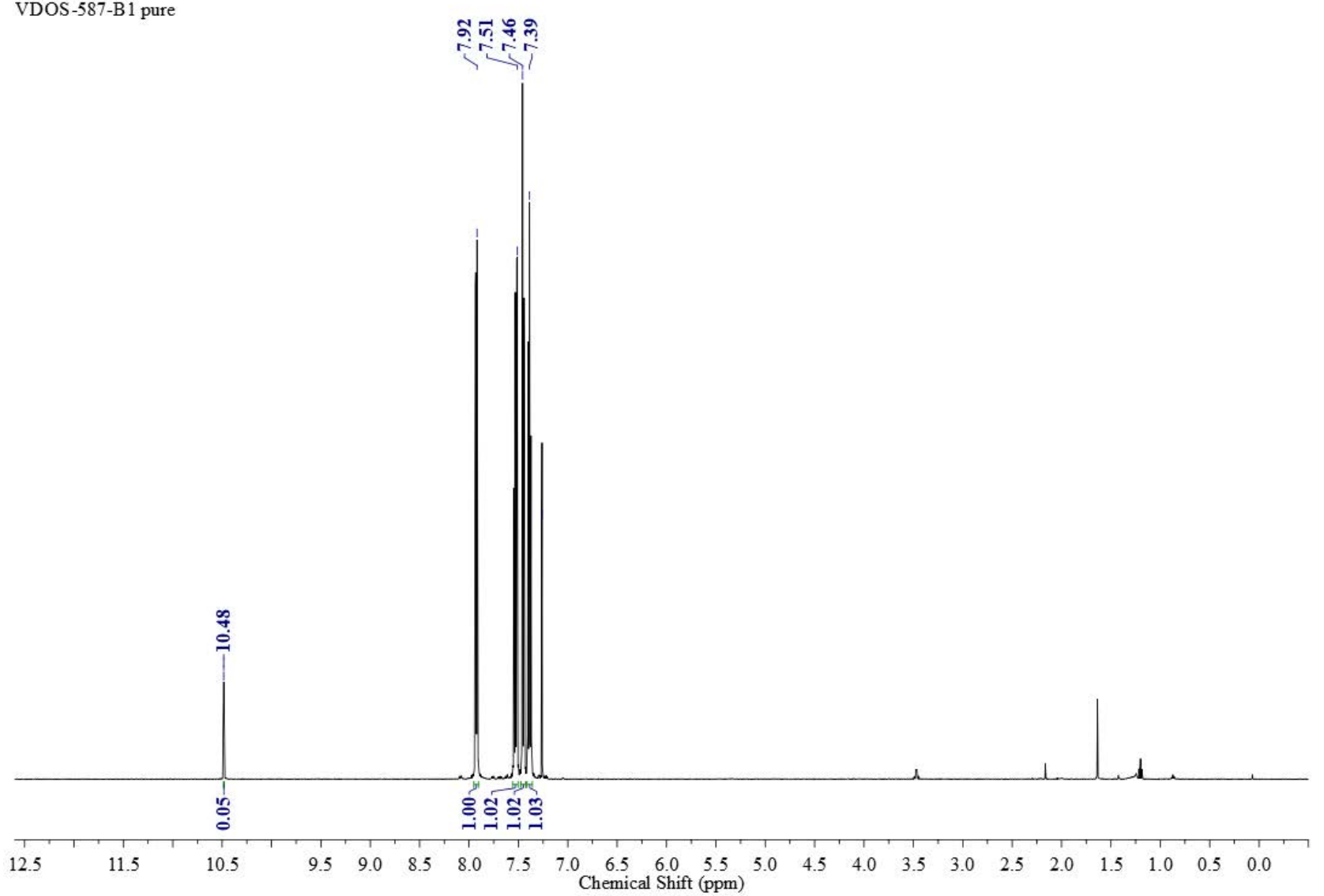


Entry 4:

VDOS-587-11 mmol-C1

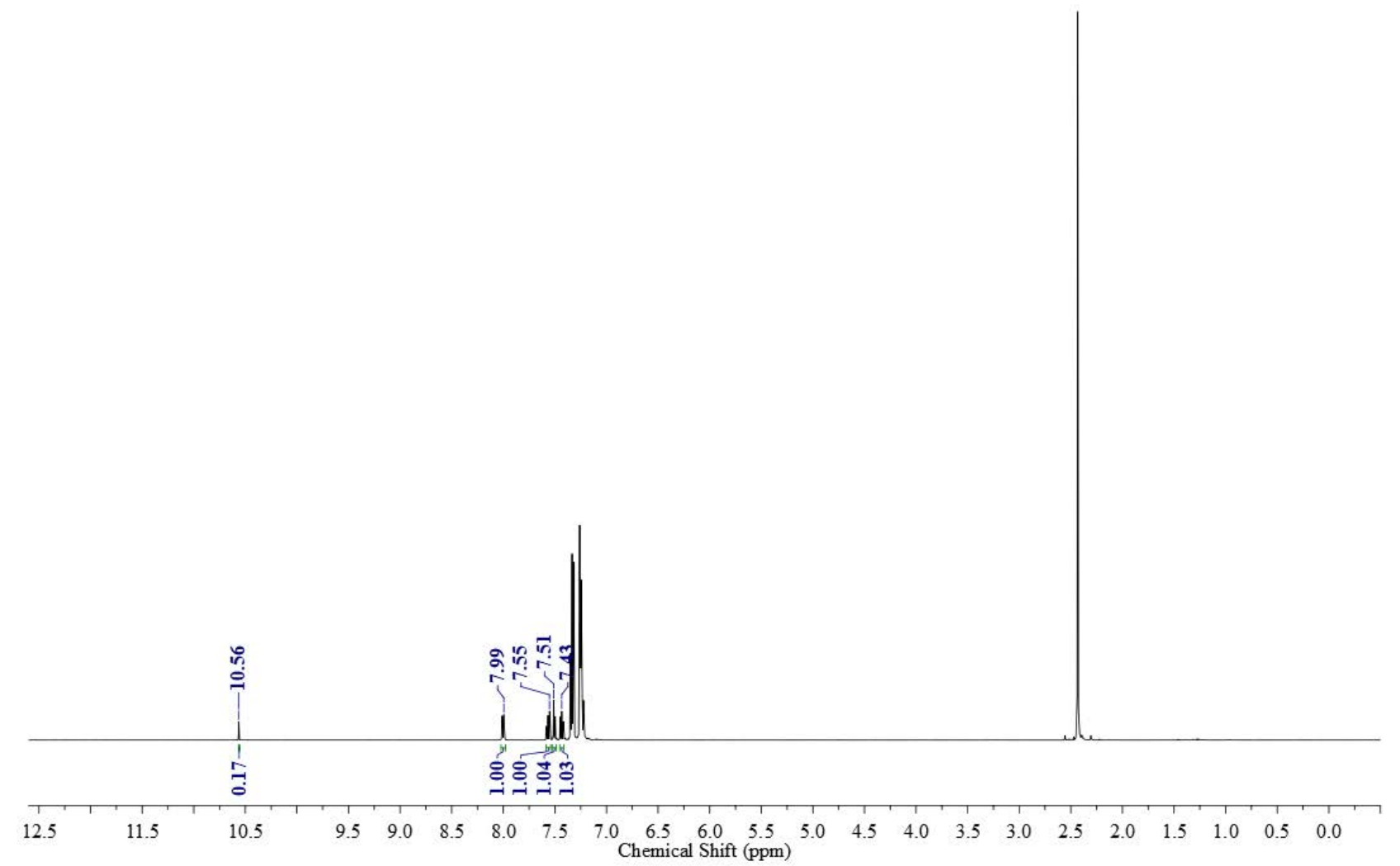

Entry 5:

VDOS-587-11mmol, D1

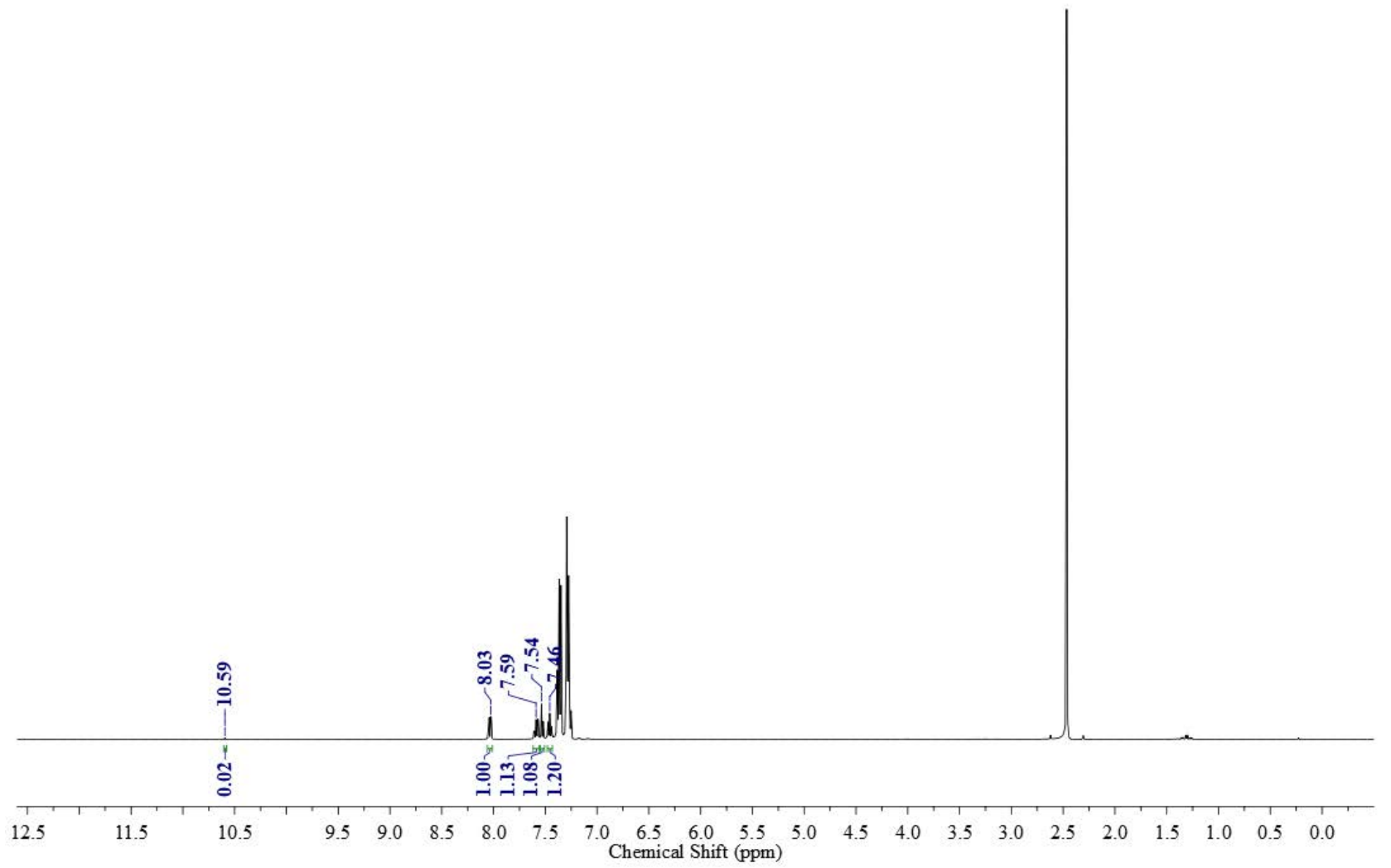


After Purification:

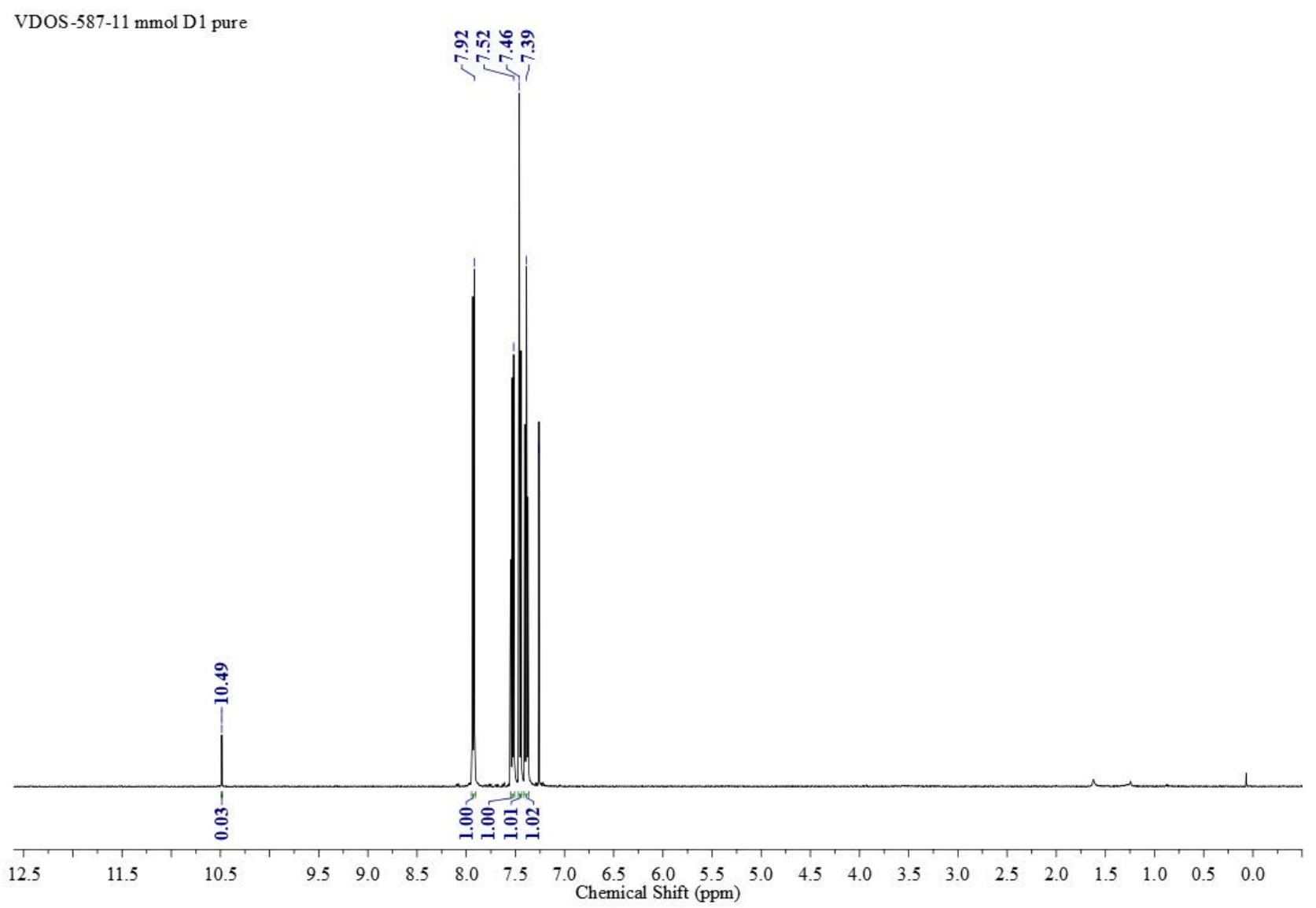

Entry 6:

VDOS-587-11 mmol E1

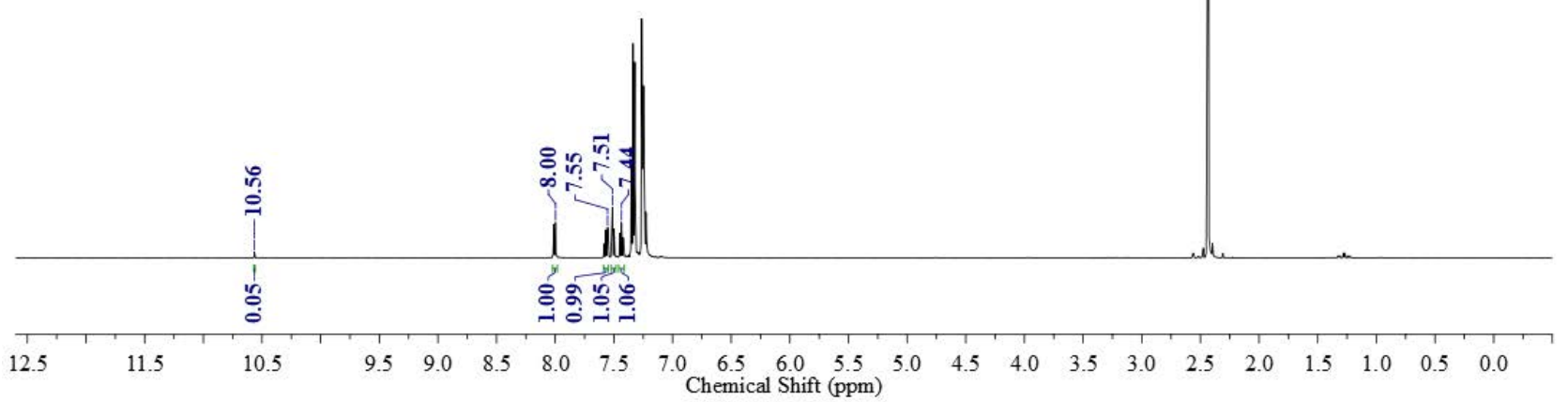


Entry 7:

VDOS-587-11 mmol-F1

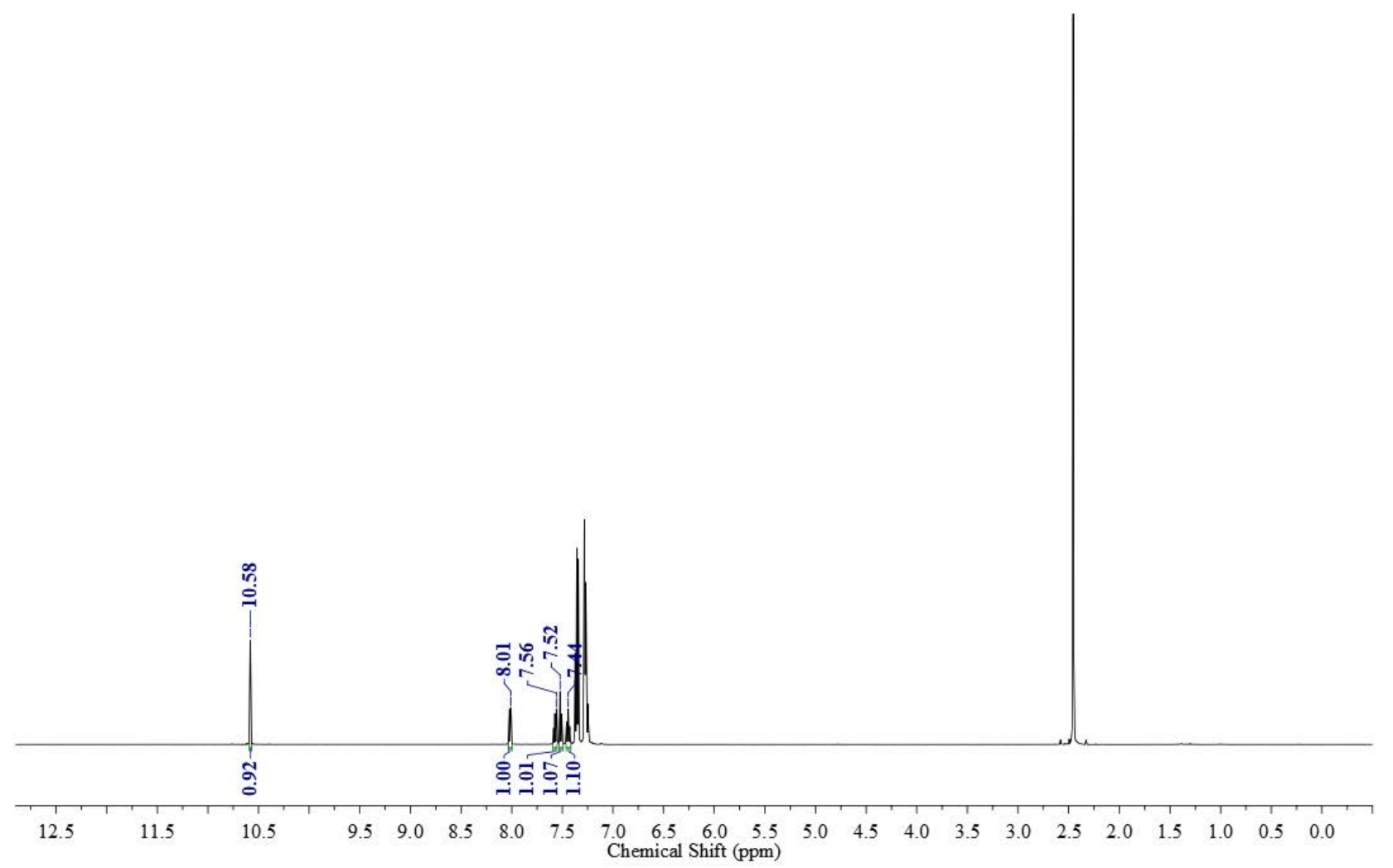

Entry 8:

VDOS -587-11 mmol-G1

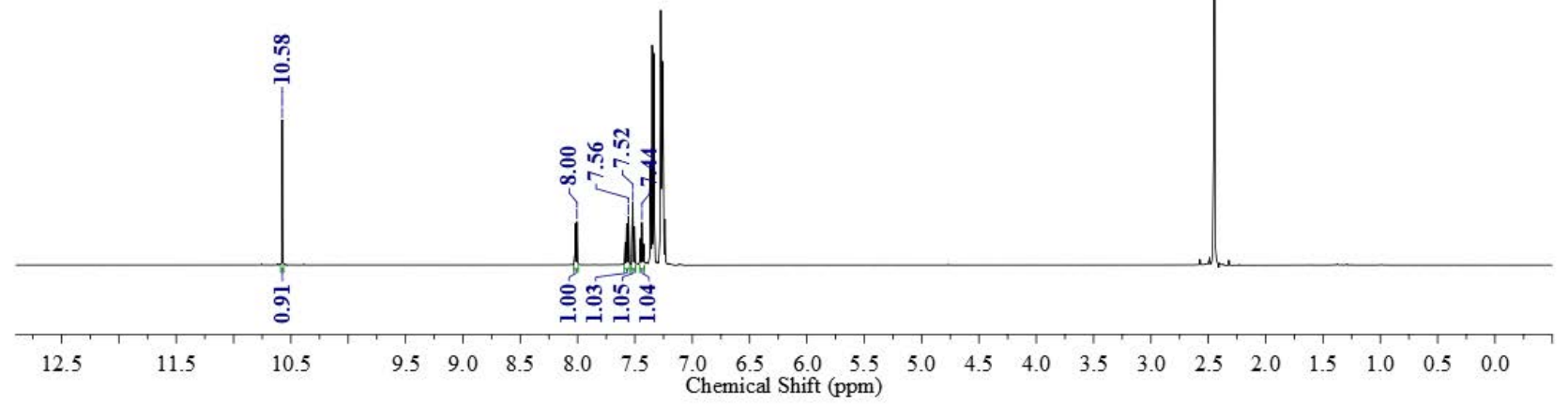


Entry 9:

vDOS-655-A

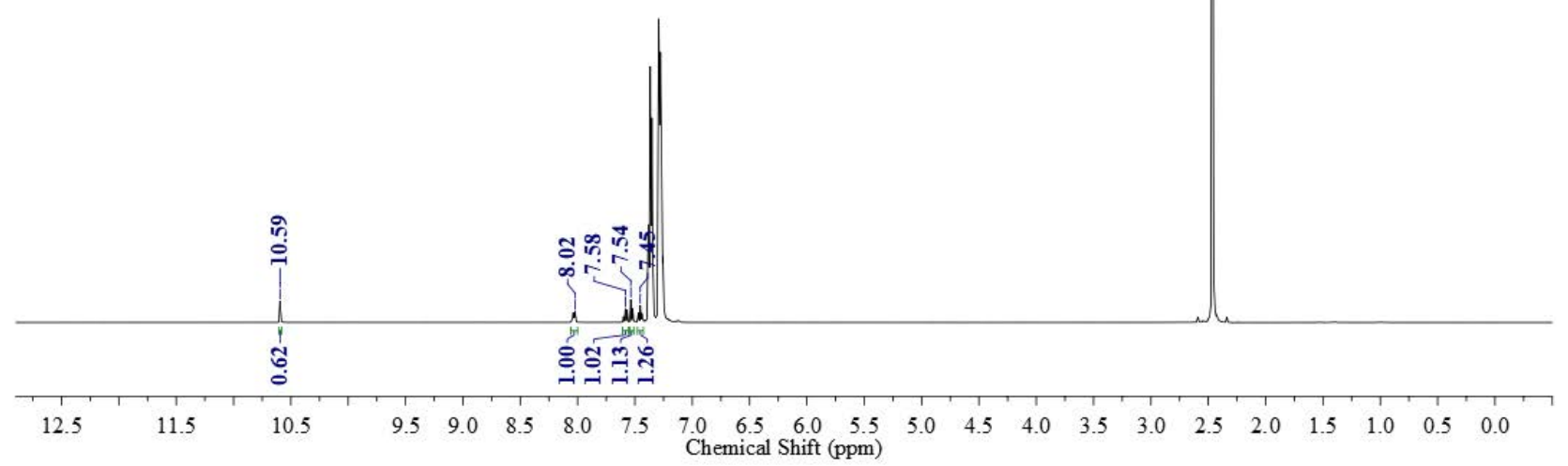

Entry 10:

vDOS-655-B

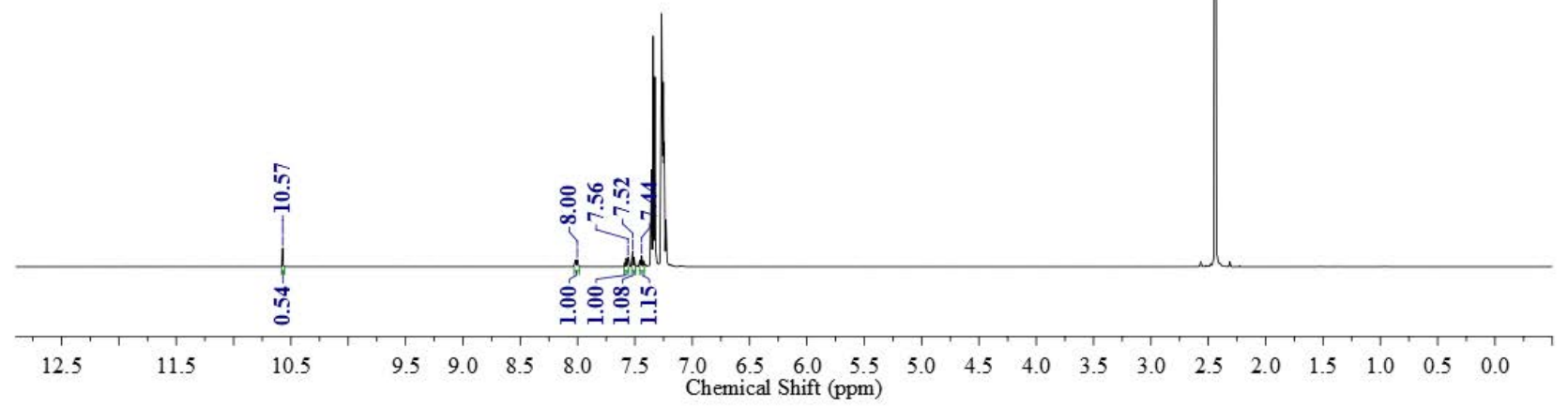


Entry 11:

vDOS-655-C

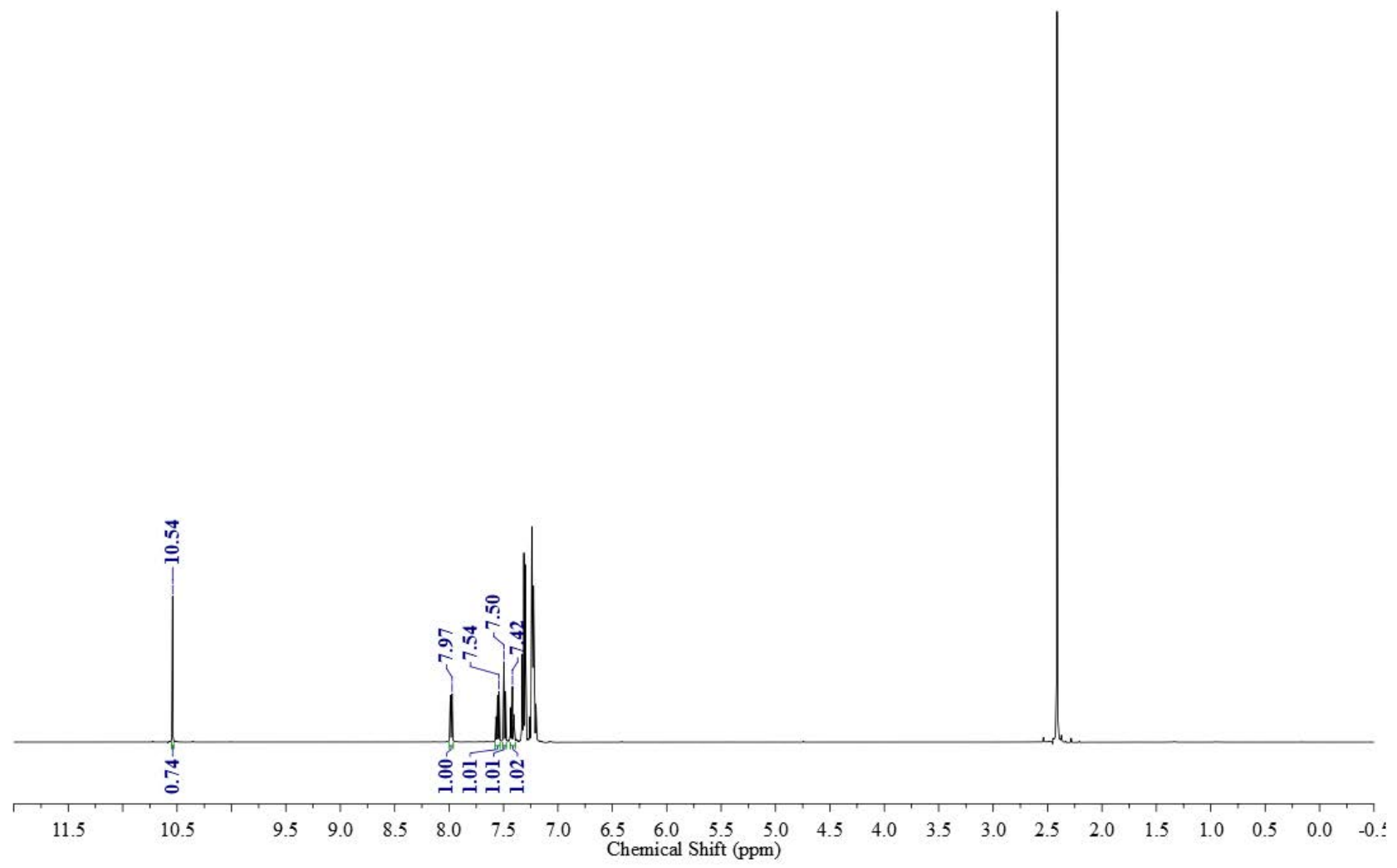

Entry 12:

VDOS-655-D

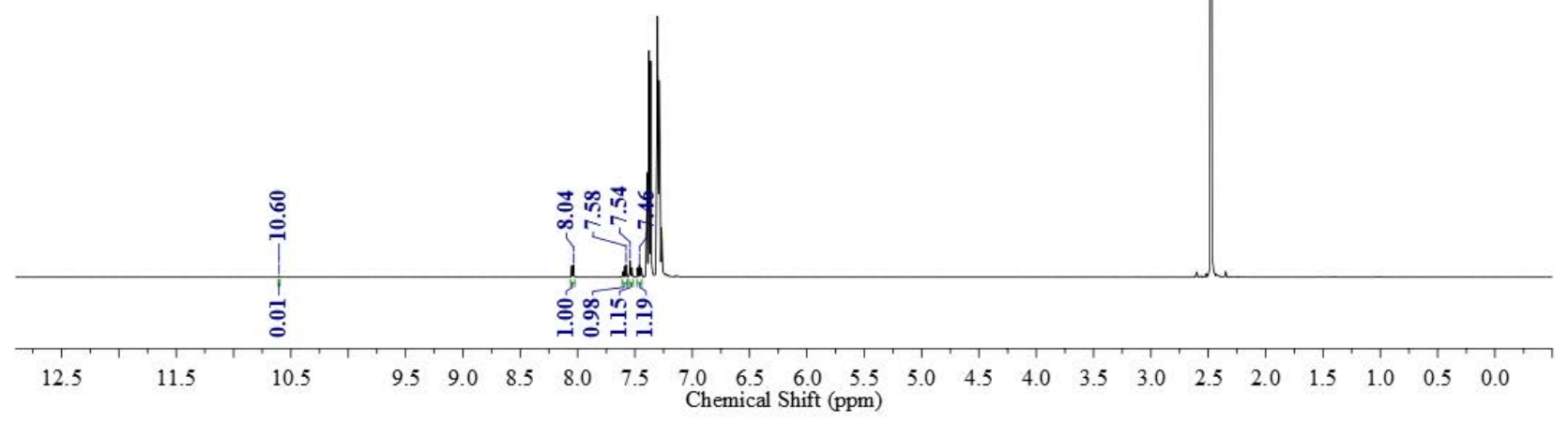


After Purification:

VDOS-655-D1 very pure

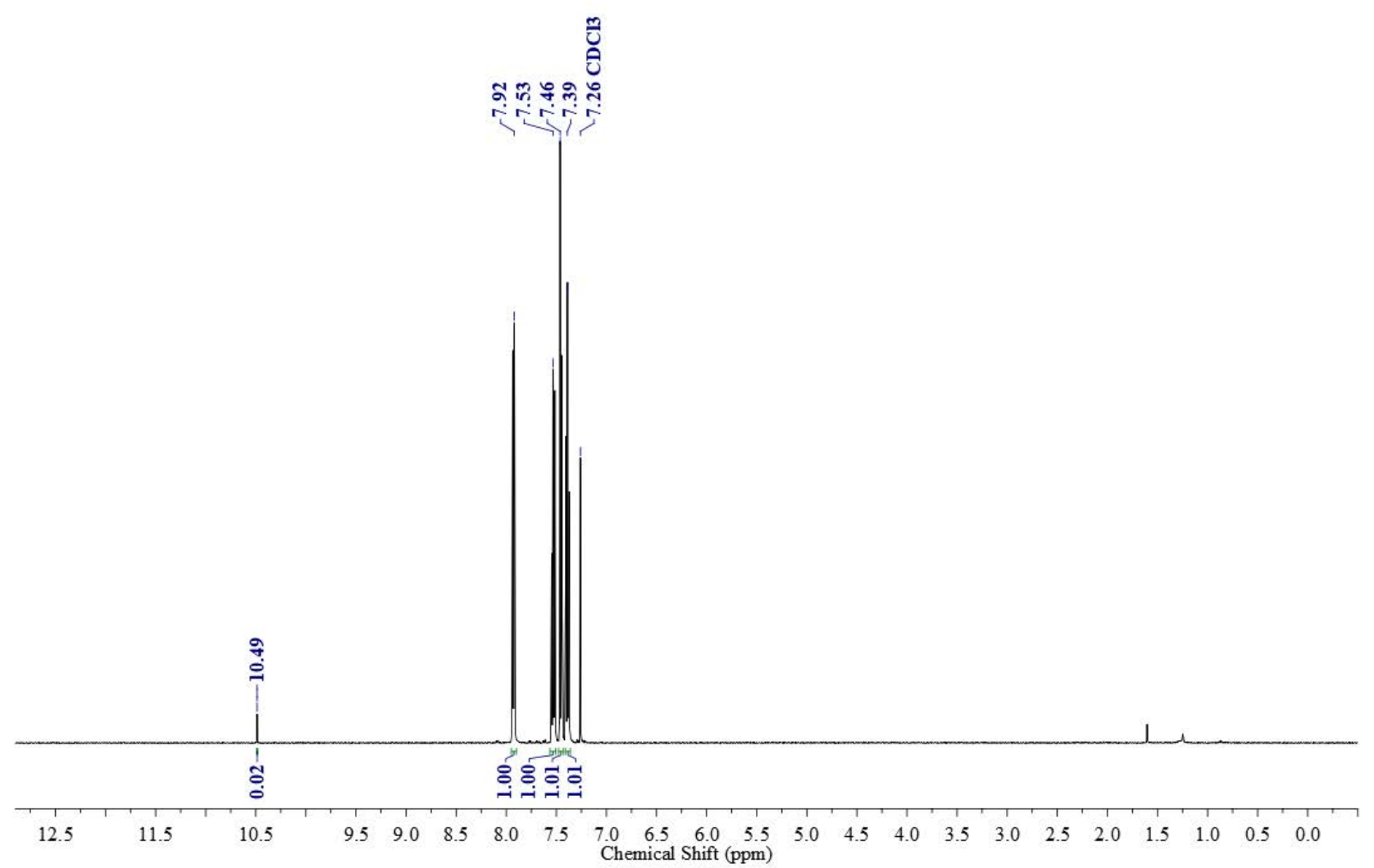

Entry 13:

VDOS $-655-\mathrm{H}$

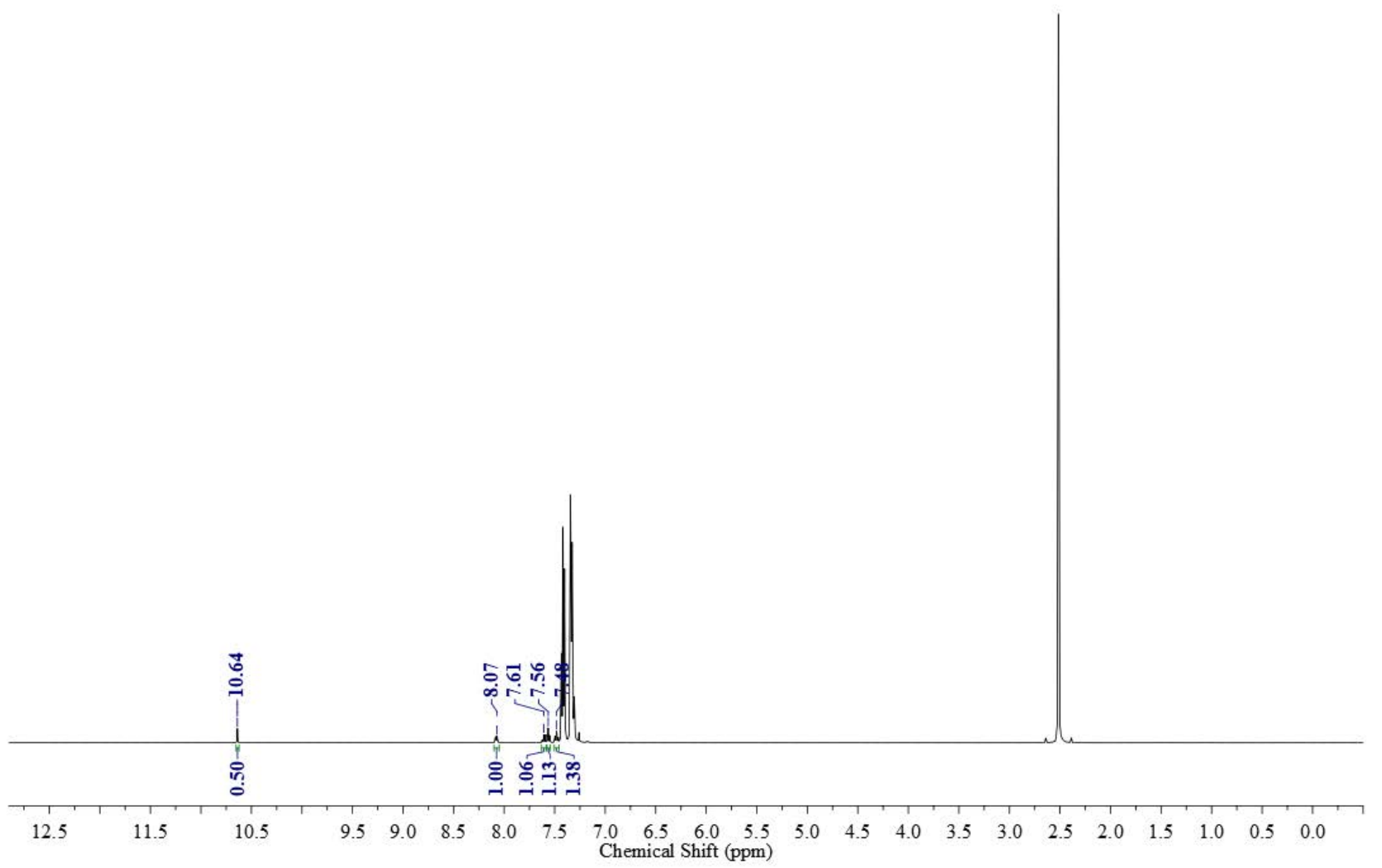


Entry 14:

vDOS-655-E

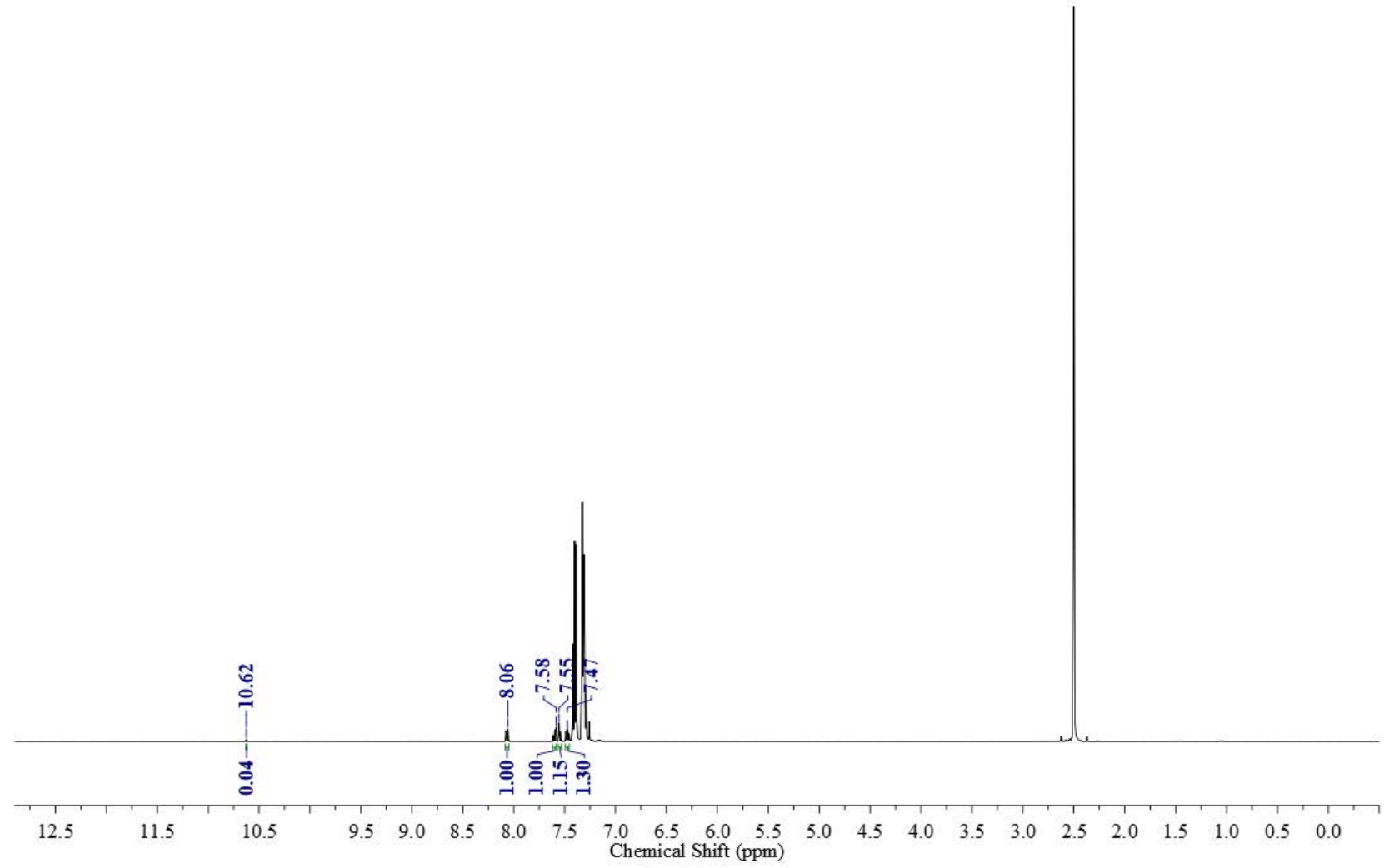

Entry 15:

VDOS-655-E3

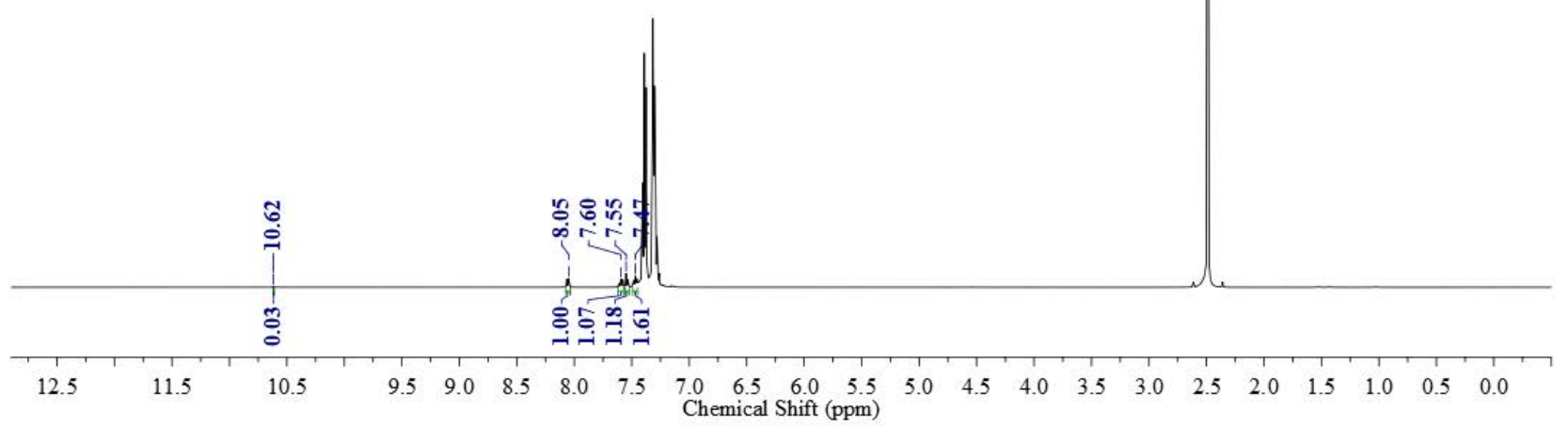


After Purification:

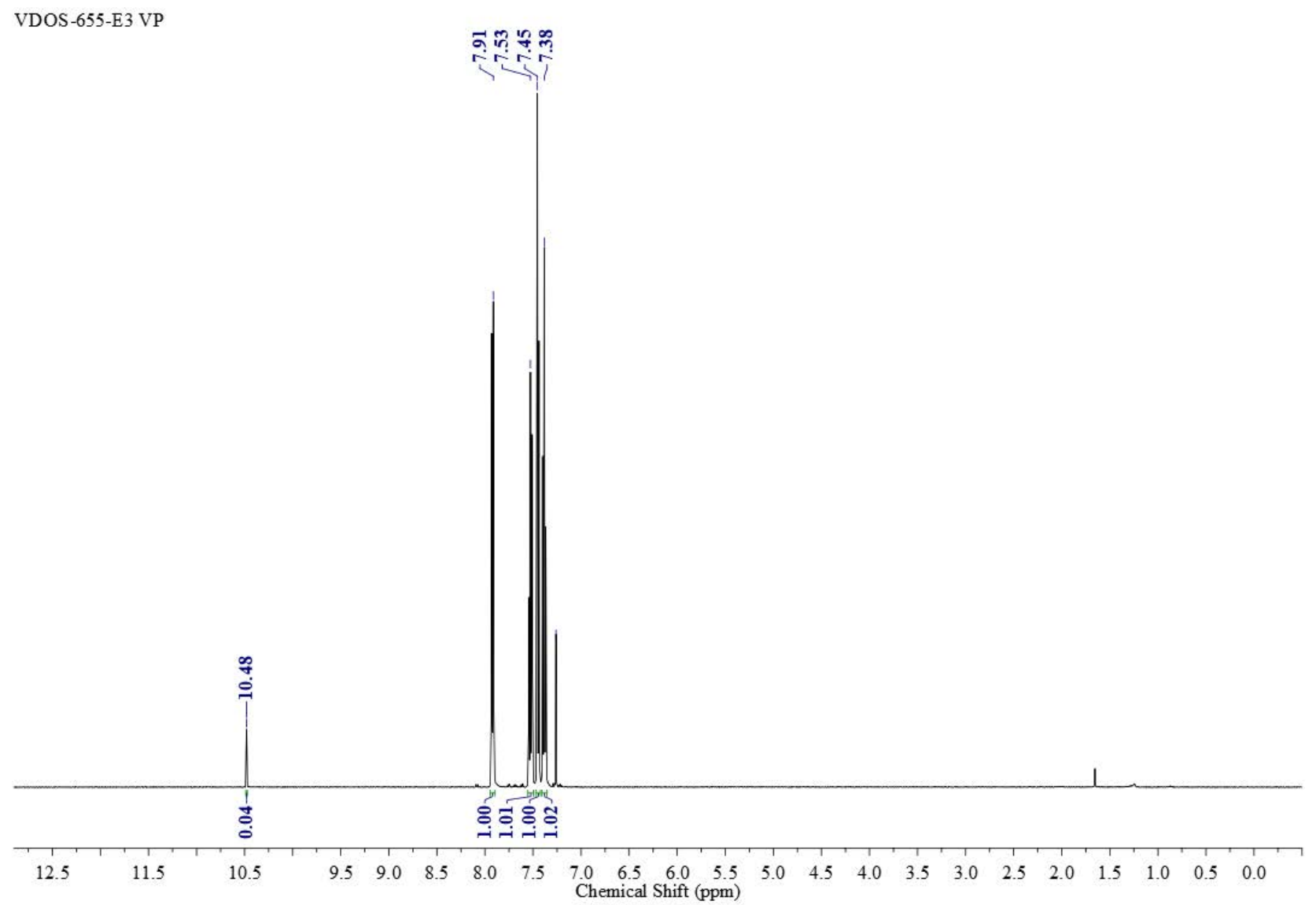

Entry 16:

vDOS-655-G

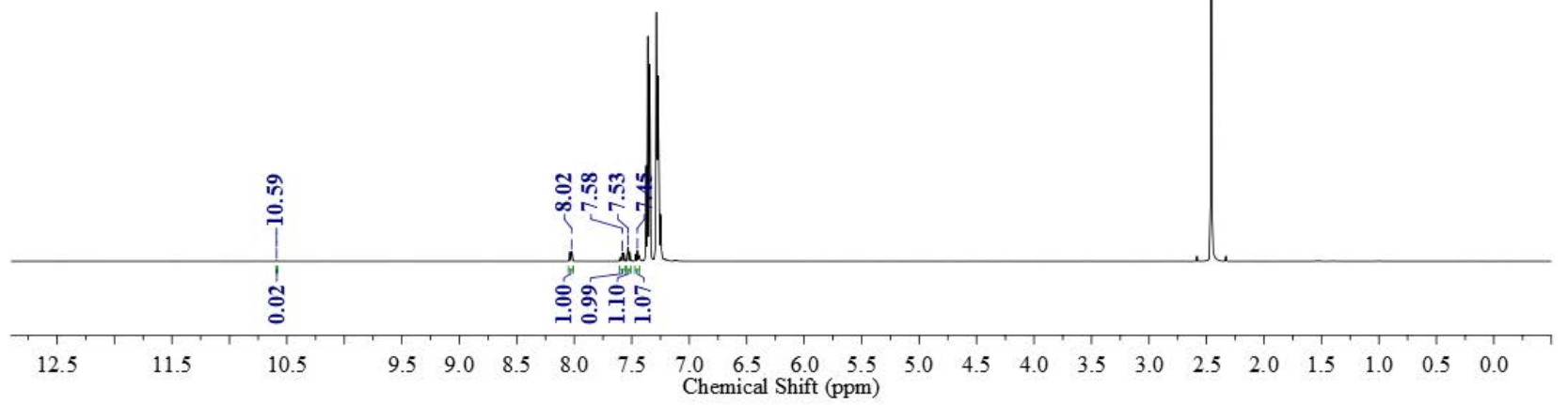


After Purification:

VDOS-655-G1 pure

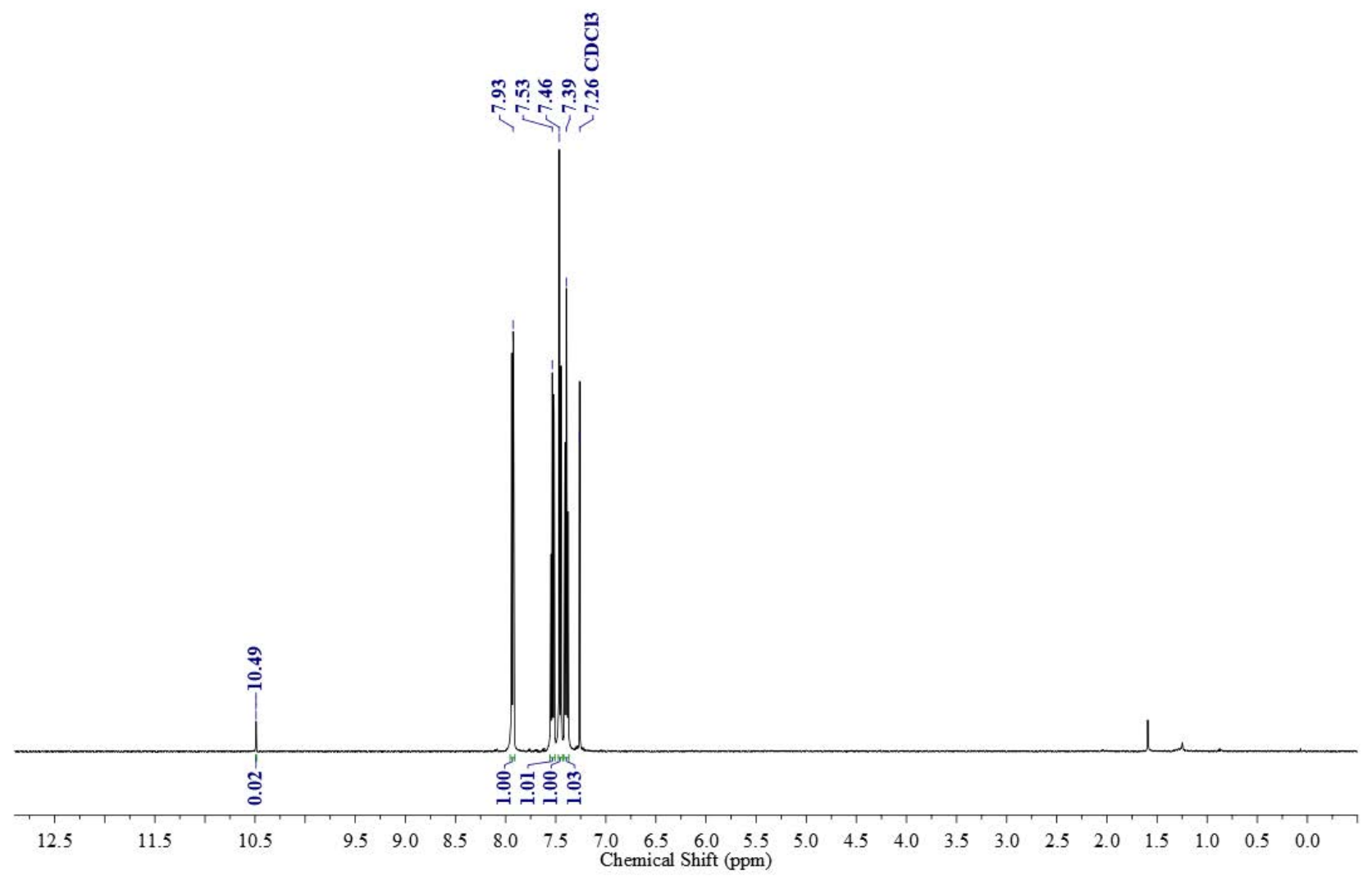

Entry 17:

VDOS-655-J1

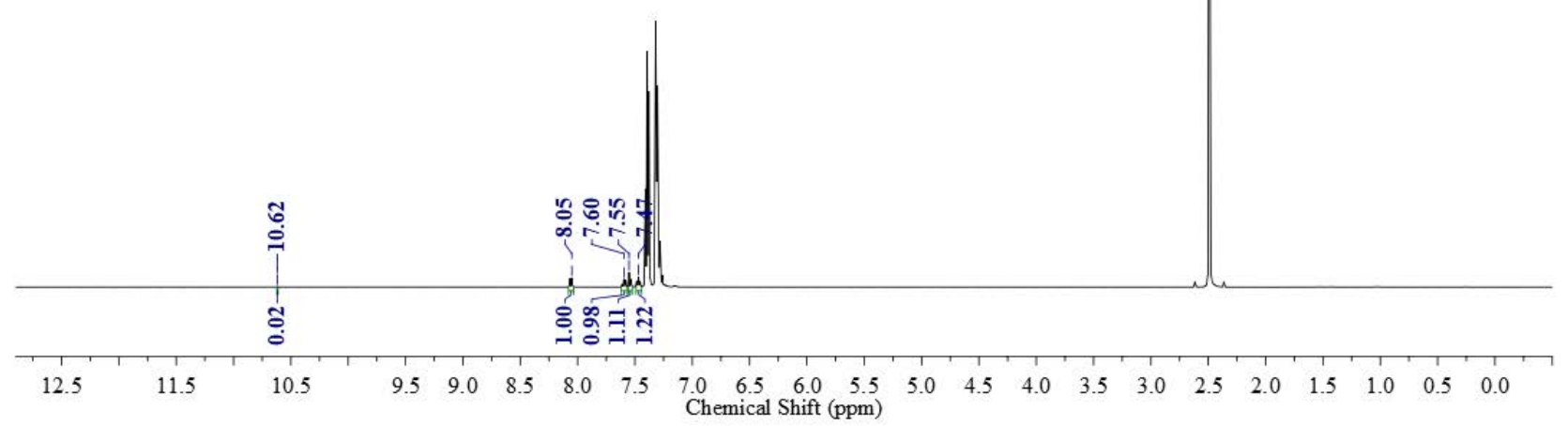


After Purification:

vDOS-655-J1 vP

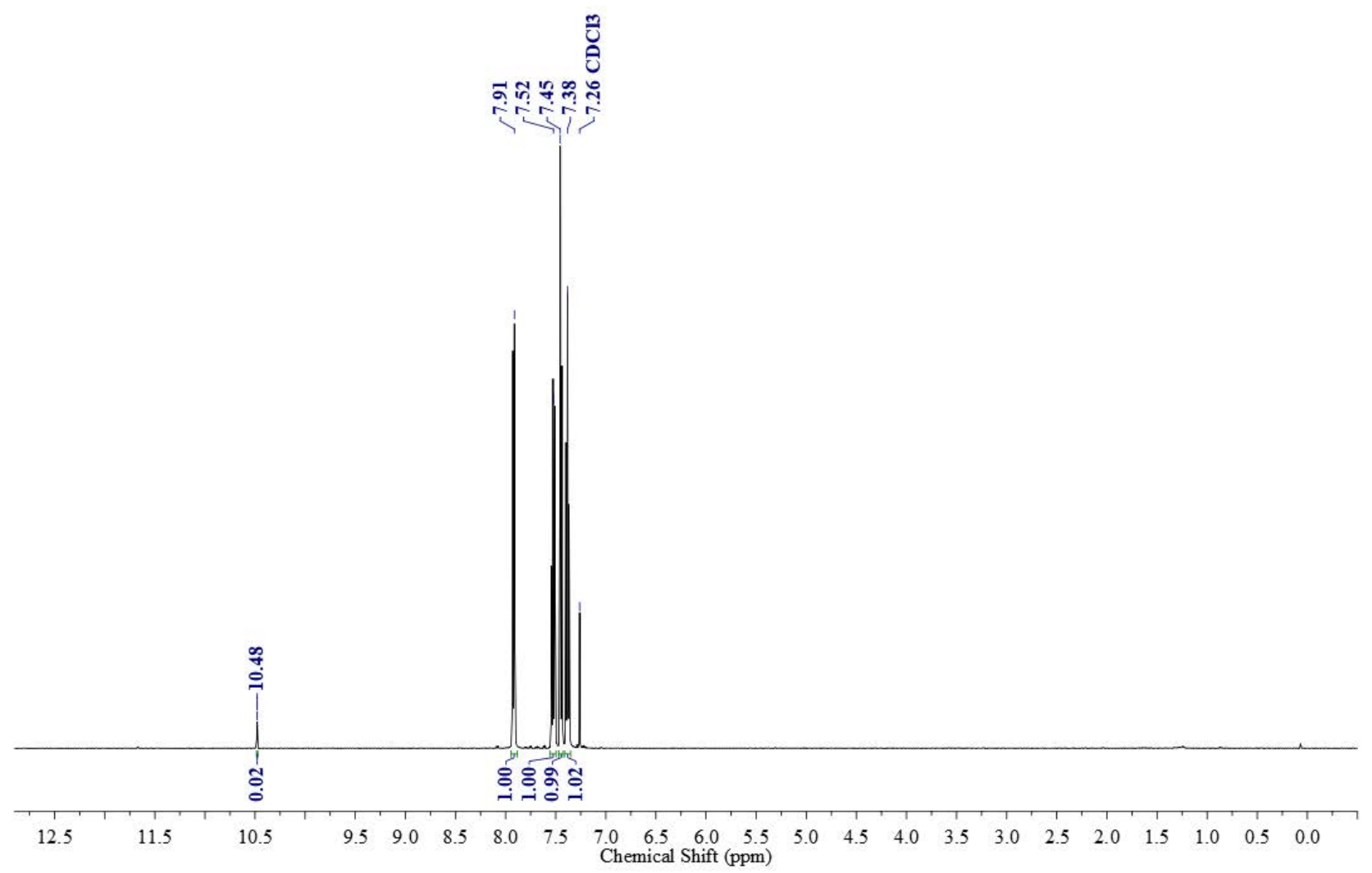

Entry 18:

vDOS-655-I2

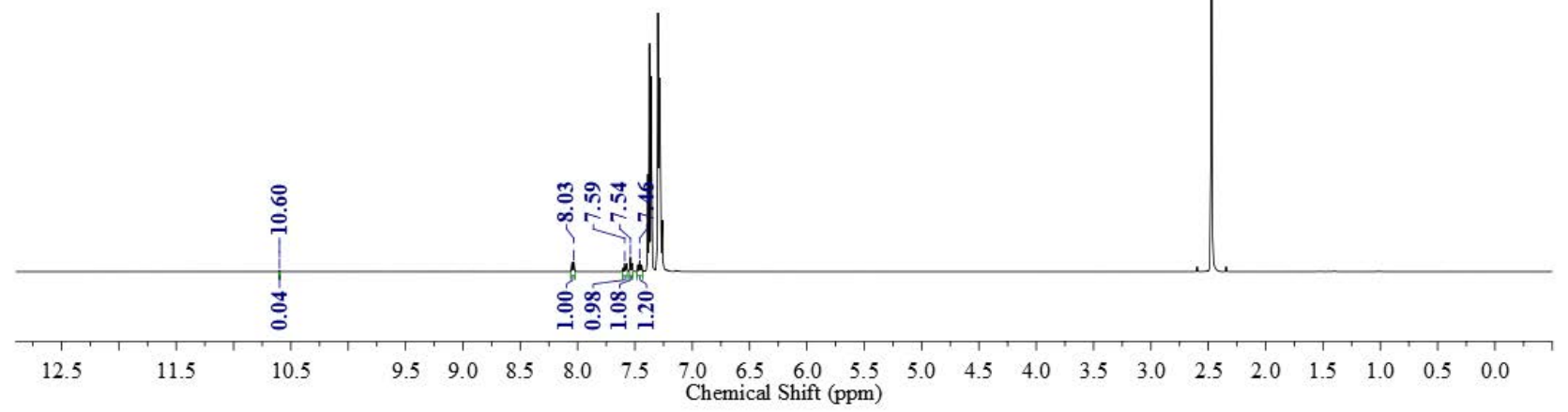


After Purification:

VDOS-655-I2 pure

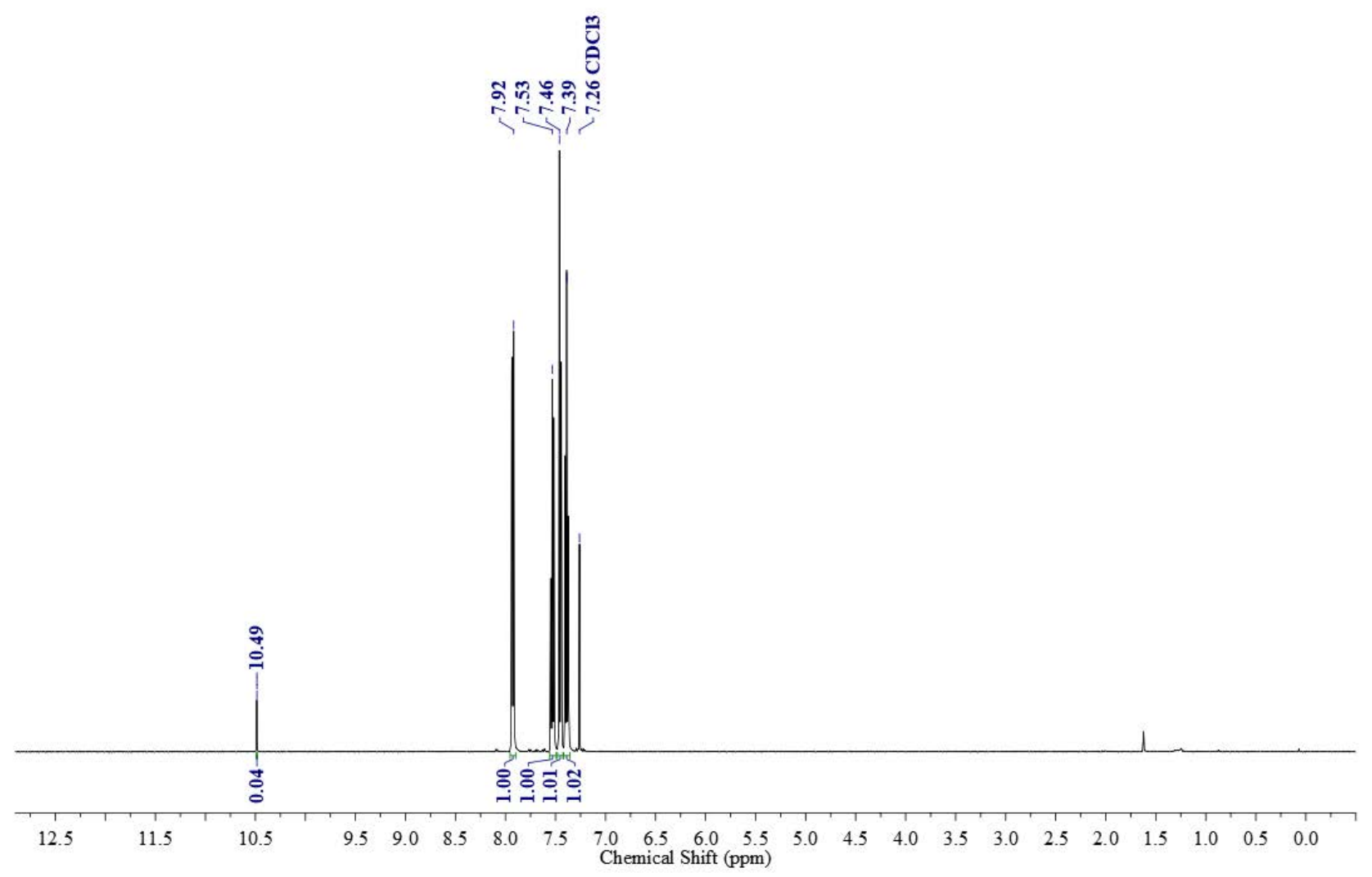




\section{Reaction with benzoin}

ILZ-053-Evap.1.fid Z-053-Evap

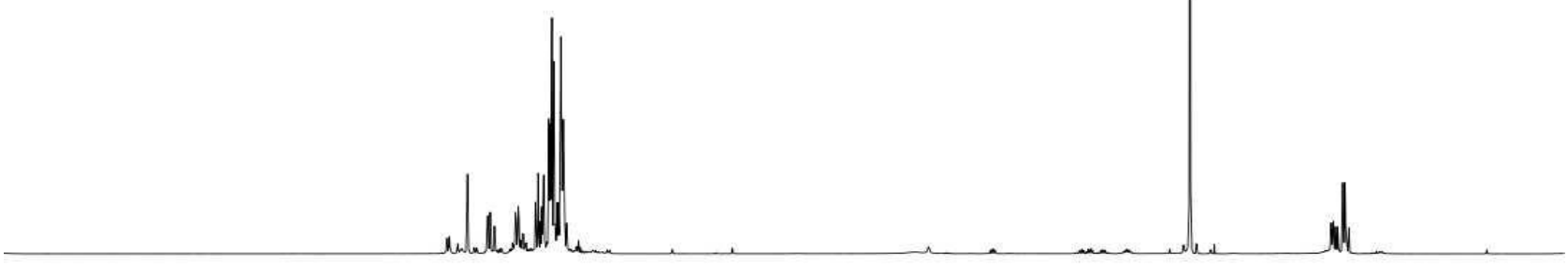

$\begin{array}{llllllllllllllllllllllll}11.0 & 10.5 & 10.0 & 9.5 & 9.0 & 8.5 & 8.0 & 7.5 & 7.0 & 6.5 & 6.0 & 5.5 & 5.0 & 4.5 & 4.0 & 3.5 & 3.0 & 2.5 & 2.0 & 1.5 & 1.0 & 0.5 & 0.0 & 1\end{array}$

Crude HPLC of the reaction:

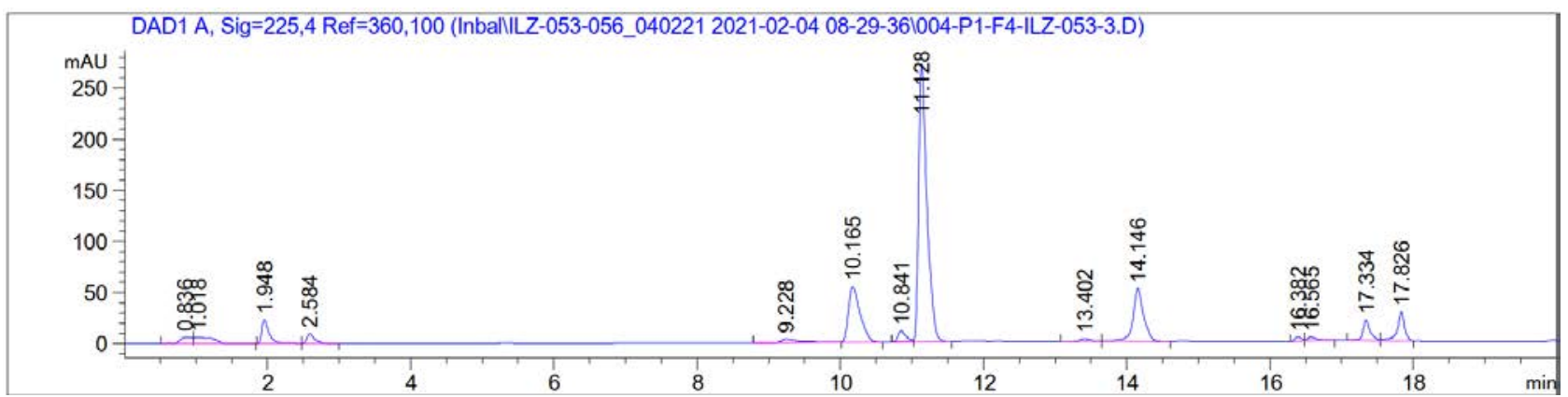

HPLC of the 3-Chlorobenzaldehyde:

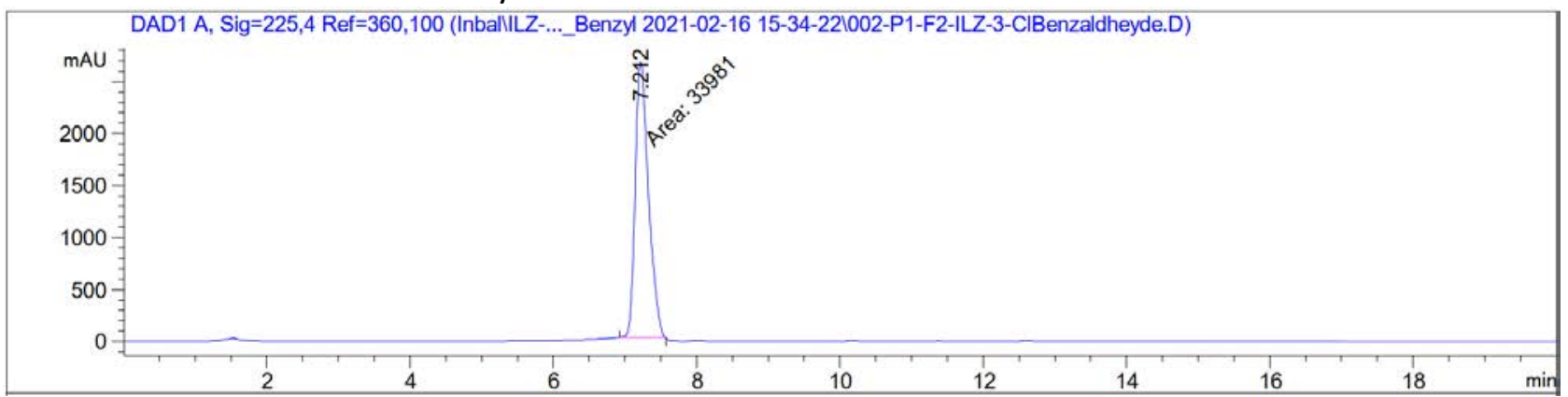


Reactions with 2,6-Methoxyphenyl Boronic-acid

1 eq. of Phenyl Boronic-acid

ILZ-056-Evap.1.fid

6-Evap

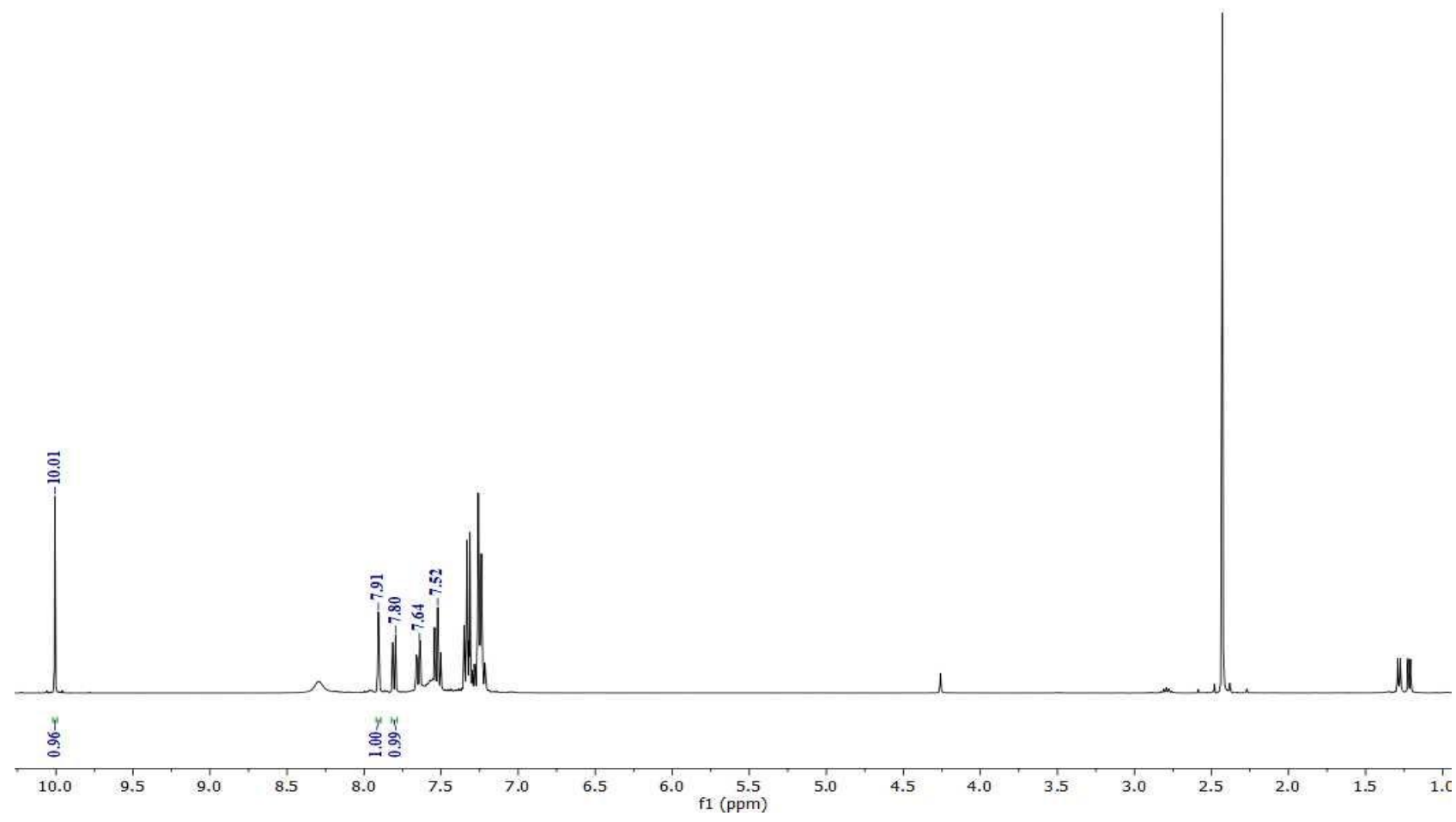

0.2 eq. and 0.12 eq. of 2,6-Methoxyphenyl Boronic-acid with water

0.12 eq. of 2,6-Methoxyphenyl Boronic-acid ILZ-057-1-Evap.1.fid

IlZ-057-1-Evap

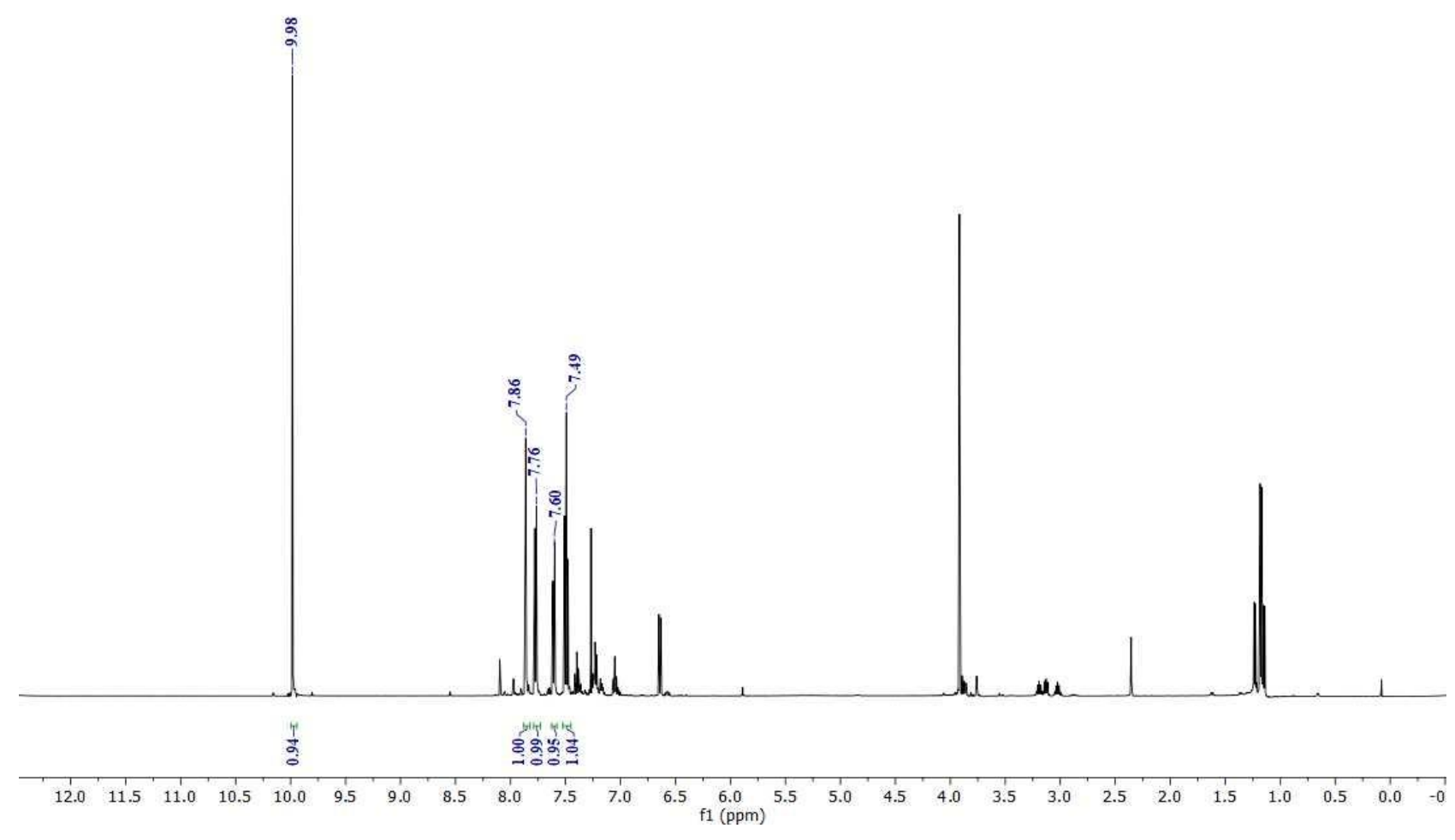


0.2 eq. of 2,6-Methoxyphenyl Boronic-acid

ILZ-057-2-Evap.1.fid

ILZ-057-2-Evap

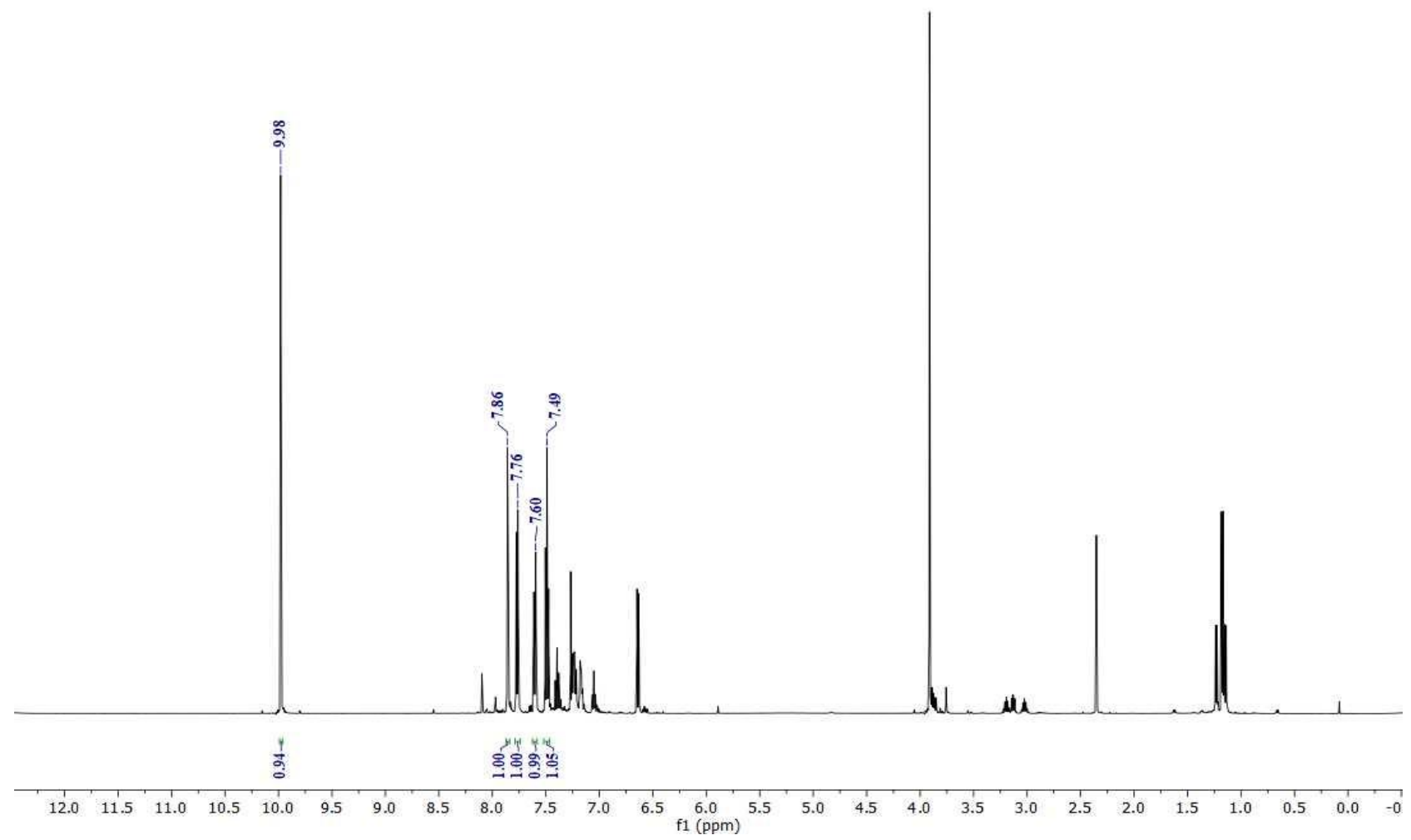

Different percentage of 2,6-Methoxyphenyl Boronic-acid without water

0.1 eq. of 2,6-Methoxyphenyl Boronic-acid

ILZ-061-1E.1.fid

ILZ-061-1E

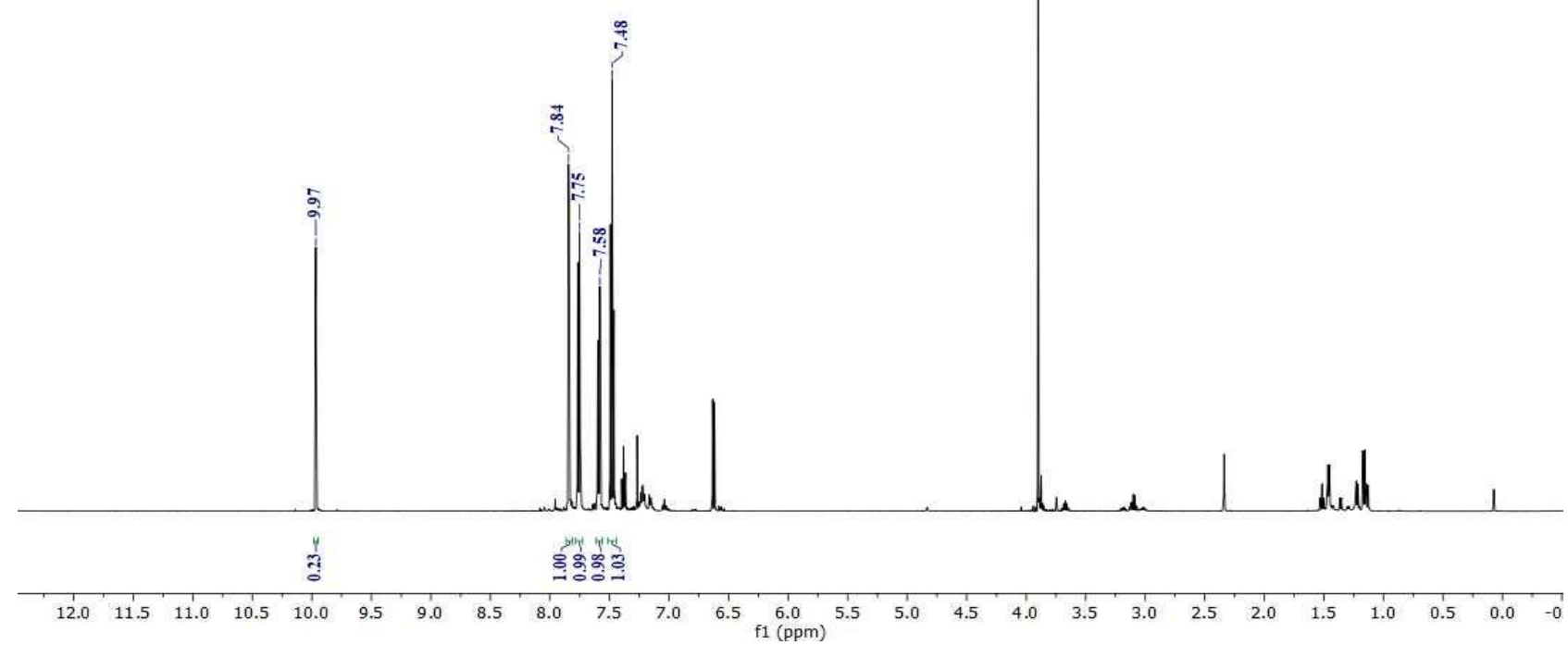




\section{2 eq. of 2,6-Methoxyphenyl Boronic-acid}

ILZ-061-2E.1.fid

ILZ-061-2E

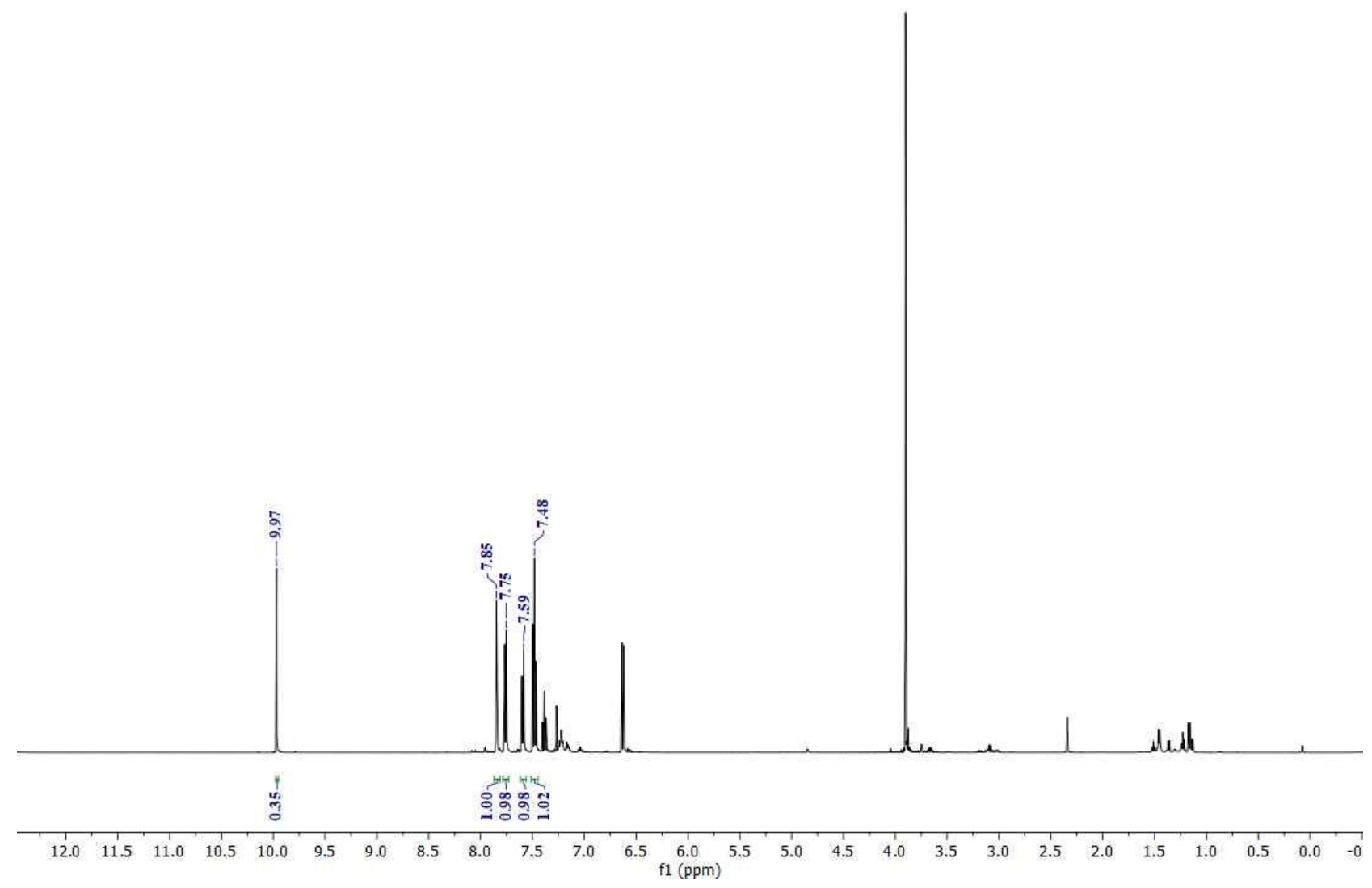

0.4 eq. of 2,6-Methoxyphenyl Boronic-acid

ILZ-061-3E.1.fid

ILZ-061-3E

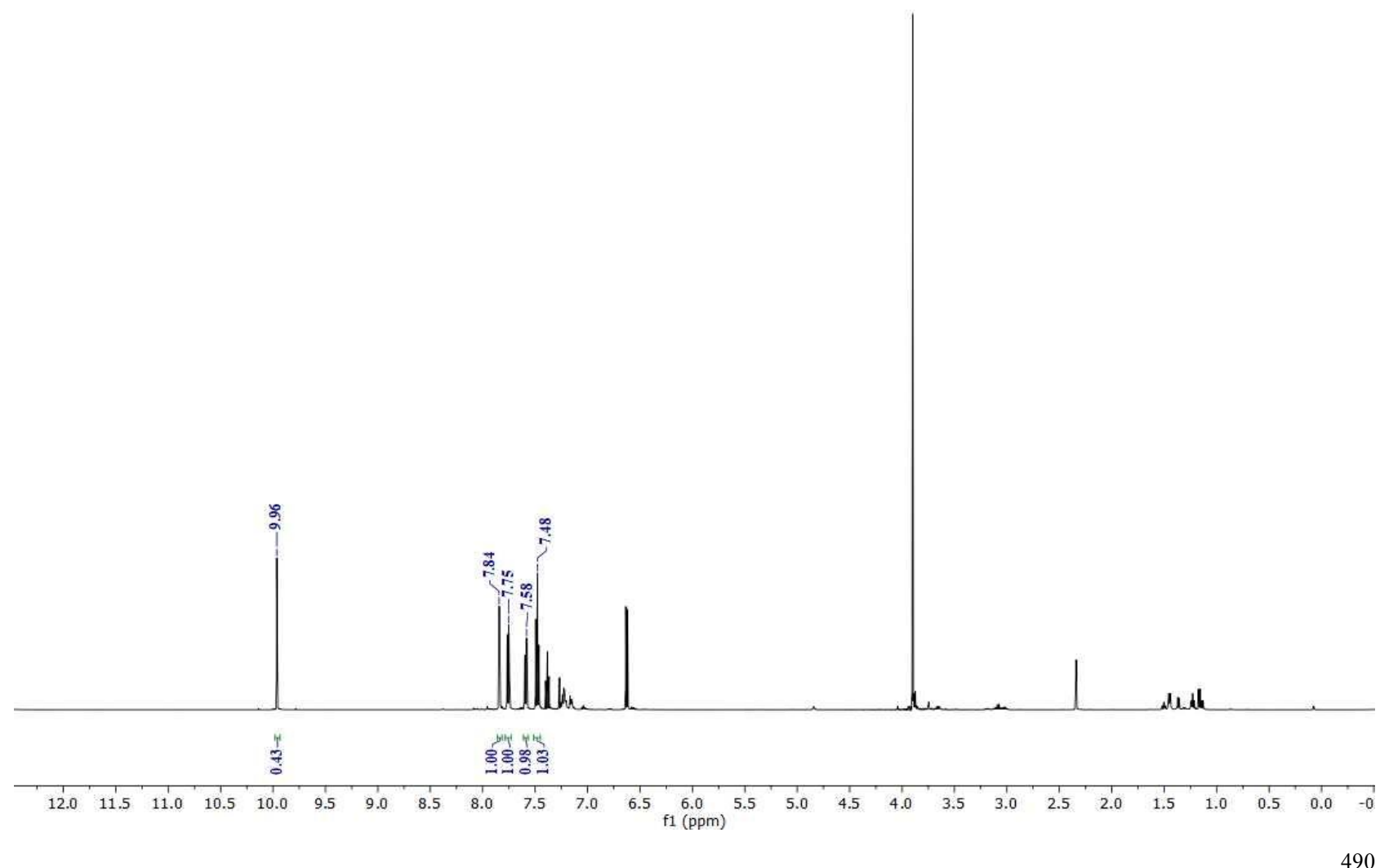




\section{References}

1. Arduengo, A. J.; Krafczyk, R.; Schmutzler, R.; Craig, H. A.; Goerlich, J. R.; Marshall, W. J.; Unverzagt, M., Imidazolylidenes, imidazolinylidenes and imidazolidines. Tetrahedron 1999, 55 (51), 14523-14534.

2. Beillard, A.; Bantreil, X.; Métro, T.-X.; Martinez, J.; Lamaty, F., Mechanochemistry for facilitated access to N,Ndiaryl NHC metal complexes. New J. Chem. 2017, 41 (3), 1057-1063.

3. Beillard, A.; Bantreil, X.; Métro, T.-X.; Martinez, J.; Lamaty, F., A more sustainable and efficient access to IMes $\cdot \mathrm{HCl}$ and IPr$\cdot \mathrm{HCl}$ by ball-milling. Green Chemistry 2018, 20 (5), 964-968.

4. Flanigan, D. M.; Romanov-Michailidis, F.; White, N. A.; Rovis, T., Organocatalytic Reactions Enabled by NHeterocyclic Carbenes. Chem. Rev. 2015, 115 (17), 9307-9387.

5. Delany, E. G.; Connon, S. J., Highly chemoselective intermolecular cross-benzoin reactions using an ad hoc designed novel N-heterocyclic carbene catalyst. Organic \& Biomolecular Chemistry 2018, 16 (5), 780-786.

6. Mahatthananchai, J.; Bode, J. W., The effect of the N-mesityl group in NHC-catalyzed reactions. Chem. Sci. 2012, 3 (1), 192-197.

7. Thomson, J. E.; Campbell, C. D.; Concellón, C.; Duguet, N.; Rix, K.; Slawin, A. M. Z.; Smith, A. D., Probing the Efficiency of N-Heterocyclic Carbene Promoted O- to C-Carboxyl Transfer of Oxazolyl Carbonates. The Journal of Organic Chemistry 2008, 73 (7), 2784-2791.

8. Kerr, M. S.; Read de Alaniz, J.; Rovis, T., An Efficient Synthesis of Achiral and Chiral 1,2,4-Triazolium Salts: Bench Stable Precursors for N-Heterocyclic Carbenes. The Journal of Organic Chemistry 2005, 70 (14), 5725-5728.

9. Romanov-Michailidis, F.; Besnard, C.; Alexakis, A., N-Heterocyclic Carbene-Catalyzed Annulation of $\alpha$-Cyano1,4-diketones with Ynals. Org. Lett. 2012, 14 (18), 4906-4909.

10. Thomson, J. E.; Rix, K.; Smith, A. D., Efficient N-Heterocyclic Carbene-Catalyzed O- to C-Acyl Transfer. Org. Lett. 2006, 8 (17), 3785-3788.

11. Cao, Y.; Xi, Y.; Teng, X.; Li, Y.; Yan, X.; Chen, L., Alkoxy substituted D- $\pi-A$ dimethyl-4-pyrone derivatives: Aggregation induced emission enhancement, mechanochromic and solvatochromic properties. Dyes and Pigments 2017, 137, 75-83.

12. Neel, A. J.; Milo, A.; Sigman, M. S.; Toste, F. D., Enantiodivergent Fluorination of Allylic Alcohols: Data Set Design Reveals Structural Interplay between Achiral Directing Group and Chiral Anion. J. Am. Chem. Soc. 2016, 138 (11), 3863-3875.

13. Crampton, R.; Woodward, S.; Fox, M., Bis-Sulfamyl Imines: Potent Substrates for Asymmetric Additions of Arylboroxines under Rhodium Catalysis. Adv. Synth. Catal. 2011, 353 (6), 903-906.

14. Xiao, Q.; Tian, L.; Tan, R.; Xia, Y.; Qiu, D.; Zhang, Y.; Wang, J., Transition-Metal-Free Electrophilic Amination of Arylboroxines. Org. Lett. 2012, 14 (16), 4230-4233.

15. Geng, H.; Chen, X.; Gui, J.; Zhang, Y.; Shen, Z.; Qian, P.; Chen, J.; Zhang, S.; Wang, W., Practical synthesis of C1 deuterated aldehydes enabled by NHC catalysis. Nat. Catal. 2019, 2 (12), 1071-1077.

16. Ibrahim, M. Y. S.; Denmark, S. E., Palladium/Rhodium Cooperative Catalysis for the Production of Aryl Aldehydes and Their Deuterated Analogues Using the Water-Gas Shift Reaction. Angew. Chem. Int. Ed. 2018, 57 (32), 10362-10367.

17. Dong, J.; Wang, X.; Wang, Z.; Song, H.; Liu, Y.; Wang, Q., Formyl-selective deuteration of aldehydes with D2O via synergistic organic and photoredox catalysis. Chem. Sci. 2020, 11 (4), 1026-1031.

18. Liu, W.; Zhao, L.-L.; Melaimi, M.; Cao, L.; Xu, X.; Bouffard, J.; Bertrand, G.; Yan, X., Mesoionic Carbene (MIC)Catalyzed H/D Exchange at Formyl Groups. Chem 2019, 5 (9), 2484-2494.

19. Zhang, M.; Yuan, X.-A.; Zhu, C.; Xie, J., Deoxygenative Deuteration of Carboxylic Acids with D2O. Angew. Chem. Int. Ed. 2019, 58 (1), 312-316.

20. Kerr, W. J.; Reid, M.; Tuttle, T., Iridium-Catalyzed Formyl-Selective Deuteration of Aldehydes. Angew. Chem. Int. Ed. 2017, 56 (27), 7808-7812.

21. Li, X.; Wu, S.; Chen, S.; Lai, Z.; Luo, H.-B.; Sheng, C., One-Pot Synthesis of Deuterated Aldehydes from Arylmethyl Halides. Org. Lett. 2018, 20 (7), 1712-1715.

22. Macco, A. A.; De Brouwer, R. J.; Nossin, P. M. M.; Godefroi, E. F.; Buck, H. M., Asymmetric induction in the synthesis of thiophene-containing steroidlike molecules via olefinic cyclization. 2. Evidence for precoiling as model description for the cyclization. The Journal of Organic Chemistry 1978, 43 (8), 1591-1593.

23. Isbrandt, E. S.; Vandavasi, J. K.; Zhang, W.; Jamshidi, M. P.; Newman, S. G., Catalytic Deuteration of Aldehydes with D2O. Synlett 2017, 28 (20), 2851-2854.

24. Sawama, Y.; Miki, Y.; Sajiki, H., N-Heterocyclic Carbene Catalyzed Deuteration of Aldehydes in D2O. Synlett 2020, 31 (07), 699-702. 